\section{ORGDP}

OAK RIDGE

GASEOUS

DIFFUSION

PLANT

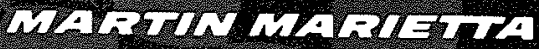

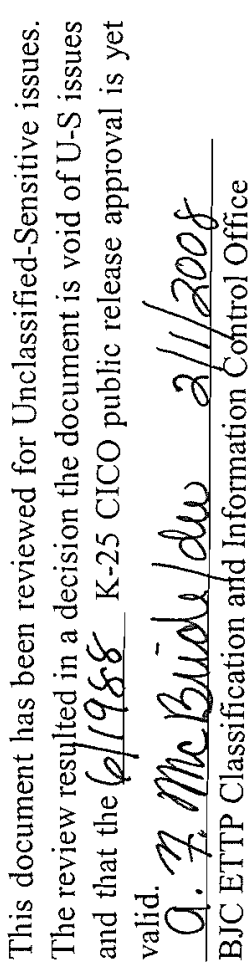

RCRA FACILITY INVESTIGATION PLAN

K-1.004 AREA LAB DRAIN AND

THE K-1007-B POND

OAK RIDGE GASEOUS DIFFUSION PLANT

OAK RIDGE, TENNESSEE

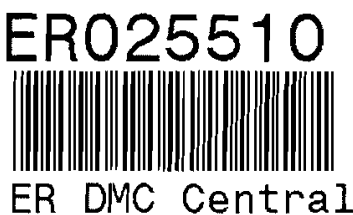




\section{DISCLAIMER}

This report was prepared as an account of work sponsored by an agency of the Unired States Government. Neither the United States Government nor any ayency thereof, nor any of their employees, makes any warranty, express or implied, or assumes any legal liability or responsibility for the accuracy, completeness, or usefulness of any information, apparatus, product, or process disciosed, or represents that its use would not infringe privately owned rights. Reference herein to any specific commercial product, process, or service by trade name, trademark, manufacturer, of otherwise, does not necessarily constitute or imply its endorsement, secommendation, or favoring by the United States Government or any agency thereot. The views and opinions of authors expressed herein do not necessarily state or reflect those of the United States Government or any agency thereot. 


\title{
RCRA FACILITY INVESTIGATION PLAN \\ K-1004 AREA LAB DRAIN AND \\ THE K-1007-8 POND \\ OAK RIDGE GASEOUS DIFFUSION PLANT \\ OAK RIDGE, TENNESSEE
}

\author{
Prepared by the \\ Oak Ridge Gaseous Diffusion Plant \\ Oak Ridge, Tennessee 37831 \\ operated by \\ MARTIN MARIETTA ENERGY SYSTEMS, INC. \\ for the \\ U. 5. DEPARTMENT OF ENERGY \\ under contract DE-ACO5-840R21400
}




$$
-
$$

$\omega$ 
TABLE OF CONTENTS

Page

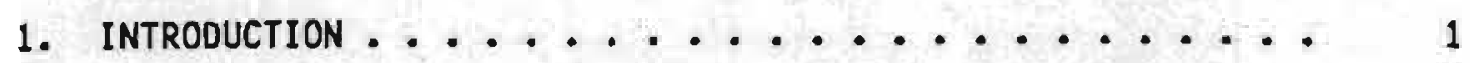

2. OBJECTIVES OF RCRA FACILITY INVESTIGATION PLANNING ..... 2

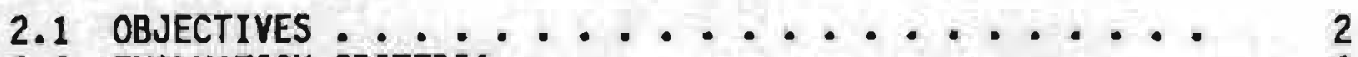

2.2 EVALUATION CRITERIA $\ldots \ldots \ldots \ldots$

2.3 SCHEDULE FOR SPECIFIC RFI ACTIVITIES ........ 2

2.4 FEASIBLE ALTERNATIVES $\ldots \ldots \ldots \ldots . \ldots . \ldots 4$

2.5 RISK ASSESSMENT ................... 4

3. DESCRIPTION OF CURRENT CONDITIONS .............. 6

3.1 GEOGRAPHICAL INFORMATION ............ 6

3.2 HISTORICAL INFORMATION ............ 6

3.3 OPERATIONAL INFORMATION ............... 11

4. CHARACTERIZATION OF THE CONTAMINANT SOURCE ....... 12

$4.1 k-1004$ AREA LAB DRAIN .................. 12

4.2 EXISTING $x-1007-B$ POND DATA $\ldots \ldots \ldots \ldots . \ldots 14$

5. CHARACTERIZATION OF THE ENVIRONMENTAL SETTING ..... 19

5.1 HYOROGEOLOGY ....................... 19

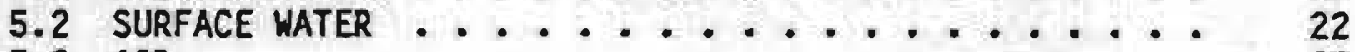

5.3 AIR $\ldots \ldots \ldots \ldots \ldots \ldots \ldots . \ldots \ldots$

6. IDENTIFICATION OF POTENTIAL PATHWAYS AND RECEPTORS . . . . 23

6.1 POTENTIAL PATHWAYS OF MIGRATION .......... 23

5.2 POTENTIAL RECEPTORS .................. 25

6.3 SUMMARY AND CONCLUSIONS $\ldots \ldots \ldots . \ldots 26$

7. EXISTING MONITORING DATA .............. 27

8. SAMPLING PLAN ....................... 29

8.1 SAMPLING AND ANALYTICAL STRATEGY . . . . . . 29

8.2 SAMPLING ANALYSIS ............... 30

8.3 FIELD SAMPLING ............... 37

8.4 ANALYTICAL PROTOCOL $\ldots \ldots \ldots 40$

8.5 SAMPLE ANALYSIS $\ldots \ldots \ldots \ldots \ldots$ 
9. DATA MANAGEMENT PROCEDURES ................ 42

10. HEALTH AND SAFETY PROCEDURES ............. 43

10.1 INTRODUCTION .................. 43

10.2 KNOWN HAZARDS AND RISKS ............ 43

10.3 LEVEL OF PROTECTION ............... 44

10.4 DESIGNATION OF WORK AREA ZONES .......... 44

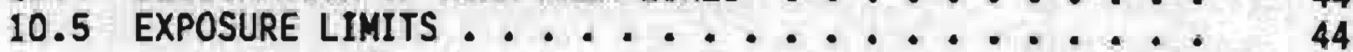

11. REFERENCES ......................... 46

APPENDIX A

APPENDIX B

APPENDIX C

APPENDIX D 


\section{INTRODUCTION}

Within the confines of the Oak Ridge Gaseous Diffusion Plant (ORGDP) are hazardous waste treatment, storage, and disposal facilfties; some are in operation while others are no longer in use. These solid waste management units (SKMUS) are subject to assessment by the U.S. Environmental Protection Agency (EPA). The RCRA Facflity Investigation (RFI) Plans are scheduled to be submitted for all units during calendar years 1987 and 1988. The RFI Plan - General Document (K/HS-132) includes information applicable to all the ORGDP SMWUs and serves as a reference document for the site-specific RFI Plans.

This document is the site-specific RFI Plan for the K-1004 Area Lab Drain (ALD) and the $X-1007-B$ Pond. This plan is based upon requirements described in the draft document, RFI Guidance, Vols. I-IV, December 1987 (EPA 530/5W-87-001). This unit is regulated by section 3004(U) of the 1984 Hazardous and Solid Waste Amendments (HSWA) to the Resource Conservation Recovery Act (RCRA). Contained within this document are geographical, historical, operational, geological, and hydrological data specific to the $K-1004$ ALD and the $K-1007-B$ Pond. The potential for release of contamination through the various media to receptors is addressed. A sampling plan is proposed to further detemine the extent (if any) of release of contamination to the surrounding environment. Included are health and safety procedures to be followed when implementing the sampling plan. Quality control (QC) procedures for remedial action occurring on the Oak Ridge Reservation (ORR) are presented in The Environmental Surveillance Procedures Quality Control Program, Martin Marietta Energy Systems, Inc., (ESH/Sub/87-21706/1), and quality assurance (QA) guidelines for ORGDP investigations are contained in the $K-25$ Remedial Actions Program Quality Assurance Plan, K/HS-231. 


\section{OBJECTIVES OF RCRA FACILITY INYESTIGATION PLANNING}

\subsection{OBJECTIVES}

Th1s RFI Plan will fdentify actions necessary to determine the nature and extent of releases of hazardous and/or radioactive contamination from the K-1004 ALD and the K-1007-B Pond. The plan sumarizes existing site information and addresses the potential for contamination of the soil, groundwater, surface water, air, and biota.

\subsection{EVALUATION CRITERIA}

In order to prepare and implement a comprehensive sampling plan and to effectively evaluate analytical sampling results, evaluation criteria must first be established. Criteria for evaluating the extent of release of contaminants are based on existing state and federal regulatory guidance and best technical judgment.

The primary media of interest for this site are surface water, groundwater, and soll. Soll, surface water, and fish samples will be collected as part of this RFI and analyzed for contaminants as described in Section 8 of this document. Groundwater will be sampled as part of the RFI in accordance with the protocol of the ORGDP Groundwater Protection program. The sampling methodology and analytical procedures are designed to characterize the contaminants of interest at or below levels sumarized in Table 2.2 of K/HS-132.

\subsection{SCHEDULE FOR SPECIFIC RFI ACTIVITIES}

A 1 ist of the sampling and analysis activities that will be perfomed for this RFI and the duration of each activity is shown in Table 2.1. 
Table 2.1. Duration of RFI activities for the $K-1004$ Area Lab Orain and the $K-1007-B$ Pond

\section{Activities}

Duration

1. Site preparation and sample location

K-1004 Area Lab Drain

(a) Soll sampies

(b) Surface water samples

1 week

2 weeks

(c) Groundwater samples

8 weeks

(includes well construction)

2. Collection of samples

K-1004 Area Lab Drain

(a) Soll samples

(b) Surface water samples

(c) Groundwater samples

1 week

6 weeks

52 weeks

\section{K-1007-B Pond}

(a) Surface water samples

(b) Fish samples

26 weeks

1 week

3. Analysts of samples.
(a) Soll samples
(b) Surface water samples
(c) Groundwater samples ${ }^{a}$
(d) Fish samples

4. Compllation of data and data presentation

5. Evaluation of results and recomendations (may include preliminary risk assessments)

4 weeks

16 weeks

66 weeks

8 weeks

18 weeks

12 weeks

6. Preparation of RFI report and submittal to EPA

8 weeks

7. Additional sampling phases (If needed)

TBD

aGroundwater sample analysis will occur concurrently with groundwater sample collection 


\subsection{FEASIBLE ALTERNATIVES}

Knowledge of feasible corrective measures has been used in preparing this RFI plan. Based on existing geologic, hydrologic, and contaminant source data, potential corrective measures for this site have been identified and are shown in Table 2.2. These corrective measures will be re-evaluated after the RFI report is completed.

\subsection{RISK ASSESSHENT}

The enviromental and public health risks associated with possible site contamination and the remedial action alternatives listed in Table 2.2 will be evaluated. This evaluation will consist of a characterization of contaminant sources, the environmental setting, the magnitude of release, pathways to human exposures, and characterization of risks. The site. sampling plan has been designed to provide data necessary for performing risk assessment. 
Table 2.2. Potential corrective measures for the $K-1004$ Area Lab Orain and the K-1007-B Pond

General Response Action

Technologies

Monitoring

Removal of source

Containment from surface water

Containment from groundwater

Treatment of groundwater

Isolate pond

In-situ treatment
Surveillance monitoring and analys is

Excavate and treat or dispose of contaminated soll or sediment at an approved landfill or place in long term starage

Cap - synthetic membrane, clay, asphalt, multimedia cap, concrete, or chemical sealants and stabillzers

Subsurface collection drains french drains, tile drains, pipe drains

Vertical containment barriers - soll bentonite slurry wall, cementbentonite siurry wall, vibrating beam, grout curtains, steel sheet piling

Groundwater diversion pumping - well polnts, deep wells, suction wells, ejector wells

Collect the groundwater and pump to a wastewater treatment plant or onsite treatment by aeration, filtration, carbon adsorption, or blological processes

Prevent access to pond by fencing, post warning and no fishing signs

Grout I ines and replace existing lines with new lines 


\section{DESCRIPTION OF CURRENT CONDITIONS}

\subsection{GEOGRAPHICAL INFORMATION}

The area of concern in this RFI is located on the south side of ORGDP near Portals 2 and 3 . The scope of this RFI includes:

- process drains assoctated with Buildings $K-1004-A, B, C, O, J$, and $L(K-1004$ group), $K-1003, K-1005$, and $K-1006$;

- retention pits around $K-1004-A, B, C$, and 0 ;

- K-1007-B Pond; and

- a portion of Storm Drain (SD) 100 that drains this area of the ORGDP.

Flow in the drains and retention pits eventually enters the SD-100 System which extends to the north and west of the $K-1007$ Computer Center and then discharges to the K-1007-B Holding Pond (Figures 3.1 and 3.2). This process/storm drain system is referred to as the ALD. The components of this RFI are located both inside and outside of the security perimeter fence. Complete geographical information is located in section 3.1 of K/HS-132.

\subsection{HISTORICAL INFORMATION}

The components of this RFI were constructed or installed in the mid to late 19405. Buildings $K-1005, K-1006$, and the $K-1004$ group contain chemical laboratories which were originally used in support of various uranium enrichment programs at ORGDP and have rematned in use to support other programs. The $K-1003$ building has been a medical center for ORGDP personnel since the 1940 s. 


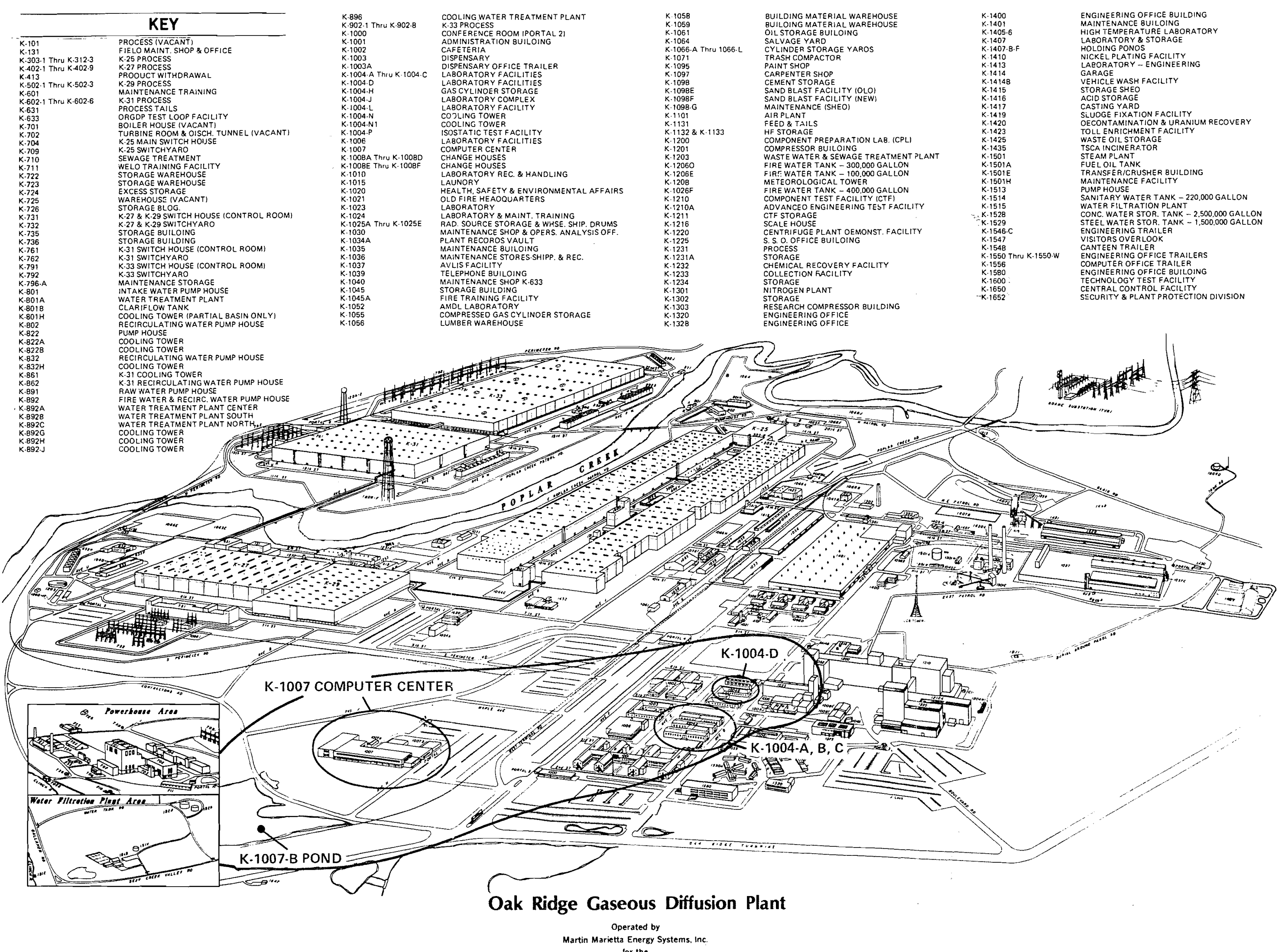
Operated by
Martin Marietta Energy Systems. Inc
lor the
U. S. Department ol Energy

Fig. 3.1. ORGDP location map for the K-1004 ALD/K-1007-B POND 

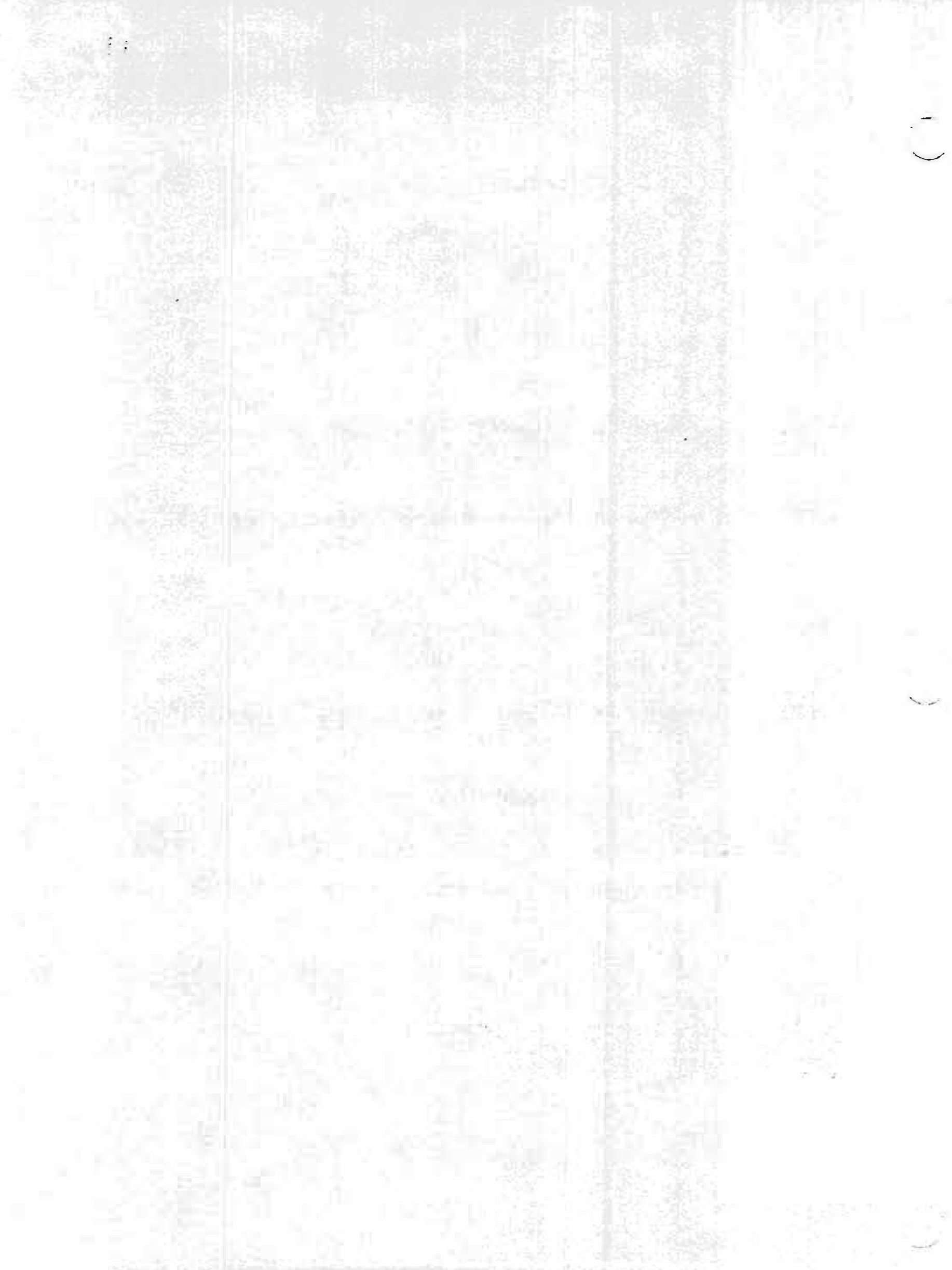

$\cdots$

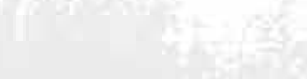




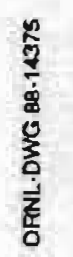

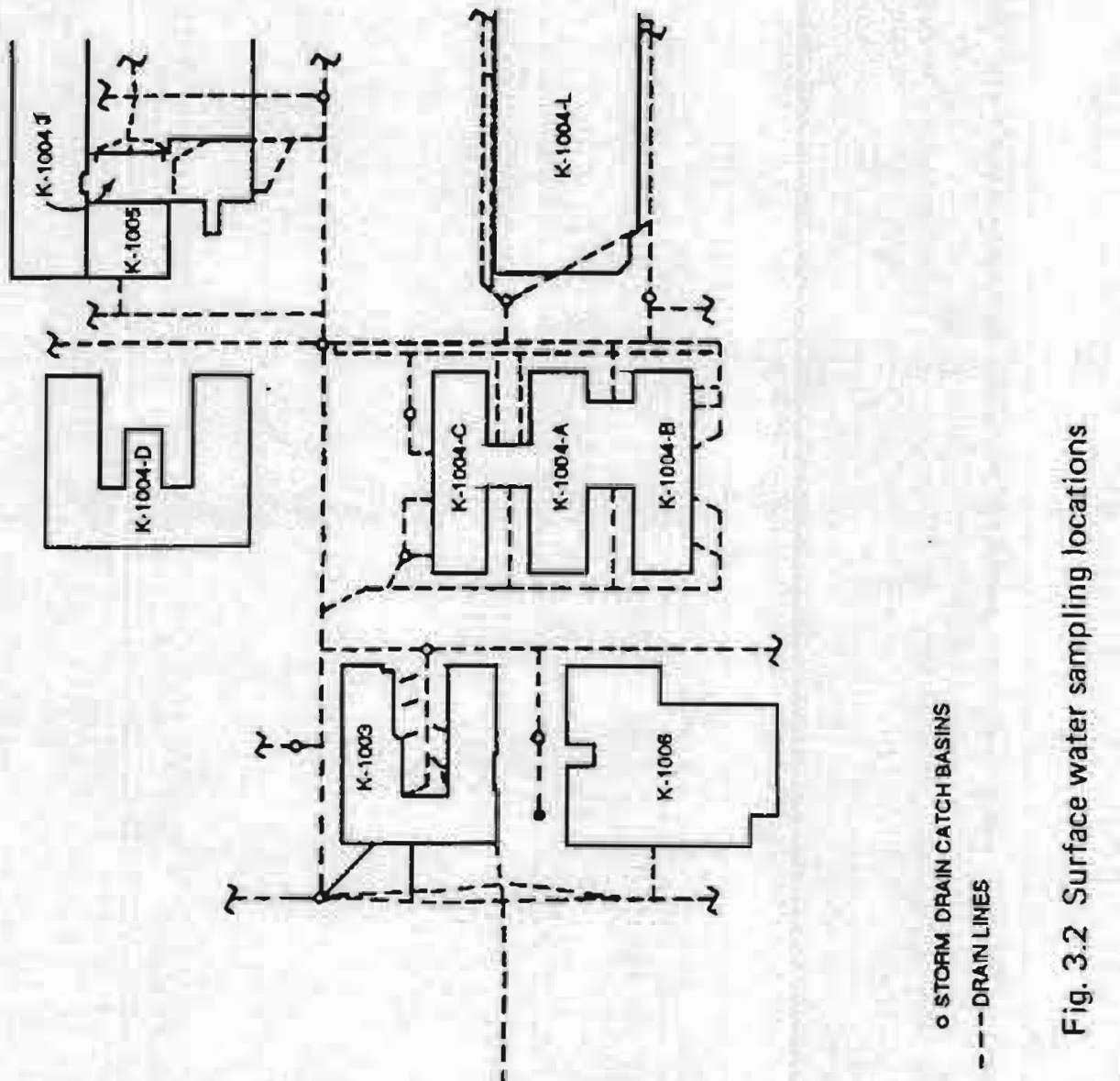




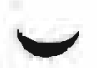


The drains which exit these bulldings are vitrified clay pipes or ductile cast iron, normally 6 or 8 inches in diameter. They increase in slze (to 12 inches or 15 inches) until they enter the 48-inch diameter SD-100 system. Because of leaks in the lines, the process drains inside Buliding K-1004-A, B, and C were replaced with polypropylene and were connected to the old lines outside of the bullding.

The drains from Bulidings K-1004-A, B, C, and D entered retention pits prior to combining with the SD-100 System. Only one of the original pits is currently visible north of Bullding $K-1004-A, B$, and C fFigure 3.3) and remains in use. Personnel interviews indicated that six more pits existed around Bullding $K-1004-A, B$, and $C$, and six pits were in use at Building $K-1004-D$. These pits have been removed or filled in and piping to the pits rerouted; some of the pit locations are now covered with asphalt or are underneath equipment. Engineering drawings did not verify these locations and show pits at the locations designated in Figure 3.3.

From the beginning of ORGDP operations until 1985, chemical byproducts of routine analytical operations carried out in ORGDP laboratories were discharged through the $K-1004$ ALD to the $K-1007-B$ Pond at a rate of approximately 2,200 gallons/year (personal conversation, Jimny Stone, formerly of ORGDP Environmental Management). The K-1007-B Pond covers approximately 25 acres and has been permitted to discharge to Poplar Creek under National Pollutant Discharge Elimination System (NPDES) regulations since 1974. A Best Management Practice (BMP) for the Pond was implemented in 1985 to control the release of hazardous waste through the K-1004 ALD. As a result of the BMP, chemical wastes from laboratory operations are now collected for proper treatment or disposal. Complete historical information is located in Section 3.2 of K/HS-132. 
10

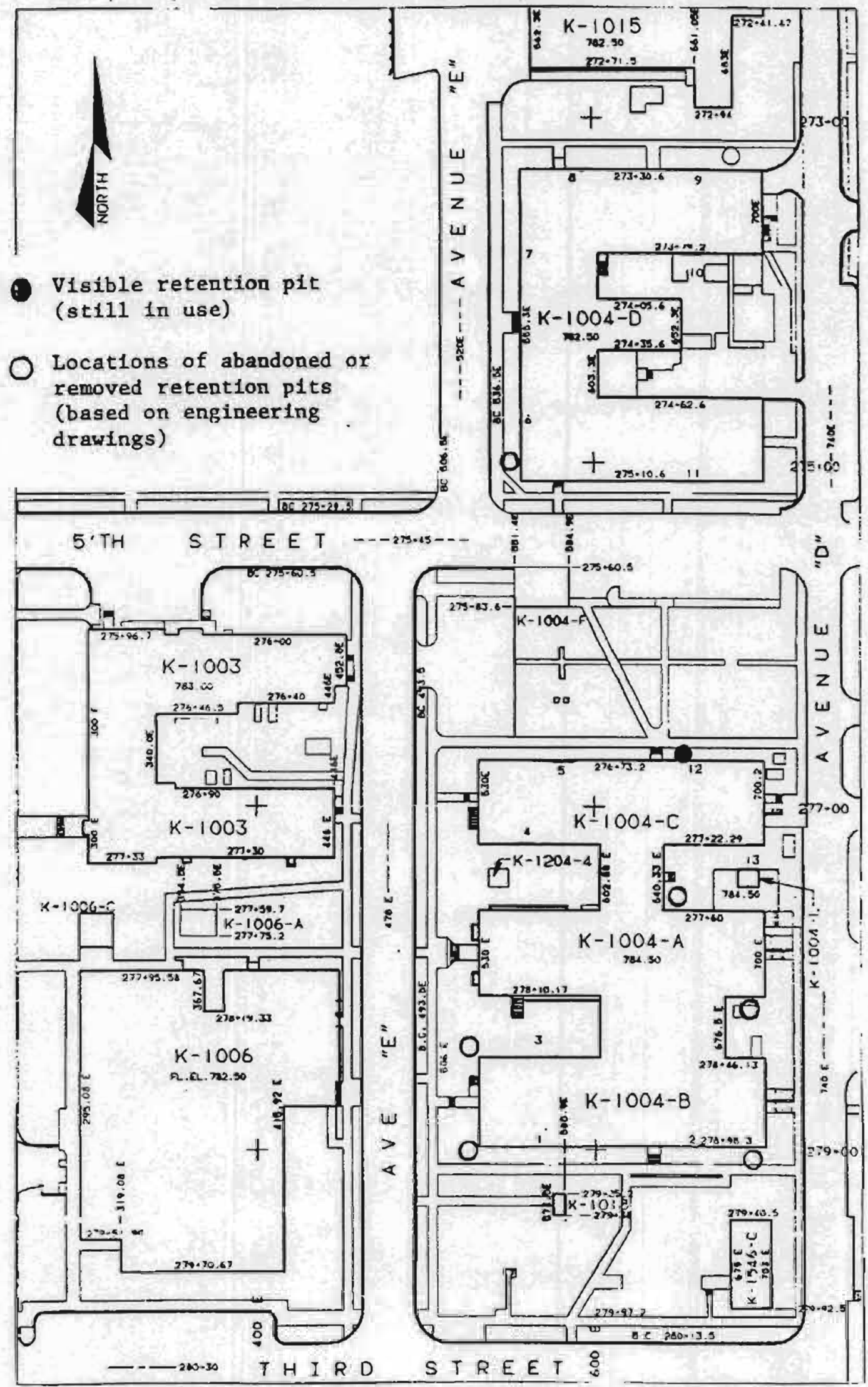

Fig. 3.3. Retention pit location map 


\subsection{OPERATIONAL INFORMATION}

Until implementation of the BMP in 1985, many chemicals used and wastes produced in the laboratories were discharged to the K-1004 ALO. Information from interviews provided laboratory-specific detalis on chemicals and procedures, but much of the information on chenicals is general to the $\mathrm{K}-1004$ laboratory area. Hazardous substances which are suspected to have entered the K-1004 ALD from these laboratories are listed in Section 4. Uranium-containing solutions from the analytical laboratories were not routinely disposed of in the ALD. Concern for safety, uranium accountability, and environmental issues required the implementation of operational procedures to control the handing of uranium solutions. Containers placed in the laboratories were used for collecting these solutions which were transported to the uranium recovery facility. Thus, only accidental releases of uranfum solutions would have entered the ALD.

Wastes from K-1004-A, B, C, and 0 Laboratories were discharged to concrete-lined retention pits prior to entering the SD-100 System. These pits were approximately $3^{\prime} \times 2^{\prime} \times 3^{\prime}$ deep and had redwood baffles in the center. Waste solutions entering one side of the pit were temporarily retained and diluted by the contents of the pit prior to splliing over the baffle into the other side of the pit and being discharged to the SD-100 system. Only one of these pits remains in use (Figure 3.3 ).

In 1985, a BMP was implemented to stop the release of hazardous waste to the K-1007-B Pond. As a result of this plan, a Lab Waste Disposal System was implemented in the K-1004 laboratories. Hazardous wastes produced in this area are presently collected in the laboratories and are treated, stored, or disposed of at RCRA-permitted facilities. anly a water and soap mixture generated from the cleaning of glassware in the laboratories is currently pemitted to be discharged through the $K-1004$ ALD. 


\section{CHARACTERIZATION OF THE CONTAMINANT SOURCE}

The scope of this RFI addresses two contaminant sources--the K-1004 $A L D$ and the $K-1007-B$ Pond. Records of the quantities and concentrations of the varlous waste solutions carried in the ALD and discharged to the $K-1007-8$ pond are not avallable.

\subsection{K-1004 AREA LAB DRAIN (ALD)}

Hazardous substances which may have entered the $K-1004$ ALO from various surrounding laboratories are listed in Table 4.1. In addition to those chemicals listed in Table 4.1, other bufldings in the area discharged chemicals to the ALD. The $K-1003$ Medical Center discharged $x$-ray fixer and developer chemicals (including silver chlorlde) and azide cleaning agent to the drain lines. The $K-1005$ Laboratory housed equipment used for experimental work on the separation of uranium from spent reactor fuel. These experiments used only gram quantities of fuel in the experiments and, as described in Section 3, any uranium-containing wastes were collected and sent to the uranium recovery facllity. The Applled Technology Division (ATD) Facility in the $K-1005$ bullding and the K-1004-J Laboratory used silicon nitrite-treated cooling water. Because of the hazardous nature of silicon nitrite, ORGDP Environmental Management required that this waste be deposited in containers for special disposal. ERL 2258, which is the tradename for an epoxy based resin and associated hardeners, was used in the fabrication of centrifuge parts at the ATD Fac11ity. ERL 2258 is a mixture of Bis-(2,3-epoxy-cyclopentyl) ether and Diglycidyl ether of Bisphenol A (DGEBA). The resin was combined with an aromatic amine hardener, such as $2 Z L-0820$, a mixture of metaphenylenediamine, Bis-(4-aminopheny1) methane, and DGEBA. These materials could have reached the $K-1004$ ALD. 
Table 4.1. Hazardous substances which may have entered K-1004 ALD

- acetic acid

- acetone

- acetonitrile

- alcohols, NOS*

- ammonium hydroxide

- benzene

- bromoform

- cadmium (from washing Cdcoated UF 6 cylinders)

- chloroform

- carbon tetrachioride

- chromates

- code BE turbine lubricating oil (non-PCB) (from leaks and spills)

- copper

- dichloropropane

- diethyiene glycol-dibutyl ether (Carbitol)

- freon

- glycols

- saturated hydrocarbons

- hydrochloric acid

- hydrofluoric acid

- hypophosphorous acid
- mercury

- methyl ethyl ketone

- methylene chioride

- neptunium

- nickel compounds

- nitric acíd

- Oakite (a caustic cleaning agent)

- PCBs

- phosphoric actd

- potasstum hydroxide

- pyridine

- rhenium

- sodium hyoroxide

- sulfuric acid

- technetium

- tetrachloroethylene

- toluene

- trichloroacetic acid

- trichloroethylene

- tungsten

- uranium

- silver chloride

* Not otherwise specified. 


\subsection{EXISTING K-1007-B POND DATA}

\subsubsection{Sampling Scheme}

Data were obtained from 36 sediment samples taken in August 1987 from the bottom of K-1007-B Pond and connecting smaller ponds. The sampling locations were selected to provide uniform coverage over the pond and are shown in Figure 4.1. The samples were obtained in a random order.

\subsubsection{Data Summary}

For each analyte in the sediment data, the following sumary statistics are provided in Appendix A:

- number of samples

- number of samples less than detection limit

- smallest detection limit value reported (see explanation below)

- minimum value reported

- maximum value reported

- lower bound on pond average

- upper bound on pond average

- units

Many of the compounds in Appendix A have more than one detection limit. Multiple detection limits occur because the detection limits are reported on a dry weight basis and the samples contain varying amounts of moisture. For each analyte, the number of samples less than detection limit represents all results which were reported as less than any detection 1imit. Because there are numerous cases where multiple detection 1 imits are reported, the smallest detection 1 imit reported is shown in Appendix A (see footnote 3). All of the data, including detection limit values, were used in finding the minimum and maximum values and the lower and upper arithmetic bounds on the pond average for each analyte. The upper bound is the average calculated using the value of the detection limit. The lower bound is the average using zero instead of the value of the detection limit. 

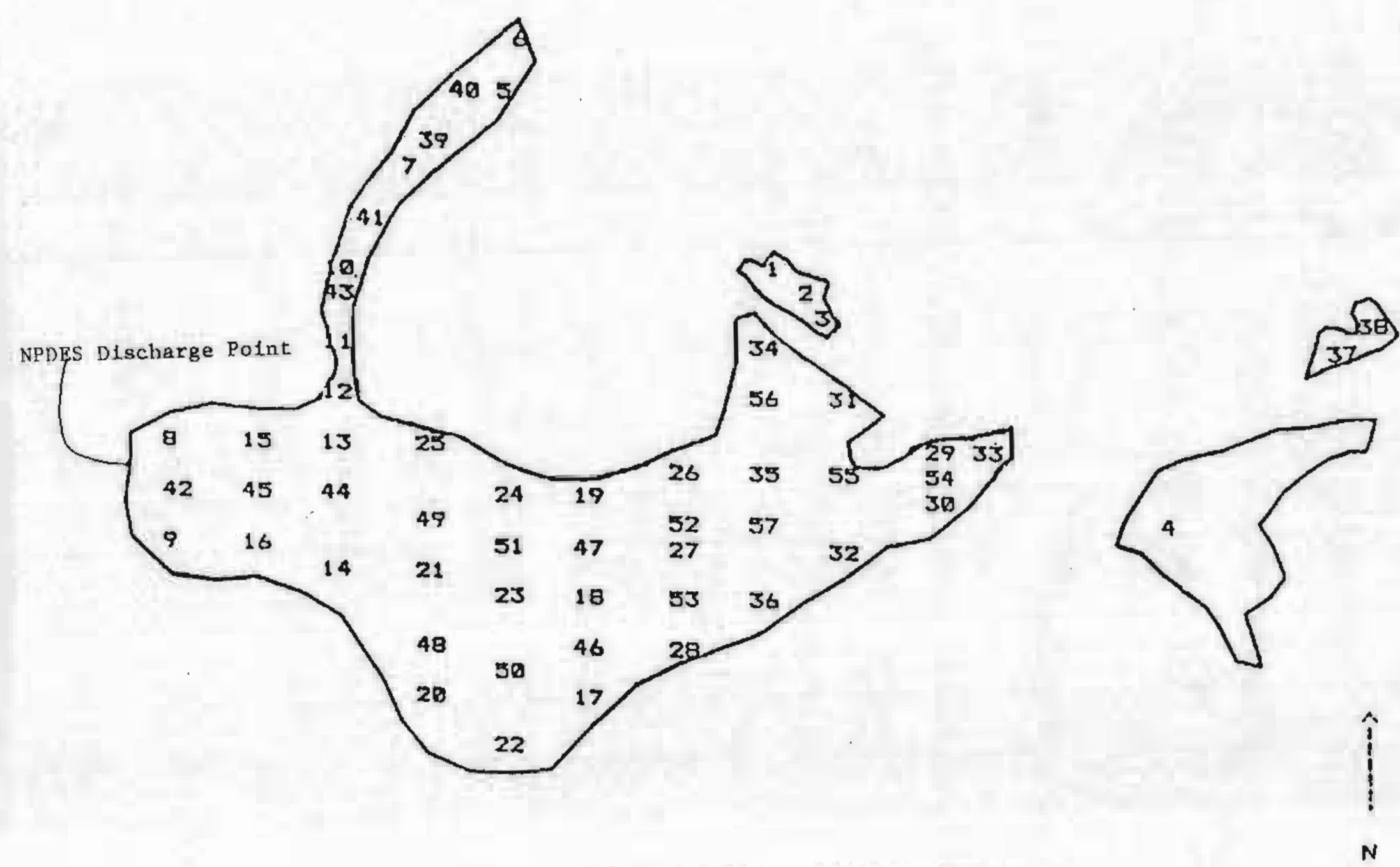

Fig. 4.1, K-1007-B Pond sediments sample locations 
Several analytes were found to have results exceeding the prescribed maximum soll limits shown in Table 2.2 of K/HS-132. These analytes are shown in Table 4.2 along with the number of samples taken for these analytes, the number of results reported that are greater than the maximum 1 Imit, the actual results involved, and the $K-1007-8$ sampling location (Figure 4.1). EP toxicity data and limits are also shown for these analytes.

\subsubsection{Spatial Plots of Analytes}

Appendix $B$ contains plots of the analytes according to their sampling locations in the $\mathrm{K}-1007-B$ Pond. Plots are oresented for regulated metals and for all organics with any detectable result.

The symbols plotted are as follows:

- All results reported as less than a detection limit are shown as a "<" (no distinction for multiple detection limits).

- Any result exceeding the K/HS-132 Table 2.2 maximum limit for solt is indicated on the plots as "*u.

- Quartile numbers, " ${ }^{*},{ }^{*}$ ", " $3 *$, and " 4 ", represent the magnitude of the data which remain after deleting results that were less than a detection limit or greater than the maximum K/HS-132 Table 2.2 soll limit.

Several spatial patterns are evident for the results contained in the third and fourth quartiles. Samples taken along the southeast bank of $\mathrm{K}-1007-B$ (sample locations $17,28,32$, and 36 on Figure 4.1.) were consistently higher for some analytes. The northern leg of $K-1007-B$ (sample locations $5,6,7,10,11,12,13,39,40,41$, and 43) and a small area in the middle of the pond (sample locations 18, 21, and 23) often showed higher results. Higher values were consistently reported 
Table 4.2. Sediment results greater than K/HS-132 Table 2.2 maximum limits for soll

\begin{tabular}{|c|c|c|c|c|c|c|}
\hline ANALYTE & $N^{1}$ & NGTMAX ${ }^{2}$ & $\begin{array}{l}\text { SAHPLE } \\
\text { RESULT } \\
\text { ug/g }\end{array}$ & LOCATION & $\begin{array}{c}\text { EP TOX } \\
\mathrm{ug} / \mathrm{m} \text { l }\end{array}$ & $\begin{array}{l}\text { EP TOX } \\
\text { LIMIT } \\
\text { ug/ml. }\end{array}$ \\
\hline CHROMIUM & 36 & 14 & $\begin{array}{l}110 \\
130 \\
170 \\
170 \\
200 \\
230 \\
390 \\
370 \\
290 \\
540 \\
260 \\
530 \\
520 \\
190\end{array}$ & $\begin{array}{l}1 \\
2 \\
3 \\
35 \\
27 \\
18 \\
5 \\
6 \\
23 \\
7 \\
21 \\
10 \\
11 \\
13\end{array}$ & $\begin{array}{l}<0.01 \\
<0.01\end{array}$ & 5 \\
\hline COPPER & 36 & 3 & $\begin{array}{l}110 \\
170 \\
110\end{array}$ & $\begin{array}{l}1 \\
3 \\
10\end{array}$ & & \\
\hline LEAD & 36 & 4 & $\begin{array}{l}220 \\
110 \\
110 \\
120\end{array}$ & $\begin{array}{l}15 \\
5 \\
7 \\
10\end{array}$ & $<0.05$ & 5 \\
\hline MERCURY & 36 & 4 & $\begin{array}{l}4.2 \\
4.5 \\
4.3 \\
4.1\end{array}$ & $\begin{array}{l}18 \\
23 \\
21 \\
13\end{array}$ & 0.0003 & 0.2 \\
\hline NAPHTHALENE & 36 & 4 & $\begin{array}{l}2500 \\
2700 \\
2200 \\
3900\end{array}$ & $\begin{array}{l}26 \\
22 \\
25 \\
12\end{array}$ & & \\
\hline ZINC & 36 & 1 & 540 & 10 & & : \\
\hline \multicolumn{7}{|c|}{ 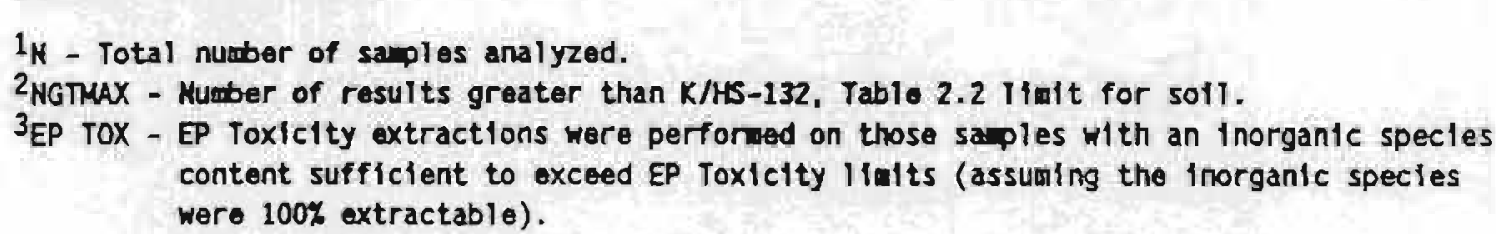 } \\
\hline
\end{tabular}


for samples taken in the small pond located northeast of K-1007-B. In most of the plots considered, higher sample results were found in the northern leg of K-1007-B, the southeast bank of $K-1007-B$, the middle of $K-1007-B$, and in the small pond to the northeast.

In the case of naphthalene, some of the reported detection limits are greater than the maximum limits given in K/HS-132 Table 2.2. These values are shown as "*" on this plot. 


\section{CHARACTERIZATION OF THE ENVIRONMENTAL SETTING}

The K-1004 ALD and the K-1007-B Pond is situated in an area of little topographic expression and very low slopes of about 2 percent. Maximum surface rellef over the length of the ALD is approximately 30 feet. The drain discharges into the $K-1007-8$ Pond with the end of the pipe underwater so the pond backs up into the drain line for some indeterminate distance.

Drill hole data are not currently avallable for this RFI area; however, there are numerous groundwater monitoring wells in other areas of ORGDP which penetrate both the same geologic formation and the solls that underlie the subject area. The well logs have been published by Geraghty and Miller in Hydrogeology of the Oak Ridge Gaseous Diffusion Plant Site. July 1986, and Phase II - Detection Monitoring, June 1987. The areal geology of ORGDP is shown in Figure 5.1 and has been campiled from three major sources: (1) Hydrogeology of the Oak Ridge Diffusion Plant; (2) recent unpublished work by R. H. Ketelle, Oak Ridge National Laboratory; and (3) "Geologic Map of the Oak Ridge Area, Tennessee," by W. M. McMaster, U.S. Geological Survey, 1958. The following geologic descriptions and discussions of hydrogeology are based on these sources, and specific data, permeablitities, etc., are referenced as applicable.

\subsection{HYOROGEOLOGY}

The K-1004 ALD is underlain by the Chickamauga Iimestone which, in the ORGDP area, consists mainly of gray to blue-gray or green, very fine-grained (micritic) limestone interbedded with calcareous shale and shaley llmestone. The limestone may be relatively "pure" or argillaceous (contains clay), and it is generally medium to thinly bedded with bedding planes comprised of very thin shaley partings. Chert may occur in certain horizons of the Chickamauga as zones of nodules or as thin, irregular layers. The calcareous shales are mostly gray to green and contain occasional very thin beds of micritic limestone. 


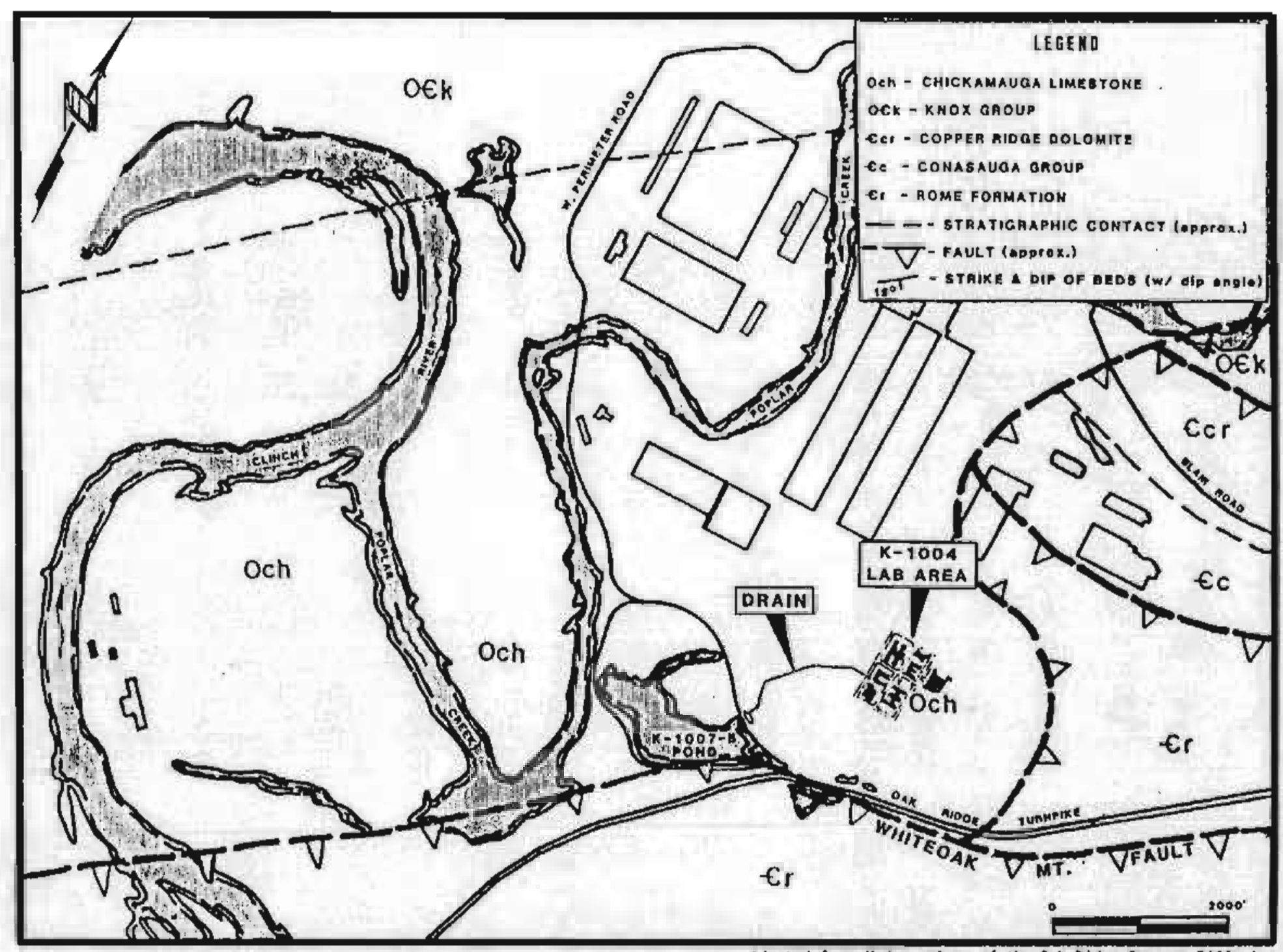

Alspled from: liydrogeolagy of llie oak Rldas Gienus Diflus lon unpuifitshed mop liy R.ti. Xetelle, ORILL.

Fig. 6.1. Areal geology of ORGDP 
The dominant geologic structural feature of the area is the Whiteoak Mountain fault, the trace of which is indicated to traverse part of the $K-1007-B$ Pond just south of the discharge end of the $K-1004$ ALD (Figure 5.1). The ALD is in the footwall of this fault which has thrust the Rome formation over the Chickamauga, and the bedrock here has been subjected to extensive defomation and fracturing so that dips (inclination of beds) are likely to be highly variable. The strike of the inclined strata will be generally northeast-southwest, approximately parallel to the strike of the fault. There are no known bedrock outcrops in this area and bedrock attitudes are inferred from observations in nearby areas.

There are no avallable descriptions of the unconsolidated (soil) zone in this vicinity. The Chickamauga limestone in the ORGOP area is generally overlain with residual solls of varying depths up to about 30 feet, although here the soll depth probably does not exceed 20 feet. These solls are typically red to brown, silty clays with scattered small fragments of chert or weathered limestone. There may also be surficial deposits of alluvial sediments in portions of the area.

Groundwater storage and movement in the Chickamauga bedrock occur in a system of interconnecting channels developed mainly within the carbonate units by solution-enlargement along joints, fractures, and bedding planes. Fractures in the more insoluble shales tend to be "tight" and water movement is thereby restricted. The shaley units channel groundwater flow along bedding planes, parallel to strike. The direction of groundwater flow in the bedrock beneath this area is southwestward along strike toward Poplar Creek. There are no permeabllity data presently available for bedrock beneath this RFI area; however, tests of wells in the Chickamauga limestone in nearby areas have indicated the average hydraulic conductivity of that formation to be approximately $3.5 \times 10^{-3} \mathrm{~cm} / \mathrm{sec}$. This value is presumed to be representative of the Chickamauga beneath the subject area. 
Groundwater flow direction in the unconsolidated zone is probably to the southwest toward the K-1007-B Pond. No site-specific permeability data are avallable for the solls of this area, but tests on similar solis within the ORGDP site suggest a probable range of hydraulic conductivity to be from $10^{-5}$ to $10^{-6} \mathrm{~cm} / \mathrm{sec}$.

Hydraulic gradients in both bedrock and the unconsolidated zone should be very low with correspondingly low flow rates, and there is ittle interflow between the two aquifer systems. There are no known outcrops, sinks, or other features which would enhance infiltration in this area.

\subsection{SURFACE WATER}

Much of the surface runoff in the area covered by the ALD is diverted to the 5D-100 system which eventually discharges into the $K-1007-B$ Pond (see figure 3.2 ). This area is almost fiat but slopes very gently to the southwest, and any storm water not intercepted by the SD-100 System will al so flow over the surface toward the $K-1007-8$ Pond. The pond drains into Poplar Creek through a weir which is also an NPDES discharge point. This weir prevents aquatic fauna in Poplar Creek from entering the pond.

Most of this area is above the 100-year flood level according to flood data developed by the Tennessee Valley Authority (TVA) and presented in Figure 3.5 of K/HS-132. However, because the discharge end of the ALD is already underwater, any flood will cause water in the K-1007-B Pond to back up further into the drain 11ne. There are no springs or other surface waters which would be affected by the drainage system.

\subsection{AIR}

No site-specific air quality data are available for the SWMU. Martin Marietta Energy Systems, Inc., has an ongoing study of the atr quality and meteorological conditions, and general data for ORGDP are available in K/HS-132. 


\section{IDENTIFICATION OF POTENTIAL PATHWAYS AND RECEPTORS}

Assessment of a hazardous waste disposal, treatment, or storage site is required to evaluate the site's potential for health or safety risks to the environment, public, and personnel. Determination of such risks must be based on evaluations of both the potential pathways of contaminant migration from toxic releases and the possible receptors of the contamination. Information used in the evaluation of the pathways which might release contaminants from the $K-1004$ ALD and the $K-1007-B$ Pond has been obtained from (1) existing analyses of sediment data from the K-1007-B Pond, (2) NPDES monitoring data from the K-1007-8 Pond, (3) data from surface water sampling from SD-100, and (4) interviews with personnel familiar with operations in this area of ORGDP. K/HS-132 will serve as a general reference concerning the potential pathways and receptors for ORGDP.

The possibility of pipe or pit leakage presents the potential for soll and groundwater contamination at this site. Residual contamination in the SD-100 system could also be transported from the area via surface water. Fish in the $\mathrm{K}-1007-B$ Pond could contain bioaccumulated contaminants which entered the pond via the ALD or by groundwater or surface water discharge. A Phase I investigation of soil, surface water, groundwater, and pond biota is proposed to assess the extent (if any) of contamination.

\subsection{POTENTIAL PATHWAYS OF MIGRATION}

\section{1 .1 Groundwater}

The possibility of leakage from the ALD and retention pit presents the potential for groundwater contamination. The low hydraulic gradient and low permeability suggest that any groundwater contamination in the vicinity of the $k-1007$ Computer center will move slowly through the unconsolidated zone to the nearby discharge area (i.e., Poplar Creek and 
the $k-1007-B$ Pond). Since previously installed wells in the unconsolidated zone in the K-1004 Laboratory area were found to be dry, wells in this area will be completed to bedrock. Assessment of the nature and extent of possible groundwater contamination will be carried out in accordance with the ORGDP Groundwater Protection Program.

\section{1 .2 Surface Water}

Residual contamination in the storm sewer lines may be contaminating surface water flow in the storm sewer system. Surface water grab samples will be collected from catch basins in the SO-100 System and analyzed to assess the nature and extent of posstble surface water contamination. Water samples will also be collected at the NPDES discharge point and analyzed to assess the impact of contamination in the $\mathrm{K}-1007-\mathrm{B}$ pond on Poplar Creek.

\section{1 .3 S011}

The practice of discharging wastes into the ALD during the history of this area, and the possibllity of leakage from the ALD and K-1004 retention pits present the potential for soll contamination. The extent of residual contamination surrounding the known retention pit will be assessed by analysis of soil samples as described in Section 8 of this document. A Phase II soil sampling plan around the $K-1004-A, B, C$, and $D$ Laboratories will be proposed should groundwater analyses reveal a contaminant plume. A Phase II soil sampling plan along the ALD may also be proposed should surface water and groundwater analyses reveal unacceptable contaminant levels. This phased sampling procedure will ensure a more realistic site characterization and will allow determination of general contamination locations, as well as contaminants of interest, should contamination exist. 


\section{1 .4 Fish}

Contaminants entering the $K-1007-B$ Pond from the ALD and environmental pathways could potentially bioaccumulate in the pond's fish population. Fish samples will be collected and analyzed specifically for mercury and polychiorinated biphenyis (PCB), contaminants which readily bioaccumulate.

\subsection{POTENTIAL RECEPTORS}

\subsubsection{Human Populations}

The security controls required by the Department of Energy (DOE) on entrance to ORGDP prevent public access to most of the area covered in the scope of this document. The $K-1007-B$ Pond is outside of the security fence; however, contamination warning signs are posted around the pond and this area is not frequented by the public.

Of the 25 potable water wells within one mile of ORGDP, none of the wells are in proximity to the ALO or the K-1007-B Pond, and none are belleved to occupy the same hydrogeologlcal environment as the groundwater at the site. Further, of the 10 public water supplies which withdraw from the $\mathrm{Clinch} \mathrm{-} \mathrm{Tennessee} \mathrm{River} \mathrm{system} \mathrm{(into} \mathrm{which} \mathrm{Poplar} \mathrm{Creek} \mathrm{drains),}$ none of these are nearer than 8 miles to the ORR. While direct discharge of surface runoff and site groundwater presents the potential for contamination, distance and dilution effects make pollution of public water supplies of low probability. Finally, the effects of distance and dilution also make unlikely the possfbility that contamination of surface water and groundwater would reach the waters used downstream in the Clinch - Tennessee River system for recreational and industrial use. 


\subsubsection{Fauna and Flora}

K/HS-132 discusses the rare, threatened, and endangered plant and animal species which are thought to inhabit the area. To date, there has been no report that any of these species in the vicinity of the ALD, the K-1007-B Pond, or surrounding areas are directly threatened by any contamination present there. However, other local fauna, namely fish in the pond, are potentially threatened by contamination emanating from the components of this site. Fish in Poplar Creek cannot enter the pond due to the NPDES weir located at the pond's only outfall to Poplar Creek and are not belleved to be threatened by contamination in the pond. The risk of contamination released from the site to the local fiora and fauna will be assessed subsequent to the RFI.

\subsection{SUMMARY AND CONCLUSIONS}

The nature of the material discharged through the ALD, disposed of in the K-1007-B Pond, and the site hydrogeology indicate the potential for so1l, groundwater, surface water, and biota contamination. Evaluation of the potential pathways of contaminant migration and possible receptors shows sufficient potential for environmental contamination and warrants an investigation of the site. 


\section{EXISTING MONITORING DATA}

NPDES data were collected from January 1976 through March 1988 with monthly averages avallable for January 1976 through October 1986, and Individual sample results were reported from November 1986 through March 1988. The sample results collected from November 1986 through March 1988 were averaged for each month in order to be consistent with the monthly averages reported from January 1976 through October 1986. Table 7.1 sumarizes these monthly averages showing the frequency and type of sample, number of monthly averages, number of reported results and/or monthly averages less than detection limit, lower and upper arithmetic bounds (as defined in 4.2) on the average of the monthly averages, and the minimum and maximum monthly averages. Also shown are the existing permit limits expressed in terms of concentration of the analyte in the sample and quantity discharged per day.

Figures $C .1-C .22$ in Appendix $C$ present plots of the monthly averages for each analyte over time, with the overall average (the upper

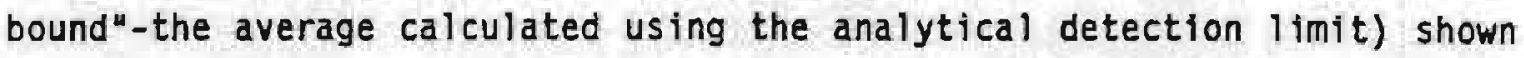
for reference purposes. Also shown for reference purposes only are permit limits for individual samples, since no limits were avallable for monthly averages. Because the data collected from November 1986 through March 1988 are reported as sample results and not monthly averages, plots are presented which show these results over time along with the average result and appropriate permit level in terms of concentrations and quantity discharged ( $\mathrm{kg} /$ day) per day.

Data from the SO-100 System are given in Appendix 0 . Point 07 in the SD-100 System has historically shown elevated levels of some contaminants and is located near the visible retention pit (see Figure 3.3 ). 
TABLE 7.1 NPDES hONTHLY AVERAGE DATA SUMMARY

\begin{tabular}{|c|c|c|c|c|c|c|c|c|c|c|c|}
\hline \multirow{3}{*}{ AMALYTEE } & \multicolumn{7}{|c|}{ OVERALI AVERAGE ${ }^{3}, 4$} & \multicolumn{2}{|c|}{ DAILY MXXIMUH } & \multicolumn{2}{|c|}{ MOUITCR IMG REOUIREMENTS } \\
\hline & 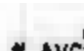 & & UPPER & LOMER & NDATHLY & VERAGE 6,4 & & OISCHARGE & LIHITATIONS & - MEASUREMETT & SAHPLE \\
\hline & IAvG & $4 \mathrm{LTL}^{\circ}$ & BOUND & POAND & M1.4 & mx & UNITS & MG/4 & KG/OAY & FREQUENCY & IYPE \\
\hline ALUMIMAS & 1 & 0 & 0,4700 & 0.4700 & 0.4700 & 0.4700 & MG/L & & & & \\
\hline BERTLLIUM & 2 & 2 & 0.0010 & 0.0000 & 0.0010 & 0.0010 & MG/L & 0.002 & & & \\
\hline CADHILM & 2 & 2 & 0.0020 & 0.0000 & 0.0020 & 0.0020 & $M E / L$ & 0.010 & & & \\
\hline CESIUM & $\overline{3}$ & $\overline{3}$ & 100.0000 & 0.0000 & 100.0000 & 100.0000 & $\mathrm{PCL} / \mathrm{L}$ & & & & \\
\hline CMEMICAL CXXYGEN DEMAHD & 150 & 52 & 12.5157 & 9.0902 & 0.1100 & 25.0000 & MG/L & 25.000 & 151.000 & WEEKLY & Conposite \\
\hline CHRONILA & 148 & 155 & 0.0135 & 0.0033 & 0.0040 & 0.1000 & $M G / L$ & 0.050 & 0.300 & LEEKLY & COMPOSITE \\
\hline COPPER & 2 & 1 & 0.0405 & 0.0055 & 0.0100 & 0.0110 & $M G / L$ & 0.290 & & & \\
\hline$C R+3$ & 1 & i & 0.0100 & 0.0000 & 0.0100 & 0.0100 & $\mathrm{MG} / \mathrm{L}$ & & & & \\
\hline$C R+6$ & 1 & 1 & 0.0100 & 0.0000 & 0.0100 & 0.0100 & MG/L & & & & \\
\hline DISSOLVED OXYGEY & 135 & 0 & 9.7306 & 9.7306 & 4.6000 & 18.0000 & $\mathrm{MG} / \mathrm{L}$ & $>5.000$ & & MEEKLY & GRAB \\
\hline FLLORIDE & 147 & 79 & 0.1924 & 0.1285 & 0.0110 & 0.9400 & $M G / L$ & 1.500 & 9.100 & WEEKLY & COHPOSITE \\
\hline LEAD & 2 & 2 & 0.0070 & 0.0000 & 0.0040 & 0.0100 & MG/L & 0.930 & & & \\
\hline MERLJRY & 2 & 2 & 0.0010 & 0.0000 & 0.0010 & 0.0010 & MG/L & 0.011 & & & \\
\hline METHYLEHE CHLOR IOE & 2 & 2 & 0.0100 & 0.0000 & 0.0100 & 0.0100 & $M G / L$ & & & & \\
\hline MEPTUMIIUM & 3 & $\mathbf{3}$ & 1.0000 & 0.0000 & 1.0000 & 1.0000 & PCI/L & & & & \\
\hline NITRATE - N & 2 & 2 & 0.1050 & 0.0000 & 0.1000 & 0.1100 & MG $\Omega$ & 32.500 & & & \\
\hline OIL 1 GREASE & 52 & 93 & 2.2635 & 0.0928 & 2.0000 & 7.0000 & $M G / L$ & 15.000 & 91.000 & MEEKLY & GRA \\
\hline PEPCHLOROET HTLENE & 2 & 2 & 0.0125 & 0.0000 & 0.0100 & 0.0150 & $\mathrm{KG} / \mathrm{L}$ & 0.210 & & & \\
\hline $\mathrm{PH}^{1}$ & $11 \overline{6}$ & $\overline{0}$ & 8.3439 & B.3439 & 6.800 & 9.0000 & & & & DAlLY & GRAB \\
\hline PLuTONILA & 3 & 3 & 1.0000 & 0.0000 & 1.0000 & 1.0000 & $\mathrm{PCI} / \mathrm{L}$ & & & & \\
\hline SELENILM & 2 & 2 & 0.0050 & 0.0000 & 0.0050 & 0.0050 & $\mathrm{HE} / \mathrm{L}$ & 0.310 & & & \\
\hline SILVER & 2 & 2 & 0.0100 & 0.0000 & 0.0100 & 0.0100 & Mo/t & 0.027 & & & \\
\hline SULFATE & 1 & 0 & 23.8000 & 23.8000 & 23,8000 & 23.9000 & $\mathrm{MG} / \mathrm{L}$ & & & & \\
\hline TECHAETILM & 3 & $\mathbf{3}$ & 120.0000 & 0.0000 & 02.0000 & 169.0000 & PCt/ & & & & \\
\hline TEMPERATLRE & 1 & 0 & 4.5875 & 4.5375 & 4.5875 & 4.5875 & DEG $\mathrm{C}$ & & & & \\
\hline TOTAL HALCMETHAHE & 1 & $i$ & 0.0100 & 0.0000 & 0.0100 & 0.0100 & $M G / L$ & 2.050 & & & \\
\hline TOTAL SUSPENEEO SO TOS & 151 & 66 & 12.8544 & 7.8048 & 5.0000 & 28.0000 & MG/L & 50.000 & 303.000 & WEEKLY & CONPOSITE \\
\hline TRICHLOROETHAME & 2 & 2 & 0.0100 & 0.0000 & 0.0100 & 0.0100 & $M G / L$ & & & & \\
\hline TRICHLOROET KYLENE & 2 & 2 & 0.0100 & 0.0000 & 0.0100 & 0.0100 & MG/L & 0.610 & & & \\
\hline URANILA & 16 & 21 & 0.0049 & 0.0022 & 0.0012 & 0.0252 & $M G / L$ & & & & \\
\hline 2IHC & 1 & 1 & 0.0200 & 0.0000 & 0.0200 & 0.0200 & MG $/ L$ & 1.520 & & & \\
\hline
\end{tabular}

1 HOT TO BE LESS THAN G.D OR GREATER THAM 10.0

2 * LESS THAM DETECIION LIMIT IHELUDES MOHTHLY AVERMGES FOR 1976-19B3 AND IMDIVIDUAL SAMPLE RESULTS FCR 1984-19GS

3 OVERALL AVERAGE OF MOHTHLY AVERAGES FDR 1976-1988. THE UPPER BOUNO IS CALCULATED gY AVERAGIHG THE MOHTHLY AVERAGES WHICH 1HCLLDE LESS THAM DETECTION LIMLT VALUES INPUTTED AS THE DETECTIOH LIMIT. THE LOHER BOUNO IS CALCULATEO BY AVERAGIHG THE MOMTHLY AVERAGES WHICM INCLUDE LESS THAH DETECTION LIMIT VALUES INPUTTED AS O.

5 THE MUMER OF SIGMLFICANT FIGURES IS SOFTMARE DEPEHOENT AHD DOES NOT NECESSARILY REPRESENT AHALYTICAL PRECTSION.

5 THE MUMBER OF MONFHLY AVERAGES.

6 THE HIMIMUM AND MAXIMLM VALGES OF MONTHLY AVERAGES FOR 1976-1988. THE MIHIHAM VALUE IS NOT REPCRTED LESS TMAN THE DETECTIOH LIMIT. 


\section{SAMPLING PLAN}

\subsection{SAMPLING AND ANALYTICAL STRATEGY}

Prior to 1985, all non-uranium containing wastes from ORGDP laboratories were disposed of via the ALD which discharged to the K-1007-B Pond. An informal survey conducted prior to the implementation of the BMP for the K-1007-B Pond indicated that acidic, basic, and neutral inorganic and organic reagents were disposed of via the ALD. Incidental quantities of uranium and other transuranics may also have accidentally entered the ALD.

The ALD, associated retention pits, and the K-1007-B Pond are considered to be potential contaminant release sources. Direct characterization (soil sampling) of the area immediately adjacent to the ALD and the $\mathrm{K}-1004$ Laboratories is considered impractical due to the density of structures in the area as well as the length of line that would have to be investigated. As an alternative to extensive soil sampling, groundwater monitoring wells will be installed to evaluate groundwater contamination resulting from any leaks. However, the area surrounding the visible retention pit is generaliy accessible and samping of the soil directly adjacent to this pit will be performed as part of this RFI. Residual contamination in the ALD WIIl be assessed by collection and analysis of surface water grab samples from so 100 catch basins. In addition, archived sediment samples from the K-1007-B Pond which were high in total contaminant metal species will undergo EP toxicity analyses.

It will be necessary to examine the biota in the $k-1007-B$ pond and the outflow from the pond to Poplar Creek. Surface water samples will be collected at the NPDES discharge point (see Figure 4.1). The parameters measured as a part of the NPOES program will be expanded to include all 
metals, volatile and semi-volatile organics, and gross alpha, beta and gamma. The $K-1007-B$ Pond sediment has been previously characterized and, since the sediment samples were archived, further analysis will include EP toxic analysis in those samples found to be high in total contaminant metal species. The results of the initial sediment characterization are discussed in Section 4 of this document. Fish samples will be collected from $K-1007-B$ and analyzed for mercury and PCBs.

If the results of this phase I investigation reveal unacceptable contaminant levels, a Phase II investigation will be initiated to locate or further define the contamination source.

\subsection{SAMPLING STRATEGY}

\subsubsection{Soll Sampling}

Sampling will occur in steps consisting of soil sampling, chemical analysis, statistical analysis, and reduction of the resultant data. Sampling phases will continue until there is sufficient information on the extent of contamination in the soll around the retention pit and decisions can be made concerning remedial actions (if appropriate).

The first phase soil sampling is designed to determine whether there have been leaks from the visible retention pit located outside of Bullding $x-1004-C$ (see Figure 3.3). Two corings to bedrock will be made on both sides of the discharge drainage line as close as possible to the exit point from the pit (Figure 8.1). A background coring will also be taken (Figure 8.1).

From each drilling to bedrock, a soil sample will be taken: (a) from every distinct layer of soil, (b) from boundaries between soil layers, and (c) at regular intervals of four feet of depth in thick homogeneous layers. For these thicker layers, soll from 2 consecutive two-foot split barrels will be composited, with care not to composite across soil layer 

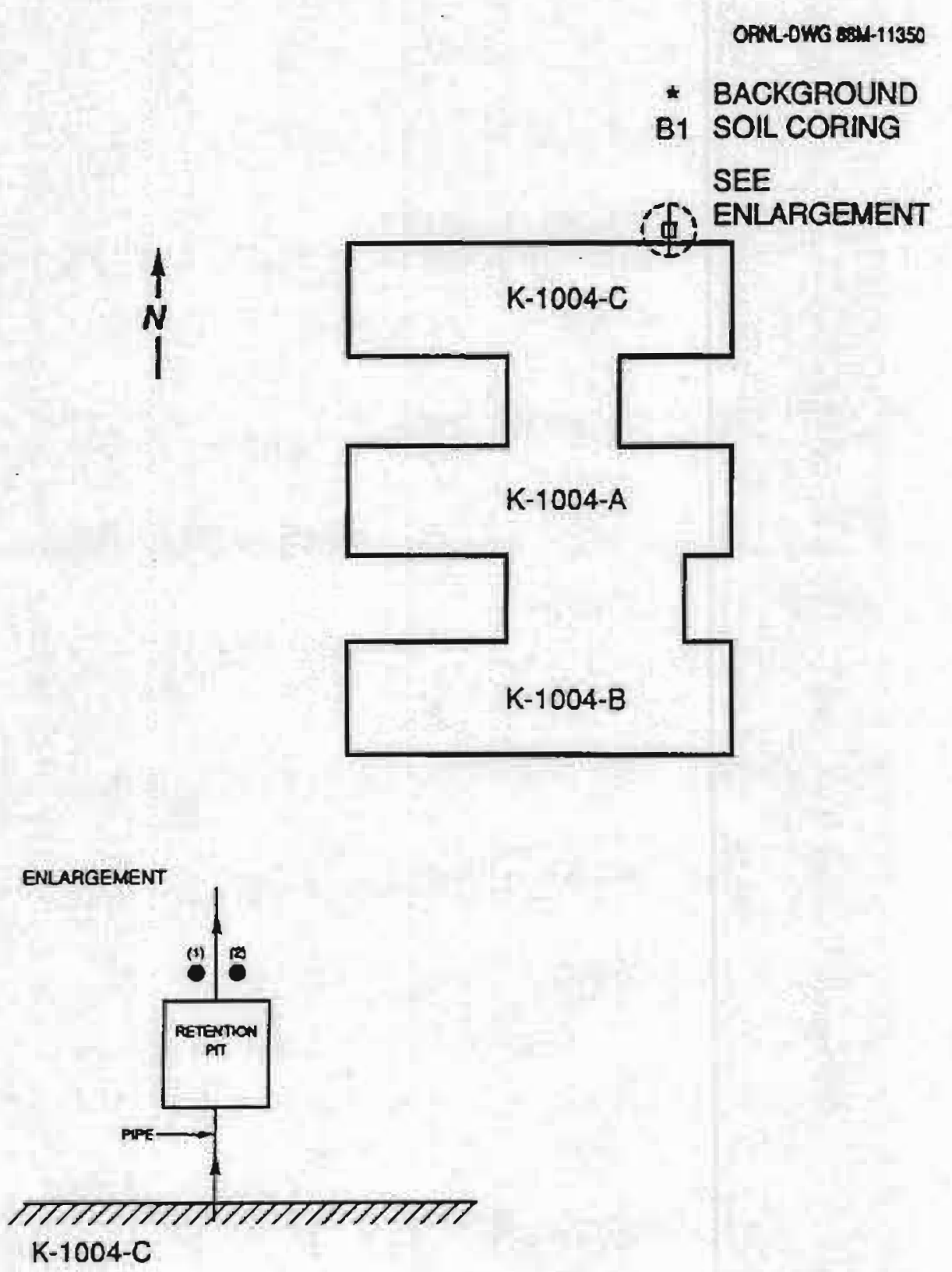

Fig. 8.1. Soil sampling locations 
types or layer boundaries. In addition, a sample will also be taken from the same elevation as the invert of the discharge drainage iine (figure 8.2). These individual samples will be divided with a portion of each sample from the coring going into a single coring composite, and a portion of each sample individually saved in case better resolution on the composite or a backup analysis is needed. The analysis order will be randomized after the total number of samples is determined.

\subsubsection{Groundwater Sampling}

Installation of five shallow groundwater monttoring wells is proposed, and analysis of groundwater from these wells will indicate if leaks from the ALD have contaminated the uppermost aquifer in the vicinity of the $\mathrm{K}-1007$ computer Center. The wells are to be placed adjacent to the ALD as shown in Figure 8.3. Four bedrock wells are also proposed to monitor groundwater which may have been contaminated from leaks in the ALD and the retention pits in the vicinity of the $K-1004$ laboratories. These wells have been placed hydraulically downgradient from the $\mathrm{K}-1004$ laboratories in an array which should intercept any groundwater contaminant plume emanating from this area (Figure 8.4). The unconsolidated zone wells will be incorporated into the ORGDP Groundwater Protection Program, and they will be sampled according to the standard RCRA protocol. The bedrock wells will be installed as a part of this RFI, but installation, sampling, and analysis of these wells will follow the protocol of the ORGDP Groundwater Protection Program.

\subsubsection{Surface Water Sampling}

Surface water grab samples will be taken from eleven catch basins in the 50100 system following three discrete rainfall events. These points are SD-100-04, SD-100-06, SD-100-07, SD-100-08, SD-100-09, SD-100-10, SD-100-11, SO-100-12, SD-100-13, SD-100-14, and SD-104 as shown in Figure 8.5 . 


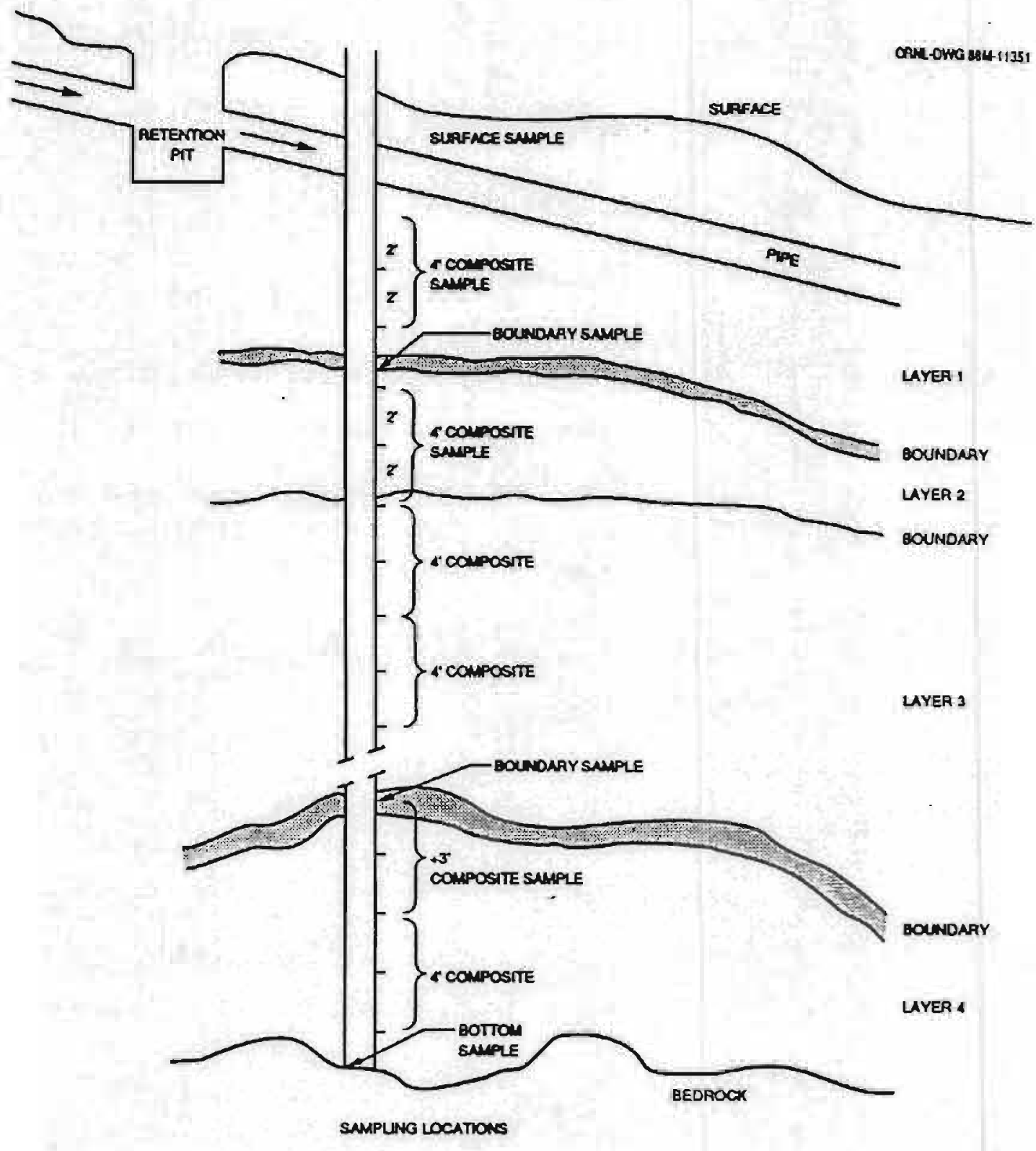

Fig. 8.2. Schematic cross-section showing soil sample locations 


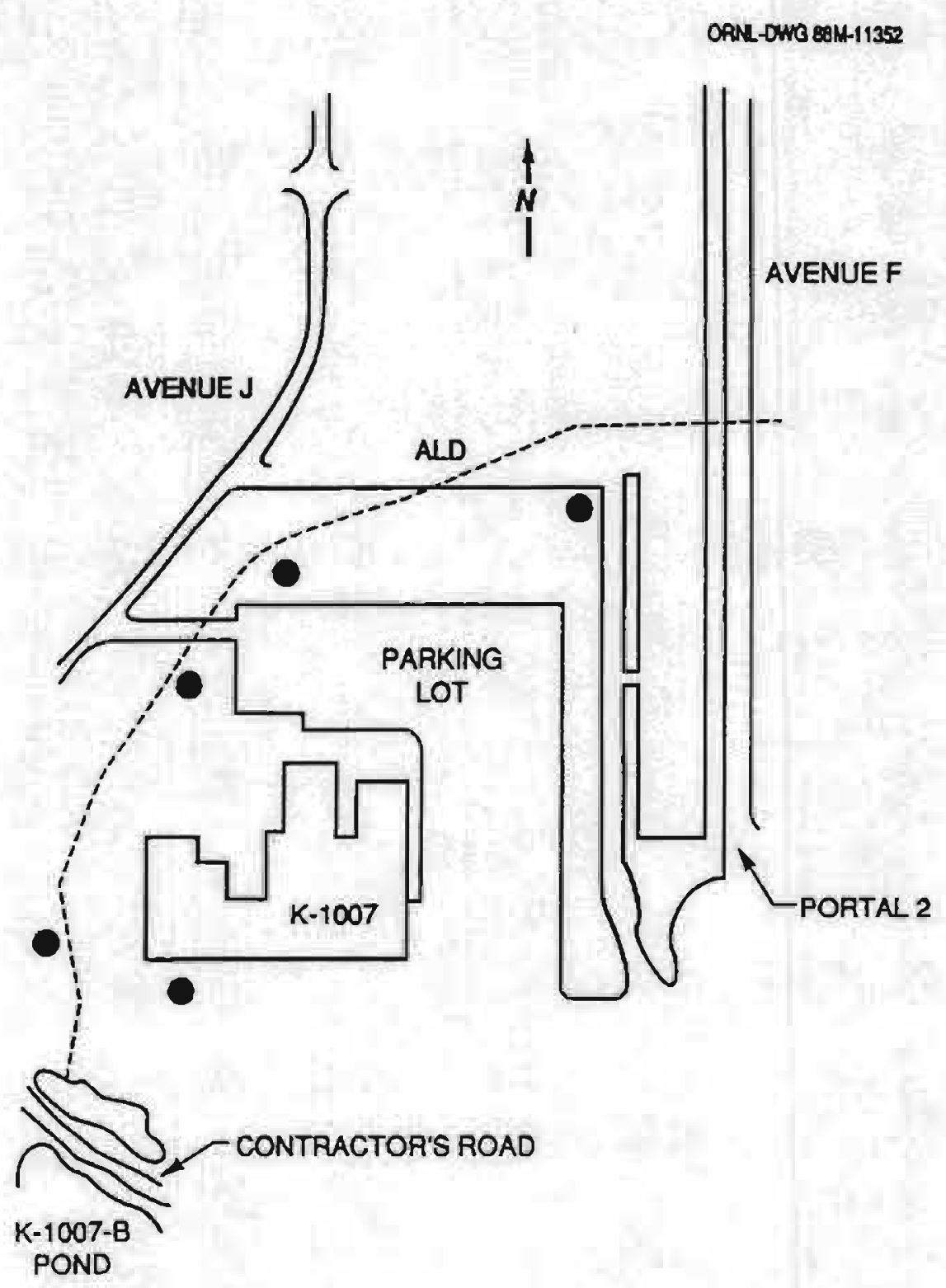

Fig. 8.3. Unconsolidated zone groundwater monitoring well locations 


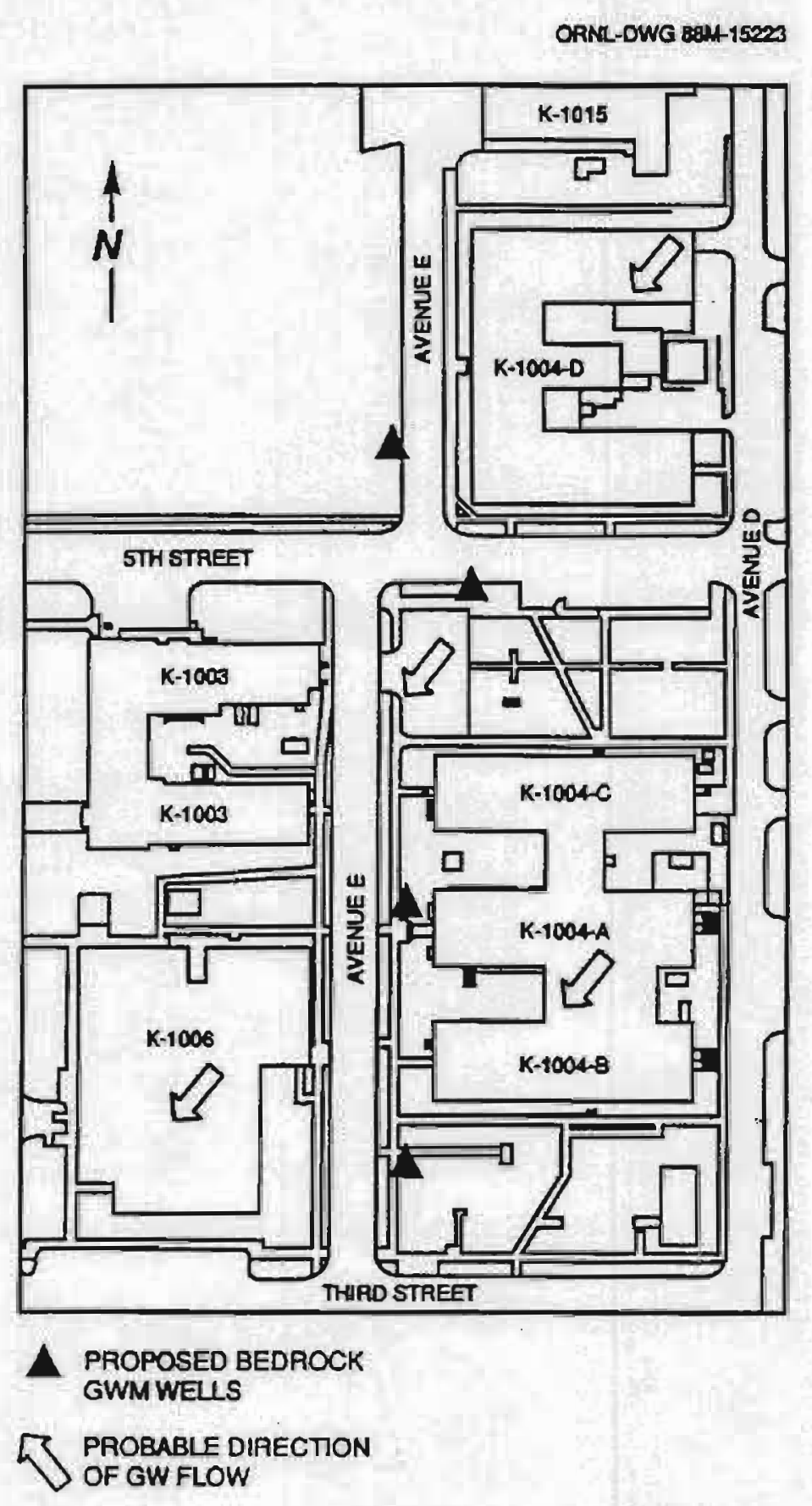

Fig. 8.4. Bedrock groundwater monitoring well locations 


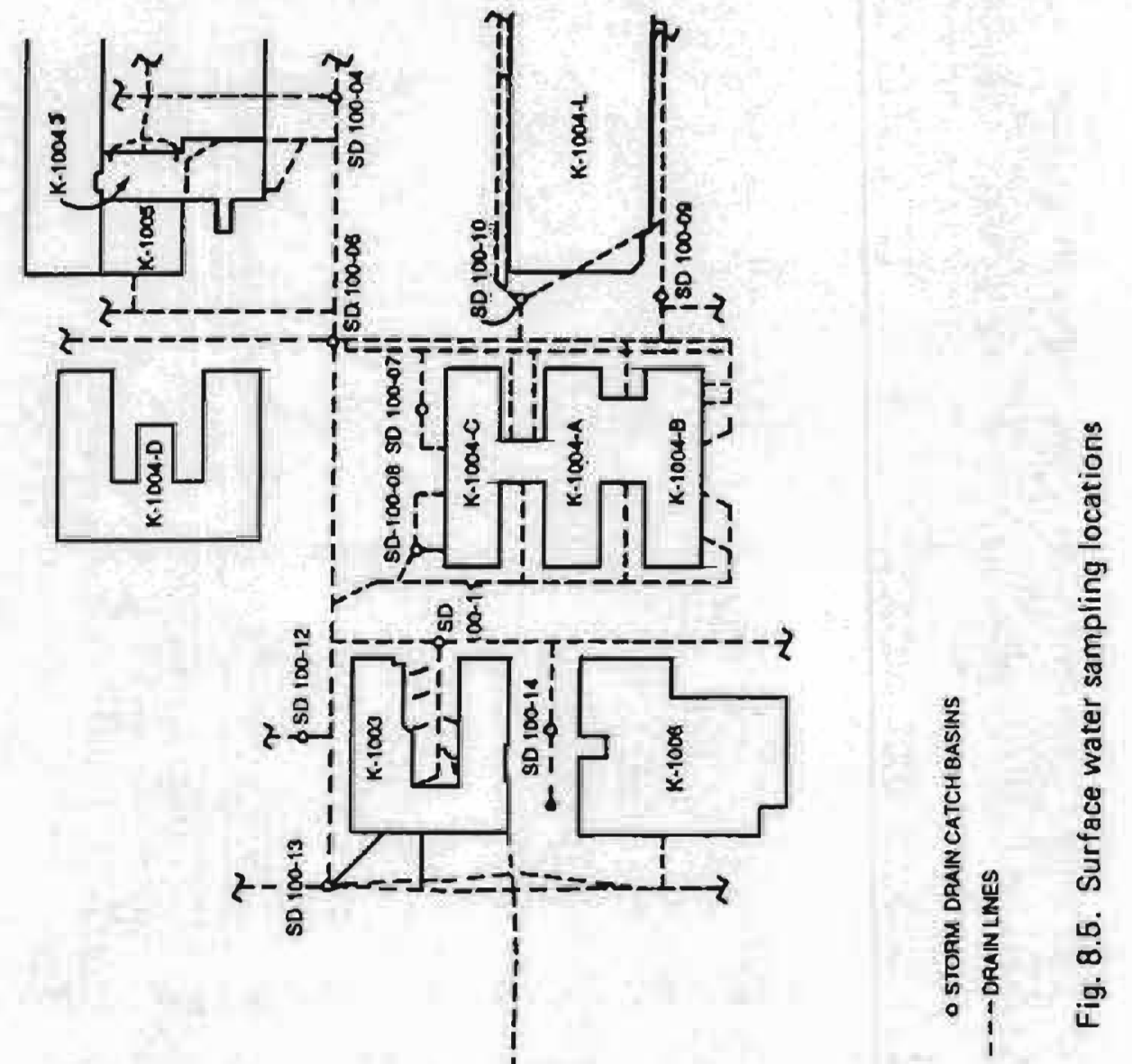


Surface water grab samples will also be collected from the NPDES discharge point where the $K-1007-B$ Pond discharges to Poplar Creek (see Figure 4.1). Samples will be collected as a part of NPDES monitoring over a six month period. The analysis order will be randomized.

\subsubsection{Fish Sampling}

Eight biuegill (Lepomis macrochivus) and eight carp (Cyprinus carpio) will be collected by electrofishing. Only fish of a size likely to be taken by sport fisherman (bluegill, 50-150 g; carp, $>500 \mathrm{~g}$ ) will be included in the collections.

\subsection{FIELD SAMPLING}

All field sampling procedures discussed in Section 8.3 are more fully described and documented in The Environmental Surveillance Procedures Quality Control Program, Martin Marfetta Energy Systems, Inc., (ESH/SUB/87-21706/1).

\subsubsection{Site Preparation}

In order to sample around the retention pit, it will be necessary to obtain the requisite permits through Martin Marietta Energy Systems Engineering. The location of drilling locations for soll sampling should be surveyed and marked prior to the arrival of the drilling crew; locations will also be recorded in the site's administrative record.

Prior to any groundwater sampling it will be necessary to install the groundwater monitoring wells discussed in Section 8.2. These wells should be constructed in accordance with the specifications contained in RCRA Ground-Water Monitoring Technical Enforcement Guidance Document (EPA/OSWER-9950.1). 


\subsubsection{Equipment and Supplies}

The drillers will provide all necessary drflling equipment (hollow core auger, split-barrel sampler, etc.). The following field sampling supplies will be required:

- nonionic detergent, Micro (International Products Corp.)

- delonized water

- $\{$ sopropyl alcohol

- volatile organtc analysis (VOA) bottles

- glass contalners, pre-cleaned, with Teflon-lined lids, one quart capacity

- bound logbook

- chain-of-custody seals

- sample labels

- chain-of-custody forms

- stainless steel trays

- alumtnum foll - heavy duty

- stainless steel spatulas

- boat

- motor (optiona1)

- electroshocker

- ice chest 


\subsubsection{Soll Sampling Procedure}

Collection of samples from this site will follow ASTM Method 0 1586-84 Penetration Test Split-Barrel Sampling of Soils. The drilling will be performed by private drilling contractors. In this method, a hollow core auger will be used to renove the soil above each segment to be sampled, and the split-barrel sampler will be driven into the soil through the center of the auger. This technique will obtain a sample that is undisturbed by the drilling operation. Using a split-barrel sampler of two-foot length, samples will be removed from each position in two-foot segments. Samples will be collected to refusal. At each two-foot increment, the split-barrel sampler will be removed from the drilling rig (to be performed by the drilling crew) and separated to expose the sample. The core samples will be composited as described in section 8.2.1.

Between samples, the equipment used for sample transfer will be cleaned with nonionic detergent and water and rinsed with defonized water and isopropyl alcohol. The split-barrel samplers will be detergent cleaned and rinsed with water by the drilling company.

After sampling of each coring is complete, drilled holes will be filled with a grout column (see Section 7.1.3 of K/HS-132) to prevent any contamination of the groundwater. From 10 percent of the core segments, duplicate samples will be submitted to the laboratory to fulfill duplicate requirements per Section 7.1 of K/HS-132. Sample containers will be labeled with the site identification, date, time, sample identification, and the sampler's name. Sampling date, site identification, time, sample identification, sampler's name, and coordinates of the sample will be recorded in the logbook. In addition to the required entries, any other pertinent information and/or observations will be recorded. The logbook used for these records will contain a map of the area and a copy of the sampling plan. The sample containers will be sealed and transported to the laboratory under chain-of-custody protocol as referenced in section 7.4 of $\mathrm{K} / \mathrm{HS}-132$. 


\subsubsection{Groundwater Sampling Procedures}

Sampling of the groundwater monitoring well system described in Section 8.2 will occur in accordance with the ORGDP Groundwater Protection Program and will follow all the associated protocols.

\subsubsection{Surface Water Sampling Procedures}

Sampling of surface water as described in Section 8.1 will require the expansion of the analysis parameters (to include all parameters listed in Table 2.1, K/HS-132) for the K-1007-B Pond NPDES discharge point. Expansion of the analysis parameters should comence with initial groundwater sampling and follow the already established NPDES sampling schedule.

\subsubsection{Fish Sampling Procedure}

Fish collected at the site will be placed on ice in a labeled ice chest and returned to the laboratory for processing. At the laboratory the fish will be tagged with a unique four digit tag wired to the lower jaw. Each fish will then be weighed, measured, and filleted with a stainless steel filet knife and the skin removed from the filet. A 1-2 g sample of the anterior dorsal portion of the axial muscle filet w11l be excised for the determination of mercury; the remainder of the fllet will be retained for PCB analysis. The remaining filet will either be utilized as a duplicate sample or archived. If archived, the filet of large fish (carp) wlll be ground three times in a hand meat grinder (1.e., Chop Rite Corp., Model 5) to homogenize the tissue and samples will be wrapped in heavy-duty aluminum foil prior to freezing.

\section{B.4 ANALYTICAL PROTOCOL}

Groundwater samples will be collected quarterly as required in the ORGOP Groundwater Protection Program. Surface water sample collection from the $K-1007-B$ Pond will follow established NPDES sampling schedules. Surface water sample collection from the SD-100 system will be collected 
as described in Section 8.2.3. All water samples will be analyzed for the parameters 1isted in Table 2.1 of K/HS-132. Fish samples will be collected as described in Section 8.3 .6 and analyzed for mercury and PCBs.

Soll samples will be collected and composited as discussed in section 8.2.1. Because of the variety of chenicals disposed of via the ALD, It will be necessary to analyze soll samples for radioactivity (gross alpha, beta, and gamma), the inorganic elements listed in Table 7.4 of K/HS-132 (includes all regulated metals and uranium), volatile and semi-volatile organics, 11sted in Table 7.6 of K-HS-132. EP toxicity extractions w111 only be performed on those samples (including archived samples from Initial sediment sampling) whose total regulated inorganic species content exceeds the limits contained in Table 2.2 of $\mathrm{K} / \mathrm{HS}-132$. An EP toxicity type extraction and subsequent radionuclide analysis will also be necessary for those samples whose total radioactivity exceeds maximum acceptable levels (based on best technical judgement).

\subsection{SAMPLE ANALYSIS}

Groundwater and surface water analyses will follow standard EPA protocol as outlined in Methods for Chemical Analys is of Water and Wastes (EPA-600/4-79-020). Soll analysis will follow standard EPA protocol as outlined in Test Methods for Evaluating Solid Waste: Physical/Chemical Methods (SW-846). Fish sample analysis will follow EPA procedure 600/4-81-055 for PC8s as outilined in Interim Methods for the Sampling and Analysis for Priority Pollutants in Sediments and Fish Tissue (EPA, 1980). and for mercury the analytical procedure will follow SW-846, EPA-7471. The QA/QC requirements outlined in Section 7.3 of K/HS-132 w111 be adhered to for all analyses. 


\section{DATA MANAGEMENT PROCEDURES}

The results of sample analyses will be presented in a clear and logical format to best lllustrate any patterns in the data. These will include tabular, graphic, and other visual displays such as maps and contour plots, as appropriate to the data.

Statistical analyses will provide for treatment of duplicate laboratory analyses and results which are reported as less than detection imit and for examination for statistical outliers. Where possible, values which are reported as less than detection 1 imits will be handled according to RCRA Ground-Water Monitoring Enforcement Guidance Document, OSWER-9950.1, September 1986, which directs calculation through the use of Cohen's statistical methodology. This is found in Tables for Maximum Likelihood Estimates from Single Truncated and Singly Censored Samples, Technometrics, Volume 3, pages 535-541, 1961. Otherwise, the detection limit will be used in the statistical analysis. 
10. HEALTH AND SAFETY PROCEDURES

\subsection{INTRODUCTION}

Special requirements and procedures to protect the health and safety of the investigating team, ORGOP site personnel, and the general public during this RFI are addressed in this section. K/HS-132 details the health, safety, environmental, security, plant protection, and emergency response organizations which are in place at ORGDP. These organizations provide the support to ORGDP Iine organizations to meet the requirements for health and safety during the RFIs. They provide the communications, response, and reporting for any plant emergency; on-site medical facilities with medical survelllance, treatment, monitoring, and periodic physical examinations; health physics and industrial hygiene surveillance hazard evaluation and control; operational safety accident prevention and control; plant security and visitor control.

In addition, K/HS-132 identifies the organizational responsibilities for health and safety at SWMUs during the RFIs. The document includes the methodology for establishing the work zones of each SWMU, the level of protection required in the exclusion zone, decontamination procedures, personnel exposure limits, monitoring requirements, and respiratory protection requirements.

\subsection{KNOWN HAZARDS AND RISKS}

Since a variety of chemicals and wastes were discharged to the $K-1007-B$ pond via the ALD the potential exists for contamination by all classes of hazardous compounds (metals, radioactivity [gross alpha, beta, and gamma], volatile and semi-volatile organics, and PCBS). 


\subsection{LEVEL OF PROTECTION}

The level of personnel protection is designated below for soil and surface water sampling. Monitoring parameters are indicated for soil sampling.

\section{Level of Designation}

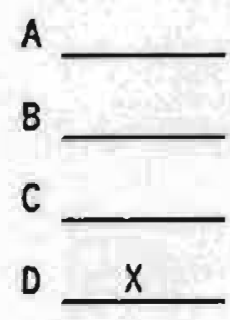

\section{Monitoring Parameters}

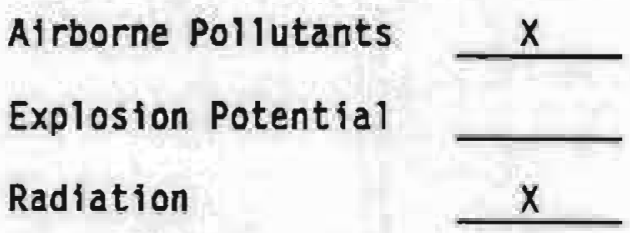

\subsection{DESIGNATION OF WORK AREA ZONES}

The three zones (Exclusion, Contamination Reduction, and Support) will be established for the work activity area in accordance with the methodology developed in Section 9 of K/HS-132. As work activity requires, the exclusion zone will move to encompass areas of drilling and sampling of soll or surface water.

\subsection{EXPOSURE LIMITS}

The personnel protection recomended for work activity in the exclusion zone of the ALD and the $X-1007-B$ Pond SWMU is Level $D$.

Employee exposure to airborne pollutants (e.g., organies) throughout the course of the investigation will be monitored through the use of air monitoring equipment. If pollutants or unusual odors are detected, work will be stopped and the area will be evacuated. The ORGDP Industrial Hygiene Department will be contacted to detemine the necessary actions to mitigate health and safety concerns. 
The responsiblitity for limiting the exposure of the workers to nonhazardous levels of radiation resides in the Site Health and Safety officer (SHSO) using instruments described in Section 9 of K/HS-132. The SHSO will monitor for radiation in the air and adjacent to sample drillings and/or diggings with a radiation meter capable of measuring 0.1 $\mathrm{mR} / \mathrm{hr}$. Should the reading exceed $0.1 \mathrm{mR} / \mathrm{hr}$, the SHSO will order work to be stopped and the crew removed from the exclusion zone. The SHSO will request the presence of a health physicist on site who will assess the potential hazaro of the conditions and determine whether or not work should continue.

Sampling personnel must be aware that equipment used for soil sampling could become contaminated with radloactive material. Personal safety shoes and other protective equipment could become contaminated. surveys should be performed on such equipment in the soil sampling areas before and after each operation. Each survey should include monitoring all applicable personnel and equipment. Equipment that is found to be contaminated above the guidelines for unrestricted release (alpha-5,000 $\mathrm{dpm} / 100 \mathrm{~cm}^{2}$ surface, $1,000 \mathrm{dpm} / 100 \mathrm{~cm}^{2}$ transferrable, and $0.1 \mathrm{mR} / \mathrm{hr}$ beta and gamma) w11l be decontaminated. Should the reading exceed an action level of $2 \mathrm{mR} / \mathrm{hr}$ (set by ORGDP Health Physics as an action point), the SHSO will order work to be stopped and the crew will be removed. The SHSO will request the presence of a health physicist on site who will assess the potential hazard of the conditions and determine whether or not work should continue. 


\section{REFERENCES}

American Society for Testing and Materials, ASTM Method D 1586-84, Penetration Test Spl1t-Barrel Sampling of Solls

Cohen, "Tables for Maximum Likelihood Estimates from Single Censored Samples," Technometrícs, Vol. 3: 535-541, (1961)

EPA (Environmental Protection Agency), Methods for Chemical Analysis of Hater and Hastes, EPA-600/4-79-020

EPA (Environmental Protection Agency), December 1987, RCRA Facility Investigation Guidance, EPA 530/SW-87-001, Vols. I-IV, OSWER Directive $9502.00 .6 \mathrm{C}$

EPA (Environmental Protection Agency), September 1986, RCRA Ground Water Monitoring Enforcement Guidance Document, OSWER Directive 9950.1

EPA, Test Methods for Evaluating Solid Waste: Physical/Chemical Methods, $(S W-846)$.

EPA, Interim Methods for the Sampling and Analysis for Priority Pollutants in Sediments and Fish Tissue, 1980

EPA, RCRA Grondwater Monitoring Technical Enforcement Guidance Document (EPA/OSWER-9950.1)

Geraghty and Mlller, Hydrogeology of the Oak Ridge Gaseous Diffusion Plant Site, July 1986

Geraghty and Miller, Phase II-Detection Monitoring, June 1987

Ketelle, R. H., Oak Ridge National Laboratory, Recent Unpublished Geological Work

Martin Marletta Energy Systems. Inc., The Environmental Survelllance Procedures Quality Control Program, ESH/SUB/87-21706(1)

McMaster, W. H., "Geologic Map of the Oak Ridge Area, Tennessee," U.S. Geological Survey, 1958

Oak Ridge Gaseous Diffusion Plant. RCRA Facllity Investigation Plan. General Document, Oak Ridge, Tennessee, K/HS-132, March 1987

Oax Ridge Gaseous Diffusion Plant, ORGDP Remedial Actions Program Quality Assurance Plan, K/HS-231

Oak Ridge Gaseous Diffusion Plant, ORGDP Remedial Actions Program Data Management Plan, K/HS-232 


\section{APFENDIX A}




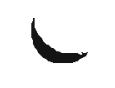

$\checkmark$ 
K-1007-B POND SEDIMENT DATA SEMARY

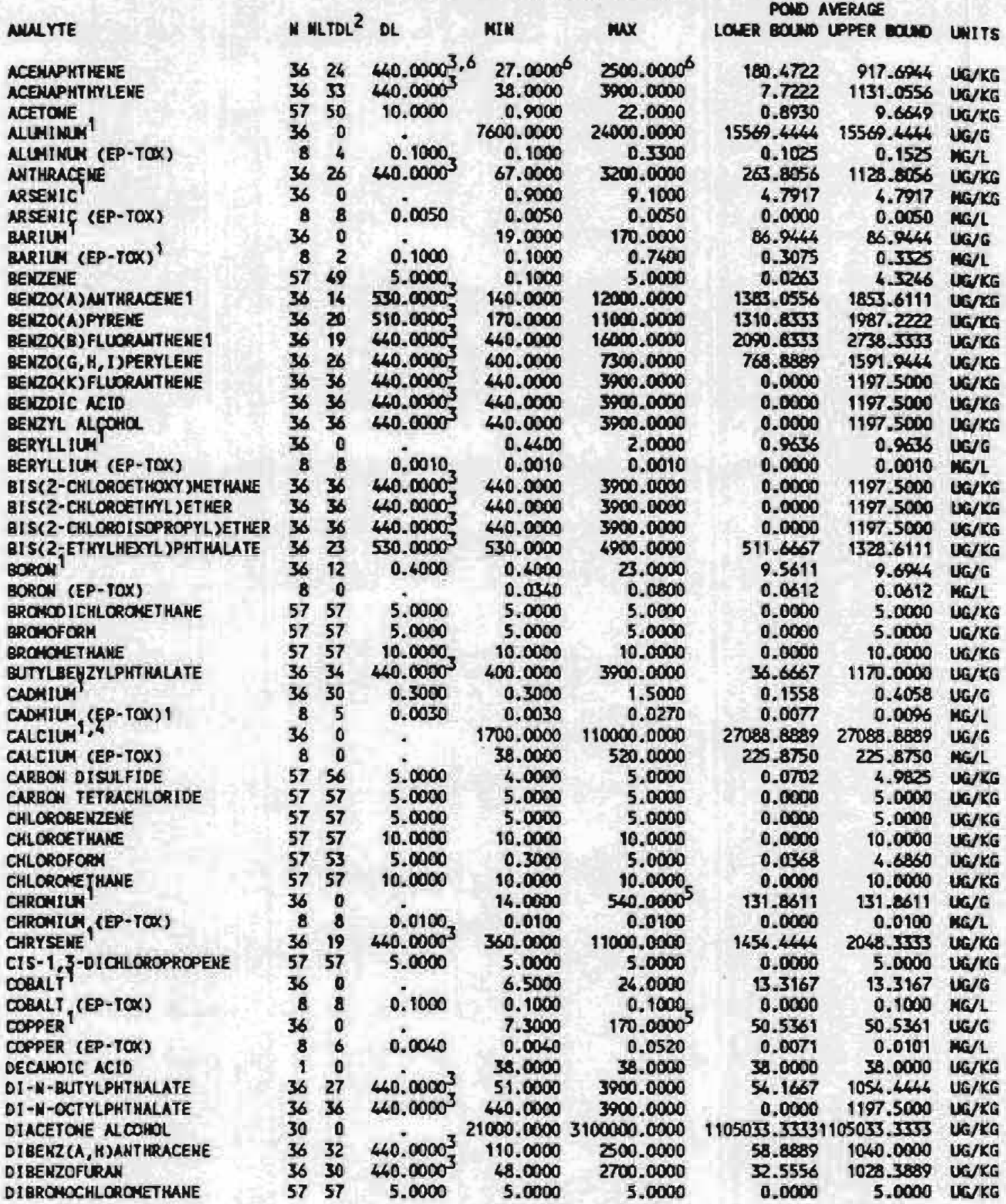


K-1007 POND DATA SUMURY (COITIUED)

\begin{tabular}{|c|c|c|c|c|c|c|c|c|}
\hline AHATTE & W & LTDL & DL & MIX & $\mathbf{m} \mathbf{x}$ & LOUER BOWO & LPPER GANO & WWITS \\
\hline DIETKYLPHTHALATE & 36 & 32 & $450.00000_{3}^{3}$ & 30.0000 & 3000.0000 & 20.2778 & 1009.4446 & VE/LEO \\
\hline DIMETKYLPHTHALATE & 36 & 36 & $460.0000^{3}$ & 460.0000 & 3900.0000 & 0.0000 & 1997.5000 & Lateg \\
\hline DOOECANOTE ACID & 2 & 0 & & 72.0000 & 110.0000 & 91.0000 & 91.0000 & varks \\
\hline ERDRIN & & 8 & 0.1000 & 0.1000 & 0.1000 & 0.0000 & 0.1000 & LE/L \\
\hline ETHYLBERZENE, & 57 & 57 & $5.0000_{3}$ & 5.0000 & 5.0000 & 0.0000 & 5.0000 & LTAKG \\
\hline FLLORANTHEKE & 36 & 10 & $50.0000^{3}$ & 200.0000 & 18000.0000 & 2262.2222 & 2613.0556 & le/kg \\
\hline FLUORENE, & $\begin{array}{l}36 \\
36\end{array}$ & 30 & $\begin{array}{l}460.0000^{3} \\
2.0000\end{array}$ & $\begin{array}{r}130.0000 \\
2.0000\end{array}$ & $\begin{array}{r}2700.0000 \\
30.0000\end{array}$ & $\begin{array}{r}103.6111 \\
5.5000\end{array}$ & 1054.6464 & La/ke \\
\hline HEPTANOIC ACID & 2 & 0 & & 95.0000 & 120.0000 & 107.5000 & 107.5000 & Danos \\
\hline HEXUCHLOROBENRENE & 36 & 36 & $460.0000_{3}^{3}$ & 40.0000 & 3000.0000 & 0.0000 & 1197.5000 & Ua/tso \\
\hline HEXACHLOROSUTADIENE & 36 & 36 & $40.0000^{3}$ & 440.0000 & 3000.0000 & 0.0000 & 1197.5000 & varixo \\
\hline HEXICHLOCOCYCLOPENTADIENE & 36 & 36 & $440.0000_{3}^{3}$ & 460.0000 & 3000.0000 & 0.0000 & 1197.5000 & verices \\
\hline MEXICHLORCET WLLE & 36 & 36 & $440.0000^{3}$ & 460.0000 & 3900.0000 & 0.0000 & 1197.5000 & varices \\
\hline HEXANOIC ACIO & 2 & 0 & 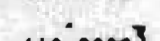 & 58.0000 & 110.0000 & 84.0200 & 84.0000 & unalces \\
\hline $\begin{array}{l}\text { IMDEYP }(1,2,3-C D) \text { PYRENE } \\
\text { IRON }\end{array}$ & $\begin{array}{l}36 \\
36\end{array}$ & $\begin{array}{r}24 \\
0\end{array}$ & $460.0000^{3}$ & $\begin{array}{r}390.0000 \\
15000.0000\end{array}$ & $\begin{array}{r}6900,00000 \\
44000.0000\end{array}$ & $\begin{array}{r}699.7222 \\
2647.2222\end{array}$ & $\begin{array}{r}1464.6444 \\
26472.2722\end{array}$ & Ua/ke \\
\hline IRON (EP-TOX) 1 & 8 & 4 & $0.0500_{3}$ & 0.0500 & 0.1800 & 0.0639 & 0.0899 & marl \\
\hline ISOPHORONE & 36 & 36 & $440.0000^{3}$ & 460.0000 & 3900.0000 & 0.0000 & 1197.5000 & varies \\
\hline $\begin{array}{l}\text { LEN' } \\
\text { LEAD (EP-YOX })^{1}\end{array}$ & $\begin{array}{r}36 \\
8\end{array}$ & $\begin{array}{l}0 \\
5\end{array}$ & 0.0500 & $\begin{array}{l}5.5000 \\
0.0500\end{array}$ & $\begin{array}{c}220.0000^{3} \\
0.0580\end{array}$ & $\begin{array}{r}50.2261 \\
0.0210\end{array}$ & $\begin{array}{r}50.2361 \\
0.0522\end{array}$ & $\begin{array}{l}\text { uavo } \\
\text { merL }\end{array}$ \\
\hline LINOANE, & 8 & 7 & 0.0500 & 0.0500 & 0.0800 & 0.0100 & 0.0537 & UarL \\
\hline LITHILA & 36 & 0 & $\cdot$ & 8.4000 & 53.0000 & 18.4233 & 18.4833 & UE/G \\
\hline LITHIUA (EP-TOX) ${ }^{1}$ & 8 & 2 & 0.0040 & 0.0040 & 0.0071 & 0.0042 & 0.0052 & $M G / L$ \\
\hline MACIESIUA & 36 & 0 & . & 1000.0000 & 13000.00000 & 5497.2222 & 5497.2222 & LeVG \\
\hline MANESIUA, (EP-TOX) & 8 & 0 & . & 7.4000 & 92.0000 & 41.5375 & 41.5375 & HerL \\
\hline MAKSNNESE & 36 & 0 & & 340.0000 & 1300.0000 & 687.2222 & 687.22222 & $U G / G$ \\
\hline MANGANESE (EP-TOX) ${ }^{\prime}$ & 8 & 2 & 0.0100 & 0.0100 & $19.00000_{5}$ & 6.1000 & 6.1025 & $M G / L$ \\
\hline MERCURY' & 36 & 21 & 1.0000 & 1.00 & $000^{5}$ & 1.2500 & 1.8333 & UG/G \\
\hline MERCURY (EP-TOK) ${ }^{1}$ & $\mathbf{B}$ & 3 & 0.0002 & 0.0002 & 0.0006 & 0.0003 & 0.0003 & man \\
\hline METHOCYCHLOR & B & 8 & 0.5000 & 0.5000 & 0.5000 & 0.0000 & 000 & Uarl \\
\hline RETHYLENE CHLORIOE & 57 & 55 & 5,0000 & 0.2000 & 5.0000 & 0.0070 & 4.8316 & va/KG \\
\hline HOLYBDENLA' & 36 & 12 & 1.0000 & 1.0000 & 2.7000 & 1.2250 & 1.5583 & UEVG \\
\hline MOLYBOENUH (EP-TOX) & 8 & 8 & 0.0100 & 0.0100 & 0.0100 & 0.0000 & 0.0100 & Marl \\
\hline MYRISTIC ACID & 6 & 0 & & 200.0000 & 3600.0000 & 1416. & 1416.6667 & verke \\
\hline W-KI TROSO-DI - N-PROPYLN & 36 & 35 & $460.00000^{3}$ & 440.0000 & 3900.0000 & 55.5556 & $1225.27 \mathrm{rB}$ & UE/KG \\
\hline N-MI TROSCO IPIENTLAKI ME & 36 & 36 & $440.0000_{2}^{3}$ & 440.0000 & $3900.00000_{2}$ & 0,0000 & 1197.5000 & UG/KE \\
\hline MUPИTHQLENE' & 36 & 33 & $440.0000^{3}$ & 45.0000 & $3900.0000^{5}$ & 9.1944 & 1146.6944 & Le/Ke \\
\hline NICKEL' & 36 & 0 & & 12.0000 & 260.0 & 7.5556 & 7.5556 & UEV \\
\hline NIOXEL (EP-TOK) $)^{1}$ & 8 & 6 & 0 & 0.0500 & $\cos$ & 0.4307 & 0.4557 & $\mathrm{ma} / \mathrm{L}$ \\
\hline HIOBIUN & 36 & 32 & 0.7000 & 0.7000 & 2.0000 & 0.1547 & 0.7769 & Levg \\
\hline MIOBIUA (EP-TEX) & 8 & 8 & $0.0070_{3}$ & 0.0070 & 0.0070 & 0.0000 & 0.0070 & ME/L \\
\hline MI TROBENZZEE & 36 & 36 & $460.00000^{5}$ & 460.0000 & 3900.0000 & & 1197.5 & LG/KG \\
\hline OCtaMoIC ACID & 5 & 0 & . & 76.0000 & 150.0000 & 119.2000 & 119.2000 & Ua/KG \\
\hline PALMITIC ACID & 19 & 0 & - & 1800.0000 & 12000 & 3621 & 4636 & USJKC \\
\hline$P C B$ & 9 & 0 & & 1000.00 & 33000.0 & 10188.8889 & 10188 & UE/KG \\
\hline PCB (AROCLOR-1016) & 36 & 36 & $0.5000^{3}$ & 0.5000 & 80,0000 & 0.0000 & $\pi .7917$ & UG/KG \\
\hline PCH (AROCLOR-1221) & 36 & 36 & 0.500 & 0.5000 & 80.0000 & 0.0000 & $\pi .7917$ & UE/KG \\
\hline PCA (AROCLOR-1232) & 36 & 36 & 0.500 & 0.50 & 80.00000 & 0.0000 & 7.7917 & Ve/Ke \\
\hline PEB (AROCL CR-1242) & 36 & 36 & $0.500 x$ & 0.50 & 80.0000 & 0.0000 & $\pi .7917$ & UETKS \\
\hline PCB (AROCLOR-1248), & 36 & 31 & & 0.5000 & 12000.0000 & 963.0556 & 1029.7361 & Le/ka \\
\hline PCB $($ AROCLCR-1254) & 36 & 11 & & 1.0000 & 16900.0000 & 1946.1667 & 1988.6389 & LuKkg \\
\hline PCB (AROCLOR-1260) & 36 & 36 & 1.000 & 1.0000 & 270.0000 & 13.0556 & 159.7500 & UGVKG \\
\hline PENTACHLOROPHENOL & 36 & 35 & $2100.0000^{3}$ & 2100.0000 & 19000.0000 & 97.2222 & $5 \pi 7 . \pi 7 B$ & UG/KG \\
\hline
\end{tabular}

PAEE 2 


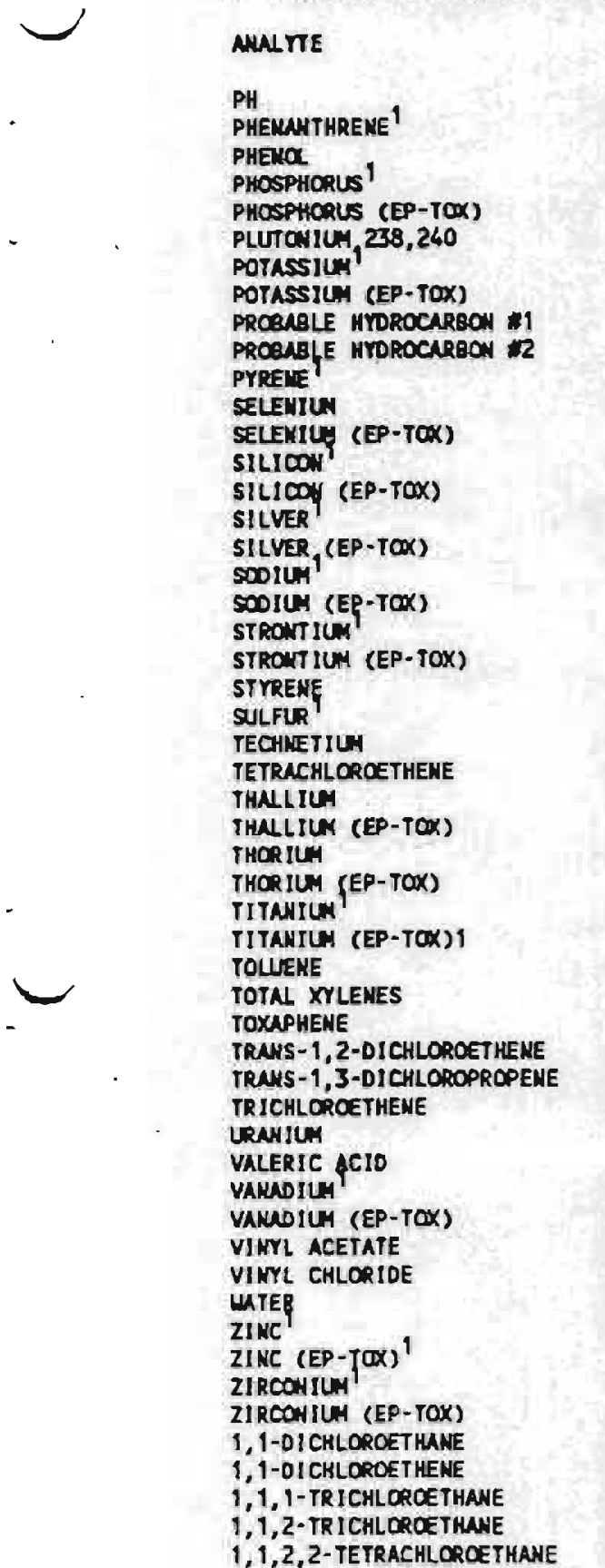

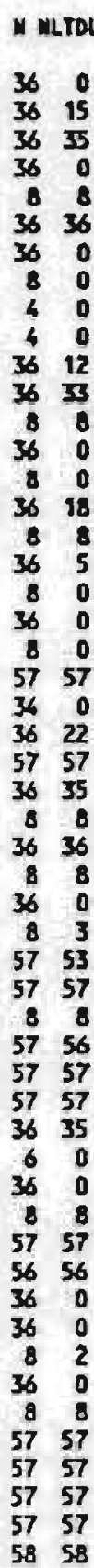

MIN $m$

POD AVERACE

PACE 3

9.0000

6.2000

$530.0000^{3}$ $440.0000^{3}$

0.2000

1.0000

-

-

$550.0000^{3}$

0.5000

0.0050

-

0.6000

0.0100

2.0000

.

$\therefore$

5.0000

28.0000

5.0000

1.0000

0.0100

20.0000

0.2000

0.0030

5.0000

5.0000

1.0000

5. 0000

5. 0000

5.0000

3.0000

.

0.5000

10. 0000

10.0000

.

0.0200

0.0050

0.0050

5.0000

5.0000

5.0000

5.0000
92.0000

440.0000

170.0000

0.2000

1.0000

850.0000

1.0000

5.0000

6.0000

350.0000

0.5000

0.0050

290.0000

1.7000

0.6000

0.0100

2.0000

4. 7000

4. 7000

0.0760

5.0000

260.0000

28.0000

5.0000

1.0000

0.0100

20.0000

0.2000

45.0000

0.0030

0.3000

5.0000

1.0000

0.2000

5.0000

5.0000

3.0000

150.0000

12. 0000

0.5000

10.0000

10.0000

8.4000

3.7000

0.0200

4.8000

0.0050

5.0000

5.0000

5.0000

5.0000

5.0000
13000.0000

5900.0000

1600.0000

0.2000

1.0000

3500.0000

4.5000

9.0000

8.0000

25000.0000

0.5000

0.0060

700.0000

3.7000

41.0000

0.0100

230.0000

5.8000

100.0000

0.3700

5.0000

820000.0000

304,0000

5.0000

1.0000

0.0100

20.0000

0.2000

250.0000

0.0220

5.0000

5.0000

1.0000

5.0000

5.0000

5.0000

25.0000

330000.0000

420000

0.5000

10.0000

10.0000

76.0000

$540.00000^{5}$

2.1000

20.0000

0.0050

5.0000

5.0000

5.0000

5.0000

5.0000
LOWER BOWD UPPER BOWD UWITS
8.0000

1367.0833

163.8889

666.3889

0.0000

0.0000

1893.0556

3.4125

7.0000

7.0000

3354.6444

0.0417

0.0000

555.83133

2.8750

6. 0.0:83

0.0000

107.2500

5.3125

21.5528

0.2130

0.0000

109975.2941

41.3889

0.0000

0.0278

0.0000

0.0000

0.0000

$112.1 \% 4$

0.0066

0.0526

0.0000

0.0000

0.0035

0.0000

0.0000

0.6944

55351.6667

24.7500

0.0000

0.0000

0.0000

36.8556

17.2972

0.6740

10.4028

0.0000

0.0000

0.0000

0.0000

0.0000

0.0000
8.0000

1969.8611 UETKC

1353.6111 VETKR

666.3889 VEVG

$0.2000 \mathrm{HEN}$

$1.0000 \mathrm{PCI} / \mathrm{C}$

1893.0556 VETG

$3.4125 \mathrm{ma} / \mathrm{L}$

7.0000 Verke

7.0000 UTKK

3781.3885 UTERTS

0.5000 nenes

0.0050 MEAL

555.8535 UF/G

$2.8750 \mathrm{MO} / \mathrm{L}$

6.3083 UEJ

$0.0100 \mathrm{MCN}$

$107.5270 \mathrm{UG} / \mathrm{G}$

$5.3125 \mathrm{mall}$

21.5528 La/o

$0.2130 \mathrm{kG} / \mathrm{L}$

5.0000 LE/KE

00875.2941 UE/KE

$58.5000 \mathrm{PCI} / \mathrm{G}$

5.0000 UEAKG

$1.0000 \mathrm{no} / \mathrm{Ko}$

$0.0100 \mathrm{mall}$

20.0000 UTV

$0.2000 \mathrm{HG} / \mathrm{L}$

112.1944 UEJG

0.007 redL

4.7018 verks

5.0000 ve/Ko

1.0000 vell

4.9158 La/Ka

$5.0000 \mathrm{HokKO}$

5.0000 varko

3.6111 UEJG

55351.6667 varks

24.7500 UE/G

$0.5000 \mathrm{kall}$

10.0000 ve/ros

10.0000 va/20

$36.8556 x$

172.2972 UEAG

$0.6790 \mathrm{HER}$

10.4028 UE/G

$0.0050 \mathrm{MG} / \mathrm{L}$

5.0000 UE/KC.

5.0000 UG/KG

5.0000 va/KG

$5.0000 \mathrm{LG} / \mathrm{KG}$
5.0000 LEE/KS 
K-1007 POAD DATA SUFURY (CONT INUED)

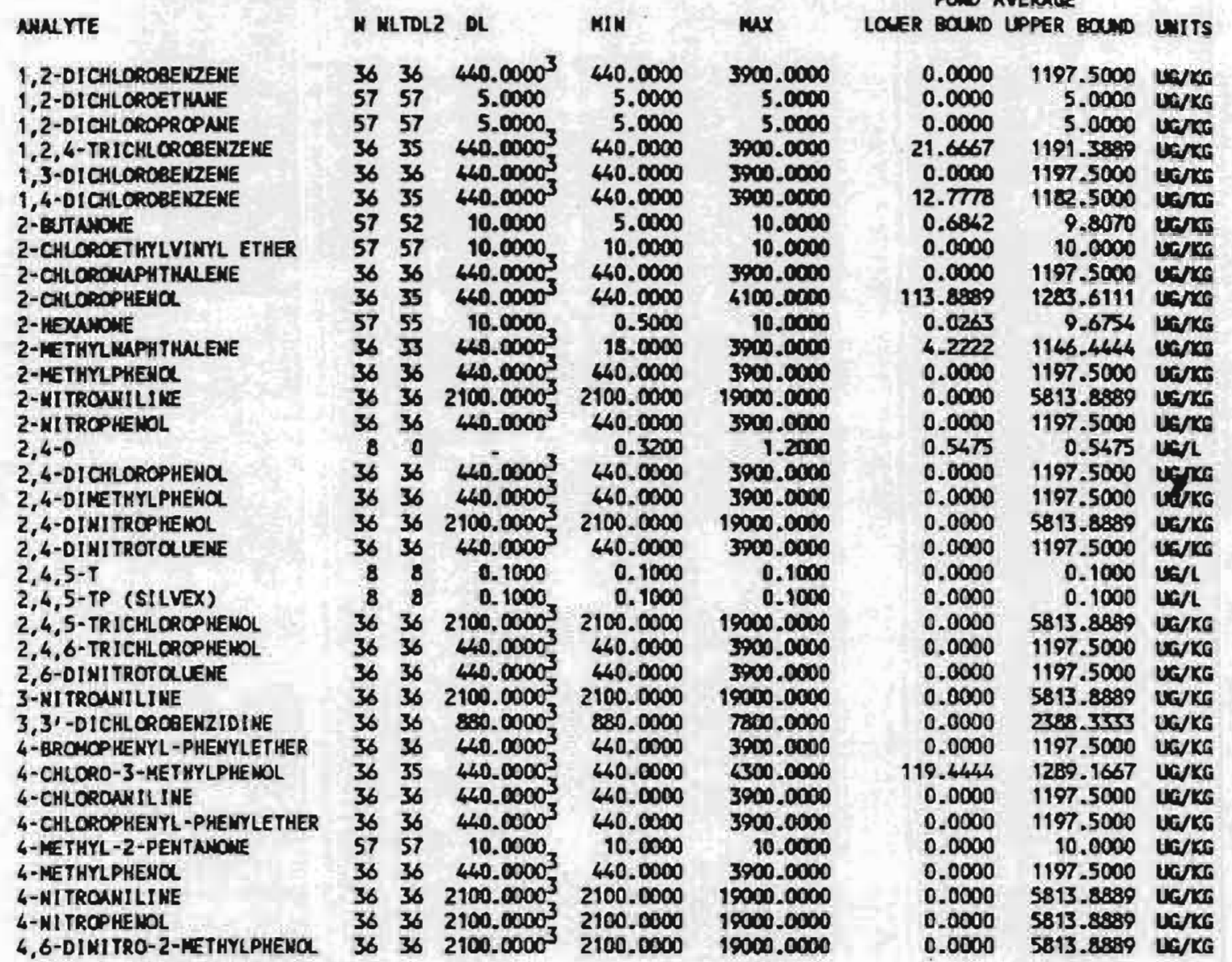
POND AVERICE PACE 4
BOWD LPPER GOWD UITS

1 PLOT OF IDOIVIDAML RESULTS AVAILABLE

2 NLTOL = MURBER OF RESULTS LESS THNY DETECTION LIMIT

3 SHALLEST OF MULTPLLE DETECTION LIMITS.

4 ONE RESLRT IS REPCRTED NS GREATER THN DETECTION LIHIT

5 RESLIT IS GREATER THAN KYHS-132 TABLE 2.2 WUX LIMIT FLR SOIL

6 THE MUMBER OF SIGHIFICAMT FIQURES IS SOFYHARE DEPENDENT AND IS WOI NECESSARILY INDICATIVE OF THE AMALYTICAL PRECISION. 
APPENDIX B 
$a$

7

7 


\section{K-1007 POND - FLUORANTHENE UG/KG}

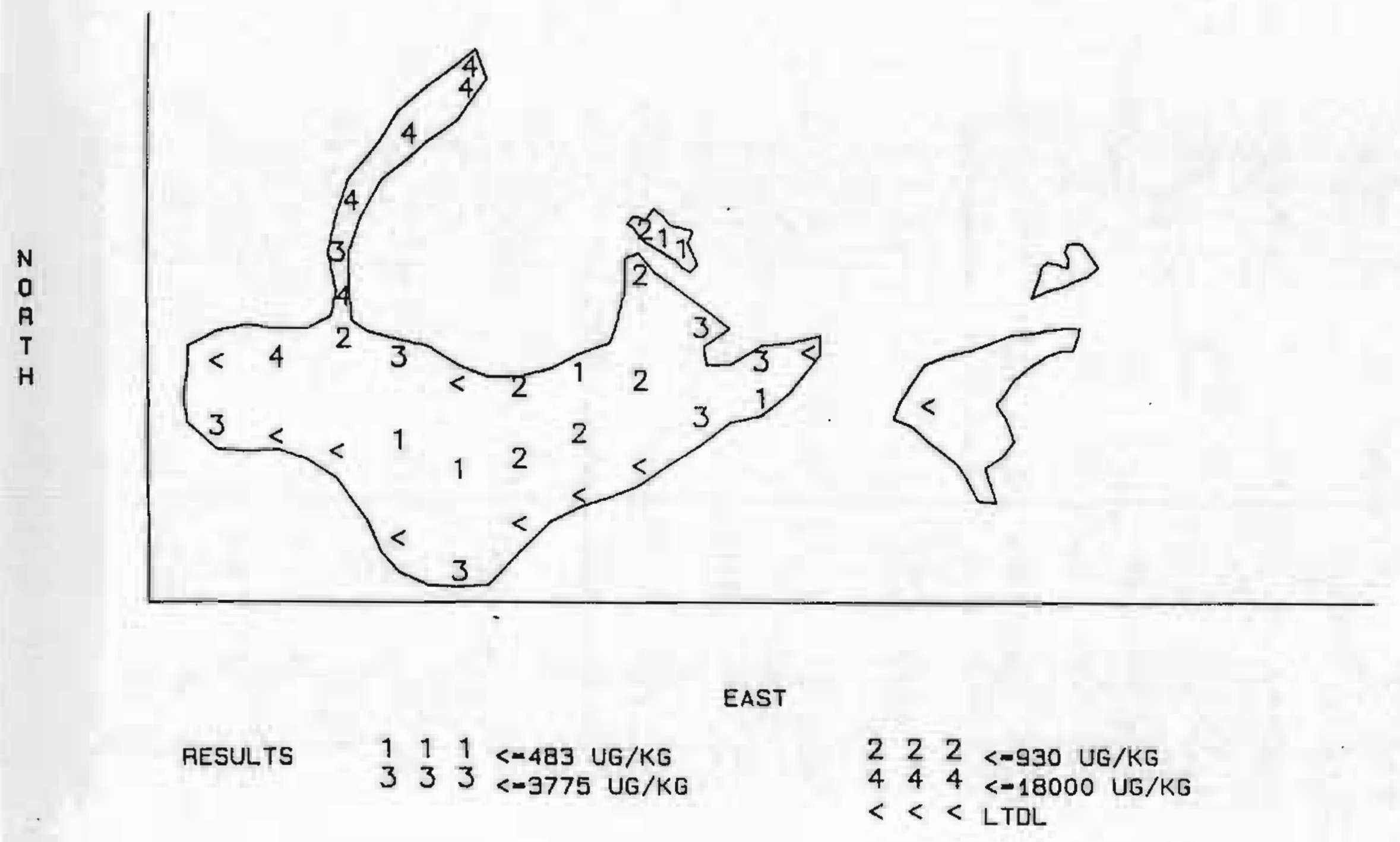

NO K/HS-132 TAELE 2.2 MAX LINIT FOR SOIL

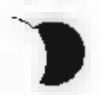




$$
\text { K-1007 POND - CHRYSENE UG/KG }
$$
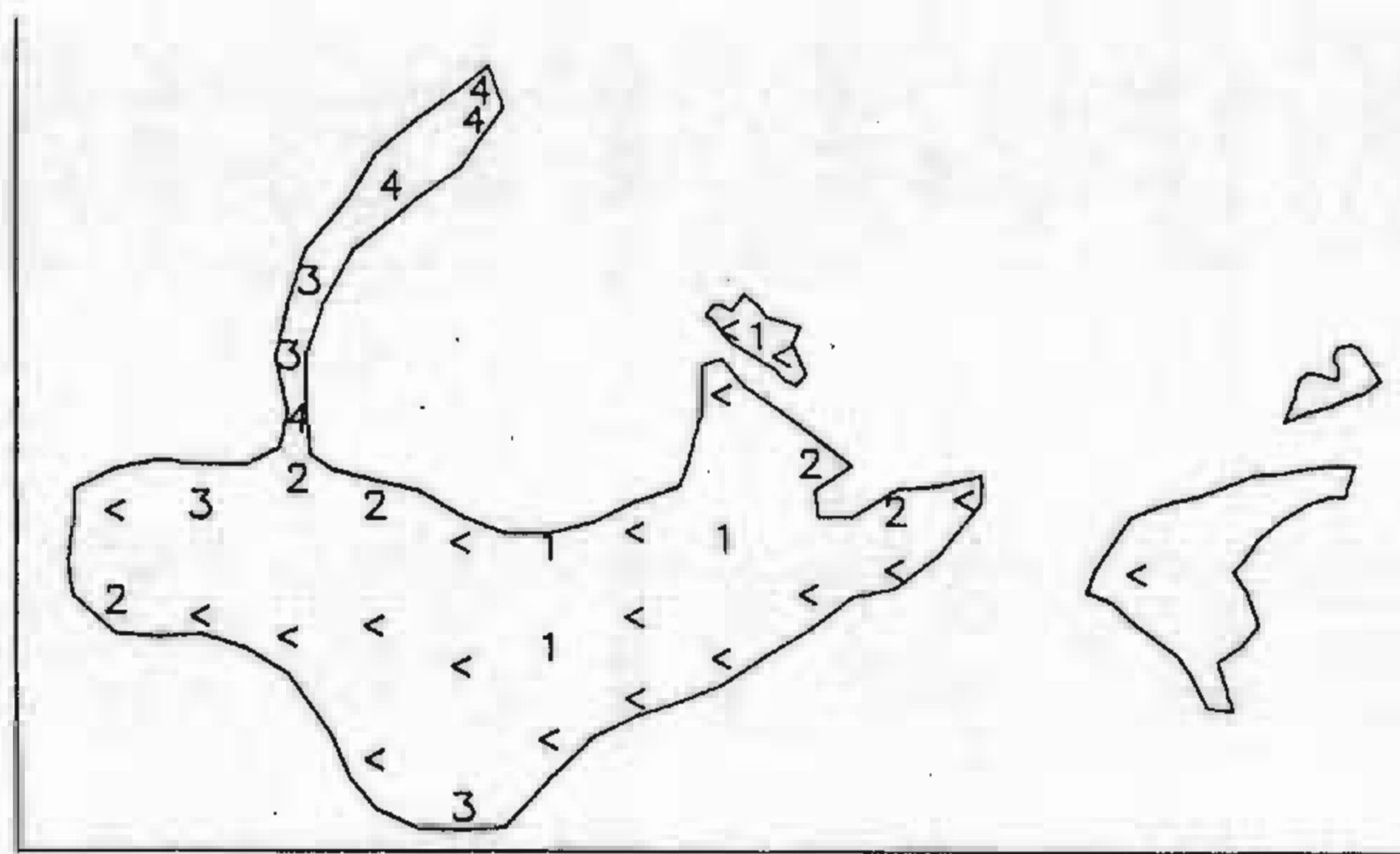

$$
\begin{aligned}
& \text { EAST } \\
& \begin{array}{lllll}
\text { RESULTS } & 1 & 1 & 1 & <-640 \mathrm{UG} / \mathrm{KG} \\
& 3 & 3 & 3 & <-3850 \mathrm{UG} / \mathrm{KG}
\end{array} \\
& \begin{array}{llll}
2 & 2 & 2 & <=1400 \text { Ur } / K G \\
4 & 4 & 4 & <=11000 \text { Ur } / K G \\
< & < & <\text { sTOL }
\end{array}
\end{aligned}
$$

NO K/HS-I32 TABLE 2.2 MAX LIMIT FOR SOIL 
K-1007 POND - BENZO(日)FLUORANTHENE UG/KG

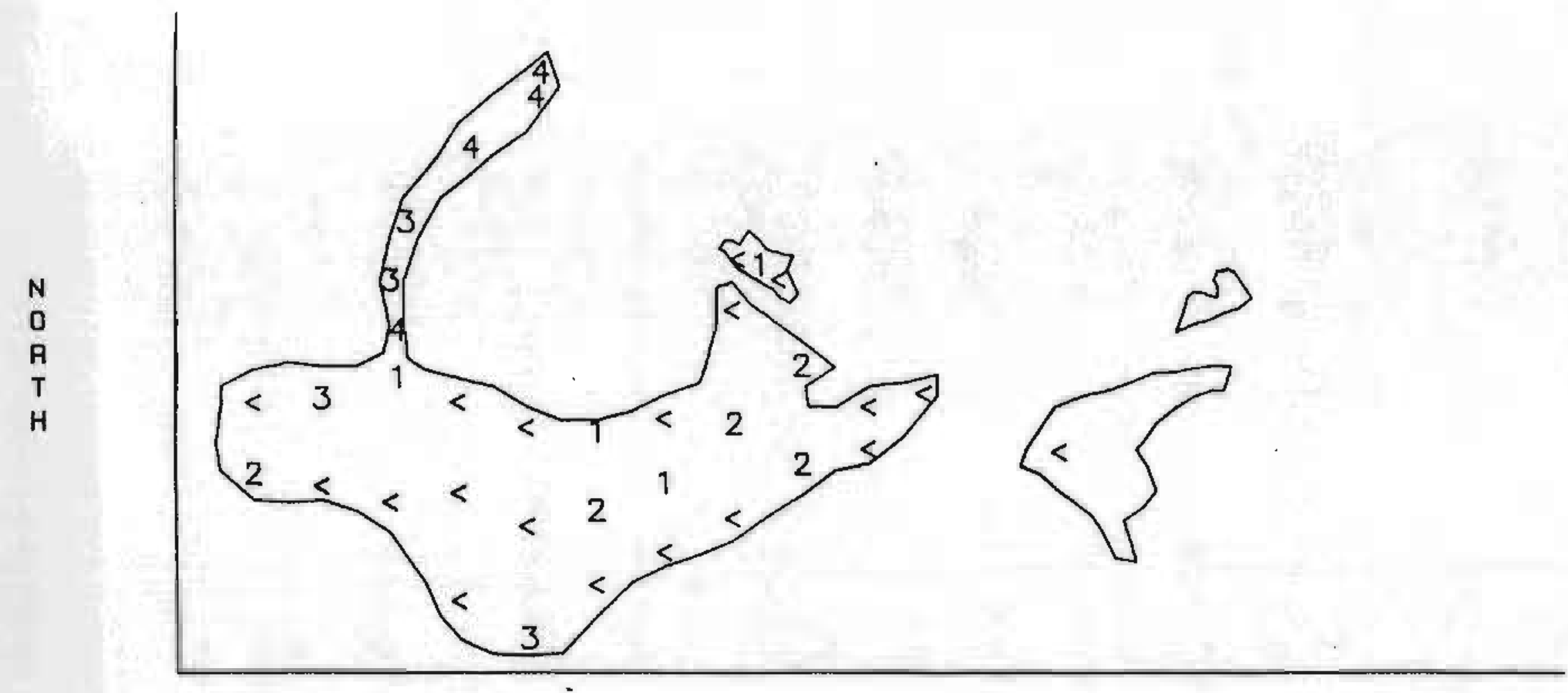

EAST

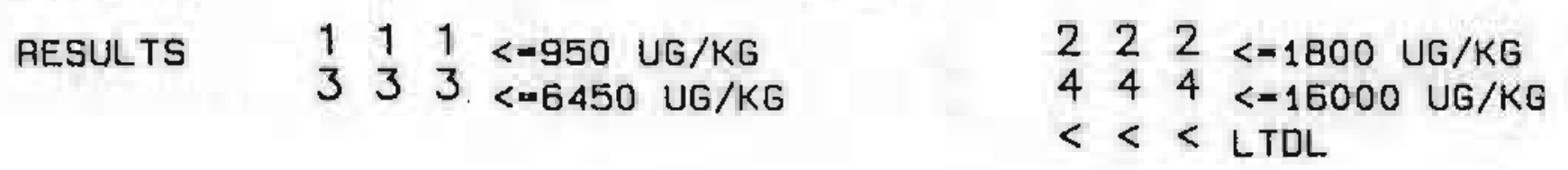

NO K/HS-132 TABLE 2.2 NAX LIMIT FOR SOIL

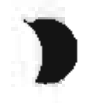


K-1007 POND - BENZO(A)ANTHRACENE UG/KG
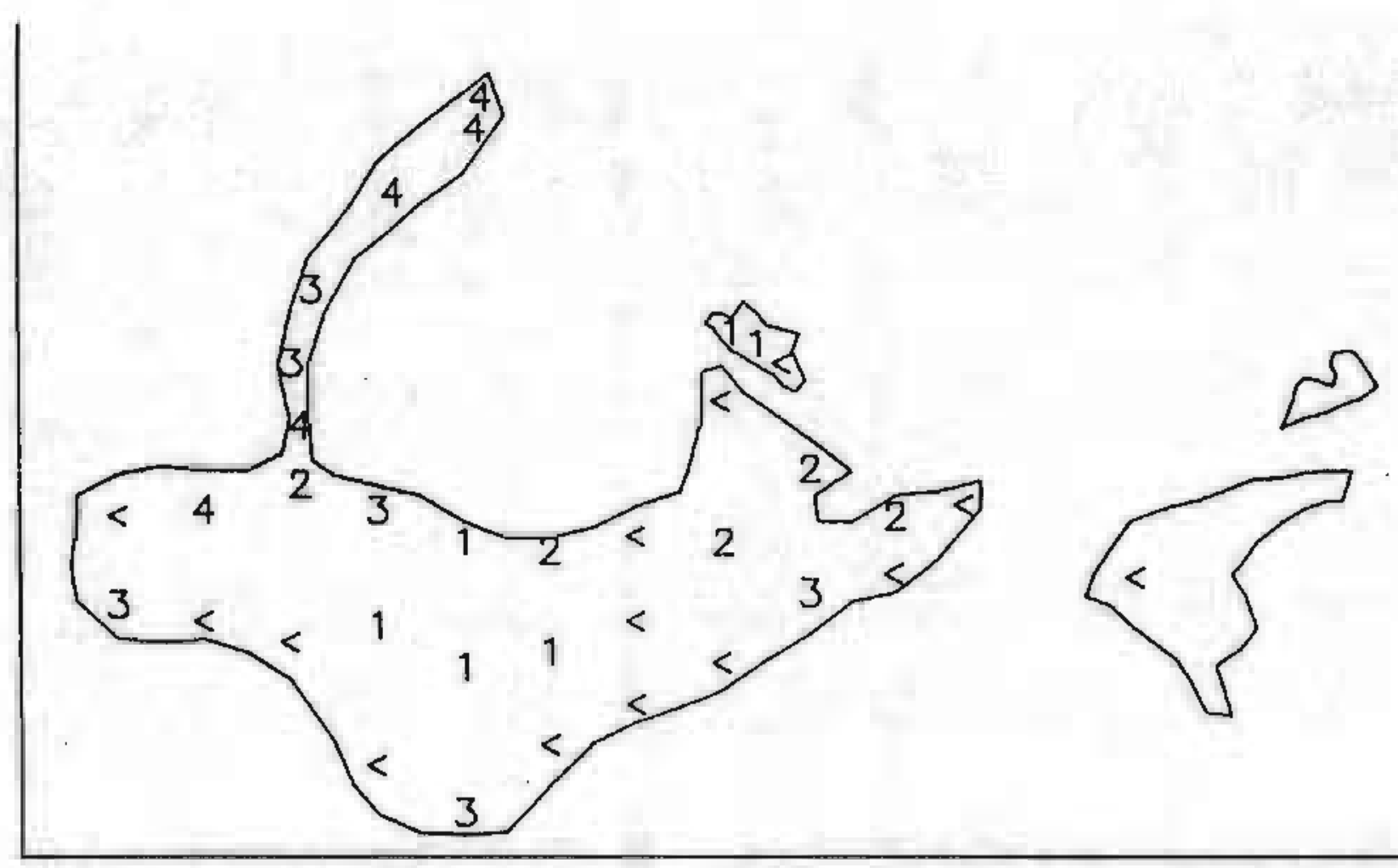

EAST

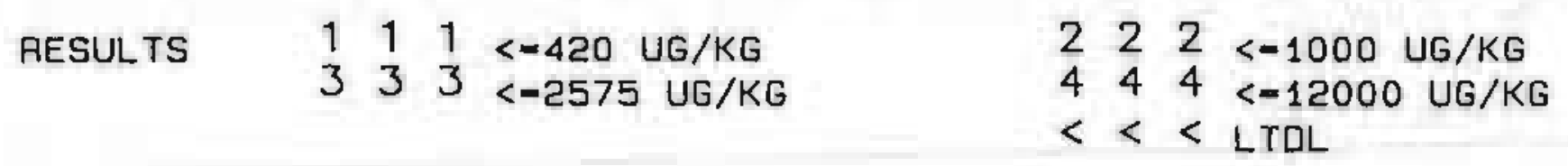

NO K/HS-132 TABLE 2.2 MAX LIMIT FOR SOIL 


$$
\text { K-1007 POND - ZINC }(\dot{E} P-T O X)-E X T R A C T M G / L
$$

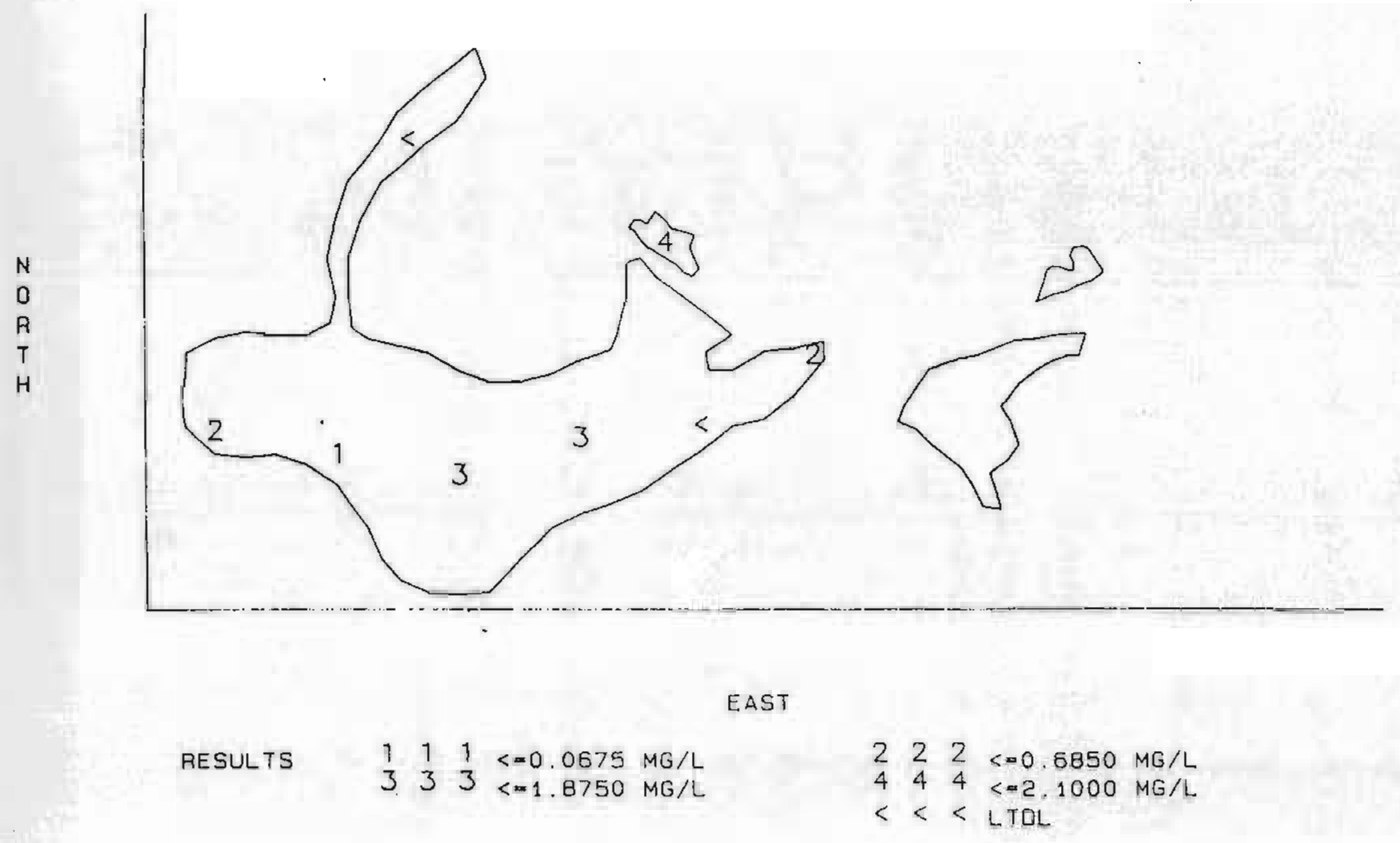

NO ESTABL ISHED EP-TOX LEVEL 


$$
\text { K-1007 POND - ZINC UG/G }
$$

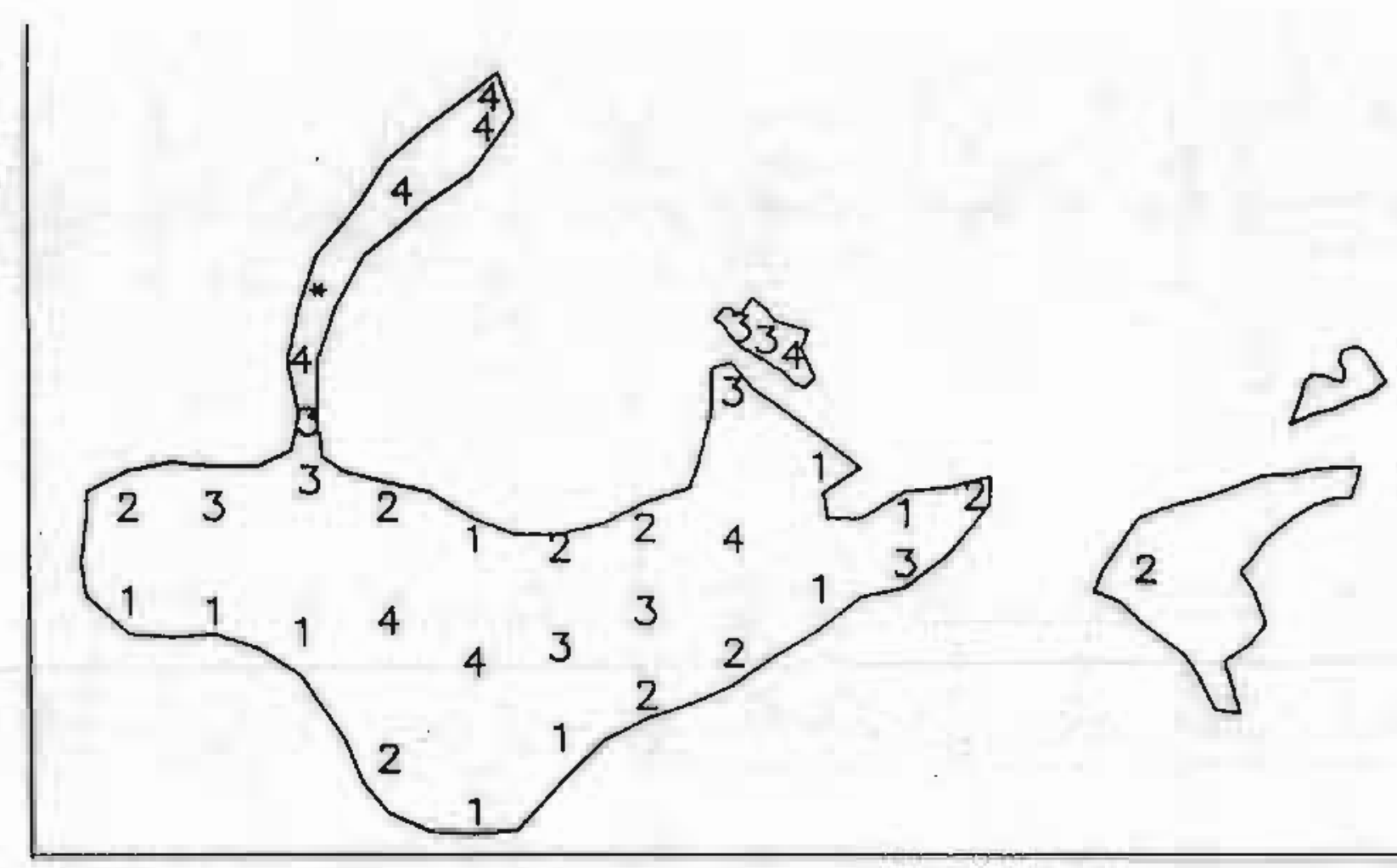

EAST

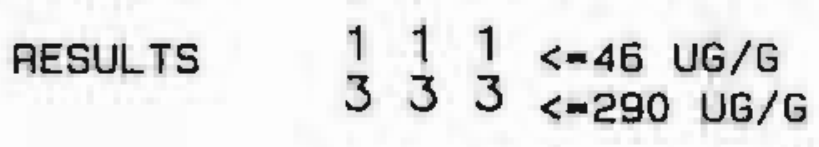

$$
\begin{array}{llll}
2 & 2 & 2 & <=66 \text { UT } / G \\
4 & 4 & 4 & <=460 \text { UT } / G \\
* & * & * & >500 \text { UT } / G \text { LIMIT }
\end{array}
$$

K/HS-132 TABLE 2.2 MAX LIMIT FOR SOIL-50O UG/G 
K-1007 POND - SULFUR UG/KG

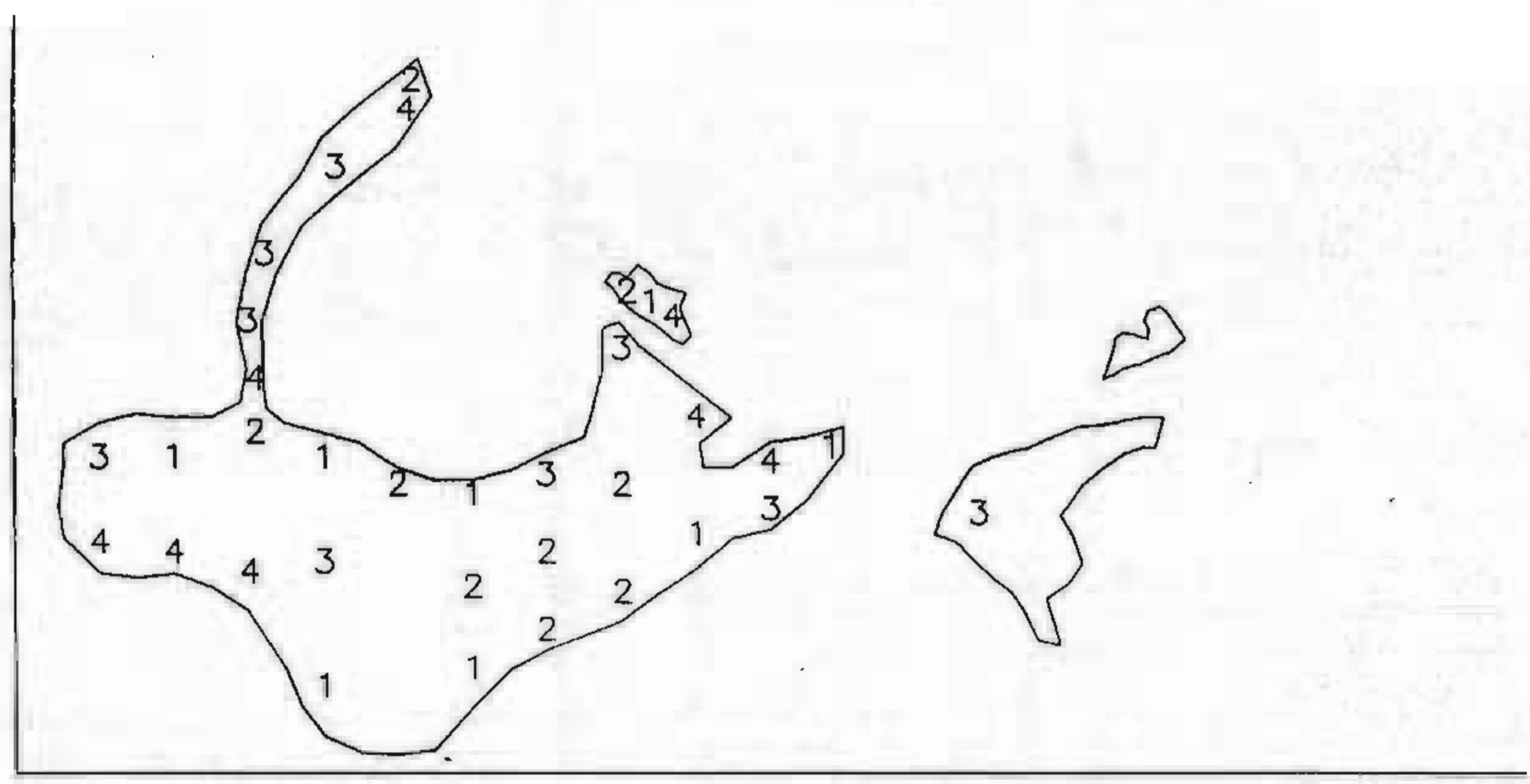

EAST

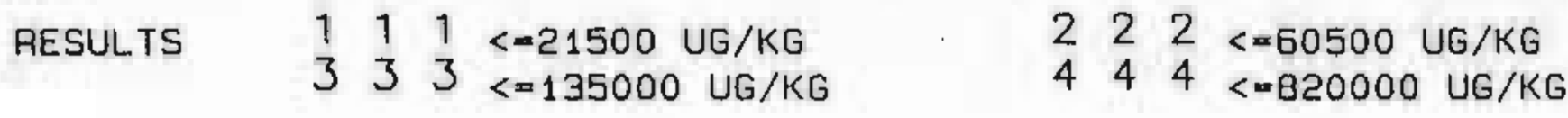

K/HS-132 TABLE 2.2 MAX LIMIT FOR SOILEN.A. 


$$
\text { K-1007 POND - STRONTIUM UG/G }
$$

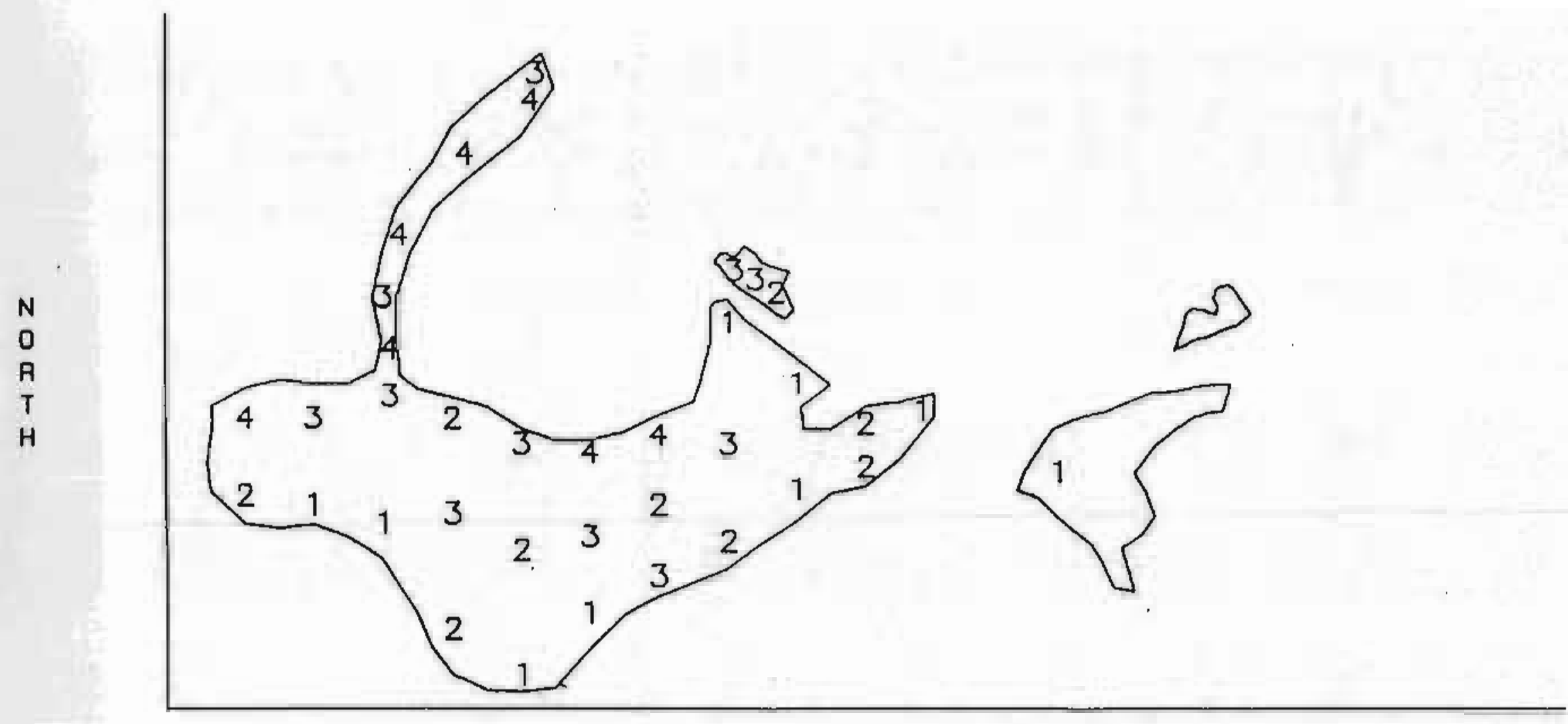

EAST

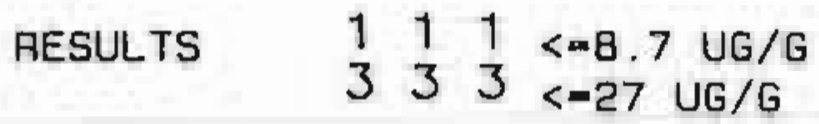

$$
\begin{aligned}
& \begin{array}{llll}
2 & 2 & 2 & <15.5 \text { UG } / G \\
4 & 4 & 4 & <=100 \text { UG } / G
\end{array}
\end{aligned}
$$




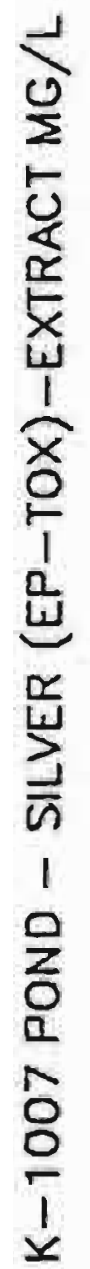

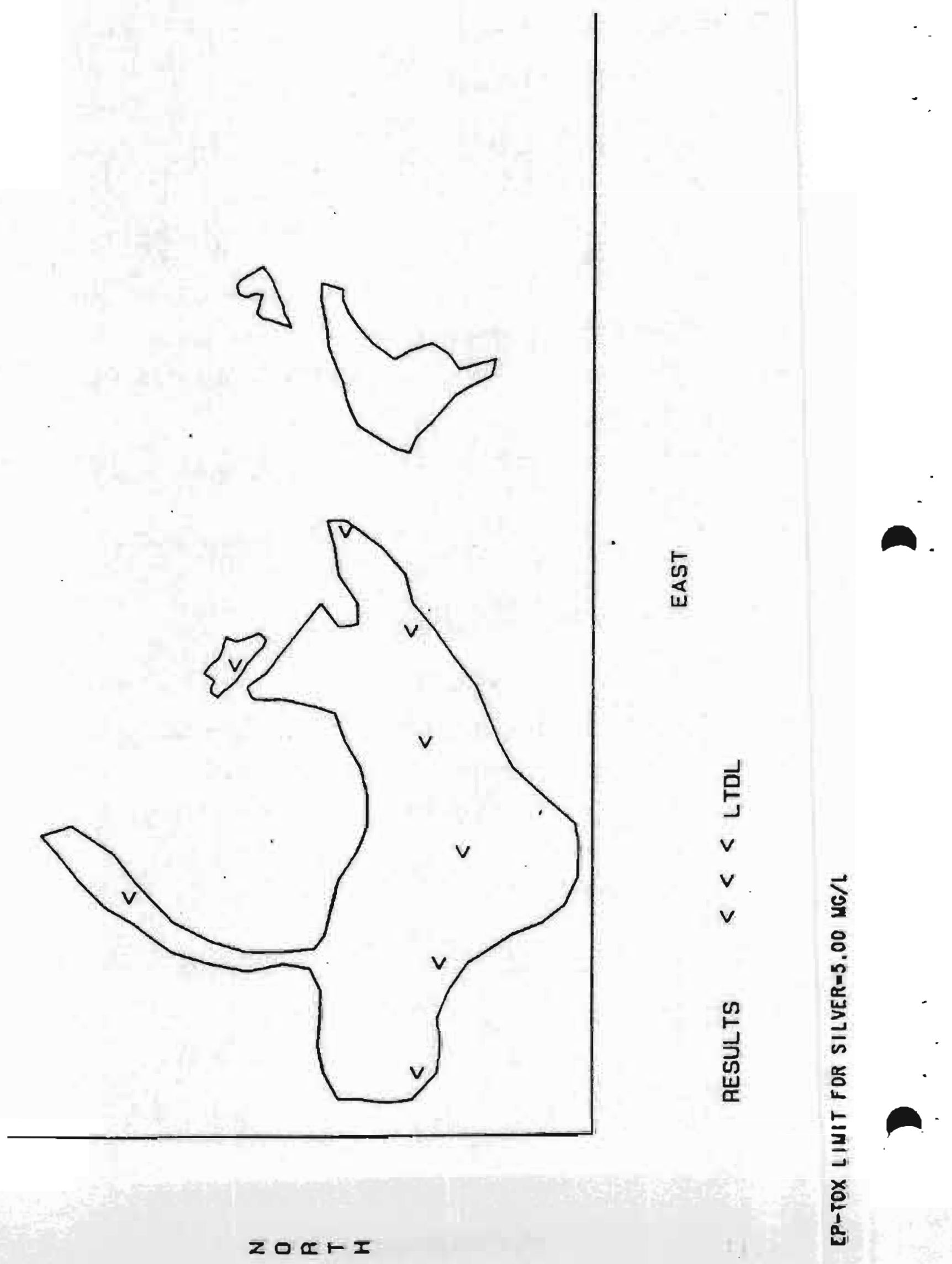


K-1007 POND - SILVER UG/G

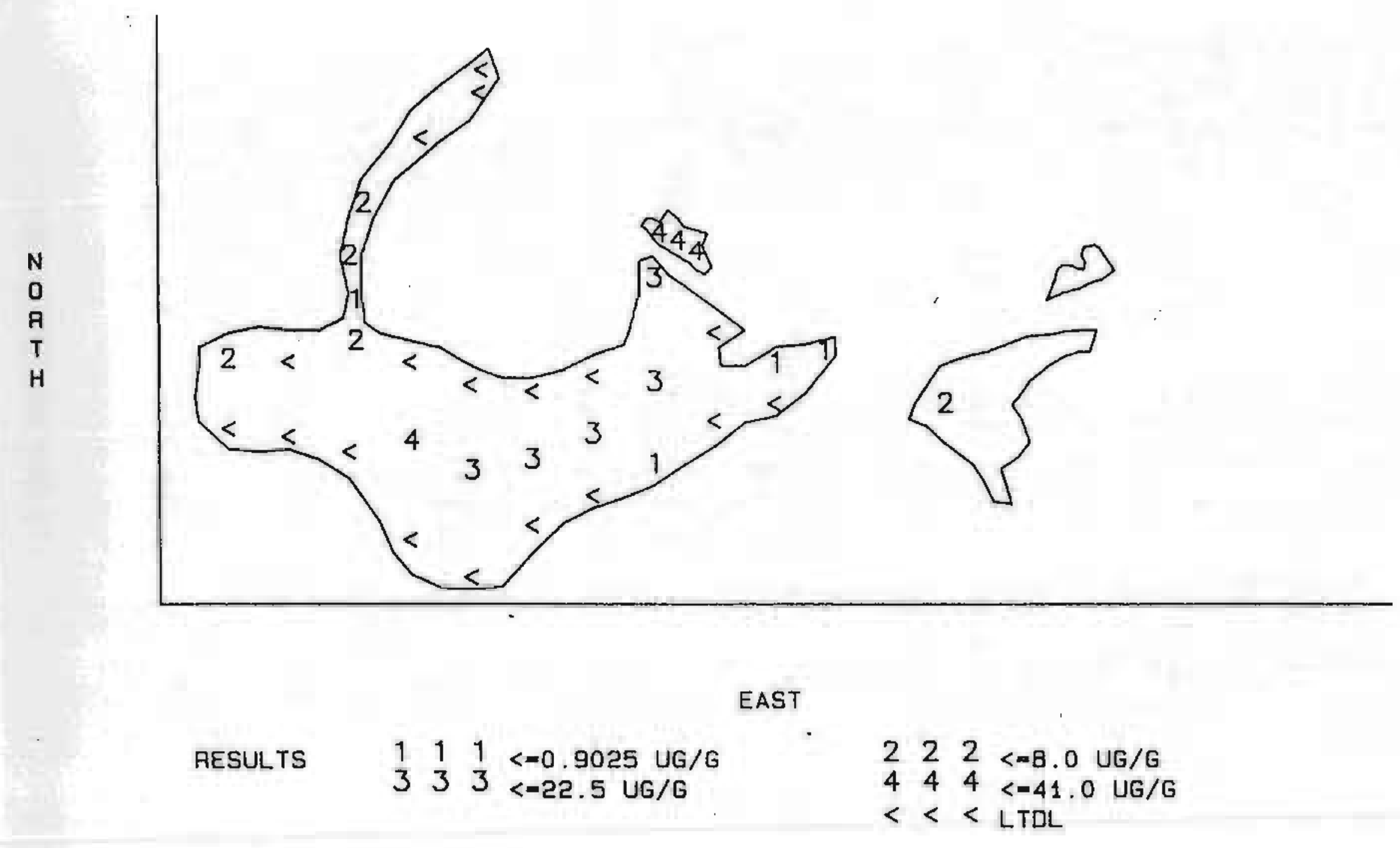




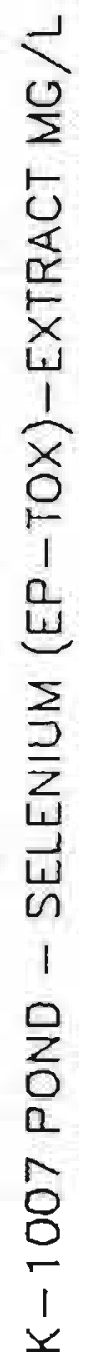

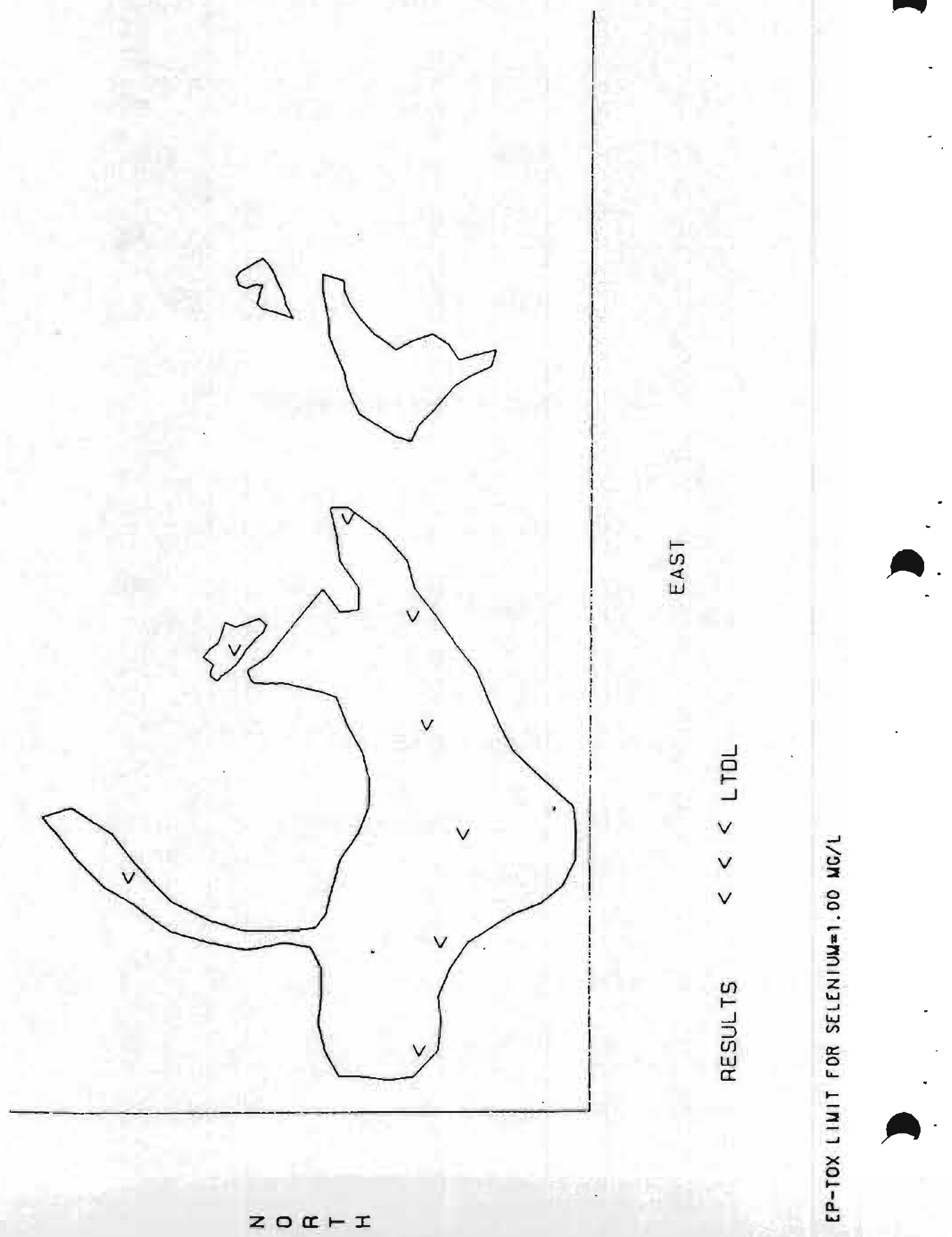


K- 1007 POND - NICKEL $($ EP-TOX)-EXTRACT MG/L

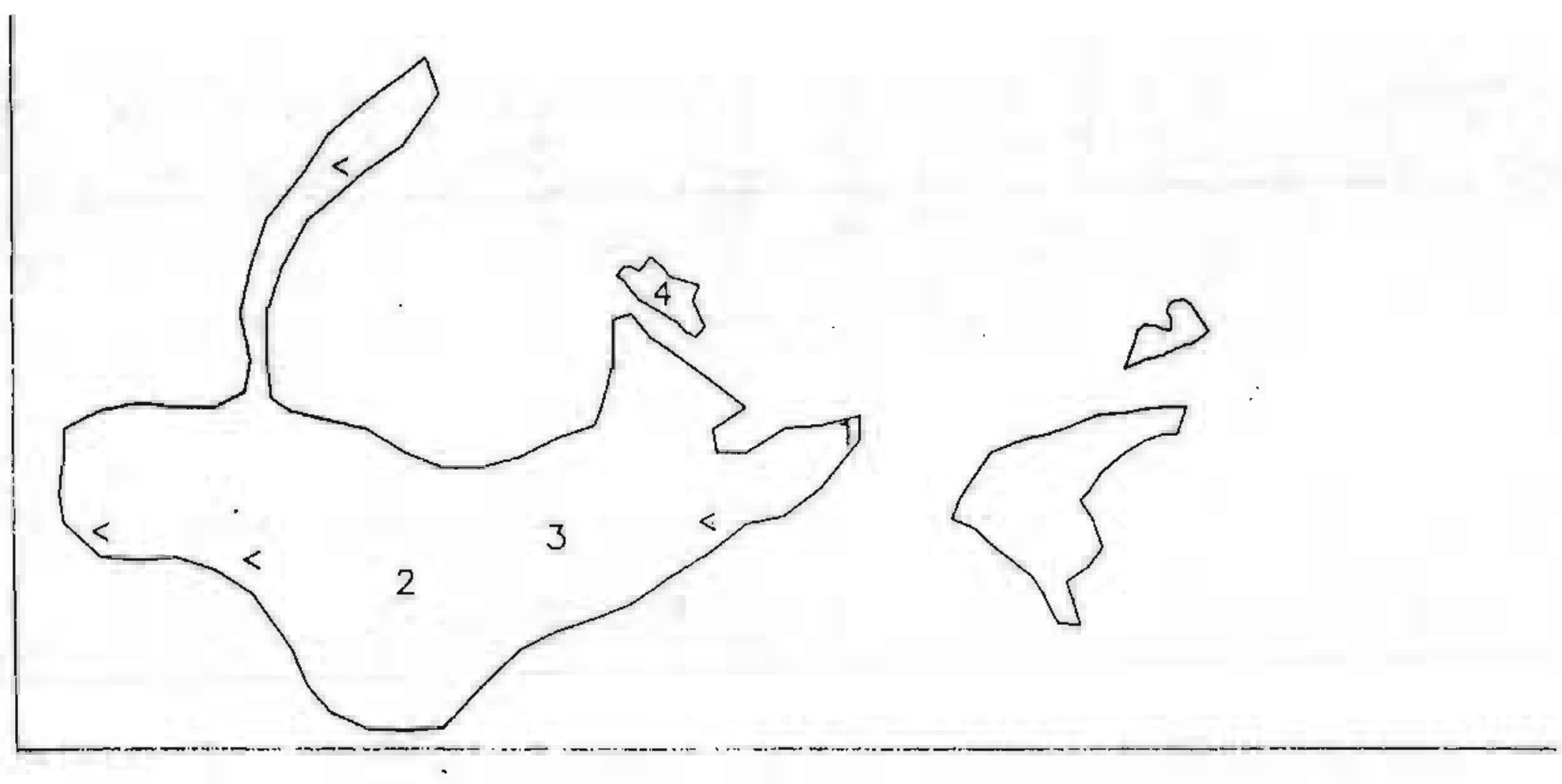

EAST

RESULTS $\quad \begin{array}{llll}1 & 1 & 1 & =0.056 \mathrm{MG} / \mathrm{L} \\ 3 & 3 & 3 & =0.74 \mathrm{MG} / \mathrm{L}\end{array}$ $\begin{array}{llll}2 & 2 & 2 & =0.65 \mathrm{MG} / \mathrm{L} \\ 4 & 4 & 4 & =2.00 \mathrm{MG} / \mathrm{L} \\ < & < & <\mathrm{LTOL}\end{array}$ 
K-1007 POND - NICKEL UG/G

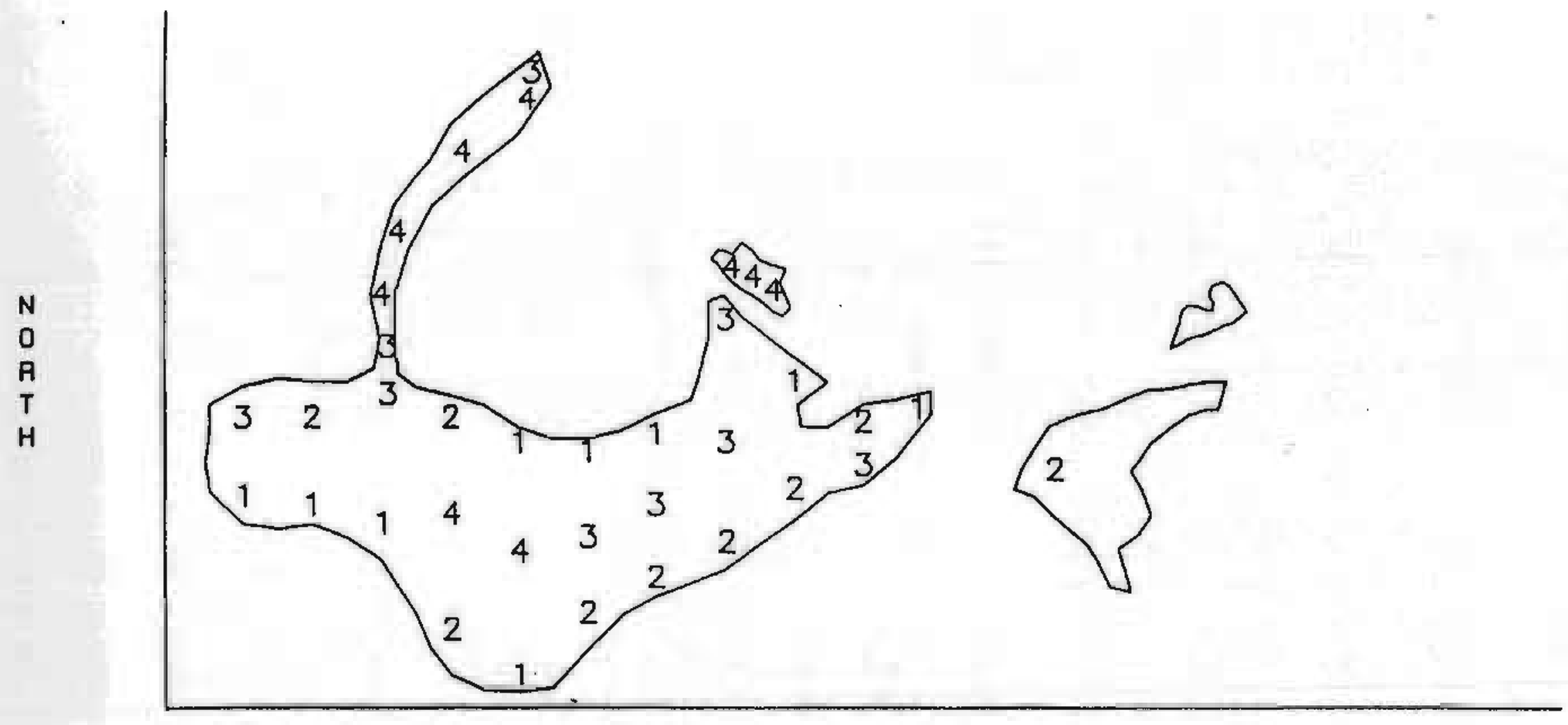

EAST

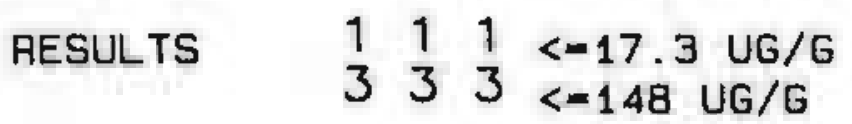
$\begin{array}{llll}2 & 2 & 2 & <-31.5 \text { UG/G } \\ 4 & 4 & 4 & <-260 \text { UG/G }\end{array}$

K/HS-132 TABLE 2.2 MAX LIMIT FOR SOIL=400 UG/G 


$$
\text { K-1007 POND - MERCURY (EP-TOX)-EXTRACT MG/L }
$$
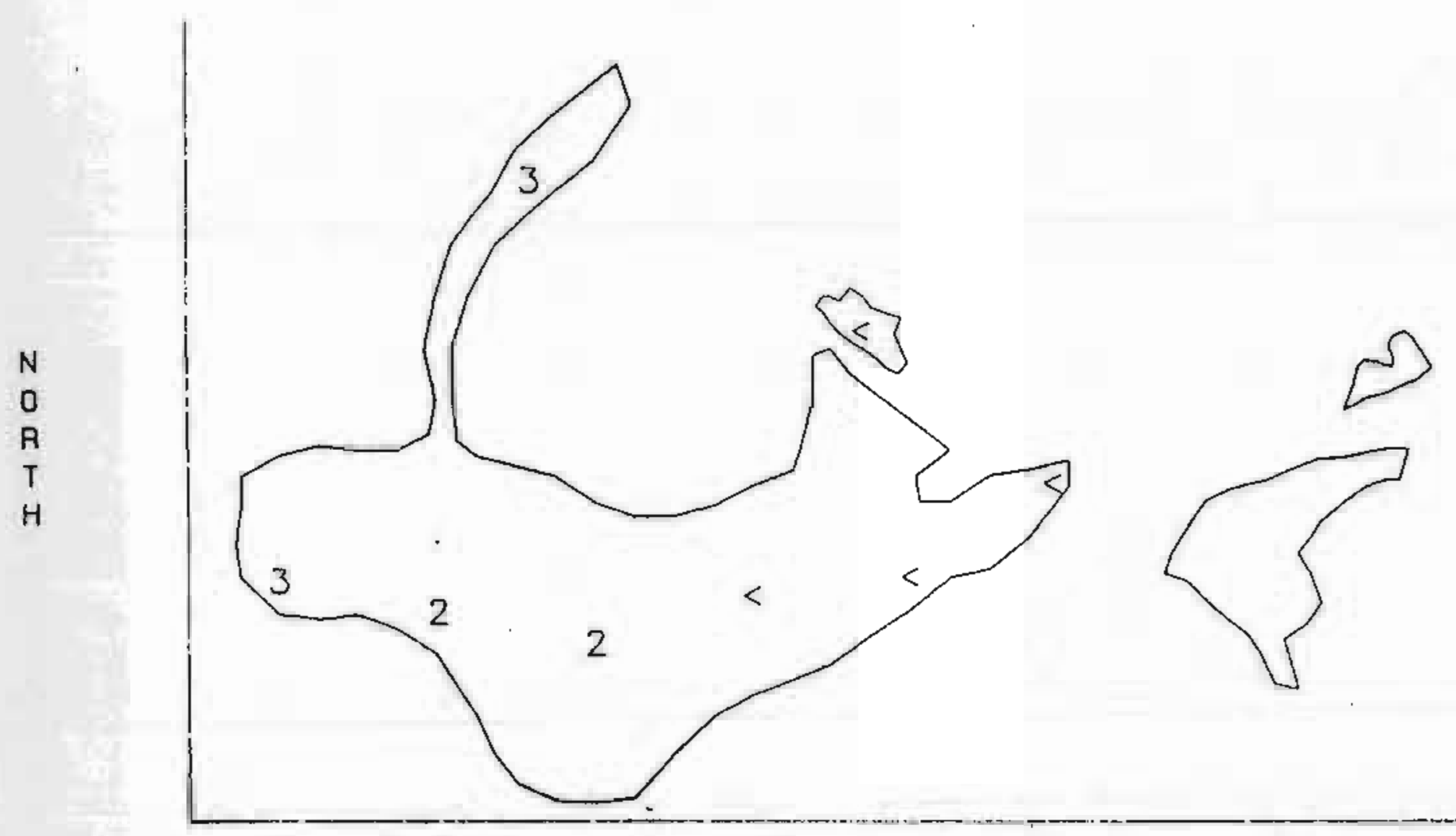

EAST

RESULTS

$$
\begin{array}{lll}
5<<<=0.0002 \mathrm{MG} / \mathrm{L} & 222<=0.0005 \mathrm{MG} / \mathrm{L} \\
333<=0.0006 \mathrm{MG} / \mathrm{L} & <<<\mathrm{LTDL}
\end{array}
$$

EP-TOX LINIT FOR MERCURY $=0.2 \mathrm{NG} / \mathrm{L}$ 
K-1007 POND - MERCURY UG/G

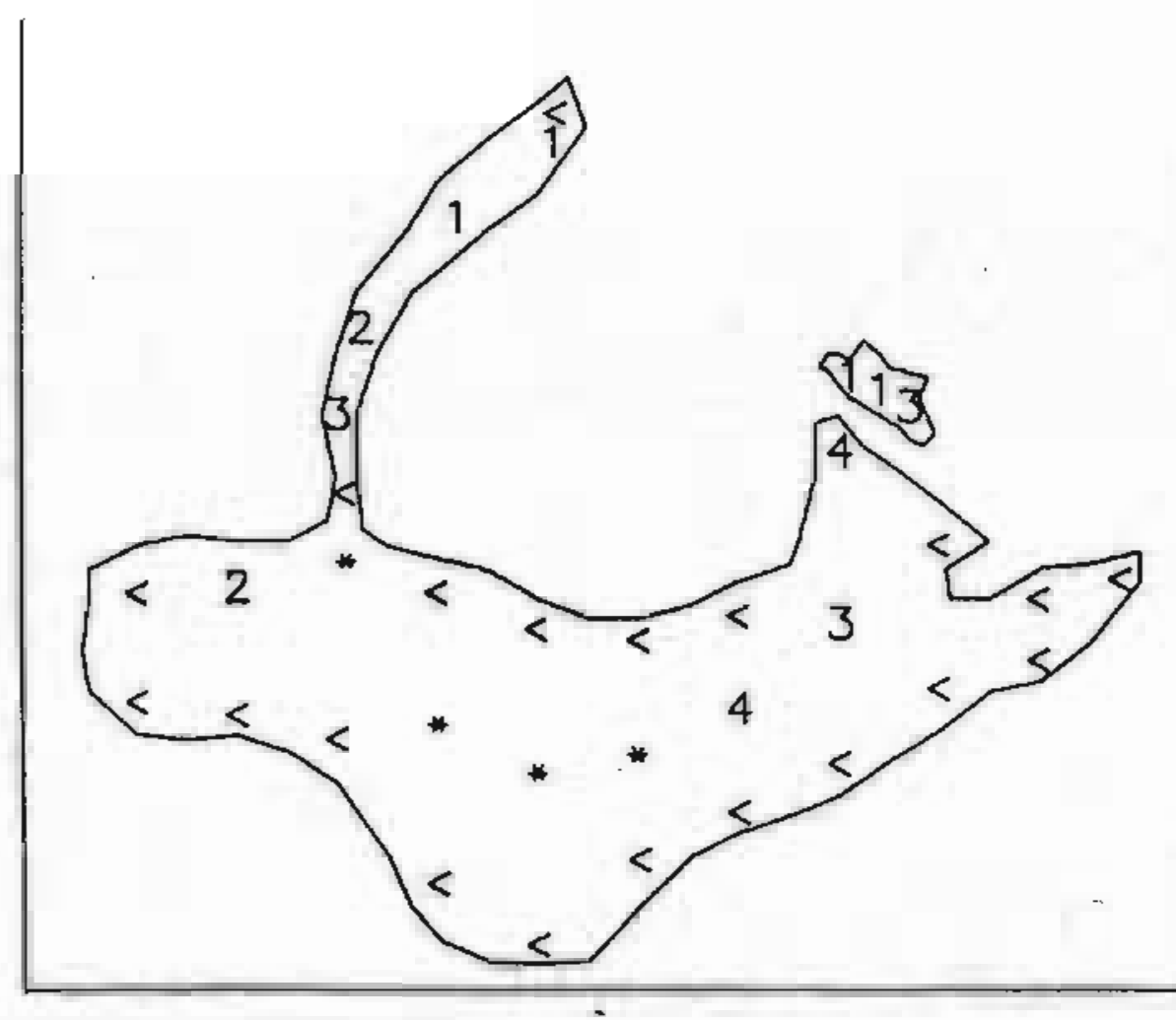

EAST

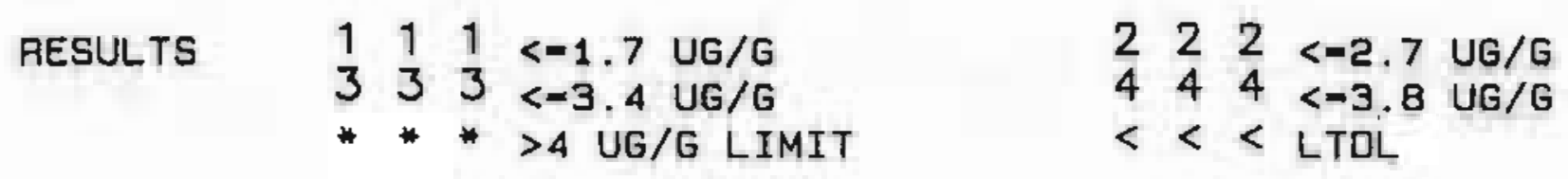

K/HS-132 TAELE 2.2 MAX LINIT FOR SOIL=4 UG/G 

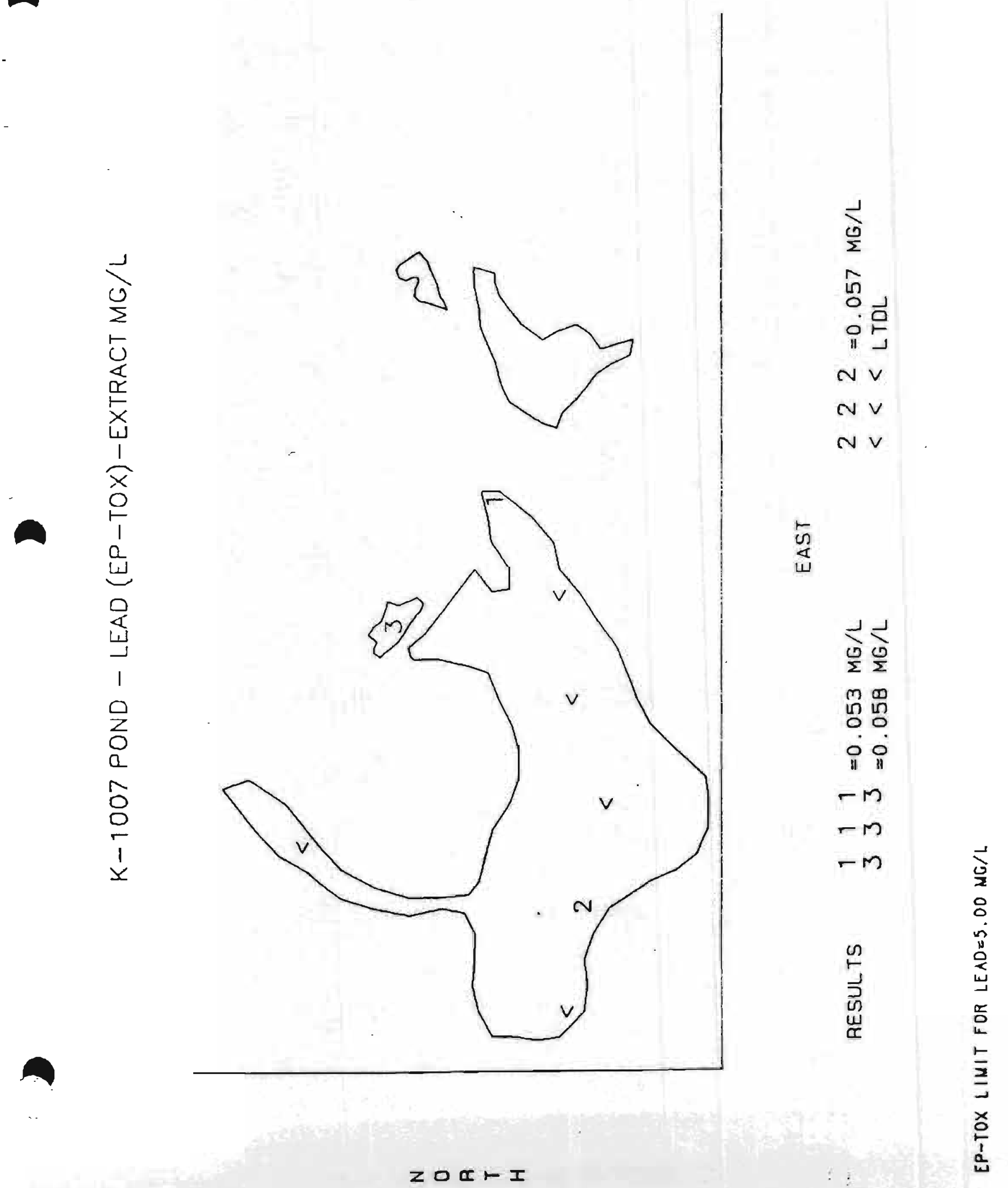


$$
\text { K-1007 POND - LEAD UG/G }
$$

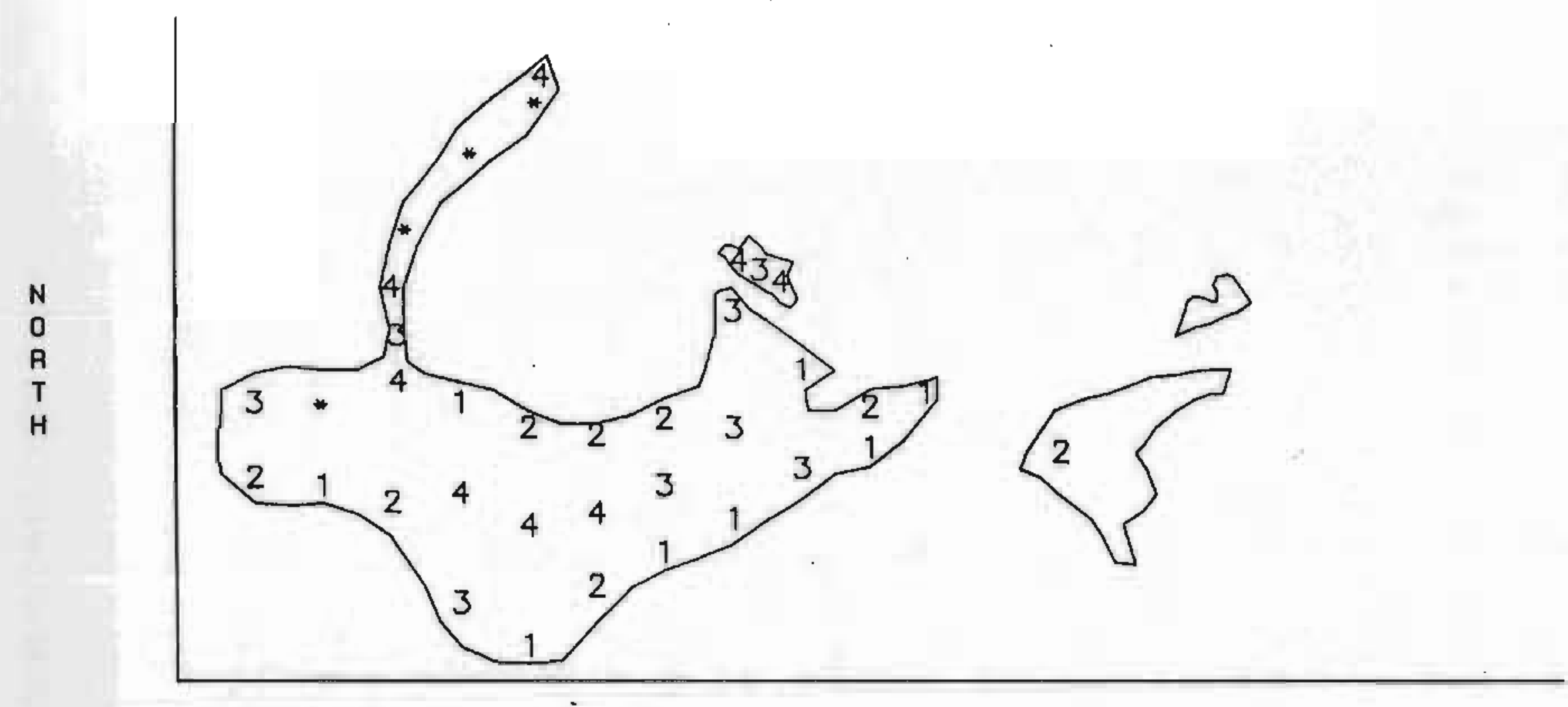

\section{EAST}

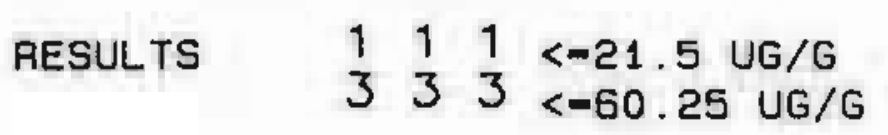

$$
\begin{array}{llll}
2 & 2 & 2 & <-33.0 \text { UG } / G \\
4 & 4 & 4 & <-97.0 \text { UG } / G \\
* & * & * & >100 \text { UG } / G \text { LIMIT }
\end{array}
$$




$$
\text { K-1007 POND - IRON (EP-TOX)-EXTRACT MG/L }
$$

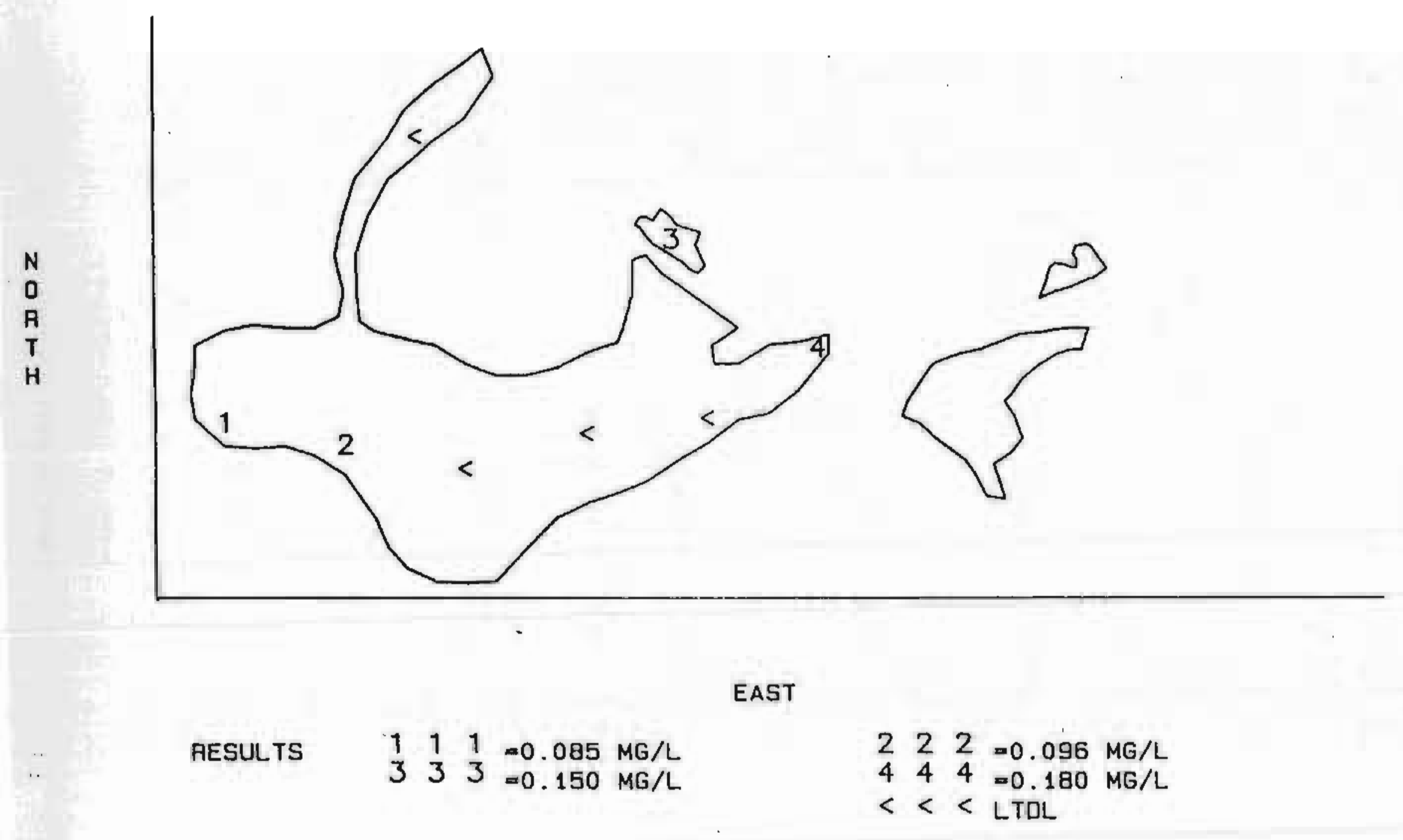

NO ESTABLISHED EP-TOX LEVEL 


$$
\text { K-1007 POND - IRON UG/G }
$$

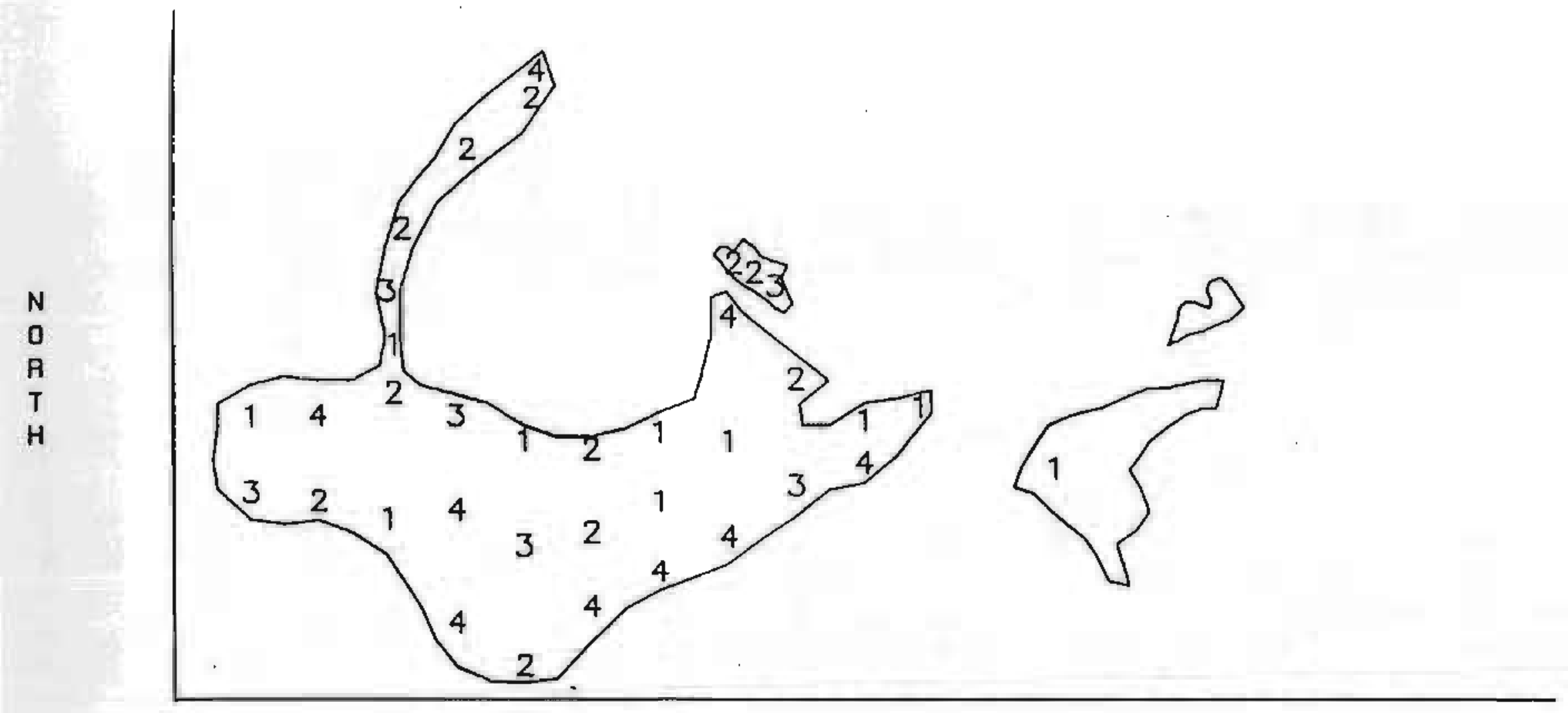

EAST

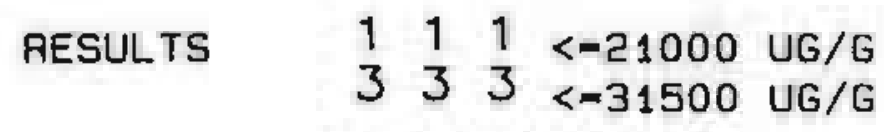

$\begin{array}{lllll}2 & 2 & 2 & <-27000 & \text { UG } / G \\ 4 & 4 & 4 & <-44000 \text { UG } / G\end{array}$

NO K/HS-132 TABLE 2.2 MAX LIMIT FOR SOIL 
0े

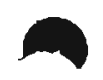

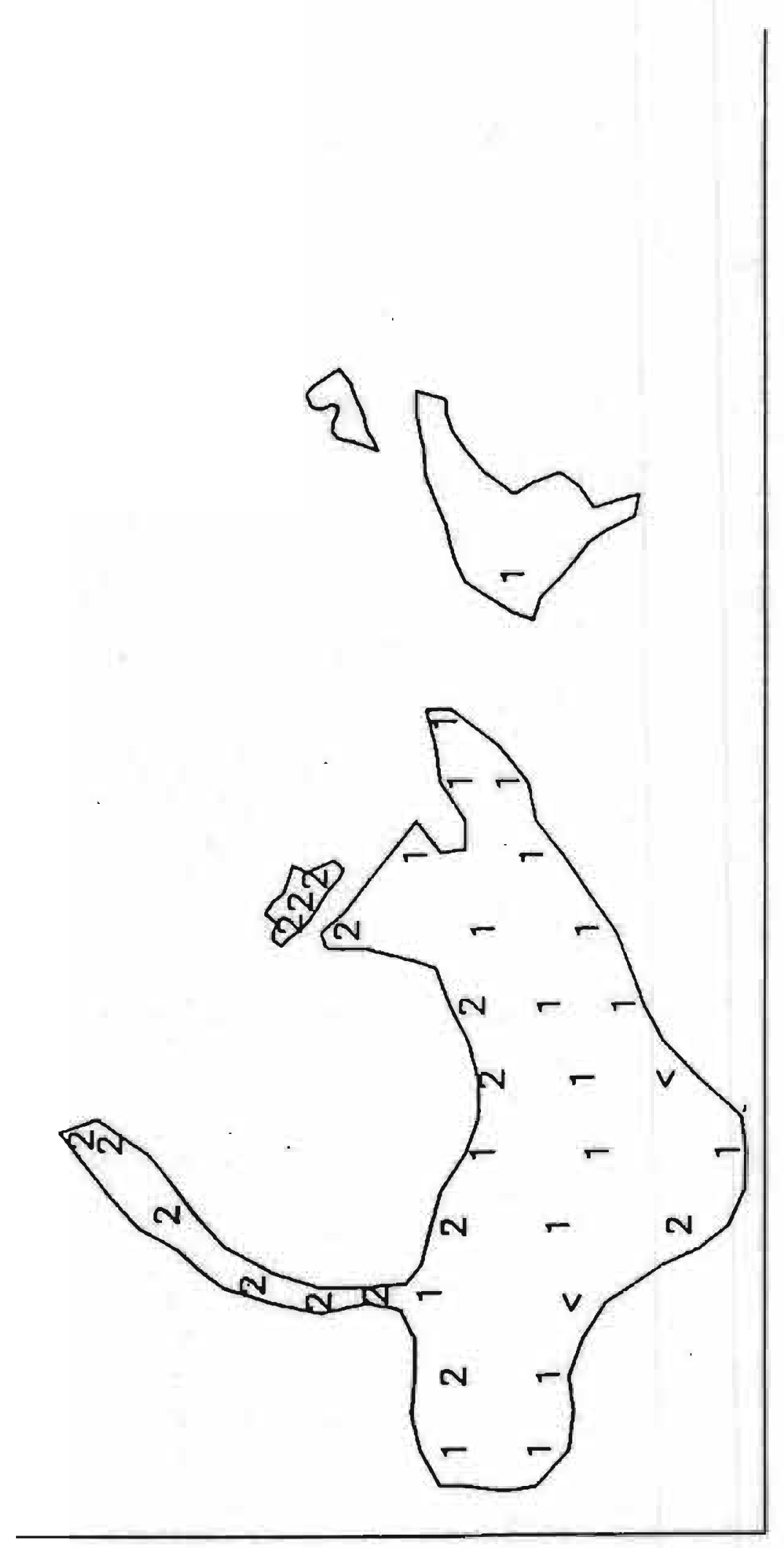

Zロ巛トI

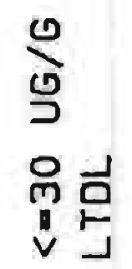
N V $N V$ N V

卣

$\frac{0}{3}$

i

$r$

-

$-$

on 


\section{K-1007 POND - COPPER UG/G}

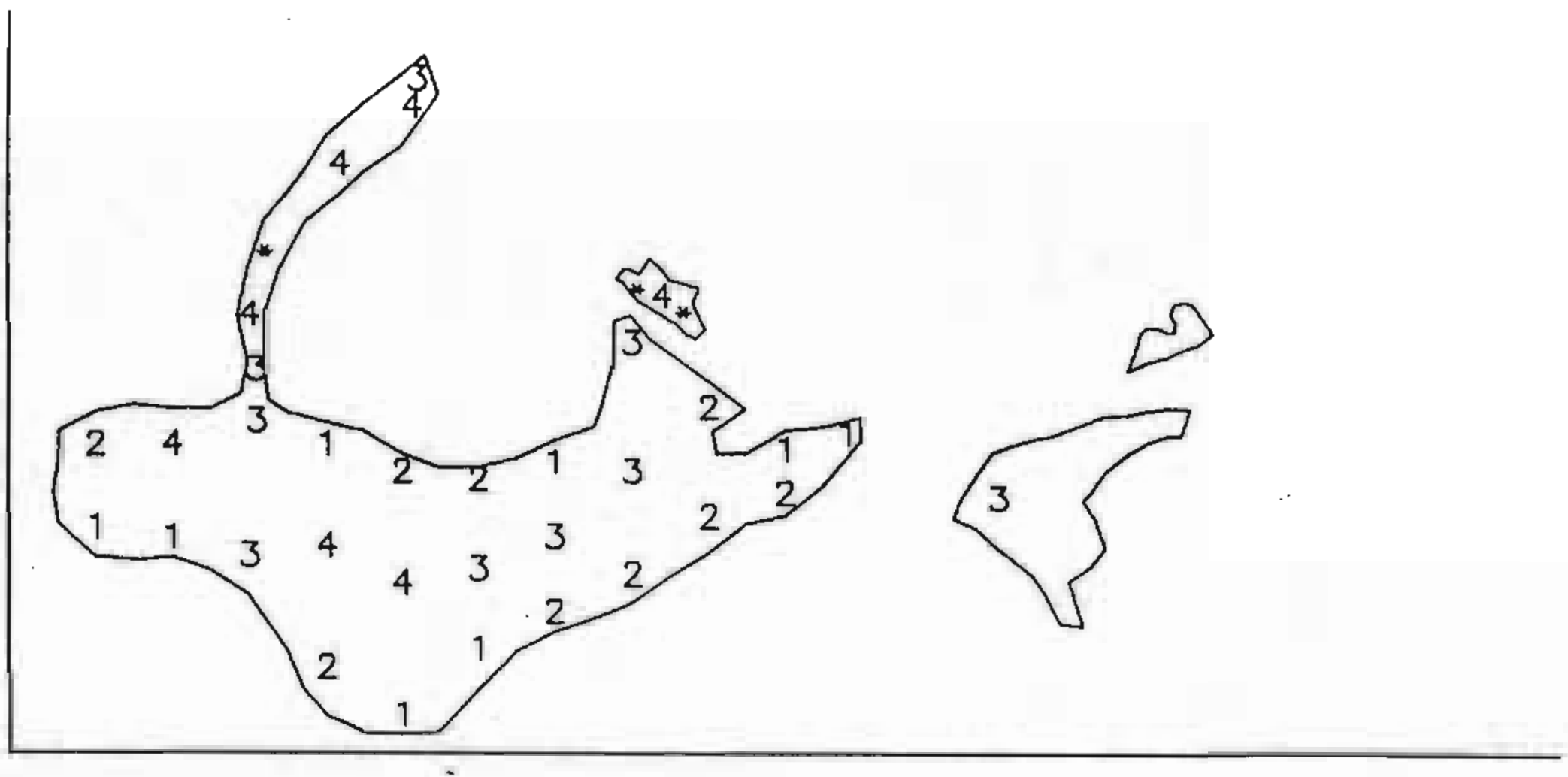

EAST

$\begin{array}{lllll}\text { RESULTS } & 1 & 1 & 1 & 3-15.5 \text { UG /G }\end{array}$
$222<-34$ UG $/ G$
$444<-100$ UG/G
$* * *>100$ UG/G LIMIT

K/HS-132 TABLE 2.2 MAX LIMIT FOR SOIL=100 UG/G 


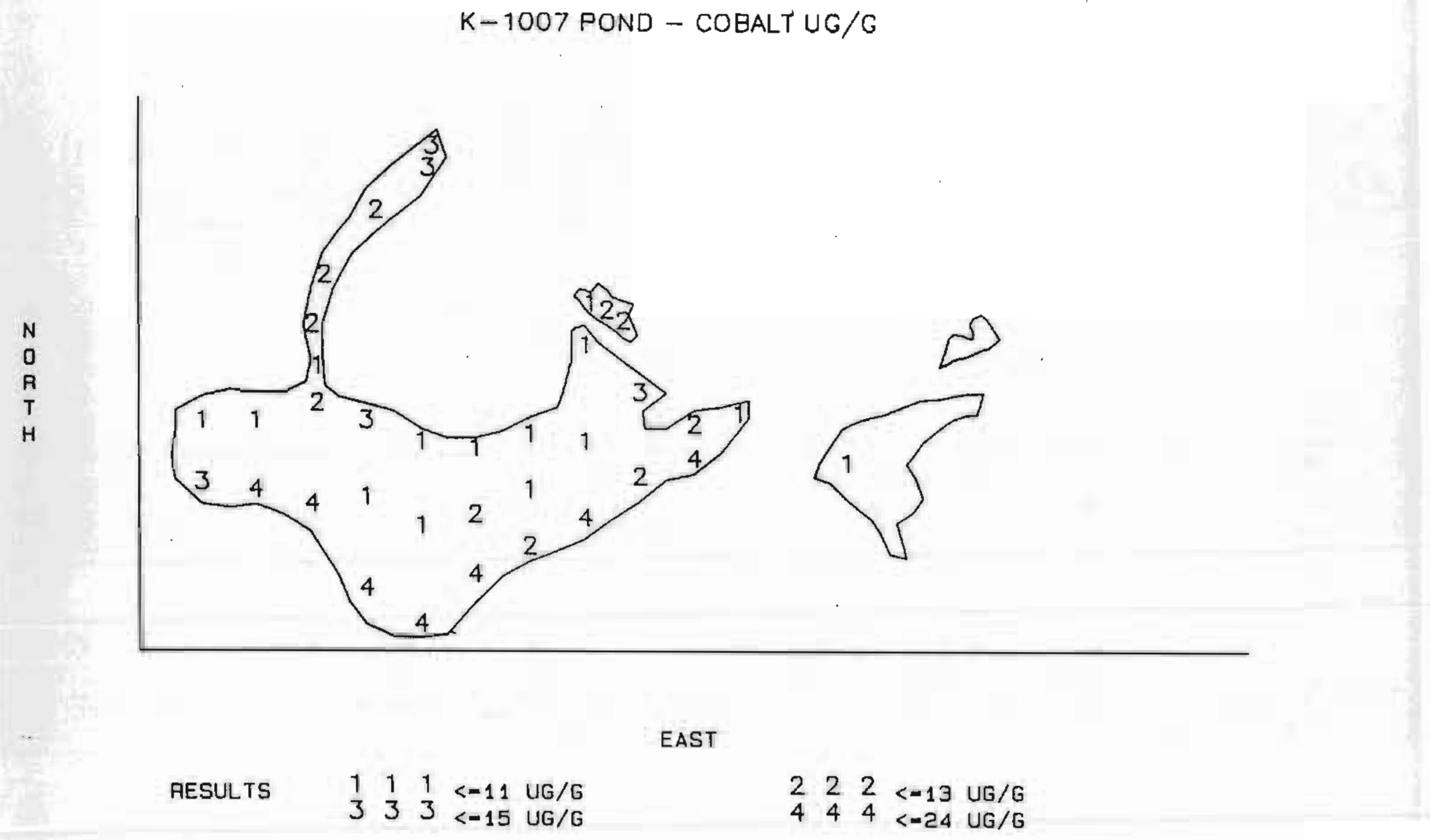

NO K/HS-132 TABLE 2.2 NAX LIHIT FOR SOIL 


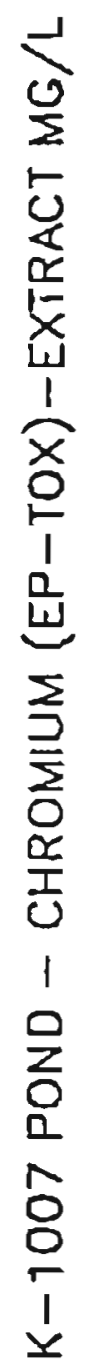

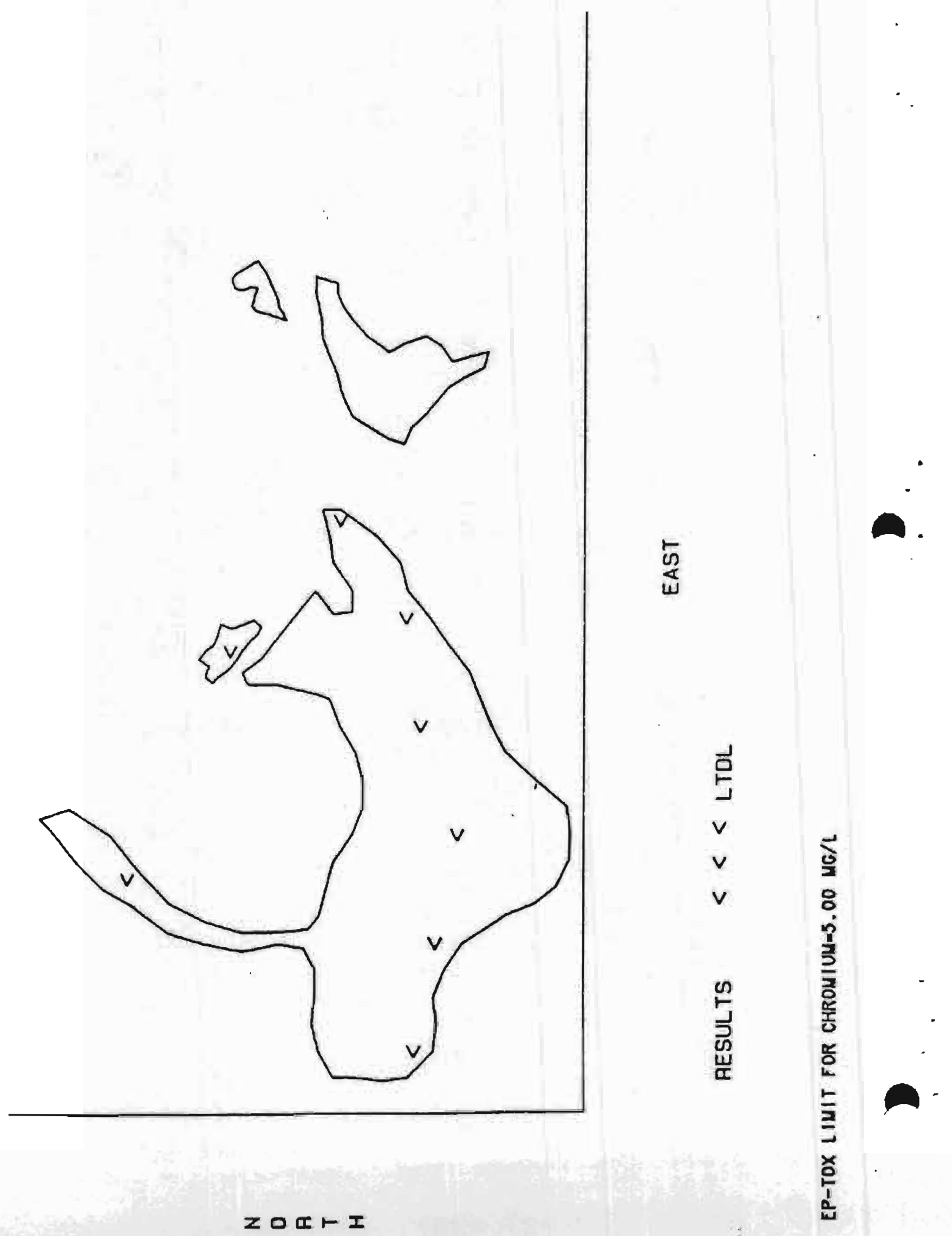


K-1007 POND - CHROMIUM UG/G

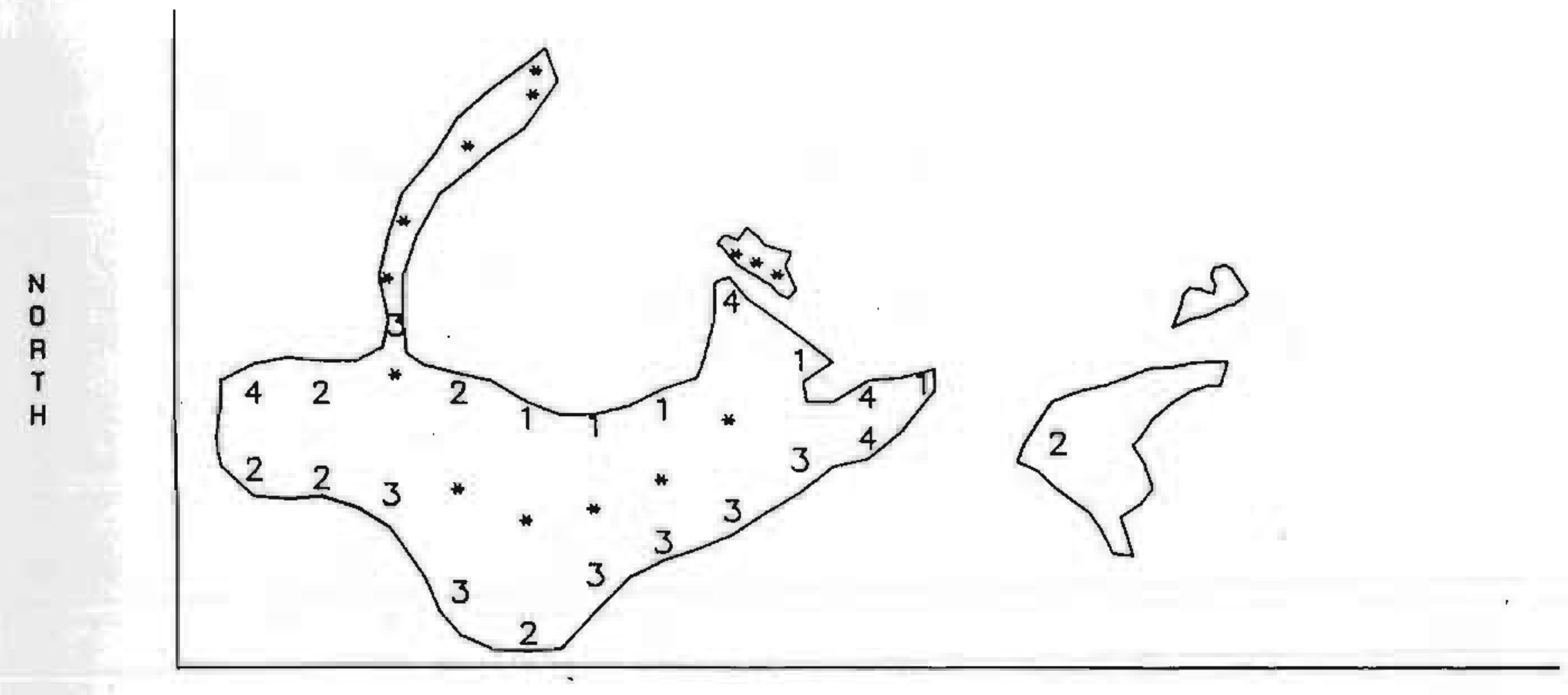

EAST

\begin{tabular}{|c|c|c|c|c|c|c|}
\hline RESULTS & $\begin{array}{lll}1 & 1 & 1 \\
3 & 3 & 3\end{array}$ & $\begin{array}{l}<-20.75 \text { UG/G } \\
<-37 \text { UG/G }\end{array}$ & $\begin{array}{l}2 \\
4 \\
*\end{array}$ & $\begin{array}{r}2 \\
4 \\
*\end{array}$ & $\begin{array}{l}2 \\
4 \\
*\end{array}$ & $\begin{array}{l}<-23.5 \text { UG/G } \\
<=72 \text { UG/G } \\
>100 \text { UG } / G \text { LIMIT }\end{array}$ \\
\hline
\end{tabular}


K-1007 POND - CADMIUM (EP-TOX) - EXTRACT MG/L

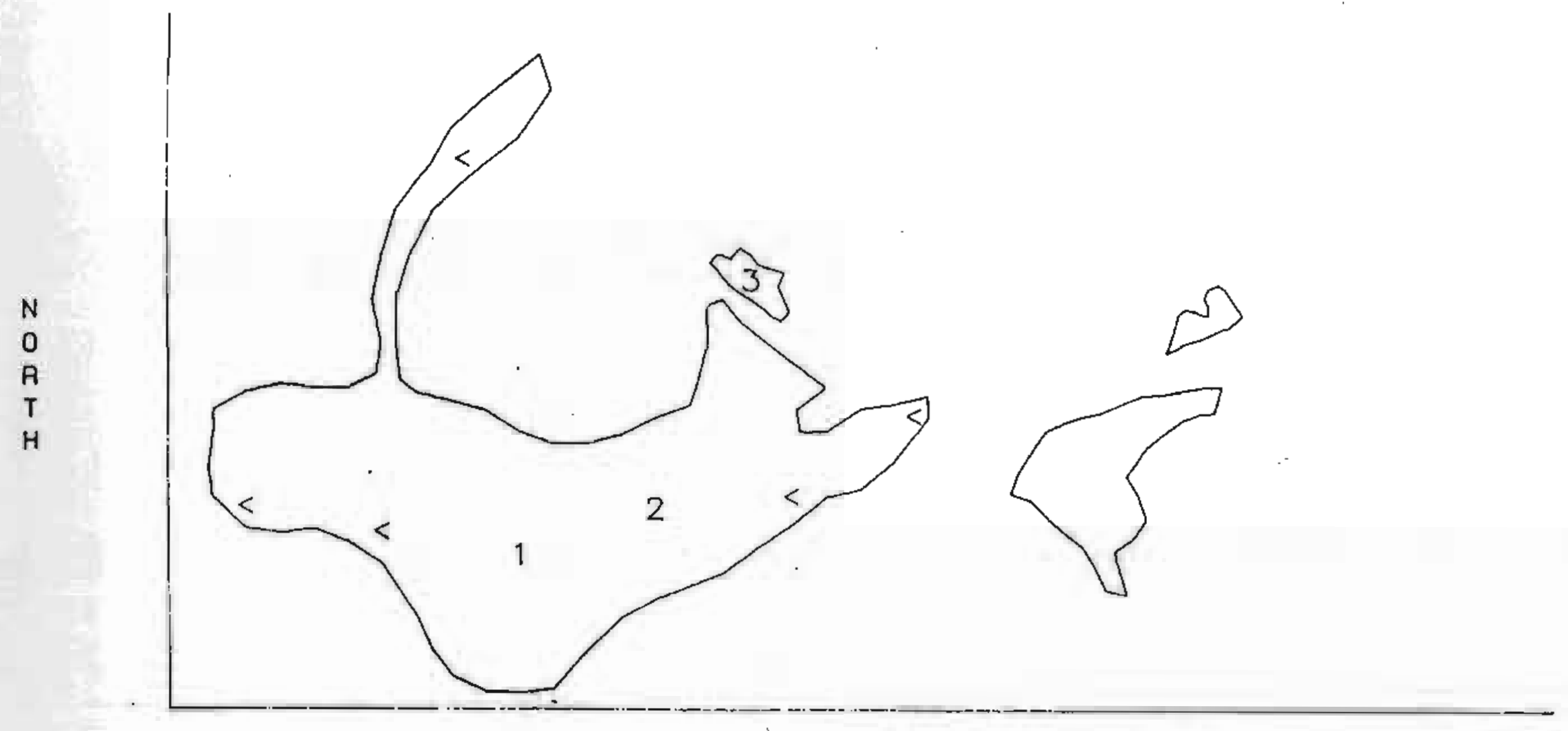

EAST

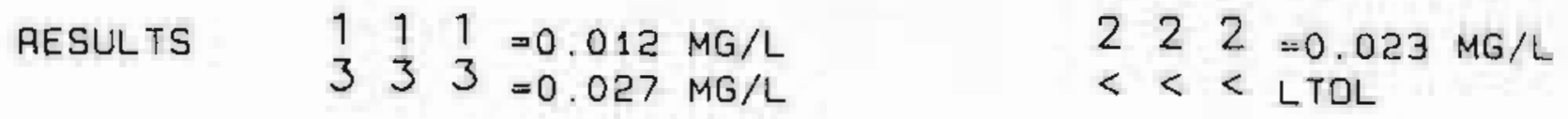

EP-TOX LINIT FOR CAONIUM $=1.00 \mathrm{NG} / \mathrm{L}$

D 
K-1007 POND - CADMIUM MG/L

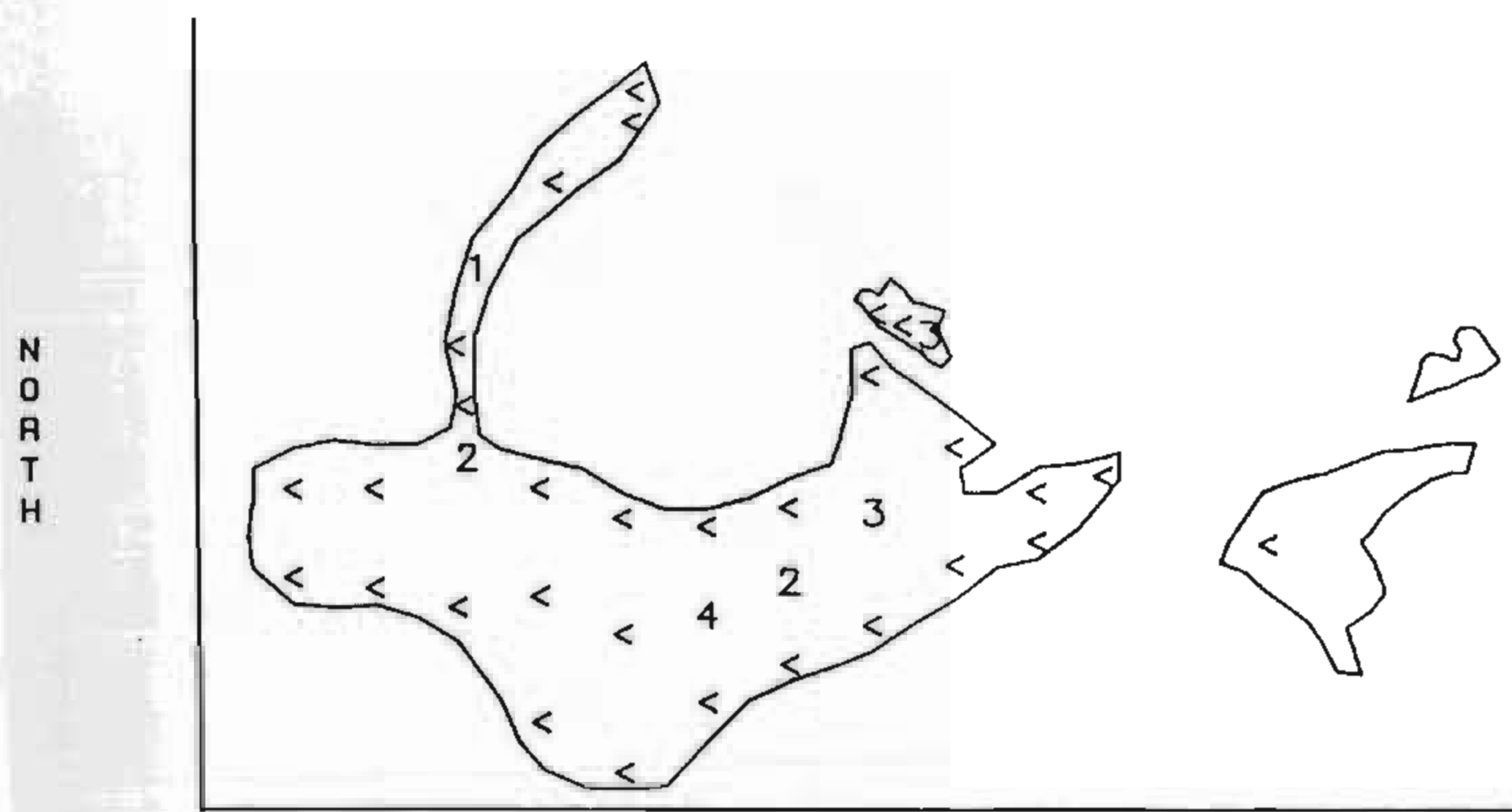

EAST

RESULTS $\quad \begin{array}{llll}1 & 1 & 1 & <-0.5675 \mathrm{MG} / \mathrm{L} \\ & 3 & 3 & 3<-1.2000 \mathrm{MG} / \mathrm{L}\end{array}$

$222<-1.0100 \mathrm{MG} / \mathrm{L}$

$444<-1.5000 \mathrm{MG} / \mathrm{L}$

$<<<$ LTDL

K/HS-132 TABLE 2.2 UAX LIMIT FOR SOIL=2O UG/G 


$$
\text { K-1007 POND - BORON UG/G }
$$

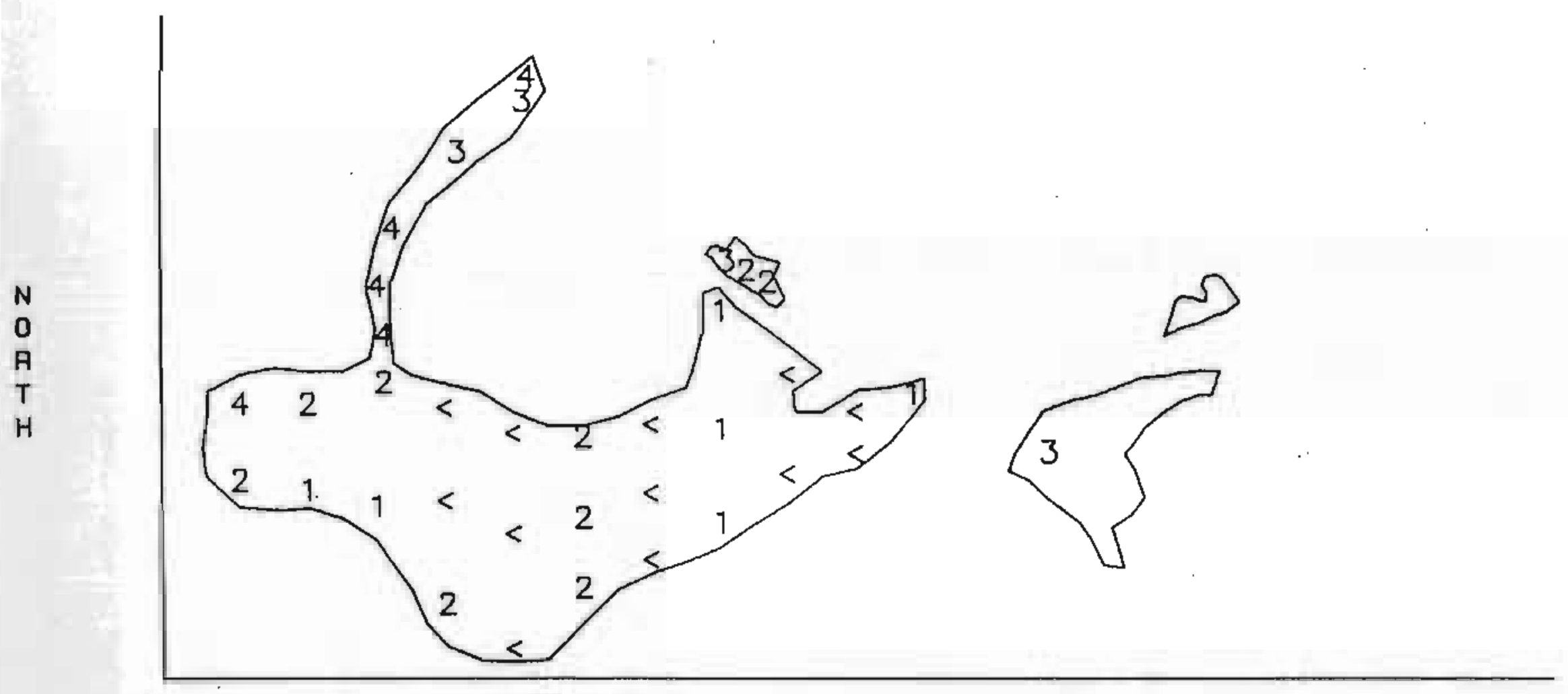

EAST

$$
\begin{aligned}
& \begin{array}{lllll}
\text { RESULTS } & 1 & 1 & 1 & <-7 \text { UG/G } \\
& 3 & 3 & 3 & <-20 \text { UG/G }
\end{array} \\
& \begin{array}{llll}
2 & 2 & 2 & <-18 \cup G / G \\
4 & 4 & 4 & <-29 \cup G / G
\end{array} \\
& <<<\text { LTOL }
\end{aligned}
$$




$$
\text { K-1007 POND - BERYLLIUM UG/G }
$$

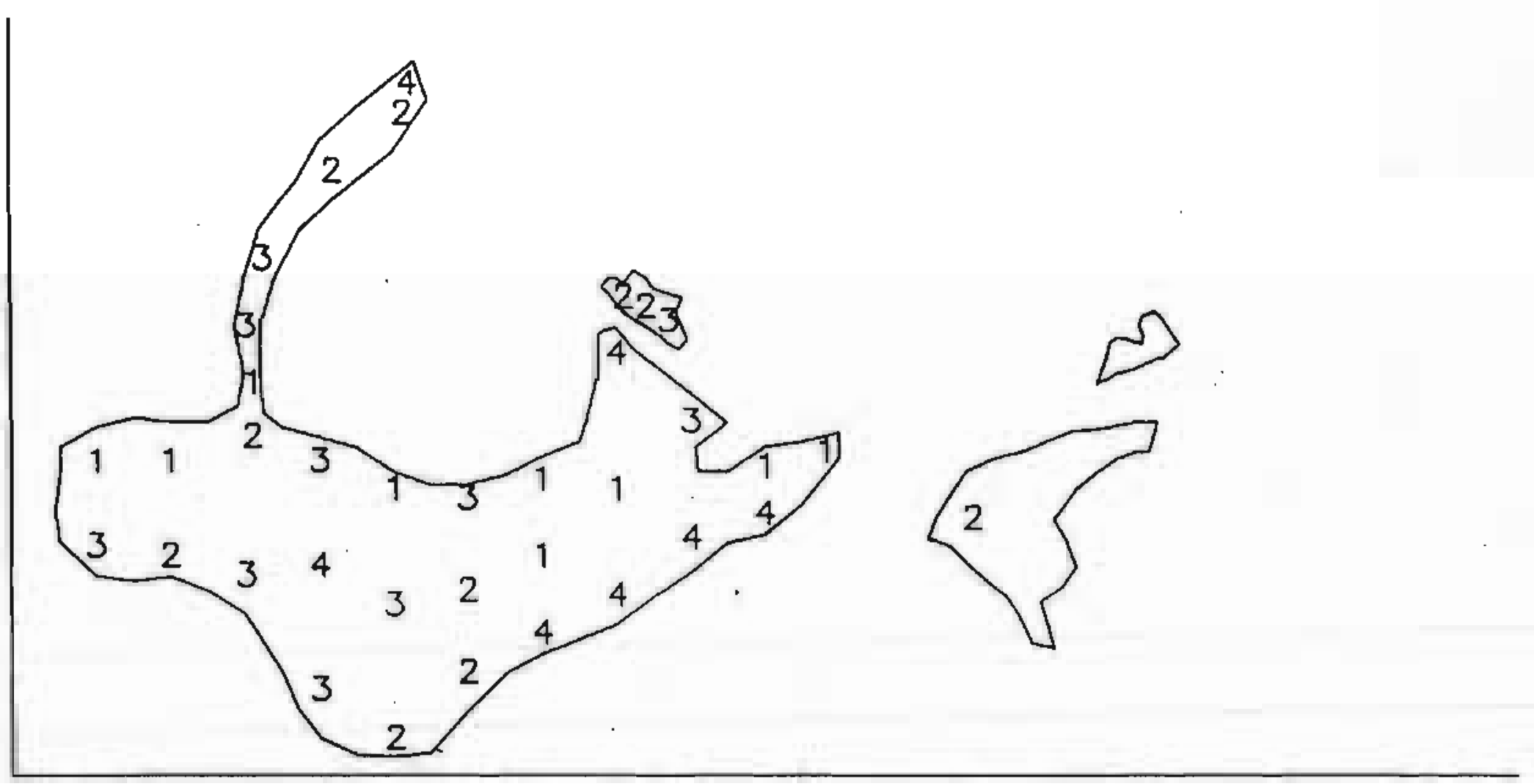

\section{EAST}
RESULTS
$\begin{array}{llll}1 & 1 & 1 & <-0.7525 \text { UG } / G \\ 3 & 3 & 3 & <-1.100 \text { UG/G }\end{array}$
$\begin{array}{llll}2 & 2 & 2 & <-0.9200 \text { UG/G } \\ 4 & 4 & 4 & <-2.000 \text { UG/G }\end{array}$ 


$$
\text { K-1007 POND - BARIUM (EP-TOX)-EXTRACT MG/L }
$$

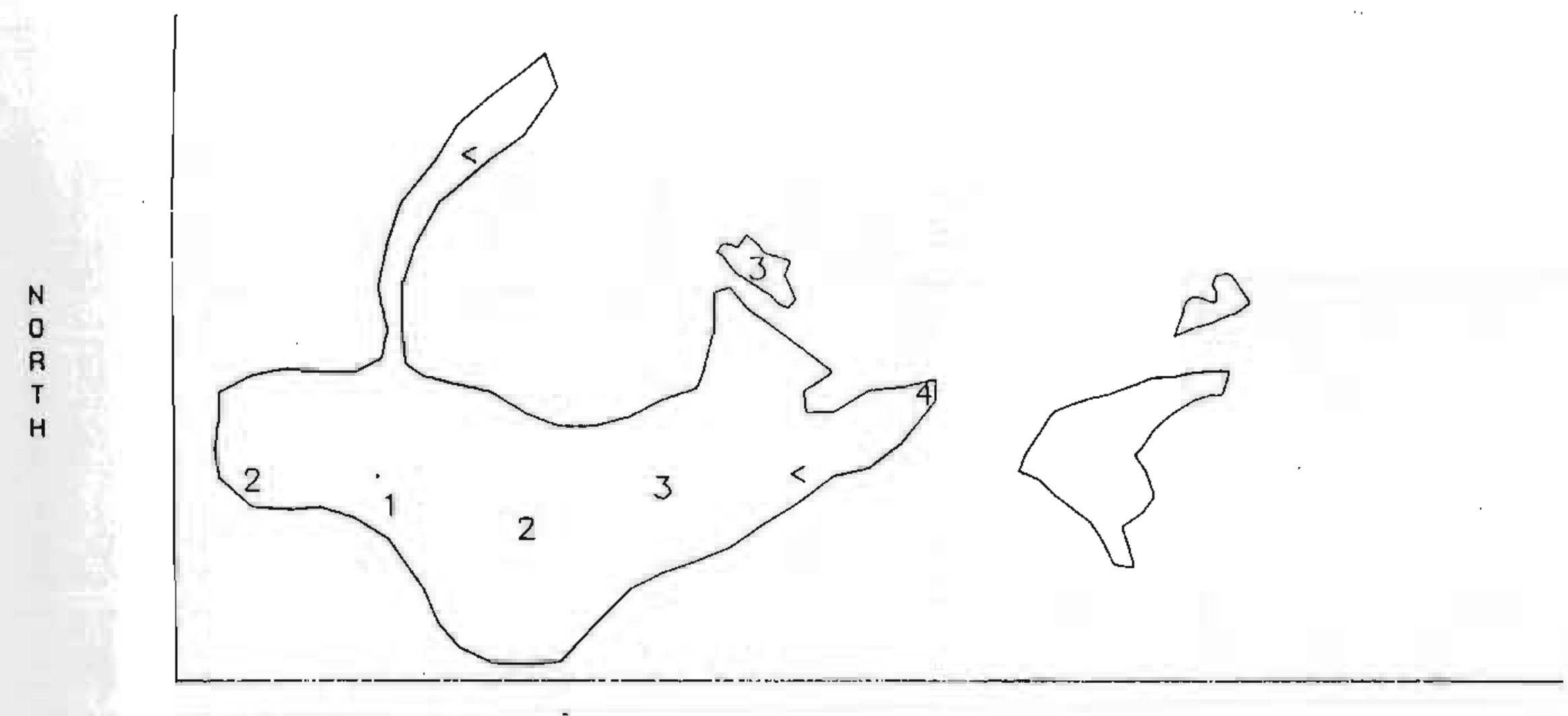

EAST

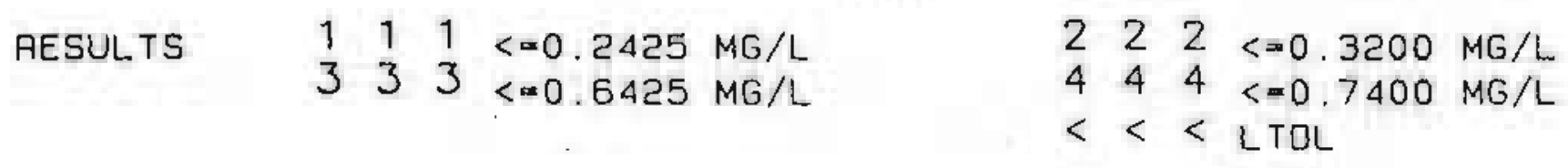

EP-TOX LIMIT FOR BARIUM=100 MG/L

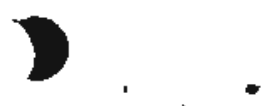




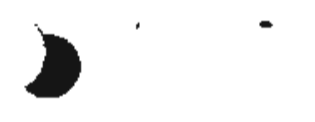

$$
\text { K-1007 POND - BARIUM UG/G }
$$

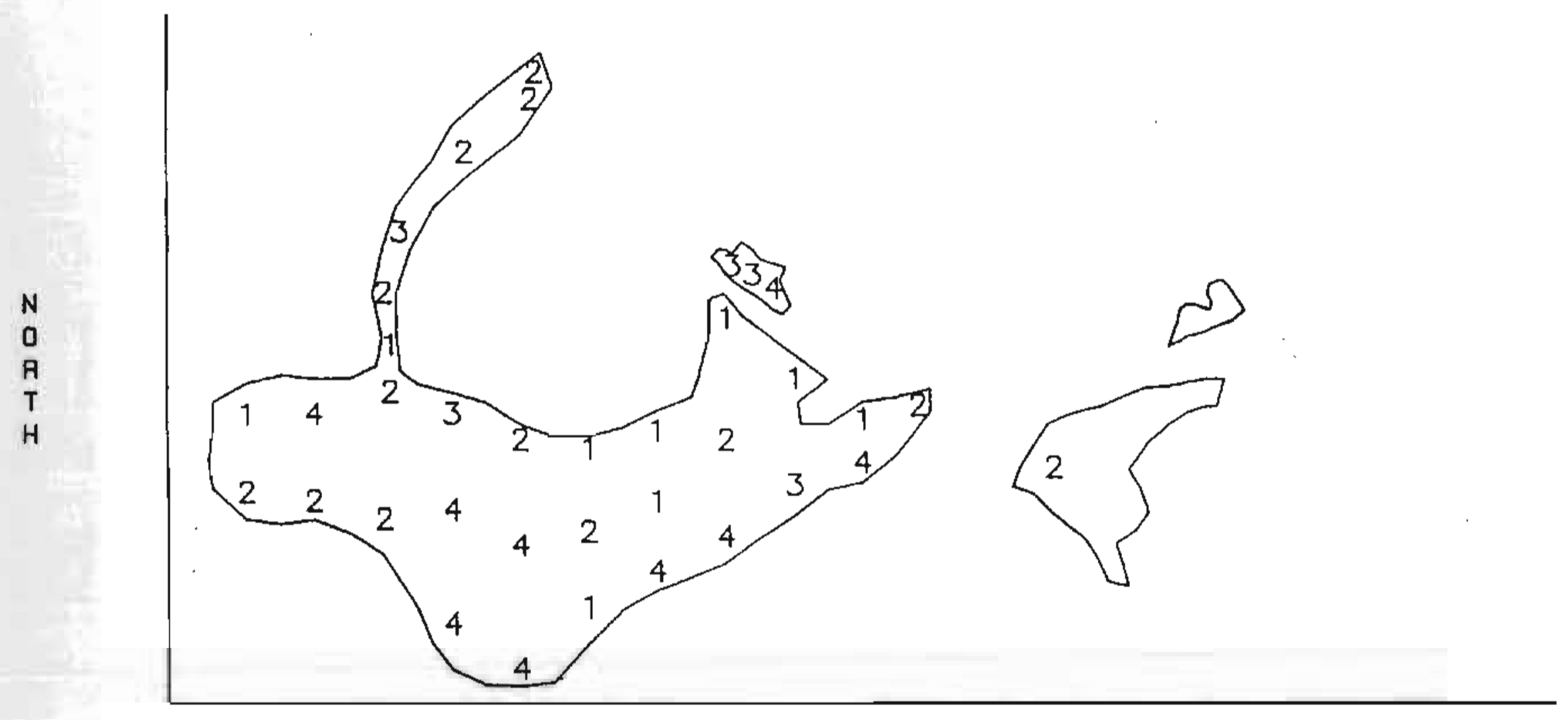

EAST

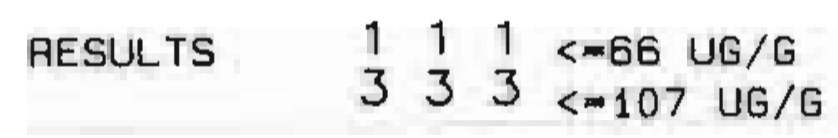$$
\begin{array}{llll}
2 & 2 & 2 & <-82 \text { UG } / G \\
4 & 4 & 4 & <-170 \text { UG } / G
\end{array}
$$ 


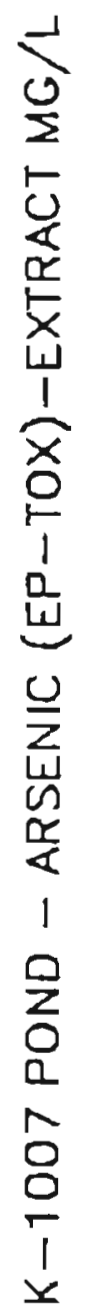

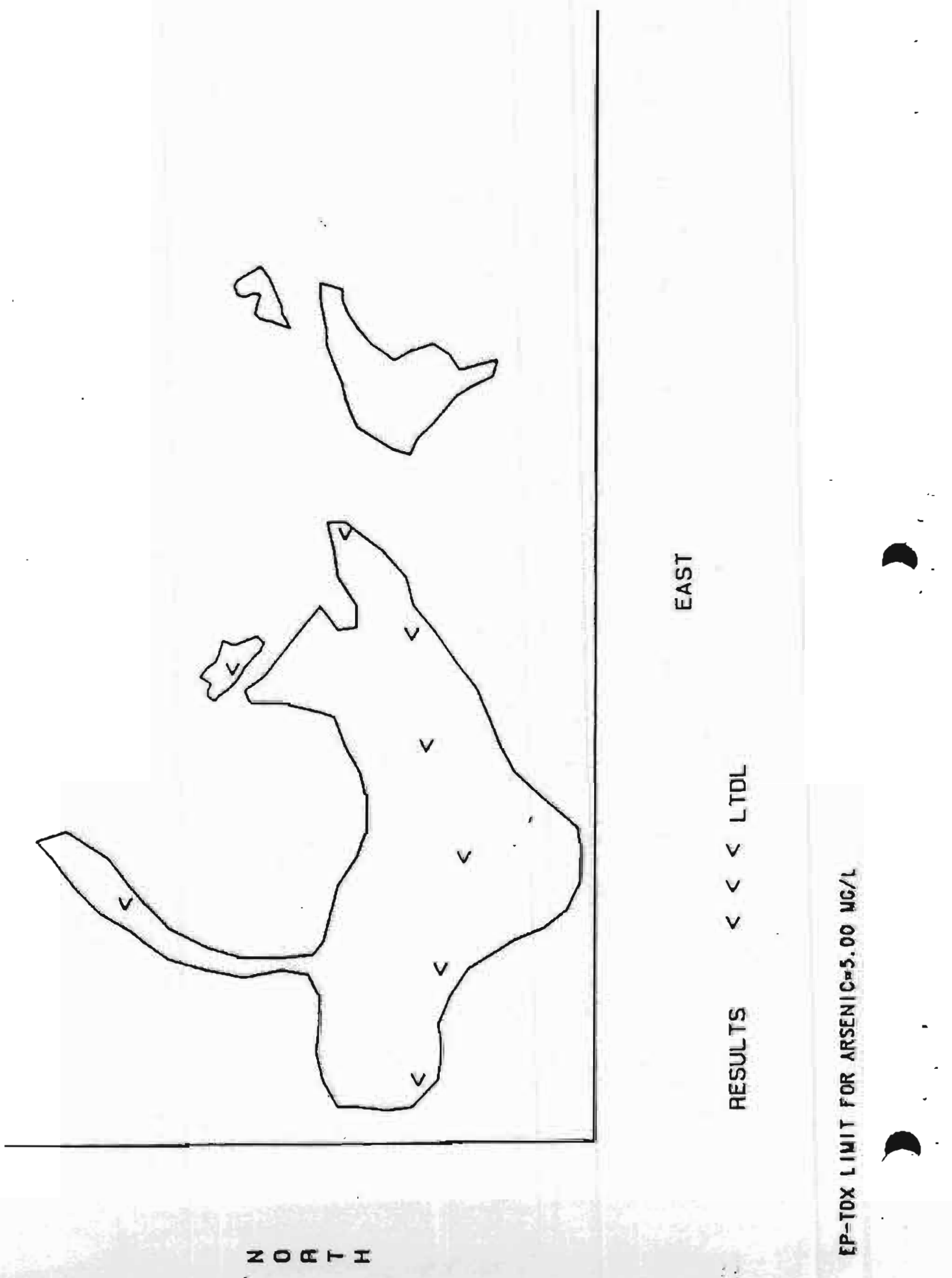




$$
\text { K-1007 POND - ARSENIC MG/KG }
$$

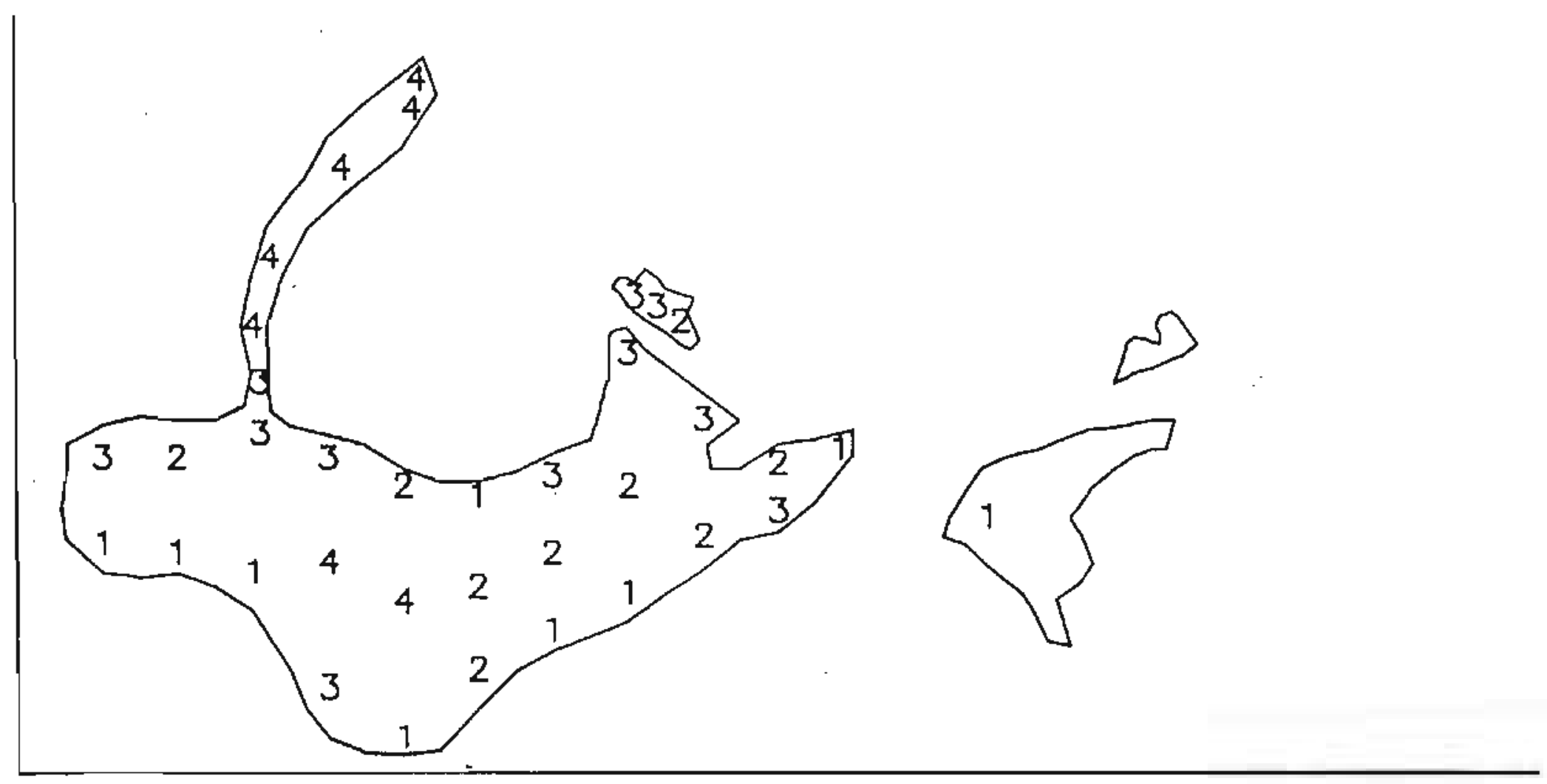

EAST

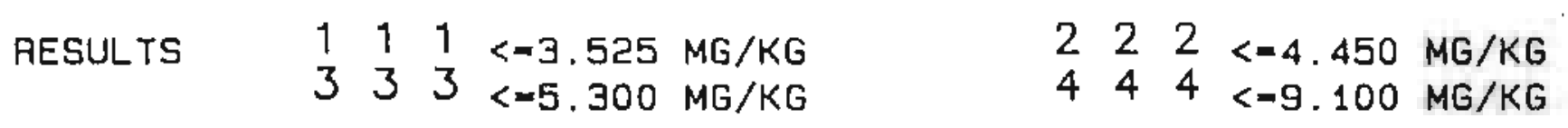




$$
\text { K-1007 POND - 2,4-D UG/L }
$$

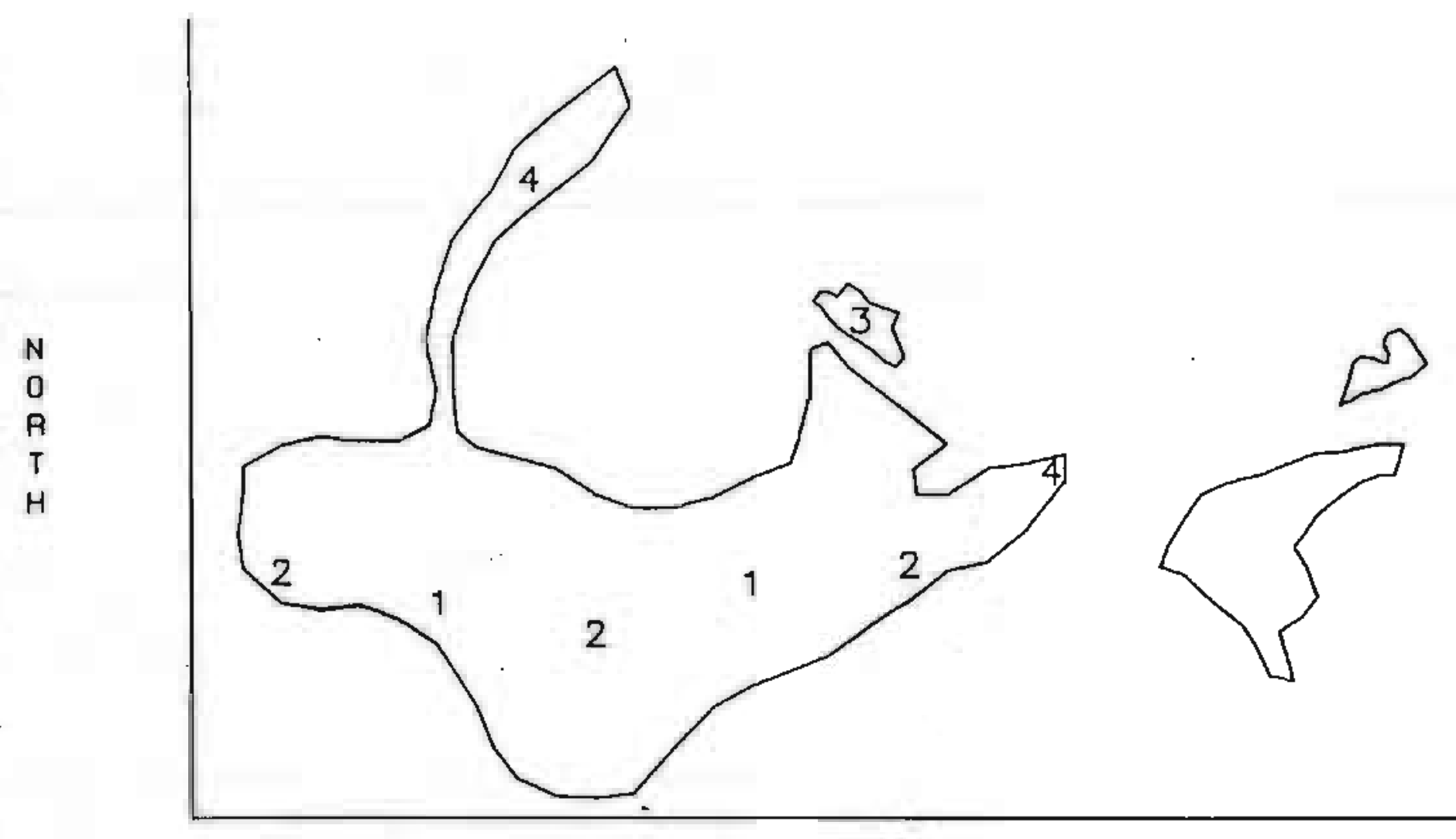

EAST

$$
\begin{array}{lllllllll}
\text { RESULTS } & 1 & 1 & 1 & 3=0.3325 \mathrm{UG} / \mathrm{L} & 2 & 2 & 2 & <-0.4200 \mathrm{UG} / \mathrm{L} \\
& 3 & 3 & 3 & 4 & 4 & 4.6925 \mathrm{UG} / \mathrm{L} & 4 & 4
\end{array}
$$

NO K/HS-132 TABLE 2.2 MAX LIMIT FOR WATER 


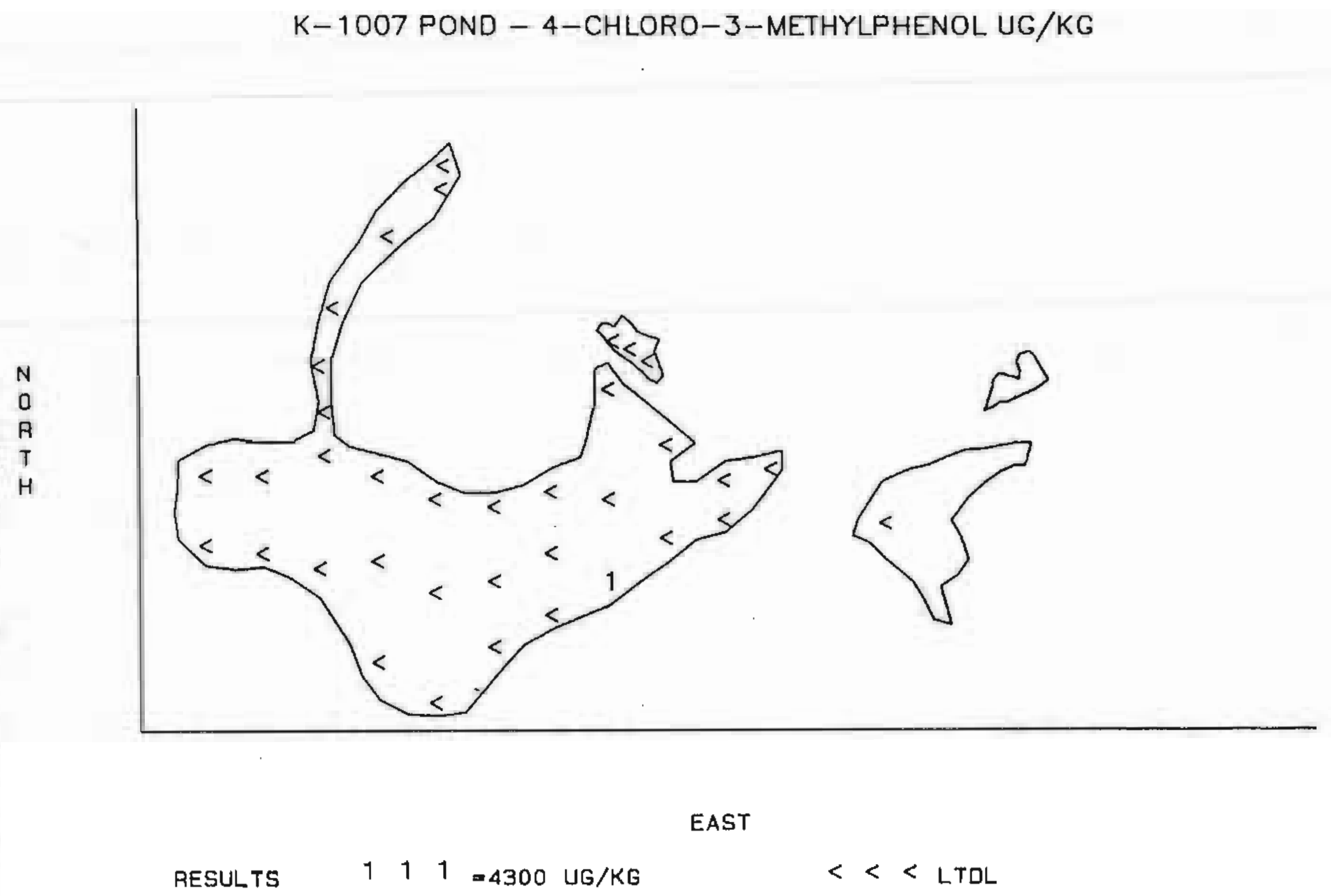

NO K/HS-132 TABLE 2.2 MAX LIMIT FOR SOIL 


$$
\text { K-1007 POND - 2-METHYLNAPHTHALENE UG/KG }
$$
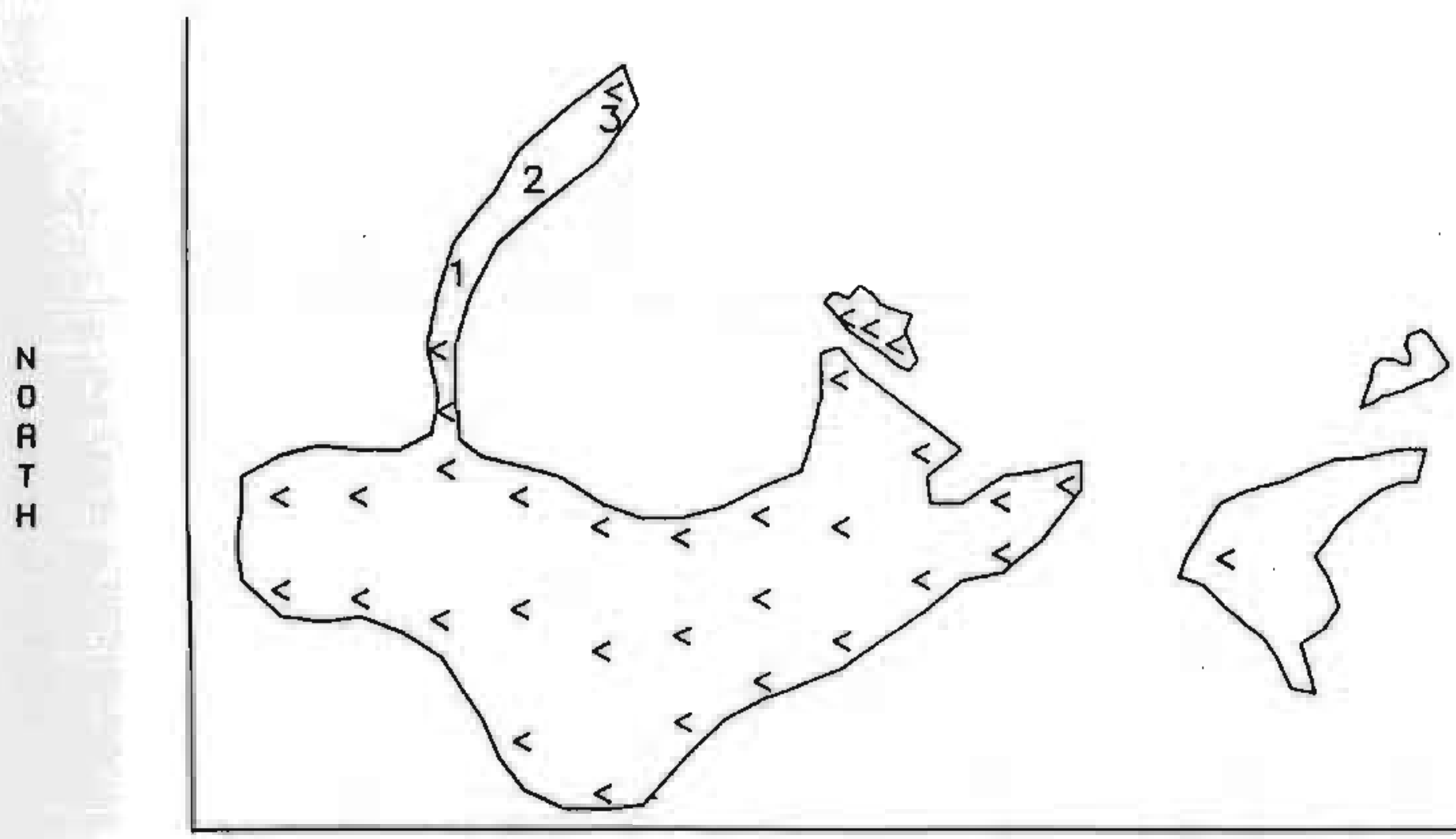

EAST

RESULTS

$$
\begin{array}{llllll}
1 & 1 & 1 & =18.0 & \text { UG } / K G \\
3 & 3 & 3 & =92.0 & \text { UG } / K G
\end{array}
$$$$
\begin{array}{llll}
2 & 2 & 2 & =42.0 \text { UG } / K G \\
< & < & <\text { LTDL }
\end{array}
$$

NO K/HS-132 TABLE 2.2 WAX LINIT FOR SOIL 


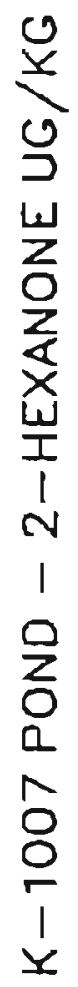

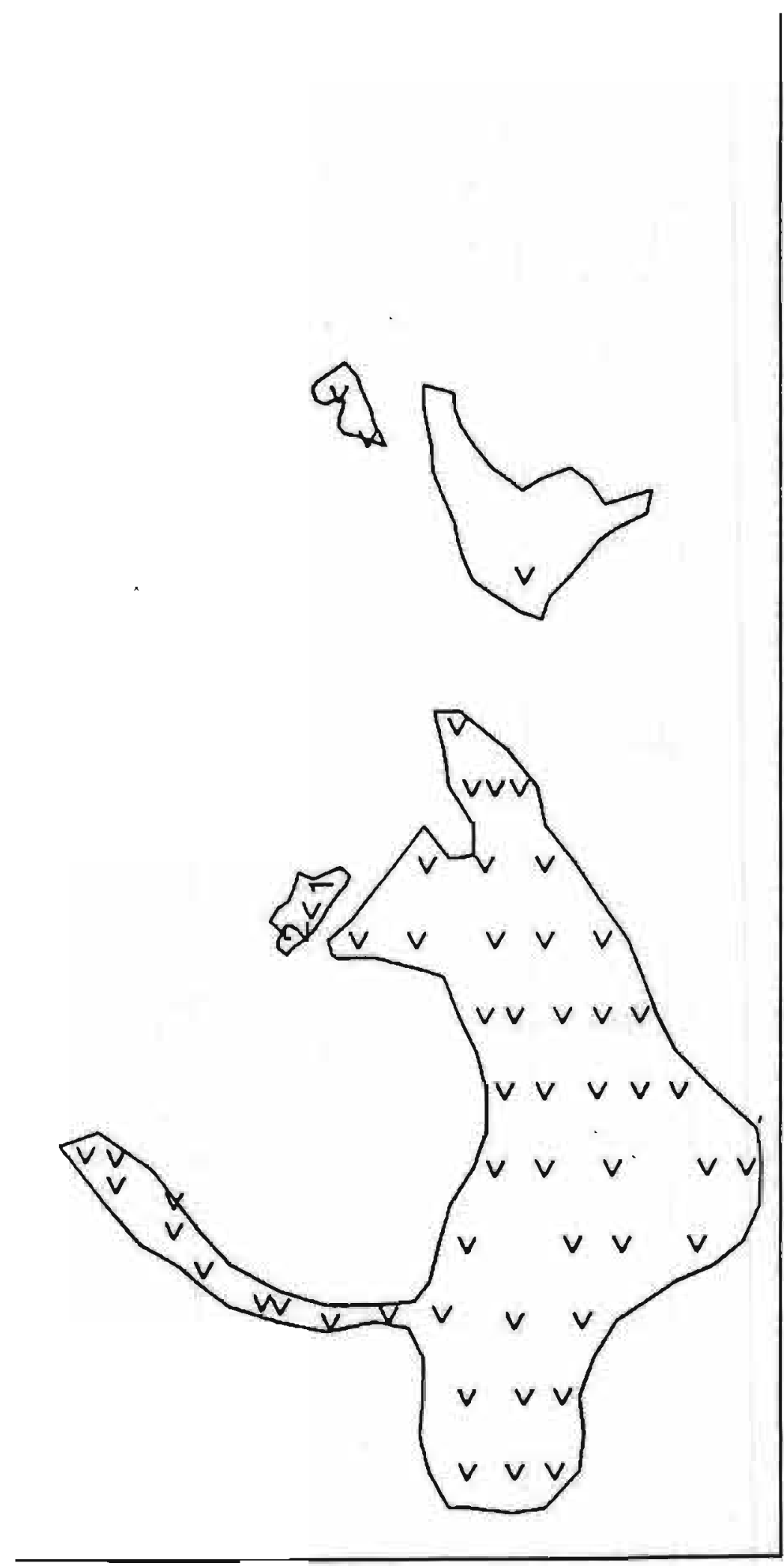

㫐

高

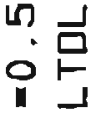

- v

- V

- v

홈 


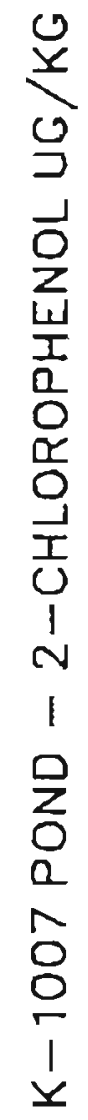

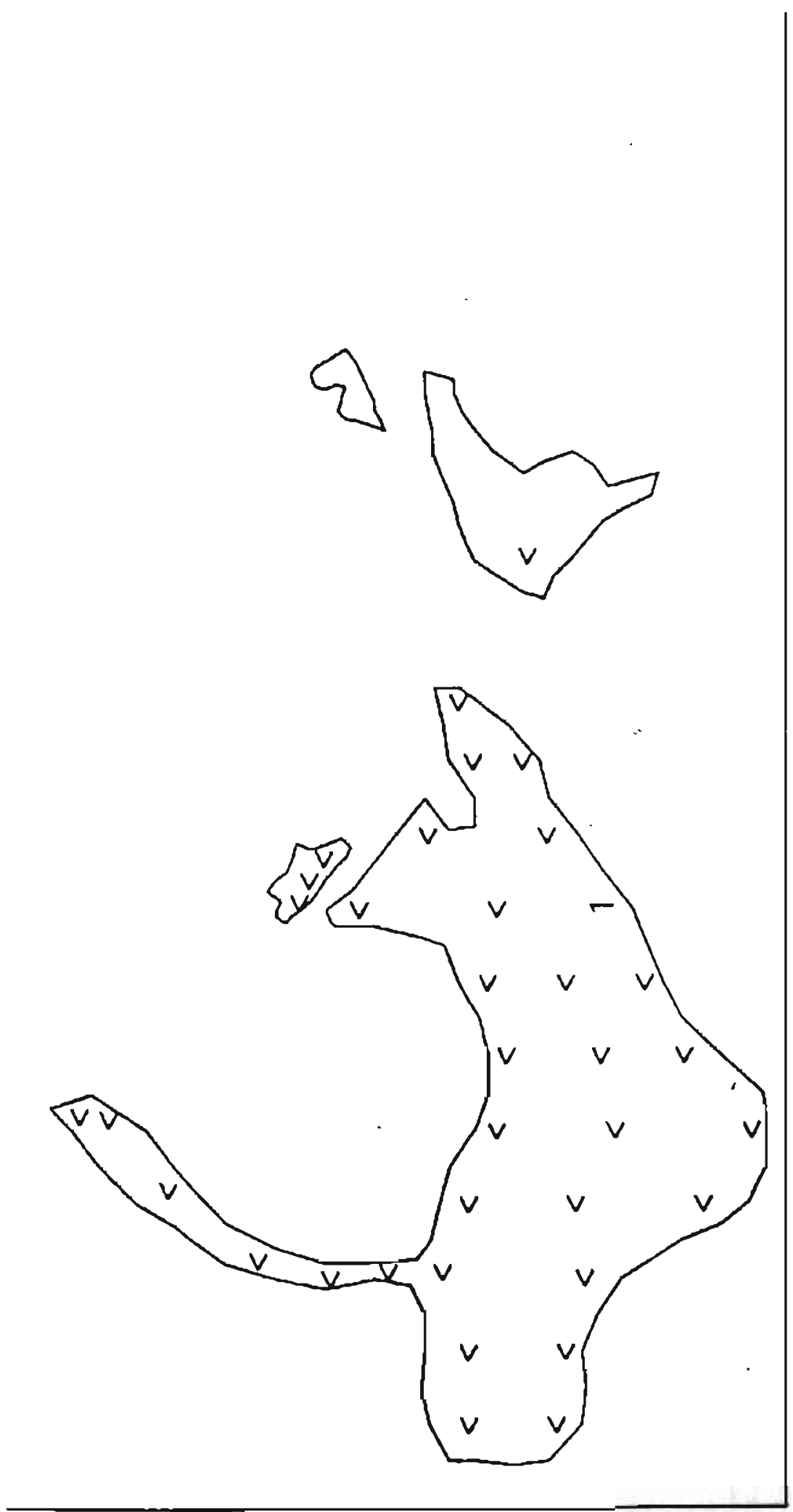

它

ㅁ
\[ \]
$v$
$v$
$v$

高

\begin{tabular}{l}
0 \\
0 \\
\hdashline \\
$\square$
\end{tabular}

$-$

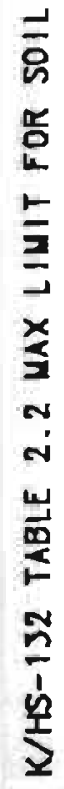

20匹トI 
0
$y$
0
0
$u$
$z$
0
$z$
5
5
0
1
2
1
0
2
0
0
1
0
0
1
1
$x$
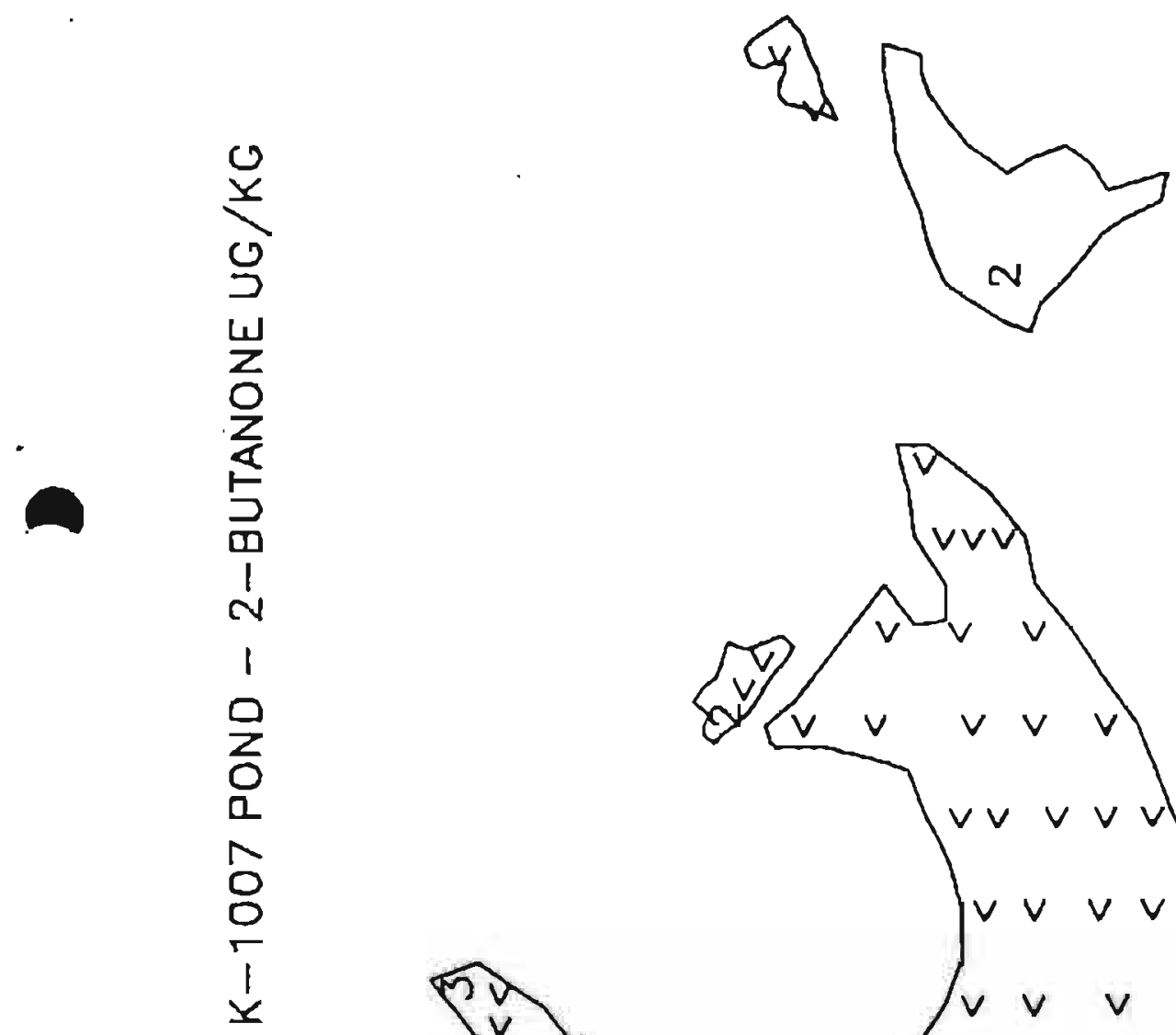

zoarI
0
$y$
0
0
0
0

$N$

$N$

$N$

枈

익

00

in

$-m$

$-M$

$-M$

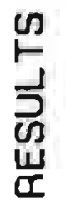




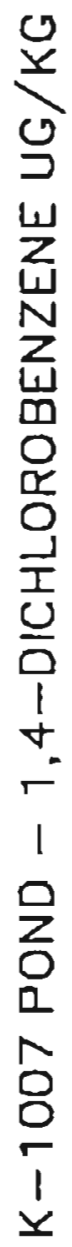
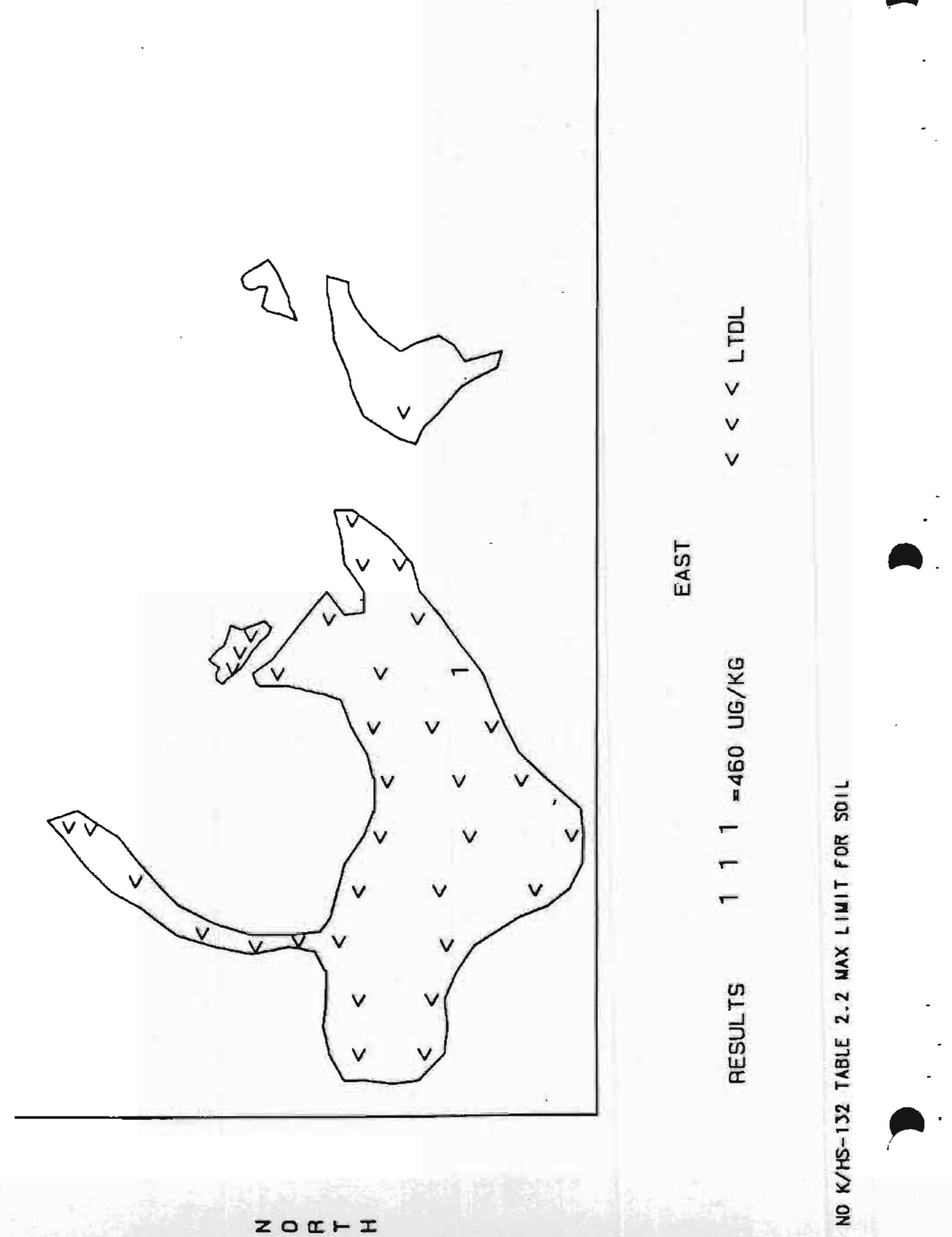

zox I 

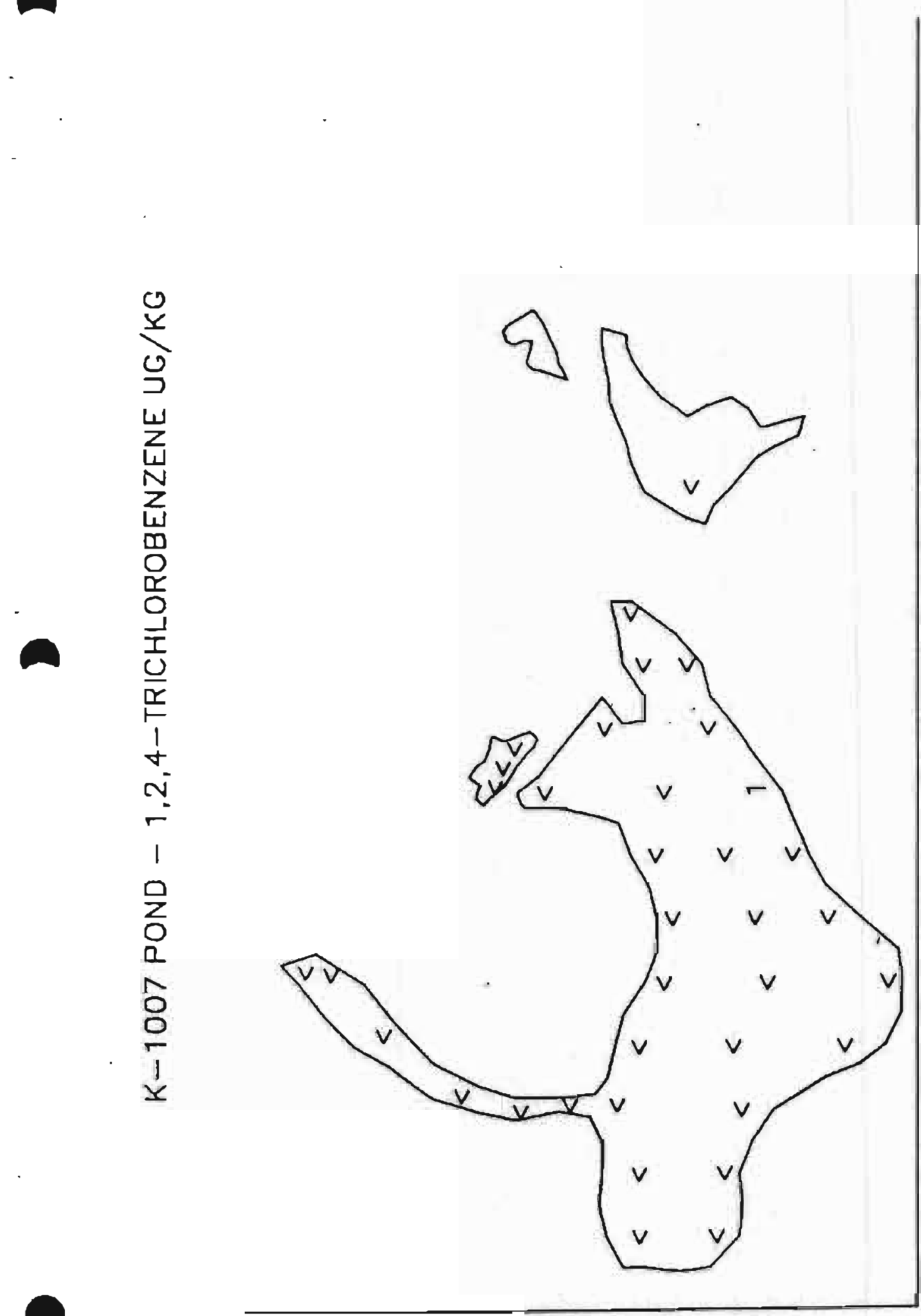

它

$\frac{0}{3}$

임

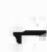

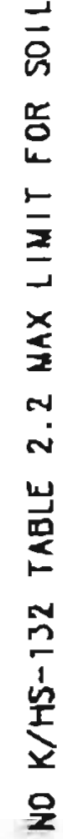


K-1007 POND - PYRENE UG/KG

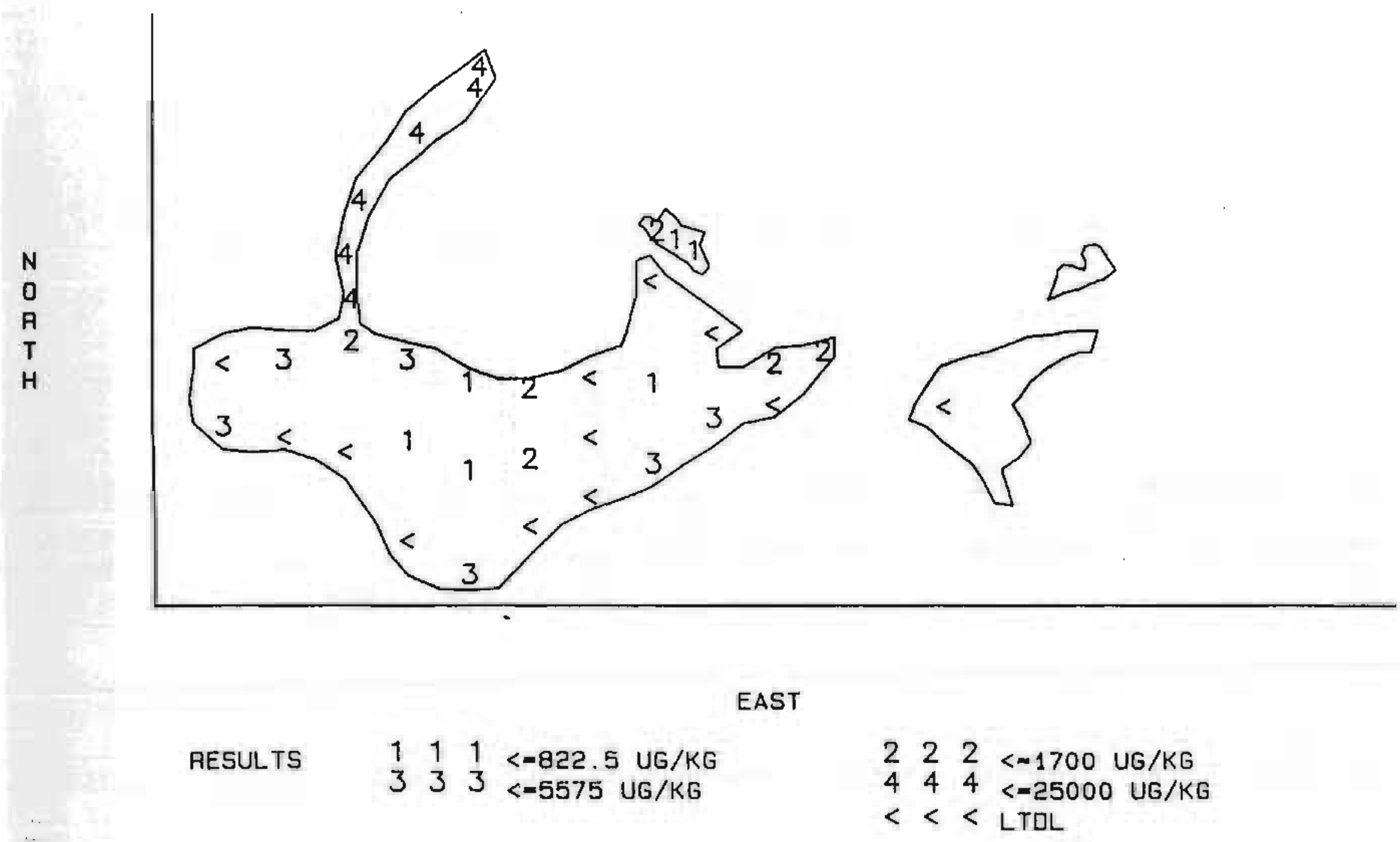

NO K/HS-132 TABLE 2.2 MAX LIMIT FOR SOIL

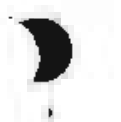


K-1007 POND - PHENANTHRENE UG/KG

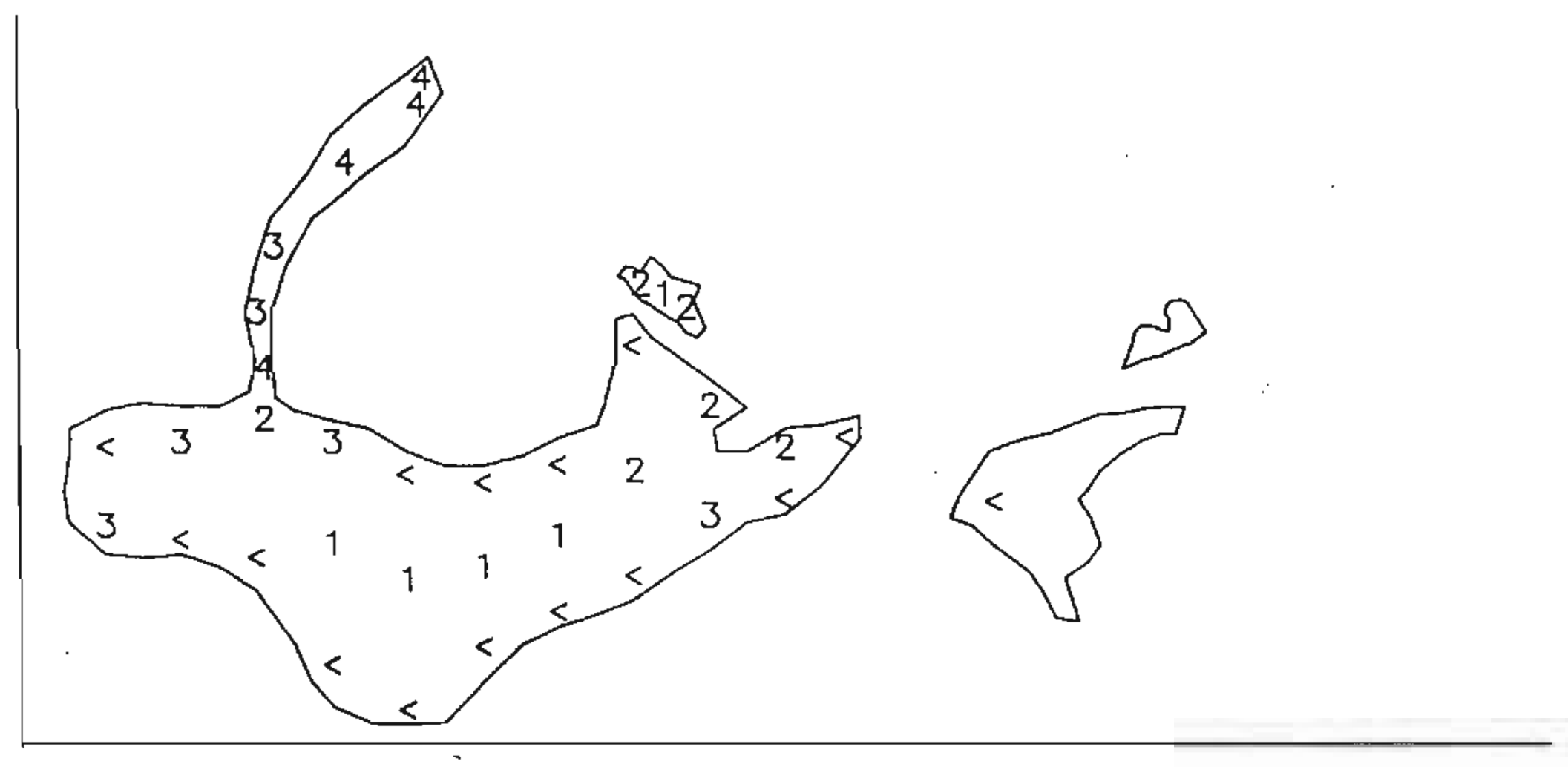

EAST

$\begin{array}{llllll}\text { RESULTS } & 1 & 1 & 1<-195 \text { UG/KG } & 2 & 2<<-630 \mathrm{UG} / \mathrm{KG} \\ & 3 & 3 & 3<-2300 \mathrm{UG} / \mathrm{KG} & 4 & 4<<13000 \mathrm{UG} / \mathrm{KG} \\ & & <<<\mathrm{LTDL}\end{array}$


K-1007 POND - PCB (AROCLOR-1254) UG/KG

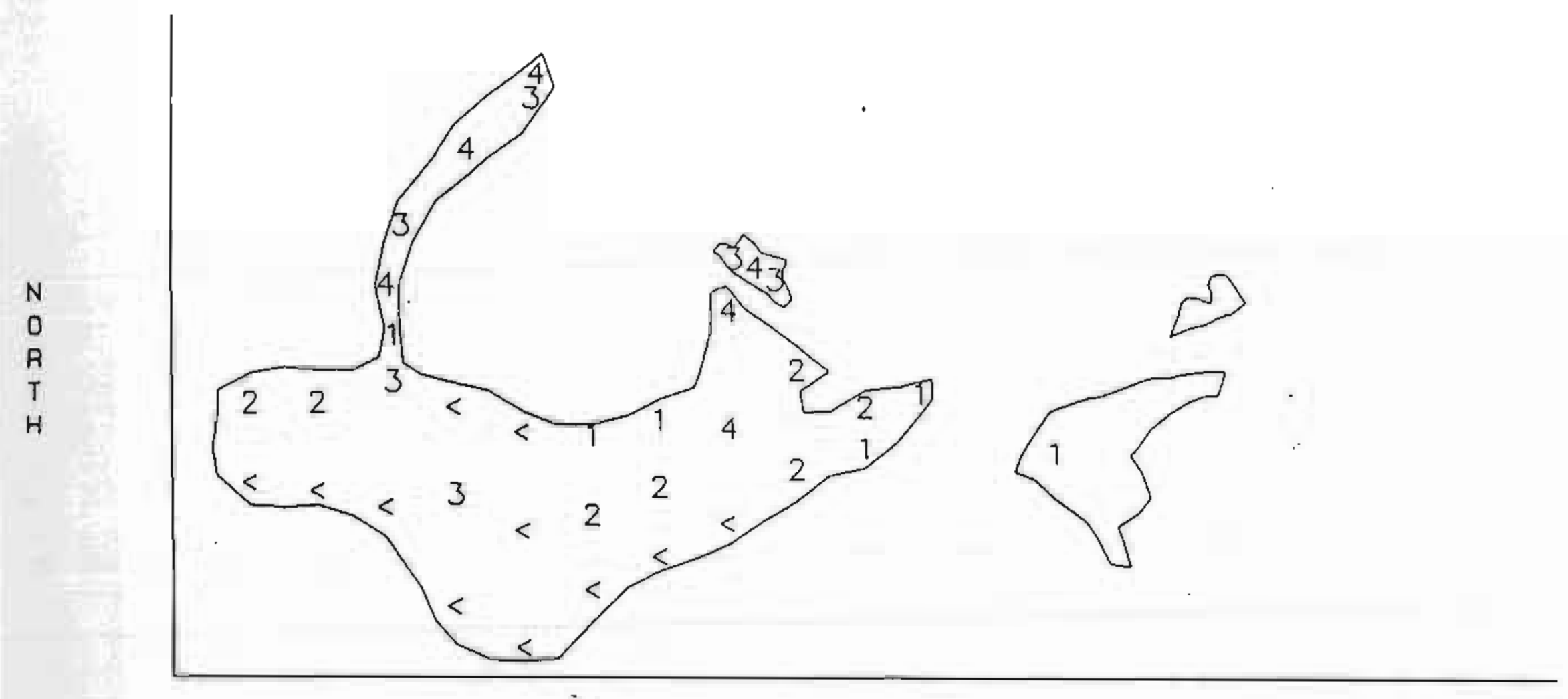

EAST

$\begin{array}{lllll}\text { RESULTS } & 1 & 1 & 1 & <-532.5 \text { UG } / K G \\ & 3 & 3 & 3 & <-3650 \text { UG/KG }\end{array}$

$\begin{array}{llll}2 & 2 & 2 & <=2600 \text { UG } / K G \\ 4 & 4 & 4 & <=16900 \text { UG } / K G \\ < & < & <\text { LTDL }\end{array}$

K/HS-132 TABLE 2.2 MAX LIMIT FOR SOIL=100,000 UG/KG 


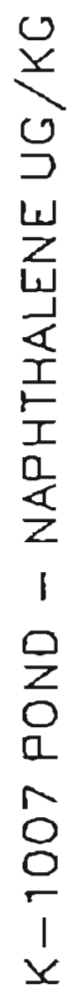
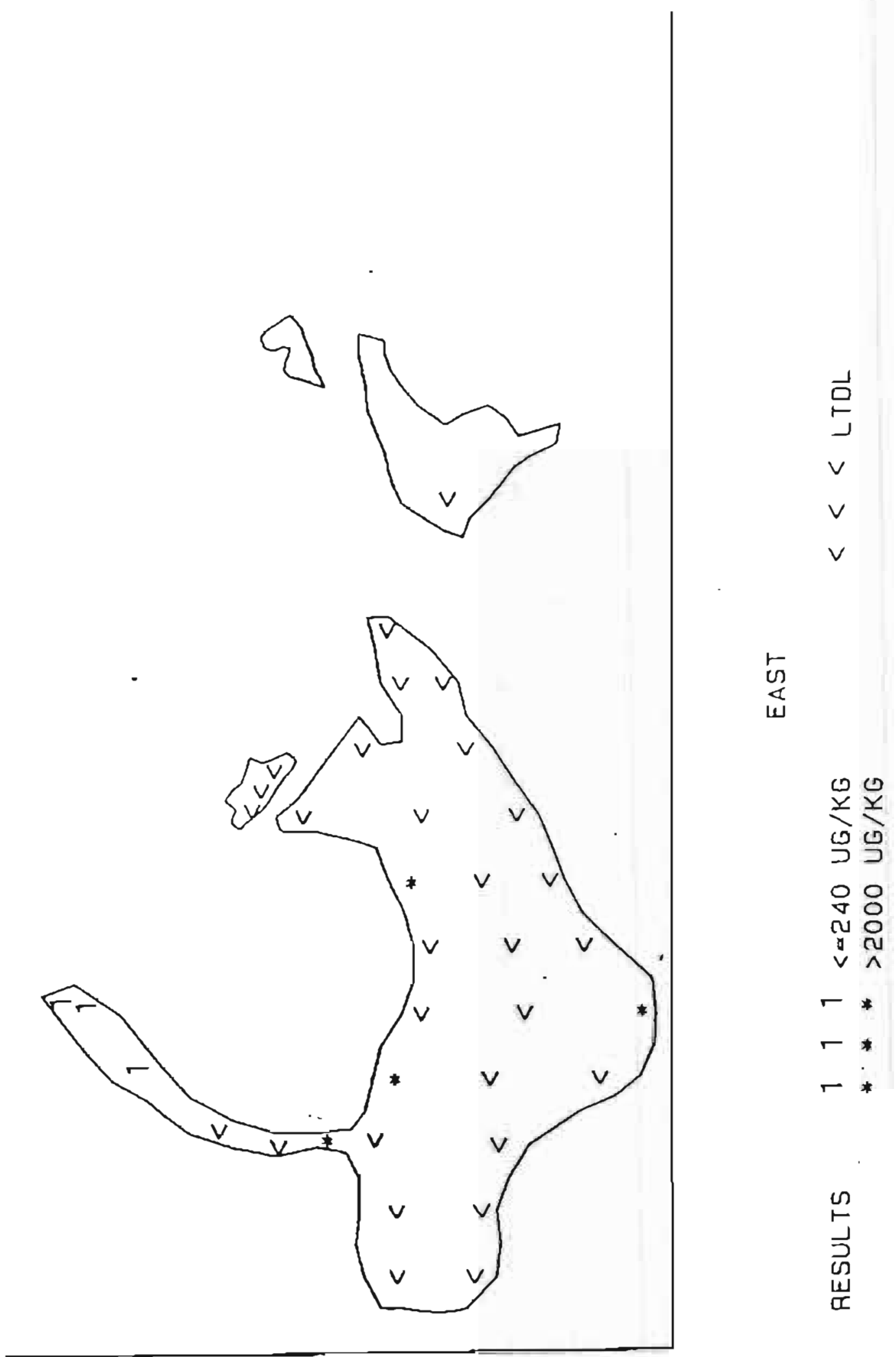

zoxトI 


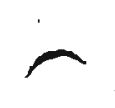

O XIINEAdV

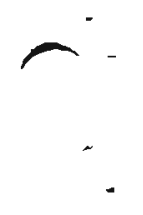


7

$-$

-

$\rightarrow$

7 
Figure C.1

NPDES DATA - CHEMICAL OXYGEN DEMAND MG /L

Monthly Average Concentration

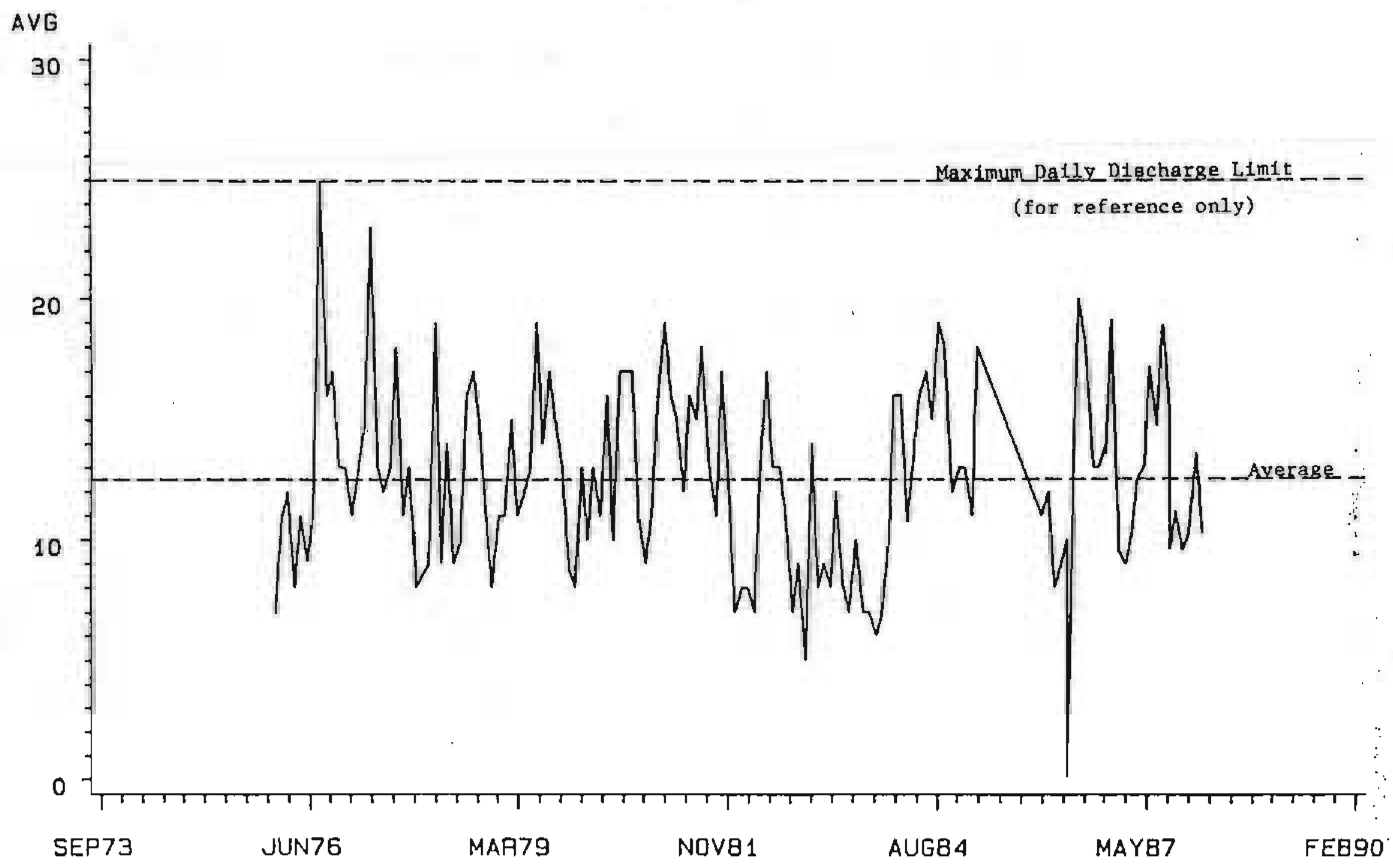

SAMPLE DATE 
Figure C.2

\section{NPDES DATA - CHROMIUM MG $/ L$}

Monthly Average Concentration

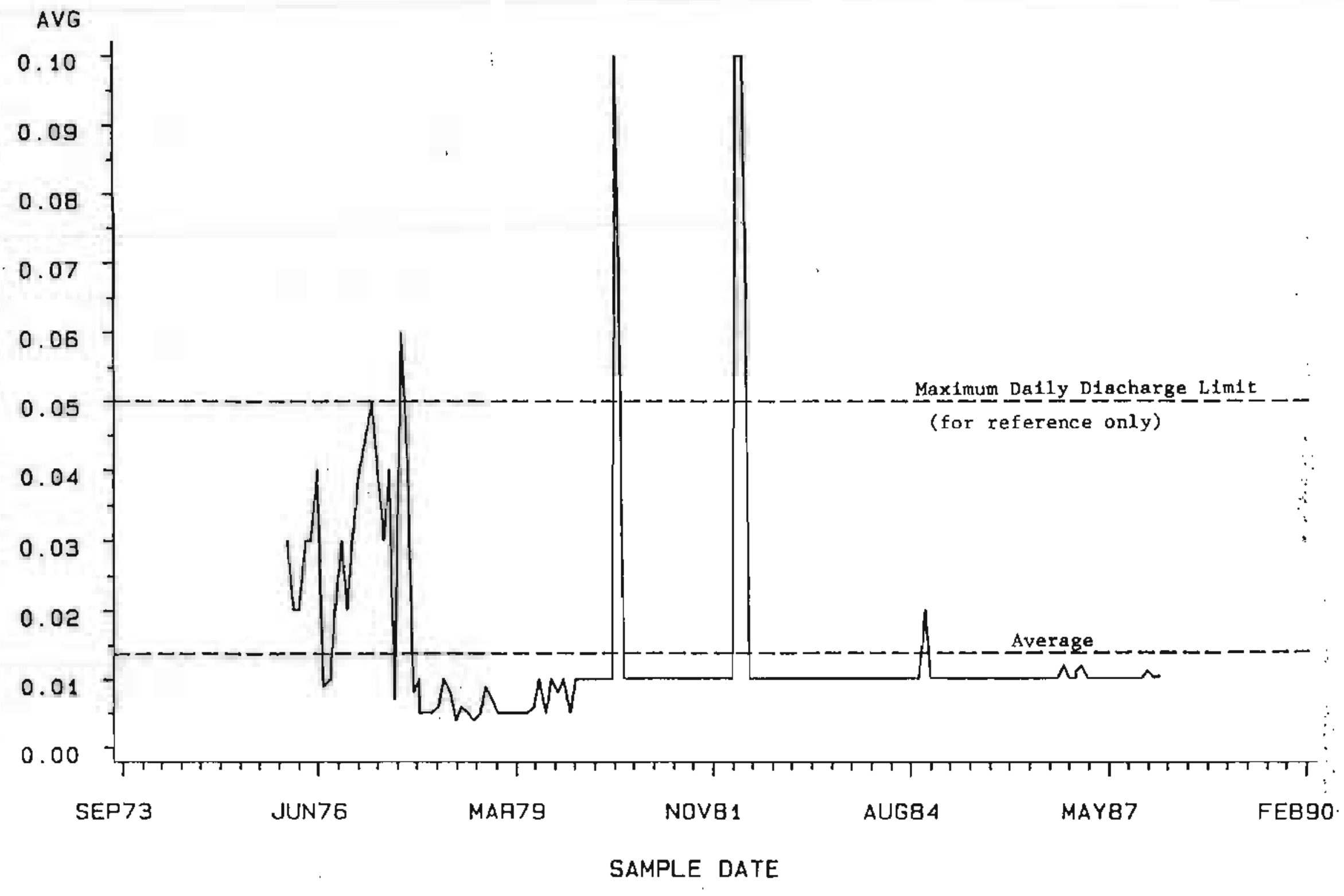

( 
Figure C. 3

NPDES DATA - DISSOLVED OXYGEN MG/L

Monthly Average Concentration

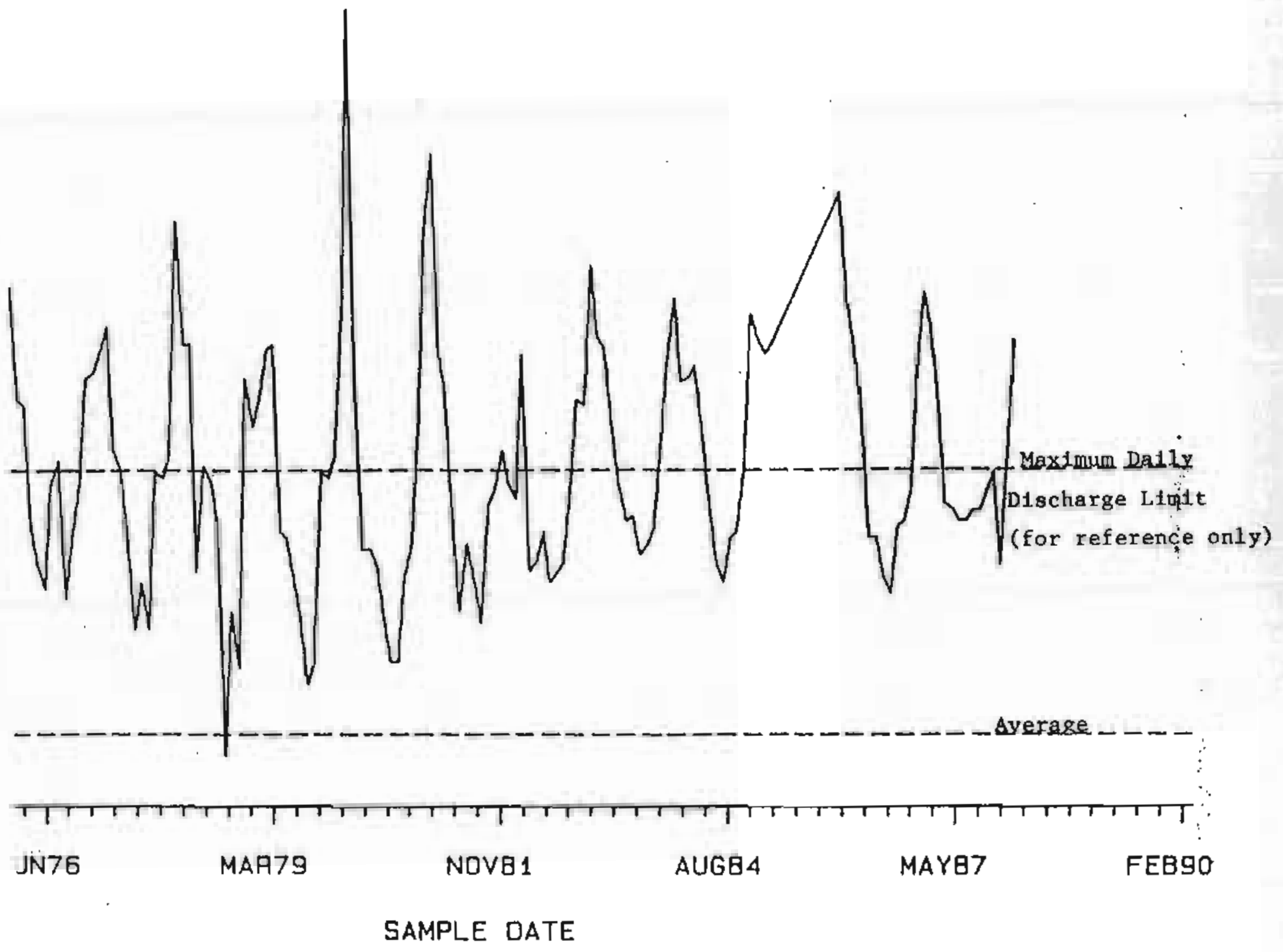




\section{NPDES DATA PIgure C.ं4 FL MG/L}

Monthly Average Concentrations

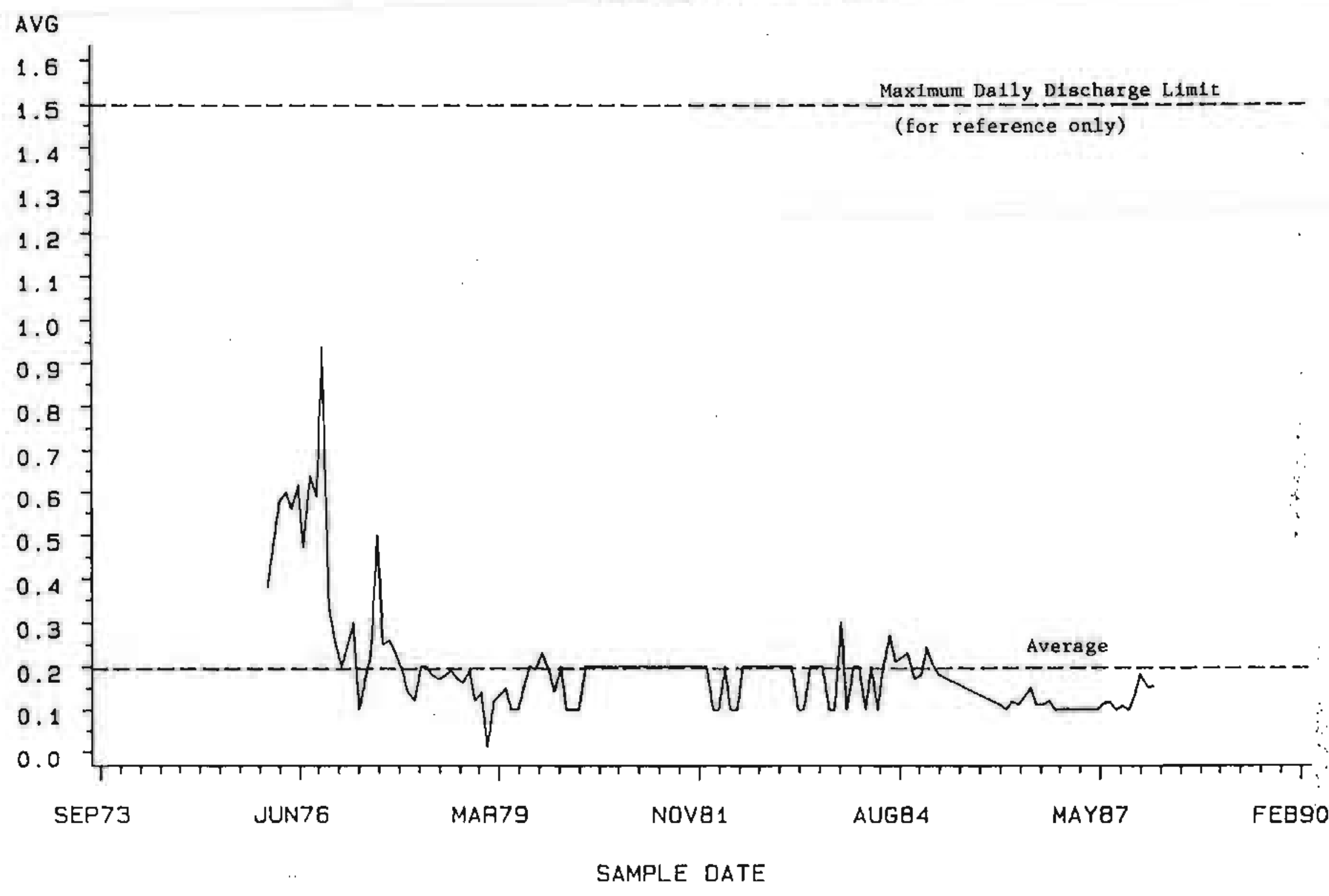

( 
Figure C.5

NPDES DATA - OIL AND GREASE MG/L

Monthly Average Concentrations

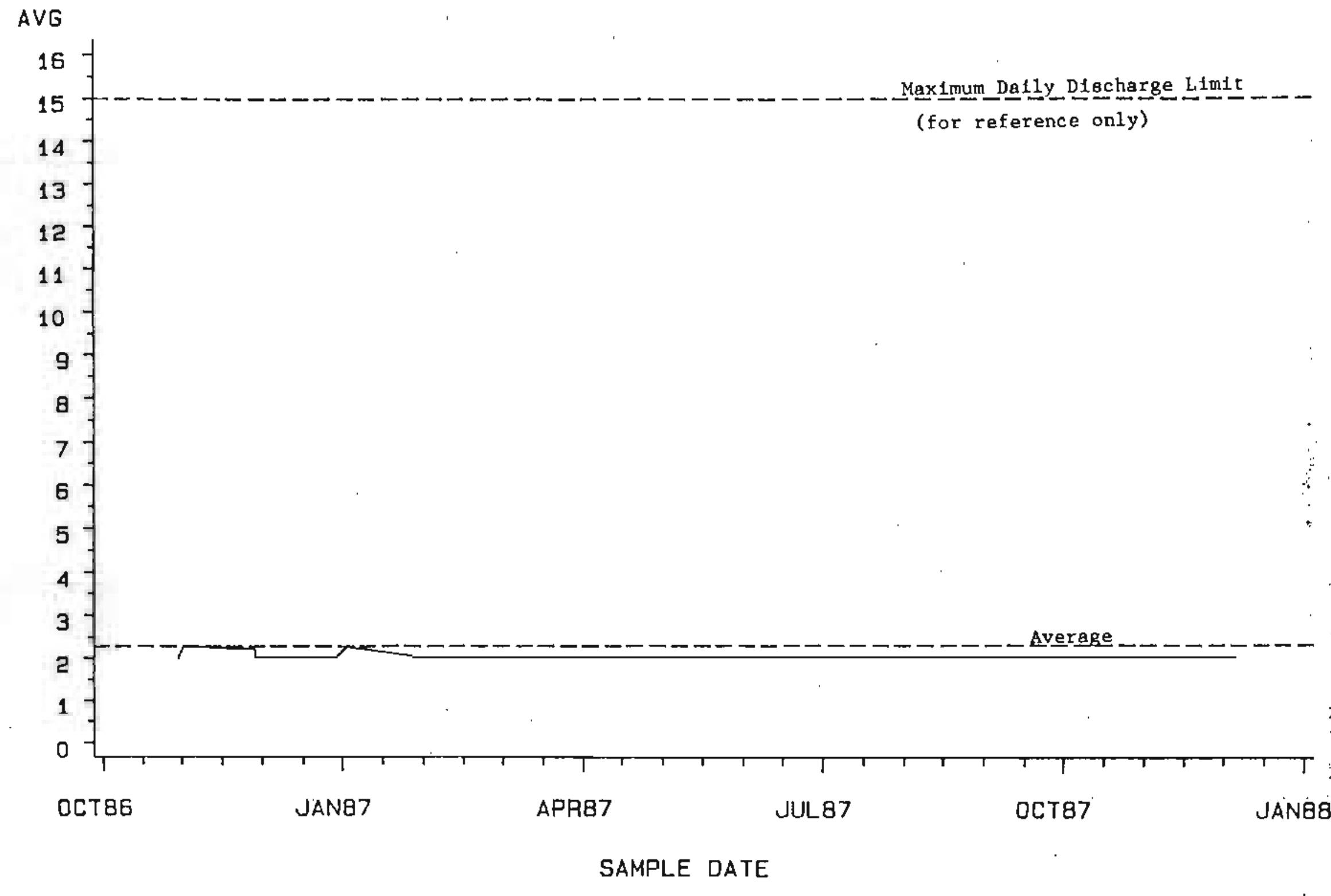


Figure 0.6

NPDES DATA - PERCHLOROETHYLENE MG/L

Monthly Average Concentrations

\section{AVG}

0.30
0.28
0.26
0.24
0.22
0.20
0.18
0.16
0.14
0.12
0.10
0.08
0.06
0.04
0.02
0.00

FEB84

MAYE 4

AUGB 4

Maximum Da11y Discharge Limits

(for reference only)

Average

SAMPLE DATE

( 
Figure $\mathrm{C} .7$

NPDES DATA - TOTAL SUSPENDED SOLIDS MG/L

Monthly Average Concentrations

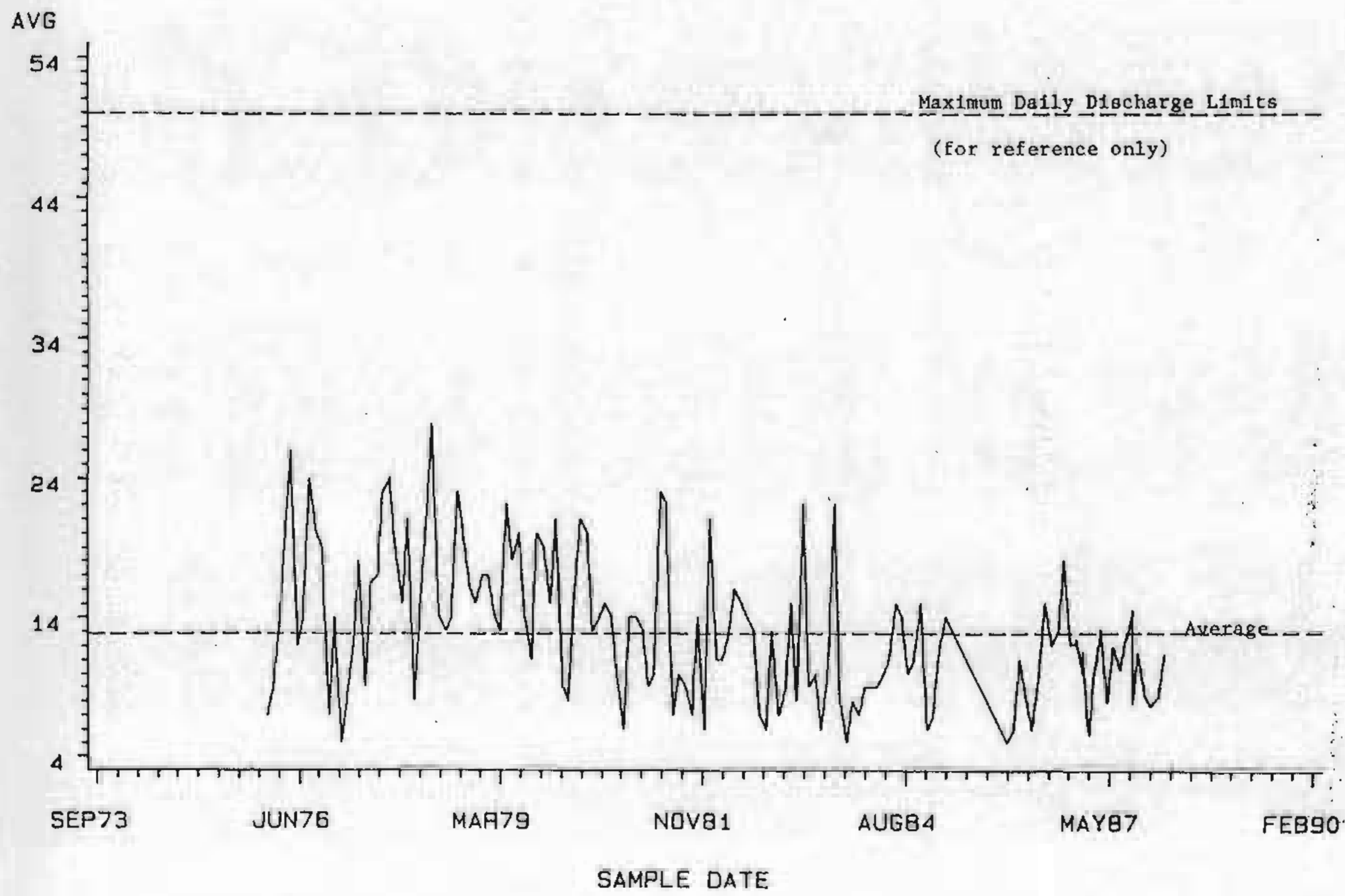


Figure C.8

NPDES DATA - URANIUM MG/L

Monthly Average Concentrations

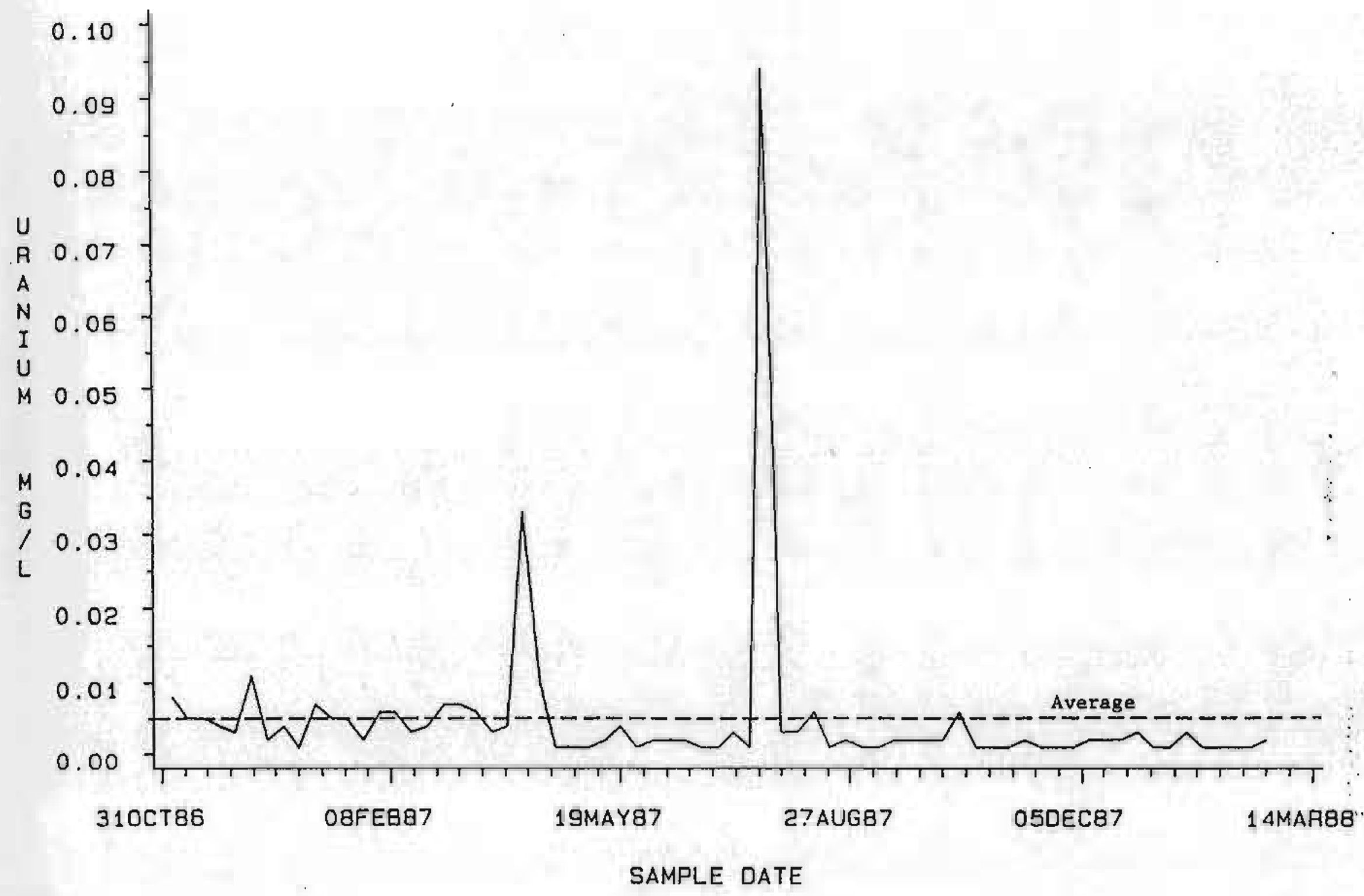

C 
Figure C.9

NPDES DATA - PH

MonthIy Average Concentrations

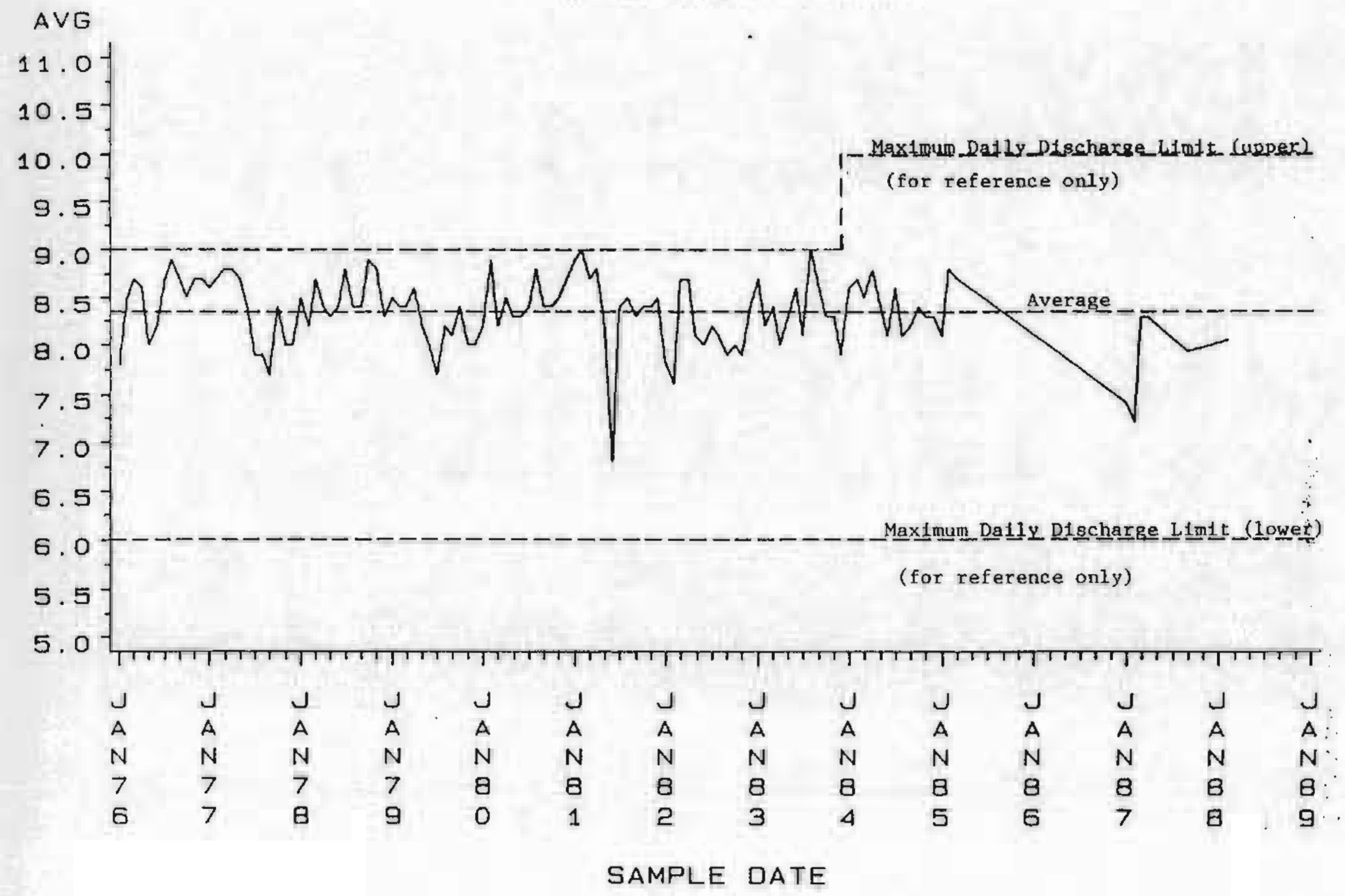


Figure C.10

NPDES DATA - TEMPERATURE DEG C

Monthly Average Concentrations

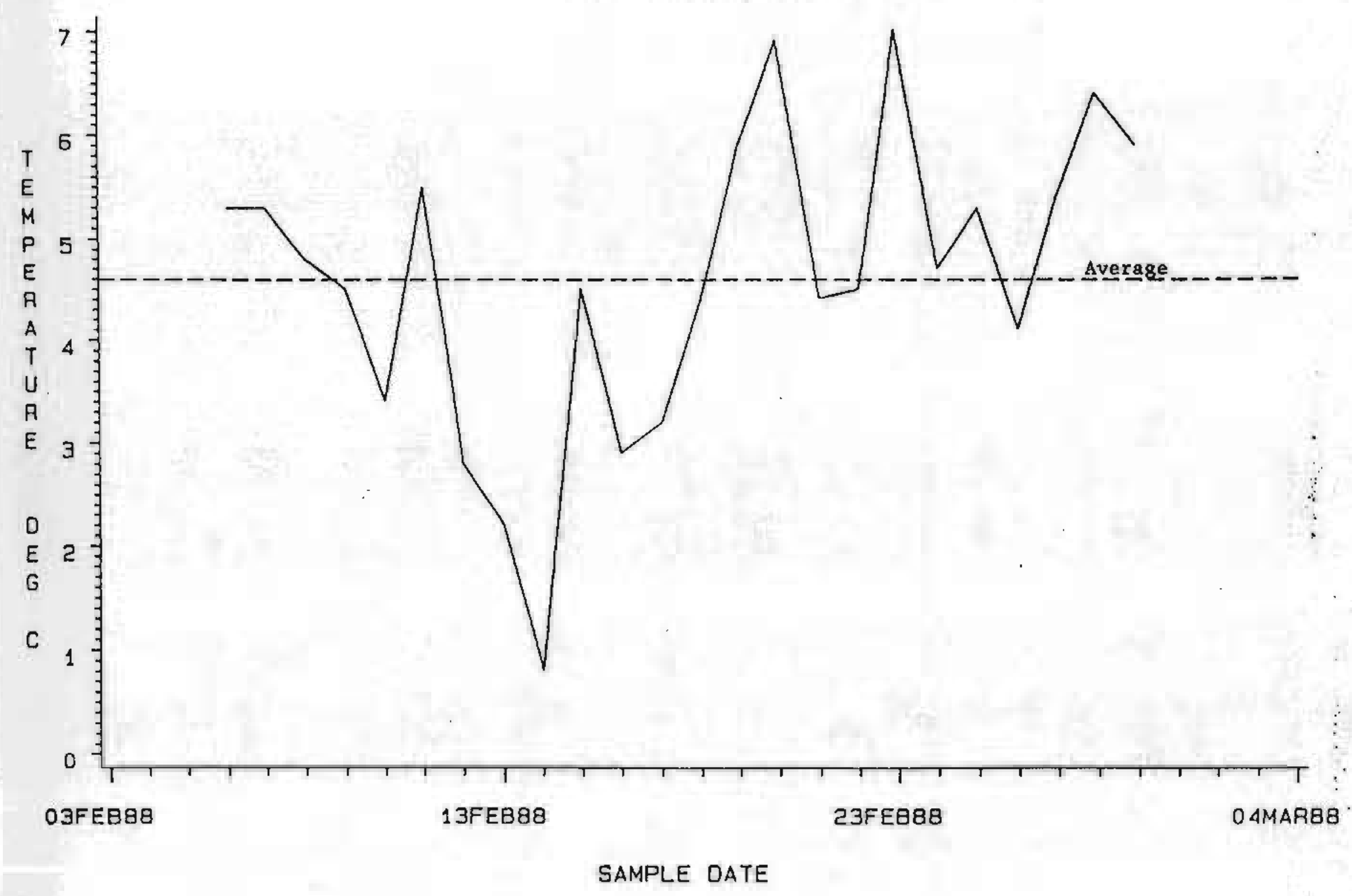

( 
Figure C.11

NPDES DATA - CHEMICAL OXYGEN DEMAND MG/L

Individual Sample Results

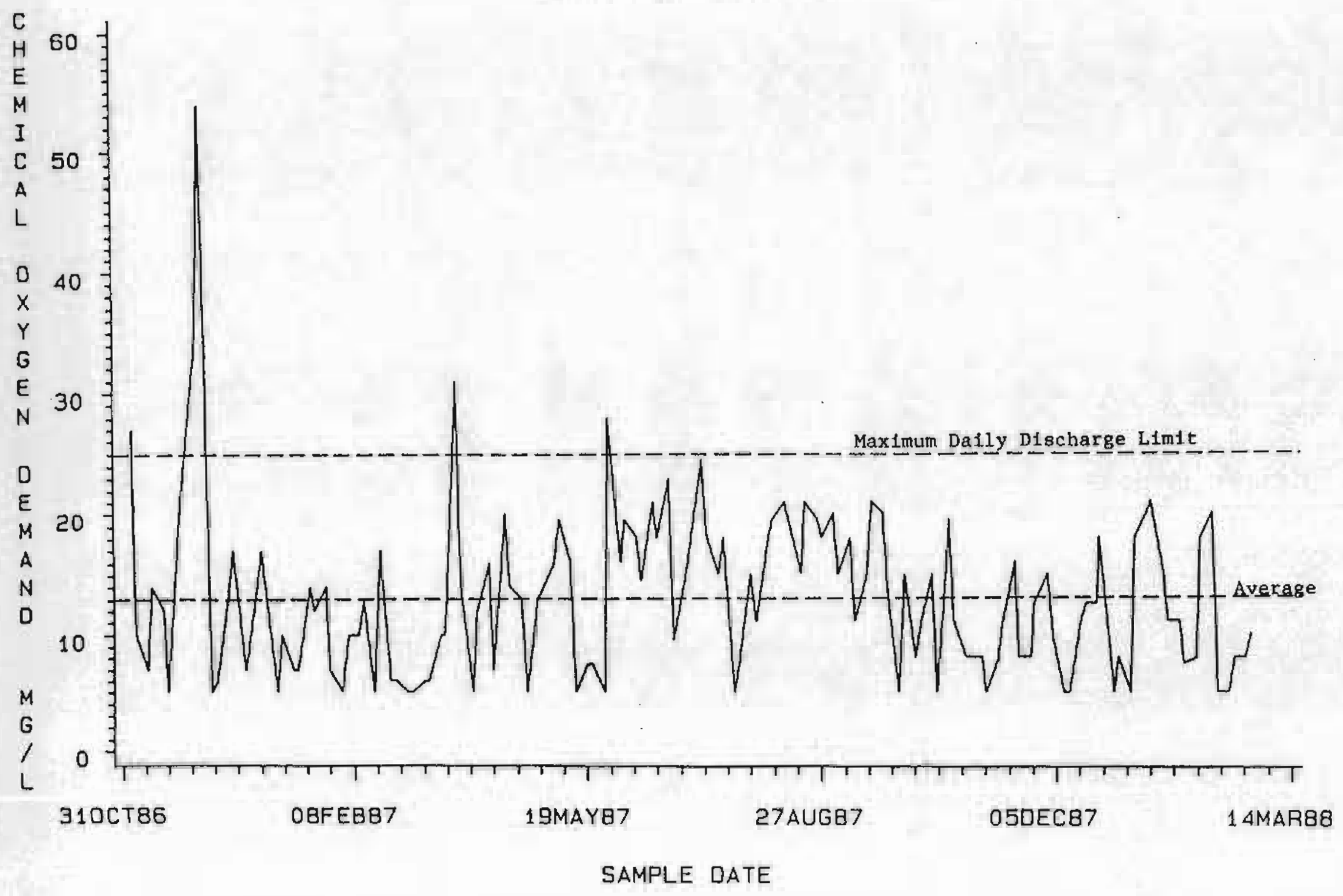


Individual Sample Results

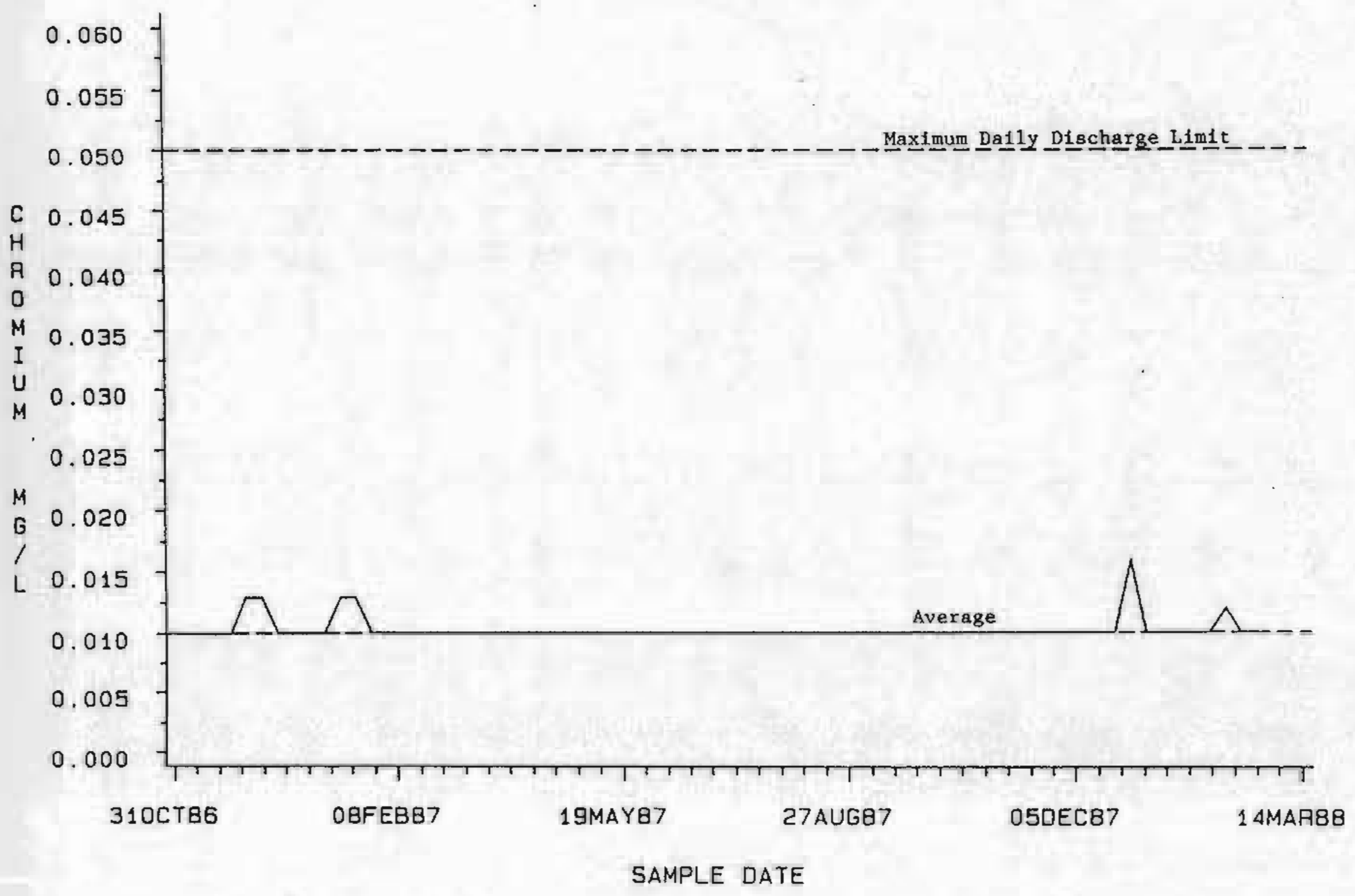

( 


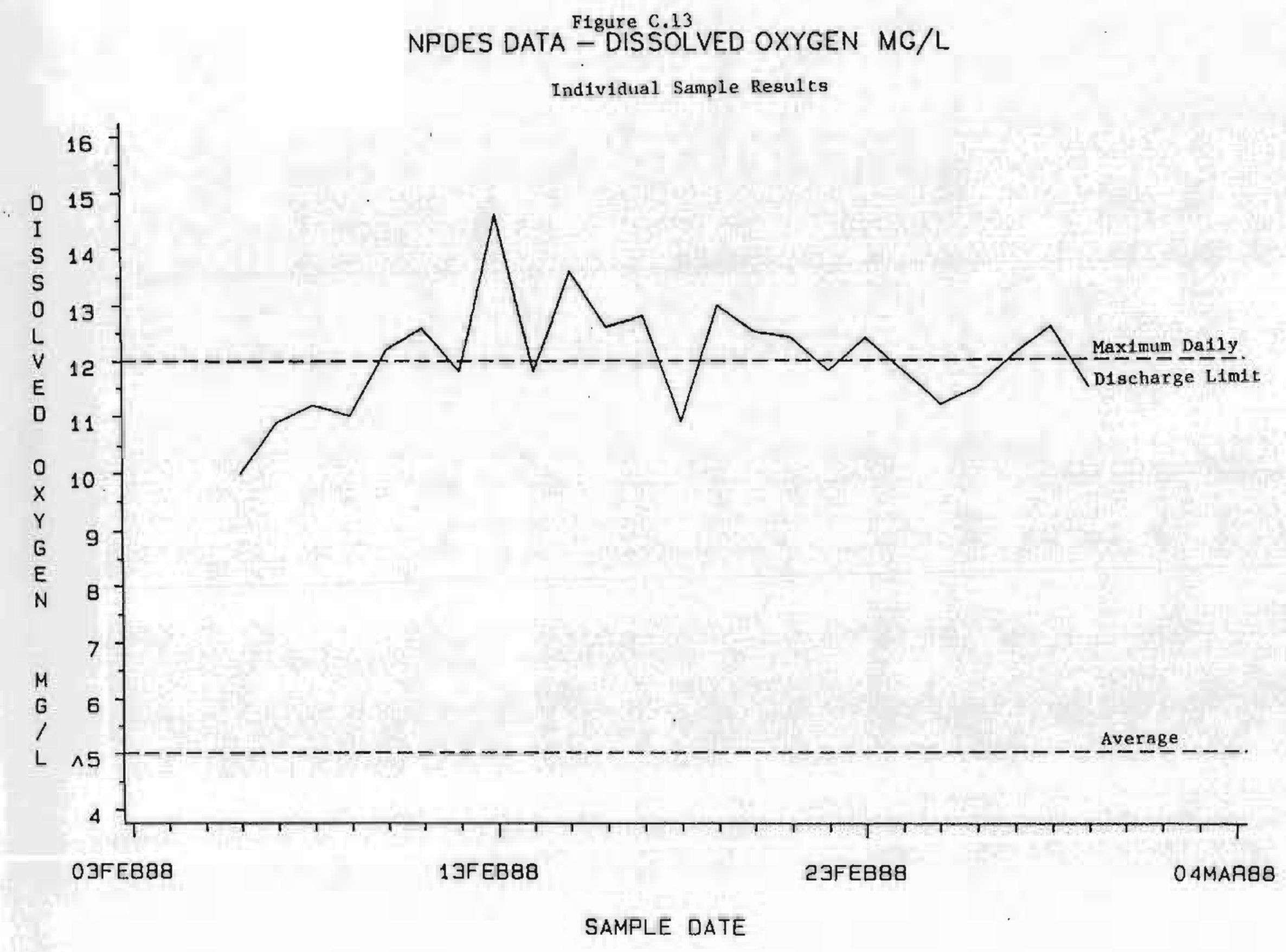


Individual Sample Results

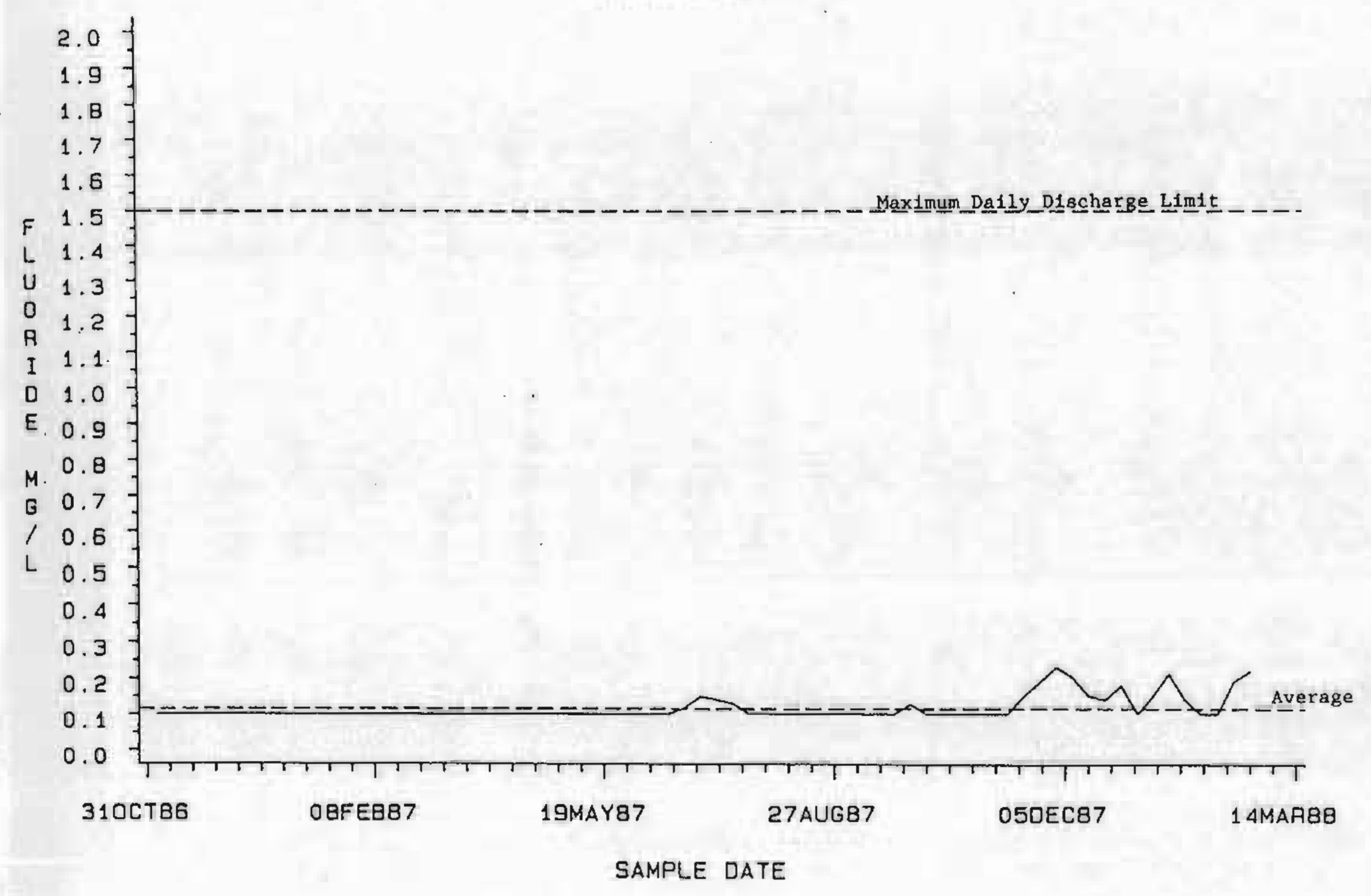

( 
NPDES DATA - OIL AND GREASE MG/L

Individual Sample Results

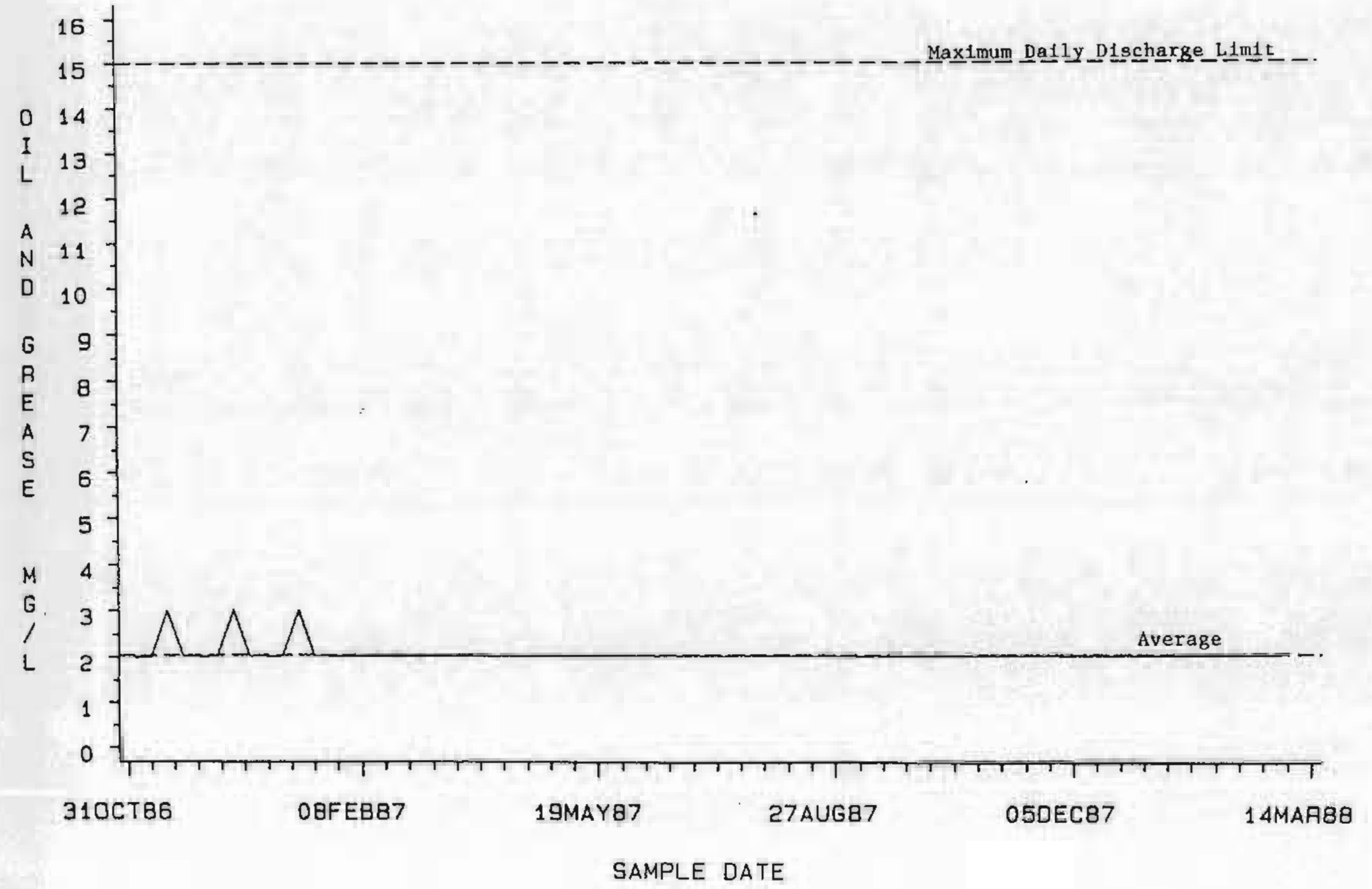


Figure C. 16

NPDES DATA - PH

Individual Sample Results

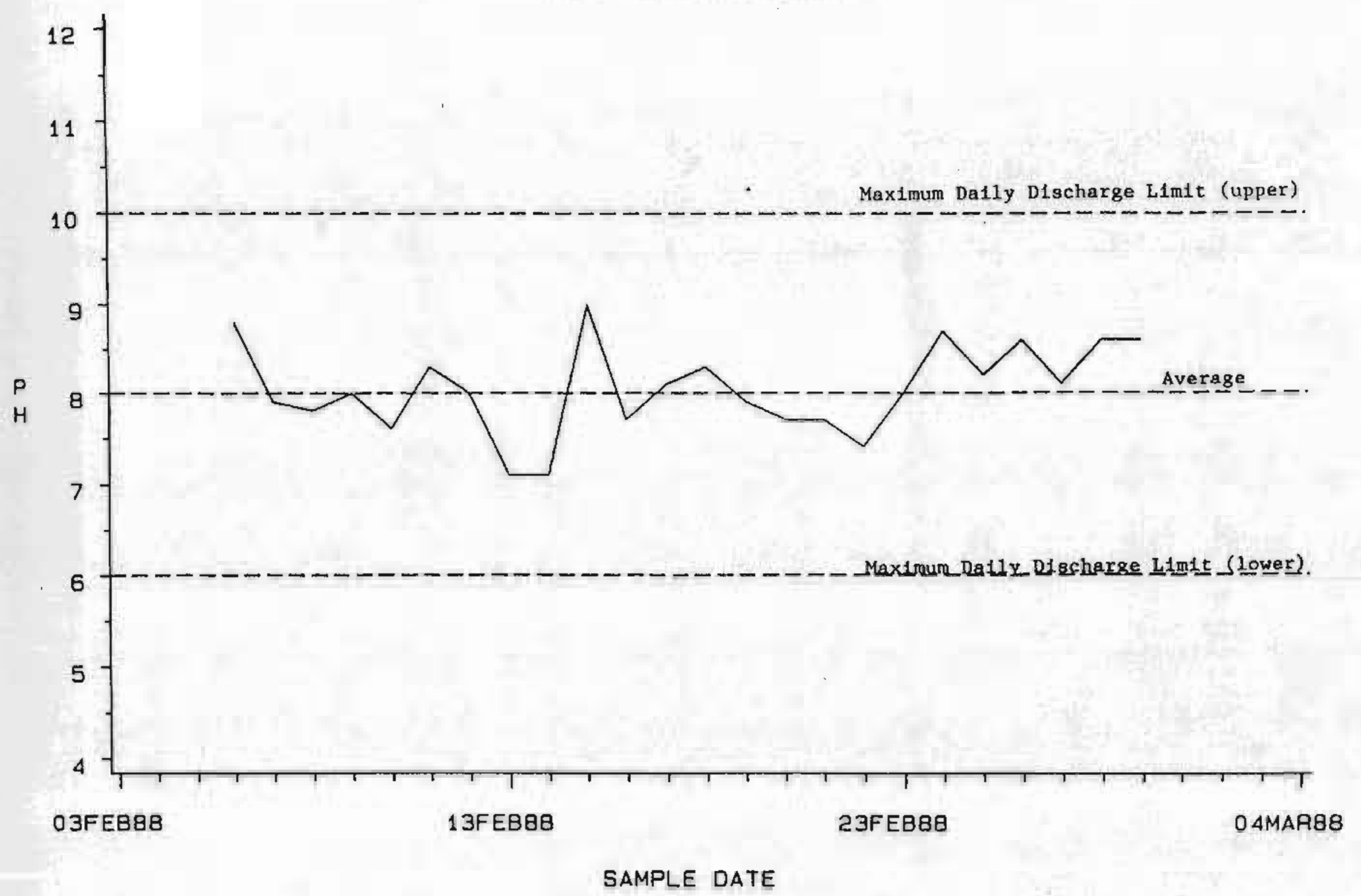

(

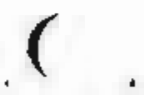


NPDES DATA - TOTAL SUSPENDED SOLIDS MG/L

Indfvidua1 Sample Reaults

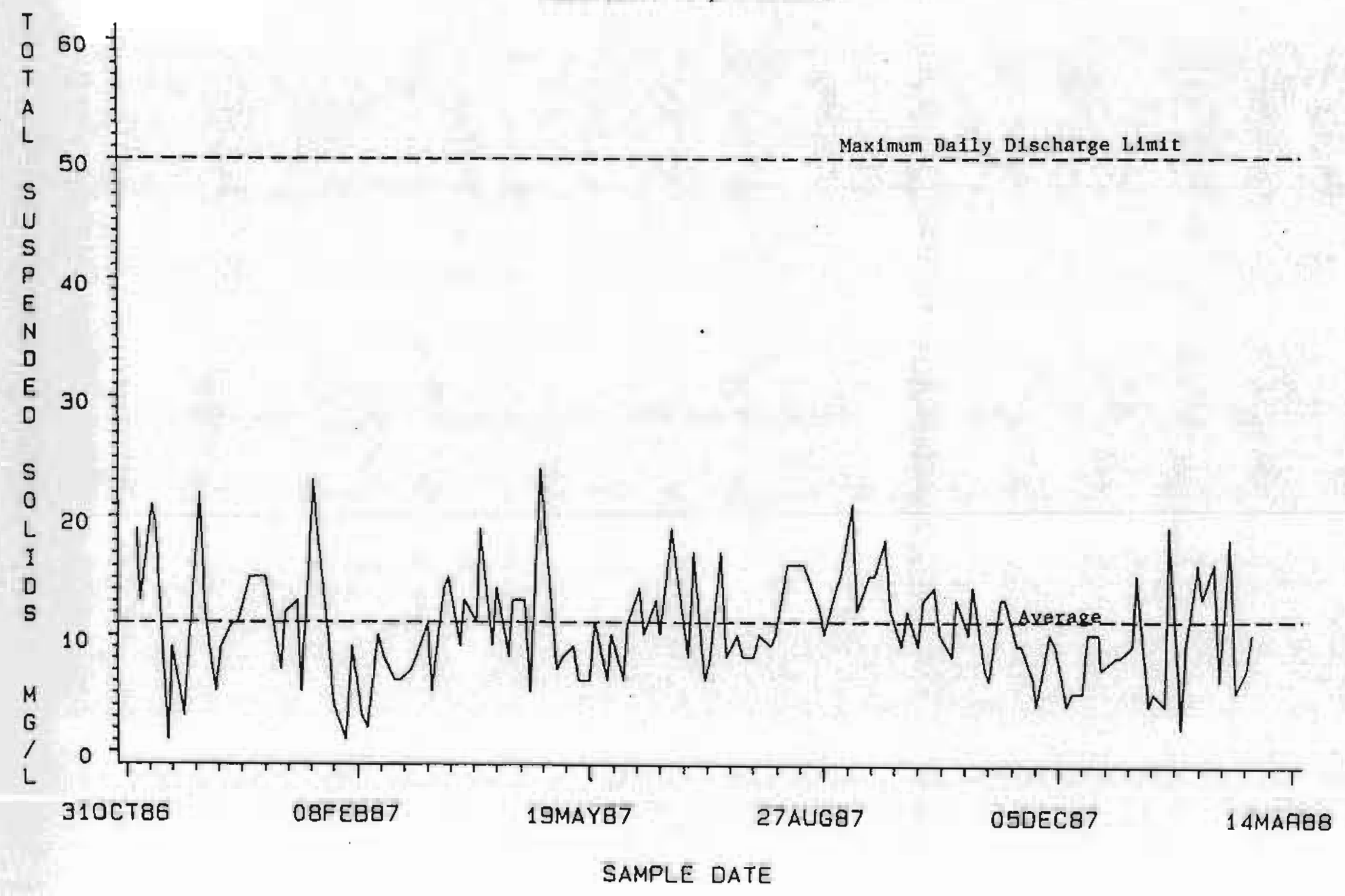




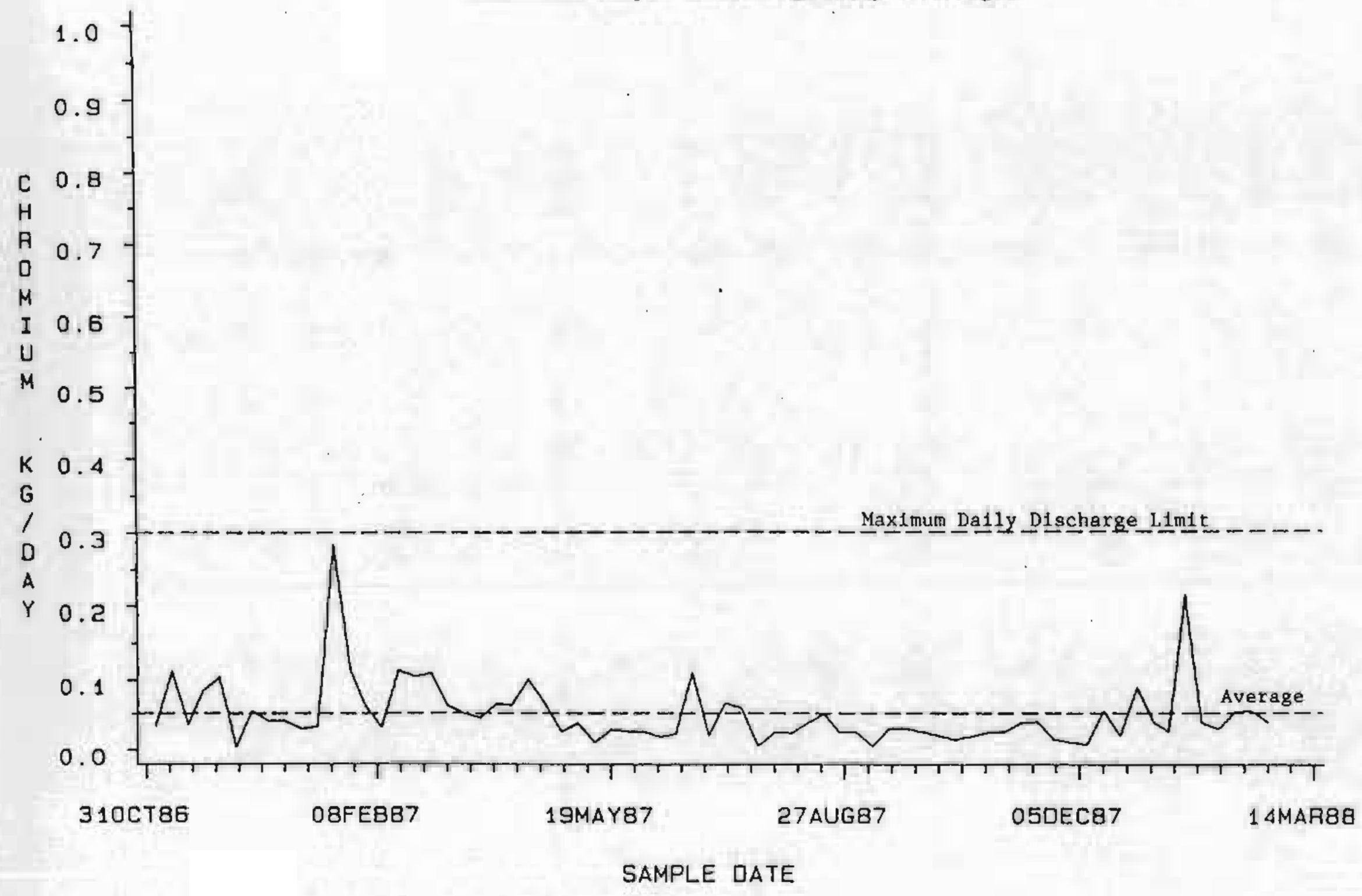


NPDES DATA - CHEMICAL OXYGEN DEMAND KG/DAY

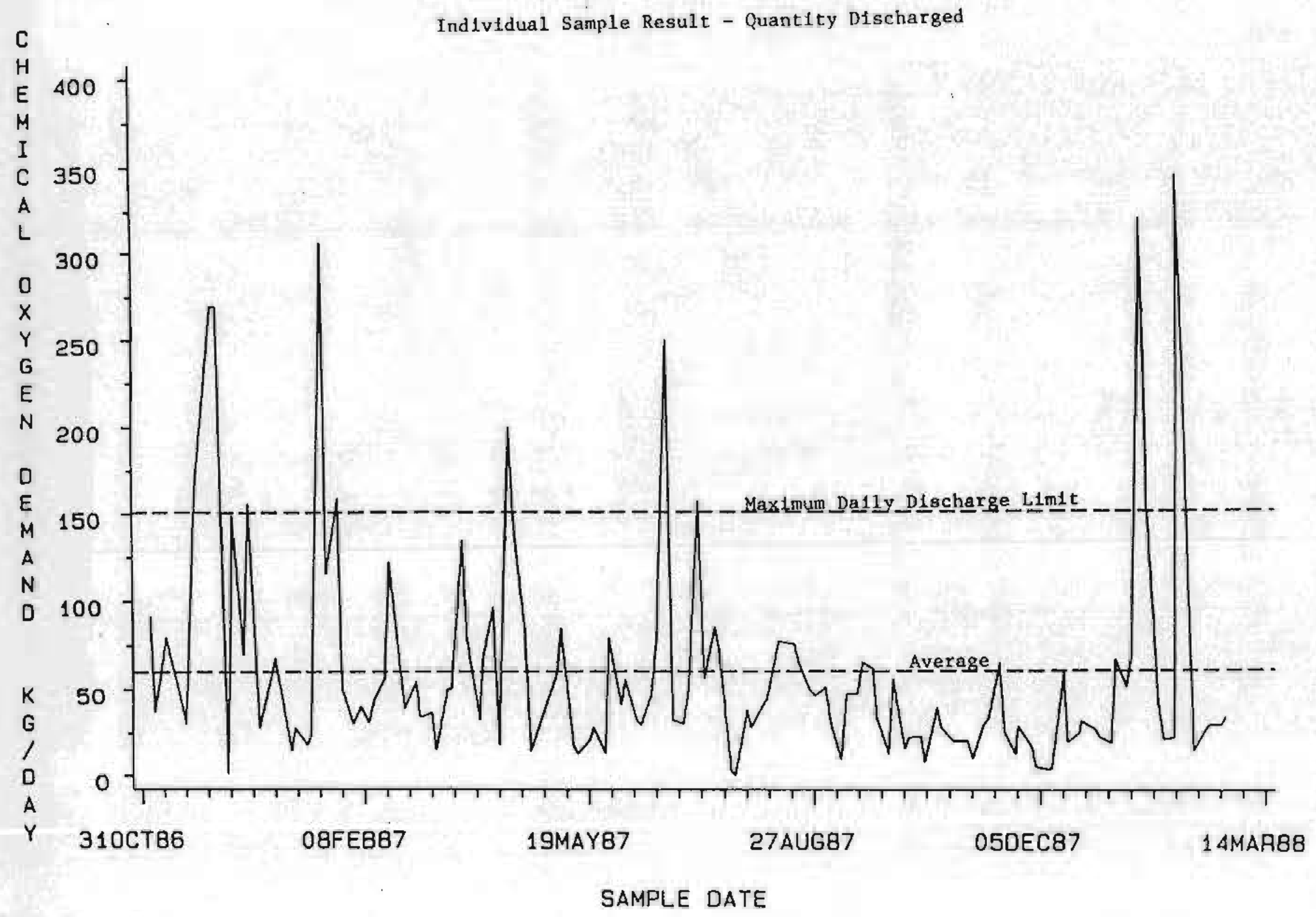


Figure C. 20

NPDES DATA - FLUORIDE KG/DAY

Individual Sample Resule - Quantfty D1scharged

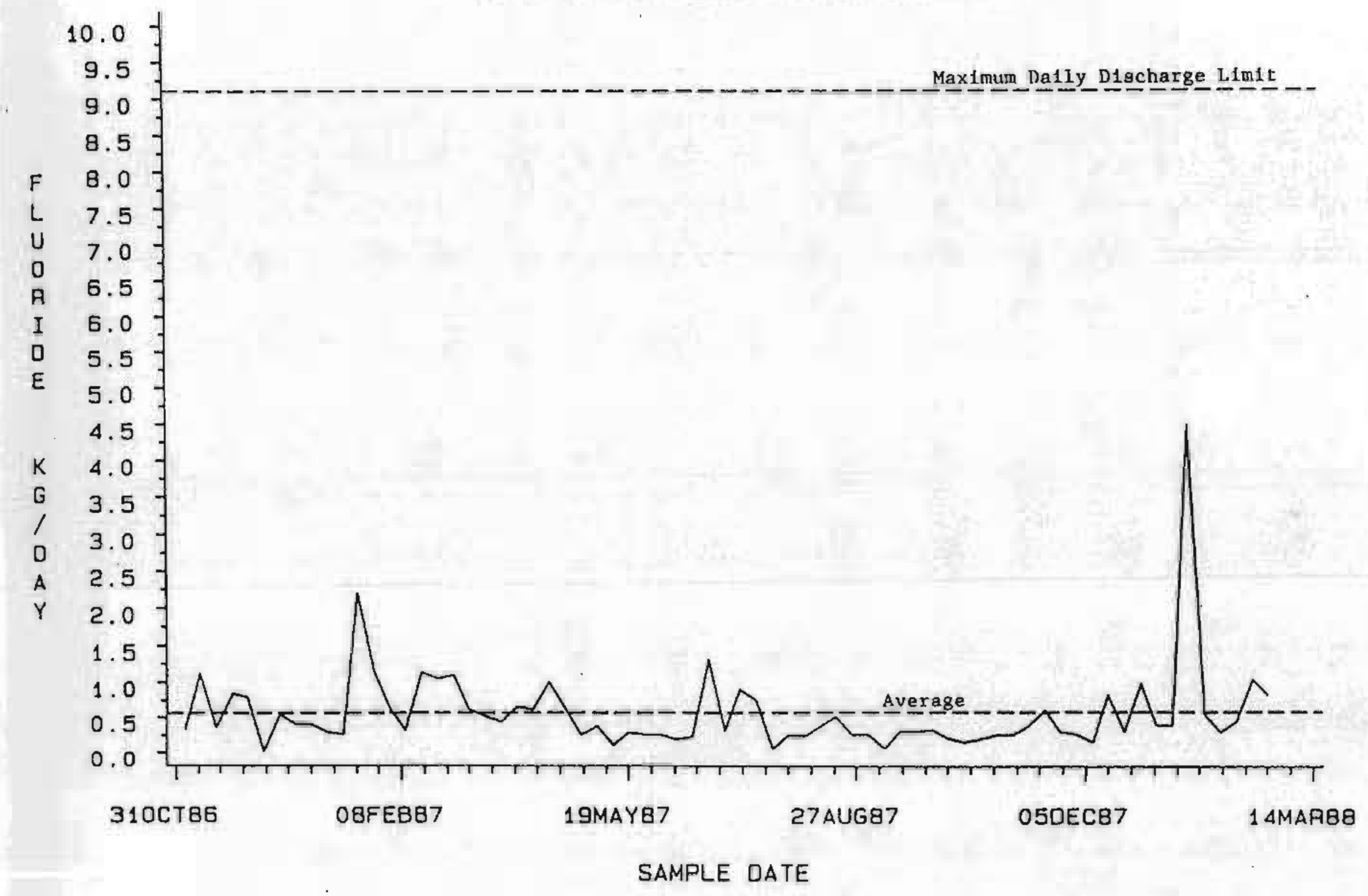

( 
Figure C.21

NPDES DATA - OIL AND GREASE KG/DAY

Individual Sample Result - Quantity Discharged

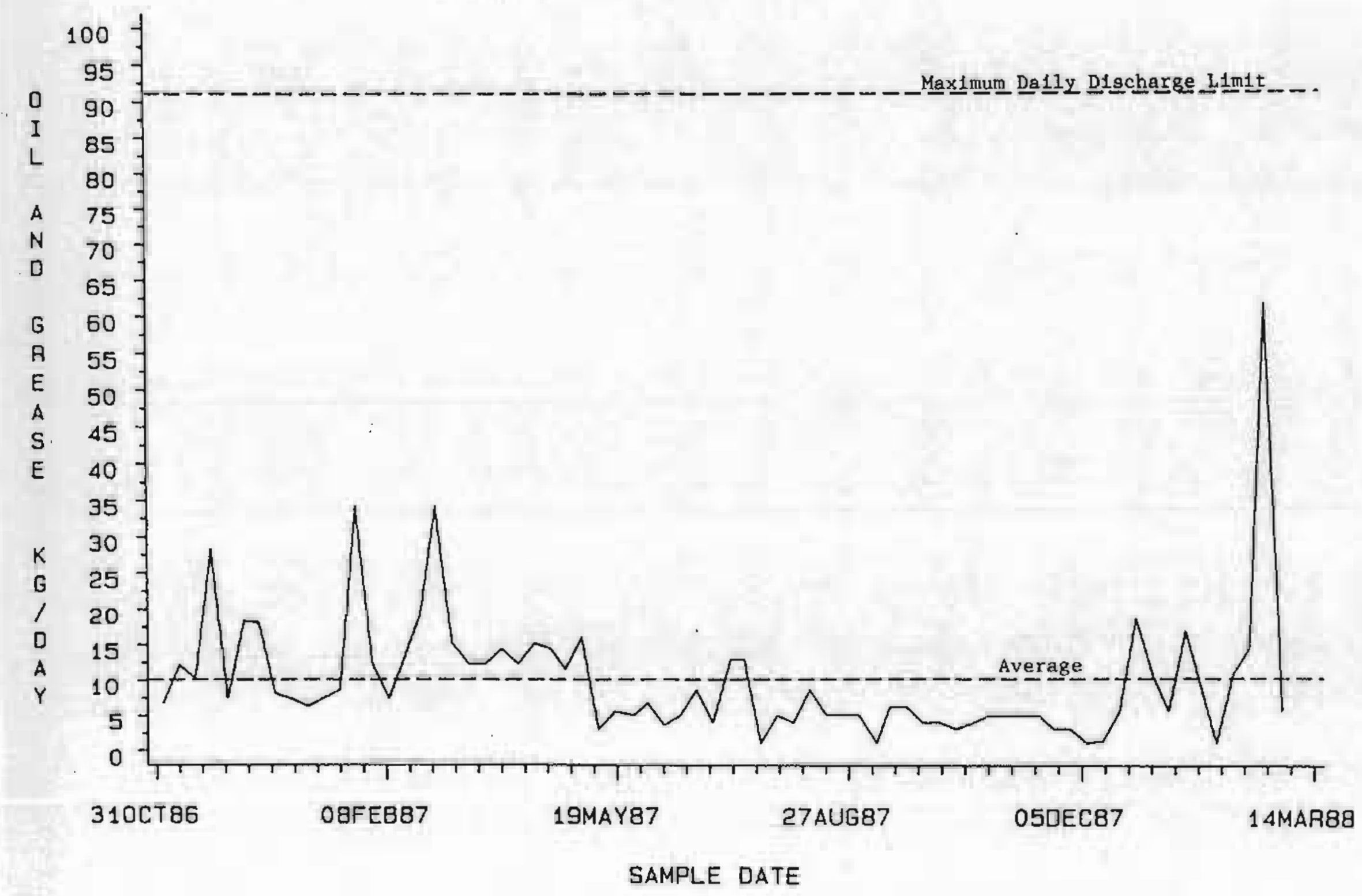




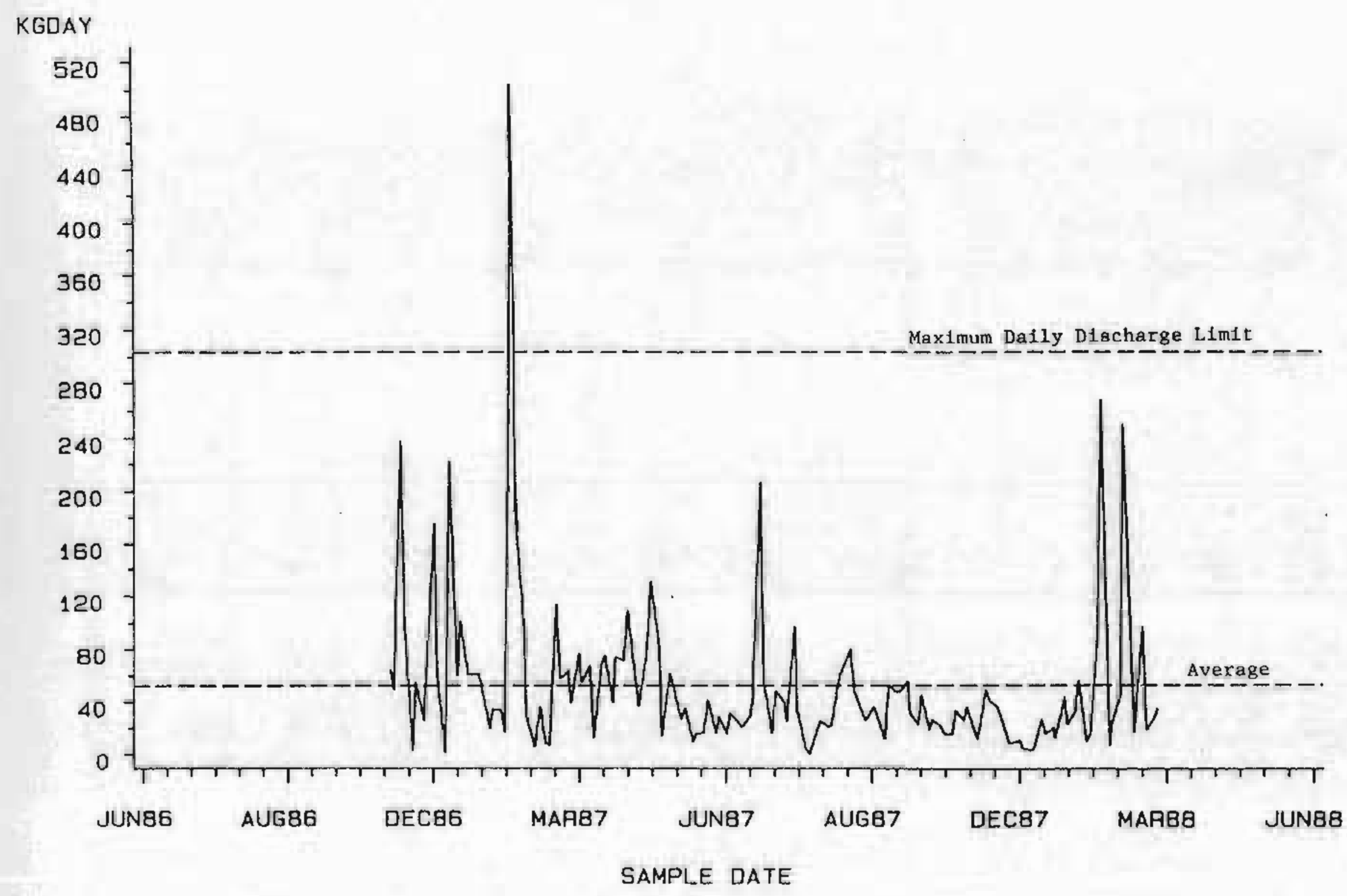


5:

c. 
Storm Drain Data for SD-100

Location Date Test Gompound Results UnIts

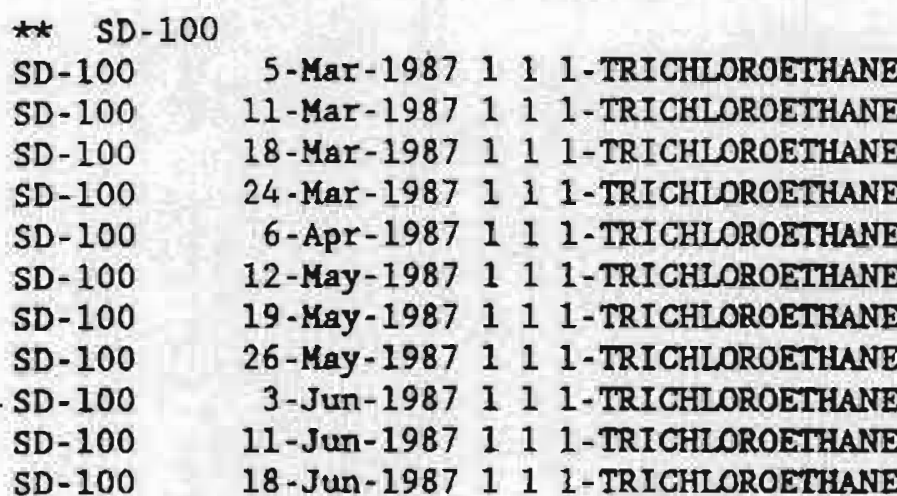

** SD - 100-01

SD-100-01 12-May-1987 11 1-TRICHLOROETHANE

SD-100-01 18- $\mathrm{Aay-1987} 11$ 1 1 -TRICHLOROETHANE

SD-100-01 26-May-1987 11 1-TRICHLOROETHANE

SD-100-01 3-Jun-1987 111 1-TRICRLOROETHANE

SD-100-01 11-JUn-1987 11 1-TRICHLOROETHANE

SD-100-01 18-Jun-1987 11 1-TRICHLOROETHANE

$\begin{array}{ll}7 & u g / L \\ 5 & u g / L \\ <5 & u g / L \\ <5 & u g / L \\ <5 & u g / L \\ <5 & u g / L \\ <5 & u g / L \\ <5 & u g / L \\ <5 & u g / L \\ <5 & u g / L \\ <5 & u g / L\end{array}$

** SD $-100-02$

SD-200-02 12-May-1987 11 1-TRICHLOROETHANE

SD-100-02 18-May-1987 111 1-TRICHLOROETHANE

SD-100-02 26-May-1987 111 1-TRICHLOROETHANE

SD-100-02 3-Jun-1987 I I 1-TRICHLOROETHANE

SD-100-02 11-Jun-1987 11 1-TRICHLOROETHANE

SD-100-02 18-Jun-1987 I I 1-TRICHLOROETHANE

$<5$

$<5$

$<5$

$<5$

$<5$

$<5$

$u_{g} / \mathrm{L}$

ug/L

$\mathrm{ug} / \mathrm{L}$

$u g / L$

$\mathrm{ug} / \mathrm{L}$

$\mathrm{ug} / \mathrm{L}$

* SD $-100-03$

SD-100-03 12-May-1987 11 1-TRICHLOROETHANE

SD-100-03 18-May-1987 I 1 1-TRICHLOROETHANE

SD-100-03 26-May-1987 11 1-TRICHLOROETHANE

SD-100-03 3-Jun-1987 11 1-TRICHLOROETHANE

SD-100-03 11-Jun-1987 1 1 1 -TRIGHLOROETHANE

SD-100-03 18-Jun-1987 11 1-TRICHLOROETHANE

** SD - 100-03D

SD-100-03D 12-May-1987 11 1-TRICHLOROETHANE

SD-100-03D 26-Kay-1987 111 1-TRICHLOROETHANE

$<5$

$<5$

$<5$

$<5$

$<5$

$<5$

$u g / L$

ug/L

ug/L

$u g / L$

$\mathrm{ug} / \mathrm{L}$

$\mathrm{ug} / \mathrm{L}$

$<5$

$<5$

$<5$

$<5$

$<5$

$<5$

$\mathrm{ug} / \mathrm{L}$

ug/L

$\mathrm{ug} / \mathrm{L}$

ug/L

ug/L

$u g / L$

** SD $-100-04$

SD-100-04 12-May-1987 111 1-TRICHLOROETHANE

SD-100-04 18-May-1987 111 1-TRICHLOROETHANE

SD-100-04 26-May-1987 \& 1 l-TRICHLOROETHANE

SD-100-04 3-Jun-1987 111 1-TRICHLOROETKANE

SD-100-04 11-Jun-1987 111 1-TRICHLOROETHANE

SD-100-04 18-Jun-1987 111 1-TRICHLOROETHANE

$<5$

$<5$

$\mathrm{ug} / \mathrm{L}$

$\mathrm{ug} / \mathrm{L}$

$<s$

ug/L

$<5 \quad$ ug/L

$<5 \quad$ ug/L

$<5 \quad$ ug/L

$<5$

$<5$

ug/L

$\mathrm{ug} / \mathrm{L}$

$\star \star$ SD $100-06$

SD-100-06 12-May-1987 111 1-TRICHLOROETHANE

$<5$

$\mathrm{ug} / \mathrm{L}$

SD-100-06 18-May-1987 111 1-TRICHLOROETHANE

$<5$

$\mathrm{ug} / \mathrm{L}$

SD $-100-06$

26-May-1987 11 I -TRICHLOROETHANE

$<5$

$\mathrm{ug} / \mathrm{L}$

SD-100-06

3.Jun-1987 11 I-TRICHLOROETHANE

$<5$

$u_{\mathrm{g}} / \mathrm{L}$ 
Storm Draln Data for SD-100

Location Date Test Compound

SD-100-06 11-Jun-1987 I I 1-TRICHLOROETHANE

SD-100-06 18-Jun-1987 11 1 1 -TRICHLOROETHANE

** SD-100-06D

SD-100-06D 3-Jun-1987 11 1-TRICHLOROETHANE

** SD-100-07

SD-100-07 12-May-1987 I 1 1-TRICHLOROETHANE

SD-100-07 19-May-1987 1 I 1-TRICHLOROETHANE

SD-100-07 26-May-1987 11 1 1-TRICHLOROETHANE

SD-100-07 3-Jun-1987 111 -IRICHIOROETHAKE

SD-100-07 11-Jun-1987 \& 1 1-TRICHLOROETHANE

SD-100-07 18-Jun-1987 111 1-TRICHLOROETHANE

** SD-100-07D

SD-100-07D 18-JLn-1987 11 1 1-TRICHLOROETHANE

$\begin{array}{ll}\text { Results } & \text { Units } \\ <5 & \text { ug/L } \\ <5 & \text { ug/L } \\ & \\ <5 & \text { ug/L }\end{array}$

190

540

690

1800

270

1400

$4 \mathrm{~g} / \mathrm{L}$

ug/L

$\mathrm{ug} / \mathrm{L}$

$\mathrm{ug} / \mathrm{L}$

ug/L

$u g / L$

1100

ug/L

** SD $-100-09$

SD-100-09 12-May-1987 \& 11 -TRICHLOROETHANE

SD-100-09 18-May-1987 111 1-TRICHLOROETHANE

SD-100-09 26-May-1987 1 I 1 -TRICHLOROETHANE

SD-100-09 3-Jun-1987 I 1 1-TRICHLOROETHANE

SD-100-09 11-Jun-1987 11 1-TRICHLOROETHANE

SD-100-09 18-Jun-1987 111 1-TRICHLOROETHANE

** SD $-100-10$

SD-100-10 12-May-1987 I 1 1-TRICHLOROETHANE

SD-100-10 19-May-1987 111 -TRICHLOROETHANE

SD-100-10 26-May-1987 I I I-TRICHLOROETHANE

SD-100-10 3-Jun-1987 11 1-TRICHLOROETHANE

SD-100-10 11-Jun-1987 111 -TRICHLOROETHANE

SD-100-10 18-Jun-1987 111 -TRICHLOROETHANE

** SD-100-11

SD-100-11 12-May-1987 111 -TRICHLOROETHANE

SD-100-11 19-May-1987 I I 1-TRICHLOROETHANE

SD-100-11 26-May-1987 11 1-TRICHLOROETHANE

SD-100-11 3-Jun-1987 11 1-TRICHLOROETHANE

SD-100-11 11-Jun-1987 1 I 1-TRICHLOROETHANE

SD-100-11 18-Jun-1987 1 I 1-TRICHLOROETHANE

** SD - 100-12

SD-100-12 12-May-1987 11 I-TRICHLOROETHANE

SD-100-12 19-May-1987 11 I -TRICHLOROETHANE

SD-100-12 26-May-1987 11 I-TRICHLOROETHANE

SD-100-12 3-Jun-1987 11 1-TRICHLOROETHANE

SD-100-12 11-Jun-1987 11 1 1 -TRICHLOROETHANE

SD-100-12 18-Jun-1987 11 1-TRICHLOROETTANE
$<5$

$<5$

$<5$

$<5$

$<5$

$<5$

$<5$

$<5$

$<5$

$<5$

$<5$

$<5$

$<5$

$<5$

$<5$

$<5$

$<5$

$<5$

$<5$

$<5$

$<5$

$<5$

$<5$

$<5$

$<5$

5

5

$<5$
ug/L

ug/L

$\mathrm{ug} / \mathrm{L}$

$4 \mathrm{~g} / \mathrm{L}$

ug/L

ug/L

ug/L

ug/L

ug/L

$\mathrm{ug} / \mathrm{L}$

ug/L

$4 \mathrm{~g} / \mathrm{L}$

ug/L

ug/L

ug/L

ug $/ L$

ug/L

ug/L

ug/L

$\mathrm{ug} / \mathrm{L}$

$u g / L$

$\mathrm{ug} / \mathrm{L}$

ug/L

$u g / L$

** SD $-100-13$

SD-100-13 12-May-1987 11 1-TRICHLOROETHANE

SD-100-13 19-May-1987 111 1-TRICHLOROETHANE

SD-100-13 26-May-1987 1 \& 1 -TRICHLOROETHANE

SD-100-13 3-Jun-1987 I 1 1-IRICHLOROETHANE 
Storm Drain Data for SD-100

Location Date Test Compound

$\begin{array}{ll}\text { Results } & \text { Units } \\ <5 & \mathrm{ug} / \mathrm{L} \\ <5 & \mathrm{ug} / \mathrm{L} \\ & \\ & \\ <5 & \mathrm{ug} / \mathrm{L} \\ <5 & \mathrm{ug} / \mathrm{L} \\ <5 & \mathrm{ug} / \mathrm{L} \\ <5 & \mathrm{ug} / \mathrm{L} \\ <5 & \mathrm{ug} / \mathrm{L} \\ <5 & \mathrm{ug} / \mathrm{L}\end{array}$

SD-100-13 11-JUn-1987 111 1-TRICHLOROETHANE

SD-100-13 18-JUn-1987 111 1-TRICHLOROETHANE

** SD $-100-14$

SD-100-14 12-May-1987 11 1-TRICHLOROETHANE

SD-100-14 19-May-1987 11 1-TRICHLOROETHANE,

SD-100-14 26-May-1987 1 i 1 -TRICHLOROETHANE

SD-100-14 3-Jun-1987 1 1 1 -TRICHLOROETHAKE

SD-100-14 11-Jun-1987 11 1 l-TRICHLOROETHANE

SD-100-14 18-Jun-1987 I 1 l-TRICHLOROETHANE

$\star \quad S D-100-C$

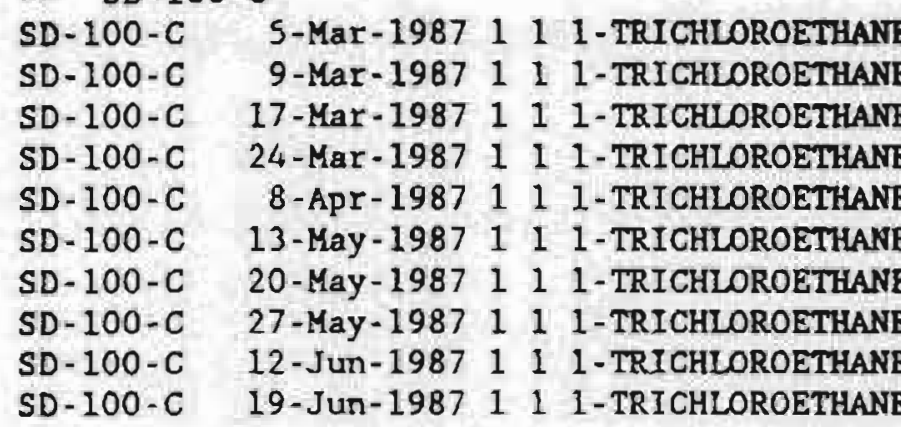

5

$<5$

$<5$

$<5$

$<5$

$<5$

$<5$

$<5$

$<5$

$<5$

$\mathrm{ug} / \mathrm{L}$

$\mathrm{ug} / \mathrm{L}$

$\mathrm{ug} / \mathrm{L}$

$u g / L$

ug/L

$u g / L$

$\mathrm{ug} / \mathrm{L}$

$4 \mathrm{~g} / \mathrm{L}$

$u g / L$

ug/L

** SD-100-D

SD-100-D 6-APY-1987 1 I I-TRICHLOROETHANE

SD-100-D 11-Jun-1987 111 1-TRICHLOROETHAKE

$<5$

$<5$

$4 \mathrm{~g} / \mathrm{L}$

ug/L

** SD -100

SD- 100 5-Mar-1987 1122 2-TETRACHLOROETHANE

SD-100 11-Mar-1987 1112 2-TETRACHLOROETHANE

SD-100 18-Mar-198711 12 2-TETRACHLOROETHANE

SD-100 24-Mar-1987 11122 2-TETRACHLOROETHANE

SD-100 6-APY-1987 1112 2-TETRACHLOROETHANE

SD-100 12-May-1987 1122 2-IETRACHLOROETHANE

SD-100 19-May-1987 1122 2-TETRACHLOROETHANE

SD-100 26-May-1987 1122 -TETRACHLOROETHANE

SD-100 3-Jun-1987 1112 2-TETRACHLOROETHANE

SD-100 11-JUn-1987 1122 2-TETRACHLOROETHANE

SD-100 18-Jun-1987 11122 -TETRACHLOROETHANE

** SD $-100-01$

SD-100-01 12-May-1987 1122 2-TETRACHLOROETHANE

SD-100-01 18-May-1987 1122 2-TETRACHLOROETHANE

SD-100-01 26-May-1987 1122 2-TETRACHLOROETHANE

SD-100-01 3-Jun-1987 11122 2-TETRACHLOROETHANE

SD-100-01 11-Jun-1987 11 1 2 2-TETRACHLOROETHANE

SD-100-01 18-Jun-1987 1112 2-TETRACHLOROETHANE

*ᄎ SD-100-02

SD-100-02 12-May-1987 1122 2-TETRACHLOROETHANE

SD-100-02 18-May-1987 1122 2-TETRACHLOROETHANE

SD-100-02 26-May-1987 1 i 2 2-TETRACHLOROETHANE

SD-100-02 3-Jun-1987 112 2 2-TETRACHLOROETHANE

SD-100-02 11-Jun-1987 1122 2-TETRACHLOROETHANE

$<5$

ug/L

ug/L

ug/L

ug/L

ug/L

$\mathrm{ug} / \mathrm{L}$

$u g / L$

$\mathrm{ug} / \mathrm{L}$

$\mathrm{ug} / \mathrm{L}$

$\mathrm{ug} / \mathrm{L}$

$\mathrm{ug} / \mathrm{L}$

$<5$

$<5$

ug/L

ug/L

$\mathrm{ug} / \mathrm{L}$

$\mathrm{ug} / \mathrm{L}$

$\begin{array}{ll}<5 & \text { ug/L } \\ <5 & \text { ug/L }\end{array}$

$<5$

ug/L

$<5 \quad$ ug/L

$<5 \quad$ ug/L

$<5 \quad$ ug/L

$<5$

$\mathrm{ug} / \mathrm{L}$ 
Storm Drain Data for SD- 100

Location Date Test Compound

SD-100-02 18-Jun-1987 1122 2-TETRACHLOROETHANE

* SD-100-03

SD-100-03 12-May-198711 12 2-TETRACKLOROETHANE

SD-100-03 18-Kay-1987 1112 2-TETRACHLOROETHANE

SD-100-03 26-May-1987 1112 2-TETRACHILROETHANE

SD-100-03 3-Jun-1987 1122 2-TETRACHLOROETHANE

SD-100-03 11-JUn-1987 1122 2-TETRACHLOROETHANE

SD-100-03 18-Jun-1987 1122 2-TETRACHLOROETHANE

*t SD-100-03D

SD-100-03D 12-May-1987 1122 2-TETRACHLOROETHANE

SD-100-03D 26-May-1987 1112 2-TETRACHLOROETHANE

t* SD $100-04$

SD-100-04 12-May-1987 1122 2-TEIRACHLOROETHANE

SD-100-04 18-May-1987 1112 2-TETRACHLOROETHANE

SD-100-04 26-May-1987 1112 2-TETRACHLOROETHANE

SD-100-04 3-Jun-1987 1112 2-TETRACHLOROETHANE

SD-100-04 11-Jun-1987 11122 2-TETRACHLOROETHANE

SD-100-04 18-Jun-1987 112 2-TETRACHLOROETHANE

** SD $-100-06$

SD-100-06 12-May-1987 1122 2-TETRACHLOROETHANE

SD-100-06 18-May-1987 11122 2-TETRACHLOROETHANE

SD-100-06 26-May-1987 I 122 2-TETRACHLOROETHANE

SD-100-06 3-Jun-1987 1112 2-TETRACHLOROETHANE

SD-100-06 11-Jun-1987 11122 2-TETRACHLOROETHANE

SD-100-06 18-Jun-1987 11122 2-TETRACHLOROETHANE

** SD-100-06D

SD-100-06D 3-Jun-1987 1122 2-TETRACHLOROETHANE

** SD-100-07

SD-100-07 12-May-1987 I 122 2-TETRACHLOROETHANE

SD-100-07 19-May-1987 1112 2-TETRACHLOROETHANE

SD-100-07 26-May-1987 1112 2-TETRACHLOROETHANE

SD-100-07 3-Jun-1987 11122 2-TETRACHLOROETHANE

SD-100-07 11-Jun-1987 1122 2-TETRACHLOROETHANE

SD-100-07 18-Jun-1987 1122 2-TETRACHLOROETHANE

\begin{tabular}{|c|c|}
\hline Results & Units \\
\hline$<5$ & ug/L \\
\hline$<5$ & $u g / L$ \\
\hline$<5$ & $\mathrm{ug} / \mathrm{L}$ \\
\hline$<5$ & $\mathrm{ug} / \mathrm{L}$ \\
\hline$<5$ & $4 \mathrm{~g} / \mathrm{L}$ \\
\hline$<5$ & $\mathrm{ug} / \mathrm{L}$ \\
\hline$<5$ & $\mathrm{ug} / \mathrm{L}$ \\
\hline$<s$ & $\mathrm{ug} / \mathrm{L}$ \\
\hline$<5$ & $\mathrm{ug} / \mathrm{L}$ \\
\hline$<5$ & $\mathrm{ug} / \mathrm{L}$ \\
\hline$<5$ & ug/L \\
\hline$<5$ & $\mathrm{ug} / \mathrm{L}$ \\
\hline$<5$ & $u_{g} / \mathrm{L}$ \\
\hline$<s$ & $\mathrm{ug} / \mathrm{L}$ \\
\hline$<5$ & $\mathrm{ug} / \mathrm{L}$ \\
\hline$<5$ & ug/L \\
\hline$<5$ & $\mathrm{ug} / \mathrm{L}$ \\
\hline$<5$ & $\mathrm{ug} / \mathrm{L}$ \\
\hline$<5$ & $\mathrm{ug} / \mathrm{L}$ \\
\hline$<5$ & ug/L \\
\hline$<5$ & $\mathrm{ug} / \mathrm{L}$ \\
\hline$<5$ & $u g / L$ \\
\hline$<5$ & ug/L \\
\hline$<5$ & $\mathrm{ug} / \mathrm{L}$ \\
\hline$<5$ & $\mathrm{ug} / \mathrm{L}$ \\
\hline$<5$ & $\mathrm{ug} / \mathrm{L}$ \\
\hline$<5$ & $\mathrm{ug} / \mathrm{L}$ \\
\hline$<5$ & $\mathrm{ug} / \mathrm{L}$ \\
\hline
\end{tabular}


Lacation Date Test Compound Results Units

** SD-100-07D

SD-100-07D 18-Jun-1987 1122 2-TETRACHLOROETHANE

$\begin{array}{ll}<5 & 4 g / L \\ & \\ <5 & 4 g / L \\ <5 & 4 g / L \\ <5 & 4 g / L \\ <5 & 4 g / L \\ <5 & 4 g / L \\ <5 & 4 g / L\end{array}$

** SD-100-09

SD-100-09 12-May-1987 1122 2-TETRACHLOROETHANE

SD-100-09 18-May-1987 1122 2-TETRACHLOROETHANE

SD-100-09 26-May-1987 11122 2-TETRACHLOROETHANE

SD-100-09 3-Jun-1987 11122 2-TETRACHLOROETHANE

SD-100-09 11-Jun-1987 1112 2-TETRACHLOROETHANE

SD-100-09 18-JUn-1987 1122 2-TETRACHLOROETHANE

* SD $-100-10$

SD-100-10 12-May-1987 11122 2-TETRACHLOROETHANE

SD-100-10 19-May-1987 1112 2-TETRACHLOROETHANE

SD-100-10 26-May-1987 1122 2-TETRACHLOROETHANE

SD-100-10 3-Jun-1987 1122 2-TETRACHLOROETHANE

SD-100-10 11-Jun-1987 1112 2-TETRACHLOROETHANE

SD-100-10 18-Jun-1987 112 2-TETRACHLOROETHANE

* SD-100-11

SD-100-11 12-May-1987 1122 2-TETRACHLOROETHANE

SD-100-11 19-May-1987 1112 2-TETRACHLOROETHANE

SD-100-11 26-May-1987 1112 2-TETRACHLOROETHANE

SD-100-11 3-Jun-1987 1112 2-TETRACHLOROETHANE

SD-100-11 11-Jun-1987 112 2-TETRACHLOROETHANE

SD-100-11 18-Jun-1987 1122 2-TETRACHLOROETHANE

$<5$

$<5$

$<5$

$<5$

$<5$

$<5$

$u g / \mathrm{L}$

$\mathrm{ug} / \mathrm{L}$

$\mathrm{ug} / \mathrm{L}$

ug/L

ug/L

$u g / L$

$<5$

ug/L

$<5$

$u g / L$

$\mathrm{ug} / \mathrm{L}$

$<5$

ug/L

$<5$

$\mathrm{ug} / \mathrm{L}$

$<5$

$\mathrm{ug} / \mathrm{L}$

** SD-100-12

SD-100-12 12-May-1987 1122 2-TETRACHLOROETHANE SD-100-12 19-May-1987 I 12 2-TETRACHLOROETHANE SD-100-12 26-May-1987 1112 2-TETRACHLOROETHANE

SD-100-12 3-Jun-1987 1112 2-TETRACHLOROETHANE

SD-100-12 11-JUח-1987 1112 2-TETRACHLOROETHANE

SD-100-12 18-Jun-1987 1112 2-TETRACHLOROETHANE

$<5$

$48 / 2$

$<5$

ug/L

ug/L

$<5 \quad \mathrm{ug} / \mathrm{l}$

$<5 \quad \mathrm{ug} / \mathrm{L}$

$<5 \quad u g / L$

** SD $-100-13$

SD-100-13 12-May-1987 1122 2-TETRACHLOROETHANE

SD-100-13 19-May-1987 1112 2-TETRACHLOROETHANE

SD-100-13 26-May-1987 I I 2 2-TETRACHLOROETHANE

SD-100-13 3-Jun-1987 1112 2-TETRACHLOROEIHANE

SD-100-13 11-Jun-1987 1112 2-TETRACHLOROETHANE

SD-100-13 18-Jun-1987 11122 2-TETRACHLOROEIHANE

$<5$

$\mathrm{ug} / \mathrm{L}$

$<5$

ug $/ \mathrm{L}$

$\mathrm{ug} / \mathrm{L}$

$\operatorname{ug} / \mathrm{L}$

$<5$

ug/L

$<5$

$\mathrm{ug} / \mathrm{L}$

* SD-100-14

SD-100-14 12-May-1987 1122 2-TETRACHLOROETHANE

SD-100-14 19-May-1987 1122 2-TETRACHLOROETHANE

$<5$

ug/L

SD-100-14 26-May-1987 11 I 2 2-TETRACHLOROETHANE

$<5$

ug/L

SD-100-14 3-Jun-1987 1112 2-TETRACHLOROETHANE

SD-100-14 11-JUn-1987 1112 2-TETRACHLOROEIHANE

SD-100-14 18-Jun-1987 1122 2-TETRACHLOROETHANE

$<5$

ug/L

$\mathrm{ug} / \mathrm{L}$

$\mathrm{ug} / \mathrm{L}$

$<5$

$<5$

ug/L 
Location
** SD-100-C

Results Units

SD-100-C 5-Mar-1987 11122 2-TETRACHLOROETHANE

SD-100-C 9-Mar-198711122-TETRACKLOROETHANE

SD-100-C 17-Mar-1987 1122 2-TETRACHLOROETHANE

SD-100-C 24-Mar-1987 I 122 2-TETRACHLOROETHANE

SD-100-C 8-Apr-1987 1112 2-TETRACHLOROETHANE

SD-100-C 13-May-1987 112 2-TETRACHLOROETHANE

SD-100-C 20-May-1987 11122 2-TETRACELOROETHANE

SD-100-C 27-May-1987 1112 2-TETRACHLOROETHANE

SD-100-C 12-Jun-1987 1122 2-TETRACHLOROBTHANE

SD-100-C 19-Jun-1987 1122 2-TETRACHLOROETHANE

** SD-100-D

SD-100-D 6-Apr-1987 1112 2-TETRACRLOROETHANE

SD-100-D 11-Jun-1987 112 2-TETRACHIOROETHANE $<s$

$<5$

$<5$

$<5$

$<5$

$<5$

$<5$

$<5$

$<5$

$<5$

$<5$

$<5$

ug/L

ug/L

$<5$

$<5$

$<5$

$<5$

$<5$

$<5$

$<5$

$<5$

$<5$

$<5$

$<5$

$<5$

$<5$

$<5$

$<5$

$<5$

$<5$

$<5$

$<5$

$<5$

$<5$

$<5$

$<5$

$<5$

$<5$

$<5$

$<5$

$<5$

$u g / L$

ug/L

$\mathrm{ug} / \mathrm{I}$

ug/L

ug/L

$\mathrm{ug} / \mathrm{L}$

$u_{g} / L$

ug/L

$4 \mathrm{~g} / \mathrm{L}$

ug/L

ug/L

$u g / L$

$\mathrm{ug} / \mathrm{L}$

$\mathrm{ug} / \mathrm{L}$

ug/ $\mathrm{L}$

$u \mathrm{~g} / \mathrm{L}$

ug/L

ug/L

ug $/ \mathrm{L}$

$\mathrm{ug} / \mathrm{L}$

$\mathrm{ug} / \mathrm{L}$

ug/L

ug/L

$\mathrm{ug} / \mathrm{L}$

$\mathrm{ug} / \mathrm{L}$

$\mathrm{ug} / \mathrm{L}$

ug/L

$\mathrm{ug} / \mathrm{L}$

ug/L

$\mathrm{ug} / \mathrm{L}$

$\mathrm{Ug} / \mathrm{I}$

$4 \mathrm{~g} / \mathrm{L}$ ug $/ 2$

** SD-100-03

SD-100-03 12-May-1987 \& 1 2-TRICELOROETHANE

SD-100-03 18-May-1987 I I 2-TRICHLOROETHANE

SD-100-03 26-May-1987 111 2-TRICHLOROETHANE

SD-100-03 11-Jun-1987 I 1 2-TRICHLOROETHANE $u g / L$

ug/L

$u g / L$

$\cup \mathrm{g} / \mathrm{L}$

ug $/ \mathrm{L}$ 
Storm Drain Data for SD-100

Location Date Test Compound

Results Units

SD-100-03 18-Jun-1987 112 2-IRICHLOROETHANE

$<5$

ug/L

* SD - 100-03D

SD-100-03D 12-May-1987 112 2-TRICHLOROETHAYE

SD-100-03D 26-May-1987 11 2-TRICHLOROETHANE

$<5$

$<5$

$\mathrm{ug} / \mathrm{L}$

** SD-100-04

SD-100-04 12-May-1987 I 1 2-TRICHLOROETAANE

SD-100-04 18-May-1987 1112 -TRICHLOROETHANE

SD-100-04 26-May-1987 11 2-TRICHLOROETHANE

SD-100-04 3-Jun-1987 112 2-TRICHLOROETHANE

SD-100-04 11-JUn-1987 11 1 2-TRICHLOROETHANE

SD-100-04 18-Jun-1987 11 1 2 -IRICHLOROETHANE

$\begin{array}{ll}<5 & \mathrm{ug} / \mathrm{L} \\ <5 & \mathrm{ug} / \mathrm{L} \\ <5 & \mathrm{ug} / \mathrm{L} \\ <5 & \mathrm{ug} / \mathrm{L} \\ <5 & \mathrm{ug} / \mathrm{L} \\ <5 & \mathrm{ug} / \mathrm{L}\end{array}$

** SD $-100-06$

SD-100-06 12-May-1987 1 1 2-TRICHLOROETHANE

SD-100-06 18-May-1987 11 1 2 -TRICHLOROETHANE

SD-100-06 26-May-1987 11 2-TRICHLOROETHANE

SD-100-06 3-Jun-1987 11 1 2-TRICHLOROETHANE

SD-100-06 11-JUn-1987 112 2-TRICHLOROETHANE

SD-100-06 18-Jun-1987 1112 2-IRICHLOROETHANE

** SD-100-06D

SD-100-06D 3-Jun-1987 I I 2-TRICHLOROETHANE

$<5$

$<5$

$<5$

$<5$

$<5$

$<5$

$<5$

* SD-100-07

SD-100-07 12-May-1987 11 2 2-TRICHLOROETHANE SD-100-07 19-May-1987 11 2 2-TRICHLOROETHANE SD-100-07 26-May-1987 11 1 2-TRICHLOROETHANE SD-100-07 3-Jun-1987 11 1 2 -TRICHLOROETHANE SD-100-07 11-Jun-1987 1112 -TRICHLOROETHANE SD-100-07 18-Jun-1987 1112 2-TRICHLOROETHANE

** SD - 100-07D

SD-100-07D 18-Jun-1987 112 2-TRICHLOROETHANE

** SD-100-09

SD-100-09 12-May-1987 112 2-TRICHLOROETHAIE

SD-100-09 18-May-1987 11 2-TRICHLOROETHANE

SD-100-09 26-May-1987 111 2-TRICHLOROETHANE

SD-100-09 3-Jun-1987 11 1 2-TRICHLOROETHANE

SD-100-09 11-Jun-1987 11 2 2-TRICHLOROETHANE

SD-100-09 18-Jun-1987 11 2-TRICHLOROETHANE

* SD $-100-10$

SD-100-10 12-May-1987 111 2-TRICHLOROETHNE

SD-100-10 19-May-1987 11 I 2-TRICHLOROETHANE

SD-100-10 26-May-1987 11 2-TRICHLOROETHANE

SD-100-10 3-Jun-1987 11 2 2 -TRICHLOROETHANE

SD-100-10 11-Jun-1987 1112 -TRICHLOROETHANE

SD-100-10 18-Jun-1987 112 2-TRICHLOROETHANE 
Location

SD-100-11 12-May-1987 11 2-TRICHLOROETHANE

SD-100-11 19-May-1987 112 2-TRICHLOROETHANE

SD-100-11 26-May-1987 112 -TRICHLOROETHANE

SD-100-11 3-Jun-1987 I 1 2-TRICHLOROETHANE

SD-100-11 11-Jun-1987 11 2-TRICHLOROETHANE

SD-100-11 18-Jun-1987 I I 2-TRICHIOROETHANE

* SD $-100-12$

SD-100-12 12-May-1987 11 2-IRICHLOROETHANE SD-100-12 19-May-1987 11 2-TRICHLOROETHANE

SD-100-12 26-May-1987 11 2-TRICHLOROETHANE

SD-100-12 3-Jun-1987 11 2-TRICHLOROETHANE

SD-100-12 11-Jun-1987 11 2-TRICHLOROETTANE

SD-100-12 18-Jun-1987 11 i 2-TRICHLOROETHANE

** SD $-100-13$

SD-100-13 12-May-1987 11 1 2 -TRICHLOROETHANE

SD-100-13 19-May-1987 112 2-TRICHLOROETHANE

SD-100-13 26-May-1987 11 2 2-TRICHLOROETHANE

SD-100-13 3-Jun-1987 11 2 2 -TRICHLOROETHANE

SD-100-13 11-Jun-1987 1112 2-TRICHLOROETHANE

SD-100-13 18-Jun-1987 11 2-TRICHLOROETHANE

** SD $-100-14$

SD-100-14 12-May-1987 112 -TRICHLOROETHANE

SD-100-14 19-May-1987 I I. 2-TRICHLOROETHANE

SD-100-14 26-May-1987 11 2-TRICHLOROETHANE

SD-100-14 3-Jun-1987 1112 -TRICHLOROETHANE

SD-100-14 11-Jun-1987 11 2-TRICHLOROETHANE

SD-100-14 18-Jun-1987 112 2-TRICHLOROETHANE

** SD-100-C

SD-100-C 5-Mar-1987 11 1 2-TRICHLOROETHANE

SD-100-C 9-Mar-1987 112 2-TRICHLOROETHANE

SD-100-C 17-Mar-1987 11 1 2-TRICHLOROETHANE

SD-100-C 24-Mar-1987 1 I 2-TRICHLOROETHANE

SD-100-C 8-APY-1987 1112 2-TRICHLOROETHANE

SD-100-C 13-May-1987 1112 2-TRICHLOROETHANE

SD-100-C 20-May-1987 11 1 2-IRICHLOROETHANE

SD-100-C 27-May-1987 1 I 2 -TRICHLOROETHANE

SD-100-C 12-JUn-1987 11 1 2-TRICHLOROETHANE

SD-100-C 19-Jun-1987 11 1 2 -TRICHLOROETHANE

* $\quad S D-100-D$

SD-100-D 6-APT-1987 I1 2-TRICHLOROETHANE

SD-100-D 11-Jun-1987 112 2-TRICHLOROETHANE

** SD -100

SD-100 5-Mar-1987 11 -DICHLOROETHANE

$\begin{array}{ll}\text { Results } & \text { Units } \\ & \\ <5 & u g / L \\ <5 & u g / L \\ <5 & u g / L \\ <5 & u g / L \\ <5 & u g / L \\ <5 & u g / L\end{array}$

$<5$

$<5$

$<5$

$<5$

$<5$

$<5$

$u g / L$

ug/L

ug/L

$\mathrm{ug} / \mathrm{L}$

$4 \mathrm{~g} / \mathrm{L}$

ug/L

$\begin{array}{ll}<5 & u g / L \\ <5 & u g / L \\ <5 & u g / L \\ <5 & u g / L \\ <5 & u g / L \\ <5 & u g / L\end{array}$

$<5$

$<5$

$<5$

$<5$

$<5$

$<5$

ug/L

ug/L

$\mathrm{ug} / \mathrm{L}$

ug/L

ug/L

$4 \mathrm{~g} / \mathrm{L}$

$<5$

$<5$

$<5$

$<5$

$<5$

$<5$

$<5$

$<5$

$<5$

$<5$

ug/L

ug/L

ug/L

ug/L

ug/L

ug/L

ug/L

ug/L

ug/L

$u g / L$

$<5$

$<5$

$\mathrm{ug} / \mathrm{L}$

ug/L

$<5$

ug/L 
* SD-100-01

SD-100-01 12-May-1987 \& 1-DICHLOROETHANE SD-100-01 18-May-1987 1 1-DICHLOROETHANE SD-100-01 26-May-1987 1 1-DICHLOROETHANE SD-100-01 3-Jun-1987 1 1-DICHLOROETHANE SD-100-01 11-Jun-1987 1 1-DICHLOROETHANE SD-100-01 18-Jun-1987 1 1-DICHLOROETHANE

SD-100-03D 12-May-1987 1 1-DICHLOROETHANE SD-100-03D 26-May-1987 I 1-DICHLOROETHANE

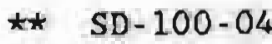

SD-100-04 12-May-1987 \& 1-DICHLOROETHANE SD-100-04 18-May-1987 1 1-DICHLOROETHANE SD-100-04 26-May-1987 1 1-DICHLOROETHANE SD-100-04 3-Jun-1987 1 1-DICHLOROETHANE SD-100-04 11-Jun-1987 \& 1-DICHLOROETHANE SD-100-04 18-Jun-1987 \& l-DICHLOROETHANE

** SD $-100-06$

SD-100-06 12-May-1987 1 1-DICHLOROETHANE SD-100-06 18-May-1987 1 1-DICHLOROETHANE SD-100-06 26-May-1987 I L-DICHLOROETHANE
$<5$

$<5$

$<5$

$<5$

$<5$

$<5$

$<5$

$<5$

$<5$

$<5$

$<5$

$<5$

$<5$

$<5$

$<5$

$<5$

$<5$

$<5$

$<5$

$$
\begin{aligned}
& u g / L \\
& u g / L \\
& u g / L \\
& u g / L \\
& u g / L \\
& u g / L \\
& u g / L \\
& u g / L \\
& u g / L \\
& u g / L
\end{aligned}
$$

ug/L

$u g / L$

$\mathrm{ug} / \mathrm{L}$

$\mathrm{ug} / \mathrm{L}$

ug/L

ug/L

$$
\begin{aligned}
& u g / L \\
& u g / L \\
& u g / L \\
& u g / L \\
& u g / L \\
& u g / L
\end{aligned}
$$

$<5 \quad$ ug/L

$<5$

$<5$

$<5$

$<5$

$<5$

$<5$

ug/L $\mathrm{ug} / \mathrm{L}$ ug/L ug/L $\mathrm{ug} / \mathrm{L}$ ug/L

$\begin{array}{ll}<5 & \text { ug/L } \\ <5 & u g / L \\ <5 & u g / L\end{array}$


Storm Drain Data for SD-100

Location Date Test Compound

SD-100-06 3-Jun-1987 I 1-DICHLOROETHANE

SD-100-06 11-Jun-1987 I 1-DICHLOROETHANE

SD-100-06 18-Jun-1987 1 1-DICHLOROETHANE

* SD-100-06D

SD-100-06D 3-Jun-1987 1 1-DICHLOROETHANE

** SD-100-07

SD-100-07 12-May-1987 1 1-DICHLOROETHANE

SD-100-07 19-May-1987 I 1-DICHLOROETHANE

SD-100-07 26-May-1987 1 1-DICHLOROETHANE

SD-100-07 3-Jun-1987 I 1-DICHLOROETHANE

SD-100-07 11-JUח-1987 1 1-DICHLOROETHANE

SD-100-07 18-Jun-1987 1 1-DICHLOROETHANE

*t SD $-100-070$

SD-100-07D 18-Jun-1987 I 1-DICHLOROETHANE

* SD-100-09

SD-100-09 12-May-1987 I 1-DICHLOROETHANE

SD-100-09 18-May-1987 1 1-DICHLOROETHANE

SD-100-09 26-May-1987 1 1-DICHLOROETHANE

SD-100-09 3-Jun-1987 \& 1-DICHLOROETHANE

SD-100-09 11-Jun-1987 1 1-DICHLOROETHANE

SD-100-09 18-Jun-1987 I 1 -DICHLOROETHANE

* SD $-100-10$

SD-100-10 12-May-1987 1 1-DICHLOROETHANE

SD-100-10 19-May-1987 I L-DICHLOROETHANE

SD-100-10 26-May-1987 1 1-DICHLOROETHANE

SD-100-10 3-Jun-1987 \& 1-DICHLOROETHANE

SD-100-10 12-Jun-1987 1 I-DICHLOROETHANE

SD-100-10 18-Jun-1987 1 1-DICHLOROETHANE

** SD-100-11

SD-100-11 12-May-1987 1 1-DICHLOROETHANE

SD-100-11 19-May-1987 \& 1-DICHLOROETHANE

SD-100-11 26-May-1987 11 -DICHLOROETHANE

SD-100-11 3-Jun-1987 I 1-DICHLOROETHANE

SD-100-11 11-JUח-1987 1 1-DICHLOROETHANE

SD-100-11 18-Jun-1987 1 1-DICHLOROETHANE

** SD-100-12

SD-100-12 12-May-1987 \& 1 -DICHLOROETHANE

SD-100-12 19-May-1987 1 1-DICHLOROETHANE

SD-100-12 26-May-1987 1 1-DICHLOROETHANE

SD-100-12 3-Jun-1987 \& 1-DICHLOROETHANE

SD-100-12 11-Jun-1987 I 1-DICHLOROETHANE

SD-100-12 18-Jun-1987 1 I-DICHLOROETHANE
Results Units

$\begin{array}{ll}<5 & u g / L \\ <5 & u g / L \\ <5 & u g / L\end{array}$

$<5$

$\mathrm{ug} / \mathrm{L}$

$<5$

$<5$

$<5$

$<5$

$<5$

$<5$

ug/ $/ \mathrm{L}$

$4 \mathrm{~g} / \mathrm{L}$

ug/L

$4 \mathrm{~g} / \mathrm{L}$

ug/L

ug/L

$<5$

UE/L

$<5$

$<5$

$<5$

$<5$

$<5$

$<5$

$<5$

$<5$

$<5$

$<5$

$<5$

$<5$

$\mathrm{ug} / \mathrm{L}$

$\mathrm{ug} / \mathrm{L}$

ug/L

ug/L

$u g / L$

ug/L

ug/L

ug/L

ug/L

ug/L

$u g / L$

$u g / L$

$<5$

$<5$

$<5$

$<5$

$<5$

$<5$

ug/L

$\mathrm{ug} / \mathrm{L}$

ug/L

Ug/L

$u g / L$

ug/ $/ \mathrm{L}$

$<5$

$<5$

$<5$

$<5$

$<5$

$<5$
Ug/L

ug/L

ug/L

$u g / L$

ug/L

ug/ $/$ 
** SD $-100-13$

SD-100-13 12-May-1987 I 1-DICHLOROETHANE

SD-100-13 19-May-1987 1 1-DICHLOROEIHANE

SD-100-13 26-May-1987 1 1-DICHLOROETHANE

SD-100-13 3-Jun-1987 1 1-DICHLOROETHANE

SD-100-13 11-Jun-1987 1 1-DICHLOROETHANE

SD-100-13 18-Jun-1987 I 1-DICHLOROEIHANE

** SD-100-14

SD-100-14 12-May-1987 I 1-DICHLOROETHANE SD-100-14 19-May-1987 I 1-DICHLOROETHANE

SD-100-14 26-May-1987 1 1-DICHLOROETHANE

SD-100-14 3-Jun-1987 11 -DICHLOROETHANE

SD-100-14 11-Jun-1987 I 1-DICHLOROETHANE

SD-100-14 18-Jun-1987 I 1-DICHLOROETHANE

** SD-100-C

SD-100-C 5-Mar-1987 1 1-DICHLOROETHANE SD-100-C 9-Mar-1987 I I-DICHLOROETHANE SD-100-C 17-Mar-1987 1 L-DICHLOROETHANE SD-100-C 24-Mar-1987 1 1-DICHLOROETHANE SD-100-C 8-Apr-1987 1 1-DICHLOROETHANE SD-100-C 13-May-1987 1 1-DICHLOROETHANE SD-100-C 20-May-1987 \& 1-DICHLOROETHANE SD-100-C 27-May-1987 I 1-DICHLOROETHANE SD-100-C 12-Jun-1987 I 1-DICHLOROETHANE SD-100-C 19-Jun-1987 1 1-DICHLOROETHANE

** SD-100-D

SD-100-D 6-Apr-1987 11 -DICHLOROETHANE SD-100-D 11-Jun-1987 1 1-DICHLOROETHANE

$\star \star \quad S D-100$

SD-100 5-Mar-1987 1 1-DICHLOROETHENE

SD-100 11-Mar-1987 1 1-DICHLOROETHENE

SD-100 18-Mar-1987 11 1-DICHLOROETRENE

SD-100 24-Mar-1987 I 1 -DICHLOROETHENE

SD -100

6-Apr-1987 I 1-DICHLOROETHENE

$S D-100$

12-May-1987 1 1-DICHLOROETHENE

$S D-100$

19-May-1987 1 1-DICHLOROETHENE

SD -100

26-May-1987 I 1-DICHLOROETHENE

SD -100

3-Jun-1987 I 1-DICHLOROETHENE

SD -100

11-Jun-1987 1 1-DICHLOROETHENE

SD -100

18-Jun-1987 I 1-DICHLOROETIENE

** SD-100-01

SD-100-01 12-May-1987 1 1-DICHLOROETHENE SD-100-01 18-May-1987 I 1-DICHLOROETHENE SD-100-01 26-May-1987 11 -DICHLOROETHENE SD-100-01 3-Jun-1987 11 -DICHLOROETHENE SD-100-01 11-JUn-1987 \& 1-DICHLOROETHENE

$\begin{array}{ll}<5 & \mathrm{ug} / \mathrm{L} \\ <5 & \mathrm{ug} / \mathrm{L} \\ <5 & \mathrm{ug} / \mathrm{L} \\ <5 & \mathrm{ug} / \mathrm{L} \\ <5 & \mathrm{ug} / \mathrm{L} \\ <5 & \mathrm{ug} / \mathrm{L}\end{array}$

$<S$

$<5$

$<5$

$<5$

$<5$

$<5$

$u g / L$ ug/L $u \mathrm{~g} / \mathrm{L}$ ug/L ug/L $48 /$

$<5$

$<5$

$<5$

$<5$

$<5$

$<5$

$<5$

$<5$

$<5$

$<5$

ug/L $\mathrm{ug} / \mathrm{L}$ $48 / 2$ $\mathrm{ug} / \mathrm{L}$ $4 \mathrm{~g} / \mathrm{L}$ ug/L - ug/L $u g / L$ ug/L ug/L

$<5$ $<5$

ug/L $\mathrm{ug} / \mathrm{L}$

$<5$

$<5$

$<5$

$<5$

$<5$

$<5$

$<5$

$<5$

$<5$

$<5$

$<5$

ug/L ug/ $/$ ug/L ug/L $\mathrm{ug} / \mathrm{L}$ ug $/$. ug $/ \mathrm{L}$ ug/L ug/L $\mathrm{ug} / \mathrm{L}$ ug/L

$<5$

ug/L ug/L ug/L ug/L ug $/ 2$ 


$\begin{array}{lccc}\text { Location } & \text { Date } & \text { Test Compound } \\ \text { SD-100-01 } & 18 \text {-Jun-1987 } & 1 & 1 \text {-DICHLOROETHENE } \\ \text { ** SD-100-02 } & & \\ \text { SD-100-02 } & 12 \text {-May-1987 } & 1 & 1 \text {-DICHLOROETHENE } \\ \text { SD-100-02 } & 18 \text {-May-1987 } & 1 & 1 \text {-DICHLOROETHENE } \\ \text { SD-100-02 } & 26 \text {-May-1987 } & 1 & 1 \text {-DICHLOROETHENE } \\ \text { SD-100-02 } & \text { 3-Jun-1987 } & 1 & 1 \text {-DICHLOROETHENE } \\ \text { SD-100-02 } & 11 \text {-Jun-1987 } & 1 & 1 \text {-DICHLOROETHENE } \\ \text { SD-100-02 } & 18 \text {-Jun-1987 } & 1 & 1 \text {-DICHLOROETHENE }\end{array}$

** SD-100-03

SD-100-03 12-May-1987 \& 1-DICHLOROETHENE SD-100-03 18-May-1987 1 1-DICHLOROETHENE SD-100-03 26-May-1987 1 1-DICHLOROETHENE SD-100-03 3-Jun-1987 I 1-DICHLOROETHENE SD-100-03 11-JUn-1987 I 1-DICHLOROETHENE SD-100-03 18-Jun-1987 \& 1-DICHLOROETHENE

** SD-100-03D

SD-100-03D 12-May-1987 1 1-DICHLOROETHENE SD-100-03D 26-May-1987 1 1-DICHLOROETHENE

$\begin{array}{cc}\text { Results } & \text { Units } \\ <5 & \text { ug/L } \\ & \\ <5 & u g / L \\ <5 & u g / L \\ <5 & u g / L \\ <5 & u g / L \\ <5 & u g / L \\ <5 & u g / L\end{array}$

$<5$

$<5$

$<5$

$<5$

$<5$

$<5$

$$
\begin{aligned}
& u g / L \\
& u g / L \\
& u g / L \\
& u g / L \\
& u g / L \\
& u g / L
\end{aligned}
$$

$<5 \quad u g / L$

** SD $-100-04$

SD-100-04 12-May-1987 1 1-DICHLOROETHENE

SD-100-04 18-May-1987 I I-DICHLOROETHENE

SD-100-04 26-May-1987 I 1-DICELOROETHENE

SD-100-04 3-Jun-1987 1 1-DICKLOROETRENE

SD-100-04 11-Jun-1987 1 1-DICHLOROETHENE

SD-100-04 18-Jun-1987 \& 1-DICHLOROETHENE

* SD-100-06

$\begin{array}{ll}<5 & u g / L \\ <5 & u g / L \\ <5 & u g / L \\ <5 & u g / L \\ <5 & u g / L \\ <5 & u g / L\end{array}$

SD-100-06 12-May-1987 1 1-DICLLOROETHENE

SD-100-06 18-May-1987 1 1-DICHLOROETHENE

SD-100-06 26-May-1987 \& 1-DICHLOROETHENE

SD-100-06 3-Jun-1987 1 1-DICHLOROETHENE

SD-100-06 11-Jun-1987 1 1-DICHLOROETHENE

SD-100-06 18-Jun-1987 1 1-DICHLOROETKENE

** SD-100-06D

SD-100-06D 3-JUn-1987 1 1-DICHLOROETHENE

** SD-100-07

SD-100-07 12-May-1987 1 1-DICHLOROETHENE

SD-100-07 19-May-1987 1 1-DICHLOROETHENE

SD-100-07 26-May-1987 1 1-DICHLOROETHENE

SD-100-07 3-Jun-1987 1 1-DICHLOROETHENE

SD-100-07 11-Jun-1987 I 1-DICHLOROETHENE

SD-100-07 18-Jun-1987 I 1-DICHLOROETHENE

** SD-100-07D

SD-100-07D 18-Jun-1987 1 1-DICHLOROETHENE
$<5$

$<5$

$<5$

$<5$

$<5$

$<5$

$<5$

\section{ug/L \\ ug/L \\ ug/L \\ $u g / L$ \\ ug/L \\ ug/L}

ug/L

5
$<5$

$<5$
6

6
7

$<5$

$<5$

ug/L

ug/L

ug/L

ug/L

$\mathrm{ug} / \mathrm{L}$

ug/L

6

ug/L 
Units

* SD- $100-09$

SD-100-09 12-May-1987 \& 1-DICHLOROETHENE SD-100-09 18-May-1987 I 1-DICHLOROETHENE SD-100-09 26-May-1987 1 1-DICHLOROETHENE SD-100-09 3-Jun-1987 \& 1-DICHLOROETHENE SD-100-09 11-Jun-1987 \& 1-DICHLOROETHENE SD-100-09 18-Jun-1987 1 1-DICHLOROETHENE

** SD $-100-10$

SD-100-10 12-May-1987 I 1-DICHLOROETHENE SD-100-10 19-May-1987 1 1-DICHLOROETHENE SD-100-10 26-May-1987 I 1-DICHLOROETHENE SD-100-10 3-Jun-1987 \& 1-DICHLOROETHENE SD-100-10 11-Jun-1987 \& i-DICHLOROETHENE SD-100-10 18-Jun-1987 I 1 -DICHLOROETHENE

$\begin{array}{ll}<5 & \text { ug/L } \\ <5 & \text { ug/L } \\ <5 & \text { ug/L } \\ <5 & \text { ug/L } \\ <5 & \text { ug/L } \\ <5 & \text { ug/L }\end{array}$

** SD $-100-11$

SD-100-11 12-May-1987 1 1-DICHLOROETHENE SD-100-11 19-May-1987 1 1-DICHLOROETHENE SD-100-11 26-May-1987 1 1-DICHLOROETHENE SD-100-11 3-Jun-1987 1 1-DICHLOROETHENE SD-100-11 11-JUn-1987 1 1-DICHLOROETHENE SD-100-11 18-Jun-1987 1 1-DICHLOROETHENE

** SD - 100-12

SD-100-12 12-May-1987 1 1-DICHLOROETHENE SD-100-12 19-May-1987 \& 1-DICHLOROETHENE SD-100-12 26-May-1987 1 1-DICHLOROETHENE SD-100-12 3-Jun-1987 I 1 -DICHLOROETHENE SD-100-12 11-Jun-1987 1 1-DICHLOROETHENE SD-100-12 18-Jun-1987 1 1-DICHLOROETHENE

ug/L

** SD $-100-13$

SD-100-13 12-May-1987 I L-DICHLOROETHENE SD-100-13 19-May-1987 \& 1-DICHLOROETHENE SD-100-13 26-May-1987 I 1-DICHLOROETHENE SD-100-13 3-Jun-1987 1 1-DICHLOROETHENE SD-100-13 11-Jun-1987 I 1-DICHLOROETHENE SD-100-13 18-Jun-1987 1 1-DICHLOROETHENE

太* SD $-100-14$

SD-100-14 12-May-1987 \& 1-DICHLOROETHENE SD-100-14 19-May-1987 1 1-DICHLOROETHENE SD-100-14 26-May-1987 I 1-DICHLOROETHENE SD-100-14 3-Jun-1987 \& 1 -DICHLOROETHENE SD-100-14 11-Jun-1987 1 1-DICHLOROETHENE SD-100-14 18-Jun-1987 \& 1-DICHLOROETHENE

* $5 D-100-C$

SD-100-C 5-Mar-1987 1 1-DICHLOROETHENE
$<5$

$<5$

$<5$

$<5$

$<5$

$<5$

$$
\begin{aligned}
& u g / L \\
& u g / L \\
& u g / L \\
& u g / L \\
& u g / L \\
& u g / L
\end{aligned}
$$

ug/L $u g / \mathrm{L}$ ug/L ug/L ug/L ug/L

$<5$

$<5$

$<5$

$<5$

$<5$

$<5$

$\mathrm{ug} / \mathrm{L}$ ug/L ug/L $u g / L$ ug $/ \mathrm{L}$ $u g / L$ $<5$ ug/L 


\begin{tabular}{|c|c|c|c|c|}
\hline Location & Dete & Test Compound & Results & Units \\
\hline SD-100-C & $9-\operatorname{Mar}-1987$ & I 1-DICHLOROETHENE & $<5$ & ug/L \\
\hline SD-100-C & 17-Mar- 1987 & 1 1-DICHLOROETHENE & $<5$ & ug/L \\
\hline SD-100-C & $24-\mathrm{Mar}-1987$ & 1 1-DICHLOROETHENE & $<5$ & ug/L \\
\hline$S D-100-C$ & B-Apr-1987 & 1 1-DICHLOROETHENE & $<5$ & $4 g / L$ \\
\hline SD-100-C & 13-May - 1987 & 1 1-DICHLOROETHENE & $<5$ & $\mathrm{ug} / \mathrm{L}$ \\
\hline SD-100-C & 20-May-1987 & 1 1-DICHLOROETHENE & $<$ & ug $/ \mathrm{L}$ \\
\hline SD $-100-C$ & 27-May-1987 & 1 1-DICHLOROETHENE & $<5$ & $\mathrm{ug} / \mathrm{L}$ \\
\hline$S D-100-C$ & $12-J$ un -1987 & 1 1-DICHLOROETHESE & $<5$ & $\mathrm{ug} / \mathrm{L}$ \\
\hline SD-100-C & $19-J$ un- 1987 & 1 1-DICHLOROETHENE & $<5$ & $\operatorname{ug} / \mathrm{L}$ \\
\hline \multicolumn{5}{|c|}{ * SD-100-D } \\
\hline$S D-100-D$ & 6-Apr- 1987 & 1 1-DICHLOROETHENE & $<5$ & $u g / L$ \\
\hline SD-100-D & $11-J$ un -1987 & 1 1-DICHLOROETHENE & $<5$ & ug/L \\
\hline \multicolumn{5}{|l|}{ ** SD-100 } \\
\hline SD-100 & 5-Mar-1987 & I 24 -TRICHLOROBENZENE & $<10$ & ug/L \\
\hline SD -100 & $11-\operatorname{Mar}-1987$ & 12 4-TRICHLOROBENZENE & $<10$ & $u g / L$ \\
\hline SD- 100 & 12-May- 1987 & I 2 4-TRICHLOROBENZENE & $<10$ & $u g / L$ \\
\hline SD -100 & $26-$ May -1987 & 12 4-TRICHLOROBENZENE & $<5.0$ & ug/L \\
\hline \multicolumn{5}{|c|}{ * SD-100-01 } \\
\hline$S D-100-01$ & 12-May - 1987 & 124 -TRICHLOROBENZENE & $<10$ & $\mathrm{ug} / \mathrm{L}$ \\
\hline SD-100-01 & 26-May- 1987 & 124 -TRICHLOROBENZENE & $<5.0$ & ug/L \\
\hline \multicolumn{5}{|c|}{ *t SD-100-02 } \\
\hline SD-100-02 & $12-$ May -1987 & 124 -TRICHLOROBENZENE & $<10$ & $\mathrm{ug} / \mathrm{L}$ \\
\hline SD $-100-02$ & $26-$ May -1987 & 124 -TRICHLOROBENZENE & $<5.0$ & $48 / \mathrm{L}$ \\
\hline \multicolumn{5}{|c|}{ ** SD-100-03 } \\
\hline SD - 100-03 & 12 - May - 1987 & 124 -TRICHLOROBENZENE & $<10$ & $\mathrm{ug} / \mathrm{L}$ \\
\hline SD $-100-03$ & $26-$ May -1987 & 124 -TRICHLOROBENZENE & $<5.0$ & $\mathrm{ug} / \mathrm{L}$ \\
\hline \multicolumn{5}{|c|}{$\star \star \quad$ SD $-100-03 D$} \\
\hline SD-100-03D & $12-$ May -1987 & 124 -TRICHLOROBENZENE & $<10$ & ug $/ \mathrm{L}$ \\
\hline$S D-100-03 D$ & $26-$ May -1987 & 124 -TRICHLOROBENZENE & $<5.0$ & $\mathrm{ug} / \mathrm{L}$ \\
\hline \multicolumn{5}{|c|}{$* * \quad$ SD $-100-04$} \\
\hline SD-100-04 & $12-$ May -1987 & 124 -TRICHLOROBENZENE & $<10$ & $\operatorname{ug} / \mathrm{L}$ \\
\hline SD - $100-04$ & $26-$ May -1987 & 124 -TRICHLOROBENZENE & $<5.0$ & ug/l \\
\hline \multicolumn{5}{|c|}{ * SD $-100-06$} \\
\hline$S D-100-06$ & $12-$ May- 1987 & 124 -TRICHLOROBENZENE & $<10$ & $\mathrm{ug} / \mathrm{L}$ \\
\hline$S D \cdot 100-06$ & $26-$ May- 1987 & 124 -TRICHLOROBENZENE & $<5.0$ & ug/L \\
\hline \multicolumn{5}{|c|}{ * SD-100-07 } \\
\hline$S D-100-07$ & 12-May- 1987 & 124 -TRICHLOROBENZENE & $<10$ & $\mathrm{ug} / \mathrm{L}$ \\
\hline SD-100-07 & $26-$ May- 1987 & 124 -TRICHLOROBENZENE & $<5.0$ & $\mathrm{ug} / \mathrm{L}$ \\
\hline \multicolumn{5}{|c|}{ ** SD-100-09 } \\
\hline$S D-100-09$ & 12-May- 1987 & 124 -TRICHLOROBENZENE & $<10$ & $4 \mathrm{~g} / \mathrm{L}$ \\
\hline SD $-100-09$ & $26-$ May- 1987 & 124 -TRICHLOROBENZENE & $<5.0$ & ug/L \\
\hline
\end{tabular}


** SD $-100-10$

SD-100-10 12-May-1987 12 4-TRICHLOROBENZENE SD-100-10 26-May-1987 I 2 4-IRICHLOROBENZENE

$\begin{array}{rr}<10 & 4 \mathrm{~g} / \mathrm{L} \\ <5.0 & \mathrm{ug} / \mathrm{L}\end{array}$

* SD $-100-11$

SD-100-11 12-May-1987 12 4-TRICHLOROBERZRNE SD-100-11 26-May-1987 I 2 4-TRICHLOROBENZINE

* SD $-100-12$

SD-100-12 12-May-1987 12 4-TRICHLOROBENZENE SD-100-12 26-May-1987 12 4-TRICHLOROBENZENE

** SD $-100-13$

SD-100-13 12-May-1987 12 4-TRICHLOROBENZENE SD-100-13 26-May-1987 12 4-TRICHLOROBENZENE

** SD $-100-14$

SD-100-14 12-May-1987 12 4-TRICHLOROBENZENE SD-100-14 26-May-1987 12 4-TRICHLOROBENZENE

$\star$ SD $-100-C$

SD-100-C 5-Mar-1987 I 2 4-TRICHLOROBENZENE SD-100-C 9-Mar-1987 12 4-TRICHLOROBENZENE SD-100-C 17-Mar-1987 12 4-TRICHLOROBENZENE SD-100-C 24-Mar-1987 12 4-IRICHLOROBENZENE SD-100-C 8-Apt-1987 12 4-TRICHLOROBENZENE SD-100-C 13-May-1987 12 4-TRICHLOROBENZENE SD-100-C 20-May-1987 12 4-TRICHLOROBENZENE SD-100-C 27-May-1987124-TRICHLOROBENZENE SD-100-C 12-JUn-1987 12 4-TRICHLOROBERZENE SD-100-C 19-JUn-1987 I 2 4-TRICHLOROBENZENE

* SD -100

SD -100

SD -100

5-Mar-1987 1 2-DICHLOROBENZENE

SD -100 11-Mar-1987 12-DICHLOROBENZENE SD $\cdot 100$ 12-May-1987 I 2-DICHLOROBENZERE 26 -May-1987 12 2-DICHLOROBENZENE

* SD $-100-01$

SD-100-01 12-May-1987 1 2-DICHLOROBENZENE SD-100-01 26-May-1987 I 2-DICHLOROBENZENE

** SD-100-02

SD-100-02 12-May-1987 \& 2-DICHLOROBENZBNE SD-100-02 26-May-1987 1 2-DICHLOROBENZENE

** SD-100-03

SD-100-03 12-May-1987 12 2-DIGHLOROBENZENE SD-100-03 26-May-1987 12 2-DICHLOROBENZENE 
Storm Drain Data for SD-100

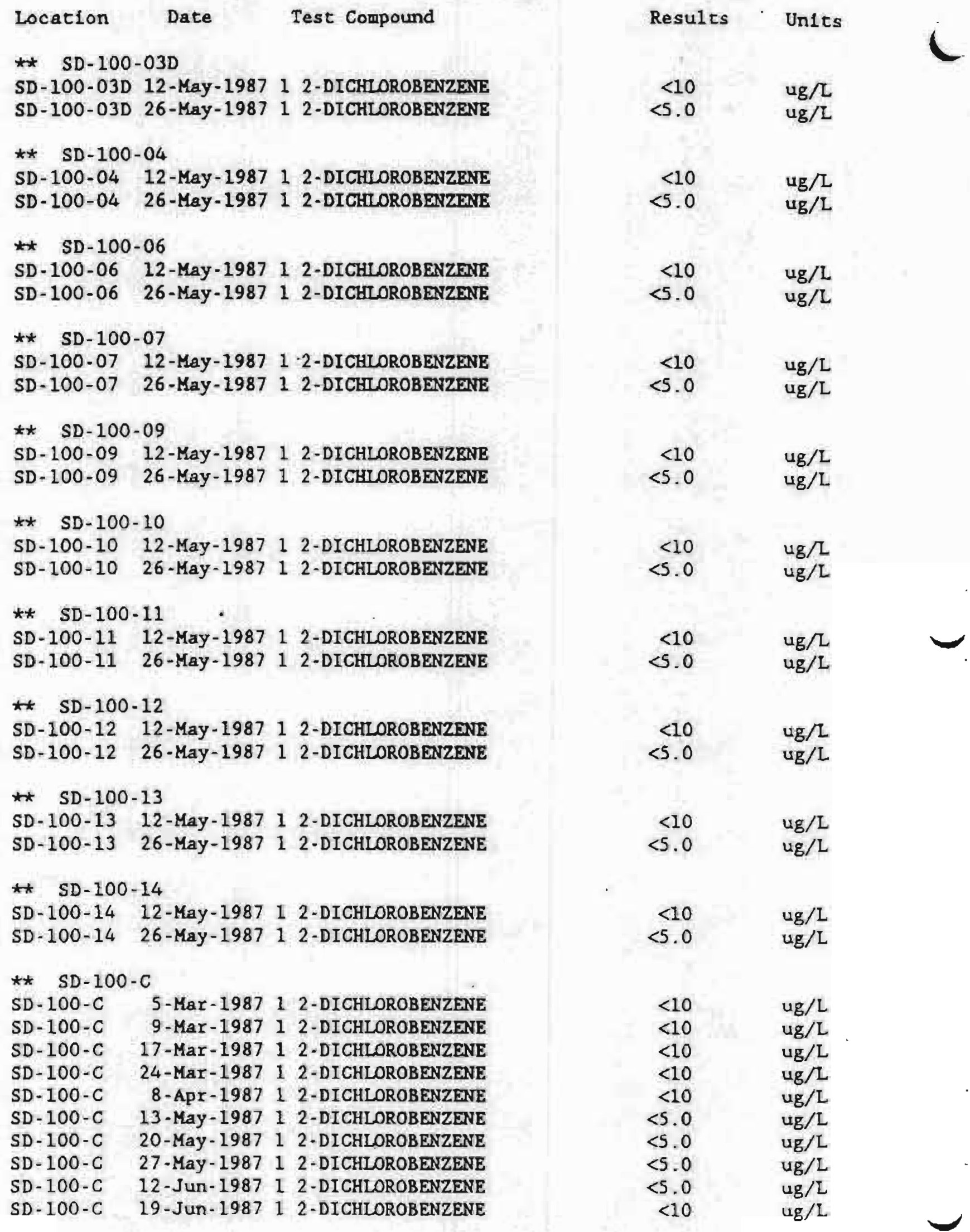


Storm Drain Data for SD-100

Location Date Test Compound Results Untes

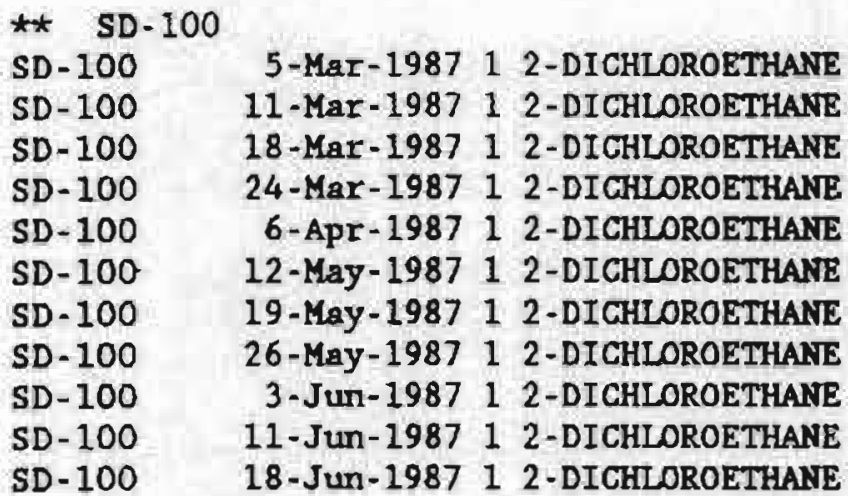

** SD-100.01

SD-100-01 12-May-1987 1 2-DICHLOROETHANE

SD-100-01 18-May-1987 \& 2-DICHLOROETHANE

SD-100-01 26-May-1987 1 2-DICHLOROETHANE

SD-100-01 3-Jun-1987 \& 2-DICHLOROETHANE

SD-100-01 11-Jun-1987 \& 2-DICHLOROETHANE

SD-100-01 18-Jun-1987 I 2-DICHLOROETHANE

** SD $-100-02$

SD-100-02 12-May-1987 \& 2-DICHLOROETHANE

SD-100-02 18-May-1987 \& 2-DICHLOROETHANE

SD-100-02 26-May-1987 I 2-DICHLOROETHANE

SD-100-02 3-Jun-1987 I 2-DICHLOROETHANE

SD-100-02 11-Jun-1987 1 2-DICHLOROETHANE

SD-100-02 18-Jun-1987 12 2-DICHLOROETHANE

** SD $-100-03$

SD-100-03 12-May-1987 I 2-DICHLOROETHANE

SD-100-03 18-May-1987 1 2-DICHLOROETHANE

SD-100-03 26-May-1987 I 2-DICHLOROETHANE

SD-100-03 3-Jun-1987 \& 2-DICHLOROETHANE

SD-100-03 11-Jun-1987 1 2-DICHLOROETHANE

SD-100-03 18-JUn-1987 1 2-DICHLOROETHANE

* SD-100-03D

SD-100-03D 12-May-1987 I 2-DICHLOROETHANE

SD-100-03D 26-May-1987 I 2-DICHLOROETHANE

$\begin{array}{ll}<5 & \mathrm{ug} / \mathrm{L} \\ <5 & \mathrm{ug} / \mathrm{L} \\ <5 & \mathrm{ug} / \mathrm{L} \\ <5 & \mathrm{ug} / \mathrm{L} \\ <5 & \mathrm{ug} / \mathrm{L} \\ <5 & \mathrm{ug} / \mathrm{L} \\ <5 & \mathrm{ug} / \mathrm{L} \\ <5 & \mathrm{ug} / \mathrm{L} \\ <5 & \mathrm{ug} / \mathrm{L} \\ <5 & \mathrm{ug} / \mathrm{L} \\ <5 & \mathrm{ug} / \mathrm{L}\end{array}$

$<5$

$<5 \quad$ ug/L

$<5$ ug/L

$<5 \quad$ ug/L

$<5 \quad 4 g / L$

$<5 \quad u g / 2$

$<5$

$<5$

$<5$

$<5$

$<5$

$<5$

ug/L

$u g / L$

$\mathrm{ug} / \mathrm{L}$

ug/L

$u g / L$

$u g / L$

$<5$

ug/L

$<5 \quad u g / L$

$<5 \quad$ ug/L

$<5 \quad u g / L$

$<5 \quad \mathrm{ug} / \mathrm{L}$

$<5 \quad$ ug/L

** SD-100-04

SD-100-04 12-May-1987 1 2-DICHLOROETHANE

SD-100-04 18-May-1987 1 2-DICHLOROETHANE

SD-100-04 26-May-1987 12 2-DICHLOROETHANE

SD-100-04 3-Jun-1987 1 2-DICHLOROETHANE

SD-100-04 11-Jun-1987 1 2-DICHLOROETHANE

SD-100-04 18-Jun-1987 i 2-DICKLOROETHANE

$\begin{array}{ll}<5 & \mathrm{ug} / \mathrm{L} \\ <5 & \mathrm{ug} / \mathrm{L} \\ & \\ & \\ <5 & \mathrm{ug} / \mathrm{L} \\ <5 & \mathrm{ug} / \mathrm{L} \\ <5 & \mathrm{ug} / \mathrm{L} \\ <5 & \mathrm{ug} / \mathrm{L} \\ <5 & \mathrm{ug} / \mathrm{L} \\ <5 & \mathrm{ug} / \mathrm{L}\end{array}$




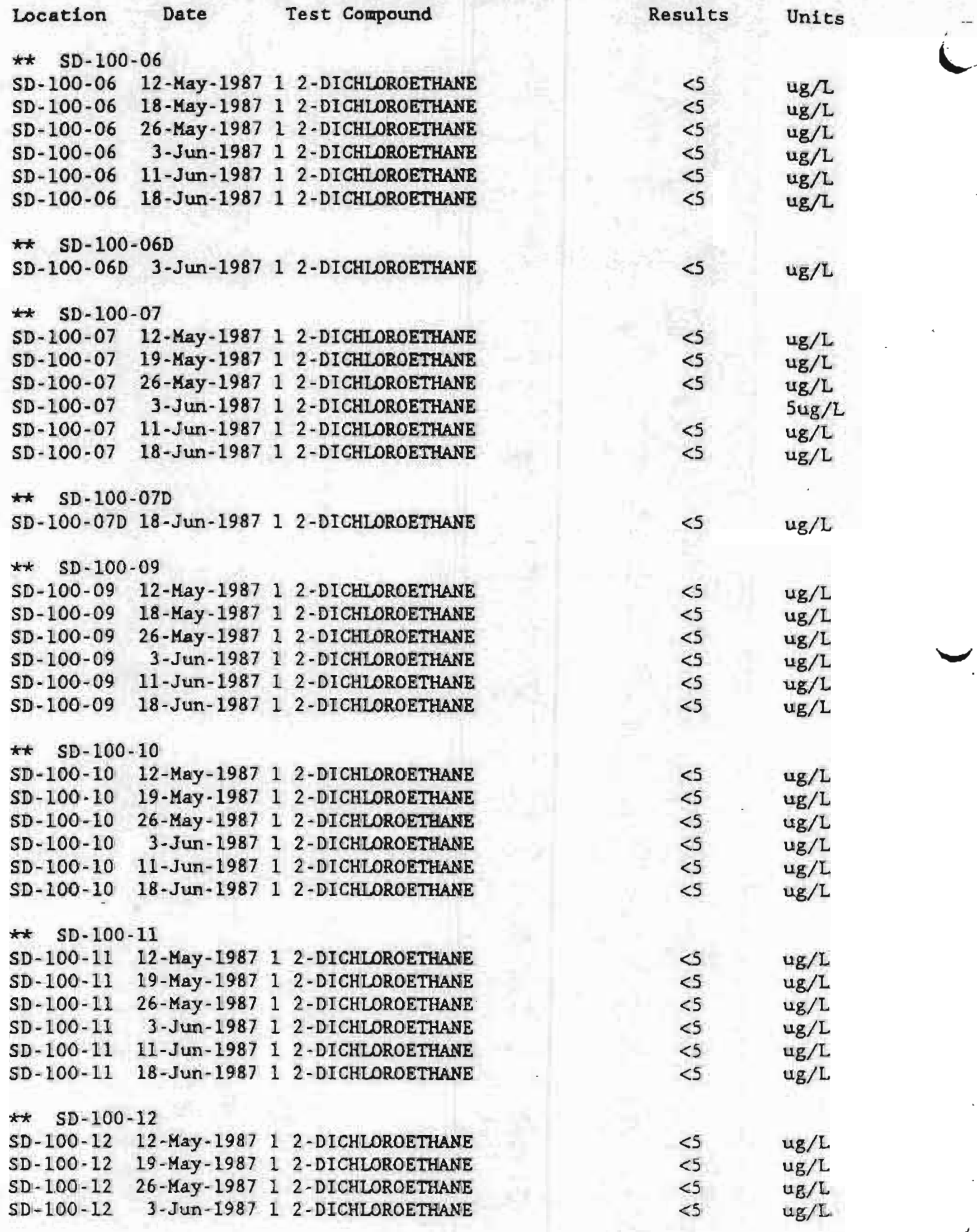


Storm Drain Data for SD-100

Location Date Test Compound Results Units

SD-100-12 11-Jun-1987 1-2-DICHLOROETHANE

SD-100-12 18 -Jun-1987 1 2-DICHLOROETHANE

* SD $-100-13$

SD-100-13 12-May-1987 12 -DICHLOROETHANE

SD-100-13 19-May-1987 I 2-DICHLOROETHANE

SD-100-13 26-May-1987 I 2-DICHLOROETHANE

SD-100-13 3-Jun-1987 1 2-DICHLOROETHANE

SD-100-13 11-Jun-1987 1 2-DICHLOROETHANE

SD-100-13 18-Jun-1987 12 2-DICHLOROETHANE

$\begin{array}{ll}<5 & u g / L \\ <5 & u g / L \\ & \\ <5 & u g / L \\ <5 & u g / L \\ <5 & u g / L \\ <5 & u g / L \\ <5 & u g / L \\ <5 & u g / L \\ & \\ & \\ <5 & u g / L \\ <5 & u g / L \\ <5 & u g / L \\ <5 & u g / L \\ <5 & u g / L \\ <5 & u g / L\end{array}$

* SD-100-14

SD-100-14 12-May-19B7 1 2-DICHLOROETHANE

SD-100-14 19-May-1987 I 2-DICHLOROETHANE

SD-100-14 26-May-1987 I 2-DICHLOROETHANE

SD-100-14 3-Jun-1987 1 2-DICHLOROETHANE

SD-100-14 11-Jun-1987 I 2-DICHLOROETHANE

SD-100-14 18-Jun-1987 1 2-DICHLOROETHANE

** SD $-100-C$

SD-100-C 5-Mar-1987 12 -DICHLOROETHANE

SD-100-C 9-Mar-1987 1 2-DICHLOROETHANE

SD-100-C 17-Mar-1987 I 2-DICHLOROETHANE

SD-100-C 24-Mar-1987 1 2-DICHLOROETHANE

SD-100-C B-API-1987 I 2-DICHLORORTHANE

SD-100-C 13-May-1987 \& 2-DICHLOROETHANE

SD-100-C 20-May-1987 12 -DICHLOROETHANE

SD-100-C 27-May-1987 I 2-DICHLOROETHANE

SD-100-C 12-Jun-1987 I 2-DICHLOROETHANE

SD-100-C 19-Jun-1987 1 2-DICHLOROETHANE

$\begin{array}{ll}<5 & u g / L \\ <5 & u g / L \\ <5 & u g / L \\ <5 & u g / L \\ <5 & u g / L \\ <5 & u g / L \\ <5 & u g / L \\ <5 & u g / L \\ <5 & u g / L \\ <5 & u g / L\end{array}$

* SD-100-D

SD-100-D 6-Apr-1987 12 -DICHLOROETHANE

SD-100-D 11-Jun-1987 1 2-DICHLOROETHANE

$<5 \quad u g / L$

$<5 \quad$ ug/L

** SD -100

SD-100 5-Mar-1987 12 2-DICHLOROPROPANE

SD-100 11-Mar-1987 I 2-DICHLOROPROPANE

SD-100 18-Mar-1987 1 2-DICHLOROPROPANE

SD-100 24-Mar-1987 1 2-DICHLOROPROPANE

SD-100 6-APY-1987 1 2-DICHLOROPROPANE

SD-100 12-May-1987 12 2-DICHLOROPROPANE

SD-100 19-May-1987 \& 2-DTCHLOROPROPANE

SD-100 26-May-1987 1 2-DICHLOROPROPANE

SD-100 3-Jun-1987 I 2-DICHLOROPROPANE

SD-100 11-Jun-1987 1 2-DICHLOROPROPANE

SD-100 18-Jun-1987 12 2-DICHLOROPROPANE

ug/L

ug/L

ug/L

ug/L

$\mathrm{ug} / \mathrm{L}$

ug/L

$u g / L$

$\mathrm{ug} / \mathrm{L}$

$\mathrm{ug} / \mathrm{L}$

ug/L

*t SD-100-01

SD-100-01 12-May-1987 1 2-DICHLOROPROPANE

$<5$

$\mathrm{ug} / \mathrm{L}$

SD-100-01 18-May-1987 \& 2-DICHLOROPROPANE

$<5$

ug/L

ug/L 
Storm Drain Data for SD-100

\begin{tabular}{lrll} 
Location & \multicolumn{1}{c}{ Date } & Test Compound \\
& & & \\
SD-100-01 & 26 -May-1987 & 1 & 2-DICHLOROPROPANE \\
SD-100-01 & 3-Jun-1987 & 1 & 2-DICHLOROPROPANE \\
SD-100-01 & 11-Jun-1987 & 2-DICHLOROPROPAN \\
SD-100-01 & 18 -Jun-1987 & 2 & 2-DICHLOROPROPANE
\end{tabular}

Results Units

SD - 100-02

SD-100-02 12-May-1987 I 2-DICHLOROPROPANE

SD-100-02 18-May-1987 1 2-DICHLOROPROPANE

SD-100-02 26-May-1987 I 2-DICHLOROPROPANE

SD-100-02 3-Jun-1987 1 2-DICHLOROPROPANE

SD-100-02 11-Jur-1987 I 2-DICHLOROPROPANE

SD-100-02 18-Jun-1987 I 2-DICHLOROPROPANE

$\begin{array}{ll}<5 & u g / L \\ <5 & u g / L \\ <5 & u g / L \\ <5 & u g / L \\ & \\ & \\ <5 & u g / L \\ <5 & u g / L \\ <5 & u g / L \\ <5 & u g / L \\ <5 & u g / L \\ <5 & u g / L\end{array}$

** SD $-100-03$

SD-100-03 12-May-1987 1 2-DICHLOROPROPANE

SD-100-03 18-May-1987 I 2-DICHLOROPROPANE

SD-100-03 26-May-1987 \& 2-DICHLOROPROPANE

SD-100-03 3-Jun-1987 1 2-DICHLOROPROPANE

SD-100-03 11-Jun-1987 1 2-DICHLOROPROPANE

SD-100-03 18-Jun-1987 I 2-DICHIOROPROPANE

$<5$

$<5$

$<5$

$<5$

$<5$

$<5$

** SD-100-03D

SD-100-03D 12-May-1987 I 2-DICHLOROPROPANE

SD-100-03D 26-May-1987 1 2-DICHLOROPROPANE

$<5$

$<5$

$u g / L$

$u g / L$

ug $/ \mathrm{L}$

ug/L

$\mathrm{ug} / \mathrm{L}$

ug/L

** SD $100-04$

SD-100-04 12-May-1987 I 2-DICHLOROPROPANE

SD-100-04 18-May-1987 1 2-DICHLOROPROPANE

SD-100-04 26-May-1987 1 2-DICHLOROPROPANE

SD-100-04 3-Jun-1987 1 2-DICHLOROPROPANE

SD-100-04 11-Jum-1987 I 2-DICHLOROPROPANE

SD-100-04 18-Jun-1987 12 2-DICHLOROPROPANE

$\begin{array}{ll}<5 & \mathrm{ug} / \mathrm{L} \\ <5 & \mathrm{ug} / \mathrm{L} \\ <5 & \mathrm{ug} / \mathrm{L} \\ <5 & \mathrm{ug} / \mathrm{L} \\ <5 & \mathrm{ug} / \mathrm{L} \\ <5 & \mathrm{ug} / \mathrm{L}\end{array}$

** SD $-100-06$

SD-100-06 12-May-1987 I 2-DICHLOROPROPANE

SD-100-06 18-May-1987 1 2-DICHLOROPROPANE

SD-100-06 26-May-1987 12 -DICHLOROPROPANE

SD-100-06 3-JUn-1987 1 2-DICHLOROPROPANE

SD-100-06 11-Jun-1987 12 2-DICHLOROPROPANE

SD-100-06 18-Jun-1987 12 2-DICHLOROPROPANE

** SD-100-06D

SD-100-O6D 3-Jun-1987 12 2-DICHLOROPROPANE

$<5$

$<5$

$<5$

$<5$

$<5$

$<5$

$u g / L$

$u g / L$

** SD-100-07

SD-100-07 12-May-1987 1 2-DICHLOROPROPANE

SD-100-07 19-May-1987 I 2-DICHLOROPROPANE

SD-100-07 26-May-1987 1 2-DICHLOROPROPANE

SD-100-07 3-Jun-1987 12 2-DICRLOROPROPANE

SD-100-07 11-JUn-1987 1 2-DICHLOROPROPANE

SD-100-07 18-JUn-1987 1 2-DICHLOROPROPANE

$<5$

$\mathrm{ug} / \mathrm{L}$

$<5$

ug/ $/$

$<5 \quad u g / L$

$<5 \quad$ ug/L

$<5 \quad u g / L$

$<5 \quad u g / L$

$<5$ ug/L 
Location Date Test Compound Results Units

* SD-100-07D

SD-100-07D 18-Jun-1987 1 2-DICHLOROPROPANE

$<5$

ug/L

** SD-100-09

SD-100-09 12-May-1987 I 2-DICHLOROPROPANE

SD-100-09 18-May-1987 1 2-DICHLOROPROPANE

SD-100-09 26-Kay-1987 1 2-DICHLOROPROPANE

SD-100-09 3-Jun-1987 1 2-DICHLOROPROPANE

SD-100-09 11-Jun-1987 I 2-DICHLOROPROPANE

SD-100-09 18-Jun-1987 1 2-DIGHLROPROPANE

** SD $-100-10$

$\begin{array}{ll}<5 & u g / L \\ <5 & u g / L \\ <5 & u g / L \\ <5 & u g / L \\ <5 & u g / L \\ <5 & u g / L\end{array}$

SD-100-10 12-May-1987 I 2-DICHLOROPROPANE

SD-100-10 19-May-1987 I 2-DICHLOROPROPANE

SD-100-10 26-May-1987 12 2-DICHLOROPROPANE

SD-100-10 3-Jun-1987 1 2-DICHLOROPROPANE

SD-100-10 11-Jun-1987 I 2-DICHLOROPROPANE

SD-100-10 18-Jun-1987 1 2-DICHLOROPROPANE

* SD-100-11

SD-100-11 12-May-1987 1 2-DICHLOROPROPANE

SD-100-11 19-May-1987 1 2-DICHLOROPROPANE

SD-100-11 26-May-1987 1 2-DICHLOROPROPANE

SD-100-11 3-Jun-1987 1 2-DICHLOROPROPANE

SD-100-11 11-Jun-1987 1 2-DICHLOROPROPANE

SD-100-11 18-Jun-1987 1 2-DICHLOROPROPANE

$<5$

$<5$

$<5$

$<5$

$<5$

$<5$

$<5$

$<5$

$<5$

$<5$

$<5$

$<5$

$<5$

$<5$

$<5$

$<5$

$<5$

$<5$

SD-100-12 18-Jun-1987 1 2-DICHLOROPROPANE

* SD $-100-13$

SD-100-13 12-May-1987 1 2-DICHLOROPROPANE

SD-100-13 19-May-1987 1 2-DICHLOROPROPANE

SD-100-13 26-May-1987 I 2-DICHLOROPROPANE

SD-100-13 3-Jun-1987 I 2-DICHLOROPROPANE

SD-100-13 11-Jun-1987 1 2-DICHLOROPROPANE

SD-100-13 18-Jun-1987 I 2-DICHLOROPROPANE

* SD - 100-14

SD-100-14 12-May-1987 1 2-DICHLOROPROPANE

SD-100-14 19-May-1987 1 2-DICHLOROPROPANE

SD-100-14 26-May-1987 1 2-DICHLOROPROPANE

SD-100-14 3-Jun-1987 12 2-DICHLOROPROPANE

SD-100-14 11-Jun-1987 I 2-DICHLOROPROPANE

SD-100-14 18-Jun-1987 12 2-DICHLOROPROPANE 


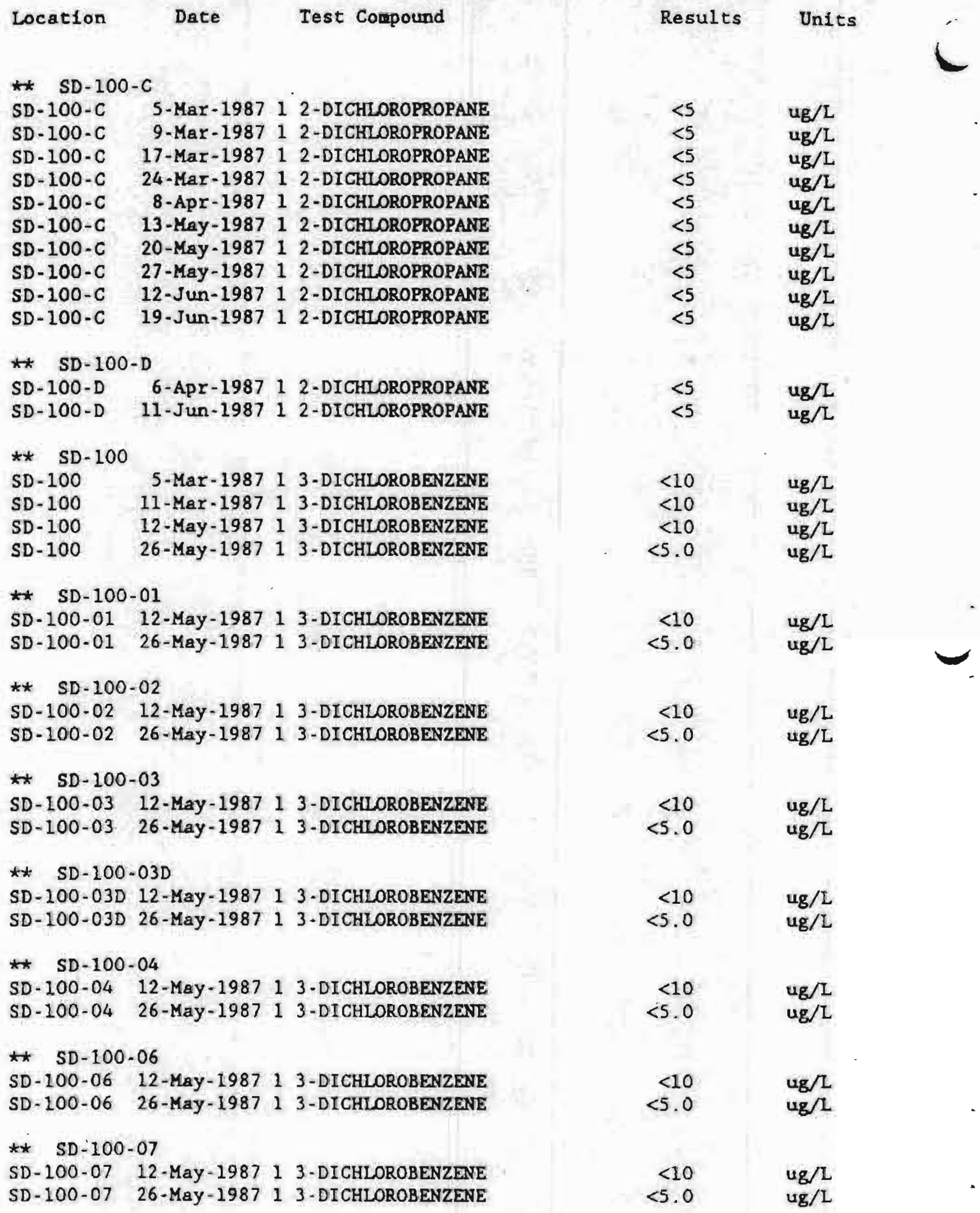


* SD-100-09

SD-100-09 12-May-1987 I 3-DICHLOROBENZENE

SD-100-09 26-May-1987 \& 3-DICHLOROBENZENE

$<10$

$<5.0$

ug/L

* SD $-100-10$

SD-100-10 12-May-1987 1 3-DICHLOROBENZENE

SD-100-10 26-Hay-1987 1 3-DICHLOROBENZENE

$<10$

$<5.0$

$\mathrm{ug} / \mathrm{L}$

* SD-100-11

SD-100-11 12-May-1987 1 3-DICHLOROBENZENE

SD-100-11 26-May-1987 1 3-DICHLOROBENZERE

$<10$

$<5.0$

ug $/ L$

** SD $-100-12$

SD-100-12 12-May-1987 I 3-DICHLOROBENZEAE

SD-100-12 26-May-1987 I 3-DICHLOROBENZENE

$\begin{array}{rr}<10 & \mathrm{ug} / \mathrm{L} \\ <5.0 & \mathrm{ug} / \mathrm{L} \\ & \\ <10 & \mathrm{ug} / \mathrm{L} \\ <5.0 & \mathrm{ug} / \mathrm{L}\end{array}$

$\star * \quad$ SD $-100-13$

SD-100-13 12-May-1987 1 3-DICHLOROBENZENE

SD-100-13 26-May-1987 I 3-DICHLOROBENZENE

** SD-100-14

SD-100-14 12-May-1987 1 3-DICHLOROBENZENE

SD-100-14 26-May-1987 1 3-DICHLOROBENZERE

$<10$

$<5.0$

$u g / L$

* SD-100-C

SD-100-C 5-Mar-1987 I 3-DICHIOROBENZENE

SD-100-C 9-Mar-1987 1 3-DICHLOROBENZENE

SD-100-C 17-Mar-1987 1 3-DICLLOROBENZENE

SD-100-C 24-Mar-1987 1 3-DICHLOROBENZENE

SD-100-C 8-APY-1987 1 3-DICHLOROBENZENE

SD-100-C 13-Kay-1987 I 3-DICHLOROBENZENE

SD-100-C 20-May-1987 1 3-DICRLOROBENZENE

SD-100-C 27-May-1987 \& 3-DICHLOROBENZENE

SD-100-C 12-Jun-1987 \& 3-DICHLOROBENZENE

$S D-100-C$ 19-JUR-1987 I 3-DICHLOROBENZENE

** SD -100

SD-100 5-Mar-1987 I 4-DICHLOROBENZENE

SD-100 11-Mar-1987 1 4-DICHLOROBENZENE

SD-100 12-May-1987 1 4-DICHLOROBENZENE

SD-100 26-May-1987 1 4-DICHLOROBENZENE

* SD $-100-01$

SD-100-01 12-May-1987 14-DICHLOROBENZENE

SD-100-01 26-May-1987 1 4-DICHLOROBENZENE

* SD - $100-02$

SD-100-02 12-May-1987 1 4-DICHLOROB ENZENE

SD-100-02 26-May-1987 1 4-DICHLOROBENZENE

$\begin{array}{cc}<10 & u g / L \\ <10 & u g / L \\ <10 & u g / L \\ <10 & u g / L \\ <10 & u g / L \\ <5.0 & u g / L \\ <5.0 & u g / L \\ <5.0 & u g / L \\ <5.0 & u g / L \\ <10 & u g / L \\ & \\ & \\ <10 & u g / L \\ <10 & u g / L \\ <10 & u g / L \\ <5.0 & u g / L \\ & \\ <10 & u g / L \\ <5.0 & u g / L \\ & \\ <10 & u g / L \\ <5.0 & u g / L\end{array}$


Storm Drain Data for SD -100

Location Date Test Compound Results Units

* SD $-100-03$

SD-100-03 12-May-1987 1 4-DICHLOROBENZENE

$<10$

$<5.0$

$\mathrm{ug} / \mathrm{L}$

SD-100-03 26-May-1987 1 4-DICHLOROBENZENE

* SD-100-03D

SD-100-03D 12 - May-1987 1 4-DTCHLOROBENZENE

SD-100-03D 26-May-1987 1 4-DICHLOROBENZENE

$\begin{array}{ll}<10 & \mathrm{ug} / \mathrm{L} \\ <5.0 & \mathrm{ug} / \mathrm{L}\end{array}$

* SD $-100-04$

SD-100-04 12-May-1987 1 4-DICHLOROBENZENE

SD-100-04 26-May-1987 I 4-DICLLOROBENZENE

$<10$

$<5.0$

ug/ $/ \mathbf{1}$

ug/L

** SD $-100-06$

SD-100-06, 12-May-1987 1 4-DICHLOROBEN2ENE

SD-100-06 26-May-1987 I 4-DICHLOROBENZENE

$\begin{array}{ll}<10 & \text { ug } / \mathrm{L} \\ <5.0 & \text { ug } / \mathrm{L}\end{array}$

** SD $-100 \cdot 07$

SD-100-07 12-M8Y-1987 1 4-DICHLOROBENZENE

SD-100-07 26-May-1987 1 4-DICHLOROBENZENE

$<10$

$<5.0$

$u g / L$

$4 \mathrm{~g} / \mathrm{L}$

** SD-100-09

SD-100-09 12-May-1987 1 4-DICHLOROBENZENE

SD-100-09 26-May-1987 1 4-DICKLOROBENZENE

$<10$

$<5.0$

ug/L

** SD $-100-10$

SD-100-10 12-May-1987 1 4-DICHLOROBENZENE

SD-100-10 26-May-1987 1 4-DICHLOROBENZENE

$<10$

$<5.0$

ug/L

* SD-100-11

SD-100-11 12-May-1987 I 4-DICHLOROBENZENE

SD-100-11 26-May-1987 I 4-DICHLOROBENZFNE

$\begin{array}{ll}<10 & \text { ug } / \mathrm{L} \\ <5.0 & \mathrm{ug} / \mathrm{L}\end{array}$

** SD $-100-12$

SD-100-12 12-May-1987 1 4-DICHLOROBENZENE

SD-100-12 26-May-1987 1 4-DICHLOROBENZENE

$\star \star \quad$ SD $-100-13$

SD-100-13 12-May-1987 1 4-DICHLOROBENZENE

SD-100-13 26-May-1987 I 4-DICHLOROBENZENE

$<10 \quad$ ug/L

** SD $-100-14$

SD-100-14 12-May-1987 1 4-DICHLOROBENZENE

SD-100-14 26-May-1987 \& 4-DICHLOROBENZENE

** SD $-100-C$

SD-100-C 5-Max-1987 I 4-DICHLOROBENZENE

SD-100-C 9-MaI-1987 \& 4-DICHLOROBENZENE

SD-100-C 17-Mar-1987 1 4-DICHLOROBENZENE

SD-100-C 24-Mar-1987 I 4-DICHLOROBENZENE

SD-100-C 8-APL-1987 \& 4-DICHLOROBENZENE

SD-100-C 13-May-1987 I 4-DICHIOROBENZENE

$<10 \quad$ ug/L

$<5.0 \quad$ ug $/ \mathrm{L}$

$<5.0 \quad \mathrm{ug} / \mathrm{L}$

$<10 \quad$ ug/L

$<5.0 \quad \mathrm{ug} / \mathrm{L}$

$<10 \quad$ ug $/ \mathrm{L}$

$<10 \quad$ ug $/ \mathrm{L}$

$<10 \quad$ ug/L

$<10 \quad$ ug/L

$<10 \quad 4 \mathrm{~g} / \mathrm{L}$

$<5.0 \quad$ ug/l 


\begin{tabular}{|c|c|c|c|c|}
\hline Location & Date & Test Compound & Results & Units \\
\hline SD $-100-C$ & 20-May - 1987 & 1 4-DICHLOROBENZENE & $<5.0$ & $\mathrm{ug} / \mathrm{L}$ \\
\hline$S D-100-C$ & 27-May-1987 & 14 -DICHLOROBENZENE & $<5.0$ & $u g / L$ \\
\hline SD $-100-C$ & 12-Jun-1987 & 1 4-DICHLOROBENZENE & $<5.0$ & $\mathrm{ug} / \mathrm{L}$ \\
\hline$S D-100-C$ & $19-J u n-1987$ & 1 4-DICHLOROBENZENE & $<10$ & $u_{g} / L$ \\
\hline \multicolumn{5}{|l|}{$\star \star \quad S D-100$} \\
\hline SD -100 & 5-Mar-1987 & 246 -TRICHLOROPHENOL & $<10$ & $\mathrm{ug} / \mathrm{L}$ \\
\hline SD-100 & 11-Mar-1987 & 246 -TRICHLOROPHENOL & $<10$ & $u g / L$ \\
\hline SD -100 & 12-May-1987 & 246 -TRICHLOROPHENOL & $<10$ & $\mathrm{ug} / \mathrm{L}$ \\
\hline SD -100 & 26-May - 1987 & 246 -TRICHLOROPHEHOL & $<5.0$ & $\mathrm{ug} / \mathrm{L}$ \\
\hline \multicolumn{5}{|c|}{ ** SD-100-01 } \\
\hline SD-100.01 & 12-May-1987 & 246 -TRICHLOROPHENOL & $<10$ & $\mathrm{ug} / \mathrm{L}$ \\
\hline$S D-100-01$ & $26-\mathrm{May}-1987$ & 24 6-TRICHLOROPHENOL & $<5.0$ & $\mathrm{ug} / \mathrm{l}$ \\
\hline \multicolumn{5}{|c|}{$\star \star \quad S D-100-02$} \\
\hline$S D-100-02$ & 12-May-1987 & 246 -TRICHLOROPHENOL & $<10$ & $u g / I$ \\
\hline$S D-100-02$ & 26-May-1987 & 246 -TRICHLOROPHENOL & $<5.0$ & $\mathrm{ug} / \mathrm{L}$ \\
\hline \multicolumn{5}{|c|}{$\star \star$ SD $-100-03$} \\
\hline$S D-100-03$ & 12-May- 1987 & 246 -TRICHLOROPHENOL & $<10$ & $\mathrm{ug} / \mathrm{L}$ \\
\hline SD $-100-03$ & 26-May-1987 & 246 -TRICHLOROPHENOZ & $<5.0$ & $\mathrm{ug} / \mathrm{L}$ \\
\hline \multicolumn{5}{|c|}{$\star \star \quad S D-100-03 D$} \\
\hline$S D-100-03 D$ & $12-$ May -1987 & 246 -TRICHLOROPHENOL & $<10$ & $u g / L$ \\
\hline$S D-100-03 D$ & 26-May- 1987 & 246 -TRICHLOROPHENOL & $<5.0$ & $\mathrm{ug} / \mathrm{L}$ \\
\hline \multicolumn{5}{|c|}{ ** SD-100-04 } \\
\hline$S D-100-04$ & 12-May-1987 & 246 -TRICHLOROPHENOL & $<10$ & $\mathbf{u g} / \mathrm{L}$ \\
\hline SD - $100-04$ & $26-$ May -1987 & 246 -TRICHLOROPHENOL & $<5.0$ & $\mathrm{ug} / \mathrm{L}$ \\
\hline \multicolumn{5}{|c|}{$\star \star \quad S D-100-06$} \\
\hline SD-100-06 & 12-Мay-1987 & 246 -TRICHLOROPHENOL & $<10$ & $4 g / L$ \\
\hline SD-100-06 & $26-$ May-1987 : & 246 -TRICHLOROPHENOL & $<5.0$ & ug/L \\
\hline \multicolumn{5}{|c|}{ ** SD $-100-07$} \\
\hline SD-100-07 & $12-$ May- 1987 & 246 -TRICHLOROPHENOL & $<10$ & ug/L \\
\hline SD-100-07 & 26-May-1987 & 246 -TRICHLOROPHENOL & $<5.0$ & $u g / L$ \\
\hline \multicolumn{5}{|c|}{ * SD-100-09 } \\
\hline SD-100-09 & 12-May-1987 ? & 246 -TRICHLOROPHENOL & $<10$ & $\mathrm{ug} / \mathrm{L}$ \\
\hline SD $-100-09$ & 26-May-1987 2 & 246 -TRICHLOROPHENOL & $<5.0$ & $\mathrm{ug} / \mathrm{L}$ \\
\hline \multicolumn{5}{|c|}{ ** SD - 100-10 } \\
\hline SD $-100-10$ & 12-May-1987? & 246 -TRICHLOROPHENOL & $<10$ & ug/L \\
\hline SD- $100-10$ & $26-$ May-1987? & 246 -TRICHLOROPHENOL & $<5.0$ & $\mathrm{ug} / \mathrm{L}$ \\
\hline \multicolumn{5}{|c|}{ ** SD - 100-11 } \\
\hline SD-100-11 & 12-May-1987 2 & 246 -TRICHLOROPHENOL & $<10$ & $4 \mathrm{~g} / \mathrm{L}$ \\
\hline SD-100-11 & $26-$ May-1987 2 & 246 -TRICHLOROPHENOL & $<5.0$ & $\mathrm{ug} / \mathrm{L}$ \\
\hline
\end{tabular}


Location
* SD-100- 12

SD-100-12 12-May-1987 24 6-TRICHLOROPHINOL SD-100-12 26-May-1987 246 -IRICHLOROPHEMOL

$<10$

Results Units

** SD $-100-13$

SD-100-13 12-May-1987 24 6-TRICHLOROPHENOL

SD-100-13 26-May-1987 24 6-TRICHLOROPHENOL

** SD-100-14

SD-100-14 12-May-1987 24 6-TRICHLOROPHENOL

SD-100-14 26-May-1987 246 -TRICHLOROPHENOL

* SD-100-C

SD-100-C 5-Mar-1987 246 -TRICHLOROPHENOL

SD-100-C 9-Mar-1987 246 -TRICHLOROPHENOL

SD-100-C 17-Mar-1987 24 6-TRICHLOROPHENOL

SD-100-C 24-Mar-1987246-TRICHLOROPHENOL

SD-100-C 8-APr-19872 2 6-TRICHLOROPHENOL

SD-100-C 13-May-1987 24 6-TRICHLOROPHENOL

SD-100-C 20-May-1987 24 6-TRICHLOROPHENOL

SD-100-C 27-May-1987 24 6-TRICHLOROPHENOL

SD-100-C 12-Jun-1987 24 6-TRICHLOROPHENOL

SD-100-C 19-Jun-1987246-TRICHLOROPHENOL

** SD -100

SD- 100 5-Mar-1987 2 4-DICHLOROPHENOL

SD-100 11-Mar-1987 2 4-DICHLOROPHENOL

SD-100 12-May-1987 2 4-DICHLOROPHENOL

SD-100 26-May-1987 2 4-DICHLOROPHENOL

** SD-100-01

SD-100-01 12-May-1987 2 4-DICHLOROPHENOL SD-100-01 26-May-1987 2 4-DICHLOROPHENOL

* $\quad$ SD $-100-02$

SD-100-02 12-May-1987 2 4-DICHLOROPHENOL

SD-100-02 26-May-1987 2 4-DICHLOROPHENOL

** SD-100-03

SD-100-03 12-May-1987 2 4-DICHLOROPHENOL SD-100-03 26-May-1987 2 4-DICHLOROPHENOL

** SD $-100-03 D$

SD-100-03D 12-May-1987 2 4-DICHLOROPHENOL SD-100-030 26-May-1987 2 4-DICHLOROPHENOL

* SD-100-04

SD-100-04 12-May-1987 2 4-DICKLOROPHENOL SD-100-04 26-May-1987 2 4-DICHLOROPHENOL

$\begin{array}{ll}\text { Results } & \text { UnIt } \\ & \\ & \\ <10 & \mathrm{ug} / \mathrm{L} \\ <5.0 & \mathrm{ug} / \mathrm{L} \\ & \\ & \\ <10 & \mathrm{ug} / \mathrm{L} \\ <5.0 & \mathrm{ug} / \mathrm{L} \\ & \\ & \\ & \\ <5.0 & \mathrm{ug} / \mathrm{L} \\ & \mathrm{ug} / \mathrm{L} \\ & \\ <10 & \mathrm{ug} / \mathrm{L} \\ <10 & \mathrm{ug} / \mathrm{L} \\ <10 & \mathrm{ug} / \mathrm{L} \\ <10 & \mathrm{ug} / \mathrm{L} \\ <10 & \mathrm{ug} / \mathrm{L} \\ <5.0 & \mathrm{ug} / \mathrm{L} \\ <5.0 & \mathrm{ug} / \mathrm{L} \\ <5.0 & \mathrm{ug} / \mathrm{L} \\ <5.0 & \mathrm{ug} / \mathrm{L} / \mathrm{L} \\ <5.0 & \mathrm{ug} / \mathrm{L} / \mathrm{L} \\ <10 & \mathrm{ug} / \mathrm{L} \\ & \\ & \\ & \end{array}$


Storm Drain Data for SD-100

Results Units

** SD - 100-06

SD-100-06 12-May-1987 2 4-DICHLORORHENOL

SD-100-06 26-May-1987 2 4-DICHLOROPHENOL

$<10$

$<5.0$

ug $/$ L

* SD-100-07

SD-100-07 12-May-1987 2 4-DICHLOROPHENOL

SD-100-07 26-May-1987 2 4-DICHLOROPLIBYOL

$<10$

$<5.0$

$u g / L$

ug/L

** SD-100-09

SD-100-09 12-May-1987 2 4-DICHLOROPHEHOL

SD-100-09 26-Kay-1987 2 4-DICHLOROPHBSOL

** SD $-100-10$

SD-100-10 12-May-1987 2 4-DICHLOROPHEMOL

SD-100-10 26-May-1987 2 4-DICHLOROPHBHOL

** SD-100-11

SD-100-11 12-May-1987 2 4-DICHLOROPHENOL

SD-100-11 26-Kay-1987 2 4-DICHLOROPHENOL

** SD $-100-12$

SD-100-12 12-May-1987 2 4-DICHLOROPHENOL

SD-100-12 26-May-1987 2 4-DICHLOROPHENOL

** SD $-100-13$

SD-100-13 12-May-1987 2 4-DICHLOROPHENOL

SD-100-13 26-May-1987 2 4-DICHLOROPHENOL

$<10$

$<5.0$

$u g / L$

$\mathrm{ug} / \mathrm{L}$

$<10$

$<5.0$

ug/L

ug/L

$<10$

$<5.0$

$4 \mathrm{~g} / \mathrm{L}$

ug/L

$<10$

ug/L

$<5.0$

$u g / L$

$<10$

$<5.0$

ug/L

$u g / L$

** SD $-100-14$

SD-100-14 12-May-1987 2 4-DICHLOROPHENOL SD-100-14 26-May-1987 2 4-DICHLOROPHENOL

$<10$

$<.0$

ug/L

$u g / L$

** SD-100-C

SD-100-C 5-Mar-19872 4-DICHLOROPHENOL

$<10$

$<10$

$<10$

$<10$

$<10$

$<5.0$

$<5.0$

$<5.0$

$<5.0$

$<10$

$4 \mathrm{~g} / \mathrm{L}$

SD-100-C 17-Mar-19872 4-DICHLOROPHENOL

SD-100-C 24-Mar-19872 4-DICHLOROPHENOL

SD-100-C 8-Apr-1987 2 4-DICHLOROPEENOL

SD-100-C 13-May-1987 2 4-DICHLOROPEEITOL

SD-100-C 20-May-1987 2 4-DICHLOROPHENOL

SD-100-C 27-May-1987 2 4-DICHLOROPHENOL'

SD-100-C 12-Jun-1987 2 4-DICHLORAPHENOL

SD-100-C 19-Jun-1987 2 4-DICHLOROPHEMOL

** SD -100

SD-100 5-Mar-1987 2 4-DIMETHYLPHENOL.

SD-100 11-MaI-1987 2 4-DIMETHYLPHEWOL.

SD-100 12-May-1987 2 4-DIMETHYLPHENOL

SD-100 26-May-1987 2 4-DIMETHYLPHENOL

$<10 \quad$ ug $/ \mathrm{L}$

$<10 \quad$ ug $/ \mathrm{L}$

$<10 \quad$ ug $/ \mathrm{L}$

$<5.0 \quad u g / L$ 
** SD-100-01

SD-100-01 12-May-1987 2 4-DIMETHYLPHENOL

SD-100-01 26-May-1987 2 4-DIMETHYLPHEQHOL

* SD $-100-02$

SD-100-02 12-May-1987 2 4-DIMETHYLPHENOL SD-100-02 26-May-1987 2 4-DIMETHYLPEEHOL

** SD $-100-03$

SD-100-03 12-May-1987 2 4-DIMETHYLPHENOL SD-100-03 26-May-1987 2 4-DIMETHYLPHENOL

** SD-100-03D

SD-100-03D 12-May-1987 2 4-DIMETHYLPHENOL

SD-100-03D 26-May-1987 2 4-DIMETHYLPHENOL

** SD- $100-04$

SD-100-04 12-May-1987 2 4-DIMETHYLPHENOL

SD-100-04 26-May-1987 2 4-DIMETHYLPHENOL

* SD-100-06

SD-100-06 12-May-1987 2 4-DIMETHYLPHENOL

SD-100-06 26-May-1987 2 4-DIMETHYLPHENOL

** SD -100.07

SD-100-07 12-May-1987 2 4-DIMETHYLPHENOL SD-100-07 26-May-1987 2 4-DIMETHYLPHEYOL

** SD-100-09

SD-100-09 12-May-1987 2 4-DIMETHYLPHEROL SD-100-09 26-May-1987 2 4-DIMETHYLPHEAIOL

* SD $-100-10$

SD-100-10 12-May-1987 2 4-DIMETHYLPHENOL SD-100-10 26-May-1987 2 4-DIMETHYLPHENOL

* SD $-100-11$

SD-100-11 12-May-1987 2 4-DIMETHYLPHENOL. SD-100-11 26-May-1987 2 4-DIMETHYLPHENOL

* SD-100-12

SD-100-12 12-May-1987 2 4-DIMETHYLPHENOL SD-100-12 26-May-1987 2 4-DIMETHYLPHENOL

* $*$ SD $-100-13$

SD-100-13 12-May-1987 2 4-DIMETHYLPHESOL SD-100-13 26-May-1987 2 4-DIMETHYLPHENOL

** SD $-100-14$

SD-100-14 12-May-1987 2 4-DIMETHYLPHENOL SD-100-14 26-May-1987 2 4-DIMETHYLPHENOL

\begin{tabular}{|c|c|}
\hline $\begin{array}{l}<10 \\
<5.0\end{array}$ & $\begin{array}{l}u g / L \\
u g / L\end{array}$ \\
\hline $\begin{array}{l}<10 \\
<5.0\end{array}$ & $\begin{array}{l}u g / 2 \\
\text { ug } / 2\end{array}$ \\
\hline $\begin{array}{l}<10 \\
<50\end{array}$ & \\
\hline & \\
\hline$<10$ & $4 \mathrm{~g} / \mathrm{l}$ \\
\hline$<5.0$ & $\mathrm{ug} / \mathrm{L}$ \\
\hline$<10$ & $\mathrm{ug} / \mathrm{L}$ \\
\hline$<5.0$ & $\mathrm{ug} / \mathrm{l}$ \\
\hline$<10$ & $\mathrm{ug} / \mathrm{L}$ \\
\hline$<5.0$ & $\mathrm{ug} / \mathrm{L}$ \\
\hline$<10$ & ug/L \\
\hline$<5.0$ & $4 \mathrm{~g} / \mathrm{L}$ \\
\hline$<10$ & $u_{\mathcal{g}} / \mathrm{L}$ \\
\hline$<5.0$ & $4 \mathrm{~g} / \mathrm{L}$ \\
\hline$<10$ & $u g / L$ \\
\hline$<5.0$ & $\mathrm{ug} / \mathrm{L}$ \\
\hline$<10$ & ug/L \\
\hline$<5.0$ & $\mathrm{ug} / \mathrm{L}$ \\
\hline$<10$ & $\mathrm{ug} / \mathrm{L}$ \\
\hline$<5.0$ & $\mathrm{ug} / \mathrm{L}$ \\
\hline$<10$ & ug $/ \mathrm{L}$ \\
\hline$<5,0$ & $\mathrm{ug} / \mathrm{L}$ \\
\hline$<10$ & ug $/ \mathrm{L}$ \\
\hline$<5.0$ & \\
\hline
\end{tabular}


Storm Drain Data for SD-100

Location Date Test Compound

Resules Units

** SD-100-C

SD-100-C 5-Mar-1987 2 4-DIMETHYLPHENOL

SD-100-C 9-Mar-1987 2 4-DIMETHYLPHENOL

$<10$

$<10$

SD-100-C 17-Mar-1987 2 4-DIMETHYLPHENOL

$<10$

SD-100-C 24-Mar-1987 2 4-DIMETHYLPHENOL

$<10$

SD-100-C 8-Apr-1987 2 4-DIMETHYLPHEMOL

$<10$

SD-100-C 13-May-1987 2 4-DIMETHYLPHENOL

$<5.0$

SD-100-C 20-May-1987 2 4-DIMETHYLPHENOL

SD-100-C 27-May-1987 2 4-DIMETHYLPHENOL

SD-100-C 12-Jun-1987 2 4-DIMETHYLPHEHOL

SD-100-C 19-Jun-1987 2 4-DIMETHYLPHENOL

** SD -100

SD-100 5-Mar-19872 4-DINITROPHENOL

SD-100 11-Mar-19872 4-DINITROPHENOL

SD-100 12-May-1987 2 4-DINITROPHENOL

SD-100 26-May-1987 2 4-DINITROPHENOL

** SD-100-01

SD-100-01 12-May-1987 2 4-DINITROPHENOL

SD-100-01 26-May-1987 2 4-DINITROPHENOL

* SD $-100-02$

SD-100-02 12-May-1987 2 4-DINITROPHENOL

SD-100-02 26-May-1987 2 4-DINITROPHENOL

** SD-100-03

SD-100-03 12-May-1987 2 4-DINITROPHENOL

SD-100-03 26-May-1987 2 4-DINITROPHENOL

** SD-100-03D

SD-100-03D 12-May-1987 2 4-DINITROPHENOL

SD-100-03D 26-May-1987 2 4-DINITROPHENOL

** SD $-100-04$

SD-100-04 12-May-1987 2 4-DINITROPHENOL

SD-100-04 26-May-1987 2 4-DINITROPHENOL

* SD-100-06

SD-100-06 12-May-1987 2 4-DINITROPHENOL

SD-100-06 26-May-1987 2 4-DINITROPHENOL

** SD-100-07

SD-100-07 12-May-1987 2 4-DINITROPHENOL SD-100-07 26-May-1987 2 4-DINITROPHENOL

** SD $-100-09$

SD-100-09 12-May-1987 2 4-DINITROPHENOL

SD-100-09 26-May-198724-DINITROPHENOL

$<5.0$

$<5.0$

$<10$

ug/L

$\mathrm{ug} / \mathrm{L}$

ug/L

ug/L

ug/L

ug/L

$\mathrm{ug} / \mathrm{L}$

ug/L

ug/L

ug/L

$<10$

$<10$

$<10$

$<25.0$

$u g / L$

ug/L

ug/L

$u g / L$

$<10$

$<5.0$

ug/L

ug/L

$<10$

$<5.0$

$\operatorname{ug} / \mathrm{L}$

$\mathrm{ug} / \mathrm{L}$

$<10$

ug/ $/$

$<5.0$

$u g / L$

$<10$

$<5.0$

ug $/ \mathrm{L}$

$\mathrm{ug} / \mathrm{L}$

$<10$

$<5.0$

ug/L

$u g / L$

$<10 \quad$ ug $/$.

$<25.0 \quad$ ug/L

$<10 \quad$ ug/

$<5.0 \quad \mathrm{ug} / \mathrm{L}$

$<10 \quad$ ug/L

$<5.0 \quad \mathrm{ug} / \mathrm{L}$ 
Location Date Test Compound

\begin{tabular}{|c|c|}
\hline Results & Units \\
\hline$<10$ & $u g / L$ \\
\hline$<25.0$ & $\mathrm{ug} / \mathrm{L}$ \\
\hline$<10$ & $u g / L$ \\
\hline$<25.0$ & $48 / 2$ \\
\hline$<10$ & ug $/ \mathrm{L}$ \\
\hline$<25.0$ & $\mathrm{ug} / \mathrm{L}$ \\
\hline$<10$ & ug $/ \mathrm{L}$ \\
\hline$<25$ & $\mathrm{ug} / \mathrm{L}$ \\
\hline$<10$ & $\mathrm{ug} / \mathrm{L}$ \\
\hline$<25.0$ & $\mathrm{ug} / \mathrm{L}$ \\
\hline$<10$ & $\mathrm{ug} / \mathrm{L}$ \\
\hline$<10$ & Ug/L \\
\hline$<10$ & $u g / L$ \\
\hline$<10$ & $\mathrm{ug} / \mathrm{L}$ \\
\hline$<10$ & $u g / L$ \\
\hline$<5.0$ & $\mathrm{ug} / \mathrm{L}$ \\
\hline$<5.0$ & $\lg / 1$ \\
\hline$<5.0$ & $u g / L$ \\
\hline$<25.0$ & $u g / L$ \\
\hline$<10$ & ug/L \\
\hline$<10$ & $u g / L$ \\
\hline$<10$ & $\mathrm{ug} / \mathrm{L}$ \\
\hline$<10$ & ug/L \\
\hline$<5.0$ & $\mathrm{ug} / \mathrm{L}$ \\
\hline$<10$ & $u_{g} / \mathrm{L}$ \\
\hline$<5.0$ & $\mathrm{ug} / \mathrm{L}$ \\
\hline$<10$ & ug/L \\
\hline$<5.0$ & $\mathbf{u g} / \mathrm{L}$ \\
\hline$<10$ & $\mathrm{ug} / \mathrm{L}$ \\
\hline$<5.0$ & $u g / L$ \\
\hline
\end{tabular}

** SD $-100-10$

SD-100-10 12-May-1987 2/4-DINITROPHENOL

SD-100-10 26-May-1987 2 4-DINITROPHENOL

** SD $-100-11$

SD-100-11 12-May-1987 2 4-DINITROPHENOL

SD-100-11 26-May-1987 2 4-DINITROPHENOL

* $*$ SD-100-12

SD-100-12 12-May-1987 2 4-DINITROPHENOL

SD-100-12 26-May-1987 2 4-DINITROPHENOL

** SD $-100-13$

SD-100-13. 12-May-1987 2 4-DINITROPHENOL SD-100-13 26-May-1987 2 4-DINITROPHENOL

** SD-100-14

SD-100-14 12-May-1987 2 4-DINITROPHENOL SD-100-14 26-May-1987 2 4-DINITROPHENOL.

* SD-100-C

SD-100-C 5-Mar-1987 2 4-DINITROPHENOL

SD-100-C 9-Kar-1987 2 4-DINITROPHENOL

SD-100-C 17-Mar-1987 2 4-DINITROPHENOL

SD-100-C 24-Mar-1987 2 4-DINITROPHENOL

SD-100-C 8-APr-1987 2 4-DINITROPHEMOL

SD-100-C 13-May-1987 2 4-DINITROPHENOL

SD-100-C 20-May-1987 2 4-DINITROPHENOL

SD-100-C 27-May-1987 2 4-DINITROPHENOL

SD-100-C 12-Jun-1987 2 4-DINITROPHENOL

SD-100-C 19-Jun-1987 2 4-DINITROPHENOL

$\star \star$ SD -100

SD-100 5-Mar-1987 2 4-DINITROTOLUENE

SD-100 11-Max-1987 2 4-DINITROTOLUENE

SD-100 12-May-1987 2 4-DINITROTOLUENE

SD-100 26-May-1987 2 4-DINITROTOLUENE

* SD-100-01

SD-100-01 12-May-1987 2 4-DINITROTOLUENE

SD-100-01 26-May-1987 24-DINITROTOLUENE

** SD-100-02

SD-100-02 12-May-1987 2 4-DINITROTOLUENE

SD-100-02 26-May-1987 2 4-DINITROTOLUENE

* $\quad$ SD $-100-03$

SD-100-03 12-May-1987 2 4-DINITROTOLUENE

SD-100-03 26-May-1987 2 4-DINITROTOLUENE

\author{
-
}


Stome Drain Data for SD- 100

Location Date Test Compound

Results Unfts

** SD-100-03D

SD-100-03D 12-May-1987 2 4-DINITROTOLUENE

SD-100-03D 26-May-1987 2 4-DINITROTOLUENE

* SD $-100-04$

SD-100-04 12-May-1987 2 4-DINITROTOLUENE

SD-100-04 26-May-1987 2 4-DINITROTOLUENE

** SD $-100-06$

SD-100-06 12-May-1987 2 4-DINITROTOLUENE

SD-100-06 26-Hay-1987 2 4-DINITROTOLUENE

** SD $-100-07$

SD-100-07 12-May-1987 2 4-DINITROTOLUENE

SD-100-07 26-May-1987 2 4-DINITROTOLUENE

** SD-100-09

SD-100-09 12-May-1987 2 4-DINITROTOLUENE

SD-100-09 26-May-1987 2 4-DINITROTOLUENE

** SD $-100-10$

SD-100-10 12-May-1987 2 4-DINITROTOLUENE

SD-100-10 26-May-1987 2 4-DINITROTOLUENE

** SD-100-11

SD-100-11 12-May-1987 2 4-DINITROTOLUENE

SD-100-11 26-May-1987 2 4-DINITROTOLUENE

* SD-100-12

SD-100-12 12-May-1987 2 4-DINITROTOLUENE

SD-100-12 26-May-1987 2 4-DINITROTOLUENE

** SD - 100-13

SD-100-13 12-May-1987 2 4-DINITROTOLUENE

SD-100-13 26-May-1987 2 4-DINITROTOLUENE

* SD $-100-14$

SD-100-14 12-May-1987 2 4-DINITROTOLUENE

SD-100-14 26-May-1987 2 4-DINITROTOLUENE

** SD-100-C

SD-100-C 5-Mar-1987 2 4-DINITROTOLUENE

SD-100-C 9-Mar-19872 4-DINITROTOLUENE

SD-100-C 17-Mar-19872 4-DINITROTOLUENE

SD-100-C 24-Kar-1987 2 4-DINITROTOLUENE

SD-100-C 8-Apr-1987 2 4-DINITROTOLUENE

SD-100-C 13-Kay-1987 2 4-DINITROTOLUENE

SD-100-C 20-May-1987 2 4-DINITROTOLUENE

SD-100-C 27-Kay-1987 2 4-DINITROTOLUENE

SD-100-C 12-Jun-1987 2 4-DINITROTOLUENE

SD-100-C 19-JUn-1987 2 4-DINITROTOLUENE

\begin{tabular}{|c|c|}
\hline $\begin{array}{l}<10 \\
<5.0\end{array}$ & $\begin{array}{l}u g / L \\
u g / L\end{array}$ \\
\hline$<10$ & $4 \mathrm{~g} / \mathrm{L}$ \\
\hline$<5.0$ & $4 \mathrm{~g} / \mathrm{L}$ \\
\hline$<10$ & $\mathrm{ug} / \mathrm{L}$ \\
\hline$<5.0$ & $\mathrm{ug} / \mathrm{L}$ \\
\hline$<0$ & ug/L \\
\hline$<5.0$ & $\mathrm{ug} / \mathrm{L}$ \\
\hline$<10$ & $u g / L$ \\
\hline$<5.0$ & ug/L \\
\hline$<10$ & ug/L \\
\hline$<5.0$ & $u g / L$ \\
\hline$<10$ & $u g / L$ \\
\hline$<5.0$ & $\mathrm{ug} / \mathrm{L}$ \\
\hline$<10$ & ug/L \\
\hline$<5.0$ & $\mathrm{ug} / \mathrm{L}$ \\
\hline$<10$ & $u_{g} / \mathrm{L}$ \\
\hline$<5.0$ & $\mathrm{ug} / \mathrm{L}$ \\
\hline$<10$ & $u g / L$ \\
\hline$<5.0$ & $4 \mathrm{~g} / \mathrm{L}$ \\
\hline$<10$ & $u_{g} / L$ \\
\hline$<10$ & $\mathrm{ug} / \mathrm{L}$ \\
\hline$<10$ & $\mathrm{ug} / \mathrm{L}$ \\
\hline$<10$ & $u g / L$ \\
\hline$<10$ & $\mathrm{ug} / \mathrm{L}$ \\
\hline$<5.0$ & ug/L \\
\hline$<5.0$ & ug/L \\
\hline$<5.0$ & $4 \mathrm{~g} / \mathrm{L}$ \\
\hline$<5.0$ & $\mathrm{ug} / \mathrm{L}$ \\
\hline$<10$ & $u g / L$ \\
\hline
\end{tabular}




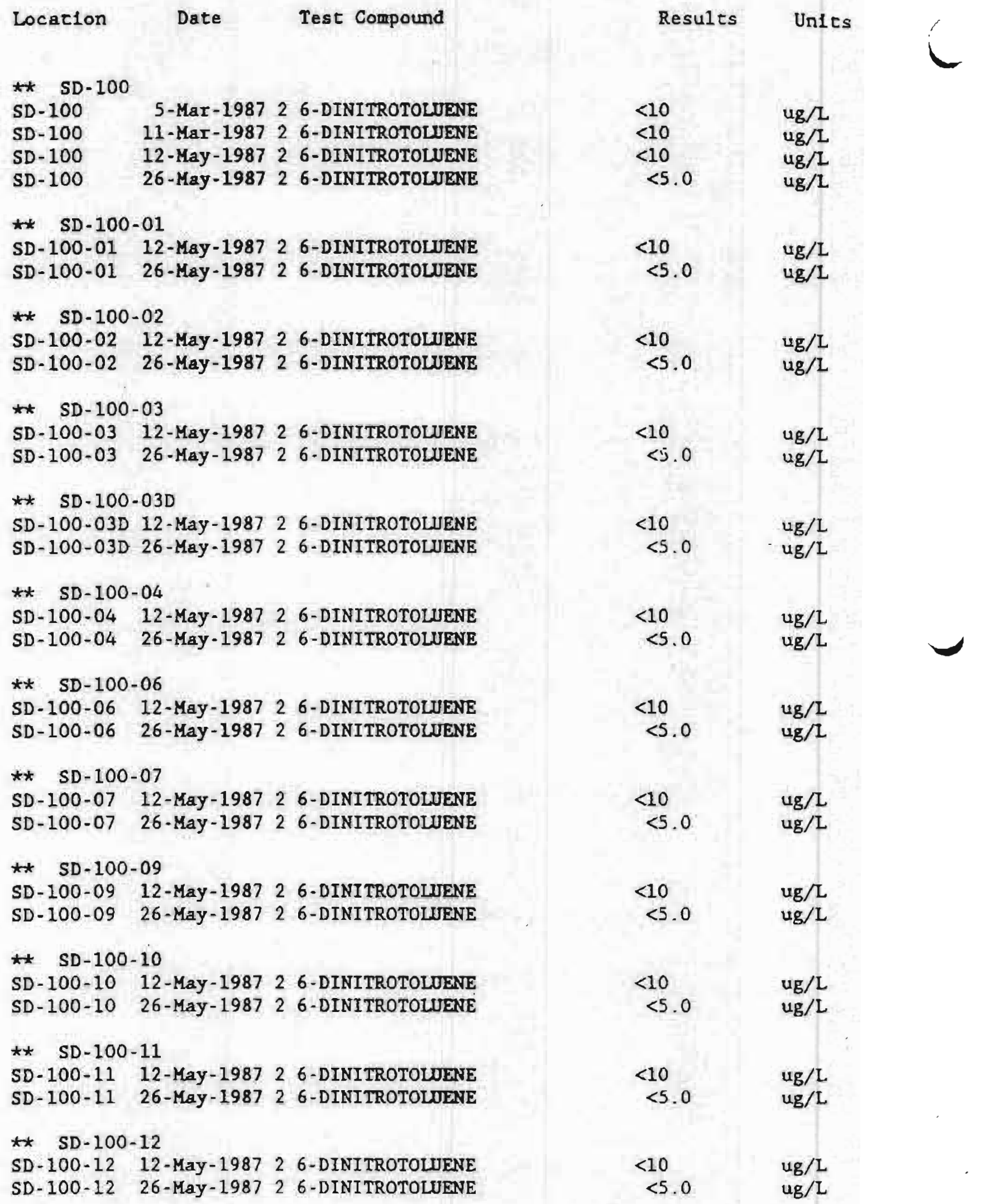


Location Date Test Compound

Results Units

** SD $-100-13$

SD-100-13 12-May-1987 2 6-DINITROTOLUENE

SD-100-13 26-May-1987 2 6-DINITROTOLUENE

$<10$

$<5.0$

ug/L

** SD $-100-14$

SD-100-14 12-May-1987 2 6-DINITROTOLUENE SD-100-14 26-May-1987 2 6-DINITROTOLUENE

$<10$

$<5.0$

ug/L

** SD-100-C

SD-100-C 5-YaY-1987 2 6-DINITROTOLDENE

$<10$

SD-100-C 9-Mar-1987 2 6-DINITROTOLUENE

SD-100-C 17-MaI-1987 2 6-DINITROTOLDENE

$<10$

$<10$

$<10$

$S D-100 \cdot C$

24-Mar-1987 2 6-DINITROTOLUENE

$<10$

SD $-100-C$

B-Apr-1987 2 6-DINITROTOLOENE

SD $-100-C$

13-May-1987 2 6-DINITROTOLUENE

$S D-100-C$

20-May-1987 2 6-DINITROTOLUENE

SD $-100-C$

27-May-1987 2 6-DINITROTOLUENE

SD $-100-C$

12-Jun-1987 2 6-DINITROTOLDENE

19-JUn-1987 2 6-DINITROTOLUENE

$<5.0$

$<5.0$

$<5.0$

$<5.0$

$<10$

$\mathrm{ug} / \mathrm{L}$

$u g / L$

*t SD -100

.SD -100

SD -100

5-Mar-1987 2-CHLOROETHYLVINYL ETHER

SD -100

11-Mar-1987 2-CHLOROETHYLVINYL ETHER

SD -100

18-Mar-1987 2-CHLOROETHYLVINYL ETHER

SD -100

24-Mar-1987 2-CHLOROETHYLVINYL ETHER

SD -100

6-Apr-1987 2-CHLOROETHYLVINYL ETHER

SD -100

12-May-1987 2-CHLOROETHYLVINYL ETHER

SD -100

19-May-1987 2-CRLOROETHYLVINYL ETHER

26-May-1987 2-CHLOROETHYLVINYL ETHER

3-Jun-1987 2-CHLOROETHYLVINYL ETHER

SD -100

SD- 100

11-Jun-1987 2-CHLOROETHYLVINYL ETHER

SD -100

18-Jun-1987 2-CHLOROETHYLVINYL ETHER

** SD-100-01

SD-100-01 12-May-1987 2-CHLOROETHYLVINYL ETHER

SD-100-01 18-May-1987 2-CHLOROETHYLVINYL ETHER

SD-100-01 26-May-1987 2-CHLOROETHYLVINYL ETHER

SD-100-01 3-Jun-1987 2-CHLOROETHYLVINYL ETHER

SD-100-01 11-Jun-1987 2-CKLOROETHYLVINYL ETHER

SD-100-01 18-Jun-1987 2-CHLOROETHYLVINYL ETHER

** SD-100-02

SD-100-02 12-May-1987 2-CHLOROETHYLVINYL ETHER

SD-100-02 18-May-1987 2-CHLOROETHYLVINYL ETHER

SD-100-02 26-May-1987 2-CHLOROETHYLVINYL ETHER

SD-100-02 3-Jun-1987 2-CHLOROETHYLVINYL ETHER

SD-100-02 11-Jun-1987 2-CHLOROETHYLVINYL ETHER

SD-100-02 18-Jun-1987 2-CHLOROETHYLVINYL ETHER

* SD $-100-03$

SD-100-03 12-May-1987 2-CHLOROETHYLVINYL ETHER

$\begin{array}{ll}<10 & \mathrm{ug} / \mathrm{L} \\ <10 & \mathrm{ug} / \mathrm{L} \\ <10 & \mathrm{ug} / \mathrm{L} \\ <10 & \mathrm{ug} / \mathrm{L} \\ <10 & \mathrm{ug} / \mathrm{L} \\ <10 & \mathrm{ug} / \mathrm{L} \\ <10 & \mathrm{ug} / \mathrm{L} \\ <10 & \mathrm{ug} / \mathrm{L} \\ <10 & \mathrm{ug} / \mathrm{L} \\ <10 & \mathrm{ug} / \mathrm{L} \\ <10 & \mathrm{ug} / \mathrm{L}\end{array}$

$<10$

$<10$

$<10$

$<10$

$<10$

ug/L

$u g / L$

$4 \mathrm{~g} / \mathrm{L}$

$\mathrm{ug} / \mathrm{L}$

ug/L

ug $/ L$

ug/L

ug/L

$\mathrm{ug} / \mathrm{L}$

ug/L

$<10$

ug/L

ug/L

ug/L

$48 / 2$

ug/L

$\mathrm{ug} / \mathrm{L}$

$<10$

$<10$

$<10$

$<10$

$<10$

$<10$

ug/L

ug/L

ug/L

ug/L

ug/L

ug/L

$<10$

ug/L 


\begin{tabular}{|c|c|c|c|c|c|}
\hline Location & Date & Test Compound & & Results & Undts \\
\hline SD $-100-03$ & 18-May- 1987 & 2-GHLOROETHYLVINYL & ETHER & $<10$ & ug/L \\
\hline SD $-100-03$ & 26-May-1987 & 2-CHLOROETHYLVINYL & ETHERR & $<10$ & $\mathrm{ug} / \mathrm{L}$ \\
\hline SD $-100-03$ & 3-Jun-1987 & 2-CHLOROETHYLVINYL & ETHER & $<10$ & $\mathrm{ug} / \mathrm{L}$ \\
\hline SD $-100-03$ & 11 - Jun- 1987 & 2-CHLOROETHYLVINIL & ETHER & $<10$ & $u g / L$ \\
\hline SD $-100-03$ & 18-Jun-1987 & 2- GHLOROETHYLVINYL & ETHER & $<10$ & $\mathrm{ug} / \mathrm{L}$ \\
\hline \multicolumn{6}{|c|}{$\star * \quad S D-100-03 D$} \\
\hline SD-100-03D & 12-May-1987 & 2-CHLOROETHYLVINYL & ETHER & $<10$ & $\mathrm{ug} / \mathrm{L}$ \\
\hline$S D=100-03 D$ & $26-\mathrm{May}-1987$ & 2- CHLOROETHYLVINYL & ETHER & $<10$ & $u g / L$ \\
\hline \multicolumn{6}{|c|}{$\star * \quad S D-100-04$} \\
\hline SD-100-04 & 12-Мay-1987 & 2. CHLOROETHYLVINYL & ETHER & $<10$ & ug/L \\
\hline SD $-100-04$ & $18-$ May- 1987 & 2-CHLOROETHYLVINYL & ETHER & $<10$ & ug/L \\
\hline SD - $100-04$ & 26 - May- 1987 & 2-CHLOROEIHYLVINYL & ETHER & $<10$ & ug/L \\
\hline SD $-100-04$ & 3-Jun-1987 & 2-CHLOROETHYLVINYL & ETHER & $<10$ & $\mathrm{ug} / \mathrm{L}$ \\
\hline SD-100-04 & 11 -Jun-1987 & 2-CHLOROETHYLVINYL & ETHER & $<10$ & $\mathrm{ug} / \mathrm{L}$ \\
\hline SD-100-04 & $18-J$ un- 1987 & 2- CHLOROETHYLVINYL & ETHER & $<10$ & $\mathrm{ug} / \mathrm{L}$ \\
\hline \multicolumn{6}{|c|}{ *夫 SD-100-06 } \\
\hline SD-100-06 & $12-$ May- 1987 & 2-CHLOROETHYLVINYL & ETHER & $<10$ & ug/L \\
\hline SD $-100-06$ & $18-$ May -1987 & 2-CHLOROETHYLVINYL & ETHER & $<10$ & $\mathrm{ug} / \mathrm{L}$ \\
\hline SD $-100-06$ & 26-May - 1987 & 2-CHLOROETHYLVINYL & ETHER & $<10$ & $u g / L$ \\
\hline SD-100-06 & 3-Jun-1987 & 2-CHLOROETHYLVINYL & ETHER & $<10$ & ug/L \\
\hline$S D-100-06$ & $11-J u n-1987$ & 2-CHLOROETHYLVINYL & ETHER & $<10$ & ug $/ \mathrm{L}$ \\
\hline SD-100-06 & $18-J u n-1987$ & 2-CHLOROETHYLVINYL & ETHER & $<10$ & $u g / L$ \\
\hline \multicolumn{6}{|c|}{ ** SD-100-06D } \\
\hline SD-100-06D & 3-Jun- 1987 & 2-CHLOROETHYLVINYL & ETHER & $<10$ & $4 \mathrm{~g} / \mathrm{h}$ \\
\hline \multicolumn{6}{|c|}{ ** SD-100-07 } \\
\hline SD-100-07 & 12-May- 1987 & 2-CHLOROETHYLVINYL & ETHER & $<10$ & ug/L \\
\hline SD -100.07 & 19-May-1987 & 2-CHLOROETHYLVINYL & ETHER & $<10$ & $4 \mathrm{~g} / \mathrm{L}$ \\
\hline SD-100-07 & $26-$ May -1987 & 2- CHLOROETHYLVINYL. & ETHER & $<10$ & $\mathrm{ug} / \mathrm{L}$ \\
\hline SD-100-07 & $3-J u n-1987$ & 2-CHLOROETHYLVINYL & ETHER & $<10$ & $\mathrm{ug} / \mathrm{L}$ \\
\hline SD-100-07 & $11-J u n-1987$ & 2- CHLOROETHYLVINYL & ETHER & $<10$ & $u g / L$ \\
\hline SD-100-07 & $18-J$ un- 1987 & 2-CHLOROETHYLVINYL & ETHER & $<10$ & $u g / L$ \\
\hline \multicolumn{6}{|c|}{ * SD-100-07D } \\
\hline \multicolumn{2}{|c|}{ SD-100-07D $18-J$ un -1987} & 2-CHLOROETHYLVINYL & ETHER & $<10$ & $\mathrm{ug} / \mathrm{L}$ \\
\hline \multicolumn{6}{|c|}{ ** SD-100-09 } \\
\hline SD-100-09 & 12-May- 1987 & 2-CHLOROETHYLVINYL & ETHER & $<10$ & $\mathrm{ug} / \mathrm{L}$ \\
\hline SD-100-09 & $18-$ May - 1987 & 2-CHLOROETHYLVINYL & ETHER & $<10$ & $\mathrm{ug} / \mathrm{L}$ \\
\hline SD-100-09 & 26-May - 1987 & 2-CHLOROETHYLVINYL & ETHER & $<10$ & ug/L \\
\hline SD $-100-09$ & 3-Jun- 1987 & 2-CHLOROETHYLVINYL & ETHER & $<10$ & $\mathrm{ug} / \mathrm{L}$ \\
\hline SD-100-09 & $11-J$ un -1987 & 2-CHLOROETHYLVINYL & ETHER & $<10$ & $u g / L$ \\
\hline SD $-100-09$ & $18-J$ un -1987 & 2-CHLOROETHYLVINYL & ETHER & $<10$ & $\mathrm{ug} / \mathrm{L}$ \\
\hline \multicolumn{6}{|c|}{$\star *$ SD $-100 \cdot 10$} \\
\hline SD $-100-10$ & 12-May- 1987 & 2-CHLOROETHYLVINYL & ETHER & $<10$ & $\mathrm{ug} / \mathrm{L}$ \\
\hline SD $-100-10$ & 19-May-1987 & 2-CHLOROETHYLVINYL & ETHER & $<10$ & $u g / L$ \\
\hline
\end{tabular}


Location Date Test Campound

Results Units

SD-100-10 26-May-1987 2-CHLOROETHYLVINYL ETHER

SD-100-10 3-Jun-1987 2-CHLOROETHYLVINYL ETHER

SD-100-10 11-Jun-1987 2-CHLOROETHYLVINYL EIHER

SD-100-10 18-Jun-1987 2-CHLOROETHYLVINYL ETHER

* SD $-100-11$

SD-100-11 12-Kay-1987 2-CYLOROETHYLINYL ETHER

SD-100-11 19-Kay-1987 2-CHLOROETHYLVINYL ETHER

SD-100-11 26-Kay-1987 2-GHLOROETHYLINYL ETHER

SD-100-11 3-Jun-1987 2-CHLOROETHYLVINYL ETHER

SD-100-11 11-Jun-1987 2-CHLOROETHYLVINYL ETHER

SD-100-11 28-Jun-1987 2-CHLOROETHYLVINYL ETHER'

* $\quad S D-100-12$

SD-100-12 12-May-1987 2-CHLOROETHYLVINYL ETHER

SD-100-12 19-May-1987 2-CHLOROETHYLVINYL ETHER

SD-100-12 26-May-1987 2-CHLOROETHYLVINYL ETHER

SD-100-12 3-JUn-1987 2-CHLOROETHYLVINYL ETHER

SD-100-12 11-Jun-1987 2-CHLOROETHYLVINYL ETHER

SD-100-12 18-JUn-1987 2-CHLOROETHYLVINYL ETHER

* SD $-100-13$

SD-100-13 12-May-1987 2-CHLOROETHYLVINYL ETHER

SD-100-13 19-May-1987 2-CHLOROETHYLVINYL ETHER

SD-100-13 26-May-1987 2-CHLOROETHYLVINYL ETHER

SD-100-13 3-Jun-1987 2-CHLOROETHYLVINYL ETHER

SD-100-13 11-Jun-1987 2-CHLOROETHYLVINYL. ETHER

SD-100-13 18-Jun-1987 2-CHLOROETHYLVINYL ETHER

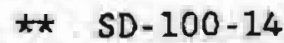

SD-100-14 22-May-1987 2-CHLOROETHYLVINYL ETHER

SD-100-14 19-May-1987 2-CKLOROETHYLVINYL ETHER

SD-100-14 26-May-1987 2-CHLOROETHYLVINYL ETHER

SD-100-14 3-Jun-1987 2-CHLOROETHYLVINYL ETHER

SD-100-14 11-Jun-1987 2-GHLOROETHYLVINYL ETHER

SD-100-14 18-Jun-1987 2-CHLOROEIHYLVINYL ETHER

* $S D-100-C$

SD-100-C 5-MaI-1987 2-CHLOROETHYLVINYL ETHER

SD-100-C 9-Mar-1987 2-CHLOROETHYLUINYL ETHER

SD-100-C 17-Mar-1987 2-CHLOROETHYLVINYL ETHER

SD-100-C 24-Mar-1987 2-CHLOROETHYLVINYL ETHER

SD-100-C 8-ApI-1987 2-CHLOROETHYLVINYL ETHER

SD-100-C 13-May-1987 2-CHLOROETHYLVINYL ETHER

SD-100-C 20-May-1987 2-CHLOROETHYLVINYL ETHER

SD-100-C 27-May-1987 2-CHLOROETHYLVINYL ETHER

SD-100-C 12-Jun-1987 2-CHLOROETHILVINYL ETHER

SD-100-C 19-Jun-1987 2-CHLOROETHYLVINYL ETHER
$<10$

$<10$

$<10$

$<10$

$<10$

$<10$

$<10$

$<10$

$<10$

$<10$

$<10$

$<10$

$<10$

$<10$

$<10$

$<10$

$<10$

$<10$

$<10$

$<10$

$<10$

$<10$

$<10$

$<10$

$<10$

$<10$

$<10$

$<10$

$<10$

$<10$

$<10$

$<10$

$<10$

$<10$

$<10$

$<10$

$<10$

$<10$

$<10$ $u g / L$

$\mathrm{ug} / \mathrm{L}$

$\mathrm{ug} / \mathrm{L}$

ug $/$

ug $/ \mathrm{L}$

$u_{B} / L$

ug $/ L$

ug $/$

ug/L

ug $/ \mathrm{L}$

ug/L

$4 \mathrm{~g} / \mathrm{L}$

ug/L

ug/L

ug/L

$\mathrm{ug} / \mathrm{L}$

ug $/ \mathrm{L}$

$u g / L$

ug/L

$\mathrm{ug} / \mathrm{L}$

ug $\Omega$

$\mathrm{ug} / \mathrm{L}$

ug/L

ug/L

$u g / L$

$\mathrm{ug} / \mathrm{L}$

$4 \mathrm{~g} / \mathrm{L}$.

ug/L

$u g / L$.

$u g / L$

$u g / L$.

$\mathrm{ug} / \mathrm{L}$

$4 \mathrm{~g} / 2$

$u g / L$

ug $/$ L

ug $/$ L

Ug/L.

$u g / L$

$\star \star \quad S D-100-D$

SD-100-D 6-Apr-1987 2-CHLOROETHYLVINYL ETHER 
Storm Drain Data for SD-100

\begin{tabular}{|c|c|c|c|c|}
\hline Location & Date & Test Compound & Results & Unfts \\
\hline SD-100-D & $11-J u n-1987$ & 2-CHLOROETHYLVIXYL ETHER & $<10$ & $\operatorname{ug} / 1$ \\
\hline \multicolumn{5}{|l|}{$\star * \quad S D-100$} \\
\hline SD -100 & 5-Mar-1987 & 2. CHLORONAPHTHALENE & $<10$ & ug $/ \mathrm{L}$ \\
\hline$S D-100$ & $11-\operatorname{Mar}-1987$ & 2-GLLORONAPHTHALENE & $<10$ & $\lg / \bar{L}$ \\
\hline SD -100 & $12-$ May -1987 & 2-CHLORONAPHTHALENE & $<10$ & $\lg \Omega$ \\
\hline SD- 100 & $26-$ May-1987 & 2-CHLORONAPHTHALENE & $<5.0$ & $\mathrm{ug} / \mathrm{L}$ \\
\hline \multicolumn{5}{|c|}{ ** SD-100-01 } \\
\hline SD-100-01 & 12-May-1987 & 2- CHIORONAPHIHALENE & $<10$ & $u g / 2$ \\
\hline SD-100-01 & 26-May-1987 & 2- CHLORONAPHTHALENE & $<5.0$ & $u g / L$ \\
\hline \multicolumn{5}{|c|}{$\star \star \quad S D-100-02$} \\
\hline SD-100-02 & $12-$ May- 1987 & 2 - CHLORONAPHTHALENE & $<10$ & $u g / 2$ \\
\hline SD $-100-02$ & $26-$ May -1987 & 2-CHLORONAPHTHALFNE & $<5.0$ & $\mathrm{ug} / \mathrm{L}$ \\
\hline \multicolumn{5}{|c|}{$\star * \quad S D-100-03$} \\
\hline$S D-100-03$ & 12-May-1987 & 2-CHLORONAPHTHALENE & $<10$ & $\mathrm{ug} / \mathrm{L}$ \\
\hline SD $-100-03$ & 26-May- 1987 & 2 - CHLORONAPKTHALENE & $<5.0$ & $\mathrm{ug} / \mathrm{L}$ \\
\hline \multicolumn{5}{|c|}{$\star \star \quad S D-100-03 D$} \\
\hline$S D-100-03 D$ & 12-May-1987 & 2 - CHLORONAPHTHALENE & $<10$ & ug $/ L$ \\
\hline$S D-100-03 D$ & 26 -May- 1987 & 2-CHLORONAPHTHALENE & $<5.0$ & $\mathrm{ug} / \mathrm{L}$ \\
\hline \multicolumn{5}{|c|}{$\star \star \quad S D-100-04$} \\
\hline$S D-100-04$ & 12-May-1987 & 2- CHLORONAPHTHALENE & $<10$ & $\mathrm{ug} / \mathrm{L}$ \\
\hline SD $-100-04$ & 26-May-1987 & 2-CHLORONAPHTHALENE & $<5.0$ & $u g / L$ \\
\hline \multicolumn{5}{|c|}{ ** SD-100-06 } \\
\hline SD-100-06 & 12-May - 1987 & 2. CHLORONAPHTHALENE & $<10$ & $u g / L$ \\
\hline SD $-100-06$ & 26-Мay-1987 & 2- CHLORONAPHTHALENE & $<5.0$ & $\mathrm{ug} / \mathrm{L}$ \\
\hline \multicolumn{5}{|c|}{ ** SD $-100-07$} \\
\hline SD - $100-07$ & 12-May-1987 & 2 - CHLORONAPHTHALENE & $<10$ & $\mathrm{ug} / \mathrm{L}$ \\
\hline SD $-100-07$ & 26-May-1987 & 2 - CHLORONAPHTHALENE & $<5.0$ & ug/L \\
\hline \multicolumn{5}{|c|}{ * SD-100-09 } \\
\hline SD $-100-09$ & 12-May-1987 & 2 - CHLORONAPHTHALENE & $<10$ & $u g / L$ \\
\hline SD $-100-09$ & 26-May - 1987 & 2- CHLORONAPHTHALENE & $<5.0$ & $\mathrm{ug} / \mathrm{L}$ \\
\hline \multicolumn{5}{|c|}{$\star \star \quad S D-100-10$} \\
\hline SD $-100-10$ & 12-May-1987 & 2-CHLORONAPHTHALENE & $<10$ & $\mathrm{ug} / \mathrm{L}$ \\
\hline SD $-100-10$ & $26-$ May -1987 & 2-CHLORONAPHTHALENE & $<5.0$ & $\mathrm{ug} / \mathrm{L}$ \\
\hline \multicolumn{5}{|c|}{$* * \quad S D-100-11$} \\
\hline$S D-100-11$ & 12 -May- 1987 & 2- CKLORONAPHTHALENE & $<10$ & $u g / L$ \\
\hline$S D-100-11$ & $26-$ May -1987 & 2 - CKLORONAPHTHALENE & $<5.0$ & $\mathrm{ug} / \mathrm{L}$ \\
\hline \multicolumn{5}{|c|}{ ** SD $-100-12$} \\
\hline$S D-100-12$ & 12-May-1987 & 2-CHLORONAPHTHALENE & $<10$ & $u_{5} / L$ \\
\hline SD $-100 \cdot 12$ & $26-$ May - 1987 & 2 - CRLORONAPHTHALENE & $<5.0$ & $\mathrm{ug} / \mathrm{L}$ \\
\hline
\end{tabular}


Storm Drain Data for SD-100

Location

Date

Test Compound

Results

Units

** SD $-100-13$

SD-100-13 12-Mzy-1987 2-CHLORONAPHTHALENE

SD-100-13 26-May-1987 2-CHLORONAPHTHALENE

$<10$

$<5.0$

ug/

** SD-100-14

SD-100-14 12-May-1987 2-GHLORONAPHTHAI ENE

SD-100-14 26-May-1987 2-CHLORONAPHTHALENE

$<10$

$<5.0$

$\operatorname{ug} / 2$

** SD-100-C

SD-100-C 5-Max-1987 2-CHLORONAPHTHAIENE

SD-100-C 9-Mar-1987 2-CHLORONAPHTHALENE

SD-100-C 17-Mar-1987 2-CHLORONAPHTHALENE

SD-100-C 24-Mar-1987 2-CHLORONAPHTHAIENE

SD-100-C 8-APr-1987 2-CHLORONAPHTHALENE

SD-100-C 13-May-1987 2-CHLORONAPHTHALLNE

SD-100-C 20-May-1987 2-CHLORONAPHTHALENE

SD-100-C 27-May-1987 2-CHLORONAPHTHALENE

SD-100-C 12-Jun-1987 2-CHLORONAPHTHALENE

SD-100-C 19-Jun-1987 2-CHLORONAPHTHALENE

* $\quad$ SD -100

SD -100

5-Mar-1987 2-CHLOROPHENOL.

SD -100

11-Mar-1987 2-CHLOROPHENOL

SD -100

12-May-1987 2-CHLOROPHENOL

SD -100

26-May- 1987 2-CHLOROPHENOL

* SD-100-01

SD-100-01 12-Kay-1987 2-CHLOROPHENOL

SD-100-01 26-Kay-1987 2-CHLOROPHEANOL

** SD-100-02

SD-100-02 12-May-1987 2-CHLOROPHENOL

SD-100-02 26-May-1987 2-CHLOROPHENOL

** SD $-100-03$

SD-100-03 12-May-1987 2-CHLOROPHENOL

SD-100-03 26-May-1987 2-GHLOROPHENOL.

** SD - 100-03D

SD-100-03D 12-May-1987 2-GHLORORHENOL

SD-100-03D 26-May-1987 2-CHLOROPHENOL

** SD $-100-04$

SD-100-04 12-May-1987 2-CHLOROPHENOL

SD-100-04 26-May-1987 2-CHLOROPHENOL

** SD $-100-06$

SD-100-06 12-May-1987 2-CHLOROPHENOL

SD-100-06 26-May-1987 2-GHLOROPHENOL 
Location Date Test Compound

* SD-100-07

SD-100-07 12-May-1987 2-CHLOROPHENOL

SD-100-07 26-May-1987 2-CHLOROPHENOL

$<10$

$<5.0$

Results

Units

* SD-100-09

SD-100-09 12-May-1987 2-CHLOROPHENOL SD-100-09 26-May-1987 2-CHLOROPHENOL

* SD $-100-10$

SD-100-10 12-May-1987 2-CZIOROPHENOL SD-100-10 26-May-1987 2-GHLROPHENOL

** SD $-100-11$

SD-100-11 12-May-1987 2-CHLOROPHENOL SD-100-11 26-May-1987 2-CHLOROPHENOL

** SD-100-12

SD-100-12 12-May-1987 2-CHLOROPHENOL SD-100-12 26-May-1987 2-CHLOROPHENOL

** SD-100-13

SD-100-13 12-May-1987 2-CHLOROPHENOL SD-100-13 26-May-1987 2-CHLOROPHENOL

$<10$

$<5.0$

$u g / L$

$u_{\mathrm{g} / \mathrm{L}}$

$<10$

$<5,0$

$<10$

$<5.0$

$<10$

$<5.0$

$u g / L$

ug/L

** SD $-100-14$

$\mathrm{ug} / \mathrm{L}$

$u g / L$

SD-100-16 12-May-1987 2-CHLOROPHENOL

SD-100-14 26-May-1987 2-CHLOROPHENOL

* SD-100-C

SD-100-C 5-Mar-1987 2-CHLOROPHENOL

SD-100-C 9-Mar-1987 2-CHLOROPHENOL

SD-100-C 17-Mar-1987 2-CHLOROPHENOL

SD-100-C 24-Mar-1987 2-CHLOROPHENOL

SD-100-C B-Apr-1987 2-CHLOROPHENOL

SD-100-C 13-May-1987 2-CHLOROPHENOL

SD-100-C 20-May-1987 2-CKLOROPHENOL

SD-100-C 27-May-1987 2-CHLOROPHENOL

SD-100-C 12-Jun-1987 2-CHLOROPHENOL

SD-100-C 19-Jun-1987 2-CHLOROPHENOL

$<10$

$<5.0$

$<10$

$<10$

$<10$

$<10$

$<10$

$<5.0$

$<5.0$

$<5.0$

$<5.0$

$<10$

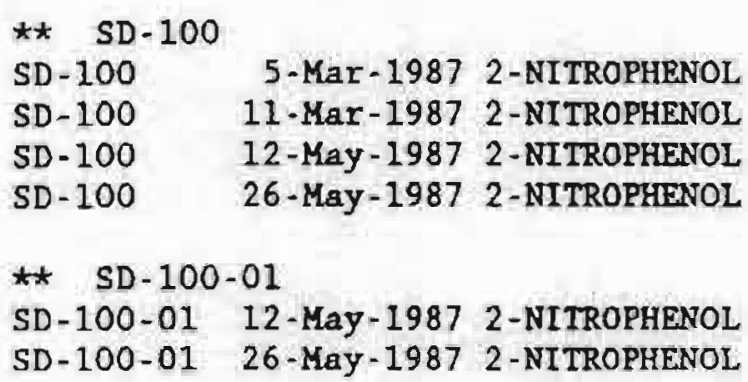

$<10$

$<10$

$<10$

$<5.0$

$<10$

$<5.0$

\section{ug/L \\ ug/L \\ ug/L \\ ug/L}

ug/L

$u g / L$

$\mathrm{ug} / \mathrm{L}$

ug/L

ug/L

ug/L

$u g / L$

ug $/ \mathrm{L}$

ug/L

ug/L

ug/L

$\mathrm{ug} / \mathrm{L}$

$u g / L$

ug/L

ug/ 1

$4 \mathrm{~g} / \mathrm{L}$

$4 \mathrm{~g} / \mathrm{L}$

ug/L

ug/L

$4 \mathrm{~g} / \mathrm{L}$ 
Location Date Test Compound

Results Units

** SD-100-02

SD-100-02 12-May-1987 2-NITROPHENOL

SD-100-02 26-May-1987 2-NITROPHENOL

$<10$

$<5.0$

ug/L

** SD-100-03

SD-100-03 12-May-1987 2-NITROPHENOZ.

SD-100-03 26-May-1987 2-NITROPHENOL

$<10$

$<5.0$

ug/L

** SD-100-03D

SD-100-03D 12-May-1987 2-MTTROPHENOL SD-100-03D 26-May-1987 2-NITROPHENOL

* SD-100-04

SD-100-04 12-May-1987 2-NITROPHENOL

SD-100-04 26-May-1987 2-NITROPHENOL

** SD-100-06

SD-100-06 12-M8Y-1987 2-NITROPHENOL

SD-100-06 26-May-1987 2-NITROPHENOL

** SD-100-07

SD-100-07 12-May-1987 2-NITROPHENOL

SD-100-07 26-May-1987 2-NITROPH8NOL

** SD-100-09

SD-100-09 12-May-1987 2-NITROPHENOL

SD-100-09 26-May-1987 2-NITROPHENOL

$<10$

$<5.0$

$u g / L$

$<10$

$<5.0$

ug/L

$u g / L$

$<10$

$<5.0$

ug/L

$\mathrm{ug} / \mathrm{L}$

$<10$

$<5.0$

ug/L

$\mathrm{ug} / \mathrm{L}$

** SD-100-10

SD-100-10 12-May-1987 2-NITROPHENOL SD-100-10 26-May-1987 2-NITROPHENOL

$<10$

$<5.0$

ug/L

$u g / L$

** SD $-100-11$

SD-100- 11 12-May-1987 2-NITROPEENOL

SD-100-11 26-May-1987 2-NITROPHENOL

$<10$

$<5.0$

ug/

** SD $-100-12$

SD-100-12 12-May-1987 2-NITROPHENOL

SD-100-12 26-May-1987 2-NITROPHENOL

$<10$

$<5.0$

ug/L

ug/L

$\star \star \quad S D \cdot 100 \cdot 13$

SD-100-13 12-May-1987 2-NITROPHENOL

SD-100-13 26-May-1987 2-NITROPHENOL

$<10$

$<5.0$

$\mathrm{ug} / \mathrm{L}$

$<5.0$

ug/L

** SD - 100-14

SD-100-14 12-May-1987 2-NITROPHENOL

SD-100-14 26-May-1987 2-NITROPHENOL

$<10$

$<5.0$

ug/L

** SD-100-C

SD-100-C S-Mar-1987 2-NITROPHENOL

SD-100-C 9-Mar-1987 2-NITROPHENOL

$<10$

$<10$

$\mathrm{ug} / \mathrm{L}$

$u g / L$

$\mathrm{ug} / \mathrm{L}$

ug/L 
$S D-100-C$

$S D-100-C$

SD $-100-C$

SD $-100-C$

$S D-100-C$

$S D-100-C$

SD $-100-C$

$S D-100-C$
17-Mar-1987 2-NITROPHENOL

24-Mar-1987 2-NITROPHENOL

8-Apr-1987 2-NITROPHENOL 13-May-1987 2-NITROPHENOL 20-May-1987 2-NITROPHENOL 27-May-1987 2-NITROPHENOL 12-JUn-1987 2-NITROPHENOL 19-Jun-1987 2-NITROPHENOL

** SD -100

SD -100

SD -100

SD -100

SD -100
5-Mar-1987 3 3'-DICHLOROBENZIDINE 11-MaI-1987 3 3'-DICHLOROBENZIDINE 12-May- $198733^{\prime}$-DICHLOROBENZIDINE 26-May-1987 $33^{\prime}$-DICHLOROBENZIDINE

** SD-100-01

SD-100-01 12-May-1987 3 3'-DICHLOROBENZIDINE SD-100-01 26-May-1987 3 3'-DICHLOROBENZIDINE

** SD-100-02

SD-100-02 12-May-19873 $33^{\prime}$-DICHLOROBENZIDINE SD-100-02 26-May-1987 3 3'-DICHLOROBENZIDTNE

* SD-100-03

SD-100-03 12-May-1987 3 3'-DICHLOROBENZIDINE SD-100-03 26-May-1987 3 3'-DICHLOROBENZIDINE

*t SD-100-03D

SD-100-03D 12-May-1987 3 3'-DICHLOROBENZIDINE

SD-100-03D 26-May-1987 3 3'-DICHLOROBENZIDINE

* SD $-100-04$

SD-100-04 12-May-1987 3 3'-DICHLOROBEN2IDINE

SD-100-04 26-May-1987 3 3'-DICHLOROBEN2IDINE

* SD $-100-06$

SD-100-06 12-May-1987 3 3'-DICKLOROBENZIDINE SD-100-06 26-May-1987 3 3'-DICHLOROBENZIDINE

** SD-100-07

SD-100-07 12-May-1987 3 3'-DICHLOROBENZIDINE SD-100-07 26-May-1987 3 3'-DICHLOROBENZIDINE

** SD-100-09

SD-100-09 12-May-1987 3 3'-DICHLOROBENZIDINE SD-100-09 26-May-1987 3 3'-DICHLOROBENZIDINE

** SD $-100-10$

SD-100-10 12-May-1987 3 3'-DICHLOROBENZIDINE SD-100-10 26-May-1987 3 3'-DICKLOROBENZIDINE

$\begin{array}{ll}<10 & \mathrm{ug} / \mathrm{L} \\ <10 & \mathrm{ug} / \mathrm{L} \\ <10 & \mathrm{ug} / \mathrm{L} \\ <5.0 & \mathrm{ug} / \mathrm{L} \\ <5.0 & \mathrm{ug} / \mathrm{L} \\ <5.0 & \mathrm{ug} / \mathrm{L} \\ <5.0 & \mathrm{ug} / \mathrm{L} \\ <10 & \mathrm{ug} / \mathrm{L} \\ & \\ & \\ <20 & \mathrm{ug} / \mathrm{L} \\ <20 & \mathrm{ug} / \mathrm{L} \\ <20 & \mathrm{ug} / \mathrm{L} \\ <10.0 & \mathrm{ug} / \mathrm{L} \\ & \\ & \\ <20 & \mathrm{ug} / \mathrm{L} \\ <10.0 & \mathrm{ug} / \mathrm{L} \\ & \\ & \\ <20 & \mathrm{ug} / \mathrm{L} \\ <10.0 & \mathrm{ug} / \mathrm{L} \\ <10.0 & \mathrm{ug} / \mathrm{L} \\ & \\ & \end{array}$


** SD-100-11

SD-100-11 12-Mey-1987 3 3'-DICHLOROBENZIDINE

SD-100-11 26-May-1987 3 3'-DICHLOROBENZIDINE

$<20$

$<10.0$

$4 \mathrm{~g} / \mathrm{L}$

* SD-100-12

SD-100-12 12-May-1987 3 3'-DICHLOROBENZIDTNE

SD-100-12 26-Kay-1987 3 3'-DICHLOROBEN2IDINE

$<20$

$<10.0$

$\operatorname{ug} / \mathrm{L}$

ug $/ \mathrm{L}$

* SD $-100-13$

SD-100-13 12-May-1987 3 3'-DICHLOROBENZIDINE

SD-100-13 26-May-1987 3 3'-DICHLOROBENZIDTNE

$<20$

$<10.0$

$u g /$

$u g / L$

* SD-100-14

SD-100-14 12-May-1987 3 3'-DICHLOROBENZIDIRE

SD-100-14 26-May-1987 3 3'-DICHLOROBENZIDINE

$<20$

$<10.0$

$\mathrm{ug} / \mathrm{L}$

$\mathrm{ug} / \mathrm{L}$

* SD-100-C

SD-100-C 5-Mar-1987 3 3'-DICHLOROBENZIDINE

$<20$

$<20$

SD-100-C

9-Mar-1987 3 3'-DICHLOROBENZIDINE

SD $-100-C$

17-Mar-1987 3 3'-DICHLOROBENZIDINE

$<20$

SD $-100-C$

24-Mar-1987 3 3'-DICHLOROBENZIDINE

$<20$

SD $-100-C$

8-Apr-1987 3 3'-DICHLOROBENZIDINE

$<20$

SD- $100-C$

13-May-1987 3 3'-DICHLOROBENZIDINE

SD $-100-C$

20-Mzy-1987 3 3'-DICHLOROBENZIDINE

SD-100-C

27-May-1987 3 3'-DICHLOROBENZIDINE

$S D-100-C$ 12-Jun-1987 3 3'-DICHLOROBENZIDINE

SD $-100-C$

19-3Ln-1987 $33^{\prime}$-DICHLOROBENZIDINE

$<10.0$

$<10.0$

$<10.0$

$<10.0$

$<20$

$\mathrm{ug} / \mathrm{L}$

$u g / L$

ug/L

$u g / L$

ug/L

$\mathrm{ug} / \mathrm{L}$

$u g / L$

ug $/ \mathrm{L}$

$\operatorname{ug} / \mathrm{L}$

$u g / L$

** SD 100

SD -100

5-Mar-I987 4 6-DINITRO-2-METHYLPHENOL

SD -100 11-Mar-1987 4 6-DINITRO-2-METHYLPHENOL

SD -100 12-May-1987 4 6-DINITRO-2-METHYLPHENOL

$<50$

$<50$

$<50$

ug/ 2

$u g / L$

$\mathrm{ug} / \mathrm{L}$

SD -100 26-May-1987 4 6-DINITRO-2-METHMLPHENOL

$<25.0$

** SD-100-01

SD-100-01 12-May-1987 4 6-DINITRO-2-METHY.PHENOL

SD-100-01 26-May-1987 4 6-DINITRO-2-METHYLPHENOL

$\mathrm{ug} / \mathrm{L}$

* SD $-100-02$

SD-100-02 12-May-1987 4 6-DINITRO-2-METHYLPHENOL

SD-100-02 26-May-1987 4 6-DINITRO-2-METHYLPHENOL

** SD-100-03

SD-100-03 12-May-1987 4 6-DINITRO-2-METHYLPHENOL

SD-100-03 26-May-1987 4 6-DINITRO-2-METHXLPHENOL

** SD-100-03D

SD-100-03D 12 -May-1987 46 -DINITRO-2-METHYLPHENOL

SD-100-O3D 26-May-1987 4 6-DINITRO-2-METHYLPHENOL
$<50$

$<25.0$

$<$ so

$<25.0$
$<50$

$<25.0$ $\mathrm{ug} / \mathrm{L}$

$u g / L$

$<50$

$<25.0$

ug/L

ug/ $L$

ug/ $L$

$\mathrm{ug} / \mathrm{L}$ 
* SD-100-04

SD-100-04 12-May-1987 \& 6-DINITRO-2-METHYLPHENOL SD-100-04 26-May-1987 4 6-DINITRO-2-METHYLPHENOL

$<50$

$<25.0$

ug/L

$\mathrm{ug} / \mathrm{L}$

* SD-100-06

SD-100-06 12-May-1987 4 6-DINITRO-2-METHYLPLENOL SD-100-06 26-May-1987 \& 6-DINITRO-2-KETHYLPHENOL

$<50$

$<25.0$

ug/L

$u_{B} / L$

* SD-100-07

SD-100-07 12-May-1987 4 6-DINITRO-2-METKYLPHEHOL SD-100-07 26-May-1987 4 6-DINITRO-2-METHYLPHENOL

$<50$

$<25.0$

ug/L

$\mathrm{ug} / \mathrm{L}$

* SD-100-09

SD-100-09 12-May-1987 4 6-DINITRO-2-METHYLPHENOL SD-100-09 26-May-1987 4 6-DINITRO-2-METHYLPHENOL

$<50$

$<25.0$

ug $/$

ug/L

* SD $-100-10$

SD-100-10 12-May-1987 \& 6-DINITRO-2-METHYLPHENOL

SD-100-10 26-May-1987 4 6-DINITRO-2-METHYLPHENOL

$<50$

$<25.0$

ug $/ \mathrm{L}$

$4 \mathrm{~g} / \mathrm{L}$

* SD $-100-11$

SD-100-11 12-May-1987 \& 6-DINITRO-2-METHYLPHENOL

SD-100-11 26-May-1987 4 6-DINITRO-2-METHYLPHENOL

$<50$

$<25.0$

ug/L

$48 / 2$

* SD $-100-12$

SD-100-12 12-May-1987 4 6-DINITRO-2-METHYLPHEROL

SD-100-12 26-May-1987 4 6-DINITRO-2-METHYLPHENOL

$<50$

$<25.0$

ug/L

ug/L

** SD $-100-13$

SD-100-13 12-May-1987 4 6-DINITRO-2-METHYLPHENOL SD-100-13 26-May-1987 4 6-DINITRO-2-METHYLPHEISOL

$\begin{array}{ll}<50 & \text { ug/L } \\ <25.0 & \text { ug/L }\end{array}$

** SD $-100-14$

SD-100-14 12-May-1987 4 6-DINITRO-2-METHYYPHENOL

SD-100-14 26-May-1987 4 6-DINITRO-2-METHYLPHENOL

$<50$

$<25.0 \quad \mathrm{ug} / \mathrm{L}$

** SD $-100-C$

SD-100-C 5-Mar-1987 4 6-DINITRO-2-METHYLPHENOL

SD-100-C 9-MaY-1987 4 6-DINITRO-2-METHYLPHENOL

SD-100-C 17-Mar-1987 4 6-DINITRO-2-METHYLPHEYOL

SD-100-C 24-Mat-1987 4 6-DINITRO-2-METHYLPHENOL

SD-100-C B-ApT-1987 4 6-DINITRO-2-METHYLPHENOL

$S D-100-C$

$S D \cdot 100-C$ 13-May-1987 4 6-DINITRO-2-METHYLPHENOL 20-May-1987 4 6-DINITRO-2-METHYLPHENOL

$S D-100-C$ 27-May-1987 4 6-DINITRO-2-MEIHYLPHENOI

SD $-100-C$

$S D-100-C$

12-Jun-1987 4 6-DINITRO-2-METHYZPHEYOL

19-Jun-1987 4 6-DINITRO-2-METHYZPHENOL

** SD -100

SD-100 5-Mar- 1987 4-BROMOPHENYL-PHENYLETHER

SD- 100

11-Mar-1987 4-BROMOPHENYL-PHENYLETHER

$<10$

ug/L

$u g / L$

$\mathrm{ug} / \mathrm{L}$

$\mathrm{ug} / \mathrm{L}$

$\mathrm{ug} / \mathrm{L}$

$u g / L$

$\mathrm{ug} / \mathrm{L}$

ug/L

$\begin{array}{ll}<25.0 & 48 / L \\ <50 & 4 g / L\end{array}$

$\begin{array}{ll}<25.0 & u g / L \\ <50 & u g / L\end{array}$

ug/L

$0 \mathrm{~g} / \mathrm{L}$ 
Storm Drain Data for SD-100

Results Units

SD-100 12-May-1987 4-BROMOPHENYL-PHENYLETHER

SD-100 26-May-1987 4-BROMOPHENYL-PHENYLETHER

* SD-100-01

SD-100-01 12-MaY-1987 4-BROHOPHENYL-PHENYLETHER

SD-100-01 26-MaY-1987 4-BROHOPHENYL-PHENYLETHER

* SD $-100-02$

SD-100-02 12-May-1987 4-BROMOPHENYL-PHENYLETHER

SD-100-02 26-May-1987 4-BROMOPHENYL-PHENYLETHER

** SD -100.03

SD-100-03 12-May-1987 4-BROMOPHENRL-PHENYLETHER

SD-100-03 26-May-1987 4-BROKOPHENYL-PHENYLETHER

** SD-100-03D

SD-100-03D 12-May-1987 4-BROMOPHENYL-PHENYLETHER

SD-100-03D 26-May-1987 4-BROMOPHENYL-PHENYLETHER

** SD $-100-04$

SD-100-04 12-May-1987 4-BROMOPHENYL-PHENYLETHER

SD-100-04 26-May-1987 4-BROMOPHENXL-PHENYLETHER

** SD $-100-06$

SD-100-06 12-May-1987 4-BROMOPHENYL-PHENYLETHER

SD-100-06 26-Hay-1987 4-BROMOPHENYL-PHENYLETHER

* SD-100-07

SD-100-07 12-May-1987 4-BROMOPHENYL-PHENYLETHER

SD-100-07 26-May-1987 4-BROHOPHENYL-PHENYLETHER

** SD - 100-09

SD-100-09 12-M8y-1987 4-BROMOPHENYL-PHENYLETHER

SD-100-09 26-May-1987 4-BROMOPHENYL-PHENYLETHER

** SD $-100-10$

SD-100-10 12-May-1987 4-BROMOPHENYL-PHENYLETRER

SD-100-10 26-May-1987 4-BROMOPHENYL-PHENYLETHER

** SD $-100-11$

SD-100-11 12-May-1987 4-BROMOPHENYL-PHENYLETHER

SD-100-11 26-May-1987 4-BROMOPHENYL-PHENXLETHER

$\star * \quad S D-100-12$

SD-100-12 12-May-1987 4-BROMOPHENYL-PHENYLETHER

SD-100-12 26-May-1987 4-BROMOPHENYL-PHENYLETHER

$\star \star \quad S D-100-13$

SD-100-13 12-May-1987 4-BROMOPHENYL-PHENYLETHER

SD-100-13 26-May-1987 4-BROMOPHENYL-PHENYLETHER

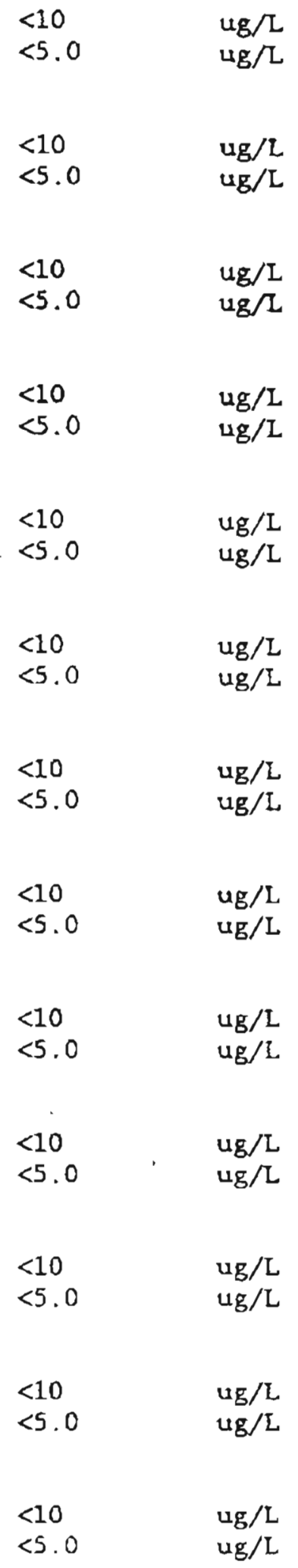


Storm Draln Data for SD-100

Location Date Test Compound

Results Units

* SD-100-14

SD-100-14 12-MBy-1987 4-BROMOPHENYL-PHEMTLETHER

SD-100-14 26-May-1987 4-BROMOPHENYL-PHENYLETHER

$<10$

$<5.0$

$u g / L$

ug/L

** SD-100-C

SD-100-C S-Mar-1987 4-BROMOPHENYL-PHENYT STHER

$<10$

$<10$

$<10$

SD-100-C 9-Mar-1987 4-BROMOPHENYL-PHENYLETHER

SD-100-C 17-Mar-1987 4-BROMOPHENYL-PHENYLETHER

SD-100-C 24-Mar-1987 4-BROMOPHENYL-PLENYLETHER

SD-100-C 8-Apr-1987 4-BROMOPHENYL-PHENYLETHER

SD-100-C 13-May-1987 4-BROMOPHENYL-PHENXLITHER

SD-100-C 20-May-1987 4-BROMOPHENYL-PHENYLETHER

SD $-100 \cdot C$

27-Kay-1987 4-BROMOPHENYL-PHERYI ETHER

SD $-100-C$

12-Jun-1987 4-BROMOPHENYL-PHENYLIETHER

SD $-100-C$

19-Jun-1987 4-BROMOPHENYL-PHENYIETHER

$\star \star$ SD -100

SD -100

5-Mar-1987 4-CHLORO-3-METHYLPHENOL

SD -100

11-Mar-1987 4-CHLORO-3-METHYLPHENOL

SD -100

12-May-1987 4-CHLORO-3-METHYLPHENOL

SD -100

26-May-1987 4-GHLORO-3-METHYLPHENOL

t* SD-100-01

SD-100-01 12-May-1987 4-CHLORO-3-METHYLPHENOL

SD-100-01 26-May-1987 4-ChLORO-3-METHYLPHEROL

** SD-100-02

SD-100-02 12-May-1987 4-CHLORO-3-METHYLPHENOL

SD-100-02 26-MaY-1987 4-CHLORO-3-METHYLPHENOL

* SD $-100-03$

SD-100-03 12-May-1987 4-CHLORO-3-METHYLPHENOL

SD-100-03 26-May-1987 4-CHLORO-3-METHYLPHENOL

** SD-100-03D

SD-100-O3D 12-May-1987 4-CHLORO-3-METHYLPHENOL

SD-100-O3D 26-May-1987 4-CHLORO-3-METHYLPHENOL

* SD-100-04

SD-100-04 12-May-1987 4-CHLORO-3-METHYLPHENOL

SD-100-04 26-May-1987 4-CHLORO-3-METHYLPHENOL

$<10$

$<10$

$<5.0$

$<5.0$

$<5.0$

$<5.0$

$<10$

$\operatorname{ug} / \mathrm{L}$

$u g / L$

ug/L

$\mathrm{ug} / \mathrm{L}$

ug/L

$u g / L$

$u \mathrm{~g} / \mathrm{L}$

ug/L

$\mathrm{ug} / \mathrm{L}$

$\mathrm{ug} / \mathrm{L}$

$<10$

$<10$

$<10$

$<5.0$

ug/L

ug/L

Ug/L

ug/L

$<10$

$<5.0$

$\operatorname{ug} / \mathrm{L}$

$\mathrm{ug} / \mathrm{L}$

$<10$

$<5.0$

ug/L

$\mathrm{ug} / \mathrm{L}$

$<10$

$<5.0$

$\mathrm{ug} / \mathrm{L}$

$u g / L$

$<10$

$<5.0$

ug/L

$\mathrm{ug} / \mathrm{L}$

$<10$

$<5.0$

$\operatorname{ug} / \mathrm{L}$

$\mathrm{ug} / \mathrm{L}$

** SD-100-06

SD-100-06 12-May-1987 4-CHLORO-3-METHYLPHENOL

SD-100-06 26-May-1987 4-CHLORO-3-METHYLPHENOL

$<10$

$<5.0$

ug/L

** SD-100-07

SD-100-07 12-May-1987 4-CHLORO-3-METHYLPHENOL

SD-100-07 26-May-1987 4-CHLORO-3-METHYLPHENOL

$<10$

$<5.0$

ug/L

ug/L 
** SD $-100-09$

SD-100-09 12-May-1987 4-GHLORO-3-METHYLPHENOL SD-100-09 26-May-1987 4-GHLORO-3-METHYLPHENOL

$<10$

$<5.0$

$\mathrm{ug} / \mathrm{L}$

* SD-100-10

SD-100-10 12-May-1987 4-CHLORO-3-METHYLPHEHOL SD-100-10 26-May-1987 4-CHLORO-3-METHYLPHEAOL

$<10$

$<5.0$

ug/L

ug/L

** SD-100-11

SD-100-11 12-May-1987 4-GHLORO-3-METHYLPHENOL SD-100-11 26-May-1987 4-CHLORO-3-METHYLPHENOL

$<10$

$<5.0$

ug/L

* SD $-100-12$

SD-100-12 12-Kay-1987 4-GHLORO-3-KETHYLPHENOL SD-100-12 26-May-1987 4-CHLORO-3-METHYLPHENOL

$<10$

$<5.0$

$\mathrm{ug} / \mathrm{L}$

* SD $-100-13$

SD-100-13 12-May-1987 4-CHLORO-3-METHYLPHENOL SD-100-13 26-May-1987 4-CHLORO-3-METHYLPHENOL

** SD $-100-14$

SD-100-14 12-May-1987 4-CHLORO-3-METHYLPHENOL SD-100-14 26-May-1987 4-CHLORO-3-METHYLPHENOL

$<10$

$<5.0$

$4 \mathrm{~g} / \mathrm{L}$

$<10 \quad$ ug/L

$<5.0$

ug/L

$\star \star \quad S D-100-C$

SD-100-C 5-Mar-1987 4-CHLORO-3-METHYLPHENOL SD-100-C 9-Mar-1987 4-CHLORO-3-METHYLPHENOL SD-100-C 17-Mar-1987 4-CHLORO-3-METHYLPHENOL SD-100-C 24-Mar-1987 4-CHLORO-3-METHYLPHENOL SD-100-C 8-Apr-1987 4-CHLORO-3-METHYLPHENOL SD-100-C 13-May-1987 4-CELORO-3-METHYLPHETTOL SD-100-C 20-May-1987 4-CHLORO-3-METHYLPHENOL SD-100-C 27-May-1987 4-CHLORO-3-METHYLPHENOL SD-100-C 12-JUn-1987 4-CHLORO-3-METHYLPHENOL SD-100-C 19-Jun-1987 4-CHLORO-3-METHYLPHBNOL

* SD -100

SD -100

5-Mar-1987 4-CKLOROPHENYL-PHENYLETHER

$S D-100$ I1-MaY-1987 4-CHLOROPHENYL-PHENYLETHER

SD -100 12-May-1987 4-CHLOROPHENYL-PHENYLETHER

SD -100 26-May-1987 4-CHLOROPHENXL-PHENYLETHER

** SD-100-01

SD-100-01 12-May-1987 4-CHLOROPHENYL-PHENYLFTHER SD-100-01 26-May-1987 4-CHLOROPHENYL-PHENYLETHER

** SD $-100-02$

SD-100-02 12-May-1987 4-CHLOROPHENYL-PHENYLETHER

SD-100-02 26-May-1987 4-GHLOROPHENYL-PHENYLETHER

$<10 \quad$ ug/L

$<5.0$

ug/L $u g / L$

$<10$

ug $/ 2$

$<10$ ug/L

$<10 \quad \mathrm{ug} / \mathrm{L}$

$<10 \quad$ ug/L

$<10 \quad \mathrm{ug} / \mathrm{L}$

$<5.0 \quad \mathrm{ug} / \mathrm{L}$

$<5.0 \quad$ ug/L

$<5.0 \quad u g / L$

$<5.0 \quad \mathrm{ug} / \mathrm{L}$

$<10 \quad u g / L$

$<10 \quad \mathrm{ug} / \mathrm{L}$

$<10 \quad \mathrm{ug} / \mathrm{L}$

$<10 \quad u g / L$

$<5.0 \quad \mathrm{ug} / \mathrm{L}$

$<10 \quad u g / L$

$<5.0 \quad \mathrm{ug} / \mathrm{L}$

ug/L 
* $S D-100-03$

SD-100-03 12-May-1987 4-CHLOROPHENYL-PHENYLETHER

SD-100-03 26-May-1987 4-CHLOROPHENYL-PLERYIETHER

$<10$

$<5.0$

$\mathrm{ug} / \mathrm{L}$

* SD-100.03D

SD-100-03D 12-May-1987 4-CHLOROPHENYL-PHENYZETHER SD-100-03D 26-May-1987 4-CHLOROPHENYL-PHEWYLETHER

$<10$

$<5.0$

$u g / L$

** SD $-100-04$

SD-100-04 12-MBy-1987 4-CHLOROPHENYL-PHETYLETHER

SD-100-04 26-May-1987 4-CHLOROPHEMY-PHETYLETHER

$<10$

$<5.0$

$\mathrm{ug} / \mathrm{L}$

** SD $-100-06$

SD-100-06 12-MAY-1987 4-CHLOROPHENYL-PHENYLETHER

SD-100-06 26-May-1987 4-CHLOROPHENYL-PHENYLETHER

$<10$

$<5.0$

ug/L

ug/L

** SD-100-07

SD-100-07 12-May-1987 4-CHLOROPHENYL-PHENYLETHER

SD-100-07 26-May-1987 4-CHLOROPHENYL-PHENYLETHER

$<10$

$<5.0$

$\mathrm{ug} / \mathrm{L}$

** SD-100-09

SD-100-09 12-May-1987 4-CHLOROPHENYL-PHENYZETHER

SD-100-09 26-May-1987 4-CHLOROPHENYL-PHENYLETHER

$<10$

$<5.0$

ug/L

$\mathrm{ug} / \mathrm{L}$

** SD $-100-10$

SD-100-10 12-May-1987 4-CHLOROPHENYL-PHENYLETHER

SD-100-10 26-May-1987 4-CHLOROPHENYL-PHENYIETHER

$<10$

$<5.0$

ug/L

** SD $-100-11$

SD-100-11 12-May-1987 4-CHLOROPHENYL-PHENYLETHRR

SD-100-11 26-May-1987 4-CHLOROPHENYL-PHENYLETHER

$<10$

$<5.0$

ug/L

$\mathrm{ug} / \mathrm{L}$

** SD $-100-12$

SD-100-12 12-May-1987 4-CHLOROPHENYL-PHENYLETHER

SD-100-12 26-May-1987 4-CHLOROPHENYL-PHENYLETHER

$<10$

$<5.0$

ug/L

** SD $-100-13$

SD-100-13 12-May-1987 4-CHLOROPHENYL-PHENYLETHER

SD-100-13 26-May-1987 4-CHLOROPHENYL-PHENYLETHER

$<10$

$<5.0$

ug/L

** SD-100-14

SD-100-14 12-May-1987 4-GHLOROPHENYL-PHENYLETHER

SD-100-14 26-May-1987 4-CHLOROPHENYL-PHENYLETHER

$<10$

$<5.0$

ug/L

** SD-100-C

SD-100-C 5-Mar-1987 4-CHLOROPHENYL-PHENYLETHER

$<10$

$<10$

$S D-100-C$

9-Mar-1987 4-CHLOROPHENYL-PHENYLETHER

SD $-100-C$

17-MaY-1987 4-CHLOROPHENYL-PHENYLETHER

SD $-100-C$

24-Mar-1987 4-CHLOROPHENYL-PHENYLETHER

SD $-100-C$

8-APT-1987 4-CHLOROPHENYL-PHENYLETHER

SD $-100-C$

13-May-1987 4-CHLOROPHENYL-PHENYLETHER

$<10$

$<10$

$<10$

$<5.0$

ug/L

ug/L

ug/L

ug $/ 2$

ug/L

$\mathrm{ug} / \mathrm{L}$

ug/L

ug/L

ug/L 


\begin{tabular}{|c|c|c|c|c|}
\hline Location & Date & Test Compound & Results & Units \\
\hline$S D-100-C$ & 20-May-1987 & 4- CHLOROPHENYL-PHENYLETHER & $<5.0$ & $\mathrm{ug} / \mathrm{L}$ \\
\hline SD-100-C & 27-May-1987 & 4-CHLOROPHENYL-PHENYLETHER & $<5.0$ & $u g / L$ \\
\hline$S D-100-C$ & 12-Jun-1987 & 4-CHLOROPHENYL-PHENYLETHER & $<5.0$ & ug/L \\
\hline SD-100-C & $19-J$ un-1987 & 4 - CHLOROPHENYL-PHEINYLETHER & $<10$ & $\operatorname{ug} / \mathrm{L}$ \\
\hline \multicolumn{5}{|l|}{ * SD -100} \\
\hline SD -100 & 5-MaI-1987 & 4-NITROPHEROL & $<50$ & $u_{g} / L$ \\
\hline SD- 100 & 11-Mar-1987 & 4-NITROPHENOL & $<50$ & $u g / L$ \\
\hline SD- 100 & 12-May-1987 & 4-NITROPHENOL & $<50$ & $u g / 2$ \\
\hline SD- 100 & $26-$ May -1987 & 4-NITROPHENOL & 25.0 & $\mathrm{ug} / \mathrm{L}$ \\
\hline \multicolumn{5}{|c|}{ ** SD - 100-01 } \\
\hline SD-100-01 & $12-$ May -1987 & 4-NITROPHENOL & $<50$ & $\mathrm{ug} / \mathrm{L}$ \\
\hline SD-100-01 & $26-\mathrm{May}-1987$ & 4-NITROPHENOL & $<25.0$ & $\mathrm{ug} / \mathrm{L}$ \\
\hline \multicolumn{5}{|c|}{$\star$ * SD $-100-02$} \\
\hline SD $-100-02$ & $12-\mathrm{May}-1987$ & 4-NITROPHENOL & $<50$ & ug/L \\
\hline SD $-100-02$ & $26-$ May -1987 & 4-NITROPHENOZ & $<25.0$ & $\mathrm{ug} / \mathrm{L}$ \\
\hline \multicolumn{5}{|c|}{$*$ SD $-100-03$} \\
\hline SD $-100-03$ & 12-May-1987 & 4-NITROPHENOL & $<50$ & $u g / L$ \\
\hline SD $-100-03$ & $26-$ May -1987 & 4-NITROPHENOL & $<25.0$ & $\mathrm{ug} / \mathrm{L}$ \\
\hline \multicolumn{5}{|c|}{ ** SD $-100-03 D$} \\
\hline SD-100-03D & $12-\mathrm{May}-1987$ & 4-NITROPHENOL & $<50$ & ug $/ \mathrm{L}$ \\
\hline$S D-100-03 D$ & 26-May-1987 & 4-NITROPHENOL & $<25.0$ & $\mu g / L$ \\
\hline \multicolumn{5}{|c|}{ * SD-100-04 } \\
\hline SD-100-04 & 12-May- 1987 & 4-NITROPHIAOL & $<50$ & $u g / L$ \\
\hline SD-100-04 & $26 \cdot$ May -1987 & 4-NITROPHERTOL & $<25.0$ & ug/L \\
\hline \multicolumn{5}{|c|}{$\star \star \quad S D-100-06$} \\
\hline SD $-100 \cdot 06$ & 12-May - 1987 & 4-NITROPHENOL & $<50$ & $u g / L$ \\
\hline SD-100-06 & 26-May - 1987 & 4-NITROPHENOL & $<25.0$ & $\mathrm{ug} / \mathrm{L}$ \\
\hline \multicolumn{5}{|c|}{$\star * \quad S D-100-07$} \\
\hline SD. 100.07 & 12-May-1987 & 4-NITROPHENOL & $<50$ & $\mathrm{ug} / \mathrm{L}$ \\
\hline SD $-100-07$ & $26-$ May -1987 & 4-NITROPHENOL & $<25.0$ & $\mathrm{ug} / \mathrm{L}$ \\
\hline \multicolumn{5}{|c|}{ ** SD-100-09 } \\
\hline$S D-100-09$ & 12-May-1987 & 4-NITROPHENOL & $<50$ & $u g / L$ \\
\hline SD-100-09 & $26-$ May -1987 & 4-NITROPHENOL & $<25.0$ & $\operatorname{Lg} / \mathrm{L}$ \\
\hline \multicolumn{5}{|c|}{ ** $S D-100-10$} \\
\hline SD- $100-10$ & $12 \cdot$ May -1987 & 4-NITROPHENOL & $<50$ & $u g / L$ \\
\hline SD $-100-10$ & $26-$ May -1987 & 4-NITROPHENOL & 25.0 & ug $/ \mathrm{L}$ \\
\hline \multicolumn{5}{|c|}{ * SD $-100-11$} \\
\hline SD $\cdot 100-11$ & 12 - May -1987 & 4-NITROPHENOL & $<50$ & ug/l \\
\hline SD-100-11 & $26-$ May-1987 & 4-NITROPHENOL & $<25.0$ & ug/L \\
\hline
\end{tabular}


Location Date Test Compound

** SD-100-12

SD-100-12 12-May-1987 4-NITROPHEHOL

SD-100-12 26-May-1987 4-NITROPHENOL

$<50$

$<25.0$

ug/L

* SD $-100-13$

SD-100-13 12-May-1987 4-NITROPHEROL

SD-100-13 26-May-1987 4-NITROPHEYOL

$<50$

$<25.0$

ug/L

$u g / L$

* SD-100-14

SD-100-14 12-May-1987 4-NITROPHENOL

SD-100-14 26-May-1987 4-NITROPHENOL

$<50$

$<25.0$

$u g / L$

$u g / L$

** SD $-100-C$

SD-100-C 5-Mar-1987 4-NITROPHFNOL

$<50$

$<50$

SD-100-C 9-Mar-1987 4-NITROPHENOL

$<50$

SD-100-C 17-Mar-1987 4-NITROPHENOL

$<50$

SD-100-C 24-Mar-1987 4-NITROPHENOL

$<50$

SD-100-C 8-Apr-1987 4-NITROPHENOL

SD-100-C 13-May-1987 4-NITROPHENOL

SD-100-C 20-May-1987 4-NITROPHENOL

SD-100-C 27-May-1987 4-NITROPHENOL

SD-100-C 12-JUn-1987 4-NITROPHENOL

SD-100-C 19-Jun-1987 4-NITROPHENOL

$<25.0$

$<25.0$

$<25.0$

$<25.0$

$<50$

ug $/ \mathrm{L}$

ug/L

$\mathrm{ug} / \mathrm{L}$

ug/L

ug/L

$\mathrm{ug} / \mathrm{L}$

$u g / L$

$\mathrm{ug} / \mathrm{L}$

$\cup \mathrm{g} / \mathrm{L}$

ug/L

* SD-100-01

SD-100-01 12-May-1987 4-TERT-BUTYLPHENOL

$0.6 \mathrm{~B}$

ug/L

** SD $-100-07$

SD-100-07 12-May-1987 4-TERT-BUTYLPHENOL

38

$\mathrm{ug} / \mathrm{L}$

** SD -100

SD-100 5-Mar-1987 ACENAPHTHENE

SD-100 11-Mar-1987 ACENAPHTHENE

SD-100 12-May-1987 ACENAPHTHENE

SD-100 26-Kay-1987 ACENAPHTHENE

$<10$

$<10$

$<10$

$<5.0$

$\mathrm{ug} / \mathrm{L}$

$u g / L$

$u g / L$

$\mathrm{ug} / \mathrm{L}$

** SD-100-01

SD-100-01 12-May-1987 ACENAPHTHENE

SD-100-01 26-May-1987 ACENAPHTHENE
$<10$

$<5.0$

$<10$

$<5.0$

$<10$

$<5.0$

$<10$

$<5.0$ $\mathrm{ug} / \mathrm{L}$

ug/L

ug/L

ug/L

ug/L

$u_{g} / L$

* SD-100-03D

SD-100-03D 12-May-1987 ACENAPHTHENE

SD-100-03D 26-May-1987 ACENAPHTHENE 
** SD $-100-04$

SD-100-04 12-MAY-1987 ACENAPHTHENE SD-100-04 26-May-1987 ACENAPHTHENE

$\begin{array}{ll}<10 & u g / L \\ <5.0 & u g / L \\ <10 & u g / L \\ <5.0 & u g / L \\ & \\ <10 & u g / L \\ <5.0 & u g / L \\ & \\ <10 & u g / L \\ <5.0 & u g / L \\ & \\ <10 & u g / L \\ <5.0 & u g / L \\ & \\ <10 & u g / L \\ <5.0 & u g / L \\ & \\ <10 & u g / L \\ <5.0 & u g / L \\ <10 & u g / L \\ <5.0 & u g / L\end{array}$

* SD $-100-06$

SD-100-06 12-May-1987 ACENAPHTHENE

SD-100-06 26-May-1987 ACENAPHTHENE

** SD $-100-07$

SD-100-07 12-May-1987 ACENAPHTHENE SD-100-07 26-May-1987 ACENAPHTHENE

$\star \star \quad$ SD $-100-09$

SD-100-09 12-May-1987 ACENAPHTHENE

SD-100-09 26-May-19B7 ACENAPHTHENE

** SD $-100-10$

SD-100-10 12-May-1987 ACENAPHTHENE

SD-100-10 26-May-1987 ACENAPHTHENE

** SD-100-11

SD-100-11 12-Mky-1987 ACENAPHTHENE

SD-100-11 26-May-1987 ACENAPHTHIFNE

** SD $-100-12$

SD-100-12 12-May-1987 AGENAPHTHENE

SD-100-12 26-May-1987 ACENAPHTHENE

$\star \star \quad S D-100-13$

SD-100-13 12-Kay-1987 ACENAPHTHENE SD-100-13 26-May-1987 ACENAPHTHENE

** SD $-100-14$

SD-100-14 12-May-1987 ACENAPHTHENE

SD-100-14 26-May-1987 ACENAPHTHENE

$<10$

$<5.0$

ug/L

$\mathrm{ug} / \mathrm{L}$

** SD $-100-\mathrm{C}$

SD-100-C 5-Mar-1987 ACENAPHTHENE

SD-100-C 9-Mar-1987 ACENAPHTHENE

SD-100-C 17-Mar-1987 ACENAPHTHENE

SD-100-C 24-Mar-1987 ACENAPHTHENE

SD-100-C 8-APr-1987 ACENAPHTHENE

SD-100-C 13-May-1987 ACENAPHTHENE

SD-100-C 20-May-1987 ACENAPHTHENE

SD-100-C 27-May-1987 ACENAPHTHENE

SD-100-C 12-JUN-1987 ACENAPHTHENE

SD-100-C 19-JUn-1987 ACENAPHTHENE

$<10 \quad$ ug $/ \mathrm{L}$

$<10 \quad$ ug $/ L$

$<10 \quad u g / L$

$<10 \quad u g / L$

$<10 \quad u g / L$

$<5.0 \quad \mathrm{ug} / \mathrm{L}$

$<5.0 \quad u g / L$

$<5.0 \quad$ ug/L

$<5.0 \quad \mathrm{ug} / \mathrm{L}$

$<10 \quad$ ug/L

** SD -100

SD-100 S-MaY-1987 ACENAPHTHYLENE

$<10$

$u g / L$ 
Storm Drain Data for SD-100

$\begin{array}{lcc}\text { Location } & \text { Date } & \text { Test Compound } \\ \text { SD-100 } & \text { 11-Mar-1987 ACENAPHTHYLENE } \\ \text { SD-100 } & \text { 12-May-1987 ACENAPHTHYLENE } \\ \text { SD-100 } & \text { 26-May-1987 ACENAPHTHYLENE }\end{array}$

** SD-100-01

SD-100-01 12-May-1987 ACENAPHTHYLENE

SD-100-01 26-May-1987 ACENAPHTHYLENE

* SD-100-02

SD-100-02 12-May-1987 ACENAPHTHYLENE

SD-100-02 26-May-1987 ACENAPHTHYLENE

* SD-100-03

SD-100-03 12-May-1987 ACENAPHTHYLENE

SD-100-03 26-May-1987 ACENAPHTHYLENE

** SD-100-03D

SD-100-03D 12-May-1987 ACENAPHTHYLENE

SD-100-03D 26-Kay-1987 ACENAPHTHYLENE

** SD-100-04

SD-100-04 12-May-1987 ACENAPHTHYLENE

SD-100-04 26-May-1987 ACENAPHTHYLENE

** SD-100-06

SD-100-06 12-May-1987 ACENAPHTHYLENE

SD-100-06 26-May-1987 ACENAPHTHYLENE

** SD-100-07

SD-100-07 12-Moy-1987 ACENAPHTHYLENE

SD-100-07 26-May-1987 ACENAPHTHYLENE

** SD-100-09

SD-100-09 12-May-1987 ACENAPHTHYLENE

SD-100-09 26-May-1987 ACENAPHTHYLENE

** SD - 100-10

SD-100-10 12-May-1987 ACENAPHTHYLENE

SD-100-10 26-May-1987 ACENAPHTHYLENE

** SD-100-11

SD-100-11 12-May-1987 ACENAPHTHYLENE

SD-100-11 26-May-1987 ACENAPHTHYLENE

** SD $-100-12$

SD-100-12 12-May-1987 ACENAPHTHYLENE

SD-100-12 26-May-1987 ACENAPHTHYLENE

\begin{tabular}{|c|c|}
\hline Results & Units \\
\hline$<10$ & $\mathrm{ug} / \mathrm{L}$ \\
\hline$<10$ & $4 \mathrm{~g} / \mathrm{L}$ \\
\hline$<5.0$ & $u_{B} / L$ \\
\hline$<10$ & ug/L \\
\hline & ug/L \\
\hline$<10$ & ug/L \\
\hline$<5.0$ & ug/L \\
\hline$<10$ & $u g / L$ \\
\hline$<5.0$ & ug/L \\
\hline$<10$ & $\mathrm{ug} / \mathrm{L}$ \\
\hline$<5.0$ & $\mathrm{ug} / \mathrm{L}$ \\
\hline$<10$ & $\mathrm{ug} / \mathrm{L}$ \\
\hline$<5.0$ & $\mathrm{ug} / \mathrm{L}$ \\
\hline$<10$ & $u_{g} / L$ \\
\hline$<5.0$ & $\mathrm{ug}_{\mathrm{g}} / \mathrm{L}$ \\
\hline$<10$ & $\mathrm{ug} / \mathrm{L}$ \\
\hline$<5.0$ & ug/l \\
\hline$<10$ & ug/L \\
\hline$<5.0$ & ug/L \\
\hline$<10$ & $\mathrm{ug} / \mathrm{L}$ \\
\hline$<5.0$ & $\mathrm{ug} / \mathrm{L}$ \\
\hline $\begin{array}{l}<10 \\
<5.0\end{array}$ & ug/L \\
\hline & $\mathrm{ug} / \mathrm{L}$ \\
\hline$<10$ & ug/L \\
\hline$<5.0$ & ug/L \\
\hline
\end{tabular}


Storm Drain Data for SD-100

\begin{tabular}{|c|c|c|c|c|}
\hline \multirow{2}{*}{\multicolumn{5}{|c|}{$\begin{array}{l}\text { Location Date } \\
\star \star \quad \text { SD-100-13 }\end{array}$}} \\
\hline & & & & \\
\hline SD $-100-13$ & 12-May-1987 & ACENAPHTHYLENE & $<10$ & $\mathrm{ug} / \mathrm{L}$ \\
\hline$S D-100-13$ & 26-\$ay-1987 & ACENAPHTHYLENE & $<5.0$ & $\mathrm{ug} / \mathrm{L}$ \\
\hline \multicolumn{5}{|c|}{$\star \star \quad S D-100-14$} \\
\hline SD $-100-14$ & 12-May-1987 & ACENAPHTHYLENE & $<10$ & $\mathrm{ug} / \mathrm{L}$ \\
\hline SD-100-14 & 26-May-1987 & ACENAPHTHYLENE & $<5.0$ & $\mathrm{ug} / \mathrm{L}$ \\
\hline \multicolumn{5}{|c|}{$\star \star \quad S D-100-C$} \\
\hline$S D-100-C$ & 5-Mar-1987 & ACENAPHTHYLENE & $<10$ & ug/L \\
\hline$S D-100-C$ & $9-\operatorname{Mar}-1987$ & ACENAPHTHYLLENE & $<10$ & $\mathrm{ug} / \mathrm{L}$ \\
\hline$S D-100-C$ & 17-Mar-1987 & ACENAPHTHYLENE & $<10$ & $u g / L$ \\
\hline$S D-100 \cdot C$ & 24-Mar-1987 & ACENAPHTHYLENE & $<10$ & $u g / L$ \\
\hline SD-100-C & 8-Apr-1987 & ACENAPHTHYLENE & $<10$ & $\mathrm{ug} / \mathrm{L}$ \\
\hline$S D-100-C$ & 13-May-1987 & ACENAPHTHYLENE & $<5.0$ & $\mathrm{ug} / \mathrm{L}$ \\
\hline$S D-100-C$ & 20-May-1987 & ACENAPHTHYLENE & $<5.0$ & $\mathrm{ug} / \mathrm{L}$ \\
\hline$S D-100-C$ & 27-May - 1987 & ACENAPHTHYLENE & $<5.0$ & $4 \mathrm{~g} / \mathrm{L}$ \\
\hline SD-100-C & $12-J u n-1987$ & ACENAPHTHYLENE & $<5.0$ & $\mathrm{ug} / \mathrm{L}$ \\
\hline$S D-100-C$ & 19-Jun-1987 & ACENAPHTHYLENE & $<10$ & $\mathrm{ug} / \mathrm{L}$ \\
\hline
\end{tabular}

** SD -100

SD-100 5-Mar-1987 AIKALINITY

SD-100 11-Mar-1987 ALKALINITY

SD-100 18-Mar-1987 ALKALINITY

SD-100 24-Mar-1987 AIKALINITY

SD-100 3-ApI-1987 ALKALINITY

SD-100 6-Apr-1987 AIKALINITY

SD-100 12-May-1987 AIKALINITY

SD-100 19-May-1987 ALKALINITY

SD-100 26-May-1987 ALKALINITY

SD-100 3-Jun-1987 ALKALINITY

SD-100 II-Jun-1987 ALKALINITY

SD-100 18-Jun-1987 ALKALINITY

$\begin{array}{ll}97 & \mathrm{mg} / \mathrm{L} \\ 97 & \mathrm{mg} / \mathrm{L} \\ 100 & \mathrm{mg} / \mathrm{L} \\ 100 & \mathrm{mg} / \mathrm{L} \\ 93 & \mathrm{mg} / \mathrm{L} \\ 97 & \mathrm{mg} / \mathrm{L} \\ 98 & \mathrm{mg} / \mathrm{L} \\ 104 & \mathrm{mg} / \mathrm{L} \\ 102 & \mathrm{mg} / \mathrm{L} \\ 102 & \mathrm{mg} / \mathrm{L} \\ 97 & \mathrm{mg} / \mathrm{L} \\ 97 & \mathrm{mg} / \mathrm{L}\end{array}$

** SD-100-01

SD-100-01 12-May-1987 ALKALINITY

SD-100-01 18-May-1987 ALKALINITY

SD-100-01 26-May-1987 AIKALINITY

SD-100-01 3-Jun-1987 AIKALINITY

SD-100-01 11-Jun-1987 ALKALINITY

SD-100-01 18-Jun-1987 ALKALINITY

$\begin{array}{ll}63 & \mathrm{mg} / \mathrm{L} \\ 76 & \mathrm{mg} / \mathrm{L} \\ 91 & \mathrm{mg} / \mathrm{L} \\ 98 & \mathrm{mg} / \mathrm{L} \\ 161 & \mathrm{mg} / \mathrm{L} \\ 110 & \mathrm{mg} / \mathrm{L}\end{array}$

** SD - 100-02

SD-100-02 12-May-1987 AIKALINITY

SD-100-02 18-May-1987 ALKALINITY

SD-100-02 26-May-1987 ALKALINITY

SD-100-02 3-Jun-1987 ALKALINITY

SD-100-02 11-Jun-1987 ALKALINITY

SD-100-02 18-Jun-1987 ALKALINITY

$\begin{array}{ll}99 & \mathrm{mg} / \mathrm{L} \\ 104 & \mathrm{mg} / \mathrm{L} \\ 103 & \mathrm{mg} / \mathrm{L} \\ 103 & \mathrm{mg} / \mathrm{L} \\ 99 & \mathrm{mg} / \mathrm{L} \\ 96 & \mathrm{mg} / \mathrm{L}\end{array}$


Storm Drain Data for SD-100

Location Date Test Compound

Results Units

* SD-100-03

SD-100-03 12-May-1987 AIKALINITY

SD-100-03 18-May-1987 ALKALINITY

SD-100-03 26-May-1987 AIKALINITY

SD-100-03 3-Jun-1987 ALKALINTTY

SD-100-03 11-Jun-1987 ALKALINITY

SD-100-03 18-Jun-1987 ALKALINITY

98

102

100

$\mathrm{mg} / \mathrm{L}$

$m g / L$

$104 \mathrm{mg} / \mathrm{L}$

$99 \mathrm{mg} / \mathrm{L}$

$98 \mathrm{mg} / \mathrm{L}$

** SD-100-03D

SD-100-03D 12-Kay-1987 ALKALIRITY

SD-100-03D 26-May-1987 ALKAIINTTY

101

105

$\mathrm{mg} / \mathrm{L}$

$\mathrm{mg} / \mathrm{L}$

** SD- $100-04$

SD-100-04 12-May-1987 ALKALINITY

SD-100-04 18-May-1987 ALKALINITY

SD-100-04 26-May-1987 ALKALINITY

SD-100-04 3-Jun-1987 AIKAIINITY

SD-100-04 11-Jun-1987 ALKALINITY

SD-100-04 18-Jun-1987 ALKALTNITY

98

Ing $/ \mathrm{L}$

ming/L

$\mathrm{mg} / \mathrm{L}$

$\mathrm{mg} / \mathrm{L}$

mg/L

$\mathbf{m g} / \mathrm{L}$

** SD $100-06$

SD-100-06 12-May-1987 ALKALINITY

SD-100-06 18-May-1987 ALKALINITY

SD-100-06 26-May-1987 ALKALINITY

SD-100-06 3-Jun-1987 ALKALINITY

SD-100-06 11-Jun-1987 ALKALINITY

SD-100-06 18-Jun-1987 ALKAIMIITY

$\mathrm{mg} \Omega$

$\mathrm{mg} / \mathrm{L}$

$\mathrm{ng} / \mathrm{L}$

mg/L

mg $/ \mathrm{L}$

$\mathrm{mg} / \mathrm{L}$

** SD-100-06D

SD-100-06D 3-JUn-1987 ALKALINITY

104

$\operatorname{mg} / \mathrm{L}$

** SD-100-07

SD-100-07 12-May-19B7 ALRALINITY

SD-100-07 19-May-1987 ALKALINITY

SD-100-07 26-May-1987 ALKALINITY

SD-100-07 3-Jun-1987 ALKALINITY

SD-100-07 11-Jun-1987 ALKALINITY

SD-100-07 18-Jun-1987 ALKALINITY

$<1$

74

83

344

40

56

$\mathrm{mg} / \mathrm{L}$

$\operatorname{mg} / L$

$\operatorname{mg} / L$

$m g / L$

$m g / L$

$\mathrm{mg} / \mathrm{L}$

** SD $-100-07 D$

SD-100-07D 18-Jun-1987 AIKALINITY

56

$\mathrm{ng} / \mathrm{L}$

** SD-100-09

SD-100-09 12-May-1987 ALKALINITY

SD-100-09 18-Kay-1987 ALKALINITY

SD-100-09 26-May-1987 ALKALINITY

SD-100-09 3-Jun-1987 AUKALINITY

SD-100-09 11-Jun-1987 ALKALINITY

100

98

101

102

95

SD-100-09

18-Jun-1987 ALKALINITY

$$
\begin{aligned}
& \mathrm{mg} / \mathrm{L} \\
& \mathrm{mg} / \mathrm{L} \\
& \mathrm{mg} / \mathrm{L} \\
& \mathrm{mg} / \mathrm{L} \\
& \mathrm{mg} / \mathrm{L} \\
& \mathrm{mg} / \mathrm{L}
\end{aligned}
$$


Storm Drain Data for SD-100

\begin{tabular}{|c|c|c|c|c|}
\hline Location & Date & Test Compound & Results & Units \\
\hline \multicolumn{5}{|c|}{$\star$ * SD $-100-10$} \\
\hline SD $-100-10$ & 12-May - 1987 & ALKALINITY & 99 & $\mathbf{m g} / \mathrm{L}$ \\
\hline SD $-100-10$ & 19- May- 1987 & ALKALINITY & 102 & $\mathrm{mg} / \mathrm{L}$ \\
\hline SD- $100-10$ & $26-$ May - 1987 & ALKALINITY & 102 & $\mathbf{m g} / L$ \\
\hline SD $-100-10$ & 3-Jun-1987 & ALKALINITY & 104 & $\operatorname{mg} / \mathrm{L}$ \\
\hline SD $-100-10$ & $11-J u n-1987$ & ALKALINITY & 100 & $\mathrm{mg} / \mathrm{L}$ \\
\hline SD-100-10 & $18-J u n-1987$ & ALKALIRITY & 97 & $\mathrm{mg} / \mathrm{L}$ \\
\hline \multicolumn{5}{|c|}{ t* SD-100-11 } \\
\hline SD-100-11 & 12-May- 1987 & ALRALINITY & 103 & $\mathbf{m g} / \mathrm{L}$ \\
\hline SD $-100-11$ & $19-M a y-1987$ & ALKALINITY & 105 & $\mathrm{mg} / \mathrm{L}$ \\
\hline SD $-100-11$ & $26-$ May- 1987 & ALKALINITY & 105 & $\mathbf{m g} / \mathbf{L}$ \\
\hline SD-100-11 & 3-Jun- 1987 & ALKALINITY & 105 & $\mathrm{mg} / \mathrm{L}$ \\
\hline SD-100-11 & 11-Jun-1987 & AIKALINITY & 104 & $\mathrm{mg} / \mathrm{L}$ \\
\hline SD - $100 \cdot 11$ & $18-J u n-1987$ & ALKALINITY & 105 & $\mathbf{m g} / \mathrm{L}$ \\
\hline \multicolumn{5}{|c|}{$* *$ SD $-100-12$} \\
\hline SD $-100-12$ & 12-May-1987 & ALKALINITY & 100 & $\operatorname{mg} / \mathrm{L}$ \\
\hline SD - $100-12$ & 19-May-1987 & ALKALINITY & 102 & $\mathrm{mg} / \mathrm{L}$ \\
\hline SD $-100-12$ & $26-\mathrm{May}-1987$ & ALRALINITY & 102 & $\mathrm{mg} / \mathrm{L}$ \\
\hline SD - $100-12$ & 3-Jun-1987 & ALKALINITY & 107 & mg $/ \mathrm{L}$ \\
\hline SD $-100-12$ & 11-Jun- 1987 & ALKALINITY & 100 & $\mathrm{mg} / \mathrm{L}$ \\
\hline$S D-100-12$ & 18 -Jun- 1987 & ALKALINITY & 100 & $\mathrm{mg} / \mathrm{L}$ \\
\hline \multicolumn{5}{|c|}{$\star \star \quad$ SD $-100-13$} \\
\hline SD $-100-13$ & 12-May- 1987 & ALKALINITY & 98 & $\pm \mathrm{mg} / \mathrm{L}$ \\
\hline$S D-100-13$ & 19-May-1987 & AUKALINITY & 102 & $\mathrm{mg} / \mathrm{L}$ \\
\hline$S D=100-13$ & 26-May-1987 & ALKALYNITY & 103 & $\mathrm{mg} / \mathrm{L}$ \\
\hline SD $-100-13$ & 3-Jun- 1987 & ALKALINITY & 104 & $\operatorname{mg} / \mathrm{L}$ \\
\hline SD $-100-13$ & 11-Jun- 1987 & ALKALIRITY & 97 & $\mathrm{mg} / \mathrm{L}$ \\
\hline SD - $100-13$ & 18 -Jun- 1987 & ALKALINITY & 92 & $\mathrm{mg} / \mathrm{L}$ \\
\hline
\end{tabular}

** SD-100-14

SD-100-14 12-May-1987 ALRALINITY

SD-100-14 19-May-1987 ALKALINITY

SD-100-14 26-May-1987 ALKALINITY

SD-100-14 3-Jun-1987 ALKALINITY

SD-100-14 11-Jun-1987 ALKALINITY

SD-100-14 18-Jun-1987 ALKALINITY 
Storm Drain Data Eor SD-100

\begin{tabular}{|c|c|c|c|c|}
\hline Location & Date & - Test Compound & Results & Units \\
\hline SD-100-C & $12-J u n-1987$ & ALKALINITY & 102 & $\operatorname{mg} / \mathrm{L}$ \\
\hline SD- $100-C$ & $19-J$ un -1987 & AIKALINITY & 97 & mg/L \\
\hline \multicolumn{5}{|c|}{$* * \quad S D-100-D$} \\
\hline$S D-100-D$ & 6-Apr-1987 & ALKALINITY & 96 & $\mathrm{mg} / \mathrm{L}$ \\
\hline SD $-100-D$ & $11-J u n-1987$ & AIKALINITY & 97 & $\mathrm{mg} / \mathrm{L}$ \\
\hline \multicolumn{5}{|c|}{ ** SD-100-13 } \\
\hline SD $-100-13$ & 12-May-1987 & ALKYL KETONE & so & $\mathrm{ug} / \mathrm{L}$ \\
\hline \multicolumn{5}{|c|}{ * SD -100} \\
\hline SD - 100 & 5-Mar-1987 & ALPHA & $<2$ & $\mathrm{pCI} / \mathrm{L}$ \\
\hline SD -100 & 11 -Mar- 1987 & ALPHA & 2.5 & $\mathrm{pC1} / \mathrm{L}$ \\
\hline SD- 100 & 18 -Mar- 1987 & ALPHA & $<2$ & $\mathrm{pCi} / \mathrm{L}$ \\
\hline SD- 100 & $24-$ Mar- 1987 & ALPHA & 3.6 & $\mathrm{pC} 1 / \mathrm{L}$ \\
\hline$S D-100$ & 3-Apr-1987 & ALPHA & $<3$ & $\mathrm{pCl} / \mathrm{L}$ \\
\hline SD- 100 & 6-Apr-1987 & ALPUA & $<1$ & $\mathrm{pCi} / \mathrm{L}$ \\
\hline SD- 100 & 12-May-1987 & ALPHA & $<1$ & $\mathrm{pCl} / \mathrm{L}$ \\
\hline SD -100 & 19-May-1987 & ALPHA & $<2$ & $\mathrm{pCi} / \mathrm{L}$ \\
\hline SD- 100 & $26-$ May -1987 & ALPHA & $<2$ & $\mathrm{pC} i / \mathrm{L}$ \\
\hline$S D-100$ & 3-Jun-1987 & ALPHA & $<2$ & $\mathrm{pCi} / \mathrm{L}$ \\
\hline SD- 100 & $11-J u n-1987$ & ALPHA & $<2$ & $\mathrm{pCi} / \mathrm{L}$ \\
\hline SD -100 & $18-J u n-1987$ & ALPHA & $<1$ & $\mathrm{pCi} / \mathrm{L}$ \\
\hline \multicolumn{5}{|c|}{$\star \star \quad S D-100-01$} \\
\hline SD $-100-01$ & $12-$ May - 1987 & ALPEA & $<1$ & $\mathrm{pCI} / \mathrm{L}$ \\
\hline SD-100-01 & $18-$ May -1987 & ALPHA & $<2$ & $\mathrm{pCI} / \mathrm{L}$ \\
\hline SD-100-01 & 26-May-1987 & ALPBA & 3 & $\mathrm{PCI} / \mathrm{L}$ \\
\hline SD-100-01 & 3-Jun-1987 & ALPHA & $<2$ & $\mathrm{pCi} / \mathrm{L}$ \\
\hline$S D-100-01$ & 11-Jun- 1987 & ALPHA & $<1$ & $\mathrm{pGi} / \mathrm{L}$ \\
\hline SD-100-01 & $18-J u n-1987$ & ALPHA & 3.5 & $\mathrm{pCi} / \mathrm{L}$ \\
\hline \multicolumn{5}{|c|}{$\star \quad$ SD $-100-02$} \\
\hline$S D-100-02$ & 12-May- 1987 & ALPYA & 1 & $\mathrm{pCi} / \mathrm{L}$ \\
\hline SD-100-02 & $18-$ May -1987 & ALPHA & $<2$ & $\mathrm{pC} \mathbf{i} / \mathrm{L}$ \\
\hline SD $-100-02$ & 26-May-1987 & ALPHA & 2 & $\mathrm{pC} i / L$ \\
\hline SD-100-02 & 3-Jun-1987 & ALPHA & $<2$ & $\mathrm{pCi} / \mathrm{L}$ \\
\hline SD-100-02 & 11-Jun-1987 & ALPKA & $<1$ & $\mathrm{pCi} / \mathrm{L}$ \\
\hline$S D-100-02$ & $18-J u n-1987$ & ALPHA & 1.8 & $\mathrm{pCl} / \mathrm{L}$ \\
\hline \multicolumn{5}{|c|}{$\star \star \quad S D-100-03$} \\
\hline$S D-100-03$ & 12-May - 1987 & ALPHA & $<1$ & $\mathrm{pCl} / \mathrm{L}$ \\
\hline SD -100.03 & 18-May-1987 & AIPHA & $<2$ & $\mathrm{pCl} / \mathrm{L}$ \\
\hline SD $-100-03$ & 26-May-1987 & AIPHA & $<2$ & $\mathrm{pC1} / \mathrm{L}$ \\
\hline$S D-100-03$ & 3-Jun-1987 & ALPHA & 2.1 & $\mathrm{PCl} / \mathrm{L}$ \\
\hline SD $-100-03$ & 11-Jun-1987 & ALPHA & $<1$ & $\mathrm{pCI} / \mathrm{L}$ \\
\hline$S D=100-03$ & $18-J$ un- 1987 & ALPHS & $<1$ & $\mathrm{pCl} / \mathrm{L}$ \\
\hline
\end{tabular}


Storm Drain Data for SD- 100

\begin{tabular}{|c|c|c|}
\hline pcatic & Date & \\
\hline ** SD -100 & $-03 D$ & \\
\hline$S D-100-C$ & 12-May- 1987 & \\
\hline$D-100-03 D$ & 26-May- & \\
\hline * SD -100 & -0.4 & \\
\hline$D-100-04$ & $12-\mathrm{MzJ}$ & \\
\hline$D-100-04$ & $18-Y$ & \\
\hline D-100-04 & $26-M B$ & \\
\hline$D-100-04$ & 3-Jun-1987 & \\
\hline$-100-04$ & 11-Jun-1987 & \\
\hline $0-100-04$ & 18 -Jun- 1987 & \\
\hline
\end{tabular}

$\begin{array}{ll}\text { Results } & \text { UnIts } \\ & \\ <1 & \mathrm{pCI} / \mathrm{L} \\ <2 & \mathrm{pCI} / \mathrm{L} \\ & \\ 1 & \mathrm{pCI} / \mathrm{L} \\ <2 & \mathrm{pCi} / \mathrm{L} \\ 2 & \mathrm{pCi} / \mathrm{L} \\ 7.3, & \mathrm{pCI} / \mathrm{L} \\ <1 & \mathrm{pCI} / \mathrm{L} \\ <1 & \mathrm{pCI} / \mathrm{L}\end{array}$

* SD-100-06

SD-100-06 12-May-1987 ALPHA

SD-100-06 18-May-1987 ALPHA

SD-100-06 26-May-1987 ALPHA

SD-100-06 3-Jur-1987 ALPHA

SD-100-06 11-JUn-1987 ALPHA

SD-100-06 18-Jun-1987 ALPHA

* SD-100-06D

SD-100-06D 3-Jun-1987 ALPHA

\section{1}

$<2$

$<2$

$<2$

$<1$

1.5

$<2$

$\mathrm{pC} \perp / \mathrm{L}$

$\mathrm{PCI} / \mathrm{L}$

$\mathrm{PCi} / \mathrm{L}$

$\mathrm{PC} \mathrm{i} / \mathrm{L}$

$\mathrm{PCI} / \mathrm{L}$

$\mathrm{PCI} / \mathrm{L}$

* SD-100-07

SD-100-07 12-May-1987 ALPHA

SD-100-07 19-May-1987 ALPHA

SD-100-07 26-May-1987 ALPHA

SD-100-07 3-Jun-1987 ALPHA

SD-100-07 11-Jun-1987 ALPHA

SD-100-07 18-Jur-1987 ALPHA

18

29.1

26

75.1

10.6

47.8

$\mathrm{PC} \mathrm{L} / \mathrm{L}$

** SD-100-07D

SD-100-07D 18-Jun-1987 ALPHA

$42.1 \quad \mathrm{pCi} / \mathrm{L}$

* SD-100-09

SD-100-09 12-May-1987 ALPHA

SD-100-09 18-May-1987 ALPHA

SD-100-09 26-May-1987 ALPHA

SD-100-09 3-Jun-1987 ALPHA

SD-100-09 11-Jun-1987 ALPHA

SD-100-09 18-Jun-1987 ALPHA

2

$<2$

$<2$

8.0

$<2$

3.5

* SD-100-10

SD-100-10 12-May-1987 ALPHA

SD-100-10 19-May-1987 ALPHA

SD-100-10 26-May-1987 ALPHA

SD-100-10 3-Jun-1987 ALPHA

SD-100-10 11-Jun-1987 ALPHA

SD-100-10 18-Jun-1987 ALPHA 
Storm Drain Data for SD-100

\begin{tabular}{|c|c|c|c|c|}
\hline Location & Date & Test Compound & Results & Untes \\
\hline \multicolumn{5}{|c|}{$\star \star S D-100-11$} \\
\hline SD $-100-11$ & 12-Kay- 1987 & AIPHA & $<1$ & $\mathrm{pC} 1 / \mathrm{L}$ \\
\hline SD $-100-11$ & $19-$ May -1987 & ALPHA & 2.1 & $\mathrm{pCl} / \mathrm{L}$ \\
\hline SD-100-11 & 26-May-1987 & ALPHA & 2 & $\mathrm{pCl} / \mathrm{L}$ \\
\hline SD-100-11 & 3-Jun-1987 & ALPHA & $<2$ & $\mathrm{pCI} / \mathrm{L}$ \\
\hline SD-100-11 & $11-J$ un-1987 & ALPHA & $<2$ & $\mathrm{PCl} / \mathrm{L}$ \\
\hline SD-100-11 & 18-Jun-1987 & ALPHA & $<2$ & $\mathrm{pCI} / \mathrm{L}$ \\
\hline \multicolumn{5}{|c|}{$\star \star \quad S D-100-12$} \\
\hline SD $-100-12$ & 12-May- 1987 & ALPHA & 2 & $\mathrm{pCI} / \mathrm{L}$ \\
\hline$S D-100-12$ & 19-May-1987 & ALPHA & $<2$ & $\mathrm{PCi} / \mathrm{L}$ \\
\hline SD-100-12 & 26-May-1987 & ALPHA & $<2$ & $\mathrm{pCi} / \mathrm{L}$ \\
\hline SD $-100-12$ & $3-J u n-1987$ & ALPRA & $<2$ & $\mathrm{pCi} / \mathrm{L}$ \\
\hline SD $-100-12$ & 11-Jun- 1987 & ALPHA & $<2$ & $\mathrm{pCi} / \mathrm{L}$ \\
\hline$S D-100-12$ & $18-J u n-1987$ & ALPHA & $<2$ & $\mathrm{pCl} / \mathrm{L}$ \\
\hline \multicolumn{5}{|c|}{ ** SD-100-13 } \\
\hline SD $-100-13$ & 12-May- 1987 & ALPHA & $<1$ & $\mathrm{pC} 1 / \mathrm{L}$ \\
\hline SD $-100-13$ & 19-May- 1987 & ALPHA & $<2$ & $\mathrm{pCi} / \mathrm{L}$ \\
\hline SD $-100-13$ & 26-May-1987 & ALPHA & $<2$ & $\mathrm{pCi} / \mathrm{L}$ \\
\hline SD $-100-13$ & 3-Jun- 1987 & ALPHA & 2.4 & $\mathrm{pCi} / \mathrm{L}$ \\
\hline SD-100-13 & $11-J u n-1987$ & ALPHA & $<2$ & $\mathrm{pCi} / \mathrm{L}$ \\
\hline$S D-100-13$ & 18-Jun-1987 & ALPHA & $<2$ & $\mathrm{pCi} / \mathrm{L}$ \\
\hline \multicolumn{5}{|c|}{$\star \star \quad S D-100-14$} \\
\hline SD-100-14 & 12-May-1987 & ALPHA & 2 & $\mathrm{pC1} / \mathrm{L}$ \\
\hline SD $-100 \cdot 14$ & 19-Mzy-1987 & AIPHA & $<2$ & $\mathrm{pCi} / \mathrm{L}$ \\
\hline SD - $100-14$ & 26-May-1987 & ALPHA & $<2$ & $\mathrm{pCl} / \mathrm{L}$ \\
\hline SD-100-14 & 3-Jun-1987 & ALPHA & 17.1 & $\mathrm{pCi} / \mathrm{L}$ \\
\hline SD $-100-14$ & $11-J u n-1987$ & ALPHA & $<2$ & $\mathrm{pCi} / \mathrm{L}$ \\
\hline SD $-100-14$ & $18-J u n-1987$ & ALPHA & $<2$ & $\mathrm{pCl} / \mathrm{L}$ \\
\hline \multicolumn{5}{|c|}{ * SD. $100 \cdot C$} \\
\hline$S D-100 \cdot C$ & 5-Kar- 1987 & ALPHA & $<2$ & $\mathrm{pCi} / \mathrm{L}$ \\
\hline$S D-100-C$ & 9-Mat-1987 & ALPHA & 1 & $\mathrm{pCi} / \mathrm{L}$ \\
\hline$S D-100-C$ & $17-\operatorname{Mar}-1987$ & ALPHA & $<2$ & $\mathrm{pCi} / \mathrm{L}$ \\
\hline$S D-100-C$ & 24-Kar-1987 & ALPHA & 2.2 & $\mathrm{pCi} / \mathrm{L}$ \\
\hline$S D=100-C$ & 8-APT-1987 & ALPHA & 3.9 & $\mathrm{PC}+\mathrm{i} / \mathrm{L}$ \\
\hline SD-100-C & 13-May-1987 & ALPHA & 2 & $\mathrm{pCi} / \mathrm{L}$ \\
\hline$S D-100-C$ & 20-May-1987 & ALPHA & 3 & $\mathrm{PCi} / \mathrm{L}$ \\
\hline$S D-100-C$ & 27-May - 1987 & ALPHA & 3 & $\mathrm{pCI} / \mathrm{L}$ \\
\hline$S D-100-C$ & $12-J u n-1987$ & AIPHA & $<2$ & $\mathrm{PCi} / \mathrm{L}$ \\
\hline$S D-100-C$ & $19-J u n-1987$ & ALPHA & $<2$ & $\mathrm{pCl} / \mathrm{L}$ \\
\hline \multicolumn{5}{|c|}{$\star \star \quad S D-100-D$} \\
\hline SD - $100-D$ & 6-APr -1987 & ALPHA & $<1$ & $\mathrm{pCi} / \mathrm{L}$ \\
\hline $5 D-100-D$ & $11-J u n-1987$ & ALPHA & $<2$ & $\mathrm{pC} 1 / \mathrm{L}$ \\
\hline
\end{tabular}


Storm Drain Data for SD-100

\begin{tabular}{|c|c|c|c|}
\hline Location & Test Compound & Results & Units \\
\hline \multicolumn{4}{|c|}{$\star \quad$ SD-100 } \\
\hline SD- 100 & 5-Kar-1987 ALUMINUM & $<0.10$ & $\operatorname{mg} / \mathrm{L}$ \\
\hline$S D-100$ & 11-Mar-1987 ALUMINUM & 0.13 & $\mathrm{mg} / \mathrm{L}$ \\
\hline$S D-100$ & 18-Mar-1987 ALUIEINUA & $<0.10$ & $m g / L$ \\
\hline SD-100 & 24-Mar-1987 ALUMINUM & 0.11 & $\mathrm{mg} / \mathrm{L}$ \\
\hline SD- 100 & 3-Apr-1987 ALUKINUM & $<0.10$ & $\mathrm{mg} / \mathrm{L}$ \\
\hline SD- 100 & 6-Apx-1987 ALUMINUM & $<0.10$ & $\mathbf{m g} / \mathrm{L}$ \\
\hline SD- 100 & 12-May-1987 ALUMINUM & $<0.10$ & $\mathrm{mg} / \mathrm{L}$ \\
\hline$S D=100$ & 19-May-1987 ALUMIMUM & $<0.10$ & $\mathrm{mg} / \mathrm{L}$ \\
\hline SD- 100 & 26-May-1987 ALUMINUM & 0.055 & $\mathrm{mg} / \mathrm{L}$ \\
\hline$S D-100$ & 3-Jun-1987 ALUMINUM & $<0.10$ & $\operatorname{mg} / \mathrm{L}$ \\
\hline SD -100 & 11-Jun-1987 ALUMINUM & $<0.10$ & $\mathrm{Ig} / \mathrm{L}$ \\
\hline$S D=100$ & 18-Jum-1987 ALUMINUM & $<0.10$ & \\
\hline
\end{tabular}

** SD-100.01

SD-100-01 12-May-1987 ALUMTNUM

SD-100-01 18-May-1987 ALUMINUM

SD-100-01 3-Jun-1987 ALUMINUM

SD-100-01 11-Jun-1987 ALUMINUM

SD-100-01 18-Jun-1987 ALUMINUM

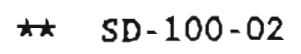

SD-100-02 12-May-1987 ALUMINUH

SD-100-02 18-May-1987 ALUMINUM

SD-100-02 3-Jun-19B7 ALUMINUM

SD-100-02 11-Jun-1987 ALIMINUM

SD-100-02 18-JUn-1987 ALUMINUM

$\begin{array}{ll}0.20 & \mathrm{mg} / \mathrm{L} \\ 1.2 & \mathrm{mg} / \mathrm{L} \\ 0.54 & \mathrm{mg} / \mathrm{L} \\ 0.21 & \mathrm{mg} / \mathrm{L} \\ 1.1 & \mathrm{mg} / \mathrm{L}\end{array}$

* SD $-100-03$

SD-100-03 12-May-1987 ALIMINUM

SD-100-03 18-May-1987 ALUMINUM

SD-100-03 26-May-1987 ALUMINUM

SD-100-03 3-Jun-1987 ALUMINUM

SD-100-03 11-Jun-1987 ALUMINUM

SD-100-03 18-Jun-1987 ALUMINUM

* SD-100-03D

SD-100-03D 12-May-1987 ALUMINUM

SD-100-03D 26-May-1987 ALUMINUM

$<0.10 \mathrm{mg} / \mathrm{L}$

$0.11 \mathrm{mg} / \mathrm{h}$

* SD-100-04.

SD-100-04 12-May-1987 ALUMINUM

SD-100-04 18-May-1987 ALUMINUM

SD-100-04 26-May-1987 ALUMINUM

SD-100-04 3-Jun-1987 ALUMINUM

SD-100-04 11-Jun-1987 ALUMINUM

$<0.10$

$<0.10$

$\mathrm{mg} / \mathrm{L}$

$<0.10$

$\mathrm{mg} / \mathrm{L}$

$<0.10$

$\mathrm{mg} / \mathrm{L}$

$<0.10$

$\mathrm{mg} / \mathrm{L}$

SD-100-04 18-Jun-1987 ALUMINUM

$\begin{array}{ll}<0.10 & \mathrm{mg} / \mathrm{L} \\ <0.10 & \mathrm{mg} / \mathrm{L} \\ 0.11 & \mathrm{mg} / \mathrm{L} \\ <0.10 & \mathrm{mg} / \mathrm{L} \\ <0.10 & \mathrm{mg} / \mathrm{L} \\ <0.10 & \mathrm{mg} / \mathrm{L} \\ & \\ <0.10 & \mathrm{mg} / \mathrm{L} \\ 0.11 & \mathrm{mg} / \mathrm{L} \\ & \\ <0.10 & \mathrm{mg} / \mathrm{L} \\ <0.10 & \mathrm{mg} / \mathrm{L} \\ 0.11 & \mathrm{mg} / \mathrm{L} \\ <0.10 & \mathrm{mg} / \mathrm{L} \\ <0.10 & \mathrm{mg} / \mathrm{L} \\ <0.10 & \mathrm{mg} / \mathrm{L}\end{array}$


Storm Drain Daca for SD-100

Lacation Date Test Compound

Results Unies

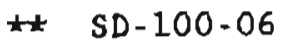

SD-100-06 12-May-1987 ALIMINUM

$<0.10$

$<0.10$

SD-100-06 18-May-1987 ALUMINUM

$<0.10$

SD-100-06 26-May-1987 ALJMINUM

$<0.10$

SD-100-06 3-Jun-1987 ALUMINUM

$<0.10$

SD-100-06 18-Jun-1987 ALIMINUM

$<0.10$

$\mathrm{mg} / \mathrm{L}$

$\mathrm{mg} / \mathrm{L}$

$\mathrm{mg} / \mathrm{L}$

$\mathrm{mg} / \mathrm{L}$

$\mathrm{mg} / \mathrm{L}$

$\mathrm{mg} / \mathrm{L}$

** SD-100-06D

SD-100-06D 3-Jun-1987 ALUMINUM

$<0.10 \quad \mathrm{mg} / \mathrm{L}$

** SD-100-07

SD-100-07 12-Kay-1987 ALUMINUM

SD-100-07 19-May-1987 ALIMINUM

SD-100-07 26-May-1987 ALUMINUM

SD-100-07 3-Jun-1987 ALUMINUM

SD-100-07 11-Jun-1987 ALUMINUM

SD-100-07 18-Jun-1987 ALUMINUM

$4.7 \quad \mathrm{mg} / \mathrm{L}$

$0.65 \mathrm{mg} / \mathrm{L}$

$0.12 \mathrm{mg} / \mathrm{L}$

$0.53 \quad \mathrm{mg} / \mathrm{L}$

$0.30 \quad \mathrm{mg} / \mathrm{L}$

$0.37 \mathrm{mg} / \mathrm{L}$

$\star$ SD $-100-07 D$

SD-100-07D 18-Jun-1987 ALUMINUM

$0.56 \mathrm{mg} / \mathrm{L}$

* SD - 100-09

SD-100-09 12-May-1987 ALUMINUM

SD-100-09 18-May-1987 ALUMINUM

SD-100-09 26-May-1987 ALUUINUM

SD-100-09 3-Jun-1987 ALUMINUM

SD-100-09 11-Jun-1987 ALUMINOM

$0.10 \mathrm{mg} / \mathrm{L}$

$<0.10 \mathrm{mg} / \mathrm{L}$

$<0.10 \quad \mathrm{mg} / \mathrm{L}$

$<0.10 \quad \mathrm{mg} / \mathrm{L}$

$<0.10 \quad \mathrm{mg} / \mathrm{L}$

** SD-100-10

SD-100-10 12-May-1987 ALUMINUM

SD-100-10 19-May-1987 ALUMINUM

SD-100-10 26-May-1987 ALUMINUM

SD-100-10 3-Jun-1987 ALUMINOM

SD-100-10 11-Jun-1987 ALUMINUM

SD-100-10 18-Jun-1987 ALUMINUM

$<0.10 \quad \mathrm{mg} / \mathrm{L}$

$<0.10 \quad \mathrm{mg} / \mathrm{L}$

$<0.10 \quad \mathrm{mg} / \mathrm{L}$

$0.60 \mathrm{mg} / \mathrm{L}$

$<0.10 \quad \mathrm{mg} / \mathrm{L}$

$<0.10 \quad \mathrm{mg} / \mathrm{L}$

** SD-100-11

SD-100-11 12-May-1987 ALUMINUM

SD-100-11 19-May-1987 ALUMINUM

SD-100-11 26-May-1987 ALUMINUM

SD-100-11 3-Jun-1987 ALUMINUM

SD-100-11 11-Jun-1987 ALUMINUM

SD-100-11 18-Jun-1987 ALUMINUM

$<0.10 \quad \mathrm{mg} / \mathrm{L}$

$<0.10 \quad \mathrm{mg} / \mathrm{L}$

$0.54 \mathrm{mg} / \mathrm{L}$

$<0.10 \quad \mathrm{mg} / \mathrm{L}$

$<0.10 \quad \mathrm{mg} / \mathrm{L}$

$<0.10 \quad \mathrm{mg} / \mathrm{L}$

** SD-100-12

SD-100-12 12-May-1987 ALUMINUN

SD-100-12 19-May-1987 ALUMINUM

SD-100-12 26-May-1987 ALUMIKUM

SD-100-12 3-Jun-1987 ALUMINUM

0.84

$\mathrm{mg} / \mathrm{L}$

$0.11 \mathrm{mg} / \mathrm{L}$

$0.12 \mathrm{mg} / \mathrm{L}$

$<0.10 \quad \mathrm{mg} / \mathrm{L}$ 
Storm Drein Data for SD-100

\begin{tabular}{|c|c|c|c|c|}
\hline Location & Date & Test Compound & Results & Units \\
\hline$S D-100-12$ & $11-J u n-1987$ & ALUMINUM & $<0.10$ & $\mathrm{mg} / \mathrm{L}$ \\
\hline SD - $100-12$ & $18-J$ un -1987 & ALUMINUM & 0.11 & $\mathrm{mg} / \mathrm{L}$ \\
\hline \multicolumn{5}{|c|}{$\star \star \quad S D-100-13$} \\
\hline$S D-100-13$ & 12-May-1987 & ALIRTHNOM & $<0.10$ & ㅍg $/ \mathrm{L}$ \\
\hline SD $-100-13$ & $19-\mathrm{May}-1987$ & ALIRTINUM & 0.049 & $\mathrm{mg} / \mathrm{L}$ \\
\hline$S D-100-13$ & 26-May - 1987 & ALIMINUM & $<0.020$ & $\mathrm{mg} / \mathrm{L}$ \\
\hline$S D-100-13$ & 11-Jun-1987 & ALURINUM & $<0.10$ & $\operatorname{mg} / \mathrm{L}$ \\
\hline$S D-100-13$ & $18-J$ un -1987 & ALUKINUM & $<0.10$ & $\mathrm{mg} / \mathrm{L}$ \\
\hline \multicolumn{5}{|c|}{$\star \star \quad S D-100-14$} \\
\hline SD $-100-14$ & $12-$ May-1987 & ALOMINUM & 0.45 & $\mathrm{mg} / \mathrm{L}$ \\
\hline SD $-100-14$ & 19-May-1987 & ALDMIINUM & 0.52 & mg/L \\
\hline SD $-100 \cdot 14$ & 26-May -1987 & ALUMINUM & 0.34 & $\mathrm{mg} / \mathrm{L}$ \\
\hline$S D-100-14$ & $3-J u n-1987$ & ALUMINUM & $<0.10$ & $\mathrm{mg} / \mathrm{L}$ \\
\hline$S D-100-14$ & $11-J u n-1987$ & ALOMINUM & $<0.10$ & $\operatorname{mg} / \mathrm{L}$ \\
\hline SD - $100-14$ & $18-J$ un -1987 & ALUMINUM & $<0.10$ & $\mathrm{mg} / \mathrm{L}$ \\
\hline \multicolumn{5}{|c|}{$* \quad S D-100-C$} \\
\hline$S D-100-C$ & 5-Mar-1987 & ALUYINUM & 0.14 & $\mathrm{mg} / \mathrm{L}$ \\
\hline$S D-100-C$ & $9-\operatorname{Mar}-1987$ & ALUMINUM & 0.12 & $\operatorname{mg} / L$ \\
\hline SD-100-C & $17-\operatorname{Mar}-1987$ & ALINCINUM & $<0.10$ & $\operatorname{mg} / \mathrm{L}$ \\
\hline SD $-100-C$ & $24-\operatorname{Mar}-1987$ & AL.UMINUM & 0.21 & $\operatorname{mg} / L$ \\
\hline$S D-100-C$ & 8-Apr-1987 & ALIRTINUM & $<0.10$ & mg/L \\
\hline$S D-100-C$ & 13-May - 1987 & ALUAINOM & $<0.10$ & $\mathbf{m g} / \mathrm{L}$ \\
\hline SD-100-C & 20-May-1987 & ALUMTNUM & $<0.10$ & mg/L \\
\hline SD- $100-C$ & $27-$ May -1987 & ALUMTNUM & $<0.10$ & $\operatorname{mg} / L$ \\
\hline SD- $100-C$ & 4-Jun-1987 & ALUAINUM & $<0.10$ & $\operatorname{mg} / \mathrm{L}$ \\
\hline$S D-100-C$ & 12-Jun- 1987 & ALIRIINUM & $<0.10$ & mg/L \\
\hline SD- $100-C$ & $19-J u n-1987$ & ALUKINUM & $<0.10$ & $\mathrm{mg} / \mathrm{L}$ \\
\hline \multicolumn{5}{|c|}{$\star \quad S D-100-D$} \\
\hline$S D-100-D$ & 6-Apr-1987 & ALUMINUM & $<0.10$ & $\operatorname{mg} / \mathrm{L}$ \\
\hline$S D \cdot 100-D$ & 11-Jun- 1987 & ALMHINUM & $<0.10$ & $\operatorname{lng} / \mathrm{L}$ \\
\hline \multicolumn{5}{|l|}{$\star \star \quad S D-100$} \\
\hline SD-100 & 5-Mar-1987 & AMMONIA & 0.26 & $\operatorname{mg} / \mathrm{L}$ \\
\hline SD -100 & 11-Mar-1987 & AMYYONIA & 0.2 & $\mathrm{mg} / \mathrm{L}$ \\
\hline$S D-100$ & $18-\operatorname{Mar}-1987$ & AMMONIA & $<0.2$ & $\mathrm{mg} / \mathrm{L}$ \\
\hline SD -100 & $24-\operatorname{Mar}-1987$ & AMRONIA & $<0.2$ & $\operatorname{mg} / \mathrm{L}$ \\
\hline$S D-100$ & 3-Арт-1987 & AMMONIA & $<0.2$ & $\mathrm{mg} / \mathrm{L}$ \\
\hline SD-100 & 6-Apr - 1987 & AMCOIIA & $<0.2$ & $\operatorname{mg} / \mathrm{L}$ \\
\hline SD -100 & 12-May-1987 & AMFONIA & $<0.2$ & $\mathrm{mg} / \mathrm{L}$ \\
\hline$S D-100$ & $19-$ May -1987 & AMMONIA & $<0.2$ & $\mathrm{ng} / \mathrm{L}$ \\
\hline SD -100 & 26-May - 1987 & AMBONIA & $<0.2$ & $\operatorname{mg} / L$ \\
\hline SD - 100 & 3-Jun-1987 & AMMONIA & $<0.2$ & $\operatorname{mg} / \mathrm{L}$ \\
\hline SD -100 & $11-J u n-1987$ & AMMONIA & $<0.2$ & $\mathrm{mg} / \mathrm{L}$ \\
\hline SD - 100 & 18-Jun-1987 & AMNONIA & $<0.2$ & $\mathrm{mg} / \mathrm{L}$ \\
\hline
\end{tabular}


Storm Drain Data for SD-100

Location Date Test Compound

Results Units

* SD-100-01

SD-100-01 12-Kay-1987 AMMONLA

SD-100-01 18-May-1987 AMIONIA

SD-100-01 26-May-1987 AMYONIA

SD-100-01 3-Jun-1987 AMEONIA

SD-100-01 11-Jun-1987 AMONIA

SD-100-01 18-Jun-1987 AMONIA

* SD-100-02

SD-100-02 12-May-1987 AMMONIA

SD-100-02 18-May-1987 AMMONIA

SD-100-02 26-May-1987 AMONIA

SD-100-02 3-Jun-1987 AMMONLA

SD-100-02 11-Jun-1987 AMONIA

SD-100-02 18-Jun-1987 AMONIA

** SD-100-03

SD-100-03 12-May-1987 AMONIA

SD-100-03 18-May-1987 AMONIA

SD-100-03 26-May-1987 AMMONIA

SD-100-03 3-Jun-1987 AMMONIA

SD-100-03 11-Jun-1987 AMONIA

SD-100-03 18-Jun-1987 AMMONIA

$<0.2 \mathrm{mg} / \mathrm{L}$

$<0.2 \mathrm{mg} / \mathrm{L}$

$<0.2 \mathrm{mg} / \mathrm{L}$

$<0.2 \mathrm{mg} / \mathrm{L}$

$<0.2 \mathrm{mg} / \mathrm{L}$

$<0.2 \mathrm{mg} / \mathrm{L}$

$<0.2 \quad \mathrm{mg} / \mathrm{L}$

$<0.2 \mathrm{mg} / \mathrm{L}$

$<0.2 \mathrm{mg} / \mathrm{L}$

$<0.2 \mathrm{mg} / \mathrm{L}$

$<0.2 \mathrm{mg} / \mathrm{L}$

$<0.2 \quad \mathrm{mg} / \mathrm{L}$

$<0.2 \mathrm{mg} / \mathrm{L}$

$<0.2 \mathrm{mg} / \mathrm{L}$

$<0.2 \mathrm{mg} / \mathrm{L}$

$<0.2 \mathrm{mg} / \mathrm{L}$

$<0.2 \mathrm{mg} / \mathrm{L}$

$<0.2 \mathrm{mg} / \mathrm{L}$

* SD-100-03D

SD-100-03D 12-May-1987 AMMONIA

SD-100-03D 26-May-1987 AMmONIA

$<0.2 \mathrm{mg} / \mathrm{l}$

$<0.2 \mathrm{mg} / \mathrm{L}$

$\star \star \quad S D-100-04$

SD-100-04 12-May-I987 AMONIA

SD-100-04 18-May-1987 AMMONIA

SD-100-04 26-May-1987 AMAONIA

SD-100-04 3-Jun-1987 AMYONIA

SD-100-04 11-JUn-1987 AMONIA

SD-100-04 18-Jun-1987 AMONIA

$<0.2 \mathrm{mg} / \mathrm{L}$

$<0.2 \mathrm{mg} / \mathrm{L}$

$<0.2 \mathrm{mg} / \mathrm{L}$

$<0.2 \mathrm{mg} / \mathrm{L}$

$<0.2 \mathrm{mg} / \mathrm{L}$

$<0.2 \mathrm{mg} / \mathrm{L}$

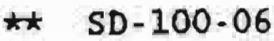

SD-100-06 12-May-1987 AMONIA

SD-100-06 18-May-1987 AMONIA

SD-100-06 26-May-1987 AMONIA

SD-100-06 3-Jun-1987 AMMONIA

SD-100-06 11-Jun-1987 AMLOUIA

SD-100.06 18-Jun-1987 AMYONIA

$<0.2 \quad \mathrm{mg} / \mathrm{I}$

$<0.2 \mathrm{mg} / \mathrm{L}$

$<0.2 \mathrm{mg} / \mathrm{L}$

$<0.2 \mathrm{mg} / \mathrm{L}$

$<0.2 \mathrm{mg} / \mathrm{L}$

$<0.2 \mathrm{mg} / \mathrm{L}$

$\star \star$ SD-100-06D

SD-100-06D 3-Jun-3987 AMMONIA

$<0.2 \mathrm{mg} / \mathrm{L}$

** SD-100-07

SD-100-07 12-May-1987 AMMONIA

$<0.2$

$\mathrm{mg} / \mathrm{L}$

SD-100-07 19-May-1987 AMMONIA

0.2

$\mathrm{mg} / \mathrm{L}$ 
Storm Drain Deta for SD-100

\begin{tabular}{|c|c|c|c|c|}
\hline Location & Date & Test Compound & Results & Units \\
\hline SD-100-07 & 26-May-1987 & AMMONIA & $<0.2$ & $\operatorname{mg} / \mathrm{L}$ \\
\hline SD- $100-07$ & 3-Jun-1987 & AMPONIA & 1.2 & $\mathrm{mg} / \mathrm{L}$ \\
\hline SD-100-07 & $11-J u n-1987$ & AMMONIA & 0.9 & $\mathrm{mg} / \mathrm{L}$ \\
\hline SD-100-07 & $18-J u m-1987$ & ARMONIA & 1.2 & $\mathrm{mg} / \mathrm{L}$ \\
\hline \multicolumn{5}{|c|}{ ** SD-100-07D } \\
\hline$S D-100-07 D$ & $18-J$ un -1987 & AMHONIA & 1.0 & $\mathrm{mg} / \mathrm{L}$ \\
\hline \multicolumn{5}{|c|}{ * SD-100-09 } \\
\hline SD-100-09 & 12-Мвy-1987 & AMMONIA & $<0.2$ & $\mathbf{m g} / \mathrm{L}$ \\
\hline SD.100-09 & $18-$ May- 1987 & ALMONIA & $<0.2$ & $\mathbf{m g} / \mathrm{L}$ \\
\hline SD $-100-09$ & $26-$ May -1987 & AMMONIA & $<0.2$ & $\mathrm{mg} / \mathrm{L}$ \\
\hline SD $-100-09$ & 3-Jun-1987 & AMMONIA & $<0.2$ & $\mathrm{mg} / \mathrm{L}$ \\
\hline SD $-100-09$ & 11-Jun- 1987 & AMMONIA & $<0.2$ & $\mathrm{mg} / \mathrm{L}$ \\
\hline SD $-100-09$ & $18-J u n-1987$ & AMLONIA & $<0.2$ & $\operatorname{mg} / L$ \\
\hline \multicolumn{5}{|c|}{ ** SD $-100-10$} \\
\hline SD $-100-10$ & 12-May- 1987 & AMMONIA & $<0.2$ & $\mathrm{mg} / \mathrm{L}$ \\
\hline SD $-100-10$ & 19-May-1987 & AMLONIA & $<0.2$ & $\operatorname{mg} / L$ \\
\hline SD- $100-10$ & 26-May-1987 & AMISONIA & $<0.2$ & $\operatorname{mg} / \mathrm{L}$ \\
\hline SD- $100-10$ & $3-3$ un -1987 & AMMONIA & $<0.2$ & $\mathbf{m g} / \mathrm{L}$ \\
\hline$S D-100-10$ & $11-J u n-1987$ & AMMONIA & $<0.2$ & $\mathrm{mg} / \mathrm{L}$ \\
\hline SD $-100-10$ & $18-J u n-1987$ & AMMONIA & $<0.2$ & $\mathrm{mg} / \mathrm{L}$ \\
\hline \multicolumn{5}{|c|}{$* * \quad S D-100-11$} \\
\hline$S D-100-11$ & $12-$ May-1987 & AMMONIA & $<0.2$ & mg/L \\
\hline SD-100-11 & 19-May-1987 & AMMONIA & 0.8 & $\mathrm{mg} / \mathrm{L}$ \\
\hline SD-100-11 & 26-May-1987 & AMMONIA & 0.2 & mg/L \\
\hline SD-100-11 & 3-Jun-1987 & AMRONIA & $<0.2$ & $\mathrm{mg} / \mathrm{h}$ \\
\hline$S D-100-11$ & $11-J u n-1987$ & AMMONIA & $<0.2$ & $\mathrm{mg} / \mathrm{l}$ \\
\hline$S D-100-11$ & $18-J u n-1987$ & AMMONIA & $<0.2$ & $\mathrm{mg} / \mathrm{L}$ \\
\hline \multicolumn{5}{|c|}{$\star$ SD $-100-12$} \\
\hline SD-100-12 & $12-$ May -1987 & AMMONIA & $<0.2$ & $\mathrm{mg} / \mathrm{l}$ \\
\hline SD $-100-12$ & 19-May-1987 & AMMONIA & $<0.2$ & $\mathrm{mg} / \mathrm{L}$ \\
\hline SD-100-12 & $26-$ May- 1987 & AMMONIA & $<0.2$ & $\mathrm{mg} / \mathrm{L}$ \\
\hline SD-100-12 & 3-Jun-1987 & AMMONIA & $<0.2$ & $\mathbf{m g} / \mathrm{L}$ \\
\hline SD $-100-12$ & $11-J u n-1987$ & AMMONIA & $<0.2$ & $\mathbf{m g} / \mathrm{L}$ \\
\hline SD - $100-12$ & $18-J$ un -1987 & AMMONIA & $<0.2$ & $\mathbf{m g} / \mathrm{L}$ \\
\hline \multicolumn{5}{|c|}{ ** SD $-100-13$} \\
\hline SD $-100-13$ & 12-May-1987 & AMMONIA & $<0.2$ & $\mathbf{m g} / \mathrm{L}$ \\
\hline SD $-100-13$ & 19-May-1987 & AMMONIA & $<0.2$ & $m g / L$ \\
\hline SD $-100-13$ & 26-May-1987 & AMMONIA & $<0.2$ & $\mathrm{mg} / \mathrm{L}$ \\
\hline SD $-100-13$ & 3-Jun-1987 & AMMONIA & $<0.2$ & $\mathrm{mg} / \mathrm{L}$ \\
\hline$S D-100-13$ & 11-Jun-1987 & AMMONIA & $<0.2$ & $\mathrm{mg} / \mathrm{L}$ \\
\hline$S D-100-13$ & $18-J u n-1987$ & AMMONIA & $<0.2$ & $\mathrm{mg} / \mathrm{L}$ \\
\hline
\end{tabular}


Storm Drain Daca for SD-100

$\begin{array}{lcr}\text { Location } & \text { Date } & \text { Test CO } \\ \text { A* SD-100-14 } & \\ \text { SD-100-14 } & 12 \text {-May-1987 } & \text { AMONIA } \\ \text { SD-100-14 } & 19 \text {-May-1987 AMMONIA } \\ \text { SD-100-14 } & 26 \text {-May-1987 AMONIA } \\ \text { SD-100-14 } & 3 \text {-Jun-1987 AMONIA } \\ \text { SD-100-14 } & 11 \text {-Jun-1987 AMMONIA } \\ \text { SD-100-14 } & 18 \text {-Jun-1987 AMYONIA }\end{array}$

Results Units

** SD-100-14

SD-100-14 12-May-1987 AMONIA

$<0.2$

$<0.2$

$<0.2$

$<0.2$

$<0.2$

$<0.2$

$\mathrm{mg} / \mathrm{L}$

$\mathrm{mg} / \mathrm{L}$

$m g / 2$

$\operatorname{mg} / \mathrm{L}$

mg $/ \mathrm{L}$

SD-100-14 18-Jun-1987 AMTONIA

mg/L

* $\quad S D-100-C$

SD-100-C 5-Mar-1987 AMMONIA

$<0.2 \quad \mathrm{mg} / \mathrm{I}$

SD-100-C 9-Mar-1987 AMMONIA

$<0.2 \quad \mathrm{mg} / \mathrm{L}$

SD-100-C 17-Mar-1987 AMMONLA

$<0.2 \quad \mathrm{mg} / \mathrm{L}$

SD-100-C 24-MaI-1987 AMMONIA

$<0.2$

SD-100-C 8-Apr-1987 AMMONIA

$<0.2$

mg $/ \mathrm{L}$

$<0.2$

$S D-100-C$

13-May-1987 AMIONIA

20-May-1987 AMMONIA

SD-100-C 27-May-1987 AMMONIA

$<0.2$

$<0.2$

SD $-100-C$

4-Jun-1987 AMMONIA

SD-100-C

12-Jun-1987 AMMONIA

$<0.2$

$<0.2$

SD-100-C

19-Jun-1987 AMMONIA

$<0.2$

$\mathrm{mg} / \mathrm{L}$

$\mathrm{mg} / \mathrm{L}$

$\mathrm{mg} / \mathrm{L}$

$m g / L$

$\mathrm{mg} / \mathrm{L}$

$\mathrm{mg} / \mathrm{L}$

** SD-100-D

SD-100-D 6-APr-1987 AMMONIA

$<0.2$

$\operatorname{mg} / \mathrm{L}$

SD-100-D 11-Jun-1987 AMMONIA

$<0.2$

$\mathrm{mg} / \mathrm{L}$

* SD -100

SD-100 5-Mar-1987 ANTHRACENE

SD-100 11-Mar-1987 ANTHRACENE

SD-100 12-May-1987 ANTHRAGENE

SD-100 26-May-1987 ANTHRACENE

$<10$

$<10$

$<10$

$<5.0$

$\operatorname{mg} / L$

** SD-100-01

SD-100-01 12-May-1987 ANTHRACENE

SD-100-01 26-May-1987 ANTHRACENE

$<10 \quad$ ug/L

$<5.0 \quad \mathrm{ug} / \mathrm{L}$

* SD-100-02

SD-100-02 12-May-1987 ANTHRACENE

SD-100-02 26-May-1987 ANTHRACENE

$<10 \quad 4 \mathrm{~g} / \mathrm{L}$

$<5.0 \quad \mathrm{ug} / \mathrm{L}$

* SD-100-03

SD-100-03 12-May-1987 ANTHRACENE

SD-100-03 26-May-1987 ANTHRAGENE

$<10 \quad \mathrm{ug} / \mathrm{L}$

$<5.0 \quad \mathrm{ug} / \mathrm{L}$

* SD-100-030

SD-100-03D 12-May-1987 ANTHRACENE

SD-100-03D 26-May-1987 ANTHRACENE

$\begin{array}{ll}<10 & \mathrm{ug} / \mathrm{L} \\ <5.0 & \mathrm{ug} / \mathrm{L} \\ & \\ <10 & \mathrm{ug} / \mathrm{L} \\ <5.0 & \mathrm{ug} / \mathrm{L}\end{array}$

** SD-100-04

SD-100-04 12-May-1987 ANTHRACENE

SD-100-04 26-May-1987 ANTHRACENE 
Storm Drain Data for SD-100

Location Date Test Compound Results Units

t SD-100-06

SD-100-06 12-Kay-1987 ANTHRACENE

SD-100-06 26-Kay-1987 ANTHRACENE

$<10 \quad$ ug $/ \mathrm{L}$

* SD-100-07

SD-100-07 12-May-1987 ANTHRACENE

SD-100-07 26-May-1987 ANTHRACENE

** SD-100-09

SD-100-09 12-May-1987 ANTHRACENE

SD-100-09 26-May-1987 ANIHRACENE

* SD-100-10

SD-100-10 12-May-1987 ANTHRACENE

SD-100-10 26-May-1987 ANTHRACENE

$<10 \quad u g / L$

10

$<5.0$

$1 \mathrm{~g} / \mathrm{L}$

$u_{g} / 2$

$<0$

$<5.0$

$4 \mathrm{~g} / \mathrm{L}$

ug $/$ L

* SD-100-11

SD-100-11 12-May-1987 ANTHRACENE

SD-100-11 26-May-1987 ANTHRACENE

* SD-100-12

SD-100-12 12-May-1987 ANTHRACENE

SD-100-12 26-May-1987 ANTHRACENE

* SD $-100-13$

SD-100-13 12-May-1987 ANTHRACENE

SD-100-13 26-May-1987 ANTHRACENE

*t SD-100-14

SD-100-14 12-May-1987 ANTHRACENE

SD-100-14 26-Hay-1987 ANTHRACENE

$<10 \quad u g / L$

$<5.0 \quad \mathrm{ug} / \mathrm{L}$

** SD-100-C

SD-100-C S-Mar-1987 ANIHRACENE

SD-100-C 9-Mar-1987 ANTHRACENE

SD-100-C 17-Mar-1987 ANTHRACENE

SD-100-C - 24-Mar-1987 ANTHRACENE

SD-100-C 8-APr-1987 ANTHRACENE

SD-100-C 13-May-1987 ANTHRACENE

SD-100-C 20-May-1987 ANTHRACENE

SD-100-C 27-May-1987 ANTHRACENE

SD-100-C 12-Jun-1987 ANTHRACENE

SD-100-C 19-Jum-1987 ANTHRACENE
$<10$

$<10$

$<10$

$<10$

$<10$

$<5.0$

$<5.0$

$<5.0$

$<5.0$

$<10$

$<0.005$

$<0.005$

$<0.005$

$<0.005$

$<0.005$ $\mathrm{ug} / \mathrm{l}$

ug/ 1

ug/L

ug $/ 2$

ug $/$

$4 g / 2$

$\mathrm{ug} / \mathrm{L}$

$u g / L$

ug/L

ug $/ \mathrm{L}$

ug/L

ug/L

$\mathrm{ug} / \mathrm{L}$

ug/I

$\mathrm{ug} / \mathrm{L}$

ug $/ \mathrm{L}$

$u g / L$

* SD-100

SD-100 5-Mar-1987 ARSENIC

SD-100 11-Mar-1987 ARSENIC

SD-100 18-Mar-1987 ARSENIC

SD-100 24-Mar-1987 ARSENIC

SD-100 3-Apr-1987 ARSENIC 
Storm Drain Data for SD-100

\begin{tabular}{|c|c|c|c|c|}
\hline Location & Date & Test Compound & Results & Units \\
\hline$S D-100$ & 6-Apr- 1987 & ARSENIC & $<0.005$ & $\mathrm{mg} / \mathrm{L}$ \\
\hline$S D-100$ & 12-Kay - 19B7 & ARSENIC & $<0.005$ & $\operatorname{mg} / \mathrm{L}$ \\
\hline SD- 100 & 19-May-1987 & ARSENIC & $<0.005$ & $\mathbf{m g} / \mathrm{L}$ \\
\hline SD- 100 & 26-May-1987 & ARSENIC & $<0.005$ & $\mathrm{mg} / \mathrm{L}$ \\
\hline SD- 100 & 3-Jun-1987 & ARSENIC & $<0.005$ & $\mathrm{mg} / \mathrm{L}$ \\
\hline SD- 100 & 11-Jun-1987 & ARSENIC & $<0.005$ & $\mathrm{mg} / \mathrm{L}$ \\
\hline SD -100 & $18-J$ un-19B7 & ARSENIC & $<0.005$ & $\mathrm{mg} / \mathrm{L}$ \\
\hline \multicolumn{5}{|c|}{$\star \quad$ SD $-100-01$} \\
\hline SD-100-01 & $12-\mathrm{May} \cdot 1987$ & ARSENIC & $<0.005$ & $\mathrm{mg} / \mathrm{L}$ \\
\hline SD $-100-01$ & 18-May-1987 & ARSENIC & $<0.005$ & $\mathbf{m g} / \mathrm{L}$ \\
\hline SD-100-01 & 26 - May-1987 & ARSENIC & $<0.005$ & $\mathrm{mg} / \mathrm{L}$ \\
\hline SD $-100-01^{\circ}$ & 3-Jun-1987 & ARSENIC & $<0.005$ & $\mathrm{mg} / \mathrm{L}$ \\
\hline$S D \cdot 100-01$ & $11-J u n-1987$ & ARSENIC & $<0.005$ & $\mathrm{mg} / \mathrm{L}$ \\
\hline$S D \cdot 100 \cdot 01$ & $18-J$ un- 1987 & ARSENIC & $<0.005$ & $\mathrm{mg} / \mathrm{L}$ \\
\hline \multicolumn{5}{|c|}{$\star \quad$ SD-100-02 } \\
\hline SD $-100-02$ & 12-May - 1987 & ARSENIC & $<0.005$ & $\mathrm{mg} / \mathrm{L}$ \\
\hline SD-100-02 & $18-$ May -1987 & ARSENIC & 0.005 & $\mathrm{mg} / \mathrm{L}$ \\
\hline$S D-100-02$ & 26-May-1987 & ARSENIC & $<0,005$ & $\mathbf{m g} / \mathrm{L}$ \\
\hline$S D-100-02$ & 3-Jun-1987 & ARSENIC & $<0.005$ & $\mathrm{mg} / \mathrm{L}$ \\
\hline$S D-100-02$ & $11-J u n-1987$ & ARSENIC & $<0.005$ & $\mathrm{mg} / \mathrm{L}$ \\
\hline SD - $100-02$ & $18-J u n-1987$ & ARSENIC & $<0.005$ & $\mathrm{mg} / \mathrm{L}$ \\
\hline \multicolumn{5}{|c|}{ ** SD-100-03 } \\
\hline$S D-100 \cdot 03$ & $12-$ May-1987 & ARSENIC & $<0,005$ & mg $/ \mathrm{L}$ \\
\hline SD $-100-03$ & 18-May-1987 & ARSENIC & $<0.005$ & $\mathrm{mg} / \mathrm{L}$ \\
\hline$S D-100-03$ & 26-May-1987 & ARSENIC & $<0.005$ & $\mathrm{mg} / \mathrm{L}$ \\
\hline SD -100.03 & $3-J u n-1987$ & ARSENIC & $<0.005$ & 血g $/ 2$ \\
\hline SD $-100-03$ & $11-J u n-1987$ & ARSENIC & $<0.005$ & $\mathrm{mg} / \mathrm{L}$ \\
\hline SD $-100-03$ & $18-J \operatorname{un}-1987$ & ARSENIC & $<0.005$ & $\mathrm{mg} / \mathrm{L}$ \\
\hline \multicolumn{5}{|c|}{$* \quad S D-100.03 D$} \\
\hline SD - 100-03D & $12-$ Kay-1987 & ARSENIC & $<0.005$ & $\mathrm{mg} / \mathrm{L}$ \\
\hline$S D-100-03 D$ & 26-May-1987 & ARSENIC & $<0.005$ & $\mathrm{mg} / \mathrm{L}$ \\
\hline \multicolumn{5}{|c|}{$\star \star \quad S D-100-04$} \\
\hline$S D-100-04$ & 12-May- 1987 & ARSENIC & $<0.005$ & $\mathbf{m g} / \mathrm{L}$ \\
\hline SD $-100-04$ & 18-May-1987 & ARSENIC & $<0.005$ & $\mathrm{mg} / \mathrm{L}$ \\
\hline$S D=100.04$ & 26-May-1987 & ARSENIC & $<0.005$ & $\mathrm{ng} / \mathrm{L}$ \\
\hline SD-100-04 & 3-Jun-1987 & ARSENIC & $<0.005$ & $\operatorname{mg} / \mathrm{L}$ \\
\hline SD - 100.04 & 11-Jun-1987 & ARSENIC & $<0.005$ & $\mathrm{mg} / \mathrm{L}$ \\
\hline SD $-100-04$ & $18-J u n-1987$ & ARSENIC & $<0.005$ & $\mathrm{mg} / \mathrm{L}$ \\
\hline \multicolumn{5}{|c|}{$\star \star \quad S D-100-06$} \\
\hline SD- 100.06 & 12-May-1987 & ARSENIC & $<0.005$ & $\operatorname{mg} / \mathrm{L}$ \\
\hline SD $-100-06$ & 18-May-1987 & ARSENIC & $<0.005$ & $\mathrm{mg} / \mathrm{L}$ \\
\hline SD - $100-06$ & 26-May-1987 & ARSENIC & $<0.005$ & $\mathrm{mg} / \mathrm{L}$ \\
\hline$S D-100-06$ & 3-Jun-1987 & ARSENIC & $<0.005$ & $\mathrm{mg} / \mathrm{L}$ \\
\hline SD-100-06 & Il-Jun-1987 & ARSENIC & $<0.005$ & $\operatorname{mg} / \mathrm{L}$ \\
\hline SD $-100-06$ & $18-J$ un - 1987 & ARSENIC & $<0,005$ & $\mathrm{mg} / \mathrm{L}$ \\
\hline
\end{tabular}


Storm Drain Data for SD-100

\begin{tabular}{|c|c|c|c|c|}
\hline Location & Date & Test Compound & Results & Units \\
\hline \multicolumn{5}{|c|}{$\star \star \quad S D-100-06 D$} \\
\hline SD-100-06D & 3-Jun-1987 & ARSENIC & $<0.005$ & $\mathrm{mg} / \mathrm{L}$ \\
\hline \multicolumn{5}{|c|}{$\star \star \quad S D-100-07$} \\
\hline SD-100-07 & 12-May-1987 & ARSENIC & 0.009 & $\mathrm{mg} / \mathrm{L}$ \\
\hline SD $-100-07$ & 19-1ваy-1987 & ARSENIC & $<0.005$ & $\mathrm{mg} / \mathrm{L}$ \\
\hline SD $-100-07$ & $26-$-4ay-1987 & ARSENIC & $<0.005$ & $\mathrm{mg} / \mathrm{L}$ \\
\hline$S D-100-07$ & 3-Jun-1987 & ARSENIC & $<0.005$ & $\mathrm{mg} / \mathrm{L}$ \\
\hline SD - $100-07$ & $11-J u n-1987$ & ARSENIC & $<0.005$ & $\mathrm{mg} / \mathrm{L}$ \\
\hline SD $-100-07$ & 18 - Jun-1987 & ARSENIC & $<0.005$ & $\mathbf{m g} / \mathrm{L}$ \\
\hline \multicolumn{5}{|c|}{ ** SD-100-07D } \\
\hline SD - $100-07 D$ & $18-J u n-1987$ & ARSENTC & $<0.005$ & $\mathrm{mg} / \mathrm{L}$ \\
\hline \multicolumn{5}{|c|}{$\star \star \quad$ SD. $100-09$} \\
\hline SD-100-09 & 12-May-1987 & ARSENIC & $<0.005$ & mg/L \\
\hline SD -100.09 & 18-May- 1987 & ARSENIC & $<0.005$ & $\mathrm{mg} / \mathrm{L}$ \\
\hline SD-100-09 & 26-May-1987 & ARSENIC & $<0.005$ & $\mathrm{mg} / \mathrm{L}$ \\
\hline SD $-100-09$ & 3-Jun-1987 & ARSENIC & $<0.005$ & $\mathrm{mg} / \mathrm{L}$ \\
\hline SD - $100-09$ & 1I-Jun- 1987 & ARSENIC & $<0.005$ & $\mathbf{m g} / \mathbf{L}$ \\
\hline SD - $100-09$ & $18-J$ un- 1987 & ARSENIC & $<0.005$ & $\mathrm{mg} / \mathrm{L}$ \\
\hline \multicolumn{5}{|c|}{$\star \star \quad S D-100-10$} \\
\hline SD - $100-10$ & 12-May-1987 & ARSENIC & $<0.005$ & $\operatorname{mg} / \mathrm{L}$ \\
\hline$S D-100-10$ & 19-May-1987 & ARSENIC & $<0.005$ & $\mathrm{mg} / \mathrm{L}$ \\
\hline SD $-100-10$ & 26-May- 1987 & ARSENIC & $<0.005$ & $\mathrm{mg} / \mathrm{L}$ \\
\hline$S D=100-10$ & $3-3 u n-1987$ & ARSENIC & $<0.005$ & $\mathrm{mg} / \mathrm{L}$ \\
\hline$S D-100-10$ & $11-$ Jun -1987 & ARSENIC & $<0.005$ & $\mathrm{mg} / \mathrm{L}$ \\
\hline SD-100-10 & $18-J$ un- 1987 & ARSENIC & $<0.005$ & $\operatorname{mg} / \mathrm{L}$ \\
\hline \multicolumn{5}{|c|}{$\star \star \quad S D-100-11$} \\
\hline SD-100-11 & 12-May- 1987 & ARSENIC & $<0.005$ & $m g / L$ \\
\hline SD-100-11 & 19-May-1987 & ARSENIC & $<0.005$ & $\mathrm{mg} / \mathrm{L}$ \\
\hline SD-100-11 & 26 -May -1987 & ARSENIC & $<0.005$ & $\operatorname{mg} / \mathrm{L}$ \\
\hline SD $-100-11$ & 3-Jun-1987 & ARSENIC & $<0.005$ & $\mathbf{m g} / \mathrm{L}$ \\
\hline SD-100-11 & 11-Jun-1987 & ARSENIC & $<0.005$ & $\mathrm{ng} / \mathrm{L}$ \\
\hline$S D-100-11$ & 18-Jun-1987 & ARSENIC & $<0.005$ & $\mathbf{n g} / \mathrm{L}$ \\
\hline \multicolumn{5}{|c|}{$\star \star \quad$ SD $-100-12$} \\
\hline SD $-100-12$ & 12 - May - 1987 & ARSEMTIC & $<0.005$ & $\mathrm{ng} / \mathrm{L}$ \\
\hline SD $-100-12$ & 19-May-1987 & ARSENIC & $<0.005$ & $\mathbf{n g} / \mathrm{L}$ \\
\hline SD - $100-22$ & $26-$ May -1987 & ARSENIC & $<0.005$ & $\mathbf{m g} / \mathrm{L}$ \\
\hline$S D-100-12$ & $3-J$ un-1987 & ARSENIC & $<0.005$ & $\mathbf{n g} / \mathrm{L}$ \\
\hline SD - $100-12$ & $11-J$ un-1987 & ARSENIC & $<0.005$ & $n g / L$ \\
\hline$S D-100-12$ & 18 - Jun- 1987 & ARSENIC & $<0.005$ & $\mathbf{m g} / \mathrm{L}$ \\
\hline \multicolumn{5}{|c|}{$\star \star \quad S D-100-13$} \\
\hline$S D-100-13$ & 12-May-1987 & ARSENIC & $<0.005$ & $\mathbf{n g} / \mathrm{L}$ \\
\hline$S D-100-13$ & $19-$ May-1987 & ARSENIC & $<0.005$ & $\mathrm{mg} / \mathrm{L}$ \\
\hline$S D-100-13$ & 26-Мay-1987 & ARSENIC & $<0.005$ & $\mathrm{ng} / \mathrm{L}$ \\
\hline
\end{tabular}


Storm Drain Data for SD-100

\begin{tabular}{|c|c|c|c|c|}
\hline Locacion & Date & Test Compound & Results & Units \\
\hline SD $-100-13$ & 3-Jun-1987 & ARSENIC & $<0.005$ & $\operatorname{mg} / \mathrm{L}$ \\
\hline SD $-100 \cdot 13$ & $11-J$ un-1987 & ARSEIIC & $<0.005$ & $\mathrm{mg} / \mathrm{L}$ \\
\hline$S D-100-13$ & 18 -Jun-1987 & ARSENIC & $<0.005$ & $\mathbf{m g} / \mathrm{L}$ \\
\hline \multicolumn{5}{|c|}{ * SD-100-14 } \\
\hline$S D-100-14$ & 12-May- 1987 & ARSERIC & $<0.005$ & $\operatorname{mg} / 1$ \\
\hline SD $-100-14$ & 19-May-1987 & ARSENIC & $<0.005$ & mg $/ \mathrm{L}$ \\
\hline SD-100-14 & $26-$ May- 1987 & ARSENIC & $<0.005$ & $\mathrm{mg} / \mathrm{L}$ \\
\hline SD - 100-14 & 3-Jun-1987 & ARSENIC & $<0.005$ & $\mathrm{mg} / \mathrm{l}$ \\
\hline SD-100-14 & 1I-Jun-1987 & ARSENIC & $<0.005$ & $\mathrm{mg} / \mathrm{L}$ \\
\hline SD-100-14 & $18-J u n-1987$ & ARSEMTC & $<0.005$ & $\operatorname{mg} / \mathrm{L}$ \\
\hline \multicolumn{5}{|c|}{$\star \star \quad S D-100-C$} \\
\hline SD $-100-C$ & 5-Kar-1987 & ARSENIC & $<0.005$ & $\mathrm{mg} / \mathrm{L}$ \\
\hline SD-100-C & 9-Mar-1987 & ARSENIC & $<0.005$ & $\mathbf{m g} / \mathrm{L}$ \\
\hline$S D-100-C$ & $17-$ Mar -1987 & ARSENIC & $<0.005$ & $m g / L$ \\
\hline SD-100-C & 24-Mar-1987 & ARSENIC & $<0.005$ & $\mathrm{mg} / \mathrm{L}$ \\
\hline SD $-100 \cdot C$ & 8-Apr-1987 & ARSENRC & $<0.005$ & mg/L \\
\hline$S D-100-C$ & 13-May-1987 & ARSENIC & $<0.005$ & $\mathrm{mg} / \mathrm{L}$ \\
\hline SD-100-C & 20- Кay-1987 & ARSENIC & $<0.005$ & $\mathrm{mg} / \mathrm{L}$ \\
\hline$S D-100-C$ & 27-May-1987 & ARSENIC & $<0.005$ & $\mathrm{mg} / \mathrm{L}$ \\
\hline SD-100-C & 4-Jun-1987 & ARSENIC & $<0.005$ & $\mathrm{mg} / \mathrm{L}$ \\
\hline$S D \cdot 100 \cdot C$ & $12-J u r-1987$ & ARSENIC & 0.005 & $\mathrm{mg} / \mathrm{L}$ \\
\hline SD - $100-C$ & 19-Jụn-1987 & ARSENIC & $<0.005$ & $\mathrm{mg} / \mathrm{L}$ \\
\hline \multicolumn{5}{|c|}{$\star * \quad S D-100-D$} \\
\hline SD-100-D & 6-Apr-1987 & ARSENIC & $<0.005$ & $\mathrm{mg} / \mathrm{L}$ \\
\hline SD-100-D & 11-Jun-1987 & ARSENIC & $<0.005$ & $\mathrm{mg} / \mathrm{L}$ \\
\hline \multicolumn{5}{|l|}{ 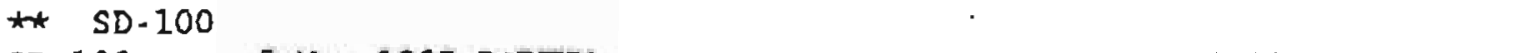 } \\
\hline SD -100 & 5-Mar-1987 & BARIUM & $<0.10$ & $\mathrm{mg} / \mathrm{L}$ \\
\hline SD -100 & $11-\operatorname{Mar}-1987$ & BARIUM & $<0.10$ & mg/L \\
\hline SD-100 & 18 -Mar- 1987 & BARTUM & $<0.10$ & $\mathrm{mg} / \mathrm{L}$ \\
\hline$S D-100$ & 24-Mar-1987 & BARIUM & $<0.10$ & $\mathrm{mg} / \mathrm{L}$ \\
\hline SD - 100 & 3-Apr-1987 & BARIUM & $<0.10$ & $\mathrm{mg} / \mathrm{L}$ \\
\hline$S D-100$ & 6-Apr-1987 & BARTUA & $<0.10$ & $\mathrm{mg} / \mathrm{L}$ \\
\hline SD - 100 & 12-May-1987 & BARIUH & $<0.10$ & $\mathrm{mg} / \mathrm{L}$ \\
\hline SD -100 & 19-May-1987 & BARIUH & $<0.10$ & $\mathrm{mg} / \mathrm{L}$ \\
\hline$S D-100$ & 26-May-1987 & BARIUM & 0.026 & 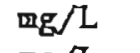 \\
\hline SD-100 & 3-Jun-1987 & BARIUH & $<0.10$ & $\mathrm{mg} / \mathrm{L}$ \\
\hline$S D=100$ & $11-J u n-1987$ & BARIUM & $<0.10$ & $\operatorname{mg} / \mathrm{L}$ \\
\hline SD -100 & $18-J$ un - 1987 & BARIUH & $<0.10$ & $\mathrm{mg} / \mathrm{L}$ \\
\hline \multicolumn{5}{|c|}{ * SD - 100-01 } \\
\hline SD-100-01 & 12-May-1987 & BARIUH & $<0.10$ & $\operatorname{mg} / \mathrm{L}$ \\
\hline$S D-100.01$ & $18-$ May - 1987 & BARIUM & 0.13 & $\mathrm{mg} / \mathrm{L}$ \\
\hline SD - $100-01$ & 3-Jun-1987 & BARIUH & $<0.10$ & $\mathrm{mg} / \mathrm{L}$ \\
\hline SD - $100-01$ & $11-J u n-1987$ & BARIUN & $<0.10$ & $\mathrm{mg} / \mathrm{L}$ \\
\hline SD - $100-01$ & $18-J$ un- 1987 & BARILA & $<0.10$ & $\mathrm{mg} / \mathrm{L}$ \\
\hline
\end{tabular}


Stora Drain Data for SD-100

\begin{tabular}{|c|c|c|c|c|}
\hline Location & Date & Test Compound & Results & Units \\
\hline \multicolumn{5}{|c|}{$\star * \quad S D-100-02$} \\
\hline$S D-100.02$ & $12-$ May- 1987 & BARIUI & $<0.10$ & $\mathrm{mg} / \mathrm{L}$ \\
\hline$S D-100.02$ & $16-$ May -1987 & BARTUY & $<0.10$ & $\operatorname{mg} / \mathrm{L}$ \\
\hline SD-100-02 & $3-J$ un -1987 & BARIUI & $<0.10$ & $\mathrm{mg} / \mathrm{L}$ \\
\hline$S D=100-02$ & $11-J u n-1987$ & BARIUM & $<0.10$ & $\pi \mathrm{g} / \mathrm{L}$ \\
\hline SD-100-02 & $18-3$ un -1987 & BARIOK & $<0.10$ & $\mathbf{m g} / \mathrm{L}$ \\
\hline \multicolumn{5}{|c|}{$\star$ * SD $-100-03$} \\
\hline$S D-100-03$ & 12-May-1987 & BARTUM & $<0.10$ & $\mathrm{mg} / \mathrm{L}$ \\
\hline$S D-100-03$ & 18-Kay-1987 & BARIUM & $<0.10$ & $\mathbf{m g} / \mathrm{L}$ \\
\hline SD $-100-03$ & 26-May-1987 & BARIUA & $<0.10$ & $\mathrm{mg} / \mathrm{L}$ \\
\hline SD-100-03 & 3-Jur-1987 & BARTUM & $<0.10$ & $\mathrm{mg} / \mathrm{L}$ \\
\hline SD $-100-03$ & 11-Jun- 1987 & BARIUM & $<0.10$ & $\mathrm{mg} / \mathrm{L}$ \\
\hline SD-100-03 & $18-J u n-1987$ & BARIUM & $<0.10$ & mg $/ \mathrm{L}$ \\
\hline \multicolumn{5}{|c|}{$\star$ \$D $-100-03 \mathrm{D}$} \\
\hline$S D-100-03 D$ & 12-May- 1987 & BARIUM & $<0.10$ & $\mathrm{mg} / \mathrm{L}$ \\
\hline$S D-100-03 D$ & $26-\mathrm{May}-1987$ & BARIUM & $<0.10$ & $\mathrm{mg} / \mathrm{L}$ \\
\hline \multicolumn{5}{|c|}{$\star S D-100-04$} \\
\hline SD-100-04 & 12-May- 1987 & BARIUR & $<0.10$ & $\mathrm{mg} / \mathrm{L}$ \\
\hline$S D \cdot 100 \cdot 04$ & 18-May-1987 & BARIUM & $<0.10$ & $\mathrm{mg} / \mathrm{L}$ \\
\hline SD-100-04 & 26- May -1987 & BARIUM & $<0.10$ & IIg $/ \mathrm{L}$ \\
\hline SD $-100-04$ & 3-Jun-1987 & BARIUM & $<0.10$ & mg/L \\
\hline SD-100-04 & $11-J u n-1987$ & BARIUM & $<0.10$ & $\operatorname{mg} / \mathrm{L}$ \\
\hline SD $-100-04$ & $18 \cdot$ Jun -1987 & BARION & $<0.10$ & $\mathrm{mg} / \mathrm{L}$ \\
\hline \multicolumn{5}{|c|}{ * SD-100-06 } \\
\hline SD $-100-06$ & $12 \cdot \mathrm{May}-1987$ & BARIUM & $<0.10$ & $\mathrm{mg} / \mathrm{L}$ \\
\hline SD $-100-06$ & 18-May- 1987 & BARIUM & $<0.10$ & $\mathrm{mg} / \mathrm{L}$ \\
\hline SD $-100-06$ & 26-May- 1987 & BARIUM & $<0.10$ & mg/L \\
\hline SD $-100-06$ & $3-J u n-1987$ & BARIUM & $<0.10$ & $\mathrm{mg} / \mathrm{L}$ \\
\hline$S D-100.06$ & $11-J u n-1987$ & BARIUM & $<0.10$ & $\mathrm{mg} / \mathrm{L}$ \\
\hline SD - 100.06 & $18-J u n-1987$ & BARIUM & $<0.10$ & $\mathrm{mg} / \mathrm{L}$ \\
\hline \multicolumn{5}{|c|}{$\star \quad$ SD $-100-06 D$} \\
\hline SD-100-D6D & 3-Jun- 1987 & BARIUM & $<0.10$ & $\mathrm{mg} / \mathrm{I}$ \\
\hline \multicolumn{5}{|c|}{$\star \star \quad S D-100-07$} \\
\hline$S D-100-07$ & 12-May-1987 & BARIUM & $<0.10$ & $\mathrm{mg} / \mathrm{L}$ \\
\hline$S D=100.07$ & 19-May -1987 & BARIUR & 0.84 & $\operatorname{mg} / \mathrm{L}$ \\
\hline SD $-100-07$ & 26-May-1987 & BARIUA & 0.11 & घg/L \\
\hline$S D-100-07$ & $3-3$ un- 1987 & BARTUL & 0.11 & $\operatorname{lng} / \mathrm{L}$ \\
\hline SD - 100-07 & 11 -Jun- 1987 & BARIUY & $<0.10$ & $\operatorname{mg} / \mathrm{L}$ \\
\hline SD $\cdot 100-07$ & $18-$ Jun -1987 & BARIUM & $<0.10$ & $\mathbf{n g} / \mathrm{L}$ \\
\hline \multicolumn{5}{|c|}{ ** SD-100-07D } \\
\hline SD - 100-07D & $18-J u n-1987$ & BARIUM & $<0.10$ & $\operatorname{mg} / \mathrm{L}$ \\
\hline
\end{tabular}


Storm Drain Data for SD-100

\begin{tabular}{|c|c|c|c|c|}
\hline Location & Date & Test Compound & Resules & Units \\
\hline * SD -100 & -09 & & & \\
\hline SD $-100-09$ & 12-May-1987 & BARIUM & $<0.10$ & mg $/ \mathrm{L}$ \\
\hline SD -100.09 & $18-$ Kay -1987 & BARIUM & $<0.10$ & $\operatorname{mg} / 2$ \\
\hline SD-100-09 & 26-Мay- 1987 & BARIUM & $<0.10$ & $\operatorname{mg} / \mathrm{L}$ \\
\hline SD-100-09 & 3-Jun-1987 & BARIUM & $<0.10$ & $\mathrm{mg} / \mathrm{L}$ \\
\hline SD-100-09 & 11 -Jun- 1987 & BARIUM & $<0.10$ & mg $/$ L \\
\hline * $\quad$ SD -100 & -10 & & & \\
\hline SD $-100-10$ & 12-May- 1987 & BARIUM & $<0.10$ & $\mathrm{mg} / \mathrm{L}$ \\
\hline$S D-100-10$ & 19-May-1987 & BARIUM & $<0.10$ & 坶 $/ \mathrm{L}$ \\
\hline SD $-100-10$ & 26-May-1987 & BARIUM & $<0.10$ & $\operatorname{mg} /$ \\
\hline SD $-100-10$ & 3-Jun-1987 & BARTUM & $<0.10$ & $\operatorname{mg} / 1$ \\
\hline$S D-100-10$ & 11-Jun-1987 & BARIUM & $<0.10$ & $\operatorname{mg} / \mathrm{L}$ \\
\hline SD $-100-10$ & $18-J u n-1987$ & BARIUM & $<0.10$ & $\operatorname{mg} / \mathrm{L}$ \\
\hline$\star \star \quad S D-100$ & -11 & & & \\
\hline SD-100-11 & 12-May- 1987 & BARIUM & $<0.10$ & $\mathrm{mg} / \mathrm{L}$ \\
\hline SD $-100 \cdot 11$ & 19-May-1987 & BARIUM & $<0.10$ & $\operatorname{mg} / \mathrm{L}$ \\
\hline SD $-100-11$ & $26-$ May -1987 & BARIUM & $<0.10$ & $\mathrm{mg} / \mathrm{L}$ \\
\hline SD-100-11 & 3-Jun-1987 & BARIUM & $<0.10$ & $\mathrm{mg} / \mathrm{L}$ \\
\hline SD $-100-11$ & 11-Jun-1987 & BARTUM & $<0.10$ & $\mathrm{mg} / 2$ \\
\hline SD $-100-11$ & 18 -Jun- 1987 & BARIUM & $<0.10$ & $\mathrm{mg} / \mathrm{L}$ \\
\hline ** SD -100 & -12 & & & \\
\hline$S D-100-12$ & 12-May - 1987 & BARIOM & $<0.20$ & $\mathrm{mg} / L$ \\
\hline SD-100-12 & 19 -May-1987 & BARTUM & 0.024 & $\mathbf{n g} / \mathrm{L}$ \\
\hline SD $-100-12$ & 26-May-1987 & BARIUM & 0.028 & $\mathbf{m g} / L$ \\
\hline SD $-100-12$ & 3-Jun-1987 & BARIUL & $<0.10$ & $\mathrm{mg} / \mathrm{L}$ \\
\hline SD-100-12 & $11-J$ un- 1987 & BARIUM & $<0.10$ & $\mathrm{mg} / \mathrm{L}$ \\
\hline SD-100-12 & $18-J u n-1987$ & BARTUM & $<0.10$ & $m g / L$ \\
\hline ** SD-100 & -13 & & & \\
\hline SD-100- 13 & 12-May-1987 & BARIUM & $<0.10$ & mg/L \\
\hline SD-100-13 & $19-$ May - 1987 & BARIUM & 0.025 & mig/L \\
\hline SD $-100-13$ & 26-May - 1987 & BARIUM & 0.025 & $\mathrm{mg} / \mathrm{L}$ \\
\hline$S . D-100-13$ & $11-J u n-1987$ & BARIUM & $<0.10$ & $\mathrm{mg} / \mathrm{L}$ \\
\hline$S D-100-13$ & $18-J u n-1987$ & RARIUM & $<0.10$ & $\operatorname{mg} / L$ \\
\hline$* * \quad S D-100$ & -14 & & & \\
\hline SD - $100-14$ & 12-May-1987 & BARTUM & $<0.10$ & $\operatorname{mg} / \mathrm{L}$ \\
\hline SD-100-14 & 19-Мay-1987 & BARIUM & $<0.10$ & $\operatorname{lng} / \mathrm{L}$ \\
\hline$S D-100-14$ & 26-May-1987 & BARIUM & 0.031 & $\operatorname{mg} / L$ \\
\hline$S D \cdot 100-14$ & $3-J$ un-1987 & BARIUM & $<0.10$ & $\operatorname{mg} /$ \\
\hline$S D-100-14$ & $11-J u n-1987$ & BARIUM & $<0.10$ & $\mathrm{mg} / \mathrm{L}$ \\
\hline SD - $100-14$ & $18-J$ แก- 1987 & BARIUM & $<0.10$ & ㄸg $/ 1$ \\
\hline ** SD -100 & & & & \\
\hline$S D-100-C$ & $5-$ Kar -1987 & BARIUM & $<0.10$ & $\mathrm{mg} / \mathrm{L}$ \\
\hline SD-100-C & $9-\operatorname{Mar}-1987$ & BARIUM & $<0.10$ & $\operatorname{mg} /$ \\
\hline SD-100-C & 17-Mar- 1987 & BARIUM & $<0.10$ & mg/L \\
\hline
\end{tabular}


Storm Drain Data for SD-100

\begin{tabular}{|c|c|c|c|c|}
\hline Locacion & Date & Test Compound & Results & Units \\
\hline SD-100-C & 24-Mar-1987 & BARIOM & $<0.10$ & $\mathrm{mg} / \mathrm{L}$ \\
\hline SD $-100-C$ & 8-Apr-1987 & BARIUM & $<0.10$ & $\mathrm{mg} / \mathrm{L}$ \\
\hline SD-100-C & 13-May-1987 & BARIUM & $<0.10$ & $\mathrm{mg}_{\mathrm{g}} / \mathrm{L}$ \\
\hline SD $-100-C$ & 20-May-1987 & BARIUM & $<0.10$ & $\mathrm{mg} / \mathrm{L}$ \\
\hline SD $-100-C$ & $27-$ May -1987 & BARIUM & $<0.10$ & $\mathrm{mg} / \mathrm{L}$ \\
\hline SD-100-C & 4-Jun-1987 & BARIUM & $<0.10$ & $\operatorname{mg} / \mathrm{L}$ \\
\hline SD $-100-C$ & 12-Jun-1987 & BARIUI & $<0.10^{\circ}$ & $\operatorname{mg} / \mathrm{L}$ \\
\hline SD-100-C & $19-J u n-1987$ & BARIUI & $<0.10$ & $\operatorname{mg} / L$ \\
\hline \multicolumn{5}{|c|}{$* \quad S D-100-D$} \\
\hline$S D-100-D$ & 6-Apr-1987 & BARIUM & $<0.10$ & $\operatorname{mg}_{\mathrm{g}} / \mathrm{L}$ \\
\hline$S D-100-D$ & $11-J$ un -1987 & BARIUM & $<0.10$ & $\mathrm{mg} / \mathrm{L}$ \\
\hline \multicolumn{5}{|c|}{$*$ SD -100} \\
\hline SD - 100 & $5-$ Mar -1987 & BENZENE & $<5$ & $\mathrm{ug} / \mathrm{L}$ \\
\hline SD -100 & $11-\operatorname{MaI}-1987$ & BENZENE & $<5$ & $\mathrm{ug} / \mathrm{L}$ \\
\hline SD- 100 & $18-\operatorname{Mar}-1987$ & BEINZENE & $<5$ & $u_{g} / L$ \\
\hline SD- 100 & $24-$ Max -1987 & BENZENE & $<5$ & $\mathrm{ug} / \mathrm{L}$ \\
\hline SD- 100 & 6-Арг-1987 & BENZENE & $<5$ & $\mathrm{ug} / \mathrm{L}$ \\
\hline SD - 100 & 12-May-1987 & BENZENE & $<5$ & $\mathrm{ug} / \mathrm{L}$ \\
\hline$S D-100$ & 19-May-1987 & BENZENE & $<5$ & $\mathrm{ug} / \mathrm{L}$ \\
\hline$S D-100$ & $26-$-ay -1987 & BENZENE & $<5$ & $\mathrm{ug} / \mathrm{L}$ \\
\hline SD - 100 & 3-Jun-1987 & BENZENE & $<5$ & $u g / L$ \\
\hline SD- 100 & $11-J u n-1987$ & BENZENE & $<5$ & $\mathrm{ug} / \mathrm{L}$ \\
\hline SD- 100 & $18-J$ un -1987 & BENZENE & $<5$ & $\mathrm{ug} / \mathrm{L}$ \\
\hline \multicolumn{5}{|c|}{$\star * \quad S D-100.01$} \\
\hline SD-100-01 & 12 -May - 1987 & BENZENE & $<5$ & $\mathrm{ug} / \mathrm{L}$ \\
\hline$S D-100-01$ & $18-$-ay -1987 & BENZENE & $<5$ & $u_{g} / L$ \\
\hline$S D-100-01$ & $26-$ Мay -1987 & BENZENE & $<5$ & ug $/ L$ \\
\hline SD-100-01 & 3-Jun-1987 & BENZENE & $<5$ & $u g / L$ \\
\hline SD-100-01 & $11-J u n-1987$ & BENZENE & $<5$ & $\mathrm{ug} / \mathrm{L}$ \\
\hline SD-100-01 & $18-J u n-1987$ & BENZENE & $<5$ & $u g / L$ \\
\hline \multicolumn{5}{|c|}{$\star \star \quad S D=100-02$} \\
\hline$S D-100-02$ & 12-May-1987 & BENZENE & $<5$ & $\mathrm{ug} / \mathrm{L}$ \\
\hline SD $-100-02$ & $18-$ May -1987 & BEAZENE & $<5$ & $48 / L$ \\
\hline$S D-100-02$ & $26-$ May -1987 & BENZENE & $<5$ & $\mathrm{ug} / \mathrm{L}$ \\
\hline SD $-100-02$ & 3-Jun-1987 & BENZENE & $<5$ & $\mathrm{ug} / \mathrm{L}$ \\
\hline SD - $100-02$ & 11 -Jun-1987 & BENZENE & $<5$ & ug/I \\
\hline SD-100-02 & $18-J u n-1987$ & BENZENE & $<5$ & $\mathrm{ug} / \mathrm{L}$ \\
\hline \multicolumn{5}{|c|}{$\star \star \quad S D-100-03$} \\
\hline SD $-100-03$ & 12-May-1987 & BENZENE & $<5$ & $u g / L$ \\
\hline SD $-100-03$ & $18-$ May -1987 & BENZENE & $<5$ & $\mathrm{ug} / \mathrm{L}$ \\
\hline SD - $100-03$ & $26-$ May -1987 & BEIZEENE & $<5$ & $\mathrm{ug} / \mathrm{L}$ \\
\hline SD -100.03 & 3-Jun-1987 & BENZENE & $<5$ & $\mathrm{ug} / \mathrm{L}$ \\
\hline SD $-100-03$ & $11-J u n-1987$ & BENZENE & $<S$ & $u_{g} / L$ \\
\hline SD- $100-03$ & $18-J u n-1987$ & BENZENE & $<5$ & $\mathrm{ug} / \mathrm{L}$ \\
\hline
\end{tabular}


Storm Draín Data for SD-100

\begin{tabular}{|c|c|c|c|c|}
\hline Location & Date & Test Compound & Results & Uolts \\
\hline \multicolumn{5}{|c|}{$* \quad S D-100-D 3 D$} \\
\hline SD-100-03D & $12-$ May -1987 & BEAZENE & $<5$ & $\mathrm{ug} / \mathrm{L}$ \\
\hline SD-100-03D & $26 \cdot$ Hay - 19B7 & BEAZERE & $<5$ & $\mathrm{ug} / \mathrm{l}$ \\
\hline \multicolumn{5}{|c|}{$\star \star \quad S D-100-04$} \\
\hline SD - $100-04$ & $12-\mathrm{May}-1987$ & BEAZZENE & $<5$ & $\mathrm{ug} / \mathrm{L}$. \\
\hline SD-100-04 & $18-$ May -1987 & BENZEANE & $<s$ & $\mathrm{ug} / \mathrm{L}$ \\
\hline SD $-100-04$ & $26-$ May- 1987 & BENZERE & $<5$ & $\mathrm{ug} / \mathrm{L}$ \\
\hline$S D=100-04$ & 3-Jun-1987 & BETLENE & $<5$ & $\mathrm{ug} / \mathrm{L}$ \\
\hline SD $-100-04$ & $11-J u n-1987$ & BEATZRAE & $<5$ & $\mathrm{ug} / \mathrm{L}$ \\
\hline SD $-100-04$ & $18-J u m-1987$ & BEMTZENE & $<5$ & $\mathrm{ug} / \mathrm{L}$ \\
\hline \multicolumn{5}{|c|}{$\star \star \quad S D-100-06$} \\
\hline$S D=100-06$ & $12-$ Kay- 1987 & BENLENE & $<5$ & $4 g / L$ \\
\hline SD - $100-06$ & 18-May - 1987 & BENZZNE & $<5$ & $\mathrm{ug} / \mathrm{L}$ \\
\hline SD - $100-06$ & 26-May-1987 & BEHZENE & $<5$ & $\mathrm{ug} / \mathrm{L}$ \\
\hline SD $-100-06$ & 3 -Jun-1987 & BENZENE & $<5$ & $u g / L$ \\
\hline$S D-100-06$ & $11-J u n-1987$ & BENZZNE & $<5$ & $\mathrm{ug} / \mathrm{L}$ \\
\hline SD - $100-06$ & 18-Jun-1987 & BENZENE & $<5$ & $\mathrm{ug} / \mathrm{L}$ \\
\hline \multicolumn{5}{|c|}{ ** SD-100-06D } \\
\hline$S D-100-06 D$ & 3-Jun-1987 & BENZENE & $<5$ & $\mathrm{ug} / \mathrm{L}$ \\
\hline \multicolumn{5}{|c|}{ ** SD-100-07 } \\
\hline SD-100-07 & $12-$ May - $19 B 7$ & BENZZWNE & 5 & ug/L \\
\hline SD - $100-07$ & $19-$ May-1987 & BENZENE & $<5$ & $\mathrm{ug} / \mathrm{L}$ \\
\hline$S D-100-07$ & 26-May-1987 & BENZDAE & 5 & $\mathrm{ug} / \mathrm{L}$ \\
\hline$S D-100-07$ & 3-Jun-1987 & BETZNIE & 5 & $\mathrm{ug} / \mathrm{L}$ \\
\hline$S D-100-07$ & 11-Jun-1987 & BENZARE & $<5$ & $\mathrm{ug} / \mathrm{L}$ \\
\hline SD-100-07 & $18-J u n-1987$ & BENZENE & $<5$ & $u g / L$ \\
\hline \multicolumn{5}{|c|}{ ** SD-100-07D } \\
\hline SD-100-07D & 18 -Jun- 1987 & BERZZRNE & $<5$ & $u g / L$ \\
\hline \multicolumn{5}{|c|}{$\star$ * SD-100-09 } \\
\hline$S D-100-09$ & $12-$ May-1987 & BENZENE & $<5$ & $\mathrm{ug} / \mathrm{L}$ \\
\hline SD -100.09 & $18-$ May-1987 & BEIZENE & $<5$ & $\mathrm{ug} / \mathrm{L}$ \\
\hline$S D-100-09$ & $26-$ May -1987 & BEARzaNE & $<5$ & $\mathrm{ug} / \mathrm{L}$ \\
\hline SD -100.09 & $3-J$ un-1987 & BENZLANB & $<5$ & $\mathrm{ug} / \mathrm{L}$ \\
\hline SD - $100-09$ & 11-Jun-1987 & BENZENE & $<5$ & $\mathrm{ug} / \mathrm{L}$ \\
\hline SD - $100-09$ & $18-J$ un-1987 & BENZEAE & $<5$ & $\mathrm{ug} / \mathrm{L}$ \\
\hline \multicolumn{5}{|c|}{$\star \star \quad S D-100-10$} \\
\hline SD - $100-10$ & 12-May - 1987 & BENZENE & $<5$ & $\mathrm{ug} / \mathrm{L}$ \\
\hline$S D-100-10$ & 19-May - 1987 & BENZRNE & $<5$ & $\mathrm{ug} / \mathrm{L}$ \\
\hline SD - $100-10$ & 26 - May - 1987 & BENZZONE & $<5$ & $\mathrm{ug} / \mathrm{L}$ \\
\hline SD- $100-10$ & $3-J u n-1987$ & BENZFNE & $<5$ & $\mathrm{ug} / \mathrm{L}$ \\
\hline SD - $100-10$ & $11-J u n-1987$ & BENZENE & $<5$ & $\mathrm{ug} / \mathrm{L}$ \\
\hline SD - $100-10$ & $18-J u n-1987$ & BENZENE & $<5$ & $\mathrm{ug} / \mathrm{L}$ \\
\hline
\end{tabular}


Location Date Test Compound Results Units

* SD-100-11

SD-100-11 12-May-1987 BENZENE

SD-100-I1 19-May-1987 BENZENE

SD-100-11 26-May-1987 BENZBNE

SD-100-11 3-Jun-1987 BENZENE

SD-100-11 11-Jun-1987 BENZENE

SD-100-11 18-Jun-1987 BENZEANE

** SD $-100-12$

SD-100-12 12-Kay-1987 BENZZRTE

SD-100-12 19-May-1987 BENZENE

SD-100-12. 26-May-1987 BENZENE

SD-100-12 3-Jun-1987 BENZENE

SD-100-12 11-JUn-1987 BENZENE

SD-100-12 18-JUn-1987 BENZENE

$\star \star \quad S D-100-13$

SD-100-13 12-May-1987 BENZENE

SD-100-13 19-May-1987 BENZENE

SD-100-13 26-May-1987 BENZENE

SD-100-13 3-Jun-1987 BENZENE

SD-100-13 11-Jun-1987 BENZENE

SD-100-13 18-Jun-1987 BENZENE

$\begin{array}{ll}<5 & u g / L \\ <5 & u g / L \\ <5 & u g / L \\ <5 & u g / L \\ <5 & u g / L \\ <5 & u g / L\end{array}$

$<$

$<5$

$<5$

$<5$

$<5$

$<5$

ug/L

$\mathrm{ug} / \mathrm{L}$

$\mathrm{ug} / \mathrm{L}$

Ug/L

$\mathrm{ug} / \mathrm{L}$

$u g / L$

** SD-100-14

SD-100-14 12-May-1987 BENZENE

SD-100-14 19-May-1987 BERZENE

SD-100-14 26-May-1987 BENZENE

SD-100-14 3-Jun-1987 BENZENE

SD-100-14 11-Jun-1987 BENZENE

SD-100-14 18-Jun-1987 BERZENE

$<5 \quad$ ug/L

$<5 \quad$ ug/L

$<5 \quad u g / t$

$<$ ug/I

$<5 \quad$ ug/L

$<5 \quad u g / L$

* SD-100-C

SD-100-C 5-Mar-1987 BEMZENE

SD-100-C 9-Mar-1987 BENZENE

SD-100-C 17-Mar-1987 BENZENE

SD-100-C 24-Mar-1987 BENZENE

SD-100-C 8-ApI-1987 BENZENE

SD-100-C 13-May-1987 BENZENE

SD-100-C 20-May-1987 BENZENE

SD-100-C 27-May-1987 BENZENE

SD-100-C 12-Jun-1987 BENZERVE

SD-100-C 19-Jun-1987 BEArzEarE

$<5 \quad$ ug/L

$<5 \quad$ ug/L

$<5 \quad$ ug/L

$<5 \quad u g / L$

$<5 \quad \mathrm{ug} / \mathrm{L}$

$<5 \quad u g / L$

* SD-100-D

SD-100-D 6-API-1987 BENZENE

SD-100-D 11-Jun-1987 BENZENE

$\begin{array}{ll}<5 & u g / L \\ <5 & u g / L \\ <5 & u g / L \\ <5 & u g / L \\ <5 & u g / L \\ <5 & u g / L \\ <5 & u g / L \\ <5 & u g / L \\ <5 & u g / L \\ <5 & u g / L \\ & \\ & \\ <5 & u g / L \\ <5 & u g / L\end{array}$


Storm Drain Data for SD-100

\begin{tabular}{|c|c|c|c|c|}
\hline Location & Date & Test Compound & Results & Onfts \\
\hline \multicolumn{5}{|l|}{ ** SD -100} \\
\hline$S D-100$ & $5-\operatorname{Mar}-1987$ & BENZIDINE & $<10$ & $4 \mathrm{~g} / \mathrm{L}$ \\
\hline SD -100 & 11 -Mar-1987 & BENZIDINE & $<10$ & $\mathrm{ug} / \mathrm{L}$ \\
\hline SD -100 & 12-May-1987 & BENZIDINE & $<10$ & $\mathrm{ug} / \mathrm{L}$ \\
\hline$S D-100$ & $26-$ May -1987 & BENZIDINE & $<5.0$ & $\mathrm{ug} / \mathrm{L}$ \\
\hline \multicolumn{5}{|c|}{$\star \star \quad S D-100-01$} \\
\hline SD-100-01 & $12-\mathrm{May}-1987$ & BENZIDINE & $<10$ & $u g / L$ \\
\hline SD-100-01 & 26-May - 1987 & BENZIDINE & $<5.0$ & $\mathrm{ug} / \mathrm{L}$ \\
\hline \multicolumn{5}{|c|}{$\star \star S D-100-02$} \\
\hline SD $-100-02$ & $12-$ May- 1987 & BENZIDINE & $<10$ & $\mathrm{ug} / \mathrm{L}$ \\
\hline SD- $100-02$ & 26-May- 1987 & BENZIDINE & $<5.0$ & $48 / \mathrm{L}$ \\
\hline \multicolumn{5}{|c|}{$\star \star \quad S D-100-03$} \\
\hline$S D-100-03$ & $12-M a y-1987$ & BENZIDINE & $<10$ & $\mathrm{ug} / \mathrm{L}$ \\
\hline$S D-100-03$ & $26-$ May -1987 & BENZIDINE & $<5.0$ & $\mathrm{ug} / \mathrm{L}$ \\
\hline \multicolumn{5}{|c|}{$\star * \quad S D-100-03 D$} \\
\hline$S D-100-03 D$ & 12-May - 1987 & BENZIDINE & $<10$ & $\mathbf{u g} / \mathrm{L}$ \\
\hline SD-100-03D & 26 -May -1987 & BENZIDINE & $<5.0$ & $u g / L$ \\
\hline \multicolumn{5}{|c|}{$\star \star \quad S D-100-04$} \\
\hline SD - $100-04$ & 12 - May -1987 & BENZIDINE & $<10$ & $\mathrm{ug} / \mathrm{L}$ \\
\hline$S D \cdot 100-04$ & 26-May-1987 & BENZIDINE & $<5.0$ & $\mathrm{ug} / \mathrm{L}$ \\
\hline \multicolumn{5}{|c|}{$\star \star \quad S D-100-06$} \\
\hline$S D-100-06$ & 12-May-1987 & BENZIDINE & $<10$ & $u g / L$ \\
\hline SD - 100.06 & 26-May-1987 & BENZIDINE & $<5.0$ & $\mathrm{ug} / \mathrm{L}$ \\
\hline \multicolumn{5}{|c|}{ ** SD-100-07 } \\
\hline SD-100-07 & $12 \cdot$ May -1987 & BENZIDINE & $<10$ & $\mathrm{ug} / \mathrm{L}$ \\
\hline SD $-100-07$ & $26-$ May -1987 & BENZIDINE & $<5.0$ & $\mathrm{ug} / \mathrm{L}$ \\
\hline \multicolumn{5}{|c|}{$\star \star \quad S D-100-09$} \\
\hline$S D-100-09$ & $12-\mathrm{May} \cdot 1987$ & BENZIDINE & $<10$ & $\mathrm{ug} / \mathrm{L}$ \\
\hline SD-100-09 & $26-$ May -1987 & BENZIDINE & $<5.0$ & $\mathrm{ug} / \mathrm{L}$ \\
\hline \multicolumn{5}{|c|}{$\star \quad$ SD $-100-10$} \\
\hline SD $-100-10$ & $12-$ May - 1987 & BENZIDLNE & $<10$ & $\mathrm{ug} / \mathrm{L}$ \\
\hline$S D-100-10$ & $26-$ Мay -1987 & BENZIDINE & $<5.0$ & $\mathrm{ug} / \mathrm{L}$ \\
\hline \multicolumn{5}{|c|}{$\star \star \quad S D-100-11$} \\
\hline SD-100-11 & $12-$ May -1987 & BENZIDINE & $<10$ & $\mathrm{ug} / \mathrm{L}$ \\
\hline SD-100-11 & 26-May-1987 & BENZIDINE & $<5.0$ & $\mathrm{ug} / \mathrm{L}$ \\
\hline \multicolumn{5}{|c|}{$* * \quad S D-100-12$} \\
\hline$S D-100-12$ & $12-$ May $\cdot 1987$ & BENZIDINE & $<10$ & $\mathrm{ug} / \mathrm{L}$ \\
\hline SD $-100-12$ & $26-$ May -1987 & BENZIDINE & $<5.0$ & $\mathrm{ug} / \mathrm{L}$ \\
\hline
\end{tabular}


Storm Drain Data for SD-100

\begin{tabular}{|c|c|c|c|c|}
\hline Location & Date & Test Compound & Results & Units \\
\hline \multicolumn{5}{|c|}{$\star \star \quad S D-100-13$} \\
\hline SD-100-13 & $12-$ May -1987 & BENZIDINE & $<10$ & $u g / L$ \\
\hline SD $-100-13$ & $26-$ May- 1987 & BENZIDINE & $<.0$ & $\mathrm{ug} / \mathrm{L}$ \\
\hline \multicolumn{5}{|c|}{$\star \quad$ SD-100-14 } \\
\hline SD $-100-14$ & $12-$ May- 1987 & BENZIDINE & $<10$ & ug $/ \mathrm{L}$ \\
\hline SD-100-14 & $26-$ May -1987 & BENZIDINE & $<5.0$ & ug $/ \mathrm{L}$ \\
\hline \multicolumn{5}{|c|}{$\star \quad S D-100-C$} \\
\hline$S D-100 \cdot C$ & 5-Mar-1987 & BENZIDINE & $<10$ & $4 g /$ \\
\hline$S D-100-C$ & 9-MaI - 1987 & BENZIDINE & $<10$ & $\mathrm{ug} / \mathrm{L}$ \\
\hline SD-100-C & $17-\operatorname{Mar}-1987$ & BENZIDINE & $<10$ & $4 g / \mathrm{L}$ \\
\hline SD-100-C & $24-\operatorname{Max}-1987$ & BENZIDINE & $<10$ & $u g / L$ \\
\hline SD $-100 \cdot C$ & 8-Apr-1987 & BENZIDINE & $<10$ & $\mathrm{ug} / \mathrm{L}$ \\
\hline$S D-100 \cdot C$ & 13-May-1987 & BENZIDINE & $<5.0$ & $\mathrm{ug} / \mathrm{L}$ \\
\hline$S D-100-C$ & 20-May - 1987 & BENZIDINE & $<5.0$ & $\mathrm{ug} / \mathrm{L}$ \\
\hline SD-100-C & 27-May-1987 & BENZIDINE & $<5.0$ & $u g / L$ \\
\hline$S D-100 \cdot C$ & 12-Jun-1987 & BENZIDINE & $<5.0$ & ug/L \\
\hline SD-100-C & $19 \cdot \mathrm{Jun}-1987$ & BENZIDINE & $<10$ & $\mathrm{ug} / \mathrm{L}$ \\
\hline \multicolumn{5}{|l|}{ ** SD-100 } \\
\hline SD -100 & $5-\operatorname{Mar}-1987$ & BENZO (A) ANTHRACENE & $<10$ & ug/L \\
\hline$S D-100$ & 11-Mar- 1987 & BENZO (A) ANTHRACENE & $<10$ & $\mathrm{ug} / \mathrm{L}$ \\
\hline SD - 100 & 12-May-1987 & BENZO (A) ANTHRACENE & $<10$ & $u g / L$ \\
\hline SD -100 & $26-$ May -1987 & BENZO (A) ANTHRACENE & $<5.0$ & $48 / 2$ \\
\hline \multicolumn{5}{|c|}{$\star \star \quad$ SD $-100-01$} \\
\hline SD $-100-01$ & 12-Kay-1987 & BENZO (A) ANTHRAGENE & $<10$ & ug/L \\
\hline SD-100-01 & $26-$ Kay -1987 & BENZO (A) ANTHRACENE & $<5.0$ & $4 g / \mathrm{L}$ \\
\hline \multicolumn{5}{|c|}{ * SD - 100-02 } \\
\hline SD $-100-02$ & $12-$ Kay - 1987 & BENZO (A) ANTHRACENE & $<10$ & ug/L \\
\hline SD $-100-02$ & $26-$ May -1987 & BENZO (A) ANTHRACENE & $<5.0$ & $\mathrm{ug} / \mathrm{L}$ \\
\hline \multicolumn{5}{|c|}{$\star * \quad S D-100.03$} \\
\hline SD $-100-03$ & 12-Kay-1987 & BENZO (A) ANTHRACENE & $<10$ & $\mathrm{ug} / \mathrm{L}$ \\
\hline SD $-100-03$ & 26-May- 1987 & BENZO (A) ANTHRACENE & $<5.0$ & $\mathrm{ug} / \mathrm{L}$ \\
\hline \multicolumn{5}{|c|}{ ** SD $-100-03 D$} \\
\hline SD $-100-03 D$ & 12-May - 1987 & BENZO (A) ANTHRACENE & $<10$ & $u g / L$ \\
\hline SD - $100-03 D$ & 26-May-1987 & BENZO (A) ANTYRACENE & $<5.0$ & $\mathrm{UE} / \mathrm{L}$ \\
\hline \multicolumn{5}{|c|}{$* * \quad S D-100-04$} \\
\hline SD-100-04 & $12-$ Kay -1987 & BENZO (A) ANTKRACENE & $<10$ & $\operatorname{ug} / \mathrm{L}$ \\
\hline SD- $100-04$ & $26-$ May -1987 & BENZO (A) ANTKRACENE & $<5.0$ & $\operatorname{ug} / \mathrm{L}$ \\
\hline \multicolumn{5}{|c|}{$\star * \quad S D-100-06$} \\
\hline SD - $100-06$ & 12-May-1987 & BENZO(A) ANTHRACENE & $<10$ & ug/L \\
\hline SD -100.06 & $26-$ May-1987 & BENZD (A) ANTHRACENE & $<5,0$ & ug/L \\
\hline
\end{tabular}


Storm Draln Data for SD-100

Location Date Test Compound

* SD-100-07

SD-100-07 12-May-1987 BENZO(A) ANTKRACENE

SD-100-07 26-May-1987 BENZO(A) ANTHRACENE

** SD $-100-09$

SD-100-09 12-May-1987 BENZO(A)ANTHRACENE

SD-100-09 26-May-1987 BENZO(A) ANTHRACENE

* $S D-100-10$

SD-100-10 12-May-1987 BEXZO(A) ANTHRACENE

SD-100-10 26-May-1987 BENZO(A) ANTHRACENE

$\star \star \quad$ SD $-100-11$

SD-100-11 12-May-1987 BENZO(A)ANTHRACENE

SD-100-11 26-May-1987 BENZO(A)ANTHRACENE

** SD-100-12

SD-100-12 12-May-1987 BENZO(A) ANTHRACENE

SD-100-12 26-May-1987 BENZO(A)ANTHRACENE

* SD - 100-13

SD-100-13 12-May-1987 BENZO(A)ANTHRACENE

SD-100-13 26-May-1987 BENZO(A)ANTHRACENE

** SD-100-14

SD-100-14 12-May-1987 BENZO(A)ANTHRACENE

SD-100-14 26-May-1987 BENZO(A)ANTHRACENE

** SD-100-C

SD-100-C 5-Mar-1987 BENZO(A)ANTHRACENE

SD-100-C 9-Mar-1987 BENZO(A)ANTHRACENE

SD-100-C 17-MaI-1987 BENZO(A)ANTHRACENE

SD-100-C 24-MaI-1987 BENZO(A)ANTHRACENE

SD-100-C 8-Apr-1987 BENZO(A)ANTHRACENE

SD-100-C 13-May-1987 BENZO(A) ANTHRACENE

SD-100-C 20-May-1987 BENZO(A) ANTHRACENE

SD-100-C 27-May-1987 BENZO(A) ANTHRACENE

SD-100-C 12-Jun-1987 BENZO(A) ANTHRACENE

SD-100-C 19-Jun-1987 BENZO (A) ANTHRACENE

** SD -100

SD-100 5-Mar-1987 BENZO(A) PYRENE

SD-100 11-Mar-1987 BEN20(A) PYRENE

SD-100 12-May-1987 BENZ0 (A) PYRENE

SD-100 26-May-1987 BENZO (A) PYRENE

** SD-100-01

SD-100-01 12-May-1987 BENZO(A) PYRENE

SD-100-01 26-May-1987 BENZO(A) PYRENE
Results Untes

$<10$

$<5.0$

$\mathrm{ug} / \mathrm{L}$

$\operatorname{ug} / 2$

$<10$

$<5.0$

ug $/ \mathrm{L}$

$4 \mathrm{~g} / \mathrm{L}$

$<10$

$<5.0$

ug/L

$\mathrm{ug} / \mathrm{L}$

$<10$

$<5.0$

ug/L

ug $/$ L

$<10$

$<5.0$

ug $/ \mathrm{L}$

ug/L

$<10$

$<0$

$\mathrm{ug} / \mathrm{I}$

$u g / L$

$<10$

$<5.0$

ug/L

ug/L

$<10$

$<10$

$<10$

$<10$

$<10$

$<5.0$

$<5.0$

$<.0$

$<5.0$

$<10$

$u g / L$

$\mathrm{ug} / \mathrm{L}$

ug/L

$u g / L$

$48 / L$

ug $/$.

$u g / L$

$u g / L$

$\mathrm{ug} / \mathrm{L}$

ug/L

410

ug/L

$<10$

ug/L

40

$\checkmark .0$

UE/L

ug/L

$<10$

$<5.0$

ug/L

ug $/$ 
Storm Drain Data for SD-100

\begin{tabular}{|c|c|c|c|c|}
\hline Location & Date & Test Compound & Results & Units \\
\hline \multicolumn{5}{|c|}{$\star \star \quad S D-100-02$} \\
\hline$S D-100-02$ & 12-May-1987 & BENZO (A) PYRENE & $<10$ & $\mathrm{ug} / \mathrm{L}$ \\
\hline SD-100-02 & $26-$ Kay -1987 & BENZO (A) PYRENE & $<5.0$ & $U g / L$ \\
\hline \multicolumn{5}{|c|}{$\star \star \quad S D-100-03$} \\
\hline$S D-100-03$ & $12-\mathrm{May}-1987$ & BENZO (A) PYRENE & $<10$ & ug $/ \mathrm{L}$ \\
\hline SD $-100-03$ & $26-\mathrm{May}-1987$ & BENZO (A) PYRENE & $<5.0$ & $\mathrm{ug} / \mathrm{L}$ \\
\hline \multicolumn{5}{|c|}{ ** SD-100-03D } \\
\hline SD-100-03D & 12-May-1987 & BENZO (A) PYRFNE & $<10$ & $\mathrm{ug} / \mathrm{L}$ \\
\hline$S D-100-03 D$ & $26-$ May- 1987 & BEN2O (A) PYRENE & $<5.0$ & $\mathrm{ug} / \mathrm{L}$ \\
\hline \multicolumn{5}{|c|}{$* \quad S D-100-04$} \\
\hline SD- $100-04$ & $12-$ May- 1987 & BENZO(A) PYRENE & $<10$ & $4 \mathrm{~g} / \mathrm{L}$ \\
\hline SD- $100-04$ & 26-May - 1987 & BENZO(A) PYRENE & $<5.0$ & $\mathrm{ug} / \mathrm{L}$ \\
\hline \multicolumn{5}{|c|}{$\star \quad$ SD $-100-06$} \\
\hline SD $-100-06$ & 12-May-1987 & BENZO(A) PYRENE & $<10$ & $\mathrm{ug} / \mathrm{L}$ \\
\hline SD- $100-06$ & $26-$ May -1987 & BENZO (A) PYRENE & $<5.0$ & $\mathrm{ug} / \mathrm{L}$ \\
\hline \multicolumn{5}{|c|}{$*$ SD-100-07 } \\
\hline$S D-100-07$ & 12-Kay-1987 & BENZO (A) FYRENE & $<10$ & $u g / L$ \\
\hline SD-100-07 & $26-$ May -1987 & BENZO (A) PYRENE & $<5.0$ & $\mathrm{ug} / \mathrm{I}$ \\
\hline \multicolumn{5}{|c|}{$\star \quad S D-100.09$} \\
\hline$S D-100-09$ & 12-May-1987 & BENZO (A) PYRENE & $<10$ & $u g / L$ \\
\hline SD-100-09 & $26-$ May -1987 & BENZO (A) PYRENE & $<5.0$ & $u g / \mathrm{L}$ \\
\hline \multicolumn{5}{|c|}{$\star \star \quad S D-100-10$} \\
\hline SD - $100-10$ & 12-May- 1987 & BENZO (A) PYRENE & $<10$ & $\operatorname{ug} / \mathrm{L}$ \\
\hline$S D-100-10$ & $26-$ May - 1987 & BENZO(A) PYRENE & $<5.0$ & ug $/ \mathrm{L}$ \\
\hline \multicolumn{5}{|c|}{ ** SD-100-11 } \\
\hline SD-100-11 & 12-May-1987 & BENZO (A) PYRENE & $<10$ & $u g / L$ \\
\hline SD-100- 11 & $26-$ May -1987 & BENZO(A) PYRENE & $<5.0$ & $u g / L$ \\
\hline \multicolumn{5}{|c|}{$\star \star \quad S D-100-12$} \\
\hline SD- $100-12$ & $12-$ May- 1987 & BENZO(A) PYRENE & $<10$ & $\mathrm{ug} / \mathrm{L}$ \\
\hline SD $-100-12$ & 26-May - 1987 & BENZO(A) PYRENE & $<5.0$ & $\mathrm{ug} / \mathrm{L}$ \\
\hline \multicolumn{5}{|c|}{$\star * \quad S D-100-13$} \\
\hline$S D-100-13$ & 12-May-1987 & BENZO(A) PYRENE & $<10$ & $\mathrm{ug} / \mathrm{L}$ \\
\hline$S D-100-13$ & $26-$ May -1987 & BENZO (A) PYRENE & $<5.0$ & $\mathrm{ug} / \mathrm{L}$ \\
\hline \multicolumn{5}{|c|}{$\star$ SD-100-14 } \\
\hline$S D-100-14$ & 12-May-1987 & BENZO (A) PYRENE & $<10$ & $4 g / L$ \\
\hline$S D-100-14$ & $26-$ May $\cdot 1987$ & BENZO (A) PYRENE & $<5.0$ & $u g / L$ \\
\hline \multicolumn{5}{|c|}{$\star \star \quad S D-100-C$} \\
\hline$S D-100-C$ & $5-\operatorname{Max}-1987$ & BENZO(A) PYRENE & $<10$ & $\mathrm{ug} / \mathrm{L}$ \\
\hline$S D-100-C$ & 9. Mar- 1987 & BENZO (A) PYRENE & $<10$ & $\mathrm{ug} / \mathrm{L}$ \\
\hline
\end{tabular}


Storm Drain Data for SD-100

Location

$$
\text { Date }
$$

Test Compound

$S D-100-C$

$S D-100-C$

$S D-100-C$

SD-100-C

$S D \cdot 100-C$

SD $-100-C$

SD $-100-C$

$S D-100-C$

17-Mar-1987 BENZO(A) PYRENE

24-Mar-1987 BENZO(A) PYREIVE

8-APr-1987 BENZO (A) PYRENE

13-MRy-1987 BENZO(A) PYRENE

20-May-1987 BENZO(A) PYRENE

27-Yay-1987 BENZO(A) PYRENE

12-Jun-1987 BENZO(A) PYRENE

19-JUR-1987 BENZO(A) PYRENE t* SD 100

SD -100

SD -100

SD -100

SD -100
5-Mar-1987 BENZO(B) FLUORANTKENE

11 -Mar-1987 BENZO(B) FLUORANTHENE 12-May-1987 BENZO (B) FLUORANTHENE 26-May-1987 BEN2D (B) FLUORANTHENE

** SD $-100-02$

SD-100-01 12-May-1987 BENZO(B) FLUORANTHENE SD-100-01 26-May-1987 BENZO(B) FLUORANTHENE

* SD-100-02

SD-100-02 12-May-1987 BENZO(B) FLUORANTHENE

SD-100-02 26-May-1987 BENZO(B) FUUORANTHENE

** SD $100-03$

SD-100-03 12-May-1987 BENZO(B) FLUORANTHENE SD-100-03 26-May-1987 BERZO(B) FLUORANTHENE

* SD-100-03D

SD-100.03D 12-May-1987 BENZO(B) FLUORANTHENE SD-100-03D 26-May-1987 BENZO(B) FLUORANTHENE * SD-100-04

SD-100-04 12-May-1987 BEN20(B) FLUORANTHENE SD-100.04 26-May-1987 BENZO(B) FLUORANTHENE

* SD-100-06

SD-100-06 12-May-1987 BENZO(B) FLUORANTHERE SD-100-06 26-May-1987 BEN20(8) FLUORANTHENE

** SD-100-07

SD-100-07 12-May-1987 BENZO (B) FLUORANTHENE

SD-100-07 26-May-1987 BENZO (B) FLUORANTHENE

* SD-100-09

SD-100-09 12-May-1987 BENZO(B) FLUORANTHENE SD-100-09 26-May-1987 BENZO (B) FUUORANTHENE

** SD-100-10

SD-100-10 12-May- 1987 BENZO (B) FLUORANTHENE

SD-100-10 26-May-1987 BENZO(B) FLUORANTHENE
Results Units

$\begin{array}{ll}<10 & \mathrm{ug} / \mathrm{L} \\ <10 & \mathrm{ug} / \mathrm{L} \\ <10 & \mathrm{ug} / \mathrm{L} \\ <5.0 & \mathrm{ug} / \mathrm{L} \\ <5.0 & \mathrm{ug} / \mathrm{L} \\ <5.0 & \mathrm{ug} / \mathrm{L} \\ <5.0 & \mathrm{ug} / \mathrm{L} \\ <10 & \mathrm{ug} / \mathrm{L}\end{array}$

$<10$

$<10$

$<10$

$<5.0$

$\mathrm{ug} / \mathrm{L}$

$\mathrm{ug} / \mathrm{L}$

ug $/ \mathrm{L}$

$\mathrm{ug} / \mathrm{L}$

$<10$

$<5.0$

$u g / L$

$\mathrm{ug} / \mathrm{L}$

$<10$

$<5.0$

ug/L

$u g / L$

$<10$

$<5.0$

$\mathrm{ug} / \mathrm{L}$

$\operatorname{ug} / \mathrm{L}$

$<10$

$<5.0$

$u g / L$

$4 \mathrm{~g} / \mathrm{L}$

$<10$

$<5.0$

$u g / L$

$u g / L$

$<10$

$<5.0$

ug/L

$\mathrm{ug} / \mathrm{L}$

$<10 \quad$ ug/L

$<5.0 \quad \mathrm{ug} / \mathrm{L}$

$<10 \quad$ ug/L

$<5.0 \quad$ ug $/ \mathrm{L}$

$<10$

$<5.0$

$\mathrm{ug} / \mathrm{L}$

$4 g / L$ 
Storm Drain Data for SD-100

Location Date Test Compound

Results Units

** SD-100-11

SD-100-11 12-May-1987 BENZO(B) FLUORANTHENE

SD-100-11 26-May-1987 BENZO(B) FLUORANTHENE

* SD $-100-12$

SD-100-12 12-May-1987 BENZO(B)FLUORANTHENE

SD-100-12 26-May-1987 BENZO(B) FLOORAFTHENE

** SD $-100-13$

SD-100-13 12-May-1987 BENZO(B) FLUORANTHENE

SD-100-13 26-May-1987 BENZO(B) FLUORANTHENE

* SD-100-14

SD-100-14 12-May-1987 BENZO(B) FLUORANTHENE

SD-100-14 26-May-1987 BEN2O(B) FLUORANTHENE

** SD- $100-C$

SD-100-C 5-Mar-1987 BENZO(B) FLUORANTHENE

SD-100-C 9-Mar-1987 BENZO(B) FLUORANTHENE

SD-100-C 17-Mar-1987 BEN20(B) FLUORANTHENE

SD-100-C 24-Mar-1987 BENZO (B) ELDORANTHENE

SD-100-C 8-Apr-1987 BENZO(B)FLUORANTHENE

SD-100-C 13-May-1987 BEN20(B)FLUORANTHENE

SD-100-C 20-May-1987 BENZO(B)FLUORANTHENE

SD-100-C 27-May-1987 BENZO(B) FLDORANTHENE

SD-100-C 12-Jun-1987 BENZO(B) FLUORANTHENE

SD-100-C 19-Jun-1987 BENZO (B) FLDORANTHENE

* SD -100

SD-100 5-Mar-1987 BENZO(G H I) PERYLENE

SD-100 11-Mar-1987 BEN20(G H I) PERYLENE

SD-100 12-May-1987 BENZO(G H I) PERYLENE

SD-100 26-May-1987 BENZO(G H I) PERYLENE

* SD $-100-01$

SD-100-01 12-May-1987 BENZO(G H I) PERYLENE

SD-100-01 26-May-1987 BENZO(G H I) PERYLENE

* SD $-100-02$

SD-100-02 12-May-1987 BENZO(G H I) PERYLENE

SD-100-02 26-May-1987 BEN20(G H I) PERYLENE

** SD-100-03

SD-100-03 12-May-1987 BENZO(G H I) PERYLENE

SD-100-03 26-May-1987 BENZO(G H I) PERYLENE

** SD - 100-03D

SD-100-03D 12-May-1987 BENZO(G H I)PERYLENE

SD-100-03D 26-May-1987 BENZO(G H I)PERYLENE

\begin{tabular}{|c|c|}
\hline $\begin{array}{l}<10 \\
<5.0\end{array}$ & $\begin{array}{l}u g / L \\
u g / L\end{array}$ \\
\hline$<10$ & $\mathrm{ug} / \mathrm{L}$ \\
\hline$<5.0$ & $u g / L$ \\
\hline$<10$ & $\mathrm{ug} / \mathrm{L}$ \\
\hline & \\
\hline$<10$ & $\mathrm{ug} / \mathrm{L}$ \\
\hline$<5.0$ & $u g / L$ \\
\hline$<10$ & ug/L \\
\hline$<10$ & $\mathrm{ug} / \mathrm{I}$ \\
\hline$<10$ & ug $/ 1$ \\
\hline$<10$ & $\mathrm{ug} / \mathrm{L}$ \\
\hline$<10$ & ug/L \\
\hline$<5.0$ & $\mathrm{ug} / \mathrm{L}$ \\
\hline$<5.0$ & $4 \mathrm{~g} / \mathrm{L}$ \\
\hline$<5.0$ & $\mathrm{ug} / \mathrm{L}$ \\
\hline$<5.0$ & $\mathrm{ug} / \mathrm{L}$ \\
\hline$<10$ & $u g / L$ \\
\hline$<10$ & $\mathrm{ug} / \mathrm{L}$ \\
\hline$<10$ & $u g / L$ \\
\hline$<10$ & ug $/ \mathrm{L}$ \\
\hline$<5.0$ & ug/L \\
\hline$<10$ & $\mathrm{ug} / \mathrm{L}$ \\
\hline$<5.0$ & $\mathrm{ug} / \mathrm{L}$ \\
\hline$<10$ & $\mathrm{ug} / \mathrm{L}$ \\
\hline$<5.0$ & $\mathrm{ug} / \mathrm{L}$ \\
\hline$<10$ & $\mathrm{ug} / \mathrm{L}$ \\
\hline$<5.0$ & $\mathrm{ug} / \mathrm{L}$ \\
\hline$<10$ & $\mathrm{ug} / \mathrm{L}$ \\
\hline$<5.0$ & $\mathrm{ug} / \mathrm{L}$ \\
\hline
\end{tabular}


Storm Drain Data for SD-100

\begin{tabular}{|c|c|c|c|c|c|}
\hline Location & Date & Test Compound & & Results & Units \\
\hline \multicolumn{6}{|c|}{ * SD-100-04 } \\
\hline$S D-100-04$ & 12-May-1987 & BENZO (G H I)PERYLENE & & $<10$ & $4 \mathrm{~g} / \mathrm{L}$ \\
\hline SD-100.04 & 26-May - 1987 & BENZO(G H I) PERYLENE & & $<5.0$ & $\mathrm{ug} / \mathrm{L}$ \\
\hline \multicolumn{6}{|c|}{$\star$ SD $-100-06$} \\
\hline SD - $100-06$ & 12-May - 1987 & BENZO(G H I) PERYLENE & & $<10$ & $u g / L$ \\
\hline SD-100-06 & $26-$ May -1987 & BENZO (G H I) PERYLENE & & $<5.0$ & $\mathrm{ug} / \mathrm{L}$ \\
\hline \multicolumn{6}{|c|}{$\star$ * SD $-100-07$} \\
\hline SD-100-07 & 12-May-1987 & BENZO (G H I) PERYLENE & $\cdot$ & $<10$ & $\mathrm{ug} / \mathrm{l}$ \\
\hline SD $-100-07$ & $26-$ May - 1987 & BENZO(G H I)PERYLENE & & $<5.0$ & $\mathrm{ug} / \mathrm{L}$ \\
\hline \multicolumn{6}{|c|}{$\star \star \quad S D-100-09$} \\
\hline SD -100.09 & 12-May-1987 & BENZO (G H I)PERYLENE & & $<10$ & ug/L \\
\hline$S D-100-09$ & 26-May - 1987 & BENZO (G H I) PERYLENE & & $<5.0$ & $u g / L$ \\
\hline \multicolumn{6}{|c|}{$* \quad$ SD-100-10 } \\
\hline$S D-100-10$ & 12-May - 1987 & BENZO(G H I) PERYLENE & & $<10$ & $\mathrm{ug} / \mathrm{L}$ \\
\hline$S D-100-10$ & $26-\mathrm{Mzy}-1987$ & BENZO(G H I) PERYLENE & & $<5.0$ & $\mathrm{ug} / \mathrm{L}$ \\
\hline \multicolumn{6}{|c|}{$\star \star \quad S D-100-1 I$} \\
\hline SD - $100-11$ & $12-$ May -1987 & BENZO(G H I) PERYLENE & & $<10$ & $\mathrm{ug} / \mathrm{L}$ \\
\hline SD-100-11 & $26-\mathrm{May}-1987$ & BENZO(G H I) PERYLENE & & $<5.0$ & $u g / L$ \\
\hline \multicolumn{6}{|c|}{$\star \star \quad S D-100-12$} \\
\hline SD-100- 12 & $12-$ May -1987 & BENZO(G H I)PERYLENE & & $<10$ & ug/L \\
\hline$S D-100-12$ & $26-$ May -1987 & BENZO(G H I) PERYLENE & & $<5.0$ & $\mathbf{u g} / \mathrm{L}$ \\
\hline \multicolumn{6}{|c|}{ ** SD-100-13 } \\
\hline$S D-100-13$ & $12-$ May -1987 & BENZO (G H I) RERYLENE & & $<10$ & $\mathrm{ug} / \mathrm{L}$ \\
\hline$S D-100-13$ & $26-$ Kay -1987 & BENZO(G H I) PERYLENE & & $<5.0$ & $\mathrm{ug} / \mathrm{L}$ \\
\hline \multicolumn{6}{|c|}{ * SD $-100-14$} \\
\hline SD-100-14 & $12-$ May - 1987 & BENZO(G H I) PERYLFNE & & $<10$ & $\mathrm{ug} / \mathrm{L}$ \\
\hline SD-100- 14 & 26-May - 1987 & BENZO(G H I) PERYLENE & & $<5.0$ & $\mathrm{ug} / \mathrm{L}$ \\
\hline \multicolumn{6}{|c|}{$\star \star \quad S D-100-C$} \\
\hline$S D-100-C$ & $5-$ Mar-1987 & BENZO (G H I) PERYLENE & & $<10$ & $\mathrm{ug} / \mathrm{L}$ \\
\hline$S D-100-C$ & $9-$ Mar-1987 & BENZO(G H I) PERYLENE & & $<10$ & ug/L \\
\hline$S D-100-C$ & $17-$ Mer -1987 & BENZO (G H I) PERYLENE & & $<10$ & $u g / L$ \\
\hline$S D-100-C$ & $24-$ Mar- 1987 & BENZO(G H I)PERYLENE & & $<10$ & $\mathbf{u g} / \mathrm{L}$ \\
\hline SD-100-C & 8-ApI-1987 & BENZO(G H I) PERYLENE & & $<10$ & $\mathbf{u g} / \mathrm{L}$ \\
\hline$S D \cdot 100 \cdot C$ & $13-$ May - 1987 & BENZO(G H I) PERYLENE & & $<5.0$ & $\mathbf{u g} / \mathrm{L}$ \\
\hline$S D-100-C$ & $20-$ May -1987 & BENZO(G H I) PERYLENE & & $<5.0$ & $\mathrm{Ug} / \mathrm{L}$ \\
\hline$S D-100-C$ & 27-May - 1987 & BENZO (G H I) PERYLENE & & $<5.0$ & $\mathrm{ug} / \mathrm{L}$ \\
\hline SD - $100-C$ & $12 \cdot J u n \cdot 1987$ & BENZO(G H I) PERYLENE & & $<5.0$ & $\mathrm{ug} / \mathrm{L}$ \\
\hline$S D-100-C$ & $19-J u n-1987$ & BENZO (G H I) PERYLENE & & $<10$ & $\mathrm{ug} / \mathrm{L}$ \\
\hline
\end{tabular}


Storm Drain Deta for SD-100

Location
* SD- 100

SD-100 5-Mat-1987 BENZO(K) FLDORANTTENE

SD-100 11-Mar-1987 BENZO (R) FLDORANTHENE

SD-100 12-May-1987 BENZO(K) FLJUORANTHENE

SD-100 26-May-1987 BENZO(K) FLUORANTHEME

* SD-100-01

SD-100-01 12-May-1987 BENZO(K) FLUORANTHENE

SD-100-01 26-May-1987 BENZO(K) FLDORANTHENE

** SD - 100-02

SD-100-02 12-May-1987 BENZO(K) FLUORANTHENE

SD-100-02 26-May-1987 BENZO(K) FLUORANTHENE

** SD $100-03$

SD-100-03 12-May-1987 BENZO(K) FLUORANTHENE

SD-100-03 26-May-1987 BEN20(K) FLUORANTHENE

* SD-100-03D

SD-100-03D 12-May-1987 BENZO(K) FLUORANTHENE

SD-100-03D 26-May-1987 BENZO(K) FLUORANTHENE

** SD-100-04

SD-100-04 12-May-1987 BENZO(K) ELUURANTHENE

SD-100-04 26-May-1987 BENZO(K) FLUORANTHENE

** SD-100-06

SD-100-06 12-May-1987 BENZO(K) FLUUORANTHENE

SD-100-06 26-May-1987 BENZO(K) FLUORANTHENE

* SD - 100-07

SD-100-07 12-May-1987 BENZO(K)FLUORANTHENE SD-100-07 26-May-1987 BENZO(K)FLUORANTHENE ** SD-100-09

SD-100-09 12-May-1987 BENZO(K) FLUORANTHENNE

SD-100-09 26-May-1987 BENZO(K) FLUORANTHENT

** SD-100-10

SD-100-10 12-May-1987 BENZO(K) FLUORANTHENE

SD-100-10 26-May-1987 BENZO(K) FLUORANTHENE

** SD-100-11

SD-100-11 12-May-1987 BENZO(K)FLUORAMTHENE

SD-100-11 26-May-1987 BENZO(K) FLUORANTHENE

* SD-100-12

SD-100-12 12-May-1987 BEN2O(K) FLUORANTHENE

SD-100-12 26-May-1987 BENZO(K) FLUORANTHENE

Results Unfts

$<10$

$<10$

$<10$

$<5.0$

$u g / L$

ug/L

Ug $/ 2$

UE/ 1

$<10$

$<5.0$

ug/L

$\mathrm{ug} / \mathrm{L}$

$<10 \quad \mathrm{ug} / \mathrm{L}$

$<5.0 \quad u g / L$

$<10 \quad \quad \mathrm{ug} / \mathrm{L}$

$<5.0 \quad \mathrm{ug} / \mathrm{L}$

$<10 \quad u g / L$

$<5.0 \quad$ ug/L

$<10 \quad \mathrm{ug} / \mathrm{L}$

$<5.0 \quad \mathrm{ug} / \mathrm{L}$

$<10 \quad \mathrm{ug} / \mathrm{L}$

$<5.0$ ug/L

$<10 \quad \mathrm{ug} / \mathrm{L}$

$<5.0 \quad \mathrm{ug} / \mathrm{L}$

$<10 \quad$ ug/L

$<5.0 \quad \mathrm{ug} \Omega$

$<10 \quad$ ug/L

$<5.0 \quad \mathrm{ug} / \mathrm{L}$

$<10 \quad$ ug/L

$<5.0 \quad \mathrm{ug} / \mathrm{L}$

$<10 \quad \mathrm{ug} / \mathrm{L}$

$<5.0 \quad \mathrm{ug} / \mathrm{L}$ 
Storm Drain Data for SD -100

Location Date Test Compound

Results Units

** SD $-100-13$

SD-100-13 12-May-1987 BENZO(K) FLUORANTHENE

SD-100-13 26-May-1987 BENZO(K) FLUORANTHENE

** SD-100-14

SD-100-14 12-May-1987 BENZO(K) FLUORANTHENE

SD-100-14 26-May-1987 BEN20(K) FLUORANTHENE

* $\quad$ SD $-100-C$

$\begin{array}{lrrl}\text { SD-100-C } & 5 \text {-Mar-1987 } & \text { BEN20(K) FLUORANTHENE } \\ \text { SD-100-C } & 9 \text {-Mar-1987 } & \text { BENZO(K) FUUORANTHENE } \\ \text { SD-100-C } & 17 \text {-Mar-1987 } & \text { BENZO(K) FUUORANTHENE } \\ \text { SD-100-C } & 24-\text { Mar-1987 } & \text { BENZO(K) FLUORANTHENE } \\ \text { SD-100-C } & 8 \text {-Apr-1987 } & \text { BENZO(K) FLUORANTHENE } \\ \text { SD-100-C } & 13 \text {-May-1987 } & \text { BENZO(K) FLUORANTHENE } \\ \text { SD-100-C } & 20 \text {-May-1987 } & \text { BENZO(K) FUUORANTHENE } \\ \text { SD-100-C } & 27 \text {-May-1987 } & \text { BENZO(K) FLUORANTHENE } \\ \text { SD-100-C } & 12-J U n-1987 & \text { BENZO(K) FLUORANTHENE } \\ \text { SD-100-C } & 19-J u n-1987 & \text { BENZO(K) FLUORANTHENE }\end{array}$

*t SD -100

SD-100 5-Mar-1987 BENZY ALCOHOL

SD-100 11-Mar-1987 BENZYL ALCOHOL

$<10 \quad$ ug/ $/ \mathrm{L}$

$<5.0 \quad \mathrm{ug} / \mathrm{L}$

$<10 \quad \mathrm{ug} / \mathrm{L}$

$<5.0 \quad \mathrm{ug} / \mathrm{L}$

$<10 \quad \mathrm{ug} / \mathrm{L}$

$<10 \quad 4 \mathrm{~g} / \mathrm{h}$

$<10 \quad u g / L$

$<10 \quad$ ug/L

$<10 \quad$ ug/L

$<5.0 \quad \mathrm{ug} / \mathrm{L}$

$<5.0 \quad u g / L$

$<5.0 \quad$ ug/L

$<5.0 \quad$ ug/L

$<10 \quad \mathrm{ug} / \mathrm{L}$

$\star$ SD $-100-C$

SD-100-C 5-Mar-1987 BENZYL ALCOHOL

SD-100-C 8-APr-1987 BENZYL ALCOHOL

IJ

$\operatorname{ug} \Omega$

$1 \quad u g / L$

$4 \quad$ ug $/ \mathcal{L}$

** SD -100

SD-100 5-Mar-1987 BERYLLIUM

SD-100 11-Mar-1987 BERYLIUM

SD-100 18-Mar-1987 BERYLIUM

SD-100 24-Mar-1987 BERYLLIUM

SD-100 3-APr-1987 BERYLLIUM

SD-100 6-APY-1987 BERYLLIUM

SD-100 12-May-1987 BERYLIIUM

SD-100 19-May-1987 BERYLIUM

SD-100 26-May-1987 BERYLLIUM

SD-100 3-Jun-1987 BERYLLIUM

SD-100 11-Jun-1987 BERYLLIUM

SD-100 18-Jun-1987 BERYLLIUM

* SD-100-01

SD-100-01 12-May-1987 BERYLIUM

SD-100-01 18-May-1987 BERYLLIUM

SD-100-01 3-Jun-1987 BERYLIUM

SD-100-01 11-JUn-1987 BERYLLIUM

SD-100-01 18-Jun-1987 BERYLIIUM

$<0.0010$

$<0.0010$

$<0.0010$

$<0.0010$

$<0.0010$

$<0.0010$

$<0.0010$

$<0.0010$

$<0.0003$

$<0.0010$

$<0.0010$

$<0.0010$

$<0.0010$

$<0.0010$

0.0010

$<0.0010$

$<0.0010$ $\mathrm{mg} / \mathrm{L}$

$\mathrm{mg} / \mathrm{L}$

ng/L

mg/L

ng/L

$\operatorname{mg} / \mathrm{L}$

$\mathrm{mg} / \mathrm{L}$

$\mathrm{mg} / \mathrm{L}$

ng/L

$\mathrm{ng} / \mathrm{L}$

ng/L

IIg/L

ng/L

mg/L

mg/L

mg/L

$\mathrm{mg} / \mathrm{L}$ 
Storm Draln Data for SD-100

Location Date Test Compound Results Units

* SD-100-02

SD-100-02 12-May-1987 BERYLLTUM

SD-100-02 18-May-1987 BERYLLTUM

SD-100-02 3-Jun-1987 BERYLLIUM

SD-100-02 11-Jun-1987 BERYLIIUM

SD-100-02 18-Jun-1987 BERYLLTU

$<0.0010$

$<0.0010$

$<0.0010$

$<0.0010$

$<0.0010$

$\mathrm{mg} / \mathrm{L}$

mg/

$\mathrm{mg} / \mathrm{L}$

$\mathrm{mg} / \mathrm{L}$

$\mathrm{mg} / \mathrm{L}$

* SD-100-03

SD-100-03 12-May-1987 BERYLLIUM

SD-100-03 18-May-1987 BERYLLIUM

SD-100-03 26-May-1987 BERYLLIUM

SD-100-03 3-Jun-1987 BERYLLIUM

SD-100-03 11-Jun-1987 BERYLIIUM

SD-100-03 18-JUn-1987 BERYLIUM

$<0.0010$

$<0.0010$

$<0.0010$

$<0.0010$

$<0.0010$

$<0.0010$

$\mathbf{m g} / \mathrm{L}$

$\mathrm{mg} / \mathrm{L}$

$\mathrm{mg} / \mathrm{L}$

$\mathrm{mg} / \mathrm{L}$

$\mathrm{mg} / \mathrm{L}$

$\mathrm{mg} / \mathrm{L}$

* SD $-100-03 D$

SD-100-03D 12-May-1987 BERYLITUM

SD-100-03D 26-May-1987 BERYLLIUM

$<0.0010 \mathrm{mg} / \mathrm{L}$

$<0.0010 \mathrm{mg} / \mathrm{L}$

** SD-100-04

SD-100-04 12-May-1987 BERYLIIUM

SD-100-04 18-May-1987 BERYLIIUM

SD-100-04 26-May-1987 BERYLIIUM

SD-100-04 3-Jun-1987 BERYLITUM

SD-100-04 11-Jun-1987 BERYLLIUM

SD-100-04 18-Jun-1987 BERYLIUM

$<0.0010 \quad \mathrm{mg} / \mathrm{L}$

$<0.0010 \mathrm{mg} / \mathrm{L}$

$<0.0010 \quad \mathrm{ng} / \mathrm{h}$

$<0.0010 \quad \mathrm{mg} / \mathrm{L}$

$<0.0010 \quad \mathrm{gg} / \mathrm{L}$

$<0.0010 \quad \mathrm{gg} / \mathrm{L}$

* SD - 100-06

SD-100-06 12-May-1987 BERYLLIUM

SD-100-06 18-May-1987 BERYLLIUM

SD-100-06 26-May-1987 BERYLLIUM

SD-100-06 3-Jun-1987 BERYLLIUM

SD-100-06 11-Jun-1987 BERYLLIUR

SD-100-06 18-Jun-1987 BERYLLIUM

$<0.0010 \quad \mathrm{gg} / \mathrm{L}$

$<0.0010 \quad \mathrm{mg} / \mathrm{L}$

$<0.0010 \quad \mathrm{gg} / \mathrm{L}$

$<0.0010 \mathrm{mg} / \mathrm{L}$

$<0.0010 \mathrm{ng} / \mathrm{L}$

$<0.0010 \quad \mathrm{mg} / \mathrm{L}$

** SD-100-06D

SD-100-06D 3-Jun-1987 BERYLLIUM

$<0.0010 \mathrm{mg} / \mathrm{L}$

* SD-100-07

SD-100-07 12-May-1987 BERYLLIUM

SD-100-07 19-May-1987 BERYLIUM

SD-100-07 26-May-1987 BERYLLIUM

SD-100-07 3-JUn-1987 BERYLIIUM

SD-100-07 11-Jun-1987 BERYLIIUM

SD-100-07 18-Jun-1987 BERYLLIUM

$<0.0010 \quad \mathrm{gg} / \mathrm{L}$

$0.0016 \mathrm{mg} / \mathrm{L}$

$<0.0010 \quad \mathrm{gg} / \mathrm{L}$

$<0.0010 \quad \mathrm{gg} / \mathrm{L}$

$<0.0010 \quad \mathrm{gg} / \mathrm{L}$

$<0.0010 \quad \mathrm{mg} / \mathrm{L}$

** SD - 100-07D

SD-100-07D 18-JUn-1987 BERYLIIUM

$<0.0010 \quad \mathrm{mg} / \mathrm{L}$ 
Storm Drain Data for SD-100

Location

Date

Test Compound

Results

Units

** SD-100-09

SD-100-09 12-Kay-1987 BERYLLIUM

SD-100-09 18-May-1987 BERYLLIUM

SD-100-09 26-Kay-1987 BERYLLIUS

SD-100-09 3-Jun-1987 BERYLLIUY

SD-100-09 11-Jun-1987 BERYLLIU

** SD-100-10

SD-100-10 12-May-1987 BERYLLIUM

SD-100-10 19-Hay-1987 BERYLIIU

SD-100-10 26-May-1987 BERYLIIUM

SD-100-10 3-Jun-1987 BERYLIUM

SD-100-10 11-JUn-1987 BERYLLIUM

SD-100-10 18-JUn-1987 BERYLLIUM

$\star$ SD-100-11

SD-100-11 12-May-1987 BERYLIUM

SD-100-11 19-May-1987 BERYLLIUM

SD-100-11 26-May-1987 BERYLLIUM

SD-100-11 3-Jun-1987 BERYLIIM

SD-100-11 11-Jun-1987 BERYLIIUM

SD-100-11 18-Jun-1987 BERYLLIUM

** SD-100-12

SD-100-12 12-May-1987 BERYLLIUM

SD-100-12 19-May-1987 BERYLIUM

SD-100-12 26-May-1987 BERYLLIUM

SD-100-12 3-JUn-1987 BERYLIIUM

SD-100-12 11-JUn-1987 BERYLLIUM

SD-100-12 18-Jun-1987 BERYLIIUM

$\begin{array}{ll}<0.0010 & \mathrm{mg} / \mathrm{L} \\ <0.0010 & \mathrm{mg} / \mathrm{L} \\ <0.0010 & \mathrm{mg} / \mathrm{L} \\ <0.0010 & \mathrm{mg} / \mathrm{L} \\ <0.0010 \quad \mathrm{mg} / \mathrm{L}\end{array}$

$<0.0010$

$<0.0010$

$<0.0010$

$<0.0010$

$<0.0010$

$<0.0010$

mg/L

$\operatorname{mg} / \mathrm{L}$

$\mathrm{mg} / \mathrm{L}$

mg/L

$\mathrm{mg} / \mathrm{L}$

$\mathrm{mg} / \mathrm{L}$

$<0.0010$

$<0.0010$

$<0.0010$

$<0.0010$

$<0.0010$

0.0012

mg/L

$\mathrm{mg} / \mathrm{L}$

$\mathrm{mg} / \mathrm{L}$

$\mathrm{mg} / \mathrm{L}$

$\mathrm{mg} / \mathrm{L}$

$\mathrm{mg} / \mathrm{L}$

** SD-100-13

SD-100-13 12-May-1987 BERYLLIUM

SD-100-13 19-May-1987 BERYLLIUM

SD-100-13 26-May-1987 BERYLLIUM

SD-100-13 11-JUn-1987 BERYLLIUM

SD-100-13 18-Jun-1987 BERYLLIUM

$<0.0010$

$<0.0003$

$<0.0003$

$<0.0010$

$<0.0010$

$<0.0010$

$\operatorname{mg} / \mathrm{L}$

$\mathbf{m g} / \mathrm{L}$

mg $\sqrt{L}$

$\mathrm{mg} / \mathrm{L}$

$\mathrm{mg} / \mathrm{L}$

mg/L

** SD $-100-14$

SD-100-14 12-May-1987 BERYLIUM

$<0.0010$

IIg $/ \mathrm{L}$

$<0.0003$

$\operatorname{mg} / \mathrm{L}$

$<0.0003$

mg/L

$<0.0010$

mg/L

$<0.0010$

$\mathrm{mg} / \mathrm{L}$

SD-100-14 19-May-1987 BERYIIUM

$<0.0010$

$\mathrm{mg} / \mathrm{L}$

$<0.0010$

mg/L

SD-100-14 26-May-1987 BERYLIIUM

$<0.0003$

$\mathrm{mg} / \mathrm{L}$

SD-100-14 3-Jun-1987 BERYLLIUY

$<0.0010$

$\mathrm{mg} / \mathrm{L}$

$<0.0010$

mg/L

SD-100-14 18-Jun-1987 BERYLLIUM

$<0.0010$

mg/L 
Storm Drain Data for SD-100

Location Date Test Compound Results Units

** SD-100-C

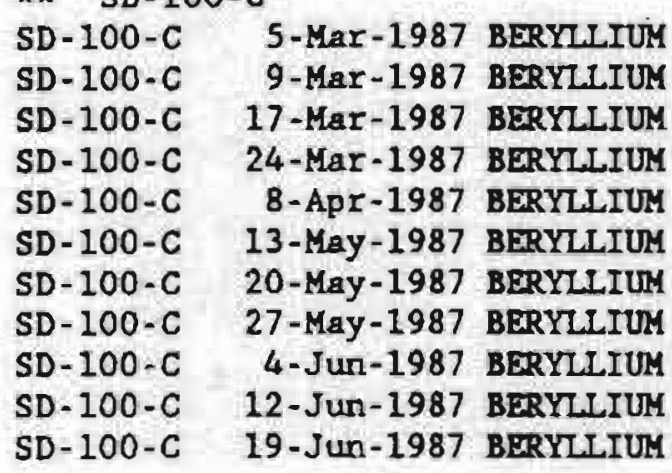

$<0.0010$

$<0.0010$

$<0.0010$

$<0.0010$

$<0.0010$

$<0.0010$

$<0.0010$

$<0.0010$

$<0.0010$

$<0.0010$

$<0.0010$

ng/L

$\mathrm{mg} / \mathrm{L}$

$\mathrm{mg} / \mathrm{L}$

$\mathrm{mg} / \mathrm{L}$

$\mathrm{mg} / \mathrm{L}$

$\mathrm{mg} / \mathrm{L}$

$\mathrm{mg} / \mathrm{L}$

$\mathrm{mg} / \mathrm{L}$

$\mathrm{mg} / \mathrm{L}$

$\mathrm{mg} / \mathrm{L}$

$\mathrm{mg} / \mathrm{L}$

*t SD-100-D

SD-100-D 6-Apr-1987 BERYLIIUM

$<0.0010 \quad \mathrm{mg} / \mathrm{L}$

SD-100-D 11-Jun-1987 BERYLLIUM

$<0.0010 \mathrm{mg} / \mathrm{L}$

** SD -100

SD-100 5-Mar-1987 BETA

SD $-100 \quad 11-$ Kar -1987 BETA

SD- $100 \quad 18-$ Mar-1987 BETA

SD-100 24-Mar-1987 BETA

SD-100 3-Apr-1987 BEIA

SD-100 6-Apr-1987 BEIA

SD-100 12-May-1987 BETA

SD-100 19-May-1987 BETA

SD-100 26-May-1987 BETA

SD-100 3-Jun-1987 BETA

SD-100 11-Jun-1987 BETA

SD-100 18-Jun-1987 BETA

4

5.5

$<2$

7.5

$<3$

6.3

2

4.3

$<3$

2.4

$<2$

4.5

3

$<2$

5

4.3

$<2$

2.4

2

$<2$

$<2$

6.2

3.0

3.5
$\mathrm{PC} i / \mathrm{L}$ $\mathrm{pCi} / \mathrm{L}$ $\mathrm{pCI} / \mathrm{L}$ $\mathrm{pCi} / \mathrm{L}$ $\mathrm{PCI} / \mathrm{L}$ PCI/L $\mathrm{PCl} / \mathrm{L}$ $\mathrm{pCi} / \mathrm{L}$ $\mathrm{pCl} / \mathrm{L}$ $\mathrm{pCi} / \mathrm{L}$ $\mathrm{PC} i / \mathrm{L}$ $\mathrm{pCi} / \mathrm{L}$

$\mathrm{pCi} / \mathrm{L}$ $\mathrm{pCi} / \mathrm{L}$ $\mathrm{pC1} / \mathrm{L}$ $\mathrm{pCi} / \mathrm{L}$ $\mathrm{PCl} / \mathrm{L}$ $\mathrm{pCi} / \mathrm{L}$

$\mathrm{PCi} / \mathrm{L}$ $\mathrm{pCi} / \mathrm{L}$ $\mathrm{PCl} / \mathrm{L}$ $\mathrm{pC1} / \mathrm{L}$ $\mathrm{PCI} / \mathrm{L}$ $\mathrm{pCI} / \mathrm{L}$ 


\section{Storm Drain Date for SD-100}

\begin{tabular}{|c|c|c|}
\hline Location & Date & \\
\hline SD -10 & -03 & \\
\hline$S D-100-03$ & 12-Kay-1987 & \\
\hline SD-100-03 & $18-\mathrm{Kay}-1987$ & \\
\hline SD- $100-03$ & $26 \cdot$ - Kay -1987 & \\
\hline SD $-100-03$ & 3-Jun-1987 & \\
\hline SD-100-03 & $11-J$ un- 1987 & \\
\hline SD $-100-03$ & $18-J u n-1987$ & \\
\hline
\end{tabular}

** SD-100-03D

SD-100-03D 12-Kay-1987 BETA SD-100-03D 26-Kay-1987 BETA

* SD-100-04

SD-100-04 12-May-1987 BETA

SD-100-04 18-May-1987 BETA

SD-100-04 26-May-1987 BETA

SD-100-04 3-Jun-1987 BETA

SD-100-04 11-Jun-1987 BETA

SD-100-04 18-Jun-1987 BETA

* SD-100-06

SD-100-06 12-May-1987 BETA

SD-100-06 18-May-1987 BETA

SD-100-06 26-May-1987 BETA

SD-100-06 3-3un-1987 BETA

SD-100-06 11-Jun-1987 BETA

SD-100-06 18-Jun-1987 BETA

** SD-100-06D

SD-100-06D 3-Jun-1987 BETA

* $\quad$ SD-100-07

SD-100-07 12-May-1987 BETA

SD-100-07 19-May-1987 BETA

SD-100-07 26-May-1987 BETA

SD-100-07 3-Jun-1987 BETA

SD-100-07 11-Jun-1987 BETA

SD-100-07 18-Jun-1987 BETA

** SD-100-07D

SD-100-07D 18-Jun-1987 BETA

* SD-100-09

SD-100-09 12-May-1987 BETA

SD-100-09 18-May-1987 BETA

SD-100-09 26-May-1987 BETA

SD-100-09 3-Jun-1987 BETA

SD-100-09 11-Jun-1987 BETA

SD-100-09 18-Jun-1987 BETA
Results Units

$\begin{array}{ll}2 & \mathrm{pCl} / \mathrm{L} \\ <2 & \mathrm{pCI} / \mathrm{L} \\ 3 & \mathrm{pCI} / \mathrm{L} \\ 4.5 & \mathrm{pCl} / \mathrm{L} \\ <2 & \mathrm{pCl} / \mathrm{L} \\ 3.1 & \mathrm{pCi} / \mathrm{L}\end{array}$

4

$2 \quad \mathrm{pCI} / \mathrm{L}$

$5 \quad \mathrm{PCi} / \mathrm{L}$

$<2 \quad \mathrm{pCi} / \mathrm{L}$

$<2 \quad \mathrm{PCi} / \mathrm{L}$

$10.2 \mathrm{pCi} / \mathrm{L}$

$<2 \quad \mathrm{pCI} / \mathrm{L}$

$4.3 \mathrm{pCi} / \mathrm{L}$

$4 \quad \mathrm{pCi} / \mathrm{L}$

$<2 \quad \mathrm{pCi} / \mathrm{L}$

C2 $\mathrm{PCi} / \mathrm{L}$

$4.4 \quad \mathrm{pCl} / \mathrm{L}$

$<2 \quad \mathrm{pC1} / \mathrm{L}$

$8.2 \mathrm{pCI} / \mathrm{L}$

$6.2 \mathrm{pCl} / \mathrm{L}$

$27 \mathrm{pCi} / \mathrm{L}$

$52.1 \quad \mathrm{pCi} / \mathrm{L}$

$24 \quad \mathrm{PCi} / \mathrm{L}$

$92.1 \mathrm{pCl} / \mathrm{L}$

$19.7 \mathrm{pC1} / \mathrm{L}$

$68.4 \mathrm{pCI} / \mathrm{L}$

$63.9 \mathrm{pCi} / \mathrm{L}$

$5 \quad \mathrm{PCI} / \mathrm{L}$

$4.4 \mathrm{pCI} / \mathrm{L}$

$<3 \quad \mathrm{PCi} / \mathrm{L}$

$12.1 \mathrm{PCI} / \mathrm{L}$

$3.2 \mathrm{pCi} / \mathrm{L}$

$4.4 \quad \mathrm{pCI} / \mathrm{L}$ 
Test Compound Results Units $\begin{array}{lll}* & \text { SD-100-10 } & \\ \text { SD-100-10 } & 12 \text {-May-1987 } & \text { BETA } \\ \text { SD-100-10 } & 19 \text { - Kay-1987 } & \text { BETA } \\ \text { SD-100-10 } & 26 \text {-May-1987 BETA } \\ \text { SD-100-10 } & 3 \text {-Jun-1987 BETA } \\ \text { SD-100-10 } & 11 \text {-Jun-1987 } & \text { BETA } \\ \text { SD-100-10 } & 18 \text {-Jun-1987 } & \text { BETA }\end{array}$

2

$<2$

$<3$

25.0

$<2$

2.7

$\mathrm{pCl} / \mathrm{L}$ $\mathrm{pCI} / \mathrm{L}$ $\mathrm{PC1} / \mathrm{L}$ $\mathrm{pCi} / \mathrm{L}$ $\mathrm{PC} 1 / \mathrm{L}$ $\mathrm{PC1} / \mathrm{L}$

** SD - 100-11

SD-100-11 12-May-1987 BETA SD-100-11 19-May-1987 BETA SD-100-11 26-May-1987 BETA SD-100-11 3-Jun-1987 BETA SD-100-11 11-Jun-1987 BETA SD-100-11 18-Jun-1987 BETA

* SD $-100-12$

SD-100-12 12-May-1987 BETA SD-100-12 19-May-1987 BETA SD-100-12 26-May-1987 BETA SD-100-12 3-Jun-1987 BETA SD-100-12 11-Jun-1987 BEIA SD-100-12 18-JUn-1987 BETA

$\begin{array}{ll}4 & \mathrm{PCi} / \mathrm{L} \\ 2.6 & \mathrm{PCi} / \mathrm{L} \\ <3 & \mathrm{PCi} / \mathrm{L} \\ <2 & \mathrm{PCi} / \mathrm{L} \\ <2 & \mathrm{PCi} / \mathrm{L} \\ 3.3 & \mathrm{PCi} / \mathrm{L}\end{array}$

*t SD-100-13

SD-100-13 12-May-1987 BETA SD-100-13 19-May-1987 BETA SD-100-13 26-May-1987 BETA SD-100-13 3-Jun-1987 BETA SD-100-13 11-Jun-1987 BETA SD-100-13 18-Jun-1987 BETA

$\begin{array}{ll}3 & \mathrm{pCl} / \mathrm{L} \\ 4.0 & \mathrm{pCi} / \mathrm{L} \\ <3 & \mathrm{PCl} / \mathrm{L} \\ <2 & \mathrm{pCi} / \mathrm{L} \\ <2 & \mathrm{pCi} / \mathrm{L} \\ 2.2 & \mathrm{pCi} / \mathrm{L}\end{array}$

** SD $-100-14$

SD-100-14 12-May-1987 BETA SD-100-14 19-May-1987 BETA SD-100-14 26-May-1987 BETA SD-100-14 3-Jun-1987 BETA SD-100-14 11-Jun-1987 BETA SD-100-14 18-Jun-1987 BETA

$\begin{array}{ll}6 & \mathrm{pCi} / \mathrm{L} \\ 3.4 & \mathrm{pCi} / \mathrm{L} \\ <3 & \mathrm{PCi} / \mathrm{L} \\ 23.6 & \mathrm{pCi} / \mathrm{L} \\ <2 & \mathrm{pCi} / \mathrm{L} \\ 2.7 & \mathrm{pCi} / \mathrm{L}\end{array}$

\begin{tabular}{|c|c|c|}
\hline$D-100-C$ & 5-Mar-1987 & \\
\hline$-100-C$ & $9-\operatorname{Mar}-1987$ & \\
\hline $100-C$ & 17-Mar-1987 & \\
\hline$-100-C$ & $24-\operatorname{Mar}-1987$ & \\
\hline$-100-C$ & 8-ApL-1987 & \\
\hline$-100-c$ & 13-May- 1987 & \\
\hline$-C$ & 20-May-1987 & \\
\hline
\end{tabular}

$\begin{array}{ll}<2 & \mathrm{pCi} / \mathrm{L} \\ 7 & \mathrm{pCI} / \mathrm{L} \\ 3.1 & \mathrm{pCi} / \mathrm{L} \\ 7.1 & \mathrm{pCI} / \mathrm{L} \\ 5.8 & \mathrm{pCi} / \mathrm{L} \\ 12 & \mathrm{pCi} / \mathrm{L} \\ <2 & \mathrm{pCi} / \mathrm{L}\end{array}$


Storm Drain Data for SD-100

Location

Date

Test Compound

Results

Units

SD-100-C 27-May-1987 BETA

SD-100-C 12-Jun-1987 BETA

SD-100-C 19-Jun-1987 BETA

$<3$

5.1

3.6

$\mathrm{pCI} / \mathrm{L}$

** SD-100-D

SD-100-D 6-Apr-1987 BETA

SD-100-D 11-Jun-1987 BETA

4. 8

$<2$

$\mathrm{pC1} / \mathrm{L}$

** SD-100-C

SD-100-C 17-Mar-1987 BHT

SD-100-C 24-Mar-1987 BHT

$8 B$

$4 B$

$\mathrm{PC1} / \mathrm{L}$

$\mathrm{pC} 1 / \mathrm{L}$

$\star \star$ SD -100

SD-100 S-Mar-1987 BIS (2-CHLOROETHOXY)METHANE

SD-100 11-Mar-1987 BIS (2-CHLOROETHOXY) METHANE

SD-100 12-May-1987 BIS (2-CHLOROETHOXY)METHANE

SD-100 26-May-1987 BIS (2-CHLOROETHOXY)METHANE

** SD-100-01

SD-100-01 12-May-1987 BIS (2-CHLOROETKOXY) METHANE

SD-100-01 26-May-1987 BIS (2-CHLOROETHOXY) METHANE

** SD-100-02

SD-100-02 12-May-1987 BIS (2-CHLOROETHOXY) METHANE

SD-100-02 26-May-1987 BIS (2-CHLOROETHOXX) METHANE

** SD $-100-03$

SD-100-03 12-May-1987 BIS (2-CHLOROETHOXY) METHANE

SD-100-03 26-May-1987 BIS (2-CHLOROETHOXY) METHANE

* SD-100-03D

SD-100-03D 12-May-1987 BIS (2-CHLOROETHOXY) KETHANE

SD-100-03D 26-May-1987 BIS (2-CHLOROETHOXY) METHANE

** SD $-100-04$

SD-100-04 12-May-1987 BIS (2-CHLOROETHOXY) METHANE

SD-100-04 26-May-1987 BIS (2-CHLOROETHOXY)METHANE

* SD-100-06

SD-100-06 12-May-1987 BIS (2-CHLOROETHOXY)METHANE

SD-100-06 26-May-1987 BIS (2-CHLOROETHOXY) METHANE

** SD-100-07

SD-100-07 12-May-1987 BIS (2-CHLOROETHOXY)METHANE

SD-100-07 26-May-1987 BIS (2-CHLOROETHOXY)METHANE

*t SD-100-09

SD-100-09 12-May-1987 BXS (2 - CHLOROETHOXY)METHANE

SD-100-09 26-May-1987 BIS(2-CHLOROETHOXY)METHANE

$\begin{array}{ll}<10 & \mathrm{ug} / \mathrm{L} \\ <10 & \mathrm{ug} / \mathrm{L} \\ <10 & \mathrm{ug} / \mathrm{L} \\ <5.0 & \mathrm{ug} / \mathrm{L} \\ & \\ <10 & \mathrm{ug} / \mathrm{L} \\ <5.0 & \mathrm{ug} / \mathrm{L} \\ & \\ <10 & \mathrm{ug} / \mathrm{L} \\ <5.0 & \mathrm{ug} / \mathrm{L} \\ & \\ <10 & \mathrm{ug} / \mathrm{L} \\ <5.0 & \mathrm{ug} / \mathrm{L} \\ & \\ <10 & \mathrm{ug} / \mathrm{L} \\ <5.0 & \mathrm{ug} / \mathrm{L} \\ & \\ <10 & \mathrm{ug} / \mathrm{L} \\ <5.0 & \mathrm{ug} / \mathrm{L} \\ <10 & \\ <5.0 & \mathrm{ug} / \mathrm{Lg} / \mathrm{L} \\ <5.0 & \mathrm{ug} / \mathrm{L} \\ <10 & \mathrm{ug} / \mathrm{L} \\ <5 & \\ & \\ & \end{array}$


Storm Drain Data for SD-100

Location Date Test Compound

Results Units

** SD-100-10

SD-100-10 12-May-1987 BIS (2-CHLOROETHOXY) METHANE

SD-100-10 26-May-1987 BIS (2-CHLOROETHOXY) METHANE

* $S D-100-11$

SD-100-11 12-May-1987 BIS (2-CHLOROETHOXY) XETHANE

SD-100-11 26-May-1987 BIS (2-CHLOROETHOXY)HETHANE

** SD-100-12

SD-100-12 12-May-1987 BIS (2-CHLOROETHOXY) METHANE

SD-100-12 26-May-1987 BIS (2-CHLOROETHOXY) METHANE

** SD $-100-13$

SD-100-13 12-May-1987 BIS (2-CHLOROETHOXY)METHANE

SD-100-13 26-May-1987 BIS (2-CHLOROETHOXY) METHANE

* SD $-100 \cdot 14$

SD-100-14 12-May-1987 BIS(2-CHLOROETHOXY)METHANE

SD-100-14 26-May-1987 BIS (2-CHLOROETHOXY)METHANE

** SD-100-C

SD-100-C 5-Mar-1987 BIS (2-CHLOROETHOXY) METHANE

SD-100-C 9-Mar-1987 BIS (2-CHLOROETHOXY)METHANE

SD-100-C 17-Mar-1987 BIS (2-CHLOROETHOXY)METHANE

SD-100-C 24-Mar-1987 BIS (2-CHLOROETHOXY)METHANE

SD-100-C 8-APR-1987 BIS (2-CHLOROETHOXY)METHANE

SD-100-C 13-May-1987 BIS (2-CHLOROETHOXY)METHANE

$S D-100-C$

20- MAY-1987 BIS (2 - CHLOROETHOXY) HEIHANE

$S D-100-C$

27-May-1987 BIS (2-CHLOROETHOXY) METHANE

$S D-100-C$

12-Jun-1987 BIS (2-GHLOROETHOXY)KETHANE

SD $-100-C$

19-Jun-1987 BIS(2-CHLOROETHOXY) HETHANE

$<10$

$<5.0$

$4 \mathrm{~g} / \mathrm{L}$

$<.0$

ug $/ \mathrm{L}$

$<10$

$<5.0$

ug/L

$\mathrm{ug} / \mathrm{L}$

$<10$

$<5.0$

ug/L

ug/L

$<10$

$<5.0$

ug $/ 2$

ug/L

$<10$

$<5.0$

ug/2

ug/L

$<10$

$<10$

$<10$

$<10$

$<10$

$<5.0$

$<5.0$

$<5.0$

$<5.0$

$<10$

$u g /$

ug/L

ug/L

ug/ $/$

ug $/ 2$

ug $/ 2$

ug $\Omega$

$4 \mathrm{~g} /$

ug/L

ug/I

** SD -100

SD -100

5-Mar-1987 BIS (2-CHLOROETHYL) ETHER

SD -100

11-Mar-1987 BIS (2-CHLOROETHY) ETHER

SD -100

12-May-1987 BIS (2-CHLOROETHYL) ETHER

SD -100

26-May- 2987 BIS (2-CHLOROETHY) ETHER

** SD-100-01

SD-100-01 12-Mzy-1987 BIS (2-CHLOROETHYL) ETHER

SD-100-01 26-Kay-1987 BIS(2-CHLOROETHY) ETHER

** SD $-100-02$

SD-100-02 12-May-1987 BIS(2-CHLOROETHYL) ETHER

SD-100-02 26-May-1987 BIS (2-CHLOROETHYL) ETTER

* SD-100-03

SD-100-03 12-May-1987 BIS (2-CHLOROETHYL) ETHER

SD-100-03 26-May-1987 BIS (2-CHLOROETMY) ETRER

$<10$

$<10$

$<10$

$<5.0$

$<10$

$<5.0$

ug $/ 2$

ug/L

ug/L

ug $/ \mathrm{L}$

$<10$

$<5.0$

$\mathrm{ug} / \mathrm{L}$

ug/L

$<10$

$<5.0$

ug/L

ug/L 
Storw Drain Daca for SD-100

Location

Date

Test Compound

Results

Units

** SD-100-03D

SD-100-03D 12-May-1987 BIS (2-CHLOROETHYL) ELHER

SD-100-03D 26-May-1987 BIS (2-CHLOROETHYL) ETHER

** SD-100-04

SD-100-04 12-May-1987 BIS (2-CHLOROETHY) ETHER

SD-100-04 26-May-1987 BIS(2-CHLOROETHY) ETHER

** SD $100-06$

SD-100-06 12-May-1987 BIS (2-CHLOROETHYL)ETHER

SD-100-06 26-May-1987 BIS (2-CHLOROETHYL) ETHIRR

** SD $-100-07$

SD-100-07 12-May-1987 BIS (2-CHLOROETHY) ETHER

SD-100-07 26-May-1987 BIS (2-CHLOROETHY) ETHER

** SD-100-09

SD-100-09 12-May-1987 BIS (2-CHLOROETHYL) ETHER

SD-100-09 26-May-1987 BIS (2-CHLOROETHYL) ETHER

** SD $-100-10$

SD-100-10 12-May-1987 BIS (2 - CHLOROETHYL) ELHER

SD-100-10 26-May-1987 BIS(2-CHLOROETHYL) ETHER

** SD-100-11

SD-100-11 12-Kay-1987 BIS (2-CHLOROETHY) ETHER

SD-100-11 26-May-1987 BIS (2-CHLOROETHYL) ETHER

* SD $-100-12$

SD-100-12 12-May-1987 BIS (2-CHLOROETHY) ETHER

SD-100-12 26-May-1987 BIS (2-CHLOROETHY) ETHER

* SD-100-13

SD-100-13 12-May-1987 BIS (2-CHLOROETHY) ETHER

SD-100-13 26-May-1987 BIS (2-CHLOROETHYL) ETHER

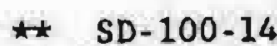

SD-100-14 12-May-1987 BIS (2-CHLOROETHYL) ETHER

SD-100-14 26-May-1987 BIS (2-CHLOROETHYL) ETHER

** SD-100-C

SD-100-C S-Mar-1987 BIS (2-CHLOROETHYL) ETHER

SD-100-C 9-Kar-1987 BIS (2-CHLOROETHYL) ETHISR

SD-100-C 17-Mar-1987 BIS (2-CHLOROETHYL) ETHER

SD-100-C 24-Mar-1987 BIS (2-CHLOROETHY) ETHER

SD-100-C 8-APr-1987 BIS (2-CHLOROETHYL) ETHER

SD-100-C 13-May-1987 BIS (2-CHLOROETHYL) ETHER

SD-100-C 20-May-1987 BIS (2-CHLOROETHY) ETHER

SD-100-C 27-May-1987 BIS (2-CHLOROETHY) ETHER

SD-100-C 12-Jun-1987 BIS (2-CHLOROETHYL) ETHER

SD-100-C 19-JUn=1987 BIS (2-CHLOROETHYL) ETHER

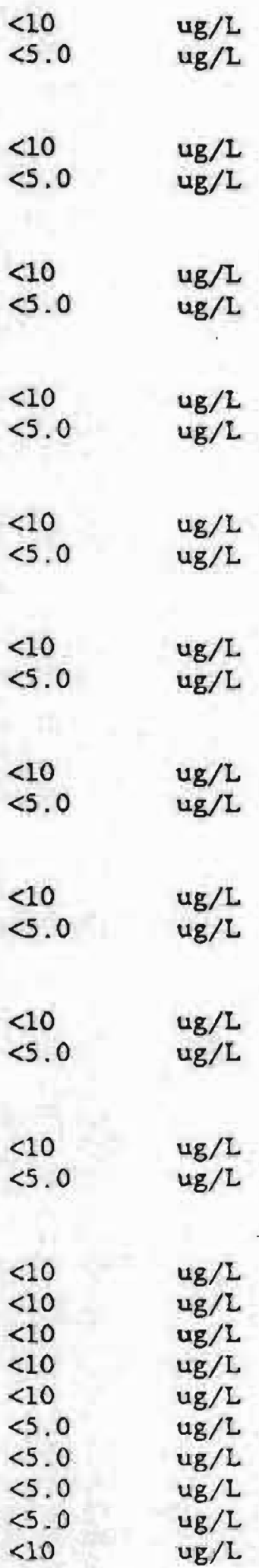


Storm Drain Date for SD-100

Location

Date

Test Compound

Results

Units

** SD -100

SD -100

5-MaT-1987 BIS (2-CHLOROISOPROPYL) ETHER

11-MaI-1987 BIS (2-GHLOROISOPROPYL) ETHER

SD -100

12-Yay-19B7 BIS (2-CHLOROISOPROPYL) ETHER

SD -100

26-May-1987 BIS (2-CHLOROISOPROPYL) ETHER

** SD-100-01

SD-100-01 26-May-1987 BIS(2-CHLOROISOPROPY) ETHER

** SD $-100-02$

SD-100-02 12- $\mathrm{Hay-1987}$ BIS (2-CHLOROISOPROPY) ETHER

SD-100-02 26-May-1987 BIS(2-CHLOROISOPROPY) ETHER

* SD $-100-03$

SD-100-03 12-May-1987 BIS (2-CHLOROISOPROPYL) ETHER SD-100-03 26-May-1987 BIS(2-CHLOROISOPROPYL) ETHER

* SD-100-03D

SD - 100-03D 12- May-1987 BIS (2 - CHLOROISOPROPYL) ETHER

SD-100-03D 26-May-1987 BIS (2-CHLOROISOPROPY) EIHER

** SD-100-04

SD-100-04 12-May-1987 BIS (2-CHLOROISOPROPYL) ETHER

SD-100-04 26-May-1987 BIS(2-CHLOROISOPROPYL) ETHER

** SD-100-06

SD-100-06 12-May-1987 BIS (2-CHLOROISOPROPYL) ETHER

SD-100-06 26-May-1987 BIS (2-CHLOROISOPROPY) ETHER

* SD $-100-07$

SD-100-07 12-May-1987 BIS (2-CHLOROISORROPYL) ETHER

SD-100-07 26-May-1987 BIS (2-CHLOROISOPROPYL) ETHER

** SD-100-09

SD-100-09 12-May-1987 BIS(2-CHLOROISOPROPYL) ETHER

SD-100-09 26-May-1987 BIS(2-CHLOROISOPROPYL) ETHER

** SD $-100-10$

SD-100-10 12-May-1987 BIS (2-CHLOROI SOPROPYL) ETHER SD-100-10 26-May-1987 BIS(2-CHLOROISOPROPYL) EITER

** SD-100-11

SD-100-11 12-May-1987 BIS (2-CHLOROISOPROPYL) ETHER

SD-100-11 26-May-1987 BIS(2-CHLOROISOPROPYL) ETHER

** SD $-100-12$

SD-100-12 12-May-1987 BIS (2 -CHLOROISOPROPY) ETHER

SD-100-12 26-May-1987 BIS(2-CHLOROISOPROPY) ETHER

$<10$

$<10$

$<10$

$<5.0$

$<5.0$

ug $/ \mathrm{L}$

$<10$

$<5.0$

ug/I

$\mathrm{ug} / \mathrm{L}$

$<10$

$<5.0$

ug/L

$u g / L$

$<10$

$<5.0$

ug/L

$u g / L$

$<10$

$<5.0$

ug/L

$u g / L$

$<10$

$<5.0$

ug/L

$u g / L$

$<10$

$<5.0$

ug/L

ug $/ L$

$<10$

$<5.0$

ug/L

ug/L

$<10$

$<5.0$

ug/L

ug/L

$<10$

$<5.0$

ug $/$

$u g / L$

$<10$

$<5.0$

ug/ $/ \mathrm{L}$

$u g / L$ 
Storm Drain Data for SD-100

Location Date Test Compound

Results Units

* SD - 100-13

SD-100-13 12-May-1987 BIS (2-CHLOROISOPROPY) ETHER

SD-100-13 26-May-1987 BIS(2-CHLOROISOPROPYL) ETHER

$\begin{array}{ll}<10 & \mathrm{ug} / \mathrm{L} \\ <5.0 & \mathrm{ug} / \mathrm{L} \\ & \\ <10 & \mathrm{ug} / \mathrm{L} \\ <5.0 & \mathrm{ug} / \mathrm{L}\end{array}$

* SD-100-14

SD-100-14 12-May-1987 BIS (2 - CHLOROISOPROPY) ETHER

SD-100-14 26-May-1987 BIS (2-CHLOROISOPROPYL) ETHER

$<5.0$

ug/L

* $\quad S D-100-C$

SD-100-C 5-Mar-1987 BIS (2-CHLROISOPROPY) ETHER

SD-100-C 9-Mar-1987 BIS (2-CHLOROISOPROPYL) ETHER

SD-100-C . 17-Mar-1987 BIS (2-CHLOROISOPROPYL)ETHER

SD-100-C 24-MaT-1987 BIS (2-CHLOROISOPROPY) ETHER

SD-100-C 8-Apr-1987 BIS.(2-CHLOROISOPROPY) ETHER

SD-100-C 13-May-1987 BIS (2-CHLOROISOPROPYL)ETHER

SD-100-C 20-May-1987 BIS (2-CKLOROI SOPROPYL)ETHER

SD-100-C 27-May-1987 BIS (2-CHLOROISOPROPY) ETHER

SD-100-C 12-Jun-1987 BIS (2-CHLOROISOPROPYL)ETHER

SD-100-C 19-Jun-1987 BIS (2-CHLOROISOPROPYL) ETHER

* SD-100-01

SD-100-01 12-May-1987 BIS(2-CHLOROISOPROPY)ETHER)

$<10$

$<10$

ug/L

$u g / L$

$<10 \quad \mathrm{ug} / \mathrm{L}$

$<10 \quad$ ug/L

$<10 \quad$ ug/L

$<5.0 \quad$ ug/

$<5.0 \quad u g / L$

$<5.0 \quad$ ug $/ \mathrm{L}$

$<5.0 \quad 4 \mathrm{~g} / \mathrm{L}$

$<10 \quad$ ug/L

* SD -100

SD- 100 5-MaY-1987 BIS (2-ETRYLHEXYL) PHTHALATE

SD-100 11-Mar-1987 BIS (2 - ETHYLHEXY) PHTHALATE

SD-100 12-May-1987 BIS (2-ETHYLHEXYL) PHTHALATE

SD-100 26-May-1987 BIS(2-ETHYLHEXYL) PHTHALATE

* SD-100-01

SD-100-01 12-May-1987 BIS(2-ETHYLHEXYL) PHTHALATE

SD-100-01 26-May-1987 BIS(2-ETHYLHEXYL) PHTHALATE

* $\quad$ SD-100-02

SD-100-02 12-May-1987 BIS (2-ETHYLHEXYL) PHTHALATE

SD-100-02 26-May-1987 BIS (2-ETHYLHEXYL) PHTHALATE

** SD $-100-03$

SD-100-03 12-May-1987 BIS(2-ETHYLHEXYL) PHTHALATE

SD-100-03 26-May-1987 BIS (2-ETHYLHEXYL) PHTHALATE

** SD-100-03D

SD-100-03D 12-May-1987 BIS (2 - ETHYLHEXXZ) PHTHALATE

SD-100-03D 26-May-1987 BIS(2-ETHYLHEXYL) PHTHALATE

* SD $-100-04$

SD-100-04 12-May-1987 BIS(2-ETHYLHEXYL) PHTHALATE

SD-100-04 26-May-1987 BIS (2-ETHYLHEXYL) PHTHALATE

$1 \mathrm{~J} \quad$ ug $/ \mathrm{L}$

** SD - $100-06$

SD-100-06 12-May-1987 BIS(2-ETHYLHEXYL) PHTHALATE

$\begin{array}{ll}<0 & \text { ug/L } \\ & \\ <10 & u g / L \\ <10 & u g / L \\ <10 & u g / L \\ <5.0 & u g / L\end{array}$

$<10 \quad$ ug $/ \mathrm{L}$

$<5.0 \quad$ ug $/ \mathrm{L}$

$<10 \quad$ ug/L

$<5.0 \quad$ ug $/ \mathrm{L}$

$<10 \quad u g / L$

$<5.0 \quad \mathrm{ug} / \mathrm{L}$

$<10 \quad$ ug/ $/$

$<5.0 \quad 4 \mathrm{~g} / \mathrm{l}$

$<5.0 \quad \mathrm{ug} / \mathrm{L}$

$<10 \quad u g / L$ 
Storm Drain Data for SD-100

Location Date Test Compound

SD-100-06 26-May-1987 BIS (2-ETHYHEXYL) PHTHALATE

** SD-100-07

SD-100-07 12-Kay-1987 BIS(2-ETHYLHEXY) PHTHAIATE

SD-100-07 26-May-1987 BIS (2-ETHYLHEXY) PHTHALATE

*t SD.100-09

SD-100-09 12-May-1987 BIS (2-ETHYL HEXY) PHTHALATE SD-100-09 26-May-1987 BIS (2-ETHYLHEXYL) PHTHALATE

* SD-100-10

SD-100-10 12-May-1987 BIS (2-ETHYLHEXYL) PHTHALATE

SD-100-10 26-May-1987 BIS (2-ETHYLHEXY) PHTHALATE

* SD-100-11

SD-100-11 12-May-1987 BIS (2-ETHYHEXYL) PHTHALATE

SD-100-11 26-May-1987 BIS (2-ETHYLHEXYL) PHTHALATE

** SD-100-12

SD-100-12 12-May-1987 BIS (2-ETHYLHEXYL) PHTHALATE

SD-100-12 26-May-1987 BIS (2-ETHYLHEXYL) PHTHALATE

** SD $-100-13$

SD-100-13 12-May-1987 BIS (2-ETHYLHEXY) PHTHAIATE

SD-100-13 26-May-1987 BIS (2-ETHYLHEXYL) PHTHALATE

** SD-100-14

SD-100-14 12-May-1987 BIS(2-ETHYLHEXY) PHTHALATE

SD-100-14 26-May-1987 BIS(2-ETHYLHEXYL) PHTHALATE

* SD $-100-\mathrm{C}$

SD-100-C 5-Mar-1987 BIS (2-ETHYLHEXYL) PHTHALATE

SD-100-C 9-Mar-1987 BIS (2-ETHYLEXYL) PHTHALATE

SD-100-C 17-Mar-1987 BIS (2-ETHYLHEXY) PHTHALATE

SD-100-C 24-Yar-1987 BIS (2-ETHYLHEXYL) PHTHALATE

SD-100-C 8-Apr-1987 BIS (2-ETHYLHEXYL) PHTHALATE

SD-100-C 13-May-1987 BIS (2-ETHYLHEXY) PHTHALATE

SD-100-C 20-May-1987 BIS (2-ETHYLHEXYL) PHTHALATE

SD-100-C 27-May-1987 BIS(2-ETHYLHEXYL) PHTHALATE

SD-100-C 12-JUn-1987 BIS (2-ETHYLEEXY) PHTHAJATE

SD-100-C 19-JUn-1987 BIS(2-ETHYLHEXYL) PHTHALATE

* SD -100

SD-100 5-Mar-1987 BORON

SD-100 11-Mar-1987 BORON

SD-100 18-Mar-1987 BORON

SD-100 24-Mar-1987 BORON

SD-100 3-APr-1987 BORON

SD-100 6-Apr-1987 BORON

SD-100 12-May-1987 BORON

SD-100 19-May-1987 BORON

\begin{tabular}{|c|c|}
\hline Results & Units \\
\hline$<5.0$ & $u_{g} / \mathrm{L}$ \\
\hline $\begin{array}{l}15 \\
6\end{array}$ & $\begin{array}{l}u g / L \\
u g / L\end{array}$ \\
\hline $\begin{array}{l}<10 \\
<5.0\end{array}$ & $\begin{array}{l}u g / L \\
u g / L\end{array}$ \\
\hline $\begin{array}{l}<10 \\
<5.0\end{array}$ & $\begin{array}{l}u g / L \\
u g / L\end{array}$ \\
\hline $\begin{array}{l}<10 \\
<5.0\end{array}$ & $\begin{array}{l}u g / L \\
u g / L\end{array}$ \\
\hline $\begin{array}{l}<10 \\
<5.0\end{array}$ & $\begin{array}{l}u g / L \\
u g / L\end{array}$ \\
\hline $\begin{array}{l}<10 \\
<5.0\end{array}$ & $\begin{array}{l}u g / L \\
u g / L\end{array}$ \\
\hline $\begin{array}{l}<10 \\
<5.0\end{array}$ & $\begin{array}{l}u g / L \\
u g / L\end{array}$ \\
\hline $\begin{array}{l}<10 \\
<10 \\
2 \mathrm{JB} \\
7 \mathrm{JB} \\
<10 \\
<5.0 \\
<5.0 \\
<5.0 \\
51 \\
3 \mathrm{~J}\end{array}$ & $\begin{array}{l}u g / L \\
u g / L \\
u g / L \\
u g / L \\
u g / L \\
u g / L \\
u g / L \\
u g / L \\
u g / L \\
u g / L\end{array}$ \\
\hline $\begin{array}{l}0.040 \\
0.014 \\
<0.0040 \\
<0.0040 \\
0.058 \\
0.099 \\
0.0084 \\
0.016\end{array}$ & $\begin{array}{l}\mathrm{mg} / \mathrm{L} \\
\mathrm{mg} / \mathrm{L} \\
\mathrm{mg} / \mathrm{L} \\
\mathrm{mg} / \mathrm{L} \\
\mathrm{mg} / \mathrm{L} \\
\mathrm{mg} / \mathrm{L} \\
\mathrm{mg} / \mathrm{L} \\
\mathrm{mg} / \mathrm{L}\end{array}$ \\
\hline
\end{tabular}


Storm Drain Data for SD-100

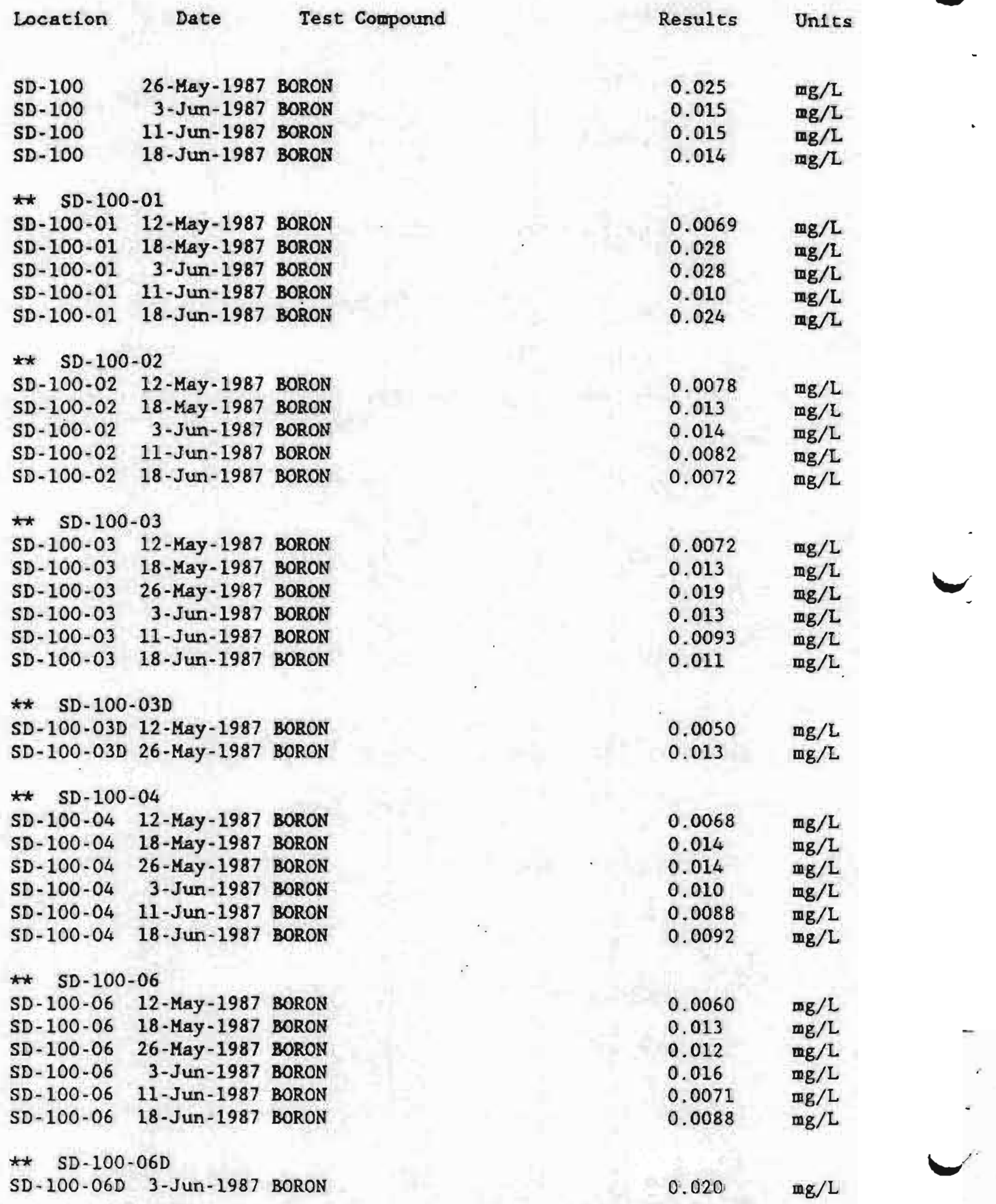


Storm Drain Data for SD-100

Location Date Test Compound Unfts

** SD-100-07

SD-100-07 12-May-1987 BORON

SD-100-07 19-May-1987 BORON

SD-100-07 26-May-1987 BORON

SD-100-07 3-Jun-1987 BORON

SD-100-07 11-Jun-1987 BORON

SD-100-07 18-Jun-1987 BORON

0.033

0.025

0.010

0.025

0.050

0.030

$\mathrm{mg} / \mathrm{L}$

$\mathrm{mg} / \mathrm{L}$

$\mathrm{mg} / \mathrm{L}$

$\mathrm{mg} / \mathrm{L}$

$\mathrm{mg} / \mathrm{L}$

$\mathrm{mg} / \mathrm{L}$

** SD - 100-07D

SD-100-07D 18-Jun-1987 BORON

$0.026 \mathrm{mg} / \mathrm{L}$

** SD-100-09

SD-100-09 12-May-1987 BORON

SD-100-09 18-May-1987 BORON

SD-100-09 26- Kay-1987 BORON

SD-100-09 3-Jun-1987 BORON

SD-100-O9 11-Jun-1987 BORON

$0.014 \mathrm{mg} / \mathrm{L}$

$0.013 \quad \mathrm{mg} / \mathrm{L}$

$0.014 \mathrm{mg} / \mathrm{L}$

$0.014 \mathrm{mg} / \mathrm{L}$

$0.0077 \mathrm{mg} / \mathrm{L}$

** SD-100-10

SD-100-10 12-May-1987 BORON

$0.011 \mathrm{mg} / \mathrm{L}$

$0.013 \mathrm{mg} / \mathrm{L}$

SD-100-10 19-May-1987 BORON

$0.012 \mathrm{mg} / \mathrm{L}$

SD-100-10 26-May-1987 BORON

$0.013 \mathrm{mg} / \mathrm{L}$

SD-100-10 11-Jun-1987 BORON

$0.0066 \mathrm{mg} / \mathrm{L}$

SD-100-10 18-Jun-1987 BORON

$0.012 \mathrm{mg} / \mathrm{L}$

* SD - 100-11

SD-100-11 12-May-1987 BORON

$0.0080 \mathrm{mg} / \mathrm{L}$

$0.014 \mathrm{mg} / \mathrm{L}$

SD-100-11 19-May-1987 BORON

0.047

$\mathrm{mg} / \mathrm{L}$

$\begin{array}{rr}\text { SD-100-11 } & 26 \text {-May-1987 BORON } \\ \text { SD-100-11 } & 3 \text {-Jun-1987 BORON }\end{array}$

0.014

$\mathrm{mg} / \mathrm{L}$

SD-100-11 11-Jun-1987 BORON

$0.0091 \mathrm{mg} / \mathrm{L}$

SD-100-11 18-Jun-1987 BORON

$0.032 \mathrm{mg} / \mathrm{L}$

* SD $-100-12$

SD-100-12 12-May-1987 BORON

SD-100-12 19-May-1987 BORON

$0.0080 \mathrm{mg} / \mathrm{L}$

$0.061 \mathrm{mg} / \mathrm{L}$

SD-100-12 26-May-1987 BORON

0.017

mg $/ \mathrm{L}$

SD-100-12 3-Jun-1987 BORON

0.035

$\mathrm{mg} / \mathrm{L}$

SD-100-12 11-Jun-1987 BORON

0.012

$\mathrm{mg} / \mathrm{L}$

SD-100-12 18-Jun-1987 BORON

$0.013 \mathrm{mg} / \mathrm{L}$

* SD $-100-13$

SD-100-13 12-May-1987 BORON

$0.0061 \mathrm{mg} / \mathrm{L}$

$0.036 \mathrm{mg} / \mathrm{L}$

SD-100-13 19-May-1987 BORON

$0.015 \mathrm{mg} / \mathrm{L}$

SD-100-13 26-May-1987 BORON

0.010

$\mathrm{mg} / \mathrm{L}$

SD-100-13 18-Jun-1987 BORON

$0.010 \mathrm{mg} / \mathrm{L}$

* SD $-100-14$

SD-100-14 12-May-1987 BORON

$0.0064 \quad \mathrm{gg} / \mathrm{L}$ 
Storm Drain Data for SD-100

\begin{tabular}{|c|c|c|c|c|}
\hline Location & Date & Test Compound & Results & Units \\
\hline SD $-100-14$ & $19-$-вy- 1987 & BORON & 0.027 & $\mathrm{mg} / \mathrm{L}$ \\
\hline SD $-100-14$ & $26-$ May -1987 & BORON & 0.025 & $\operatorname{mg} /$ / \\
\hline SD $-100-14$ & 3-Jun- 1987 & BORON & 0.053 & $\mathrm{mg} / \mathrm{L}$ \\
\hline SD $-100-14$ & 11-Jun-1987 & BORON & 0.013 & $\mathrm{mg} / \mathrm{L}$ \\
\hline SD $-100-14$ & 18-Jun- 1987 & BORON & 0.0090 & $\mathrm{mg} / \mathrm{L}$ \\
\hline \multicolumn{5}{|c|}{$\star \star \quad S D-100-C$} \\
\hline$S D-100-C$ & 5-Mar-1987 & BORON & 0.032 & mg $/$ \\
\hline SD-100-C & 9-Mar-1987 & BORON & 0.024 & $\operatorname{mg} / \bar{L}$ \\
\hline SD-100-C & 17-Mar-1987 & BORON & 0.013 & $\mathrm{mg} / \mathrm{L}$ \\
\hline SD-100-C & 24 -Mar-1987 & BORON & 0.036 & $\operatorname{mg} / \mathrm{L}$ \\
\hline SD-100-C & 8-Apr-1987 & BORON & $<0.0040$ & $\operatorname{mg} / \mathrm{h}$ \\
\hline SD-100-C & 13-May - 1987 & BORON & 0.013 & $\mathrm{mg} / \mathrm{l}$ \\
\hline SD $-100-C$ & $20-$ May-1987 & BORON & 0.014 & $\mathrm{mg} / \mathrm{L}$ \\
\hline SD- $100-C$ & $27-\mathrm{May}-1987$ & BORON & 0.012 & $\mathrm{mg} / \mathrm{h}$ \\
\hline SD-100-C & 4-Jun- 1987 & BORON & 0.020 & $\mathrm{mg} / \mathrm{L}$ \\
\hline SD- $100-C$ & $12-J u n-1987$ & BORON & 0.018 & $\operatorname{mg} / \mathrm{L}$ \\
\hline$S D-100-C$ & $19-J u n-1987$ & BORON & 0.014 & $\operatorname{mg} / 1$ \\
\hline \multicolumn{5}{|c|}{$\star \star \quad S D-100-D$} \\
\hline SD-100-D & 6 -Apr-1987 & BORON & 0.012 & $\mathrm{mg} / \mathrm{L}$ \\
\hline SD-100-D & $11-J u n-1987$ & BORON & 0.023 & $\mathrm{mg} / \mathrm{L}$ \\
\hline \multicolumn{5}{|c|}{$\star \star \quad S D-100-12$} \\
\hline SD $-100-12$ & 12-May- 1987 & BROMO CYCLOHEXENE & 80 & $\operatorname{ug} / \mathrm{L}$ \\
\hline \multicolumn{5}{|l|}{$\star \star$ SD -100} \\
\hline SD-100 & 5-Mar - 1987 & BROMODICHLOROMETHANE & 5 & ug $/ \mathrm{L}$ \\
\hline$S D-100$ & 11-Mar-1987 & BROMODICHLOROMETHANE & 5 & $\mathrm{ug} / \mathrm{l}$ \\
\hline SD- 100 & 18 -Kar -1987 & BROYODICHLOROMETHAFE & 8 & $4 g / L$ \\
\hline$S D-100$ & $24-\mathrm{Max}-1987$ & BROMODICHLOROMETHANE & 8 & $u_{g} / \mathrm{L}$ \\
\hline SD- 100 & 6-Apr-1987 & BROMODICHLOROMETHANE & 9 & $u g / 1$ \\
\hline SD -100 & 12-May-1987 & BROMODICHLOROYETHANE & 10. & ug/l \\
\hline$S D-100$ & 19-May-1987 & BROMODICHLOROMEIHANE & 6 & $u g / h$ \\
\hline$S D-100$ & $26-$ May -1987 & BROHODICHLORONETHANE & 9 & $\mathrm{ug} / \mathrm{L}$ \\
\hline$S D-100$ & 3-Jun-1987 & BROMODICHLOROMETHANE & 11 & $48 / \mathrm{L}$ \\
\hline SD -100 & $11-J$ un -1987 & BROMODICHLOROMETHANE & 5 & $u g / L$ \\
\hline SD -100 & $18-J$ un -1987 & BROMODICHLOROMETHLANE & 16 & $u g / L$ \\
\hline \multicolumn{5}{|c|}{$\star \star \quad S D-100-01$} \\
\hline$S D-100-01$ & 12-May-1987 & BROMODICHLOROMETHANE & $<5$ & $\mathrm{ug} / \mathrm{L}$ \\
\hline SD $-100-01$ & $18-\mathrm{May}-1987$ & BROMODICHLOROMETHANE & $<5$ & $\mathrm{ug} / \mathrm{L}$ \\
\hline SD-100-01 & $26-$ May -1987 & BROMODI CHLOROMETHANE & $<5$ & $\mathrm{ug} / \mathrm{L}$ \\
\hline SD -100.01 & $3-J u n-1987$ & BROHODICHLOROMETHANE & $<5$ & $\mathrm{ug} / \mathrm{L}$ \\
\hline SD-100-01 & $11-J u n-1987$ & BROMODICHLOROMETHANE & $<5$ & $\mathrm{ug} / \mathrm{L}$ \\
\hline SD $-100-01$ & $18-J u n-1987$ & BROMODICHLOROMETHANE & $<5$ & $\mathrm{ug} / \mathrm{L}$ \\
\hline \multicolumn{5}{|c|}{$\star \quad$ SD $-100-02$} \\
\hline SD $-100-02$ & $12-$ - $a y-1987$ & BROMODICHLOROMETHANE & 5 & ug/L \\
\hline$S D-100-02$ & $18-$ May -1987 & BROMODICHLOROMETHANE & 5 & $\mathrm{ug} / \mathrm{L}$ \\
\hline$S D-100-02$ & $26-$ May -1987 & BROMOD I CHLOROMETHANE & 5 & ug/L \\
\hline
\end{tabular}


Storm Drain Daca for SD-100

Location

Date

Test Compound

SD $-100-02$

SD-100-02

SD-100-02

3-Jun-1987 BROMODICHLOROMETHANE

11-Jun-1987 BROMODICKLOROHETHANE

18-Jun-1987 BROMODICHLOROMETHANE

* SD $-100-03$

SD-100-03 12-May-1987 BROMODICHLOROMETHANE SD-100-03 18-May-1987 BROMODICHLOROMETHANE SD-100-03 26-Kay-1987 BROMODICHLOROAETHANE SD-100-03 3-Jun-1987 BROMODICHLORONETHANE SD-100-03 11-Jun-1987 BROMODICHLOROMETHANE SD-100-03 18-JUn-1987 BROMODICHLOROMETHANE

* SD-100-03D

SD-100-03D 12-May-1987 BROMODICHLOROMETHANE SD-100-03D 26-May-1987 BROMODICHLOROMETHANE

** SD-100-04

SD-100-04 12-May-1987 BROMODICHLOROMETHANE SD-100-04 18-May-1987 BROMODICHLOROMETHANE SD-100-04 26-May-1987 BROMODICHLOROMETHANE SD-100-04 3-JUn-1987 BROMODICHLOROMETHANE SD-100-04 11-JUn-1987 BROMODICHLOROMETHANE SD-100-04 18-Jun-1987 BROMODICHLOROLETHANE

* SD-100-06

SD-100-06 12-May-1987 BROMODICHLOROMETHANE SD-100-06 18-May-1987 BROMODICFILOROMETHANE SD-100-06 26-May-1987 BROMODICHLOROMETHANE SD-100-06 3-Jun-1987 BROMODICHLOROMETHANE SD-100-06 11-JUn-1987 BROMODICHLOROMETHANE SD-100-06 18-Jun-1987 BROMODICHLOROMETHANE

** SD-100-06D

SD-100-06D 3-Jun-1987 BROMODICHLOROKETHANE

* SD - 100-07

SD-100-07 12-May-1987 BROMODICHLOROMETHANE

SD-100-07 19-May-1987 BROMODICHLOROMETHANE

SD-100-07 26-May-1987 BROMODICHLOROMETHANE

SD-100-07 3-JUn-1987 BROMODICHLOROMETHANE

SD-100-07 11-JUn-1987 BROMODIGHLOROMETHANE

SD-100-07 18-Jun-1987 BROMODICHLOROMETHANE

*t SD-100-07D

SD-100-O7D 18-JUn-1987 BROMODICHLOROMETHANE

** SD $-100-09$

SD-100-09 12-May-1987 BROMODICHLOROMETHANE

SD-100-09 18-May-1987 BROMODICHLOROMETHANE

SD-100-09 26-May-1987 BROMODICHLOROMETHANE

SD-100-09 3-JUn-1987 BROMODICHLOROMETHANE

$\begin{array}{ll}\text { Results } & \text { UnIts } \\ 5 & \mathrm{ug} / \mathrm{L} \\ 5 & \mathrm{ug} / \mathrm{L} \\ 5 & \mathrm{ug} / \mathrm{L} \\ & \\ 6 & \mathrm{ug} / \mathrm{L} \\ 5 & \mathrm{ug} / \mathrm{L} \\ 5 & \mathrm{ug} / \mathrm{L} \\ 5 & \mathrm{ug} / \mathrm{L} \\ 5 & \mathrm{ug} / \mathrm{L} \\ 5 & \mathrm{ug} / \mathrm{L} \\ & \\ & \mathrm{ug} / \mathrm{L} \\ 6 & \mathrm{ug} / \mathrm{L} \\ 5 & \\ & \mathrm{ug} / \mathrm{L} \\ & \mathrm{ug} / \mathrm{L} \\ 6 & \mathrm{ug} / \mathrm{L} \\ 5 & \mathrm{ug} / \mathrm{L} \\ 5 & \mathrm{ug} / \mathrm{L} \\ 5 & \mathrm{ug} / \mathrm{L} \\ 5 & \end{array}$

ug/L

ug/ $/ \mathrm{L}$ ug $/$. Ug/L $u g / L$ $4 \mathrm{~g} / \mathrm{L}$ $\mathrm{ug} / \mathrm{L}$

$<5$

ug/L $\mathrm{ug} / \mathrm{L}$ $\mathrm{ug} / \mathrm{L}$ ug/L ug/L ug/L

ug/L

$\begin{array}{ll}5 & \mathrm{ug} / \mathrm{L} \\ 5 & \mathrm{ug} / \mathrm{L} \\ 5 & \mathrm{ug} / \mathrm{L} \\ 5 & \mathrm{ug} / \mathrm{L}\end{array}$


Storm Dra1n Data for SD-100

Location

Date

Test Compound

SD - $100-09$

SD- $100-09$

11-JUN-1987 BROMODI CHLOROMETHANE

18-Jun-1987 BROMODICKLOROMETHATE

** SD-100-10

SD-100-10 12-May-1987 BROMODICHLOROAIETRAME

SD-100-10 19-MaY-1987 BROMODICHLOROMETHANE

SD-100-10 26-Mry-1987 BROMODICHLOROMETHANE

SD-100-10 3-Jun-1987 BROMODICHLOROFITIANE

SD-100-10 11-JUn-1987 BROMODICHLOROMETTIANE

SD-100-10 18-Jun-1987 BROKODICHLOROKETHANE

** SD - 100-11

SD-100-11 12-May-1987 BROMODICHLOROMETHAXE

SD-100-11 19-May-1987 BROMODICHLOROMETHANE

SD-100-11 26-May-1987 BROMODICHLOROMETHANE

SD-100-11 3-JUn-1987 BROMODICHLOROMETHANE

SD-100-11 11-JUn-1987 BROMODICHLOROMETHANE

SD-100-11 18-Jun-1987 BROMODICHLOROMETHANE

** SD $-100-12$

SD-100-12 12-May-1987 BROMODICHIOROMETTANE

SD-100-12 19-May-1987 BROMODICHLOROMETHANE

SD-100-12 26-May-1987 BROMODICHLOROMETHARE

SD-100-12 3-JUn-1987 BROMODICHLOROMETHANE

SD-100-12 11-JUn-1987 BROMODICHLOROMETHANE

SD-100-12 18-Jun-1987 BROMODICHLOROMETHANE

* SD $-100-13$

SD-100-13 12-May-1987 BROMODICHLOROMETHANE

SD-100-13 19-May-1987 BROMODICHLOROMETHUNE

SD-100-13 26-May-1987 BROMODICHLOROMETHANE

SD-100-13 3-Jun-1987 BROMODICHLOROMETHANE

SD-100-13 11-Jun-1987 BROMODICHLOROMETHANE

SD-100-13 18-Jun-1987 BROMODICHLOROMEIHANE

** SD $-100 \cdot 14$

SD-100-14 12-May-1987 BROMODICHLOROMETHANE

SD-100-14 19-May-1987 BROMODICZLOROMETHANE

SD-100-14 26-May-1987 BROMODICHLOROMETHANE

SD-100-14 3-Jun-1987 BROMODICHLOROMETHANE

SD-100-14 11-JUn-1987 BROMODICHLOROMETHANE

SD-100-14

18-JUN-1987 BROMODICELOROMETHANE

** SD-100-C

SD-100-C 5-Mar-1987 BROMODICHLOROMETHANE

SD-100-C 9-Mar-1987 BROMODICHLOROMETHANE

SD-100-C

SD $-100-C$

SD $-100-C$

SD $-100-C$

SD $-100 \cdot C$

17-Mar-1987 BROMODICHLOROMETHANE

24-MaT-1987 BROMODICHLOROMETHANE

8-APE-1987 BROMODICHLOROMETHANE

13-May-1987 BROMODICHLOROMETHANE

20-May-1987 BROMODICHLOROMETHANE
Results Units

5

ug/L

$\mathrm{ug} / \mathrm{L}$

$u g / L$

$\mathrm{ug} / \mathrm{L}$

ug/L

ug $/ \mathrm{L}$

ug/L

ug/L

ug/L

ug/L

ug/L

ug/L

$\operatorname{ug} / \mathrm{L}$

ug/L

ug/L

ug/L

$u g / L$

ug/L

ug/L

ug/L

$\begin{array}{ll}11 & \text { ug/L } \\ 5 & \text { ug/L } \\ 5 & \text { ug/L } \\ 5 & \text { ug/L } \\ 5 & \text { ug/L } \\ 5 & \text { ug/L }\end{array}$

5

$8 \quad \mathrm{ug} / \mathrm{L}$

5 ug/L

$5 u g / L$

5 ug/h

$5 \quad \mathrm{ug} / \mathrm{L}$
ug/L

ug/L

Ug/L

ug/L

ug/L

ug/L

ug/L 
Stom Drain Data for SD-100

$\begin{array}{ccc}\text { Location } & \text { Date } & \text { Test Compound } \\ \text { SD-100-C } & \text { 27-May-1987 } & \text { BROHODICHLOROMETHANE } \\ \text { SD-100-C } & \text { 12-Jun-1987 } & \text { BROHODICHLOROMETHANE } \\ \text { SD-100-C } & \text { 19-Jun-1987 } & \text { BROHODICHLOROHETHANE }\end{array}$

$\begin{array}{ll}\text { Results } & \text { Units } \\ 14 & \text { ug/L } \\ 6 & u g / L \\ 5 & u g / L\end{array}$

* SD-100-D

SD-100-D 6-Apr-1987 BROMODICHLOROMETHANE

SD-100-D 11-Jun-1987 BROMODICHLOROMETHANE

$10 \quad$ ug/ $/ 2$

$5 \quad$ ug/L

** SD -100

SD-100 S-Mar-1987 BROMOFORY

SD-100 11-Mar-1987 BROMOFORM

SD-100 18-Mar-1987 BROMOFORM

SD-100 24-Max-1987 BROMOFORM

SD-100 6-API-1987 BROKOFORH

SD-100 12-May-1987 BROMOFORM

SD-100 19-May-1987 BROMOFORM

SD-100 26-May-1987 BROHOFORM

SD-100 3-Jun-1987 BROYOFORM

SD-100 11-Jun-1987 BROHOFORM

SD-100 18-Jun-1987 BROHOFORM

$<5$

ug/L

Ug/L

$5 \mathrm{~J} \quad$ ug/L

$<5 \quad$ ug/L

$<5 \quad u g / L$

$5 \quad \mathrm{ug} / \mathrm{L}$

$5 \quad u g / L$

$<5$ ug/L

$<5 \quad u g / L$

$5 \quad$ ug/

** SD-100-01

SD-100-01 12-May-1987 8ROMOFORM

SD-100-01 18-May-1987 BROMOFORM

SD-100-01 26-May-1987 BROMOFORM

SD-100-01 3-Jun-1987 BROHOFORM

SD-100-01 11-Jun-1987 BROMOFORM

SD-100-01 18-Jun-1987 BROHOFORM

$<5$

$<5$

$<5$

$<5$

$<5$

$<5$

ug/ $/$

$u g / L$

$\mathrm{ug} / \mathrm{L}$

ug/L

$u g / L$

ug $/ \mathrm{L}$

** SD 100.02

SD-100-02 12-May-1987 BROMOFORM

$<5$

SD-100-02 18-May-1987 BROKOFORM

SD-100-02 26-May-1987 BROMOFORM

SD-100-02 3-Jun-1987 BROHOFORM

SD-100-02 11-Jun-1987 BROMOFORM

SD-100-02 18-Jun-1987 BROMOFORM

$4 \mathrm{~g} / \mathrm{L}$

$<5 \quad 4 \mathrm{~g} / \mathrm{L}$

$<5 \quad$ ug/L

$<5$

$<5$

ug/L

* SD $-100-03$

SD-100-03 12-May-1987 BROHOFORM

SD-100-03 18-May-1987 BROMOFORM

SD-100-03 26-May-1987 BROMOPORM

SD-100-03 3-Jun-1987 BROMOFORM

SD-100-03 11-Jun-1987 BROHOFORM

SD-100-03 18-Jun-1987 BROKOFORM

$<5$

$<5$

$<5$

$<5$

$<5$

$<5$

$\mathrm{ug} / \mathrm{L}$

** SD-100-03D

SD-100-03D 12-May-1987 BROMOFORM

SD-100-03D 26-May-1987 BROMOFORM

$<5$

$<5$

ug $/ 2$

$u g / L$

$u g / L$

ug $/ \mathrm{L}$

$\mathrm{ug} / \mathrm{L}$

ug/L

* SD-100-04

SD-100-04 12-May-1987 BROHOFORM

$<5$

ug/L

ug/L

ug/L 
Storm Drain Data for SD-100

\begin{tabular}{|c|c|c|c|c|}
\hline Location & Date & Test Compound & Results & Units \\
\hline SD-100-04 & 18-May - 1987 & BROKOFORY & $<5$ & $u g / L$ \\
\hline SD-100-04 & 26-May-1987 & BRONOFORY & $<5$ & $\mathrm{ug} / \mathrm{L}$ \\
\hline SD-100-04 & 3-Jun- 1987 & BROSOFORY & $<5$ & $\operatorname{ug} / \mathrm{L}$ \\
\hline SD-100-04 & 11-Jun-1987 & BRCYOFORY & $<5$ & $u g /$ \\
\hline SD-100-04 & $18-J u n-1987$ & BROSOFORY & $<5$ & $u g / L$ \\
\hline \multicolumn{5}{|c|}{$* *$ SD $-100-06$} \\
\hline SD-100-06 & $12-\mathrm{May}-1987$ & BROYOFORM & 5 & $u g / L$ \\
\hline SD- $100-06$ & $18-$ May -1987 & BROYOFORY & 5 & $4 \mathrm{~g} / \mathrm{L}$ \\
\hline SD- $100-06$ & $26-$ May - 1987 & BROMOFORY & $<5$ & $48 / 2$ \\
\hline SD-100-06 & $3-J$ un -1987 & BROMOFORH & $<5$ & $\operatorname{ug} \Omega$ \\
\hline SD. $100-06$ & 11-Jun-1987 & BROYOFORM & $<5$ & ug $/ \mathrm{L}$ \\
\hline SD-100-06 & 18-Jun-1987 & BROMOFORY & $<5$ & $48 / 2$ \\
\hline \multicolumn{5}{|c|}{$\star * \quad S D-100-06 D$} \\
\hline SD $-100-06 D$ & 3-Jun- 1987 & BROHOFORM & $<5$ & ug/L \\
\hline \multicolumn{5}{|c|}{$\star \star \quad S D-100-07$} \\
\hline SD - 100-07 & $12 \cdot$ May - 1987 & BROKOFORY & $<5$ & $u g / L$ \\
\hline SD-100-07 & $19-$ May -1987 & BROYOFORM & $<5$ & $u g / \mathrm{L}$ \\
\hline SD $-100-07$ & $26-$ May- 1987 & BROMOFORM & $<5$ & $\mathrm{ug} / \mathrm{L}$ \\
\hline SD- $100-07$ & 3-Jun-1987 & BROMOFORM & $<5$ & $u g / L$ \\
\hline SD $-100-07$ & $11-J$ un -1987 & BROMOFORM & $<5$ & $4 \mathrm{~g} / \mathrm{L}$ \\
\hline SD-100-07 & $18-J$ un -1987 & BROMOFORM & $<5$ & $\mathrm{ug} / \mathrm{L}$ \\
\hline \multicolumn{5}{|c|}{$\star * \quad S D-100-07 D$} \\
\hline SD-100-07D & $18-J u n-1987$ & BROMOFORM & $<5$ & $u g / L$ \\
\hline \multicolumn{5}{|c|}{$\star \star$ SD -100.09} \\
\hline$S D-100-09$ & 12-May-1987 & BROMOFORM & $<5$ & $\mathrm{ug} / \mathrm{L}$ \\
\hline So-100-09 & $18 \cdot$ - May -1987 & BROMOFORY & $<5$ & ug/L \\
\hline SD-100-09 & 26-May - 1987 & BROHOFORM & $<5$ & $\mathrm{ug} / \mathrm{L}$ \\
\hline SD -100.09 & 3-Jun- 1987 & BROMOFORM & $<5$ & ug $/$ \\
\hline SD-100-09 & 11 -Jun- 1987 & BROMOFORM & $<5$ & $u_{g} / L$ \\
\hline SD $-100-09$ & $18-J u n-1987$ & BROHOFORM & $<5$ & $\mathrm{ug} / \mathrm{l}$ \\
\hline \multicolumn{5}{|c|}{$\star *$ SD $-100-10$} \\
\hline$S D-100-10$ & 12-May- 1987 & BROMOFORM & $<5$ & $\mathrm{ug} / \mathrm{L}$ \\
\hline$S D-100-10$ & 19-May - 1987 & BROMOFORY & $<5$ & $\mathrm{ug} / \mathrm{L}$ \\
\hline SD $-100-10$ & $26-$ May- 1987 & BROMOFORY & $<5$ & $\mathrm{ug} / \mathrm{L}$ \\
\hline SD $-100-10$ & 3-Jun- 1987 & BROMOFORK & $<5$ & $\mathrm{ug} / \mathrm{L}$ \\
\hline SD $-100-10$ & 11 -Jur- 1987 & BROMOFORM & $<5$ & $\mathrm{ug} / \mathrm{L}$ \\
\hline SD $-100 \cdot 10$ & $18-$ Jun- 1987 & BROMOFORM & $<5$ & ug/L \\
\hline \multicolumn{5}{|c|}{$\star$ SD $-100-11$} \\
\hline SD - $100-11$ & $12-\mathrm{May}-1987$ & BROMOFORM & $<5$ & ug/L \\
\hline SD-100-11 & 19-May-1987 & BROMOFORM & 5 & $u g / L$ \\
\hline SD - 100-11 & 26-May- 1987 & BROMOFORY & $<5$ & ug/L \\
\hline SD-100-11 & $3-J u n-1987$ & BROMOFORM & $<5$ & $\mathrm{ug} / \mathrm{L}$ \\
\hline SD-100-11 & $11-J u n-1987$ & BROMOFORY & $<5$ & $u g / L$ \\
\hline SD-100-11 & $18-J u n-1987$ & BROMOFORM & $<5$ & $u g / L$ \\
\hline
\end{tabular}


Storm Drain Data for SD-100

Location Date Test Compound Results Units

** SD $-100-12$

SD-100-12 12-May-1987 BROMOFORM

SD-100-12 19-May-1987 BROYOFORY

SD-100-12 26-May-1987 BROAOFORM

SD-100-12 3-Jun-1987 BROMOFORM

SD-100-12 11-Jun-1987 BROMOFORY

SD-100-12 1B-JUn-1987 BROKOFORY

$<5$

$<5$

$<5$

$<5$

$<5$

$<5$

ug/L

$u g / L$

$4 \mathrm{~g} / \mathrm{L}$

$\mathrm{ug} / \mathrm{L}$

$4 \mathrm{~g} / \mathrm{L}$

$4 \mathrm{~g} / \mathrm{L}$

** SD-100-13

SD-100-13 12-May-1987 BROMOFORM

SD-100-13 19-May-1987 BROMOFORY

SD-100-13 26-May-1987 BROMIOFORY

SD-100-13 3-Jun-1987 BROMOFORM

SD-100-13 1I-Jun-1987 BROMOFORM

SD-100-13 18-Jun-1987 BROMOFORM

$<5$

$<5$

$<5$

$<5$

$<5$

$<5$

$u g / 1$

ug $/ 1$

$u g / L$

ug $/$.

$u g / L$

$\mathrm{ug} / \mathrm{L}$

** SD-100-14

SD-100-14 12-May-1987 BROMOFORM

SD-100-14 19-May-1987 BROMOFORM

SD-100-14 26-May-1987 BROMOFORM

SD-100-14 3-Jun-1987 BROHOFORY

SD-100-14 11-Jun-1987 BROMOFORM

SD-100-14 18-Jun-1987 BROMOFORM

$<5$

$<5$

$<5$

$<5$

$<5$

$<5$

$u g / L$

ug/L

$\mathrm{ug} / \mathrm{L}$

ug $/ 2$

$\mathrm{ug} / \mathrm{L}$

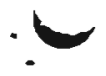

** SD-100-C

SD-100-C 5-MaI-1987 BROMOFORM

SD-100-C 9-Mar-1987 BROHOFORM

SD-100-C 17-Mar-1987 BROMOFORM

SD-100-C 24-Mar-1987 BROMOPORM

SD-100-C 8-APT-1987 BROMOFORM

SD-100-C 13-May-1987 BROMOFORM

SD-100-C 20-May-1987 BROHOFORM

SD-100-C 27-May-1987 BROMOFORM

SD-100-C 12-Jun-1987 BROMOFORM

SD-100-C 19-Jum-1987 BROMOFORH

<S ug/L

$<5 \quad 4 \mathrm{~g} / \mathrm{L}$

$<5 \quad u g / L$

$<5 \quad$ ug $/ \mathrm{L}$

$1 \mathrm{~J} \quad \mathrm{ug} / \mathrm{L}$

$7 \quad \mathrm{ug} / \mathrm{L}$

$5 \quad$ ug $/ 2$

$5 \quad \mathrm{ug} / \mathrm{L}$

$5 \quad 4 \mathrm{~g} / \mathrm{I}$

$5 \quad$ ug/L

** SD-100-D

SD-100-D 6-Apr-1987 BROMOFORM

SD-100-D 11-Jun-1987 BROMOFORM

$<5 \quad 4 \mathrm{~g} / \mathrm{L}$

$<5 \quad$ ug/l

* SD -100

SD-100 S-Mar-1987 BROMOIETHANE

SD-100 11-Mar-1987 BROMOLETHANE

SD-100 18-Mar-1987 BROMOMETHANE

SD-100 24-Mar-1987 BROMOAITHANE

SD-100 6-APY-1987 BROMOMETHANE

SD-100 12-May-1987 BROMOMETHANE

SD-100 19-May-1987 BROMOMETHANE

SD-100 26-May-1987 BROMOMETHANE

SD-100 3-Jun-1987 BROMOKETHANE

$\begin{array}{ll}<10 & \text { ug/L } \\ <10 & \mathrm{ug} / \mathrm{L} \\ <10 & \mathrm{ug} / \mathrm{L} \\ <10 & \mathrm{ug} / \mathrm{L} \\ <10 & \mathrm{ug} / \mathrm{L} \\ <10 & \mathrm{ug} / \mathrm{L} \\ <10 & \mathrm{ug} / \mathrm{L} \\ <10 & \mathrm{ug} / \mathrm{L} \\ <10 & \mathrm{ug} / \mathrm{L}\end{array}$


Storn Draln Data for SD-100

Location

Date

Test Compound

SD -100

11-JUn- 1987 BROMOMETHANE

SD -100

18-Jun-1987 BROMOHETHANE
** SD-100-01

SD-100-01 12-May-1987 BROMOMETHANE

SD-100-01 18-May-1987 BROMOMETHANE

SD-100-01 26-May-1987 BROMOMETHANE

SD-100-01 3-Jun-1987 BROYOMETHANE

SD-100-01 11-Jun-1987 BROMONETHANE

SD-100-01 18-Jun-1987 BROMOMETHANE

* SD-100-02

SD-100-02 12-May-1987 BROMOMETHANE

SD-100-02 18-May-1987 BROMOMETHANE

SD-100-02 26-May-1987 BROMOMETHANE

SD-100-02 3-Jun-1987 BROMOMETHANE

SD-100-02 11-JUn-1987 BROMOMETHANE

SD-100-02 18-Jun-1987 BROMOMETHANE

* SD-100-03

SD-100-03 12-May-1987 BROMOMETHANE

SD-100-03 18-May-1987 BROMOMETHANE

SD-100-03 26-May-1987 BROMOMETHANE

SD-100-03 3-Jun-1987 BROMOMETHANE

SD $=100-03 \quad 11$-JUn-1987 BROMOMETHLANE

SD-100-03 18-Jun-1987 BROMOMETHANE

** SD-100-03D

SD-100-03D 12-May-1987 BROMOMETHANE

SD-100-03D 26-May-1987 BROMOMETHANE

** SD-100-04

SD-100-04 12-May-1987 BROMOKETHANE

SD $=100-04 \quad 18$-May-1987 BROMOMETHANE

SD-100-04 26-May-1987 BROMOMETHANE

SD-100-04 3-Jun-1987 BROMOKETLANE

SD-100-04 11-Jun-1987 BROMOKETHANE

SD-100-04 18-Jun-1987 BROMOMETHANE

* SD $100-06$

SD-100-06 12-May-1987 BROMOMETHANE

SD-100-06 18-May-1987 BROMOMETHANE

SD-100-06 26-May-1987 BROMOMETHANE

SD-100-06 3-Jun-1987 BROMOMETHANE

SD-100-06 11-Jun-1987 BROMOMETHANE

SD-100-06 18-Jun-1987 BROMOMETHANE

* SD-100-06D

SD-100-06D 3-JUn-1987 BROMOMETHANE

$\begin{array}{cc}\text { Results } & \text { Units } \\ <10 & \begin{array}{l}u g / L \\ \text { ug/L }\end{array} \\ <10 & \\ & \\ & \\ <10 & u g / L \\ <10 & u g / L \\ <10 & u g / L \\ <10 & u g / L \\ <10 & u g / L \\ <10 & u g / L\end{array}$

$\begin{array}{ll}<10 & u g / L \\ <10 & \text { ug/L } \\ <10 & \text { ug } / \mathrm{L} \\ <10 & \text { ug/L } \\ <10 & \text { ug/L } \\ <10 & \text { ug/L }\end{array}$

$<10 \quad$ ug/L

$<10 \quad$ ug/L

$<10 \quad$ ug/L

$<10 \quad$ ug/L

$<10 \quad \mathrm{ug} / \mathrm{L}$

$<10 \quad$ ug/L

$<10 \quad$ ug/L

$<10 \quad$ ug $\sqrt{2}$

$<10 \quad \mathrm{ug} / \mathrm{L}$

$<10 \quad$ ug $/ \mathrm{L}$

$<10 \quad$ ug/L

$<10 \quad$ ug/L

$<10 \quad$ ug/L

$<10 \quad \mathrm{ug} / \mathrm{L}$

$<10$

$<10$

$<10$

$<10$

$<10$

$<10$

$\mathrm{ug} / \mathrm{L}$.

ug/L

$u_{\mathrm{g} / L}$

ug/L.

ug/L

$u g / 2$

$<10 \quad u g / L$ 
Storm Drain Data for SD-100

Location Date Test Compound Results Units

$\begin{array}{llll}* \star & \text { SD-100-07 } & \\ \text { SD-100-07 } & 12 \text {-May-1987 } & \text { BROMOMETHANE } \\ \text { SD-100-07 } & 19 \text {-May-1987 } & \text { BROMOMETHANE } \\ \text { SD-100-07 } & 26 \text {-May-1987 } & \text { BROMOMETHANE } \\ \text { SD-100-07 } & \text { 3-Jun-1987 } & \text { BROMOMETHANE } \\ \text { SD-100-07 } & 11-J u n-1987 & \text { BROMOMETHANE } \\ \text { SD-100-07 } & \text { 18-Jun-1987 } & \text { BROMOMETHANE }\end{array}$

$<10$

$u g / L$

$<10$

$<10$

$<10$

$<10$

$<10$

ug/L

$u g / L$

$\mathrm{ug} / \mathrm{L}$

ug/L

$u g / L$

** SD-100-07D

SD-100-07D 18-Jun-1987 BROMOMETHANE

$<10$

$u g / \mathrm{L}$

* SD-100-09

SD-100-09 12-MaY-1987 BROMOMETHANE

SD-100-09 18-May-1987 BROMOMETHANE

SD-100-09 26-May-1987 BROMOMETHANE

SD-100-09 3-Jun-1987 BROMOMETHANE

SD-100-09 11-Jun-1987 BROMOMETHANE

SD-100-09 18-JUn-1987 BROMOMETHANE

$<10$

$<10$

$<10$

$<10$

$<10$

$<10$

ug/L

ug $/ \mathrm{L}$

ug $/ \mathrm{L}$

ug/L

$\mathrm{ug} / \mathrm{L}$

$u g / 2$

* SD-100-10

SD-100-10 12-May-1987 BROMOMETHANE

SD-100-10 19-May-1987 BROMOMETHANE

SD-100-10 26-May-1987 BROMOMETHANE

SD-100-10 3-Jun-1987 BROMOMETHANE

SD-100-10 11-Jun-1987 BROMOMETHANE

SD-100-10 18-Jun-1987 BROMOMETHANE

$<10$

$<10$

$<10$

$<10$

$<10$

$<10$

ug/ $/$

ug/L

$4 \mathrm{~g} / \mathrm{L}$

ug $\Omega$

ug $/ \mathrm{L}$

$u g / 2$

** SD-100-11

SD-100-11 12-May-1987 BROMOMETHANE

SD-100-11 19-May-1987 BROMOMETHANE

SD-100-11 26-May-1987 BROMOMETHANE

SD-100-11 3-JUR-1987 BROMOMETHANE

SD-100-11 11-JUn-1987 BROMOMETHANE

SD-100-11 18-JUn-1987 BROMOMETHANE

$<10$

$<10$

$<10$

$<10$

$<10$

$<10$

$\mathrm{ug} / \mathrm{L}$

$48 / 2$

$4 \mathrm{~g} / \mathrm{L}$

$u g / L$

$u g / L$

ug/h

* SD-100-12

SD-100-12 12-May-1987 BROMOMETHANE

SD-100-12 19-May-1987 BROMOMETHANE

SD-100-12 26-May-1987 BROMOMETHANE

SD-100-12 3-JUn-1987 BROMOMETHANE

SD-100-12 11-JUn-1987 BROMOMETHANE

SD-100-12 18-JUn-1987 BROMOMETHANE

$<10$

$<10$

$<10$

$<10$

$<10$

$<10$

ug/L

$u g / L$

$u g / L$

$u g / L$

$\mathrm{ug} / \mathrm{L}$

ug $/ \mathrm{L}$

* SD $-100-13$

SD-100-13 12-May-1987 BROMOMETHANE

SD-100-13 19-May-1987 BROMOMETHANE

SD-100-13 26-May-1987 BROMOMETHANE

SD-100-13 3-Jun-1987 BROMOMETHANE

SD-100-13 11-JUn-1987 BROMOMETHANE

SD-100-13 18-Jun-1987 BROMOMETHANE

ug $/ \mathrm{L}$

$<10$

$<10$

$<10$

$<10$

$u g / L$

$\mathrm{ug} / \mathrm{L}$

ug/L

$\mu g / L$

$4 \mathrm{~g} / \mathrm{L}$ 
Storn Draln Data for SD-100

Location Date Test Compound

Results Units

** SD $-100-14$

SD-100-14 12-May-1987 BROMOMETHANE

SD-100-14 19-May-1987 BROKOLIETHANE

SD-100-14 26-May-1987 BROMOHETLANE

SD-100-14 3-Jun-1987 BROMOMETHANE

SD-100-14 11-Jun-1987 BROMOKETHANE

SD-100-14 18-Jun-1987 BROMOMETHANE

* SD-100-C

SD-100-C 5-Mar-1987 BROMONETHANE

SD-100-C 9-Mar-1987 BROMONETHANE

SD-100-C 17-Mar-1987 BROMOMETHANE

SD-100-C 24-Mar-1987 BROMOMETHANE

SD-100-C 8-Apr-1987 BROMOMETHANE

SD-100-C 13-May-1987 BROMOMETHANE

SD-100-C 20-May-1987 BROMOLETHANE

SD-100-C 27-May-1987 BROMOMETHANE

SD-100-C 12-Jun-1987 BROMOMETHANE

SD-100-C 19-Jun-1987 BROMOMETHANE

$\begin{array}{ll}<10 & \mathrm{ug} / \mathrm{L} \\ <10 & \mathrm{ug} / \mathrm{L} \\ <10 & \mathrm{ug} / \mathrm{L} \\ <10 & \mathrm{ug} / \mathrm{L} \\ <10 & \mathrm{ug} / \mathrm{L} \\ <10 & \mathrm{ug} / \mathrm{L}\end{array}$

** SD-100-D

SD-100-D 6-Apr-1987 BROMOHETHANE

SD-100-D 11-JUn-1987 BROMOMETHANE

** SD-100-C

SD-100-C 8-APr-1987 BUTYL ALCOHOL

SD-100-C 5-Mar-1987 BUTYL CARBITOL

SD-100-C 9-Mar-1987 BUTYL CARBITOL

SD-100-C 24-Mar-1987 BUTYL GARBITOL

$<10$

$<10$

$<10$

$<10$

$<10$

$<10$

$<10$

$<10$

$<10$

$<10$

$\operatorname{ug} /$ L

ug/L

ug/L

ug/L

$u g / L$

ug/L

$\mathrm{ug} / \mathrm{L}$

ug/L

$\mathrm{ug} / \mathrm{L}$

$u g / L$

* SD -100

SD -100

5-Mar-1987 BUTYLBENZYLPHTHALATE

SD -100

11-Mar-1987 BUTYLBENZYLPHTHALATE

SD -100

12-May-1987 BUTYLBENZYLPHTHALATE

SD -100

26-May-1987 BUTYLBENZYLPHTHALATE

* SD-100-01

SD-100-01 12-May-1987 BUTYLBENZYLPHTHALATE

SD-100-01 26-May-1987 BUTYLBENZYLPHTHALATE

$\star \star \quad S D-100-02$

SD-100-02 12-May-1987 BUTYLBENZYLPHTHALATE

SD-100-02 26-May-1987 BUTYLBENZYLPHTHALATE

** SD $-100-03$

SD-100-03 12-May-1987 BUTYLBENZYLPHTHALATE

SD-100-03 26-May-1987 BUTYLBENZYLPHTHALATE

$\star \star \quad$ SD $-100-03 D$

SD-100-030 12-May-1987 BUTYLBENZYLPHTHALATE

SD-100-03D 26-MaY-1987 BUTYLBENZYLPHTHALATE

$\begin{array}{ll}<10 & \mathrm{ug} / \mathrm{L} \\ <10 & \mathrm{ug} / \mathrm{L}\end{array}$

$870 \quad u g / L$

$930 \mathrm{ug} / \mathrm{L}$

$690 \quad \mathrm{ug} / \mathrm{L}$

$2 \quad \mathrm{ug} / \mathrm{L}$

$<10 \quad$ ug $/ 2$

$1 \mathrm{~J} \quad$ ug/L

$<10 \quad$ ug $/ \mathrm{L}$

$<5.0 \quad$ ug/L

$<10 \quad$ ug/L

$<5.0 \quad$ ug $/ \mathrm{L}$

$<10 \quad$ ug/L

$<5.0 \quad \mathrm{HB} / \mathrm{L}$

$<10 \quad$ ug/L

$<5.0 \quad$ ug $/ \mathrm{L}$

$<10 \quad$ ug/t.

$<5.0 \quad \mathrm{ug} / \mathrm{L}$ 
Storm Drain Data for SD-100

Locetion Date Test Compound

Results Units

** SD $-100-04$

SD-100-04 12-May-1987 BUTYLBENZYLPHTHALATE SD-100-04 26-MaY-1987 BUTYLBENZILPHTHALATE

$<10$

$<5.0$

$\mathrm{ug} / \mathrm{L}$

* SD -100.06

SD-100-06 12-May-1987 BUTYLBENZYLPHTHALATE

SD-100-06 26-May-1987 BUTYLBNZYLPHTHALATE

$\begin{array}{ll}<10 & \mathrm{ug} / \mathrm{L} \\ <5.0 & \mathrm{ug} / \mathrm{L} \\ & \\ <10 & \mathrm{ug} / \mathrm{L} \\ <5.0 & \mathrm{ug} / \mathrm{L} \\ & \\ <10 & \mathrm{ug} / \mathrm{L} \\ <5.0 & \mathrm{ug} / \mathrm{L} \\ & \\ <10 & \mathrm{ug} / \mathrm{L} \\ <5.0 & \mathrm{ug} / \mathrm{L}\end{array}$

** SD-100-07

SD-100-07 12-May-1987 BUTYLBENZYLPHTHALATE

SD-100-07 26-May-1987 BUTYLBENZYLPHTHALATE

* SD-100-09

SD-100-09 12-May-1987 BUTYLBENZYLPHTHALATE

SD-100-09 26-May-1987 BUTYLBENZYLPHTHALATE

** SD $-100-10$

SD-100-10 12-May-1987 BUTYLBENZYLPHTHALATE

SD-100-10 26-May-1987 BUTYLBENZYLPHTHALATE

* SD-100-11

SD-100-11 12-May-1987 BUTYLBENZYLPHTHALATE

SD-100-11 26-May-1987 BUTYLBENZYLPHTHALATE

** SD $-100-12$

SD-100-12 12-May-1987 BUTYBENZYLPHTHALATE

SD-100-12 26-May-1987 BUTYLBENZYLPHTHALATE

** SD $-100-13$

SD-100-13 12-May-1987 BUTYLBENZXLPHTHALATE

SD-100-13 26-May-1987 BUTYBBENZYLPHTHALATE

* SD $-100-14$

SD-100-14 12-May-1987 BUTYLBENZYLPHTHALATE

SD-100-14 26-May-1987 BUTYLBENZYLPHTHALATE

$\star \star$ SD $-100 \cdot C$

SD-100-C 5-Mar-1987 BUTYLBENZYLPHTHALATE

SD-100-C 9-Mar-1987 BUTYLENZYLPHTHALATE

SD-100-C 17-Mar-1987 BUTYLBENZYLPHTHALATE

SD-100-C 24-Mar-1987 BUTYLBENZYLPHTHALATE

SD-100-C 8-APT-1987 BUTYLBENZYLPHTHALATE

SD-100-C 13-May-1987 BUTYLBENZYLPHTIALATE

SD-100-C 20-May-1987 BUTYLBENZYLPHTHAIATE

SD-100-C 27-May-1987 BUTYLBENZYLPHTHALATE

SD-100-C 12-JUn-1987 BUTYLBENZYLPHTHALATE

SD-100-C 19-Jun-1987 BUTYLBENZTI.PHTHALATE

* SD-100

SD $-100 \quad 5-$ Mar -1987 CADMIUM

$<10 \quad$ ug/L

$<5.0 \quad$ ug/L

$<10 \quad$ ug $\Omega$

$<5.0 \quad \mathrm{ug} / \mathrm{L}$

$<10 \quad$ ug/L

$<5.0 \quad$ ug/L

$<10 \quad$ ug $/ 2$

$<5.0 \quad$ ug/L

$<10 \quad$ ug/

$<10 \quad$ ug/L

$<10$ ug/

$<10 \quad$ ug/L

$1 \mathrm{~J} \quad \mathrm{ug} / \mathrm{L}$

$<5.0 \quad u g / L$

$<5.0 \quad \mathrm{ug} / \mathrm{L}$

$<5.0 \quad \mathrm{ug} / \mathrm{L}$

$<5.0 \quad$ ug/L

$<10$ ug/L

$<0.0030 \mathrm{mg} / \mathrm{L}$ 


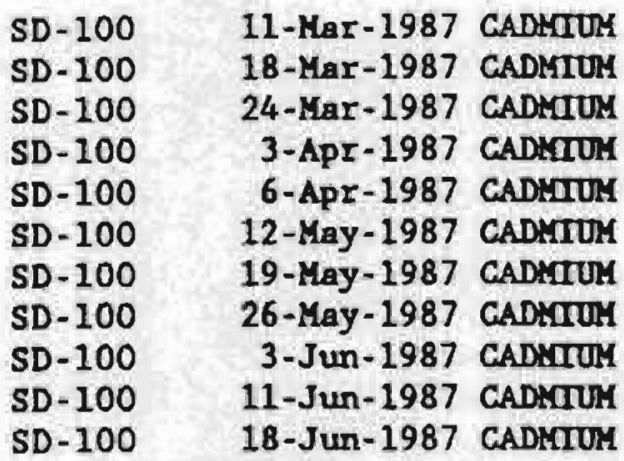

$\begin{array}{ll}<0.0030 & \mathrm{mg} / \mathrm{L} \\ <0.0030 & \mathrm{mg} / \mathrm{L} \\ <0.0030 & \mathrm{mg} / \mathrm{L} \\ <0.0030 & \mathrm{mg} / \mathrm{L} \\ <0.0030 & \mathrm{mg} / \mathrm{L} \\ <0.0030 & \mathrm{mg} / \mathrm{L} \\ <0.0030 & \mathrm{mg} / \mathrm{L} \\ <0.0030 & \mathrm{mg} / \mathrm{L} \\ <0.0030 & \mathrm{mg} / \mathrm{L} \\ <0.0030 & \mathrm{mg} / \mathrm{L} \\ <0.0030 & \mathrm{mg} / \mathrm{L}\end{array}$

* SD-100-01

SD-100-01 12-May-1987 CADMTUM SD-100-01 18-May-1987 CADMTUM SD-100-01 3-Jun-1987 CADAIUK SD-100-01 11-Jun-1987 CADHIIUY SD-100-01 28-Jun-1987 GADKTUM

** SD $-100-02$

SD-100-02 12-May-1987 CADAIIUI SD-100-02 18-May-1987 CADLTUY SD-100-02 3-Jun-1987 CADMTUM SD-100-02 11-Jun-1987 CADMIUM SD-100-02 18-Jun-1987 CADKIUM

* SD-100-03

SD-100-03 12-May-1987 CADMTUY SD-100-03 18-May-1987 CADMIUM SD-100-03 26-May-1987 CADMIUM SD-100-03 3-Jun-1987 CAD:TIU SD-100-03 11-Jun-1987 CADKTUM SD-100-03 18-Jun-1987 CADMTUM

** SD-100-03D

SD-100-03D 12-May-1987 CADACUM SD-100-03D 26-Mey-1987 CADKTUM

** SD-100-04

SD-100-04 12-May-1987 CADHIUM SD-100-04 18-May-1987 CADLIUU SD-100-04 26-May-1987 CADMIUM SD-100-04 3-Jun-1987 CADHIUY SD-100-04 11-Jun-1987 CADHITM SD-100-04 18-Jun-1987 GADMTUM

** SD $-100-06$

SD-100-06 12-May-1987 CADMTUM SD-100-06 18-May-1987 CADMTUM SD-100-06 26-May-1987 CADHIUM SD-100-06 3-Jun-1987 CADMIUM
$<0.0030$

$<0.0030$

$<0.0030$

$<0.0030$

$<0.0030$

$<0.0030$

$<0.0030$

$<0.0030$

$<0.0030$

$<0.0030$

$<0.0030$

$<0.0030$

$<0.0030$

$<0.0030$

$<0.0030$

$<0.0030$

$<0.0030$

$<0.0030$

mg/L

$\mathrm{mg} / \mathrm{L}$

$<0.0030$

$<0.0030$

$<0.0030$

$<0.0030$

$<0.0030$

$<0,0030$

mg/L mg/L. mg/L mg/L $\mathrm{mg} / \mathrm{L}$ $\mathrm{mg} / \mathrm{L}$

$<0.0030$

$<0.0030$

$<0.0030$

$\mathrm{mg} / \mathrm{L}$ $\mathrm{mg} / \mathrm{L}$ $\mathrm{mg} / \mathrm{L}$ $<0.0030$ 
Stom Drain Data for SD-100

Location Date Test Compound

Results Unfts

SD-100-06 11-JUn-1987 CADMIUM SD-100-06 18-Jun-1987 CADMIUM

$<0.0030$

$<0.0030$

$\mathrm{mg} / \mathrm{L}$

-

*t SD-100-06D

SD-100-06D 3-Jun-1987 CADKIUM

$<0.0030 \mathrm{mg} / \mathrm{L}$

* SD-100-07

SD-100-07 12-May-1987 CADMIUM

SD-100-07 19-May-1987 CADMIUM

SD-100-07 26-May-1987 CADHIUM

SD-100-07 3-Jun-1987 CADEITUM

SD-100-07 11-Jun-1987 CADYIUM

SD-100-07 18-Jun-1987 CADMIUM

$<0.0030$

$<0.0030$

$<0.0030$

0.019

$<0.0030$

0.054

$\mathrm{mg} / \mathrm{L}$

$\mathrm{mg} / \mathrm{L}$

$\mathrm{mg} / \mathrm{L}$

$\mathrm{mg} / \mathrm{L}$

mg/L

$\mathrm{mg} / \mathrm{L}$

** SD-100-07D

SD-100-07D 18-Jun-1987 GADYIUM

$0.048 \mathrm{mg} / \mathrm{L}$

* SD-100-09

SD-100-09 12-May-1987 CADMIUM

SD-100-09 18-May-1987 CADMIUM

SD-100-09 26-May-1987 GADMIUM

SD-100-09 3-Jun-1987 CADMIUM

SD-100-09 11-Jun-1987 GADHIIU

$<0.0030$

$<0.0030$

$<0.0030$

$<0.0030$

$<0.0030$

Ing $/ \mathrm{L}$

$\mathrm{mg} / \mathrm{L}$

$\mathrm{mg} / \mathrm{L}$

$\mathrm{mg} / \mathrm{L}$

$\mathrm{mg} / \mathrm{L}$

* SD $-100-10$

SD-100-10 12-May-1987 CADMIUM

SD-100-10 19-May-1987 CADMIUUM

SD-100-10 26-May-1987 CADIIUM

SD-100-10 3-Jun-1987 CADHIUM

SD-100-10 11-Jun-1987 CADKIIUM

SD-100-10 18-Jun-1987 CADYIUM

$<0.0030$

$<0.0030$

$<0.0030$

$<0.0030$

$<0.0030$

$<0.0030$

mg $/ \mathrm{L}$

$\mathrm{mg} / \mathrm{L}$

$\mathrm{mg} / \mathrm{L}$

$\mathrm{mg} / \mathrm{L}$

$\mathrm{mg} / \mathrm{L}$

* SD-100-11

SD-100-11 12-May-1987 CADWIUM

SD-100-11 19-May-1987 CADMIUM

SD-100-11 26-May-1987 GADHIUM

SD-100-11 3-Jun-1987 CADMIUM

SD-100-11 11-Jun-1987 CAOHIUM

SD-100-11 18-Jun-1987 CADHIUU

$<0.0030$

$<0.0030$

$<0.0030$

$<0.0030$

$<0.0030$

$\mathrm{mg} / \mathrm{L}$

** SD $-100-12$

SD-100-12 12-May-1987 CADKUM

SD-100-12 19-May-1987 CADMTUM

SD-100-12 26-May-1987 CADMIUM

SD-100-12 3-Jun-1987 CADMIUM

SD-100-12 11-Jun-1987 CADMIUM

SD-100-12 18-Jun-1987 CADMIUM

$<0.0030$

$\mathrm{mg} / \mathrm{L}$

$\mathrm{mg} / \mathrm{L}$

$\mathrm{mg} / \mathrm{L}$

$\mathrm{mg} / \mathrm{L}$

$\mathrm{mg} / \mathrm{L}$

$\mathrm{mg} / \mathrm{L}$

$<0.0030$

$<0.0030$

$<0.0030$

$<0.0030$

$<0.0030$

$<0.0030$

$\mathrm{mg} / \mathrm{L}$

mg/L

$\mathrm{mg} / \mathrm{L}$

$\mathrm{mg} / \mathrm{L}$

$\mathrm{mg} / \mathrm{L}$

$\mathrm{mg} / \mathrm{L}$

* SD-100-13

SD-100-13 12-May-1987 CADMIUM

SD-100-13 19-May-1987 CADMIUM

$<0.0030$

$\mathrm{mg} / \mathrm{L}$

$<0.0030$

$\mathrm{mg} / \mathrm{L}$ 
Storm Drain Data for SD-100

$\begin{array}{lcc}\text { Location } & \text { Date } & \text { Test } \\ & \\ \text { SD-100-13 } & 26 \text {-May-1987 CADMIUM } \\ \text { SD-100-13 } & 11 \text {-Jun-1987 CADMIUM } \\ \text { SD-100-13 } & 18 \text {-Jun-1987 CADMIUM }\end{array}$

Results Unies

$\star$ SD-100-14

SD-100-14 12-May-1987 GADMIUM

SD-100-14 19-May-1987 CADMIUM

SD-100-14 26-Hay-1987 CADMIUM

SD-100-14 3-Jun-1987 CADMIUM

SD-100-14 11-JUn-1987 CADMIUM

SD-100-14 18-Jun-1987 CADMIUM

$<0.0030$

$<0.0030$

$<0.0030$

mg/L

mg/L

mg/L

* SD-100-C

SD-100-C 5-Mar-1987 CADMIUM

SD-100-C 9-Mar-1987 CADMIUM

SD-100-C 17-Mat-1987 CADMIUM

SD-100-C 24-Mar-1987 GADIIUM

SD-100-C 8-Apr-1987 CADMIUM

SD-100-C 13-May-1987 CADMIUM

SD-100-C 20-May-1987 CADMIUM

SD-100-C 27-May-1987 CADMIUM

SD-100-C 4-Jun-1987 CADMIUM

SD-100-C 12-JUn-1987 CADMIUM

SD-100-C 19-JUn-1987 CADMIUM

$\begin{array}{ll}<0.0030 & \mathrm{mg} / \mathrm{L} \\ 0.0052 & \mathrm{mg} / \mathrm{L} \\ <0.0030 & \mathrm{mg} / \mathrm{L} \\ <0.0030 & \mathrm{mg} / \mathrm{L} \\ <0.0030 & \mathrm{mg} / \mathrm{L} \\ <0.0030 & \mathrm{mg} / \mathrm{L}\end{array}$

** SD-100-D

SD-100-D 6-Apr-1987 GADKIUM

SD-100-D 11-Jun-1987 CADKIUM

$<0.0030 \mathrm{mg} / \mathrm{L}$

$<0.0030 \mathrm{mg} / \mathrm{L}$

$<0.0030 \mathrm{mg} / \mathrm{L}$

$<0.0030 \mathrm{mg} / \mathrm{L}$

$<0.0030 \mathrm{mg} / \mathrm{L}$

$<0.0030 \mathrm{mg} / \mathrm{L}$

$<0.0030 \mathrm{mg} / \mathrm{L}$

$<0.0030 \mathrm{mg} / \mathrm{L}$

$<0.0030 \mathrm{mg} / \mathrm{L}$

$<0.0030 \mathrm{mg} / \mathrm{L}$

$<0.0030 \mathrm{mg} / \mathrm{L}$

$<0.0030 \mathrm{mg} / \mathrm{L}$

$<0.0030 \mathrm{mg} / \mathrm{L}$

** SD -100

SD-100 5-Mar-1987 CALCIUM

SD-100 11-Mar-1987 CALCIUM

SD-100 18-Mar-1987 CALCIUM

SD-100 24-Mar-1987 GALCIUM

SD-100 3-Apr-1987 CALCIUM

SD-100 6-Apr-1987 CALCIUM

SD-100 12-May-1987 CALCIUM

SD-100 19-May-1987 CALCIUM

SD-100 26-May-1987 CALCIUM

SD-100 3-Jun-1987 GALCIUM

SD-100 11-Jun-1987 CALCIUM

SD-100 18-Jun-1987 CALCIUM

$\begin{array}{ll}32 & \mathrm{mg} / \mathrm{L} \\ 31 & \mathrm{mg} / \mathrm{L} \\ 32 & \mathrm{mg} / \mathrm{L} \\ 33 & \mathrm{mg} / \mathrm{L} \\ 29 & \mathrm{mg} / \mathrm{L} \\ 32 & \mathrm{mg} / \mathrm{L} \\ 33 & \mathrm{mg} / \mathrm{L} \\ 32 & \mathrm{mg} / \mathrm{L} \\ 35 & \mathrm{mg} / \mathrm{L} \\ 35 & \mathrm{mg} / \mathrm{L} \\ 35 & \mathrm{mg} / \mathrm{L} \\ 35 & \mathrm{mg} / \mathrm{L}\end{array}$

** SD-100-01

SD-100-01 12-May-1987 GALCIUM

SD-100-01 18-May-1987 CALCIUM

SD-100-01 3-Jun-1987 CALCIUM

SD-100-01 11-Jun-1987 CALCIUM

SD-100-01 18-Jun-1987 GALCIUM

$\begin{array}{ll}17 & \mathrm{mg} / \mathrm{L} \\ 23 & \mathrm{mg} / \mathrm{L} \\ 27 & \mathrm{mg} / \mathrm{L} \\ 48 & \mathrm{mg} / \mathrm{L} \\ 31 & \mathrm{mg} / \mathrm{L}\end{array}$


Storm Dra1n Data for SD-100

Location Date Test Compound Results Units

* SD-100-02

$\begin{array}{rrr}\text { SD-100-02 } & 12 \text {-May-1987 } & \text { GALCIUM } \\ \text { SD-100-02 } & 18 \text {-May-1987 } & \text { CALCIUM } \\ \text { SD-100-02 } & \text { 3-Jun-1987 CALCIUM } \\ \text { SD-100-02 } & 11 \text {-Jun-1987 } & \text { CALCIUM } \\ \text { SD-100-02 } & 18 \text {-Jun-1987 GALCIUM }\end{array}$

$\mathrm{mg} / \mathrm{L}$ mg/L $\mathrm{mg} / \mathrm{L}$ $\mathrm{mg} / \mathrm{L}$ $\mathrm{mg} / \mathrm{L}$

* SD-100-03

SD-100-03 12-May-1987 CALCIUM SD-100-03 18-May-1987 GALCIUH SD-100-03 26-May-1987 CALCIUM SD-100-03 3-Jun-1987 CALCIUM SD-100-03 11-Jun-1987 CALCIUM SD-100-03 18-Jun-1987 CALCIUM

$\mathrm{mg} / \mathrm{L}$ $\mathrm{mg} / \mathrm{L}$ mg $/ L$ $m g / L$ mg/L $\operatorname{mg} / \mathrm{L}$

** SD-100-03D

SD-100-03D 12-May-1987 CALCIUM SD-100-03D 26-May-1987 CALCIUM

* SD-100-06

SD-100-06 12-May-1987 CALCIU SD-100-06 18-May-1987 CALCIUM SD-100-06 26-May-1987 CALCIUM SD-100-06 3-Jun-1987 CALCIUM SD-100-06 11-Jun-1987 CALCIUM SD-100-06 18-Jun-1987 CALCIUM

$\mathrm{mg} / \mathrm{L}$ $\mathrm{mg} / \mathrm{L}$ $\mathrm{mg} / \mathrm{L}$ $\mathrm{mg} / \mathrm{L}$ $\mathbf{m g} / \mathrm{L}$ $\operatorname{mg} / L$

** SD $-100-060$

SD-100-06D 3-Jun-1987 CALCIUM

t* SD-100-07

SD-100-07 12-May-1987 CALCIU

SD-100-07 19-May-1987 CALCIUM

SD-100-07 26-May-1987 CALCIUM

SD-100-07 3-Jun-1987 CALCIUM

SD-100-07 11-JUR-1987 CALCIUM

SD-100-07 18-Jun-1987 CALCIUM

$\begin{array}{ll}31 & \mathrm{mg} / \mathrm{L} \\ 26 & \mathrm{mg} / \mathrm{L} \\ 26 & \mathrm{mg} / \mathrm{L} \\ 23 & \mathrm{mg} / \mathrm{L} \\ 19 & \mathrm{mg} / \mathrm{L} \\ 37 & \mathrm{mg} / \mathrm{L} \\ & \\ 32 & \mathrm{mg} / \mathrm{L} \\ 32 & \mathrm{mg} / \mathrm{L}\end{array}$

** SD-100-07D

SD-100-07D 18-Jun-1987 CALCIUM $\operatorname{mg} /$

** SD-100-09

SD-100-09 12-May-1987 CALCIUM 
Storm Drain Data for SD-100

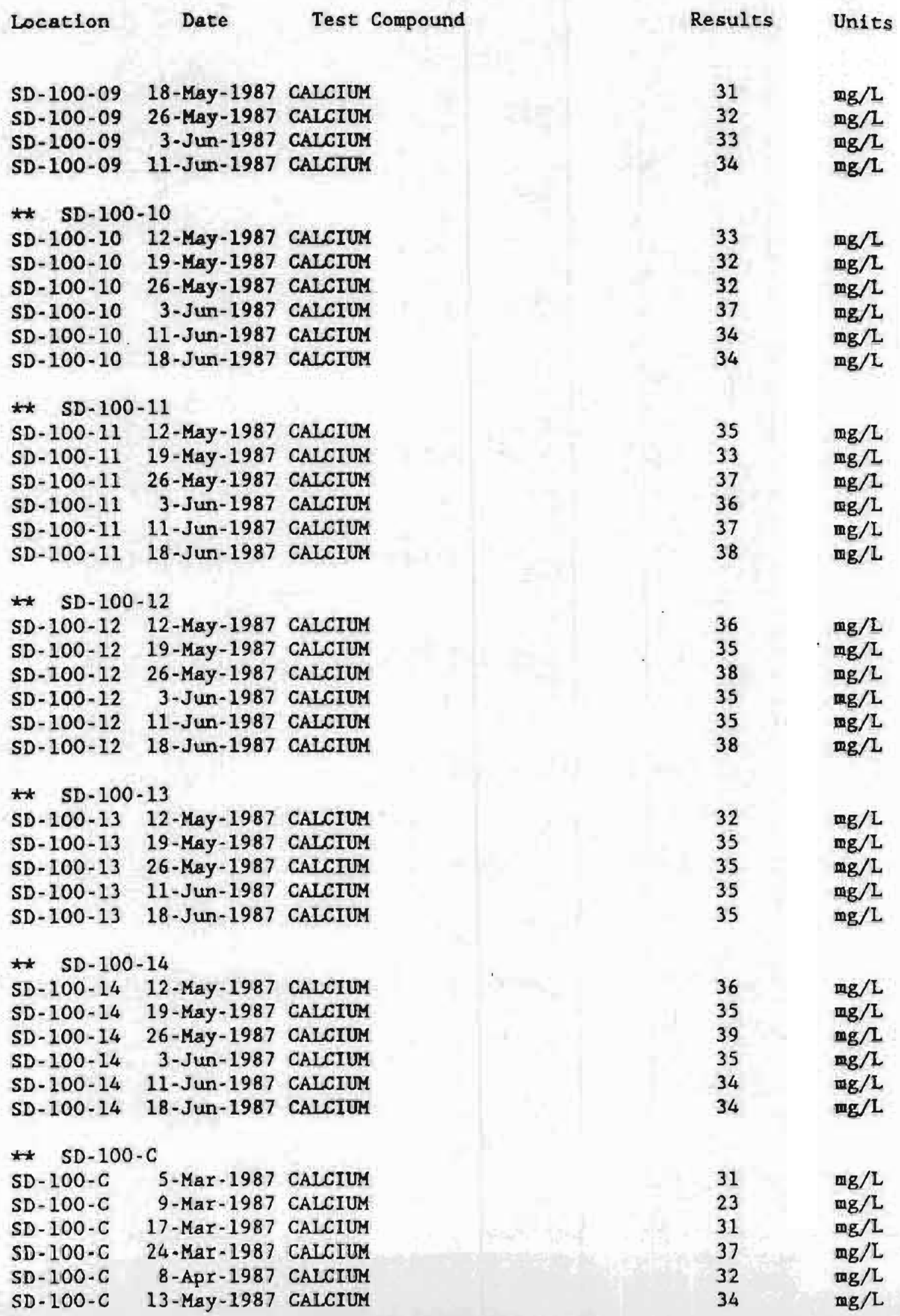


Storm Drain Data for SD-100

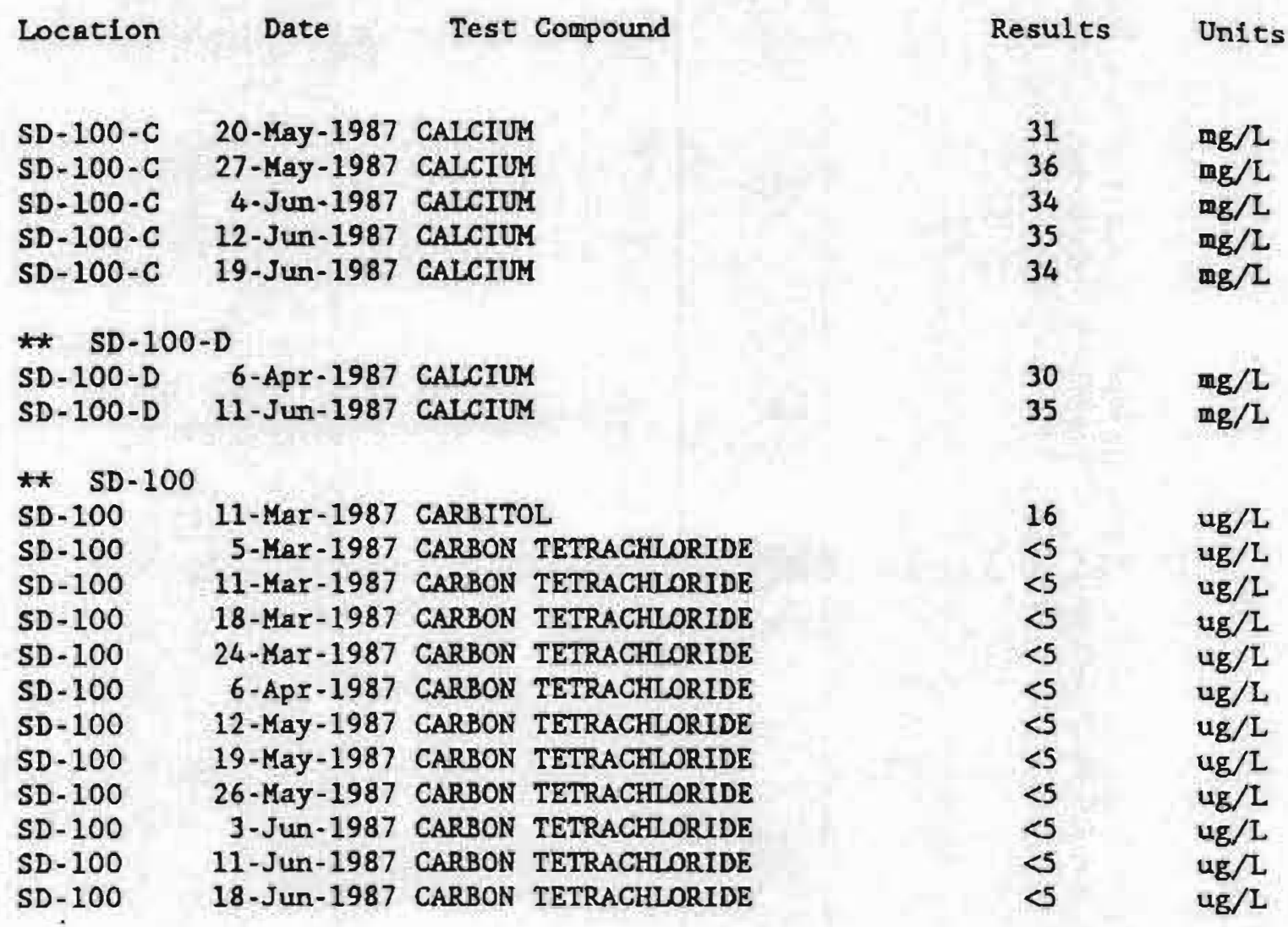

* SD-100-01

SD-100-01 12-May-1987 GARBON TETRACHLORIDE SD-100-01 18-May-1987 CARBON TETRACHLORIDE SD-100-0I 26-May-1987 CARBON TETRACHLORIDE SD-100-01 3-JLM-1987 CARBON TETRACHLORIDE SD-100-01 11-Jun-1987 CARBON TETRACHLORIDE SD-100-01 18-JUM-1987 CARBON TETRACHLORIDE

¿* SD $-100-02$

SD-100-02 12-May-1987 CARBON TETRACHLORIDE SD-100-02 18-May-1987 CARBON TETRACHLORIDE SD-100-02 26-May-1987 CARBON TETRACHLORIDE SD-100-02 3-Jun-1987 CARBON TETRACHLORIDE SD-100-02 11-Jun-1987 CARBON TETRACHLORIDE SD-100-02 18-Jun-1987 CARBON TETRACHLORIDE

* SD-100-03

SD-100-03 12-May-1987 CARBON TETRACHLORIDE SD-100-03 18-May-1987 CARBON TETRACHLORIDE SD-100-03 26-May-1987 GARBON TETRACHLORIDE SD-100-03 3-Jun-1987 GARBON TETRACHLORIDE SD-100-03 11-Jun-1987 CARBON TETRACHLORIDE SD-100-03 18-Jun-1987 CARBON TETRACHLORIDE

** SD. 100-03D

SD-100-03D 12-Yay-1987 CARBON TETRACHLORIDE SD-100-03D 26-May-1987 CARBON TETRACHLORIDE

$<5$

$<5$

$<$

$<5$

$<5$

$<5$

$<5$

$<5$

$<5$

$<5$

$<5$

$<5$

$<5$

$<5$

$<5$

$<5$

$<5$

$<5$

$<5$

$<5$
ug/I

$\mathrm{ug} / \mathrm{L}$

$\mathrm{ug} / \mathrm{L}$

ug/L

$\mathrm{ug} / \mathrm{L}$

$u g / L$

ug/L

ug $/$

$u g / L$

ug/L

ug $/ L$

$\mathrm{ug} / \mathrm{L}$

$u g / \mathrm{L}$

$4 \mathrm{~g} / \mathrm{L}$

$48 / 2$

$u g /$

$\mathrm{ug} / \mathrm{L}$

ug/L.

ug/L $\mathrm{ug} / \mathrm{L}$ 
Storm Draln Data for SD-100

Location

Date

Test Compound

Results

Units

* SD-100-04

SD-100-04 12-Kay-1987 GARBON TETRACKLORIDE

SD-100-04 18-May-1987 GARBON TETRACHLORIDE

SD-100-04 26-May-1987 CARBON TETRACHLORIDE

SD-100-04 3-Jun-1987 CARBON TETRACHLORIDE

SD-100-04 11-Jun-1987 CARBON TETRACHLORIDE

SD-100-04 18-Jun-1987 CARBON TETRACHLORIDE

$\begin{array}{ll}<5 & u g / 1 \\ <5 & u g / 1 \\ <5 & u g / L \\ <5 & u g / L \\ <5 & u g / L \\ <5 & u g / L\end{array}$

** SD-100-06

SD-100-06 12-May-1987 CARBON TETRACHLORIDE

SD-100-06 18-May-1987 CARBON TETRACHLORIDE

SD-100-06 26-May-1987 CARBON TETRACHLORIDE

SD-100-06 3-JUn-1987 CARBON TETRACHLORIDE

SD-100-06 11-Jun-1987 CARBON TETRACHLORIDE

SD-100-06 18-Jun-1987 CARBON TETRACHLORIDE

** SD-100-06D

SD-100-06D 3-JUn-1987 CARBON TETRACHLORIDE

$<5$

$<5$

$<5$

$<5$

$<5$

$<5$

$<5$

** SD-100-07

ug $/ \mathrm{h}$

ug/ $/$

ug/L

$u g / 2$

$\mathrm{ug} / \mathrm{L}$

$u g / 2$

SD-100-07 12-May-1987 CARBON IETRACHLORIDE

SD-100-07 19-May-1987 CARBON TETRACHLORIDE

SD-100-07 26-May-1987 CARBON TETRACHLORIDE

SD-100-07 3-JUn-1987 CARBON TETRACHLORIDE

SD-100-07 11-JUn-1987 CARBON TETRACHLORIDE

SD-100-07 18-JUN-1987 CARBON TETRACHLORIDE

** SD $-100-07 D$

SD-100-07D 18-JUn-1987 CARBON TETRACKLORIDE

$<5$

** SD-100-09

SD-100-09 12-May-1987 CARBON TETRACHLORIDE

SD-100-09 18-May-1987 CARBON TETRAGHLORIDE

SD-100-09 26-May-1987 CARBON TETRACHLORIDE

SD-100-09 3-Jun-1987 CARBON TETRACHLORIDE

SD-100-09 11-Jun-1987 CARBON TETRACHLORIDE

SD-100-09 18-Jun-1987 CARBON TETRACHLORIDE

13

$<5$

36

18

5

$<5$

$<5$

$<5$

$<5$

$<5$

$<5$

$<5$

** SD $-100-10$

SD-100-10 12-May-1987 CARBON TETRACHLORIDE SD-100-10 19-May-1987 CARBON TETRACHLORIDE SD-100-10 26-May-1987 GARBON TETRACHLORIDE SD-100-10 3-Jun-1987 CARBON TETRACHLORIDE

SD-100-10 11-Jun-1987 CARBON TETRACHLORIDE

SD-100-10 18-Jun-1987 GARBON TETRACHLORIDE

* SD-100-11

SD-100-11 12-May-1987 CARBON TETRACHLORIDE

SD-100-11 19-May-1987 CARBON TETRACHLORIDE

SD-100-11 26-May-1987 CARBON TETRACHLORIDE 
Storm Drain Data for SD-100

Location Date Test Compound

Results Units

SD-100-11 3-JUn-1987 CARBON TETRACHLORTDE

SD-100-11 11-Jun-1987 GARBON TETRAGHLORIDE

SD-100-11 18-Jun-1987 CARBON TETRACHLORIDE

* SD-100-12

SD-100-12 12-May-1987 GARBON TETRAGHLORIDE

SD-100-12 19-May-1987 GARBON TETRACHLORTDE

SD-100-12 26-May-1987 GARBON TETRACHLORIDE

SD-100-12 3-Jun-1987 CARBON TETRACHLORIDE

SD-100-12 11-Jun-1987 GARBON TETRACHLORIDE

SD-100-12 18-Jun-1987 GARBON TETRAGHLORIDE

$\begin{array}{ll}<5 & u g / L \\ <5 & u g / L \\ <5 & u g / L\end{array}$

* SD - 100-13

SD-100-13 12-May-1987 GARBON TETRACHLORIDE

SD-100-13 19-May-1987 GARBON TETRACHLORIDE

SD-100-13 26-May-1987 GARBON TETRACHLORIDE

SD-100-13 3-JUn-1987 CARBON TETRACHLORIDE

SD-100-13 11-JUR-1987 CARBON TETRACHLORIDE

SD-100-13 18-Jun-1987 CARBON TETRACHLORIDE

$<5$

$<5$

$<5$

$<5$

$<5$

$<5$

$\star \star \quad S D-100-14$

SD-100-14 12-May-1987 GARBON TETRACHLORIDE

SD-100-14 19-May-1987 CARBON TETRACHLORIDE

SD-100-14 26-May-1987 CARBON IETRACHLORIDE

SD-100-14 3-JUn-1987 GARBON TETRACHLORIDE

SD-100-14 11-JUn-1987 GARBON TETRACHLORIDE

SD-100-14 18-Jun-1987 GARBON TETRACHLORIDE

* $S D-100-C$

SD-100-C 5-Mar-1987 CARBON TETRACHLORIDE

SD-100-C 9-Mar-1987 CARBON TETRACHLORIDE

SD-100-C 17-Mar-1987 GARBON TETRACHLORIDE

SD-100-C 24-Mar-1987 CARBON TETRACHLORIDE

SD-100-C B-APr-1987 CARBON TETRACHLORIDE

SD-100-C 13-May-1987 GARBON IETRACHLORIDE

SD-100-C 20-May-1987 CARBON TETRACHLORIDE

SD-100-C 27-May-1987 GARBON TETRACHLORIDE

SD-100-C 12-JUn-1987 CARBON TETRACHLORIDE

SD-100-C 19-JUn-1987 CARBON TETRACHLORIDE

** SD-100-D

SD-100-D 6-Apr-1987 CARBON TETRACHLORIDE

SD-100-D 11-JUR-1987 GARBON TETRACHLORIDE
$<5$

$<5$

$<5$

$<5$

$<5$

$<5$

$<5$

$<5$

$<5$

$<5$

$<5$

$<5$

$<5$

$<5$

$<5$

$<5$

$<5$

$<5$

6

$<5$

10

$<5$

$<5$

$<5$
ug/L

ug/L

$\mathrm{ug} / \mathrm{L}$

ug/L

$u g / L$

$u g / 2$

ug/L

ug/L

ug $/ 2$

ug/L

ug/L

ug/l

$u g / L$

$u_{g} / L$

ug/I.

ug/ $L$

ug/L

ug/L

$u g / L$

ug/L

ug/L.

ug $/ 2$

** SD -100

SD-100 11-Mar-1987 CHEMICAL OXYGEN DEMAND (COD)

SD-100 18-Mar-1987 CHEMICAL OXYGEN DEMAND (COD)

SD -100

SD -100

24-MaY-1987 CHEMICAL OXYGEN DEMAND (COD)

3-ApY-1987 CHEMICAL OXYGEN DEMAND (COD)

6-APr-1987 CHEMICAL OXYGEN DEMAND (COD) 
Storm Drain Data for SD-100

Location

Date

Test Compound

SD -100

SD -100

SD- 100

SD -100

SD- 100

SD- 100
12-May-1987 CHEMITCAL OXYGEN DEMAND (COD)

19-MAY-1987 CHEMICAL OXYGEN DEMAND (COD)

26-MaY-1987 CHEMICAL OXYGEN DEYAND (COD)

3-Jun-1987 CHEYICAL OXYGEN DEMAND (COD)

11-JUn-1987 CHEIICAL OXYGEN DEYAND (COD)

18-Jun-1987 CHEMICAL OXYGEN DEMAND (COD)

** SD-100-01

SD-100-01 12-May-1987 CHEIICAL OXYGEN DEMAND (COD)

SD-100-01 18-May-1987 CHEMTGAL OXYGEN DEMAND (COD)

SD-100-01 26-Kay-1987 CHEICAI OXYGEN DEMAND (COD)

SD-100-01 3-Jun-1987 CHEMICAL OXYGEN DEMAND (COD)

SD-100-01 11-Jun-1987 CHEMICAL OXYGEN DEMAND (COD)

SD-100-01 18-Jun-1987 CHBMICAL OXXGEN DEMAND (COD)

th SD-100-02

SD-100-02 12-May-1987 CHEMTCAL OXYGEN DEMAND (COD)

SD-100-02 18-May-1987 CHEMTCAL OXYGEN DEMAND (COD)

SD-100-02 26-May-1987 CHEMICAL OXYGEN DEMAND (COD)

SD-100-02 3-JUn-1987 CHEMICAL OXYGEN DEMAND (COD)

SD-100-02 11-Jun-1987 CHEMICAL OXYGEN DEMAND (COD)

SD-100-02 18-Jun-1987 CHEMICAL OXYGEN DEMAND (COD)

** SD - 100-03

SD-100-03 12-May-1987 CHEMICAL OXYGEN DEMAND (COD)

SD-100-03 18-May-1987 CHEITCAL OXYGEN DEMAND (COD)

SD-100-03 26-MaY-1987 CHEYICAL OXYGEN DEMAND (COD)

SD-100-03 3-Jun-1987 CHEMICAL OXYGEN DEMAND (COD)

SD-100-03 11-Jun-1987 CHEMICAL OXYGEN DEMAND (COD)

SD-100-03 18-Jun-1987 CHEYICAL OXYGEN DEMAND (COD)

* SD-100-03D

SD-100-03D 12-May-1987 CHEMICAL OXYGEN DEMAND (COD)

SD-100-03D 26-May-1987 CHEMICAL OXYGEN DEMAND (COD)

* SD-100-04

SD-100-04 12-May-1987 CHEMICAL OXYGEN DEMAND (COD)

SD-100-04 18-May-1987 CHEMICAL OXYGEN DEMMAND (COD)

SD-100-04 26-May-1987 CHEMICAL OXYGEN DEMAND (COD)

SD-100-04 3-Jun-1987 CHEMICAL OXYGEN DEMAND (COD)

SD-100-04 11-Jun-1987 CHEMICAL OXYGEN DEMAND (COD)

SD-100-04 18-Jun-1987 CHEMICAL OXYGEN DEMAND (COD)

* SD-100-06

SD-100-06 12-May-1987 CHEMICAL OXYGEN DEMAND (COD)

SD-100-06 18-May-1987 CHEMICAL OXYGEN DEMAND (COD)

SD-100-06 26-May-1987 CHEMICAL OXYGEN DEMAND (COD)

SD-100-06 3-Jun-1987 CHEMICAL OXYGEN DEMAND (COD)

SD-100-06 11-JUn-1987 CHEMICAL OXYGEN DEMAND (COD)

SD-100-06 18-3un-1987 CHEMICAL OXYGEN DEMAND (COD)
Results Units

$\begin{array}{ll}<5 & \mathrm{mg} / \mathrm{L} \\ <5 & \mathrm{mg} / \mathrm{L} \\ 11.0 & \mathrm{mg} / \mathrm{L} \\ <5 & \mathrm{mg} / \mathrm{L} \\ 4.5 & \mathrm{mg} / \mathrm{L} \\ <5 & \mathrm{mg} / \mathrm{L}\end{array}$

$\begin{array}{ll}7.5 & \mathrm{mg} / \mathrm{L} \\ 32.0 & \mathrm{mg} / \mathrm{L} \\ 24.5 & \mathrm{mg} / \mathrm{L} \\ 6.5 & \mathrm{mg} / \mathrm{L} \\ 2.5 & \mathrm{mg} / \mathrm{L} \\ 11 & \mathrm{mg} / \mathrm{L}\end{array}$

$\begin{array}{ll}<5 & \mathrm{mg} / \mathrm{L} \\ 7.5 & \mathrm{mg} / \mathrm{L} \\ 7.5 & \mathrm{mg} / \mathrm{L} \\ <5 & \mathrm{mg} / \mathrm{L} \\ <5 & \mathrm{mg} / \mathrm{L} \\ <5 & \mathrm{mg} / \mathrm{L}\end{array}$

$\begin{array}{ll}<5 & \mathrm{mg} / \mathrm{L} \\ <5 & \mathrm{mg} / \mathrm{L} \\ 14.5 & \mathrm{mg} / \mathrm{L} \\ <5 & \mathrm{mg} / \mathrm{L} \\ <5 & \mathrm{mg} / \mathrm{L} \\ <5 & \mathrm{ng} / \mathrm{L}\end{array}$

$<5 \quad \mathrm{mg} / \mathrm{L}$

$<5 \quad \mathrm{mg} / \mathrm{L}$

$<5 \quad \mathrm{mg} / \mathrm{L}$

$<5 \quad \mathrm{mg} / \mathrm{L}$

<5 $\mathrm{gg} / \mathrm{L}$

$4.5 \mathrm{mg} / \mathrm{L}$

$<5 \quad \mathrm{ng} / \mathrm{L}$

$\begin{array}{ll}<5 & \mathrm{mg} / \mathrm{L} \\ 11.0 & \mathrm{mg} / \mathrm{L} \\ <5 & \mathrm{mg} / \mathrm{L} \\ <5 & \mathrm{mg} / \mathrm{L} \\ <5 & \mathrm{ng} / \mathrm{L} \\ <5 & \mathrm{mg} / \mathrm{L}\end{array}$


Storm Drain Data for SD-100

Location

Date

Test Compound

Results

Units

* SD-100-06D

SD-100-06D 3-JUn-1987 GAEMIGAL OXYGEN DEMAND (COD)

* SD-100-07

SD-100-07 12-May-1987 CHEMICAL OXYGEN DEMAND (COD)

SD-100-07 19-May-1987 CHEMICAL OXYGEN DEMAND (COD)

SD-100-07 26-May-1987 CHEMICAL OXYGEN DEMAND (COD)

SD-100-07 3-JUn-1987 GHEMICAL OXYGEN DEMAND (COD)

SD-100-07 11-Jun-1987 CHEMICAL OXYGEN DEMAND (COD)

SD-100-07 18-Jun-1987 CBRMICAL OXYGEN DEMAND (COD)

* SD-100-07D

SD-100-07D 18-Jun-1987 CHEMICAL OXYGEN DEMAND (COD)

980

$\operatorname{mg} / \mathrm{L}$

* SD $-100-09$

SD-100-09 12-May-1987 CHEMICAL OXYGEN DEMAND (COD)

SD-100-09 18-May-1987 CHEMICAL OXYGEN DEMAND (COD)

SD-100-09 26-May-1987 CHEMICAL OXYGEN DEMAND (COD)

SD-100-09 3-Jun-1987 CHEMICAI OXYGEN DEMAND (COD)

SD-100-09 11-Jun-1987 CHEMICAL OXYGEN DEMAND (COD)

SD-100-09 18-Jun-1987 CHEMICAL OXYGEN DEYAND (COD)

* SD-100-10

SD-100-10 12-May-1987 CHEMICAL OXYGEN DEMAND (COD)

SD-100-10 19-May-1987 CHEMICAL OXYGEN DEMAND (COD)

SD-100-10 26-May-1987 CHEMICAL OXYGEN DEMAND (COD)

SD-100-10 3-Jun-1987 CHEMICAL OXYGEN DEYAND (COD)

SD-100-10 11-JUn-1987 CHEMICAL OXYGEN DEMAND (COD)

SD-100-10 18-Jun-1987 CHEMICAL OXYGEN DEMAND (COD)

** SD-100-11

SD-100-11 12-May-1987 CHEMICAL OXYGEN DEMAND (COD)

SD-100-11 19-MaY-1987 CHEMICAL OXYGEN DEMAND (COD)

SD-100-11 26-May-1987 CHEMICAL OXYGEN DEMAND (COD)

SD-100-11 3-Jun-1987 CHEMICAL OXYGEN DEMAND (COD)

SD-100-11 11-JUn-1987 CHEMICAL OXYGEN DEMAND (COD)

SD-100-11 18-Jun-1987 CHEMICAL OXYGEN DEMAND (COD)

** SD $-100-12$

SD-100-12 12-May-1987 CHEMICAL OXYGEN DEMATD (COD)

SD-100-12 19-May-1987 CHEMICAL OXYGEN DEMAND (COD)

SD-100-12 26-May-1987 CHEMICAL OXYGEN DEMAND (COD)

SD-100-12 3-JUn-1987 GHFMICAL OXYGEN DEMAND (COD)

SD-100-12 11-Jun-1987 CHEMICAL OXYGEN DEMAND (COD)

SD-100-12 18-Jun-1987 CHEMICAL OXYGEN DEMAND (COD)

** SD $-100-13$

SD-100-13 12-May-1987 CHEMICAL OXYGEN DEMAND (COD)

SD-100-13 19-May-1987 CHEMICAL OXYGEN DEMAND (COD)

SD-100-13 26-May-1987 CHEMICAL OXYGEN DEMAND (COD)

SD-100-13 3-Jun-1987 CHEMICAL OXYGEN DEMAND (COD)

$\begin{array}{ll}<5 & \mathrm{mg} / \mathrm{L} \\ 835 & \mathrm{mg} / \mathrm{L} \\ 255 & \mathrm{mg} / \mathrm{L} \\ 85.5 & \mathrm{mg} / \mathrm{L} \\ 610 & \mathrm{mg} / \mathrm{L} \\ 38 & \mathrm{mg} / \mathrm{L} \\ 1270 & \mathrm{mg} / \mathrm{L} \\ & \\ 980 & \mathrm{mg} / \mathrm{L} \\ & \\ 18.0 & \mathrm{mg} / \mathrm{L} \\ 19.5 & \mathrm{mg} / \mathrm{L} \\ <5 & \mathrm{mg} / \mathrm{L} \\ <5 & \mathrm{mg} / \mathrm{L} \\ 2.5 & \mathrm{mg} / \mathrm{L} \\ 8 & \mathrm{mg} / \mathrm{L} \\ & \\ <5 & \mathrm{mg} / \mathrm{L} \\ 7.5 & \mathrm{mg} / \mathrm{L} \\ 11.0 & \mathrm{mg} / \mathrm{L} \\ <5 & \mathrm{mg} / \mathrm{L} \\ 4.5 & \mathrm{mg} / \mathrm{L} \\ <5 & \mathrm{mg} / \mathrm{L}\end{array}$

$<5$

18.0

$<5$

$<5$

2.5

$<5$

$\mathrm{mg} / \mathrm{L}$

$\mathrm{mg} / \mathrm{L}$

$\mathrm{mg} / \mathrm{L}$

$\mathrm{g} / \mathrm{L}$

$\mathrm{g} / \mathrm{L}$

$g$

$\mathrm{g} / \mathrm{L}$

$\mathrm{g} / \mathrm{L}$

$\mathrm{mg} / \mathrm{L}$

$\begin{array}{ll}<5 & \mathrm{mg} / \mathrm{L} \\ 7.5 & \mathrm{mg} / \mathrm{L} \\ 11.0 & \mathrm{ng} / \mathrm{L} \\ <5 & \mathrm{gg} / \mathrm{L} \\ 16 & \mathrm{mg} / \mathrm{L} \\ 11 & \mathrm{gg} / \mathrm{L}\end{array}$

$<5 \quad \mathrm{mg} / \mathrm{L}$

$<5 \quad \mathrm{mg} / \mathrm{L}$

$<5 \quad \mathrm{mg} / \mathrm{L}$

$7.5 \mathrm{mg} / \mathrm{L}$ 
Stora Drain Data for SD-100

Location Date Test Compound

Results Units

SD-100-13 11-JUn-1987 CHEYICAL OXYGEN DEMAND (COD)

SD-100-13 18-Jun-1987 CHEMICAL OXYGEN DEMAND (COD)

$\begin{array}{ll}4.5 & \mathrm{mg} / \mathrm{L} \\ 7 & \mathrm{mg} / \mathrm{L} \\ & \\ <5 & \mathrm{mg} / \mathrm{L} \\ 11.0 & \mathrm{mg} / \mathrm{L} \\ 35.0 & \mathrm{mg} / \mathrm{L} \\ <5 & \mathrm{mg} / \mathrm{L} \\ 4.5 & \mathrm{mg} / \mathrm{L} \\ <5 & \mathrm{mg} / \mathrm{L}\end{array}$

** SD $-100-14$

SD-100-14 12-May-1987 GHEMTGAI OXYGEN DEMAND (COD)

SD-100-14 19-May-1987 GHEMICAL OXYGEN DEMAND (COD)

SD-100-14 26-May-1987 CHEMICAL OXYGEM DEMAND (COD)

SD-100-14 3-Jun-1987 CHEMICAL OXTGEN DEMAND (COD)

SD-100-14 11-Jun-1987 GHEMICAI OXYGEN DEAAND (COD)

SD-100-14 18-Jun-1987 CHEMTCAL OXYGEN DEYAND (COD)

$\star$ SD-100-C

SD-100-C 5-MaT-1987 CHEMICAZ OXYGEN DEMAND (COD)

SD-100-C 9-Mar-1987 CHEMICAL OXYGEN DEMAND (COD)

SD-100-C 17-Mar-1987 CHEMICAI OXYGEN DEMAND (COD)

SD-100-C 24-Mar-1987 CHEMICAL OXYGEN DEMAND (COD)

SD-100-C 8-Apr-1987 CHEMICAZ OXYGEN DEYAND (COD)

SD-100-C 13-May-1987 CHEMICAL OXYGEN DEMAND (COD)

SD-100-C 20-May-1987 CHEMICAI OXYGEN DEMAND (COD)

SD-100-C 27-May-1987 GHEMICAL OXYGEN DEMAND (COD)

SD-100-C 4-JUn-1987 CHEMICAL OXYGEN DEMAND (COD)

SD-100-C 12-Jun-1987 CHEMICAL OXYGEN DEMAND (COD)

SD-100-C 19-Jun-1987 CHEMICAL OXYGEN DEMIAND (COD)

* SD $-100-D$

SD-100-D 6-APr-1987 CHEMICAL OXYGEN DEMAND (COD)

SD-100-D 11-Jun-1987 GLEMICAL OXYGEN DEMAND (COD)

** SD -100

SD-100 5-MaT-1987 CHLORIDE

SD-100 11-Mar-1987 GHLORIDE

SD-100 18-Mar-1987 GHLORIDE

SD-100 24-Mar-1987 CHLORIDE

SD-100 3-Apr-1987 CHLORIDE

SD-100 6-APY-1987 CHLORIDE

SD-100 12-May-1987 GHLORIDE

SD-100 19-May-1987 CHLORIDE

SD-100 26-May-1987 CHLORIDE

SD-100 3-Jun-1987 CHLORIDE

SD-100 11-JUn-1987 CHLORIDE

SD-100 18-Jun-1987 GHLORIDE

$\begin{array}{ll}<5 & \mathrm{mg} / \mathrm{L} \\ 7 & \mathrm{mg} / \mathrm{L} \\ 10 & \mathrm{mg} / \mathrm{L} \\ 7 & \mathrm{mg} / \mathrm{L} \\ 15 & \mathrm{mg} / \mathrm{L} \\ 14.5 & \mathrm{mg} / \mathrm{L} \\ <5 & \mathrm{mg} / \mathrm{L} \\ 7.5 & \mathrm{mg} / \mathrm{L} \\ 21 & \mathrm{mg} / \mathrm{L} \\ <5 & \mathrm{mg} / \mathrm{L} \\ 5 & \mathrm{mg} / \mathrm{L}\end{array}$

$<5 \quad \mathrm{mg} / \mathrm{L}$

$4.5 \mathrm{ng} / \mathrm{L}$

** SD $-100-01$

SD-100-01 12-May-1987 CHLORIDE

SD-100-01 18-May-1987 CHLORIDE

SD-100-01 26-May-1987 CHLORIDE

SD-100-01 3-Jun-1987 CHLORIDE

SD-100-01 11-JUN-1987 CHLORIDE

SD-100-01 18-Jun-1987 GHLORIDE

$\begin{array}{ll}10.2 & \mathrm{mg} / \mathrm{L} \\ 8.4 & \mathrm{mg} / \mathrm{L} \\ 8.2 & \mathrm{mg} / \mathrm{L} \\ 8.4 & \mathrm{mg} / \mathrm{L} \\ 8.8 & \mathrm{mg} / \mathrm{L} \\ 9.2 & \mathrm{mg} / \mathrm{L} \\ 9.9 & \mathrm{mg} / \mathrm{L} \\ 10.2 & \mathrm{mg} / \mathrm{L} \\ 10.3 & \mathrm{mg} / \mathrm{L} \\ 10.2 & \mathrm{mg} / \mathrm{L} \\ 12 & \mathrm{mg} / \mathrm{L} \\ 15.6 & \mathrm{mg} / \mathrm{L}\end{array}$

$\begin{array}{ll}1.2 & \mathrm{mg} / \mathrm{L} \\ 1.1 & \mathrm{mg} / \mathrm{L} \\ 1.3 & \mathrm{mg} / \mathrm{L} \\ 1.3 & \mathrm{mg} / \mathrm{L} \\ 1.1 & \mathrm{gg} / \mathrm{L} \\ 1.4 & \mathrm{mg} / \mathrm{L}\end{array}$




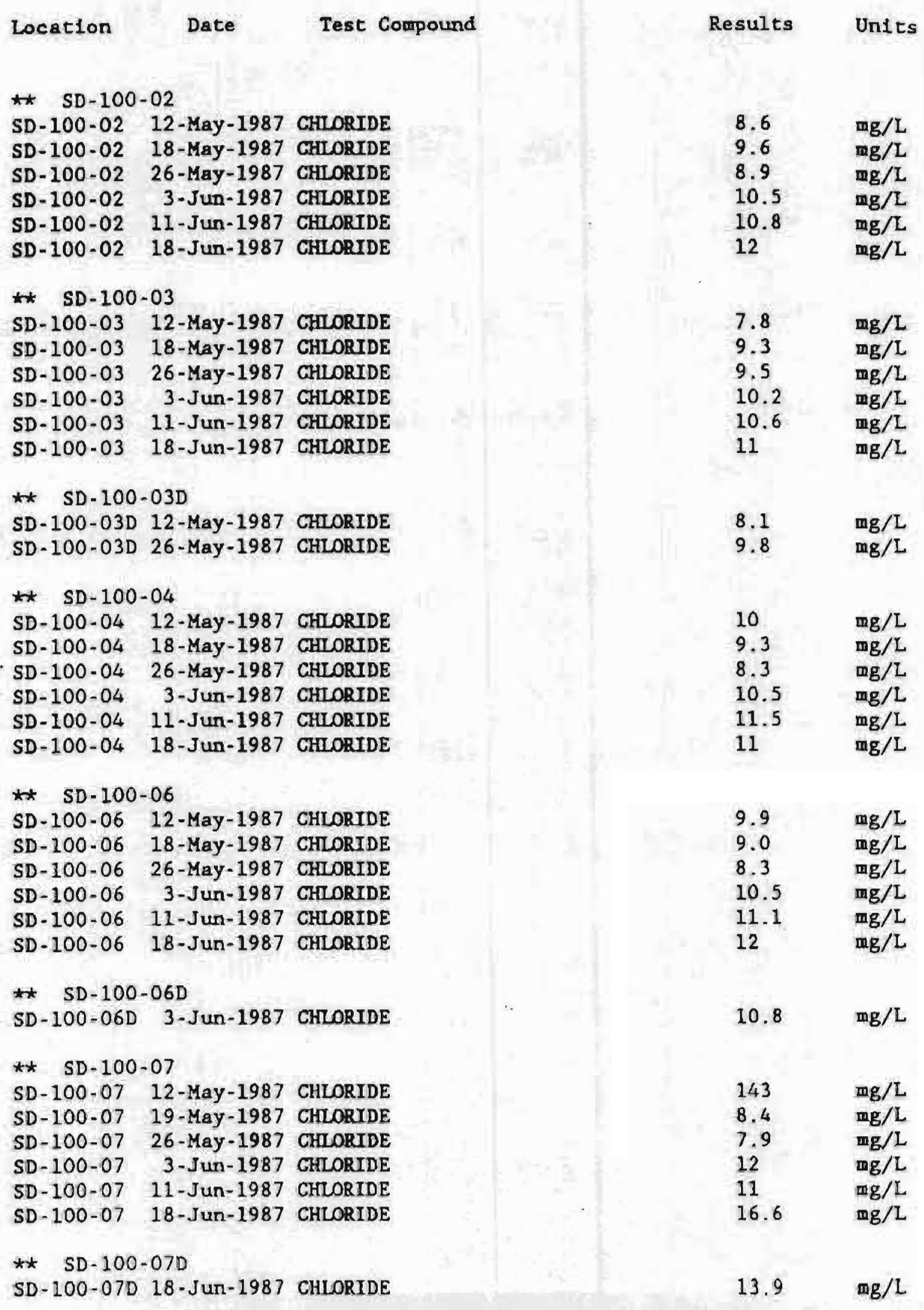


Storw Drain Data for SD-100

\begin{tabular}{|c|c|c|c|c|}
\hline Location & Date & Test Compound & Results & Units \\
\hline \multicolumn{5}{|c|}{$\star \star \quad S D-100-09$} \\
\hline SD-100-09 & 12-May-1987 & CHIORIDE & 8.5 & $\mathrm{mg} / \mathrm{L}$ \\
\hline SD $-100-09$ & $18-$ May -1987 & CHIORIDE & 9.3 & $\mathrm{mg} / \mathrm{L}$ \\
\hline SD $-100-09$ & $26-$ May -1987 & CHLORIDE & 8.1 & $\mathrm{mg} / \mathrm{L}$ \\
\hline SD $-100-09$ & 3-Jum-1987 & CHLORIDE & 9.6 & $\mathrm{mg} / \mathrm{L}$ \\
\hline SD $-100-09$ & $11 \cdot \mathrm{J}$ un-1987 & CHLORIDE & 11.1 & $\mathrm{mg} / \mathrm{L}$ \\
\hline SD-100-09 & $18-J u n \cdot 1987$ & CHLORIDE & 11 & $\mathrm{mg} / \mathrm{L}$ \\
\hline \multicolumn{5}{|c|}{$\star *$ SD $-100-10$} \\
\hline SD $-100-10$ & 12-May- 1987 & CHLORIDE & 10.6 & $\mathrm{mg} / \mathrm{L}$ \\
\hline SD $-100-10$ & $19-$ May -1987 & CKILORIDE & 9.3 & $\mathrm{mg} / \mathrm{L}$ \\
\hline SD $-100-10$ & 26 - May -1987 & CHLORIDE & 10.5 & $\mathrm{mg} / \mathrm{L}$ \\
\hline SD $-100-10$ & 3-Jun-1987 & CHLORIDE & 10.2 & $\mathrm{mg} / \mathrm{L}$ \\
\hline$S D-100-10$ & 11-Jun-1987 & CHLORIDE & 11.4 & $\mathrm{mg} / \mathrm{L}$ \\
\hline SD-100-10 & $18-J u n-1987$ & CHLORIDE & 11.4 & $\mathrm{mg} / \mathrm{L}$ \\
\hline \multicolumn{5}{|c|}{$\star \star \quad S D-100-11$} \\
\hline SD- $100-11$ & 12-May-1987 & CKLORIDE & 12.5 & $\operatorname{mg} / \mathrm{L}$ \\
\hline$S D-100-11$ & $19-$ May -1987 & CHLORIDE & 10.2 & $\mathrm{mg} / \mathrm{L}$ \\
\hline SD $-100-11$ & 26-May-1987 & CHLORIDE & 11.1 & $m g / L$ \\
\hline$S D-100-11$ & $3-J$ un -1987 & CHLORIDE & 10.8 & $\mathrm{mg} / \mathrm{L}$ \\
\hline SD $-100-11$ & $11-J$ un -1987 & CHLORIDE & 12.3 & $\mathrm{mg} / \mathrm{L}$ \\
\hline SD-100-11 & $18-J$ un -1987 & CHLORIDE & 13.5 & $\mathrm{mg} / \mathrm{L}$ \\
\hline \multicolumn{5}{|c|}{$\star \quad$ SD $-100-12$} \\
\hline SD-100-12 & 12-May-1987 & CHLORIDE & 9.5 & $\mathrm{mg} / \mathrm{L}$ \\
\hline SD $-100-12$ & 19-May-1987 & CHLORIDE & 9.3 & mg/L \\
\hline SD $-100-12$ & 26 - May -1987 & CHLORIDE & 10.2 & $\mathbf{m g} / \mathrm{L}$ \\
\hline SD $-100-12$ & 3-Jun-1987 & CHLORIDE & 10.4 & $\mathrm{mg} / \mathrm{L}$ \\
\hline$S D-100-12$ & $11-J u n-1987$ & CHLORIDE & 11.4 & $\mathrm{mg} / \mathrm{L}$ \\
\hline SD $-100-12$ & $18-J u n-1987$ & CHLORIDE & 11. & $\mathrm{mg} / \mathrm{L}$ \\
\hline \multicolumn{5}{|c|}{$\star \star \quad S D-100-13$} \\
\hline$S D-100-13$ & 12-Kay-1987 & CHLORIDE & 10 & $\mathrm{mg} / \mathrm{L}$ \\
\hline SD $-100-13$ & 19-May-1987 & CHLORIDE & 9.9 & $\mathrm{mg} / \mathrm{L}$ \\
\hline$S D-100-13$ & $26-$ May -1987 & CHLORIDE & 9.4 & $\mathrm{mg} / \mathrm{L}$ \\
\hline SD $-100-13$ & 3-Jun-1987 & CHLORIDE & 10.4 & $\mathrm{mg} / \mathrm{L}$ \\
\hline SD $-100-13$ & $11-J u n-1987$ & CHLORIDE & 11.0 & $\mathbf{m g} / \mathrm{L}$ \\
\hline$S D-100-13$ & $18-J u n-1987$ & CHLORIDE & 12 & $\mathrm{mg} / \mathrm{L}$ \\
\hline \multicolumn{5}{|c|}{$\star \star \quad S D-100-14$} \\
\hline SD-100-14 & $12-$ May - 1987 & CHLORIDE & 12 & $\operatorname{mg} / \mathrm{L}$ \\
\hline SD-100-14 & 19-May-1987 & CHLORIDB & 10.5 & $\mathbf{g} / \mathrm{L}$ \\
\hline SD $-100-14$ & $26-\mathrm{May}-1987$ & CHLORIDE & 10.4 & $m g / L$ \\
\hline SD $-100-14$ & 3.Jun-1987 & CHLORIDE & 10.2 & $\operatorname{mg} / \mathrm{L}$ \\
\hline$S D-100-14$ & $11-J u n-1987$ & CHLORIDE & 11.2 & $m g / L$ \\
\hline SD - $100-14$ & $18-J$ un -1987 & CHLORIDE & 12 & $\mathrm{mg} / \mathrm{L}$ \\
\hline \multicolumn{5}{|c|}{$\star \star \quad S D-100-C$} \\
\hline$S D-100-C$ & $5-$ Mar -1987 & CHLORIDE & 10.5 & $\mathrm{mg} / \mathrm{L}$ \\
\hline SD $-100 \cdot C$ & 9-Mar-1987 & CHLORIDE & 10.2 & $\mathrm{mg} / \mathrm{L}$ \\
\hline
\end{tabular}


Storm Drain Data for SD-100

\begin{tabular}{|c|c|c|}
\hline ocation & Date & Test \\
\hline$S D-100 \cdot C$ & 17-Kar-1987 & CHLORIDE \\
\hline$S D-100-C$ & 24-Mar-1987 & CHLORIDE \\
\hline SD-100-C & 8-Apr-1987 & CHLORIDE \\
\hline SD-100-C & 13-May-1987 & CHLORIDE \\
\hline SD-100-C & 20-May-1987 & CHLORIDE \\
\hline SD-100-C & 27-May-1987 & GHLORIDE \\
\hline SD $-100-C$ & 4-Jun-1987 & CRLORID \\
\hline$S D-100-C$ & 12-Jun-1987 & CHLORID \\
\hline$S D-100-C$ & $19-J u n-1987$ & CHIORID \\
\hline
\end{tabular}

Results Units

** SD-100-D

SD-100-D 6-APT-1987 CHLORIDE

SD-100-D 11-Jun-1987 CHLORIDE

$\begin{array}{ll}7.5 & \mathrm{mg} / \mathrm{L} \\ 9.4 & \mathrm{mg} / \mathrm{L} \\ 9.0 & \mathrm{mg} / \mathrm{L} \\ 10.2 & \mathrm{mg} / \mathrm{L} \\ 10.2 & \mathrm{mg} / \mathrm{L} \\ 10.2 & \mathrm{mg} / \mathrm{L} \\ 10.5 & \mathrm{mg} / \mathrm{L} \\ 11.7 & \mathrm{mg} / \mathrm{L} \\ 12.3 & \mathrm{mg} / \mathrm{L}\end{array}$

$9.2 \mathrm{mg} / \mathrm{L}$

$12 \mathrm{mg} / \mathrm{L}$

* SD-100-02

SD-100-02 12-May-1987 CHLORINATED HYDROCARBON

$410 \quad \mathrm{ug} / \mathrm{L}$

* SD $-100-10$

SD-100-10 12-May-1987 CHLORINATED HYDROGARBON

37

$u g / L$

** SD $-100-03$

SD-100-03 12-May-1987 CHLORINATED HYDROCARBONS

180

ug $/$ L

* SD-100-03D

SD-100-03D 12-May-1987 CHLORINATED HYDROGARBONS

270

ug/L

** SD-100-04

SD-100-04 12-May-1987 CKLORINATED HYDROCARBONS

270

$\mathrm{ug} / \mathrm{L}$

** SD-100-06

SD-100-06 12-May-1987 CHLORINATED HYDROCARBONS

180

ug/L

* SD -100

$S D-100$

5-Mar-1987 CHLORINE TOTAL RESIDUAL

SD -100

11-Mar-1987 CHLORINE TOTAL RESIDUAL

WATER

WATER

SD -100

18-Mar-1987 CHLORINE TOTAL RESIDUAL

SD- 100

24-Mar-1987 CHLORINE TOTAL RESIDUAI

SD -100

3-Apr-1987 CHLORINE TOTAL RESIDUAL

SD -100

6-APr-1987 CHLORINE TOTAL RESIDUAL

SD -100

12-May-1987 CHLORINE TOTAL RESIDUAL

SD -100

19-May-1987 CHLORINE TOTAL RESIDUAL

SD -100

26-May-1987 CHLORINE TOTAL RESIDUAL

SD -100

3-JUn-1987 CHLORINE TOTAL RESIDUAL

SD -100

11-JUn-1987 CHLORINE TOTAL RESIDUAL

WATER

WATER

WATER

WATER

WATER

WATER

WATER

WATER

SD -100

18-Jun-1987 CHLORINE TOTAL RESIDUAL WATER

$<0.1$

$\mathrm{mg} / \mathrm{L}$

$<0.1 \quad \mathrm{mg} / \mathrm{L}$

$<1.0$

$<0.1$

$<0.1$

0.2

$<0.1$

$<0.1$

$\mathrm{mg} / \mathrm{L}$

$\mathrm{mg} / \mathrm{L}$

$\mathrm{mg} / \mathrm{L}$

$\mathrm{mg} / \mathrm{L}$

$\mathrm{mg} / \mathrm{L}$

$<0.1$

ㄸg/L

$\mathrm{mg} / \mathrm{L}$

$0.2 \mathrm{mg} / \mathrm{L}$

$<0.1 \quad \mathrm{mg} / \mathrm{L}$

$<0.1 \quad \mathrm{mg} / \mathrm{L}$

* SD - 100-01

SD-100-01 12-May-1987 CHLORINE TOTAL RESIDUAL WATER

$<0.1$

$\mathrm{mg} / \mathrm{L}$

SD-100-01 18-May-1987 CHLORINE TOTAL RESIDUAL VATER

0.5

$\mathrm{mg} / \mathrm{L}$

SD-100-01 26-May-1987 CHLORINE TOTAL RESIDUAL HATER

0.7

$m g / L$

SD $-100-01$

3-JUN-1987 CHLORINE TOTAL RESIDUAL WATER

0.2

$\mathrm{mg} / \mathrm{L}$ 
Storm Drain Data for SD-100

Location

Date

Test Compound

Results

Units

SD-100-01 11-JUN-1987 CHLORINE TOTAL RESIDUAL WATER SD-100-01 18-JUR-1987 CHLORINE TOTAL RESIDUAL WATER

$<0.1$

$<0.1$

$\mathrm{mg} / \mathrm{L}$

$\mathrm{mg} / \mathrm{L}$

* SD $-100-02$

SD-100-02 12-May-1987 CHLORINE TOTAL RESIDUAL UATER

SD-100-02 18-May-1987 CHLORINE TOTAL RESIDUAL WATER

SD-100-02 26-May-1987 CHLORINE TOTAL RESIDUAL WATER

SD-100-02 3-Jun-1987 CHLORINE TOTAL RESIDUAL HATER

SD-100-02 11-JUn-1987 CHLORINE TOTAL RESIDUAL WATER

SD-100-02 18-Jun-1987 CHLORINE TOTAL RESIDOAL. WATER

$1.3 \mathrm{mg} / \mathrm{L}$

$0.6 \mathrm{mg} / \mathrm{L}$

$1.2 \mathrm{mg} / 2$

$0.6 \mathrm{mg} / \mathrm{h}$

$1.0 \mathrm{mg} / \mathrm{L}$

$1.2 \mathrm{mg} / \mathrm{L}$

* SD-100-03

SD-100-03 12-May-1987 CHLORINE TOTAL RESIDUAL HATER

SD-100-03 18-May-1987 CHLORINE TOTAL RESIDUAL WATER

SD-100-03 26-May-1987 CHLORINE TOTAL RESIDUAL WATER

SD-100-03 3-JUn-1987 CHLORINE TOTAL RESIDUAL WATER

SD-100-03 11-JUn-1987 CHLORINE TOTAL RESIDUAL WATER

SD-100-03 18-Jun-1987 CHLORINE TOTAL RESIDUAL WATER

$1.0 \mathrm{mg} / \mathrm{L}$

$0.5 \mathrm{mg} / \mathrm{L}$

$0.8 \mathrm{mg} / \mathrm{L}$

$0.8 \quad \mathrm{mg} / \mathrm{L}$

$1.1 \mathrm{mg} / \mathrm{L}$

$1.0 \mathrm{mg} / \mathrm{L}$

* SD-100-03D

SD-100-03D 12-May-1987 CHLORINE TOTAL RESIDUAL WATER

SD-100-030 26-May-1987 CHLORINE TOTAL RESIDUAL WATER

$1.0 \mathrm{mg} / \mathrm{L}$

$0.8, \mathrm{mg} / \mathrm{L}$

* SD $-100-04$

SD-100-04 12-May-1987 CHLORINE TOTAL RESIDUAL WATER

SD-100-04 18-May-1987 CHLORINE TOTAL RESIDUAL WATER

SD-100-04 26-May-1987 CHLORINE TOTAL RESIDUAL WATER

SD-100-04 3-JUn-1987 CHLORINE TOTAL RESIDUAL WATER

SD-100-04 12-JUn-1987 CHLORINE TOTAL RESIDUAL WATER

SD-100-04 18-Jun-1987 CHLORINE TOTAL RESIDUAL WATER

$1.0 \quad \mathrm{mg} / \mathrm{L}$

$0.6 \mathrm{mg} / \mathrm{L}$

$0.7 \quad \mathrm{mg} / \mathrm{L}$

$0.7 \mathrm{mg} / \mathrm{L}$

$1.2 \mathrm{mg} / \mathrm{L}$

$1.4 \mathrm{mg} / \mathrm{L}$

** SD-100-06

SD-100-06 12-May-1987 CHLORINE TOTAL RESIDUAL WATER

SD-100-06 18-May-1987 CHLORINE TOTAL RESIDUAL WATER

SD-100-06 26-May-1987 CHLORINE TOTAL RESIDUAL WATER

SD-100-06 3-Jun-1987 CHLORINE TOTAL RESIDUAL WATER

SD-100-06 11-JUn-1987 CHLORINE TOTAL RESIDUAL WATER

SD-100-06 18-Jun-1987 CHLORINE TOTAL RESIDUAL WATER

$0.3 \mathrm{mg} / \mathrm{L}$

$0.6 \mathrm{mg} / \mathrm{L}$

$0.4 \mathrm{mg} / \mathrm{L}$

$0.7 \mathrm{mg} / \mathrm{L}$

$1.1 \mathrm{mg} / \mathrm{L}$

$0.4 \mathrm{mg} / \mathrm{L}$

* SD-100-06D

SD-100-06D 3-Jun-1987 CHLORINE TOTAL RESIDUAL WATER

$0.5 \mathrm{mg} / \mathrm{L}$

** SD-100-07

SD-100-07 12-May-1987 CHLORINE TOTAL RESIDUAL WATER

SD-100-07 19-May-1987 CHLORINE TOTAL RESIDUAL WATER

SD-100-07 26-MaY-1987 CHLORINE TOTAL RESIDUAL WATER

$0.3 \mathrm{mg} / \mathrm{L}$

$<0.1 \quad \mathrm{mg} / \mathrm{L}$

$0.3 \mathrm{mg} / \mathrm{L}$

SD-100-07 3-Jun-1987 CHLORINE TOTAL RESIDUAL WATER

$7.8 \mathrm{mg} / \mathrm{L}$

SD-100-07 11-JUn-1987 CHLORINE TOTAL RESIDUAL WATER

$<0.1 \quad \mathrm{mg} / \mathrm{L}$

SD-100-07 18-JUn-1987 CHLORINE TOTAL RESIDUAL WATER $<0.1 \quad \mathrm{ag} / \mathrm{L}$ 
Storm Drain Data for SD-100

Location

Dace

Test Compound

Results

Onits

** SD-100-07D

SD-100-07D 18-Jun-1987 CHLORINE TOTAL RESIDUAL HATER

$<0.1$

$m g / L$

** SD-100-09

SD-100-09 12-May-1987 CHLORINE TOTAL RESIDUAL WATER

SD-100-09 18-May-1987 CHLORINE TOTAL RESIDUAL HATER

SD-100-09 26-MaY-1987 CHLORINE TOTAL RESIDUAL UATER

SD-100-09 3-Jun-1987 CHLORINE TOTAL RESIDUAL WATER

SD-100-09 11-JUn-1987 CHLORINE TOTAL RESIDUAL WATER

SD-100-09 18-JUn-1987 CHLORINE TOTAL RESIDUAL WATER

$\begin{array}{ll}0.2 & \mathrm{mg} / L \\ 0.2 & \mathrm{mg} / \mathrm{L} \\ 0.9 & \mathrm{mg} / \mathrm{L} \\ 0.2 & \mathrm{mg} / \mathrm{L} \\ 0.4 & \mathrm{mg} / \mathrm{L} \\ 0.4 & \mathrm{mg} / \mathrm{L}\end{array}$

« SD $-100-10$

SD-100-10 12-May-1987 CHLORINE TOTAL RESIDUAZ WATER SD-100-10 19-May-1987 CHLORINE TOTAL RESIDUAL WATER SD-100-10 26-May-1987 CHLORINE TOTAL RESIDUAL SD-100-10 3-Jun-1987 CHLORINE TOTAL RESIDUAL SD-100-10 11-Jun-1987 CHLORINE TOTAL RESIDUAL

WATER

WATER

WATER

SD-100-10

18-JUn-1987 CHLORINE TOTAL RESIDUAL

HATER

* SD $-100-11$

SD-100-11 12-May-1987 CHLORINE TOTAL RESIDUAL WATER

SD-100-11 19-May-1987 CHLORINE TOTAL RESIDUAL

SD-100-11 26-May-1987 CHLORINE TOTAL RESIDUAI

SD-100-11 3-JUn-1987 CHLORINE TOTAL RESIDUAL

WATER

WATER

WATER

SD-100-11 11-JUn-1987 CHLORINE TOTAL RESIDUAL VATER

SD-100-11 18-JUn-1987 CHLORINE TOTAL RESIDUAL WATER

$1.2 \mathrm{mg} / \mathrm{L}$

$1.0 \mathrm{mg} / \mathrm{L}$

$0.7 \mathrm{mg} / \mathrm{L}$

$1.0 \mathrm{mg} / \mathrm{L}$

$1.1 \mathrm{mg} / \mathrm{L}$

$1.4 \mathrm{mg} / \mathrm{L}$

\# SD-100-12

SD-100-12 12-May-1987 CHLORINE TOTAL RESIDUAL WATER SD-100-12 19-May-1987 CHLORINE TOTAL RESIDUAL SD-100-12 26-May-1987 CHLORINE TOTAL RESIDUAZ SD-100-12 3-Jun-1987 CHLORINE TOTAL RESIDUAL SD-100-12 11-JUn-1987 CHLORINE TOTAL RESIDUAL SD-100-12 18-Jun-1987 CHLORINE TOTAL RESIDUAL

WATER

WATER

WATER

WATER

WATER

2.8

$\mathrm{mg} / \mathrm{L}$

$<0.1 \quad \mathrm{mg} / \mathrm{h}$

$0.2 \mathrm{mg} / \mathrm{L}$

$0.7 \quad \mathrm{mg} / \mathrm{L}$

$0.2 \mathrm{mg} / \mathrm{L}$

$0.7 \mathrm{mg} / \mathrm{L}$

** SD-100-13

SD-100-13 12-May-1987 CHLORINE TOTAL RESIDUAL HATER SD-100-13 19-May-1987 CKLORINE TOTAL RESIDUAL HATER SD-100-13 26-May-1987 CHLORINE TOTAL RESIDUAL WATER SD-100-13 3-Jun-1987 CHLORINE TOTAL RESIDUAL HATER SD-100-13 11-Jun-1987 CHLORINE TOTAL RESIDUAL WATER SD-100-13 18-Jun-1987 CHLORINE TOTAL RESIDUAL WATER

$1.0 \quad \mathrm{mg} / \mathrm{L}$ $0.8 \quad \mathrm{mg} / \mathrm{L}$ $0.1 \quad \mathrm{mg} / \mathrm{L}$ $0.8 \mathrm{mg} / \mathrm{L}$ $0.6 \quad \mathrm{mg} / \mathrm{L}$ $0.9 \mathrm{mg} / \mathrm{L}$

** SD - 100-14

SD-100-14 12-May-1987 CHLORINE TOTAL RESIDUAL SD-100-14 19-May-1987 CHLORINE TOTAL RESIDUAL SD-100-14 26-May-1987 CHLORINE TOTAL RESIDUAL SD-100-14 3-Jun-1987 CHLORINE TOTAL RESIDUAL SD-100-14 11-Jun-1987 CHLORINE TOTAL RESIDUAL SD-100-14 18-Jun-1987 CHLORINE TOTAL RESIDUAL

HATER

HATER

WATER

WATER

WATER

HATER
0.9

0.4

0.5

0.7

0.9

0.4

$<0.1$

0.3

0.1

0.7

1. 2

1. 5 $\mathrm{mg} / \mathrm{L}$ $\mathrm{mg} / \mathrm{L}$ mg $/$ $\mathrm{mg} / \mathrm{L}$ $\mathrm{mg} / \mathrm{L}$. $\mathrm{ng} / \mathrm{L}$

$$
\begin{aligned}
& \mathrm{mg} / \mathrm{L} \\
& \mathrm{mg} / \mathrm{L} \\
& \mathrm{mg} / \mathrm{L} \\
& \mathrm{mg} / \mathrm{L} \\
& \mathrm{mg} / \mathrm{L} \\
& \mathrm{mg} / \mathrm{L}
\end{aligned}
$$


ug/L

\# SD-100-11

SD-100-11 12-MaY-1987 CHLORO CYCLOHEXANOL

$\mathrm{ug} / \mathrm{l}$

** SD-100-12

SD-100-12 12-May-1987 CHLORO CYCLOHEXANOL

$u g / L$

* SD $-100-13$

SD-100-13 12-May-1987 CHLORO CYCLOHEXANOL

$\mathrm{ug} / \mathrm{L}$

** SD $-100-14$

SD-100-14 12-May-1987 CHLORO CYCLOHEXANOL

$\mathrm{ug} / \mathrm{L}$

** SD-100-11

SD-100-11 12-May-1987 CHLORO CYCLOHEXETE

ug/L

* SD $-100-13$

SD-100-13 12-May-1987 CHLORO CYCLOHEXENE

* SD-100-14

SD-100-14 12-May-1987 CHLORO CYCLOHEXENE

$\mathrm{ug} / \mathrm{L}$

* SD-100

SD-100 5-Mar-1987 CHLOROBENZENE

SD-100 11-Mar-1987 CHLOROBENZENE

SD-100 18-Mar-1987 CHLOROBENZENE

SD-100 24-Mar-1987 CHLOROBENZENE

SD-100 6-Apr-1987 CHLOROBENZENE

SD-100 12-May-1987 CHLOROBENZENE

SD-100 19-Kay-1987 CHLOROBENZENE

SD-100 26-May-1987 CHLOROBENZENE

SD -100

3-JUI-1987 CHLOROBENZENE

$\begin{array}{ll}<5 & \mathrm{ug} / \mathrm{L} \\ <5 & \mathrm{ug} / \mathrm{L} \\ <5 & \mathrm{ug} / \mathrm{L} \\ <5 & \mathrm{ug} / \mathrm{L} \\ <5 & \mathrm{ug} / \mathrm{L} \\ <5 & \mathrm{ug} / \mathrm{L} \\ <5 & \mathrm{ug} / \mathrm{L} \\ <5 & \mathrm{ug} / \mathrm{L} \\ <5 & \mathrm{ug} / \mathrm{L}\end{array}$


Stom Drain Data for SD-100

Location

Date

Test Compound

Results

Units

SD-100 11-JUR-1987 CHLOROBENZENE

SD-100

18-JUn-1987 CHLOROBENZENE

** SD-100-01

SD-100-01 12-May-1987 CHLOROBENZENE

SD-100-01 18-May-1987 CHLOROBENZENE

SD-100-01 26-May-1987 CHLOROBENZENE

SD-100-01 3-Jun-1987 CHLOROBENZENE

SD-100-01 11-JUn-1987 CHLOROBENZENE

SD-100-01 18-Jun-1987 CHLOROBENZENE

* SD-100-02

SD-100-02 12-May-1987 CHLOROBENZENE

SD-100-02 18-May-1987 CHLOROBENZENE

SD-100-02 26-May-1987 CHLOROBENZENE

SD-100-02 3-Jun-1987 CHLOROBENZENE

SD-100-02 11-JUn-1987 CHROROBENZENE

SD-100-02 18-Jun-1987 CHLOROBENZENE

$\star \star$ SD $-100-03$

SD-100-03 12-May-1987 CHLOROBENZENE

SD-100-03 18-May-1987 CHLOROBENZENE

SD-100-03 26-May-1987 CHLOROBENZENE

SD-100-03 3-Jun-1987 CHLOROBENZENE

SD-100-03 11-Jun-1987 GHLOROBENZENE

SD-100-03 18-Jun-1987 GHLOROBENZENE

* SD-100-03D

SD-100-03D 12-May-1987 CHLOROBENZENE

SD-100-03D 26-May-1987 CHLOROBENZENE

$<5$

$<5$

$<5$

$<5$

$<5$

$<5$

$<5$

$<5$

$<5$

$<5$

$<5$

$<5$

$<5$

$<5$

$<5$

$<5$

$<5$

$<5$

$<5$

$<5$

$<5$

$<5$

$<5$

$<5$

$<5$

$<5$

$<5$

$<5$

$<5$

$<5$

$<5$

$<5$

$<5$

$<5$

SD-100-06

12-May-1987 CHLOROBENZENE 18-May-1987 CHLOROBENZENE

26-May-1987 CHLOROBENZENE

3-Jun-1987 CHLOROBENZENE

11-Jun-1987 CHLOROBENZENE

18-Jun-1987 CHLOROBENZENE

** SD-100-06D

SD-100-06D 3-Jun-1987 CHLOROBENZENE 
Storm Drain Data for SD-100

\begin{tabular}{|c|c|c|}
\hline Location & Date & Test Compoun \\
\hline \multicolumn{3}{|c|}{$\star \star$ SD-100-07 } \\
\hline SD $-100-07$ & $12-\mathrm{May}-1987$ & GHLOROBENZENE \\
\hline SD $-100-07$ & $19-\mathrm{May}-1987$ & GLOROBENZENE \\
\hline SD $-100-07$ & $26-\mathrm{Kay}-1987$ & CHLOROBEIIZENE \\
\hline SD-100-07 & 3-Jun-1987 & GHLOROBENZERTE \\
\hline SD-100-07 & 11-Jun-1987 & CHLOROBENZEANE \\
\hline SD $=100.07$ & $18-J u n-1987$ & CHLOROBENZENE \\
\hline
\end{tabular}

Results Units

** SD-100-07D

SD-100-07D 18-Jun-1987 CHLOROBETZFNE

* SD-100-09

SD-100-09 12-Kay-1987 CHLOROBENZENE

SD-100-09 18-May-1987 CHLOROBENZENE

SD-100-09 26-May-1987 CHLOROBENZENE

SD-100-09 3-Jun-1987 GHLOROBENZENE

SD-100-09 11-Jun-1987 CHLOROB ENZENE

SD-100-09 18-Jun-1987 CHLOROBENZENE

$<5$

$<5$

$<5$

$<5$

$<5$

$<5$

$u g / L$

$u g / L$

ug $/ 2$

$4 g / 2$

ug $/$.

ug/L

** SD $100-10$

SD-100-10 12-May-1987 CHLOROBENZENE

SD-100-10 19-May-1987 CHLOROBENZENE

SD-100-10 26-May-1987 CHLOROBENZENE

SD-100-10 3-Jun-1987 CHLOROBENZENE

SD-100-10 11-Jun-1987 CHLOROBENZENE

SD-100-10 18-Jun-1987 CHLOROBENZENE

$<5$

ug/L

$<5$

$<5$

$<5$

$<5$

$<5$

$<5$

$\mathrm{ug} / \mathrm{L}$

ug/L

ug/L

$\mathrm{ug} / \mathrm{L}$

$4 g / 2$

$\mathrm{ug} / \mathrm{L}$

$<5$

ug $/$ L

$<5$

$<5$

$<5$

$<5$

$<5$

ug/l

$\mathrm{ug} / \mathrm{L}$

$u g / L$

$\mathrm{ug} / \mathrm{L}$

ug/L

* SD-100-11

SD-100-11 12-May-1987 CHLOROBENZENE

SD-100-11 19-May-1987 CHLOROBENZENE

SD-100-11 26-May-1987 CHLOROBENZENE

SD-100-11 3-Jun-1987 CHLOROBENZENE

SD-100-11 11-Jun-1987 CHLOROBENZENE

SD-100-11 18-Jun-1987 CHLOROBEN2ENE

\section{* SD $-100-12$}

SD-100-12 12-May-1987 CHLOROBENZENE

SD-100-12 19-May-1987 CHLOROBENZENE

SD-100-12 26-May-1987 CHLOROBENZENE

SD-100-12 3-Jun-1987 CHLOROBENZENE

SD-100-12 11-JUn-1987 CHLOROBENZENE

SD-100-12 18-Jun-1987 CHLOROBENZENE

$<5$

$<5$

$<5$

$<5$

$<5$

$<5$

$\mathrm{ug} / \mathrm{L}$

ug $/ \mathrm{L}$

$\mathrm{ug} / \mathrm{L}$

$\mathrm{ug} / \mathrm{L}$

$u g / L$

$u g / L$

* SD $-100-13$

SD-100-13 12-May-1987 CHLOROBENZENE

SD-100-13 19-May-1987 CHLOROBENZENE

SD-100-13 26-May-1987 CHLOROBENZENE

SD-100-13 3-Jun-1987 CHLOROBENZENE

SD-100-13 11-Jun-1987 CHLOROBENZENE

SD-100-13 18-Jun-1987 CHLOROBENZENE

$\begin{array}{ll}<5 & u g / L \\ <5 & u g / L \\ <5 & u g / L \\ <5 & u g / L \\ <5 & u g / L \\ <5 & u g / L \\ & \\ & \\ <5 & u g / L \\ <5 & u g / L \\ <5 & u g / L \\ <5 & u g / L \\ <5 & u g / L \\ <5 & u g / L\end{array}$


Location Date Test Compound Results Units

** SD-100-14

SD-100-14 12-May-1987 CHLOROBENZENE

SD-100-14 19-May-1987 CHLOROBENZENE

SD-100-14 26-May-1987 CHLOROBENZENE

SD-100-14 3-Jun-1987 CHLOROBENZENE

SD-100-14 11-Jun-1987 CHLOROBENZENE

SD-100-14 18-Jun-1987 CHLOROBEN2ENE

t* $S D-100-C$

SD-100-C 5-Mar-1987 CHLOROBENZENE

SD-100-C 9-Mar-1987 CHLOROBENZENE

SD-100-C 17-Mar-1987 CHLOROBENZENE

SD-100-C 24-Mar-1987 CHLOROBENZENE

SD-100-C 8-APr-1987 CHLOROBENZENE

SD-100-C 13-May-1987 CHLOROBENZENE

SD-100-C 20-May-1987 CHLOROBENZENE

SD-100-C 27-May-1987 CHLOROBENZENE

SD-100-C 12-JUn-1987 CHLOROBENZENE

SD-100-C 19-Jun-1987 CHLOROBENZENE

l

** SD - $100-D$

SD-100-D 6-APT-1987 CHLOROBBNZENE

SD-100-D 11-Jun-1987 CHLOROBENZENE

* SD 100

SD -100

SD -100

SD -100

SD -100

$S D-100$

SD -100

SD -100

SD -100

SD -100

SD -100

SD-100
5-Mar-1987 CHLORORTHANE 11-Mar-1987 CHLOROETHANE 18-Mar-1987 CHLOROETHANE 24-Mar-1987 CHLOROETHANE 6-API-1987 CHLOROETHANE 12-May-1987 CHLOROETHANE 19-May-1987 CHLOROETHANE 26-May-1987 CHLOROETHANE 3-Jun-1987 CHLOROETHANE 11-JUR-1987 CHLOROETHANE 18-Jun-1987 CHLOROETIANE

* SD-100-01

SD-100-01 12-May-1987 CHLOROETHANE

SD-100-01 18-May-1987 CHLOROETHANE

SD-100-01 26-May-1987 CHLOROETHANE

SD-100-01 3-Jun-1987 CHLOROETHANE

SD-100-01 11-Jun-1987 CHLOROEIHANE

SD-100-01 18-Jun-1987 CHLOROETHANE

* SD $-100-02$

SD-100-02 12-May-1987 CHLOROETHANE

SD-100-02 18-May-1987 CHLOROETHANE

SD-100-02 26-May-1987 CHLOROETHANE

SD-100-02 3-Jun-1987 CHLOROETHANE

SD-100-02 11-Jun-1987 CHLOROETHANE

$\begin{array}{ll}<5 & u g / L \\ <5 & u g / L \\ <5 & u g / L \\ <5 & u g / L \\ <5 & u g / L \\ <5 & u g / L\end{array}$

$<5$

$<5$

$<5$

$<5$

$1 \mathrm{~J}$

$<5$

$<5$

$<5$

$<5$

$<5$

ug/L

ug/L

ug/L

ug/L

ug/L

$4 \mathrm{~g} / \mathrm{L}$

ug $/ \mathrm{L}$

ug $/ \mathrm{L}$

ug $/$.

ug $/ 2$

$<5$

$<5$

ug $/$ L

ug/ $/$

$<10$

$<10$

$<10$

$<10$

$<10$

$<10$

$<10$

$<10$

$<10$

$<10$

$<10$

$\mathrm{ug} / \mathrm{L}$

ug $\Omega$ L

ug/L

ug $/ L$

ug/L

ug $/ L$

ug/L

ug/L

ug/L

ug $/$ L

ug $/ \mathrm{L}$

$<10$

ug/L

$<10$

$<10$

$<10$

$<10$

$<10$

ug/L

$\mathrm{ug} / \mathrm{L}$

ug/L

$4 \mathrm{~g} / \mathrm{L}$

ug/L

$<10$

$<10$

ug/L

$<10$

$<10$

ug/L

ug/L

ug/L

$<10$ 
Storm Drain Data for SD-100

$\begin{array}{lcc}\text { Location } & \text { Date } & \text { Test COMPO } \\ \text { SD-100-02 } & 18 \text {-Jun-1987 } & \text { CHLOROETHANE } \\ & & \\ \text { A } & \\ \text { SD-100-03 } & & \\ \text { SD-100-03 } & 12-\text { May-1987 } & \text { CHLOROETHANE } \\ \text { SD-100-03 } & 18 \text {-May-1987 } & \text { CHLOROETHANE } \\ \text { SD-100-03 } & 26 \text {-May-1987 CHLOROETHANE } \\ \text { SD-100-03 } & 3 \text {-Jun-1987 CHLOROETHANE } \\ \text { SD-100-03 } & 11 \text {-Jun-1987 CHLOROETHANE } \\ \text { SD-100-03 } & 18 \text {-Jun-1987 CHLOROETHANE }\end{array}$

$\begin{array}{cc}\text { Results } & \text { Undts } \\ & \\ <10 & \mathrm{ug} / \mathrm{L} \\ & \\ & \\ <10 & \mathrm{ug} / \mathrm{L} \\ <10 & \mathrm{ug} / \mathrm{L} \\ <10 & \mathrm{ug} / \mathrm{L} \\ <10 & \mathrm{ug} / \mathrm{L} \\ <10 & \mathrm{ug} / \mathrm{L} \\ <10 & \mathrm{ug} / \mathrm{L}\end{array}$

** SD-100-03D

SD-100-03D 12-May-1987 CHLOROETHANE SD-100-03D 26-May-1987 CHLOROETHANE

$u g / L$ $u g / L$

** SD-100-04

SD-100-04 12-May-1987 CHLOROETHANE SD-100-04 18-May-1987 CHLOROETHLNE SD-100-04 26-May-1987 CHLOROETHANE SD-100-04 3-Jun-1987 CHLOROETHANE SD-100-04 11-Jun-1987 CHLOROETHANE SD-100-04 18-Jun-1987 CKLOROETHANE

* SD $-100-06$

SD-100-06 12-May-1987 CHLOROETHANE SD-100-06 18-May-1987 CHLOROETHANE SD-100-06 26-May-1987 CHLOROETHANE SD-100-06 3-Jun-1987 CHLOROETHANE SD-100-06 11-Jun-1987 CHLOROETHANE SD-100-06 18-JUn-1987 CHLOROETHANE

* SD-100-06D

SD-100-06D 3-Jun-1987 CHLOROETHANE

$<10$

ug $/ \mathrm{L}$ $\mathrm{ug} / \mathrm{L}$ $\mathrm{ug} / \mathrm{L}$ ug/L ug $\Omega$ ug $/ \mathrm{L}$

$<10$

$<10$

$<10$

$<10$

$<10$

$<10$

ug/ $/ \mathrm{L}$

ug/L

$4 \mathrm{~g} / \mathrm{L}$

$4 \mathrm{~g} / \mathrm{L}$

ug/L

$\mathrm{ug} / \mathrm{L}$

*t SD-100-07

SD-100-07 12-May-1987 CHLOROETHANE SD-100-07 19-May-1987 CHLOROETLANE SD-100-07 26-May-1987 CHLOROETHANE SD-100-07 3-Jun-1987 CHLOROETHANE SD-100-07 11-Jun-1987 CHLOROETHANE SD-100-07 18-Jun-1987 CHLOROETHANE

ug/L

* SD-100-07D

SD-100-07D 18-Jun-1987 CHLOROETHANE

$<10$

ug $/ 2$

** SD $-100-09$

SD-100-09 12-May-1987 CHLOROETHANE

SD-100-09 18-May-1987 CHLOROETHANE

SD-100-09 26-May-1987 CHLOROETHANE

SD-100-09 3-JUn-1987 CHLOROETHANE

SD-100-09 11-JUn-1987 CHLOROETHANE

SD-100-09 18-Jun-1987 CHLOROETHANE

$\begin{array}{ll}<10 & \mathrm{ug} / \mathrm{L} \\ <10 & \mathrm{ug} / \mathrm{L} \\ <10 & \mathrm{ug} / \mathrm{L} \\ <10 & \mathrm{ug} / \mathrm{L} \\ <10 & \mathrm{ug} / \mathrm{L} \\ <10 & \mathrm{ug} / \mathrm{L}\end{array}$


Storn Drain Data for SD-100

Locstion Date Test Compound Results Units

$\begin{array}{lll}\text { Wt } & \text { SD-100-10 } \\ \text { SD-100-10 } & 12 \text {-May-1987 } & \text { CHLOROETHANE } \\ \text { SD-100-10 } & 19 \text {-May-1987 CHLOROETHANE } \\ \text { SD-100-10 } & 26 \text {-May-1987 CHLOROETHANE } \\ \text { SD-100-10 } & \text { 3-Jun-1987 } & \text { CHLOROETHANE } \\ \text { SD-100-10 } & 11 \text {-Jun-1987 CHLOROETHANE } \\ \text { SD-100-10 } & 18 \text {-Jun-1987 CHLOROETHANE }\end{array}$

** SD-100-11

SD-100-11 12-May-1987 CHLOROETHANE

SD-100-11 19-May-1987 CHLOROETHANE

SD-100-11 26-May-1987 CHLOROETHANE

SD-100-11 3-Jun-1987 CHLOROETHANE

SD-100-11 11-Jun-1987 CHLOROETHANE

SD-100-11 18-JUn-1987 CHLOROETHANE

* SD-100-12

SD-100-12 12-May-1987 CHLOROETHANE

SD-100-12 19-May-1987 CHLOROETHANE

SD-100-12 26-May-1987 CHLOROETHANE

SD-100-12 3-JUn-1987 CHLOROETLANE

- SD-100-12 11-JUn-1987 CHLOROETHANE

SD-100-12 18-JUn-1987 CHLOROETHANE

* SD-100-13

SD-100-13 12-May-1987 CHLOROETHANE

SD-100-13 19-May-1987 CHLOROETHANE

SD-100-13 26-May-1987 CHLOROETHANE

SD-100-13 3-Jun-1987 CHLOROETHANE

SD-100-13 11-JUn-1987 CHLOROETHANE

SD-100-13 18-JUn-1987 CHLOROETHANE

* SD $-100-14$

SD-100-14 12-May-1987 CHLOROETHANE

SD-100-14 19-May-1987 CHLOROETHANE

SD-100-14 26-May-1987 CHLOROETHANE

SD-100-14 3-Jun-1987 CHLOROETHANE

SD-100-14 11-Jun-1987 CHLOROETHANE

SD-100-14 18-Jun-1987 CHLOROETHANE

** SD-100-C

SD-100-C 5-Mar-1987 CHLOROETHANE

SD-100-C 9-Mar-1987 CHLOROETHANE

SD-100-C 17-Mar-1987 CHLOROETHANE

SD-100-C 24-Mar-1987 CHLOROETHANE

SD-100-C 8-APL-1987 CHLOROETHANE

SD-100-C 13-May-1987 CHLOROETHANE

SD-100-C ' 20-May-1987 CHLOROETHANE

SD-100-C 27-May-1987 CHLOROETHANE

SD-100-C 12-JUn-1987 CHLOROETHANE

$\begin{array}{ll}<10 & \mathrm{ug} / \mathrm{L} \\ <10 & \mathrm{ug} / \mathrm{L} \\ <10 & \mathrm{ug} / \mathrm{L} \\ <10 & \mathrm{ug} / \mathrm{L} \\ <10 & \mathrm{ug} / \mathrm{L} \\ <10 & \mathrm{ug} / \mathrm{L} \\ & \\ & \\ <10 & \mathrm{ug} / \mathrm{L} \\ <10 & \mathrm{ug} / \mathrm{L} \\ <10 & \mathrm{ug} / \mathrm{L} \\ <10 & \mathrm{ug} / \mathrm{L} \\ <10 & \mathrm{ug} / \mathrm{L} \\ <10 & \mathrm{ug} / \mathrm{L} \\ & \mathrm{u} \\ & \mathrm{ug} / \mathrm{L} \\ <10 & \mathrm{ug} / \mathrm{L} \\ <10 & \mathrm{ug} / \mathrm{L} \\ <10 & \mathrm{ug} / \mathrm{L} \\ <10 & \mathrm{ug} / \mathrm{L} \\ <10 & \mathrm{ug} / \mathrm{L} \\ <10 & \\ & \\ & \mathrm{ug} / \mathrm{L} \\ <10 & \mathrm{ug} / \mathrm{L} \\ <10 & \mathrm{ug} / \mathrm{L} \\ <10 & \mathrm{ug} / \mathrm{L} \\ <10 & \mathrm{ug} / \mathrm{L} \\ <10 & \mathrm{ug} / \mathrm{L} \\ <10 & \end{array}$

$<10$

$<10$

$<10$

$<10$

$<10$

$<10$

$\mathrm{ug} / \mathrm{L}$

ug $/ \mathrm{L}$

ug/L

$4 \mathrm{~g} / \mathrm{L}$

ug/L

$\mathrm{ug} / \mathrm{L}$

$<10 \quad 4 \mathrm{~g} / \mathrm{L}$

$<10 \quad$ ug $/ \mathrm{L}$

$<10 \quad \mathrm{ug} / \mathrm{L}$

$<10 \quad \mathrm{ug} / \mathrm{L}$

$<10 \quad$ ug/L

$<10 \quad \mathrm{ug} / \mathrm{L}$

$<10 \quad \mathrm{ug} / \mathrm{L}$

$<10 \quad \mathrm{ug} / \mathrm{L}$

$<10 \quad \mathrm{ug} / \mathrm{L}$ 
Storm Drain Data for SD-100

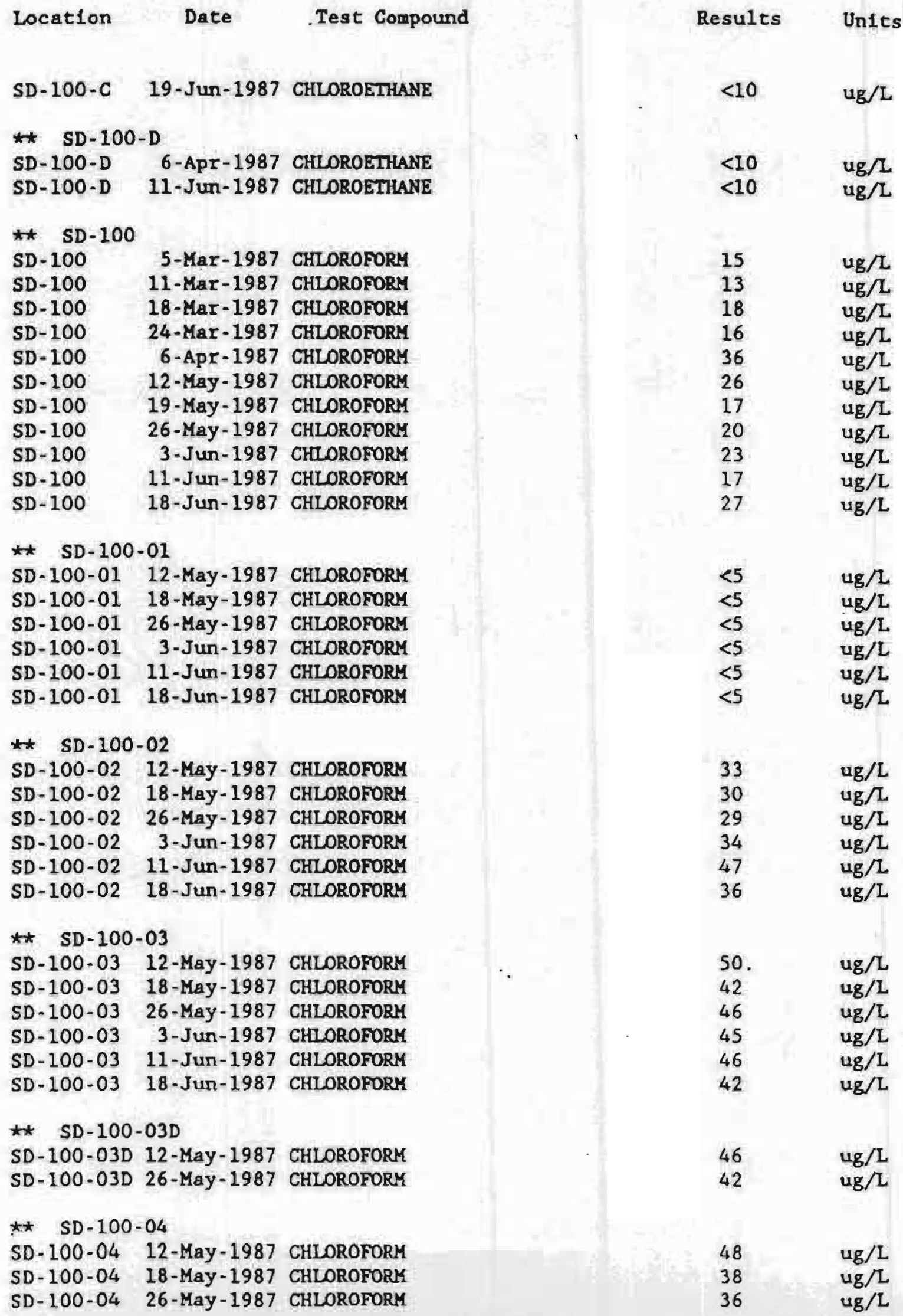


Location Date Test Compound Resules UnIts

$\begin{array}{rrr}\text { SD-100-10 } & 3 \text {-Jun-1987 } & \text { COPPER } \\ \text { SD-100-10 } & 11 \text {-Jun-1987 } & \text { COPPER } \\ \text { SD-100-10 } & 18-J u n-1987 & \text { COPPER }\end{array}$

* SD-100-11

SD-100-11 12-May-1987 COPPER

SD-100-11 19-May-1987 COPPER

SD-100-11 26-May-1987 COPPER

SD-100-11 3-Jun-1987 COPPER

SD-100-11 11-Jun-1987 COPPER

SD-100-11 18-Jun-1987 COPPER

** SD $-100-12$

SD-100-12 12-May-1987 COPPER

SD-100-12 19-May-1987 COPPER

SD-100-12 26-May-1987 COPPER

SD-100-12 3-Jun-1987 COPPER

SD-100-12 11-Jun-1987 COPPER

SD-100-12 18-Jun-1987 COPPER

*t SD-100-13

SD-100-13 12-May-1987 COPPER

SD-100-13 19-May-1987 COPPER

SD-100-13 26-May-1987 COPPER

SD-100-13 II-Jun-1987 COPPER

SD-100-13 18-Jun-1987 COPPER

* SD $-100-14$

SD-100-14 12-May-1987 COPPER

SD-100-14 19-May-1987 COPPER

SD-100-14 26-Kay-1987 COPPER

SD-100-14 3-Jun-1987 COPPER

SD-100-14 11-Jun-1987 COPPER

SD-100-14 18-Jun-1987 COPPER

* $\quad$ SD-100-C

SD-100-C 5-Mar-1987 COPPER

SD-100-C 9-Mar-1987 COPPER

SD-100-C 17-Mar-1987 COPPER

SD-100-C 24-Mar-1987 COPPER

SD-100-C 8-Apr-1987 COPPER

SD-100-C 13-May-1987 COPPER

SD-100-C 20-MaY-1987 COPPER

SD-100-C 27-May-1987 COPPER

SD-100-C 4-Jun-1987 COPPER

SD-100-C 12-Jun-1987 COPPER

SD-100-C 19-Jun-1987 COPPER

$\star$ SD-100-D

SD-100-D 6-APY-1987 COPPER

SD-100-D 11-Jun-1987 COPPER $\begin{array}{ll}0.054 & \mathrm{mg} / \mathrm{h} \\ <0.0040 & \mathrm{mg} / \mathrm{L} \\ <0.0040 \quad \mathrm{mg} / \mathrm{L}\end{array}$

$<0.0040 \mathrm{mg} / \mathrm{L}$

$<0.0040 \mathrm{mg} / \mathrm{L}$

$<0.0040 \mathrm{mg} / \mathrm{L}$

$0.0075 \mathrm{mg} / \mathrm{L}$

$<0.0040 \mathrm{mg} / \mathrm{L}$

$<0.0040 \mathrm{mg} / \mathrm{L}$

$\begin{array}{ll}0.60 & \mathrm{mg} / \mathrm{L} \\ 0.017 & \mathrm{mg} / \mathrm{L} \\ 0.055 & \mathrm{mg} / \mathrm{L} \\ 0.013 & \mathrm{mg} / \mathrm{L} \\ 0.044 & \mathrm{mg} / \mathrm{L} \\ 0.025 & \mathrm{mg} / \mathrm{L}\end{array}$

$<0.0040 \mathrm{mg} / \mathrm{L}$

$0.011 \mathrm{mg} / \mathrm{L}$

$<0.0040 \mathrm{mg} / \mathrm{L}$

$<0.0040 \mathrm{mg} / \mathrm{L}$

$<0.0040 \mathrm{mg} / \mathrm{L}$

$0.019 \mathrm{mg} / \mathrm{L}$

$0.030 \mathrm{mg} / \mathrm{L}$

$0.029 \mathrm{mg} / \mathrm{L}$

$0.010 \mathrm{mg} / \mathrm{L}$

$0.013 \mathrm{mg} / \mathrm{L}$

$<0.0040 \mathrm{mg} / \mathrm{L}$

0.016

$<0.0040 \mathrm{mg} / \mathrm{L}$

$<0.0040 \mathrm{mg} / \mathrm{L}$

$0.0090 \mathrm{mg} / \mathrm{L}$

$<0.0040 \mathrm{mg} / \mathrm{L}$

$<0.0040 \mathrm{mg} / \mathrm{L}$

$<0.0040 \mathrm{mg} / \mathrm{L}$

$<0.0040 \mathrm{mg} / \mathrm{L}$

$<0.0040 \mathrm{mg} / \mathrm{L}$

$<0.0040 \mathrm{mg} / \mathrm{L}$

$<0.0040 \mathrm{mg} / \mathrm{L}$

$0.0055 \mathrm{mg} / \mathrm{L}$ $<0.0040 \mathrm{mg} / \mathrm{L}$ 


$\begin{array}{ll}0.014 & \mathrm{mg} / \mathrm{L} \\ 0.11 & \mathrm{mg} / \mathrm{L} \\ 0.05 & \mathrm{mg} / \mathrm{L} \\ 0.08 & \mathrm{mg} / \mathrm{L} \\ 0.07 & \mathrm{mg} / \mathrm{L} \\ 0.004 & \mathrm{mg} / \mathrm{L} \\ .041 & \mathrm{mg} / \mathrm{L} \\ 0.02 & \mathrm{ng} / \mathrm{L} \\ 0.016 & \mathrm{mg} / \mathrm{L} \\ <0.002 & \mathrm{mg} / \mathrm{L} \\ 0.12 & \mathrm{mg} / \mathrm{L} \\ .08 & \mathrm{mg} / \mathrm{L}\end{array}$

** SD-100-02

SD-100-02 12-Mny-1987 CYANIDE

SD-100-02 18-May-1987 CYANIDE

SD-100-02 26-May-1987 CYANIDE

SD-100-02 3-Jun-1987 CYANIDE

SD-100-02 I1-Jun-1987 CYAMTDE

SD-100-02 18-Jun-1987 CYANIDE

$\begin{array}{ll}<0.002 & \mathrm{mg} / \mathrm{L} \\ 0.002 & \mathrm{mg} / \mathrm{L} \\ 0.002 & \mathrm{mg} / \mathrm{L} \\ <0.002 & \mathrm{mg} / \mathrm{L} \\ .02 & \mathrm{mg} / \mathrm{L} \\ <0.002 & \mathrm{mg} / \mathrm{L}\end{array}$

* SD $-100-03$

SD-100-03 12-May-1987 CYANIDE

SD-100-03 18-May-1987 CYANIDE

SD-100-03 26-May-1987 CYANIDE

SD-100-03 3-Jun-1987 CYANIDE

SD-100-03 11-Jun-1987 CYANIDE

SD-100-03 18-Jun-1987 CYANIDE

$\begin{array}{ll}<0.002 & \mathrm{mg} / \mathrm{L} \\ <0.002 & \mathrm{ng} / \mathrm{L} \\ 0.002 & \mathrm{ng} / \mathrm{L} \\ <0.002 & \mathrm{mg} / \mathrm{L} \\ <0.002 & \mathrm{mg} / \mathrm{L} \\ <0.002 & \mathrm{mg} / \mathrm{L}\end{array}$

** SD-100-03D

SD-100-03D 12-May-1987 CYARIDE

SD-100-03D 26-May-1987 CYANTDE

$\begin{array}{ll}<0.002 & \mathrm{mg} / \mathrm{L} \\ 0.002 & \mathrm{mg} / \mathrm{L} \\ <0.002 & \mathrm{mg} / \mathrm{L} \\ <0.002 & \mathrm{mg} / \mathrm{L} \\ <0.002 & \mathrm{mg} / \mathrm{L} \\ <0.002 & \mathrm{mg} / \mathrm{L}\end{array}$

* SD-100-04

SD-100-04 12-May-1987 CYANIDE

SD-100-04 18-May-1987 CYANIDE

SD-100-04 26-May-1987 CYANIDE

SD-100-04 3-Jun-1987 CYANIDE

SD-100-04 11-Jun-1987 CYANIDE

SD-100-04 18-Jun-1987 CYANIDE

$<0.002 \quad \mathrm{gg} / \mathrm{L}$

$<0.002 \mathrm{mg} / \mathrm{L}$

$<0.002 \mathrm{mg} / \mathrm{L}$

$<0.002 \mathrm{mg} / \mathrm{L}$

$0.002 \mathrm{gg} / \mathrm{L}$

$<0.002 \mathrm{mg} / \mathrm{L}$

$<0.002 \mathrm{mg} / \mathrm{L}$ 
** SD-100-06

SD-100-06 12-May-1987 CYANIDE

SD-100-06 18-May-1987 CYANIDE

SD-100-06 26-May-1987 CYANIDE

SD-100-06 3-Jun-1987 CYANIDE

SD-100-06 11-Jun-1987 CYANIDE

SD-100-06 18-Jun-1987 CXANIDE

$\begin{array}{ll}<0.002 & \mathrm{mg} / \mathrm{L} \\ <0.002 & \mathrm{mg} / \mathrm{L} \\ <0.002 & \mathrm{mg} / \mathrm{L} \\ <0.002 & \mathrm{mg} / \mathrm{L} \\ <0.002 & \mathrm{mg} / \mathrm{L} \\ <0.002 & \mathrm{mg} / \mathrm{L}\end{array}$

** SD-100-06D

SD-100-06D 3-Jun-1987 CXANIDE

** SD-100-07

SD-100-07 12-May-1987 CYANIDE

SD-100-07 19-May-1987 CYANIDE

SD-100-07 26-May-1987 CYANIDE

SD-100-07 3-Jun-1987 CYANIDE

SD-100-07 11-Jun-1987 CYANIDE

SD-100-07 18-Jun-1987 CYANIDE

** SD-100-07D

SD-100-07D 18-Jun-1987 CYANIDE

.06

$\mathrm{mg} / \mathrm{L}$

** SD $-100-09$

SD-100-09 12-May-1987 CYANIDE

SD-100-09 18-May-1987 CYANIDE

SD-100-09 26-May-1987 CYANIDE

SD-100-09 3-Jun-1987 CYANIDE

SD-100-09 11-Jun-1987 CYANIDE

SD-100-09 18-Jun-1987 CYANIDE

$\begin{array}{ll}<0.002 & \mathrm{mg} / \mathrm{L} \\ 0.002 & \mathrm{mg} / \mathrm{L} \\ <0.002 & \mathrm{mg} / \mathrm{L} \\ <0.002 & \mathrm{mg} / \mathrm{L} \\ <0.002 & \mathrm{ag} / \mathrm{L} \\ <0.002 & \mathrm{mg} / \mathrm{L}\end{array}$

** SD $-100-10$

SD-100-10 12-May-1987 CYANIDE

SD-100-10 19-May-1987 CYANIDE

SD-100-10 26-May-1987 CYANIDE

SD-100-10 3-Jum-1987 CYANIDE

SD-100-10 11-Jun-1987 CYANIDE

SD-100-10 18-Jun-1987 CYANIDE

$\begin{array}{ll}<0.002 & \mathrm{gg} / \mathrm{L} \\ <0.002 & \mathrm{gg} / \mathrm{L} \\ <0.002 & \mathrm{ng} / \mathrm{L} \\ <0.002 & \mathrm{mg} / \mathrm{L} \\ <0.002 & \mathrm{ng} / \mathrm{L} \\ .002 & \mathrm{gg} / \mathrm{L}\end{array}$

** SD $-100-11$

SD-100-11 12-May-1987 CYANIDE

SD-100-11 19-May-1987 CYANIDE

SD-100-1I 26-May-1987 CYANIDE

SD-100-11 3-Jun-1987 CYANIDE

SD-100-11 11-Jun-1987 CYANIDE

SD-100-11 18-Jun-1987 CYANIDE

$\begin{array}{ll}0.003 & \mathrm{mg} / \mathrm{L} \\ <0.002 & \mathrm{mg} / \mathrm{L} \\ 0.004 & \mathrm{mg} / \mathrm{L} \\ <0.002 & \mathrm{mg} / \mathrm{L} \\ <0.002 & \mathrm{mg} / \mathrm{L} \\ <0.002 & \mathrm{ng} / \mathrm{L}\end{array}$

** SD $-100-12$

SD-100-12 12-May-1987 CYANIDE

SD-100-12 19-May-1987 GYANIDE

SD-100-12 26-May-1987 CYANIDE

$0.003 \mathrm{mg} / \mathrm{L}$

$<0.002 \mathrm{mg} / \mathrm{L}$

SD-100-12 3-Jun-1987 CYANIDE

$0.002 \mathrm{ng} / \mathrm{L}$

$<0.002 \mathrm{mg} / \mathrm{L}$ 
Location Date Test Compound

Resules Units

SD-100-12 11-Jun-1987 CYANIDE

SD-100-12 18-Jun-1987 CYANIDE

$<0.002 \mathrm{mg} / \mathrm{L}$

$<0.002 \mathrm{mg} / \mathrm{L}$

* SD-100-13

SD-100-13 12-May-1987 CYANIDE

SD-100-13 19-May-1987 GYANIDE

SD-100-13 26-May-19B7 GYANIDE

SD-100-13 3-Jun-1987 CYANIDE

SD-100-13 11-JUn-19B7 CYANIDE

SD-100-13 18-Jun-1987 CYANIDE

$<0.002 \mathrm{mg} / \mathrm{L}$

$0.004 \mathrm{mg} / \mathrm{L}$

$<0.002 \mathrm{mg} / \mathrm{L}$

$<0.002 \mathrm{mg} / \mathrm{L}$

$<0.002 \mathrm{mg} / \mathrm{L}$

$<0.002 \quad \mathrm{mg} / \mathrm{L}$

* SD-100-14

SD-100-14 12-May-1987 CYANIDE

$0.002 \mathrm{mg} / \mathrm{L}$

SD-100-14 19-May-1987 CYANIDE

0.03

$\mathrm{mg} / \mathrm{L}$

SD-100-14 26-May-1987 CYANIDE

SD-100-14 3-Jun-1987 CYANIDE

SD-100-14 11-Jun-1987 CYANIDE

SD-100-14 18-Jun-1987 CYANIDE

$<0.002$

$<0.002$

$\mathrm{mg} / \mathrm{L}$

$<0.002$

* SD-100-C

$\begin{array}{lrr}\text { SD-100-C } & \text { 5-Mar-1987 CYANIDE } \\ \text { SD-100-C } & 9 \text {-Mar-1987 CYANIDE } \\ \text { SD-100-C } & 17 \text {-Mar-1987 CYANIDE } \\ \text { SD-100-C } & 24 \text {-Mar-1987 CYANIDE } \\ \text { SD-100-C } & 8 \text {-APr-1987 CYANIDE } \\ \text { SD-100-C } & 13 \text {-May-1987 CYANIDE } \\ \text { SD-100-C } & 20 \text {-May-1987 CYANIDE } \\ \text { SD-100-C } & 27 \text {-May-1987 CYANIDE } \\ \text { SD-100-C } & 4-J \text { Un-1987 CYANIDE } \\ \text { SD-100-C } & 12-J u n-1987 & \text { CYANDDE } \\ \text { SD-100-C } & 19-\text { Jun-1987 CYANIDE }\end{array}$

$0.08 \mathrm{mg} / \mathrm{L}$

$0.01 \mathrm{mg} / \mathrm{L}$.

$0.03 \mathrm{mg} / \mathrm{L}$

$0.1 \mathrm{mg} / \mathrm{L}$

$0.03 \mathrm{mg} / \mathrm{L}$

$0.16 \mathrm{mg} / \mathrm{L}$

$0.11 \mathrm{mg} / \mathrm{L}$

$0.07 \mathrm{mg} / \mathrm{L}$

$.018 \mathrm{mg} / \mathrm{L}$

$0.04 \mathrm{mg} / \mathrm{L}$

$0.2 \mathrm{mg} / \mathrm{L}$

* SD-100-D

SD-100-D 6-Apr-1987 CYANIDE

$0.003 \mathrm{mg} / \mathrm{L}$

0.12 mg $/ \mathrm{L}$

** SD-100-07

SD-100-07 12-May-1987 DECANOIC ACID

5

ug/L

** SD-100

SD-100 5-Mar-1987 DI-N-BUTYLPHTHALATE

SD-100 11-Max-1987 DI-N-BUTYLPHTHALATE

SD-100 12-May-1987 DI-N-BUTYLPHTHALATE

SD-100 26-May-1987 DI-N-BUTYLPHTHALATE

** SD $-100-01$

SD-100-01 12-May-19B7 DI-N-BUTYLPHTHALATE

SD-100-01 26-May-1987 DI-N-BUTYLPHTHALATE

$<10 \quad$ ug $/ 2$

$<5.0 \quad$ ug $/ \mathrm{L}$

* SD-100-02

SD-100-02 12-May-1987 DI-N-BUTYLPHTHAIATE

$<10$

ug/L 
Storm Drain Data for SD- 100

Location

Date

Test Compound

Results Units

SD-100-02 26-May-1987 DI-N-BUTYLPHTHALATE

$<5.0$

ug/L

** SD $-100-03$

SD-100-03 12-Kay-1987 DI-N-BUTYLPHTHALATB

SD-100-03 26-May-1987 DI-N-BUTYLPHTHALATE

* SD-100-03D

SD-100-03D 12-May-1987 DI-N-BUTYLPHTHALATE

SD-100-03D 26-May-1987 DI-N-BUTYLPHTHALATE

** SD-100-04

SD-100-04 12-May-1987 DI-N-BUTYLPHTHALATE

SD-100-04 26-May-1987 DI-N-BUTYLPHTHALATE

** SD-100-06

SD-100-06 12-May-1987 DI -N-BUTYLPHTHALATE

SD-100-06 26-May-1987 DI-N-BUTYLPHTHALATE

* SD-100-07

SD-100-07 12-May-1987 DI-N-BUTYLPHTHALATE

SD-100-07 26-May-1987 DI-N-BUTYLPHTHALATE.

** SD-100-09

SD-100-09 12-May-1987 DI-N-BUTYLPHTHALATE

SD-100-09 26-May-1987 DI-N-BUTYLPHTHALATE

** SD $-100-10$

SD-100-10 12-Kay-1987 DI-N-BUTYLPHTHALATE

SD-100-10 26-May-1987 DI-N-BUTYLPHTHALATE

** SD $-100-11$

SD-100-11 12-May-1987 DI-N-BUTYLPHTHALATE

SD-100-11 26-May-1987 DI-N-BUTYLPHTHALATE

* SD-100-12

SD-100-12 12-May-1987 DI-N-BUTYPHTHALATE

SD-100-12 26-May-1987 DI-N-BUTYLPHTHALATE

$\star \star \quad S D-100-13$

SD-100-13 12-May-1987 DI-N-BUTYPHTHALATE

SD-100-13 26-May-1987 DI-N-BUTYLPHTHALATE

** SD-100-14

SD-100-14 12-May-1987 DI-N-BUTYLPHTHALATE

SD-100-14 26-May-1987 DI-N-BUTYLPHTHALATE

** SD-100-C

SD-100-C 5-Mar-1987 DI-N-BUTYLPHTHALATE

SD-100-C 9-Mar-1987 DI-N-BUTYLPHTHALATE

SD-100-C 17-Mat-1987 DI-N-BUTYZPHTHALATE

SD-100-C 24-Mat-1987 DI-N-BUTYLPHTHALATE

$<10 . \quad 4 g / 2$

$<5.0 \quad$ ug $/ \mathrm{L}$

$<10 \quad \mathrm{ug} / \mathrm{L}$

$<5.0 \quad$ ug/L

$<10 \quad u g / L$

$<5.0 \quad \mathrm{ug} / \mathrm{L}$

$0.4 \mathrm{JB} \quad \mathrm{ug} / \mathrm{L}$

$<5.0 \quad \mathrm{ug} / \mathrm{L}$

$<10 \quad 4 \mathrm{~g} / \mathrm{L}$

$<5.0 \quad \mathrm{ug} / \mathrm{L}$

$<10 \quad u g / L$

$<5.0 \quad \mathrm{ug} / \mathrm{L}$

$<10 \quad \mathrm{ug} / \mathrm{L}$

$<5.0 \quad \mathrm{ug} / \mathrm{L}$

$<10 \quad$ ug $/ \mathrm{L}$

$<5.0$ ug $/ \mathrm{L}$

$<10 \quad \mathrm{ug} / \mathrm{L}$

$<5.0 \quad \mathrm{ug} / \mathrm{L}$

$<10 \quad 4 \mathrm{~g} / \mathrm{L}$

$<5.0 \quad \mathrm{ug} / \mathrm{L}$

$<10 \quad 4 g / L$

$<5.0 \quad \mathrm{ug} / \mathrm{L}$

$<10 \quad$ ug/L

$<10$ ug/L

$<10 \quad$ ug $/ 2$

$<10 \quad \mathrm{ug} / \mathrm{L}$ 
Location Date Test Compound

SD-100-C 8-Apt-1987 DI-N-8UTYLPHTHALATE SD-100-C 13-May-1987 DI-N-BUTYLPHTHALATE SD-100-C 20-May-1987 DI-8V-BUTYLPHTLALATE SD-100-C 27-May-1987 DI-N-BUTYLPHTHALATE SD-100-C 12-Jun-1987 DI-B-BUTYLPHTHALATE SD-100-C 19-Jun-1987 DI-N-BUTYLPHTHALATE

* SD-100

SD -100

SD -100

5-Mar-1987 DI-LI-OCTYRPHTHALATE

SD -100

11-Mar-1987 DI-N-OCTYLPHTHALATE

12-May-1987 DI-N-OCTYLPHTHAIATE

SD -100
26-May-1987 DI-N-OCTYLPHTHALATE

* SD-100-01

SD-100-01 12-May-1987 DI-N-OCTYLPHTHALATE SD-100-01 26-May-1987 DI-N-OCTYLPHTHALATE

* SD-100-02

SD-100-02 12-May-1987 DI-N-OCTYLPHTHALATE

SD-100-02 26-May-1987 DI-N-OCTYLPHTHALATE

* SD-100-03

SD-100-03 12-May-1987 DI-N-OCTYLPHTLALATE SD-100-03 26-May-1987 DI-N-OCTYLPHTHALATE

* SD-100-03D

SD-100-03D 12-May-1987 DI-N-OCTYLPHTHALATE SD-100-03D 26-May-1987 DI-N-OCTYLPHTHALATE

** SD $-100-04$

SD-100-04 12-May-1987 DI-N-OCTYLPHTHALATE SD-100-04 26-May-1987 DI-N-OCTYLPHTHAIATE

* SD-100-06

SD-100-06 12-May-1987 DI-N-OCTYLPHTHALATE SD-100-06 26-May-1987 DI-N-OCTYLPHTHALATE

** SD-100-07

SD-100-07 12-May-1987 DI-N-OCTYLPHTHALATE

SD-100-07 26-May-1987 DI-N-OCTYLPHTHALATE

* SD-100-09

SD-100-09 12-May-1987 DI-N-OGTYLPHTHALATE SD-100-09 26-Kay-1987 DI-N-OCTYLPHTHALATE

** SD $-100-10$

SD-100-10 12-May-1987 DI-N-OCTYLPHTHALATE

SD-100-10 26-May-1987 DI-N-OCTYLPHTHALATE

* SD $-100-11$

SD-100-11 12-May-1987 DI-N-OCTYLPHTHALATE
Results Units

$\begin{array}{ll}<10 & \mathrm{ug} / \mathrm{L} \\ <5.0 & \mathrm{ug} / \mathrm{L} \\ 1 \mathrm{~J} & \mathrm{ug} / \mathrm{L} \\ <5.0 & \mathrm{ug} / \mathrm{L} \\ <5.0 & \mathrm{ug} / \mathrm{L} \\ <10 & \mathrm{ug} / \mathrm{L}\end{array}$

$<10 \quad$ ug $/ 2$

$1 \mathrm{~J} \quad 4 \mathrm{~g} / \mathrm{L}$

$<10 \quad 48 /$

$<5.0 \quad \mathrm{ug} / \mathrm{L}$

$<10 \quad u g / 2$

$<5.0 \quad \mathrm{ug} / \mathrm{L}$

$0.2 \mathrm{JB} \quad \mathrm{ug} / \mathrm{L}$

$<5.0 \quad \mathrm{ug} / \mathrm{L}$

$0.2 \mathrm{JB} \quad \mathrm{ug} / \mathrm{L}$

$<5.0 \quad \mathrm{ug} / \mathrm{L}$

$<10 \quad \mathrm{ug} / \mathrm{L}$

$<5.0 \quad \mathrm{ug} / \mathrm{L}$

$<I 0 \quad \mathrm{ug} / \mathrm{L}$

$<5.0 \quad \mathrm{ug} / \mathrm{L}$

$<10 \quad$ ug/L

$<5.0 \quad \mathrm{ug} / \mathrm{L}$

$<10 \quad \mathrm{ug} / \mathrm{L}$

$<5.0 \quad \mathrm{ug} / \mathrm{L}$

$<10 \quad u g / 2$

$<5.0 \mathrm{ug} / \mathrm{L}$

$<10 \quad$ ug/L

$<5.0 \quad \mathrm{ug} / \mathrm{L}$

$<10 \quad$ ug $\Omega$ 
Storm Drain Daca for SD-100

Location Date Test Compound

Results Units

SD-100-11 26-May-1987 DI-N-OCTYLPTHALATE

$<5.0 \quad$ ug/2

** SD-100-12

SD-100-12 12-May-1987 DI-N-OCTYLPHTHATATE

SD-100-12 26-May-1987 DI-N-OCTYLPHTHAIATE

** SD $-100-13$

SD-100-13 12-May-1987 DI-N-OCTYLPHTHALATE

SD-100-13 26-May-1987 DI-N-OCTYLPHTHAIATE

* SD $-100-14$

SD-100-14 12-May-1987 DI-N-OCTYLPHTHALATE

SD-100-14 26-May-1987 DI-N-OCTYLPHTHALATE

** SD-100.C

SD-100-C 5-Max-1987 DI -N-OCTYLPHTHALATE

SD-100-C 9-Mar-1987 DI-N-OCTYLPHTHALATE

SD-100-C 17-Mar-1987 DI-N-OCTYLPHTHALATE

SD-100-C 24-Mar-1987 DI-N-OCTYLPHTHALATE

SD-100-C 8-Apr-1987 DI-N-OCTYLPHTHALATE

SD-100-C 13-May-1987 DI-N-OCTYLPHTHALATE

SD-100-C 20-May-1987 DI-N-OCTYPHTHALATE

SD-100-C 27-May-1987 DI-N-OCTYLPHTHALATE

SD-100-C 12-JUn-1987 DI-N-OCTYLPHTHALATE

SD-100-C 19-Jun-1987 DI-N-OCTYLPHTHAIATE

** SD -100

SD-100 11-Mar-1987 DIACETONE ALCOHOL

SD-100 12-May-1987 DLACETONE ALCOHOL

$<10 \quad$ ug/ $/ 2$

$<5.0 \quad \mathrm{ug} / \mathrm{L}$

$<10 \quad$ ug/L

$<5.0 \quad$ ug $/ \mathrm{L}$

$<10 \quad$ ug/L

$<5.0 \quad$ ug $/ \mathrm{L}$

$<10 \quad$ ug/L

$<10 \quad \mathrm{ug} / \mathrm{L}$

$<10 \quad$ ug/L

$1 \mathrm{~J} \quad$ ug/L

$<10 \quad$ ug/L

$<5.0 \quad \mathrm{ug} / \mathrm{L}$

$<5.0 \quad \mathrm{ug} / \mathrm{L}$

$<5.0 \quad \mathrm{ug} / \mathrm{L}$

$<5.0 \quad \mathrm{ug} / \mathrm{L}$

$<10 \quad \mathrm{ug} / \mathrm{L}$

* $\quad$ SD $-100-11$

SD-100-11 12-May-2987 DIACETONE ALCOHOL

$120 \mathrm{ug} / \mathrm{L}$

$9 \mathrm{ug} / \mathrm{L}$

* SD $-100-13$

SD-100-13 12-May-1987 DIACETONE ALCOHOL

4

$u g / \mathrm{L}$

** SD - 100-14

SD-100-14 12-May-1987 DIACETONE ALCOHOL

$6 \quad u g / 2$

** SD-100-C

SD-100-C 17-Mar-1987 DIACETONE ALCOHOL

SD-100-C 24-Mar-1987 DLACETONE ALCOHOL

5B ug/L

$4 B \quad \mathrm{Hg} / \mathrm{L}$

** SD -100

SD-100 5-Mat-1987 DIBENZ(A H)ANTHRACENE

SD-100 11-Mar-1987 DIBENZ(A H)ANTHRACENE

SD-100 12-May-1987 DIBENZ (A H) ANTHRACENE

SD-100 26-Kay-1987 DIBENZ(A H)ANTHRACENE

$<10 \quad \mathrm{ug} / \mathrm{L}$

$<10 \quad$ ug/L

$<10 \quad$ ug/L

$<5.0 \quad$ ug/L

** SD $-100-01$

SD-100-01 12-May-1987 DIBENZ(A H)ANTHRAGENE

$<10$

ug/L 
Storm Drain Data for SD-100

Location Date Test Compound

Results Units

SD-100-01 26-May-1987 DIBENZ(A H)ANTFRACENE

** SD-100-02

SD-100-02 12-MaY-1987 DIBENZ(A H)ANTHRACENE

SD-100-02 26-May-1987 DIBENZ(A H) ANTHRACENE

** SD $-100-03$

SD-100-03 12-May-1987 DIBENZ (A H) ANTHRACENE

SD-100-03 26-Yay-1987 DIBENZ (A H)ANTHRACENE

** SD-100-03D

SD-100-03D 12-May-1987 DIBENZ(A H) ANTHRACENE

SD-100-03D 26-May-1987 DIBENZ(A H) ANTHRACENE

* SD $-100-04$

SD-100-04 12-Kay-1987 DIBENZ (A H) ANTHRACENE

SD-100-04 26-May-1987 DIBENZ(A H) ANTHRACENE

** SD-100-06

SD-100-06 12-May-1987 DIBENZ(A H)ANTHRACENE

SD-100-06 26-May-1987 DIBEN2 (A H) ANTHRACENE

** SD-100-07

SD-100-07 12-May-1987 DIBENZ (A H) ANTHRACENE

SD-100-07 26-May-1987 DIBENZ (A H) ANTHRACENE

** SD-100-09

SD-100-09 12-May-1987 DIBENZ (A H) ANTHRACENE

SD-100-09 26-May-1987 DIBENZ (A H)ANTHRACENE

** SD $-100-10$

SD-100-10 12-May-1987 DIBENZ(A H) ANTHRACENE

SD-100-10 26-May-1987 DIBENZ(A H)ANTHRACENE

* SD-100-11

SD-100-11 12-May-1987 DIBENZ(A H) ANTHRACENE

SD-100-11 26-May-1987 DIBENZ(A H) ANTHRAGENE

** SD-100-12

SD-100-12 12-May-1987 DIBENZ (A H) ANTHRACENE

SD-100-12 26-May-1987 DIBENZ(A H) ANTHRACENE

* $\quad$ SD $-100-13$

SD-100-13 12-May-1987 DIBENZ(A H) ANTHRACENE

SD-100-13 26-May-1987 DIBENZ(A H)ANTHRACENE

** SD $-100-14$

SD-100-14 12-May-1987 DIBENZ(A H) ANTHRACENE

SD-100-14 26-May-1987 DIBENZ (A H) ANTHRACENE

$<5.0 \quad u \mathrm{~g} / \mathrm{L}$

$<10 \quad$ ug/L

$<5.0 \quad \mathrm{ug} / \mathrm{L}$

$<10 \quad$ ug/L

$<5.0 \quad$ ug $/ \mathrm{L}$

$<10 \quad$ ug/L

$<5.0 \quad$ ug/L

$<10 \quad u g / 2$

$<5.0 \quad \mathrm{ug} / \mathrm{L}$

$<10 \quad$ ug/L

$<5.0 \quad u g / L$

$<10 \quad 4 \mathrm{~g} / \mathrm{L}$

$<5.0 \quad \mathrm{ug} / \mathrm{L}$

$<10 \quad$ ug/L

$\leqslant .0 \quad u g / L$

$<10 \quad$ ug/L

$<5.0 \quad$ ug $/ \mathrm{L}$

$<10 \quad$ ug/L

$<5.0 \quad$ ug/L

$<10 \quad$ ug/L

$<5.0 \quad \mathrm{ug} / \mathrm{L}$

$<10 \quad$ ug/L

$<5.0 \quad$ ug $/ \mathrm{L}$

$<10$ ug/L

$<5.0 \quad$ ug $/ \mathrm{L}$ 
Storm Drain Data for SD-100

Location Date Test Compound Results Units

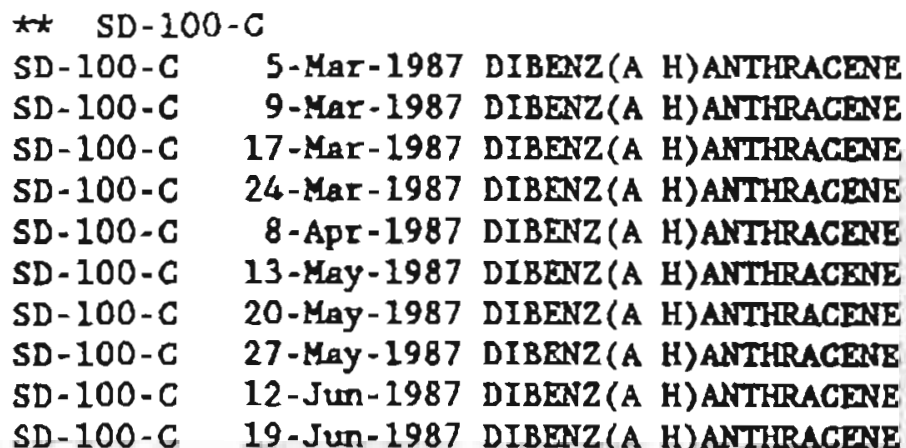

** SD-100

SD-100 5-Mar-1987 DIBROMOCHLOROMETRANE

SD-100 11-Mar-1987 DIBROMOCHLOROMETHANE

SD-100 18-Mar-1987 DIBROMOCHLOROMETHANE

SD-100 24-Mar-1987 DIBROMOCHLOROMETHANE

SD-100 6-APF-1987 DIBROMOCHLOROMETHANE

SD-100 12-May-1987 DIBROMOCHLOROMETHANE

SD-100 19-May-1987 DIBROMOCHLOROMETHANE

SD-100 26-May-1987 DIBROMOCHLOROMETHANE

SD-100 3-Jun-1987 DIBROMOCHLOROMETHAVE

SD-100 11-JUN-1987 DIBROMOCHLOROMETHANE

SD-100 18-Jun-1987 DIBROMOCHLOROMETHANE

* SD-100-01

SD-100-01 12-May-1987 DIBROMOCHLOROMETHARE

SD-100-01 18-May-1987 DIBROMOCHLOROMETHANE

SD-100-01 26-May-1987 DIBROMOCHLOROMETHANE

SD-100-01 3-Jun-1987 DIBROMOCHLOROMETTANE

SD-100-01 11-Jun-1987 DIBROMOCHLOROMETHANE

SD-100-01'18-JUn-1987 DIBROMOCHLOROMETHANE
$<10$

$<10$

$<10$

$<10$

$<10$

$<5.0$

$<5.0$

$<5.0$

$<5.0$

$<10$

$<5$

7

5

10

9

5

14

$<$

$<5$

$<5$

$<5$

$<$

$<5$

$<5$

$<5$

$<5$

$<5$

$<5$

$<5$

$<5$

$<5$

$<5$

$<5$

$<5$

$<5$

$<5$
ug/L

ug $/ L$

ug $/ \mathrm{L}$

ug $/ \mathrm{L}$

ug/L

ug/L

$\mathrm{ug} / \mathrm{L}$

ug $/ \mathrm{L}$

$\mathrm{ug} / \mathrm{L}$

ug/L

ug/

ug/L

ug/L

ug/L

ug/L

ug/L

$u \mathrm{~g} / \mathrm{L}$

ug/L

ug/L

ug/L

ug $/$.

ug $/ \mathrm{L}$ ug/L $\mathrm{ug} / \mathrm{L}$ ug/L $\mathrm{ug} / \mathrm{L}$ $\mathrm{ug} / \mathrm{L}$

ug/L ug $/ \mathrm{L}$ ug $/ \mathrm{L}$ ug/L $\mathrm{ug} / \mathrm{L}$ ug $/ \mathrm{L}$

* SD - 100-03
SD-100-03 18-May-1987 OIBROMOCHLOROMETIANE

SD-100-03 26-May-1987 DIBROMOCHLOROMETHANE

SD-100-03 3-JUח-1987 DIBROMOCHLOROMETHANE

SD-100-03 11-JUn-1987 DIBROMOCHLOROMETHANE

SD-100-03 18-JUR-1987 DIBROMOCHLOROMETHANE

$$
\begin{aligned}
& u g / L \\
& u g / L \\
& u g / L \\
& u g / L \\
& u g / L \\
& u g / L
\end{aligned}
$$

* SD - $100-03 D$

SD-100-03D 12-May-1987 DIBROMOCHLOROMETHANE 
Storm Drain Data for SD-100

Locstion

Date

Test Compound

Results

Units

SD-100-03D 26-May-1987 DIBROMOCHLOROMETHANE

* SD-100-04

SD-100-04 12-May-1987 DIBROKOCFLOROAETAANE

SD-100-04 18-May-1987 DIBROMOCELOROMETHANE

SD-100-04 26-May-1987 DIBROMOCHLOROMETHANE

SD-100-04 3-Jun-1987 DIBROMOCHLOROMETHANE

SD-100-04 11-Jum-1987 DIBROMOCHLOROMETHANE

SD-100-04 18 -Jun-1987 DIBROMOCHLOROMETHANE

** SD-100-06

SD-100-06 12-May-1987 DIBROMOCHLOROMETHANE

SD-100-06 18-May-1987 DIBROMOCHLOROMETHANE

SD-100-06 26-May-1987 DIBROMOCHLOROMETHANE

SD-100-06 3-Jur-1987 DIBROMOCHLOROMETHANE

SD-100-06 11-Jun-1987 DIBROMOCHLOROMETHANE

SD-100-06 18-Jun-1987 DIBROMOCHLOROMETHANE

* SD-100-06D

SD-100-06D 3-JUn-1987 DIBROMOCHLOROAETHANE

$\star \star \quad S D-100-07$

SD-100-07 12-May-1987 DIBROMOCHLOROMETHANE

SD-100-07 19-May-1987 DIBROMOCHLOROMETHANE

SD-100-07 26-MaY-1987 DIBROMOCHLOROMETHANE

SD-100-07 3-JUn-1987 DIBROMOCHLOROMETHANE

SD-100-07 11-Jun-1987 DIBROMOCHLOROMETHANE

SD-100-07 18-JUn-1987 DIBROMOCHLOROMETAANE

* SD-100-07D

SD-100-07D 18-JUn-1987 DIBROMOCHLOROMETHANE

* SD-100-09

SD-100-09 12-MaY-1987 DIBROMOCHLOROMETHAITE

SD-100-09 18-May-1987 DIBROMOCHLOROMETHANE

SD-100-09 26-May-1987 DIBROMOCHLOROHETHANE

SD-100-09 3-Jun-1987 DIBROMOCHLOROMEIHANE

SD-100-09 11-Jun-1987 DIBROMOCHLOROMETHANE

SD-100-09 18-Jun-1987 DI8ROKOCHLOROMETHANE

** SD-100-10

SD-100-10 12-May-1987 DIBROMOCHLOROMETHANE

SD-100-10 19-May-1987 DIBROMOCHLOROMEIHANE

SD-100-10 26-May-1987 DIBROMOCHLOROMETHANE

SD-100-10 3-Jun-1987 DIBROMOCHLOROMETHANE

SD-100-10 11-Jun-1987 DIBROMOCHLOROMETHANE

SD-100-10 18-Jun-1987 DI BROMOCHLOROMETHANE

$\star \star \quad S D-100-11$

SD-100-11 12-May-1987 DIBROMOCHLOROMETHANE

SO-100-11 19-May-1987 DIBROMOCHLOROMETHANE

\begin{tabular}{|c|c|}
\hline$<5$ & $\mathrm{ug} / \mathrm{L}$ \\
\hline$<5$ & $u g / L$ \\
\hline$<5$ & $\mathrm{ug} / \mathrm{L}$ \\
\hline$<5$ & $\mathrm{ug} / \mathrm{L}$ \\
\hline$<5$ & $\mathrm{ug} / \mathrm{L}$ \\
\hline$<5$ & $\mathrm{ug} / \mathrm{L}$ \\
\hline$<5$ & $\mathrm{ug} / \mathrm{L}$ \\
\hline 15 & $u g / L$ \\
\hline 10 & $\mathrm{ug} / \mathrm{L}$ \\
\hline 5 & $u g / L$ \\
\hline$<5$ & $4 \mathrm{~g} / \mathrm{L}$ \\
\hline$<5$ & $\mathrm{ug} / \mathrm{L}$ \\
\hline$<5$ & $\mathrm{ug} / \mathrm{L}$ \\
\hline$<5$ & $\mathrm{ug} / \mathrm{L}$ \\
\hline$<5$ & $u g / L$ \\
\hline$<5$ & $u g / L$ \\
\hline$<5$ & $\mathrm{ug} / \mathrm{L}$ \\
\hline$<5$ & $\mathrm{ug} / \mathrm{L}$ \\
\hline$<5$ & $\mathrm{ug} / \mathrm{L}$ \\
\hline$<5$ & $\mathrm{ug} / \mathrm{L}$ \\
\hline$<5$ & $u g / L$ \\
\hline$<5$ & $u g / L$ \\
\hline$<5$ & ug/L \\
\hline$<5$ & $\mathbf{u g} / \mathrm{L}$ \\
\hline$<5$ & ug/L \\
\hline$<5$ & $u g / L$ \\
\hline$<s$ & ug/L \\
\hline$<5$ & $\mathrm{ug} / \mathrm{l}$ \\
\hline$<5$ & ug/l \\
\hline$<5$ & $48 / L$ \\
\hline$<5$ & $u g / L$ \\
\hline$<5$ & $\mathrm{ug} / \mathrm{L}$ \\
\hline$<5$ & $\mathrm{ug} / \mathrm{L}$ \\
\hline$<5$ & ug \\
\hline 5 & \\
\hline
\end{tabular}


Storm Drain Data for SD-100

\begin{tabular}{lrl} 
Location & \multicolumn{1}{c}{ Date } & Test Compound \\
& & \\
SD-100-11 & $26-$ May-1987 & DIBROMOGHLOROLITHANE \\
SD-100-11 & $3-J u n-1987$ & DIBROMOCHLOROMETHANE \\
SD-100-11 & $11-J u n-1987$ & DIBROMOCHLOROKLTHANE \\
SD-100-11 & $18-J u n-1987$ & DIBROMOCHLOROMISTHANE
\end{tabular}

Results Units

* SD-100-12

SD-100-12 12-May-1987 DIBROYOCHLOROMETHANE

SD-100-12 19-May-1987 DIBROMOCHLOROKETHANE

SD-100-12 26-May-1987 DIBROMOCHLOROLITIHANE

SD-100-12 3-Jun-1987 DIBROMOCHLOROIRTHANE

SD-100-12 11-JUn-1987 DIBROMOCHLOROLETHANE

SD-100-12 18-Jun-1987 DIBROMOCHLOROMETHANE

** SD $-100-13$

SD-100-13 12-May-1987 DIBROMOCHLOROMETHANE

SD-100-13 19-May-1987 DIBROMOCHLOROMETHANE

SD-100-13 26-May-1987 DIBROMOCHLOROMETHANE

SD-100-13 3-JUN-1987 DIBROMOCHLOROMETEANE

SD-100-13 11-Jun-1987 DIBROMOCHLOROMETHANE

SD-100-13 18-Jun-1987 DIBROMOCHLOROMETHANE

** SD $-100-14$

SD-100-14 12-May-1987 DIBROMOCHLOROMETHLANE

SD-100-14 19-May-1987 DIBROMOCHLOROMETHANE

SD-100-14 26-May-1987 DIBROMOCHLOROMETHANE

SD-100-14 3-Jun-1987 DIBROMOCHIOROHETHANE

SD-100-14 11-Jun-1987 DIBROMOCHLOROMIETHANE

SD-100-14 18-Jun-1987 DIBROMOCHLOROMETHANE

5

$<5$

$<5$

$<5$

$<5$

5

$<5$

$<5$

$<5$

$<5$

5

$<5$

$<5$

$<5$

$<5$

$<5$

$<5$

5

$<5$

$<5$

$<5$

$<5$

5

$<5$

$<5$

$<5$

$4 \mathrm{~J}$

$<5$

7

11

5

5

$S D-100-C$ 27-May-1987 DIBROMOCHLOROMETHANE 12-Jun-1987 DIBROMOCHLOROMETHANE 19-Jun-1987 DIBROMOCHLOROMETHANE

** SD $-100-D$

SD-100-D 6-API-1987 DIBROMOCHLOROMETHANE

SD-100-D 11-JUn-1987 DIBROMOCHLOROMETHANE

$<5$

5

$u g / L$

$u g / L$

$\mathrm{ug} / \mathrm{L}$

ug/L

$\mathrm{ug} / \mathrm{L}$

$u g / L$

* SD $100-11$

SD-100-11 12-May-1987 DICHLORO CYCLOHEXANE

ug $/ 2$

ug $/ 2$

ug $/ L$

ug $/$ L

ug $/ \mathrm{L}$

ug/L

** SD-100-12

SD-100-12 12-May-1987 DICHLORO CYCLOHEXANE

ug/L $u g / L$ ug/L ug $/ L$ $\mathrm{ug} / \mathrm{L}$ ug/L $\mathrm{ug} / \mathrm{L}$ ug/L $u g / L$ ug $/ 2$

$\log \pi$ ug/L

$u g / L$ 
Stom Drain Data for SD-100

\begin{tabular}{|c|c|c|c|c|}
\hline Location & Date & Test Compound & Results & Units \\
\hline \multicolumn{5}{|c|}{ ** SD-100-13 } \\
\hline$S D-100-13$ & $12-$ May -1987 & DICHLORO CYCLOHEXANB & 200 & ug $/ \mathrm{L}$ \\
\hline \multicolumn{5}{|c|}{ * SD-100-14 } \\
\hline SD-100-14 & $12-$ May -1987 & DICHLORO CYCLOREXANE & 420 & $\mathrm{ug} / \mathrm{L}$ \\
\hline \multicolumn{5}{|l|}{$\star \star \quad S D-100$} \\
\hline SD - 100 & $5-\operatorname{Mar}-1987$ & DIETHYLPHTHALATE & $<10$ & $\mathrm{ug} / \mathrm{L}$ \\
\hline SD- 100 & 11-Mar- 1987 & DIETHYLPHTHALATE & $<10$ & $\mathbf{u g} / \mathrm{L}$ \\
\hline$S D-100$ & 12-May-1987 & DIETHYLPHTHALATE & $<10$ & $\mathrm{ug} / \mathrm{L}$ \\
\hline SD-100 & 26-May-1987 & DIETHYLPHTHALATE & $<5.0$ & $\mathbf{u g} / \mathrm{L}$ \\
\hline \multicolumn{5}{|c|}{ ** SD-100-01 } \\
\hline SD-100-01 & 12-May-1987 & DIETKYLPHTHALATE & $<10$ & ug/L \\
\hline SD $-100-01$ & 26-May - 1987 & DIETHYLPHTHALATE & $<5.0$ & $\mathrm{ug} / \mathrm{L}$ \\
\hline \multicolumn{5}{|c|}{ * SD-100-02 } \\
\hline$S D-100-02$ & 12-May-1987 & DIETHYLPHTHALATE & $<10$ & $\mathrm{ug} / \mathrm{L}$ \\
\hline SD-100-02 & $26 \cdot$ - May-1987 & DIETHYLPHTHALATE & $<5.0$ & $u g / L$ \\
\hline \multicolumn{5}{|c|}{$\star$ * SD $-100-03$} \\
\hline SD $-100-03$ & 12-May-1987 & DIETHYLPHTHALATE & $<10$ & $\mathrm{ug} / \mathrm{L}$ \\
\hline SD $-100-03$ & $26-$ Мay- 1987 & DIETHYLPHTHALATE & $<5.0$ & $\mathrm{ug} / \mathrm{L}$ \\
\hline \multicolumn{5}{|c|}{ * SD-100-03D } \\
\hline SD-100-03D & 12-May-1987 & DIETHYLPHTHALATE & $<10$ & ug/L \\
\hline SD-100-03D & $26-$ May-1987 & DIETHYLPHTHALATE & $<.0$ & ug/L \\
\hline \multicolumn{5}{|c|}{$\star *$ SD $-100-04$} \\
\hline SD- $100-04$ & $12-$ Kay -1987 & DIETHYLPHTHALATE & $<10$ & ug/L \\
\hline SD $-100-04$ & $26-$ Kay -1987 & DIETHYLPHTHALATE & $<5.0$ & $u g / L$ \\
\hline \multicolumn{5}{|c|}{$\star$ ¿ SD-100-06 } \\
\hline SD-100-06 & $12-$ May- 1987 & DIETHYLPHTHALATE & $<10$ & $\mathrm{ug} / \mathrm{L}$ \\
\hline SD - $100-06$ & 26-May-1987 & DIETHYLPHTHALATE & $<5.0$ & ug/L \\
\hline \multicolumn{5}{|c|}{ ** SD-100-07 } \\
\hline SD-100-07 & 12 - May-1987 & DIETHYLPHTHALATE & $8 \mathrm{~J}$ & ug/L \\
\hline SD $-100-07$ & $26-$ May-1987 & DIETHYLPHTHALATE & $<5.0$ & ug/L \\
\hline \multicolumn{5}{|c|}{ * SD-100-09 } \\
\hline$S D=100-09$ & 12-May-1987 & DIETHYLPHTHALATE & $<10$ & ug/L \\
\hline SD $-100-09$ & 26-May-1987 & DIETHYLPHTHALATE & $<.0$ & $\mathrm{ug} / \mathrm{L}$ \\
\hline \multicolumn{5}{|c|}{ ** SD $-100-10$} \\
\hline SD- $100-10$ & 12 - May - 1987 & DYETHYLPHTHALATE & $<10$ & ug/L \\
\hline SD - $100-10$ & $26-$ May -1987 & DIETHYLPHTHALATE & $<5.0$ & $\mathrm{ug} / \mathrm{L}$ \\
\hline \multicolumn{5}{|c|}{$\star \star \quad S D-100-11$} \\
\hline SD-100-11 & 12 - May - 1987 & DIETHYLPHTHALATE & $<10$ & ug/L \\
\hline$S D=100 \cdot 11$ & 26-May- 1987 & DIETHYLPHTHALATE & $<5.0$ & ug/L \\
\hline
\end{tabular}


Storm Drain Deta for SD-100

Location Date Test Compound

Results Units

$\star \star$ SD $-100-12$

SD-100-12 12-Kay-1987 DIETHYLPHTHALATE

SD-100-12 26-Kay-1987 DIETHYLPHTHALATE

** SD $-100-13$

SD-100-13 12-Kay-1987 DIETHYLPHTHALATE

SD-100-13 26-May-1987 DIETHYLHTHALATE

* SD $-100-14$

SD-100-14 12-May-1987 DIETHYLPHTHALATE

SD-100-14 26-May-1987 DIETHYLPHTHALATE

** SD-100-C

SD-100-C 5-Mar-1987 DIETHYLPHTHALATE

SD-100-C 9-Mar-1987 DIETHYLPHTHALATE

SD-100-C 17-Mar-1987 DIETHYLPHTHALATE

SD-100-C 24-Mar-1987 DIETHYLPHTHALATE

SD-100-C 8-Apr-1987 DIETHYLPHTHALATE

SD-100-C 13-May-1987 DIETHYLPHTHALATE

SD-100-C 20-May-1987 DIETHYLPETHALATE

SD-100-C 27-May-1987 DIETHYLPHTHALATE

SD-100-C 12-Jun-1987 DIETHYLPHTHALATE

SD-100-C 19-Jun-1987 DIETHYLPHTHALATE

* SD -100

SD-100 5-Mar-1987 DIMETHYLPHTHALATE

SD-100 11-Mar-1987 DIMETHYLPHTHALATE

SD-100 12-May-1987 DIMETHYLPHTHALATE

SD-100 26-May-1987 DIMETHYLPHTHAIATE

** SD $-100-01$

SD-100-01 12-May-1987 DIMETHYLPHTHALATE

SD-100-01 26-May-1987 DIMETHYLPHTHALATE

** SD-100-02

SD-100-02 12-May-1987 DIMETHYLPHTHALATE

SD-100-02 26-May-1987 DIMETKYLPHTHALATE

* SD-100-03

SD-100-03 12-May-1987 DIMETKYLPHTHALATE

SD-100-03 26-May-1987 DIMETHYLPHTHALATE

** SD-100-03D

SD-100-03D 12-May-1987 DIMETHYLPHTHALATE

SD-100-03D 26-May-1987 DIMETHYLPHTHAIATE

* SD-100-04

SD-100-04 12-May-1987 DIMETHYLPHTHALATE

SD-100-04 26-May-1987 DIMETHYLPHTHALATE

$\begin{array}{ll}<10 & \mathrm{ug} / \mathrm{L} \\ <5.0 & \mathrm{ug} / \mathrm{L} \\ & \\ <10 & \mathrm{ug} / \mathrm{L} \\ <5.0 & \mathrm{ug} / \mathrm{L} \\ & \\ & \\ <10 & \mathrm{ug} / \mathrm{L} \\ <5.0 & \mathrm{ug} / \mathrm{L} \\ & \\ & \\ <10 & \mathrm{ug} / \mathrm{L} \\ <10 & \mathrm{ug} / \mathrm{L} \\ <10 & \mathrm{ug} / \mathrm{L} \\ <10 & \mathrm{ug} / \mathrm{L} \\ <10 & \mathrm{ug} / \mathrm{L} \\ <5.0 & \mathrm{ug} / \mathrm{L} \\ <5.0 & \mathrm{ug} / \mathrm{L} \\ <5.0 & \mathrm{ug} / \mathrm{L} \\ <5.0 & \mathrm{ug} / \mathrm{L} \\ <10 & \mathrm{ug} / \mathrm{L}\end{array}$


Starm Drain Data for SD-100

Location Date Test Compound

Results Units

* SD-100-06

SD-100-06 12-Kay-1987 DIKETHYLPHTHALATE

SD-100-06 26-May-1987 DIRETHYLPHTHALATE

** SD $-100-07$

SD-100-07 12-May-1987 DIMETHYLPHTHALATE

SD-100-07 26-May-1987 DIYETHYLPHTHAIATE

** SD 100.09

SD-100-09 12-May-1987 DIMETHYLPHTHALATE

SD-100-09 26-May-1987 DIMETHYLPHTHALATE

* SD $-100-10$

SD-100-10 12-May-1987 DIMETHYLPHTHALATE

SD-100-10 26-May-1987 DIMETHYLPHTHALATE

** SD $-100-11$

SD-100-11 12-May-1987 DIMETHYLPHTHALATE

SD-100-11 26-May-1987 DIMETHYLPHTHALATE

** SD - 100-12

SD-100-12 12-May-1987 DIMETHYLPHTHALATE

SD-100-12 26-May-1987 DIMETHYLPHTHALATE

* SD-100-13

SD-100-13 12-May-1987 DIMETHYLPHTHALATE

SD-100-13 26-May-1987 DIMETHYLPHTHALATE

* SD- $100-14$

SD-100-14 12-May-1987 DIMETHYLPHTHALATE

SD-100-14 26-May-1987 DIMETHYLPHTHALATE

$\star$ SD $-100-\mathrm{C}$

SD-100-C 5-KaI-1987 DIMETHYLPHTHALATE

SD-100-C 9-Mar-1987 DIMETHYLPHTHALATE

SD-100-C 17-Mar-1987 DIMETHYLPHTHALATE

SD-100-C 24-Mar-1987 DIMETHYLPHTHALATE

SD-100-C 8-APT-1987 DIMETHYLPHTHALATE

SD-100-C 13-May-1987 DIMETHYLPHTHALATE

SD-100-C 20-May-1987 DIMETHYLPHTHALATE

SD-100-C 27-May-1987 DIMETHYLPHTHALATE

SD-100-C 12-Jun-1987 DIMETHYLPHTHALATE

SD-100-C 19-JUn-1987 DIMETHYLPHTHALATE

* SD-100

SD-100 S-Mar-1987 DISSOLVED OXYGEN

SD-100 11-Mar-1987 DISSOLVED OXYGEN

SD-100 18-Mar-1987 DISSOLVED OXYGEN

SD-100 24-Mar-1987 DISSOLVED OXYGEN

SD-100 3-APY - 1987 DISSOLVED OXYGEN

SD-100 6-API-1987 DISSOLVED OXYGEN

\begin{tabular}{|c|c|}
\hline $\begin{array}{l}<10 \\
<5.0\end{array}$ & $\begin{array}{l}u g / L \\
u g / L\end{array}$ \\
\hline $\begin{array}{l}<10 \\
<5.0\end{array}$ & $\begin{array}{l}u g / L \\
u g / L\end{array}$ \\
\hline $\begin{array}{l}<10 \\
<5.0\end{array}$ & $\begin{array}{l}\mathrm{ug} / \mathrm{L} \\
\mathrm{ug} / \mathrm{L}\end{array}$ \\
\hline $\begin{array}{l}<10 \\
<5.0\end{array}$ & $\begin{array}{l}\mathrm{ug} / \mathrm{L} \\
\mathrm{ug} / \mathrm{L}\end{array}$ \\
\hline $\begin{array}{l}<10 \\
<5.0\end{array}$ & $\begin{array}{l}\mathrm{ug} / \mathrm{L} \\
\mathrm{ug} / \mathrm{L}\end{array}$ \\
\hline $\begin{array}{l}<10 \\
<5.0\end{array}$ & $\begin{array}{l}\mathrm{ug} / \mathrm{L} \\
\mathrm{ug} / \mathrm{l}\end{array}$ \\
\hline $\begin{array}{l}<10 \\
<5.0\end{array}$ & $\begin{array}{l}\operatorname{ug} / \\
\operatorname{ug} /\end{array}$ \\
\hline $\begin{array}{l}<10 \\
<5.0\end{array}$ & $\begin{array}{l}\mathrm{ug} / \mathrm{L} \\
\mathrm{ug} / \mathrm{l}\end{array}$ \\
\hline$<10$ & ug $/ \mathrm{L}$ \\
\hline$<10$ & $\operatorname{ug} / 2$ \\
\hline$<10$ & $u g / L$ \\
\hline$<10$ & ug/L \\
\hline$<10$ & $u_{B} \Omega$ \\
\hline$<5.0$ & $4 \mathrm{~B} / \mathrm{I}$ \\
\hline$<5.0$ & $\mathrm{ug} / \mathrm{L}$ \\
\hline$<5.0$ & ug $/ \mathrm{L}$ \\
\hline$<5.0$ & $u g / L$ \\
\hline$<10$ & $u g / L$ \\
\hline & \\
\hline 10.0 & Ppq \\
\hline 11.2 & Ppm \\
\hline 7.3 & Ppm \\
\hline 7.2 & PPW \\
\hline 7.2 & ppa \\
\hline 8.3 & Ppa \\
\hline
\end{tabular}


Storz Drain Data for SD-100

Location Date Test Compound Results Units

SD-100-04 3-Jun-1987 CHLOROFORY

SD-100-04 11-Jun-1987 CHLOROFORH

SD-100-04 18-JUn-1987 CHOOROFORM

48

45

43

$4 \mathrm{~g} / \mathrm{L}$

$\operatorname{ug} / \mathrm{L}$

* SD-100-06

SD-100-06 12-May-1987 CHLOROFORM

SD-100-06 18-Mag-1987 CHLOROFORM

SD-100-06 26-May-1987 CHLOROFORM

SD-100-06 3-Jun-1987 CHLOROFORM

SD-100-06 11-Jun-1987 CHLQROFORM

SD-100-06 18-Jun-1987 GHOROFORM

$\begin{array}{ll}31 & \mathrm{ug} / \mathrm{L} \\ 29 & \mathrm{ug} / \mathrm{L} \\ 33 & \mathrm{ug} / \mathrm{L} \\ 36 & \mathrm{ug} / \mathrm{L} \\ 46 & \mathrm{ug} / \mathrm{L} \\ 42 & \mathrm{ug} / \mathrm{L}\end{array}$

** SD $-100-06 D$

SD-100-06D 3-Jun-1987 CHLOROFORM

38

$u g / L$

* SD-100-07

SD-100-07 12-May-1987 CHLOROFORM

SD-100-07 19-May-1987 CHLOROFORM

SD-100-07 26-May-1987 CHLOROPORM

SD-100-07 3-Jun-1987 GHLROFORM

SD-100-07 11-3 un-1987 CHLOROFORM

SD-100-07 18-Jun-1987 CHLOROFORM

$1700 \quad 4 \mathrm{~g} / \mathrm{L}$

380 ug/

$220 \mathrm{ug} / \mathrm{L}$

$21 \mathrm{ug} / \mathrm{L}$

$3500 \quad$ ug/L

$<5 \quad \mathrm{ug} / \mathrm{L}$

** SD-100-07D

SD-100-07D 18-Jun-1987 CHLOROFORY

$u g / 2$

* SD-100-09

SD-100-09 12-May-1987 CHLOROFORM

SD-100-09 18-May-1987 CKLOROFORM

SD-100-09 26-May-1987 CHLOROFORM

SD-100-09 3-Jun-1987 CHLOROFORY

SD-100-09 11-Jun-1987 CHLOROFORM

SD-100-09 18-Jun-1987 CHLOROFORM

$\begin{array}{ll}27 & \mathrm{ug} / L \\ 45 & \mathrm{ug} / L \\ 39 & \mathrm{ug} / L \\ 38 & \mathrm{ug} / L \\ 40 & \mathrm{ug} / L \\ 36 & \mathrm{ug} / \mathrm{L}\end{array}$

** SD $-100-10$

SD-100-10 12-May-1987 CHLOROFORM

SD-100-10 19-May-1987 CHLOROFORM

SD-100-10 26-May-1987 CHLOROFORM

SD-100-10 3-JUn-1987 CHLOROFORY

SD-100-10 11-Jun-1987 CHLOROFORY

SD-100-10 18-Jun-1987 CHLOROFORY

$\begin{array}{ll}57 & \mathrm{ug} / \mathrm{L} \\ 44 & \mathrm{ug} / \mathrm{L} \\ 44 & \mathrm{ug} / \mathrm{L} \\ 44 & \mathrm{ug} / \mathrm{L} \\ 51 & \mathrm{ug} / \mathrm{L} \\ 35 & \mathrm{ug} / \mathrm{L}\end{array}$

** SD-100-11

SD-100-11 12-May-1987 CHLOROFORM

SD-100-11 19-May-1987 CHLOROFORM

SD-100-11 26-May-1987 CHLOROFORM

SD-100-11 3-Jun-1987 CHLOROFORM

SD-100-11 11-JUn-1987 CHLOROFORM

SD-100-11 18-Jun-1987 CHLOROFORM

$\begin{array}{ll}52 & u g / L \\ 14 & u g / L \\ 21 & u g / L \\ 35 & u g / L \\ 54 & u g / L \\ 47 & u g / L\end{array}$


Storm Drain Data for SD-100

Location

Date

Test Compound

Result

Units

* SD-100-12

SD-100-12 12-May-1987 CHLOROFORM

SD-100-12 19-May-1987 GHLOROFORY

SD-100-12 26-May-1987 CHLOROPORY

SD-100-12 3-Jun-1987 CHLOROFORM

SD-100-12 11-Jun-1987 CHLOROFORM

SD-100-12 18-Jun-1987 GHLROFORK

$\begin{array}{ll}44 & u g / L \\ 57 & u g / L \\ 52 & u g / L \\ 31 & u g / L \\ 67 & u g / L \\ 62 & u g / L\end{array}$

** SD $-100-13$

SD-100-13 12-May-1987 CHLOROFORM

SD-100-13 19-May-1987 CHLOROFORM

SD-100-13 26-May-1987 CHLOROPORM

SD-100-13 3-Jun-1987 CHLOROFORM

SD-100-13 11-Jun-1987 CHLOROFORM

SD-100-13 18-Jun-1987 CHLOROFORM

$\begin{array}{ll}49 & \mathrm{ug} / \mathrm{L} \\ 40 & \mathrm{ug} / \mathrm{L} \\ 42 & \mathrm{ug} / \mathrm{L} \\ 36 & \mathrm{ug} / \mathrm{L} \\ 40 & \mathrm{ug} / \mathrm{L} \\ 59 & \mathrm{ug} / \mathrm{L}\end{array}$

** SD $-100-14$

SD-100-14 12-May-1987 CHLOROFORM

SD-100-14 19-May-1987 CHLOROFORM

SD-100-14 26-May-1987 CHLOROFORM

SD-100-14 3-Jun-1987 CHLOROFORM

SD-100-14 11-JUn-1987 CHLOROFORM

SD-100-14 18-Jun-1987 CHLOROFORM

$\begin{array}{ll}56 & \text { ug/L } \\ 32 & \text { ug/L } \\ 40 & \text { ug/L } \\ 40 & \text { ug/L } \\ 48 & \text { ug/L } \\ 43 & \text { ug/L }\end{array}$

** SD $-100-C$

SD-100-C 5-Mar-1987 CHLOROFORM

SD-100-C 9-Mar-1987 CHLOROFORM

SD-100-C 17-Mat-1987 CHLOROFORM

SD-100-C 24-Mar-1987 CHLOROFORM

SD-100-C 8-Apr-1987 CHLOROFORM

SD-100-C 13-May-1987 CHLOROFORM

SD-100-C 20-May-1987 CHLOROFORM

SD-100-C 27-May-1987 CHLOROFORM

SD-100-C 12-Jun-1987 CHLOROFORM

SD-100-C 19-Jun-1987 CHLOROFORM

** SD-100-D

SD-100-D 6-APx-1987 CHLOROFORM

$\operatorname{ug} \sqrt{1}$

* SD-100

SD -100

5-Mar-1987 CHLOROMETHANE

$<10$

ug $/$ L

$\mathrm{ug} / \mathrm{L}$

ug/L

ug/L

$\mathrm{ug} / \mathrm{L}$

$\mathrm{ug} / \mathrm{L}$

$\mathrm{ug} / \mathrm{L}$

$\mathrm{ug} / \mathrm{L}$

ug/L

ug/L

SD -100

11-Mar-1987 CHLORONETHANE

SD -100

18-Mar-1987 GHLOROMETHANE

SD -100

24-Mar-1987 CHLOROMETHANE

SD -100

6-Apr-1987 CHLOROMETHANE

SD -100

12-May-1987 CHLOROMETHANE

SD -100

19-May-1987 CHLOROMETHANE

SD -100

26-May-1987 CHLOROMETHANE

SD -100

3-JUn-1987 CHLOROMETHANE

SD- 100

11-Jun-1987 CHLOROHETHANE

$\operatorname{ug} \Omega$

$4 \mathrm{~g} / \mathrm{L}$

ug/L

$\mathrm{ug} / \mathrm{L}$

$u g / L$

$4 \mathrm{~g} / \mathrm{L}$

$u g / L$

ug/L

ug/L

$u g / L$ 
Storm Drain Data for SD-100

Location Dace Test Compound

Results Units

SD-100 18-Jun-1987 CHLOROMETHANE

$<10 \quad \mathrm{ug} / \mathrm{L}$

** SD-100-01

SD-100-01 12-May-1987 CHLOROMETHANE

SD-100-01 18-May-1987 CHLOROMITTHANE

SD-100-01 26-May-1987 CHLORONETHANE

SD-100-01 3-Jun-1987 CHLOROMETHANE

SD-100-01 - 11-Jun-1987 GHLOROKETHANE

SD-100-01 18-Jun-1987 CHLOROMETHANE

$40 \quad 4 g / L$

$<10 \quad u g / L$

$<10 \quad 48 / \mathrm{L}$

$<10 \quad$ ug/L

$<10 \quad 4 \mathrm{~g} / \mathrm{L}$

$<10 \quad$ ug/L

** SD $-100-02$

SD-100-02 12-May-1987 CHLOROMETHANE

SD-100-02 18-May-1987 CHLOROMETHANE

SD-100-02 26-May-1987 CHLOROMETHANE

SD-100-02 3-Jun-1987 CHLOROMETHANE

SD-100-02 11-Jun-1987 CHLOROMETHANE

SD-100-02 18-Jun-1987 CHLOROMETHANE

$<10 \quad$ ug/L

$<10 \quad \mathrm{ug} / \mathrm{L}$

$<10 \quad$ ug $/ \mathrm{L}$

$<10 \quad$ ug/L

$<10 \quad$ ug/L

$<10 \quad$ ug/L

* SD-100-03

SD-100-03 12-Kay-1987 CHLOROMETHANE

SD-100-03 18-May-1987 CHLOROMETHANE

SD-100-03 26-May-1987 CHLOROMETHANE

SD-100-03 3-Jun-1987 CHLOROMETHANE

SD-100-03 11-Jun-1987 CHLOROMETHANE

SD-100-03 18-Jun-1987 CHLOROMETHANE

* SD-100-03D

SD-100-03D 12-May-1987 CHLOROMETHANE

SD-100-03D 26-May-1987 CHLOROMETHANE

$<10 \quad \mathrm{ug} / \mathrm{L}$

$<10 \quad \mathrm{ug} / \mathrm{L}$

$<10 \quad$ ug/L

$<10 \quad u g / L$

$<10 \quad \mathrm{ug} / \mathrm{L}$

$<10 \quad$ ug/L

$010 \quad u g / L$

$<10 \quad \mathrm{ug} / \mathrm{L}$

** SD-100-04

SD-100-04 12-May-1987 CHLOROMETHANE

SD-100-04 18-May-1987 CHLOROMETHANE

SD-100-04 26-May-1987 CHLOROMETHANE

SD-100-04 3-Jun-1987 CHLOROMETHANE

SD-100-04 11-Jum-1987 GILOROMETHANE

SD-100-04 18-Jun-1987 CHLOROMETHANE

$<10 \quad 4 \mathrm{~g} / \mathrm{L}$

$<10 \quad \mathrm{ug} / \mathrm{L}$

$<10 \quad u g / L$

$<10 \quad \mathrm{ug} / \mathrm{L}$

$<10 \quad$ ug/L

$<10 \quad \mathrm{ug} / \mathrm{L}$

** SD-100-06

SD-100-06 12-MAY-19B7 CELOROMETHANE

SD-100-06 18-May-1987 CHLOROMETHANE

SD-100-06 26-May-1987 CHLOROHETHANE

SD-100-06 3-J un-1987 CHLOROMETHANE

SD-100-06 11-Jun-1987 CHLOROMETHANE

SD-100-06 18-Jun-1987 CHLOROKETHANE

$<10 \quad \mathrm{ug} / \mathrm{L}$

$<10 \quad \mathrm{ug} / \mathrm{L}$

$<10 \quad$ ug/L

$<10 \quad u g / 1$

$<10 \quad \mathrm{ug} / \mathrm{L}$

$<10 \quad 4 \mathrm{~g} / \mathrm{L}$

** SD-100-06D

SD-100-06D 3-Jun-1987 CHLOROMETHANE

$<10 \quad$ ug/L

* SD - 100-07

SD-100-07 12-May-1987 CHLOROMETHANE

$<10$

ug/L 
Storm Drain Data for SD-100

\begin{tabular}{lrr} 
Location & \multicolumn{1}{c}{ Date } & Test Compou \\
& & \\
SD-100-07 & 19-May-1987 & CHLOROMETHANE \\
SD-100-07 & $26-$ May-1987 CHLOROMETHANE \\
SD-100-07 & $3-J u m-1987$ & GHLOROMETHANE \\
SD-100-07 & $11-J u n-1987$ & GLOROMETHANE \\
SD-100-07 & $18-J u m-1987$ & GLOROMETHANE
\end{tabular}

Results Units

* SD-100-07D

SD-100-07D 18-Jun-1987 GHLOROMETHANE

** SD $-100-09$

SD-100-09 12-May-1987 CHLOROMETHANE

SD-100-09 18-May-1987 CHIOROMETHANE

SD-100-09 26-May-1987 CHLOROMETHANE

SD-100-09 3-Jun-1987 CHLOROMETHLANE

SD-100-09 11-Jun-1987 CHLOROMETHANE

SD-100-09 18-Jun-1987 CHLOROMETHANE

* SD $-100 \cdot 10$

SD-100-10 12-May-1987 CHLOROMETHANE

SD-100-10 19-May-1987 CHLOROMETHANE

SD-100-10 26-May-1987 CHLOROMETHANE

SD-100-10 3-Jun-1987 CHLOROMETHANE

SD-100-10 11-Jun-1987 GHLOROMETHANE

SD-100-10 18-Jun-1987 CHLOROMETHANE

** SD $-100-11$

SD-100-11 12-May-1987 CHLOROMETHANE

SD-100-11 19-Yay-1987 CHLOROMETHANE

SD-100-11 26-May-1987 CHLOROMETHANE

SD-100-11 3-Jun-1987 CHLOROMETHANE

SD-100-11 11-Jun-1987 CHLOROMETHANE

SD-100-11 18-Jun-1987 CHLOROMETHANE

* SD-100-12

SD-100-12 12-Kay-1987 CHLOROMETHANE

SD-100-12 19-May-1987 CHLOROMETHANE

SD-100-12 26-Hay-1987 CHLOROMETHANE

SD-100-12 3-Jun-1987 CHLOROMETHANE

SD-100-12 11-Jun-1987 CHLOROMETHANE

SD-100-12 18-Jun-1987 CHLOROMETHANE

* SD-100-13

SD-100-13 12-May-1987 CHLOROMETHANE

SD-100-13 19-May-1987 CHLOROMETHANE

SD-100-13 26-May-1987 CHLOROMETHANE

SD-100-13 3-Jun-1987 CHLOROMETHANE

SD-100-13 11-Jun-1987 CHLOROMETHANE

SD-100-13 18-Jun-1987 CHLOROMETHANE

$<10 \quad$ ug $/ \mathrm{h}$

$<10$

$<10$

$<10$

$<10$

$<10$

$<10$

$\mathrm{ug} / \mathrm{L}$

$<10$

$<10$

$<10$

$<10$

ug/L

$4 \mathrm{~g} / \mathrm{L}$

$\mathrm{ug} / \mathrm{L}$

$4 \mathrm{~g} / \mathrm{L}$

$<10$

$<10$

$<10$

$<10$

$<10$

$<10$

ug $/$

$u g / L$

$4 \mathrm{~g} / \mathrm{L}$

ug/L

$u g / L$

ug/L

** SD $-100-14$

SD-100-14 12-May-1987 CHLOROMETHANE

$<10$

ug/L

$\mathrm{ug} / \mathrm{L}$

$\mathrm{ug} / \mathrm{L}$

ug/L

ug $/ \mathrm{L}$

ug/L

$<10$

ug $/$ L

$<10$

$<10$

$<10$

$<10$

$<10$

ug $/ \mathrm{L}$

ug/L

ug $/$

ug $/ \mathrm{L}$

ug/L

$<10$

ug $/ \mathrm{L}$

$<10$

$<10$

$<10$

$<10$

$<10$

$u g / L$

$\mathrm{ug} / \mathrm{L}$

Ug/L

ug $/ 2$

$4 \mathrm{~g} / \mathrm{L}$

$<10$

$<10$

$<10$

ug 2

$<10$

$u \mathrm{~g} / \mathrm{L}$

ug/L

$<10$

ug/L

ug/L

$<10$

ug/L

$u g / 2$ 
Stom Drain Data for SD-100

\begin{tabular}{|c|c|c|c|c|}
\hline Location & Dete & Test Compound & Results & Units \\
\hline$S D-100-14$ & 19-May-1987 & CKLOROMETHANE & $<10$ & $\operatorname{ug} / \mathrm{L}$ \\
\hline$S D-100-14$ & 26-May-1987 & CHLOROAETHANE & $<10$ & $\mathrm{ug} / \mathrm{L}$ \\
\hline SD-100-14 & 3-Jun-1987 & CHLOROSETHANE & $<10$ & $\lg / \mathrm{L}$ \\
\hline SD $-100-14$ & $11 \cdot J u n-1987$ & CHLOROKIETHANE & $<10$ & Ug/L \\
\hline SD-100-14 & 18.Jun-1987 & CRIOROIETHANE & $<10$ & $\mathrm{ug} / \mathrm{L}$ \\
\hline \multicolumn{5}{|c|}{$\star * \quad S D-100-C$} \\
\hline$S D=100-C$ & 5-Mar-1987 & CHLOROMETHANE & $<10$ & $\mathrm{ug} / \mathrm{L}$ \\
\hline SD-100-C & $9-\operatorname{Mar}-1987$ & CHLOROMETHANE & $<10$ & $\mathrm{ug} / \mathrm{l}$ \\
\hline$S D \cdot 100-C$ & 17 -Mar- 1987 & CHLOROMETHANE & $<10$ & $4 g / 2$ \\
\hline SD-100-C & $24-\operatorname{Mar}-1987$ & CHLOROIETHANE & $<10$ & $4 \mathrm{~g} / \mathrm{L}$ \\
\hline SD-100-C & 8-Apr-1987 & CHILOROMEIHANE & $<10$ & $\mathrm{ug} / \mathrm{L}$ \\
\hline SD-100-C & 13-May-1987 & CHLOROMETHANE & $<10$ & $u g / L$ \\
\hline SD-100-C & 20-May-1987 & CHLOROMETHANE & $<10$ & $\operatorname{ug} / \mathrm{L}$ \\
\hline SD-100-C & 27-May - 1987 & CHLOROMETHANE & $<10$ & $\mathrm{ug} / \mathrm{I}$ \\
\hline$S D-100-C$ & 12 -Jun- 1987 & CHLOROMETHANE & $<10$ & $4 g / L$ \\
\hline$S D-100-C$ & 19-Jun-1987 & GHLOROMETHANE & $<10$ & $\mathrm{ug} / \mathrm{L}$ \\
\hline \multicolumn{5}{|c|}{$\star \star S D-100-D$} \\
\hline SD-100-D & 6-API- 1987 & CHLOROHETHANE & $<10$ & $4 \mathrm{~g} / \mathrm{L}$ \\
\hline SD-100-D & $11-J u n-1987$ & CHLOROMETHANE & $<10$ & $u g / L$ \\
\hline
\end{tabular}

\begin{tabular}{|c|c|c|}
\hline \multicolumn{3}{|c|}{ * $\quad$ SD-1 } \\
\hline$S D-100$ & - Mar-1987 & $\mathrm{CH}$ \\
\hline$D-100$ & $11-\operatorname{Mar}-1987$ & CHROMIO \\
\hline$D=100$ & $18-\operatorname{Mar}-1987$ & CHROAIU \\
\hline$D-100$ & $24-\mathrm{Mar}-1987$ & CHROMIUS \\
\hline$S D-100$ & 3-Арт -1987 & CHROMIUY \\
\hline$S D-100$ & 6-Apr-1987 & CHROMIUR \\
\hline$S D-100$ & 12-May -1987 & CHROMUM \\
\hline$S D-100$ & 19-May-1987 & CHROHIOM \\
\hline SD -100 & 26-May-1987 & CHROMIUM \\
\hline$S D-100$ & 3-Jun-1987 & GHROMIUM \\
\hline$D=100$ & 11-Jun-1987 & CHROMIUM \\
\hline & 18 -Jun-1987 & \\
\hline
\end{tabular}

$<0.010 \mathrm{mg} / \mathrm{L}$

$<0.010 \mathrm{mg} / \mathrm{L}$

$<0.010 \mathrm{mg} / \mathrm{L}$

$<0.010 \mathrm{mg} / \mathrm{L}$

$<0.010 \mathrm{mg} / \mathrm{L}$

$<0.010 \mathrm{mg} / \mathrm{L}$

$<0.010 \mathrm{mg} / \mathrm{L}$

$<0.010 \mathrm{mg} / \mathrm{L}$

$<0.020 \mathrm{mg} / \mathrm{L}$

$<0.010 \quad \mathrm{mg} / \mathrm{L}$

$<0.010 \mathrm{mg} / \mathrm{L}$

$<0.010 \mathrm{mg} / \mathrm{L}$

* SD-100-01

SD-100-01 12-May-1987 CHROMIUM

$<0.010 \mathrm{mg} / \mathrm{L}$

SD-100-01 18-May-1987 ChROMIUM

SD-100-01 3-Jun-1987 CEROMIOM

SD-100-01 11-Jun-1987 CHROHIUM

SD-100-01 18-Jun-1987 CHROMIUM

$<0.010 \mathrm{mg} / \mathrm{L}$

$<0.010 \mathrm{mg} / \mathrm{L}$

$<0.010 \mathrm{mg} / \mathrm{L}$

$<0.010 \mathrm{mg} / \mathrm{L}$

* SD-100-02

SD-100-02 12-May-1987 CHROMIUM

SD-100-02 18-May-1987 CHROMIUM

$<0.010 \mathrm{mg} / \mathrm{L}$

$<0.010 \mathrm{mg} / \mathrm{L}$

SD-100-02 3-Jun-1987 CHROMIUM

$<0.010 \quad \mathrm{mg} / \mathrm{L}$

SD-100-02 11-Jun-1987 CHROMIUM

$<0.010 \mathrm{mg} / \mathrm{L}$

SD-100-02 18-Jun-1987 CHROMIUM

$<0.010 \mathrm{mg} / \mathrm{L}$ 
Storm Drain Data for SD-100

Location

Date

. Test Compound

Results

Units

** SD $-100-03$

SD-100-03 12-May-1987 GHROMTUM

SD-100-03 18-May-1987 GROMIUM

SD-100-03 26-May-1987 CAROMIOM

SD-100-03 3-Jun-1987 CHROMIUM

SD-100-03 11-Jun-1987 GHROMIUY

SD-100-03 18-Jun-1987 GHROKIUM

** SD-100-03D

SD-100-03D 12-May-1987 GTROMIUM

SD-100-03D 26-May-1987 CRROHIIUM

* SD-100-04

SD-100-04 12-May-1987 GIROMIUM

SD-100-04 18-May-1987 GUROMIUM

SD-100-04 26-May-1987 GHROMIUM

SD-100-04 3-Jun-1987 GHROMIUM

SD-100-04 11-Jun-1987 CHROMIUM

SD-100-04 18-Jun-1987 GHROMIUM

* SD-100-06

SD-100-06 12-May-1987 GRROMIUM

SD-100-06 18-May-1987 GrROYIUM

SD-100-06 26-May-1987 CHROMIUM

SD-100-06 3-Jun-1987 CHROMIUM

SD-100-06 11-Jun-1987 CHROMIUM

SD-100-06 18-Jun-1987 GHROKIUM

* SD-100-06D

SD-100-06D 3-Jun-1987 CHROMTUM

** SD-100-07

SD-100-07 12-May-1987 GHROMTUM

SD-100-07 19-May-1987 CHROMIUM

SD-100-07 26-May-1987 CHROMIUM

SD-100-07 3-Jun-1987 CHROMTUM

SD-100-07 11-Jun-1987 CHROMIUM

SD-100-07 18-Jun-1987 GAROMIUM

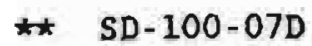

SD-100-07D 18-Jun-1987 CIROHLIM

$<0.010$

$<0.010$

$\mathrm{mg} / \mathrm{L}$

$<0.010$

$\mathrm{mg} / \mathrm{L}$

$<0.010$

$\mathrm{mg} / \mathrm{L}$

$<0.010$

mg $\Omega$

$<0.010$

$\operatorname{mg} / \mathrm{L}$

$<0.010$

mg/l

$<0.010$

$\mathrm{mg} / \mathrm{L}$

$<0.010$

$<0.010$

$<0.010$

$<0.010$

$-<0.010$

$<0.010$

mg $/$.

$\mathrm{mg} / \mathrm{L}$

mg $/ \mathrm{L}$

$\mathrm{mg} / \mathrm{L}$

$\mathrm{mg} / \mathrm{L}$

$\mathrm{mg} / \mathrm{L}$

$<0.010$

$<0.010$

$<0.010$

mg $/ \mathrm{L}$

$<0.010$

$\mathrm{mg} / \mathrm{L}$

$<0.010$

$\mathrm{mg} / \mathrm{L}$

$<0.010$

mg/L

$\mathrm{mg} / \mathrm{L}$

$<0.010 \mathrm{mg} / \mathrm{L}$

0.19

0.081

$\mathrm{mg} / \mathrm{L}$

0.011

ㄸg $/ \mathrm{L}$

0.093

$\mathrm{mg} / \mathrm{L}$

$<0.010$

$\mathrm{mg} / \mathrm{L}$

0.037

$\operatorname{mg} / \mathrm{L}$

* SD-100-09

SD-100-09 12-May-1987 CHROMIUM

SD-100-09 18-May-1987 CHROMIUM

SD-100-09 26-May-1987 CHROKIUM

SD-100-09 3-Jun-1987 CHROMIUM

SD-100-09 11-JUR-1987 CHROMIUM

$0.038 \mathrm{ng} / \mathrm{h}$

$<0.010 \mathrm{mg} / \mathrm{L}$

$<0.010 \mathrm{mg} / \mathrm{L}$

$<0.010 \mathrm{mg} / \mathrm{L}$

$<0.010 \quad$ تgg $/ \mathrm{L}$

$<0.010 \mathrm{mg} / \mathrm{L}$

* SD-100-10

SD-100-10 12-May-1987 CHROMIUM

$<0.010$

$\mathrm{mg} / \mathrm{L}$ 
Storm Drain Data for SD-100

Location

Dace

Test Compound

Results

Units

SD-100-10

SD-100-10

SD $-100-10$

SD $-100-10$

SD $-100-10$
19-May-1987 CHROMIUM

26-May-1987 CHROMTUM

3-Jun-1987 CHROMIOM

11-Jun-1987 CHROMION

18-Jun-1987 CHROMIOM

* SD-100-11

SD-100-11 12-May-1987 CHROMIUM

SD-100-11 19-May-1987 CHROKIOM

SD-100-11 26-May-1987 CHROMIUM

SD-100-11 3-Jun-1987 CHROMIUM

SD-100-11 11-Jun-1987 CHROMIUM

SD-100-11 18-Jun-1987 CHROHIUM

* SD-100-12

SD-100-12 12-May-1987 CHROMIUM

SD-100-12 19-May-1987 CHROMIUM

SD-100-12 26-May-1987 CHROMIUM

SD-100-12 3-Jun-1987 CHROLIUH

SD-100-12 11-Jun-1987 CHROMTUM

SD-100-12 18-Jun-1987 CHROMIUM

** SD $-100-13$

SD-100-13 12-May-1987 CHROMITU

SD-100-13 19-May-1987 CHROHITM

SD-100-13 26-May-1987 CHROHIIU

SD-100-13 11-Jun-1987 CHROKTUM

SD-100-13 18-Jun-1987 GHROMIUM

** SD $-100-14$

SD-100-14 12-May-1987 CKROHIUM

SD-100-14 19-May-1987 GHROHIUM

SD-100-14 26-May-1987 CHROHTUM

SD-100-14 3-Jun-1987 CHROHTUM

SD-100-14 11-Jun-1987 CHROHTUM

SD-100-14 18-Jun-1987 CRROFIUM

* $\quad$ SD $-100-C$

SD-100-C 5-Mar-1987 CHROHIU

SD-100-C 9-Mar-1987 CHROMIUM

SD-100-C 17-Mar-1987 CHROHTU

SD-100-C 24-Mar-1987 CHROMTUM

SD-100-C 8-Apr-1987 CHROMTUM

SD-100-C 13-May-1987 CHROMIUM

SD-100-C 20-May-1987 CHROMIUM

SD-100-C 27-May-1987 CHROMIUM

SD-100-C 4-Jun-1987 CMROMIUM

SD-100-C 12-Jun-1987 CHROHIUM

SD-100-C 19-Jun-1987 CHROMIUM

$<0.010$

$<0.010$

$<0.010$

$<0.010$

$<0.010$

$\mathrm{mg} / \mathrm{L}$

$\operatorname{mg} / \mathrm{L}$

$\mathrm{mg} / \mathrm{L}$

$\mathrm{mg} / \mathrm{L}$

mg/L

$<0.010$

$<0.010$

$<0.010$

$<0.010$

$<0.010$

$<0.010$

$\mathrm{mg} / \mathrm{L}$

$\operatorname{mg} / \mathrm{L}$

$\mathrm{mg} / \mathrm{L}$

$\mathrm{mg} / \mathrm{L}$

$\mathrm{mg} / \mathrm{L}$

$\mathrm{mg} / \mathrm{L}$

$<0.010$

$<0.010$

$<0.010$

$<0.010$

$<0.010$

$<0.010$

$\mathrm{mg} / \mathrm{L}$

$\mathrm{mg} / \mathrm{L}$

$\mathrm{mg} / \mathrm{L}$

$\mathrm{mg} / \mathrm{L}$

$\mathrm{mg} / \mathrm{L}$

$\mathrm{mg} / \mathrm{L}$

$<0.010$

$<0.010$

$<0.010$

$<0.010$

$<0.010$

mg $/ \mathrm{L}$

$\mathrm{mg} / \mathrm{L}$

$\mathrm{mg} / \mathrm{L}$

$\mathrm{mg} / \mathrm{L}$

$\mathrm{mg} / \mathrm{L}$

$<0.010$

$\mathrm{mg} / \mathrm{L}$

$<0.010$

$<0.010$

$\mathrm{mg} / \mathrm{L}$

$<0.010$

$<0.010$

$\mathrm{mg} / \mathrm{L}$

mg/L

$<0.010$

$\mathrm{mg} / \mathrm{L}$

$\mathrm{mg} / \mathrm{L}$

$<0.010$

$\mathrm{mg} / \mathrm{L}$

$<0.010$

$<0.010$

$\mathrm{mg} / \mathrm{L}$

$<0.010$

$\mathrm{mg} / \mathrm{I}$

$<0.010$

$\mathrm{mg} / \mathrm{L}$

$<0.010$

mg/L

$<0.010$

$<0.010$

$<0.010$

$<0.010$

$\mathrm{mg} / \mathrm{L}$

$\mathrm{mg} / \mathrm{L}$

$\mathrm{mg} / \mathrm{L}$

$\mathrm{mg} / \mathrm{L}$

$\mathrm{mg} / \mathrm{L}$

$<0.010 \mathrm{mg} / \mathrm{L}$ 
Storm Drain Data for SD-100

Location

Date

Test Compound

Results Units

** SD-100-D

SD-100-D 6-ApF-1987 CHROMIUM

SD-100-D 11-Jun-1987 CHROMIUM

$\begin{array}{ll}<0.010 & \mathrm{mg} / \mathrm{L} \\ <0.010 & \mathrm{mg} / \mathrm{L} \\ & \\ <10 & \mathrm{ug} / \mathrm{L} \\ <10 & \mathrm{ug} / \mathrm{L} \\ <10 & \mathrm{ug} / \mathrm{L} \\ <5.0 & \mathrm{ug} / \mathrm{L} \\ & \\ <10 & \mathrm{ug} / \mathrm{L} \\ <5.0 & \mathrm{ug} / \mathrm{L}\end{array}$

** SD-100

SD-100 5-Mar-1987 CHRYSEANE

SD-100 11-Mer-1987 CRRYSENE

SD-100 12-May-1987 CHRYSENE

SD-100 26-May-1987 CHRYSENE

** SD-100-01

SD-100-01 12-Kay-1987 CHRYSENE

SD-100-01 26-May-1987 CHRYSENE

* SD-100-02

SD-100-02 12-May-1987 CHRYSENE

SD-100-02 26-May-1987 CHRYSENE

$<10 \quad$ ug/L

$<5.0 \quad$ ug/L

* SD $-100-03$

SD-100-03 12-May-1987 CHRYSENE

SD-100-03 26-May-1987 CHRYSENE

* SD-100-03D

SD-100-03D 12-May-1987 CHRYSENE

SD-100-03D 26-May-1987 CHRYSENE

* SD-100-04

SD-100-04 12-May-1987 CHRYSENE

SD-100-04 26-May-1987 CHRYSENE

* SD-100-06

SD-100-06 12-May-1987 CHRYSENE

SD-100-06 26-May-1987 CHRYSENE

** SD-100-07

SD-100-07 12-May-1987 CHRYS ENE

SD-100-07 26-May-1987 CHRYSENE

$<10 \quad \mathrm{ug} / \mathrm{L}$

$<5.0 \quad$ ug/L

$<10 \quad 4 \mathrm{~g} / \mathrm{L}$

$<5.0 \quad 4 \mathrm{~g} / \mathrm{L}$

$<10 \quad$ ug/L

$<5.0 \quad \mathrm{ug} / \mathrm{l}$

$<10 \quad$ ug/L

$<5.0 \quad$ ug/L

** SD-100-09

SD-100-09 12-May-1987 CHRYSENE

SD-100-09 26-May-1987 CHRYSENE

* SD-100-10

SD-100-10 12-May-1987 CHRYSENE

SD-100-10 26-May-1987 CHRYSENE

$<10 \quad 48 / \mathrm{L}$

$<5.0 \quad 4 \mathrm{~g} / \mathrm{L}$

$<10 \quad$ ug/L

$<5.0 \quad \mathrm{ug} / \mathrm{L}$

** SD-100-11

SD-100-11 12-May-1987 CHRYSENE

SD-100-11 26-May-1987 CHRYSENE

$<10 \quad \mathrm{ug} / \mathrm{L}$

$<5.0 \quad \mathrm{ug} / \mathrm{h}$

$<10 \quad$ ug/L

$<5.0 \quad$ ug $/ \mathrm{L}$ 
Storm Draln Data for SD-100

\begin{tabular}{|c|c|c|c|c|}
\hline Location & Dace & Test Compound & Results & Units \\
\hline \multicolumn{5}{|c|}{$\star \star \quad S D-100-12$} \\
\hline$S D-100-12$ & 12-May-1987 & CHRYSENE & $<10$ & ug/L \\
\hline SD $-100-12$ & 26-May-1987 & CHRYSENE & $<5.0$ & ug $/ \mathrm{L}$ \\
\hline \multicolumn{5}{|c|}{$\star \star \quad S D-100-13$} \\
\hline$S D-100-13$ & 12-May-1987 & CHRYSENE & $<10$ & $u g / L$ \\
\hline$S D-100-13$ & 26-May - 1987 & CHRYSENE & $<5.0$ & $u g / L$ \\
\hline \multicolumn{5}{|c|}{ ** SD $-100-14$} \\
\hline$S D-100-14$ & 12-May-1987 & CHRYSENE & $<10$ & $\mathrm{ug} / \mathrm{L}$ \\
\hline SD-100-14 & $26-\mathrm{May}-1987$ & CHRYSENE & $<5.0$ & $4 g / L$ \\
\hline \multicolumn{5}{|c|}{$\star \star \quad S D-100-C}$. \\
\hline$S D-100 \cdot C$ & 5-Mar-1987 & CHRYSENE & $<10$ & $4 \mathrm{~g} / \mathrm{L}$ \\
\hline SD $-100-C$ & $9-\operatorname{Mar}-1987$ & CHRYSENE & $<10$ & $\lg / \mathrm{L}$ \\
\hline SD- $100-C$ & 17-Mar-1987 & CHRYSENE & $<10$ & $u g / L$ \\
\hline$S D-100-C$ & $24-$ Mar- 1987 & CHRYSENE & $<10$ & $\mathrm{ug} / \mathrm{L}$ \\
\hline$S D-100-C$ & 8-ApF-19B7 & CHRYSENE & $<10$ & $u g / L$ \\
\hline SD- $100-C$ & 13-May-1987 & CHRYSENE & $<5.0$ & $\mathrm{ug} / \mathrm{L}$ \\
\hline SD-100-C & 20- May - 1987 & CHRYSENE & $<5.0$ & $u g / L$ \\
\hline SD-100-C & 27-May-1987 & CHRYSENE & $<5.0$ & $4 \mathrm{~g} / \mathrm{L}$ \\
\hline SD-100-C & 12-Jun-1987 & CHRYSENE & $<5.0$ & $4 \mathrm{~g} / \mathrm{L}$ \\
\hline$S D-100-C$ & $19-5$ un- 1987 & CHRYSENE & $<10$ & $\mathbf{u g} / \mathrm{L}$ \\
\hline
\end{tabular}

\begin{tabular}{|c|c|c|c|c|}
\hline \multicolumn{5}{|l|}{ **. SD -100} \\
\hline SD-100 & $11-\operatorname{Mar}-1987$ & CIS-I 3-DICHLOROPROPENE & $<5$ & $\log / \mathrm{L}$ \\
\hline SD -100 & $18-$ Mar -1987 & CIS-1 3-DICHLOROPRORENE & $<5$ & $4 \mathrm{Lg} / \mathrm{L}$ \\
\hline$S D=100$ & $24-\operatorname{MaI}-1987$ & CIS-1 3-DICHLOROPROPENE & $<5$ & $\mathrm{ug} / \mathrm{L}$ \\
\hline SD- 100 & 6-Apr-1987 & CIS-1 3-DICHLOROPROPENE & $<5$ & $\lg / \mathrm{L}$ \\
\hline SD- 100 & 12-Kay -1987 & CIS-1 3-DICHLOROPROPENE & $<5$ & $u g / L$ \\
\hline SD- 100 & $19-\mathrm{Mzg}-1987$ & CIS-1 3-DICHLOROPROPENE & $<5$ & $\operatorname{ug} / \mathrm{L}$ \\
\hline SD- 100 & 26 - May- 1987 & CIS-1 3-DICHLOROPROPENE & $<5$ & $u g / L$ \\
\hline SD -100 & 3-Jun-1987 & CIS-1 3-DICHLOROPROPENE & $<5$ & $u g / L$ \\
\hline SD -100 & 11-Jun-1987 & CIS-I 3-DICHLOROPROPENE & $<5$ & $4 g / L$ \\
\hline$S D-100$ & $18-J u n-1987$ & CIS-I 3-DICHLOROPROPENE & $<5$ & $\mathrm{ug} / \mathrm{l}$ \\
\hline \multicolumn{5}{|c|}{ ** SD-100-01 } \\
\hline$S D-100-01$ & 12-May-1987 & CIS-1 3-DICHLOROPROPENE & $<5$ & $\mathrm{ug} / \mathrm{L}$ \\
\hline SD-100-01 & $18-$ Kay -1987 & CIS - I 3-DICHLOROPROPENE & $<5$ & ug $/ \mathrm{L}$ \\
\hline SD- $100-01$ & 26 -May - 1987 & CIS-1 3-DICHLOROPROPENE & $<5$ & $\mathbf{u g} / \mathrm{L}$ \\
\hline SD-100-01 & 3-Jun-1987 & CIS-1 3-DICHLOROPROPENE & $<5$ & $\mathrm{ug} / \mathrm{L}$ \\
\hline SD-100-01 & $11-J u n-1987$ & CIS-I 3-DICHLOROPROPENE & $<5$ & $\mathrm{ug} / \mathrm{L}$ \\
\hline SD $-100-01$ & 18-Jum- 1987 & CIS-1 3-DICHLOROPROPENE & $<5$ & $48 / \mathrm{L}$ \\
\hline \multicolumn{5}{|c|}{$\star \quad$ SD $-100-02$} \\
\hline$S D-100-02$ & 12- May - 1987 & CIS-1 3-DICHLOROPROPENE & $<5$ & ug/L \\
\hline SD - $100-02$ & 18-May-1987 & CIS-1 3-DICHLOROPROPENE & $<5$ & ug/L \\
\hline SD $-100-02$ & 26-May- 1987 & CIS-1 3-DICHLOROPROPENE & $<5$ & $\mathrm{ug} / \mathrm{L}$ \\
\hline SD- $100-02$ & 3-Jun-1987 & CIS-I 3-DICHLOROPROPENE & $<5$ & $\mathrm{ug} / \mathrm{L}$ \\
\hline SD- $100-02$ & $11-J u n-1987$ & CIS-1 3-DICHLOROPROPENE & $<5$ & ug/L \\
\hline
\end{tabular}


Storm Drain Data for SD-100

Location

Date

Test Compound

Results Units

SD-100-02 18-Jun-1987 CIS-1 3-DICHLOROPROPENE

** SD $-100-03$

SD-100-03 12-Hay-1987 CIS-1 3-DICHIOROPROPENE SD-100-03 18-May-1987 CIS-1 3-DICHLOROPROPENE SD-100-03 26-May-1987 GIS-1 3-DICHLOROPROPENE

SD-100-03 3-Jun-1987 CIS-1 3-DICHLOROPROPENE SD-100-03 11-Jun-1987 CIS-1 3-DICLLOROPROPENE SD-100-03 18-JUn-1987 CIS-1 3-DICHLOROPROPENE

** SD-100-03D

SD-100-03D 12-May-1987 CIS-1 3-DICHLOROPROPENE SD-100-03D 26-Kay-1987 CIS-1 3-DICHLOROPROPENE

** SD-100-04

SD-100-04 12-May-1987 CIS-1 3-DICHLOROPROPENE

SD-100-04 18-May-1987 CIS-1 3-DICHLOROPROPENE

SD-100-04 26-May-1987 CIS-I 3-DICHLOROPROPENE

SD-100-04 3-Jum-1987 CIS-1 3-DICHLOROPROPENE

SD-100-04 11-Jun-1987 CIS-1 3-DICHLOROPROPENE

SD-100-04 18-Jun-1987 CIS-1 3-DICKLOROPROPENE

* SD-100-06

SD-100-06 12-May-1987 CIS-1 3-DICHLOROPROPENE

SD-100-06 18-May-1987 CIS-1 3-DICHLOROPROPENE

SD-100-06 26-Kay-1987 CIS-1 3-DICHLOROPROPENE

SD-100-06 3-Jun-1987 CIS-1 3-DICHLOROPROPENE

SD-100-06 11-Jun-1987 CIS-1 3-DICHLOROPROPENE

SD-100-06 18-Jun-1987 CIS-1 3-DICHLOROPROPENE

** SD-100-06D

SD-100-06D 3-JUn-1987 CIS-1 3-DICHLOROPROPENE

$<5$

$\mathrm{ug} / \mathrm{L}$

$\begin{array}{ll}<5 & u g / L \\ <5 & u g / L \\ <5 & u g / L \\ <5 & u g / L \\ <5 & u g / L \\ <5 & u g / L\end{array}$

$<5$

$<$

ug/L

ug $/ 2$

$<5$

$<5$

$<5$

$<5$

$<$

$<$

ug/L

ug/L

$u g / L$

ug/L

4g/L

$\mathrm{ug} / \mathrm{L}$

$<$

$<$

$<5$

$<5$

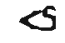

$<5$

$4 g / L$

ug/L

$\mu g / L$

$\mathrm{ug} / \mathrm{L}$

$u g / L$

$4 \mathrm{~g} / \mathrm{L}$

* SD - 100-07

SD-100-07 12-May-1987 CIS-1 3-DICHLOROPROPENE

SD-100-07 19-May-1987 CIS-1 3-DICHLOROPROPENE

SD-100-07 26-May-1987 CIS-1 3-DICHLOROPROPENE

SD-100-07 3-Jun-1987 CIS-1 3-DICHLOROPROPENE

SD-100-07 11-Jun-1987 CIS-1 3-DICHLOROPROPENE

SD-100-07 18-Jun-1987 CIS-1 3-DICHLOROPROPENE

$<$

$u g / \mathrm{L}$

** SD-100-07D

SD-100-OTD 18-JUn-1987 CIS-1 3-DICHLOROPROPENE

$u g / L$

** SD-100-09

SD-100-09 12-May-1987 CIS-1 3-DICHLOROPROPENE

SD-100-09 18-May-1987 CIS-1 3-DICHLOROPROPENE

SD-100-09 26-May-1987 CIS-1 3-DICHLOROPROPENE

SD-100-09 3-JUn-1987 CIS-1 3-DICHLOROPROPENE

SD-100-09 11-Jun-1987 CIS-1 3-DICHLOROPROPENE

SD-100-09 18-Jun-1987 CIS-1 3-DICHLOROPROPENE

$<5$

$<$

$<5$

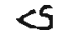

$<5$

$<5$

$<5$

$<5$

$<5$

$<5$

<s

$<5$

$<5$

ug/L

$\mathrm{ug} / \mathrm{L}$

ug $/ \mathrm{L}$

Ug/L

ug/L

$u g / L$

$u g / L$

ug/L

ug $/ L$

ug/L

ug/L

$u g / L$ 
Storm Draln Data for SD-100

Location Date Test Compound

Results Units

* SD-100-10

SD-100-10 12-May-1987 CIS-1 3-DICLLOROPROPENE

SD-100-10 19-Kay-1987 CIS-1 3-DICHLORORROPBNE

SD-100-10 26-May-1987 CIS-1 3-DICHLORORROPENE

SD-100-10 3-Jun-1987 CIS-1 3-DICHLOROPROPENE

SD-100-10 11-Jun-1987 CIS-1 3-DICHLOROPROPENE

SD-100-10 18-Jun-1987 CIS-1 3-DICHLOROPROPENE

*t SD-100-11

SD-100-11 12-May-1987 CIS-1 3-DICHLOROPROPEITE

SD-100-11 19-May-1987 CIS-1 3-DICHLOROPROPENE

SD-100-11 26-May-1987 CIS-1 3-DICHLOROPROPENE

SD-100-11 3-Jun-1987 CIS-1 3-DICHLOROPROPENE

SD-100-11 11-Jun-1987 CIS-1 3-DICHLOROPROPENE

SD-100-11 18-Jun-1987 CIS-1 3-DICHLOROPROPENE

$\begin{array}{ll}<5 & \mathrm{ug} / \mathrm{L} \\ <5 & \mathrm{ug} / \mathrm{L} \\ <5 & \mathrm{ug} / \mathrm{L} \\ <5 & \mathrm{ug} / \mathrm{L} \\ <5 & \mathrm{ug} / \mathrm{L} \\ <5 & \mathrm{ug} / \mathrm{L}\end{array}$

** SD $-100-12$

SD-100-12 12-May-1987 CIS-1 3-DICELOROPROPENE

SD-100-12 19-May-1987 CIS-1 3-DICHLOROPROPENE

SD-100-12 26-May-1987 CIS-1 3-DICHLOROPROPENE

SD-100-12 3-Jun-1987 CIS-1 3-DICHLOROPROPENE

SD-100-12 11-Jun-1987 CIS-1 3-DICHLOROPROPERE

SD-100-12 18-Jun-1987 CIS-1 3-DICHLOROPROPEIJE

**. SD $-100-13$

SD-100-13 12-May-1987 CIS-1 3-DICHLOROPROPETE

SD-100-13 19-May-1987 CIS-1 3-DICHLOROPROPETE

SD-100-13 26-May-1987 CIS-1 3-DICHLOROPROPENE

SD-100-13 3-Jun-1987 CIS-1 3-DICHLOROPROPENE

SD-100-13 11-Jun-1987 CIS-1 3-DICHLOROPROPENE

SD-100-13 18-Jun-1987 CIS-1 3-DICHLOROPROPENE

$\star \star \quad S D-100-14$

SD-100-14 12-May-1987 CIS-1 3-DICHLOROPROPENE

SD-100-14 19-May-1987 CIS-1 3-DICHLOROPROPENE

SD-100-14 26-May-1987 CIS-1 3-DICHLOROPROPENE

SD-100-14 3-Jun-1987 CIS-1 3-DICHLOROPROPEVE

SD-100-14 11-JUn-1987 CIS-1 3-DICHLOROPROPENE

SD-100-14 18-Jun-1987 CIS-1 3-DICHLOROPROPENE

$<$

ug $/ \mathrm{L}$

$\mathrm{ug} / \mathrm{L}$

ug/L

$u g / L$

$u g / L$

$\mathrm{ug} / \mathrm{L}$

$\begin{array}{ll}<5 & \mathrm{ug} / \mathrm{L} \\ <5 & \mathrm{ug} / \mathrm{L} \\ <5 & \mathrm{ug} / \mathrm{L} \\ <5 & \mathrm{ug} / \mathrm{L} \\ <5 & \mathrm{ug} / \mathrm{L} \\ <5 & \mathrm{ug} / \mathrm{L}\end{array}$

$\begin{array}{ll}<5 & \mathrm{ug} / \mathrm{L} \\ <5 & \mathrm{ug} / \mathrm{L} \\ <5 & \mathrm{ug} / \mathrm{L} \\ <5 & \mathrm{ug} / \mathrm{L} \\ <5 & \mathrm{ug} / \mathrm{L} \\ <5 & \mathrm{ug} / \mathrm{L}\end{array}$

* $\quad$ SD $-100-C$

SD-100-C 5-Mar-1987 CIS-1 3-DICHLOROPROPERE

SD-100-C 9-Mar-1987 CIS-1 3-DICHLOROPROPENE

SD-100-C 17-Mar-1987 CIS-1 3-DICHLOROPROPENE

SD-100-C 24-Mar-1987 CIS-1 3-DICHLOROPROPENE

SD-100-C 8-APr-1987 CIS-1 3-DICHLOROPROPENE

SD-100-C 13-May-1987 CIS-1 3-DICHLOROPROPENE

SD-100-C 20-May-1987 CIS-1 3-DICHLOROPROPENE

SD-100-C 27-May-1987 CIS-I 3-DICHLOROPROPENE

$S D-100-C$

12-Jun-1987. CIS-1 3-DICALOROPROPENE

$\begin{array}{ll}<5 & \mathrm{ug} / \mathrm{L} \\ <5 & \mathrm{ug} / \mathrm{L} \\ <5 & \mathrm{ug} / \mathrm{L} \\ <5 & \mathrm{ug} / \mathrm{L} \\ <5 & \mathrm{ug} / \mathrm{L} \\ <5 & \mathrm{ug} / \mathrm{L}\end{array}$

$\begin{array}{ll}<5 & \mathrm{ug} / \mathrm{L} \\ <5 & \mathrm{ug} / \mathrm{L} \\ <5 & \mathrm{ug} / \mathrm{L} \\ <5 & \mathrm{ug} / \mathrm{L} \\ <5 & \mathrm{ug} / \mathrm{L} \\ <5 & \mathrm{ug} / \mathrm{L} \\ <5 & \mathrm{ug} / \mathrm{L} \\ <5 & \mathrm{ug} / \mathrm{L} \\ <5 & \mathrm{ug} / \mathrm{L}\end{array}$


Storm Drain Data for SD-100

Location

Date

Test Compound

SD $-100-$

19-JUn-1987 CIS-1 3-DICHLOROPROPENE

* SD-100-D

SD-100-D 6-APr-1987 CIS-1 3-DICHLOROPROPEINE SD-100-D 11-JUn-1987 CIS-1 3-DICHLOROPROPENE

** SD-100

SD -100

5-Mar-1987 COBALT

SD -100

11-Mar-1987 COBALT

SD -100

18-Mar-1987 COBALT

SD -100

24-Mar-1987 COBALT

SD -100

SD -100

3-Apr-1987 COBALT

SD -100

6-Apr-1987 COBALT

SD -100

12-May-1987 COBALT

SD -100

19-May-1987 COBALT

SD -100

26-May-1987 COBALT

SD -100

3-Jun-1987 COBALT

SD -100

11-Jun-1987 COBALT

18-Jun-1987 COBALT

* SD-100-01

SD-100-01 12-May-1987 COBALT SD-100-01 18-May-1987 COBALT SD-100-01 3-Jun-1987 COBALT SD-100-01 11-Jun-1987 COBALT SD-100-01 18-Jun-1987 COBALT

* SD-100-02

SD-100-02 12-May-1987 COBALT SD-100-02 18-May-1987 COBALT SD-100-02 3-Jun-1987 COBALT SD-100-02 11-Jun-1987 COBALT SD-100-02 18-Jur-1987 COBALT

* SD $-100-03$

SD-100-03 12-May-1987 COBALT SD-100-03 18-May-1987 COBALT SD-100-03 26- $\mathrm{Kay}-1987$ COBALT SD-100-03 3-Jun-1987 COBALT SD-100-03 11-Jun-1987 COBALT SD-100-03 18-Jun-1987 COBALT

** SD-100-03D

SD-100-03D 12-May-1987 COBALT SD-100-03D 26-Kay-1987 COBALT

* SD-100-04

SD-100-04 12-May-1987 COBALT SD-100-04 18-May-1987 COBALT SD-100-04 26-May-1987 COBALT SD-100-04 3-Jun-1987 COBALT
Results Units

$\begin{array}{ll}<5 & u g / L \\ <5 & u g / 2 \\ <5 & u g / L\end{array}$

$<0.10$

$<0.10$

$<0.10$

$<0.10$

$<0.10$

$<0.10$

$<0.10$

$<0.10$

$<0.0050$

$<0.10$

$<0.10$

$<0.10$

$<0.10$

$<0.10$

$<0.10$

$<0.10$

$<0.10$

$<0.10$

$<0,10$

$<0.10$

$<0.10$

$<0.10$

$\mathrm{mg} /$

$\mathrm{mg} / \mathrm{L}$

$\mathrm{mg} / \mathrm{L}$

ㄸg $/$ L

$\mathrm{mg} / \mathrm{L}$

$<0.10$

$<0.10$

$<0.10$

$<0.10$

$<0.10$

$<0.10$

$\mathrm{mg} / \mathrm{L}$

$\mathrm{mg} / \mathrm{L}$

$\mathrm{mg} / \mathrm{L}$

$\mathrm{mg} / \mathrm{L}$

mg/L

$<0.10 \mathrm{mg} / \mathrm{L}$

$<0.10 \mathrm{mg} / \mathrm{L}$

$<0.10$

$\mathrm{mg} / \mathrm{L}$

$<0.10$

mg/L

$<0.10$

$\mathrm{mg} / \mathrm{L}$

$<0.10$

$\mathrm{mg} / \mathrm{L}$ 
Storm Drain Dats for SD -100

Location Date Test Compound Results Units

SD-100-04 11-Jun-1987 COBALT

SD-100-04 18-Jun-1987 GOBALT

** SD-100-06

SD-100-06 12-May-1987 COBALT

SD-100-06 18-May-1987 COBALT

SD-100-06 26-Kay-1987 COBALT

SD-100-06 3-Jun-1987 COBALT

SD-100-06 11-Jun-1987 COBALT

SD-100-06 18-Jun-1987 CO\&ALT

** SD-100-06D

SD-100-06D 3-Jun-1987 COBALT

* SD-100-07

SD-100-07 12-May-1987 COBALT

SD-100-07 19-May-1987 COBALT

SD-100-07 26-May-1987 COBALT

SD-100-07 3-Jun-1987 COBALT

SD-100-07 11-Jun-1987 COBALT

SD-300-07 18-Jun-1987 COBALT

* SD-100-07D

SD-100-07D 18-Jun-1987 COBALT

* SD-100-09

SD-100-09 12-May-1987 COBALT

SD-100-09 1B-May-1987 COBALT

SD-100-09 26-May-1987 CORALT

SD-100-09 3-Jun-1987 COBALT

SD-100-09 11-Jun-1987 COBALT

* SD-100-10

SD-100-10 12-May-1987 COBALT

SD-100-10 19-Kay-1987 COBALT

SD-100-10 26-May-1987 COBALT

SD-100-10 3-Jun-1987 COBALT

SD-100-10 11-Jun-1987 COBALT

SD-100-10 18-Jun-1987 COBALT

* SD-100-11

SD-100-11 12-May-1987 COBALT

SD-100-11 19-May-1987 COBALT

SD-100-11 26-Kay-1987 COBALT

SD-100-11 3-Jun-1987 COBALT

SD-100-11 11-Jun-1987 COBALT

SD-100-11 18-Jun-1987 COBALT

$\star \star \quad S D-100-12$

SD-100-12 12-May-1987 CORALT

SD-100-12 19-May-1987 COBALT

$\begin{array}{ll}<0.10 & \mathrm{mg} / \mathrm{L} \\ <0.10 & \mathrm{mg} / \mathrm{L} \\ & \\ <0.10 & \mathrm{mg} / \mathrm{L} \\ <0.10 & \mathrm{mg} / \mathrm{L} \\ <0.10 & \mathrm{mg} / \mathrm{L} \\ <0.10 & \mathrm{mg} / \mathrm{L} \\ <0.10 & \mathrm{mg} / \mathrm{L} \\ <0.10 & \mathrm{mg} / \mathrm{L}\end{array}$

$<0.10 \mathrm{mg} / \mathrm{L}$

$<0.10 \mathrm{mg} / \mathrm{L}$

$<0.10 \mathrm{mg} / \mathrm{L}$

$<0.10 \mathrm{mg} / \mathrm{L}$

$<0.10 \quad \mathrm{mg} / \mathrm{L}$

$<0.10 \mathrm{mg} / \mathrm{L}$

$<0.10 \mathrm{mg} / \mathrm{L}$

$<0.10 \mathrm{mg} / \mathrm{L}$

$<0.10 \quad \mathrm{ng} / \mathrm{L}$

$<0.10 \quad \mathrm{gg} / \mathrm{L}$

$<0.10 \mathrm{mg} / \mathrm{L}$

$<0.10 \mathrm{mg} / \mathrm{L}$

$<0.10 \mathrm{mg} / \mathrm{L}$

$<0.10 \mathrm{mg} / \mathrm{L}$

$<0.10 \mathrm{mg} / \mathrm{L}$

$<0.10 \mathrm{mg} / \mathrm{L}$

$<0,10 \mathrm{mg} / \mathrm{L}$

$<0.10 \mathrm{mg} / \mathrm{L}$

$<0.10 \mathrm{mg} / \mathrm{L}$

$<0.10 \mathrm{mg} / \mathrm{L}$

$<0.10 \mathrm{ng} / \mathrm{L}$

$<0.10 \mathrm{mg} / \mathrm{L}$

$<0.10 \mathrm{mg} / \mathrm{L}$

$<0.10 \mathrm{mg} / \mathrm{L}$

$<0.10 \mathrm{mg} / \mathrm{L}$

$<0.10 \quad \mathrm{gg} / \mathrm{L}$

$<0.0050 \mathrm{ng} / \mathrm{L}$ 
Storm Drain Data for SD-100

Location

Date

Test Compound
Results Units

$\begin{array}{ll}<0.0050 & \mathrm{mg} / \mathrm{L} \\ <0.10 & \mathrm{mg} / \mathrm{L} \\ <0.10 & \mathrm{mg} / \mathrm{L} \\ <0.10 & \mathrm{mg} / \mathrm{L}\end{array}$

** SD-100-13

SD-100-13 12-May-1987 COBALT

SD-100-13 19-Kay-1987 COBALT

SD-100-13 26-May-1987 COBALT

SD-100-13 11-Jun-1987 COBAIT

SD-100-13 18-Jun-1987 COBALT

* SD-100-14

SD-100-14 12-May-1987 COBALT

SD-100-14 19-May-1987 COBAIT

SD-100-14 26-May-1987 COBALT

SD-100-14 3-Jum-1987 COBALT

SD-100-14 11-Jun-1987 COBALT

SD-100-14 I8-Jun-1987 COBALT

* SD-100-C

SD-100-C S-Mar-1987 COBALT

SD-100-C 9-Mar-1987 COBALT

SD-100-C 17-Mar-1987 COBALT

SD-100-C 24-Mar-1987 COBALT

SD-100-C 8-Apr-1987 COBALT

SD-100-C 13-May-1987 COBALT

SD-100-C 20-May-1987 CORALT

SD-100-C 27-May-1987 COBALT

SD-100-C 4-Jun-1987 COBALT

SD-100-C 12-Jun-1987 COBALT

SD-100-C 19-Jun-1987 COBALT

* SD-100-D

SD-100-D 6-APT-1987 COBALT

SD-100-D 11-Jun-1987 COBALT

$\star \star \quad S D-100$

SD-100 5-Mar-1987 COHDUCTIVITY

SD-100 11-Mar-1987 COADUCTIVITY

SD-100 18-Mar-1987 COLDUCTIVITY

SD-100 24-Mar-1987 COMDUCTIVITY

SD-100 3-Apr-1987 CONDOCTIVITY

SD-100 6-Apr-1987 CORDUCTIVITY

SD-100 12-May-1987 CONDUCTIVITY

SD-100 19-May-1987 CONDUCTIVITY

SD-100 26-May-1987 CONDUCTIVITY

SD-100 3-Jun-1987 CONDUCTIVITY

SD-100 11-Jun-1987 CONDUCTIVITY

SD-100 18-Jun-1987 CONDUCTIVITY
$<0.10$

$<0.0050$

$<0.0050$

$<0.10$

$<0.10$

$<0.10$

$<0.10$

$<0.0050$

$<0.10$

$<0.10$

$<0.10$

$<0.10$

$<0.10$

$<0.10$

$<0.10$

$<0.10$

$<0.10$

$<0.10$

$<0.10$

$<0.10$

$<0.10$

$<0.10$

$<0.10$

$<0.10$

281

246

266

260

262

268

299

295

299

301

318

278

\section{$\operatorname{mg} / L$ \\ $\mathrm{mg} / \mathrm{L}$ \\ $\mathrm{mg} / \mathrm{L}$ \\ mg/L \\ $\mathrm{mg} / \mathrm{L}$}

mg/L

$\mathrm{mg} / \mathrm{L}$

mg $/ \mathrm{L}$

mg/L

$\mathrm{mg} / \mathrm{h}$

世g/ $/ 2$

$\mathrm{mg} / \mathrm{L}$

$\mathrm{mg} / \mathrm{L}$

mg/L

$\mathrm{mg} / \mathrm{L}$

$\mathrm{mg} / \mathrm{L}$

$\mathrm{mg} / \mathrm{L}$

mg/L

$\mathrm{mg} / \mathrm{L}$

$\operatorname{mg} / L$

$\operatorname{mg} / L$

IIg $/ 2$

$\mathrm{mg} / \mathrm{L}$

$\mathrm{mg} / \mathrm{L}$

uaho/cm umho/cm unho/cm unho/cm umho/cm tomho/cm unho/ct umho/c⿴囗十 wnho/cm unho/ $\mathrm{cm}$ umho/cm umho/cul 
Location Date Test Compound Results Units

* SD-100-01

SD-100-01 12-Kay-1987 CONDUCTIVITY

SD-100-01 18-May-1987 CONDUCTIVITY

SD-100-01 26-May-1987 CONDUCTIVITY

SD-100-01 3-Jun-1987 CONDUCTIVITY

SD-100-01 11-Jun-1987 CONDUCTIVITY

SD-100-01 18-Jun-1987 CONDUCTIVITY

$\begin{array}{ll}158 & \text { umho/cm } \\ 154 & \text { unho/cm } \\ 190 & \text { umho/cm } \\ 207 & \text { unho/cm } \\ 343 & \text { umho/cm } \\ 210 & \text { unho/cm }\end{array}$

* SD - 100-02

SD-100-02 12-May-1987 CONDUCTIVITY

SD-100-02 18-May-1987 CONDUCTIVITY

SD-100-02 26-May-1987 CONDUCTIVITY

SD-100-02 3-Jun-1987 CONDUCTIVITY

SD-100-02 11-Jun-1987 CONDUCTIVITY

SD-100-02 18-Jun-1987 CONDUCTIVITY

umho/cu umho/cri unho/cm umho/cm unho/cm * SD-100-03

SD-100-03 12-May-1987 CONDUCTIVITY SD-100-03 18-May-1987 CONDUCTIVITY SD-100-03 26-May-1987 CONDUCTIVITY

SD-100-03 3-Jun-1987 CONDUCTIVITY

SD-100-03 11-Jun-1987 CONDUCTIVITY

SD-100-03 18-Jun-1987 CONDUCTIVITY

umho $/ \mathrm{cm}$

* SD-100-03D

SD-100-03D 12-May-1987 CONDUCTIVITY SD-100-03D 26-May-1987 CONDUCTIVITY

* SD-100-04

SD-100-04 12-May-1987 CONDUCTIVITY

SD-100-04 18-May-1987 CONDUCTIVITY

SD-100-04 26-May-1987 CONDUCTIVITY

SD-100-04 3-Jun-1987 CONDUCTIVITY

SD-100-04 11-Jun-1987 CONDUCTIVITY

SD-100-04 18-Jun-1987 CONDUCTIVITY

* SD-100-06

SD-100-06 12-May-1987 CONDUCTIVITY

SD-100-06 18-May-1987 CONDUCTIVITY

SD-100-06 26-May-1987 CONDUCTIVITY

SD-100-06 3-Jun-1987 CONDUCTIVITY

SD-100-06 11-Jun-1987 CONDUCTIVITY

SD-100-06 18-Jun-1987 CONDUCTIVITY

** SD-100-06D

SD-100-D6D 3-Jun-1987. CONDUCTIVITY

* SD-100-07

SD-100-07 12-May-1987 CONDUCTIVITY

SD-100-07 19-May-1987 CONDUCTIVITY

SD-100-07 26-May-1987 CONDUCTIVITY
302

umho/cm

unho/cm

380

206000

292

300

313

280

umho/cm unho/cm urho $/ \mathrm{cm}$ unho/cm umho/cm umho/cm

284

285

294

305

312

281

3970

1180

umho/cm unho/cm unho/cm umho/cm umho/cm unho/cm

254 umho/cm umho/cm

unho/cm umho/cm umho $/ \mathrm{cm}$ unho/cm umho/cm who $/ \mathrm{cm}$

umbo/cm umho/cm 
Location Date Test Compound Results Uafts

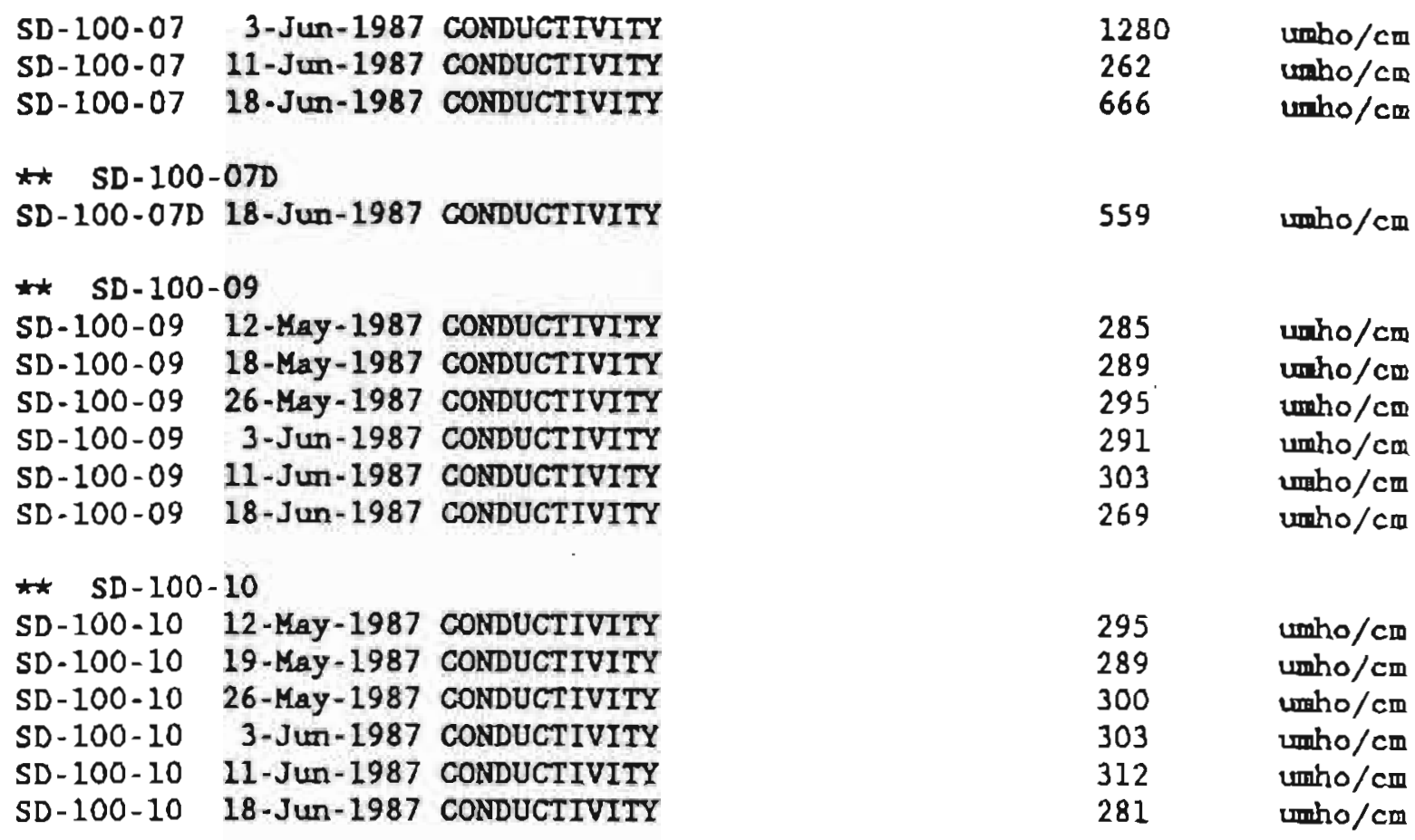

* SD $-100-11$

SD-100-11 12-May-1987 CONDUCTIVITY

SD-100-11 19-May-1987 CORDUCTIVITY

SD-100-11 26-May-1987 CONDUCTIVITY

SD-100-11 3-Jun-1987 CONDUCTIVITY

SD-100-11 11-Jun-1987 CONDUCTIVITY

SD-100-11 18-Jun-1987 CONDUCTIVITY

SD-100-12 19-May-1987 CONDUCTIVITY

SD-100-12 26-May-1987 CONDUCTIVITY

SD-100-12 3-Jun-1987 CONDUCTIVITY

unho/cm who $/ \mathrm{cm}$ umho/cm unho/cm umho/cm unho/cm

\section{unho/cm} wisho/cm unho/cm umho/cm umho/cm umho/cm

who/cm unho/cm umho/cm unho/cm umho/cm unho/cm

who/cm unho/cm unho/cm 
Location Date Test Compound Results Onits

$\begin{array}{lrl}\text { SD-100-14 } & \text { 3-Jun-1987 } & \text { CONDUCTIVITY } \\ \text { SD-100-14 } & 11 \text {-Jun-1987 CONDUCTIVITY } \\ \text { SD-100-14 } & 18-J u n-1987 & \text { CONDUCTIVITY }\end{array}$

** SD-100-C

SD-100-C 5-Mar-1987 CONDUCTIVITY

SD-100-C 9-Mar-1987 CONDUCTIVITY

SD-100-C 17-MaI-1987 CONDUCTIVITY

SD-100-C 24-Mar-1987 CONDUCTIVITY

SD-100-C 8-Apr-1987 CONDUCTIVITY

SD-100-C 13-May-1987 CONDUCTIVITY

SD-100-C 20-Kay-1987 CONDUCTIVITY

SD-100-C 27-May-1987 CONDUCTIVITY

SD-100-C 4-Jun-1987 CONDUCTIVITY

SD-100-C 12-Jun-1987 CONDUCTIVITY

SD-100-C 19-Jun-1987 CONDUCTIVITY

** SD-100-D

SD-100-D 6-Apr-1987 CONDUCTIVITY

SD-100-D 11-Jun-1987 CONDUCTIVITY

** SD -100

$\begin{array}{lrl}\text { SD-100 } & \text { 5-Mar-1987 } & \text { COPPER } \\ \text { SD-100 } & 11-\text { Mar-1987 } & \text { COPPER } \\ \text { SD-100 } & 18 \text {-Mar-1987 COPPER } \\ \text { SD-100 } & 24-\mathrm{Mar}-1987 & \text { COPPER } \\ \text { SD-100 } & \text { 3-Apr-1987 COPPER } \\ \text { SD-100 } & 6 \text {-Apr-1987 COPPER } \\ \text { SD-100 } & 12-\mathrm{May}-1987 & \text { COPPER } \\ \text { SD-100 } & 19-\mathrm{May}-1987 & \text { COPPER } \\ \text { SD-100 } & 26 \text {-May-1987 COPPER } \\ \text { SD-100 } & \text { 3-Jun-1987 COPPER } \\ \text { SD-100 } & 11-\mathrm{Jun}-1987 \text { COPPER } \\ \text { SD-100 } & 18-J u n-1987 & \text { COPPER }\end{array}$

** SD-100-01

SD-100-01 12-May-1987 COPPER

SD-100-01 18-May-1987 COPPER

SD-100-01 3-Jun-1987 COPPER

SD-100-01 11-Jun-1987 COPPER

SD-100-01 18-Jun-1987 COPPER

* SD-100-02

SD-100-02 12-May-1987 COPPER

SD-100-02 18-May-1987 COPPER

SD-100-02 3-Jun-1987 COPPER

SD-100-02 11-Jun-1987 COPPER

SD-100-02 18-Jun-1987 COPPER
317

312

281

254

225

258

311

276

296

300

281

301

291

289

266

318

0.0050

$<0.0040$

$<0.0040$

0.0090

0.0069

0.013

$<0.0040$

$<0.0040$

$<0.0040$

$<0.0040$

$<0.0040$

$<0.0040$

$<0.0040$

$<0.0040$

$<0.0040$

$<0.0040$

$<0.0040$

$\mathrm{mg} / \mathrm{L}$

$\mathrm{mg} / \mathrm{L}$

mg $/ \mathrm{L}$

$\mathrm{mg} / \mathrm{L}$

$\mathrm{mg} / \mathrm{L}$

$<0.0040$

$<0.0040$

$<0.0040$

$<0.0040$

$<0.0040$

$\operatorname{mg} / \mathrm{L}$

$\mathrm{mg} / \mathrm{L}$

$\mathrm{mg} / \mathrm{L}$

$\mathrm{mg} / \mathrm{L}$

$\operatorname{mg} / \mathrm{L}$

* SD-100-03

SD-100-03 12-May-1987 COPPER 


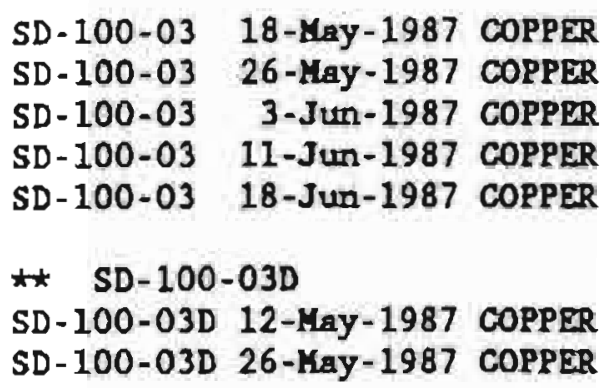

$$
\begin{array}{ll}
<0.0040 & \mathrm{mg} / \mathrm{L} \\
<0.0040 & \mathrm{mg} / \mathrm{L} \\
<0.0040 & \mathrm{mg} / \mathrm{L} \\
<0.0040 & \mathrm{mg} / \mathrm{L} \\
<0.0040 & \mathrm{mg} / \mathrm{L} \\
& \\
& \\
<0.0040 & \mathrm{mg} / \mathrm{L} \\
<0.0040 & \mathrm{mg} / \mathrm{L}
\end{array}
$$

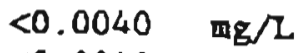

$<0.0040$

$<0.0040$

ㅁg $/ \mathrm{L}$ $<0.0040$ mg $/ \mathrm{L}$ 0.0040 $<0.0040$ $\mathrm{mg} / \mathrm{L}$ $\log / \mathrm{L}$ mg/ 1 $<0.0040 \mathrm{mg} / \mathrm{L}$

$<0.0040 \mathrm{mg} / \mathrm{L}$

$\begin{array}{ll}0.059 & \mathrm{mg} / \mathrm{L} \\ 0.022 & \mathrm{mg} / \mathrm{L} \\ <0.0040 & \mathrm{mg} / \mathrm{L} \\ 0.14 & \mathrm{mg} / \mathrm{L} \\ 0.012 & \mathrm{mg} / \mathrm{L} \\ 0.11 & \mathrm{mg} / \mathrm{L}\end{array}$

$0.099 \mathrm{mg} / \mathrm{L}$

$0.061 \mathrm{ng} / \mathrm{L}$ $0.043 \quad \mathrm{mg} / \mathrm{L}$ $<0.0040 \mathrm{mg} / \mathrm{L}$ $0.047 \mathrm{mg} / \mathrm{L}$ $0.013 \mathrm{mg} / \mathrm{L}$

$<0.0040 \mathrm{mg} / \mathrm{L}$ $<0.0040 \mathrm{mg} / \mathrm{L}$ $<0.0040 \mathrm{mg} / \mathrm{L}$ 
Storm Drain Data for SD-100

Location

Date

Test Compound

Results

Units

SD -100

12-May-1987 DISSOLVED OXYGEN

SD -100

19-May-1987 DISSOLVED OXYGEA

SD -100

26-May-1987 DISSOLVED OXYGEN

SD -100

3-Jun-1987 DISSOLVED OXYGEN

SD -100

11-Jun-1987 DISSOLVED OXYGEN

SD- 100

18-Jun-1987 DISSOLVED OXYGEN

* SD-100-01

SD-100-01 12-May-1987 DISSOLVED OXYGEN SD-100-01 18-May-1987 DISSOLVED OXYGEN SD-100-01 26-May-1987 DISSOLVED OXYGEN SD-100-01 3-Jun-1987 DISSOLVED OXYGEN SD-100-01 11-Jun-1987 DISSOLVED OXYGEN SD-100-01 18-JUn-1987 DISSOLVED OXYGEN

** SD-100-02

SD-100-02 12-May-1987 DISSOLVED OXYGEN SD-100-02 18-May-1987 DISSOLVED OXYGEN SD-100-02 26-Kay-1987 DISSOLVED OXYGEN SD-100-02 3-Jun-1987 DISSOLVED OXYGEN SD-100-02 11-Jun-1987 DISSOLVED OXYGEN SD-100-02 18-Jun-1987 DISSOLVED OXYGEN

* SD-100-03

SD-100-03 12-May-1987 DISSOLVED OXYGEN SD-100-03 18-May-1987 DISSOLVED OXYGEN SD-100-03 26-May-1987 DISSOLVED OXYGEN SD-100-03 3-Jun-1987 DISSOLVED OXYGEN SD-100-03 11-JUn-1987 DISSOLVED OXYGEN SD-100-03 18-Jun-1987 DISSOLVED OXYGEN

** SD-100-03D

SD-100-03D 12-May-1987 DISSOLVED OXYGEN SD-100-03D 26-May-1987 DISSOLVED OXYGEN

9.0 PP⿴

$8.6 \quad \mathrm{ppm}$

9.5 Ppm

$9.5 \quad \mathrm{ppm}$

$9.4 \quad \mathrm{ppm}$

8.9 Ppm

10.5 ppm

$8.5 \quad \mathrm{Ppm}$

7.5 ppm

$7.4 \quad$ ppm

8.6 PPm

$8.5 \quad \mathrm{PPI}$

10.5 Ppm

$7.5 \quad$ PPm

* SD-100-04

SD-100-04 12-May-1987 DISSOLVED OXYGEN SD-100-04 18-May-1987 DISSOLVED OXYGEN SD-100-04 26-May-1987 DISSOLVED OXYGEN SD-100-04 3-JUn-1987 DISSOLVED OXYGEN SD-100-04 11-Jun-1987 DISSOLVED OXYGEN SD-100-04 18-Jun-1987 DISSOLVED OXYGEN

$9.2 \quad \mathrm{ppm}$

$10.0 \quad$ PPW

7.6 рpu

8.0 Ppm

$8.5 \quad \mathrm{PPm}$

$8.5 \quad \mathrm{ppm}$

* SD-100-06

SD-100-06 12-May-1987 DISSOLVED OXYGEN SD-100-06 18-May-1987 DISSOLVED OXYGEN SD-100-06 26-May-1987 DISSOLVED OXYGEN SD-100-06 3-JUR-1987 DISSOLVED OXYGEN SD-100-06 11-JUn-1987 DISSOLVED OXYGEN SD-100-06 18-Jun-1987 DISSOLVED OXYGEN

$8.8 \quad \mathrm{ppm}$

$8.4 \quad \mathrm{ppm}$

$7.8 \quad$ PPm

$7.5 \quad$ PPm

$8.8 \quad$ PPm

9.2 PpI 
Stom Drain Dete for SD- 100

Location

Date

Test Compound

Results

Units

** SD-100-06D

SD-100-06D 3-Jun-1987 DISSOLVED OXYGEN

7.5

PPm

* SD-100-07

SD-100-07 12-MaY-1987 DISSOLVED OXYGEN

SD-100-07 19-Mry-1987 DISSOLVED OXYGEN

SD-100-07 26-May-1987 DISSOLVED OXYGEN

SD-100-07 3-Jun-1987 DISSOLVED OXYGEN

SD-100-07 11-Jun-1987 DISSOLVED OXYGEN

SD-100-07 18-Jun-1987 DISSOLVED OXYGEN

** SD-100-07D

SD-100-07D 18-JUn-1987 DISSOLVED OXYGEN

$5.7 \quad$ ppm

*t SD $-100-09$

SD-100-09 12-May-1987 DISSOLVED OXYGEN

SD-100-09 18-May-1987 DISSOLVED OXYGEN

SD-100-09 26-May-1987 DISSOLVED OXYGEN

SD-100-09 3-Jun-1987 DISSOLVED OXYGEN

SD-100-09 11-JUn-1987 DISSOLVED OXYGEN

SD-100-09 18-Jun-1987 DISSOLVED OXYGEN

** SD $-100-10$

SD-100-10 12-May-1987 DISSOLVED OXYGEN

SD-100-10 19-May-1987 DISSOLVED OXYGEN

SD-100-10 26-May-1987 DISSOLVED OXYGEN

SD-100-10 3-Jun-1987 DISSOLVED OXYGEN

SD-100-10 11-JUn-1987 DISSOLVED OXYGEN

SD-100-10 18-Jun-1987 DISSOLVED OXYGEN

$7.8 \quad$ ppm

$7.4 \quad \mathrm{PPI}$

$7.8 \quad \mathrm{ppm}$

$7.5 \quad$ Ppm

$5.6 \quad \mathrm{Ppm}$

$5.7 \quad \mathrm{Ppm}$

** SD-100-11

SD-100-11 12-May-1987 DISSOLVED OXYGEN

SD-100-11 19-May-1987 DISSOLVED OXYGEN

SD-100-11 26-May-1987 DISSOLVED OXYGEN

SD-100-11 3-Jun-1987 DISSOLVED OXYGEN

SD-100-11 11-JUR-1987 DISSOLVED OXYGEN

SD-100-11 18-Jun-1987 DISSOLVED OXYGEN

8.0 Ppm

10.0 PPO

$8.0 \quad \mathrm{ppm}$

$7.5 \quad \mathrm{ppm}$

$7.5 \quad \mathrm{PPM}$

8.5 ppm

$10.5 \quad \mathrm{Ppm}$

$8.4 \quad \mathrm{ppm}$

$9.0 \quad \mathrm{Ppm}$

$8.5 \quad \mathrm{Ppm}$

9.5 Ppm

9.4 PPI

** SD $-100-12$

SD-100-12 12-May-1987 DISSOLVED OXYGEN

SD-100-12 19-May-1987 DISSOLVED OXYGEN

SD-100-12 26-May-1987 DISSOLVED OXYGEN

SD-100-12 3-Jun-1987 DISSOLVED OXYGEN

SD-100-12 11-Jun-1987 DISSOLVED OXYGEN

SD-100-12 18-Jun-1987 DISSOLVED OXYGEN

8. 2 Ppm

7.8 PPm

6.8 ppm

6.7 Ppm

8.4 ppm

7.8 ppm

$\star$ ¿ SD $-100-13$

SD-100-13 12-May-1987 DISSOLVED OXYGEN

SD-100-13 19-May-1987 DISSOLVED OXYGEN

SD-100-13 26-May-1987 DISSOLVED OXYGEN

SD-100-13 3-Jun-1987 DISSOLVED OXYGEN

$\begin{array}{ll}6.0 & \mathrm{ppm}- \\ 6.4 & \mathrm{ppm} \\ 5.5 & \mathrm{ppm} \\ 15.0 & \mathrm{ppm} \\ 7.0 & \mathrm{ppm} \\ 6.6 & \mathrm{ppm}\end{array}$

$\begin{array}{ll}8.5 & \text { Pрس } \\ 8.0 & \text { Ppm } \\ 5.9 & \text { ppm } \\ 6.0 & \text { Ppm }\end{array}$


Storm Dra1n Deta for SD-100

\begin{tabular}{|c|c|c|c|}
\hline Location & Date & Test Compound & Results \\
\hline $\begin{array}{l}\text { SD }-100-13 \\
\text { SD-100-13 }\end{array}$ & $\begin{array}{l}11-J u n-1987 \\
18-J u n-1987\end{array}$ & $\begin{array}{l}\text { DISSOLVED OXYGEN } \\
\text { DISSOLVED OXYGEN }\end{array}$ & $\begin{array}{l}8.8 \\
8.4\end{array}$ \\
\hline \multicolumn{4}{|c|}{ * SD-100-14 } \\
\hline$S D-100-14$ & $12-$ May -1987 & DISSOLVED OXYGEN & 7.5 \\
\hline$S D-100-14$ & $19-$ May- 1987 & DISSOLVED OXYGEN & 9.0 \\
\hline SD-100-14 & $26-$ May -1987 & DISSOLVED OXYGEN & 9.2 \\
\hline SD $-100-14$ & 3-Jun-1987 & DISSOLVED OXYGEN & 7.0 \\
\hline SD-100-14 & $11-J u n-1987$ & DISSOLVED OXYGEN & 6.0 \\
\hline SD-100-14 & $18-J u n-1987$ & DISSOLVED OXYGEN & 8.3 \\
\hline
\end{tabular}

** SD-100-D

SD-100-D 6-APT-1987 DISSOLVED OXYGEN SD-100-D 11-Jun-1987 DISSOLVED OXYGEN

8.3 ppm

8.5 ppm

** SD $-100-09$

SD-100-09 12-May-1987 DODEGANOIC ACID

4

$u g / L$

** SD -100

SD-100 5-Mar-1987 ETHYLBENZENE

SD-100 11-Mar-1987 ETHYLB ENZENE

SD-100 18-Mar-1987 ETHYLBEN2ENE

SD-100 24-Mar-1987 ETHYLBENZENE

SD-100 6-Apr-1987 ETHYBBENZENE

SD-100 L2-May-1987 ETHYLBENZENE

SD-100 19-May-1987 ETHYLBENZENE

SD-100 26- Yay-1987 ETHYLBERZENE

SD-100 3-Jun-1987 ETHYLBERZENE

SD-100 11-Jun-1987 ETHY BENZENE

SD-100 18-Jun-1987 ETHYLB ENZENE

* SD-100-01

SD-100-01 12-Yay-1987 ETHYLBERZENE

SD-100-01 18-May-1987 ETHYLBENZENE

SD-100-01 26-May-1987 ETHYLBDERENE

SD-100-01 3-Jun-1987 ETHYTBEN2ENE

SD-100-01 11-JUn-1987 ETHYLBENZENE

SD-100-01 18-Jun-1987 ETHYLBENZENE

* SD $-100-02$

SD-100-02 12-May-1987 ETHYLB ENZENE

SD-100-02 18-May-1987 ETHYLBETZETE

SD-100-02 26-May-1987 ETHYLB BNZENE

SD-100-02 3-Jun-1987 ETHYLBENZENE

SD-100-02 11-Jun-1987 ETHYLBERENE

SD-100-02 18-Jun-1987 ETHYLBENZENE

$\begin{array}{ll}<5 & u g / L \\ <5 & u g / L \\ <5 & u g / L \\ <5 & u g / L \\ <5 & u g / L \\ <5 & u g / L \\ <5 & u g / L \\ <5 & u g / L \\ <5 & u g / L \\ <5 & u g / L \\ <5 & u g / L\end{array}$

** SD $-100-03$

SD-100-03 12-May-1987 ETHYLBEAYZNE

SD-100-03 18-May-1987 ETHYLBENZENE

SD-100-03 26-May-1987 ETHYLBENZENE

$<5$

$<5$

$<5$

$<5$

$<5$

$<5$

ug $\Omega$

ug $\Omega$

$\mathrm{ug} / \mathrm{L}$

ug $/ \mathrm{L}$

ug $/$

ug/L

$<5$

ug/

$<5 \quad \mathrm{ug} / \mathrm{L}$

$<5$ ug/L

$<5$ ug/L

$<5 \quad u g / l$

$<5 \quad 4 g / 2$

$<5$

$<5$

ug/L

$<5$

ug $/$

$4 \mathrm{~g} / \mathrm{L}$ 
Storw Drain Data for SD-100

Location

Date

Test Compound

Results Units

SD-100-03 3-Jun-1987 ETHYLBENZENE

SD-100-03 11-Jun-1987 ETHYLBENZENE

SD-100-03 18-Jun-1987 ETHYLBENZENE

* SD-100-03D

SD-100-03D 12-May-1987 ETHYLBENZENE

SD-100-03D 26-May-1987 EIFYLBENZENE

** SD-100-04

SD-100-04 12-May-1987 ETHYLBENZENE

SD-100-04 18-May-1987 ETHYLBENZENE

SD-100-04 26-May-1987 ETHYLBENZENE

SD-100-04 3-Jun-1987 ETHYLBENZENE

SD-100-04 11-JUn-1987 ETHYLBENZENE

SD-100-04 18-JUn-1987 ETHYLBENZENE

* SD-100-06

SD-100-06 12-May-1987 ETHYLBENZENE

SD-100-06 18-May-1987 ETHYLBENZENE

SD-100-06 26-May-1987 ETHYLBENZENE

SD-100-06 3-Jun-1987 ETHYLBENZENE

SD-100-06 11-Jun-1987 ETHYLBENZENE

SD-100-06 18-Jun-1987 ETHYLBENZENE

** SD-100-06D

SD-100-06D 3-Jun-1987 ETHYLBENZENE

** SD - 100-07

SD-100-07 12-May-1987 ETHYLBENZENE

SD-100-07 19-May-1987 ETHYLBENZENE

SD-100-07 26-May-1987 ETHYLBENZENE

SD-100-07 3-Jun-1987 ETHYLBENZENE

SD-100-07 11-Jun-1987 ETHYBENZENE

SD-100-07 18-Jun-1987 ETIYLBENZENE

** SD-100-07D

SD-100-07D 18-Jun-1987 ETHYLBENZENE
$<5$

$<5$

$<5$

$<5$

$<5$

$<5$

$<5$

$<5$

$<5$

$<5$

$<5$

$<5$

$<5$

$<5$

$<5$

$<5$

$<5$

$<5$

$<5$

$<5$

5

$<5$

$<5$

$<5$

5

$<5$

$<5$

$<5$

$<5$

$<5$

$<5$

$<5$

$<5$

$<5$

$<5$
ug/L

ug/L

$4 \mathrm{~g} / 2$

ug/L

ug $/ 2$

ug $/ 2$

ug $/ \mathrm{L}$

ug/L

ug/L

ug/L

ug/L

ug/L

ug/L

ug/L

$u g / L$

ug/L

ug/L

ug/L

ug/L

ug/L

ug/L

ug/L

ug $/ \mathrm{L}$

$u g / L$

4g/2

$4 g / 2$

ug/ $/$

ug/L

ug/L

ug/L

ug/L

** SD $-100-10$

SD-100-10 12-May-1987 ETHYLBENZENE

SD-100-10 19-May-1987 ETHYLBENZENE

SD-100-10 26-May-1987 ETHYLBENZENE

SD-100-10 3-Jun-1987 ETHYLBENZENE
ug/L

$u g / L$

$u g / L$

ug/L 
Storm Drain Data for SD -100

Location Date Test Compound

Results Units

SD-100-10 11-Jun-1987 ETHYLBENZENE

SD-100-10 18-Jun-1987 ETHYLBEANZENE

$\begin{array}{ll}<5 & \text { ug/L } \\ <5 & \text { ug } / L\end{array}$

* SD-100-11

SD-100-11 12-May-1987 ETHYLERZENE

SD-100-11 19-May-1987 ETHYLBENZENE

SD-100-11 26-May-1987 ETHYLBENZENE

SD-100-11 3-Jun-1987 ETHYLBEMZENE

SD-100-11 11-Jun-1987 ETHYRBENZENE

SD-100-11 18-Jun-1987 ETHYLBENZENE

$<5$

$<5$

$<5$

$<5$

$<5$

$<5$

ug $/ \mathrm{L}$

** SD-100-12

SD-100-12 12-May-1987 ETHYLBENZENE

SD-100-12 19-May-1987 ETHYLBENZENE

SD-100-12 26-May-1987 ETHYLBENZENE

SD-100-12 3-Jun-1987 ETHYLBENZENE

SD-100-12 11-Jun-1987 ETHYLBENZENE

SD-100-12 18-Jun-1987 ETHYLBENZENE

ug/L

ug/L

$\mathrm{ug} / \mathrm{L}$

ug/L

ug $/ 2$

$u g / L$

* SD $-100-13$

SD-100-13 12-May-1987 ETHYLBENZENE

SD-100-13 19-May-1987 ETHYLBENZENE

SD-100-13 26-May-1987 ETHYBBENZENE

SD-100-13 3-JUH-1987 ETHYLBENZENE

SD-100-13 11-Jun-1987 ETHYLBENZENE

SD-100-13 18-Jun-1987 ETHYLBENZENE

$\begin{array}{ll}<5 & u g / L \\ <5 & u g / L \\ <5 & u g / L \\ <5 & u g / L \\ <5 & u g / L \\ <5 & u g / L \\ & \\ & \\ <5 & u g / L \\ <5 & u g / L \\ <5 & u g / L \\ <5 & u g / L \\ <5 & u g / L \\ <5 & u g / L\end{array}$

* SD $-100-14$

SD-100-14 12-May-1987 ETHYLBENZENE

SD-100-14 19-May-1987 ETHYLBENZENE

SD-100-14 26-May-1987 ETHYLBENZENE

SD-100-14 3-Jun-1987 ETHYLBENZENE

SD-100-14 11-JUn-1987 ETHYLBENZENE

SD-100-14 18-Jun-1987 ETHYLBENZENE

$\begin{array}{ll}<5 & \mathrm{ug} / \mathrm{L} \\ <5 & \mathrm{ug} / \mathrm{L} \\ <5 & \mathrm{ug} / \mathrm{L} \\ <5 & \mathrm{ug} / \mathrm{L} \\ <5 & \mathrm{ug} / \mathrm{L} \\ <5 & \mathrm{ug} / \mathrm{L}\end{array}$

* $\mathrm{SD}-100-\mathrm{C}$

SD-100-C 5-Mar-1987 ETHYLBENZENE

SD-100-C 9-Mar-1987 ETHYLBENZENE

SD-100-C 17-Mar-1987 ETHYLBENZENE

SD-100-C 24-Mar-1987 ETHYLBENENE

SD-100-C 8-ApT-1987 ETHYLBENZENE

SD-100-C 13-May-1987 ETHYLBENZENE

SD-100-C 20-May-1987 ETHYLBENZENE

SD-100-C 27-May-1987 ETHY BENZENE

SD-100-C 12-Jun-1987 ETHYLBENZENE

SD-100-C 19-Jun-1987 ETHYLBENZENE

$<5$

ug/L

$\mathrm{ug} / \mathrm{L}$

$\mathrm{ug} / \mathrm{L}$

$\mathrm{ug} / \mathrm{L}$

$4 \mathrm{~g} / \mathrm{L}$

ug $/ \mathrm{L}$

ug/L

$u g / L$.

$\begin{array}{ll}<5 & \text { ug } / \mathrm{L} \\ <5 & \text { ug } / \mathrm{L}\end{array}$

* SD-100-D

SD-100-D 6-Apr-1987 ETHYLBENZENE

SD-100-D 11-Jun-1987 ETHYLBENZENE

$<5$

$<5$

ug/L

$u_{G} / L$ 
Stom Draln Data for SD -100

\begin{tabular}{|c|c|c|c|c|}
\hline Location & Date & Test Compound & Resules & Units \\
\hline \multicolumn{5}{|l|}{$\star * \quad$ SD -100} \\
\hline SD-100 & 5-Mar-1987 & FLUORANTHENE & $<10$ & ug/L \\
\hline SD- 100 & 11-Mar-1987 & FLUORANTHENE & $<10$ & $\mathrm{ug} / \mathrm{L}$ \\
\hline SD- 100 & $12-$ May- 1987 & FLUORANITHENE & $<10$ & $\mathrm{ug} / \mathrm{L}$ \\
\hline SD -100 & 26-May-1987 & FUUORANITEENE & $<5.0$ & $\mathrm{ug} / \mathrm{L}$ \\
\hline \multicolumn{5}{|c|}{$\star *$ SD $-100-01$} \\
\hline SD-100-01 & 12-Kay-1987 & FLUORANTHENE & $<10$ & $\mathrm{ug} / \mathrm{L}$ \\
\hline SD-100-01 & $26-12 a y-1987$ & FUORANTHEANE & $<5.0$ & $\mathrm{ug} / \mathrm{L}$ \\
\hline \multicolumn{5}{|c|}{$\star \star$ SD $-100-02$} \\
\hline SD-100-02 & $12-$ May-1987 & FWORANTHENE & $<10$ & $u g / L$ \\
\hline SD $-100-02$ & $26-$ May-1987 & FUUORANTHENE & $<5.0$ & $\mathrm{ug} / \mathrm{L}$ \\
\hline \multicolumn{5}{|c|}{$\star \star$ SD $-100-03$} \\
\hline SD $-100-03$ & $12-$ Kay-1987 & FLUORANTHENE & $<10$ & $u g / L$ \\
\hline SD $-100-03$ & $26-\mathrm{May}-1987$ & FLUORANTKENE & $<5.0$ & $u_{g} / L$ \\
\hline \multicolumn{5}{|c|}{$*$ SD-100-03D } \\
\hline SD-100-03D & $12-$ May-1987 & FLUORANTHENE & $<10$ & ug/L \\
\hline$S D-100-03 D$ & $26-$ May -1987 & FUORANTHENE & $<5.0$ & $\mathrm{ug} / \mathrm{L}$ \\
\hline \multicolumn{5}{|c|}{$\star \star$ SD $-100-04$} \\
\hline$S D-100.04^{-}$ & $12-\mathrm{May}-1987$ & FUUORANTHENE & $<10$ & $4 g / L$ \\
\hline SD-100-04 & $26-$ May - 1987 & FUUORANTHENE & $<5.0$ & ug/L \\
\hline \multicolumn{5}{|c|}{$\star \star$ SD $-100-06$} \\
\hline SD $-100-06$ & $12-\mathrm{May}-1987$ & FUUORANTHETE & $<10$ & $u g / L$ \\
\hline SD $-100-06$ & $26-$ May -1987 & FLUORANTHENE & $<5.0$ & $\mathrm{ug} / \mathrm{L}$ \\
\hline \multicolumn{5}{|c|}{$\star * \quad$ SD $-100-07$} \\
\hline$S D-100-07$ & 12-May-1987 & FUORANTHENE & $0.7 \mathrm{~J}$ & $u g / L$ \\
\hline SD $-100-07$ & $26-$ May-1987 & FLUORANTHENE & $<5.0$ & $\mathrm{ug} / \mathrm{L}$ \\
\hline \multicolumn{5}{|c|}{$\star * \quad$ SD $-100-09$} \\
\hline SD-100-09 & 12-May - 1987 & FLUORANTHENE & $<10$ & ug/L \\
\hline SD-100-09 & $26-$ May -1987 & FLUORANTHENE & $<5.0$ & $u g / L$ \\
\hline \multicolumn{5}{|c|}{$\star * \quad S D-100-10$} \\
\hline SD- $100-10$ & 12-Kay-1987 & FLUORANTHENE & $<10$ & $4 \mathrm{~g} / \mathrm{L}$ \\
\hline SD $-100-10$ & $26-$ May -1987 & FLUORANTHENE & $<5.0$ & $u g / L$ \\
\hline \multicolumn{5}{|c|}{$\star \star \quad S D-100-11$} \\
\hline$S D-100-11$ & 12-May - 1987 & FUUORANTHENE & $<10$ & $u g / L$ \\
\hline$S D-100-11$ & 26-May-1987 & FLUORANTHENE & $<5.0$ & $\mathrm{ug} / \mathrm{L}$ \\
\hline \multicolumn{5}{|c|}{$\star \star \quad S D-100-12$} \\
\hline SD $-100-12$ & 12-May-1987 & FLUORANTHENE & $<10$ & $u g / L$ \\
\hline SD $-100-12$ & 26-May-1987 & FUUORANTHENE & $<5.0$ & $\mathrm{ug} / \mathrm{L}$ \\
\hline
\end{tabular}


Storm Drain Data for SD-100

Location

Date

Test Compound

Results

Units

* SD-100-13

SD-100-13 12-May-1987 FLUORANTHENE

SD-100-13 26-MAY-1987 FLUORAKTHENE

$<10$

$<5.0$

ug/L

** SD-100-14

SD-100-14 12-May-1987 FLUORANTHENE

SD-100-14 26-May-1987 FLORAMTHENE

$<10$

$<5.0$

$\mathrm{ug} / \mathrm{L}$

** SD-100-C

SD-100-C 5-Mar-1987 FLUORANTHENE

SD-100-C 9-Mar-1987 FLUORANTHENE

SD-100-C 17-Mar-1987 FLUORANTHENE

SD-100-C 24-Mar-1987 FLUORANTHENE

SD-100-C 8-APr-1987 FLUORANTHENE

SD-100-C 13-May-1987 FLUORANTHENE

$S D-100-C$

20-May-1987 FLUORANTHENE

$S D-100-C$

27-May-1987 FLDORANTHENE

SD $-100-C$

12-JUn-1987 FLUORANTHENE

SD $-100-C$

19-Jun-1987 FLUORANTHENE

$<10$

$<10$

$<10$

$<10$

$<10$

$<5.0$

$<5.0$

$<5.0$

$<5.0$

$<10$

ug/L

$\star$ SD -100

SD -100

5-Mat-1987 FLUORENE

SD -100

11-Mar-1987 FUUORENE

SD -100

12-May-1987 FLUORENE

SD -100

26-May-1987 FLUORENE

** SD-100-01

SD-100-01 12-May-1987 FLUORENE

SD-100-01 26-May-1987 FLUORENE

* SD - 100-02

SD-100-02 12-May-1987 FLUORENE

SD-100-02 26-May-1987 FLUORENE

* SD - 100-03

SD-100-03 12-May-1987 FLUORENE

SD-100-03 26-May-1987 FLUORENE

** SD-100-03D

SD-100-03D 12-May-1987 FLUORENE

SD-100-03D 26-May-1987 FWORENE

** SD-100-04

SD-100-04 12-May-1987 FUUORENE

SD-100-04 26-May-1987 FLUORENE

$<10$

$<10$

$<10$

$<5.0$

$\mathrm{ug} / \mathrm{L}$

* SD-100-06

SD-100-06 12-May-1987 FWORENE

SD-100-06 26-May-1987 FLUORENE

$<10 \quad$ ug/

$<5.0 \quad$ ug/L

$<10 \quad$ ug/L

$<5.0 \quad \mathrm{ug} / \mathrm{L}$

$<10 \quad$ ug $/ \mathrm{L}$

$<5.0 \quad$ ug $/ \mathrm{L}$

$<10 \quad$ ug/L

$<5.0 \quad \mathrm{ug} / \mathrm{L}$

$<10 \quad$ ug/L

$<5.0 \quad \mathrm{ug} / \mathrm{L}$

$<10 \quad u g / L$

$<5.0 \quad$ ug $/ \mathrm{L}$ 
Storm Drain Data for SD-100

Location Date Test Compound

Results Units

** SD $-100-07$

SD-100-07 12-Kay-1987 FLDORENE

SD-100-07 26-May-1987 FUORENE

** SD $-100-09$

SD-100-09 12-Kay-1987 FLUORERE

SD-100-09 26-Yay-1987 FLUORERE

** SD $-100-10$

SD-100-10 12-May-1987 FLUORENE

SD-100-10 26-Kay-1987 FLUORENE

** SD-100-11

SD-100-11 12-May-1987 FLUORENE

SD-100-11 26-Kay-1987 FLUORENE

* SD $-100-12$

SD-100-12 12-May-1987 FLUORENE

SD-100-12 26-May-1987 FLUORENE

* SD $-100-13$

SD-100-13 12-May-1987 FLUORENE

SD-100-13 26-May-1987 FLUORENE

$<10$

$<5.0 \quad \mathrm{ug} / \mathrm{L}$

ug $/ 2$

$<10$

$<5.0$

$\mathrm{ug} / \mathrm{L}$

ug/L

$<10$

$<5.0$

ug $/ \mathrm{L}$

ug/L

$<10 \quad$ ug/L

$<5.0 \quad u g / L$

$<10$

$<5.0 \quad \mathrm{ug} / \mathrm{L}$

** SD-100-14

SD-100-14 12-May-1987 FLUORENE

SD-100-14 26-May-1987 FLUORDNE

$<10$

$<5.0$

ug/L

ug/L

$<10 \quad$ ug $/ \mathrm{L}$

$<5.0 \quad$ ug/L

* $\quad$ SD-100-C

SO-100-C 5-KaY-1987 FLUORENE

SD-100-C 9-Mar-1987 FLUORENE

SD-100-C 17-Mar-1987 FLUORENE

SD-100-C 24-Kar-1987 FLUORENE

SD-100-C 8-Apr-1987 FLUORENE

SD-100-C 13-May-1987 FLJORENE

SD-100-C 20-May-1987 FLUORENE

SD-100-C 27-Kay-1987 FLUORENE

SD-100-C 12-JUn-1987 FLUORENE

SD-100-C 19-Jun-1987 FLUORENE

$<10 \quad \mathrm{ug} / \mathrm{l}$

$<10 \quad u g / L$

$<10$ ug/L

$<10 \quad$ ug/L

$<10 \quad$ ug/l

$<5.0 \quad \mathrm{ug} / \mathrm{L}$

$<5.0 \quad$ ug/L

$<5.0 \quad u g / L$

$<5.0 \quad u g / L$

$<10 \quad u g / L$

* SD -100

SD -100

5-Mar-1987 FUUORIDE

SD -100

11-KaT-1987 FLUORIDE

SD -100

18-MaY-1987 FUUORIDE

SD -100

24-Max-1987 FLUORIDE

SD -100

3-APT-1987 FLUORIDE

SD -100

6-API-1987 FLUORIDE

SD -100

12-May-1987 FLUORIDE

SD -100

19-May-1987 FUURIDE

SD -100

26-Kay-1987 FLUORIDE

SD -100

3-Jun-1987 FLUORIDE

$0.1 \quad \mathrm{gg} / \mathrm{L}$

$<0.1 \quad \mathrm{ag} / \mathrm{L}$

$<1.0 \quad \mathrm{gg} / \mathrm{L}$

$0.1 \quad \mathrm{ng} / \mathrm{L}$

$0.4 \mathrm{mg} / \mathrm{L}$

$0.1 \mathrm{mg} / \mathrm{L}$

$<0.1 \quad \mathrm{mg} / 1$

$<0.1 \quad \mathrm{mg} / \mathrm{L}$

$<0.1 \quad \mathrm{mg} / \mathrm{L}$

$<0.1 \quad \mathrm{mg} / \mathrm{L}$ 
Storm Drain Deta for SD-100

Location

Date

Test Compound

Results

Units

SD -100

11-Jun-1987 FLUORIDE

SD -100

18-Jun-1987 FLUORIDE

$<0.1$

$<0.1$

$\mathrm{mg} / \mathrm{L}$

त* SD-100-01

SD-100-01 12-May-1987 FLUORIDE

SD-100-01 18-May-1987 FLUORIDE

SD-100-01 26-May-1987 FLUORIDE

SD-100-01 3-Jun-1987 FLUORIDE

SD-100-01 11-Jun-1987 FLOORIDE

SD-100-01 18-Jun-1987 FLUORIDE

$0.2 \mathrm{mg} / \mathrm{L}$

$0.1 \mathrm{mg} / \mathrm{L}$

$0.2 \mathrm{mg} / \mathrm{L}$

$0.1 \mathrm{mg} / \mathrm{L}$

$<0.1 \quad \mathrm{mg} / \mathrm{L}$

$0.1 \mathrm{mg} / \mathrm{L}$

* SD-100-02

SD-100-02 12-Mey-1987 FLUORIDE

SD-100-02 18-May-1987 FLUORIDE

SD-100-02 26-May-1987 FLUORIDE

SD-100-02 3-JUn-1987 FLUORIDE

SD-100-02 11-Jun-1987 FLUORIDE

SD-100-02 18-Jun-1987 FUORIDE

$0.1 \quad \mathrm{mg} / \mathrm{L}$

$<0.1 \mathrm{mg} / \mathrm{L}$

$0.1 \mathrm{mg} / \mathrm{L}$

$<0.1 \quad \mathrm{mg} / \mathrm{L}$

$<0.1 \quad \mathrm{mg} / \mathrm{L}$

$<0.1 \mathrm{mg} / \mathrm{L}$

** SD $-100-03$

SD-100-03 12-May-1987 FLUORIDE

SD-100-03 18-May-1987 FLUORIDE

SD-100-03 26-May-1987 FLUORIDE

SD-100-03 3-Jun-1987 FLUORIDE

SD-100-03 11-Jun-1987 FLUORIDE

SD-100-03 18-Jun-1987 FLUORIDE

$0.1 \mathrm{mg} / \mathrm{L}$

$<0.1 \quad \mathrm{mg} / \mathrm{L}$

$<0.1 \quad \mathrm{mg} / \mathrm{L}$

$<0.1 \quad \mathrm{mg} / \mathrm{L}$

$<0.1 \quad \mathrm{mg} / \mathrm{L}$

$<0.1 \mathrm{mg} / \mathrm{L}$

* SD-100-03D

SD-100-03D 12-May-1987 FLUORIDE

SD-100-03D 26-May-1987 FLUORIDE

$0.1 \mathrm{mg} / \mathrm{L}$

$0.1 \mathrm{mg} / \mathrm{L}$

* SD-100-04

SD-100-04 12-May-1987 FLUORIDE

SD-100-04 18-May-1987 FLUORIDE

SD-100-04 26-May-1987 FLUORIDE

SD-100-04 3-Jun-1987 FLUORIDE

SD-100-04 11-Jun-1987 FLUORIDE

SD-100-04 18-Jun-1987 FLUORIDE

$0.1 \mathrm{mg} / \mathrm{L}$

$<0.1 \quad \mathrm{mg} / \mathrm{L}$

$<0.1 \mathrm{mg} / \mathrm{L}$

$<0.1 \quad \mathrm{mg} / \mathrm{L}$

$<0.1 \quad \mathrm{ng} / \mathrm{L}$

$<0.1 \mathrm{mg} / \mathrm{L}$

* SD-100-06

SD-100-06 12-May-1987 FLUORIDE

SD-100-06 18-May-1987 FUORIDE

SD-100-06 26-May-1987 FLOORIDE

SD-100-06 3-Jun-1987 FLUORIDE

SD-100-06 11-JUn-1987 FLUORIDE

SD-100-06 18-Jun-1987 FLUORIDE

$0.1 \mathrm{mg} / \mathrm{L}$

$<0.1 \mathrm{mg} / \mathrm{L}$

$<0.1 \mathrm{mg} / \mathrm{L}$

$x$

$<0.1 \quad \mathrm{mg} / \mathrm{L}$

$<0.1 \quad \mathrm{mg} / \mathrm{L}$

** SD-100-06D

SD-100-06D 3-Jun-1987 FLUORIDE

$<0.1 \quad \mathrm{mg} / \mathrm{L}$ 
Storm Drain Data for SD-100

Location

Date

Test Coupound

Results

Units

* $\quad$ SD $-100-07$

SD-100-07 12-May-1987 FLUORIDE

SD-100-07 19-Kay-1987 FUORIDE

SD-100-07 26-May-1987 FLUORIDE

SD-100-07 3-Jun-1987 FLUORIDE

SD-100-07 11-Jun-1987 FLUORIDE

SD-100-07 18-Jun-1987 FLURIDE

* SD-100-07D

SD-100-07D 18-Jun-1987 FLUORIDE

** SD $-100-09$

SD-100-09 12-May-1987 FLUORIDE

SD-100-09 18-May-1987 FUUORIDE

SD-100-09 26-Kay-1987 FUORIDE

SD-100-09 3-Jun-1987 FLUORIDE

SD-100-09 11-Jun-1987 FLUORIDE

SD-100-09 18-Jun-1987 FLUORIDE

** SD-100-10

SD-100-10 12-May-1987 FUUORIDE

SD-100-10 19-May-1987 FUUORIDE

SD-100-10 26-May-1987 FLUORIDE

SD-100-10 3-JUn-1987 FLUORIDE

SD-100-10 11-Jun-1987 FLUORIDE

SD-100-10 18-Jun-1987 FLUORIDE

* $\$$ SD $-100-11$

SD-100-11 12-May-1987 FLUORIDE

SD-100-11 19-May-1987 FLUORIDE

SD-100-11 26-May-1987 FLUORIDE

SD-100-11 3-Jun-1987 FWORIDE

SD-100-11 11-Jun-1987 FLUORIDE

SD-100-11 18-Jun-1987 FLUORIDE

\# SD-100-12

SD-100-12 12-May-1987 FUUORIDE

SD-100-12 19-May-1987 FLUORIDE

SD-100-12 26-May-1987 FLUORIDE

SD-100-12 3-Jun-1987 FLUORIDE

SD-100-12 11-Jun-1987 FUUORIDE

SD-100-12 18-Jun-1987 FUORIDE

$0.1 \quad \mathrm{mg} / \mathrm{L}$

$0.1 \quad \mathrm{ng} / \mathrm{L}$

$<0.1 \quad \mathrm{mg} / \mathrm{L}$

$0.1 \mathrm{mg} / \mathrm{L}$

$0.3 \mathrm{mg} / \mathrm{L}$

$0.4 \quad \mathrm{mg} / 2$

$0.4 \quad \mathrm{mg} / \mathrm{L}$

$0.1 \mathrm{mg} / \mathrm{L}$

$0.1 \quad \mathrm{mg} / \mathrm{L}$

$<0.1 \quad \mathrm{mg} / \mathrm{L}$

$<0.1 \quad \mathrm{mg} / \mathrm{L}$

$0.1 \mathrm{mg} / \mathrm{L}$

$<0.1 \quad \mathrm{mg} / \mathrm{L}$

$<0.1 \quad \mathrm{mg} / \mathrm{L}$

$<0.1 \quad \mathrm{ng} / \mathrm{L}$

$<0.1 \quad \mathrm{mg} / \mathrm{l}$

$<0.1 \quad \mathrm{mg} / \mathrm{L}$

$<0.1 \quad \mathrm{mg} / \mathrm{L}$

$<0.1 \quad \mathrm{mg} / \mathrm{L}$

$<0.1 \mathrm{mg} / \mathrm{L}$

$<0.1 \quad \mathrm{mg} / \mathrm{L}$

$<0.1 \quad \mathrm{~g} / \mathrm{L}$

$<0.1 \quad \mathrm{mg} / \mathrm{L}$

$<0.1 \mathrm{ng} / \mathrm{L}$

$<0.1 \quad \mathrm{gg} / \mathrm{L}$

$<0.1 \quad \mathrm{gg} / \mathrm{L}$

$<0.1 \quad \mathrm{mg} / \mathrm{L}$

$<0.1 \quad$ ng $/ \mathrm{L}$

$<0.1 \quad \mathrm{gg} / \mathrm{L}$

$<0.1 \quad \mathrm{gg} / \mathrm{L}$

$0.1 \quad \mathbf{g} / \mathrm{L}$

** SD-100-13

SD-100-13 12-May-1987 FLUORIDE

SD-100-13 19-May-1987 FLUORIDE

SD-100-13 26-May-1987 FLUORIDE

SD-100-13 3-Jun-1987 FLUORIDE

SD-100-13 11-Jun-1987 FLUORIDE

SD-100-13 18-Jun-1987 FLUORIDE

$<0.1 \quad \mathrm{gg} / \mathrm{L}$

$<0.1 \quad \mathrm{mg} / \mathrm{L}$

$<0.1 \quad$ ag/L

$<0.1 \quad \mathrm{ng} / \mathrm{L}$

$<0.1 \quad \mathrm{gg} / \mathrm{L}$

$0.2 \quad \mathrm{E} / \mathrm{L}$ 
Location Date Test Compound

Results Units

** SD-100-14

$\begin{array}{lrl}\text { SD-100-14 } & 12-\mathrm{May}-1987 & \text { FUUORIDE } \\ \text { SD-100-14 } & 19-\mathrm{May}-1987 & \text { FUUORIDE } \\ \text { SD-100-14 } & 26-\mathrm{May}-1987 & \text { FLUORIDE } \\ \text { SD-100-14 } & \text { 3-Jun-1987 } & \text { FUORIDE } \\ \text { SD-100-14 } & 11-\mathrm{Jun}-1987 & \text { FUUORIDE } \\ \text { SD-100-14 } & 18-J u n-1987 & \text { FUUORIDE }\end{array}$

$<0.1 \quad \mathrm{mg} / \mathrm{L}$

$<0.1 \quad \mathrm{mg} / \mathrm{L}$

$<0.1 \quad \mathrm{mg} / \mathrm{L}$

$<0.1 \quad \mathrm{mg} / \mathrm{L}$

$<0.1 \quad \mathrm{mg} / \mathrm{L}$

$<0.1 \quad \mathrm{mg} / \mathrm{L}$

* $\quad S D-100-C$

SD-100-C 5-Mar-1987 FLOORIDE

$0.1 \mathrm{mg} / \mathrm{L}$

SD-100-C 9-Mar-1987 FLUORIDE

0.1

0.1

$\mathrm{mg} / \mathrm{L}$

SD-100-C

17-Mar-1987 FLUORIDE

SD-100-C 24-MaY-1987 FLOORIDE

SD-100-C 8-Apr-1987 FLUORIDE

SD-100-C 13-May-1987 FLUORIDE

SD-100-C 20-May-1987 FLUORIDE

SD-100-C 27-May-1987 FLUORIDE

SD-100-C 4-Jun-1987 FLUORIDE

SD-100-C 12-Jun-1987 FLUORIDE

SD-100-C 19-Jun-1987 FLUORIDE

0.1

$\mathrm{mg} / \mathrm{L}$

0.2

$<0.1$

$<0.1$

0.1

$<0.1$

$<0.1$

$<0.1$

$\mathrm{mg} / \mathrm{L}$

$\mathbf{m g} / \mathrm{L}$

$\mathrm{mg} / \mathrm{L}$

** SD-100-D

SD-100-D 6-Apr-1987 FLUORIDE

0.2

$\mathrm{mg} / \mathrm{L}$

$\mathrm{mg} / \mathrm{L}$

$\mathrm{mg} / \mathrm{L}$

$\mathrm{mg} / \mathrm{L}$

$m g / L$

SD-100-D 11-Jun-1987 FLUORIDE

$<0.1$

$\mathrm{mg} / \mathrm{L}$

* SD -100

$\mathrm{mg} / \mathrm{L}$

SD-100 5-Mar-1987 HARDHESS

SD $-100 \quad 11$-Mar -1987 HARDNESS

124

130

18-MaY - 1987 HARDNESS

SD -100

24-Kar - 1987 HARDNESS

SD -100

3-Apr-1987 HARDNESS

SD -100

6-APr - 1987 HARDNESS

SD -100

12-MaY-1987 HARDNESS

SD -100

19-Mry-1987 HARDNESS

SD-100 26-May-1987 HARDNESS

SD-100 3-Jun-1987 HARDNESS

SD-100 11-Jun-1987 HARDNESS

SD-100 18-JUn-1987 HARDNESS

144

118

118

125

126

133

586

128

132

** SD-100-01

SD-100-01 12-May-1987 HARDNESS

SD-100-01 18-May-1987 HARDNESS

SD-100-01 26-May-1987 MARDNESS

SD-100-01 3-Jun-1987 HARDNESS

SD-100-01 11-JUn-1987 HARDNESS

SD-100-01 18-Jun-1987 HARDNESS
70

90

85

112

150

106

126

133

127 $\operatorname{mg} / \mathrm{L}$

$\mathrm{mg} / \mathrm{L}$

$\mathrm{mg} / \mathrm{L}$

mg/L

$\mathrm{mg} / \mathrm{L}$

$m g / L$

mg/L

$\mathrm{mg} / \mathrm{L}$

$\mathrm{mg} / \mathrm{L}$

$\mathrm{mg} / \mathrm{L}$

$\mathrm{mg} / \mathrm{L}$

$\mathrm{mg} / \mathrm{L}$

* $S D-100.02$

SD-100-02 12-May-1987 HARDNESS

SD-100-02 18-Mzy-1987 HARDNESS

SD-100-02 26-May-1987 HARDNESS 
Stora Drain Data for SD-100

Locaclon

Date

Test Compound

Results Units

SD-100-02 3-Jun-1987 HARDNESS

SD-100-02 11-Jun-1987 HARDNESS

SD-100-02 18-Jun-1987 HARDNESS

136

130

133

** SD-100-03

SD-100-03 12-May-1987 HARDNESS

SD-100-03 18-Kay-1987 HARDNESS

SD-100-03 26-May-1987 HARDNESS

SD-100-03 3-Jun-1987 HARDNESS

SD-100-03 11-Jun-1987 HARDNESS

SD-100-03 18-Jun-1987 HARDNESS

** SD-100-03D

SD-100-O3D 12-Kay-1987 HARDNESS

SD-100-03D 26-May-1987 HARDNESS

** SD $-100-04$

SD-100-04 12-May-1987 HARDNESS

SD-100-04 18-May-1987 HARDNESS

SD-100-04 26-MaY-1987 HARDNESS

SD-100-04 3-Jun-1987 HARDNESS

SD-100-04 11-Jun-1987 HARDNESS

SD-100-04 18-Jun-1987 HARDNESS

** SD-100-06

SD-100-06 12-May-1987 HARDNESS

SD-100-06 18-May-1987 HARDNESS

SD-100-06 26-Kay-1987 HARDNESS

SD-100-06 3-Jun-1987 HARDNESS

SD-100-06 11-Jun-1987 HARDNESS

SD-100-06 18-JUn-1987 HARDNESS

* SD-100-06D

SD-100-06D 3-Jun-1987 HARDNESS

** SD-100-07

SD-100-07 12-May-1987 HARDNESS

SD-100-07 19-May-1987 HARDNESS

SD-100-07 26-May-1987 HARDNESS

SD-100-07 3-Jun-1987 HARDNESS

SD-100-07 11-Jun-1987 HARDNESS

SD-100-07 18-Jun-1987 BARDNESS

** SD-200-07D

SD-100-07D 18-JUn-1987 HARDNESS

* SD-100-09

SD-100-09 12-May-1987 HARDNESS

SD-100-09 18-May-1987 HARDNESS

SD-100-09 26-May-1987 LARDNESS

SD-100-09 3-Jun-1987 HARDNESS
127

127

130

146

125

130

130

118

127

130

128

130

130

130

127

131

126

134

130

128

137

$\mathrm{mg} / \mathrm{L}$

137

100

114

102

71

48

45

$\mathrm{mg} / \mathrm{L}$ $\mathrm{mg} / \mathrm{L}$

$\mathrm{ng} / \mathrm{L}$

$\mathrm{mg} / \mathrm{L}$

ng/L

ng/L

ng/L

$\mathrm{mg} / \mathrm{L}$

$\mathrm{mg} / \mathrm{L}$

$\mathrm{mg} / \mathrm{L}$

mg/L

mg/L

Ing $/ L$

$\operatorname{mg} / \mathrm{L}$

$\mathrm{mg} / \mathrm{L}$

mg/L

$m g / L$

wg/L $\mathrm{mg} / \mathrm{L}$

$\mathrm{mg} / \mathrm{L}$

$\mathrm{mg} / \mathrm{L}$

IIg/L

$\mathrm{mg} / \mathrm{L}$

$\mathrm{mg} / \mathrm{L}$

ng/L

$\operatorname{ng} / \mathrm{L}$

$\mathrm{mg} / \mathrm{L}$

ng/L

mg $/ L$

$\mathrm{g} / \mathrm{L}$

ng $/ L$

$\mathrm{mg} / \mathrm{L}$

$\mathrm{mg} / \mathrm{L}$ 
Storm Drain Data for SD-100

Results Units

SD-100-09 11-JUn-1987 HARDNESS

SD-100-09 18-Jun-1987 HARDNESS

$123 \mathrm{mg} / \mathrm{L}$

$124 \mathrm{mg} / \mathrm{L}$

* SD $-100-10$

SD-100-10 12-May-1987 HARDNESS

SD-100-10 19-Kay-1987 HARDNESS

SD-100-10 26-May-1987 KARDNESS

SD-100-10 3-Jun-1987 HARDNESS

SD-100-10 11-JUm-1987 MARDNESS

SD-100-10 18-Jun-1987 HARDNBSS

$143 \mathrm{mg} / \mathrm{L}$

$131 \mathrm{mg} / \mathrm{L}$

$129 \mathrm{mg} / \mathrm{L}$

$131 \mathrm{mg} / \mathrm{L}$

$125 \mathrm{mg} / \mathrm{L}$

$128 \mathrm{mg} / \mathrm{L}$

** SD $-100-11$

SD-100-11 12-May-1987 HARDNESS

SD-100-11 19-May-1987 HARDNBSS

SD-100-11 26-May-1987 HARDNESS

SD-100-11 3-Jun-1987 HARDNESS

SD-100-11 11-Jun-1987 HARDNESS

SD-100-11 18-Jun-1987 HARDNESS

171

133

139

149

135

138

** SD $-100-12$

SD-100-12 12-Kay-1987 HARDNESS

SD-100-12 19-May-1987 HARDNESS

SD-100-12 26-May-1987 HARDNESS

129

133

SD-100-12 3-Jun-1987 HARDNESS

353

SD-100-12 11-Jun-1987 HARDNESS

129

SD-100-12 18-JUn-1987 HARDNESS

130

$\mathrm{mg} / \mathrm{L}$

$\mathrm{mg} / \mathrm{L}$

$\mathrm{mg} / \mathrm{L}$

ㅍg/L

$\mathrm{mg} / \mathrm{L}$

mg/L

** SD $-100-13$

SD-100-13 12-May-1987 HARDNESS

SD-100-13 19-May-1987 HARDNESS

SD-100-13 26-May-1987 HARDNESS

SD-100-13 3-Jun-1987 HARDNESS

SD $-100-13$ 11-JUn-1987 HARDNESS

SD-100-13 18-JUn-1987 HARDNESS

$\begin{array}{ll}132 & \mathrm{mg} / \mathrm{L} \\ 127 & \mathrm{mg} / \mathrm{L} \\ 131 & \mathrm{mg} / \mathrm{L} \\ 136 & \mathrm{mg} / \mathrm{L} \\ 123 & \mathrm{mg} / \mathrm{L} \\ 125 & \mathrm{mg} / \mathrm{L}\end{array}$

* SD $-100-14$

SD-100-14 12-May-1987 HARDNESS

SD-100-14 19-May-1987 MARDNESS

SD-100-14 26-May-1987 HARDNESS

SD-100-14 3-JUn-1987 HARDNESS

SD-100-14 11-Jun-1987 HARDNESS

SD-100-14 18-Jun-1987 HARDNESS

171

133

134

135

123

127

$\operatorname{mg} / \mathrm{L}$

$\mathrm{mg} / \mathrm{L}$

$\mathrm{mg} / \mathrm{L}$

$\mathrm{mg} / \mathrm{L}$

$\mathrm{mg} / \mathrm{L}$

$\operatorname{mg} / \mathrm{L}$

** SD-100-C

SD-100-C 5-Mar-1987 HARDNESS

SD-100-C 9-Mar-1987 HARDNESS

SD-100-C 17-Mar-1987 HARDNESS

SD-200-C 24-Mar-1987 HARDNESS

SD-100-C 8-APT-1987 HARONESS

SD-100-C 13-May-1987 HARDNESS

SD-100-C 20-May-1987 HARDNESS

$\begin{array}{ll}134 & \mathrm{mg} / \mathrm{L} \\ 108 & \mathrm{mg} / \mathrm{L} \\ 130 & \mathrm{mg} / \mathrm{L} \\ 170 & \mathrm{mg} / \mathrm{L} \\ 119 & \mathrm{mg} / \mathrm{L} \\ 129 & \mathrm{mg} / \mathrm{L} \\ 125 & \mathrm{mg} / \mathrm{L}\end{array}$


Storm Drain Deta for SD-100

Location

Date

Test Compound

Results

Units

SD-100-C

27-MAY-1987 HARDNESS

SD $-100-C$

4-Jun-1987 HARDNESS

SD-100-C

12-JUn-1987 HARDNESS

SD-100-C 19-Jun-1987 HARDNESS

130

175

133

mg $/ \mathrm{L}$

$\mathrm{mg} / \mathrm{L}$

$\mathrm{mg} / \mathrm{L}$

$\mathrm{mg} / \mathrm{L}$

* SD-100-D

SD-100-D 6-Apr-1987 HARDNESS

119

127

$\mathrm{mg} / \mathrm{L}$

SD-100-D 11-Jun-1987 HARDNESS

$\mathrm{mg} / \mathrm{L}$

** SD-100

SD-100 5-Mar-1987 HEXACHLOROBENZENE

SD-100 11-Mar-1987 HEXACHLOROBENZENE

SD-100 12-May-1987 HEXACHLOROBENZENE

SD-100 26-May-1987 HEXACHLOROBENZENE

** SD-100-01

SD-100-01 12-May-1987 HEXACHLOROBENZENE

SD-100-01 26-May-1987 HEXACHLOROBENZENE

* SD $-100-02$

SD-100-02 12-May-1987 HEXACHLOROBENZENE SD-100-02 26-May-1987 HEXACHLOROBENZENE

* SD-100-03

SD-100-03 12-May-1987 HEXACHLOROBENZENE

SD-100-03 26-May-1987 HEXACHLOROBENZENE

* SD-100-03D

SD-100-03D 12-May-1987 HEXACHLOROBENZENE

SD-100-03D 26-May-1987 HEXACHLOROBENZENE

* SD-100-04

SD-100-04 12-May-1987 HEXACHLOROBENZENE

SD-100-04 26-May-1987 HEXACHLOROBENZENE

** SD $-100-06$

SD-100-06 12-May-1987 HEXACHLOROBENZENE SD-100-06 26-May-1987 HEXACHLOROBENZENE

** SD-100-07

SD-100-07 12-May-1987 HEXACHLOROBENZENE

SD-100-07 26-May-1987 HEXACHLOROBENZENE

** SD $-100-09$

SD-100-09 12-May-1987 HEXACHLOROBENZENE

SD-100-09 26-May-1987 HEXACHLOROBENZENE

** SD $-100-10$

SD-100-10 12-May-1987 HEXACHLOROBENZENE

SD-100-10 26-May-1987 HEXACHLOROBENZENE

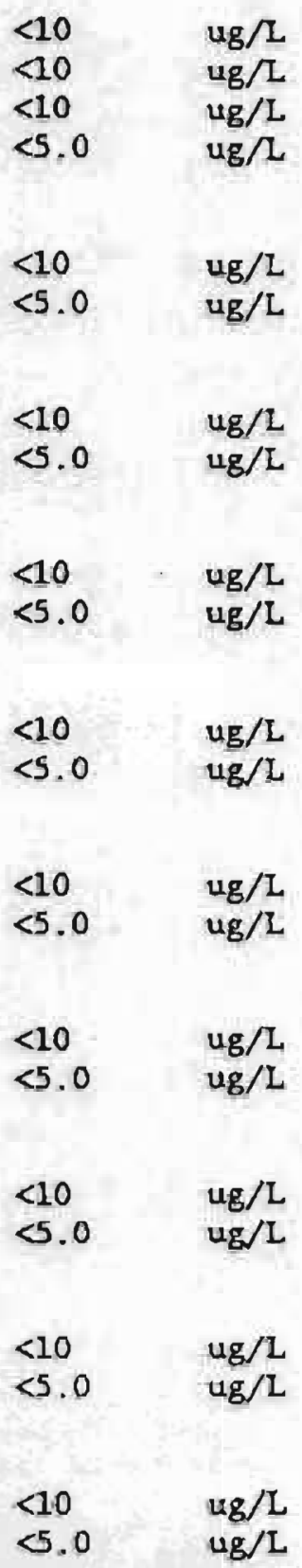


Storm Drain Data for SD-100

\begin{tabular}{|c|c|c|c|c|}
\hline Locatlon & Date & Test Compound & Results & Units \\
\hline \multicolumn{5}{|c|}{$\star *$ SD $-100-11$} \\
\hline$S D-100-11$ & 12-May-1987 & HEXACHLOROB ENZENE & $<10$ & $u g / L$ \\
\hline SD-100-11 & $26-$-Lay - 1987 & HEXACHLOROBENZENE & $<5.0$ & $u g / L$ \\
\hline \multicolumn{5}{|c|}{$*$ SD-100-12 } \\
\hline$S D-100-12$ & 12-Kay - 1987 & HEXACHLOROBENZENE & $<10$ & $\mathrm{ug} /$ \\
\hline SD $-100-12$ & $26-$ Мвy -1987 & HEXACHLOROBEXZENE & $<5.0$ & $u g / 2$ \\
\hline \multicolumn{5}{|c|}{$\star \star \quad S D-100-13$} \\
\hline SD $-100-13$ & $12-$ May -1987 & HEXACHLOROBENZENE & $<10$ & $u g / L$ \\
\hline SD $-100-13$ & 26-May-1987 & HEXACHLOROBENZENE & $<5.0$ & $u g / L$ \\
\hline \multicolumn{5}{|c|}{ \# SD-100-14 } \\
\hline SD $-100-14$ & 12-May - 1987 & HEXACHLOROBENZENE & $<10$ & ug $/ \mathrm{L}$ \\
\hline SD $-100-14$ & $26-$ May- 1987 & HEXACHLOROBENZENE & $<5.0$ & $\mathrm{ug} / \mathrm{L}$ \\
\hline \multicolumn{5}{|c|}{$\star$ SD $-100-\mathrm{C}$} \\
\hline$S D-100-C$ & 5-Kar-1987 & HEXACHLOROBEN ZEAE & $<10$ & $\mathrm{ug} / \mathrm{L}$ \\
\hline$S D-100-C$ & 9-Mar-1987 & HEXACHLOROBENZENE & $<10$ & $\mathrm{ug} / \mathrm{L}$ \\
\hline SD- $100-C$ & 17-Mar- 1987 & HEXACHLOROBENZENE & $<10$ & $\mathrm{ug} / \mathrm{L}$ \\
\hline$S D-100-C$ & $24-\operatorname{Mar}-1987$ & HEXACKLOROBENZENE & $<10$ & $\mathrm{ug} / 1$ \\
\hline SD-100-C & 8-Apr-1987 & HEXACHLOROBENZENE & $<10$ & $\mathrm{ug} / \mathrm{L}$ \\
\hline SD-100-C & 13-May - 1987 & HEXACHLOROBENZENE & $<5.0$ & $\mathrm{ug} / \mathrm{L}$ \\
\hline$S D-100-C$ & 20-May-1987 & HEXACHLOROBENZENE & $<5.0$ & $\mathrm{ug} / \mathrm{I}$ \\
\hline$S D-100-C$ & 27-May-1987 & HEXACHLOROBEAZENE & $<5.0$ & $u g / 2$ \\
\hline$S D-100-C$ & 12-Jun-1987 & HEXACHLOROBENZENE & $<5.0$ & ug $/ \mathrm{L}$ \\
\hline SD-100-C & 19-Jum-1987 & HEXACHLOROBENZEAE & $<10$ & ug $/ \mathrm{L}$ \\
\hline \multicolumn{5}{|l|}{ * SD-100 } \\
\hline SD -100 & 5-Mar-1987 & HEXACHLOROBUTADIENE & $<10$ & $\mathrm{ug} / \mathrm{L}$ \\
\hline SD-100 & 11 -Mar- 1987 & HEXACHLOROBUTADIENE & $<10$ & $u g / L$ \\
\hline$S D-100$ & 12-May- 1987 & HEXACHLOROBUTADIENE & $<10$ & $\mathrm{ug} / \mathrm{L}$ \\
\hline$S D-100$ & 26-May-1987 & HEXACHLOROBUTADIENE & $<.0$ & ug/L \\
\hline \multicolumn{5}{|c|}{$\star$ * SD-100-01 } \\
\hline SD $-100-01$ & 12-May-1987 & HEXACHLOROBUTADIENE & $<10$ & $u g / L$ \\
\hline SD $-100-01$ & $26-$ Kay-1987 & HEXACHLOROBUTADIENE & $<5.0$ & $u g / L$ \\
\hline \multicolumn{5}{|c|}{$\star \quad S D-100-02$} \\
\hline SD $-100-02$ & 12-May-1987 & HEXACHLOROBUTADIENE & $<10$ & $u g / L$ \\
\hline SD-100-02 & 26-May-1987 & HEXACHLOROBUTADIENE & $<5.0$ & ug/L \\
\hline \multicolumn{5}{|c|}{$\star \star \quad S D-100-03$} \\
\hline SD- $100 \cdot 03$ & 12-May- 1987 & HEXACHLOROBUTADIENE & $<10$ & $u g / L$ \\
\hline SD $-100-03$ & 26-May-1987 & HEXACHLOROBUTADIENE & $<5.0$ & ug/L \\
\hline \multicolumn{5}{|c|}{ ** SD $-100 \cdot 03 D$} \\
\hline$S D-100-03 D$ & 12-May-1987 & HEXACHLOROBUTADTENE & $<10$ & ug/L \\
\hline SD-100-03D & $26-$ May - 1987 & HEXACHLOROBUTADIENE & $<5.0$ & $\mathrm{ug} / \mathrm{L}$ \\
\hline
\end{tabular}


Storm Drain Data for SD -100

Location

Date

Test Compound

Results Units

** SD-100-04

SD-100-04 12-May-1987 HEXACHLOROBUTADIENE SD-100-04 26-May-1987 HEXACHLOROBUTADIEIE

* SD-100-06

SD-100-06 12-May-1987 HEXACHLOROBUTADIENE

SD-100-06 26-May-1987 HEXACHLOROBUTADIENE

* SD-100-07

SD-100-07 12-May-1987 HEXACHLOROBUTADIERE

SD-100-07 26-May-1987 HEXACHLOROBUTADIERE

* SD-100-09

SD-100-09 12-May-1987 HEXACHLOROBUTADIBNE

SD-100-09 26-May-1987 HEXACHLOROBUTADIENE

** SD $-100-10$

SD-100-10 12-May-1987 HEXACHLOROBUTADIENE

SD-100-10 26-May-1987 HEXAGHLOROBUTADIEAR

** SD-100-11

SD-100-11 12-May-1987 HEXACHLOROBUTADIERE

SD-100-11 26-May-1987 HEXACHLOROBUTADIETE

* SD $-100-12$

SD-100-12 12-May-1987 HEXAGHLOROBUTADIETE

SD-100-12 26-May-1987 HEXACHLOROBUTADIEIE

** SD $-100-13$

SD-100-13 12-May-1987 HEXACHLOROBUTADIDNE

SD-100-13 26-May-1987 HEXACHLOROBUTADIENE

* SD $-100-14$

SD-100-14 12-May-1987 HEXACHLOROBUTADIETE

SD-100-14 26-May-1987 HEXACHLOROBUTADIENE

** SD $-100-C$

SD-100-C 5-Mar-1987 HEXACHLOROBUTADIEIE

SD-100-C 9-Mar-1987 HEXACHLOROBUTADIPIE

SD-100-C 17-Mar-1987 HEXACHLOROBUTADIEIE

SD-100-C 24-Mar-1987 HEXACHLOROBUTADIERE

SD-100-C 8-APT-1987 HEXACHLOROBUTADIEIE

SD-100-C 13-May-1987 HEXACHLOROBUTADIETE

SD-100-C 20-MaY-1987 HEXACHLOROBUTADIENE

SD-100-C 27-May-1987 HEXACHLOROBUTADIENE

SD-100-C 12-JUn-1987 HEXACHLOROBUTADIENE

SD-100-C 19-J Un-1987 HEXACHLOROBUTADIENE

** SD -100

SD-100 5-Mar-1987 HEXACHLOROCYCLOPETTADIENE

SD-100 11-Mar-1987 HEXACHLOROCYCLOPETTADIENE

40

$<5.0$

$<10$

5.0

$4 \mathrm{~g} / \mathrm{L}$

ug/L

10

$<.0$

Ug/

ug/ $/ \mathrm{L}$

$<10$

$<5.0$

ug/L

$\mathrm{Lg} / \mathrm{L}$

$<10$

$<5.0$

ug/

ug/ $/$

$<10$

$<.0$

ug $/ \mathrm{L}$

$u g / L$

$<10$

$<.0$

ug $/$ L

ug $/ \mathrm{L}$

$<10$

$<5.0$

ug/L

$u g / L$

$<10$

$<5.0$

ug/L

ug/L

$<10$

$<10$

$<10$

$<10$

$<10$

<s. 0

$<5.0$

$<5.0$

$<5.0$

$<10$

ug/L

ug/L

$u g / L$

ug $/ 2$

ug $/$ L

$u g / L$

$u g / L$

ug/L

uE $/ \mathrm{L}$

$\mathrm{ug} / \mathrm{L}$

$<10$

$<10$

ug/L

ug/L 
Store Drain Data for SD-100

Location Dat

Test Compound

Results Units

SD -100

12-May- 1987 HEXACHLOROCYCLOPENTADIENE

SD-100 26-May-1987 HEXACHLOROCYCLOPENTADIENE

$<10$

$<5.0$

ug/L

$\therefore \quad$ SD-100

$26-1$

* SD $-100-01$

SD-100-01 12-May-1987 HEXACHLOROCYCLOPENTADIENE

SD-100-01 26-May-1987 HEXAGHLOROCYCLOPENTADIENE

* SD-100-02

SD-100-02 12-May-1987 HEXACHLOROCYCLOPENTADIENE

SD-100-02 26-May-1987 HEXACHLOROCYCLOPENTADIENE

** SD $-100-03$

SD-100-03 12-May-1987 HEXACHLOROCYCLOPENTADIENE

SD-100-03 26-May-1987 HEXACHLOROCYCLOPENTADIENE

** SD-100-03D

SD-100-03D 12-May-1987 HEXACHLOROCYCLOPENTADIENE

SD-100-03D 26-May-1987 HEXACHLOROCYCLOPENTADIENE

** SD $-100-04$

SD-100-04 12-May-1987 HEXACHLOROCYCLOPENTADIENE

SD-100-04 26-May-1987 HEXACHZOROCYCLOPENTADIENE

* SD-100-06

SD-100-06 12-May-1987 HEXACHLOROCYCLOPENTADIENE

SD-100-06 26-May-1987 HEXACHLOROCYCLOPENTADIENE

** SD $-100-07$

SD-100-07 12-May-1987 HEXACHLOROCYCLOPENTADIENE

SD-100-07 26-May-1987 HEXACHLOROCYCLOPENTADIENE

** SD $-100-09$

SD-100-09 12-May-1987 HEXACHLOROCYCLOPENTADIENE

SD-100-09 26-May-1987 HEXACHLOROCYCLOPENTADIENE

* SD-100-10

SD-100-10 12-May-1987 HEXACHLOROCYCLOPENTADIENE

SD-100-10 26-MaY-1987 HEXACHLOROCYCLOPENTADIENE

$\star$ ¿ SD $-100-11$

SD-100-11 12-May-1987 HEXACHLOROCYCLOPENTADIENE

SD-100-11 26-May-1987 HEXACHLOROCYCLOPENTADIENE

$\begin{array}{ll}<10 & \mathrm{ug} / \mathrm{L} \\ <5.0 & \mathrm{ug} / \mathrm{L}\end{array}$

$<10 \quad$ ug $/ \mathrm{L}$

$<5.0 \quad \mathrm{ug} / \mathrm{L}$

$<10 \quad 4 \mathrm{~g} / \mathrm{L}$

$<5.0 \quad$ ug/L

$<10 \quad$ ug/L

$<5.0 \quad \mathrm{ug} / \mathrm{L}$

$<10 \quad 4 \mathrm{~g} / \mathrm{L}$

$<5.0 \quad 4 \mathrm{~g} / \mathrm{L}$

$<10 \quad 4 \mathrm{~g} / \mathrm{L}$

$<5.0 \quad \mathrm{ug} / \mathrm{L}$

$<10 \quad$ ug/L

$<5.0 \quad$ ug/ $/ \mathrm{L}$

$<10 \quad \mathrm{ug} / \mathrm{L}$

$<5.0 \quad$ ug/L

$<10 \quad$ ug/L

$<5.0 \quad \mathrm{ug} / \mathrm{L}$

* SD-100-12

SD-100-12 12-May-1987 HEXACHLOROCYCLOPENTADIENE

SD-100-12 26-May-1987 HEXACHLOROCYCLOPENTADIENE

** SD $-100-13$

SD-100-13 12-May-1987 HEXACHLOROCYCLOPENTADIENE

SD-100-13 26-May-1987 HEXACHLOROCYCLOPENTADIENE

$\begin{array}{ll}<10 & \mathrm{ug} / \mathrm{L} \\ <5.0 & \mathrm{ug} / \mathrm{L} \\ & \\ <10 & \mathrm{ug} / \mathrm{L} \\ <5.0 & \mathrm{ug} / \mathrm{L} \\ & \\ & \\ & \\ & \\ <5.0 & \mathrm{ug} / \mathrm{L} \\ \mathrm{ug} / \mathrm{L}\end{array}$


Storm Drain Data for SD-100

Location

Date

Test Compound

Results

Unlts

* SD $-100-14$

SD-100-14 12-May-1987 HEXACHLOROCYCLOPENTADIENE SD-100-14 26-May-1987 HEXACHLOROCYCLOPENTADIENE

$<10$

$<5.0$

ug/L

ug/L

** SD-100-C

SD-100-C 5-MaT-1987 HEXACHLOROCYCLOPEATTADI TRE SD-100-C 9-Mar-1987 HEXACHLOROCYCLOPENTADIENE

SD-100-C 17-Mar-1987 HEXACHLOROCYCLOPENTADIENE

SD-100-C 24-Mar-1987 HEXAGHLOROCYCLOPENTADIENE

SD-100-C 8-APr-1987 HEXAGHLOROCYCLOPENTADIENE

SD-100-C 13-May-1987 HEXACHLOROCYCLOPENTADIENE

SD-100-C 20-May-1987 HEXACHLOROCYCLOPENTADIENE

SD-100-C 27-May-1987 HEXACHLOROCYCLOPENTADIENE

SD-100-C 12-JUn-1987 HEXACHLOROCYCLOPENTADIENE

SD-100-C 19-Jun-1987 HEXACHLOROCYCLOPENTADIENE

* SD-100

SD -100

5-Mar-1987 HEXAGHLOROETHANE

SD -100

11-Mar-1987 HEXACHLOROETHANE

$\mathrm{SD}-100$

12-MaY-1987 HEXACHLOROETHANE

SD-100

26-MaY-1987 HEXACHLOROETHANE

* SD-100-01

SD-100-01 12-May-1987 HEXACHLOROETHANE

SD-100-01 26-May-1987 HEXACHLOROETHANE

** SD-100-02

SD-100-02 12-May-1987 HEXACHLOROETHANE

SD-100-02 26-May-1987 HEXACHLOROETHANE

** SD-100-03

SD-100-03 12-May-1987 HEXACHLOROETHANE

SD-100-03 26-May-1987 HEXAGHLOROETHANE

$<10$

$<10$

$<10$

$<10$

$<10$

$<5.0$

$<5.0$

$<5.0$

$<5.0$

$<10$

$4 \mathrm{~g} / \mathrm{L}$

ug/L

$u g / L$

$\mathrm{ug} / \mathrm{L}$

ug/L

ug/L

$4 \mathrm{~g} / \mathrm{L}$

$\mu \mathrm{g} / \mathrm{L}$

$\mathrm{ug} / \mathrm{L}$

$4 \mathrm{~g} / \mathrm{L}$

$<10$

$<10$

$<10$

$<5.0$

ug/L

$\mathrm{ug} / \mathrm{L}$

$u g / L$

$\mathrm{ug} / \mathrm{L}$

$<10$

$<5.0$

$u g / L$

$u g / L$

$<10$

$<5.0$

$u g / L$

ug/L

** SD-100-03D

SD-100-03D 12-May-1987 HEXACHLOROETHANE SD-100-03D 26-May-1987 HEXACHLOROETHANE

** SD-100-04

SD-100-04 12-May-1987 HEXACHLOROETHANE

SD-100-04 26-May-1987 HEXACHLOROETHANE

** SD-100-06

SD-100-06 12-May-1987 HEXACHLOROETHANE

SD-100-06 26-May-1987 HEXACHLOROETHANE

$<10$

$<5.0$

ug/L

ug/L

$<10 \quad$ ug/L

$<5.0 \quad$ ug/L

$<10 \quad$ ug $/ \mathrm{L}$

$<5.0 \quad$ ug $/ \mathrm{L}$

$<10 \quad$ ug/L

$<5.0 \quad$ ug/L

** SD -100.07

SD-100-07 12-May-1987 HEXACHLOROETHANE

SD-100-07 26-May-1987 HEXACHLOROETHANE

$<10 \quad$ ug/L

$<5.0 \quad$ ug $/ \mathrm{L}$ 
Storm Drain Data for SD-100

Location Date Test Compound

Results Units

* SD-100-09

SD-100-09 12-May-1987 HEXACHLOROETHANE

SD-100-09 26-May-1987 HEXACHLOROETHANE

$<10$

$<5.0$

ug/L

* SD $-100-10$

SD-100-10 12-May-1987 HEXACHLOROETHANE

SD-100-10 26-May-1987 HEXACHLOROETHANE

** SD - 100-11

SD-100-11 12-Kay-1987 HEXACHLOROETHANE

SD-100-11 26-MaY-1987 HEXACHLOROETHANE

** SD $-100-12$

SD-100-12 12-May-1987 HEXACHLOROETHANE

SD-100-12 26-May-1987 HEXACHLOROETHANE

* SD-100-13

SD-100-13 12-May-1987 HEXACHLOROETHANE

SD-100-13 26-May-1987 HEXACHLOROETHANE

** SD $-100-14$

SD-100-14 12-May-1987 HEXACHLOROETHANE

SD-100-14 26-May-1987 HEXACHLOROETHANE

$<10 \quad$ ug/

$<5.0 \quad \mathrm{ug} / \mathrm{L}$

$<10 \quad$ ug/L

$<5.0 \quad u g / L$

$<10 \quad$ ug/L

$<5.0 \quad \mathrm{ug} / \mathrm{L}$

$<10 \quad$ ug/L

$<5.0 \quad$ ug/L

$<10 \quad \mathrm{ug} / \mathrm{L}$

$<5.0 \quad \mathrm{ug} / \mathrm{L}$

** SD-100-C

SD-100-C 5-Mar-1987 HEXACHLOROETHANE

$<10 \quad$ ug/L

$<10 \quad \mathrm{ug} / \mathrm{L}$

$<10 \quad$ ug/L

$<10 \quad$ ug/L

$<10 \quad 4 g / 2$

$<5.0 \quad$ ug/

$<5.0 \quad$ ug $/ \mathrm{L}$

$<5.0 \quad$ ug/L

$<5.0 \quad$ ug/L

$<10 \quad$ Ug/L

** SD 100

SD-100 5-Mar-1987 INDENO(1 2 3-CD) PYREANE

SD-100 11-Mar-1987 INDENO(1 2 3-CD) PYRFNE

SD-100 12-May-1987 INDENO(1 2 3-CD) PYRENE

SD-100 26-May-1987 INDENO(1 2 3-CD) PYREIE

** SD $-100-01$

SD-100-01 12-May-1987 INDENO(1 2 3-CD) PYRRNE

SD-100-01 26-May-1987 INDENO(1 2 3-CD) PYRENE

** SD $-100-02$

$\begin{array}{llll}\text { SD-100-02 } & 12-\text { May-1987 INDENO(1 } & 2 & 3-C D) \text { PYRENE } \\ \text { SD-100-02 } & 26-\text { May-1987 INDENO(1 } & 2 & 3-C D) \text { PYRENE }\end{array}$

$<10 \quad$ ug/ $/ 2$

$<10 \quad \mathrm{ug} / \mathrm{L}$

$<10 \quad$ ug/L

$<5.0 \quad$ ug/L

$<10 \quad$ ug $/ \mathrm{L}$

$<5.0 \quad$ ug/L

$<10 \quad$ ug/L

$<5.0 \quad$ ug/L 
Storm Drain Data for SD-100

Location

Date

Test Compound

Results Units

** SD - 100-03

SD-100-03 12-May-1987 INDENO(1 2 3-CD) PYRERE

SD-100-03 26-Kay-1987 INDENO(1 2 3-CD) PYRERE

$<10$

$<5.0$

ug $/ 2$

* SD-100-03D

SD-100-03D 12-May-1987 INDENO(1 2 3-CD) PYRERE

SD-100-03D 26-May-1987 INDENO(1 2 3-CD) PYRERE

* SD-100-04

SD-100-04 12-May-1987 INDENO(1 2 3-CD) PYRFNE

SD-100-04 26-May-1987 INDENO(1 2 3-CD) PYRERIE

* SD-100-06

SD-100-06 12-May-1987 INDENO(1 2 3-CD) PYRENE

SD-100-06 26-May-1987 INDENO(1 2 3-CD) PYREANE

* SD-100-07

SD-100-07 12-May-1987 INDENO(1 2 3-CD) PYREANE

SD-100-07 26-May-1987 INDENO(1 2 3-CD) PYRENE

* SD-100-09

SD-100-09 12-May-1987 INDENO(1 2 3-CD) PYRENE

SD-100-09 26-May-1987 INDENO(1 2 3-CD) PYRERE

* SD $-100-10$

SD-100-10 12-May-1987 INDENO(1 2 3-CD) PYRERE

SD-100-10 26-May-1987 INDENO(1 2 3-CD) PYRENE

* SD $-100-11$

SD-100-11 12-May-1987 INDEANO(1 2 3-CD) PYRERE

SD-100-11 26-May-1987 INDENO(1 2 3-CD) PYRENE

** SD-100-12

SD-100-12 12-May-1987 INDENO(1 2 3-CD) PYRERE SD-100-12 26-May-1987 INDENO(1 2 3-CD) PYRENE

* SD $-100-13$

SD-100-13 12-May-1987 INDENO(1 2 3-CD) PYRENE

SD-100-13 26-May-1987 INDENO(1 2 3-CD) PYRENE

** SD-100-14

SD-100-14 12-May-1987 INDENO(1 2 3-CD) PYREIE

SD-100-14 26-May-1987 INDENO(1 2 3-CD) PYRENTE

$<10 \quad \mathrm{ug} / \mathrm{L}$

$<5.0 \quad \mathrm{ug} / \mathrm{L}$

$<10$

$<5.0$

$u g / 2$

ug/1

$<10 \quad$ ug/L

$<5.0 \quad \mathrm{ug} / \mathrm{h}$

$<10 \quad$ ug/L

$<5.0 \quad \mathrm{ug} / \mathrm{L}$

$<10 \quad \mathrm{ug} / \mathrm{l}$

$<5.0 \quad \mathrm{ug} / \mathrm{L}$

$<10 \quad$ ug/L

$<5.0 \quad \mathrm{ug} / \mathrm{L}$

$<10 \quad 4 \mathrm{~g} / \mathrm{L}$

$<5.0 \quad 4 \mathrm{~g} / \mathrm{L}$

$<10 \quad \mathrm{ug} / \mathrm{h}$

$<5.0 \quad \mathrm{ug} / \mathrm{L}$

$<10 \quad \mathrm{ug} / \mathrm{L}$

$<5.0 \quad$ ug $/ \mathrm{L}$

$<10 \quad u g / L$

$<5.0 \quad$ ug $/ \mathrm{L}$

* SD-100-C

SD-100-C 5-Mar-1987 INDENO(1 2 3-CD) PYRENE

SD-100-C 9-MaT-1987 INDENO(1 22 3-CD) PYRENE

SD-100-C 17-Mar-1987 INDENO(1 2 3-CD) PYRENE

SD-100-C 24-Mar-1987 INDENO(1 2 3-CD) PYRENE

SD-100-C 8-Apr-1987 INDENO(1 2 3-CD) PYRENE

SD-100-C 13-May-1987 INDENO(1 2 3-CD) PYRENE

$<10 \quad$ ug/L

$<10 \quad$ ug/L

$<10 \quad 4 \mathrm{~L} / \mathrm{L}$

$<10 \quad$ ug/L

$<10 \quad \mathrm{ug} / \mathrm{L}$

$<5.0 \quad$ ug $/ \mathrm{L}$ 
Storm Drain Daca for SD-100

Location

Date

Test Compound

Results Units

SD-100-C

SD $-100-C$

SD- $100-C$

SD- $100-C$
20-MaY-1987 INDENO(1 2 3-CD) PYREAE

27-May-1987 INDENO(1 2 3-CD) PYRENE

12-Jun-1987 INDENO (1 2 3-CD) PYRENE

19-Jun-1987 INDENO(1 2 3-CD) PYRENE
* SD -100
SD -100

SD -100

SD -100

SD -100

SD -100

SD -100

SD -100

SD -100

SD -100

SD -100

SD -100

SD -100
5-Mar-1987 IRON

11-Mar-1987 IRON

18-Mar-1987 IRON

24-Max-1987 IRON

3-Apr-1987 IRON

6-APT-1987 IRON

12-May-1987 IRON

19-May-1987 IRON

26-May-1987 IRON

3-Jun-1987 IRON

11-Jun-1987 IRON

18-Jun-1987 IRON
$<5.0$

$<5.0$

$<5.0$

$<10$

0.55

0.32

0.12

0.16

0.13

0.16

0.18

0.13

0.14

0.19

0.073

0.086

0.97

4.8

1.8

0.75

3.1

0.066

$<0.050$

$<0.050$

$<0.050$

$<0.050$

0.082

0.050

$<0.050$

$<0.050$

$<0.050$

$<0.050$

0.083

0.053

$\mathrm{mg} / \mathrm{L}$

SD-100-030 12-May-1987 IRON

SD-100-03D 26-May-1987 IRON

* SD -100.04

SD-100-04 12-May-1987 IRON

SD-100-04 18-May-1987 IRON

SD-100-04 26-May-1987 IRON

SD-100-04 3-Jun-1987 IRON

SD-100-04 11-Jun-1987 IRON ug $/ \mathrm{L}$

ug $/ 2$

ug/L

ug/L mg $/ \mathrm{L}$

$\mathrm{mg} / \mathrm{L}$

$\mathrm{mg} / \mathrm{L}$

$\mathrm{mg} / \mathrm{L}$

$\mathrm{mg} / \mathrm{L}$

$\mathrm{mg} / \mathrm{L}$

$\operatorname{mg} / \mathrm{L}$

$\mathrm{mg} / \mathrm{L}$

mg/L

mg/L

ng/L

mg/L

$\begin{array}{lrl}\text { SD-100-01 } & 12-\text { May-1987 } & \text { IRON } \\ \text { SD-100-01 } & 18 \text {-May-1987 } & \text { IRON } \\ \text { SD-100-01 } & \text { 3-Jun-1987 } & \text { IRON } \\ \text { SD-100-01 } & 11 \text {-Jun-1987 } & \text { IRON } \\ \text { SD-100-01 } & 18 \text {-Jun-1987 } & \text { IRON } \\ & & \\ \text { SD-100-02 } & & \\ \text { SD-100-02 } & 12-\text { May-1987 IRON } \\ \text { SD-100-02 } & 18 \text {-May-1987 IRON } \\ \text { SD-100-02 } & 3 \text {-Jun-1987 IRON } \\ \text { SD-100-02 } & 11 \text {-Jun-1987 IRON } \\ \text { SD-100-02 } & 18-J u n-1987 & \text { IRON }\end{array}$

mg/L

$\mathrm{mg} / \mathrm{L}$

$m g / L$

mg $/ 2$

mg $几$

SD-100-03 26-May-1987 IRON

SD-100-03 11-Jun-1987 IRON

SD-100-03 18-Jun-1987 IRON

$\mathrm{mg} / \mathrm{L}$
$\mathrm{mg} / \mathrm{L}$
$\mathrm{gg} / \mathrm{L}$
$\mathrm{mg} / \mathrm{L}$
$\mathrm{gg} / \mathrm{L}$

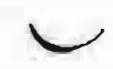


Storm Draln Data for SD-100

\begin{tabular}{|c|c|c|c|c|}
\hline Location & Date & Test Compound & Results & Units \\
\hline SD $-100-04$ & $18-J u n-1987$ & IRON & 0.097 & $m g / L$ \\
\hline \multicolumn{5}{|c|}{$\star \quad S D-100.06$} \\
\hline SD-100-06 & $12-$ Kay- 1987 & IRON & 0.13 & $\mathrm{mg} / \mathrm{L}$ \\
\hline SD $-100-06$ & 18-May- 1987 & IRON & $<0.050$ & $\mathrm{mg} / \mathrm{L}$ \\
\hline$S D-100-06$ & 26-May-1987 & IRON & $<0.050$ & $\mathrm{mg} / \mathrm{L}$ \\
\hline SD-100-06 & 3-Jun-1987 & IRON & 0.085 & $\mathrm{mg} / \mathrm{L}$ \\
\hline SD-100-06 & 11-Jun-1987 & IRON & $<0.050$ & $\mathrm{mg} / \mathrm{L}$ \\
\hline SD-100-06 & $18-J u n-1987$ & IRON & 0.33 & $\operatorname{mg} / \mathrm{L}$ \\
\hline \multicolumn{5}{|c|}{$\star \star \quad S D-100-06 D$} \\
\hline SD-100-06D & 3-Jun-1987 & IRON & $<0.050$ & $\mathrm{mg} / \mathrm{L}$ \\
\hline \multicolumn{5}{|c|}{ * SD $-100-07$} \\
\hline SD- $100-07$ & 12- - $a y-1987$ & IRON & 11 & $\mathrm{mg} / \mathrm{L}$ \\
\hline SD -100.07 & $19-$ May - 1987 & IRON & 1.8 & $m g / L$ \\
\hline SD $-100-07$ & 26-Мay- 1987 & IRON & 0.44 & $\mathrm{mg} / \mathrm{L}$ \\
\hline$S D \cdot 100-07$ & 3-Jun- 1987 & IRON & 2.3 & $\mathrm{mg} / \mathrm{L}$ \\
\hline SD $-100-07$ & 11-Jun-1987 & IRON & 0.61 & $\operatorname{mg} / \mathrm{L}$ \\
\hline SD- $100-07$ & $18-J u n-1987$ & IRON & 2.8 & mg/L \\
\hline \multicolumn{5}{|c|}{$\star * \quad S D-100-07 D$} \\
\hline SD $-100-07 D$ & $18-J u n-1987$ & IRON & 2.9 & $\mathbf{m g} / \mathrm{L}$ \\
\hline \multicolumn{5}{|c|}{ * SD $-100-09$} \\
\hline SD-100-09 & $12-$ May- 1987 & IRON & 0.31 & $\mathrm{mg} / \mathrm{L}$ \\
\hline SD $-100-09$ & 18 -May- 1987 & IRON & 0.11 & $\mathrm{mg} / \mathrm{L}$ \\
\hline SD -100.09 & $26-$ May- 1987 & IRON & 0.051 & $\operatorname{mg} / \mathrm{L}$ \\
\hline SD -100.09 & 3-Jun-1987 & IRON & 0.25 & $\mathrm{mg} / \mathrm{L}$ \\
\hline SD -100.09 & $11-J$ un -1987 & IRON & 0.063 & $\mathrm{mg} / \mathrm{L}$ \\
\hline \multicolumn{5}{|c|}{$\star \star S D-100-10$} \\
\hline SD- $100-10$ & 12-May-1987 & IRON & $<0.050$ & $\mathrm{mg} / \mathrm{L}$ \\
\hline$S D \cdot 100 \cdot 10$ & 19-May- 1987 & IRON & $<0.050$ & $\mathrm{mg} / \mathrm{L}$ \\
\hline SD $-100-10$ & $26-\mathrm{May}-1987$ & IRON & 0.054 & $\mathrm{mg} / \mathrm{l}$ \\
\hline SD $-100-10$ & 3-Jun-1987 & IRON & 0.35 & $\mathrm{mg} / \mathrm{L}$ \\
\hline$S D-100-10$ & 11 -Jun- 1987 & IRON & $<0.050$ & $m g / L$ \\
\hline$S D-100-10$ & $18-J u n-1987$ & IRON & $<0.050$ & $\mathrm{mg} / \mathrm{L}$ \\
\hline \multicolumn{5}{|c|}{$*$ SD $-100-11$} \\
\hline$S D-100-11$ & 12-May-1987 & IRON & $<0.050$ & $\operatorname{mg} / \mathrm{L}$ \\
\hline SD $-100 \cdot 11$ & 19-May-1987 & IRON & 0.32 & $\mathbf{m g} / \mathrm{L}$ \\
\hline SD-100-11 & 26-May-1987 & IRON & 0.59 & $\operatorname{mg} / \mathrm{L}$ \\
\hline SD $-100-11$ & 3-Jun-1987 & IRON & 0.063 & $\operatorname{mg} / \mathrm{L}$ \\
\hline SD $-100-11$ & $11-J u n-1987$ & IRON & 0.14 & $m g / L$ \\
\hline SD $-100-11$ & $18-J u n-1987$ & IRON & $<0.050$ & $\operatorname{mg} / \mathrm{L}$ \\
\hline \multicolumn{5}{|c|}{$\star \quad S D-100-12$} \\
\hline SD- $100 \cdot 12$ & $12-$ May- 1987 & IRON & 1.0 & $\mathbf{m g} / \mathbf{L}$ \\
\hline$S D-100-12$ & 19-May-1987 & IRON & 0.074 & $\mathrm{mg} / \mathrm{L}$ \\
\hline SD $-100-12$ & 26-May-1987 & IRON & 0.14 & $\operatorname{mg} / \mathrm{L}$ \\
\hline
\end{tabular}




$\begin{array}{ll}\text { Location Date } & \begin{array}{c}\text { Storm Drain Data for SD-100 } \\ \text { Test Compound }\end{array}\end{array}$

Results Units

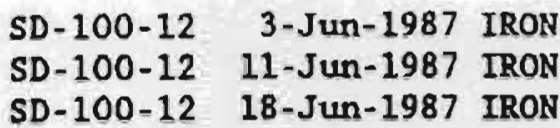

$<0.050 \mathrm{mg} / \mathrm{L}$

$0.090 \mathrm{mg} / \mathrm{L}$

$0.16 \mathrm{mg} / \mathrm{L}$

** SD - 100-13

SD-100-13 12-May-1987 IRON

$0.063 \mathrm{mg} / \mathrm{L}$

SD-100-13 19-May-1987 IRON

0.17

SD-100-13 26-May-1987 IRON

0.030

$\mathrm{mg} / \mathrm{L}$

SD-100-13 11-Jun-1987 IRON

$<0.050$

$\mathrm{mg} / \mathrm{L}$

SD-100-13 18-Jun-1987 IRON

0.11

$\mathrm{mg} / \mathrm{L}$

* SD-100-14

$\begin{array}{lrl}\text { SD-100-14 } & 12-\text { May-1987 } & \text { IRON } \\ \text { SD-100-14 } & 19-\text { May-1987 } & \text { IRON } \\ \text { SD-100-14 } & 26-\text { May-1987 } & \text { IRON } \\ \text { SD-100-14 } & 3-\text { Jun-1987 IRON } \\ \text { SD-100-14 } & 11-J u n-1987 & \text { IRON } \\ \text { SD-100-14 } & 18-J u n-1987 & \text { IRON }\end{array}$

$0.74 \quad \mathrm{mg} / \mathrm{L}$

$3.1 \quad \mathrm{mg} / \mathrm{L}$

$0.76 \quad \mathrm{mg} / \mathrm{L}$

$<0.050 \quad \mathrm{mg} / \mathrm{L}$

$<0.050 \mathrm{mg} / \mathrm{L}$

$<0.050 \mathrm{mg} / \mathrm{L}$

** SD-100-C

$\begin{array}{lrl}\text { SD-100-C } & 5 \text {-Mar-1987 IRON } \\ \text { SD-100-C } & 9 \text {-Mar-1987 IRON } \\ \text { SD-100-C } & \text { I7-Mar-1987 } & \text { IRON } \\ \text { SD-100-C } & 24-\text { Mar-1987 IRON } \\ \text { SD-100-C } & 8-\text { Apr-1987 } & \text { IRON } \\ \text { SD-100-C } & 13-\text { May-1987 IRON } \\ \text { SD-100-C } & 20-\text { May-1987 IRON } \\ \text { SD-100-C } & 27-\text { May-1987 IRON } \\ \text { SD-100-C } & 4-\text { Jun-1987 } & \text { IRON } \\ \text { SD-100-C } & 12-J u n-1987 & \text { IRON } \\ \text { SD-100-C } & 19-J u n-1987 & \text { IRON }\end{array}$

$0.42 \mathrm{mg} / \mathrm{L}$

$0.28 \mathrm{mg} / \mathrm{L}$

$0.11 \mathrm{mg} / \mathrm{L}$

$0.37 \mathrm{mg} / \mathrm{L}$

$<0.050 \mathrm{mg} / \mathrm{L}$

$0.12 \mathrm{mg} / \mathrm{L}$

$0.079 \mathrm{mg} / \mathrm{L}$

$<0.050 \mathrm{mg} / \mathrm{L}$

$<0.050 \quad \mathrm{mg} / \mathrm{L}$

$<0.050 \mathrm{mg} / \mathrm{L}$

$0.059 \mathrm{mg} / 2$

* SD-100-D

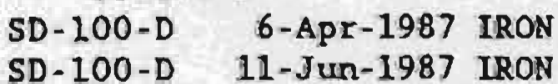

$0.14 \mathrm{mg} / \mathrm{L}$

$0.068 \mathrm{mg} / \mathrm{L}$

** SD -100

SD-100 5-Mar-1987 ISOPHORONE

SD-100 11-Mar-1987 ISOPHORONE

SD-100 12-May-1987 ISOPHORONE

SD-100 26-May-1987 ISOPHORONE

$<10 \quad \mathrm{ug} / \mathrm{L}$

$<10 \quad$ ug $/ 2$

$<10 \quad$ ug $/ \mathrm{L}$

$<5.0 \quad$ ug/L

** SD $-100-01$

SD-100-01 12-May-1987 ISOPHORONE

SD-100-01 26-May-1987 ISOPHORONE

$<10 \quad \mathrm{ug} / \mathrm{h}$

$<5.0 \quad \mathrm{ug} \Omega$

* SD - 100-02

SD-100-02 12-May-1987 ISOPHORONE

SD-100-02 26-May-1987 ISOPHORONE

$\begin{array}{ll}<10 & \text { ug } / \mathrm{L} \\ <5.0 & \text { ug } / \mathrm{L}\end{array}$ 
Storm Drain Data for SD-100

\begin{tabular}{|c|c|c|c|c|}
\hline Location & Date & Test Compound & Results & Units \\
\hline \multicolumn{5}{|c|}{ ** SD-100-03 } \\
\hline $\begin{array}{l}\text { SD- } 100-03 \\
\text { SD- } 100-03\end{array}$ & $\begin{array}{l}12-\text { May- } 1987 \\
26-\text { May-1987 }\end{array}$ & $\begin{array}{l}\text { ISOPHORONE } \\
\text { ISOPHORONE }\end{array}$ & $\begin{array}{l}<10 \\
<5.0\end{array}$ & $\begin{array}{l}u g / L \\
u g / L\end{array}$ \\
\hline \multicolumn{5}{|c|}{$\star \star \quad S D-100-03 D$} \\
\hline $\begin{array}{l}\text { SD }-100-03 D \\
\text { SD }-100-03 D\end{array}$ & $\begin{array}{l}12-\text { May-1987 } \\
26 \text { - Мay- } 1987\end{array}$ & $\begin{array}{l}\text { ISOPHORONE } \\
\text { ISOPHORONE }\end{array}$ & $\begin{array}{l}<10 \\
<5.0\end{array}$ & $\begin{array}{l}u g / L \\
u g / L\end{array}$ \\
\hline \multicolumn{5}{|c|}{$\star *$ SD $-100-04$} \\
\hline $\begin{array}{l}S D-100-04 \\
S D-100-04\end{array}$ & $\begin{array}{l}12-\text { May-1987 } \\
26-\text { May-1987 }\end{array}$ & $\begin{array}{l}\text { ISOPHORONE } \\
\text { ISOPHORONE }\end{array}$ & $\begin{array}{l}<10 \\
<5.0\end{array}$ & $\begin{array}{l}u g / L \\
u g / L\end{array}$ \\
\hline \multicolumn{5}{|c|}{$\star \quad$ SD $-100-06$} \\
\hline $\begin{array}{l}S D-100-06 \\
S D-100-06\end{array}$ & $\begin{array}{l}12-\text { May-1987 } \\
26-\text { May-1987 }\end{array}$ & $\begin{array}{l}\text { ISOPHORONE } \\
\text { ISOPHORONE }\end{array}$ & $\begin{array}{l}<10 \\
<5.0\end{array}$ & $\begin{array}{l}\mathrm{ug} / \mathrm{L} \\
\mathrm{ug} / \mathrm{L}\end{array}$ \\
\hline \multicolumn{5}{|c|}{$\star$ SD $-100-07$} \\
\hline SD $-100-07$ & $12-$ May -1987 & ISOPHORONE & $<10$ & $\mathrm{ug} / \mathrm{L}$ \\
\hline SD $-100-07$ & $26-$ May- 1987 & ISOPHORONE & $<5.0$ & $\mathrm{ug} / \mathrm{L}$ \\
\hline \multicolumn{5}{|c|}{ ** SD $-100-09$} \\
\hline SD- $100-09$ & 12-May-1987 & ISOPHORONE & $<10$ & $u g / L$ \\
\hline SD $-100-09$ & $26-$ May -1987 & ISOPHORONE & $<5.0$ & $\mathrm{ug} / \mathrm{L}$ \\
\hline \multicolumn{5}{|c|}{$\star * \quad S D-100-10$} \\
\hline SD $-100-10$ & 12-May-1987 & ISOPHORONE & $<10$ & $u g / L$ \\
\hline SD $-100-10$ & $26-$ May -1987 & ISOPHORONE & $<5.0$ & $\mathrm{ug} / \mathrm{L}$ \\
\hline \multicolumn{5}{|c|}{$\star \star$ SD $-100-11$} \\
\hline$S D-100-11$ & $12-$ May- 1987 & ISOPHORONE & $<10$ & $u_{g} / L$ \\
\hline SD-100-11 & 26-May- 1987 & ISOPHORONE & $<5.0$ & $\mathrm{ug} / \mathrm{L}$ \\
\hline \multicolumn{5}{|c|}{$* \quad S D-100-12$} \\
\hline SD - $100-12$ & 12-May- 1987 & ISOPHORONE & $<10$ & $\mathrm{ug} / \mathrm{L}$ \\
\hline SD $-100-12$ & $26-$ Kay-1987 & ISOPHORONE & $<5.0$ & $\mathrm{ug} / \mathrm{L}$ \\
\hline \multicolumn{5}{|c|}{ * SD $-100-13$} \\
\hline SD $-100-13$ & 12-May-1987 & ISOPHORONE & $<10$ & ug/L \\
\hline SD $-100-13$ & $26-$ May -1987 & ISOPHORONE & $<5.0$ & $\mathrm{ug} / \mathrm{L}$ \\
\hline \multicolumn{5}{|c|}{ * SD-100-14 } \\
\hline SD $-100-14$ & 12-May -1987 & ISOPHORONE & $<10$ & $u g / L$ \\
\hline SD- $100-14$ & $26-$ May - 1987 & ISOPHORONE & $<5.0$ & ug/L \\
\hline \multicolumn{5}{|c|}{$\star$ SD-100-C } \\
\hline SD - $100-C$ & $5-\operatorname{Mar}-1987$ & ISOPHORONE & $<10$ & ug/L \\
\hline SD $-100 \cdot C$ & $9-M a r-1987$ & ISOPHORONE & $<10$ & $48 / \mathrm{L}$ \\
\hline$S D-100-C$ & $17-$ Mar- 1987 & ISOPHORONE & $<10$ & $1 \mathrm{gg} / \mathrm{L}$ \\
\hline$S D-100-C$ & $24 \cdot \operatorname{Maz}-1987$ & ISOPHORONE & $<10$ & $4 g / \mathrm{h}$ \\
\hline SD $-100-C$ & 8-APT-1987 & ISOPHORONE & $<10$ & $\lg / \mathrm{L}$ \\
\hline SD- $100-C$ & $13-$ May -1987 & ISOPHORONE & $<5.0$ & $\mathrm{ug} / \mathrm{L}$ \\
\hline
\end{tabular}




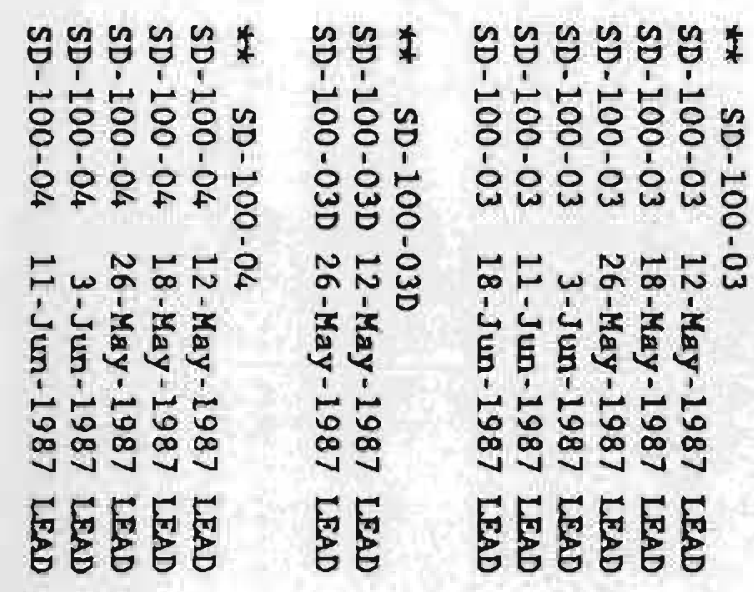

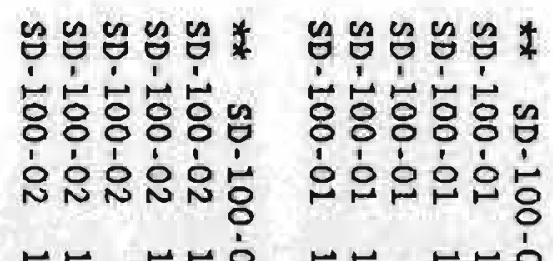

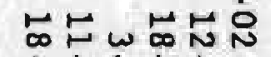

崖它寅密

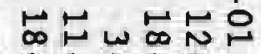

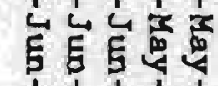

505

的它主

范它它

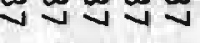

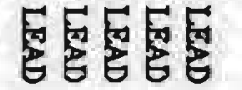
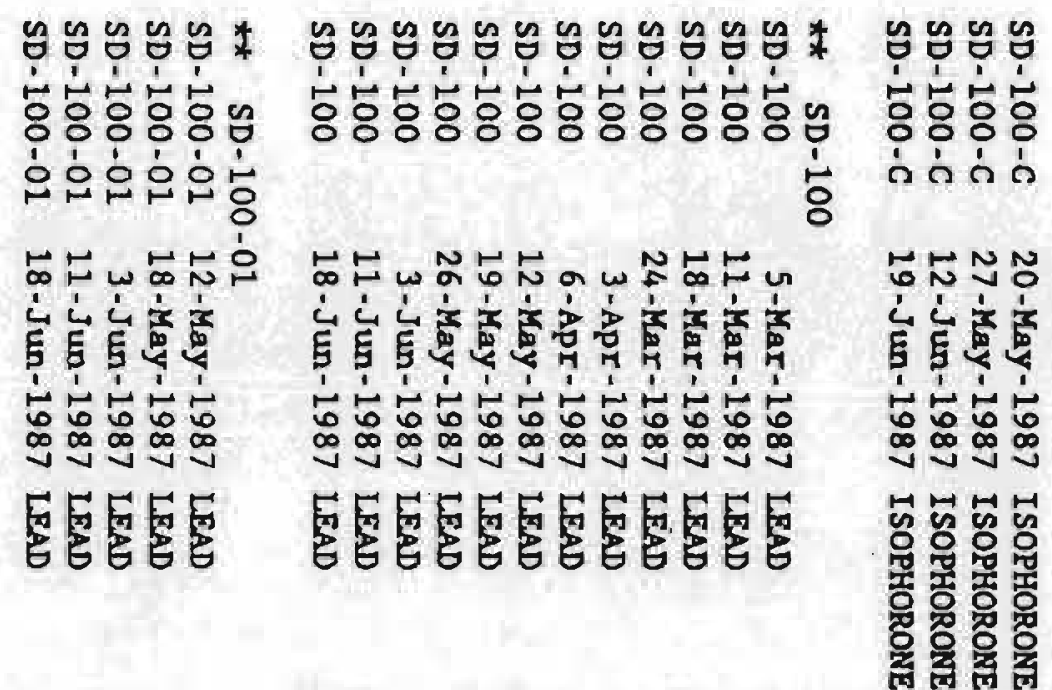

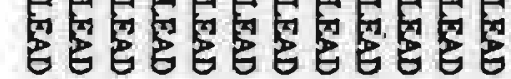

ôốốo

승

ốốốô

승승ㅅㅇㅇ

ôôốô

ôốốốôốốốô

앵융융응융

ํㅡㅇ응

냉앵능융융용

냉ํํㅇํํㅇํㅕㅇ영

냉영녕녕영

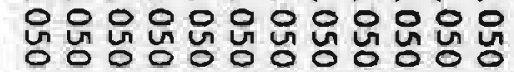

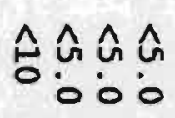

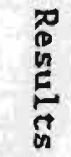

量品是最最 量

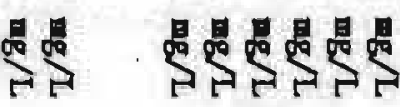

思是是昌遏

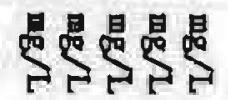

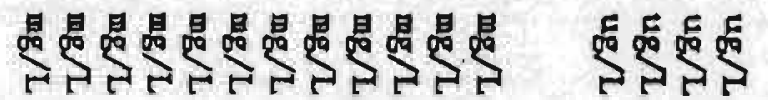

点 
Storm Drain Data for SD-100

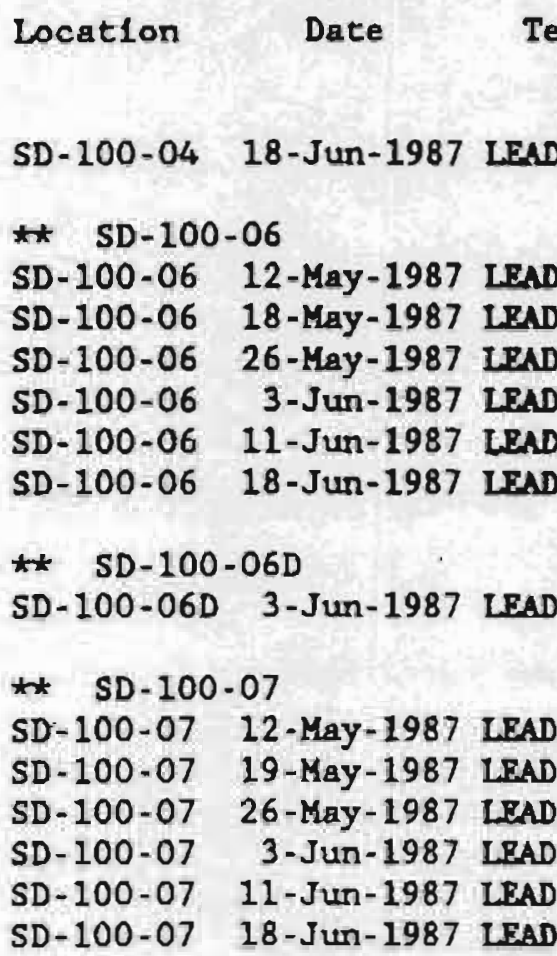

Results Units

Test Compound

$<0.050 \mathrm{mg} / \mathrm{L}$

$<0.050$

$<0.050$

$<0.050$

$<0.050$

$<0.050$

$<0.050$

$\mathrm{mg} / 1$

$\operatorname{mg} / \Omega$

$\mathrm{mg} / \mathrm{L}$

$\mathrm{mg} / \mathrm{L}$

$\mathrm{mg} / \mathrm{l}$

$\mathrm{mg} / \mathrm{l}$

$<0.050 \quad \mathrm{mg} / \mathrm{L}$

$<0.050$

$<0.050$

$<0.050$

$<0.050$

$<0.050$

0.20

$\mathrm{mg} / \mathrm{L}$

$\mathrm{mg} / \mathrm{L}$

$\mathrm{mg} / \mathrm{L}$

$\mathrm{mg} / \mathrm{L}$

$\mathrm{mg} / \mathrm{L}$

$\mathrm{mg} / \mathrm{L}$

** SD-100-07D

SD-100-07D 18-Jun-1987 LEAD

0.18

$\operatorname{mg} / \mathrm{L}$

* SD-100-09

SD-100-09 12-May-1987 LEAD

SD-100-09 18-May-1987 LEAD

SD-100-09 26-May-1987 LEAD

SD-100-09 3-JUn-1987 LEAD

SD-100-09 11-Jun-1987 IEAD

$<0.050$

$<0.050$

$<0.050$

$<0.050$

$<0.050$

$\operatorname{mg} / \mathrm{L}$

$\mathrm{mg} / \mathrm{L}$

$\mathrm{mg} / \mathrm{L}$

$\mathrm{mg} / \mathrm{L}$

$\mathrm{mg} / \mathrm{L}$

* SD-100-10

SD-100-10 12-May-1987 LEAD

$<0.050$

$<0.050$

$<0.050$

SD-100-10 26-May-1987 LEAD

$<0.050$

SD-100-10 3-Jun-1987 ISAD

$<0.050$

$<0.050$

$\mathrm{mg} / \mathrm{L}$

$\mathrm{mg} / \mathrm{L}$

$\mathrm{mg} / \mathrm{L}$

$\mathrm{mg} / \mathrm{L}$

SD-100-10 11-Jun-1987 IEAD

SD-100-10 18-Jun-1987 IEAD

$\mathrm{mg} / \mathrm{L}$

$\mathrm{mg} / \mathrm{L}$

** SD - 100-11

SD-100-11 12-May-1987 LEAD

SD-100-11 19-May-1987 IFAD

SD-100-II 26-May-1987 ISAD

SD-100-11 3-JUn-1987 IEAD

SD-100-11 11-JUn-1987 IEAD

SD-100-11 18-Jun-1987 LEAD

$<0.050$

$<0.050$

$<0.050$

$<0.050$

$<0.050$

$<0.050$

$\operatorname{mg} / \mathrm{L}$

$\operatorname{mg} / \mathrm{L}$

mg/L

$\mathrm{mg} / \mathrm{L}$

$\mathrm{mg} / \mathrm{L}$

$\mathrm{Ig} / \mathrm{L}$

** SD $-100-12$

SD-100-12 12-May-1987 LEAD

0.061

$<0.050$

$\mathrm{mg} / \mathrm{L}$

SD-100-12 19-May-1987 LEAD

$<0.050$

$\mathrm{mg} / \mathrm{L}$

$m g / L$ 
Storm Drain Data for SD-100

\begin{tabular}{|c|c|c|c|c|}
\hline Location & Date & Test Compound & Results & Units \\
\hline $\begin{array}{l}\text { SD }-100-12 \\
\text { SD }-100-12 \\
\text { SD }-100-12\end{array}$ & $\begin{array}{r}3-J u n-1987 \\
11-J u n-1987 \\
18-J u n-1987\end{array}$ & $\begin{array}{l}\text { LEAD } \\
\text { LEAD } \\
\text { LEAD }\end{array}$ & $\begin{array}{l}<0.050 \\
<0.050 \\
<0.050\end{array}$ & $\begin{array}{l}\mathrm{mg} / \mathrm{L} \\
\mathrm{mg} / \mathrm{L} \\
\mathrm{mg} / \mathrm{L}\end{array}$ \\
\hline \multicolumn{5}{|c|}{$\star$ SD $-100-13$} \\
\hline SD $-100-13$ & $12-$ May - 1987 & LEAD & $<0.050$ & $\mathrm{mg} / \mathrm{L}$ \\
\hline SD $-100-13$ & $19-$ Kay - 1987 & $\overline{\text { LEAD }}$ & $<0.050$ & $\mathrm{mg} / \mathrm{L}$ \\
\hline SD $-100-13$ & 26-May-1987 & LEAD & $<0.050$ & $\operatorname{mg} / 2$ \\
\hline SD $-100-13$ & 11-Jun-1987 & LEAD & $<0.050$ & $\mathrm{mg} / \mathrm{L}$ \\
\hline$S D-100-13$ & $18-J u n-1987$ & LEAD & $<0.050$ & $\mathrm{mg} / \mathrm{L}$ \\
\hline \multicolumn{5}{|c|}{$*$ SD $-100-14$} \\
\hline SD $-100-14$ & 12-Hay-1987 & LEAD & $<0.050$ & $\mathrm{mg} / \mathrm{L}$ \\
\hline$S D-100-14$ & $19-$ May-1987 & LEAD & $<0.050$ & $\mathrm{mg} / \mathrm{L}$ \\
\hline SD $-100-14$ & $26-$ Kay -1987 & LEAD & $<0.050$ & $\mathrm{mg} / \mathrm{L}$ \\
\hline SD $-100-14$ & 3-Jun-1987 & LEAD & $<0.050$ & $\mathrm{mg} / \mathrm{L}$ \\
\hline SD $-100-14$ & $11-J u n-1987$ & LEAD & $<0.050$ & $\mathrm{mg} / \mathrm{L}$ \\
\hline SD-100-14 & $18-J$ un -1987 & LEAD & $<0.050$ & $\mathrm{mg} / \mathrm{L}$ \\
\hline \multicolumn{5}{|c|}{$\star$ * SD-100-C } \\
\hline$S D-100-C$ & $5-\operatorname{Mar}-1987$ & LEAD & $<0.050$ & $\mathrm{mg} / \mathrm{L}$ \\
\hline$S D-100-C$ & 9-Mar-1987 & LEAD & $<0.050$ & $\mathrm{mg} / \mathrm{L}$ \\
\hline$S D-100-C$ & 17-Mar-1987 & LFAD & $<0.050$ & $\mathrm{mg} / \mathrm{t}$ \\
\hline SD- $100-C$ & $24-$ Mar -1987 & IEAD & $<0.050$ & $\mathrm{mg} / \mathrm{L}$ \\
\hline$S D-100-C$ & 8-Apr-1987 & LEAD & $<0.050$ & $\mathrm{mg} / \mathrm{L}$ \\
\hline SD-100-C & 13-May-1987 & LEAD & $<0.050$ & $\mathrm{mg} / \mathrm{L}$ \\
\hline SD- $100-C$ & 20-May-1987 & LEAD & $<0.050$ & $\mathrm{mg} / \mathrm{L}$ \\
\hline$S D-100-C$ & 27- -ау - 1987 & IEAD & $<0.050$ & $\operatorname{mg} / \mathrm{L}$ \\
\hline$S D-100-C$ & 4-Jun-1987 & LEAD & $<0.050$ & $\operatorname{mg} / \mathrm{L}$ \\
\hline SD $-100-C$ & $12-J u n-1987$ & LEAD & $<0.050$ & $\mathrm{mg} / \mathrm{L}$ \\
\hline$S D-100-C$ & $19-J u n-1987$ & LFAD & $<0.050$ & mg/L \\
\hline \multicolumn{5}{|c|}{ * SD-100-D } \\
\hline$S D-100 \cdot D$ & 6-Apr-1987 & LEAD & $<0.050$ & $\operatorname{mg} / \mathrm{L}$ \\
\hline SD $-100-D$ & 11 -Jun- 1987 & LEAD & $<0.050$ & $\mathrm{mg} / \mathrm{L}$ \\
\hline \multicolumn{5}{|l|}{ *t SD -100} \\
\hline SD -100 & 5-Mar- 1987 & LITHIUM & 0.0051 & $\operatorname{mg} \Omega$ \\
\hline SD -100 & 11-Mar-1987 & LITHIUM & 0.0056 & $\mathrm{mg} / \mathrm{L}$ \\
\hline SD -100 & $18-\operatorname{Mar}-1987$ & LITHIUM & 0.0053 & $\mathrm{mg} / \mathrm{L}$ \\
\hline SD- 100 & $24-\operatorname{Max}-1987$ & LITHIUM & 0.0061 & $\mathrm{mg} / \mathrm{L}$ \\
\hline SD -100 & 3-Apr-1987 & LITHIUM & $<0.0040$ & $\operatorname{mg} / \mathrm{L}$ \\
\hline SD- 100 & 6-Apr-1987 & LITHIUM & $<0.0040$ & $\mathrm{mg} / \mathrm{L}$ \\
\hline SD -100 & 12-May- 1987 & LITIIUM & 0.0049 & $\mathrm{mg} / \mathrm{L}$ \\
\hline SD-100 & 19-May-1987 & LITHIUM & 0.0053 & $\mathrm{mg} / \mathrm{L}$ \\
\hline SD -100 & 26-May- 1987 & LITHIUM & 0.0050 & $\operatorname{mg} / \mathrm{L}$ \\
\hline$S D-100$ & $3-J$ un- 1987 & LITHIUM & $<0.0040$ & $\operatorname{mg} / \mathrm{L}$ \\
\hline$S D-100$ & 11-Jun-1987 & LITHIUM & $<0.0040$ & $\mathrm{mg} / \mathrm{L}$ \\
\hline SD -100 & $18-J u n-1987$ & LITHIUM & 0.0049 & $\mathrm{mg} / \mathrm{L}$ \\
\hline
\end{tabular}


* SD-100-01

SD-100-01 12-May-1987 LITHTUM SD-100-01 18-May-1987 IITHIUM SD-100-01 3-Jun-1987 LITHTUM SD-100-01 11-Jun-1987 LITHIUM SD-100-01 18-Jen-1987 LITHIUM

* $\mathrm{SD}=100-02$

SD-100-02 12-May-1987 LITHIUM SD-100-02 18-May-1987 LITHIUM SD-100-02 3-Jun-1987 LITHIUM SD-100-02 11-Jun-1987 LITHIUM SD-100-02 18-Jun-1987 LITHIUM

** SD-100-03

SD-100-03 12-May-1987 LITHIUM SD-100-03 18-May-1987 LITHIUM SD-100-03 26-May-1987 LITHIUM SD-100-03 3-Jun-1987 LITHIUM SD-100-03 11-Jun-1987 LITHIUM SD-100-03 18-Jun-1987 LITHIUM

* SD-100-03D

SD-100-03D 12-May-1987 LITHIUY SD-100-O3D 26-May-1987 LITHIUM

* SD-100-04

SD-100-04 12-May-1987 LITHIUM SD-100-04 18-May-1987 LITHIUM SD-100-04 26-May-1987 LITHIUM SD-100-04 3-Jun-1987 LITHIUM SD-100-04 11-Jun-1987 LITHIUM SD-100-04 18-Ju-1987 LITHIUM

* SD-100-06

SD-100-06 12-May-1987 LITHIUM SD-100-06 18-May-1987 LITHIUM SD-100-06 26-May-1987 LITHIUM SD-100-06 3-JUn-1987 LITHIUM SD-100-06 11-Jun-1987 LITHIUM SD-100-06 18-Jun-1987 LITHTUM

* * SD- $100-06 D$

SD-100-060 3-Jun-1987 LITHIUM

** SD-100-07

SD-100-07 12-May-1987 LITHIUM

SD-100-07 19-May-1987 LITHIUM

SD-100-07 26-May-1987 LITHIUM

SD-100-07 3-Jun-1987 LITHIUM

SD-100-07 11-Jun-1987 LITHIUM
$<0.0040$

0.0051

$<0.0040$

$<0.0040$

0.0042

$\mathrm{mg} / \mathrm{L}$

$\mathrm{mg} / \mathrm{L}$

$\mathrm{mg} / \mathrm{L}$

$\mathrm{mg} / \mathrm{L}$

$\mathrm{mg} / \mathrm{L}$

0.0056

$<0.0040$

$<0.0040$

$<0.0040$

$<0.0040$

$\mathrm{mg} / \mathrm{L}$

$\mathrm{mg} / \mathrm{L}$

$\mathrm{mg} / \mathrm{L}$

$\mathrm{mg} / \mathrm{L}$

$\mathrm{mg} / \mathrm{L}$

0.0051

0.0055

0.0089

$<0.0040$

0.0044

0.0053

$\mathrm{mg} / \mathrm{L}$

$\mathrm{mg} / \mathrm{L}$

mg/L

$\mathrm{mg} / \mathrm{L}$

$\mathrm{mg} / \mathrm{L}$

$\mathrm{mg} / \mathrm{L}$

0.0054

0.0079

mg/L

$\mathrm{mg} / \mathrm{L}$

0.0052

0.0061

0.0072

$<0.0040$

0.0044

0.0051

mg/L

$\mathrm{mg} / \mathrm{L}$

$\mathrm{mg} / \mathrm{L}$

mg/L

IIg/L

$\mathrm{mg} / \mathrm{L}$

0.0050

0.0055

$\mathrm{mg} / \mathrm{L}$

0.0073

$<0.0040$

$<0.0040$

0.0048

$\mathrm{mg} / \mathrm{L}$

$\mathrm{mg} / \mathrm{L}$

$\mathrm{mg} / \mathrm{L}$

mg $/ 2$

$\mathrm{mg} / \mathrm{L}$

$<0.0040 \mathrm{mg} / \mathrm{L}$

0.010

mg/L

0.0067

mg/L

0.0041

0.0045

$\mathrm{mg} / \mathrm{L}$

$\mathrm{mg} / \mathrm{L}$

$<0.0040$ 
Storm Drain Data for SD-100

Location Date Test Compound

Results Units

SD-100-07 18-JUn-1987 LITHIUM

$0.0060 \mathrm{mg} / \mathrm{L}$

* SD-100-07D

SD-100-07D 18-Jun-1987 LITHIUM

$<0.0040 \mathrm{mg} / \mathrm{L}$

** SD-100-09

SD-100-09 12-May-1987 LITHIUM

$0.0053 \mathrm{mg} / \mathrm{L}$

SD-100-09 18-May-1987 LITHIUM

0.0060

SD-100-09 26-May-1987 LITHIUM

SD-100-09 3-JUn-1987 LITHTUM

0.0073

$<0.0040$

SD-100-09 11-Jun-1987 LITHIUM

$<0.0040$

$\mathrm{mg} / \mathrm{L}$

$\mathrm{mg} / \mathrm{L}$

$\mathrm{mg} / \mathrm{L}$

* SD - 100-10

SD-100-10 12-May-1987 LITHIUM

SD-100-10 19-May-1987 LITHIUM

SD-100-10 26-May-1987 LITHIUM

SD-100-10 3-Jun-1987 LITHIUM

SD-100-10 11-Jun-1987 LITHIUM

SD-100-10 18-Jun-1987 LITHIUM

$\begin{array}{ll}0.0051 & \mathrm{mg} / \mathrm{L} \\ 0.0061 & \mathrm{mg} / \mathrm{L} \\ 0.0071 & \mathrm{mg} / \mathrm{L} \\ <0.0040 & \mathrm{mg} / \mathrm{L} \\ <0.0040 & \mathrm{mg} / \mathrm{L} \\ <0.0040 & \mathrm{mg} / \mathrm{L}\end{array}$

* SD-100-11

SD-100-11 12-May-1987 LITHIUM

SD-100-11 19-May-1987 LITHTUM

SD-100-11 26-May-1987 LITHIUY

SD-100-11 3-Jun-1987 LITHIUM

SD-100-11 11-Jun-1987 LITHIUM

SD-100-11 18-Jun-1987 LITHIUM

$0.0054 \mathrm{mg} / \mathrm{L}$

$0.0071 \mathrm{mg} / \mathrm{L}$

$0.0090 \mathrm{mg} / \mathrm{L}$

$<0.0040 \mathrm{mg} / \mathrm{L}$

$<0.0040 \mathrm{mg} / \mathrm{L}$

$0.0064 \mathrm{mg} / \mathrm{L}$

* SD $-100-12$

SD-100-12 12-May-1987 LITHIUM

$0.0057 \mathrm{mg} / \mathrm{L}$

SD-100-12 19-May-1987 LITHIUM

$0.0050 \mathrm{mg} / \mathrm{L}$

SD-100-12 26-May-1987 LITHIUM

$0.0046 \mathrm{mg} / \mathrm{L}$

SD-100-12 3-Jun-1987 LITHIUM

SD-100-12 11-Jun-1987 LITHIUM

SD-100-12 18-JUn-1987 LITHIUT

$0.0046 \mathrm{mg} / \mathrm{L}$

$<0.0040 \mathrm{mg} / \mathrm{L}$

$0.0054 \mathrm{mg} / \mathrm{L}$

** SD - 100-13

SD-100-13 12-May-1987 LITHIUM

SD-100-13 19-May-1987 LITHIUM

SD-100-13 26-May-1987 LITHIUM

SD-100-13 11-JUn-1987 LITHTUM

SD-100-13 18-Jun-1987 LITHIUM

$\begin{array}{ll}0.0048 & \mathrm{mg} / \mathrm{L} \\ 0.0057 & \mathrm{mg} / \mathrm{L} \\ 0.0052 & \mathrm{mg} / \mathrm{L} \\ 0.0047 & \mathrm{mg} / \mathrm{L} \\ 0.0044 & \mathrm{mg} / \mathrm{L}\end{array}$

* SD-100-14

SD-100-14 12-May-1987 LITHIUM

SD-100-14 19-May-1987 LITHIUM

SD-100-14 26-May-1987 LITHIUM

SD-100-14 3-Jun-1987 LITHIUM

SD-100-14 11-Jun-1987 LITHIUM

SD-100-14 18-Jun-1987 LITHIUM

$0.0060 \mathrm{mg} / \mathrm{l}$

$0.010 \mathrm{mg} / \mathrm{L}$

$0.0051 \mathrm{mg} / \mathrm{L}$

$0.0047 \mathrm{mg} / \mathrm{L}$

$<0.0040 \mathrm{mg} / \mathrm{L}$

$<0.0040 \mathrm{mg} / \mathrm{L}$ 


\begin{tabular}{|c|c|c|}
\hline$S D-100-C$ & $5-$ Max -1987 & LITHIUM \\
\hline$D-100-C$ & $9-\operatorname{Max}=1987$ & LITHIUM \\
\hline SD- $100-C$ & 17-Kar-1987 & LITHIUM \\
\hline$S D-100-C$ & $24-\mathrm{Kar}-1987$ & IтTнгм \\
\hline SD - $100-C$ & 8-Apr-1987 & LITHIUМ \\
\hline SD-100-C & 13-May - 1987 & LTHIUM \\
\hline SD-100-C & 20-May-1987 & LITHIUA \\
\hline SD-100-C & 27-May-1987 & LITHIUS \\
\hline$S D-100-C$ & 4-Jun-1987 & LITHTU \\
\hline SD- $100-C$ & $12-J u n-1987$ & LITHTUM \\
\hline & & \\
\hline
\end{tabular}

太 SD-100-D

SD-100-D 6-Apr-1987 LITHIUM

SD-100-D 11-Jun-1987 LITHIUM

** SD-100

SD- 100 5-Mar-1987 MAGNESIIM

SD-100 11-Mar-1987 MACNESIUM

SD $-100 \quad 18-$ Mar-1987 MAGNESIUM

SD-100 24-Mar-1987 MAGNESIUM

SD-100 3-Apr-1987 KAGNESIUM

SD-100 6-APr-1987 MAGNESIUM

SD-100 12-May-1987 KAGNESIUM

SD-100 19-May-1987 MAGNESIUM

SD-100 26-May-1987 MAGNESIUM

SD-100 3-Jun-1987 HAGNESIUM

SD-100 11-JUn-1987 KAGNESIUM

SD-100 18-Jun-1987 MAGNESIUM

* SD-100-01

SD-100-01 12-May-1987 KAGAESIUM

SD-100-01 18-May-1987 MAGNESIUM

SD-100-01 3-Jun-1987 MAGNESIUM

SD-100-01 11-Jum-1987 MAGNESIUM

SD-100-01 18-Jun-1987 MAGNESIUM

* SD-100-02

SD-100-02 12-May-1987 MAGNESIUM

SD-100-02 18-May-1987 MAGIESIUM

SD-100-02 3-Jun-1987 MAGNESIUM

SD-100-02 11-JUn-1987 KAGNESIUM

SD-100-02 18-Jun-1987 HAGNESIUM

$\star \star \quad S D-100-03$

SD-100-03 12-May-1987 MAGNESIUM

SD-100-03 18-May-1987 MAGNESIUM

SD-100-03 26-May-1987 MAGNESIUM

SD-100-03 3-Jun-1987 MAGNESIUM

SD-100-03 11-Jun-1987 MAGNESIUM

$\begin{array}{ll}0.0050 & \mathrm{mg} / \mathrm{L} \\ <0.0040 & \mathrm{mg} / \mathrm{L} \\ 0.0041 & \mathrm{mg} / \mathrm{L} \\ 0.0044 & \mathrm{mg} / \mathrm{L} \\ <0.0040 & \mathrm{mg} / \mathrm{L} \\ 0.0052 & \mathrm{mg} / \mathrm{L} \\ 0.0076 & \mathrm{mg} / \mathrm{L} \\ 0.0054 & \mathrm{mg} / \mathrm{L} \\ <0.0040 & \mathrm{mg} / \mathrm{L} \\ <0.0040 & \mathrm{mg} / \mathrm{L} \\ <0.0040 & \mathrm{mg} / \mathrm{L}\end{array}$

$<0.0040 \mathrm{mg} / \mathrm{L}$

$0.0041 \mathrm{mg} / \mathrm{L}$

$\begin{array}{ll}9.6 & \mathrm{mg} / \mathrm{L} \\ 8.6 & \mathrm{mg} / \mathrm{L} \\ 8.8 & \mathrm{mg} / \mathrm{L} \\ 10 & \mathrm{mg} / \mathrm{L} \\ 8.2 & \mathrm{mg} / \mathrm{L} \\ 8.8 & \mathrm{mg} / \mathrm{L} \\ 11 & \mathrm{mg} / \mathrm{L} \\ 9.0 & \mathrm{mg} / \mathrm{L} \\ 9.4 & \mathrm{mg} / \mathrm{L} \\ 9.8 & \mathrm{mg} / \mathrm{L} \\ 10 & \mathrm{mg} / \mathrm{L} \\ 10 & \mathrm{mg} / \mathrm{L}\end{array}$

5.1

6.7

6. 8

7.6

8.0

11

9.7

9.7

10

9.8

$\mathbf{m g} /$ L

$\operatorname{Irg} \Omega$

$\mathrm{mg} / \mathrm{L}$

$\mathrm{mg} / \mathrm{L}$

$\mathrm{mg} / \mathrm{L}$

11

8.9

9.6

9.6

10 $\operatorname{mg} / \mathrm{L}$

$\mathrm{mg} / \mathrm{L}$

$\operatorname{mg} / \mathrm{L}$

$\mathrm{mg} / \mathrm{L}$

$\mathrm{mg} / \mathrm{L}$

$\mathrm{mg} / \mathrm{L}$

$\mathrm{mg} / \mathrm{L}$

$\mathrm{mg} / \mathrm{L}$

$\operatorname{mg} / \mathrm{L}$

$\mathrm{mg} / \mathrm{L}$ 
Storm Drain Data for SD-100

Location Date Test Compound

Results Units

SD-100-03 18-JUn-1987 MAGIESTUM

$10 \mathrm{mg} / \mathrm{L}$

* SD - 100-03D

SD-100-03D 12-May-1987 MAGNESTUM

SD-100-03D 26-May-1987 MAGNESIUM

$10 \mathrm{mg} / \mathrm{L}$

$9.4 \mathrm{mg} / \mathrm{L}$

* SD-100-04

SD-100-04 12-May-1987 KAGNESIUM

SD-100-04 18-May-1987 MAGNESTUM

SD-100-04 26-May-1987 MAGNESTUA

SD-100-04 3-Jun-1987 MAGNESTUM

SD-100-04 11-Jun-1987 MAGNESIUM

SD-100-04 18-Jun-1987 KAGNESIUM

$11 \mathrm{mg} / \mathrm{L}$

$8.8 \mathrm{mg} / \mathrm{L}$

$9.6 \mathrm{mg} / \mathrm{L}$

$9.6 \quad \mathrm{mg} / \mathrm{L}$

$10 \quad \mathrm{mg} / \mathrm{L}$

$10 \mathrm{mg} / \mathrm{L}$

* SD-100-06

SD-100-06 12-May-1987 MAGNESIUM

SD-100-06 18-May-1987 MAGNESIUM

SD-100-06 26-May-1987 KAGNESIUM

SD-100-06 3-Jun-1987 MAGNESIUM

SD-100-06 11-Jun-1987 MAGNESTUM

SD-100-06 18-Jun-1987 MAGNESIUM

** SD-100-06D

SD-100-06D 3-Jun-1987 MAGNESTUM

11

8.9

9.6

9.6

10

10

$\mathrm{mg} / \mathrm{L}$

$\mathrm{mg} / \mathrm{L}$

$\mathrm{mg} / \mathrm{L}$

$\mathrm{mg} / \mathrm{L}$

$\mathrm{mg} / \mathrm{L}$

$\mathrm{mg} / \mathrm{L}$

$9.6 \mathrm{mg} / \mathrm{L}$

* SD-100-07

SD-100-07 12-May-1987 MAGNESIUM

SD-100-07 19-May-1987 MAGNESIUM

SD-100-07 26-May-1987 MAGNESIUM

SD-100-07 3-JUn-1987 KAGNESIUM

SD-100-07 11-Jun-1987 MAGNESIUM

SD-100-07 18-Jun-1987 MAGNESTUM

** SD $-100-07 D$

SD-100-07D 18-JUn-1987 MAGNESIUM

$7.2 \mathrm{mg} / \mathrm{L}$

** SD-100-09

SD-100-09 12-May-1987 MAGNESTUM

SD-100-09 18-May-1987 MAGNESIUM

SD-100-09 26-May-1987 MAGNESIUM

SD-100-09 3-Jun-1987 MAGNESTUM

SD-100-09 11-Jun-1987 MAGNESTUM

$10 \mathrm{mg} / \mathrm{L}$

$7.5 \mathrm{mg} / \mathrm{L}$

$7.6 \quad \mathrm{mg} / \mathrm{L}$

$6.3 \mathrm{mg} / \mathrm{L}$

$5.7 \quad \mathrm{mg} / \mathrm{L}$

$8.3 \mathrm{mg} / \mathrm{L}$

* SD $-100-10$

SD-100-10 12-May-1987 MAGNESIUM

SD-100-10 19-May-1987 MAGNESIUM

SD-100-10 26-May-1987 MAGNESIUM

SD-100-10 3-Jun-1987 MAGNESIUM

SD-100-10 11-Jun-1987 MAGNESIUM

SD-100-10 18-Jun-1987 MAGNESIUM

11

9.1

9.6

9.3

10

11

9.1

9.6

10

10

9.9 $\mathrm{mg} / \mathrm{L}$

$\mathrm{mg} / \mathrm{L}$

$\mathrm{mg} / \mathrm{L}$

$\mathrm{mg} / \mathrm{L}$

$\mathrm{mg} / \mathrm{L}$

mg/L

$\mathrm{mg} / \mathrm{L}$

แg/L

$\mathrm{mg} / \mathrm{L}$

$\mathrm{mg} / \mathrm{L}$

mg/L 
* SD-100-11

SD-100-11 12-May-1987 MAGNESIUM

SD-100-11 19-May-1987 MAGNESIUM

SD-100-11 26-May-1987 MAGNESTUM

SD-100-11 3-Jun-1987 MAGNESIUM

SD-100-11 11-Jun-1987 MAGNESIUM

SD-100-11 18-JUn-1987 MAGNESIUM

* SD-100-12

SD-100-12 12-May-1987 MAGNESIUM

SD-100-12 19-May-1987 MAGNESTUM

SD-100-12 26-May-1987 MAGNESIUM

SD-100-12 3-Jun-1987 MAGNESIUM

SD-100-12 11-Jun-1987 MAGNESIUM

SD-100-12 18-JUn-1987 MAGNESIUM

* SD $-100-13$

SD-100-13 12-May-1987 MAGNESIUM

SD-100-13 19-May-1987 MAGNESIUM

SD-100-13 26-May-1987 MAGNESIUM

SD-100-13 11-JUn-1987 KAGNESIUY

SD-100-13 18-Jun-1987 KAGNESIUM

** SD $-100-14$

SD-100-14 12-May-1987 MAGNES IUM

SD-100-14 19-May-1987 MAGNESIUM

SD-100-14 26-May-1987 MAGNESIUM

SD-100-14 3-Jun-1987 MAGNESTUM

SD-100-14 11-Jun-1987 MAGNESIUM

SD-100-14 18-Jun-1987 MAGNESIUM

11

9.4

11

10

11

11

14

9.1

10

9.3

9.9

10

11

9.0

9.4

10

9.7

11

10

10

9.4

9.6

9.9

\#* SD-100-C

SD-100-C 5-Mar-1987 MAGNESIUM

SD-100-C 9-Mar-1987 MAGNESIUM

SD-100-C 17-Mar-1987 MAGNESIUM

SD-100-C 24-Mar-1987 MAGNESIUM

SD-100-C 8-Apr-1987 MAGNESIUM

SD-100-C 13-May-1987 MAGNESIUM

SD-100-C 20-May-1987 MAGNESIUM

SD-100-C 27-May-1987 MAGNES TUM

SD-100-C 4-Jun-1987 MAGNESIUM

SD-100-C 12-Jun-1987 MAGNESIUM
SD- $100-C$

19-Jun-1987 MAGNESIUY

** SD - 100-D

SD-100-D 6-Apr-1987 MAGNESIUM

SD-100-D 11-Jun-1987 MAGNESIUM

** SD -100

SD-100 5-Mar-1987 MANGANESE

SD-100 11-Mar-1987 MANGANESE
$8.6 \mathrm{mg} / \mathrm{L}$

$6.5 \mathrm{mg} / \mathrm{L}$

$8.5 \mathrm{mg} / \mathrm{L}$

$12 \mathrm{mg} / \mathrm{L}$

$9.4 \quad \mathrm{mg} / \mathrm{L}$

$11 \mathrm{mg} / \mathrm{L}$

$8.9 \mathrm{mg} / \mathrm{L}$

$11 \mathrm{mg} / \mathrm{L}$

$9.5 \mathrm{mg} / \mathrm{L}$

$9.8 \mathrm{mg} / \mathrm{L}$

$9.8 \mathrm{mg} / \mathrm{L}$

$8.7 \mathrm{mg} / \mathrm{L}$

$10 \mathrm{mg} / \mathrm{L}$

$0.080 \mathrm{mg} / \mathrm{L}$

$0.057 \mathrm{mg} / \mathrm{L}$ 
Storm Drain Data for SD-100

Location Date Test Compound Results Units

\begin{tabular}{|c|c|c|}
\hline SD-100 & $18-\operatorname{Max}-1987$ & MANGANESE \\
\hline SD -100 & 24-Mar-1987 & MANGANESE \\
\hline SD -100 & 3-Apr- 1987 & MANGANESE \\
\hline-100 & 6-Apr-1987 & MANGANESE \\
\hline-100 & 12-May-1987 & MANGANESE \\
\hline 100 & 19-May-1987 & MANGANESE \\
\hline 100 & 26-May-1987 & MANGANESE \\
\hline-100 & 3-Jun-1987 & MANGANESE \\
\hline-100 & 11 -Jun- 1987 & MANGANESE \\
\hline-100 & $18-J$ un-1987 & MANGANES \\
\hline
\end{tabular}

** SD-100-01

SD-100-01 12-May-1987 MANGANESE

SD-100-01 18-May-1987 MANGANESE

SD-100-01 3-Jun-1987 MANGANESE

SD-100-01 11-Jun-1987 MANGANESE

SD-100-01 18-Jun-1987 MANGANESE

* SD-100-02

SD-100-02 12-May-1987 MANGANESE

SD-100-02 18-May-1987 MANGANESE

SD-100-02 3-Jun-1987 MANGANESE

SD-100-02 11-Jun-1987 MANGANESE

SD-100-02 18-Jun-1987 MANGANESE

$0.034 \mathrm{mg} / \mathrm{L}$

$0.034 \mathrm{mg} / \mathrm{L}$

$0.039 \mathrm{mg} / \mathrm{L}$

$0.059 \mathrm{mg} / \mathrm{L}$

$0.025 \mathrm{mg} / \mathrm{L}$

$0.021 \mathrm{mg} / \mathrm{L}$

$0.019 \mathrm{mg} / \mathrm{L}$

$0.018 \mathrm{mg} / \mathrm{L}$

$0.010 \mathrm{mg} / \mathrm{L}$

$0.022 \mathrm{mg} / \mathrm{L}$

$\begin{array}{ll}0.40 & \mathrm{mg} / \mathrm{L} \\ 2.0 & \mathrm{mg} / \mathrm{L} \\ 1.1 & \mathrm{mg} / \mathrm{L} \\ 0.48 & \mathrm{mg} / \mathrm{L} \\ 1.2 & \mathrm{mg} / \mathrm{L}\end{array}$

$<0.010 \mathrm{mg} / \mathrm{L}$

$<0.010 \mathrm{mg} / \mathrm{L}$

$<0.010 \mathrm{mg} / \mathrm{L}$

$<0.010 \mathrm{mg} / \mathrm{L}$

$<0.010 \mathrm{mg} / \mathrm{L}$

* SD-100-03

SD-100-03 12-May-1987 MANGANESE

SD-100-03 18-May-1987 MANGANESE

SD-100-03 26-May-1987 MANGANESE

SD-100-03 3-Jun-1987 MANGANESE

SD-100-03 11-Jun-1987 MANGANESE

SD-100-03 18-Jun-1987 MANGANESE

$0.019 \mathrm{mg} / \mathrm{L}$

$0.012 \mathrm{mg} / \mathrm{L}$

$0.015 \mathrm{mg} / \mathrm{L}$

$0.015 \mathrm{mg} / \mathrm{L}$

$<0.010 \mathrm{mg} / \mathrm{L}$

$<0.010 \mathrm{mg} / \mathrm{L}$

** SD-100-03D

SD-100-03D 12-May-1987 MANGANESE

SD-100-03D 26-May-1987 MANGANESE

$0.018 \mathrm{mg} / \mathrm{L}$

$0.015 \mathrm{mg} / \mathrm{L}$

* SD $-100-04$

SD-100-04 12-May-1987 MANGANESE

SD-100-04 18-May-1987 MANGANESE

SD-100-04 26-May-1987 MANGANESE

SD-100-04 3-3un-1987 MANGANESE

SD-100-04 11-Jun-1987 MANGANESE

SD-100-04 18-Jun-1987 MANGANESE

$<0.010 \mathrm{mg} / \mathrm{L}$

$<0.010 \mathrm{mg} / \mathrm{L}$

$<0.010 \mathrm{mg} / \mathrm{L}$

$<0.010 \mathrm{mg} / \mathrm{l}$

$<0.010 \quad \mathrm{mg} / \mathrm{L}$

$<0.010 \mathrm{mg} / \mathrm{L}$

** SD $-100-06$

SD-100-06 12-May-1987 MANGANESE

SD-100-06 18-May-1987 MANGANESE

SD-100-06 26-May-1987 MANGANESE

SD-100-06 3-JUI-1987 MANGANESE

SD-100-06 11-Jun-1987 MANGANESE

$\begin{array}{ll}0.013 & \mathrm{mg} / \mathrm{L} \\ <0.010 & \mathrm{mg} / \mathrm{L} \\ <0.010 & \mathrm{mg} / \mathrm{L} \\ 0.013 & \mathrm{gg} / \mathrm{L} \\ <0.010 & \mathrm{~g} / \mathrm{L}\end{array}$


Storm Drain Data for SD-100

Location

Date

Test Compound

Results

Units

SD-100-06 18-Jun-1987 MANGANESE

$<0.010 \mathrm{mg} / \mathrm{L}$

* SD-100-06D

SD-100-06D 3-Jun-1987 MANGANESE

$<0.010 \mathrm{mg} / \mathrm{L}$

** SD-100-07

SD-100-07 12-Kay-1987 MANGANESE

SD-100-07 19-May-1987 MANGANESE

SD-100-07 26-May-1987 MANGANESE

SD-100-07 3-Jun-1987 MANGANESE

SD-100-07 11-Jun-1987 MANGANESE

SD-100-07 18-Jun-1987 MANGANESE

$\begin{array}{ll}0.33 & \mathrm{mg} / \mathrm{L} \\ 0.020 & \mathrm{mg} / \mathrm{L} \\ 0.017 & \mathrm{mg} / \mathrm{L} \\ 0.037 & \mathrm{mg} / \mathrm{L} \\ 0.025 & \mathrm{mg} / \mathrm{L} \\ 0.049 & \mathrm{mg} / \mathrm{L}\end{array}$

* SD-100-07D

SD-100-07D 18-Jun-1987 MANGANESE

$0.050 \mathrm{mg} / \mathrm{L}$

* SD $100-09$

SD-100-09 12-May-1987 MANGANESE

SD-100-09 18-May-1987 MANGANESE

SD-100-09 26-May-1987 MANGANESE

SD-100-09 3-Jun-1987 MANGANESE

SD-100-09 11-Jun-1987 MANGANESE

** SD-100-10

SD-100-10 12-May-1987 MANGANESE

SD-100-10 19-May-1987 MANGANESE

SD-100-10 26-Kay-1987 MANGANESE

SD-100-10 3-Jun-1987 MANGANESE

SD-100-10 11-Jun-1987 MANGANESE

SD-100-10 18-Jun-1987 MANGANESE

$\begin{array}{ll}0.015 & \mathrm{mg} / \mathrm{L} \\ <0.010 & \mathrm{mg} / \mathrm{L} \\ <0.010 & \mathrm{mg} / \mathrm{L} \\ <0.010 & \mathrm{mg} / \mathrm{L} \\ <0.010 & \mathrm{mg} / \mathrm{L}\end{array}$

* SD $-100-11$

SD-100-11 12-May-1987 MANGANESE

SD-100-11 19-May-1987 MANGANESE

SD-100-11 26-May-1987 MANGANESE

SD-100-11 3-Jun-1987 MANGANESE

SD-100-11 11-Jun-1987 MANGANESE

SD-100-11 18-Jun-1987 MANGANESE

$\begin{array}{ll}<0.010 & \mathrm{mg} / \mathrm{L} \\ <0.010 & \mathrm{mg} / \mathrm{L} \\ <0.010 & \mathrm{mg} / \mathrm{L} \\ 0.034 & \mathrm{mg} / \mathrm{L} \\ <0.010 & \mathrm{mg} / \mathrm{L} \\ <0.010 & \mathrm{mg} / \mathrm{L}\end{array}$

* SD $-100-12$

SD-100-12 12-May-1987 MANGANESE

SD-100-12 19-May-1987 MANGANESE

SD-100-12 26-May-1987 MANGANESE

SD-100-12 3-Jun-1987 MANGANESE

SD-100-12 11-Jun-1987 MANGANESE

SD-100-12 18-Jun-1987 MANGANESE

$<0.010$

$<0.010$

0.046

$<0.010$

0.013

$<0.010$

mg $/ \mathrm{L}$

$\mathrm{mg} / \mathrm{L}$

$\mathrm{mg} / \mathrm{L}$

$\mathrm{mg} / \mathrm{L}$

$\mathrm{mg} / \mathrm{L}$

$\mathrm{mg} / \mathrm{L}$

* SD-100-13

SD-100-13 12-May-1987 MANGANESE

SD-100-13 19-May-1987 MANGANESE

SD-100-13 26-May-1987 MANGANESE

0.16

0.0069

0.039

$<0.010$

0.017

0.025

$<0.010$

0.0059

0.0034
mg/L $\mathrm{mg} / \mathrm{L}$

$\mathrm{mg} / \mathrm{L}$

$\mathrm{mg} / \mathrm{L}$

$\mathrm{mg} / \mathrm{L}$

$\mathrm{mg} / \mathrm{L}$

$\mathrm{mg} / \mathrm{L}$ $\mathrm{mg} / \mathrm{L}$ $\mathrm{mg} / \mathrm{L}$ 
Storm Drain Data for SD-100

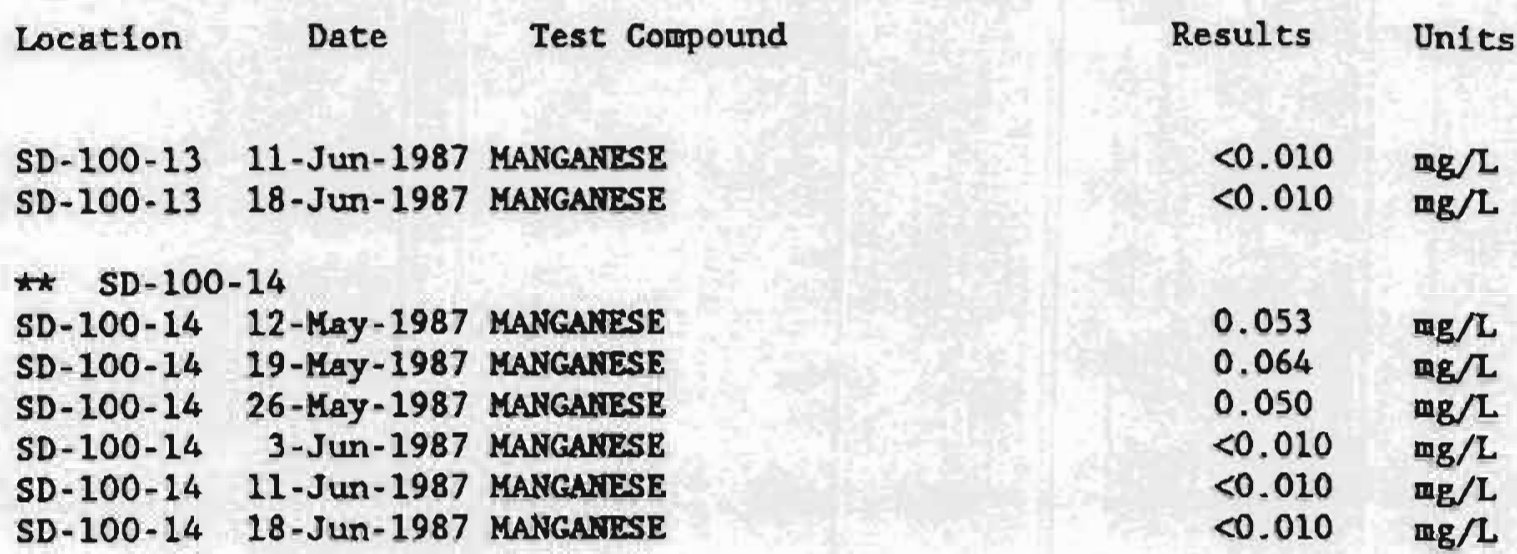

\begin{tabular}{|c|c|c|}
\hline SD $-100-C$ & S-Mar-1987 & MANGANE \\
\hline & 87 & MANGANE \\
\hline & $17-\mathrm{M}$ & MANGANE. \\
\hline & $24-1$ & MANG \\
\hline & $r-1987$ & MANG \\
\hline SD- 10 & 13-May - 1987 & MANGANES \\
\hline$S D-100-C$ & 20-Kay-1987 & MANGANES \\
\hline SD -100 & 27-May-1987 & KANGAN \\
\hline SD $-100-$ & 4-Jun-1987 & MANGA \\
\hline & $12-3$ & MANG \\
\hline & & \\
\hline
\end{tabular}

$0.076 \mathrm{mg} / \mathrm{L}$

$0.047 \mathrm{mg} / \mathrm{L}$

$0.047 \mathrm{mg} / \mathrm{L}$

$0.056 \mathrm{mg} / \mathrm{L}$

$0.024 \mathrm{mg} / \mathrm{L}$

$0.020 \mathrm{mg} / \mathrm{L}$

$0.015 \mathrm{mg} / \mathrm{L}$

$<0.010 \mathrm{mg} / \mathrm{L}$

$<0.010 \mathrm{mg} / \mathrm{L}$

$<0.010 \mathrm{mg} / \mathrm{L}$

$<0.010 \mathrm{mg} / \mathrm{L}$

$\begin{array}{lll}\text { A } & \text { SD-100-D } \\ \text { SD-100-D } & 6-\text { ApI-1987 MANGANESE } \\ \text { SD-100-D } & 11 \text {-JUn-1987 MANGAKESE }\end{array}$

$0.056 \mathrm{mg} / \mathrm{L}$

$0.011 \mathrm{mg} / \mathrm{L}$

* SD-100

SD-100 5-Mar-1987 MERCURY

SD-100 11-Kar-1987 MERCURY

SD-100 18-MaI-1987 MERCURY

SD-100 24-Mar-1987 MERCORY

SD-100 3-Apr-1987 MERCURY

SD-100 6-APr-1987 MERCURY

SD-100 12-May-1987 MERCURY

SD-100 19-May-1987 MERCURY

SD-100 26-May-1987 MERCURY

SD-100 3-Jun-1987 MERCURY

SD-100 11-Jun-1987 MERCURY

SD-100 18-Jun-1987 MERGURY

$<0.0002 \mathrm{mg} / \mathrm{L}$

$<0.0002 \mathrm{mg} / \mathrm{L}$

$<0.0002 \mathrm{mg} / \mathrm{L}$

$<0.0002 \mathrm{mg} / \mathrm{L}$

$<0.0002 \mathrm{mg} / \mathrm{L}$

$<0.0002 \mathrm{mg} / \mathrm{L}$

$<0.0002 \mathrm{mg} / \mathrm{L}$

$<0.0002 \mathrm{mg} / \mathrm{L}$

$<0.0002$ шg/L

$<0.0002 \cdot \mathrm{mg} / \mathrm{L}$

$<0.0002 \mathrm{mg} / \mathrm{L}$

$<0.0002 \mathrm{mg} / \mathrm{L}$

** SD-100-01

SD-100-01 12-May-1987 MERCURY

SD-100-01 18-May-1987 MERCURY

SD-100-01 26-Kay-1987 MERCORY

SD-100-01 3-Jun-1987 MERCURY

SD-100-01 11-Jun-1987 MERCURY

SD-100-01 18-Jun-1987 MERCURY

$<0.0002$

$<0.0002$

$<0.0002$

$\mathrm{mg} / \mathrm{L}$

$<0.0002$

$\mathrm{mg} / \mathrm{L}$

$<0.0002$

$\mathrm{mg} / 2$

$\mathrm{mg} / \mathrm{L}$

$<0.0002$

$\mathrm{mg} / \mathrm{L}$

$m g / L$ 
Storm Drain Data for SD-100

Locaction

Dete

Test Compound

Results

Units

* SD-100-02

SD-100-02 12-May-1987 MERCURY

SD-100-02 18-May-1987 MERRCURY

SD-100-02 26-May-1987 MRRCURY

SD-100-02 3-Jun-1987 MERCJRY

SD-100-02 11-Jun-1987 MERCURY

SD-100-02 18-Jun-1987 MERRCURY

** SD $-100-03$

SD-100-03 12-May-1987 MERCOURY

SD-100-03 18-MRy-1987 MERTCURY

SD-100-03 26-May-1987 MERCURY

SD-100-03 3-JUn-1987 MERCURY

SD-100-03 11-Jun-1987 MERCURY

SD-100-03 18-JUn-1987 MERCURY

* SD-100-03D

SD-100-03D 12-May-1987 MERCURY

SD-100-03D 26-May-1987 MERCURY

$<0.0002$

$<0.0002$

$<0.0002$

$<0.0002$

$<0.0002$

$<0.0002$

$\mathrm{mg} / \mathrm{L}$

$\mathrm{mg} / \mathrm{L}$

$\mathrm{mg} / \mathrm{L}$

$\mathrm{mg} / \mathrm{L}$

mg/L

$\mathrm{mg} / \mathrm{L}$

* SD-100-04

SD-100-04 12-May-1987 MERCURY

SD-100-04 18-May-1987 MERCJRY

SD-100-04 26-May-1987 MERCORY

SD-100-04 3-Jun-1987 MERCURY

SD-100-04 11-Jun-1987 MERCURY

SD-100-04 18-Jun-1987 MERCURY

$<0.0002$

$<0.0002$

$<0.0002$

$<0.0002$

$<0.0002$

$<0.0002$

mg/L

$\mathrm{mg} / \mathrm{L}$

mg/L

$\mathrm{mg} / \mathrm{L}$

$\mathrm{mg} / \mathrm{L}$

$\mathrm{mg} / \mathrm{L}$

$<0.0002$

$<0.0002$

$\mathrm{mg} / \mathrm{L}$

$\operatorname{mg} / \mathrm{L}$

$<0.0002$

$\mathrm{mg} / \mathrm{L}$

$<0.0002$

$\mathrm{mg} / \mathrm{L}$

$<0.0002$

$\mathrm{mg} / \mathrm{L}$

$<0.0002$

$\mathrm{mg} / \mathrm{L}$

$<0.0002$

$\mathrm{mg} / \mathrm{L}$

$<0.0002$

$\mathrm{mg} / \mathrm{L}$

** SD $-100-06$

SD-100-06 12-May-1987 MERCURY

SD-100-06 18-May-1987 MERCURY

SD-100-06 26-May-1987 MERCURY

SD-100-06 3-Jun-1987 MERCURY

SD-100-06 11-Jun-1987 MERCURY

$S D=100-06 \quad 18-J$ Un-1987 MERCURY

$<0.0002$

$<0.0002$

$<0.0002$

$<0.0002$

$<0.0002$

$<0.0002$

$\mathrm{mg} / \mathrm{L}$

$\mathrm{mg} / \mathrm{L}$

$\mathrm{mg} / \mathrm{L}$

$\mathrm{mg} / \mathrm{L}$

$\mathrm{mg} / \mathrm{L}$

$\mathrm{mg} / \mathrm{L}$

$\star \star$ SD $-100-06 D$

SD-100-06D 3-Jun-1987 MERCORY

$<0.0002 \mathrm{mg} / \mathrm{L}$

** SD $-100-07$

SD-100-07 12-May-1987 MERGURY

SD-100-07 19-May-1987 MERCURY

SD-100-07 26-May-1987 MERCORY

SD-100-07 3-Jun-1987 MERCURY

SD-100-07 11-Jun-1987 MERCURY

SD-100-07 18-Jun-1987 MERCURY

$0.493 \mathrm{mg} / \mathrm{L}$

$0.036 \mathrm{mg} / \mathrm{L}$

$0.058 \mathrm{mg} / \mathrm{L}$

$0.33 \mathrm{mg} / \mathrm{L}$

$0.0031 \mathrm{mg} / \mathrm{L}$

$0.051 \mathrm{mg} / \mathrm{L}$

** SD-100-07D

SD-100-07D 18-Jun-1987 MERCURY

$0.065 \mathrm{mg} / \mathrm{L}$ 
Storm Drain Data for SD-100

Location
** SD-100-09

SD-100-09 12-May-1987 MERCORY

SD-100-09 1B-May-1987 MERCURY

SD-100-09 26-May-1987 MERCURY

SD-100-09 3-Jun-1987 MERCURY

SD-100-09 11-Jun-1987 LERRCURY

SD-100-09 18-Jun-1987 KEERCORY

Results Units

* SD-100-10

SD-100-10 12-May-1987 MERCURY

SD-100-10 19-Kay-1987 MERCURY

SD-100-10 26-May-1987 MERCURY

SD-100-10 3-Jun-1987 MERCURY

SD-100-10 11-Jun-1987 MERCURY

SD-100-10 18-Jun-1987 MERCURY

0.0007

0.0009

0.0008

0.0021

0.0005

0.0009

mg/L

$\mathrm{mg} / \mathrm{L}$

$\mathrm{mg} / \mathrm{L}$

$\mathrm{mg} / \mathrm{L}$

$\mathrm{mg} / \mathrm{L}$

$\mathrm{mg} / \mathrm{L}$

** SD-100-11

SD-100-11 12-May-1987 MERCURY

SD-100-11 19-May-1987 MERCURY

SD-100-11 26-May-1987 MERCURY

SD-100-11 3-Jun-1987 MERGURY

SD-100-11 11-Jun-1987 MERCURY

SD-100-11 18-Jun-1987 MERCURY

$<0.0002$

$<0.0002$

$<0.0002$

0.0004

$<0.0002$

$<0.0002$

$\mathrm{mg} / \mathrm{L}$

$\mathrm{mg} / \mathrm{L}$

$\mathrm{mg} / \mathrm{L}$

$\mathrm{mg} / \mathrm{L}$

$\mathrm{mg} / \mathrm{L}$

$\mathrm{mg} / \mathrm{L}$

$<0.0002$

$<0.0002$

$<0.0002$

$<0.0002$

$<0.0002$

$<0.0002$

$\mathrm{mg} / \mathrm{L}$

$\mathrm{mg} / \mathrm{L}$

$\mathrm{mg} / \mathrm{L}$

$\mathrm{mg} / \mathrm{L}$

$\mathrm{mg} / \mathrm{L}$

$\mathrm{mg} / \mathrm{L}$

** SD $-100-12$

SD-100-12 12-May-1987 MERCURY

SD-100-12 19-May-1987 MIRRCURY

SD-100-12 26-May-1987 MERCURY

SD-100-12 3-Jum-1987 MERCURY

SD-100-12 11-Jun-1987 KERCURY

SD-100-12 18-Jun-1987 MERCURY

$<0.0002$

$<0.0002$

$<0.0002$

$<0.0002$

$<0.0002$

$<0.0002$

$\mathrm{mg} / \mathrm{L}$

$\mathrm{mg} / \mathrm{L}$

$\mathrm{mg} / \mathrm{L}$

$\mathrm{mg} / \mathrm{L}$

$\mathrm{mg} / \mathrm{L}$

ㄸg/L

** SD-100-13

SD-100-13 12-May-1987 MERCURY

SD-100-13 19-May-1987 MERCURY

SD-100-13 26-May-1987 MERCURY

SD-100-13 3-Jun-1987 MERCURY

SD-100-13 11-Jun-1987 MERCURY

SD-100-13 18-Jun-1987 MERCORY

$<0.0002$

0.0003

$<0.0002$

$<0.0002$

$<0.0002$

0.0002

$\mathrm{mg} / \mathrm{L}$

ㅁg/L

$\mathrm{mg} / \mathrm{L}$

$\mathrm{mg} / \mathrm{L}$

$\mathrm{mg} / \mathrm{L}$

$\mathrm{mg} / \mathrm{L}$

** SD-100-14

SD-100-14 12-May-1987 MERCURY

SD-100-14 19-May-1987 MERRCURY

SD-100-14 26-May-1987 MERCURY

SD-100-14 3-Jun-1987 MERCURY

SD-100-14 11-Jun-1987 MERCURY

SD-100-14 18-Jun-1987 MERCURY

$<0.0002$

$<0.0002$

$\mathrm{mg} / \mathrm{L}$

$<0.0002$

$\mathrm{mg} / \mathrm{L}$

$<0.0002$

$\mathrm{mg} / \mathrm{L}$

$<0.0002$

$\mathrm{mg} / \mathrm{L}$

$<0.0002$

$\mathrm{mg} / \mathrm{L}$

* SD $-100-C$

SD-100-C 5-Mar-1987 MERCURY

SD-100-C 9-Mar-1987 MERCURY

$<0.0002$

$<0.0002$

mg/L

$\mathrm{mg} / \mathrm{L}$ 
Storm Drain Data for SD-100

Location

Dace

Test Compound

Results

Units

$\begin{array}{lrl}\text { SD-100-C } & 17 \text {-Mar-1987 } & \text { MERCURY } \\ \text { SD-100-C } & 24-\text { Mar-1987 } & \text { MERCURY } \\ \text { SD-100-C } & 8 \text {-Apr-1987 } & \text { MERCURY } \\ \text { SD-100-C } & 13 \text {-May-1987 } & \text { MERCURY } \\ \text { SD-100-C } & 20 \text {-May-1987 } & \text { MERCURY } \\ \text { SD-100-C } & 27-\text { May-1987 } & \text { MERCURY } \\ \text { SD-100-C } & 4-J u n-1987 & \text { MERCURY } \\ \text { SD-100-C } & 12-J u n-1987 & \text { MERCURY } \\ \text { SD-100-C } & 19-J u n-1987 & \text { MERCURY }\end{array}$

$<0.0002$

$<0.0002$

$<0.0002$

$<0.0002$

$<0.0002$

$<0.0002$

$<0.0002$

$<0.0002$

$<0.0002$

$\mathrm{mg} / \mathrm{L}$

$\operatorname{mg} / \mathrm{L}$

$m g / L$

mg $/ \mathrm{L}$

$\mathrm{mg} / \mathrm{L}$

$\mathrm{mg} / \mathrm{L}$

$m g / L$

$\mathbf{m g} / \mathrm{L}$

$\mathrm{mg} / \mathrm{L}$

** SD-100-D

SD-100-D 6-Apr-1987 MERCURY

$<0.0002$

$m g / L$

SD-100-D 11-Jun-1987 MERCURY

$<0.0002$

mg/L

** SD 100

SD-100 5-Mar-1987 METHYLENE CHLORIDE

SD-100 11-Mar-1987 METHYLENE CHLORIDE

SD-100 18-Mar-1987 METHYLENE CHLORIDE

SD-100 24-Mar-1987 METHYLENE CHLORIDE

SD -100

6-APE-1987 METHYLENE CHLORIDE

SD -100

12-May-1987 METHYLENE CHLORIDE

SD -100

19-May-1987 METHYLENE CHLORIDE

SD -100

26-MaY-1987 METHYLFNE CHLORIDE

SD -100

3-JUn-1987 METHYLENE CHLORIDE

SD -100

11-JUn-1987 METHYLENE GHLORIDE

SD -100

18-JUn-1987 METHYLENE CHLORIDE

* SD-100-01

SD-100-01 12-Kay-1987 METHYLENE CHLORIDE SD-100-01 18-May-1987 METHYENE CHLORIDE SD-100-01 26-May-1987 METHYLENE CHLORIDE

SD-100-01 3-Jun-1987 METHYLENE CHLORIDE

SD-100-01 11-Jun-1987 METHYLENE CHLORIDE

SD-100-01 18-Jun-1987 METHYLENE GHLORIDE

no SD $-100-02$

SD-100-02 12-May-1987 METHYLNE CHLORIDE

SD-100-02 18-May-1987 METHYLENE CHLORIDE

SD-100-02 26-May-1987 METHYLENE CHLORIDE

SD-100-02 3-Jun-1987 METHYLENE CHLORIDE

SD-100-02 11-Jun-1987 METHYLENE CHLORIDE

SD-100-02 18-JUn-1987 METHYLENE CHLORIDE

5

12

$<5$

$<5$

15

$<5$

11

$<5$

27

38

ug/L

ug/L

ug/L

ug/L

$4 \mathrm{~g} / \mathrm{L}$

$u g / L$

$u g / 2$

ug/I

$48 / 2$

ug/L

ug/L

$<5$

$<5$

$<5$

$<5$

$<5$

$<5$

$\mathrm{ug} / \mathrm{L}$

$\mathrm{ug} / \mathrm{L}$

ug/L

$\mathrm{ug} / \mathrm{L}$

ug/L

ug/L

** SD $-100-03$

SD-100-03 12-May-1987 METHYLENE CHLORIDE

SD-100-03 18-May-1987 METHYLENE CHLORIDE

SD-100-03 26-May-1987 METHYLENE CHLORIDE

SD-100-03 3-Jun-1987 METHYLENE CHLORIDE

SD-100-03 11-JUn-1987 METHYLENE CHLORIDE

SD-100-03 18-JUR-1987 METHYLENE CHLORIDE

$\begin{array}{ll}<5 & \mathrm{ug} / \mathrm{L} \\ <5 & \mathrm{ug} / \mathrm{L} \\ <5 & \mathrm{ug} / \mathrm{L} \\ <5 & \mathrm{ug} / \mathrm{L} \\ <5 & \mathrm{ug} / \mathrm{L} \\ <5 & \mathrm{ug} / \mathrm{L} \\ & \\ & \\ <5 & \mathrm{ug} / \mathrm{L} \\ <5 & \mathrm{ug} / \mathrm{L} \\ <5 & \mathrm{ug} / \mathrm{L} \\ <5 & \mathrm{ug} / \mathrm{L} \\ <5 & \mathrm{ug} / \mathrm{L} \\ <5 & \mathrm{ug} / \mathrm{L}\end{array}$


Storm Drain Data for SD-100

Location Date Test Compound Results Units

** SD-200-03D

SD-100-03D 12-May-1987 METHYLENE CHLORIDE

SD-100-03D 26-May-1987 METHYLENE GHLORIDE

** SD $-100-04$

SD-100-04 12-May-1987 METHYLENE CHLORIDE

SD-100-04 18-May-1987 KETHYLENE CHLORIDE

SD-100-04 26-May-1987 METHYLENE CHLORIDE

SD-100-04 3-Jun-1987 METTYLENE CHLORIDE

SD-100-04 11-Jun-1987 METHYLENE CHLORIDE

SD-100-04 18-Jun-1987 METHYLENE CHLORIDE

** SD $-100-06$

SD-100-06 12-May-1987 METHYLRNE CHLORIDE

SD-100-06 18-May-1987 METHYLENE CHLORIDE

SD-100-06 26-May-1987 METHYLENE CHLORIDE

SD-100-06 3-JUn-1987 METHYLENE CHLORIDE

SD-100-06 11-JUn-1987 METHYLENE CHLORIDE

SD-100-06 18-Jun-1987 METHYLENE CHLORIDE

$\begin{array}{ll}<5 & u g / L \\ <5 & 4 \mathrm{ug} / \mathrm{L} \\ & \\ & \\ <5 & \mathrm{ug} / \mathrm{L} \\ <5 & \mathrm{ug} / \mathrm{L} \\ <5 & \mathrm{ug} / \mathrm{L} \\ <5 & \mathrm{ug} / \mathrm{L} \\ <5 & \mathrm{ug} / \mathrm{L} \\ <5 & \mathrm{ug} / \mathrm{L}\end{array}$

* SD-100-06D

SD-100-06D 3-JUn-1987 METHYLENE CHLORIDE

** SD-100-07

SD-100-07 12-MaY-1987 METHYLENE CHLORIDE

SD-100-07 19-May-1987 METHYLENE CHLORIDE

SD-100-07 26-May-1987 METHYLENE CHLORIDE

SD-100-07 3-Jun-1987 METHYLENE CHLORIDE

SD-100-07 11-JUn-1987 METHYLENE CHLORIDE

SD-100-07 18-Jun-1987 METHYLENE CHLORIDE

* SD-100-07D

SD-100-07D 18-JUn-1987 METHYLENE CHLORIDE

$<5 \quad$ ug/L

** SD-100-09

SD-100-09 12-MaY-1987 METHYLENE CHLORIDE

SD-100-09 18-May-1987 METHYLENE CHLORIDE

SD-100-09 26-May-1987 METHYLENE CHLORIDE

SD-100-09 3-JUn-1987 METHYLENE CHLORIDE

SD-100-09 11-JUn-1987 METHYLENE CHLORIDE

SD-100-09 18-JUN-1987 METHYLENE GHLORIDE

$\begin{array}{ll}<5 & u g / L \\ <5 & u g / L \\ 5 & u g / L \\ 5 & u g / L \\ <5 & u g / L \\ <5 & u g / L\end{array}$

5

ug/l

$\begin{array}{ll}<5 & 4 g / L \\ <5 & u g / L \\ <5 & u g / L \\ <5 & 4 g / L \\ <5 & u g / L \\ <5 & 4 g / L\end{array}$

* SD $-100-10$

SD-100-10 12-May-1987 METHYLENE CHLORIDE

SD-100-10 19-MaY-1987 METHYLENE CHLORIDE

SD-100-10 26-May-1987 METHYLENE CHLORIDE

SD-100-10 3-J Un-1987 HETHYLENE CHLORIDE

SD-100-10 11-JUn-1987 METHYLENE CHLORIDE

SD-100-10 18-JUn-1987 METHYLENE CHLORIDE 
Storm Drain Data for SD-100

Location

Date

Test Compound

Results

Units

* SD-100-11

SD-100-11 12-May-1987 METHYLPIE CHLORIDE

SD-100-11 19-May-1987 METIYLENE GHORIDE

SD-100-11 26-May-1987 METTYLBVE CHLORIDE

SD-100-11 3-Jun-1987 METHYLINE CHLORIDE

SD-100-11 11-Jun-1987 METHYLRNE CHLORIDE

SD-100-11 18-Jun-1987 METHYLANE GLORIDE

** SD $-100-12$

SD-100-12 12-Kay-1987 METHYLENE CHLORIDE

SD-100-12 19-May-1987 METHYLNE CHLORIDE

SD-100-12 26-May-1987 METHYLFNE CHLORIDE

SD-100-12 3-Jun-1987 METHYLENE CHLORIDE

SD-100-12 11-JUn-1987 METHYLENE CHLORIDE

SD-100-12 18-Jun-1987 METHYLENE CHLORIDE

** SD $-100-13$

SD-100-13 12-May-1987 METHYLFNE CHLORIDE

SD-100-13 19-May-1987 METHYLENE CHLORIDE

SD-100-13 26-May-1987 METHYLENE CHLORIDE

SD-100-13 3-Jun-1987 METHYLFNE CHLORIDE

SD-100-13 11-JUn-1987 METHYLENE CHLORIDE

SD-100-13 18-Jun-1987 METHYYLENE CHLORIDE

$\begin{array}{ll}<5 & u g / L \\ <5 & u g / L \\ 5 & u g / L \\ 11 & u g / L \\ 5 & u g / L \\ <5 & u g / L\end{array}$

* SD-100-14

SD-100-14 12-May-1987 METHYLENE CHLORIDE

SD-100-14 19-May-1987 METHYLENE CHLORIDE

SD-100-14 26-Ylay-1987 METHYLENE CHLORIDE

SD-100-14 3-Jun-1987 METHYLENE CHLORIDE

SD-100-14 11-JUn-1987 METHYLENE CHLORIDE

SD-100-14 18-Jun-1987 METHYLFNE CHLORIDE

$\begin{array}{ll}<5 & u g / L \\ <5 & u g / L \\ <5 & u g / L \\ <5 & u g / L \\ <5 & u g / L \\ <5 & 4 g / L\end{array}$

$\begin{array}{ll}<5 & u g / L \\ 5 & u g / L \\ 87 & u g / L \\ 100 & u g / L \\ 33 & u g / L \\ 7 & u g / L\end{array}$

$<5$

$<5$

ug/L

$<5 \quad$ ug/

$<5 \quad$ ug/

$<5$

$<5$

ug $/$ L

ug/L

** SD- $100-C$

SD-100-C 5-Mar-1987 METHYLENE CHLORIDE

SD-100-C 9-MaT-1987 METHXLENE CHLORIDE

SD-100-C 17-Mar-1987 METHYLENE CHLORIDE

SD-100-C 24-MaI-1987 METHYLENE CHLORIDE

SD-100-C 8-APT-1987 METHYLENE CHLORIDE

SD-100-C 13-May-1987 METHYLINE CHLORIDE

SD-100-C 20-May-1987 METHYLDNE CHLORIDE

SD-100-C 27-May-1987 METTYLENE CHLORTDE

SD-100-C 12-JUn-1987 METHYLENE CHLORIDE

SD-100-C 19-Jun-1987 METHYLENE CHLORIDE

* SD-100-D

SD-100-D 6-APY-1987 METHYLENE CHLORIDE

SD-100-D 11-JUn-1987 METHYLENE CHLORIDE

6

$<5$

ug/L

5

$5 \mathrm{~J}$

$<5$

12

6

11

5

73

ug/L

ug $/ \mathrm{L}$

ug/L

ug/L

ug/L

ug/L

ug/L

ug/L

ug $/ \mathrm{L}$

* SD -100

SD-100 5-Mar-1987 MOLYBDENUM

SD-100 11-MaT-1987 MOLYBDENUM

$<0.010 \quad \mathrm{mg} / \mathrm{L}$ 
Storm Drain Data for SD-100

\begin{tabular}{|c|c|c|c|}
\hline Location & Date & Test Compound & Results \\
\hline SD -100 & $18-\mathrm{Kar}-1987$ & MOLYBDENUY & $<0.010$ \\
\hline SD- 100 & 24-Mar- 1987 & MOLYBDENUY & $<0.010$ \\
\hline SD- 100 & 3-Apr-1987 & MOLYBDENUM & $<0.010$ \\
\hline$S D-100$ & 6-Apr-1987 & MOLYBDENOX & 0.015 \\
\hline SD- 100 & 12-May-1987 & MOLYBDENOM & $<0.010$ \\
\hline SD- 100 & 19-Kay-1987 & MOLYBDEANUM & $<0.010$ \\
\hline SD-100 & 26-May-1987 & MOLYBDENUM & $<0.010$ \\
\hline SD- 100 & 3-Jun-1987 & MOLYBDENOM & $<0.010$ \\
\hline$S D-100$ & 11-Jun-1987 & MOLYBDENTM & $<0.010$ \\
\hline SD -100 & $18-J u n-1987$ & MOLYBDENUM & $<0.010$ \\
\hline
\end{tabular}

* SD-100-01

SD-100-01 12-May-1987 MOLYBDENUM

SD-100-01 18-May-1987 MOLYBDENTM

SD-100-01 3-Jun-1987 MOLYBDENUM

SD-100-01 11-Jun-1987 MOLYBDENUM

SD-100-01 18-Jun-1987 MOLYBDENUY

$\begin{array}{ll}<0.010 & \mathrm{mg} / \mathrm{L} \\ 0.017 & \mathrm{mg} / \mathrm{L} \\ 0.025 & \mathrm{mg} / \mathrm{L} \\ <0.010 & \mathrm{mg} / \mathrm{L} \\ <0.010 & \mathrm{mg} / \mathrm{L}\end{array}$

* SD $-100-02$

SD-100-02 12-May-1987 MOLYBDENUM

SD-100-02 18-May-1987 MOLYBDENUM

SD-100-02 3-Jun-1987 MOLYBDENUM

SD-100-02 11-Jun-1987 MOLYBDENOM

SD-100-02 18-Jun-1987 MOLYBDENUM

$<0.010 \mathrm{ng} / \mathrm{L}$

$0.010 \mathrm{mg} / \mathrm{L}$

$0.016 \mathrm{mg} / \mathrm{L}$

$<0.010 \mathrm{mg} / \mathrm{L}$

$<0.010 \mathrm{ng} / \mathrm{L}$

* SD-100-03

SD-100-03 12-Kay-1987 MOLYBDENOM

SD-100-03 18-May-1987 MOLYBDENOM

SD-100-03 26-Kay-1987 KOLYBDENUM

SD-100-03 3-Jun-1987 MOLYBDETM

SD-100-03 11-Jun-1987 MOLYBDENOM

SD-100-03 18-Jun-1987 MOLYBDENUM

$<0.010 \quad \mathrm{gg} / \mathrm{L}$

$0.012 \mathrm{mg} / \mathrm{L}$

$0.029 \mathrm{mg} / \mathrm{L}$

$<0.010 \quad \mathrm{gg} / \mathrm{L}$

$<0.010 \mathrm{mg} / \mathrm{L}$

$<0.010 \quad \mathrm{ng} / \mathrm{L}$

* SD-100-03D

SD-100-03D 12-May-1987 MOLYBDEANUM

$<0.010 \quad \mathrm{mg} / \mathrm{L}$

SD-100-03D 26-May-1987 MOLYBDENUM

$0.016 \mathrm{ng} / \mathrm{L}$

** SD- $100-04$

SD-100-04 12-May-1987 MOLYBDENUM

SD-100-04 18-May-1987 MOLYBDENUT

SD-100-04 26-May-1987 MOLYBDENOM

SD-100-04 3-Jun-1987 MOLYBDENUM

SD-100-04 11-Jun-1987 MOLYBDENUM

SD-100-04 18-Jun-1987 MOLYBDENUM

$<0.010 \mathrm{mg} / \mathrm{L}$

$<0.010 \mathrm{ng} / \mathrm{L}$

$<0.010 \quad \mathrm{gg} / \mathrm{L}$

$0.012 \quad \mathrm{ng} / \mathrm{L}$

$<0.010 \quad \mathrm{ng} / \mathrm{L}$

$<0.010 \mathrm{mg} / \mathrm{L}$

** SD - 100-06

SD-100-06 12-May-1987 MOLYBDENUM

$<0.010 \mathrm{mg} / \mathrm{L}$

SD-100-06 18-May-1987 MOLYBDENUM

$<0.010$

$\mathrm{ag} / \mathrm{L}$

SD-100-06 26-May-1987 MOLYBDENUM

0.012

$m g / L$

$S D-100-06$

3-JUn-1987 MOLYBDENUM

SD-100-06

11-Jun-1987 MOLYBDENUM

0.014

$<0.010$

$\mathrm{mg} / \mathrm{L}$

$\mathrm{mg} / \mathrm{L}$ 
Storm Drain Data for SD-100

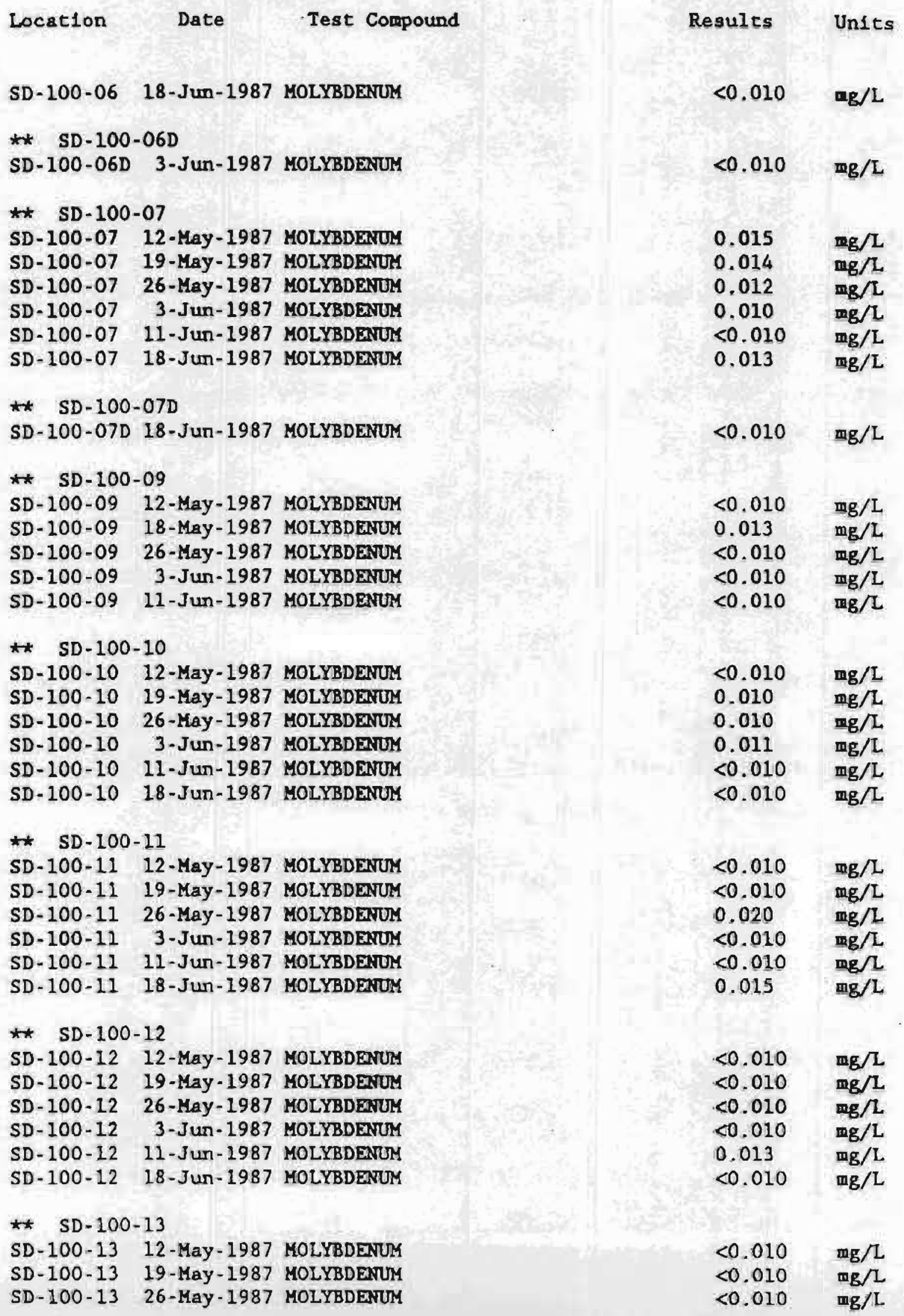


Stora Drain Data for SD-100

\begin{tabular}{|c|c|c|}
\hline ocation & Date & Test \\
\hline $\begin{array}{l}S D-100-13 \\
S D-100-13\end{array}$ & $\begin{array}{l}11-J u n-1987 \\
18-J u n-1987\end{array}$ & \\
\hline \multicolumn{3}{|c|}{ ** SD $-100-14$} \\
\hline SD- $100-14$ & $12-\mathrm{K}_{8}$ & MOL \\
\hline D $-100-14$ & 19-Mzy - 1987 & MOLY \\
\hline SD-100-14 & 26-May-1987 & MOLYI \\
\hline SD-100-14 & 3-Jun-1987 & MOLYB \\
\hline$S D-100-14$ & 11-Jun-1987 & \\
\hline & 8-Jun-1987 & \\
\hline
\end{tabular}

$\begin{array}{lr}\text { Results } & \text { Units } \\ <0.010 & \mathrm{mg} / \mathrm{L} \\ <0.010 & \mathrm{mg} / \mathrm{L} \\ & \\ & \\ <0.010 & \mathrm{mg} / \mathrm{L} \\ 0.023 & \mathrm{mg} / \mathrm{L} \\ <0.010 & \mathrm{mg} / \mathrm{L} \\ <0.010 & \mathrm{mg} / \mathrm{L} \\ <0.010 & \mathrm{mg} / \mathrm{L} \\ <0.010 & \mathrm{mg} / \mathrm{L}\end{array}$

\begin{tabular}{|c|c|c|}
\hline$S D-100-C$ & $5-\mathrm{Mar}-1987$ & MOLYBDENUM \\
\hline$S D-100-C$ & 9 -Mar-1987 & MOLYBDENUM \\
\hline$S D-100-C$ & 17-Mar- 1987 & MOLYBDENUM \\
\hline$D-100-C$ & $24-\operatorname{Mar}-1987$ & MOLYBDENUM \\
\hline$D-100-C$ & 8-Арг-1987 & MOLYBDENUM \\
\hline BD $-100-C$ & 13-May-1987 & MOLYBDENUM \\
\hline SD- $100-C$ & 20-May-1987 & MOLYBDENUM \\
\hline SD- $100 \cdot C$ & 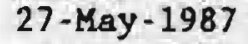 & MOLYBDENUM \\
\hline$S D-100-C$ & 4-Jun-1987 & MOLYBDENUM \\
\hline$S D-100-C$ & $12-J u n-1987$ & MOLYBDENUM \\
\hline & $19-J u n-1987$ & MOLYBDE \\
\hline
\end{tabular}

$<0.010$

$<0.010$

$<0.010$

$<0.010$

$<0.010$

$<0.010$

0.018

0.021

0.010

0.022

$<0.010$

$\mathrm{mg} / \mathrm{L}$

$\mathrm{mg} / \mathrm{L}$

$\mathrm{mg} / \mathrm{L}$

$\mathrm{mg} / \mathrm{L}$

$\mathrm{mg} / \mathrm{L}$

$\mathrm{mg} / \mathrm{L}$

$\mathrm{mg} / \mathrm{L}$

$\operatorname{mg} / \mathrm{L}$

$\mathrm{mg} / \mathrm{L}$

$\mathrm{mg} / \mathrm{L}$

$S D-100-C$

19-Jun-1987 MOLYBDENUM

$\mathrm{mg} / \mathrm{L}$

* $\$$ SD $-100-D$

SD-100-D 6-APL-1987 MOLYBDENUM

SD-100-D 11-JUn-1987 MOLYBDENUM

$<0.010$

$<0.010$

$\mathrm{mg} / \mathrm{L}$

$\mathrm{mg} / \mathrm{L}$

* SD-100-07

SD-100-07 12-May-1987 MYRISTIC ACID

85

$\mathrm{ug} / \mathrm{L}$

$\star$ SD -100

SD-100 5-Mar-1987 N-NITROSO-DI-N-PROPYLAMINE

SD-100 11-Mar-1987 N-NITROSO-DI-N-PROPYLAMINE

SD-100 12-May-1987 N-NITROSO-DI-N-PROPYLAMINE

SD-100 26-May-1987 N-NITROSO-DI-N-PROPYLAMINE

* SD-100-01

SD-100-01 12-May-1987 N-NITROSO-DI-N-PROPYLAMINE

SD-100-01 26-May-1987 N-NITROSO-DI-N-PROPYLAMINE

* SD $100-02$

SD-100-02 12-Kay-1987 N-MITROSO-DI-N-PROPYLAMINE

SD-100-02 26-May-1987 N-NITROSO-DI-N-PROPYLAMINE

* SD-100-03

SD-100-03 12-May-1987 N-NITROSO-DI-N-PROPYLAMINE

SD-100-03 26-May-1987 N-NITROSO-DI-N-PROPYLAMINE

$<10$

$<5.0$

ug/L

$<10$

$<10$

$<10$

$<5.0$

$\mathrm{ug} / \mathrm{L}$

$u g / L$

ug/L

ug/L

** SD-100-03D

SD-100-O3D 12 -May-1987 N-NITROSO-DI-N-PROPYLAMINE

$<10$

ug/L 
** SD-100-04

SD-100-04 12-May- 1987 N-NITROSO-DI-N-PROPYLAKTHE SD-100-04 26-May-1987 N-NITROSO-DI-N-PROPYLAKIIRE

SD-100-06 12-May-1987 N-NITROSO-DI-N-PROPYLARTHE SD-100-06 26-May-1987 N-NITROSO-DI-N-PROPYLAYIIE

** SD-100-07

SD-100-07 12-May-1987 N-NITROSO-DI-N-PROPYLAMTNE SD-100-07 26-May-1987 N-NITROSO-DI-N-PROPYLAMTEE

* SD-100-09

SD-100-09 12-May-1987 N-NITROSO-DI-N-PROPYLAMINE SD-100-09 26-Hay-1987 N-NITROSO-DI-N-PROPYLAYINE

** SD $-100-10$

SD-100-10 12-May-1987 N-NITROSO-DI-N-PROPYLAMIIIE SD-100-10 26-Kay-1987 N-NITROSO-DI-N-PROPYLAMIIE

* SD $-100-11$

SD-100-11 12-Kay-1987 N-NITROSO-DI -N-PROPYLARTIE SD-100-11 26-May-1987 N-NITROSO-DI-N-PROPYLAKTINE

** SD $-100-12$

SD-100-12 12-May-1987 N-NITROSO-DI-N-PROPYLAKIIE SD-100-12 26-May-1987 N-NITROSO-DI-N-PROPYLAMINE

** SD $-100-13$

SD-100-13 12-May-1987 N-NITROSO-DI-N-PROPYLAYINE SD-100-13 26-May-1987 N-NITROSO-DI-N-PROPYLAKINE

* SD-100-14

SD-100-14 12-May-1987 N-NITROSO-DI -N-PROPYLANTNE SD-100-14 26-May-1987 N-NITROSO-DI-N-PROPYLAMENE

* $S D-100-C$

SD-100-C 5-Mar-1987 N-NITROSO-DI-N-PROPYLARTIT

SD-100-C 9-Mar-1987 N-NITROSO-DI-N-PROPYLAMTRE

SD-100-C 17-Mar-1987 N-NITROSO-DI-N-PROPYLAMIIRE

SD-100-C 24-Mar-1987 N-NITROSO-DI-N-PROPYLAMTEE

$S D-100-C$

8-API-1987 N-NITROSO-DI - N-PROPYLAHINE

SD $-100-C$ 13-May- 1987 N-NITROSO-DI-N-PROPYLAIINE

$S D-100-C$ 20-May-1987 N-NITROSO-DI - N - PROPYLAMTNE

$S D-100-C$ 27-May- 1987 N-NITROSO-DI-N-PROPYLAMTNE

$S D-100-C$ 12-JUn- 1987 N-NITROSO-DI-N-PROPYLAMINE SD-100-C 19-Jun-1987 N-NITROSO-DI-N-PROPYLAMINE

$<10$

$<5.0$

$<10$

$<5.0$

$<10$

$<5.0$

$<10$

$<5.0$

$<10$

$<5.0$

$<10$

$<5.0$

$<10$

$<5.0$

$<10$

$<5.0$

$<10$

$<10$

$<10$

$<10$

$<10$

$<5.0$

$<5.0$

$<5.0$

$<5.0$

$<10$
ug/L

ug/L

$u g / L$

ug/L ug/L

ug/L ug/L

ug/L ug/L

ug/L $u g / L$

ug $/$ L ug/L

ug/L ug/L

ug $/$ ug/L

ug/L ug/L ug/L $\mathrm{ug} / \mathrm{L}$ ug/L ug/L $4 \mathrm{~g} / \mathrm{L}$ ug/L ug/L ug/L 
** SD -100

SD- 100 5-KaT-1987 N-NITROSODIMETHYLAMTNE SD-100 11-Mar-1987 N-NITROSODIMETHYLAMUNE SD-100 12-May-1987 N-NITROSODIMETHYLAMINE SD-100 26-May-1987 N-NITROSODIMETHYLAMINE

* SD-100-01

SD-100-01 12-Kay-1987 N-NITROSODIMETHYLAKINE SD-100-01 26-Hay-1987 N-NITROSODIMETHYLAMINE

** SD $-100-02$

SD-100-02 12-Kay-1987 N-NITROSODIMETHYLAMINE SD-100-02 26-May-1987 N-NITROSODIMETHYLAMINE

* SD-100-03

SD-100-03 12-May-1987 N-NITROSODIMETHYLAMINE SD-100-03 26-May-1987 N-NITROSODIMETHYLAMINE

* SD-100-03D

SD-100-03D 12-Kay-1987 N-NITROSODIMETHYLAKTNE SD-100-03D 26-May-1987 N-NITROSODIMETHYLAMINE

** SD-100-04

SD-100-04 12-May-1987 N-NITROSODIMETHYLAMLIE SD-100-04 26-May-1987 N-NITROSODIMETHYLAMINE

** SD $-100-06$

SD-100-06 12-May-1987 N-NITROSODIMETHYLAMINB

SD-100-06 26-May-1987 N-NITROSODIMETHYLAMINE

** SD-100-07

SD-100-07 12-Kay-1987 N-NITROSODIMETHYLAMINE SD-100-07 26-May-1987 N-NITROSODIMETHYLAMINE

$\star \star$ SD-100-09

SD-100-09 12-May-1987 N-NITROSODIMETHYLAYINE

SD-100-09 26-Kay-1987 N-NITROSODIMETHYLAMINE

* SD $-100-10$

SD-100-10 12-May-1987 N-NITROSODIMETHYLAMINE

SD-100-10 26-May-1987 N-NITROSODIMETHYLAMUNE

* SD-100-11

SD-100-11 12-May-1987 N-NITROSODIMETHYLAMINE

SD-100-11 26-May-1987 N-NITROSODIMETHYLAHINE

** SD - 100-12

SD-100-12 12-Hay-1987 N-NITROSODIMETHYLAMINE

SD-100-12 26-May-1987 N-NITROSODIMETHYLAMINE

$<10$

$<10$

$<10$

$<5.0$

$<10$

$<5.0$

$<10$

$<5.0$

$<10$

$<5.0$

$<10$

$<5.0$

$<10$

$<5.0$

$<10$

$<5.0$

$<10$

$<5.0$

$<10$

$<5.0$

ug/L

ug/L

$<10$

$<5.0$

ug $/ L$

ug $/ 2$

$<10$

$<5.0$

ug/L ug/L

$<10$

$<5.0$
ug/L ug/L 
« SD-100-13

SD-100-13 12-May-1987 N-NITROSODIMETHYLAMINE

SD-100-13 26-Kay-1987 N-NITROSODIMETHYAMINE

* SD-100-14

SD-100-14 12-May-1987 N-NITROSODIMETHYLAMINE

SD-100-14 26-May-1987 N-NITROSODIMETHYAMINE

* SD-100-C

SD-100-C 5-Mar-1987 N-NITROSODIMETHYLAMINE

SD-100-C 9-Mar-1987 N-NITROSODIMETHYLAMINE

SD-100-C 17-MaI-1987 N-NITROSODIMETHYLAMTNE

SD-100-C 24-MaI-1987 N-NITROSODIMETHYLAMINE

SD-100-C 8-Apr-1987 N-NITROSODIMETHYLAMINE

SD-100-C 13-May-1987 N-NITROSODIMETHYLAMINE

SD-100-C 20-May-1987 N-NITROSODIMETHYLAMINE

SD-100-C 27-May-1987 N-NITROSODIMETTYLAMINE

SD-100-C 12-JUn-1987 N-NITROSODIMETFYLAMINE

SD $-100-C$

19-Jun-1987 N-NITROSODIMETHYLAMINE

太* SD -100

SD-100 5-Mar-1987 N-NITROSODIPHENYLAMINE

SD-100 11-Mar-1987 N-NITROSODIPHENYLAMINE

SD-100 12-May-1987 N-NITROSODIPHENYLAMINE

SD-100 26-May-1987 N-NITROSODIPHENYLAKINE

** SD $-100-01$

SD-100-01 12-May-1987 N-NITROSODIPHENYLAMIYE

SD-100-01 26-May-1987 N-NITROSODIPHEAYLAMTNE

** SD $100-02$

SD-100-02 12-May-1987 N-NITROSODIPHENYLAYINE

SD-100-02 26-May-1987 N-NITROSODIPHENYLAYINE

** SD $-100-03$

SD-100-03 12-May-1987 N-NITROSODIPHENYLAMTNE

SD-100-03 26-May-1987 N-NITROSODIPHENYLAYINE

* SD-100-03D

SD-100-03D 12-May-1987 N-NITROSODIPHENYLAMINE

SD-100-03D 26-May-1987 N-NITROSODIPHENYLAKINE

** SD-100-04

SD-100-04 12-May-1987 N-NITROSODIPHENYLAKINE

SD-100-04 26-May-1987 N-NITROSODIPHENYLAMTNE

** SD $-100-06$

SD-100-06 12-May-1987 N-NITROSODIPHERYLAMINE

SD-100-06 26-May-1987 N-NITROSODIPHENYLAMINE

10

$<5.0$

$<10$

$<5.0$

$<10$

$<10$

$<10$

$<10$

$<10$

$<5.0$

$<5.0$

$<5.0$

$<5.0$

$<10$

$<10$

$<10$

$<10$

$<5.0$

$<10$

$8 \mathrm{JB}$

$\mathrm{ug} / \mathrm{I}$

ug/L

$<10$

$4 \mathrm{JB}$

ug/L

ug/L

$<10$

6 B

ug/L

$48 / L$

$<10$

7 B

ug/L

ug/L

$<10$

7 B

$4 g / L$

ug/L

$<10$

$<5.0$
ug/L

ug/L 
Storm Drain Data for SD-100

\begin{tabular}{|c|c|c|c|c|}
\hline Location & Date & Test Compound & Results & Units \\
\hline \multicolumn{5}{|c|}{$\star \star \quad S D-100-07$} \\
\hline $\begin{array}{l}\text { SD }-100-07 \\
\text { SD-100-07 }\end{array}$ & $\begin{array}{l}12-\text {-ay }-1987 \\
26-\text { May }-1987\end{array}$ & $\begin{array}{l}\text { N-NITROSODIPHENYLAMNE } \\
\text { N-NITROSODIPHEAYLAKINE }\end{array}$ & $\begin{array}{l}<10 \\
<5.0\end{array}$ & \\
\hline \multicolumn{5}{|c|}{$*$ SD $-100-09$} \\
\hline $\begin{array}{l}S D \cdot 100-09 \\
S D-100-09\end{array}$ & $\begin{array}{l}12-\text { Kay }-1987 \\
26 \text { - Kay-1987 }\end{array}$ & $\begin{array}{l}\text { N-NITROSODI PHETYLANINE } \\
\text { N-NIIROSODI PHENYLAYINE }\end{array}$ & $\begin{array}{l}<10 \\
<5.0\end{array}$ & \\
\hline \multicolumn{5}{|c|}{ * SD $-100-10$} \\
\hline $\begin{array}{l}S D-100-10 \\
S D-100-10\end{array}$ & $\begin{array}{l}12-\text { Kay-1987 } \\
26-\text { May-1987 }\end{array}$ & $\begin{array}{l}\text { N-NITROSODI PHENYLAKINE } \\
\text { N-NITROSODIPHENYLAYINE }\end{array}$ & $\begin{array}{l}<10 \\
6 \text { B }\end{array}$ & ug \\
\hline \multicolumn{5}{|c|}{$\star *$ SD $-100-11$} \\
\hline$S D-100-11$ & 12-May-1987 & N-NITROSODIPHEAYYAMINE & $<10$ & \\
\hline SD- $100-11$ & 26-May- 1987 & N-NITROSODIPHENYLAMINE & 58 & \\
\hline
\end{tabular}

** SD $-100-12$

SD-100-12 12-May-1987 N-NITROSODIPHENYLAYINE SD-100-12 26-\$ay-1987 N-NITROSODIPHENYLAIINE

* SD - $100-13$

SD-100-13 12-May-1987 N-NITROSODIPHENYIAMINE SD-100-13 26-May-1987 N-NITROSODIPHENYLAMINE

* SD-100-14

SD-100-14 12-May-1987 N-NITROSODIPHENYLAKINE SD-100-14 26-May-1987 N-NITROSODIPHENYLAYINE

** SD $-100-C$

SD-100-C 5-Mar-1987 N-NITROSODIPHEANRAMINE

SD-100-C 9-Mar-1987 N-NITROSODIPHENYLAINE

SD-100-C 17-Mar-1987 N-NITROSODIPHENYLAKINE

SD-100-C 24-Mar-1987 N-NITROSODIPHENYLAMINE

SD-100-C 8-Apr-1987 N-NITROSODIPHENYLAMINE

SD-100-C 13-May-1987 N-NITROSODIPHENYLANINE

SD-100-C 20-May-1987 N-NITROSODIPHENYLAYINE

SD-100-C 27-May-1987 N-NITROSODIPHENYLAKINE

SD-100-C 12-Jun-1987 N-NITROSODI PHENYLAKINE

SD-100-C 19-Jun-1987 N-NITROSODIPHENYLAYINE

** SD -100

SD-100 5-Mar-1987 NAPHTHALENE

SD-100 11-Mar-1987 NAPHTHALENE

SD-100 12-May-1987 NAPHTHALENE

SD-100 26-May-1987 NAPHTHALENE

$<10$

$4 \mathrm{JB}$

$u g / L$

$u g / L$

$<10$

$4 \mathrm{JB}$

ug/L

ug/L

$<10 \quad 4 \mathrm{~g} / \mathrm{L}$

$6 \mathrm{~B} \quad \mathrm{ug} / \mathrm{L}$

$<10$

$<10$

$<10$

$<10$

$<10$

$<5.0$

14 B

6 B

$<5.0$

$<10$

$\mathrm{ug} / \mathrm{L}$

ug/L

$\mathrm{ug} / \mathrm{L}$

$u g / L$

ug/L

ug/L.

ug/L

$u g / L$

ug/L

ug/L

** SD $-100-01$

SD-100-01 12-May-1987 NAPHTHALENE

SD-100-01 26-May-1987 NAPHTHALENE

$<10$

$<10$

$<10$

$<5.0$

$u g / 2$

ug/

$\mathrm{ug} / \mathrm{L}$

$\mathrm{ug} / \mathrm{L}$

$<10$

$<5.0$

$\mathrm{ug} / \mathrm{L}$

ug/L 
Untes

** SD - 100-02

SD-100-02 12-Yay-1987 NAPHTHALENE SD-100-02 26-May-1987 NAPHTHALENE

** SD $-100-03$

SD-100-03 12-May-1987 NAPHTHALBAE

SD-100-03 26-May-1987 NAPHTHAJENE

$<10$

$<5.0$

ug/L

ug $/ \mathrm{L}$

** SD-100-03D

SD-100-03D 12-May-1987 NAPHTHALENE

SD-100-03D 26-May-1987 NAPHTHALENE

* SD- $100-04$

SD-100-04 12-May-1987 NAPHTHALENE

SD-100-04 26-May-1987 NAPHTRALENE

** SD-100-06

SD-100-06 12-May-1987 NAPHTHALENE

SD-100-06 26-May-1987 NAPHTHALENE

* SD-100-07

SD-100-07 12-May-1987 NAPHTHAIENE

SD-100-07 26-May-1987 NAPHTHALENE

** SD-100-09

SD-100-09 12-May-1987 NAPHTHAI BNE

SD-100-09 26-May-1987 NAPHTHALENE

** SD $-100-10$

SD-100-10 12-Kay-1987 NAPHTHALENE

SD-100-10 26-May-1987 NAPHTHALENE

* SD-100-11

SD-100-11 12-May-1987 NAPHTHALENE

SD-100-11 26-May-1987 NAPHTHALENE

* SD-100-12

SD-100-12 12-May-1987 NAPHTHALENE

SD-100-12 26-Kay-1987 NAPHTMALENE

** SD $-100-13$

SD-100-13 12-Kay-1987 NAPHTHAIENE

SD-100-13 26-May-1987 NAPHTHAIENE

* SD-100-14

SD-100-14 12-May-1987 NAPHTRALENE

SD-100-14 26-May-1987 NAPHTHALENE

** SD $-100-C$

SD-100-C 5-Mar-1987 NAPHTHALENE

SD-100-C 9-Mar-1987 NAPHTHALENE
$<10$

$<5.0$

$<10$

$<5.0$

$<10$

$<5.0$

$<10$

$1 \mathrm{~J}$

$<10$

$<5.0$

ug/L

ug/L

$<10$

$<.0$

ug/L

ug/L

$<10$

$<5.0$

ug/L

ug/L

$<10$

$<5.0$

ug/L

ug/L

$<10$

$<5.0$

ug $/ \mathrm{L}$

$u g / L$

$<10$

$<5.0$

ug/L

ug/L

$<10$

$4 \mathrm{~g} / \mathrm{L}$

ug/L 


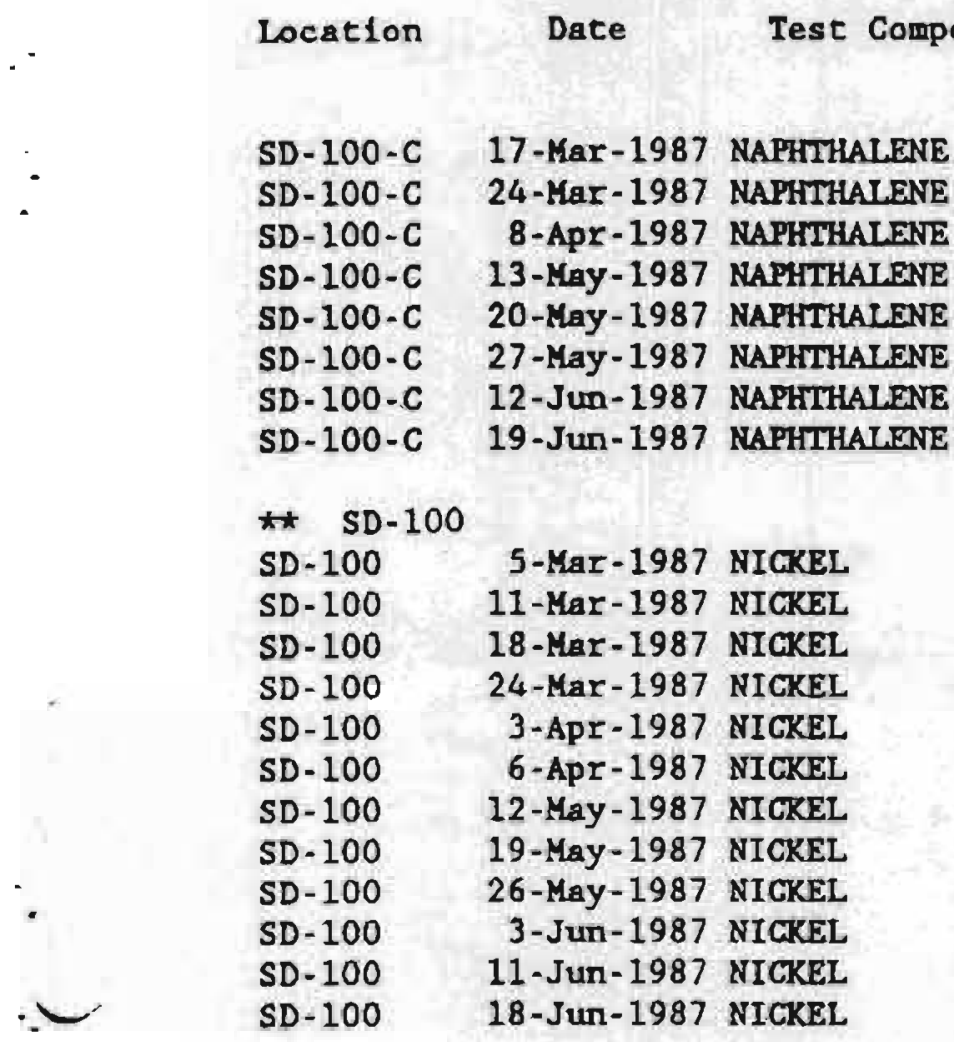

Results Units

* SD-100-01

SD-100-01 12-May-1987 NIGKEL SD-100-01 18-May-1987 NICKEL SD-100-01 3-Jun-1987 NICKEL SD-100-01 11-Jun-1987 NICKEI SD-100-01 18-Jun-1987 NICKEL

$<10$

$<10$

$<10$

$<5.0$

$<5.0$

$<5.0$

$<5.0$

$<10$

$<0.050$

$<0.050$

$<0.050$

$<0.050$

$<0.050$

$<0.050$

$<0.050$

$<0.050$

$<0.010$

$<0.050$

$<0.050$

$<0.050$

$<0.050$

$<0.050$

$<0.050$

$<0.050$

$<0.050$

$<0.050$

$<0.050$

$<0.050$

$<0.050$

$<0.050$

$<0.050$

$<0.050$

$<0.050$

$<0.050$

$<0.050$

$<0.050$

$<0.050$

$<0.050$

$\mathrm{mg} / \mathrm{L}$

SD-100-03D 12-May-1987 NICKEL

SD-100-03D 26-May-1987 NICKEL

ug/L $\mathrm{ug} / \mathrm{L}$ ug/L $\mathrm{ug} / \mathrm{L}$ ug/L ug/L $u g / L$ ug/L

$\mathrm{mg} / \mathrm{L}$ $\mathrm{mg} / \mathrm{L}$ $\mathrm{mg} / \mathrm{L}$ $\mathrm{mg} / \mathrm{L}$ $\mathrm{mg} / \mathrm{L}$ mg/L $\mathrm{mg} / \mathrm{L}$ mg $/ \mathrm{L}$ mg/L $\mathrm{mg} / \mathrm{L}$ $\mathrm{mg} / \mathrm{I}$ $\mathrm{mg} / \mathrm{L}$

$\mathrm{mg} / \mathrm{L}$ $\mathrm{mg} / \mathrm{h}$ $\mathrm{mg} / \mathrm{L}$ $\mathrm{mg} / \mathrm{L}$ $\mathrm{mg} / \mathrm{L}$

$\mathrm{mg} / \mathrm{L}$ mg $/ \mathrm{L}$ Im $/ L$ $\mathrm{mg} / \mathrm{L}$ $\mathrm{mg} / \mathrm{L}$

$\mathrm{mg} / \mathrm{L}$ $\mathrm{mg} / \mathrm{L}$ $\mathrm{mg} / \mathrm{L}$ $\mathrm{mg} / \mathrm{L}$ $\mathrm{mg} / \mathrm{L}$ $\mathbf{m g} / \mathrm{L}$

* SD- 100.04

SD-100-04 12-May-1987 NIGKEL
$<0.050 \quad \mathrm{mg} / \mathrm{L}$ 


\begin{tabular}{lrrr} 
Location & \multicolumn{1}{c}{ Date } & Test \\
& & \\
SD-100-04 & 18 -May-1987 & NICKEL \\
SD-100-04 & $26-$ May-1987 & NICKEL \\
SD-100-04 & 3 -Jun-1987 & NICKEI \\
SD-100-04 & 11 -Jun-1987 & NICKEL \\
SD-100-04 & 18 -Jun-1987 & NICKEI
\end{tabular}

Results Units

* SD-100-06

SD-100-06 12-May-1987 NICKEL

Compound

18-May-1987 NICKEL

SD-100-06

26-May-1987 NICXEZ

SD-100-06

3-Jun-1987 NICKEL

SD- $100-06$

3-Jun-1987 NTCrer

SD-100-06 11-Jun-1987 NCKEL

* SD-100-06D

SD-100-06D 3-Jun-1987 NICKEL

$<0.050$

$<0.050$

$<0.050$

$<0.050$

$<0.050$

** SD-100-07

SD-100-07 12-Kay-1987 NICXEL

SD-100-07 19-May-1987 NICKEL

SD-100-07 26-May-1987 NICKEL

SD-100-07 3-Jun-1987 NICKEL

SD-100-07 11-Jun-1987 NICXEL.

SD-100-07 18-Jun-1987 NICKEL

$<0.050$

$<0.050$

$<0.050$

$<0.050$

$<0.050$

$<0.050$

$\mathrm{mg} / \mathrm{L}$

$\mathrm{mg} / \mathrm{L}$

$\mathrm{mg} / \mathrm{L}$

$\mathrm{mg} / \mathrm{L}$

$\mathrm{mg} / \mathrm{L}$

* SD-100-07D

SD-100-07D 18-Jun-1987 NICKEL

0.11

$\mathrm{mg} / \mathrm{L}$

$\mathrm{mg} / \mathrm{L}$

$\mathrm{mg} / \mathrm{L}$

$\mathrm{mg} / \mathrm{L}$

$\mathrm{mg} / \mathrm{L}$

$\mathrm{mg} / \mathrm{L}$

$<0.050$

$\mathrm{mg} / \mathrm{L}$

0.13

0.22

0.063

0.094

0.10

0.090

$\mathrm{mg} / \mathrm{L}$

mg/L

$\mathrm{mg} / \mathrm{L}$

$\mathrm{mg} / \mathrm{L}$

$\mathrm{mg} / \mathrm{L}$

$\mathrm{mg} / \mathrm{L}$

* SD-100-09

SD-100-09 12-May-1987 NICKEL

SD-100-09 18-May-1987 NICKEL

SD-100-09 26- $\mathrm{Hay-1987} \mathrm{NICXEL}$

SD-100-09 3-Jun-1987 NICXEL

SD-100-09 11-Jun-1987 NICKEL

$<0.050$

$<0.050$

$<0.050$

$<0.050$

$<0.050$

$\mathrm{mg} / \mathrm{L}$

** SD - 100-10

SD-100-10 12-Kay-1987 NICKEL

SD-100-10 19-May-1987 NICKEL

SD-100-10 26-Kay-1987 NIGKEI

SD-100-10 3-Jun-1987 NICKEL

SD-100-10 11-Jun-1987 NICKEL

SD-100-10 18-Jun-1987 NICKEL

$<0.050$

$<0.050$

$<0.050$

$<0.050$

$<0.050$

$<0.050$

$\mathrm{mg} / \mathrm{L}$

$\mathrm{mg} / \mathrm{L}$

$\mathrm{mg} / \mathrm{L}$

$\mathrm{mg} / \mathrm{L}$

mg/L

* SD-100-11

SD-100-11 12-Kay-1987 NICKEL

SD-100-11 19-Kay-1987 NICKEL

SD-100-11 26-May-1987 NICKEL

SD-100-11 3-Jun-1987 NICKEL

SD-100-11 11-Jun-1987 NICKEL

SD-100-11 18-Jun-1987 NICKEL

$<0.050$

$<0.050$

$<0.050$

$<0.050$

$<0.050$

$<0.050$ $\mathrm{mg} / \mathrm{L}$

rg $/ L$

mg/L

mg $/ \mathrm{L}$

$\mathrm{mg} / \mathrm{L}$.

mg/L

घg $/ \mathrm{L}$

$\mathrm{mg} / \mathrm{L}$

$\mathrm{mg} / \mathrm{L}$

$\mathrm{mg} / \mathrm{L}$

$\mathrm{mg} / \mathrm{L}$

$\mathrm{mg} / \mathrm{L}$ 
Location Date Test Gompound

Results Units

* SD-100-12

$\begin{array}{lrl}\text { SD-100-12 } & 12 \text {-May-1987 } & \text { NICKEL } \\ \text { SD-100-12 } & 19 \text {-May-1987 } & \text { NICKEL } \\ \text { SD-100-12 } & 26 \text { - May-1987 } & \text { NICKEL } \\ \text { SD-100-12 } & \text { 3-Jun-1987 } & \text { NICKEL } \\ \text { SD-100-12 } & 11 \text {-Jun-1987 } & \text { NICKEL } \\ \text { SD-100-12 } & 18-J u n-1987 & \text { NICKEL }\end{array}$

0.17

$<0.010$

$<0.010$

$<0.050$

$<0.050$

$<0.050$

* SD-100-13

SD-100-13 12-May-1987 NICKEL

SD-100-13 19-May-1987 NICKEL

SD-100-13 26-May-1987 NICKEL

SD-100-13 11-Jun-1987 NICKEL

SD-100-13 18-Jun-1987 NICKEL

$<0.050$

$<0.010$

$<0.010$

$<0.050$

$<0.050$

$\mathrm{mg} / \mathrm{L}$

$\operatorname{mg} / \mathrm{L}$

$\mathrm{mg} / \mathrm{L}$

$\mathrm{mg} / \mathrm{L}$

$\mathrm{mg} / \mathrm{L}$

$\mathrm{mg} / \mathrm{L}$

** SD-100-14

SD-100-14 12-May-1987 NICKEL

SD-100-14 19-May-1987 NICKEL

SD-100-14 26-May-1987 NICKEL

SD-100-14 3-Jun-1987 NICKEL

SD-100-14 11-Jun-1987 NICKEL

$S D=100-14 \quad 18-J u n-1987$ NICKEL

$<0.050$

$<0.050$

$<0.010$

$<0.050$

$<0.050$

$<0.050$

$\mathrm{mg} / \mathrm{L}$

$\mathrm{mg} / \mathrm{L}$

$\operatorname{mg} / \mathrm{L}$

$\mathrm{mg} / \mathrm{L}$

$\mathrm{mg} / \mathrm{L}$

** SD-100-C

SD-100-C 5-Mar-1987 NICKEL

SD-100-C 9-Mar-1987 NICKEL

SD-100-C 17-Mar-1987 NIGKEL

SD-100-C 24-Mar-1987 NIGKII

SD-100-C 8-Apr-1987 NICKEL

SD-100-C 13-May-1987 NICKEL

SD-100-C 20-May-1987 NICKEL

SD-100-C 27-May-1987 NICKEL

SD-100-C 4-Jun-1987 NIGXEL

SD-100-C 12-Jun-1987 NICKEI

SD-100-C 19-Jun-1987 NIGKEL

$<0.050$

$<0.050$

$<0.050$

$<0.050$

$<0.050$

$<0.050$

$<0.050$

$<0.050$

$<0.050$

$<0.050$

$<0.050$

$\mathrm{mg} / \mathrm{L}$

$\operatorname{mg} / \mathrm{L}$

$\mathrm{mg} / \mathrm{L}$

$\mathrm{mg} / \mathrm{L}$

$\mathrm{mg} / \mathrm{L}$

$\mathrm{mg} / \mathrm{L}$

** SD-100-D

SD-100-D 6-Apr-1987 NIGKEL

SD-100-D 11-Jun-1987 NIGKEL

$<0.050$

$<0.050$

$\mathrm{mg} / \mathrm{L}$

$\mathrm{mg} / \mathrm{L}$

* SD -100

SD- $100 \quad 5$-Mar-1987 NIOBIUM

$<0.0070$

$<0.0070$

11-Mar-1987 NIOBTUM

SD -100

18-Mar-1987 NIOBIUM

0.0081

SD -100

24-Mar-1987 NIOBIUM

SD -100

3-Apr-1987 NIOBIUM

$<0.0070$

$\mathrm{mg} / \mathrm{L}$

$\operatorname{mg} / L$

$\mathrm{mg} / \mathrm{L}$

$\mathrm{mg} / \mathrm{h}$

$\mathrm{mg} / \mathrm{L}$

$\mathrm{mg} / \mathrm{L}$

$\mathrm{mg} / \mathrm{L}$

mg/L

$\mathrm{mg} / \mathrm{L}$

$\mathrm{mg} / \mathrm{L}$

$\mathrm{mg} / \mathrm{L}$

SD -100

6-Apr-1987 NIOBIUM

SD -100

12-May-1987 NIOBIUM

SD -100

19-May-1987 NIOBIUM

SD -100

26-May- 1987 NIOBIUM

$<0.0070$

$<0.0070$

$<0.0070$

$<0.0070$

$<0.0070$

3-Jun-1987 NIOBIUM

$<0.0070$ 
Location Date Test Compound

Results

Units

SD-100 11-Jun-1987 NIOBIUM

$<0.0070$

$\mathrm{mg} / \mathrm{L}$

SD-100 18-JUn-1987 NIOBIUM

$<0.0070$

$\mathrm{mg} / \mathrm{L}$

** SD-100-01

SD-100-01 12-May-1987 NIOBTUM

SD-100-01 18-May-1987 RIOBIUM

SD-100-01 3-Jun-1987 NIOBIUM

SD-100-01 11-Jun-1987 NIOBTUM

SD-100-01 18-Jun-1987 NIOBIUA

$<0.0070$

$<0.0070$

$<0.0070$

$<0.0070$

$<0.0070$

$\mathrm{mg} / \mathrm{L}$

mg $/ \mathrm{L}$

$\mathrm{mg} / \mathrm{h}$

$\mathrm{mg} / \mathrm{L}$

$\mathrm{mg} / \mathrm{L}$

* SD-100-02

SD-100-02 12-May-1987 NIOBIUM

SD-100-02 18-May-1987 NIOBIUM

SD-100-02 3-JUn-1987 NIOBIUM

SD-100-02 11-Jun-1987 NIOBIUM

SD-100-02 18-Jun-1987 NIOBIUM

$<0.0070$

$<0.0070$

$\mathrm{mg} / \mathrm{L}$

$<0.0070$

$\mathrm{mg} / \mathrm{L}$

$<0.0070$

$\mathrm{mg} / \mathrm{L}$

$<0.0070$

mg/L

$m g / L$

** SD $-100-03$

SD-100-03 12-Kay-1987 NIOBIUM

$<0.0070$

$<0.0070$

SD-100-03 18-May-1987 NIOBIUM

SD-100-03 26-May-1987 NIOBIUM

0.0072

$<0.0070$

SD-100-03 3-Jun-1987 NIOBIUM

$<0.0070$

SD-100-03 18-Jun-1987 NIOBIUM

$<0.0070$

mg $/ \mathrm{L}$

mg $/ \mathrm{L}$

$\mathrm{mg} / \mathrm{L}$

$\mathrm{mg} / \mathrm{L}$

mg/L

$\mathrm{mg} / \mathrm{L}$

** SD-100-03D

SD-100-03D 12-May-1987 NIOBIUM

SD-100-03D 26-Kay-1987 NIOBIUM

$<0.0070$

mg/L

$<0.0070$

$\mathrm{mg} / \mathrm{L}$

** SD-100-04

SD-100-04 12-May-1987 NIOBIUM

SD-100-04 18-May-1987 NIOBIUM

SD-100-04 26-May-1987 NIOBIUM

SD-100-04 3-Jun-1987 NIOBIUM

SD-100-04 11-Jun-1987 NIOBIUM

SD-100-04 18-Jun-1987 NIOBIUM

$<0.0070$

$<0.0070$

$<0.0070$

$<0.0070$

$<0.0070$

$<0.0070$

$\mathrm{mg} / \mathrm{L}$

ing $/ L$

mg/L

mg/L

$\mathrm{mg} / \mathrm{L}$

mg/ $L$

** SD-100-06

SD-100-06 12-Kay-1987 NIOBIUM

SD-100-06 18-Kay-1987 NIOBIUM

$<0.0070$

$<0.0070$

$<0.0070$

$<0.0070$

SD- $100-06$

26-May-1987 NIOBTUM

3-JUn-1987 NIOBIUM

$<0.0070$

$<0.0070$

$\mathrm{mg} / \mathrm{L}$

$\mathrm{mg} / \mathrm{L}$

mg/L.

$\mathrm{mg} / \mathrm{L}$

$\mathrm{mg} / \mathrm{L}$

SD-100-06 18-Jun-1987 NIOBIUM

* SD $=100-06 D$

SD-100-06D 3-Jun-1987 NIOBIUM

$<0.0070$

$\mathrm{mg} / \mathrm{L}$

* SD-100-07

SD-100-07 12-May-1987 NIOBIUM

SD-100-07 19-May-1987 NIOBIUM

$<0.0070$

$<0.0070$

$\mathrm{mg} / \mathrm{L}$

$\mathrm{mg} / \mathrm{L}$ 
SD-100-07 26-Kay-1987 NIOBIUM SD-100-07 3-Jun-1987 NIOBIUM SD-100-07 11-Jun-1987 NIOBIUM SD-100-07 18-JUn-1987 NIOBIUM

** SD-100-07D

SD-100-07D 18-Jun-1987 NTOBIUM

** SD-100-09

SD-100-09 12-May-1987 NIOBIUM

SD-100-09 18-May-1987 NIOBIUM

SD-100-09 26-May-1987 NIOBIUM

SD-100-09 3-Jun-1987 NIOBIUM

SD-100-09 11-Jun-1987 NIOBIUM

** SD-100-10

SD-100-10 12-Kay-1987 NIOBIUM

SD-100-10 19-May-1987 NIOBIUM

SD-100-10 26-Kay-1987 NIOBIUM

SD-100-10 3-Jun-1987 NIOBIUM

SD-100-10 11-Jun-1987 NIOBIUM

SD-100-10 18-Jun-1987 NIOBIUM

** SD-100-11

SD-100-11 12-May-1987 NIOBIUM

SD-100-11 19-May-1987 NIOBIUM

SD-100-11 26-May-1987 NIOBIUM

SD-100-11 3-Jun-1987 NIOBIUM

SD-100-11 11-JUn-1987 NIOBIUM

SD-100-11 18-Jun-1987 NIOBIUM

* SD-100-12

SD-100-12 12-Kay-1987 NIOBIUM

SD-100-12 19-Kay-1987 NIOBIUM

SD-100-12 26-Kay-1987 NIOBIUM

SD-100-12 3-Jun-1987 NIOBIUM

SD-100-12 11-Jun-1987 NIOBIUM

SD-100-12 18-Jun-1987 NIOBIUM

** SD-100-13

SD-100-13 12-Kay-1987 NIOBIUT

SD-100-13 19-Kay-1987 NIOBIUY

SD-100-13 26-May-1987 NIOBIUM

SD-100-13 11-Jun-1987 NIOBIUM

SD-100-13 18-Jun-1987 NIOBIUM

$+\quad$ SD-100.14

SD-100-14 12-Kay-1987 NIOBIUM

SD-100-14 19-May-1987 NIOBIUM

SD-100-14 26-May-1987 NIOBIUM

SD-100-14 3-Jun-1987 NIOBIUM

$\begin{array}{ll}0.013 & \mathrm{mg} / \mathrm{L} \\ 0.067 & \mathrm{mg} / \mathrm{L} \\ <0.0070 & \mathrm{mg} / \mathrm{L} \\ <0.0070 & \mathrm{mg} / \mathrm{L} \\ & \\ & \\ & \\ & \\ & \mathrm{mg} / \mathrm{L} \\ <0.0070 & \\ <0.0070 & \mathrm{mg} / \mathrm{L} \\ <0.0070 & \mathrm{mg} / \mathrm{L} \\ <0.0070 & \mathrm{mg} / \mathrm{L} \\ <0.0070 & \mathrm{mg} / \mathrm{L} \\ & \mathrm{mg} / \mathrm{L} \\ <0.0070 & \mathrm{mg} / \mathrm{L} \\ <0.0070 & \mathrm{mg} / \mathrm{L} \\ <0.0070 & \mathrm{mg} / \mathrm{L} \\ <0.0070 & \mathrm{mg} / \mathrm{L} \\ <0.0070 & \mathrm{mg} / \mathrm{L} \\ <0.0070 & \mathrm{mg} / \mathrm{L}\end{array}$

$<0.0070$

$<0.0070$

$<0.0070$

$<0.0070$

$<0.0070$

$<0.0070$

mg $/$ L

$\mathrm{mg} / \mathrm{L}$

mg/L

mg/L

mg $/$ L

$\mathrm{mg} / \mathrm{L}$

$<0.0070$

$<0.0070$

$<0.0070$

$<0.0070$

$<0.0070$

$<0.0070$

$\mathrm{mg} / \mathrm{L}$

$\mathrm{mg} / \mathrm{L}$

$\mathrm{mg} / \mathrm{L}$

$\mathrm{mg} / \mathrm{l}$

mg/L

$\mathrm{mg} / \mathrm{L}$

$<0.0070$

$<0.0070$

$<0.0070$

$<0.0070$

$<0.0070$

mg/L

$\mathrm{mg} / \mathrm{L}$

mg/L

mg $/ \mathrm{L}$

ㅍg/L

$<0.0070$

$<0.0070$

$<0.0070$

$<0.00>0$
mg/L

mg/L

$\mathrm{mg} / \mathrm{L}$

$\mathrm{mg} / \mathrm{L}$ 
Storm Drain Data for SD-100

Location

Date

Test Compound

Results

Units

SD-100-14 11-Jun-1987 NIOBIUM

$<0.0070$

SD-100-14

18-JUn-1987 NIOBIUM

$<0.0070$

$\mathrm{mg} / \mathrm{L}$

$\mathrm{mg} / \mathrm{L}$

* SD-100-C

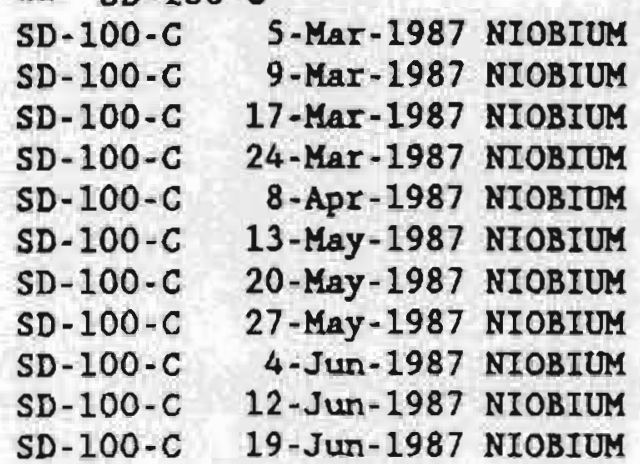

0.0097

$<0.0070$

$<0.0070$

$<0.0070$

$<0.0070$

$<0.0070$

$<0.0070$

$<0.0070$

$<0.0070$

$<0.0070$

$<0.0070$

$\mathrm{mg} / \mathrm{L}$

$\mathrm{mg} / \mathrm{L}$

$\mathrm{mg} / \mathrm{L}$

$\mathrm{mg} / \mathrm{L}$

$\mathrm{mg} / \mathrm{L}$

$\mathrm{mg} / \mathrm{L}$

$\mathrm{mg} / \mathrm{L}$

$\mathrm{mg} / \mathrm{L}$

$\mathrm{mg} / \mathrm{L}$

$\mathrm{mg} / \mathrm{L}$

$\mathrm{mg} / \mathrm{L}$

* SD-100-D

SD-100-D 6-ApI-1987 NIOBIUM

$<0.0070$

$<0.0070$

mg/L

$\mathrm{mg} / \mathrm{L}$

* SD- 100

SD-100 5-Mar-1987 NITRATE

SD $-100 \quad 11$-Mar-1987 NITRATE

SD-100 18-Mar-1987 NITRATE

SD-100 24-Mar-1987 NITRATE

SD-100 3-Apr-1987 NITRATE

SD-100 6-Apr-1987 NITRATE

SD-100 12-May-1987 NITRATE

SD-100 19-May-1987 NITRATE

SD-100 26-May-1987 NITRATE

SD-100 3-Jun-1987 NITRATE

SD- $100 \quad 11$-Jun-1987 NITRATE

SD-100 18-Jun-1987 NITRATE

1.9

3.3

1.3

1.8

1.7

1.4

2.4

2.0

2.1

2.4

2.6

2.2

** SD - 100-01

SD-100-01 12-May-1987 NITRATE

SD-100-01 18-May-1987 NITRATE

SD-100-01 26-May-1987 NITRATE

SD-100-01 3-Jun-1987 NITRATE

SD-100-01 11-Jun-1987 NITRATE

SD-100-01 18-JUn-1987 NITRATE

$<0.5$

$<0.5$

$<0.5$

4.3

4.6

$<0.5$

mg $/ \mathrm{L}$

$\mathrm{mg} / \mathrm{L}$

$\mathrm{mg} / \mathrm{L}$

$\mathrm{mg} / \mathrm{L}$

mg/L

$\mathrm{mg} / \mathrm{L}$

mg/L

$\mathrm{mg} / \mathrm{L}$

$\mathrm{mg} / \mathrm{L}$

$\mathrm{mg} / \mathrm{L}$

$\mathrm{mg} / \mathrm{L}$

$\mathrm{mg} / \mathrm{L}$

* SD $-100-02$

SD-100-02 12-May-1987 NITRATE

SD-100-02 18-May-1987 NITRATE

SD-100-02 26-May-1987 NITRATE

SD-100-02 3-Jun-1987 NITRATE

SD-100-02 11-Jun-1987 NITRATE

SD-100-02 18-Jun-1987 NITRATE

$\begin{array}{ll}2.2 & \mathrm{mg} / \mathrm{L} \\ 2.1 & \mathrm{mg} / \mathrm{L} \\ 2.5 & \mathrm{mg} / \mathrm{L} \\ 2.6 & \mathrm{mg} / \mathrm{L} \\ 2.6 & \mathrm{mg} / \mathrm{L} \\ 2.5 & \mathrm{mg} / \mathrm{L}\end{array}$

$\mathrm{mg} / \mathrm{L}$

$\mathrm{mg} / \mathrm{L}$

$\mathrm{mg} / \mathrm{L}$

$\mathbf{m g} / \mathrm{L}$

$\mathrm{mg} / \mathrm{L}$

$\mathrm{mg} / \mathrm{L}$

$\mathrm{mg} / \mathrm{L}$

$\mathrm{mg} / \mathrm{L}$

$\mathrm{mg} / \mathrm{L}$

$\mathrm{mg} / \mathrm{L}$

$\mathrm{mg} / \mathrm{L}$ 
Location Date Test Compound

Resules Units

** SD - 100-03

SD-100-03 12-May-1987 NITRATE

SD-100-03 18-May-1987 NITRATE

SD-100-03 26-MaY-1987 NITRATE

SD-100-03 3-Jun-19B7 NITRATE

SD-100-03 11-Jum-1987 NITRATE

SD-100-03 18-Jun-1987 NITRATE

1.9

2. 0

2.3

2.4

2.6

2.5

* SD-100-03D

SD-100-03D 12-May-1987 NITRATE

SD-100-03D 26-May-1987 NITRATE

1.9

2.3

** SD $-100-04$

SD-100-04 12-May-1987 NITRATE

SD-100-04 18-Kay-1987 NITRATE

SD-100-04 26-May-1987 NITRATE

SD-100-04 3-Jun-1987 NITRATE

SD-100-04 1I-Jun-1987 NITRAIE

SD-100-04 1B-Jun-1987 NITRATE

2.2

2.0

1.9

2.4

2.7

2.6

$\mathrm{mg} / \mathrm{L}$

$\mathrm{mg} / \mathrm{L}$

$\mathrm{mg} / \mathrm{L}$

$\mathrm{mg} / \mathrm{L}$

$\mathrm{mg} / \mathrm{L}$

mg/L

** SD- $100-06$

SD-100-06 12-May-1987 NITRATE

SD-100-06 18-May-19B7 NITRATE

SD-100-06 26-May-1987 NITRATE

SD-100-06 3-Jun-1987 NITRATE

SD-100-06 11-Jun-1987 NITRATE

SD-100-06 18-Jun-1987 NITRATE

2.7

2.1

1.8

2.9

2.6

2.6

$\mathrm{ng} / \mathrm{L}$

$\mathbf{\square g} / \mathrm{L}$

* SD-100-06D

SD-100-06D 3-Jun-1987 NITRATE

$2.8 \quad \mathrm{mg} / \mathrm{L}$

** SD-100-07

SD-100-07 12-May-1987 NITRATE

SD-100-07 19-May-1987 NITRATE

SD-100-07 26-May-1987 NITRATE

SD-100-07 3-Jun-1987 NITRATE

SD-100-07 11-Jun-1987 NITRATE

SD-100-07 18-Jun-1987 NITRATE

11

32.4

18.2

240

26

$\mathrm{mg} / \mathrm{L}$

$\mathrm{mg} / \mathrm{L}$

$\mathrm{mg} / \mathrm{L}$

$\mathrm{mg} / \mathrm{L}$

$m g / L$

$\mathrm{mg} / \mathrm{L}$

* SD-100-07D

SD-100-07D 18-JUn-1987 NITRATE

74

$\mathrm{mg} / \mathrm{L}$

mg $/ \mathrm{L}$

mg $/ \mathrm{L}$

$\mathrm{mg} / \mathrm{L}$

$\mathrm{mg} / \mathrm{L}$

mg $/ 2$

** SD - 100-09

SD-100-09 12-May-19B7 NITRATE

SD-100-09 18-May-1987 NITRATE

SD-100-09 26-May-1987 NITRATE

SD-100-09 3-Jun-1987 NITRATE

SD-100-09 11-Jun-1987 NITRATE

94

$\mathrm{mg} / \mathrm{L}$

$\mathrm{mg} / \mathrm{L}$

$\mathrm{mg} / \mathrm{L}$

ag $/ \mathrm{L}$

$\mathrm{mg} / \mathrm{L}$

$\mathrm{mg} / \mathrm{L}$

SD-100-09 18-Jun-1987 NITRATE

2.6

$\mathrm{mg} / \mathrm{L}$

2.1

$\mathrm{mg} / \mathrm{L}$

1.8

2.5

2.9

$\mathbf{m g} / \mathrm{L}$

Rg/L

2.0

$\mathrm{mg} / \mathrm{L}$

$\operatorname{mg} / \mathrm{L}$ 
** SD-100-10

SD-100-10 12-May-1987 NITRATE

SD-100-10 19-Kay-1987 NITRATE

SD-100-10 26-Kay-1987 NITRATE

SD-100-10 3-Jun-1987 NITRATE

SD-100-10 11-Jun-1987 NITRATE

SD-100-10 18-Jun-1987 NITRATE

** SD - 100- 11

SD-100-11 12-May-1987 NITRATE

SD-100-11 19-May-1987 NITRATE

SD-100-11 26--May-1987 NITRATE

SD-100-11 3-Jun-1987 NITRATE

SD-100-11 11-Jun-1987 NITRATE

SD-100-11 18-Jun-1987 NITRATE

** SD $-100-12$

SD-100-12 12-May-1987 NITRATE

SD-100-12 19-May-1987 NITRATE

SD-100-12 26-May-1987 NITRATE

SD-100-12 3-Jun-1987 NITRATE

SD-100-12 11-Jun-1987 NITRATE

SD-100-12 18-Jun-1987 NITRATE

* SD $-100-13$

SD-100-13 12-May-1987 NITRATE

SD-100-13 19-May-1987 NITRATE

SD-100-13 26-May-1987 NITRATE

SD-100-13 3-Jun-1987 VITRATE

SD-100-13 11-JUn-1987 NITRATE

SD-100-13 18-Jun-1987 NITRATE

* SD $-100-14$

SD-100-14 12-May-1987 NITRATE

SD-100-14 19-May-1987 NITRATE

SD-100-14 26-Kay-1987 NITRATE

SD-100-14 3-Jun-1987 NITRATE

SD-100-14 11-Jun-1987 NITRATE

SD-100-14 18-Jun-1987 NITRATE

* $\quad S D-100-C$

SD-100-C 5-Mar-1987 NITRATE

SD-100-C 9-Mar-1987 NITRATE

SD-100-C 17-Mar-1987 NITRATE

SD-100-C 24-Mar-1987 NITRATE

SD-100-C 8-Apr-1987 NITRATE

SD-100-C 13-May-1987 NITRATE

SD-100-C 20-May-1987 NITRATE

SD-100-C 27-May-1987 NITRATE

SD-100-C 4-Jun-1987 NITRATE

SD-100-C 12-Jun-1987 NITRATE
2.6

2.3

2.5

2.8

2.7

2.5

2.8

2.6

2.6

2.9

3.0

2.9

2.5

2.2

2.5

2.8

2.6

2.5

2.5

4.0

2.5

2.9

2. 6

6.7

mg/L

mg/L

$\mathrm{mg} / \mathrm{L}$

$\mathrm{mg} / \mathrm{L}$

$\mathrm{mg} / \mathrm{L}$

$\mathrm{mg} / \mathrm{L}$

mg $/ L$

$\mathrm{mg} / \mathrm{l}$

mg/L

$\mathrm{mg} / \mathrm{L}$

$\mathrm{mg} / \mathrm{L}$

mg/L

mg/L

$\mathrm{mg} / \mathrm{L}$

$\mathrm{mg} / \mathrm{L}$

$\mathrm{mg} / \mathrm{L}$

$\mathrm{mg} / \mathrm{L}$

Ig/L

2.8

7.0

2.4

2.7

2.6

2.5

$\mathrm{mg} / \mathrm{L}$

$\mathrm{mg} / \mathrm{L}$

$\mathrm{g} / \mathrm{L}$

ng $/ 2$

mg $/ L$

mg $/ L$

1.4

2.1

1. 3

2. 0

1. 2

2.1

2. 1

1. 9

2. 2

2. 3
mg/L

mg/L

mg/L

mg/L

mg/L

mg/L

mg/L

ng/L

IIg/L

$\mathrm{mg} / \mathrm{L}$

mag/ $\mathrm{L}$

$\mathrm{mg} / \mathrm{L}$

$\mathrm{mg} / \mathrm{L}$

ng/L

$\mathrm{mg} / \mathrm{L}$

$\mathrm{ng} / \mathrm{L}$ 
Storm Drain Data for SD-100

\begin{tabular}{|c|c|c|c|c|}
\hline Location & Date & Test Compound & Results & Units \\
\hline SD-100-C & $19-J u n-1987$ & NITRATE & 2.0 & $\mathrm{mg} / \mathrm{L}$ \\
\hline \multicolumn{5}{|c|}{ * SD-100-D } \\
\hline$S D-100-D$ & 6-Apr-1987 & NITRATE & 1.4 & $\mathbf{m g} / \mathrm{L}$ \\
\hline SD-100-D & 11-Jun-1987 & NITRATE & 2.5 & $\mathrm{mg} / \mathrm{L}$ \\
\hline \multicolumn{5}{|l|}{$\star * \quad$ SD -100} \\
\hline SD -100 & 5-Mar-1987 & NITROBENZENE & $<10$ & $\mathrm{ug} / \mathrm{L}$ \\
\hline SD -100 & 11 -Mar-1987 & NITROBENZENE & $<10$ & $\mathrm{ug} / \mathrm{L}$ \\
\hline SD -100 & 12-May-1987 & NITROBENZENE & $<10$ & $u g / L$ \\
\hline SD- 100 & $26-$ May -1987 & NITROBENZENE & $<5.0$ & $\mathrm{ug} / \mathrm{L}$ \\
\hline \multicolumn{5}{|c|}{ \#* SD-100-01 } \\
\hline$S D-100-01$ & 12-May-1987 & NITROBENZENE & $<10$ & $4 \mathrm{~g} / \mathrm{L}$ \\
\hline SD-100-01 & $26-$ May- 1987 & NITROBENZENE & $<5.0$ & $\mathrm{ug} / \mathrm{L}$ \\
\hline \multicolumn{5}{|c|}{$\star \star \quad$ SD $-100-02$} \\
\hline SD $-100-02$ & 12-May-1987 & NITROBENZENE & $<10$ & $u g / L$ \\
\hline SD $-100-02$ & 26-May-1987 & NITROBENZENE & $<5.0$ & $\mathrm{ug} / \mathrm{L}$ \\
\hline \multicolumn{5}{|c|}{ * SD $-100-03$} \\
\hline SD $-100-03$ & $12-$ May $=19.87$ & NITROBENZENE & $<10$ & ug/L \\
\hline SD $-100-03$ & 26-May-1987 & NITROBENZENE & $<5.0$ & $4 \mathrm{~g} / \mathrm{L}$ \\
\hline \multicolumn{5}{|c|}{ ** SD-100-03D } \\
\hline$S D-100-03 D$ & $12-$ May -1987 & NITROBENZENE & $<10$ & $\mathrm{ug} / \mathrm{L}$ \\
\hline SD-100-03D & $26-$ May -1987 & NITROBENZENE & $<5.0$ & $\mathrm{ug} / \mathrm{L}$ \\
\hline \multicolumn{5}{|c|}{$\star$ SD -100.04} \\
\hline SD $-100-04$ & 12- May-1987 & NITROBENZENE & $<10$ & ug/L \\
\hline SD-100-04 & 26 - May- 1987 & NITROBENZENE & $<5.0$ & $\mathrm{ug} / \mathrm{L}$ \\
\hline \multicolumn{5}{|c|}{ ** SD $-100-06$} \\
\hline SD -100.06 & $12-$ May- 1987 & NITROBENZENE & $<10$ & ug/L \\
\hline SD $-100-06$ & 26-May- 1987 & NITROBENZENE & $<5.0$ & $u_{g} / \mathrm{L}$ \\
\hline \multicolumn{5}{|c|}{$\star$ SD $-100-07$} \\
\hline SD $-100-07$ & $12-$ May-1987 & NITROBENZENE & $<10$ & $4 \mathrm{~g} / \mathrm{L}$ \\
\hline SD $-100-07$ & 26-May-1987 & NITROBENZENE & $<5,0$ & ug/I \\
\hline \multicolumn{5}{|c|}{ * SD-100-09 } \\
\hline SD $-100-09$ & 12-Kay-1987 & NITROBENZENE & $<10$ & $\operatorname{ug} \sqrt{2}$ \\
\hline SD $-100-09$ & 26- Hay-1987 & NITROBENZENE & $<5.0$ & $\mathrm{ug} / \mathrm{L}$ \\
\hline \multicolumn{5}{|c|}{ ** SD $-100-10$} \\
\hline SD $-100-10$ & $12-$ May -1987 & NITROBENZENE & $<10$ & ug $/ \mathrm{L}$ \\
\hline SD $-100-10$ & 26-May-1987 & NITROBENZENE & $<5.0$ & ug/L \\
\hline \multicolumn{5}{|c|}{$\star * \quad S D-100-11$} \\
\hline SD-100-11 & 12-May-1987 & NITROBENZENE & $<10$ & $u g / L$ \\
\hline$S D-100-11$ & $26-$ May -1987 & NITROBENZENE & $<5.0$ & $\mathrm{ug} / \mathrm{L}$ \\
\hline
\end{tabular}


Location Date Test Compound

Results Units

** SD-100-12

SD-100-12 12-May-1987 NITROBENZENE

SD-100-12 26-May-1987 NITROBENZENE

$<10$

$<5.0$

$<10$

$<5.0$

SD-100-13 12-May-1987 NITROBENZENE

SD-100-13 26-May-1987 NITROBENZENE

t* SD $-100-14$

SD-100-14 12-May-1987 NITROBENZENE

SD-100-14 26-May-1987 NITROBENZENE

t* SD-100-C

SD-100-C 5-MaT-1987 NITROBENZENE

SD-100-C 9-Mar-1987 NITROBENZENE

SD-100-C 17-Mar-1987 NITROBENZENE

SD-100-C 24-Mar-1987 NITROBENZENE

SD-100-C 8-API-1987 NITROBENZENE

SD-100-C 13-May-1987 NITROBENZENE

SD-100-C 20-May-1987 NITROBENZENE

SD-100-C 27-May-1987 NITROBENZENE

SD-100-C 12-Jun-1987 NITROBENZENE

SD-100-C 19-JUת-1987 NITROBENZENE

** SD $-100-07$

SD-100-07 12-May-1987 OCTANOIC ACID

** SD -100

SD -100

5-Mar-1987 OIL \& GREASE

SD -100

11-Mar-1987 OIL \& GREASE

SD -100

18-Mar-19B7 OIL \& GREASE

SD -100

24-Mar-1987 OIL \& GRBASE

SD -100

3-Apr-1987 OIL \& GREASE

SD -100

6-API-1987 OIL \& GRFASE

SD -100

12-May-1987 OIL \& GREASE

SD -100

19-May-1987 OIL \& GREASE

SD -100

SD -100

26-May-1987 OIL \& GREASE

3-Jun-1987 OIL \& GRRASE

SD -100

11-Jun-1987 OIL \& GREASE

SD -100

18-Jun-1987 OIL \& GREASE

* SD-100-01

SD-100-01 12-May-1987 OIL \& GREASE

SD-100-01 18-May-1987 OIL \& GREASE

SD-100-01 26-May-2987 OIL \& GREASE

SD-100-01 3-Jun-1987 OIL \& GREASE

SD-100-01 11-Jun-1987 OIL \& GREASE

SD-100-01 18-Jun-1987 OIL \& GREASE
25

$<10$

$<10$

$<10$

$<10$

$<10$

$<5.0$

$<5.0$

$<5.0$

$<5.0$

$<10$

$<2$

$<2$

$<2$

$<2$

$<2$

$<2$

$<2$

$<2$

$<2$

2

$<2$

$<2$

3

$<2$

$<2$

$<2$

$<2$

$<2$ $u g / 2$

ug/L

ug $/ \mathrm{L}$

ug $/$ L

$\mathrm{ug} / \mathrm{L}$

ug/L

ug/L

ug/L

ug/L

$\mathrm{ug} / \mathrm{L}$

ug/L

ug/L

ug $/$

ug/L

ug/L

ug/L

mg/L

ng/L

$\mathrm{mg} / \mathrm{L}$

$\mathrm{mg} / \mathrm{L}$

mg/L

$\mathrm{ng} / \mathrm{L}$

mg/L

mg/L

$m g / L$

mg/L

$\mathrm{mg} / \mathrm{L}$

ng/L

$\operatorname{mg} / \mathrm{L}$

IIB $/ \mathrm{L}$

mg/L

mg/L

ag/L

mg/L 
** SD-100-02

SD-100-02 12-May-1987 OIL \& GREASE SD-100-02 18-May-1987 OIL \& GREASE SD-100-02 26-May-1987 OIL \& GREASE SD-100-02 3-Jun-1987 OIL \& GREASE SD-100-02 11-Jun-1987 OIL \& GREASE SD-100-02 18-Jun-1987 OIL \& GREASE

** SD-100-03

SD-100-03 12-May-1987 OIL \& GREASE SD-100-03 18-May-1987 OIL \& GREASE SD-100-03 26-May-1987 OIL \& GREASE SD-100-03 3-Jun-1987 OIL \& GREASE SD-100-03 11-JUn-1987 OIL \& GREASE SD-100-03 18-Jun-1987 OIL \& GREASE

** SD-100-03D

SD-100-03D 12-May-1987 OIL \& GREASE SD-100-03D 26-May-1987 OIL \& GREASE

* SD-100-04

SD-100-04 12-May-1987 OIL \& GREASE SD-100-04 18-May-1987 OIL \& GREASE SD-100-04 26-May-1987 OIL \& GREASE SD-100-04 3-Jun-1987 OIL \& GREASE SD-100-04 11-Jun-1987 OIL \& GREASE SD-100-04 18-Jun-1987 OIL \& GREASE

** SD. $100-06$

SD-100-06 12-May-1987 OIL \& GREASE SD-100-06 18-May-1987 OIL \& GREASE SD-100-06 26-May-1987 OII \& GREASE SD-100-06 3-Jun-1987 OIL \& GREASE SD-100-06 11-Jun-1987 OIL \& GREASE SD-100-06 18-Jun-1987 OIL \& GREASE

** SD-100-06D

SD-100-06D 3-Jun-1987 OIL \& GREASE

** SD-100-07

SD-100-07 12-May-1987 OIL \& GREASE SD-100-07 19-May-1987 OIL \& GREASE SD-100-07 26-May-1987 OIL \& GREASE SD-100-07 3-Jun-1987 OIL \& GREASE SD-100-07 11-Jun-1987 OIL \& GREASE SD-100-07 18-JUn-1987 OIL \& GREASE

** SD - 100-07D

SD-100-07D 18-Jun-1987 OIL \& GREASE
3

$<2$

$<2$

$<2$

$<2$

$<2$

$<2$

$<2$

$<2$

$<2$

7

$<2$

$<2$

$<2$

mg/L mg/L

5

$<2$

$<2$

$<2$

$<2$

$<2$

3

$<2$

$<2$

$<2$

$<2$

$<2$

$<2$

mg/L

$\mathrm{mg} / \mathrm{L}$

ng/L

$\mathrm{mg} / \mathrm{L}$

mg/L

mg $/ L$

$\mathrm{mg} / \mathrm{L}$

$\mathrm{mg} / \mathrm{L}$

mg/L

$\mathrm{mg} / \mathrm{L}$

$\mathrm{mg} / \mathrm{L}$

mg/L

mg/L $\mathrm{mg} / \mathrm{L}$ $\mathrm{mg} / L$ mg/L $\operatorname{mg} / \mathrm{L}$

$\mathrm{Ing} / \mathrm{L}$ $\mathrm{mg} / \mathrm{L}$ mg/L $\mathrm{mg} / \mathrm{L}$ $\mathrm{mg} / \mathrm{L}$ $\mathrm{mg} / \mathrm{L}$

mg/L $\mathrm{mg} / \mathrm{L}$ ug $/$ L $\mathrm{mg} / \mathrm{L}$ $\mathrm{mg} / \mathrm{L}$ mg/L

mg/l 
** SD-100-09

SD-100-09 12-May-1987 OIL \& GREASE SD-100-09 18-Kay-1987 OIL \& GREASE SD-100-09 26-May-1987 OIL \& GREASE SD-100-09 3-Jun-1987 OIL \& GREASE SD-100-09 11-Jun-1987 OIL \& GREASE SD-100-09 18-Jun-1987 OIL \& GREASE

6

$<2$

$<2$

$<2$

$<2$

$<2$

** SD $-100-10$

SD-100-10 12-May-1987 OIL \& GREASE SD-100-10 19-May-1987 OIL \& GREASE SD-100-10 26-May-1987 OIL \& GREASE SD-100-10 3-Jun-1987 OIL \& GREASE SD-100-10 11-Jun-1987 OIL \& GREASE SD-100-10 18-Jur-1987 OIL \& GREASE

** SD $-100-11$

SD-100-11 12-May-1987 OIL \& GREASE SD-100-11 19-May-1987 OIL \& GREASE SD-100-11 26-May-1987 OIL \& GREASE SD-100-11 3-Jun-1987 OIL \& GREASE SD-100-11 11-Jun-1987 OIL \& GREASE SD-100-11 18-Jun-1987 OIL \& GREASE

** SD $-100-12$

SD-100-12 12-May-1987 OIL \& GREASE SD-100-12 19-May-1987 OIL \& GREASE SD-100-12 26-May-1987 OIL \& GREASE SD-100-12 3-Jun-1987 OIL \& GREASE SD-100-12 11-Jun-1987 OIL \& GREASE SD-100-12 18-Jun-1987 OIL \& GREASE

** SD $-100-13$

SD-100-13 12-May-1987 OIL \& GREASE SD-100-13 19-May-1987 OIL \& GREASE SD-100-13 26-May-1987 OIL \& GREASE SD-100-13 3-Jun-1987 OIL \& GREASE SD-100-13 11-Jun-1987 OIL \& GREASE SD-100-13 IB-Jun-1987 OIL \& GREASE

* $\quad$ SD $-100-14$

SD-100-14 12-May-1987 OIL \& GREASE SD-100-14 19-May-1987 OIL \& GREASE SD-100-14 26-May-1987 OIL \& GREASE SD-100-14 3-3un-1987 OIL \& GREASE SD-100-14 11-Jun-1987 OIL \& GREASE SD-100-14 18-Jun-1987 OIL \& GREASE

** SD-100-C

$S D-100 \cdot C$ 5-Mar-1987 OIL \& GREASE SD-100-C 9-Mar-1987 OIL \& GRFASE
$<2$

$<2$

$<2$

$<2$

$<2$

$<2$

$<2$

$<2$

$<2$

$<2$

$<2$

$<2$

$<2$

3

$<2$

$<2$

$<2$

$<2$

$<2$

$<2$

$<2$

$<2$

$<2$

$<2$

$<2$

$<2$

$<2$

$<2$

$<2$

$<2$

$<2$

$<2$ mg $/ L$

IE/ $/$

Ig $/ 2$

$\mathrm{mg} / \mathrm{L}$

mg/L

$\operatorname{mg} /$

$\mathrm{mg} / \mathrm{L}$

mg $/$

$\mathrm{mg} / \mathrm{L}$

$\mathrm{mg} / \mathrm{L}$

mg $/$ L

mg $/ \mathrm{L}$

$\mathrm{mg} / \mathrm{L}$

$\mathrm{mg} / \mathrm{L}$

mg/L

$\mathrm{mg} / \mathrm{L}$

mg $/ \mathrm{L}$

$\mathrm{mg} / \mathrm{L}$

mg $/ 2$

ag/L

IIg $/ \mathrm{L}$

ag $/ \mathrm{L}$

ng/L

ag/L

mg/L

$\mathrm{mg} / \mathrm{L}$

IE/ $/ L$

$n g / L$

听/

mo/L

m $/ 2$

$\mathrm{LE} / \mathrm{L}$

ng $/ L$

$\mathrm{ng} / \mathrm{L}$

$\mathrm{mg} / \mathrm{L}$

ug $/ \mathrm{L}$

$m_{B} / L$ $\mathrm{mg} / \mathrm{L}$. 
Location Date Test Compound Results Units

SD-100-C 17-MaI-1987 OIL \& GREASE

SD-100-C 24-Kar-1987 OIL \& GREASE

SD-100-C 8-APT-1987 OIL \& GREASE

SD-100-C 13-May-1987 OIL \& GREASE

SD-100-C 20-May-1987 OIL \& GREASE

SD-100-C 27-Kay-1987 OIL \& GREASE

SD-100-C 4-J un-1987 OIL GREASE

SD-100-C 12-Jun-1987 OIL \& GREASE

SD-100-C 19-Jun-1987 OIL \& GREASE

\begin{tabular}{l|l}
$<2$ & $\mathrm{mg} / \mathrm{L}$ \\
2 & $\mathrm{mg} / \mathrm{L}$ \\
$<2$ & $\mathrm{mg} / \mathrm{L}$ \\
$<2$ & $\mathrm{mg} / \mathrm{L}$ \\
$<2$ & $\mathrm{mg} / \mathrm{L}$ \\
$<2$ & $\mathrm{mg} / \mathrm{L}$ \\
$<2$ & $\mathrm{mg} / \mathrm{L}$ \\
$<2$ & $\mathrm{mg} / \mathrm{L}$ \\
$<2$ & $\mathrm{mg} / \mathrm{L}$
\end{tabular}

** SD - 100-D

SD-100-D 6-ApT-1987 OIL \& GREASE

SD-100-D I1-Jun-1987 OIL \& GREASE

$<2$

$<2$

$\mathrm{mg} / \mathrm{L}$

$\mathrm{mg} / \mathrm{L}$

** SD $-100-04$

SD-100-04 12-May-1987 PALMITIC ACID

3

$\operatorname{ug} / \mathrm{L}$

* SD - 100-07

SD-100-07 12-May-1987 PALMITIC ACID

ug/L

** SD-100-09

SD-100-09 12-May-1987 PALMITIC ACID

$u g / L$

*t SD -100

SD -100

5-Mar-1987 PCB (AROCLOR-1016)

SD -100

11-Mar-1987 PCB (AROCLOR-1016)

SD -100

12-May-1987 PCB (AROCLOR-1016)

SD -100

26-May-1987 PCB (AROCLOR-1016)

** SD-100-01

SD-100-01 12-May-1987 PCB (AROCLOR-1016)

SD-100-01 26-May-1987 PCB (AROCLOR-1016)

$<0.5$

$<0.5$

$\mathrm{ug} / \mathrm{L}$

$<1$

$<1$

$<0.5$

$<0.5$

ug/L

$u g / L$

ug $/ \mathrm{L}$

$u g / L$

** SD $-100-02$

SD-100-02 12-May-1987 PCB (AROCLOR-1016)

SD-100-02 26-May-1987 PCB (AROCLOR-1016)

$<0.5$

$<0.5$

ug/L

** SD $-100-03$

SD-100-03 12-May-1987 PCB (AROCLOR-1016)

SD-100-03 26-May-1987 PCB (AROCLOR-1016)

$<0.5$

$<0.5$

$\mathrm{ug} / \mathrm{L}$

$u g / L$

* SD-100-03D

SD-100-03D 12-May-1987 PCB (AROCLOR-1016)

SD-100-03D 26-May-1987 PCB (AROCLOR-1016)

$<0.5$

$\mathrm{ug} / \mathrm{L}$

$<0.5$

$\mu g / 2$

** SD-100-04

SD-100-D4 12-May-1987 PCB (AROCLOR-1016)

SD-100-04 26-May-1987 PCB (AROCLOR-1016)

$<0.5$

$\mathrm{ug} / \mathrm{L}$

$<0.5 \quad \mathrm{ug} / \mathrm{L}$

** SD-100-06

SD-100-06 12-May-1987 PCB (AROCLOR-1016)

$<0.5$

ug $/$ 


\begin{tabular}{|c|c|c|c|c|c|}
\hline Location & Date & & est Compound & Results & Units \\
\hline SD $-100-06$ & $26-$ Kay -1987 & PCB & (AROCLOR - 1016) & $<0.5$ & $\mathrm{ug} / \mathrm{L}$ \\
\hline \multicolumn{6}{|c|}{ ** SD-100-07 } \\
\hline $\begin{array}{l}\text { SD }-100-07 \\
\text { SD }-100-07\end{array}$ & $\begin{array}{l}12-\text { May-1987 } \\
26-\text { May-1987 }\end{array}$ & $\begin{array}{l}\text { PCB } \\
\text { PCB }\end{array}$ & $\begin{array}{l}\text { (AROCLOR-1016) } \\
\text { (AROCLOR-1016) }\end{array}$ & $\begin{array}{l}<0.5 \\
<0.5\end{array}$ & $\begin{array}{l}u_{g} / L \\
u g / L\end{array}$ \\
\hline \multicolumn{6}{|c|}{$\star \star \quad S D-100-09$} \\
\hline SD $-100-09$ & $12-$ Kay -1987 & PCB & (AROCLOR-1016) & $<0.5$ & $\mathrm{ug} / \mathrm{L}$ \\
\hline SD $-100-09$ & $26-$ May -1987 & PCB & (AROCLOR - 1016) & $<0.5$ & $u g / L$ \\
\hline \multicolumn{6}{|c|}{ * SD $-100-10$} \\
\hline SD $-100-10$ & 12-May-1987 & $\mathrm{PCB}$ & (AROCLOR-1016) & $<0.5$ & ug/L \\
\hline$S D-100-10$ & $26-\mathrm{May}-1987$ & PCB & (AROCLOR - 1016) & $<0.5$ & $\mathrm{ug} / \mathrm{L}$ \\
\hline \multicolumn{6}{|c|}{$* * \quad S D-100-11$} \\
\hline SD $-100-11$ & $12-$ May-1987 & PCB & (AROCLOR-1016) & $<0.5$ & $\mathbf{u g} / \mathrm{L}$ \\
\hline SD $-100-11$ & 26-May-1987 & PCB & (AROCLOR-1016) & $<0.5$ & $\mathrm{ug} / \mathrm{L}$ \\
\hline \multicolumn{3}{|c|}{ ** SD $-100-12$} & & & \\
\hline SD $-100-12$ & $12-$ May -1987 & PCB & (AROCLOR-1016) & $<0.5$ & $\mathrm{ug} / \mathrm{L}$ \\
\hline$S D-100-12$ & $26-$ May -1987 & PCB & (AROCLOR-1016) & $<0.5$ & $\mathrm{ug} / \mathrm{L}$ \\
\hline \multicolumn{6}{|c|}{ ** SD-100-13 } \\
\hline SD- $100-13$ & $12-$ May-1987 & PCB & (AROCLOR-1016) & $<0.5$ & ug/L \\
\hline SD $-100-13$ & 26 - May - 1987 & PCB & (AROCLOR-1016) & $<0.5$ & $\mathrm{ug} / \mathrm{L}$ \\
\hline \multicolumn{6}{|c|}{ ** SD-100-14 } \\
\hline SD-100-14 & 12-May -1987 & PCB & (AROCLOR-1016) & $<0.5$ & $\mathrm{ug} / \mathrm{L}$ \\
\hline SD $-100-14$ & 26 - May -1987 & PCB & (AROCLOR-1016) & $<0.5$ & ug/L \\
\hline \multicolumn{6}{|c|}{ ** SD-100-C } \\
\hline SD $-100-C$ & $5-\operatorname{Mar}-1987$ & PCB & (AROCLOR - 1016) & $<1$ & $u g /$ \\
\hline$S D-100-C$ & $9-\operatorname{Mar}-1987$ & PCB & (AROCLOR-1016) & $<1$ & $u g / L$ \\
\hline SD-100-C & $17-$ Mar- 1987 & PCB & (AROCLOR - 1016) & $<0.5$ & ug/L \\
\hline$S D-100 \cdot C$ & $24-$ Mar- 1987 & PCB & (AROCLOR-1016) & $<0.5$ & ug/L \\
\hline SD-100-C & $8-A p r-1987$ & PCB & (AROCLOR-1016) & $<0.5$ & $\mathbf{u g} / \mathrm{L}$ \\
\hline SD-100-C & 13- Мay- 1987 & PCB & (AROCLOR - 1016). & $<0.5$ & ug/L \\
\hline SD-100-C & 20-May-1987 & PCB & (AROCLOR-1016) & $<0.5$ & ug/L \\
\hline SD-100-C & $27-$ Mzy- 1987 & PCB & (AROCLOR-1016) & $<0.5$ & $\mathrm{ug} / \mathrm{L}$ \\
\hline$S D-100-C$ & 12-Jun-1987 & PCB & (AROCLOR-1016) & $<0.5$ & ug $/ \mathrm{L}$ \\
\hline$S D-100-C$ & $19-J u n-1987$ & PCB & (AROCLOR-1016) & $<1$ & $4 \mathrm{~g} / \mathrm{L}$ \\
\hline \multicolumn{6}{|l|}{$\star \star \quad S D-100$} \\
\hline SD-100 & $5-\operatorname{Mar}-1987$ & PCB & (AROCLOR - 1221) & $<1$ & ug/L \\
\hline SD -100 & $11-\mathrm{Mar}-1987$ & PCB & (AROCLOR-1221) & $<1$ & ug/L \\
\hline$S D-100$ & $12-$ May - 1987 & PCB & (AROCLOR-1221) & $<0.5$ & $\mathrm{ug} / \mathrm{L}$ \\
\hline SD -100 & 26-May - 1987 & PCB & (AROCLOR-1221) & $<0.5$ & $\mathrm{ug} / \mathrm{L}$ \\
\hline \multicolumn{6}{|c|}{$\star \star \quad S D \cdot 100-01$} \\
\hline SD $-100-01$ & 12-May-1987 & $\mathrm{PCB}$ & (AROCLOR - 1221) & $<0.5$ & $\mathrm{ug} / \mathrm{L}$ \\
\hline SD - $100-01$ & 26-May-1987 & PCB & (AROCLOR-1221) & $<0.5$ & ug/L \\
\hline
\end{tabular}


Location Date Test Compound Results UnIts

\begin{tabular}{|c|c|c|c|c|c|}
\hline $\begin{array}{l}\text { SD }-100-02 \\
\text { SD }-100-02\end{array}$ & $\begin{array}{l}12-\text { May-1987 } \\
26-\text { May-1987 }\end{array}$ & $\begin{array}{l}\text { PCB } \\
\text { PCB }\end{array}$ & $\begin{array}{l}\text { (AROCLOR-1221) } \\
\text { (AROCLOR-1221) }\end{array}$ & $\begin{array}{l}<0.5 \\
<0.5\end{array}$ & $\begin{array}{l}\mathrm{ug} / \mathrm{L} \\
\mathrm{ug} / \mathrm{L}\end{array}$ \\
\hline \multicolumn{6}{|c|}{$\star *$ SD-100-03 } \\
\hline SD $-100 \cdot 03$ & 12-May-1987 & PCB & (AROCLOR-1221) & $<0.5$ & ug/L \\
\hline SD $-100-03$ & $26-\mathrm{May} \cdot 1987$ & PCB & (AROCLOR-1221) & $<0.5$ & ug/L \\
\hline \multicolumn{6}{|c|}{$\star \quad$ SD-100-03D } \\
\hline SD-100-03D & $12-$ May-1987 & PCB & (AROCLOR-1221) & $<0.5$ & ug $/ \mathrm{L}$ \\
\hline SD-100-03D & 26-Kay-1987 & PCB & (AROCLOR-1221) & $<0.5$ & $\mathrm{ug} / \mathrm{L}$ \\
\hline \multicolumn{6}{|c|}{$\star \quad$ SD-100-04 } \\
\hline SD-100-04 & $12-\mathrm{May}-1987$ & PCB & (AROCLOR-1221) & $<0.5$ & $u g / \mathrm{L}$ \\
\hline SD-100-04 & 26-Kay-1987 & PCB & (AROCLOR - 1221) & $<0.5$ & $\mathrm{ug} / \mathrm{L}$ \\
\hline \multicolumn{6}{|c|}{$\star \quad S D-100-06$} \\
\hline SD- $100-06$ & 12-May- 1987 & PCB & (AROCLOR-1221) & $<0.5$ & $u g / L$ \\
\hline SD - $100-06$ & 26-May-1987 & PCB & (AROCLOR-1221) & $<0.5$ & ug/L \\
\hline \multicolumn{6}{|c|}{$\star \star \quad S D-100-07$} \\
\hline SD-100-07 & 12-May-1987 & PCB & (AROCLOR-1221) & $<0.5$ & $\mathrm{ug} / \mathrm{L}$ \\
\hline$S D-100-07$ & 26-May-1987 & PCB & (AROCLOR-1221) & $<0.5$ & ug $/ \mathrm{L}$ \\
\hline \multicolumn{6}{|c|}{ ** SD-100-09 } \\
\hline$S D-100-09$ & 12-May-1987 & PCB & (AROCLOR-1221) & $<0.5$ & $u g / 2$ \\
\hline SD $-100-09$ & 26-May-1987 & PCB & (AROCLOR-1221) & $<0.5$ & ug $/ \mathrm{L}$ \\
\hline \multicolumn{6}{|c|}{$\star *$ SD $-100-10$} \\
\hline SD - $100-10$ & 12-May-1987 & PCB & (AROCLOR-1221) & $<0.5$ & $u g / L$ \\
\hline SD $-100-10$ & 26-May-1987 & PCB & (AROCLOR-1221) & $<0.5$ & $\mathrm{ug} / \mathrm{L}$ \\
\hline \multicolumn{6}{|c|}{$\star$ SD $-100-11$} \\
\hline SD - $100-11$ & 12-May-1987 & PCB & (AROCLOR-1221) & $<0.5$ & ug/l \\
\hline SD - $100-11$ & $26-\mathrm{May}-1987$ & PCB & (AROCLOR-1221) & $<0.5$ & $\mathrm{ug} / \mathrm{L}$ \\
\hline \multicolumn{6}{|c|}{$\star \quad$ SD $-100-12$} \\
\hline SD- $100-12$ & 12-May-1987 & PCB & (AROCLOR-1221) & $<0.5$ & $u g / L$ \\
\hline SD $-100-12$ & $26-$ May -1987 & PCB & (AROCLOR-1221) & $<0.5$ & ug/L \\
\hline \multicolumn{6}{|c|}{$*$ SD $-100-13$} \\
\hline$S D-100-13$ & 12- May- 1987 & PCB & (AROCLOR-1221) & $<0.5$ & ug $/ \mathrm{L}$ \\
\hline SD $-100-13$ & 26-Мay-1987 & PCB & (AROCLOR-1221) & $<0.5$ & $4 \mathrm{~g} / \mathrm{L}$ \\
\hline \multicolumn{6}{|c|}{ * SD-100-14 } \\
\hline SD $-100-14$ & $12-$ May- 1987 & PCB & (AROCLOR-1221) & $<0.5$ & $u g / L$ \\
\hline$S D-100-14$ & $26-$ May- 1987 & PCB & (AROCLOR-1221) & $<0.5$ & $u g / L$ \\
\hline \multicolumn{6}{|c|}{ * SD - 100-C } \\
\hline SD - $100 \cdot C$ & 5-Mar-1987 & PCB & (AROCLOR - 1221) & $<1$ & ug/ \\
\hline
\end{tabular}




\begin{tabular}{|c|c|c|c|c|c|}
\hline Location & Date & & est Compound & Results & Units \\
\hline SD $-100-C$ & $9-\operatorname{Mar}-1987$ & PCB & (AROCLOR-1221) & $<1$ & $u g / L$ \\
\hline SD-100-C & $17-$ Mar - 1987 & PCB & (AROCLOR-1221) & $<0.5$ & $\mathrm{ug} / \mathrm{L}$ \\
\hline$S D-100-C$ & $24-$ Mar- 1987 & PCB & (AROCLOR-1221) & $<0.5$ & $u g / L$ \\
\hline SD-100-C & 8-Apr-1987 & PCB & (AROCLOR-1221) & $<0.5$ & $u g / 2$ \\
\hline SD $-100-C$ & 13-May- 1987 & PCB & (AROCLOR-1221) & $<0.5$ & $\mathrm{ug} / \mathrm{I}$ \\
\hline SD-100-C & 20-May-1987 & PCB & (AROCLOR - 1221) & $<0.5$ & ug/L \\
\hline$S D-100-C$ & 27-May-1987 & PCB & (AROCLOR-1221) & $<0.5$ & $u g / L$ \\
\hline SD-100-C & 12-Jun-1987 & PCB & (AROCLOR-1221) & $<0.5$ & $u g / L$ \\
\hline SD-100-C & 19-Jun-1987 & PCB & (AROCLOR-1221) & $<1$ & $4 g / 2$ \\
\hline \multicolumn{6}{|l|}{ ** SD -100} \\
\hline SD- 100 & $5-\operatorname{Mar}-1987$ & PCB & (AROCLOR-1232) & $<1$ & $48 / 2$ \\
\hline SD -100 & 11-Mar-1987 & PCB & (AROCLOR-1232) & $<1$ & $\mathrm{ug} / \mathrm{L}$ \\
\hline SD- 100 & 12-May-1987 & PCB & (AROCLOR-1232) & $<0.5$ & $\mathrm{ug} /$ \\
\hline SD -100 & 26-May-1987 & PCB & (AROCLOR-1232) & $<0.5$ & $\mathrm{ug} / \mathrm{I}$ \\
\hline \multicolumn{6}{|c|}{ ** SD-100-01 } \\
\hline$S D-100-01$ & 12-May-1987 & PCB & (AROCLOR-1232) & $<0.5$ & $\operatorname{ug} / \mathrm{L}$ \\
\hline SD-100-01 & 26-May-1987 & PCB & (AROCLOR-1232) & $<0.5$ & $\mathrm{ug} / \mathrm{L}$ \\
\hline \multicolumn{6}{|c|}{ ** SD-100-02 } \\
\hline SD- $100-02$ & 12-May-1987 & PCB & (AROCLOR-1232) & $<0.5$ & ug/l \\
\hline SD-100-02 & $26-$ May -1987 & PCB & (AROCLOR-1232) & $<0.5$ & $\mathrm{ug} / \mathrm{L}$ \\
\hline \multicolumn{6}{|c|}{$\star \quad S D-100-03$} \\
\hline SD $-100-03$ & 12-May-1987 & PCB & (AROCLOR - 1232) & $<0.5$ & $\mathrm{ug} / \mathrm{L}$ \\
\hline SD $-100-03$ & 26-May-1987 & PCB & (AROCLOR-1232) & $<0.5$ & $\mathrm{ug} / \mathrm{L}$ \\
\hline \multicolumn{6}{|c|}{ ** SD-100-03D } \\
\hline SD-100-03D & $12-$ Kay-1987 & PCB & (AROCLOR-1232) & $<0.5$ & $u g / L$ \\
\hline SD - 100-03D & 26-May - 1987 & PCB & (AROCLOR-1232) & $<0.5$ & $4 g / L$ \\
\hline \multicolumn{6}{|c|}{$\star \star \quad S D-100-04$} \\
\hline SD-100-04 & 12-May-1987 & PCB & (AROCLOR-1232) & $<0.5$ & $48 / \mathrm{L}$ \\
\hline SD-100-04 & $26-$ May-1987 & PCB & (AROCLOR-1232) & $<0.5$ & $\mathrm{ug} / \mathrm{L}$ \\
\hline \multicolumn{6}{|c|}{$\star \star S D-100-06$} \\
\hline SD -100.06 & $12-$ May-1987 & PCB & (AROCLOR-1232) & $<0.5$ & $\operatorname{ug} / \mathrm{L}$ \\
\hline SD $-100-06$ & $26-$ May -1987 & PCB & (AROCLOR-1232) & $<0.5$ & ug/L \\
\hline \multicolumn{6}{|c|}{$\star *$ SD $-100-07$} \\
\hline SD- $100-07$ & 12-May-1987 & PCB & (AROCLOR-1232) & $<0.5$ & ug/L \\
\hline$S D-100-07$ & $26-$ May -1987 & PCB & (AROCLOR-1232) & $<0.5$ & $\mathrm{ug} / \mathrm{L}$ \\
\hline \multicolumn{6}{|c|}{ ** SD-100.09 } \\
\hline SD -100.09 & 12-May-1987 & $\mathrm{PCB}$ & (AROCLOR - 1232) & $<0.5$ & ug $/ L$ \\
\hline SD $-100-09$ & 26-May- 1987 & $\mathrm{PCB}$ & (AROCLOR-1232) & $<0.5$ & $\mathrm{ug} / \mathrm{L}$ \\
\hline \multicolumn{6}{|c|}{ ** SD-100-10 } \\
\hline SD $-100-10$ & 12-May-1987 & PCB & (AROCLOR - 1232) & $<0.5$ & ug/L \\
\hline SD $-100-10$ & 26-May-1987 & PCB & (AROCLOR-1232) & $<0.5$ & ug/L \\
\hline
\end{tabular}


Results Units

** SD-100-11

SD-100-11 12-May-1987 PCB (AROCLOR-1232)

SD-100-11 26-May-1987 PCB (AROCLOR-1232)

$<0.5$

$<0.5$

ug/L

** SD-100-12

SD-100-12 12-May-1987 PCB (AROCLOR-1232)

SD-100-12 26-May-1987 PCB (AROCLOR-1232)

$<0.5$

$u g / L$

$\star \star \quad$ SD $-100-13$

SD-100-13 12-May-1987 PCB (AROCLOR-1232)

SD-100-13 26-May-1987 PCB (AROCLOR-1232)

$<0.5$

ug $/$ L

ug $/ 2$

$<0.5$

$<0.5$

ug/L

$u g / L$

* SD $-100-14$

SD-100-14 12-May-1987 PCB (AROCLOR-1232)

SD-100-14 26-May-1987 PCB (AROCLOR-1232)

$<0.5$

ug/ $/$

$<0.5$

ug/L

** SD $-100-C$

SD-100-C 5-Mar-1987 PGB (AROCLOR-1232)

SD-100-C 9-Mar-1987 PCB (AROCLOR-1232)

SD-100-C 17-Mar-1987 PCB (AROCLOR-1232)

SD-100-C 24-Mar-1987 PCB (AROCLOR-1232)

SD-100-C 8-APr-1987 PCB (AROCLOR-1232)

SD-100-C 13-May-1987 PCB (AROCLOR-1232)

SD-100-C 20-Kay-1987 PCB (AROCLOR-1232)

SD-100-C 27-May-1987 PCB (AROCLOR-1232)

SD-100-C 12-Jun-1987 PCB (AROCLOR-1232)

SD-100-C 19-Jun-1987 PCB (AROCLOR-1232)

$<1$

$<1$

$<0.5$

$<0.5$

$<0.5$

$<0.5$

$<0.5$

$<0.5$

$<0.5$

$<1$

ug/L

$\mathrm{ug} / \mathrm{L}$

ug $/ L$

ug/L

ug/L

$u g / L$

ug/L

ug/L

ug/L

$u g / 2$

* SD 100

SD-100 5-Mar-1987 PCB (AROCLOR-1242)

SD-100 11-Mar-1987 PCB (AROCLOR-1242)

SD-100 12-Kay-1987 PCB (AROCLOR-1242)

SD-100 26-May-1987 PC8 (AROCLOR-1242)

$<1$

$<1$

$<0.5$

$<0.5$

ug/L

ug/L

ug $/ 2$

ug/L

** SD - 100-01

SD-100-01 12-May-1987 PCB (AROCLOR-1242)

SD-100-01 26-May-1987 PCB (AROCLOR-1242)

$<0.5$

ug $/ L$

$<0.5$

ug/L

* SD $-100-02$

SD-100-02 12-May-1987 PCB (AROCLOR-1242)

$<0.5$

ug/L

SD-100-02 26-May-1987 PCB (AROCLOR-1242)

$<0.5$

$48 / \mathrm{L}$

** SD - 100-03

SD-100-03 12-May-1987 PCB (AROCLOR-1242)

SD-100-03 26-May-1987 PCB (AROCLOR-1242)

$<0.5$

ug/L

$<0.5$

$\mathrm{ug} / \mathrm{L}$

** SD - 100-03D

SD-100-03D 12-May-1987 PCB (AROCLOR-1242)

$<0.5$

ug/L

SD-100-03D 26-May-1987 PCB (AROCLOR-1242)

$<0.5$

ug/L 
* SD $-100-04$

SD-100-04 12-May-1987 PCB (AROCLOR-1242)

$<0.5$

SD-100-04 26-May-1987 PCB (AROCLOR-1242)

$<0.5$

$\mu \mathrm{g} / \mathrm{L}$

** SD $-100-06$

SD-100-06 12-May-1987 PCB (AROCLOR-1242)

SD-100-06 26-May-1987 PCB (AROCLOR-1242)

$<0.5$

$<0.5$

ug/L

** SD-100-07

SD-100-07 12-May-1987 PCB (AROCLOR-1242)

SD-100-07 26-May-1987 PCB (AROCLOR-1242)

$<0.5$

$<0.5$

$4 \mathrm{~g} / \mathrm{L}$

$\mathrm{ug} / \mathrm{L}$

* SD $-100-09$

SD-100-09 12-May-1987 PCB (AROCLOR-1242)

SD-100-09 26-May-1987 PCB (AROCLOR-1242)

$<0.5$

$<0.5$

$\mathrm{ug} / \mathrm{L}$

$u g / L$

* SD-100-10

SD-100-10 12-May-1987 PCB (AROCLOR-1242)

SD-100-10 26-May-1987 PCB (AROCLOR-1242)

$<0.5$

$<0.5$

ug/L

ug/L

* SD $-100-11$

SD-100-11 12-May-1987 PCB (AROCLOR-1242)

SD-100-11 26-May-1987 PCB (AROCLOR-1242)

$<0.5$

$<0.5$

* SD-100-12

SD-100-12 12-May-1987 PCB (AROCLOR-1242)

SD-100-12 26-May-1987 PCB (AROCLOR-1242)

$<0.5$

$<0.5$

ug/L

** SD-100-13

SD-100-13 12-May-1987 PCB (AROCLOR-1242)

SD-100-13 26-May-1987 PCB (AROCLOR-1242)

$<0.5$

$<0.5$

ug $\sqrt{L}$

ug/L

** SD $-100-14$

SD-100-14 12-May-1987 PGB (AROCLOR-1242)

SD-100-14 26-May-1987 PCB (AROCLOR-1242)

$<0.5$

$<0.5$

ug $/ 2$

** SD-100-C

SD $-100-C$

5-Mar-1987 PCB (AROCLOR-1242)

SD $-100-C$

9-Mar-1987 PCB (AROCLOR-1242)

SD $-100-C$

17-Mar-1987 PCB (AROCLOR-1242)

SD $-100-C$

24-Mar-1987 PCB (AROCLOR-1242)

SD $-100-C$

8-Apr-1987 PCB (AROCLOR-1242)

SD $-100-C$

13-May-1987 PCB (AROCLOR-1242)

SD $-100 \cdot C$

20-May-1987 PCB (AROCLOR-1242)

$S D-100-C$

27-May-1987 PGB (AROCLOR-1242)

SD $-100-C$

12-Jun-1987 PCB (AROCLOR-1242)

SD-100-C

19-Jun-1987 PCB (AROCLOR-1242)

$<1$

$<1$

$<0.5$

$<0.5$

$<0.5$

$<0.5$

$<0.5$

$<0.5$

$<0.5$

$<1$

ug/L

** SD -100

SD -100

5-Mar-1987 PCB (AROCLOR-1248)

SD -100

11-MaI-1987 PCB (AROCLOR-1248)

$<1$

$<1$

$\mathrm{ug} / \mathrm{L}$

$\mathrm{ug} / \mathrm{L}$

ug/L

$\mathrm{ug} / \mathrm{L}$

$\mathrm{ug} / \mathrm{L}$

ug/L

ug $/ \mathrm{l}$

$\mathrm{ug} / \mathrm{L}$

$4 \mathrm{~g} / \mathrm{L}$

ug/L

$\mathrm{ug} / \mathrm{L}$

ug/L

$\mathrm{ug} / \mathrm{L}$ 


\begin{tabular}{|c|c|c|c|c|c|}
\hline Location & Date & & est Compound & Results & Units \\
\hline $\begin{array}{l}S D-100 \\
S D-100\end{array}$ & $\begin{array}{l}12-\text { May- } 1987 \\
26-\text { May- } 1987\end{array}$ & $\begin{array}{l}\text { PCB } \\
\text { PCB }\end{array}$ & $\begin{array}{l}\text { (AROCLOR-1248) } \\
\text { (AROCLOR-1248) }\end{array}$ & $\begin{array}{l}<0.5 \\
<0.5\end{array}$ & $\begin{array}{l}\mathrm{ug} / \mathrm{L} \\
\mathrm{ug} / \mathrm{L}\end{array}$ \\
\hline \multicolumn{6}{|c|}{$\star$ SD $-100-01$} \\
\hline SD $-100-01$ & $12-$ May -1987 & PCB & (AROCLOR-1248) & $<0.5$ & $\operatorname{ug} /$ \\
\hline SD-100-01 & 26-May-1987 & PCB & (AROCLOR-1248) & $<0.5$ & $4 \mathrm{~g} / \mathrm{L}$ \\
\hline \multicolumn{6}{|c|}{$\star \star$ SD $-100-02$} \\
\hline$S D-100-02$ & $12-$ May -1987 & PCB & (AROCLOR-1248) & $<0.5$ & ug/L \\
\hline$S D-100-02$ & $26-$ May -1987 & PCB & (AROCLOR-1248) & $<0.5$ & ug $\Omega$ \\
\hline \multicolumn{6}{|c|}{ * SD $-100-03$} \\
\hline SD $-100-03$ & $12-$ May -1987 & $\mathrm{PCB}$ & (AROCLOR-1248) & $<0.5$ & ug/L \\
\hline SD $-100-03$ & 26-May-1987 & PCB & (AROCLOR-1248) & $<0.5$ & ug $/ \mathrm{h}$ \\
\hline \multicolumn{6}{|c|}{$\star$ * SD-100-03D } \\
\hline$S D-100-03 D$ & $12-$ May -1987 & PCB & (AROCLOR-1248) & $<0.5$ & $u g / L$ \\
\hline$S D-100-03 D$ & $26-$ May -1987 & PCB & (AROCLOR - 1248) & $<0.5$ & ug/L \\
\hline \multicolumn{6}{|c|}{ * SD $-100-04$} \\
\hline$S D-100-04$ & $12-$ May -1987 & $\mathrm{PCB}$ & (AROCLOR-1248) & $<0.5$ & $u g / L$ \\
\hline SD-100-04 & $26-\mathrm{May}-1987$ & PCB & (AROCLOR-1248) & $<0.5$ & $\operatorname{ug} / 2$ \\
\hline \multicolumn{6}{|c|}{ ** SD $-100-06$} \\
\hline$S D-100.06$ & 12-May-1987 & $\mathrm{PCB}$ & (AROCLOR-1248) & $<0.5$ & $u_{g} / 2$ \\
\hline SD-100-06 & $26-$ May -1987 & PCB & (AROCLOR-1248) & $<0.5$ & $\mathrm{ug} / \mathrm{L}$ \\
\hline \multicolumn{6}{|c|}{$\star$ SD $-100-07$} \\
\hline SD-100-07 & $12-$ Kay - 1987 & PCB & (AROCLOR-1248) & $<0.5$ & $\mathrm{ug} / \mathrm{L}$ \\
\hline SD- $100-07$ & 26-May-1987 & PCB & (AROCLOR-1248) & $<0.5$ & $\mathrm{ug} / \mathrm{L}$ \\
\hline \multicolumn{6}{|c|}{$\star \star S D-100.09$} \\
\hline SD-100-09 & 12-Kay-1987 & PCB & (AROCLOR-1248) & $<0.5$ & $\mathrm{ug} / \mathrm{L}$ \\
\hline SD - $100-09$ & $26-$ May -1987 & PCB & (AROCLOR-1248) & $<0.5$ & $\mathrm{ug} / \mathrm{L}$ \\
\hline \multicolumn{6}{|c|}{$\star \star S D-100-10$} \\
\hline$S D-100-10$ & 12-May - 1987 & PCB & (AROCLOR-1248) & $<0.5$ & $\operatorname{ug} / \mathrm{L}$ \\
\hline SD $-100-10$ & $26-$ May -1987 & PCB & (AROCLOR-1248) & $<0.5$ & $u g / L$ \\
\hline \multicolumn{6}{|c|}{$\star$ * SD $-100-11$} \\
\hline$S D-100-11$ & $12-$ May- 1987 & $\mathrm{PCB}$ & (AROCLOR-1248) & $<0.5$ & $u g / L$ \\
\hline SD-100-11 & $26-$ May- 1987 & PCB & (AROCLOR-1248) & $<0.5$ & $\mathrm{ug} / \mathrm{L}$ \\
\hline \multicolumn{6}{|c|}{$\star * \quad S D-100-12$} \\
\hline SD- $100-12$ & $12-\mathrm{May}-1987$ & PCB & (AROCLOR-1248) & $<0.5$ & $\mathrm{ug} / \mathrm{L}$ \\
\hline SD- $100-12$ & $26-$ May -1987 & PCB & (AROCLOR-1248) & $<0.5$ & $\mathrm{ug} / \mathrm{L}$ \\
\hline \multicolumn{6}{|c|}{$\star \star \quad S D-100-13$} \\
\hline SD - $100-13$ & 12-May-1987 & PCB & (AROCLOR-1248) & $<0.5$ & ug/L \\
\hline SD $-100-13$ & $26-$ May -1987 & PCB & (AROCLOR-1248) & $<0.5$ & ug \\
\hline
\end{tabular}


** SD-100-14

SD-100-14 12-May-1987 PCB (AROCLOR-1248)

$<0.5$

SD-100-14 26-May-1987 PCB (AROCLOR-1248)

$<0.5$

ug/l

$u g / 2$

** SD-100-C

SD-100-C 5-Mar-1987 PCB (AROCLOR-1248)

$<1$

$<1$

SD-100-C 9-Mar-1987 PCB (AROCLOR-1248)

SD-100-C 17-Mar-1987 PCB (AROCLOR-1248)

SD-100-C 24-Mar-1987 PCB (AROCLOR-1248)

SD-100-C 8-API-1987 PCB (AROCLOR-1248)

SD $-100-C$

13-May-1987 PCB (AROCLOR-1248)

SD $-100-C$

20-May-1987 PCB (AROCLOR-1248)

$S D-100-C$

27-May-1987 PCB (AROCLOR-1248)

$S D-100-C$

12-Jun-1987 PCB (AROCLOR-1248)

SD $-100-C$

19-Jun-1987 PCB (AROCLOR-1248)

$<0.5$

$<0.5$

$<0.5$

$<0.5$

$<0.5$

$<0.5$

$<0.5$

$<1$

ug $/ 2$

ug $/ 2$

ug $\Omega$

ug $/ \mathrm{L}$

ug $/ \mathrm{L}$

$\mathrm{ug} / \mathrm{l}$

$4 \mathrm{~g} / \mathrm{L}$

ug/L

ug $/ \mathrm{L}$

ug/ $/$

* SD-100

SD -100

5-Mar-1987 PCB (AROCLOR-1254)

11-Mar-1987 PCB (AROCLOR-1254)

SD -100

12-May - 1987 PCB (AROCLOR-1254)

SD -100

26-May-1987 PCB (AROCLOR-1254)

$<1$

$<1$

$<1$

$<1$

ug $/$

$u g / L$

ug/L

$\mathrm{ug} / \mathrm{L}$

** SD-100-01

SD-100-01 12-May-1987 PCB (AROCLOR-1254)

$<1$

$<1$

ug/L

SD-100-01 26-May-1987 PCB (AROCLOR-1254)

ug $/ \mathrm{L}$

* SD-100-02

SD-100-02 12-May-1987 PCB (AROCLOR-1254)

SD-100-02 26-May-1987 PCB (AROCLOR-1254)

$<1$

$4 \mathrm{~g} / \mathrm{L}$

$<1$

$u g / L$

* SD $-100-03$

SD-100-03 12-Kay-1987 PCB (AROCLOR-1254)

SD-100-03 26-May-1987 PCB (AROCLOR-1254)

$<1$

ug/L

$<1$

ug/L

** SD - 100-03D

SD-100-03D 12-Kay-1987 PCB (AROCLOR-1254)

SD-100-03D 26-May-1987 PCB (AROCLOR-1254).

$<1$

$<1$

ug/L

** SD-100-04

SD-100-04 12-May-1987 PCB (AROCLOR-1254)

SD-100-04 26-May-1987 PCB (AROCLOR-1254)

$<1$

$<1$

ug/L

$u g / t$

* SD $-100-06$

SD-100-06 12-May-1987 PCB (AROCLOR-1254)

SD-100-06 26-May-1987 PCB (AROCLOR-1254)

$<1$

$<1$

ug/L

** SD-100-07

SD-100-07 12-May-1987 PCB (AROCLOR-1254)

SD-100-07 26-May-1987 PCB (AROCLOR-1254)

$<1$

$<1$

$u g / \mathrm{L}$ 
Location Date Test Compound

Results Units

** SD-100-09

SD-100-09 12-May-1987 PCB (AROCLOR-1254)

SD-100-09 26-May-1987 PCB (AROCLOR-1254)

$<1$

ug/L

$<1$

ug/L

** SD $-100-10$

SD-100-10 12-May-1987 PCB (AROCLOR-1254)

SD-100-10 26-May-1987 PCB (AROCLOR-1254)

$<1$

$<1$

$\operatorname{Lg} / 2$

** SD-100-11

SD-100-11 12-May-1987 PCB (AROCLOR-1254)

SD-100-11 26-May-1987 PCB (AROCLOR-1254)

$<1$

$<1$

$\operatorname{ug} / \mathrm{L}$

** SD-100-12

SD-100-12 12-May-1987 PCB (AROCLOR-1254)

SD-100-12 26-May-1987 PCB (AROCLOR-1254)

$<1$

$<1$

$\mathrm{ug} / \mathrm{L}$

ug/L

** SD $-100-13$

SD-100-13 12-May-1987 PCB (AROCLOR-1254)

SD-100-13 26-May-1987 PCB (AROCLOR-1254)

$<1$

$\mathrm{ug} / \mathrm{L}$

$<1 \quad$ ug/L

** SD $-100-14$

SD-100-14 12-May-1987 PCB (AROCLOR-1254)

SD-100-14 26-May-1987 PCB (AROCLOR-1254)

$<1$

$\mathrm{ug} / \mathrm{L}$

$<1$

ug/L

** SD $-100-C$

SD-100-C 5-Mar-1987 PCB (AROCLOR-1254)

SD-100-C 9-Mar-1987 PCB (AROCLOR-1254)

SD-100-C 17-Mar-1987 PCB (AROCLOR-1254)

SD-100-C 24-Kar-1987 PCB (AROCLOR-1254)

SD-100-C 8-Apr-1987 PCB (AROCLOR-1254)

SD-100-C 13-Kay-1987 PCB (AROCLOR-1254)

SD-100-C 20-May-1987 PCB (AROCLOR-1254)

SD-100-C 27-May-1987 PCB (AROCLOR-1254)

SD-100-C 12-Jun-1987 PCB (AROCLOR-1254)

SD-100-C 19-Jun-1987 PCB (AROCLOR-1254)

$<1$

$<1$

$<1$

$<1$

$<1$

$<1$

$<1$

$<1$

$<1$

$<1$

ug/L

ug/L

$\operatorname{ug} / \mathrm{L}$

ug/L

ug $/ \mathrm{L}$

$\mathrm{ug} / \mathrm{L}$

$u g / L$

ug $/$

ug/L

ug $/ \mathrm{l}$

** SD -100

SD-100 5-Mar-1987 PCB (AROCLOR-1260)

SD-100 11-Mar-1987 PCB (AROCLOR-1260)

SD-100 12-May-1987 PCB (AROCLOR-1260)

SD-100 26-Kay-1987 PCB (AROCLOR-1260)

$<1$

$<1$

$<1$

$<1$

ug/

$u g \Omega$

ug $/ \mathrm{L}$

ug $/ 1$

* SD $-100-01$

SD-100-01 12-Kay-1987 PCB (AROCLOR-1260)

SD-100-01 26-May-1987 PCB (AROCLOR-1260)

$<1$

ug/L

$<1 \quad u g / L$

* SD-100-02

SD-100-02 12-May-1987 PCB (AROCLOR-1260)

$<1$

$\mathrm{ug} / \mathrm{L}$

SD-100-02 26-May-1987 PCB (AROCLOR-1260)

$<1$

$\mathrm{ug} / \mathrm{L}$ 
** SD -100.03

SD-100-03 12-May-1987 PCB (AROCLOR-1260)

SD-100-03 26-Kay-1987 PCB (AROCLOR-1260)

SD-100-11 12-May-1987 PCB (AROCLOR-1260)

SD-100-11 26-May-1987 PCB (AROCLOR-1260)

* SD - 100-12

SD-100-12 12-May-1987 PCB (AROCLOR-1260)

SD-100-12 26-May-1987 PCB (AROCLOR-1260)

* SD $-100-13$

SD-100-13 12-May-1987 PCB (AROCLOR-1260)

SD-100-13 26-May-1987 PCB (AROCLOR-1260)

* SD-100-14

SD-100-14 12-May-1987 PCB (AROCLOR-1260)

SD-100-14 26-May-1987 PCB (AROCLOR-1260)

** SD-100-C

SD-100-C 5-Mar-1987 PCB (AROCLOR-1260)

SD-100-C 9-Mar-1987 PCB (AROCLOR-1260)

SD-100-C 17-Mar-1987 PCB (AROCLOR-1260)

SD-100-C 24-Mar-1987 PCB (AROCLOR-1260)

SD-100-C 8-APr-1987 PCB (AROCLOR-1260)

SD-100-G 13-May-1987 PCB (AROCLOR-1260)
$<1$

$<1$

$<1$

$<1$

$<1$

$<1$

$<1$

$<1$

$<1$

$<1$

$<1$

$<1$

$<1$

$<1$

$\begin{array}{ll}<1 & \mathrm{ug} / \mathrm{L} \\ <1 & \mathrm{ug} / \mathrm{L}\end{array}$

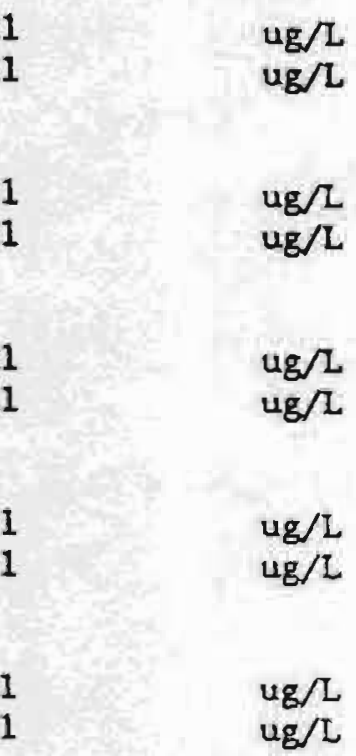

ug/L

$4 \mathrm{~g} / \mathrm{L}$

ug/L

1 ug/L

$<1 \quad$ ug/L

$<1 \quad 4 \mathrm{~g} / \mathrm{L}$

$<1 \quad$ ug $/ \mathrm{L}$

$<1 \quad$ ug/L

$<1 \quad$ Ug/L

$\begin{array}{ll}<1 & \mathrm{ug} / \mathrm{L} \\ <1 & \mathrm{ug} / \mathrm{L} \\ <1 & \mathrm{ug} / \mathrm{L} \\ <1 & \mathrm{ug} / \mathrm{L} \\ <1 & \mathrm{ug} / \mathrm{L} \\ <1 & \mathrm{ug} / \mathrm{L}\end{array}$


Units

SD $-100-C$

SD-100-C

SD $-100-C$

SD $-100-C$
20- May-1987 PCB (AROCLOR-1260)

27- May-1987 PCB (AROCLOR-1260)

12-Jun-1987 PCB (AROCLOR-1260)

19-Jun-1987 PCB (AROCLOR-1260)

** SD -100

SD -100

SD -100

SD -100

SD -100
5-Mar-1987 PENTACHLORORHENOL 11-Mar-1987 PENTACHLOROPHENOL 12-May-1987 PENIACHLOROPHENOL 26-May-1987 PENTACHLOROPHENOL

** SD-100-01

SD-100-01 12-May-1987 PENTACHLOROPHENOL SD-100-01 26-May-1987 PENTACHLOROPHENOL

* SD-100-02

SD-100-02 12-May-1987 PENTACHLOROPHENOL SD-100-02 26-May-1987 PENTACHLOROPHENOL

* SD - 100-03

SD-100-03 12-May-1987 PENTACHLOROPHENOL SD-100-03 26-May-1987 PENTACHLOROPHENOL

** SD-100-03D

SD-100-03D 12-May-1987 PENTACHLOROPHENOZ SD-100-03D 26-May-1987 PERTAGHLOROPHENOL

* SD-100-04

SD-100-04 12-May-1987 PERTACHLOROPHENOL SD-100-04 26-May-1987 PENTAGHLOROPHENOL

* $*$ SD - 100-06

SD-100-06 12-May-1987 PENTACHLOROPHENOL SD-100-06 26-May-1987 PENTACHLOROPHENOL

* * SD - 100-07

SD-100-07 12-May-1987 PENTACHLOROPHENOL SD-100-07 26-May-1987 PENTACHLOROPHENOL

** SD-100-09

SD-100-09 12-May-1987 PENTACHLOROPHENOL SD-100-09 26-May-1987 PENTACHLOROPHENOL

** SD $-100-10$

SD-100-10 12-May-1987 PENTACHLOROPHENOL SD-100-10 26-May-1987 PENTACHLOROPHENOL

* SD $-100-11$

SD-100-11 12-May-1987 PENTACHLOROPHENOL SD-100-11 26-May-1987 PENTACHLOROPHENOL

$<1$

$<1$

$<1$

$<1$

$<50$

$<50$

$<50$

$<25.0$

$<50$

$<25.0$

ug/L $u g / L$

$<50$

$<25.0$

ug/L

ug/L

$<50$

$<25.0$

ug/L ug/L

$<50$

$<25.0$

ug/L ug/L

$<50$

$<25.0$

ug/L ug/L

$<50$

$<25.0$

ug/L ug/L

$<50$

$<25.0$

ug/L ug/L

$<0$

$<25.0$

ug/L $\mathrm{ug} / \mathrm{L}$

$<50$

$<25.0$

ug $/ \mathrm{L}$ ug/L

$<50$

$<25.0$
ug/L 
Units

** SD - 100-12

SD-100-12 12-May-1987 PENTACHLOROPHENOL SD-100-12 26-May-1987 PENTACHLOROPHENOL

$<50$

$<25.0$

ug/L ug/L

* SD-100-13

SD-100-13 12-Kay-1987 PENTACHLOROPHENOL

SD-100-13 26-Kay-1987 PENTACHLOROPHENOL

$<50$

Q25.0

ug/L

ug/L

** SD-100-14

SD-100-14 12-May-1987 PENTACHLOROPHENOL

SD-100-14 26-May-1987 PEITACHLOROPHENOL

$<50$

$<25.0$

$\operatorname{ug} / 2$

$\mathrm{ug} / \mathrm{L}$

*t SD-100-C

SD-100-C 5-MaT-1987 PENTACHLOROPHENOL

$<50$

9-Mar-1987 PENTACHLOROPHENOL

SD $-100-C$

17-MaT-1987 PENTACHLOROPHENOL

SD $-100-C$

24-Mar-1987 PENTACHLOROPHENOL

SD $-100-C$

8-APY-1987 PENTACHLOROPHEMOL

SD $-100-C$

13-May-1987 PENTACHLOROPHENOL

$S D=100-C$

20-MaY-1987 PENTACHLOROPHENOL

$S D-100-C$

27-May-1987 PENTACHLOROPHENOL

SD- $100-C$

12-JUN-1987 PENTACHLOROPHENOL

SD $-100-C$

19-Jun-1987 PENTACHLOROPHENOL

$<50$

$<50$

$<50$

$<50$

$<25.0$

$<25.0$

$<25.0$

$<25.0$

$<50$

ug/L

$\mathrm{ug} / \mathrm{L}$

ug/L

ug/L

ug/L

ug/L

ug/L

ug/L

ug/L

ug/L

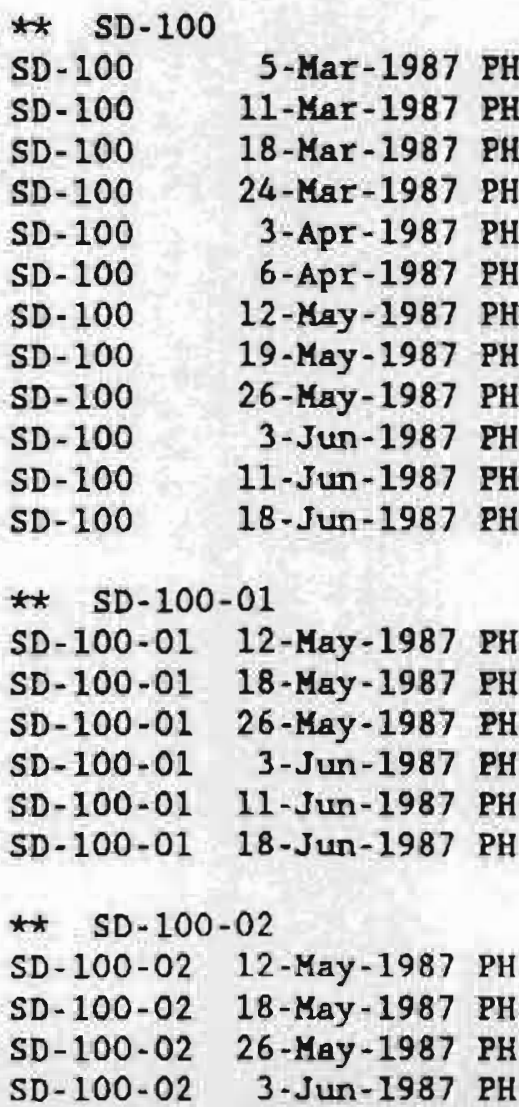

7. 3

7.9

1.1

8. 2

7.8

7.7

8.2

8.1

7.9

8.3

8.3

B. 6

7.8

7.7

7.5

7.8

8. 0

8. 6

8. 3

8.2

7.4

8. 2 
Storm Drain Data for SD-100

Location Date Test Compound Results Units

SD-100-02 11-Jun-1987 PH SD-100-02 18-Jun-1987 PH

8.3

8.6

* SD-100-03

SD-100-03 12-May-1987 PH

SD-100-03 18-May-1987 PH

SD-100-03 26-May-1987 PH

SD-100-03 3-Jun-1987 PH

SD-100-03 11-Jun-1987 PH

SD-100-03 18-Jun-1987 PH

8.2

8.1

7.8

8.1

8.3

8.6

* SD-100-03D

SD-100-03D 12-May-1987 PH

8.2

SD-100-03D 26-May-1987 PH

7.8

* SD-100-04

SD-100-04 12-May-1987 PH

SD-100-04 18-May-1987 PH

SD-100-04 26-May-1987 PH

SD-100-04 3-Jun-1987 PH

SD-100-04 11-Jun-1987 PH

SD-100-04 18-Jun-1987 PH

6.9

7.0

7.6

7.8

8.1

8.4

** SD $-100-06$

SD-100-06 12-May-1987 PH

SD-100-06 18-May-1987 PH

SD-100-06 26-May-1987 PH

SD-100-06 3-Jun-1987 PH

SD-100-06 11-Jun-1987 PH

SD-100-06 18-Jun-1987 PH

8.1

8.2

7.5

8.3

8.2

8.5

** SD-100-06D

SD-100-06D 3-Jun-1987 PB

2.0

** SD-100-07

SD-100-07 12-May-1987 PH

SD-100-07 19-Мay-1987 PH

SD-100-07 26-May-1987 PH

SD-100-07 3-Jun-1987 PH

SD-100-07 11-Jun-1987 PH

SD-100-07 18-Jun-1987 PH

2.7

7.1

6.8

7.9

6.6

6.6

** SD-100-07D

SD-100-07D 18-Jun-1987 PH

6.4

** SD $-100-09$

SD-100-09 12-May-1987 PH

8.2

SD-100-09 18-May-1987 PH

7.5

SD-100-09 26-May-1987 PH

7.7

SD-100-09 3-Jun-1987 PH

8.2

SD-100-09 11-Jun-1987 PH

8.1 
Storm Drain Data for SD-100

Location

Dace

Test Compound

Results Units

SD-100-09 18-Jun-1987 PH

8.2

* SD-100-10

SD-100-10 12-Kay-1987 PH

7.9

SD-100-10 19-May-1987 PH

8.0

SD-100-10 26-May-1987 PH

7.5

SD-100-10 3-Jun-1987 PH

SD-100-10 11-Jun-1987 PH

8.0

7.8

SD-100-10 18-Jun-1987 PH

8.2

* SD-100-11

SD-100-11 12-May-1987 PH

8. 3

SD-100-11 19-May-1987 PH

8.2

SD-100-11 26-May-1987 PH

8.0

SD-100-11 3-Jun-1987 PH

8.3

SD-100-11 11-Jun-1987 PH

8.3

SD-100-11 18-Jun-1987 PH

8.5

** SD-100-12

SD-100-12 12-May-1987 PH

8.3

SD-100-12 19-May-1987 PH

SD-100-12 26-May-1987 PH

8.2

7.6

SD-100-12 3-Jur-1987 PH

8.3

SD-100-12 11-Jun-1987 PH

8.3

SD-100-12 18-Jun-1987 PH

8.5

** SD-100-13

SD-100-13 12-May-1987 PH

SD-100-13 19-May-1987 PH

SD-100-13 26-May-1987 PH

SD-100-13 3-Jun-1987 PH

SD-100-13 11-Jun-1987 PH

SD-100-13 18-Jun-1987 PH

8.0

8.1

7.9

8.3

8.3

8.4

* SD-100-14

SD-100-14 12-May-1987 PH

SD-100-14 19-May-1987 PH

SD-100-14 26-May-1987 PH

SD-100-14 3-Jun-1987 PH

8.2

8.0

7.6

8.1

SD-100-14 11-Jun-1987 PH

8.2

SD-100-14 18-Jun-1987 PH

8.4

\# SD-100-C

SD-100-C S-Mar-1987 PH

7.2

SD-100-C 9-Mar-1987 PH

8.4

SD-100-C 17-Mar-1987 PH

8. 2

SD $-100-C$

24-Mar-1987 PH

7.9

SD $-100-C$

8-Apr-1987 PH

8.1

SD $-100-C$

23-May-1987 PH

7.9

SD-100-C 20-May-1987 PH

7.9

SD-100-C 27-May-1987 PH

7.9 


Location Date Test Compound Results Units

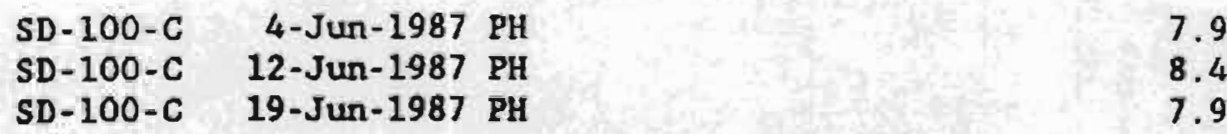

** SD - 100-D

SD-100-D 6-Apr-1987 PH $\quad 8.0$

SD-100-D 11-Jun-1987 PH $\quad 8.4$

** SD -100

SD-100 5-Mar-1987 PHENANTHRENE

SD-100 11-Mar-1987 PHERANTHRENE

SD-100 12-May-1987 PHENANTHRENE

SD-100 26-May-1987 PHENANTHRENE

$\star$ SD-100-01

SD-100-01 12-May-1987 PHENANTHRENE

SD-100-01 26-May-1987 PHENANTHRENE

$<10$

$<10$

$<10$

$<5.0$

ug/L

$\mathrm{ug} / \mathrm{L}$

ug/L

$\mathrm{ug} / \mathrm{L}$

$<10$

$<5.0$

ug/L

ug/L

** SD $-100-02$

SD-100-02 12-May-1987 PHENANTHRENE

SD-100-02 26-May-1987 PHENANTHRENE

** SD $-100-03$

SD-100-03 12-May-1987 PHENANTHRENE

SD-100-03 26-May-1987 PHENANTHRENE

$<10$

$<5.0$

$u g / L$

$\mathrm{ug} / \mathrm{L}$

$\star$ SD-100-03D

SD-100-03D 12-May-1987 PHENANTHRENE

SD-100-03D 26-May-1987 PHENANTHRENE

$<10$

$<5.0$

ug/L

ug/L

\# SD-100-04

SD-100-04 12-May-1987 PHENANTHRENE

SD-100-04 26-May-1987 PHENANTHRENE

$<10$

$<5.0$

ug/L

$<10 \quad$ ug/L

$<5.0 \quad$ ug $/ \mathrm{L}$

** SD $-100-06$

SD-100-06 12-May-1987 PHENANTHRENE

SD-100-06 26-May-1987 PHENANTHRENE

$<10$

$<5.0$

ug/L

** SD - 100-07

SD-100-07 12-May-1987 PHENANTHRENE

SD-100-07 26-May-1987 PHENANTHRENE

$<10$

$<5,0$

ug/L

$\mathrm{ug} / \mathrm{L}$

* SD - 100-09

SD-100-09 12-May-1987 PHENANTHRENE

SD-100-09 26-May-1987 PHENANTHRENE

$<10$

$<5.0$

ug/L

* SD-100-10

SD-100-10 12-May-1987 PHENANTHRENE

SD-100-10 26-May-1987 PHENANTHRENE

$<10$

$<5.0$

ug/L

ug/L 
** SD $-100-11$

SD-100-11 12-May-1987 PHENANTHRENE SD-100-11 26-May-1987 PHERTANTHRENE

$<10$

$<5.0$

ug/L

** SD $-100-12$

SD-100-12 12-May-1987 PHENANTHRENE SD-100-12 26-May-1987 PHENANTHRENE

$<10$

$<5.0$

ug/L

ug/L

** SD-100-13

SD-100-13 12-May-1987 PHENANTHRENE

SD-100-13 26-May-1987 PHENANTHRENE

$<10$

$<5.0$

ug/L

$u g / L$

** SD $-100-14$

SD-100-14 12-May-1987 PHENANTHRENE

SD-100-14 26-May-1987 PHENANTHRENE

$<10$

$<5.0$

ug/L

$\mathrm{ug} / \mathrm{L}$

* SD-100-C

SD-100-C 5-Mar-1987 PHEANANTHRENE

SD-100-C 9-Mar-1987 PHENANTHRENE

SD-100-C 17-Mar-1987 PHENANTHRENE

SD-100-C 24-Mar-1987 PHENANTHRENE

SD-100-C 8-APY-1987 PHENANTHRENE

SD-100-C 13-May-1987 PHENANTHRENE

SD-100-C 20-May-1987 PHENANTHRENE

SD-100-C 27-May-1987 PHENANTHRENE

SD-100-C 12-Jun-1987 PHENANTHRENE

SD-100-C 19-Jun-1987 PHENANTHRENE

$<10$

$<10$

$<10$

$<10$

$<10$

$<5.0$

$<5.0$

$<5.0$

$<5.0$

$<10$

$\mathrm{ug} / \mathrm{L}$

ug/L

ug/L

$u g / 2$

$\mathrm{ug} / \mathrm{L}$.

ug $/ 2$

$\mathrm{ug} / \mathrm{L}$

$u g / L$

ug/L

$\mathrm{ug} / \mathrm{L}$

* SD -100

SD-100 5-Mar-1987 PHENOL

SD-100 11-Mar-1987 PHENOL

SD-100 12-May-1987 PHENOL

SD-100 26-May-1987 PHENOL

2* SD-100-01

SD-100-01 12-May-1987 PHENOL

SD-100-01 26-May-1987 PHENOL

$<10$

$<10$

$<10$

$<5.0$

ug/L

ug/L

ug/L

$u g / L$

* SD-100-02

SD-100-02 12-May-1987 PHENOL

SD-100-02 26-May-1987 PHENOL

$<10$

$<5.0$

ug/L

ug/L

* SD $-100-03$

SD-100-03 12-May-1987 PHENOL

SD-100-03 26-May-1987 PHENOL

$<10$

$<5.0$

$\mathrm{ug} / \mathrm{L}$

$\mathrm{ug} / \mathrm{L}$

* SD - 100-03D

SD-100-03D 12-May-1987 PHENOL

SD-100-03D 26-May-1987 PHENOL

$<10$

$<5.0$

$<10$

$<5.0$
ug/L

ug/L

ug/L

$u g / L$ 


\begin{tabular}{|c|c|c|c|c|}
\hline Location & Date & Test Compound & Results & Units \\
\hline \multicolumn{5}{|c|}{ ** SD $-100-04$} \\
\hline $\begin{array}{l}\text { SD-100-04 } \\
\text { SD-100-04 }\end{array}$ & $\begin{array}{l}\text { 12-May-1987 } \\
\text { 26-May-1987 }\end{array}$ & $\begin{array}{l}\text { PHENOL } \\
\text { PHENOL }\end{array}$ & $\begin{array}{l}<10 \\
<5.0\end{array}$ & $\begin{array}{l}u g / L \\
u g / L\end{array}$ \\
\hline \multicolumn{5}{|c|}{$\star$ SD $-100-08$} \\
\hline $\begin{array}{l}\text { SD-100-06 } \\
\text { SD-100-0.6 }\end{array}$ & $\begin{array}{l}12-\text { Kay }-1987 \\
26-\text { Kay }-1987\end{array}$ & $\begin{array}{l}\text { PHENOL } \\
\text { PHENOL }\end{array}$ & $\begin{array}{l}<10 \\
<5.0\end{array}$ & $\begin{array}{l}\text { ug } / \mathrm{L} \\
\text { ug } / \mathrm{L}\end{array}$ \\
\hline \multicolumn{5}{|c|}{$\star$ * SD $-100-07$} \\
\hline SD $-100-07$ & 12-Kay-1987 & PHENOL & $<10$ & ug $/ \mathrm{L}$ \\
\hline SD $-100-07$ & 26-May-1987 & PHENOL & $<5.0$ & ug/L \\
\hline \multicolumn{5}{|c|}{$\star$ * SD $-100-09$} \\
\hline SD $-100-09$ & 12-May-1987 & PHENOL & $<10$ & $\mathrm{ug} / \mathrm{h}$ \\
\hline SD $-100-09$ & 26-May-1987 & PHENOL & $<5.0$ & $\mathrm{ug} / \mathrm{L}$ \\
\hline \multicolumn{5}{|c|}{$\star$ SD $-100-10$} \\
\hline$S D-100-10$ & $12-$ May-1987 & PHENOL & $<10$ & ug $/ \mathrm{L}$ \\
\hline$S D-100-10$ & $26-$ May- 1987 & PHENOL & $<5.0$ & $\mathrm{ug} / \mathrm{L}$ \\
\hline \multicolumn{5}{|c|}{$\star \star$ SD $-100-11$} \\
\hline$S D-100-11$ & 12-Kay-1987 & PHENOL & $<10$ & ug $/ \mathrm{L}$ \\
\hline$S D-100-11$ & $26-$ Kay-1987 & PHENOL & $<5.0$ & ug/L \\
\hline \multicolumn{5}{|c|}{$\star \star S D-100-12$} \\
\hline$S D-100-12$ & 12-Kay-1987 & PHENOZ & $<10$ & $\mathrm{ug} / \mathrm{L}$ \\
\hline SD-100-12 & $26-\mathrm{May}-1987$ & FHENOL & $<5.0$ & $\mathrm{ug} / \mathrm{L}$ \\
\hline \multicolumn{5}{|c|}{$\star \star$ SD $-100-13$} \\
\hline$S D-100-13$ & $12-$ May -1987 & PHENOL & $<10$ & ug/L \\
\hline SD- $100-13$ & $26-$ May -1987 & PHENOL & $<5.0$ & $\mathrm{ug} / \mathrm{L}$ \\
\hline \multicolumn{5}{|c|}{$\star \star \quad S D-100-14$} \\
\hline$S D-100-14$ & 12-May- 1987 & PHENOL & $<10$ & $\mathrm{ug} / \mathrm{L}$ \\
\hline SD $-100-14$ & 26 -Kay- 1987 & PHENOL & $<5.0$ & ug/L \\
\hline \multicolumn{5}{|c|}{ ** SD $-100-C$} \\
\hline$S D-100-C$ & 5-Mar-1987 & PHENOL & $<10$ & $u g / L$ \\
\hline SD- $100-C$ & $9-$ Kar-1987 & PHENOL & $<10$ & $u g / L$ \\
\hline SD $-100-C$ & 17 -Kar -1987 & PHENOL & $<10$ & ug/L \\
\hline SD $-100-C$ & 24-Mar-1987 & PHENOL & $<10$ & ug/L \\
\hline SD - $100-C$ & 8-Apr-1987 & PHENOL & $<10$ & ug/L \\
\hline SD $-100-C$ & 13-May-1987 & PHENOL & $<5.0$ & UE/I \\
\hline$S D-100-C$ & 20-May-1987 & PHENOL & $<5.0$ & $u g / 2$ \\
\hline $\mathrm{SD}-100 \cdot \mathrm{C}$ & 27-May-1987 & PHENOL & $<5.0$ & ug/L \\
\hline SD $-100-C$ & 12-Jun-1987 & PHENOZ & $<5.0$ & ug/L \\
\hline SD $-100-C$ & $19-J u n-1987$ & PHENOL & $<10$ & $\mathrm{ug} / \mathrm{L}$ \\
\hline \multicolumn{5}{|l|}{$\star \star \quad S D-100$} \\
\hline SD - 100 & $5-\operatorname{Mar}-1987$ & PHOSPHORUS & 0.55 & $\mathrm{mg} / \mathrm{L}$ \\
\hline SD - 100 & $11-\operatorname{Mar}-1987$ & PHOS PHORUS & 0.64 & $\operatorname{mg} / \mathrm{L}$ \\
\hline
\end{tabular}


** SD-100-01

SD-100-01 12-May-1987 PHOSPHORUS SD-100-01 18-May-1987 PHOSPHORUS SD-100-01 3-Jun-1987 PHOSPHORUS SD-100-01 11-Jun-1987 PHOSPHORUS SD-100-01 18-Jun-1987 PHOSPHORUS

$<0.20$

$<0.2$

$<0.20$

$<0.20$

$<0.20$

$<0.20$

0.40

0.22

0.56

0.48

SD-100-02 11-Jun-1987 PHOSPHORUS

SD-100-02 18-Jun-1987 PHOSPHORUS

t* SD-100-03

SD-100-03 12-Kay-1987 PHOSPHORUS SD-100-03 18-Kay-1987 PHOSPHORUS SD-100-03 26-May-1987 PHOSPHORUS SD-100-03 3-Jun-1987 PHOSPHORUS SD-100-03 11-Jun-1987 PHOSPHORUS SD-100-03 18-Jun-1987 PHOSPHORUS

* SD-100-03D

SD-100-03D 12- Hay-1987 PHOSPHORUS SD-100-03D 26-Kay-1987 PHOSPHORUS

** SD $-100-04$

SD-100-04 12-Kay-1987 PHOSPHORUS

SD-100-04 18-May-1987 PHOSPHORUS

SD-100-04 26-May-1987 PHOSPHORUS

SD-100-04 3-Jun-1987 PHOSPHORUS

SD-100-04 11-Jun-1987 PHOSPHORUS

SD-100-04 18-Jun-1987 PHOSPHORUS

** SD-100-06

SD-100-06 12-May-1987 PHOSPHORUS

SD-100-06 18-May-1987 PHOSPHORUS

SD-100-06 26-May-1987 PHOSPHORUS

SD-100-06 3-Jun-1987 PHOSPHORUS

SD-100-06 11-Jun-1987 PHOSPHORUS
0.35

0.35

0.32

0.47

0.37

0.38

0.30

0.49

0.25

0.30

0.36

0.38

0.56

0.39

0.26

0.31

0.27

0.39

0.24 $\mathrm{mg} / \mathrm{L}$
$\mathrm{mg} / \mathrm{L}$
$\mathrm{mg} / \mathrm{L}$
$\mathrm{mg} / \mathrm{L}$
$\mathrm{mg} / \mathrm{L}$
$\mathrm{mg} / \mathrm{L}$
$\mathrm{mg} / \mathrm{L}$
$\mathrm{mg} / \mathrm{L}$
$\mathrm{mg} / \mathrm{L}$
$\mathrm{mg} / \mathrm{L}$

mg/L

$\mathrm{mg} / \mathrm{L}$

$\mathrm{mg} / \mathrm{L}$

$\mathrm{mg} / \mathrm{L}$

匹g/L

$\mathrm{mg} / \mathrm{L}$

听/L

$\mathrm{mg} / \mathrm{L}$

$\mathrm{mg} / \mathrm{L}$

$\mathrm{mg} / \mathrm{L}$

ng/L

$\mathrm{mg} / \mathrm{L}$

me/L

mg/L

$\mathrm{mg} / \mathrm{L}$

$\mathrm{mg} / \mathrm{L}$

mg/L

mg/L

ng/L

$\operatorname{mg} / \mathrm{L}$

ng/L

ng $/ \mathrm{L}$

$-g / L$

$\mathrm{gg} / \mathrm{L}$

$\mathrm{mg} / \mathrm{L}$

$\mathrm{mg} / \mathrm{L}$

ag/L

ag/L

mg/L 
Storm Drain Data for SD-100

\begin{tabular}{|c|c|c|c|c|}
\hline Location & Date & Test Compound & Results & Units \\
\hline$S D-100-06$ & $18-J u n-1987$ & PHOSPHORUS & 0.27 & \\
\hline \multicolumn{5}{|c|}{$\star * \quad S D-100-06 D$} \\
\hline SD $-200-06 D$ & 3-Jun-1987 & PHOSPHORUS & 0.43 & \\
\hline \multicolumn{5}{|c|}{$* * \quad S D-100-07$} \\
\hline SD -100.07 & 12-May-1987 & PHOSPHORUS & 1.8 & \\
\hline SD-100-07 & $19-$ May-1987 & PHOSPHORUS & 2.4 & $\operatorname{mg}$ \\
\hline SD $-100-07$ & 26-May-1987 & PHOSPHORUS & 0.27 & $\mathrm{mg}$ \\
\hline SD -100.07 & 3-Jun-1987 & PHOSPHORUS & 1.0 & \\
\hline SD- 100.07 & $11-J u n-1987$ & PHOSPHOROS & 16 & mg \\
\hline SD $-100-07$ & $18-J u n-1987$ & PHOSPHORUS & $<0.20$ & \\
\hline \multicolumn{5}{|c|}{ ** SD-100-07D } \\
\hline$S D-100-070$ & $18-J u n-1987$ & PHOSPHORUS & $<0.20$ & $\mathrm{mg}$ \\
\hline \multicolumn{5}{|c|}{$\star$ SD-100-09 } \\
\hline SD-100-09 & 12-May-1987 & PHOSPHORUS & 0.25 & $\mathrm{mg} / \mathrm{L}$ \\
\hline SD-100-09 & 18-May-1987 & PHOSPHORUS & 0.28 & $\mathrm{mg} /$ \\
\hline SD-100-09 & 26-May - 1987 & PHOSPHORUS & 0.33 & $\mathrm{mg} / \mathrm{L}$ \\
\hline SD-100-09 & 3-Jun- 1987 & PHOSPHORUS & 0.33 & mg/ \\
\hline SD-100-09 & 11-Jun-1987 & PHOSPHORUS & 0.51 & \\
\hline
\end{tabular}

* SD-100-10

SD-100-10 12-May-1987 PHOSPHORUS

SD-100-10 19-May-1987 PHOSPHORUS

SD-100-10 26-May-1987 PHOSPHORUS

SD-100-10 3-Jun-1987 PHOSPHORUS

SD-100-10 11-Jun-1987 PHOSPHORUS

SD-100-10 18-Jun-1987 PHOSPHORUS

$<0.20$

0.36

$<0.2$

0.50

0.41

0.51

$\mathrm{mg} / \mathrm{L}$

$\mathrm{mg} / \mathrm{L}$

$\mathrm{mg} / \mathrm{L}$

$\mathrm{mg} / \mathrm{I}$

mg $/ L$

* SD-100-11

SD-100-1-1 12-May-1987 PHOSPHORUS

SD-100-11 19-May-1987 PHOSPHORUS

SD-100-11 26-May-1987 PHOSPHORUS

SD-100-11 3-Jun-1987 PHOSPHORUS

SD-100-11 11-Jun-1987 PHOSPHORUS

SD-100-11 18-Jun-1987 PHOSPHORUS

0.36

$<0.2$

$<0.2$

0.29

0.42

0.58

mg/L

* SD-100-12

SD-100-12 12-May-1987 PHOSPHORUS

SD-100-12 19-May-1987 PHOSPHORUS

SD-100-12 26-May-1987 PHOSPHORUS

SD-100-12 3-Jun-1987 PHOSPHORUS

SD-100-12 11-Jun-1987 PHOSPHORUS

SD-100-12 18-Jun-1987 PHOSPHORUS

0.47

0.36

0.37

0.37

0.32

0.33

mg/L

$\mathrm{mg} / \mathrm{L}$

$\mathrm{mg} / \mathrm{L}$

$\mathrm{mg} / \mathrm{L}$

$m g / L$

$\mathrm{mg} / \mathrm{L}$

** SD $-100-13$

SD-100-13 12-May-1987 PHOSPHORUS

SD-100-13 19-May-1987 PHOSPHORUS

SD-100-13 26-May-1987 PHOSPHORUS

$0.32 \mathrm{mg} / \mathrm{L}$

0.66

0.41

$\mathrm{mg} / \mathrm{L}$

$\mathbf{m g} / \mathrm{L}$

$\mathrm{mg} / \mathrm{L}$

mg/L

$\mathrm{mg} / \mathrm{L}$

mg/L

$\mathbf{m g} / \mathrm{L}$ 
** SD-100-14

SD-100-14 12-Mzy-1987 PHOSPHORUS SD-100-14 19-May-1987 PHOSPHOROS SD-100-14 26-Kay-1987 PHOSPHORUS SD-100-14 3-Jun-1987 PHOSPHORUS SD-100-14 11-Jun-1987 PHOSPHORUS SD-100-14 18-Jun-1987 EHOSPHORUS

* SD-100-C

SD-100-C 5-Mar-1987 PHOSPHORUS

0.26

0.36

SD-100-C 9-Mar-1987 PHOSPHORUS

SD-100-C 17-Mar-1987 PHOSPHORUS

SD-100-C 24-Mar-1987 PHOSPHORUS

SD-100-C 8-Apr-1987 PHOSPHORUS

SD-100-C 13-May-1987 PHOSPHORUS

SD-100-C 20-May-1987 PHOSPHORUS

SD-100-C 27-May-1987 PHOSPHORUS

SD-100-C 4-Jun-1987 PHOSPHORUS

SD-100-C 12-Jun-1987 PHOSPHORUS

SD-100-C 19-Jun-1987 PHOSPHORUS

0.44

0.29

$<0.20$

$<0.20$

$<0.20$

$<0.20$

0.42

0.47

0.46

** SD-100-D

SD-100-D 6-APr-1987 PHOSPHOROS

SD-100-D 11-Jun-1987 PHOSPHORUS

$<0.20$

0.60

$\mathrm{mg} / \mathrm{L}$

$\mathrm{mg} / \mathrm{L}$

mg/L

$\mathrm{mg} / \mathrm{L}$

$\mathrm{mg} / \mathrm{L}$

$\mathrm{mg} / \mathrm{L}$

$\mathrm{mg} / \mathrm{L}$

$\mathrm{mg} / \mathrm{L}$

$\mathrm{mg} / \mathrm{L}$

$\mathrm{mg} / \mathrm{L}$

$\mathrm{mg} / \mathrm{L}$

** SD -100

$\mathrm{mg} / \mathrm{L}$

$\mathrm{mg} / \mathrm{L}$

SD -100 5-MaI-1987 POTASSIUM

SD-100 11-Mar-1987 POTASSIUY

SD-100 18-Mar-1987 POTASSIUM

SD-100 24-Mar-1987 POTASSIUM

SD-100 3-Apr-1987 POTASSIUM

SD-100 6-Apr-1987 POTASSIUM

SD-100 12-May-1987 POTASSIUM

SD-100 19-May-1987 POTASSTUM

SD-100 26-May-1987 POTASSIUM

SD-100 3-Jun-1987 POTASSIUM

SD-100 11-Jun-1987 POTASSIUM

SD-100 18-Jun-1987 POTASSIUM

1.6

1.6

0.73

3.8

2.9

$<0.60$

1.5

0.78

1.8

1.5

$<0.60$

** SD-100-0I

SD-100-01 12-May-1987 POTASSIUM

SD-100-01 18-May-1987 POTASSIUM

SD-100-01 3-Jun-1987 POTASSIUM

SD-100-01 11-JUn-1987 POTASSIUM

SD-100-01 18-Jun-1987 POTASSIUM
1.3

1.3

$<0.6$

1.2

3.3

$<0.60$ $\mathrm{mg} / \mathrm{L}$

$\mathrm{mg} / \mathrm{L}$

$\mathrm{mg} / \mathrm{L}$

$\mathrm{mg} / \mathrm{L}$

$\mathrm{mg} / \mathrm{L}$

$\mathrm{mg} / \mathrm{L}$

$\mathrm{mg} / \mathrm{L}$

$\mathrm{mg} / \mathrm{L}$

$\mathrm{mg} / \mathrm{L}$

$\mathrm{mg} / \mathrm{L}$

$\mathrm{mg} / \mathrm{L}$

$\mathrm{mg} / \mathrm{L}$

$\mathrm{mg} / \mathrm{L}$

mg/L

$\mathrm{mg} / \mathrm{L}$

mg/L

$\mathrm{mg} / \mathrm{L}$

** SD-100-02

SD-100-02 12-May-1987 POTASSTUM

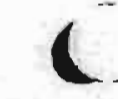


Location Date Test Compound Results Units

SD-100-02 18-May-1987 POTASSIUM

SD-100-02 3-Jun-1987 POTASSIUM

SD-100-02 11-Jun-1987 POTASSIUM

SD-100-02 18-Jun-1987 POTASSIUM

** SD $-100-03$

SD-100-03 12-May-1987 POTASSIUN

SD-100-03 18-Kay-1987 POTASSIUM

SD-100-03 26-May-1987 POTASSIOM

SD-100-03 3-Jun-1987 POTASSIUM

SD-100-03 11-Jun-1987 POIASSIUM

SD-100-03 18-Jun-1987 POTASSIUM

** SD-100-03D

SD-100-03D 12-May-1987 POTASSIUM

SD-100-03D 26-May-1987 POTASSIUM

** SD $-100-04$

SD-100-04 12-May-1987 POTASSIUM

SD-100-04 18-May-1987 POTASSIUM

SD-100-04 26-May-1987 POTASSIUM

SD-100-04 3-Jun-1987 POTASSIUM

SD-100-04 11-Jun-1987 POTASSIUM

SD-100-04 18-Jun-1987 PÓTASSIUM

** SD $-100-06$

SD-100-06 12-May-1987 POTASSIUM

SD-100-06 18-Hay-1987 POTASSIUM

SD-100-06 26-May-1987 POTASSIUM

SD-100-06 3-Jun-1987 POTASSIUM

SD-100-06 11-Jun-1987 POTASSIUM

SD-100-06 18-Jun-1987 POTASSIUM

* SD-100-06D

SD-100-06D 3-JUn-1987 POTASSIUM

** SD-100-07

SD-100-07 12-Mry-1987 POTASSIUM

SD-100-07 19-May-1987 POTASSIUM

SD-100-07 26-May-1987 POTASSIUM

SD-100-07 3-JUn-1987 POTASSIUM

SD-100-07 11-Jun-1987 POTASSIUM

SD-100-07 18-Jun-1987 POTASSIUM

** SD-100-07D

SD-100-07D 18-Jü-1987 POTASSIUM

** SD-100-09

SD-100-09 12-Kay-1987 POTASSIUM

SD-100-09 18-May-1987 POTASSIUM

SD-100-09 26-May-1987 POTASSIUM
1.6

2.0

1.3

1.8

$<0.60$

0.72

$<0.60$

1.7

1.4

0.94

$<0.60$

$<0.60$

$<0.60$

$<0.60$

$<0.60$

1.7

0.94

0.99

1.1

0.66

$<0.60$

1.4

0.89

0.79

1.7

$\mathrm{mg} / \mathrm{L}$

4.6

$<0.60$

$<0.60$

0.65

4.0

$<0.60$

$\mathrm{mg} / \mathrm{L}$

$\mathrm{mg} / \mathrm{L}$

$\mathrm{mg} / \mathrm{L}$

$\mathrm{mg} / \mathrm{L}$

$\mathrm{mg} / \mathrm{L}$

$\mathrm{mg} / \mathrm{L}$

2.7

$\mathrm{mg} / \mathrm{L}$

$<0.60$

1. 5

$<0.60$
mg/L Ing/L $\operatorname{mg} / \mathrm{L}$ 
Storm Drain Data for SD- 100

Location Date Test Compound

SD-100-09 3-Jun-1987 POTASSIUM

SD-100-09 11-Jun-1987 POTASSIUM

1.8

0.84

mg $/ 2$

* SD $-100-10$

SD-100-10 12-May-1987 POTASSIUM

SD-100-10 19-May-1987 POTASSIUM

SD-100-10 26-May-1987 POTASSIUM

SD-100-10 3-Jun-1987 POTASSIUM

SD-100-10 11-Jun-1987 POTASSIUM

SD-100-10 18-Jun-1987 POTASSIUM

$<0.60$

1.2

0.93

1.6

1.3

2.1

Units

* $\quad$ SD-100-11

SD-100-11 12-May-1987 POTASSIUM

SD-100-11 19-May-1987 POTASSIUM

SD-100-11 26-May-1987 POTASSIUM

SD-100-11 3-Jun-1987 POTASSIUM

SD-100-11 11-Jun-1987 POTASSIUY

SD-100-11 18-Jun-1987 POTASSIUM

1.1

1.9

2.2

1.6

1.4

1.2

$m g / L$

** SD $-100-12$

SD-100-12 12-May-1987 POTASSIUM

SD-100-12 19-May-1987 POTASSIUM

SD-100-12 26-Hay-1987 POTASSIUM

SD-100-12 3-Jun-1987 POTASSIUM

SD-100-12 11-Jun-1987 POTASSIUM

SD-100-12 18-Jun-1987 POTASSIUM

0.87

0.77

$<0.60$

3.9

2.1

0.89

$\operatorname{mg} / 1$

$m g / L$

mg/2

$\mathrm{mg} / \mathrm{L}$

$\mathrm{mg} / \mathrm{L}$

$\operatorname{mg} / 2$

* SD $-100-13$

SD-100-13 12-May-1987 POTASSIUM

SD-100-13 19-May-1987 POTASSIUM

SD-100-13 26-May-1987 POTASSIUM

SD-100-13 11-Jun-1987 POTASSIUM

SD-100-13 18-Jun-1987 POTASSIUM

1.1

2.7

$<0.60$

1.1

1.6

$\mathrm{mg} / \mathrm{L}$

$\mathrm{mg} / \mathrm{L}$

$\mathrm{mg} / \mathrm{L}$

$\mathrm{mg} / \mathrm{L}$

$\mathrm{mg} / \mathrm{L}$

$\mathrm{mg} / \mathrm{L}$

* SD $-100-14$

SD-100-14 12-May-1987 POTASSIUM

SD-100-14 19-May-1987 POTASSIUM

SD-100-14 26-May-1987 POTASSIUM

SD-100-14 3-Jun-1987 POTASSIUM

SD-100-14 11-Jun-1987 POTASSTUM

SD-100-14 18-Jun-1987 POTASSIUM

$<0.60$

5.7

0.94

3.5

2.2

1.8

$\mathrm{mg} / \mathrm{L}$

$\mathrm{mg} / \mathrm{L}$

$\mathrm{mg} / \mathrm{L}$

$\mathrm{mg} / \mathrm{L}$

$\mathrm{mg} / \mathrm{L}$

mg/L

* SD $-100-C$

SD-100-C 5-Mar-1987 POTASSIUM

SD-100-C 9.Mar-1987 POTASSIUM

SD-100-C 17-Mar-1987 POTASSIUM

SD-100-C 24-Mar-1987 POTASSIUM

SD-100-C 8-APY-1987 POTASSIUM

SD-100-C 13-May-1987 POTASSIUM

SD-100-C 20-May-1987 POTASSIUM

SD-100-C 27-May-1987 POTASSIUM

1.9

0.88

1.8

4.5

1.9

0.91

$<0.60$

1.2 $\operatorname{mg} / \mathrm{L}$

$\operatorname{mg} / \mathrm{L}$

$m g / L$

$\mathrm{mg} / \mathrm{L}$

$\mathrm{mg} / \mathrm{L}$

ng/L

ag/ 1

$\mathrm{mg} / \mathrm{l}$

$\mathrm{mg} / \mathrm{L}$.

$n g / L$

ng/L

ag/ $/$

ng/ $\mathrm{L}$

$\mathrm{ng} / \mathrm{L}$

$\mathrm{mg} / \mathrm{L}$

$\mathrm{ng} / \mathrm{L}$

ng/L

ng $/ L$

ng/L 
$u g / L$ ug/L ug/L $u g / L$

$<10$

$<5.0$

ug/

ug/L

$<10$

$<5.0$

ug/L

$\mathrm{ug} / \mathrm{L}$

$<10$

$<5.0$

ug/L ug/L

$<10$

$<5.0$

ug/L ug/L

$<10$

$<5.0$

ug/L $u g / L$

$<10$

$<5.0$

ug/L ug $/$

$<10$

$<5.0$

$4 g / 2$ ug/L

$<10$

$<5.0$

$4 \mathrm{~g} / \mathrm{L}$

ug/L

$<10$

$<5.0$

ug/ $/$ $\mathrm{ug} / \mathrm{L}$ 
* SD-100-11

SD-100-11 12-Klay-1987 PYRENE

SD-100-11 26-May-1987 PYRENE

$<10$

$<5.0$

10

$<5.0$

$<10$

$<5.0$

$<10$

$<5.0$

$<10$

$<10$

$<10$

$<10$

$<10$

$<5.0$

$<5.0$

$<5.0$

$<5.0$

$<10$

$<0.005$

$<0.005$

$<0.005$

$<0.005$

$<0.005$

$<0.005$

$<0.005$

$<0.005$

$<0.005$

$<0.005$

$<0.005$

$<0.005$

$<0.005$

$<0.005$

$<0.005$

$<0.005$

$<0.005$

$<0.005$
ug/L

ug/L

ug/L

$\mathrm{ug} / \mathrm{L}$

ug/L

$u g / L$

ug/L

ug/L

ug $/ L$

ug/L

ug/L

$\mathrm{ug} / \mathrm{L}$

ug/L

ug/L

$4 \mathrm{~g} / \mathrm{L}$

$u g / L$

ug/L

ug/L

$\mathrm{mg} / \mathrm{L}$

$\mathrm{mg} / \mathrm{L}$

mg/L

ng/L

mg/L

$\mathrm{mg} / \mathrm{L}$

$\mathrm{mg} / \mathrm{L}$

ag/L

II $/ \mathrm{L}$

Eg/L

파 $/ L$

mg/L

ag/L

mg/L

ng/L

mg/L

ag/L

IIg/L 
** SD-100-02

SD-100-02 12-May-1987 SELENIUM SD-100-02 18-May-1987 SELENIUM SD-100-02 26-May-1987 SBLENIUM SD-100-02 3-Jun-1987 SELENIUM SD-100-02 11-JUn-1987 SELENIUM SD-100-02 18-Jun-1987 SELENIUM

$\begin{array}{ll}<0.005 & \mathrm{mg} / \mathrm{L} \\ <0.005 & \mathrm{mg} / \mathrm{L} \\ <0.005 & \mathrm{mg} / \mathrm{L} \\ <0.005 & \mathrm{mg} / \mathrm{L} \\ <0.005 & \mathrm{mg} / \mathrm{L} \\ <0.005 & \mathrm{mg} / \mathrm{L} \\ & \\ <0.005 & \mathrm{mg} / \mathrm{L} \\ <0.005 & \mathrm{mg} / \mathrm{L} \\ <0.005 & \mathrm{mg} / \mathrm{L} \\ <0.005 & \mathrm{mg} / \mathrm{L} \\ <0.005 & \mathrm{mg} / \mathrm{L} \\ <0.005 & \mathrm{mg} / \mathrm{L}\end{array}$

$<0.005$

$<0.005$

mg/L

$\mathrm{mg} / \mathrm{L}$

$<0.005$

$<0.005$

$<0.005$

$<0.005$

$<0.005$

$<0.005$

mg $/ \mathrm{L}$

따 $/ \mathrm{L}$

$\mathrm{mg} / \mathrm{L}$

$\mathrm{mg} / \mathrm{L}$

$\mathrm{mg} / \mathrm{L}$

$\mathrm{mg} / \mathrm{L}$

$<0.005$

$<0.005$

$<0.005$

$<0.005$

$<0.005$

$<0.005$

mg/L

$\mathrm{mg} / \mathrm{L}$

mg/L

$\mathrm{mg} / \mathrm{L}$

m/L

$\mathrm{mg} / \mathrm{L}$

$<0.005$

$\operatorname{mg} / \mathrm{L}$

$<0.005$

$<0.005$

$<0.005$

$<0.005$

$<0.005$

$<0.005$

Ing/L

$\mathrm{mg} / \mathrm{L}$

mg/L

II $/ \mathrm{L}$

$\mathrm{mg} / \mathrm{L}$

$\mathrm{ng} / \mathrm{L}$

$<0.005$

$\mathrm{mg} / \mathrm{L}$ 
** SD-100-09

$\begin{array}{rrr}\text { SD-100-09 } & 12 \text {-May-1987 } & \text { SELENIUM } \\ \text { SD-100-09 } & 18 \text {-May-1987 } & \text { SELENIUM } \\ \text { SD-100-09 } & 26 \text {-May-1987 } & \text { SELENIUM } \\ \text { SD-100-09 } & 3-J u n-1987 & \text { SELENIUM } \\ \text { SD-100-09 } & 11-J u n-1987 & \text { SELENIUM } \\ \text { SD-100-09 } & 18-J u n-1987 & \text { SELENIUM }\end{array}$

$<0.005$

$<0.005$

$<0.005$

$<0.005$

$<0.005$

$<0.005$

$<0.005$

$<0.005$

$<0.005$

$<0.005$

$<0.005$

$<0.005$

SD-100-10 11-Jun-1987 SELENIUM

* SD $-100-11$

SD-100-11 12-May-1987 SELENIUM

SD-100-11 19-May-1987 SELBNIUM

SD-100-11 26-May-1987 SELENIUM

SD-100-11 3-Jun-1987 SELENIUM

SD-100-11 11-Jun-1987 SELERIUM

SD-100-11 18-JUn-1987 SELENIUM

** SD-100-12

SD-100-12 12-Kay-1987 SELENIUM

SD-100-12 19-Kay-1987 SELENIUM

SD-100-12 26-Kay-1987 SELENIUM

SD-100-12 3-Jun-1987 SELENIUM

SD-100-12 11-Jun-1987 SELENIUM

SD-100-12 18-Jun-1987 SELENIUM

** SD $-100-13$

SD-100-13 12-Kay-1987 SELFNIUM

SD-100-13 19-May-1987 SELENIUM

SD-100-13 26-Kay-1987 SELENIUM

SD-100-13 3-Jun-1987 SELFNIUM

SD-100-13 11-Jun-1987 SELENIUM

SD-100-13 18-Jun-1987 SELENIUM

« SD $-100-14$

SD-100-14 12-May-1987 SELENIUM

SD-100-14 19-Kay-1987 SELENTUM

SD-100-14 26-Kay-1987 SELENIUM

SD-100-14 3-JUn-1987 SELENIUM

SD-100-14 11-Jun-1987 SELENIUM

SD-100-14 18-Jun-1987 SELENIUM

** SD-100-C

SD-100-C S-Kar-1987 SELENIUM

$\begin{array}{lll}\text { SD-100-C } & \text { S-Mar-1987 } & \text { SELENIUM } \\ \text { SD-100-C } & 9-\text { Mar-1987 SELENIUM }\end{array}$
$<0.005$

$<0.005$

$<0.005$

$<0.005$

$<0.005$

$<0.005$

$<0.005$

$<0.005$

$<0.005$

$<0.005$

$<0.005$

$<0.005$

$<0.005$

$<0.005$

$<0.005$

$<0.005$

$<0.005$

$<0.005$

$<0.005$

$<0.005$

$<0.005$

$<0.005$

$<0.005$

$<0.005$

$<0.005$

$<0.005$ $\mathrm{mg} / \mathrm{L}$

$\mathrm{mg} / \mathrm{L}$

$\operatorname{mg} / \mathrm{L}$

mg/L

$\mathrm{mg} / \mathrm{L}$

mg/L

mg/L

$\mathrm{mg} / \mathrm{L}$

$\mathrm{mg} / \mathrm{L}$

$\mathrm{mg} / \mathrm{L}$

$\mathrm{mg} / \mathrm{L}$

mg/L

$\operatorname{mg} /$

mg/L

$\mathrm{mg} / \mathrm{L}$

$\mathrm{mg} / \mathrm{L}$

$\mathrm{mg} / \mathrm{L}$

$\mathrm{mg} / \mathrm{L}$

mg/L

$\mathrm{mg} / \mathrm{L}$

$\mathrm{mg} / \mathrm{L}$

$\mathrm{mg} / \mathrm{L}$

$\mathrm{mg} / \mathrm{L}$

$\mathrm{mg} / \mathrm{L}$

mg/L

mg/L

$\mathrm{mg} / \mathrm{L}$

$\mathrm{mg} / \mathrm{L}$

$\mathrm{mg} / \mathrm{L}$

$\mathrm{mg} / \mathrm{L}$

II $/ \mathrm{L}$

$\mathrm{mg} / \mathrm{L}$

tig $/ \mathrm{L}$

mg/L

$\mathrm{mg} / \mathrm{L}$

$\mathrm{mg} / \mathrm{L}$

$\mathrm{mg} / \mathrm{L}$

$\mathrm{mg} / \mathrm{L}$ 


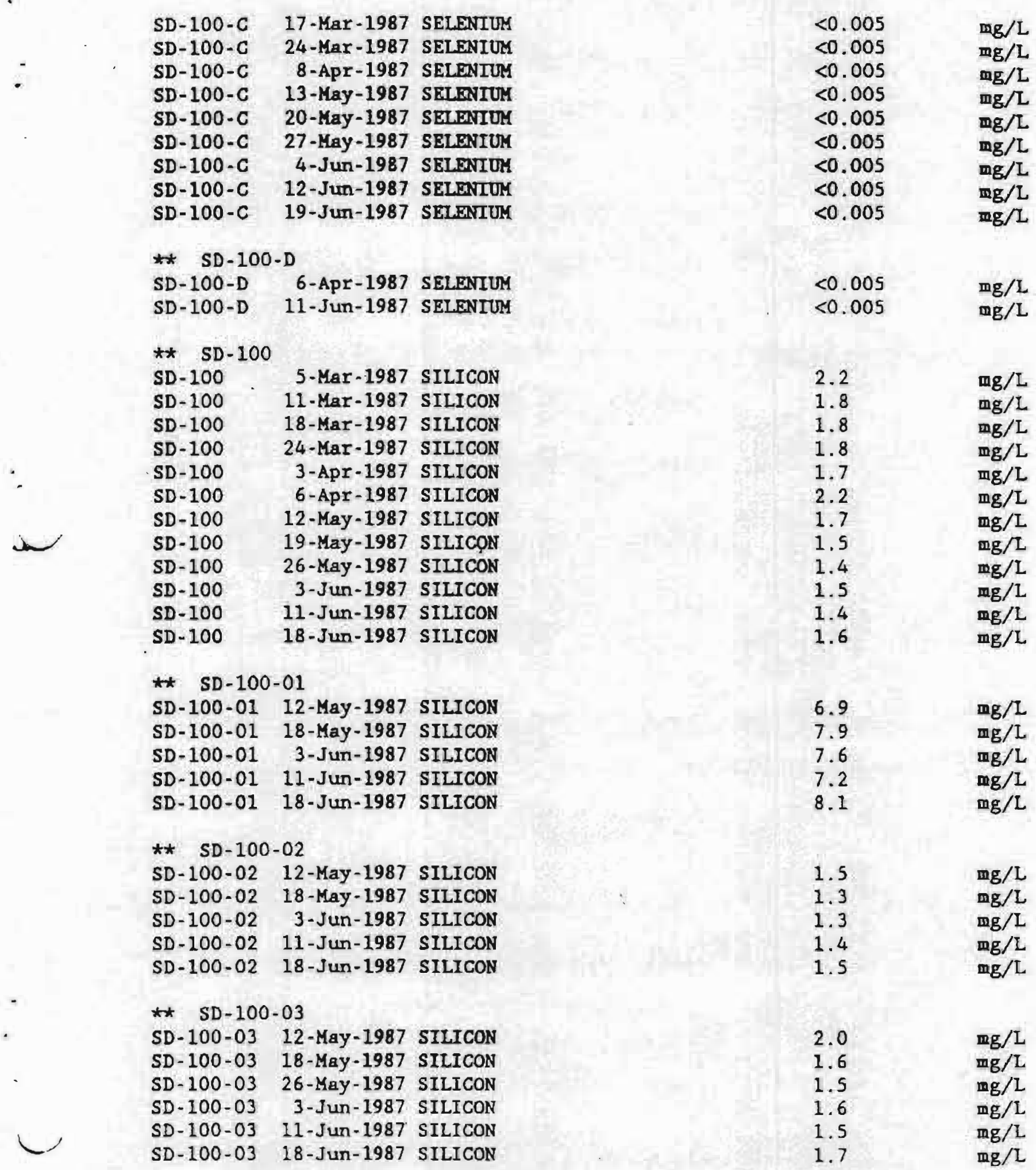


Location Date Test Compound

Results Units

** SD - 100-03D

SD-100-03D 12-May-1987 SILICON

SD-100-03D 26-May-1987 SILICON

2.0

$\mathrm{mg} /$.

1.5

$\mathrm{mg} / \mathrm{L}$

* SD $-100-04$

SD-100-04 12-Mry-1987 SILICON

SD-100-04 18-May-1987 SILICON

SD-100-04 26-May-1987 SILICON

SD-100-04 3-Jun-1987 SILICON

SD-100-04 11-Jun-1987 SILICON

SD-100-04 18-Jun-1987 SILICON

1.7

1.4

1.3

1.4

1.4

1.6

$\mathbf{m g} / \mathrm{L}$

$\mathrm{mg} / \mathrm{L}$

mg/L

$\mathrm{mg} / \mathrm{L}$

$\mathrm{mg} / \mathrm{L}$

$\mathrm{mg} / \mathrm{L}$

* SD-100-06

SD-100-06 12-May-1987 SILICON

SD-100-06 18-May-1987 SILICON

SD-100-06 26-May-1987 SILICON

SD-100-06 3-Jun-1987 SILICON

SD-100-06 11-Jun-1987 SILICON

SD-100-06 18-Jun-1987 SILICON

1.7

1.4

1.3

1.5

1.4

1.6

mg/L

$\mathrm{mg} / \mathrm{L}$

$\mathrm{mg} / \mathrm{L}$

mg $/ \mathrm{L}$

$\mathrm{mg} / \mathrm{L}$

$\mathrm{mg} / \mathrm{L}$

* SD $-100-06 \mathrm{D}$

SD-100-06D 3-Jun-1987 SILICON

1.5

wg $/ \mathrm{L}$

** SD-100-07

SD-100-07 12-May-1987 SILICON

SD-100-07 19-May-1987 SILICON

SD-100-07 26-May-1987 SILICON

SD-100-07 3-Jun-1987 SILICON

SD-100-07 11-Jun-1987 SILICON

SD-100-07 18-Jun-1987 SILICON

1.6

1.1

2.1

1.0

2.6

* SD-100-07D

SD-100-07D 18-Jun-1987 SILICON

2.6

$\mathbf{m g} / \mathbf{L}$

* SD-100-09

SD-100-09 12-May-1987 SILICON

SD-100-09 18-May-1987 SILICON

SD-100-09 26-May-1987 SILICON

SD-100-09 3-Jun-1987 SILICON

SD-100-09 11-Jun-1987 SILICON

1.5

1.3

1.2

1.3

1.4

$\mathbf{m g} / \mathrm{L}$

$\mathrm{mg} / \mathrm{L}$

$\mathrm{mg} / \mathrm{L}$

$\mathrm{mg} / \mathrm{L}$

$\mathrm{mg} / \mathrm{L}$

$\mathrm{mg} / \mathrm{L}$

* SD-100-10

SD-100-10 12-May-1987 SILICON

SD-100-10 19-May-1987 SILICON

1.5

1.3

1.2

2.3

SD-100-10 3-Jun-1987 SILICON

1.5

SD-100-10 11-Jun-1987 SILICON

1. 5

mg/L

$\mathrm{mg} / \mathrm{L}$

$\mathrm{mg} / \mathrm{L}$

$\mathrm{mg} / \mathrm{L}$

$\mathrm{mg} / \mathrm{L}$

SD-100-10 18-Jun-1987 SILICON

mg/L

$\mathrm{mg} / \mathrm{L}$

$\mathrm{ng} / \mathrm{L}$

$\mathrm{mg} / \mathrm{L}$

$\operatorname{mg} / \mathrm{L}$

$m g / L$

* SD-100-11

SD-100-11 12-May-1987 SILICON

1.7

$\mathrm{mg} / \mathrm{L}$ 
Storm Dra1n Data for SD-100

$\begin{array}{lrlll}\text { Location } & \text { Date } & \text { Test Compound } & \text { Results } & \text { UnIts } \\ & & & \\ \text { SD-100-11 } & 19-\mathrm{May}-1987 & \text { SILICON } & 1.5 & \mathrm{mg} / \mathrm{L} \\ \text { SD-100-11 } & 26-\mathrm{May}-1987 & \text { SILICON } & 2.3 & \mathrm{mg} / \mathrm{L} \\ \text { SD-100-11 } & 3-\mathrm{Jun}-1987 & \text { SILICON } & 1.5 & \mathrm{mg} / \mathrm{L} \\ \text { SD-100-11 } & 11-J u n-1987 & \text { SILICON } & 1.6 & \mathrm{mg} / \mathrm{L} \\ \text { SD-100-11 } & 18-J u n-1987 & \text { SILICON } & 1.7 & \mathrm{mg} / \mathrm{L}\end{array}$

* SD-100-12

SD-100-12 12-May-1987 SILICON

$2.9 \mathrm{mg} / \mathrm{L}$

SD-100-12 19-May-1987 SILICON

SD-100-12 26-May-1987 SILICON

1.4

1.4

1.4

SD-100-12 3-Jun-1987 SILICON

SD-100-12 11-Jun-1987 SILICON

SD-100-12 18-Jun-1987 SILICON

1.5

1.7

** SD - 100-13

SD-100-13 12-May-1987 SILICON

SD-100-13 19-May-1987 SILICON

SD-100-13 26-May-1987 SILICON

SD-100-13 11-Jun-1987 SILICON

SD-100-13 18-Jun-1987 SILICON

1.6

1.5

1.4

1.5

1.6

$\mathrm{mg} / \mathrm{L}$

$\mathrm{mg} / \mathrm{L}$

$\mathrm{mg} / \mathrm{L}$

$\mathrm{mg} / \mathrm{L}$

$\mathrm{mg} / \mathrm{L}$

* SD $-100-14$

SD-100-14 12-May-1987 SILICON

SD-100-14 19-May-1987 SILICON

SD-100-14 26-May-1987 SILICON

SD-100-14 3-Jun-1987 SILICON

SD-100-14 11-Jun-1987 SIIICON

SD-100-14 18-Jun-1987 SILICON

1.8

1.6

1.7

1.3

1.4

1.5

$\mathrm{mg} / \mathrm{L}$

$\mathrm{ng} / \mathrm{L}$

$\mathbf{m g} / \mathrm{L}$

$\mathrm{mg} / \mathrm{L}$

$m g / L$

* $\mathrm{SD}-100-\mathrm{C}$

SD-100-C 5-Kar-1987 SILICON

SD-100-C 9-Mar-1987 SILICON

SD-100-C 17-Mar-1987 SILICON

SD-100-C 24-Mar-1987 SILICON

SD-100-C 8-Apr-1987 SILICON

SD-100-C 13-May-1987 SILICON

SD-100-C 20-May-1987 SILICON

SD-100-C 27-May-1987 SILICON

SD-100-C 4-Jun-1987 SILICON

SD-100-C 12-Jun-1987 SILICON

SD-100-C 19-Jun-1987 SILICON

$2.1 \quad \mathrm{mg} / \mathrm{L}$

$1.7 \quad \mathrm{gg} / \mathrm{L}$

$1.6 \mathrm{mg} / \mathrm{L}$

$2.0 \mathrm{mg} / \mathrm{L}$

$2.7 \quad \mathrm{ng} / \mathrm{L}$

$1.8 \quad \mathrm{gg} / \mathrm{L}$

$1.4 \mathrm{gg} / \mathrm{L}$

$1.5 \mathrm{mg} / \mathrm{L}$

$1.5 \mathrm{mg} / \mathrm{L}$

$1.5 \mathrm{mg} / \mathrm{L}$

$1.5 \mathrm{gg} / \mathrm{L}$

** SD-100-D

SD-100-D 6-Apr-1987 SILICON

SD-100-D 11-JUn-1987 SILICON

2.3

1.5

$m g / 2$

$\mathrm{mg} / \mathrm{L}$.

* SD -100

SD -100

5-Mar-1987 SILVER

SD -100

11-Mar-1987 SILVER

SD -100

18-Mar-1987 SILVER

SD -100

24-Mar-1987 SILVER

$<0.010$

$\mathrm{mg} / \mathrm{L}$

$<0.010$

mg/L

$<0.010$

$\mathrm{mg} / \mathrm{L}$

$<0.010$

$\mathbf{n g} / \mathrm{L}$ 
Storm Drain Data for SD-100

Location

Date

Test Compound
Results

Units

\begin{tabular}{|c|c|c|}
\hline SD -100 & 3-Apr-1987 & SILVE \\
\hline SD -100 & 6-Apr-1987 & SILVE \\
\hline SD -100 & 12-Kay-1987 & SILVE \\
\hline SD -100 & 19-Кау - 1987 & SILVE \\
\hline SD -100 & 26-Kay-1987 & SILVE \\
\hline$S D-100$ & 3-Jun-1987 & SILVE \\
\hline SD- 100 & 11-Jun-1987 & SILVE \\
\hline SD -100 & & SIZVE \\
\hline \multicolumn{3}{|c|}{ ** SD-100-01 } \\
\hline SD $-100-01$ & $12-$ May - 1987 & SILVE \\
\hline SD $-100-01$ & 18-1ay-1987 & SILVE \\
\hline$S D-100-01$ & $a-1987$ & SILVE \\
\hline$S D-100-01$ & $1-1987$ & SILVE \\
\hline SD $-100-01$ & $18-J$ & SILVED \\
\hline \multicolumn{3}{|c|}{$\star \star \quad S D-100-02$} \\
\hline SD $-100-02$ & 12-Maj & SILVE \\
\hline$S D-100-02$ & 18-May-1987 & SILVE \\
\hline SD $-100-02$ & 3-Jun- 1987 & SILVE \\
\hline SD- $100-02$ & 11-Jun- 1987 & SILVE \\
\hline SD - $100-02$ & 18-Jun- 1987 & SILVE \\
\hline \multicolumn{3}{|c|}{$\star \quad$ SD $=100-03$} \\
\hline SD $-100-03$ & 12-Мay-1987 & SILVE \\
\hline SD - $100-03$ & 18-May-1987 & SILVEI \\
\hline$S D-100-03$ & 26-May-1987 & SILVER \\
\hline SD-100.03 & 3-Jun-1987 & SILVER \\
\hline SD $-100-03$ & 11-Jun-1987 & SILVER \\
\hline$S D-100-03$ & $18-J u n-1987$ & SILVE \\
\hline
\end{tabular}

* SD-100-03D

SD-100-03D 12-Kay-1987 SILVER SD-100-03D 26-May-1987 SILVER

** SD-100-04

SD-100-04 12-May-1987 SILVER

SD-100-04 18-May-1987 SILVER

SD-100-04 26-May-1987 SILVER

SD-100-04 3-Jun-1987 SILVER

SD-100-04 11-Jun-1987 SILVER

SD-100-04 18-Jun-1987 SILVER

** SD-100-06

SD-100-06 12-May-1987 SILVER

SD-100-06 18-May-1987 SILVER

SD-100-06 26-May-1987 SILVER

SD-100-06 3-JUn-1987 SILVER

SD-100-06 11-Jun-1987 SILVER

SD-100-06 18-Jun-1987 SILVER
$<0.010$

$<0.010$

$<0.010$

$<0.010$

0.018

$<0.010$

$<0.010$

$<0.010$

$<0.010$

$<0.010$

$<0.010$

$<0.010$

$<0.010$

$<0.010$

$<0.010$

$<0.010$

$<0.010$

$<0.010$

$<0.010$

$<0.010$

$<0.010$

$<0.010$

$<0.010$

$<0.010$

$<0.010$

$<0.010$

$<0.010$

$<0.010$

$<0.010$

$<0.010$

$<0.010$

$<0.010$

0.12

$<0.010$

$<0.010$

$<0.010$

$<0.010$

$<0.010$ $\mathrm{mg} / \mathrm{L}$

mg/L

$\mathrm{mg} / \mathrm{L}$

$\mathrm{mg} / \mathrm{L}$

$\mathrm{mg} / \mathrm{L}$

$\mathrm{mg} / \mathrm{L}$

$\mathrm{mg} / \mathrm{L}$

$\mathrm{mg} / \mathrm{L}$

$\mathrm{mg} / \mathrm{L}$

mg/L

mg/L

$\mathrm{mg} / \mathrm{L}$

$\mathrm{mg} / \mathrm{L}$

$\mathrm{mg} / \mathrm{L}$

mg/L

mg/L

mg/L

$\mathrm{mg} / \mathrm{L}$

$\mathrm{mg} / \mathrm{L}$

$\mathrm{mg} / \mathrm{L}$

$\mathrm{mg} / \mathrm{L}$

$\mathrm{mg}_{\mathrm{g}} / \mathrm{L}$

mg/L

$\mathrm{mg} / \mathrm{L}$

$\mathrm{mg} / \mathrm{L}$

$\mathrm{mg} / \mathrm{L}$

$\mathrm{mg} / \mathrm{l}$

mg/L

mg/L

$\mathrm{mg} / \mathrm{I}$

mg/L

mg/L

mg/L

$\mathrm{mg} / \mathrm{L}$

$\mathrm{mg} / \mathrm{L}$

$\mathrm{mg} / \mathrm{L}$

mg/ $L$

$\mathrm{mg} / \mathrm{L}$ 
Location Date Test Compound Results UnIts

** SD-100-06D

SD-100-06D 3-Jun-1987 SILVER

$<0.010 \quad \mathrm{mg} / \mathrm{L}$

** SD-100-07

SD-100-07 12-May-1987 SILVER

SD-100-07 19-May-1987 SILVER

SD-100-07 26-May-1987 SILVER

SD-100-07 3-Jun-1987 SILVER

SD-100-07 11-JUn-1987 SILVER

SD-100-07 18-Jun-1987 SILVER

0.010

0.012

$<0.010$

$<0.010$

$<0.010$

$<0.010$

$\mathrm{mg} / \mathrm{L}$

$\mathrm{mg} / \mathrm{L}$

$\mathbf{m g} / \mathrm{L}$

mg $/ \mathrm{L}$

mg $/ L$

$\mathrm{mg} / \mathrm{L}$

** SD-100-07D

SD-100-07D 18-Jun-1987 SILVER

$<0.010$

$\operatorname{mg} / \mathrm{L}$

* SD -100.09

SD-100-09 12-May-1987 SILVER

SD-100-09 18-May-1987 SILVER

SD-100-09 26-May-1987 SILVER

SD-100-09 3-Jun-1987 SILVER

SD-100-09 11-Jun-1987 SILVER

$<0.010$

$<0.010$

$<0.010$

$<0.010$

$<0.010$

mg/L

$\mathrm{mg} / \mathrm{L}$

$\mathrm{mg} / \mathrm{L}$

$\mathrm{mg} / \mathrm{L}$

$\mathrm{mg} / \mathrm{L}$

** SD-100-10

SD-100-10 12-May-1987 SILVER

SD-100-10 19-May-1987 SILVER

SD-100-10 26-May-1987 SILVER

SD-100-10 3-Jun-1987 SILVER

SD-100-10 11-Jun-1987 SILVER

SD-100-10 18-Jun-1987 SILVER

$<0.010$

$<0.010$

$<0.010$

$<0.010$

$<0.010$

$<0.010$

mg/L

$\mathrm{mg} / \mathrm{L}$

$\mathrm{mg} / \mathrm{l}$

mg/L

mg $/ \mathrm{L}$

$\mathrm{mg} / \mathrm{L}$

** SD-100-11

SD-100-11 12-Kay-1987 SILVER

$<0.010$

0.019

SD-100-11 19-May-1987 SILVER

SD-100-11 26-May-1987 SILVER

SD-100-11 3-JUn-1987 SILVER

$<0.010$

$<0.010$

SD-100-11 11-Jun-1987 SILVER

SD-100-11 18-Jun-1987 SILVER

$<0.010$

$<0.010$

$\mathrm{mg} / L$

$m g / L$

$m g / L$

$\mathrm{mg} / \mathrm{L}$

$\mathrm{mg} / \mathrm{L}$

$\mathrm{mg} / \mathrm{L}$

* SD - 100-12

SD-100-12 12-May-1987 SILVER

SD-100-12 19-May-1987 SILVER

SD-100-12 26-May-1987 SILVER

SD-100-12 3-Jun-1987 SILVER

SD-100-12 11-Jun-1987 SILVER

SD-100-12 18-Jun-1987 SILVER

$<0.010$

$<0.0060$

$<0.0060$

$<0.010$

$<0.010$

$<0.010$

$\mathrm{mg} / \mathrm{L}$

$\mathrm{mg} / \mathrm{L}$

$\mathrm{mg} / \mathrm{L}$

$\mathrm{mg} / \mathrm{L}$

$\mathrm{mg} / \mathrm{L}$

$\mathrm{mg} / \mathrm{L}$

*t SD-100-13

SD-100-13 12 -May-1987 SILVER

SD-100-13 19-May-1987 SILVER

SD-100-13 26-May-1987 SILVER

SD-100-13 11-Jun-1987 SILVER

SD-100-13 18-Jun-1987 SILVER

$<0.010$

$<0.0060$

$<0.0060$

$<0.010$

$<0.010$

$\mathrm{mg} / \mathrm{L}$

$\mathrm{mg} / \mathrm{L}$

$\mathrm{mg} / \mathrm{L}$

$\mathrm{mg} / \mathrm{L}$

$\mathrm{mg} / \mathrm{L}$ 
$<0.010$

$<0.010$

$<0.0060$

$<0.010$

$<0.010$

$<0.010$

$<0.010$

$<0.010$

$<0.010$

$<0.010$

$<0.010$

$<0.010$

$<0.010$

$<0.010$

$<0.010$

$<0.010$

$<0.010$

$<0.010$

$<0.010$

16

7.5

7.2

7.2

6.4

5.5

7.3

7.3

5. 9

5.9

6.1

6.9

2.6

2.7

2.9

3. 6

3.1

5. 8

5. 3

5. 2 $\mathrm{mg} / \mathrm{h}$
$\mathrm{mg} / \mathrm{L}$
$\mathrm{mg} / \mathrm{L}$
$\mathrm{mg} / \mathrm{L}$
$\mathrm{mg} / \mathrm{L}$
$\mathrm{mg} / \mathrm{L}$

mg/L

$\mathrm{mg} / \mathrm{L}$

$\mathrm{mg} / \mathrm{L}$

mg/L

$\mathrm{mg} / \mathrm{L}$

$\mathrm{mg} / \mathrm{L}$

$\mathrm{mg} / \mathrm{L}$

$\mathrm{mg} / \mathrm{h}$

$\mathrm{mg} / \mathrm{L}$

$\mathrm{mg} / \mathrm{L}$

mg $/ \mathrm{L}$

mg $/$ L

$\mathrm{mg} / \mathrm{L}$

$\mathrm{mg} / \mathrm{L}$

$\mathrm{ng} / \mathrm{L}$

$\mathrm{mg} / \mathrm{L}$

$\mathrm{mg} / \mathrm{L}$

mg/L

mg/L

$\mathrm{mg} / \mathrm{L}$

mg/L

$\mathrm{mg} / \mathrm{L}$

$\mathrm{mg} / \mathrm{L}$

$\mathrm{mg} / \mathrm{L}$

mg/L

mg/L

mg $/ 2$.

mg/L

$\mathrm{mg} / \mathrm{L}$

$\mathrm{mg} / \mathrm{L}$

$\mathrm{mg} / \mathrm{L}$

$\mathrm{mg} / \mathrm{L}$

$\mathrm{mg} / \mathrm{L}$ 


\begin{tabular}{|c|c|c|c|c|}
\hline Location & Date & Test Compound & Results & Units \\
\hline $\begin{array}{l}\text { SD }-100=02 \\
\text { SD }-100-02\end{array}$ & $\begin{array}{l}11-J u n-1987 \\
18-J u n-1987\end{array}$ & $\begin{array}{l}\text { SODIUM } \\
\text { SODIUM }\end{array}$ & $\begin{array}{l}5.5 \\
5.0\end{array}$ & $\begin{array}{l}\mathrm{mg} / \mathrm{L} \\
\mathrm{mg} / \mathrm{L}\end{array}$ \\
\hline \multicolumn{5}{|c|}{$* \star \quad S D-100.03$} \\
\hline SD $-100-03$ & $12-$ May -1987 & SODIUM & 5.6 & $\mathrm{ng} / \mathrm{L}$ \\
\hline SD $-100-03$ & $18-$ May-1987 & SODIUM & 5.7 & $\mathrm{mg} / \mathrm{L}$ \\
\hline SD $-100-03$ & 26-May- 1987 & SODIUM & 5.9 & $\mathbf{n g} / \mathrm{L}$ \\
\hline SD $-100-03$ & 3-Jนn-1987 & SODIUM & 5.4 & $\operatorname{mg} / \mathrm{L}$ \\
\hline SD $-100-03$ & $11-J u n-1987$ & SODIUM & 5.5 & $\log / \mathrm{L}$ \\
\hline$S D=100-03$ & 18 -Jun-1987 & SODIUM & 5.5 & $\mathrm{mg} / \mathrm{L}$ \\
\hline
\end{tabular}

** SD-100-03D

SD-100-03D 12-May-1987 SODIUM

SD-100-03D 26-May-1987 SODIUM

$5.5 \mathrm{mg} / \mathrm{L}$

$5.8 \mathrm{mg} / \mathrm{L}$

* SD $-100-04$

SD-100-04 12-May-1987 SODIUM

SD-100-04 18-May-1987 SODIUM

SD-100-04 26-Kay-1987 SODIUM

SD-100-04 3-Jun-1987 SODIUM

SD-100-04 11-Jun-1987 SODIUM

SD-100-04 18-Jun-1987 SODIUM

$5.6 \quad \mathrm{mg} / \mathrm{L}$

$5.3 \mathrm{mg} / \mathrm{L}$

$5.7 \mathrm{mg} / \mathrm{L}$

$5.2 \quad \mathbf{m g} / \mathrm{L}$

$5.4=\mathrm{mg} / \mathrm{L}$

$5.4 \mathrm{mg} / \mathrm{L}$

** SD $-100-06$

SD-100-06 12-May-1987 SODIUM

SD-100-06 18-May-1987 SODIUM

SD-100-06 26-May-1987 SODIUM

SD-100-06 3-Jun-1987 SODIUM

SD-100-06 11-Jun-1987 SODIUM

SD-100-06 18-Jun-1987 SODIUM

$5.6 \mathrm{mg} / \mathrm{L}$

$5.6 \mathrm{ag} / \mathrm{L}$

$5.7 \mathrm{mg} / \mathrm{I}$

$5.4 \mathrm{mg} / \mathrm{L}$

$5.4 \mathrm{ng} / \mathrm{L}$

$5.4 \mathrm{mg} / \mathrm{L}$

** SD - 100-06D

SD-100-06D 3-Jun-1987 SODIUM

$5.3 \mathrm{mg} / \mathrm{L}$

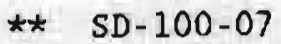

SD-100-07 12-May-1987 SODIUM

SD-100-07 19-May-1987 SODIUM

SD-100-07 26-May-1987 SODIUM

SD-100-07 3-Jun-1987 SODIUM

SD-100-07 11-Jun-1987 SODIUM

SD-100-07 18-Jun-1987 SODIUM

$5.5 \mathrm{mg} / \mathrm{L}$

$7.7 \quad \mathrm{mg} / \mathrm{L}$

$8.0 \mathrm{mg} / \mathrm{L}$

$230 \mathrm{mg} / \mathrm{L}$

$8.1 \quad \mathrm{gg} / \mathrm{L}$

$150 \mathrm{mg} / \mathrm{L}$

* SD-100-07D

SD-100-07D 18-Jun-1987 SODIUM

$130 \quad \mathrm{mg} / \mathrm{L}$

** SD $-100-09$

SD-100-09 12-May-1987 SODIUM

SD-100-09 18-May-1987 SODIUM

SD-100-09 26-May-1987 SODIUM

SD-100-09 3-Jun-1987 SODIUM

SD-100-09 11-Jun-1987 SODIUM

$\begin{array}{ll}6.4 & \mathrm{mg} / \mathrm{L} \\ 5.8 & \mathrm{mg} / \mathrm{L} \\ 5.7 & \mathrm{mg} / \mathrm{L} \\ 5.2 & \mathrm{mg} / \mathrm{L} \\ 5.4 & \mathrm{mg} / \mathrm{L}\end{array}$


Units

\begin{tabular}{|c|c|c|}
\hline SD - IO & & \\
\hline$S D-100-10$ & $12-$ May - 1987 & SODI \\
\hline SD $-100-10$ & 19-May - 1987 & \\
\hline SD $-100-10$ & 26-May-1987 & \\
\hline SD $-100-10$ & 3-Jun-1987 & \\
\hline$S D-100-10$ & 11-Jun-1987 & \\
\hline$S D-100-10$ & 18-Jun-1987 & \\
\hline SD -100 & -11 & \\
\hline SD-100-11 & 12-May-1987 & \\
\hline SD-100-11 & 19-May-1987 & \\
\hline SD $-100 \cdot 11$ & $26-\mathrm{May}-1987$ & \\
\hline SD - $100-11$ & 3-Jun-1987 & \\
\hline$S D-100-11$ & 11-Jun-1987 & \\
\hline D-100-11 & $18-\mathrm{Jun}-1987$ & \\
\hline
\end{tabular}

\section{7}

5.6

5.7

5.4

5.4

5.1

8.1

7.7

6.9

5.9

5.9

6.5

* SD-100-12

SD-100-12 12-May-1987 SODIUM

SD-100-12 19-May-1987 SODIUK

SD-100-12 26-May-1987 SODIUM

SD-100-12 3-Jun-1987 SODIUM

SD-100-12 11-Jun-1987 SODIUM

SD-100-12 18-Jun-1987 SODIUY

* SD-100-13

SD-100-13 12-May-1987 SODIUM

SD-100-13 19-May-1987 SODTUM

SD-100-13 26-May-1987 SODIOM

SD-100-13 11-Jun-1987 SODIUM

SD-100-13 18-Jun-1987 SODIUM

* SD-100-14

SD-100-14 12-May-1987 SODIUM

SD-100-14 19-May-1987 SODIUM

SD-100-14 26-May-1987 SODIUM

SD-100-14 3-Jun-1987 SODIUM

SD-100-14 11-Jun-1987 SODTUM

SD-100-14 18-Jun-1987 SODIOM
5.7

4.9

5.3

5.1

5.3

5.2

6.8

6.5

5.9

5.4

6.2

6.2

6.4

5.6

5.2

5.2

5.1

8.1

4.1

6.6

6.8

8.7

7.5

7.0

6.5

5. 8

5.8 $\mathrm{mg} / \mathrm{L}$

IIg/L

mg/L

mg/L

$\mathrm{mg} / \mathrm{L}$

mg/L

$\mathrm{mg} / \mathrm{L}$

$\mathrm{mg} / \mathrm{L}$

mg/L

$\mathrm{mg} / \mathrm{L}$

$\mathrm{mg} / \mathrm{L}$

$\mathrm{mg} / \mathrm{L}$

$\mathrm{mg} / \mathrm{L}$

$\mathrm{mg} / \mathrm{L}$

$\mathrm{mg} / \mathrm{L}$

mg $/ L$

$\mathrm{mg} / \mathrm{L}$

$\mathrm{mg} / \mathrm{L}$

mg/L

$\mathrm{mg} / \mathrm{L}$

mg/L

$\mathrm{mg} / \mathrm{L}$

mg/L

$\mathrm{mg} / \mathrm{L}$

$\mathrm{mg} / \mathrm{L}$

II $B / L$

mg/L

mg/L

mg/L

$\mathrm{mg} / \mathrm{L}$

$\mathrm{mg} / \mathrm{L}$

$\mathrm{mg} / \mathrm{L}$

$\mathrm{mg} / \mathrm{L}$

$\mathrm{mg} / \mathrm{L}$

$\mathrm{mg} / \mathrm{L}$

$\mathrm{mg} / \mathrm{L}$

$\mathrm{mg} / \mathrm{L}$

$\mathrm{mg} / \mathrm{L}$

ang $/ \mathrm{L}$ 
Location Date Test Compound Results Units

SD-100-C 19-Jun-1987 SODIUM

$6.4 \quad \mathrm{mg} / \mathrm{L}$

trk SD-100-D

SD-100-D 6-Apr-1987 SODIUM

$5.5 \mathrm{mg} / \mathrm{L}$

SD-100-D 11-Jun-1987 SODIU:

$6.0 \mathrm{mg} / \mathrm{L}$

* SD-100

SD-100 5-MaT-1987 STRONTTUM

5-Mar-1987 STRONTIUM
11-Mar-1987 STrONTIUM

$0.076 \mathrm{mg} / \mathrm{L}$

$\begin{array}{lll}\text { SD }-100 & 11-\mathrm{Mar}-1987 & \text { STRONTIUM } \\ \text { SD }-100 & 18-\mathrm{Mar}-1987 & \text { STRONTIUM }\end{array}$

SD-100 24-Mar-1987 STRONTIUM

0.075

0.078

0.076

SD-100 3-Apr-1987 STRONTIUM

SD-100 6-Apr-1987 STRONTIUM

SD-100 12-May-1987 STRONTIUM

SD-100 19-May-1987 STRONTIUM

SD-100 26-May-1987 STRONTIUM

SD. $100 \quad 3$-JUn-1987 STRONTIUM

SD-100 11-Jun-1987 STRONTIUM

SD-100 18-Jun-1987 STRONTIUM

0.064

$0.064 \mathrm{mg} / \mathrm{L}$

$0.070 \mathrm{mg} / \mathrm{L}$

0.065

$0.077 \mathrm{mg} / \mathrm{L}$

$0.072 \mathrm{mg} / \mathrm{L}$

$0.076 \quad \mathrm{mg} / \mathrm{L}$

$0.075 \mathrm{mg} / \mathrm{L}$

* SD $-100^{\circ}-01$

SD-100-01 12-May-1987 STRONTIUM

SD-100-01 18-May-1987 STRONTIUM

SD-100-01 3-Jun-1987 STRONTIUM

SD-100-01 11-Jun-1987 STRONTIUM

SD-100-01 18-Jun-1987 STRONTIUM

$0.030 \mathrm{mg} / \mathrm{L}$

$0.039 \mathrm{mg} / \mathrm{L}$

$0.046 \mathrm{mg} / \mathrm{L}$

$0.056 \quad \mathrm{mg} / \mathrm{L}$

$0.052 \mathrm{mg} / \mathrm{L}$

** SD $-100-02$

SD-100-02 12-May-1987 STRONTIUM

SD-100-02 18-May-1987 STRONTIUM

$0.071 \mathrm{mg} / \mathrm{L}$

SD-100-02 3-Jun-1987 STRONTIUM

$0.068 \quad \mathrm{mg} / \mathrm{L}$

SD-100-02 11-Jun-1987 STRONTIUM

$0.070 \quad \mathrm{mg} / \mathrm{L}$

SD-100-02 18-Jun-1987 STRONTIUM

$0.075 \mathrm{mg} / \mathrm{L}$

$0.068 \mathrm{mg} / \mathrm{L}$

** SD $-100-03$

SD-100-03 12-May-1987 STRONTIUM

SD-100-03 18-May-1987 STRONTIUM

$0.070 \quad \mathrm{mg} / \mathrm{L}$

$0.066 \mathrm{mg} / \mathrm{L}$

SD-100-03 26-May-1987 STRONTIUM

$0.074 \mathrm{mg} / \mathrm{L}$

SD-100-03 3-Jun-1987 STRONTIUA

SD-100-03 11-Jun-1987 STRONTIUM

0.072

$\mathrm{mg} / \mathrm{L}$

$0.076 \mathrm{mg} / \mathrm{L}$

SD-100-03 18-Jun-1987 STRONTIUM

$0.076 \mathrm{mg} / \mathrm{L}$

** SD-100-03D

SD-100-03D 12-May-1987 STRONTIUM

SD-100-03D 26-May-1987 STRONTIUM

$0.069 \mathrm{mg} / \mathrm{L}$

$0.072 \mathrm{mg} / \mathrm{L}$

*t SD-100-04

SD-100-04 12-May-1987 STRONTIUM

SD-100-04 18-May-1987 STRONTIUM

$0.070 \mathrm{mg} / \mathrm{L}$

SD-100-04 26-May-1987 STRONTIUM

0.065

0.073

$\mathrm{mg} / \mathrm{L}$

SD-100-04 3-Jun-1987 STRONTIUM

0.069

$\mathrm{mg} / \mathrm{L}$

mg/L 
SD-100-04 11-JUm-1987 STRONTIUM SD-100-04 18-Jun-1987 STRONTIUM

* SD-100-06

SD-100-06 12-May-1987 STRONTIUM SD-100-06 18-May-1987 STRONTIUM SD-100-06 26-May-1987 STRONTIUM SD-100-06 3-Jun-1987 STRONTIUM SD-100-06 11-Jun-1987 STRONTIUM SD-100-06 18-Jun-1987 STRONTIUY

* SD-100-06D

SD-100-06D 3-Jun-1987 STRONTIUY

* SD-100-07

SD-100-07 12-May-1987 STRONTIUM SD-100-07 19-May-1987 STRONTIUM SD-100-07 26-May-1987 STRONTIUM SD-100-07 3-Jun-1987 STRONTIUM SD-100-07 11-Jun-1987 STRONTIUM SD-100-07 -18-Jun-1987 STRONTIUM

* SD-100-07D

SD-100-07D 18-Jun-1987 STRONTIUM

* SD-100-09

SD-100-09 12-May-1987 STRONTIUM SD-100-09 18-May-1987 STRONTIUM SD-100-09 26-May-1987 STRONTIUM SD-100-09 3-Jun-1987 STRONTIUM SD-100-09 11-Jun-1987 STRONTIUM

* SD-100-10

SD-100-10 12-May-1987 STRONTIUM SD-100-10 19-May-1987 STRONTIUM SD-100-10 26-May-1987 STRONTIUM SD-100-10 3-JUn-1987 STRONTIUM SD-100-10 11-JUR-1987 STRONIIUM SD-100-10 18-Jun-1987 STRONTIUY

** SD-100-11

SD-100-11 12-May-1987 STRONTIUM SD-100-II 19-May-1987 STRONTIUM SD-100-I1 26-May-1987 STRONTIUM SD-100-11 3-JUR-1987 STRONTIUM SD-100-11 11-Jun-1987 STRONTIUM SD-100-11 18-Jun-1987 STRONTIUT

** SD $-100-12$

SD-100-12 12-May-1987 STRONTIUM SD-100-12 19-May-1987 STRONTIUM
$0.076 \mathrm{mg} / \mathrm{L}$

$0.075 \mathrm{mg} / \mathrm{L}$

$0.069 \quad \mathrm{mg} / \mathrm{L}$

$0.066 \mathrm{mg} / \mathrm{L}$

$0.073 \mathrm{mg} / \mathrm{L}$

$0.070 \mathrm{mg} / \mathrm{L}$

$0.076 \mathrm{mg} / \mathrm{L}$

$0.075 \mathrm{mg} / \mathrm{L}$.

$0.070 \mathrm{mg} / \mathrm{L}$

0.066

0.056

0.058

0.045

0.039

0.067

0.056

$\operatorname{mg} / \mathrm{L}$

0.068

0.066

0.072

0.067

0.074

mg/L

mg/L

mg/L

mg/L

$\mathrm{mg} / \mathrm{L}$

$\mathrm{mg} / \mathrm{L}$

0.071

0.067

0.073

0.074

0.076

0.068

mg/L

$\mathrm{mg} / 2$

mg/L

mg/L

mg/L

0.073

0.070

0.081

0.075

0.078

0.079

$\mathrm{mg} / \mathrm{L}$

$\mathrm{mg} / \mathrm{L}$

mg/L

mg/L

mg/L

$\mathrm{mg} / \mathrm{L}$

0.069

0.071 $\mathrm{mg} / \mathrm{L}$ ng/L mg/L $\mathrm{mg} / \mathrm{L}$ $\mathrm{mg} / \mathrm{L}$ mg/L

ng/L ng/ $/$ 
Storm Drain Data for SD-100

Location

Date

Test Compound

Results Unies

SD-100-12 26-Kay-1987 STRONTIUY

SD-100-12 3-Jun-1987 STRONTIUM

SD-100-12 11-Jun-1987 STRONTIUM

SD-100-12 18-Jun-1987 STRONTIUM

$0.077 \mathrm{mg} / \mathrm{L}$

$0.076 \mathrm{mg} / \mathrm{L}$

$0.069 \mathrm{mg} / \mathrm{L}$

$0.074 \mathrm{mg} / \mathrm{L}$

** SD $-100-13$

SD-100-13 12-May-1987 STRONTIUM

SD-100-13 19-Kay-1987 STRONTIUM

SD-100-13 26-Hay-1987 STRONIIUM

SD-100-13 11-Jun-1987 STRONTIUI

SD-100-13 18-Jum-1987 STRONTIUM

$0.069 \quad \mathrm{mg} / \mathrm{L}$

$0.071 \mathrm{mg} / \mathrm{L}$

$0.077 \quad \mathrm{mg} / \mathrm{L}$

$0.077 \mathrm{mg} / \mathrm{L}$

$0.071 \mathrm{mg} / \mathrm{L}$

* $S D \cdot 100-14$

SD-100-14 12-Kay-1987 STRONTIUM

SD-100-14 19-May-1987 STRONTIUM

SD-100-14 26-May-1987 STRONTIUM

SD-100-14 3-Jun-1987 STRONTIUM

SD-100-14 11-Jun-1987 STRONTIUM

SD-100-14 18-Jun-1987 STRONTIUR

$0.076 \quad \mathrm{mg} / \mathrm{L}$

$0.074 \mathrm{mg} / \mathrm{l}$

$0.085 \mathrm{mg} / \mathrm{h}$

$0.077 \mathrm{mg} / \mathrm{L}$

$0.068 \mathrm{mg} / \mathrm{L}$

$0.068 \mathrm{mg} / \mathrm{L}$

$\star \star \quad S D-100-C$

SD-100-C S-Mar-1987 STRONTIUM

SD-100-C 9-Mar-1987 STRONTIUM

SD-100-C 17-Mar-1987 STRONTIUM

SD-100-C 24-Kar-1987 STRONTIUM

SD-100-C 8-Apr-1987 STRONTIUM

SD-100-C 13-Kay-1987 STRONTIUM

SD-100-C 20-Kay-1987 STRONIIUM

SD-100-C 27-Kay-1987 STRONTIUM

SD-100-C 4-Jun-1987 STRONTIUM

SD-100-C 12-Jun-1987 STRONTIUM

SD-100-C 19-Jun-1987 STRONTIUM

$0.075 \mathrm{mg} / \mathrm{L}$

$0.059 \quad \mathrm{mg} / \mathrm{L}$

$0.076 \mathrm{mg} / \mathrm{L}$

$0.080 \mathrm{mg} / \mathrm{L}$

$0.060 \quad \mathrm{mg} / \mathrm{L}$

$0.070 \mathrm{mg} / \mathrm{L}$

$0.066 \quad \mathrm{mg} / \mathrm{L}$

$0.071 \mathrm{mg} / \mathrm{L}$

$0.071 \quad \mathrm{mg} / \mathrm{L}$

$0.070 \mathrm{mg} / \mathrm{L}$

$0.068 \mathrm{mg} / \mathrm{L}$

* SD-100-D

SD-100-D 6-APT-1987 STRONTIUM

SD-100-D 11-Jun-1987 STRONTIUM

$0.063 \mathrm{mg} / \mathrm{L}$

$0.076 \quad \mathrm{mg} / \mathrm{L}$

* SD $-100-12$

SD-100-12 12-May-1987 SUBSTITUTED CHLOROCLOPENTANE 60 ug/L

** SD - 100-14

SD-100-14 12-May-1987 SUBSTITUTED CHLOROCYCLOPENTANE $70 \quad$ ug $/ \mathrm{L}$

\#* SD-100-C

SD-100-C 17-Mar-1987 SUBSTITUTED DIPHENYL PHOSPHATE 18 ug/L

** SD -100

SD $-100 \quad 5$-Mat -1987 SULFATE

SD-100 11-Mar-1987 SULFATE

SD-100 18-Mar-1987 SULFATE

SD-100 24-Mar-1987 SULFATE

$\begin{array}{ll}44 & \mathrm{mg} / \mathrm{L} \\ 30 & \mathrm{mg} / \mathrm{L} \\ 30 & \mathrm{mg} / \mathrm{L} \\ 37 & \mathrm{mg} / \mathrm{L}\end{array}$


SD-100-01 12-May-1987 SULFATE

6.0

5.0

4.7

5. 1

16

10

$\mathrm{ng} / \mathrm{L}$

mg/L

$\mathrm{ng} / \mathrm{L}$

$\mathbf{m g} / \mathrm{L}$

mg/L

$\operatorname{mg} / \mathrm{L}$

00-02

SD-100-02

$\mathrm{mg} / \mathrm{L}$ $\mathrm{mg} / \mathrm{L}$ $\mathrm{mg} / \mathrm{L}$ $\mathrm{ng} / \mathrm{L}$ $\mathrm{mg} / \mathrm{L}$ $\mathrm{mg} / \mathrm{L}$

ng/ $/$

$\mathrm{ng} / \mathrm{L}$

$\mathrm{Ig} / \mathrm{L}$ ag/ $/$ $\mathrm{ag} / \mathrm{L}$ $\operatorname{mg} / \mathrm{L}$ 
Location Date Test Compound

Results Units

SD-100-06 18-JUn-1987 SULFATE

26

mg/L

* SD - 100-06D

SD-100-06D 3-Jun-1987 SULFATE

$\mathrm{mg} / \mathrm{L}$

* SD-100-07

SD-100-07 12-May-1987 SULFATE

SD-100-07 19-Kay-1987 SULFATE

SD-100-07 26-May-1987 SULFATE

SD-100-07 3-Jun-1987 SULFATE

SD-100-07 11-Jun-1987 SULFATE

SD-100-07 18-Jun-1987 SULFATE

* SD - 100-07D

SD-100-07D 18-Jun-1987 SULFATE

mg/L

** SD-100-09

SD-100-09 12-Kay-1987 SULFATE

SD-100-09 18-May-1987 SULFATE

SD-100-09 26-May-1987 SULFATE

SD-100-09 3-Jun-1987 SULFATE

SD-100-09 11-Jun-1987 SULFATE

SD-100-09 18-Jun-1987 SULFATE

** SD $-100-10$

SD-100-10 12-May-1987 SULFATE

SD-100-10 19-Kay-1987 SULFATE

SD-100-10 26-Kay-1987 SULFATE

SD-100-10 3-Jun-1987 SULFATE

SD-100-10 11-Jun-1987 SULFATE

SD-100-10 18-Jun-1987 SULFATE

$\mathbf{m g} / \mathrm{L}$ $\mathrm{mg} / \mathrm{L}$ mg/L $\mathrm{mg} / \mathrm{L}$ $\mathrm{ng} / \mathrm{L}$ $\mathrm{mg} / \mathrm{L}$

** SD $-100-11$

SD-100-11 12-May-1987 SULFATE SD-100-11 19-Kay-1987 SULFATE SD-100-11 26-May-1987 SULFATE SD-100-11 3-Jun-1987 SULFATE SD-100-11 11-Jun-1987 SULFATE SD-100-11 18-Jun-1987 SULFATE

$\mathrm{mg} / \mathrm{L}$ $\mathrm{ng} / \mathrm{L}$ ng/L $\mathrm{ng} / \mathrm{L}$ $\operatorname{mg} / \mathrm{L}$ mg/L

* SD-100-12

SD-100-12 12-May-1987 SULFATE SD-100-12 19-May-1987 SULFATE SD-100-12 26-Kay-1987 SULFATE SD-100-12 3-JUn-1987 SULFATE SD-100-12 11-Jun-1987 SULFATE SD-100-12 18-Jun-1987 SULFATE

$\begin{array}{ll}29 & \mathrm{mg} / L \\ 31 & \mathrm{mg} / L \\ 32.1 & \mathrm{mg} / \mathrm{L} \\ 28 & \mathrm{ng} / \mathrm{L} \\ 30 & \mathrm{mg} / \mathrm{L} \\ 30 & \mathrm{mg} / \mathrm{L}\end{array}$

\# SD $-100-13$

SD-100-13 12-May-1987 SULFATE SD-100-13 19-May-1987 SULFATE 
Location Date Test Compound Results Units

$\begin{array}{lr}\text { SD-100-13 } & 26-\text { May-1987 SULFATE } \\ \text { SD-100-13 } & 3-J \text { Un-1987 SURFATE } \\ \text { SD-100-13 } & 11-J u n-1987 \text { SULFATE } \\ \text { SD-100-13 } & 18-J u n-1987 \text { SULFATE }\end{array}$

** SD - 100-14

SD-100-14 12-Mzy-1987 SULFATE

SD-100-14 19-Kay-1987 SULFATE

SD-100-14 26-Kay-1987 SULFATE

SD-100-14 3-Jun-1987 SULFATE

SD-100-14 11-JUn-1987 SULFATE

SD-100-14 18-Jun-1987 SULFATE

* SD-100-C

$\begin{array}{ll}29.8 & \mathrm{mg} / \mathrm{L} \\ 27 & \mathrm{mg} / \mathrm{L} \\ 26 & \mathrm{mg} / \mathrm{L} \\ 27 & \mathrm{mg} / \mathrm{L}\end{array}$

27

30

27.3

26

26

27

13.0

10.0

11.0

13.0

11.0

11.0

20.0

21.0

23.0

22

22

23.0

16.0

21.0

19.0

20

22

20.0 $\mathrm{mg} / \mathrm{l}$

$\mathrm{mg} / \mathrm{L}$

$\mathrm{mg} / \mathrm{L}$
$\mathrm{mg} / \mathrm{L}$
$\mathrm{mg} / \mathrm{L}$
$\mathrm{mg} / \mathrm{L}$
$\mathrm{mg} / \mathrm{L}$
$\mathrm{mg} / \mathrm{L}$

$\mathrm{mg} / \mathrm{L}$ $\mathrm{mg} / \mathrm{L}$ $\mathrm{mg} / \mathrm{L}$ mg/L 吗/L ag/L $\operatorname{mg} / \mathrm{L}$ $\operatorname{mg} / \mathrm{L}$ $\mathrm{mg} / \mathrm{L}$ $\mathrm{mg} / \mathrm{L}$ $m g / L$

Deg. C Deg. C Deg. C Deg. C Deg. C Deg. C Deg. $C$ Deg. C Deg. C Deg. C Deg . C Deg. C

Deg. C Deg. C Deg. C Deg, C Deg. C Deg. C 
Storm Drain Data for SD-100

Location Date Test Compound Results Units

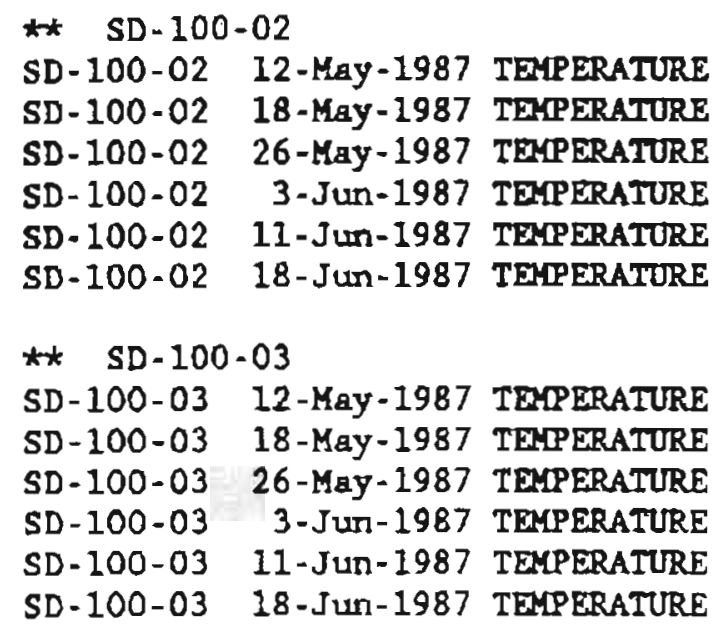

** SD-100-03D

SD-100-03D 12-May-1987 TEMPERATURE

SD-100-03D 26-May-1987 TEIPERATURE

** SD-100-04

SD-100-04 12-May-1987 TEMPERATURE

SD-100-04 18-May-1987 TEMPERATURE

SD-100-04 26-May-1987 TEMPERATURE

SD-100-04 3-Jun-1987 TEYPERATURE

SD-100-04 11-JUM-1987 TEMPERATURE

SD-100-04 18-Jun-1987 TEMPERATURE

** SD-100-06

SD-100-06 12-May-1987 TEMTERATURE

SD-100-06 18-May-1987 TEMPERATURE

SD-100-06 26-May-1987 TEMPERATURE

SD-100-06 3-Jun-1987 TEMPERATURE

SD-100-06 11-Jun-1987 TEMPERATURE

SD-100-06 18-Jun-1987 TEMPERATURE

*t SD-100-06D

SD-100-06D 3-JUN-1987 TEMPERATURE

$\star \star \quad S D-100-07$

SD-100-07 12-May-1987 TEYPERATURE

SD-100-07 19-May-1987 TEMPERATURE

SD-100-07 26-May-1987 TEMPERATURE

SD-100-07 3-JUn-1987 TEMPERATURE

SD-100-07 11-JUn-1987 TEMPERATURE

SD-100-07 18-Jun-1987 TEMPERATURE

** SD-100-07D

SD-100-07D 18-Jun-1987 TEMPERATURE

$\begin{array}{ll}17.0 & \text { Deg. C } \\ 22.0 & \text { Deg. C } \\ 21.0 & \text { Deg. C } \\ 21 & \text { Deg. C } \\ 21 & \text { Deg. C } \\ 21.0 & \text { Deg. C }\end{array}$

17.0

22.0

22.0

22

22

23.0

17.0

22.0

17.0

24.0

22.0

23

22

22.0

18.0

22.0

22.0

22

21

22.0

22

Deg. C

25.0

Deg. $C$

Deg. $C$

Deg. C

Deg. C

Deg. $C$

Deg. C

37.0

Deg. C 


Location Date Test Compound Results Units

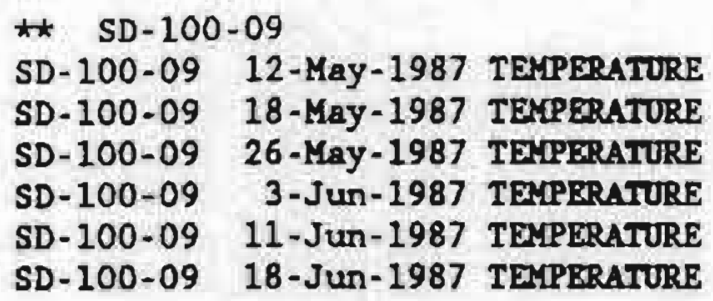

$\begin{array}{ll}22.0 & \text { Deg. C } \\ 24.0 & \text { Deg. C } \\ 23.0 & \text { Deg. C } \\ 23 & \text { Deg. C } \\ 23 & \text { Deg. C } \\ 23.0 & \text { Deg. C }\end{array}$

18.0

22.0

20.0

20

21

21.0

25.0

25.0

25.0

27

24

26.0

32.0

34.0

34.0

34

34

34.0

20.0

22.0

24.0

24

21

23.0

24.0
25.0
26.0
26
26
27.0

Deg. C Deg. C Deg. C Deg. C Deg. C Deg. C

Deg. C Deg. $C$ 
Location Date Test Compound Results Units

\begin{tabular}{|c|c|c|}
\hline SD $=100$ & 5-Mar-1987 & TETRACHLOROEII \\
\hline$D-100$ & 11-Mar-1987 & IEIRACHLOROETH \\
\hline 100 & 18-Mar-1987 & TETRACHLOROBTH \\
\hline 100 & 24-Mar-1987 & TETRACHLOROETT \\
\hline 100 & 6-Apr-1987 & TETRACHLOROETY \\
\hline-100 & 12-May-1987 & TETRACHLOROETH \\
\hline 100 & 19-Мay-1987 & TETRACKLOROETH \\
\hline 100 & 26- May - 1987 & TETRACKLOROETH \\
\hline 100 & 3-Jun-1987 & TETRACHLOROETHI \\
\hline & 11-Jun-1987 & TETRACHLOROETT \\
\hline & 18-Jun-1987 & TETRAC \\
\hline
\end{tabular}

* SD-100-01

SD-100-01 12-May-1987 TETRACHLOROETHENE

SD-100-01 18-May-1987 TETRACHLOROETHENE

SD-100-01 26-MaY-1987 TETRACHLOROETHENE

SD-100-01 3-Jun-1987 TETRACHLOROETHENE

SD-100-01 11-Jun-1987 TETRACHLOROETHENE

SD-100-01 18-Jun-1987 TETRACHLOROETHENE

SD-100-02 18-Jun-1987 TETRACHIOROETHENE

* SD-100-03

SD-100-03 12-May-1987 TETRACHLOROETHENE

SD-100-03 18-May-1987 TETRACHLOROETHENE

SD-100-03 26-May-1987 TETRACHLOROETHENE

SD-100-03 3-Jun-1987 TETRACHLOROETHENE

SD-100-03 11-Jun-1987 TETRACHLOROETHENE

SD-100-03 18-Jun-1987 TEIRACHLOROETHENE

* SD-100-03D

SD-100-03D 12-May-1987 TETRACHLOROETHENE

SD-100-03D 26-May-1987 TETRACHLOROETHENE

* SD-100-04

SD-100-04 12-May-1987 TETRACHLOROETHENE

SD-100-04 18-May-1987 TETRACHLOROETHENE

SD-100-04 26-May-1987 TETRACHLOROETHENE

SD-100-04 3-JUn-1987 TETRACHLOROETHENE

SD-100-04 11-Jun-1987 TETRACHLOROETHENE

SD-100-04 18-Jun-1987 TETRACHLOROETHENE
$<5$

$<5$

$<$

$<5$

$<5$

$<5$

$<5$

$<5$

$<5$

$<5$

$<5$

$<5$

$<5$

$<5$

$<5$

$<5$

$<5$

$<5$

$<5$

$<5$

$<5$

$<5$

$<5$

$\begin{array}{ll}u g / L \\ u g / L \\ u g / L \\ u g / L \\ u g / L \\ u g / L \\ \text { ug/L } \\ \text { ug } / L \\ \text { ug } / L \\ 5 & u g / L \\ & u g / L\end{array}$

$\mathrm{ug} / \mathrm{L}$

ug $/ \mathrm{L}$

$4 \mathrm{~g} / \mathrm{L}$

$u g / L$

$u g / L$

$\mathrm{ug} / \mathrm{L}$

$1 \mathrm{~g} / \mathrm{L}$

$1 \mathrm{~g} / \mathrm{L}$

ug/L

ug/L

ug/L

$4 \mathrm{~g} / \mathrm{L}$

ug/L

ug $/ L$

ug/L

ug/ I

$<5$

$<5$

$<5$

$<5$

$<$

$<5$

$u g /$ L

ug/ $/$

$u g / L$

ug/ $/$

ug/L

ug/L

$<5$

$<5$

$<5$

$<5$

$<5$

$<5$

$<5$

$<5$
ug/L

ug/L

ug/L ug/L ug/L ug/L ug $/ L$ ug/L

ug/L ug/L ug/L ug/L ug/L $u_{B} / L$ 
* SD $-100-06$

SD-100-06 12-May-1987 TETRAGHLOROETHENE

SD-100-06 18-May-1987 TETRAGHLOROETHENE

SD-100.06 26-May-1987 TETRACHLOROETHENE

SD-100-06 3-Jun-1987 TEIRACHLOROETHENE

SD-100-06 11-Jun-1987 TETRACHLOROETHENE

SD-100-06 18-Jun-1987 TETRACHLOROETHENE

** SD-100-06D

SD-100-06D 3-Jun-1987 TETRACHLOROETHENE

ug/L

$<5$

$<5$

$<5$

$4 \mathrm{~g} / \mathrm{L}$

$\mathrm{ug} / \mathrm{L}$

$\mathrm{ug} / \mathrm{L}$

ug/L

$u_{8} / \mathrm{L}$

** SD - 100-07

SD-100-07 12-May-1987 TETRACHLOROETHENE

SD-100-07 19-May-1987 TETRACHLOROETHENE

SD-100-07 26-May-1987 TETRACHLOROETHENE

SD-100-07 3-Jun-1987 TETRACHLOROETHENE

SD-100-07 11-Jun-1987 TETRACHLOROETHENE

SD-100-07 18-Jun-1987 TETRACHLOROETHENE

$<5$

$<5$

ug/ $/ 2$

5

$<5$

5

$<5$

$<5$

$<5$

ug/L

ug/L

$\mathrm{ug} / \mathrm{L}$

ug/L

ug/L

ug/L

* SD-100-07D

SD-100-07D 18-JUn-1987 TETRAGHLOROETHENE

5

$u g /$

** SD $-100-09$

SD-100-09 12-May-1987 TETRACHLOROETHENE

SD-100-09 18-May-1987 TETRACHLOROETHIDNE

SD-100-09 26-May-1987 TETRACHLOROETHENE

SD-100-09 3-Jun-1987 TETRACHLOROETHERNE

SD-100-09 11-Jun-1987 TETRAGHLOROETHENE

SD-100-09 18-Jun-1987 TETRACHLOROETHENE

t* SD-100-10

SD-100-10 12-May-1987 TETRACHLOROETHENE

SD-100-10 19-May-1987 TETRACHLOROETHENE

SD-100-10 26-May-1987 TETRACHLOROETHENE

SD-100-10 3-Jun-1987 TETRACHLOROETHERE

SD-100-10 11-JUn-1987 TETRACHLOROETLENE

SD-100-10 18-Jun-1987 TETRACHLOROETHENE

$\begin{array}{ll}<5 & u g / L \\ <5 & u g / L \\ <5 & u g / L \\ <5 & u g / L \\ <5 & u g / L \\ <5 & u g / L\end{array}$

* SD-100-11

SD-100-11 12-May-1987 TETRACHLOROETHENE

SD-100-11 19-May-1987 TETRACHLOROETHaNE

SD-100-11 26-May-1987 TETRACHLOROETHEHE

SD-100-11 3-Jun-1987 TETRACHLOROETHENE

SD-100-11 11-JUn-1987 TETRACHLOROETHENE

SD-100-11 18-Jun-1987 TETRACHLOROETHENE

$<5$

$<5$

$<5$

$<5$

$<5$

$<5$

$<5$

$<5$

$<5$

$<5$

$<5$

$<5$

$<5$

$<5$

$<5$

$<5$ 
Location Date Test Compound Results UnIts

SD-100-12 11-JUn-1987 TETRACHLOROETHEINE SD-100-12 18-Jun-1987 TETRACHLOROETHENE

** SD-100-13

SD-100-13 12-May-1987 TELRACHLOROETHENE

SD-100-13 19-May-1987 TETRACHLOROETHENE

SD-100-13 26-May-1987 TEIRACHLOROETHENE

SD-100-13 3-Jun-1987 TETRACHLOROETHENE

SD-100-13 11-JUn-1987 TRTRACHLOROETHENE

SD-100-13 I8-Jun-1987 TETRACHLOROETHENE

** SD-100-14

SD-100-14 12-May-1987 TETRACHLOROETHENE

SD-100-14 19-May-1987 TETRACHLOROETHENE

SD-100-14 26-May-1987 TETRACHLOROETHENE

SD-100-14 3-JUR-1987 TETRACHLOROETHENE

SD-100-14 11-Jun-1987 TETRAGHLOROETHENE

SD-100-14 18-Jun-1987 TETRACHLOROETHENE

** SD-100-C

SD-100-C 5-MaI-1987 TEIRACHLOROETHENE

SD-100-C 9-Mar-1987 TETRAGHLOROETHENE

SD-100-C 17-Mar-1987 TETRACHLOROETHENE

SD-100-C 24-Mar-1987 TETRAGHLOROETHENE

SD-100-C 8-APr-1987 TETRACHLOROETHENE

SD-100-C 13-May-1987 IETRACHLOROETHENE

SD-100-C 20-May-1987 TETRACHLOROETHENE

SD-100-C 27-May-1987 TEIRACHLOROETHENE

SD-100-C 12-JUI-1987 TETRACELOROETHENE

SD-100-C 19-JUR-1987 TETRACHIOROETHENE

** SD-100-D

SD-100-D 6-APY-1987 TETRACHLOROETHENE

SD-100-D 11-Jun-1987 TETRACHLOROETHENE
$<5$

$<5$

$<5$

$<5$

$<5$

$<5$

$<5$

$<5$

$<5$

$<5$

$<5$

$<5$

$<5$

$<5$

$<5$

$<5$

$<5$

$<5$

$<5$

$<5$

$<5$

$<5$

$<5$

$<5$

$<5$

$<5$

$<0.20$

$<0.20$

$<0.20$

$<0.20$

$<0.20$

$<0.20$

$<0.20$

$<0.20$

$<0.20$

$<0.20$

$<0.20$

$<0.20$

$<0.20$ $\mathrm{ug} / \mathrm{L}$

ug/L

ug $/ \mathrm{L}$

ug $/ \mathrm{L}$

$\mathrm{ug} / \mathrm{L}$

ug/L

$u g / L$

ug/L

48/L

ug/L

ug/L

$4 \mathrm{~g} / \mathrm{L}$

$\mathrm{Lg} / \mathrm{L}$

$\mathrm{ug} / \mathrm{L}$

ug/L

ug/L

ug/L

$u g / L$

$4 \mathrm{~g} / \mathrm{L}$

ug/L

4g/L

$4 \mathrm{~g} / \mathrm{L}$

ug/L

$\mathrm{ug} / \mathrm{L}$

$\mathrm{ug} / \mathrm{L}$

ug/L

$\mathrm{mg} / \mathrm{L}$

$\mathrm{mg} / \mathrm{L}$

$\mathrm{mg} / \mathrm{L}$

mg/L

$\mathrm{mg} / \mathrm{L}$

$m g / L$

$\mathrm{mg} / \mathrm{L}$

mg/L

$\mathrm{mg} / \mathrm{L}$

$\mathrm{mg} / \mathrm{L}$

$\mathrm{mg} / \mathrm{L}$

$\mathrm{mg} / \mathrm{L}$

** SD.100-01

SD-100-01 12-May-1987 THORIUM 
SD-100-01 3-Jun-1987 THORIUM SD-100-01 11-Jun-1987 THORIUM SD-100-01 18-Jun-1987 THORIUM

** SD-100-02

SD-100-02 12-May-1987 THORIUM SD-100-02 18-May-1987 THORIU: SD-100-02 3-Jun-1987 THORTUM SD-100-02 11-Jun-1987 THORIUM SD-100-02 18-Jur-1987 THORTOM

* SD $-100-03$

SD-100-03 12-May-1987 THORIUM SD-100-03 18-May-1987 THORIUM SD-100-03 26-May-1987 THORIUM SD-100-03 3-Jun-1987 THORIUM SD-100-03 11-Jun-1987 THORTUM SD-100-03 18-Jun-1987 THORIUM

** SD-100-03D

SD-100-03D 12-May-1987 THORIUM SD-100-03D 26-May-1987 THORIUM

* SD-100-04

SD-100-04 12-May-1987 THORTUM SD-100-04 18-May-1987 THORIUM SD-100-04 26-May-1987 THORIUM SD-100-04 3-Jun-1987 THORTUM SD-100-04 11-Jun-1987 THORIUM SD-100-04 18-Jun-1987 THORIUM

** SD-100-06

SD-100-06 12-May-1987 THORIUM SD-100-06 18-May-1987 THORIUM SD-100-06 26-May-1987 THORIUM SD-100-06 3-Jun-1987 THORIUM SD-100-06 11-Jun-1987 THORIUM SD-100-06 18-Jun-1987 THORTUM

* SD-100-06D

SD-100-06D 3-Jun-1987 THORIUA

** SD - 100-07

SD-100-07 12-May-1987 THORIOM

SD-100-07 19-May-1987 THORIUM

SD-100-07 26-May-1987 THORIUM

SD-100-07 3-Jun-1987 THORIUM

SD-100-07 11-JUn-1987 THORIUM

SD-100-07 18-Jun-1987 THORIUM
$<0.20$

$<0.20$

$<0.20$

$<0.20$

$<0.20$

$<0.20$

$<0.20$

$<0.20$

$<0.20$

$<0.20$

$<0.20$

$<0.20$

$<0.20$

$<0.20$

$<0.20$

$<0.20$

$<0.20$

$<0.20$

$<0.20$

$<0.20$

$<0.20$

$<0.20$

$<0.20$

$<0.20$

$<0.20$

$<0.20$

$<0.20$

$<0.20$

$<0.20$

$<0.20$

$<0.20$

$<0.20$

$<0.20$

$<0.20$

$<0.20$

$$
\begin{aligned}
& \mathrm{mg} / \mathrm{L} \\
& \mathrm{mg} / \mathrm{L} \\
& \mathrm{mg} / \mathrm{L}
\end{aligned}
$$

$\mathrm{mg} / \mathrm{L}$

$\mathrm{ng} / \mathrm{L}$

$\mathrm{ng} / \mathrm{L}$

IIE $/ L$

IIg/L

$\operatorname{mg} / \mathrm{L}$

$\mathrm{mg} / \mathrm{L}$

mg/L

$\mathrm{mg} / \mathrm{L}$

$\mathrm{mg} / \mathrm{L}$

$\mathrm{mg} / \mathrm{L}$

mg $/$ L

$\mathrm{mg} / \mathrm{l}$

mg/L

$\mathrm{mg} / \mathrm{L}$

$\mathrm{mg} / \mathrm{L}$

$\mathrm{ng} / \mathrm{L}$

$\mathrm{mg} / \mathrm{L}$

$\mathrm{mg} / \mathrm{L}$

$\mathrm{mg} / \mathrm{L}$ $\mathrm{mg} / \mathrm{L}$ $\mathrm{mg} / \mathrm{L}$ mg/L $\mathrm{mg} / \mathrm{L}$ mg/L

$\mathrm{ng} / \mathrm{L}$

mg/L $\mathrm{mg} / \mathrm{L}$ mg/L $\mathrm{mg} / \mathrm{L}$ mg $/ L$ ㅍg $/ \mathrm{L}$ 


Location Date Test Compound Results Units

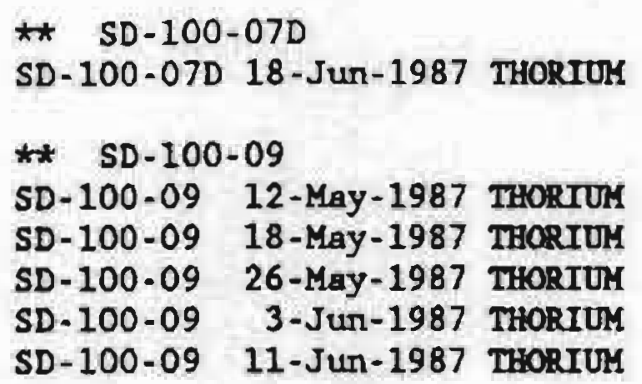

$\operatorname{mg} / \mathrm{L}$ $\mathrm{mg} / \mathrm{L}$ $\mathrm{mg} / \mathrm{L}$ $\mathrm{mg} / \mathrm{L}$ $\mathrm{mg} / \mathrm{L}$ $m g / L$

** SD-100-12

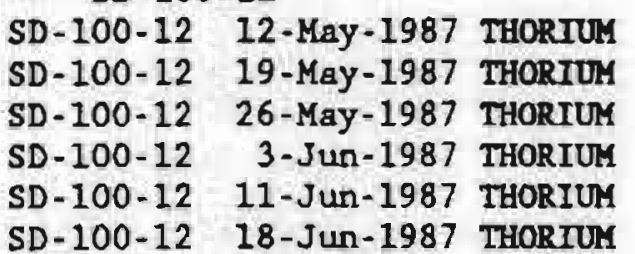

$<0.20$

$<0.20$

$<0.20$

$<0.20$

$<0.20$

$<0.20$

$\mathrm{mg} / \mathrm{L}$
$\mathrm{mg} / \mathrm{L}$
$\mathrm{mg} / \mathrm{L}$
$\mathrm{mg} / \mathrm{L}$
$\mathrm{mg} / \mathrm{L}$
$\mathrm{mg} / \mathrm{L}$

** SD $100-13$

SD-100-13 12-May-1987 THORIUM SD-100-13 19-May-1987 THORIUK SD-100-13 26-May-1987 THORIUM SD-100-13 11-Jun-1987 TEORTUM SD-100-13 18-Jun-1987 THORTUM

$\operatorname{mg} / \mathrm{l}$ $\mathrm{mg} / \mathrm{L}$ $\mathrm{mg} / \mathrm{L}$ $\mathrm{mg} / \mathrm{L}$ $\mathrm{mg} / \mathrm{L}$ $\operatorname{mg} / \mathbf{L}$

** SD-100-14

SD-100-14 12-May-1987 THORIUM

$<0.20$

$<0.20$

SD-100-14 19-May-1987 THORIUM

$<0.20$

SD-100-14 26-May-1987 THORIUM

$<0.20$

$<0.20$

SD-100-14 11-Jun-1987 THORIUM

SD-100-14 18-Jun-1987 THORIUM

$<0.20$

$\mathrm{mg} / \mathrm{L}$ $\mathrm{mg} / \mathrm{L}$ $\mathrm{mg} / \mathrm{L}$ $\mathbf{m g} / \mathrm{L}$ $\mathrm{mg} / \mathrm{L}$

** SD-100-C

SD-100-C 5-MaT-1987 THORIUM

$<0.20$

$\mathrm{mg} / \mathrm{L}$ 


\begin{tabular}{|c|c|c|}
\hline Location & Date & Test \\
\hline$S D-100 \cdot C$ & 9- Har -1987 & THORIURA \\
\hline$S D-100-C$ & 17-Mar - 1987 & THORTu \\
\hline SD-100-C & 24-Mar - 1987 & THORTaA \\
\hline$S D-100-C$ & 8-Apr -1987 & THORIOA \\
\hline$S D-100-C$ & 13-May-1987 & THORTUM \\
\hline SD $-100 \cdot C$ & 20-May-1987 & THORIUM \\
\hline$S D-100-C$ & 27-Kay - 1987 & THORTU: \\
\hline SD-100-C & 4-Jun-1987 & THORTU \\
\hline$D-100 \cdot C$ & 12-Jun-1987 & THORIU \\
\hline$D-100-C$ & 19-Jun-1987 & THORT \\
\hline
\end{tabular}

Results Units

* SD-100-D

SD-100-D 6-Apr-1987 THORIUM

SD-100-D 11-Jun-1987 THORIOT

$<0.20$

$<0.20$

$<0.20$

$<0.20$

$<0.20$

$<0.20$

$<0.20$

$<0.20$

$<0.20$

$<0.20$

$\mathrm{mg} / \mathrm{L}$
$\mathrm{mg} / \mathrm{L}$
$\mathrm{mg} / \mathrm{L}$
$\mathrm{mg} / \mathrm{L}$
$\mathrm{mg} / \mathrm{L}$
$\mathrm{mg} / \mathrm{L}$
$\mathrm{mg} / \mathrm{L}$
$\mathrm{mg} / \mathrm{L}$
$\mathrm{mg} / \mathrm{L}$
$\mathrm{mg} / \mathrm{L}$

** SD -100

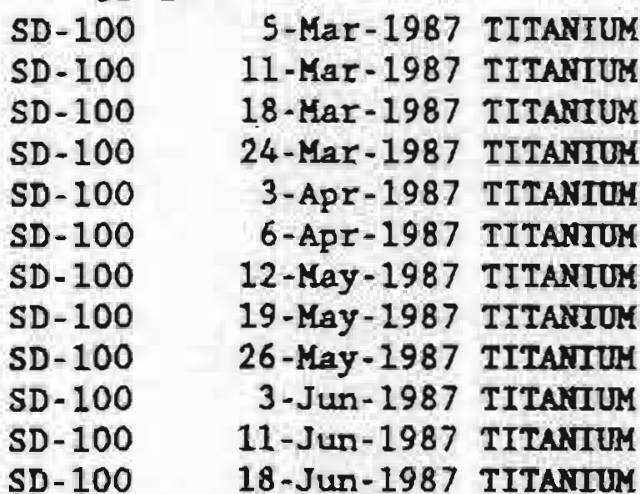

$<0.20$

$<0.20$

$\mathrm{mg} / \mathrm{L}$

$\mathrm{mg} / \mathrm{L}$

$<0.0030$

$<0.0030$

$<0.0030$

$<0.0030$

$<0.0030$

0.0043

$<0.0030$

$<0.0030$

0.0036

0.0051

0.0041

0.0061

$\mathrm{mg} / \mathrm{L}$
$\mathrm{mg} / \mathrm{L}$
$\mathrm{mg} / \mathrm{L}$
$\mathrm{mg} / \mathrm{L}$
$\mathrm{mg} / \mathrm{L}$
$\mathrm{mg} / \mathrm{L}$
$\mathrm{mg} / \mathrm{L}$
$\mathrm{mg} / \mathrm{L}$
$\mathrm{mg} / \mathrm{L}$
$\mathrm{mg} / \mathrm{L}$
$\mathrm{mg} / \mathrm{L}$
$\mathrm{mg} / \mathrm{L}$

* SD-100-01

SD-100-01 12-May-1987 TITANIUM

SD-100-01 18-May-1987 TITANIUM

SD-100-01 3-Jun-1987 TITANIUM

SD-100-01 11-Jun-1987 TITANTUM

SD-100-01 18-Jun-1987 TITANIUM

$\begin{array}{ll}0.0068 & \mathrm{mg} / \mathrm{L} \\ 0.034 & \mathrm{mg} / \mathrm{L} \\ 0.022 & \mathrm{mg} / \mathrm{L} \\ 0.0098 & \mathrm{mg} / \mathrm{L} \\ 0.030 & \mathrm{mg} / \mathrm{L}\end{array}$

** SD-100-02

SD-100-02 12-May-1987 TITANIUK

SD-100-02 18-May-1987 IITANTUH

$<0,0030$

$\mathrm{mg} / \mathrm{L}$

0.0050

0.0097

$\mathrm{mg} / \mathrm{L}$

SD-100-02 3-Jun-1987 TITARTOH

0.0033

$\mathrm{mg} / \mathrm{L}$

SD-100-02 11-Jun-1987 TITANIUY

0.0035

$\mathrm{mg} / \mathrm{L}$

SD-100-02 18-Jun-1987 TITAKTUY

$<0.0030$

0.0050

0.0073

0.0087

0.0049

0.0040

$\mathrm{mg} / \mathrm{L}$

mg/L

mg/L

mg/L

$\mathrm{mg} / \mathrm{L}$

SD-100-03 11-Jun-1987 TITANIUM

SD-100-03 18-Jun-1987 TITANIUM 
Location Date Test Compound Results Units

** SD-100-03D

SD-100-03D 12-May-1987 TITANTUM

SD-100-03D 26-May-1987 TITnNUM

$<0.0030$

$\mathrm{mg} / \mathrm{L}$

0.0053

$\mathbf{n g} / \mathrm{L}$

* SD $-100-04$

SD-100-04 12-May-1987 TITANTUM

SD-100-04 18-May-1987 TITANTUM

SD-100-04 26-May-1987 TITANTUM

SD-100-04 3-Jun-1987 TITAKTUM

SD-100-04 11-Jun-1987 TITANIUM

SD-100-04 18-Jun-1987 TITANIUM

$\begin{array}{ll}0.0030 & \mathrm{gg} / \mathrm{L} \\ <0.0030 & \mathrm{ng} / \mathrm{L} \\ <0.0030 & \mathrm{mg} / \mathrm{L} \\ 0.0061 & \mathrm{gg} / \mathrm{L} \\ 0.0045 & \mathrm{gg} / \mathrm{L} \\ 0.0042 & \mathrm{mg} / \mathrm{L}\end{array}$

* SD-100-06

SD-100-06 12-May-1987 TITANIUM

SD-100-06 18-May-1987 TITANIUM

SD-100-06 26-May-1987 TITANIUM

SD-100-06 3-Jun-1987 TITANIUM

SD-100-06 11-Jun-1987 TITANIUM

SD-100-06 18-Jun-1987 TITANTUM

$<0.0030 \quad \mathrm{ng} / \mathrm{L}$

$<0.0030 \quad$ تgg $/ \mathrm{L}$

$<0.0030 \quad \mathrm{mg} / \mathrm{L}$

$0.0064 \quad \mathrm{gg} / \mathrm{L}$

$0.0041 \mathrm{mg} / \mathrm{L}$

$0.0037 \mathrm{mg} / \mathrm{L}$

** SD-100-06D

SD-100-06D 3-Jun-1987 TITANIUM

$0.0057 \quad \mathrm{mg} / \mathrm{L}$

* SD-100-07

SD-100-07 12-May-1987 TITANIUM

SD-100-07 19-May-1987 TITANIUM

SD-100-07 26-May-1987 TITANIUM

SD-100-07 3-Jun-1987 TITANIUI

SD-100-07 11-Jun-1987 TITANIUM

SD-100-07 18-Jun-1987 TITANIUN

$0.072 \quad \mathrm{gg} / \mathrm{L}$

$0.036 \quad \mathrm{mg} / \mathrm{L}$

$0.0082 \quad \mathrm{mg} / \mathrm{L}$

$0.037 \mathrm{mg} / \mathrm{L}$

$0.0032 \quad \mathrm{gg} / \mathrm{L}$

$0.038 \quad \mathrm{gg} / \mathrm{L}$

** SD-100-07D

SD-100-07D 18-Jun-1987 TITANTUM

$0.052 \mathrm{mg} / \mathrm{L}$

** SD $-100-09$

SD-100-09 12-May-1987 IITANIUM

SD-100-09 18-May-1987 IITANIUM

SD-100-09 26-May-1987 TITARIUM

SD-100-09 3-Jun-1987 TITANIUM

SD-100-09 11-Jun-1987 TITANIUY

$\begin{array}{ll}0.0050 & \mathrm{gg} / \mathrm{L} \\ 0.0042 & \mathrm{mg} / \mathrm{L} \\ <0.0030 & \mathrm{gg} / \mathrm{L} \\ 0.0079 & \mathrm{gg} / \mathrm{L} \\ 0.0043 & \mathrm{gg} / \mathrm{L}\end{array}$

** SD $-100-10$

SD-100-10 12-May-1987 TITANIUM

SD-100-10 19-May-1987 TITANIUM

SD-100-10 26-May-1987 TITANIUM

SD-100-10 3-Jun-1987 TITANIUM

SD-100-10 11-Jun-1987 TITANIUM

SD-100-10 18-Jun-1987 TITANIUM

$\begin{array}{ll}<0.0030 & \mathrm{mg} / \mathrm{L} \\ 0.0044 & \mathrm{gg} / \mathrm{L} \\ <0.0030 & \mathrm{mg} / \mathrm{L} \\ 0.017 & \mathrm{mg} / \mathrm{L} \\ 0.0056 & \mathrm{gg} / \mathrm{L} \\ 0.0070 & \mathrm{gg} / \mathrm{L}\end{array}$

* SD-100-11

SD-100-11 12-May-1987 TITANIUM

$<0.0030$

$\nabla \mathrm{g} / \mathrm{L}$ 
Lacation Date Test Compound

SD-100-11 19-May-1987 TITANIUM

SD-100-11 26-May-1987 TITANTUM

SD-100-11 3-Jun-1987 TITANTUM

SD-100-11 11-Jun-1987 TITANIUM

SD-100-11 1B-Jun-1987 TITANIUM

* SD-100-12

SD-100-12 12-May-1987 TITANIUM

SD-100-12 19-May-1987 TITANIUM

SD-100-12 26-May-1987 TITANIUM

SD-100-12 3-Jun-1987 TITANIUM

SD-100-12 11-Jun-1987 TITANIUM

SD-100-12 18-Jun-1987 TITANIUM

* SD-100-13

SD-100-13 12-May-1987 TITANIUM

SD-100-13 19-May-1987 TITANIUM

SD-100-13 26-May-1987 TITANIUM

SD-100-13 11-Jun-1987 TITANIUM

SD-100-13 18-Jun-1987 TITANIUM

$\star \star \quad S D-100-14$

SD-100-14 12-May-1987 TITANIUM

SD-100-14 19-May-1987 TITANIUM

SD-100-14 26-May-1987 TITANIUM

SD-100-14 3-Jun-1987 TITANIUM

SD-100-14 11-Jun-1987 TITANIUM

SD-100-14 18-Jun-1987 TITANIUM

* SD $-100-C$

SD-100-C 5-Kar-1987 TITANTOM

SD-100-C 9-Mar-1987 TITANIUM

SD-100-C 17-Kar-1987 TITANIUM

SD-100-C 24-Kar-1987 TITANIUM

SD-100-C 8-Apt-1987 TITANIUM

SD-100-C 13-May-1987 TITANIUM

SD-100-C 20-May-1987 TITANIUM

SD-100-C 27-May-1987 TITANIUM

SD-100-C 4-Jun-1987 TITANIUM

SD-100-C 12-Jun-1987 TITANIUM

SD-100-C 19-Jun-1987 TITANIUI

$\star \star \quad S D-100-D$

SD-100-D 6-APr-1987 TITANIUM

SD-100-D 11-Jun-1987 TITANIUM

* SD. 100

SD-100 5-Mar-1987 TOLUENE

SD-100 11-Mar-1987 TOLUENE

SD-100 18-Mar-1987 TOLUENE

SD-100 24-Mar-1987 TOLUENE
Results Untts

$\begin{array}{ll}0.0032 & \mathrm{mg} / \mathrm{L} \\ 0.024 & \mathrm{mg} / \mathrm{L} \\ 0.0099 & \mathrm{mg} / \mathrm{L} \\ 0.0057 & \mathrm{mg} / \mathrm{L} \\ 0.0081 & \mathrm{mg} / \mathrm{L}\end{array}$

0.036

0.0048

0.0063

0.0057

0.0071

0.0088

$\mathrm{mg} / \mathrm{L}$
$\mathrm{mg} / \mathrm{L}$
$\mathrm{mg} / \mathrm{L}$
$\mathrm{mg} / \mathrm{L}$
$\mathrm{mg} / \mathrm{L}$
$\mathrm{mg} / \mathrm{L}$

$<0.0030$

$<0.0030$

0.0055

0.0051

0.0048

$\mathrm{mg} / \mathrm{L}$

$\mathrm{mg} / \mathrm{L}$

$m \mathrm{~g} / \mathrm{L}$

mg/L

IIg/

0.012

0.026

0.0082

0.0057

0.0052

0.0081

$\mathrm{mg} / \mathrm{L}$

mg/L

$\mathrm{mg} / \mathrm{L}$

$\mathrm{mg} / \mathrm{L}$

mg/L

$\mathrm{mg} / \mathrm{L}$

$<0.0030$

mg/L

$<0.0030$

$\mathrm{mg} / \mathrm{L}$

$<0.0030$

mg/L

0.0053

0.0044

$<0.0030$

$<0.0030$

$<0.0030$

0.0046

0.0051

$<0.0030$

$\mathrm{mg} / \mathrm{l}$

$\mathrm{mg} / \mathrm{L}$

mg $/ 2$

$\mathrm{mg} / \mathrm{h}$

$\mathrm{mg} / \mathrm{L}$

$\mathrm{mg} / \mathrm{L}$

IIE/L

$\mathrm{mg} / \mathrm{L}$

$<0.0030$

$m g / L$

0.0099

mg/L

$<5$

$<5$

$<5$

$<5$
ug/L

$u g / L$

ug $/ \mathrm{L}$

ug/L 
Location Date Test Compound Results Units

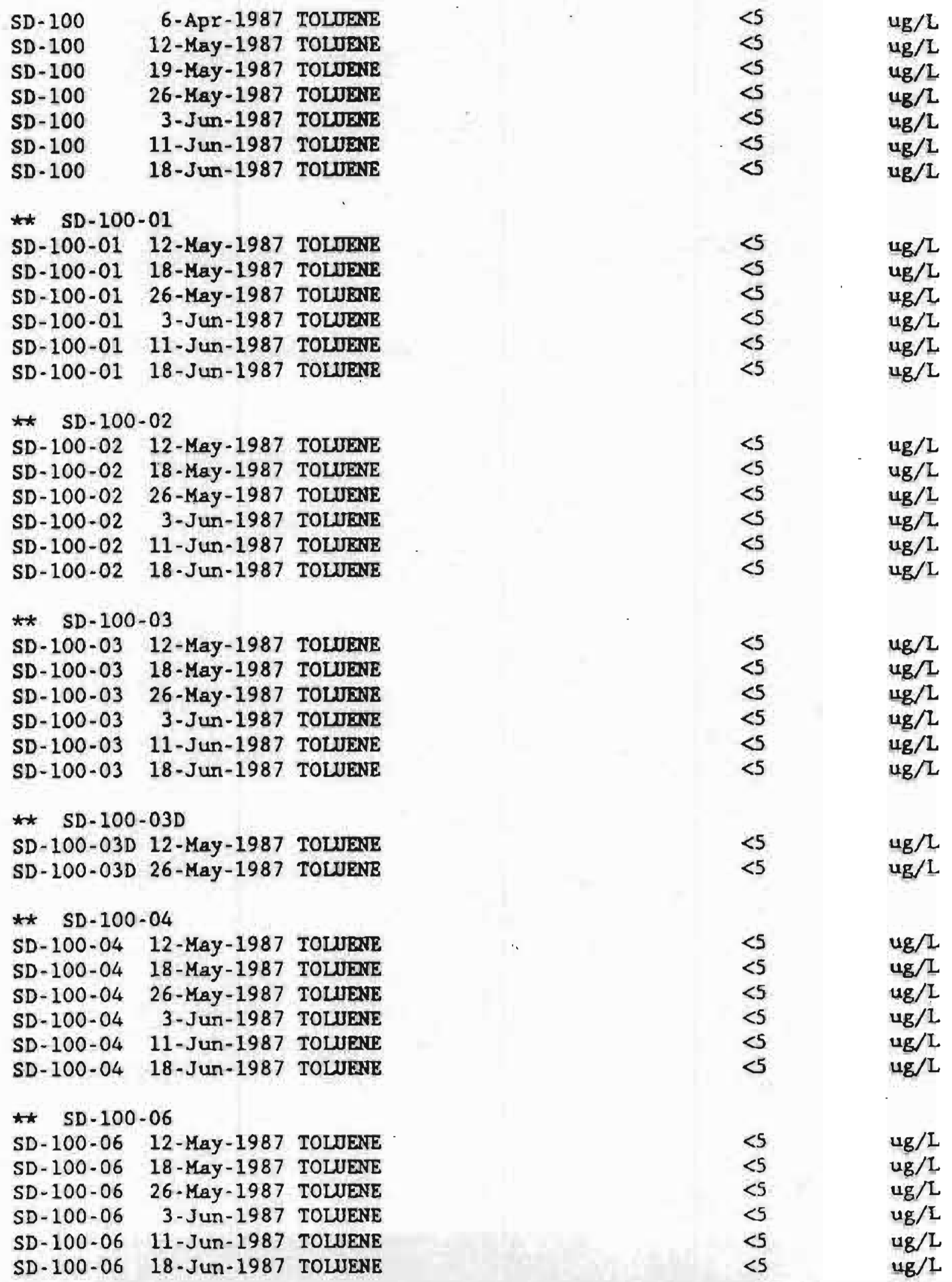




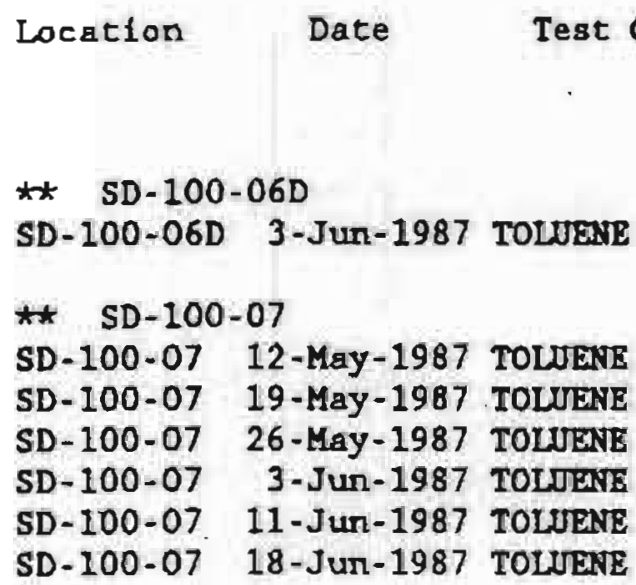

** SD-100-06D

SD-100-06D 3-Jun-1987 TOLUENE

* SD-100-07

SD-100-07 12-May-1987 TOLUENE

SD-100-07 19-May-1987 IOLUENE

SD-100-07 26-May-1987 TOLUENE

SD-100-07 3-Jun-1987 TOLUENE

SD-100-07 11-Jun-1987 TOLUENE

SD-100-07 18-JUn-1987 TOLUENE

$\begin{array}{ll}<5 & u g / L \\ 8 & \\ <5 & u g / L \\ 18 & u g / L \\ 5 & u g / L \\ 5 & u g / L \\ 50 & u g / L \\ & 4 g / L \\ & \\ 9 & \\ & 4 g / L \\ & \\ <5 & u g / L \\ <5 & u g / L \\ <5 & u g / L \\ <5 & u g / L \\ <5 & u g / L \\ <5 & u g / L\end{array}$

* SD-100-07D

SD-100-07D 18-Jun-1987 TOLUENE

$1 \mathrm{~g} / \mathrm{L}$

** SD-100-09

SD-100-09 12-May-1987 TOLUENE

SD-100-09 18-May-1987 TOLUENE

SD-100-09 26-May-1987 TOUUENE

SD-100-09 3-Jun-1987 TOLUENR

SD-100-09 11-JUn-1987 TOLUENE

SD-100-09 18-Jun-1987 TOLUENE

ug/L

** SD $-100-10$

SD-100-10 12-May-1987 TOLUENE

SD-100-10 19-May-1987 TOLUENE

SD-100-10 26-May-1987 TOLUENE

SD-100-10 3-Jun-1987 TOLDENE

SD-100-10 11-Jun-1987 TOLUENE

SD-100-10 18-Jun-1987 TOLOENE

SD-100-11 18-Jun-1987 TOLUENE

$\star$ SD-100-12

SD-100-12 12-May-1987 TOLUENE

SD-100-12 19-May-1987 TOLUENE

SD-100-12 26-May-1987 TOLUENE

SD-100-12 3-Jun-1987 TOLUENE

SD-100-12 11-Jun-1987 TOLJENE

SD-100-12 18-Jun-1987 TOLUENE

* SO $-100-13$

SD-100-13 12-May-1987 TOLUENE

SD-100-13 19-May-1987 TOLUENE

SD-100-13 26-May-1987 TOLUENE
$<5$

$<s$

$<5$

$<5$

$<5$

$<5$

$<5$

$<5$

$<5$

$<5$

$<5$

$<5$

ug/L

ug/L

ug/L

ug/L

$\mathrm{ug} / \mathrm{L}$

$\mathrm{ug} / \mathrm{L}$

ug/L

$u g / L$

$\mathrm{ug} / \mathrm{L}$

ug/L

ug/L

$u g / L$

$<5$

ug/L

ug/L

ug/L

$u g / L$

ug/L

$u g / L$

$<5$

ug/L

ug $/ \mathrm{L}$

ug/L 
$<5$

16

$<5$

$\mathrm{ug} / \mathrm{L}$

ug $/ 2$

ug/ $/ 1$

$<5$

$<5$

$<5$

$<5$

12

$<5$

$<5$

$<5$

$<5$

$<5$

$<5$

$<5$

$<5$

$<5$

$<5$

$<5$

19-Jun-1987 TOLUENE

ug/L

ug/L

$\mathrm{ug} / \mathrm{L}$

$\mathrm{ug} / \mathrm{L}$

$u g / \mathrm{L}$

ug $/ \mathrm{L}$

24-Mar-1987 TOUURE

8-Apr-1987 TOLUENE

20-May-1987 TOLUENE

27-May-1987 TOLUENE

12-Jun-1987 TOLUENE

$\mathrm{ug} / \mathrm{L}$

$u g / L$

ug/L

$u g / L$

ug/L

$4 \mathrm{~g} / \mathrm{L}$

$u g / L$

ug/L

ug/L,

ug/L

* SD-100-C

SD-100-C 9-Mar-1987 TOLUENE

SD-100-C

SD-100-C

SD-100-C

SD $-100-C$

SD-100-C

* SD-100-D

SD-100-D 6-APr-1987 TOLUENE

SD-100-D 11-Jun-1987 TOWENE

$<5$

$<5$

* SD-100

SD-100 S-MaI-1987 TOTAL DISSOLVED SOLIDS

SD-100 11-Mar-1987 TOTAL DISSOLVED SOLIDS

SD-100 18-MaT-1987 TOTAL DISSOLVED SOLIDS

SD- 100

24-Mar-1987 TOTAL DISSOLVED SOLIDS

SD -100

3-APr-1987 TOTAL DISSOLVED SOLIDS

SD- 100

6-Apr-1987 TOTAL DISSOLVED SOLIDS

SD -100

12-May-1987 TOTAL DISSOLUED SOLIDS

SD -100

19-May-1987 TOTAL DISSOLVED SOLIDS

SD -100

26-May-1987 TOTAL DISSOLVED SOLIDS

3-JUn-1987 TOTAL DISSOLVED SOLIDS

SD -100

11-Jun-1987 TOTAL DISSOLVED SOLIDS

SD -100

18-Jun-1987 TOTAL DISSOLVED SOLIDS

SD -100

182

ug $/ \mathrm{L}$

$48 / 2$

** SD-100-01

SD-100-01 12-May-1987 TOTAL DISSOLVED SOLIDS

SD-100-01 18-May-1987 TOTAL DISSOLVED SOLIDS

SD-100-01 26-May-1987 TOTAL DISSOLVED SOLIDS

SD-100-01 3-Jun-1987 TOTAL DISSOLVED SOLIDS

$\mathbf{m g} / \mathrm{L}$ $\mathrm{mg} / \mathrm{L}$ $\mathrm{mg} / \mathrm{L}$

mg/L mg/L $\mathrm{mg} / \mathrm{L}$ $\mathrm{mg} / \mathrm{L}$ $\mathbf{m g} / \mathrm{L}$ $\operatorname{mg} / \mathrm{I}$ $m g / L$ ang $/ \mathrm{L}$ $\mathbf{m g} / \mathrm{L}$

SD-100-01 18-Jun-1987 TOTAL DISSOLVED SOLIDS

148

$\mathbf{m g} / \mathrm{L}$ $\mathrm{mg} / \mathrm{L}$ $\mathrm{mg} / \mathrm{L}$ mg/L mg/L $\mathbf{n g} / \mathrm{L}$ 
** SD-100-02

SD-100-02 12-May-1987 TOTAL DISSOLVED SOLIDS

SD-100-02 18-May-1987 TOTAL DISSOLVED SOLIDS

SD-100-02 26-MaY-1987 TOTAL DISSOLVED SOLIDS

166

SD-100-02 3-Jun-1987 TOTAL DISSOLVED SOLIDS

172

SD-100-02 11-Jun-1987 TOTAL DISSOLVED SOLIDS

148

SD-100-02 18-Jun-1987 TOTAL DISSOLVED SOLIDS

164

** SD-100-03

SD-100-03 12-May-1987 TOTAL DISSOLVED SOLIDS SD-100-03 18-May-1987 TOTAL DISSOLVED SOLIDS SD-100-03 26-May-1987 TOTAL DISSOLVED SOLIDS SD-100-03 3-Jun-1987 TOTAL DISSOLVED SOLIDS SD-100-03 11-JUn-1987 TOTAL DISSOLVED SOLIDS SD-100-03 18-Jun-1987 TOTAL DISSOLVED SOLIDS

** SD-100-03D

SD-100-03D 12-May-1987 TOTAL DISSOLVED SOLIDS SD-100-03D 26-May-1987 TOTAL DISSOLVED SOLIDS

** SD-100-04

$\mathrm{mg} / \mathrm{L}$

$\mathrm{mg} / \mathrm{L}$

SD-100-04 12-May-1987 TOTAL DISSOLVED SOLIDS

SD-100-04 18-May-1987 TOTAL DISSOLVED SOLIDS

SD-100-04 26-May-1987 TOTAL DISSOLVED SOLIDS

SD-100-04 11-JUn-1987 TOTAL DISSOLVED SOLIDS

158

SD-100-04 18-Jun-1987 TOTAL DISSOLVED SOLIDS

168

* SD-100-06

SD-100-06 12-Kay-1987 TOTAL DISSOLVED SOLIDS SD-100-06 18-May-1987 TOTAL DISSOLVED SOLIDS SD-100-06 26-May-1987 TOTAL DISSOLVED SOLIDS SD-100-06 3-JUa-1987 TOTAL DISSOLVED SOLIDS SD-100-06 11-JUn-1987 TOTAL DISSOLVED SOLIDS SD-100-06 18-Jun-1987 TOTAL DISSOLVED SOLIDS

** SD-100-06D

SD-100-06D 3-Jun-1987 TOTAL DISSOLVED SOLIDS

140

138

178

178

156

194

188

$\operatorname{mg} / \mathrm{L}$ 由g/L $\mathrm{mg} / \mathrm{L}$ $\mathrm{mg} / \mathrm{L}$ mg/L $\mathrm{mg} / \mathrm{L}$

$\mathrm{mg} / \mathrm{L}$ mg/L $\mathbf{m g} / \mathrm{L}$ mg/L $\mathbf{m g} / \mathrm{L}$ $\mathrm{mg} / \mathrm{L}$

** SD-100-07

SD-100-07 12-May-1987 TOTAL DISSOLVED SOLIDS SD-100-07 19-May-1987 TOTAL DISSOLVED SOLIDS SD-100-07 26-May-1987 TOTAL DISSOLVED SOLIDS SD-100-07 3-JUn-1987 TOTAL DISSOLVED SOLIDS SD-100-07 11-Jun-1987 TOTAL DISSOLVED SOLIDS SD-100-07 18-Jun-1987 TOTAL DISSOLVED SOLIDS

* SD-100-070

SD-100-07D 18-Jun-1987 TOTAL DISSOLVED SOLIDS
886

302

104

148

896

146

1072 $\mathbf{m g} / \mathrm{L}$

$\mathbf{m g} / \mathrm{L}$ $\mathrm{mg} / \mathrm{L}$ $\mathrm{mg} / \mathrm{l}$ $\mathrm{mg} / \mathrm{L}$ mg/L $\mathrm{mg} / \mathrm{L}$

$\mathrm{mg} / \mathrm{L}$ 
Location Date Test Compound Results Units

** SD-100-09

SD-100-09 12-Kay-1987 TOTAL DISSOLVED SOLIDS

SD-100-09 18-Kay-1987 TOTAL DISSOLVED SOLIDS

SD-100-09 26-MaY-1987 TOTAL DISSOLVED SOLIDS

SD-100-09 3-Jun-1987 TOTAL DISSOLVED SOLIDS

SD-100-09 11-Jun-1987 TOTAL DISSOLVED SOLIDS

SD-100-09 18-JUI-1987 TOTAL DISSOLVED SOLIDS

* SD $-100-10$

SD-100-10 12-MaY-1987 TOTAL DISSOLVED SOLIDS

SD-100-10 19-Kay-1987 TOTAL DISSOLVED SOLIDS

SD-100-10 26-May-1987 TOTAL DISSOLVED SOLIDS

SD-100-10 3-Jun-1987 TOTAL DISSOLVED SOLIDS

SD-100-10 11-Jun-1987 TOTAZ DISSOLVED SOLIDS

SD-100-10 18-JUn-1987 TOTAL DISSOLVED SOLIDS

** SD-100-11

SD-100-11 12-May-1987 TOTAL BISSOLVED SOLIDS

SD-100-11 19-May-1987 TOTAL DISSOLVED SOLIDS

SD-100-11 26-May-1987 TOTAL DISSOLVED SOLIDS

SD-100-11 3-JUn-1987 TOTAL DISSOLVED SOLIDS

SD-100-11 11-Jun-1987 TOTAL DISSOLVED SOLIDS

SD-100-11 18-Jun-1987 TOTAL DISSOLVED SOLIDS

* $\quad$ SD $-100-12$

SD-100-12 12-May-1987 TOTAL DISSOLVED SOLIDS

SD-100-12 19-Kay-1987 TOTAL DISSOLVED SOLIDS

SD-100-12 26-May-1987 TOTAL DISSOLVED SOLIDS

SD-100-12 3-Jun-1987 TOTAL DISSOLVED SOLIDS

SD-100-12 11-Jun-1987 TPTAL DISSOLVED SOLIDS

SD-100-12 18-Jun-1987 TOTAL DISSOLVED SOLIDS

* SD-100-13

SD-100-13 12-May-1987 TOTAL DISSOLVED SOLIDS

SD-100-13 19-May-1987 TOTAL DISSOLVED SOLIDS

SD-100-13 26-May-1987 TOTAL DISSOLVED SOLIDS

SD-100-13 3-Jun-1987 TOTAL DISSOLVED SOLIDS

SD-100-13 11-Jun-1987 TOTAL DISSOLVED SOLIDS

SD-100-13 18-JUn-1987 TOTAL DISSOLVED SOLIDS

** SD-100-14

SD-100-14 12-May-1987 TOTAL DISSOLVED SOLIDS

SD-100-14 19-May-1987 TOTAL DISSOLVED SOLIDS

SD-100-14 26-May-1987 TOTAL DISSOLVED SOLIDS

SD-100-14 3-Jun-1987 TOTAL DISSOLVED SOLIDS

SD-100-14 11-Jun-1987 TOTAL DISSOLVED SOLIDS

SD-100-14 18-Jun-1987 TOTAL DISSOLVED SOLIDS

** SD $100 \cdot C$

SD-100-C 5-Mar-1987 TOTAL DISSOLVED SOLIDS

SD-100-C 9-Mar-1987 TOTAL DISSOLVED SOLIDS

$\begin{array}{ll}164 & \mathrm{mg} / \mathrm{L} \\ 162 & \mathrm{mg} / \mathrm{L} \\ 158 & \mathrm{mg} / \mathrm{L} \\ 168 & \mathrm{mg} / \mathrm{L} \\ 148 & \mathrm{mg} / \mathrm{L} \\ 204 & \mathrm{mg} / \mathrm{L}\end{array}$

130

130

146

190

158

218

$\operatorname{mg} / \mathrm{L}$

$\mathrm{mg} / \mathrm{L}$

$\mathrm{mg} / \mathrm{L}$

mg/L

$\mathrm{mg} / \mathrm{L}$

$\mathrm{mg} / \mathrm{L}$

174

164

152

166

162

188

$\mathrm{mg} / \mathrm{L}$

$\mathrm{mg} / \mathrm{L}$

$\mathrm{mg} / \mathrm{L}$

mg $/ L$

$\mathrm{mg} / \mathrm{L}$

$\mathrm{mg} / \mathrm{L}$

162

172

162

174

160

196

mg/L

$\mathrm{mg} / \mathrm{L}$

mg/L

$\mathrm{mg} / \mathrm{L}$

mg $/ \mathrm{L}$

$\mathrm{ng} / \mathrm{L}$

140

160

150

176

146

218

mg $/$ L

$\mathrm{mg} / \mathrm{L}$

$\mathrm{mg} / \mathrm{L}$

mg/L

$\mathrm{mg} / \mathrm{L}$

$\mathrm{mg} / \mathrm{L}$

172

192

170

204

164

204

$\mathrm{mg} / \mathrm{L}$

$m / L$

$\mathrm{ng} / \mathrm{L}$

$\mathrm{mg} / \mathrm{L}$

mg/L

$\mathrm{mg} / \mathrm{L}$

$\mathrm{mg} / \mathrm{L}$

$\mathrm{mg} / \mathrm{L}$ 
$\star \star \quad S D-100-11$

SD-100-11 12-May-1987 TOTAL ORGANIC GARBON (TOC) SD-100-11 19-May-1987 TOTAL ORGANIC CARBON (TOC) SD-100-11 26-May-1987 TOTAL ORGANIC GARBON (TOC) SD-100-11 3-Jun-1987 TOTAL ORGANIC GARBON (TOC) SD-100-11 11-Jun-1987 TOTAL ORGARIC CARBON (TOC) SD-100-11 18-Jun-1987 TOTAL ORGANIC CARBON (TOC)

$\star \star$ SD $-100-12$

SD-100-12 12-May-1987 IOTAL ORGANIC GARBON (TOC) SD-100-12 19-May-1987 TOTAL ORGANIC GARBON (TOC) SD-100-12 26-May-1987 TOTAL ORGANIC CARBON (TOC) SD-100-12 3-Jun-1987 TOTAL ORGANIC CARBON (TOC) SD-100-12 11-JUn-1987 TOTAL ORGANIC CARBON (TOC) SD-100-12 18-Jun-1987 TOTAL ORGANIC GARBON (TOC)

*t SD-100-13

SD-100-13 12-May-1987 TOTAL ORGANIC CARBON (TOC) SD-100-13 19-May-1987 TOTAL ORGANIC GARBON (TOC) SD-100-13 26-May-1987 TOTAL ORGANIC GARBON (TOC) SD-100-13 3-Jun-1987 TOTAL ORGANIC CARBON (TOC) SD-100-13 11-Jun-1987 TOTAL ORGANIC CARBON (TOC) SD-100-13 18-Jun-1987 TOTAL ORGANIC GARBON (TOC)

** SD-100-14

SD-100-14 12-May-1987 TOTAL ORGANIC CARBON (TOC) SD-100-14 19-May-1987 TOTAL ORGANIC CARBON (TOC) SD-100-14 26-May-1987 TOTAL ORGANIC GARBON (TOC) SD-100-14 3-Jun-1987 TOTAL ORGANIC CARBON (TOC) SD-100-14 11-Jun-1987 TOTAL ORGANIC GARBON (TOC) SD-100-14 18-JUI-1987 TOTAL ORGANIC GARBON (TOC)

* SD-100-C

SD-100-C 5-MaI-1987 TOTAL ORGANIC CARBON (TOC) SD-100-C 9-Mar-1987 TOTAL ORGANIC CARBON (TOC) SD-100-C 17-MaI-1987 TOTAL ORGANIC GARBON (TOC) SD-100-C 24-Mar-1987 TOTAL ORGANIC CARBON (TOC) $S D-100-C$ $S D-100-C$ $S D-100-C$ $S D-100-C$ $S D-100-C$ SD $-100-C$ 8-Apr-1987 TOTAL ORGANIC GARBON (TOC) 13-May-1987 TOTAL ORGANIC CARBON (TOC) 20-Kay-1987 TOTAL ORGANIC CARBON (TOC) 27-Kay-1987 TOTAL ORGANIC CARBON (TOC) 4-Jun-1987 TOTAL ORGANIC CARBON (TOC) 12-JUn-1987 TOTAL ORGANIC CARBON (TOC) SD-100-C 19-Jun-1987 TOTAL ORGANIC CARBON (TOC)

** SD-100-D

SD-100-D 6-ApI-1987 TOTAL ORGANIC CARBON (TOC) SD-100-D 11-JUn-1987 TOTAL ORGANIC CARBON (TOC)

* SD-100

SD- 100

5-Mar-1987 TOTAL SUSPENDED SOLIDS

$\begin{array}{ll}2.1 & \mathrm{mg} / \mathrm{L} \\ 3.2 & \mathrm{mg} / \mathrm{L} \\ 2.2 & \mathrm{mg} / \mathrm{L} \\ 2.7 & \mathrm{mg} / \mathrm{L} \\ 2.2 & \mathrm{mg} / \mathrm{L} \\ 3.0 & \mathrm{mg} / \mathrm{L}\end{array}$

$2.0 \quad \mathrm{mg} / \mathrm{L}$

$2.0 \mathrm{mg} / \mathrm{L}$

$2.9 \mathrm{mg} / \mathrm{L}$

$2.6 \mathrm{mg} / \mathrm{L}$

$1.5 \mathrm{mg} / \mathrm{L}$

$2.8 \mathrm{mg} / \mathrm{L}$

$2.6 \mathrm{mg} / \mathrm{L}$

$2.6 \mathrm{mg} / \mathrm{L}$

$2.2 \mathrm{mg} / \mathrm{L}$

$2.6 \mathrm{mg} / \mathrm{L}$

$1.6 \mathrm{mg} / \mathrm{L}$

$3.2 \mathrm{mg} / \mathrm{L}$

$2.1 \mathrm{mg} / \mathrm{L}$

$2.3 \mathrm{mg} / \mathrm{L}$

$2.1 \mathrm{mg} / \mathrm{L}$

$1.9 \mathrm{mg} / \mathrm{L}$

$1.4 \mathrm{mg} / \mathrm{L}$

$2.0 \mathrm{mg} / \mathrm{L}$

$12 \quad \mathrm{mg} / \mathrm{L}$

$5 \mathrm{mg} / \mathrm{L}$

$27 \mathrm{mg} / \mathrm{L}$

$5 \mathrm{mg} / \mathrm{L}$

$4 \mathrm{mg} / \mathrm{L}$

$21 \mathrm{mg} / \mathrm{L}$

$23 \mathrm{mg} / \mathrm{L}$

$23 \mathrm{mg} / \mathrm{L}$

$10.6 \mathrm{mg} / \mathrm{L}$

$23.8 \mathrm{mg} / \mathrm{L}$

$3.1 \mathrm{mg} / \mathrm{L}$

$6 \mathrm{mg} / \mathrm{L}$

$1.8 \mathrm{mg} / \mathrm{L}$

$12 \mathrm{mg} / \mathrm{L}$

ic $\cdot$ 


Location Date Rest Compound Rults Units

\begin{tabular}{|c|c|c|c|c|}
\hline SD- 100 & $11-$ Mar - 1987 & TOTAL & SUSPEANDED & SOLIDS \\
\hline SD -100 & $18-\mathrm{Kax}-1987$ & TOTAL & SUSPENDED & SOLIDS \\
\hline SD -100 & 24 -HaI- 1987 & TOTAL & SUSPENDED & SOLIDS \\
\hline SD - 100 & 3-Apr-1987 & TOTAL & SUSPERNED & SOLIDS \\
\hline SD -100 & 6-Apr-1987 & TOTAL & SUSPEANDED & SOLID \\
\hline SD -100 & 12-Kay-1987 & TOTAL & SUSPEADED & SOLIDS \\
\hline SD - 100 & 19-May-1987 & TOTAL & SUSPENDED & SOLIDS \\
\hline SD - 100 & 26 - Мay -1987 & TOTAL & SUSPERTDED & SOLIDS \\
\hline SD - 100 & 3-Jun-1987 & TOTAL & SUSPENDED & SOLIDS \\
\hline SD- 100 & 11-Jun-1987 & TOTAL & SUSPENDED & SOLTD \\
\hline SD -100 & $18-J u n-1987$ & TCTT & SUSPENDED & SOLII \\
\hline
\end{tabular}

$\begin{array}{ll}<1 & \mathrm{mg} / \mathrm{L} \\ 1 & \mathrm{mg} / \mathrm{L} \\ 2 & \mathrm{mg} / \mathrm{L} \\ 2 & \mathrm{mg} / \mathrm{L} \\ 2 & \mathrm{mg} / \mathrm{L} \\ 1 & \mathrm{mg} / \mathrm{L} \\ <1 & \mathrm{mg} / \mathrm{L} \\ 4 & \mathrm{mg} / \mathrm{L} \\ 1 & \mathrm{mg} / \mathrm{L} \\ 2 & \mathrm{mg} / \mathrm{L} \\ <4 & \mathrm{mg} / \mathrm{L}\end{array}$

* SD $-100-01$

SD-100-01 12-May-1987 TOTAL SUSPENDED SOLIDS

SD-100-01 18-May-1987 TOTAL SUSPENDED SOLIDS

SD-100-01 26-May-1987 TOTAL SUSPENDED SOLIDS

SD-100-01 3-JUn-1987 TOTAL SUSPENDED SOLIDS

SD-100-01 11-Jun-1987 TOTAL SUSPENDED SOLIDS

SD-100-01 18-Jun-1987 TOTAL SUSPENDED SOLIDS

12

148

35

37

7

45

$\mathrm{mg} / \mathrm{L}$

$\mathrm{mg} / \mathrm{L}$

mg/L

$\mathrm{mg} / \mathrm{L}$

mg/L

$\mathrm{mg} / \mathrm{L}$

* SD $-100-02$

SD-100-02 12-Hay-1987 TOTAL SUSPENDED SOLIDS

SD-100-02 18-May-1987 TOTAL SUSPENDED SOLIDS

SD-100-02 26-Kay-1987 TOTAL SUSPENDED SOLIDS

SD-100-02 3-Jun-1987 TOTAL SUSPEDED SOLIDS

SD-100-02 11-Jun-1987 TOTAL SUSPENDED SOLIDS

SD-100-02 18-Jun-1987 TOTAL SUSPENDED SOLIDS

* SD-100-03

SD-100-03 12-May-1987 TOTAL SUSPENDED SOLIDS

SD-100-03 18-May-1987 TOTAL SUSPENDED SOLIDS

SD-100-03 26-May-1987 TOTAL SUSPENDED SOLIDS

SD-100-03 3-Jun-1987 TOTAL SUSPENDED SOLIDS

SD-100-03 11-Jun-1987 TOTAL SUSPENDED SOLIDS

SD-100-03 18-Jun-1987 TOTAL SUSPENDED SOLIDS

** SD-100-03D

SD-100-03D 12-May-1987 TOTAL SUSPENDED SOLIDS

SD-100-03D 26-May-1987 TOTAL SUSPENDED SOLIDS

$\begin{array}{ll}<1 & \mathrm{mg} / \mathrm{L} \\ <1 & \mathrm{mg} / \mathrm{L} \\ <1 & \mathrm{mg} / \mathrm{L} \\ 1 & \mathrm{mg} / \mathrm{L} \\ 4 & \mathrm{mg} / \mathrm{L} \\ <4 & \mathrm{mg} / \mathrm{L}\end{array}$

3

$<1 \quad \mathrm{mg} / \mathrm{L}$

$<1 \quad$ ng $/$ L

$6 \mathrm{mg} / \mathrm{L}$

$<1 \quad$ mg/L

$<4 \quad \mathrm{mg} / \mathrm{L}$

1

$<1 \quad \mathrm{mg} / \mathrm{L}$

** SD-100-04

SD-100-04 12-May-1987 TOTAL SUSPENDED SOLIDS

SD-100-04 18-May-1987 TOTAL SUSPENDED SOLIDS

SD-100-04 26-May-1987 TOTAL SUSPENDED SOLIDS

SD-100-04 3-JUn-1987 TOTAL SUSPENDED SOLIDS

SD-100-04 11-Jun-1987 TOTAL SUSPENDED SOLIDS

SD-100-04 18-Jun-1987 TOTAL SUSPENDED SOLIDS

2

$<1$

$<1$

9

4

$<4$

** SD -100.06

SD-100-06 12-Yay-1987 TOTAL SUSPENDED SOLIDS

SD-100-06 18-May-1987 TOTAL SUSPENDED SOLIDS 
Location Date Test Compound

Resules Units

SD-100-06 26-May-1987 TOTAL SUSPERDED SOLIDS

SD-100-06 3-JUn-1987 TOTAL SUSPENDED SOLTDS

SD-100-06 11-Jun-1987 TOTAL SUSPENDED SOLTDS

SD-100-06 18-Jun-1987 TOTAL SUSPERDDED SOLIDS

** SD $-100-06 D$

SD-100-06D 3-Jun-19B7 TOTAL SUSPETDED SOLIDS

** SD-100-07

SD-100-07 12-May-1987 TOTAL SUSPEIDED SOLTDS

SD-100-07 19-May-1987 TOTAL SUSPERDE SOLIDS

SD-100-07 26-May-1987 TOTAL SUSPEIDED SOLIDS

SD-100-07 3-Jun-1987 TOTAL SUSPENDED SOLIDS

SD-100-07 11-JUM-1987 TOTAL SUSPEMDED SOLIDS

SD-100-07 18-Jun-1987 TOTAL SUSPENDED SOLIDS

** SD-100-07D

SD-100-07D 18-Jun-1987 TOTAL SUSPENDED SOLIDS

** SD-100-09

SD-100-09 12-May-1987 TOTAL SUSPENDED SOLIDS

SD-100-09 18-Kay-1987 TOTAL SUSPENDED SOLIDS

SD-100-09 26-MaY-1987 TOTAL SUSPENDED SOLIDS

SD-100-09 3-Jun-1987 TOTAL SUSPENDED SOLTDS

SD-100-09 11-JUn-1987 TOTAL SUSPENDED SOLIDS

SD-100-09 18-Jun-1987 TOTAL SUSPENDED SOLIDS

*t SD $-100-10$

SD-100-10 12-May-1987 TOTAL SUSPENDED SOUIDS

SD-100-10 19-May-1987 TOTAL SUSPENDED SOLIDS

SD-100-10 26-MaY-1987 TOTAL SUSPENDED SOLIDS

SD-100-10 3-JUn-1987 TOTAL SUSPEMDED SOLIDS

SD-100-10 11-Jun-1987 TOTAL SUSPEADED SOLIDS

SD-100-10 18-JUn-1987 TOTAL SUSPEADED SOLIDS

** SD-100-11

SD-100-11 12-May-1987 TOTAL SUSPERDED SOLIDS

SD-100-11 19-May-1987 TOTAL SUSPERDED SOLDS

SD-100-11 26-May-1987 TOTAL SUSPENDED SOLIDS

SD-100-11 3-JUn-1987 TOTAL SUSPENDED SOLIDS

SD-100-11 11-Jun-1987 TOTAL SUSPENDED SOLIDS

SD-100-11 18-Jun-1987 TOTAL SUSPENDED SOLIDS

* SD -100.12

SD-100-12 12-Kay-1987 TOTAL SUSPENDED SOLIDS

SD-100-12 19-May-1987 TOTAL SUSPENDED SOLIDS

SD-100-12 26-May-1987 TOTAL SUSPEIDED SOLIDS

SD-100-12 3-JUn-1987 TOTAL SUSPENDED SOLIDS

SD-100-12 11-Jun-1987 TOTAL SUSPENDED SOLIDS

SD-100-12 18-Jun-1987 TOTAI SUSPENDED SOLIDS

$\begin{array}{ll}<1 & \mathrm{mg} / \mathrm{L} \\ 3 & \mathrm{mg} / \mathrm{L} \\ 3 & \mathrm{mg} / \mathrm{L} \\ <4 & \mathrm{mg} / \mathrm{L} \\ & \\ 2 & \mathrm{mg} / \mathrm{L}\end{array}$

$\begin{array}{ll}909 & \mathrm{mg} / \mathrm{L} \\ 169 & \mathrm{mg} / \mathrm{L} \\ 38 & \mathrm{mg} / \mathrm{L} \\ 262 & \mathrm{mg} / \mathrm{L} \\ 21 & \mathrm{mg} / \mathrm{L} \\ 50 & \mathrm{mg} / \mathrm{L}\end{array}$

$61 \mathrm{mg} / \mathrm{L}$

8
6
5
5
7
8

$m g / L$ $\mathrm{mg} / \mathrm{L}$ mg $/ L$ $\mathrm{mg} / \mathrm{L}$ $\mathrm{mg} / \mathrm{L}$ mg/L

$<1$

mg $/$ $\mathrm{mg} / \mathrm{L}$ $\mathrm{mg} / \mathrm{L}$ $\mathrm{mg} / \mathrm{L}$ mg/L mg $/ \mathrm{L}$

$<1$

5

3

6

20

$<4$

$\mathrm{mg} / \mathrm{L}$ ㄸg $/ 2$ IIg/L $\mathrm{mg} / \mathrm{a}$ IIE/L mg/L 
Storm Draln Data for SD-100

Location Date Test Compound Results Units

* SD-100-13

SD-100-13 12-Kay-1987 TOTAL SUSPBNDED SOLIDS

SD-100-13 19-May-1987 TOTAL SUSPENDED SOLIDS

SD-100-13 26-Kay-1987 TOTAL SUSPENDED SOLIDS

SD-100-13 3-Jun-1987 TOTAL SUSPEIDED SOLIDS

SD-100-13 11-Jun-1987 TOTAL SUSPEADED SOLIDS

SD-100-13 18-Jun-1987 TOTAL SUSPENDED SOLIDS

t* SD-100-14

SD-100-14 12-MAY-1987 TOTAL SUSPENDED SOLIDS

SD-100-14 19-MaY-1987 TOTAI SUSPENDED SOLIDS

SD-100-14 26-May-1987 TOTAL SUSPENDED SOLIDS

SD-100-14 3-Jun-1987 TOTAL SUSPENDED SOLIDS

SD-100-14 11-Jun-1987 TOTAL SUSPENDED SOLIDS

SD-100-14 18-Jun-1987 TOTAI SUSPENDED SOLIDS

\#* SD-100-C

SD-100-C 5-Mar-1987 TOTAL SUSPENDED SOLIDS

SD-100-C 9-Mar-1987 TOTAL SUSPENDED SOLIDS

SD-100-C 17-Mar-1987 TOTAL SUSPENDED SOLIDS

SD-100-C 24-Mar-1987 TOTAL SUSPENDED SOLIDS

SD-100-C . 8-APT-1987 TOTAL SUSPENDED SOLIDS

SD-100-C 13-May-1987 TOTAL SUSPENDED SOLIDS

SD-100-C 20-MaY-1987 TOTAL SUSPENDED SOLIDS

SD-100-C 27-May-1987 TOTAL SUSPENDED SOLIDS

SD-100-C 4-Jun-1987 TOTAL SUSPENDED SOLIDS

SD-100-C 12-Jun-1987 TOTAL SUSPENDED SOLIDS

SD-100-C I9-Jun-1987 TOTAL SUSPERED SOLIDS

$<1$

1

69

2

2

$<4$

15

14

5

16

6

$<4$

7

5

3

10

3

6

2

7

9

$<1$

13

6

4

$<5$

$<5$

$<5$

$<5$

$<5$

$<5$

$<5$

$<5$

$<5$

$<5$

$<5$

SD -100
3-JUN-1987 TRANS - 1 2-DICHLOROETHENE

18-Jun-1987 TRANS-1 2-DICHLOROETHENE

** SD-100-01

SD-100-01 12-May-1987 TRANS-1 2-DICHLOROETHENE

SD-100-01 18-May-1987 TRANS-1 2-DICHLOROETHENE

SD-100-01 26-May-1987 TRANS-1 2-DICHLOROETHENE

SD-100-01 3-Jun-1987 TRANS-1 2-DICHLOROETHENE

$\begin{array}{ll}<5 & u g / L \\ <5 & u g / L \\ <5 & u g / L \\ <5 & u g / L\end{array}$


* SD-100-03

SD-100-03 12-May-1987 TRANS-1 2-DICHLOROETHENE SD-100-03 18-May-1987 TRANS-1 2-DICHLOROETHENE SD-100-03 26-May-1987 TRANS-1 2-DICHLOROETHENE SD-100-03 3-Jun-1987 TRANS-1 2-DICHLOROETHENE SD-100-03 11-Jun-1987 TRANS - I 2-DICHLOROETHENE SD-100-03 18-Jun-1987 TRANS-1 2-DICHLOROETHENE

* SD-100-03D

SD-100-03D 12-May-1987 TRANS-1 2-DICHLOROETHENE SD-100-03D 26-May-1987 TRANS-1 2-DICRLOROETHENE

* SD-100-04

SD-100-04 12-May-1987 TRANS-1 2-DICELOROETHENE 18-May-1987 TRANS - 1 2-DICHLOROETHENE SD- $100-04$ SD-100-04 SD-100-04 SD-100-04 26-Kay-1987 TRANS - 1 2-DICHLOROETHENE 3-Jun-1987 TRANS - 1 2-DICHLOROETHENE 11-JUn-1987 TRANS-1 2-DICHLOROETHENE 18-Jun-1987 IRANS - 1 2-DICHLOROETHENE

$\star$ SD $-100-06$

SD-100-06 12-May-1987 TRANS-1 2-DICHLOROETHENE SD-100-06 18-May-1987 TRANS-1 2-DICHLOROETHENE SD-100-06 26-May-1987 TRANS-1 2-DICHLOROETHENE SD-100-06 3-Jun-1987 IRANS-1 2-DICHLOROETHENE SD-100-06 11-Jun-1987 IRANS-1 2-DICHLOROETHENE SD-100-06 18-Jun-1987 TRANS-1 2-DICHLOROETHENE

* SD-100-06D

SD-100-06D 3-Jun-1987 TRANS-1 2-DICHLOROETHENE

** SD $-100-07$

SD-100-07 12-May-1987 TRANS-1 2-DICHLOROETHENE SD-100-07 19-Mry-1987 TRANS-1 2-DICHLOROETHENE SD-100-07 26-May-1987 TRANS- 1 2-DICHLOROETHENE SD-100-07 3-Jun-1987 TRANS-1 2-DICHLOROETHENE SD-100-07 11-JUn-1987 TRANS - 1 2-DICHLOROETHENE SD-100-07 18-Jun-1987 TRANS-1 2-DICHLOROETHENE
$<5$

$<5$

$<5$

$<5$

$<5$

$<$

$<5$

$<5$

$u g / L$
$u g / L$
$u g / L$
$u g / L$
$u g / L$
$u g / L$

ug/L ug/L ug/L ug/L ug $/ \mathrm{L}$ ug $/ 2$

ug/ $u g / L$

$<5$

$<5$

$<5$

$<5$

$<$

$<5$

$\mathrm{ug} / \mathrm{L}$ ug/L ug/L ug/L $4 \mathrm{~g} / \mathrm{L}$ ug/L

$<$

$<5$

$<$

$<5$

es

$<5$

ug $/ \mathrm{L}$ $u g / L$ $u g / L$ ug/L ug/L ug/L

$<$

ug/L

ug/ $L$ ug/L ug/L ug/L ug/L ug/L 
Location Date Test Compound

Results Unics

* SD-100-07D

SD-100-07D 18-Jun-1987 TRANS-1 2-DIGLIOROETHENE

$<$

$u g / L$

* SD-100-09

SD-100-09 12-May-1987 TRANS-1 2-DIGEROROETHETNE

SD-100-09 18-May-1987 TRANS-1 2-DICFLOROETHENE

SD-100-09 26-May-1987 TRANS-1 2-DICHLOROETHENE

SD-100-09 3-Jun-1987 TRANS-1 2-DICHLOROETHENE

SD-100-09 11-JUn-1987 TRANS-1 2-DICHLOROETHENE

SD-100-09 18-Jun-1987 TRANS-1 2-DICHLOROETHENE

* SD $-100-10$

SD-100-10 12-May-1987 TRANS-1 2-DICKLOROETHENE

SD-100-10 19-May-1987 TRANS-I 2-DICHLOROETHENE

SD-100-10 26-May-1987 TRANS-1 2-DICHLOROETHENE

SD-100-10 3-Jun-1987 TRANS-1 2-DICHLOROETHENE

SD-100-10 11-JUn-1987 TRANS-1 2-DICHLOROETHENE

SD-100-10 18-JUn-1987 TRANS-1 2-DICHLOROETHENE

* SD - $100-11$

SD-100-11 12-May-1987 TRANS-1 2-DICHLOROETHENE

SD-100-11 19-May-1987 TRARS-1 2-DICHLOROETHENE

SD-100-11 26-May-1987 TRANS-1 2-DICHIOROETHENE

SD-100-11 3-Jun-1987 TRANS-1 2-DICHZOROETHENE

SD-100-11 11-JUn-1987 TRANS-1 2-DICHLOROETHENE

SD-100-11 18-Jun-1987 TRAHS-1 2-DICHLOROETHENE

** SD-100-12

SD-100-12 12-May-1987 TRANS-1 2-DICHLOROETHENE

SD-100-12 19-May-1987 TRANS-1 2-DICHLOROETHENE

SD-100-12 26-May-1987 TRANS-1 2-DICHLOROETHENE

SD-100-12 3-Jun-1987 TRANS-1 2-DICHLOROETHENE

SD-100-12 11-JUn-1987 TRANS-1 2-DICHLOROETHENE

SD-100-12 18-Jun-1987 TRANS-1 2-DICHLOROETHENE

$\begin{array}{ll}<5 & u g / L \\ <5 & u g / L \\ <5 & u g / L \\ <5 & u g / L \\ <5 & u g / L \\ <5 & u g / L\end{array}$

$<5$

$<5$

$<5$

$<5$

$<5$

$<5$

$\mathrm{ug} / \mathrm{L}$

$-u g / L$

$u g / L$

ug/L

$u g / L$

$\mathrm{ug} / \mathrm{L}$

* SD-100-13

SD-100-13 12-May-1987 TRANS-1 2-DICHZOROETHENE

SD-100-13 19-May-1987 TRANS-1 2-DICHLOROETHENE

SD-100-13 26-May-1987 TRANS-1 2-DICHLOROETHENE

SD-100-13 3-Jun-1987 TRANS-1 2-DICHLOROETHENE

SD-100-13 11-Jun-1987 TRANS-1 2-DICHLOROETEETE

SD-100-13 18-Jun-1987 TRANS-1 2-DICHLOROETHENIE

$<5$

$<5$

$<5$

$<5$

$<5$

$<5$

ug/L

ug/I

$\mathrm{ug} / \mathrm{L}$

ug/L

ug/L

$u g / L$

* SD - 100-14

SD-100-14 12-May-1987 TRANS-1 2-DICHLOROETHENE

SD-100-14 19-May-1987 TRANS-1 2-DICHLOROETHENE

SD-100-14 26-May-1987 TRANS-1 2-DICHLOROETHENE

SD-100-14 3-Jun-1987 TRANS-1 2-DICHLOROETHENE

SD-100-14 11-Jun-1987 TRANS-1 2-DICHLORORTHENE

SD-100-14 18-Jun-1987 TRANS-1 2-DICHLOROETHENE

ug/L

$u g / L$

ug/L

$u g / L$

$u g / L$

ug/L

$<5$

$u g / L$

$u g / L$

ug/L

ug $/$.

ug/L

ug/I

$<5$

$4 \mathrm{~g} / 2$

$<5$

$<5$

$<5$

$<5$

$<5$

ug/L

$u g / L$

ug/L

$\mathrm{ug} / \mathrm{L}$

ug/L 
Storm Drain Data for SD-100

Location Date Test Compound

Results Units

* SD-100-C

SD-100-C 5-Mat-1987 TRANS-1 2-DICHLOROETHENE

SD-100-C 9-Mar-1987 TRANS-1 2-DICHLOROEIHENE

SD-100-C 17-MaT-1987 TRANS-1 2-DICHLOROBTHENE

SD-100-C 24-Kar-1987 TRANS-1 2-DICEROROETHENE

SD-100-C 8-API-1987 TRANS-1 2-DICHLOROETHENE

SD-100-C 13-May-1987 TRANS-1 2-DIGHLOROETHENE

SD-100-C 20-May-1987 TRANS - 1 2-DICtLOROETHIENE

SD-100-C 27-Kay-1987 TRANS-1 2-DICHLOROETHENE

SD-100-C 12-Jun-1987 TRANS-1 2-DICHLOROETHENE

SD-100-C 19-Jun-1987 IRANS-1 2-DICHLOROBTHENE

* SD-100-D

SD-100-D 6-Apr-1987 TRANS-1 2-DICHLOROETHENE

SD-100-D 11-Jun-1987 TRANS-1 2-DICHLOROETHEIVE

$\begin{array}{ll}<5 & u g / / \\ <5 & u g / L \\ <5 & u g / L \\ <5 & u g / L \\ <5 & u g / L \\ <5 & u g / L \\ <5 & u g / L \\ <5 & u g / L \\ <5 & u g / L \\ <5 & u g / L\end{array}$

** SD-100

$<5$

ug $/ 2$

$u g / L$

SD-100 5-Mar-1987 TRANS-1 3-DICHLOROPROPENE

SD-100 11-Mar-1987 TRANS-1 3-DICHLOROPROPENE

SD-100 18-Mar-1987 TRANS-1 3-DICHIOROPROPENE

SD-100 24-MaI-1987 TRANS-1 3-DICHLOROPROPENE

SD -100

6-APY-1987 TRANS-1 3-DICHLOROPROPENE

SD -100

12-May-1987 TRANS-1 3-DICHLOROPROPENE

SD-100

19-May-1987 TRANS-1 3-DICHLOROPROPENE

SD -100

26-May-1987 TRANS-1 3-DICHLOROPROPENE

SD -100

3-Jun-1987 TRANS - 1 3-DICHLOROPROPEATE

SD -100

11-Jun-1987 TRANS-I 3-DICHLOROPROPENE

SD -100

18-Jun-1987 TRANS-1 3-DICHLOROPROPENE

* SD-100-01

SD-100-01 12-May-1987 TRANS-1 3-DICHLOROPROPENE

SD-100-01 18-May-1987 TRANS-1 3-DICHLOROPROPENE

SD-100-01 26-May-1987 TRANS-1 3-DICHLOROPROPENE

SD-100-01 3-Jun-1987 TRANS-1 3-DICHLOROPROPENE

SD-100-01 11-Jun-1987 TRANS-1 3-DICHLOROPROPENE

SD-100-01 18-Jun-1987 TRANS-1 3-DICHLOROPROPENE
$<5$

$<5$

$<$

$<5$

$<5$

$<5$

$<5$

$<5$

$<5$

$<5$

$<5$

$<5$

$<5$

$<5$

$<5$

$<5$

$<5$

5

$<5$

$<5$

$<5$

$<5$

$<5$

$<5$

$<5$

$<5$

$<5$

$<5$
ug/L

$\mathrm{ug} / \mathrm{L}$

$\mathrm{ug} / \mathrm{L}$

ug/L

ug/L

ug/L

$\mathrm{ug} / \mathrm{L}$

$\mathrm{ug} / \mathrm{L}$

$u g / L$

$4 \mathrm{~g} / \mathrm{I}$

ug/h

$4 \mathrm{~g} / \mathrm{L}$

$1 \mathrm{~g} / \mathrm{L}$

ug/L

ug/L

$\mathrm{ug} / \mathrm{L}$

ug/ $/$

ug/L

ug/L

ug $/ \mathrm{L}$

ug $\Omega$

ug/L

ug/L

** SD $-100-03$

SD-100-03 12-May-1987 TRANS-1 3-DICHLOROPROPENE

SD-100-03 18-May-1987 TRANS-1 3-DICHLOROPROPENE

SD-100-03 26-May-1987 TRANS-1 3-DICHLOROPROPENE

SD-100-03 3-Jun-1987 TRANS- 1 3-DICHLOROPROPENE

SD-100-03 11-Jun-1987 TRANS-1 3-DICHLOROPROPENE $u g / L$

$u g / L$

ug/L

$u g / L$

ug/L 
Storm Dra1n Data for SD-100

Location

Date

Test Compound

Results

Untes

SD-100-03 18-Jun-1987 TRANS-1 3-DICHLOROPROPENE

* SD-100-03D

SD-100-03D 12-May-1987 TRANS- 1 3-DIGHLROPROPENE

SD-100-03D 26-May-1987 TRANS-1 3-DICHLOROPROPENE

** SD-100-04

SD-100-04 12-May-1987 TRANS-1 3-DICELOROPROPERE

SD-100-04 18-May-1987 TRANS-1 3-DICHLOROPROPENE

SD-100-04 26-Kay-1987 TRANS-1 3-DICHLOROPROPENE

SD-100-04 3-Jun-1987 TRANS-1 3-DICHLOROPROPENE

SD-100-04 11-Jun-1987 TRANS-1 3-DICHLOROPROPENE

SD-100-04 18-Jun-1987 TRANS-1 3-DICHLOROPROPENE

$\begin{array}{ll}<5 & u g / L \\ & \\ <5 & u g / L \\ <5 & u g / L \\ & \\ <5 & u g / L \\ <5 & u g / L \\ <5 & u g / L \\ <5 & u g / L \\ <5 & u g / L \\ <5 & 4 g / L\end{array}$

* SD-100-06

SD-100-06 12-May-1987 TRANS-1 3-DICHLOROPROPENB

SD-100-06 18-May-1987 TRANS-1 3-DICHLOROPROPENE

SD-100-06 26-May-1987 TRANS-1 3-DICHLOROPROPENE

SD-100-06 3-JUn-1987 TRANS-1 3-DICHLOROPROPENE

SD-100-06 11-JUn-1987 TRANS-1 3-DICHLOROPROPERE.

SD-100-06 18-Jun-1987 TRANS-1 3-DICHLOROPROPENE

** SD-100-06D

SD-100-06D 3-JUn-1987 TRANS-1 3-DICHLOROPROPENE

ug/L

$\mathrm{ug} / \mathrm{L}$

$\mathrm{ug} / \mathrm{L}$

$u g / L$

$<5$

$<5$

$4 \mathrm{~g} / \mathrm{L}$

** SD-100-07

SD-100-07 12-May-1987 TRANS-1 3-DICHLOROPROPZIE

SD-100-07 19-May-1987 TRANS-1 3-DICHLOROPROPENE

SD-100-07 26-May-1987 TRANS-1 3-DICHLOROPROPENE

SD-100-07 3-JUn-1987 TRANS-1 3-DICHLOROPROPENE

SD-100-07 11-JUn-1987 TRANS-1 3-DICHLOROPROPENE

SD-100-07 18-Jun-1987 TRANS-1 3-DICHLOROPROPENE

** SD-100-07D

SD-100-07D 18-Jun-1987 TRANS-1 3-DICHLOROPROPEANE

$<5$

$\operatorname{ug} / \mathrm{L}$

$<5$

ug $/ 2$

$<$

ug/

ug/L

$\mathrm{ug} / \mathrm{L}$

$<5$

$<5$

ug/L

$<5$

$u g / \mathrm{L}$

* SD-100-09

SD-100-09 12-May-1987 TRANS-1 3-DICHLOROPROPERE

SD-100-09 18-May-1987 TRANS-1 3-DICHLOROPROPEITE

SD-100-09 26-May-1987 TRANS-1 3-DICHLOROPROPENE

SD-100-09 3-Jun-1987 IRANS-1 3-DICHLOROPROPEANE

SD-100-09 11-JUn-1987 TRANS-1 3-DICHLOROPROPENE

SD-100-09 18-Jun-1987 TRANS-1 3-DICHLOROPROPENE

$<s$

$u g / 2$

$<5$

$u g / L$

$<5$

$\operatorname{ug} / \mathrm{L}$

$<5$

$\operatorname{ug} / \mathrm{L}$

$<5 \quad 4 g / \mathrm{L}$

$<5 \quad$ ug/ $/$.

$<5 \quad u g / L$

* $\mathrm{SD}-100-10$

SD-100-10 12-May-1987 TRANS-1 3-DICHLOROPROPENE

SD-100-10 19-May-1987 TRANS-1 3-DICHLOROPROPENE

SD-100-10 26-May-1987 TRANS - 1 3-DICHLOROPROPENE

SD-100-10 3-Jun-1987 TRANS - 1 3-DICHLOROPROPENE

SD-100-10 11-Jun-1987 TRANS-1 3-DICHLOROPROPENE

SD-100-10 18-Jun-1987 TRANS-1 3-DICHLOROPROPENE

$<5$

$<5$

$<5$

$<5$

$<5$

$<5$

$\mathrm{ug} / \mathrm{L}$ ug/L ug/L ug/L $u g / L$ ug/L. 
Location Date Test Compound

* SD-100-06

SD-100-06 12-May-1987 TRICELOROETHEANE

SD-100-06 18-May-1987 TRICHLOROETHERTE

SD-100-06 26-May-1987 TRICLLOROETHEAR

SD-100-06 3-Jun-1987 TRICHLOROETHENE

SD-100-06 11-Jun-1987 TRICHLOROETHEMTE

SD-100-06 18-Jun-1987 TRICHLOROETHENE

* SD $-100-06 D$

SD-100-06D 3-Jun-1983 TRICHLOROETHENE

** SD-100-07

SD-100-07 12-May-1987 TRICHLOROETHETE

SD-100-07 19-May-1987 TRICHLOROETHERE

SD-100-07 26-May-1987 TRICHLOROBTHIRE

SD-100-07 3-Jun-1987 TRICHLOROETHENE

SD-100-07 11-Jun-1987 TRICHLOROETHENE

SD-100-07 18-Jun-1987 TRICHLOROETHENE

** SD - 100-07D

SD-100-07D 18-Jun-1987 TRICHLOROETHENE

* SD-100-09

SD-100-09 12-Kay-1987 TRICHLOROETHENE

SD-100-09 18-May-1987 TRICHLOROETHENE

SD-100-09 26-May-1987 TRICHLOROETHENE

SD-100-09 3-Jun-1987 TRICHLOROETHENE

SD-100-09 11-Jun-1987 TRICHLOROETHENE

SD-100-09 18-Jun-1987 TRICHLOROETHENE

** SD $-100-10$

SD-100-10 12-May-1987 TRICHLOROETHRANE

SD-100-10 19-May-1987 TRICHLOROETHENE

SD-100-10 26-May-1987 IRICHLOROETHENE

SD-100-10 3-Jun-1987 TRICHLOROETHENE

SD-100-10 11-Jun-1987 TRICELOROETHENE

SD-100-10 18-Jun-1987 TRICHLOROETHENE

* SD-100-11

SD-100-11 12-May-1987 TRICHLOROEIHENE

SD-100-11 19-May-1987 TRICHLOROETHENE

SD-100-11 26-May-1987 TRICHLOROETHERNE

SD-100-11 3-Jun-1987 TRICHLOROETHENE

SD-100-11 11-JUn-1987 TRICHLOROETHENE

SD-100-11 18-Jun-1987 TRICHLOROETHENE

* SD $-100-12$

SD-100-12 12-Kay-1987 TRICHLOROETHENE

SD-100-12 19-May-1987 TRICHLOROETHENE

SD-100-12 26-Kay-1987 TRICHLOROETHENE

SD-100-12 3-Jun-1987 TRIGHLOROETHENE
Results Units

$\begin{array}{ll}<5 & \mathrm{ug} / \mathrm{L} \\ <5 & \mathrm{ug} / \mathrm{L} \\ 10 & \mathrm{ug} / \mathrm{L} \\ 5 & \mathrm{ug} / \mathrm{L} \\ <5 & \mathrm{ug} / \mathrm{L} \\ <5 & \mathrm{ug} / \mathrm{L} \\ & \\ & \\ 5 & \mathrm{ug} / \mathrm{L} \\ & \\ 180 & \mathrm{ug} / \mathrm{L} \\ 360 & \mathrm{ug} / \mathrm{L} \\ 390 & \mathrm{ug} / \mathrm{L} \\ 340 & \mathrm{ug} / \mathrm{L} \\ 93 & \mathrm{ug} / \mathrm{L} \\ 1100 & \mathrm{ug} / \mathrm{L}\end{array}$

910

$u g / L$

is

$<5$

$<5$

$<5$

$<5$

$<5$

$\mathrm{ug} / \mathrm{L}$

ug $/$

$u g / L$

$4 \mathrm{~g} / \mathrm{L}$

$4 \mathrm{~g} / \mathrm{L}$

ug/L

$<5$

$u g / L$

ug/L

$4 g / 2$

ug/L

ug $/ \mathrm{L}$

ug/L

$<5$

$<5$

$<$

$<5$

$<5$

$<5$

ug/L

ug/L

ug/L

ug $/ L$

ug/L

$4 \mathrm{~g} / \mathrm{L}$

$<5$

ug/L

$u g / L$

ug/L

ug/L 
Location Date Test Compound Results Units

SD-100-12 11-Jun-1987 TRICHLOROETHFNE SD-100-12 18-Jun-1987 TRICHLOROETHENE

** SD - 100-13

SD-100-13 12-May-1987 TRICHLOROETHENE

SD-100-13 19-May-1987 TRICHLOROETHEVE

SD-100-13 26-MBY-1987 TRICHLOROETHENE

SD-100-13 3-Jum-1987 TRICHLOROETHENE

SD-100-13 11-Jun-1987 TRICHLOROETHESTE

SD-100-13 18-Jun-1987 TRICHLOROETHENE

* SD-100-14

SD-100-14 12-May-1987 TRICHLOROETHENE

SD-100-14 19-May-1987 TRICHLOROETHENE

SD-100-14 26-May-1987 TRICHLOROETHENE

SD-100-14 3-Jun-1987 TRICHLOROETHENE

SD-100-14 11-Jun-1987 TRICHLOROETHENE

SD-100-14 18-Jun-1987 TRICHLOROETHENE

$\star$ SD $-100-C$

SD-100-C 5-Mar-1987 TRICHLOROETHENE

SD-100-C 9-Mar-1987 TRICHLOROETHENE

SD-100-C 17-MaI-1987 TRICHLOROETHENE

SD-100-C 24-Mar-1987 TRICHLOROETHINE

SD-100-C 8-Apr-1987 TRICHLOROETHENE

SD-100-C 13-May-1987 TRICHLOROETHENE

SD-100-C 20-May-1987 TRICHLOROETHENE

SD-100-C 27-May-1987 TRICHLOROETHENE

SD-100-C 12-Jun-1987 TRICHLOROETHENE

SD-100-C 19-Jun-1987 IRICHLOROETRENE

$\star \star \quad S D-100-D$

SD-100-D 6-Apr-1987 TRICHLOROETHENE

SD-100-D I1-Jun-1987 TRICHLOROETHENE

* SD-100

SD -100 S-Mar-1987 TURBIDITY

SD-100 11-Mar-1987 TURBIDITY

SD-100 18-Mar-1987 TURBIDITY

SD-100 24-Mar-1987 TURBIDITY

SD-100 3-Apr-1987 TURBIDITY

SD-100 6-Apr-1987 TURBIDITY

SD-100 12-May-1987 TURBIDITY

SD-100 19-May-1987 TURBIDITY

SD-100 26-May-1987 TURBIDITY

SD-100 3-Jun-1987 TURBIDITY

SD-100 11-Jun-1987 TURBIDITY

SD-100 18-Jun-1987 TURBIDITY
$<5$

$<\quad 4 \mathrm{gg} / \mathrm{I}$

$<5$

$<5$

9

5

5

5

$<$

$<5$

$<5$

$<5$

$<5$

$<5$

$<5$

$<5$

$<5$

$<5$

$<5$

$<5$

$<5$

5

$<5$

$<5$

$<5$

$<5$

$\mathrm{ug} / \mathrm{L}$

$\mathrm{ug} / \mathrm{L}$

NTU

NTU

NTU

NTU

NTU

NTU

NTU

NTY

NTU

NTU

NTU

NTU 
Storm Draln Data for SD-100

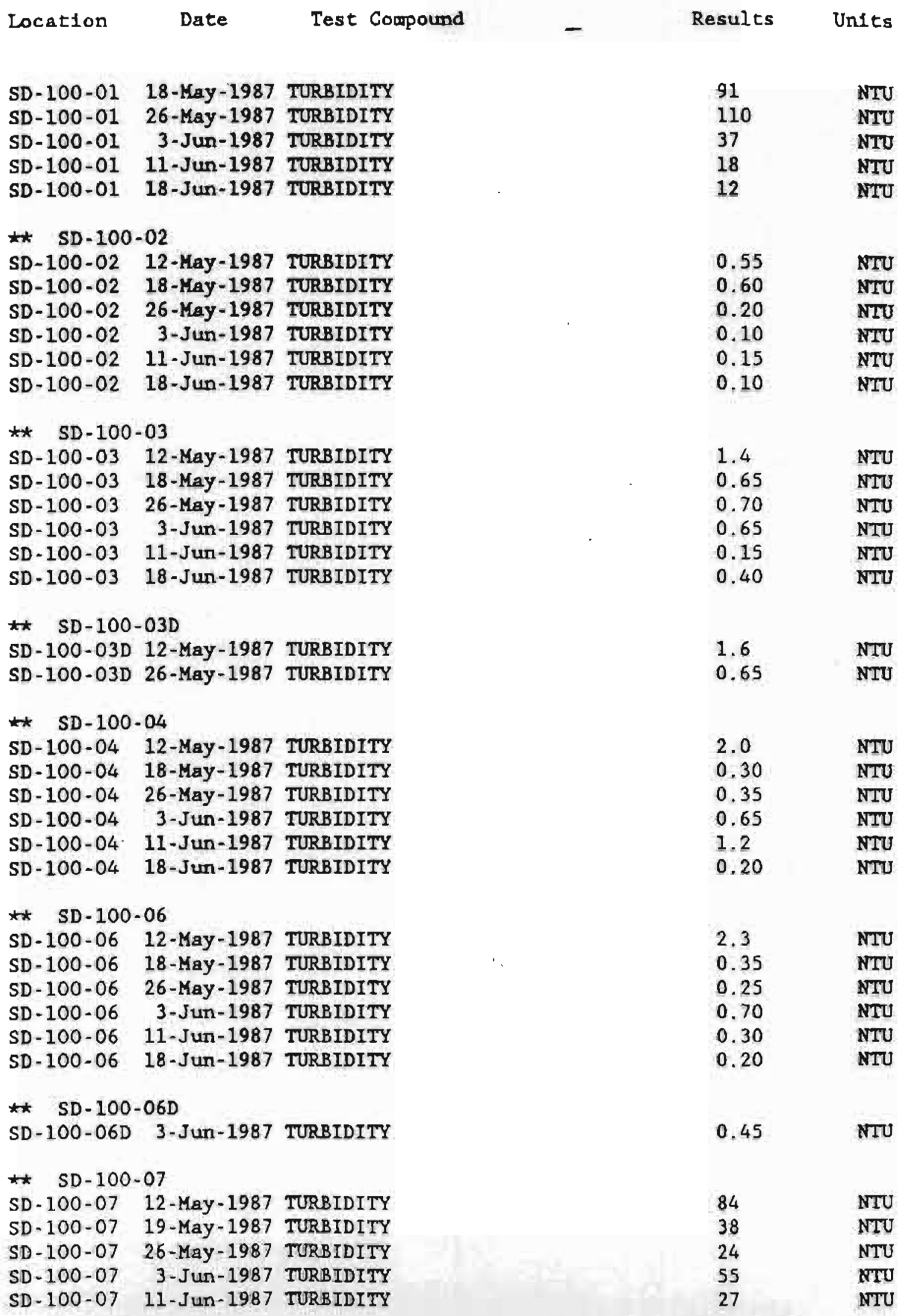


Storm Drain Data for SD-100

Location Date Test Compound

Results Units

SD-100-07 18-Jun-1987 TURBIDITY

33

NIU

* SD-100-07D

SD-100-07D 18-Jum-1987 TURBIDITY

36

NTU

* SD-100-09

SD-100-09 12-May-1987 TURBIDITY

9.0

0.20

NTU

SD-100-09 18-May-1987 TURBIDITY

0.90

NTU

SD-100-09 26-Yay-1987 TURBIDITY

10.2

NTU

SD-100-09 3-Jun-1987 TURBIDITY

0.70

NTU

SD-100-09 11-Jun-1987 TURBIDITY

SD-100-09 18-Jun-1987 TURBIDITY

$2.4 \quad$ NTU

** SD-100-10

SD-100-10 12-May-1987 TURBIDITY

SD-100-10 19-May-1987 TURBIDITY

SD-100-10 26-May-1987 TURBIDITY

SD-100-10 3-Jun-1987 TURBIDITY

SD-100-10 11-JUn-1987 TURBIDITY

SD-100-10 18-Jun-1987 TURBIDITY

$0.25 \quad$ NTU

$0.20 \quad$ NTU

0.15 NTU

1.0 NTU

$0.15 \quad$ NTU

0.15 NTU

** SD-100-11

SD-100-11 12-Kay-1987 TURBIDITY

0.85

0.70

SD-100-11 19-May-1987 TURBIDITY

6.7

SD-100-11 3-Jun-1987 TURBIDITY

SD-100-11 11-Jun-1987 TURBIDITY

1.0

5.0

0.70

NTU

NTU

SD-100-11 18-JUn-1987 TURBIDITY

$0.95 \quad$ NTU

* SD - 100-12

SD-100-12 12-May-1987 TURBIDITY

0.25

SD-100-12 19-May-1987 TURBIDITY

SD-100-12 26-May-1987 TURBIDITY

SD-100-12 3-Jun-1987 TURBIDITY

SD-100-12 11-Jun-19B7 TURBIDITY

SD-100-12 18-Jun-1987 TURBIDITY

0.55

0.25

2.1

1.9

NTU

NTU

NTU

NTU

** SD-100-13

SD-100-13 12-May-1987 TURBIDITY

0.60

0.70

0.90

SD-100-13 26-May-1987 TURBIDITY

SD-100-13 3-Jun-1987 TURBIDITY

SD-100-13 11-Jun-1987 TURBIDITY

0.50

0.40

SD-100-13 18-Jun-1987 TURBIDITY

1.7

NTU

NTU

NTU

NTU

FTU

** SD $-100-14$

SD-100-14 12-May-1987 TURBIDITY

SD-100-14 19-May-1987 TURBIDITY

SD-100-14 26-Kay-1987 TURBIDITY

SD-100-14 3-Jun-1987 TURBIDITY

SD-100-14 11-Jun-1987 TURBIDITY

$\begin{array}{ll}7.6 & \text { NTU } \\ 1.8 & \text { NTU } \\ 8.0 & \text { NTU } \\ 2.5 & \text { NTU } \\ 0.05 & \text { NTU }\end{array}$


** SD $-100-C$

SD-100-C 5-Mar-1987 TURBIDITY

SD-100-C 9-KaY-1987 TORBIDITY

SD-100-C 17-Kar-1987 TURBIDITY

SD-100-C 24-Mar-1987 TURBIDITY

SD-100-C 8-Apr-1987 TURBIDITY

SD-100-C 13-Kay-1987 TURBIDITY

SD-100-C 20-Mry-1987 TURBIDITY

SD-100-C 27-Kay-1987 TURBIDITY

SD-100-C 4-Jun-1987 TURBIDITY

SD-100-C 12-Jun-1987 TURBIDITY

SD-100-C 19-Jun-1987 TURBIDITY

** SD-100-D

SD-100-D 6-Apt-1987 TURBIDITY

SD-100-D 11-Jun-1987 TURBIDITY

** SD-100-C

SD-100-C 17-Mar-1987 UNKNOWN

$\mathrm{ug} / \mathrm{L}$

** SD-100-07

SD-100-07 12-May-1987 UNKNOWN ACIDS

** SD-100-C

SD-100-C 5-MaI-1987 UNKNOHN ALCOHOL

SD-100-C 9-Mar-1987 UNEAYWN ALCOHOL

SD-100-C 17-Mar-1987 UMNWOUN ALCOHOL

SD-100-C 8-ApT-1987 UNKNOWN ALCOHOL

SD-100-C 17-Mar-1987 UNKNOWN ALKYL/ALKOXY COMPOUND

$u g / L$

* $\quad$ SD $-100-11$

SD-100-I1 12-May-1987 UNKNOUN HEPTANE

$\mathrm{ug} / \mathrm{L}$

* $\quad$ SD-100-C

SD-100-C 19-JUn-1987 UNKNOWN HYDROCARBON

$\mathrm{ug} / \mathrm{L}$

** SD $-100-06$

SD-100-06 12-May-1987 UNKNNOWN KETONE

4g/L

** SD -100

SD-100 11-Mar-1987 UNKNOWNS

ug/ $/$

* SD-100-02

SD-100-02 12-May-1987 UNEAOWNS

ug/L

* SD-100-03

SD-100-03 12-May-1987 UNKNOWNS 


\begin{tabular}{|c|c|c|c|c|}
\hline Location & Date & Test Compound & Results & Units \\
\hline \multicolumn{5}{|c|}{$* \quad$ SD $-100-03 D$} \\
\hline SD-100-03D & 12-May-1987 & UNKNOWNS & 730 & $\operatorname{ug} / \mathrm{L}$ \\
\hline \multicolumn{5}{|c|}{ ** SD-100-04 } \\
\hline SD-100-04 & 12-May-1987 & UNKNOWNS & 470 & $\operatorname{ug} / \mathrm{I}$ \\
\hline \multicolumn{5}{|c|}{ ** SD-100-06 } \\
\hline SD-100-06 & $12-$ May- 1987 & UNKWOWNS & 64 & $\operatorname{Lg} / \mathrm{L}$ \\
\hline \multicolumn{5}{|c|}{$\star \star$ SD $-100-10$} \\
\hline$S D-100-10$ & 12-May-1987 & UNKWOWNS & 570 & $u g / 1$ \\
\hline \multicolumn{5}{|c|}{$\star \quad$ SD $-100-C$} \\
\hline$S D-100-C$ & 5-Mar- 1987 & UNRYYOWNS & 13 & $u g / L$ \\
\hline$S D-100-C$ & 8-Apr-1987 & UNKaNOWNS & 12 & $\mathrm{ug} / \mathrm{L}$ \\
\hline \multicolumn{5}{|l|}{ ** SD-100 } \\
\hline SD-100 & 5-Mar- 1987 & URANIUM & 0.004 & $\mathrm{mg} / \mathrm{L}$ \\
\hline SD- 100 & $11-\operatorname{Mar}-1987$ & URANIUM & 0.004 & $\mathrm{mg} / \mathrm{L}$ \\
\hline$S D-100$ & 18-Kar-1987 & URANIUM & 0.003 & $\mathrm{mg} / \mathrm{L}$ \\
\hline$S D-100$ & 24-Mar- 1987 & URANIUM & 0.004 & $\mathrm{mg} / \mathrm{L}$ \\
\hline SD- 100 & 3-Apr-1987 & URANIUM & 0.002 & $\mathrm{mg} / \mathrm{L}$ \\
\hline SD- 100 & 6-Apr-1987 & URANIUR & 0.002 & $\mathrm{mg} / \mathrm{L}$ \\
\hline SD-100 & 12-May-1987 & URANIUS & $<0.001$ & $\mathrm{mg} / \mathrm{L}$ \\
\hline$S D-100$ & $19-\mathrm{May}-1987$ & URANTUK & 0.002 & $\mathrm{mg} / \mathrm{L}$ \\
\hline SD- 100 & $26-\mathrm{May}-1987$ & URANIUY & $<0.001$ & $\operatorname{mg} / \mathrm{L}$ \\
\hline SD. 100 & 3-Jum-1987 & URAKIUM & 0.001 & $\mathrm{mg} / \mathrm{L}$ \\
\hline SD-100 & 11-Jun-1987 & URANIUK & 0.002 & $\mathrm{mg} / \mathrm{L}$ \\
\hline$S D-100$ & 18-Jun-1987 & URAKIUM & 0.026 & $\mathrm{mg} / \mathrm{L}$ \\
\hline
\end{tabular}

** SD-100-01

SD-100-01 12-MaY-1987 URANIUM

SD-100-01 18-May-1987 URAKIUM

SD-100-01 26-May-1987 URANIUM

SD-100-01 3-J un-1987 URANIUM

SD-100-01 11-Jun-1987 URANIUM

SD-100-01 18-Jun-1987 URANIUT

$\begin{array}{ll}<0.001 & \mathrm{mg} / \mathrm{L} \\ <0.001 & \mathrm{mg} / \mathrm{L} \\ <0.001 & \mathrm{mg} / \mathrm{L} \\ <0.001 & \mathrm{mg} / \mathrm{L} \\ 0.004 & \mathrm{mg} / \mathrm{L} \\ 0.002 & \mathrm{mg} / \mathrm{L}\end{array}$

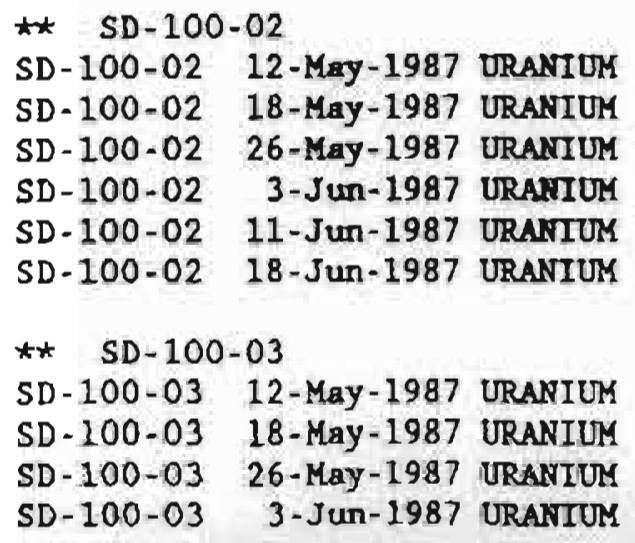

$\begin{array}{ll}<0.001 & \mathrm{mg} / \mathrm{L} \\ 0.001 & \mathrm{mg} / \mathrm{L} \\ 0.001 & \mathrm{mg} / \mathrm{L} \\ 0.001 & \mathrm{mg} / \mathrm{L}\end{array}$


Location Date Test Compound

Results Units

SD-100-03 11-Jun-1987 URANIUM

SD-100-03 18-Jun-1987 URANTUM

$0.002 \mathrm{mg} / \mathrm{L}$

$0.001 \mathrm{mg} / \mathrm{L}$

** SD-100-03D

SD-100-03D 12-May-1987 URARIOM

SD-100-03D 26-May-1987 URANIUM

$0.003 \quad \mathrm{mg} / \mathrm{L}$

$<0.001 \quad \mathrm{mg} / \mathrm{L}$

** SD-100-04

SD-100-04 12-May-1987 URANIUY

SD-100-04 18-May-1987 URAKIUT

SD-100-04 26-May-1987 URANIUT

SD-100-04 3-Jun-1987 URANIUM

SD-100-04 11-JUn-1987 URANIUM

SD-100-04 18-Jun-19B7 URANIUM

$<0.001 \quad \mathrm{mg} / \mathrm{L}$

$<0.001 \mathrm{mg} / \mathrm{L}$

$<0.001 \quad \mathrm{mg} / \mathrm{L}$

$0.003 \mathrm{mg} / \mathrm{L}$

$0.004 \quad \mathrm{mg} / \mathrm{L}$

$0.003 \quad \mathrm{mg} / \mathrm{L}$

* SD $100-06$

SD-100-06 12-May-1987 URANTUM

SD-100-06 18-May-1987 URANIUM

SD-100-06, 26-May-1987 URANIUM

SD-100-06 3-Jun-1987 URANIUM

SD-100-06 11-Jun-1987 URANIUM

SD-100-06 18-JUn-1987 URANIUM

$0.003 \mathrm{mg} / \mathrm{L}$

$<0.001 \mathrm{mg} / \mathrm{L}$

$<0.001 \quad \mathrm{mg} / \mathrm{L}$

$<0.001 \quad \mathrm{mg} / \mathrm{L}$

$0.002 \quad \mathrm{mg} / \mathrm{L}$

$0.002 \quad \mathrm{mg} / \mathrm{L}$

** SD-100-06D

SD-100-06D 3-Jun-1987 URANIUM

$<0.001 \quad \mathrm{mg} / \mathrm{L}$

** SD-100-07

SD-100-07 12-May-1987 URANTUM

SD-100.07 19-May-1987 URANIUM

SD-100-07 26-May-1987 URANIUM

SD-100-07 3-Jun-1987 URANIUM

SD-100-07 11-Jun-1987 URANIUM

SD-100-07 18-Jun-1987 URANIUM

$0.245 \mathrm{mg} / \mathrm{L}$

$0.092 \mathrm{mg} / \mathrm{L}$

$0.041 \mathrm{mg} / \mathrm{L}$

$.340 \mathrm{mg} / \mathrm{L}$

$0.006 \quad \mathrm{ng} / \mathrm{L}$

$0.050 \quad \mathrm{mg} / \mathrm{L}$

** SD $-100-07 D$

SD-100-07D 18-Jur-1987 URANIUM

$0.053 \mathrm{Eg} / \mathrm{L}$

* SD-100-09

SD-100-09 12-May-1987 URANIUM

SD-100-09 18-May-1987 URANIUM

SD-100-09 26-May-1987 URANIUM

SD-100-09 3-Jun-1987 URANTUM

SD-100-09 11-Jun-1987 URANIUM

SD-100-09 18-Jun-1987 URARIOM

$\begin{array}{ll}0.003 & \mathrm{mg} / \mathrm{L} \\ 0.005 & \mathrm{mg} / \mathrm{L} \\ 0.005 & \mathrm{mg} / \mathrm{L} \\ 0.012 & \mathrm{mg} / \mathrm{L} \\ 0.003 & \mathrm{mg} / \mathrm{L} \\ 0.003 & \mathrm{mg} / \mathrm{L}\end{array}$

* SD-100-10

SD-100-10 12-May-1987 URANIUTM

$<0.001 \quad \mathrm{mg} / \mathrm{L}$

SD-100-10 19-May-1987 URANIUT

$<0.001$

$\mathrm{ng} / \mathrm{L}$

SD-100-10 26-May-1987 URANIUT

$<0.001 \quad \mathrm{mg} / \mathrm{L}$

SD-100-10 3-Jun-1987 URANIUM

0.002

atg $/ \mathrm{L}$

SD-100-10 11-Jun-1987 URANTUY

0.002

mg/ $\mathrm{L}$ 
Location Date Test Compound Results UnIts

SD-100-10 18-Jun-1987 URANIUM

$0.003 \mathrm{mg} / \mathrm{L}$

* SD $100-11$

SD-100-11 12-Kay-1987 URANIUM

$<0.001 \quad \mathrm{ng} / \mathrm{L}$

SD-100-11 19-Kay-1987 URANIUM

$<0.001$

SD-100-11 26-Yay-1987 URANIUM

0.001

SD-100-11 3-Jun-1987 URANIUM

SD-100-11 11-Jun-1987 URANIUM

$<0.001$

0.002

SD-100-11 18-Jun-1987 URANIUM

0.004

$\mathbf{m g} / \mathrm{L}$

$\mathrm{mg} / \mathrm{L}$

$m g / L$

$\operatorname{mg} / \mathrm{L}$

** SD-100-12

SD-100-12 12-May-1987 URANIUM

SD-100-12 19-May-1987 URANIUM

SD-100-12 26-Kay-1987 URANIUM

SD-100-12 3-Jun-1987 URANIUM

SD-100-12 11-JUR-1987 URANIUM

SD-100-12 18-Jun-1987 URANIUM

$<0.001 \quad \operatorname{mg} / \mathrm{L}$

$<0.001 \quad \mathrm{mg} / \mathrm{L}$

$<0.001 \quad \mathrm{mg} / \mathrm{h}$

$0.001 \quad \mathrm{mg} / \mathrm{L}$

$0.002 \quad \mathrm{mg} / \mathrm{L}$

$0.003 \mathrm{mg} / \mathrm{L}$

** SD $-100-13$

SD-100-13 12-Kay-1987 URANIUM

SD-100-13 19-May-1987 URANIUM

SD-100-13 26-May-1987 URANIUM

SD-100-13 3-Jun-1987 URANIUN

SD-100-13 11-Jun-1987 URANIUM

SD-100-13 18-Jun-1987 URANIUM

$<0.001 \quad \mathrm{mg} / \mathrm{L}$

$<0.001 \quad \mathrm{mg} / \mathrm{L}$

$0.002 \quad \mathrm{mg} / \mathrm{L}$

$<0.001 \quad \mathrm{mg} / \mathrm{L}$

$0.003 \quad \mathrm{mg} / \mathrm{L}$

$0.005 \quad \mathrm{ag} / \mathrm{L}$

** SD - 100-14

SD-100-14 12-May-1987 URANIUM

SD-100-14 19-May-1987 URANIUM

SD-100-14 26-May-1987 URANIUM

SD-100-14 3-Jun-1987 URANIUM

SD-100-14 11-Jun-1987 URANIUM

SD-100-14 18-Jun-1987 URANIUM

$<0.001 \quad \mathrm{gg} / \mathrm{L}$

$0.002 \quad \mathrm{mg} / \mathrm{L}$

$0.001 \quad \mathrm{mg} / \mathrm{L}$

$<0.001 \quad \mathrm{mg} / \mathrm{L}$

$0.002 \quad \mathrm{mg} / \mathrm{L}$

$<0.001 \quad \mathrm{gg} / \mathrm{L}$

* SD-100-C

SD-100-C 5-KaT-1987 URANTUM

SD-100-C 9-Mer-1987 URANIUM

SD-100-C 17-Mar-1987 URANIUM

SD-100-C 24-Kar-1987 URANIUT

SD-100-C 8-ApT-1987 URANTUM

SD-100-C 13-May-1987 URAHIUM

SD-100-C 20-Kay-1987 URANIUM

SD-100-C 27-May-1987 URANIUM

SD-100-C 4-Jun-1987 URANIUM

SD-100-C 12-Jun-1987 URANIUM

SD-100-C 19-Jun-1987 URANIUM

$0.006 \quad \mathrm{mg} / \mathrm{L}$

$0.007 \quad \mathrm{mg} / \mathrm{L}$

$0.003 \quad \mathrm{gg} / \mathrm{L}$

$0.004 \quad \mathrm{ng} / \mathrm{L}$

$0.002 \quad \mathrm{gg} / \mathrm{L}$

$<0.001 \quad \mathrm{ng} / \mathrm{L}$

$<0.001 \quad \mathrm{mg} / \mathrm{L}$

$<0.001 \quad \mathrm{~g} / \mathrm{L}$

$<0.001 \quad \mathrm{gg} / \mathrm{L}$

$0.002 \quad \mathrm{mg} / \mathrm{L}$

$0.002 \quad \mathrm{mg} / \mathrm{L}$

** $S D-100-D$

SD-100-D 6-Apr-1987 URANIUM

$0.004 \quad \mathrm{~g} / \mathrm{L}$

SD-100-D 11-Jun-1987 URANIUM

$<0.001$

$\mathrm{ng} / \mathrm{L}$ 
Storm Drain Deta for SD-100

Location Date Test Compound

* SD-100

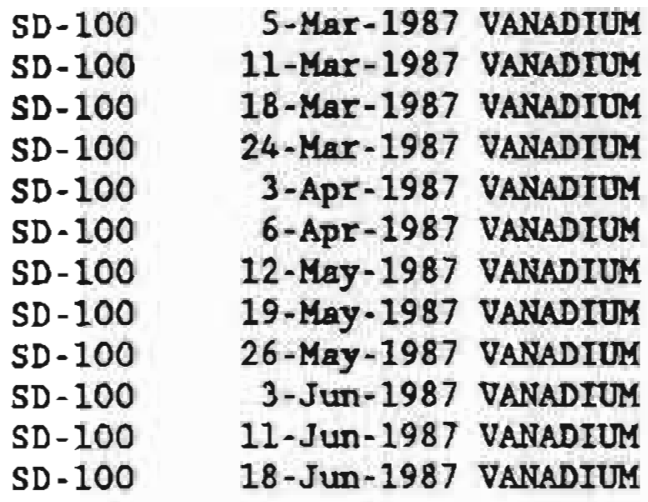

$<0.50$

$\mathrm{mg} / \mathrm{L}$

$<0.50$

$m g / L$

$<0.50$

$<0.50$

$<0.50$

$<0.50$

$<0.50$

$<0.50$

$<0.0050$

$<0.50$

$<0.50$

$<0.50$

$\mathrm{mg} / \mathrm{L}$

$\mathrm{mg} / \mathrm{L}$

mg $/ \mathrm{L}$

$\mathrm{mg} / \mathrm{L}$

$\operatorname{mg} / \mathrm{L}$

$\mathrm{mg} / \mathrm{L}$

$\mathrm{mg} / \mathrm{L}$

$\mathrm{mg} / \mathrm{L}$

$\mathrm{mg} / \mathrm{L}$

** SD-100-01

SD-100-01 12-May-1987 VANADIUM

SD-100-01 18-May-1987 VANADIUM

SD-100-01 3-Jun-1987 VANADIUM

SD-100-01 11-Jun-1987 VANADIUM

SD-100-01 18-Jun-1987 VANADIUM

$<0.50$

$<0.50$

$<0.50$

$<0.50$

$\mathrm{mg} / \mathrm{L}$

** SD $-100-02$

SD-100-02 12-May-1987 VANADIUM

SD-100-02 18-May-1987 VANADIUM

SD-100-02 3-Jun-1987 VANADIUM

SD-100-02 11-Jun-1987 VANADIUM

SD-100-02 18-Jun-1987 VANADTUM

$<0.50$

$<0.50$

$<0.50$

$<0.50$

$<0.50$

$<0.50$

$\mathrm{mg} / \mathrm{L}$

mg $/ \mathrm{L}$

mg $/ L$

$m g / L$

$\mathrm{mg} / \mathrm{L}$

** SD-100-03

SD-100-03 12-May-1987 VANADIUM

SD-100-03 18-May-1987 VANADIUM

SD-100-03 26-May-1987 VANADIUM

SD-100-03 3-Jun-1987 VANADIUM

SD-100-03 11-Jun-1987 VANADIUM

SD-100-03 18-Jun-1987 VANADIUM

$<0.50$

$<0.50$

$<0.50$

$<0.50$

$<0.50$

$<0.50$

$\mathrm{mg} / \mathrm{L}$

$\mathrm{mg} / \mathrm{L}$

$\mathrm{mg} / \mathrm{L}$

$\mathrm{mg} / \mathrm{L}$

$\mathrm{mg} / \mathrm{L}$

** SD-100-03D

SD-100-03D 12-May-1987 VANADIUI

SD-100-03D 26-May-1987 VANADIUT

$<0.50$

$<0.50$

$\mathrm{mg} / \mathrm{L}$

$\mathrm{mg} / \mathrm{L}$

$\mathrm{mg} / \mathrm{L}$

西g/L

mg/L

$\mathrm{mg} / \mathrm{L}$

* SD-100-04

SD-100-04 12-May-1987 VANADIUM

SD-100-04 18-May-1987 VANADIUM

SD-100-04 26-May-1987 VANADIUM

SD-100-04 3-Jun-1987 VANADIUM

SD-100-04 11-JUn-1987 VANADIUM

SD-100-04 18-Jun-1987 VANADIUM

$<0.50$

$<0.50$

$<0.50$

$<0.50$

$<0.50$

$\mathrm{mg} / \mathrm{L}$

$<0.50$

mg/L

*t SD - 100-06

SD-100.06 12-May-1987 VANADIUM

SD-100-06 18-May-1987 VANADIUM

$<0.50$

$<0.50$

mg/L

$\mathrm{mg} / \mathrm{L}$

$\mathbf{m g} / \mathrm{L}$

mg/L

ag/L

$\mathbf{m g} / \mathrm{L}$

agg/L

$\mathrm{mg} / \mathrm{L}$ 


Location Date Test Compound Results Units

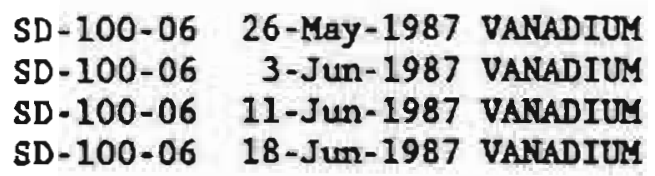

$\begin{array}{ll}<0.50 & \mathrm{mg} / \mathrm{L} \\ <0.50 & \mathrm{mg} / \mathrm{L} \\ <0.50 & \mathrm{mg} / \mathrm{L} \\ <0.50 & \mathrm{mg} / \mathrm{L} \\ & \\ <0.50 & \mathrm{mg} / \mathrm{L} \\ & \\ <0.50 & \mathrm{mg} / \mathrm{L} \\ <0.50 & \mathrm{mg} / \mathrm{L} \\ <0.50 & \mathrm{mg} / \mathrm{L} \\ <0.50 & \mathrm{mg} / \mathrm{L} \\ <0.50 & \mathrm{mg} / \mathrm{L} \\ <0.50 & \mathrm{mg} / \mathrm{L} \\ & \\ <0.50 & \mathrm{mg} / \mathrm{L} \\ & \\ <0.50 & \mathrm{mg} / \mathrm{L} \\ <0.50 & \mathrm{mg} / \mathrm{L} \\ <0.50 & \mathrm{mg} / \mathrm{L} \\ <0.50 & \mathrm{mg} / \mathrm{L} \\ <0.50 & \mathrm{mg} / \mathrm{L} \\ <0.50 & \mathrm{mg} / \mathrm{L} \\ <0.50 & \mathrm{mg} / \mathrm{L} \\ <0.50 & \mathrm{mg} / \mathrm{L} \\ <0.50 & \mathrm{mg} / \mathrm{L} / \mathrm{L} \\ <0.50 & \mathrm{mg} / \mathrm{m}\end{array}$


** SD-100-13

SD-100-13 12-May-1987 VANADIUM

SD-100-13 19-May-1987 VANADIUM

SD-100-13 26-May-1987 VANADIUM

SD-100-13 11-Jun-1987 VANADIUM

SD-100-13 18-Jun-1987 VANADIUM

* SD-100-14

SD-100-14 12-May-1987 VANADIOM

SD-100-14 19-May-1987 VANADIUM

SD-100-14 26-May-1987 VANADIUM

SD-100-14 3-Jun-1987 VANADIUM

SD-100-14 11-Jun-1987 VANADIUM

SD-100-14 18-Jun-1987 VANADIUM

** SD-100-C

SD-100-C 5-Mar-1987 VANADIUM

SD-100-C 9-Mar-1987 VANADIUM

SD-100-C 17-Mar-1987 VANADIUM

SD-100-C 24-Mar-1987 VANADIUM

SD-100-C 8-ApI-1987 VANADIUM

SD-100-C 13-Mzy-1987 VARADIUM

SD-100-C 20-May-1987 VANADIUM

SD-100-C 27-May-1987 VANADTUM

SD-100-C 4-Jun-1987 VANADIUM

SD-100-C 12-Jun-1987 VANADIUM

SD-100-C 19-Jun-1987 VANADTUM

* SD-100-D

SD-100-D 6-ApI-1987 VANADIUM

SD-100-D 11-Jun-1987 VANADIUM

** SD -100

SD -100

SD -100

5-Mar-1987 VINYL GHLORIDE

SD -100

SD -100

SD -100

SD -100

SD -100

SD -100

SD -100

SD -100

SD $\cdot 100$

11-Mar-1987 VINYL, CHLORIDE

18-Mar-1987 VIFY GHLORIDE

24-Mar-1987 VINYL CHLORIDE

6-Apr-1987 VINYL CHLORIDE

12-May-1987 VINYL CHLORIDE

19-May-1987 VINYL CHLORIDE

26-May-1987 VINYL CHLORIDE

3-Jun-1987 VINYL CHLORIDE

11-Jun-1987 VIKY CHLORIDE

18-Jur-1987 VINYZ CHLORIDE

*t SD-100-01

SD-100-01 12-May-1987 VINYL CHLORIDE

SD-100-01 18-May-1987 VINIL CHLORIDE

SD-100-01 26-May-1987 VINYL CHLORIDE

SD-100-01 3-Jun-1987 VINYL CHLORIDE

SD-100-01 11-Jun-1987 VINYL CHLORIDE

$\begin{array}{ll}<0.50 & \mathrm{mg} / \mathrm{L} \\ <0.0050 & \mathrm{mg} / \mathrm{L} \\ <0.0050 & \mathrm{mg} / \mathrm{L} \\ <0.50 & \mathrm{mg} / \mathrm{L} \\ <0.50 & \mathrm{mg} / \mathrm{L}\end{array}$

$<0.50$

$<0.50$

$\mathrm{mg} / \mathrm{L}$

$<0.0050$

$<0.50$

$<0.50$

$<0.50$

mg/ 1

$\operatorname{mg} / \mathrm{L}$

$\mathrm{mg} / \mathrm{L}$

$\mathrm{mg} / \mathrm{L}$

$\mathrm{mg} / \mathrm{L}$

$<0.50$

$<0.50$

$<0.50$

$<0.50$

$<0.50$

$<0.50$

$<0.50$

$<0.50$

$<0.50$

$<0.50$

$<0.50$

$\operatorname{mg} / \mathrm{L}$

ag $/ 1$

$\mathrm{mg} / \mathrm{L}$

mg $/ \mathrm{L}$

$\mathrm{mg} / \mathrm{L}$

$\mathrm{mg} / \mathrm{L}$

mg/L

$\mathrm{mg} / \mathrm{L}$

$\mathrm{mg} / \mathrm{L}$

$\mathbf{m g} / \mathrm{L}$

$\mathrm{mg} / \mathrm{L}$

$<0.50 \mathrm{mg} / \mathrm{L}$

$<0.50 \mathrm{mg} / \mathrm{L}$

$<10$

$<10$

$<10$

$<10$

$<10$

$<10$

$<10$

$<10$

$<10$

$<10$

$<10$

ug/L

ug/L

$u g / L$

$u g / L$

ug $/ 2$

ug/

ug $/ \mathrm{L}$

$u g / L$

ug/

ug $/ 2$

ug/L

$<10$

$<10$

$<10$

$<10$

$<10$ $\mathrm{ug} / \mathrm{L}$ $4 \mathrm{~g} / \mathrm{L}$ ug/L ug/L $u g / L$ 
Location Date Test Compound

Results Units

SD-100-01 18-Jun-1987 VINYL, CHLORIDE

$<10 \quad 48 / 2$

* SD-100-02

SD-100-02 12-May-1987 VINYL CHLORIDE

SD-100-02 18-May-1987 VINYL GHLORIDE

SD-100-02 26-May-1987 VINYL GHLORIDE

SD-100-02 3-Jun-1987 VIMY GHLORIDE

SD-100-02 11-Jun-1987 VINYL GHLORIDE

SD-100-02 18-Jun-1987 VINYL CHLORIDE

$<10$

$<10$

$<10$

$<10$

$<10$

40

ug $/ \mathrm{L}$

$\operatorname{ug} / \mathrm{L}$

$\mathrm{ug} / \mathrm{L}$

$\operatorname{Lg} / \mathrm{L}$

$\mathrm{ug} / \mathrm{L}$

$u g / L$

** SD-100-03

SD-100-03 12-May-1987 VINYL GHLORIDE

SD-100-03 18-May-1987 VINYL CHLORIDE

SD-100-03 26-May-1987 VINYL CHLORIDE

SD-100-03 3-Jun-1987 VINYL CHLORIDE

SD-100-03 11-Jun-1987 VIRYL CHLORIDE

SD-100-03 18-Jun-1987 VINYL GHLORIDE

* SD-100-03D

SD-100-03D 12-May-1987 VINYL CHLORIDE

SD-100-03D 26-May-1987 VINYL CHLORIDE

SD-100-04 18-JUn-1987 VINY GHIORTDE

* SD-100-06

SD-100-06 12-May-1987 VINYL CHLORIDE

SD-100-06 18-May-1987 VINYL GHLORIDE

SD-100-06 26-May-1987 VINYL CHLORIDE

SD-100-06 3-Jun-1987 VINYL CHLORIDE

SD-100-06 11-JUn-1987 VINYL CHLORIDE

SD-100-06 18-JWI-1987 VINYL CHLORIDE

\# SD-100-06D

SD-100-06D 3-Jun-1987 VINYL CHLORIDE

* SD $-100-07$

SD-100-07 12-May-1987 VINYL CHLORIDE

SD-100-07 19-May-1987 VINYZ CHLORIDE

SD-100-07 26-May-1987 VINYL CHLORIDE

SD-100-07 3-Jun-1987 VINYL CHLORIDE

SD-100-07 11-JUn-1987 VINYL CHLORIDE

SD-100-07 18-Jun-1987 VINYL CHLORIDE
$<10$

$<10$

$<10$

$<10$

$<10$

$<10$

$<10$

$<10$

$<10$

$<0$

$<10$

40

40

$<10$

ug/L

$\mathrm{ug} / \mathrm{L}$

$\mathrm{ug} / \mathrm{L}$

$u g / L$

$u g / L$

ug/L

ug $/ 2$

ug/L

ug/L

$u g / L$

$\mathrm{ug} / \mathrm{L}$

$\mathrm{ug} / \mathrm{L}$

ug/L

$\mathrm{ug} / \mathrm{L}$

$<10$

$<0$

$<10$

40

$<10$

$<0$

ug/L

ug/L

$u g / L$

ug/ $/$

$u g / L$

$\mathrm{ug} / \mathrm{L}$

$<10$

ug/L

$<10$

$<10$

$<10$

$<10$

$<10$

$<10$

ug $/$

ug $/ \mathrm{L}$

ug/L

ug/L

ug/L

ug/L

ug/L 
* SD-100-10

SD-100-10 12-May-1987 VINYL GHORIDE

SD-100-10 19-May-1987 VINYL CHLORIDE

SD-100-10 26-May-1987 VINYL CHLORIDE

SD-100-10 3-Jun-1987 VINYL CHLORIDE

SD-100-10 11-Jun-1987 VINYL CHLORIDE

SD-100-10 18-JUn-1987 VINYL CHLORIDE

SD-100-11 12-May-1987 VINYL CHLORIDE SD-100-11 19-May-1987 VINYL CHLORIDE SD-100-11 26-May-1987 VINY CHLORIDE SD-100-11 3-JUn-1987 VINYL CHLORIDE SD-100-11 11-JUn-1987 VINYL GHLORIDE SD-100-11 18-Jun-1987 VINYL GHLRIDE

** SD $-100 \cdot 12$

SD-100-12 12-May-1987 VINYL CHLORIDE SD-100-12 19-May-1987 VINYL CHLORIDE SD-100-12 26-May-1987 VINYL CHLORIDE SD-100-12 3-JUN-1987 VINYL GHLORIDE SD-200-12 11-Jun-1987 VINYL CHLORIDE SD-100-I2 18-Jun-1987 VINY CHLORIDE

\#* SD-100-13

SD-100-13 12-May-1987 VINYL CHLORIDE SD-100-13 19-May-1987 VINYL CHLORIDE SD-100-13 26-MaY-1987 VINYL CHLORIDE SD-100-13 3-Jun-1987 VINYL CHLORIDE SD-100-13 11-Jun-1987 VINYL CHLORIDE SD-100-13 18-Jun-1987 VINYL CHLORIDE
$<10$

$<10$

$<10$

$<10$

$<10$

$<10$

$<10$

$<10$

$<10$

$<10$

$<10$

$<10$

$<10$

$<10$

$<10$

$<10$

$<10$

$<10$ $\begin{array}{ll}\text { SD-100-14 } & 11-J \text { Un-1987 VINYL CHLORIDE } \\ \text { SD-100-14 } & 18 \text {-Jun-1987 VINYL CHLORIDE }\end{array}$
48/L ug $/ 2$ $\mathrm{ug} / \mathrm{L}$ ug/L $u g / L$ ug/L

ug/L ug/L ug/L ug/L $\mathrm{ug} / \mathrm{L}$ ug/L

ug/L ug/L $u g / L$ ug/L Ug/L ug/L

ug/L ug/L ug/L $u g / L$ ug/L $\mathrm{ug} / \mathrm{L}$

SD-100-14 12-May-1987 VINYL GHLRIDE SD-100-14 19-May-1987 VINY, CHLORIDE SD-100-14 26-May-1987 VINYL CHLORIDE SD-100-14 3-Jun-1987 VINYL CHLORIDE

*t $\quad S D-100-C$

SD-100-C 5-Mar-1987 VINYL CHLORIDE
$<10$

$<10$

$<10$

$<10$

$<10$

$<10$

$<10$

ug/L ug/L $\mathrm{ug} / \mathrm{L}$ $u g / L$ $u g / L$ ug/L

ug/L 
Storm Drain Data for SD-100

Locetion

Date

Test Compound

Results Units

SD-100-C 9-Mar-1987 VINYL CHLORIDE

SD-100-C 17-Mar-1987 VINY CHLORIDE

SD-100-C 24-Mar-1987 VINYL GHLORIDE

SD-100-G 8-APT-1987 VINYL CHLORIDE

SD-100-C 13-May-1987 VINY CHLORIDE

SD-100-C 20-May-1987 VINYL CHLORIDE

SD-100-C 27-May-1987 VINYL CHLORIDE

SD-100-C 12-Jun-1987 VINYL CHLORIDE

SD-100-C 19-Jun-1987 VINYL CHLORIDE

$<10$

$<10$

$<10$

$<10$

$<10$

$<10$

$<10$

$<10$

$<10$

ug/L

$4 \mathrm{~g} / \mathrm{L}$

$u g / L$

$u g / L$

$u g / L$

$u g / L$

$u g / L$

$u g / L$

$\mathrm{ug} / \mathrm{L}$

** SD-100-D

SD-100-D 6-Apr-1987 VINYL CHLORIDE

SD-100-D 11-Jun-1987 VINYL CHLORIDE

$<10$

$u g / L$

$<10$

ug/L

$\star \quad$ SD -100

SD $-100 \quad 5-\operatorname{Mar}-1987$ ZINC

SD-100 11-Mar-1987 2INC

SD-100 18-Mar-1987 ZINC

SD-100 24-Mar-1987 ZINC

SD-100 3-Apr-1987 ZINC

SD-100 6-Apr-1987 ZINC

SD-100 12-May-1987 2INC

SD-100 19-May-1987 ZINC

SD-100 26-May-1987 ZINC

SD-100 3-Jun-1987 ZINC

SD-100 11-Jun-1987 ZINC

SD-100 18-Jun-1987 2INC

$0.042 \mathrm{mg} / \mathrm{L}$

$0.034 \quad \mathrm{mg} / \mathrm{L}$

$0.036 \mathrm{mg} / \mathrm{I}$

$0.027 \quad \mathrm{mg} / \mathrm{L}$

$0.053 \mathrm{mg} / \mathrm{L}$

$0.020 \mathrm{mg} / \mathrm{L}$

$0.028 \mathrm{mg} / \mathrm{L}$

$0.029 \mathrm{mg} / \mathrm{L}$

$0.027 \mathrm{mg} / \mathrm{L}$

$0.024 \mathrm{mg} / \mathrm{L}$

$0.030 \mathrm{mg} / \mathrm{L}$

$0.038 \mathrm{mg} / \mathrm{L}$

** SD $-100-01$

SD-100-01 12-May-1987 ZINC

SD-100-01 18-May-1987 ZINC

SD-100-01 3-Jun-1987 ZINC

SD-100-01 11-Jun-1987 ZINC

SD-100-01 18-Jun-1987 ZINC

$<0.020 \quad \mathrm{gg} / \mathrm{L}$

$0.11 \mathrm{mg} / \mathrm{L}$

$0.042 \mathrm{mg} / \mathrm{L}$

$0.033 \quad \mathrm{mg} / \mathrm{L}$

$0.071 \mathrm{mg} / \mathrm{L}$

** SD-100-02

SD-100-02 12-May-1987 ZINC

SD-100-02 18-May-1987 2INC

SD-100-02 3-Jun-1987 ZINC

SD-100-02 11-Jun-1987 ZINC

SD-100-02 I8-Jun-1987 ZINC

$0.070 \mathrm{mg} / \mathrm{h}$

$0.061 \mathrm{mg} / \mathrm{L}$

$0.064 \mathrm{mg} / \mathrm{L}$

$0.076 \mathrm{mg} / \mathrm{L}$

$0.085 \mathrm{mg} / \mathrm{L}$

* SD-100-03

SD-100-03 12-May-1987 ZINC

SD-100-03 18-May-1987 ZINC

SD-100-03 26-May-1987 ZINC

SD-100-03 3-Jun-1987 ZINC

SD-100-03 11-Jun-1987 2INC

SD-100-03 18-Jun-1987 2INC

$0.021 \mathrm{mg} / \mathrm{L}$

$0.021 \mathrm{mg} / \mathrm{L}$

$0.027 \mathrm{mg} / \mathrm{L}$

$0.023 \mathrm{mg} / \mathrm{L}$

$0.027 \mathrm{mg} / \mathrm{L}$

$0.031 \mathrm{mg} / \mathrm{L}$ 
* SD-100-03D

SD-100-03D 12-May-1987 ZINC

SD-100-03D 26-May-1987 ZINC

$<0.020$

0.027

$\mathbf{n g} / \mathrm{L}$

* SD-100-04

SD-100-04 12-May-1987 ZINC

SD-100-04 18-May-1987 ZINC

SD-100-04 26-May-1987 ZINC

SD-100-04 3-Jum-1987 ZINC

SD-100-04 11-Jun-1987 ZINC

SD-100-04 18-Jun-1987 ZINC

0.041

0.037

0.032

0.079

0.033

0.048

$\mathrm{mg} / \mathrm{L}$

** SD - 100-06

SD-100-06 12-May-1987 ZINC

0.034

0.024

0.020

0.031

SD-100-06 26-May-1987 ZINC

SD-100-06 3-Jun-1987 ZINC

SD-100-06 11-Jun-1987 2INC

SD-100-06 18-Jun-1987 ZINC

$<0.020$

0.041

$\mathrm{mg} / \mathrm{L}$

mg/L

$\mathrm{ng} / \mathrm{L}$

$\mathrm{mg} / \mathrm{L}$

$m g / L$

要号/

** SD-100-06D

SD-100-06D 3-Jun-1987 ZINC

$0.024 \mathrm{mg} / \mathrm{L}$

* SD-100-07

SD-100-07 12-May-1987 ZINC

SD-100-07 19-May-1987 ZINC

SD-100-07 26-May-1987 ZINC

SD-100-07 3-Jun-1987 ZINC

SD-100-07 11-Jun-1987 ZINC

SD-100-07 18-Jua-1987 ZINC

0.11

0.14

0.50

0.15

0.69

$\mathrm{mg} / \mathrm{L}$

mg/L

mg/L

$\mathrm{mg} / \mathrm{L}$

$\mathbf{n g} / \mathrm{L}$

$\mathrm{mg} / \mathrm{L}$

* SD-100-07D

SD-100-07D 18-Jun-1987 ZINC

0.60

$\mathrm{mg} / \mathrm{L}$

* SD-100-09

SD-100-09 12-May-1987 2INC

SD-100-09 18-May-1987 2INC

SD-100-09 26-May-1987 ZINC

SD-100-09 3-Jun-1987 ZINC

SD-100-09 11-JUn-1987 ZINC

0.080

0.053

0.042

0.055

0.042

$\mathrm{mg} / \mathrm{L}$

$\mathbf{m g} / L$

$\mathrm{mg} / \mathrm{L}$

$\mathrm{mg} / \mathrm{L}$

的/L

$\mathrm{ng} / \mathrm{L}$

** SD-100-10

SD-100-10 12-May-1987 2INC

SD-100-10 19-May-1987 2INC

SD-100-10 26-May-1987 2INC

SD-100-10 3-Jun-1987 2INC

SD-100-10 11-Jun-1987 ZINC

SD-100-10 18-Jun-1987 2INC

$<0.020$

$<0.020$

$<0.020$

0.048

$<0.020$

$<0.020$

\section{mg $/ \mathrm{L}$ \\ $\mathbf{m g} / \mathrm{L}$ \\ $\mathbf{m g} / \mathrm{L}$ \\ $0 \Omega$ \\ ig/L}

* SD-100-11

SD-100-11 12-May-1987 ZINC

0.025

$\mathbf{m g} / \mathrm{L}$ 
Lacation Date Test Compound Results Units

SD-100-11 19-May-1987 ZINC

SD-100-11 26-May-1987 ZIXC

SD-100-11 3-Jun-1987 ZINC

SD-100-11 11-Jun-1987 ZINC

SD-100-11 18-Jun-1987 ZINC

* SD-100-12

SD-100-12 12-May-1987 ZINC

SD-100-12 19-May-1987 ZINC

SD-100-12 26-May-1987 ZINC

SD-100-12 3-Jun-1987 ZINC

SD-100-12 21-Jun-1987 ZINC

SD-100-12 18-Jun-1987 ZINC

** SD-100-13

SD-100-13 12-May-1987 ZINC

SD-100-13 19-May-1987 ZINC

SD-100-13 26-May-1987 ZINC

SD-100-13 $11-J$ un-1987 ZINC

SD-100-13 18-Jur-1987 ZINC

* SD-100-14

SD-100-14 12-May-1987 2INC

SD-100-14 19-May-1987 ZINC

SD-100-14 26-May-1987 ZINC

SD-100-14 3-Jun-1987 ZINC

SD-100-14 11-Jun-1987 ZINC

SD-100-14 18-Jun-1987 ZIAC

** SD-100-C

SD-100-C 5-Mar-1987 ZINC

SD-100-C 9-Mar-1987 ZINC

SD-100-C 17-Mar-1987 ZINC

SD-100-C 24-Mar-1987 ZINC

SD-100-C 8-Apr-1987 ZINC

SD-100-C 13-May-1987 ZINC

SD-100-C 20-May-1987 ZINC

SD-100-C 27-Hay-1987 ZINC

SD-100-C 4-Jun-1987 ZINC

SD-100-C 12-Jun-1987 ZINC

SD-100-C 19-Jun-1987 2INC

* $\quad S D-100-D$

SD-100-D 6-Apr-1987 ZINC

SD-100-D 11-Jun-1987 ZINC

$\star \star \quad S D-100$

SD-100 S-Mar-1987 ZIRCONIUM

SD-100 11-Mar-1987 2IRCONIUM

SD-100 18-Mar-1987 ZIRCONIUM

SD-100 24-Mar-1987 ZIRCONIUM
0.033

0.069

0.028

0.042

0.036

0.86

0.039

0.093

$<0.020$

0.043

0.076

$$
\begin{aligned}
& m g / L \\
& m g / L \\
& m g / L \\
& m g / L \\
& m g / L
\end{aligned}
$$

0.022

0.033

0.021

0.024

0.13

$$
\begin{aligned}
& \mathrm{mg} / \mathrm{L} \\
& \mathrm{mg} / \mathrm{L} \\
& \mathrm{mg} / \mathrm{L} \\
& \mathrm{mg} / \mathrm{L} \\
& \mathrm{mg} / \mathrm{L} \\
& \mathrm{mg} / \mathrm{L}
\end{aligned}
$$

0.10

0.092

0.11

0.040

0.036

0.049

$\mathrm{mg} / \mathrm{L}$
$\mathrm{mg} / \mathrm{L}$
$\mathrm{mg} / \mathrm{L}$
$\mathrm{mg} / \mathrm{L}$
$\mathrm{mg} / \mathrm{L}$

$\mathrm{mg} / \mathrm{L}$

$\mathrm{mg} / \mathrm{L}$

mg/L

$\mathrm{mg} / \mathrm{L}$

$\mathrm{mg} / \mathrm{L}$

$\mathrm{ng} / \mathrm{L}$

0.072

0.082

0.036

0.024

$<0.020$

0.022

0.020

$<0.020$

$<0.020$

$<0.020$

0.024

mg $/$

$\mathrm{mg} /$

mg/L

$\mathrm{mg} / \mathrm{L}$.

$\mathrm{mg} / \mathrm{L}$

mg/L

mg $\Omega$

$\mathrm{mg} / 2$

$\mathrm{ag} / \mathrm{L}$

$\mathrm{mg} / \mathrm{L}$

ag $/$

0.020

0.033

ng/L

$\mathrm{mg} / \mathrm{L}$

$<0.0050$

Rg/L

$<0.0050$

ㅁg $/$ L

$<0.0050$

$\mathrm{mg} / \mathrm{L}$

$<0.0050$ 
3-APT-1987 ZIRCONIUM

6-Apt-1987 ZIRCONTUM

12-May-1987 ZIRCONIUT

19-May-1987 ZIRCONIUM

26-May-1987 ZIRCONIUM

3-Jun-1987 ZIRCOHIUM

11-Jun-1987 ZIRCONIUM

18-Jun-1987 ZIRCOHIUM

** SD-100-01

SD-100-01 12-May-1987 ZIRCONTUH

SD-100-01 18-May-1987 ZIRCONIUM

SD-100-01 3-Jun-1987 ZIRCONTUM

SD-100-01 11-Jun-1987 ZIRCONIUM

SD-100-01 18-Jun-1987 ZIRCONIUM

* SD-100-02

SD-100-02 12-May-1987 ZIRCONTUM

SD-100-02 18-May-1987 ZIRCONIUM

SD-100-02 3-Jun-1987 ZIRCONIUM

SD-100-02 11-Jun-1987 ZIRCONIUH

SD-100-02 18-Jun-1987 2IRCONTUM

** SD-100-03

SD-100-03 12-May-1987 ZIRCONIUM

SD-100-03 18-May-1987 ZIRCONTUM

SD-100-03 26-May-1987 ZIRCONIUM

SD-100-03 3-Jun-1987 ZIRCONTUM

SD-100-03 11-JUn-1987 ZIRCONIUM

SD-100-03 18-Jun-1987 ZIRCONIUM

** SD-100-03D

SD-100-03D 12-May-1987 2IRCoNTUM

SD-100-03D 26-May-1987 ZIRCONIUM

* SD-100-04

SD-100-04 12-May-1987 ZIRCONTUY

SD-100-04 18-May-1987 ZIRCONTUM

SD-100-04 26-May-1987 ZIRCONIUM

SD-100-04 3-Jun-1987 ZIRCONIUM

SD-100-04 11-Jun-1987 ZIRCONIUH

SD-100-04 18-Jun-1987 ZIRCONIUH

** SD-100-06

SD-100-06 12-May-1987 ZIRCONIUM

SD-100-06 18-May-1987 ZIRCONTUR

SD-100-06 26-May-1987 ZIRCONIUM

SD-100-06 3-Jun-1987 ZIRCONIUM

SD-100-06 11-Jun-1987 ZIRCONIUM

SD-100-06 18-Jun-1987 ZIRCONIUM
$<0.0050$

$<0.0050$

$<0.0050$

$<0.0050$

$<0.0050$

$<0.0050$

$<0.0050$

$<0.0050$

$<0.0050$

$<0.0050$

$<0.0050$

$<0.0050$

$<0.0050$

$<0.0050$

$<0.0050$

$<0.0050$

$<0.0050$

$<0.0050$

$<0.0050$

$<0.0050$

$<0.0050$

$<0.0050$

$<0.0050$

$<0.0050$

$<0.0050$

$<0.0050$

$<0.0050$

$<0.0050$

$<0.0050$

$<0.0050$

$<0.0050$

$<0.0050$

$<0.0050$

$<0.0050$

$<0.0050$

$<0.0050$

$<0.0050$

$<0.0050$
$\mathrm{Eg} / \mathrm{L}$

$\mathrm{ng} / \mathrm{L}$

ge/ 1

ag/

zg $\Omega$

ag $/$

$-g /$

ngl $\mathrm{gg} / \mathrm{l}$
$\mathrm{gg} / \mathrm{L}$
$\mathrm{mg} / \mathrm{L}$
$\mathrm{ug} / \mathrm{L}$
$\mathrm{mg} / \mathrm{L}$
$\mathrm{mg} / \mathrm{L}$ ng/ 1

$\mathrm{ng} / \mathrm{L}$

$\operatorname{mg} / L$

$n g / L$

$\mathrm{Eg} / \mathrm{L}$

g $/ 2$

$\mathrm{ng} / \mathrm{L}$

$\mathrm{ng} / \mathrm{L}$

$n g / L$

$\operatorname{mg} / 2$

$\mathbf{m g} / \mathrm{L}$

$\mathrm{ng} / \mathrm{L}$

$\mathrm{mg} / \mathrm{L}$

$=\mathrm{g} / \mathrm{L}$

ng/ $/$

$\mathrm{ng} / \mathrm{L}$

$\mathrm{ng} / \mathrm{L}$

$\mathrm{g} / \mathrm{l}$ 
Location Date Test Compound Results Units

** SD-100-06D

SD-100-06D 3-Jun-1987 ZIRCONTUM

$<0.0050 \quad \mathrm{mg} / \mathrm{L}$

* SD-100-07

SD-100-07 12-May-1987 2IRCONTUM

SD-100-07 19-May-1987 ZIRCONTUM

SD-100-07 26-May-1987 ZIRCONIUM

SD-100-07 3-Jun-1987 ZTRCONTUM

SD-100-07 11-Jun-1987 ZIRCONIUA

SD-100-07 18-Jun-1987 2IRCONTUM

* SD-100-07D

SD-100-07D 18-Jun-1987 ZLRCONIOM

$<0.0050 \quad \mathrm{ng} / \mathrm{L}$

$0.0068 \quad \mathrm{ng} / \mathrm{L}$

$<0.0050 \mathrm{ng} / \mathrm{L}$

$0.0060 \mathrm{ng} / \mathrm{L}$

$<0.0050 \quad \mathrm{ng} / \mathrm{L}$

$<0.0050 \quad \mathrm{mg} / \mathrm{L}$

** SD $-100-09$

SD-100-09 12-May-1987 ZIRCONIUM

SD-100-09 18-May-1987 ZIRCONIUM

SD-100-09 26-May-1987 2IRCONIUM

SD-100-09 3-Jun-1987 ZIRCONIUM

SD-100-09 11-Jur-1987 ZIRCONIUM

$<0.0050 \quad \mathrm{ng} / \mathrm{L}$

$<0.0050 \quad \mathrm{mg} / \mathrm{L}$

$<0.0050 \mathrm{ng} / \mathrm{L}$

$<0.0050 \mathrm{mg} / \mathrm{L}$

$<0.0050 \mathrm{mg} / \mathrm{L}$

$<0.0050 \quad \mathrm{~g} / \mathrm{L}$

* SD- $100-10$

SD-100-10 12-May-1987 2IRCONIUM

SD-100-10 19-May-1987 ZIRCONIUM

SD-100-10 26-May-1987 ZIRCONIUM

SD-100-10 3-Jun-1987 ZIRCONIUM

SD-100-10 11-Jun-1987 2IRCONTUM

SD-100-10 18-Jun-1987 ZIRCONIUM

$<0.0050 \quad \mathrm{ng} / \mathrm{L}$

$<0.0050 \mathrm{mg} / \mathrm{L}$

$<0.0050 \quad \mathrm{gg} / \mathrm{L}$

$<0.0050 \mathrm{mg} / \mathrm{L}$

$<0.0050 \quad \mathrm{mg} / \mathrm{L}$

$<0.0050 \mathrm{ng} / \mathrm{L}$

* SD-100-11

SD-100-I1 12-May-1987 2IRCORIUM

SD-100-11 19-May-1987 ZIRCONIUM

SD-100-11 26-May-1987 ZIRCOIIUM

SD-100-11 3-Jun-1987 ZIRCONIOH

SD-100-11 11-Jun-1987 ZIRCONIUY

SD-100-11 18-Jun-1987 ZIRCOAIUM

$<0.0050 \quad \mathrm{ng} / \mathrm{L}$

$<0.0050 \quad \mathrm{mg} / \mathrm{L}$

$<0.0050 \quad \mathrm{gg} / \mathrm{L}$

$<0.0050 \quad \mathrm{mg} / \mathrm{h}$

$<0.0050 \quad \mathrm{ng} / \mathrm{L}$

$<0.0050 \mathrm{gg} / \mathrm{L}$

** SD-100-12

SD-100-12 12-May-1987 ZIRCONIUM

SD-100-12 19-May-1987 ZIRCONIUM

SD-100-12 26-May-1987 ZIRCORIUM

SD-100-12 3-Jun-1987 2IRCONTUM

SD-100-12 11-Jun-1987 ZIRCONIUM

SD-100-12 18-Jun-1987 ZIRCOHIUM

$<0.0050 \quad \mathrm{gg} / \mathrm{L}$

$0.0060 \quad \mathrm{gg} / \mathrm{L}$

$<0.0050 \mathrm{ng} / \mathrm{L}$

$<0.0050 \quad \mathrm{mg} / \mathrm{L}$

$<0.0050 \quad \mathrm{~g} / \mathrm{L}$

$<0.0050 \quad \mathrm{~g} / \mathrm{L}$

* SD-100-13

SD-100-13 12-May-1987 ZIRCONIUT

$<0.0050 \quad \mathrm{mg} / \mathrm{L}$

SD-100-13 19-May-1987 ZIRCONIUH

$<0.0050 \mathrm{mg} / \mathrm{L}$

SD-100-13 26-May-1987 ZIRCOHIUM

$<0.0050 \quad \mathrm{gg} / \mathrm{L}$

SD-100-13 11-3un-1987 ZIRCONIUM

$<0.0050 \quad \mathrm{gg} / \mathrm{L}$

SD-100-13 18-Jun-1987 2IRCONIUM

$<0.0050 \mathrm{mg} / \mathrm{L}$ 
** SD-100-14

SD-100-14 12-MaY-1987 ZIRCONIUM

SD-100-14 19-May-1987 2IRCONIUM

SD-100-14 26-May-1987 2IRCONIUM

SD-100-14 3-Jun-1987 2IRCONIUM

SD-100-14 11-Jun-1987 ZIRCONIUM

SD-100-14 18-Jun-1987 ZIRCONIUM

$\begin{array}{ll}<0.0050 & \mathrm{mg} / \mathrm{L} \\ <0.0050 & \mathrm{mg} / \mathrm{L} \\ <0.0050 & \mathrm{mg} / \mathrm{L} \\ <0.0050 & \mathrm{mg} / \mathrm{L} \\ <0.0050 & \mathrm{mg} / \mathrm{L} \\ <0.0050 & \mathrm{mg} / \mathrm{L}\end{array}$

$\star$ SD-100-C

SD-100-C 5-Mar-1987 ZIRCONIOM

SD-100-C . 9-Mar-1987 ZIRCONIUM

SD-100-C 17-Mar-1987 2IRCONIUM

SD-100-C 24-Mar-1987 ZIRCONIUI

SD-100-C 8-Apr-1987 ZIRCONIUM

SD-100-C 13-Kay-1987 2IRCONIUM

SD-100-C 20-May-1987 ZIRCONIUM

SD-100-C 27-May-1987 2IRCONIUM

SD-100-C 4-JUn-1987 ZIRCONIUM

SD-100-C 12-Jun-1987 ZIRCONYUM

SD-100-C 19-JUn-1987 ZIRCONIUM

$\begin{array}{ll}<0.0050 & \mathrm{mg} / \mathrm{L} \\ <0.0050 & \mathrm{mg} / \mathrm{L} \\ <0.0050 & \mathrm{mg} / \mathrm{L} \\ <0.0050 & \mathrm{mg} / \mathrm{L} \\ <0.0050 & \mathrm{mg} / \mathrm{L} \\ <0.0050 & \mathrm{mg} / \mathrm{L} \\ <0.0050 & \mathrm{mg} / \mathrm{L} \\ <0.0050 & \mathrm{mg} / \mathrm{L} \\ <0.0050 & \mathrm{mg} / \mathrm{L} \\ <0.0050 & \mathrm{mg} / \mathrm{L} \\ <0.0050 & \mathrm{mg} / \mathrm{L}\end{array}$


Storm Drain Data for SD-100-04

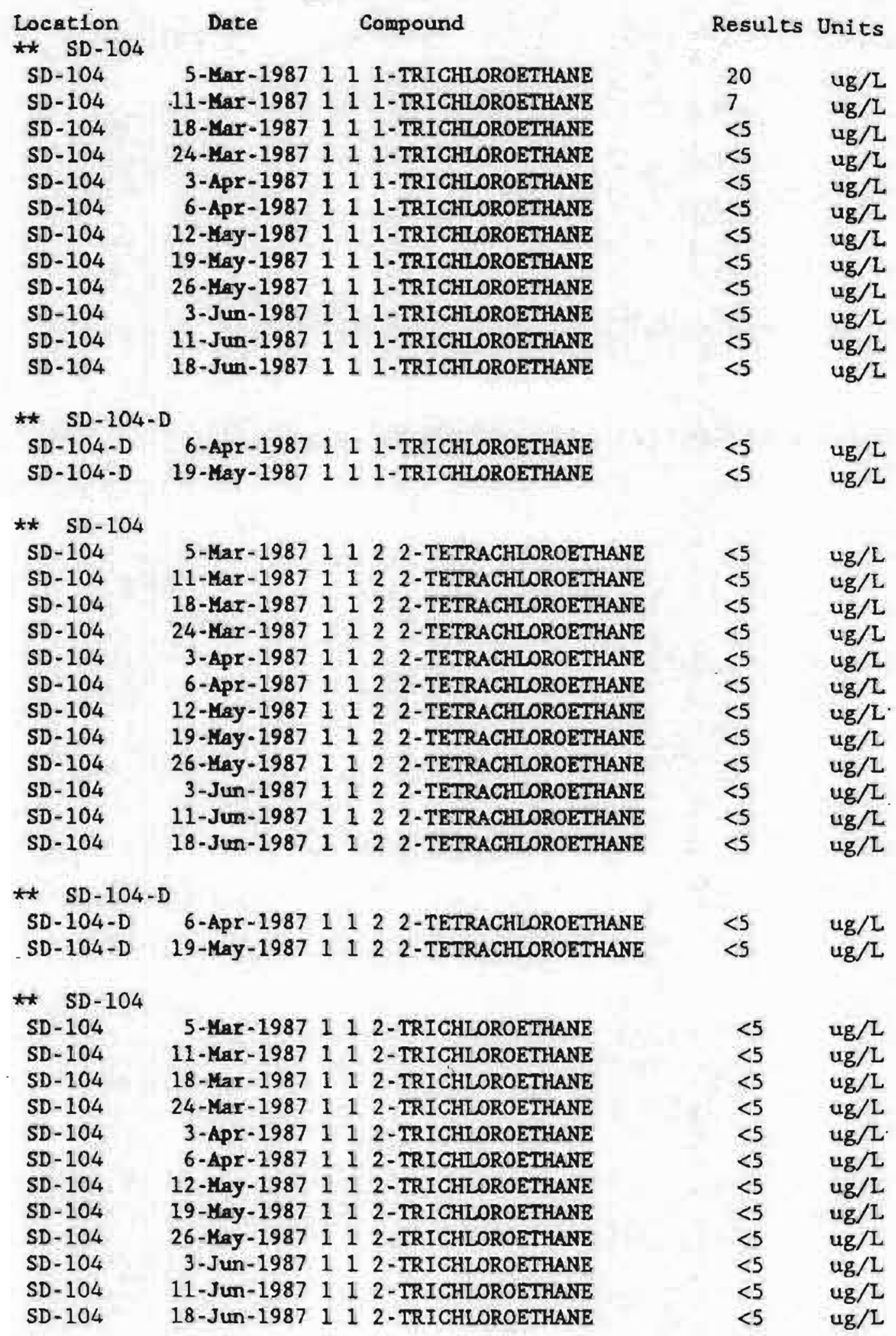


Storm Draln Data for SD-100-04

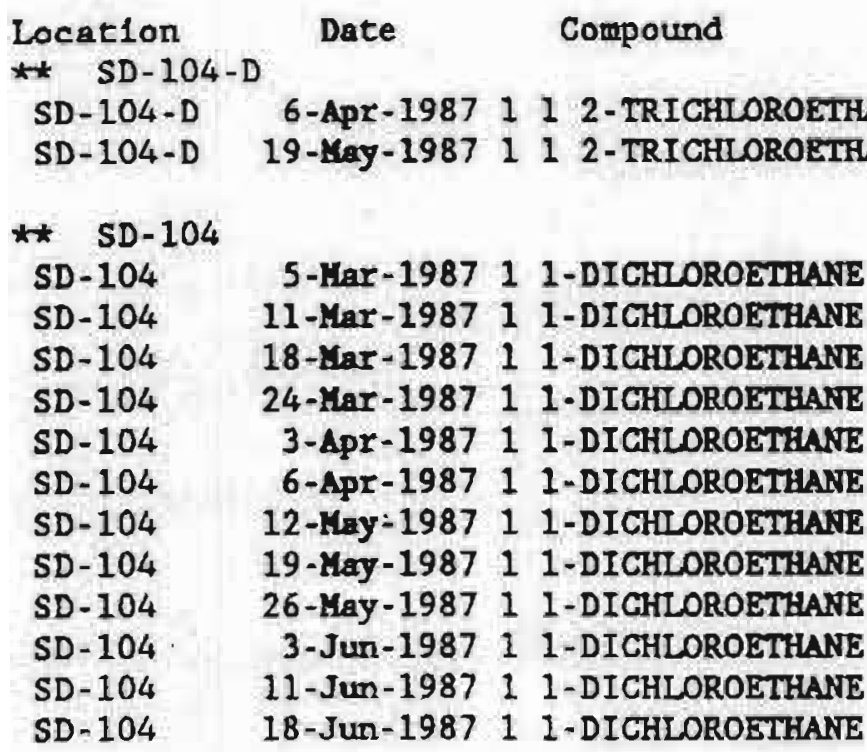

Results Units

$\begin{array}{ll}<5 & \text { ug } / \mathrm{L} \\ <5 & \mathrm{ug} / \mathrm{L}\end{array}$

$<5 \quad$ ug/L

$<5 . \quad \mathrm{ug} / \mathrm{L}$

$<5 \quad u g / L$

$<5 \quad u g / L$

$<5 \quad$ ug/L

$<5 \quad$ ug $/ \mathrm{L}$

$<5 \quad u g / L$

$<5 \quad$ ug $/ \mathrm{L}$

$<5 \quad u g / 2$

$<5 \quad 4 g /$

$<5 \quad$ ug/L

$<5 \quad u g / h$

** SD-104-D

SD-104-D 6-Apr-1987 1 1-DICHLOROETHANE

SD-104-D 19-Hay-1987 1 1-DICHLOROETHANE

$<5 \quad u g / 2$

$<5 \quad$ ug/L

** SD-104

SD-104

SD -104

5-Mar-1987 I 1-DICHLOROETHENE

SD-104

11-Mar-1987 1 1-DICHLOROETHENE

SD- 104

18-Mar-1987 1 1-DICHLOROETHENE

SD -104

24-Mar-1987 1 1-DICHLOROETHENE

SD -104

3-Apr-1987 1 1-DICHLOROETHENE

6-Apr-1987 1 1-DICHLOROETHENE

SD- 104

12-May-1987 1 1-DICHLOROETHENE

SD-104

19-May-1987 1 1-DICHLOROETHENE

SD-104

26-May-1987 1 1-DICHLOROETHENE

SD -104

3-Jun-1987 1 1-DICHLOROETHENE

SD- 104

11-Jun-1987 I 1-DICHLOROETHENE

SD-104

18-Jun-1987 1 1-DICHLOROETHENE

$<5$

$<5$

$<5$

$<5$

$<5$

$<5$

$<5$

$<5$

$<5$

$<5$

$<5$

$<5$

$$
\begin{aligned}
& u g / L \\
& u g / L \\
& u g / L \\
& u g / L \\
& u g / L \\
& u g / L \\
& u g / L \\
& u g / L \\
& u g / L \\
& u g / L \\
& u g / L \\
& u g / L
\end{aligned}
$$

** SD-104-D

SD-104-D

6-ApY-1987 1 1-DICHLOROETHENE

19-May-1987 I 1-DICHLOROETHEME

SD-104-D

* SD -104

SD -104

SD- 104

5-Mar-1987 12 4-TRICHZOROBENZENE

11-Mar-1987 12 4-TRICHLOROBENZENE

SD- 104

12-May-1987 12 4-TRICHLOROBENZENE

SD -104

26-May-1987 12 4-TRICHLOROBENZENE

SD -104

SD -104

5-Mar-1987 I 2-DICHLOROBENZENE

SD -104

11-Mar-1987 I 2-DICHLOROB ENZENE

SD -104

12-May-1987 1 2-DICHLOROBENZENE

SD -104

26-May-1987 1 2-DICHLOROBENZENE

5-MaY-1987 1 2-DICHLOROETHANE

SD -104

11-Mar-1987 I 2-DICHLOROETHANE

$<5$

ug $/$

$<5 \quad$ ug/

$<10 \quad \mathrm{ug} / \mathrm{h}$

$<12 \quad 4 g \Omega$

$<10$ ug/

$<5.0 \quad 48 / 2$

$<10 \quad$ ug $/ \mathrm{L}$

$<12 \quad \mathrm{ug} / \mathrm{L}$

$<10 \quad \mathrm{ug} / \mathrm{L}$

$<5.0 \quad \mathrm{ug} / \mathrm{L}$

$<5 \quad \mathrm{~kg} / \mathrm{L}$

$<5 \quad \mathrm{ug} / \mathrm{L}$ 
Storm Drain Data for SD-100-04

\begin{tabular}{|c|c|c|}
\hline Location & Date & Compound \\
\hline SD- 104 & 18-Mar-1987 & 1 2-DICHLOROETHA \\
\hline SD- 104 & 24-MaI- 1987 & 1 2-DICHLOROETHAN \\
\hline SD- 104 & 3-Apr-1987 & 1 2-DICHOROETHAM \\
\hline SD- 104 & 6-Apr-1987 & 1 2-DICHLOROETHA \\
\hline SD-104 & 12-May-1987 & 1 2-DICHLOROETH \\
\hline SD- 104 & 19-Мay-1987 & 1 2-DICHLOROETH \\
\hline SD- 104 & 26-May-1987 & 1 2-DICHLOROETH \\
\hline SD-104 & $3-J u n-1987$ & 2-DICHLOROETHA \\
\hline SD- 104 & $11-J u n-1987$ & 2-DICHLOROETHA \\
\hline SD- 104 & $18-J u n-1987$ & 2-DICHLOROETHA \\
\hline
\end{tabular}

Results Units

$<5$

$<5 \quad$ ug/L

<5 $\quad 4 g / 2$

<5 ug $/ 2$

$<5$ ug/

$<5 \quad$ ug/L

$<5 \quad 4 \mathrm{~g} / \mathrm{L}$

$<5 \quad 4 g / L$

$<5 \quad \mathrm{ug} / \mathrm{L}$

$<5$ ug/L

** SD-104-D

SD-104-D 6-ApX-1987 I 2-DICHLOROETHANE

SD-104-D 19-May-1987 1 2-DICHLOROETHANE

* SD -104
SD- 104

SD -104

SD- 104

SD-104

SD- 104

SD- 104

SD-104

SD- 104

SD- 104

SD- 104

SD-104

SD-104

5-MaX-1987 I 2-DICHLOROPROPANE 11-Mar-1987 1 2-DICHLOROPROPANE 18-Mar-1987 1 2-DICHLOROPROPANE 24-Mar-1987 1 2-DICHLOROPROPANE 3-Apr-1987 1:2-DICHLOROPROPANE

6-APT-1987 I 2-DICKLOROPROPANE 12-May-1987 1 2-DICHLOROPROPANE 19-May-1987 1 2-DICHLOROPROPANE 26-May-1987 1 2-DICHLOROPROPANE 3-Jun-1987 1 2-DICHLOROPROPANE 11-Jun-1987 I 2-DIGHOROPROPANE 18-Jun-1987 I 2-DICHLOROPROPANE

* SD-104-D

SD-104-D 6-Apr-1987 1 2-DICHLOROPROPANE SD-104-D 19-May-1987 \& 2-DICHLOROPROPANE

* SD-104

SD- 104

SD - 104

SD-104

SD -104

SD -104

SD -104

SD-104

SD-104

SD-104

SD- 104

$S D-104$

SD -104

SD -104

SD-104

SD- 104

SD-104
5-Mar-1987 1 3-DICHLOROBENZENE

11-Mar-1987 1 3-DICHLOROBENZENE

12-Kay-1987 1 3-DICHLOROBENZENE

26-Kay-1987 I 3-DICHLOROBENZENE

5-MaY-1987 1 4-DICHLOROBENZENE

11-KaI-1987 2 4-DICHLOROBENZENE

12-May-1987 1 4-DICHLOROBEMZEREE

26-May-1987 1 4-DICHLOROBENZDNE

5-Mar-1987 24 6-TRICHLOROPHENOL

11-KaI-1987 24 6-TRICHLOROPHENOL

12-May-1987 24 6-TRICHLOROPHENOL

26-May-1987 2 4 6-TRICHLOROPHENOL

5-Mar-1987 2 4-DICHLOROPHENOL

11-Mar-1987 2 4-DICHLOROPHENOL

12-Hay-1987 2 4-DICHLOROPHENOL

26-May-1987 2 4-DICHLOROPHENOL
$<5$

$<5$

ug/L

$\mathrm{ug} / \mathrm{L}$

$<5$

$<5$

$<5$

$<5$

$<5$

$<5$

$<5$

$<5$

$<5$

$<5$

$<5$

$<5$

$<5$

$<5$

ug/L

ug/L

$\begin{array}{ll}<10 & \mathrm{ug} / L \\ <12 & \mathrm{ug} / L \\ <10 & \mathrm{ug} / \mathrm{L} \\ <5.0 & \mathrm{ug} / \mathrm{L} \\ <10 & \mathrm{ug} / \mathrm{L} \\ <12 & \mathrm{ug} / \mathrm{L} \\ <10 & \mathrm{ug} / \mathrm{L} \\ <5.0 & \mathrm{ug} / \mathrm{L} \\ <10 & \mathrm{ug} / \mathrm{L} \\ <12 & \mathrm{ug} / \mathrm{L} \\ <10 & \mathrm{ug} / \mathrm{L} \\ <5.0 & \mathrm{ug} / \mathrm{L} \\ <10 & \mathrm{ug} / \mathrm{L} \\ <12 & \mathrm{ug} / \mathrm{L} \\ <10 & \mathrm{ug} / \mathrm{L} \\ <5.0 & \mathrm{ug} / \mathrm{L}\end{array}$


Stora Drain Data for SD-100-04

\section{Location}

SD-104

SD-104

SD-104

SD-104

SD-104

SD-104

SD-104

SD-104

SD- 104

SD-104

SD-104

SD-104

SD-104

SD- 104

SD-104

SD- 104

SD-104

SD-104

SD-104

SD-104

SD-104

SD-104

SD-104

SD-104

SD-104

SD-104

SD-104

SD-104
Date

Compound

5-Max-1987 2 4-DIMETHYLPHENOL

11-Mar-1987 2 4-DIMETHYLPHEROL

12-May-1987 2 4-DIMETHYLPHEYOL

26-May-1987 2 4-DIMETHYLPLEROLL

5-Mar-1987 2 4-DINITROPHETOL

11-Kar-1987 2 4-DIKITROPHEHOL

12-May-1987 2 4-DINITROPHEXOL

26-May-1987 2 4-DINITROPHEHOL

5-Mar-1987 2 4-DINITROTOLUERE

11-Kar-1987 2 4-DINITROTOLUENE

12-Kay-1987 2 4-DINITROTOLUERE

26-May-1987 2 4-DINITROTOLUERE

5-MaI-1987 2 6-DINITROTOLUENE

11-Kar-1987 2 6-DINITROTOLUERE

12-May-1987 2 6-DINITROTOLUENB

26-May-1987 2 6-DINITROTOUUETE

5-Mar-1987 2-CHLOROETHYLVINY ETHER

11-KaY-1987 2-CHLOROETHYLVINYL ETHER

18-Yar-1987 2-CHLOROETHYLVIMY ETHER

24-Mar-1987 2-CHLOROETHYLVIMY ETHER

3-Apr-1987 2-CHLOROETHYLVINYL ETHER

6-ApI-1987 2-CHLOROETHYLVINYL ETHER

12-May-1987 2-CHLOROETHYLINYL ETHER 19-May-1987 2-CHLOROETHYLVINYL ETHER

26-Mry-1987 2-CHLOROETHYLUIMY ETHER

3-Jun-1987 2-CHLOROETHYLVINYL ETHER

11-Jun-1987 2-CHLOROETHYLVIML ETHER

18-Jun-1987 2-CHLOROETHYLIML ETHER

* SD-104-D

SD-104-D 6-Apr-1987 2-CHLOROETHYLVINY ETHER

SD-104-D 19-May-1987 2-CHLOROETHYLVINYL ETHER

$\star \star \quad S D-104$

SD-104

SD -104

SD-104

SD-104

SD-104

SD -104

SD -104

SD-104

SD -104

SD-104

SD-104

SD- 104

SD -104

SD-104

SD-104

SD-104

5-Ma5-1987 2-CKLORONAPHTHALFNE

11-Mar-1987 2-CHLORONAPHTHALENE

12-May-1987 2-CHLORONAPHTHALGRE

26-Kay-1987 2-CHLORONAPHTHAL ERE

5-Mar-1987 2-CHLOROPHENOL

11-Mar-1987 2-CHLOROPHENOL

12-May-1987 2-CHLOROPHENOL

26-May-1987 2-CHLOROPHENOL

5-MaI-1987 2-NITROPHENOL

11-Mar-1987 2-NITROPHENOL

12-May-1987 2-NITROPHENOL

26-May-1987 2-NITROPHENOL

5-Mar-1987 3 3'-DICHLOROBEAZIDINE

11-Mar-1987 $33^{\prime}$-DICHLOROBENZIDINE

12-May-1987 3 3'-DICHLOROBENZIDINE

26-May-1987 3 3'-DICHLOROBENZIDINE
Results Onits

$\begin{array}{ll}<10 & \mathrm{ug} / \mathrm{L} \\ <12 & \mathrm{ug} / \mathrm{L} \\ <10 & \mathrm{ug} / \mathrm{L} \\ <5.0 & \mathrm{ug} / \mathrm{L} \\ <10 & \mathrm{ug} / \mathrm{L} \\ <12 & \mathrm{ug} / \mathrm{L} \\ <10 & \mathrm{ug} / \mathrm{L} \\ <25.0 & \mathrm{ug} / \mathrm{L} \\ <10 & \mathrm{ug} / \mathrm{L} \\ <12 & \mathrm{ug} / \mathrm{L} \\ <10 & \mathrm{ug} / \mathrm{L} \\ <5.0 & \mathrm{ug} / \mathrm{L} \\ <10 & \mathrm{ug} / \mathrm{L} \\ <12 & \mathrm{ug} / \mathrm{L} \\ <10 & \mathrm{ug} / \mathrm{L} \\ <5.0 & \mathrm{ug} / \mathrm{L} \\ <10 & \mathrm{ug} / \mathrm{L} \\ <10 & \mathrm{ug} / \mathrm{L} \\ <10 & \mathrm{ug} / \mathrm{L} \\ <10 & \mathrm{ug} / \mathrm{L} \\ <10 & \mathrm{ug} / \mathrm{L} \\ <10 & \mathrm{ug} / \mathrm{L} \\ <10 & \mathrm{ug} / \mathrm{L} \\ <10 & \mathrm{ug} / \mathrm{L} \\ <10 & \mathrm{ug} / \mathrm{L} \\ <10 & \mathrm{ug} / \mathrm{L} \\ <10 & \mathrm{ug} / \mathrm{L} \\ <10 & \mathrm{ug} / \mathrm{L} \\ <10 & \end{array}$

$<10 \quad u g / L$

$<10 \mathrm{ug} / \mathrm{L}$

$\begin{array}{ll}<10 & \mathrm{ug} / \mathrm{L} \\ <12 & \mathrm{ug} / \mathrm{L} \\ <10 & \mathrm{ug} / \mathrm{L} \\ <5.0 & \mathrm{ug} / \mathrm{L} \\ <10 & \mathrm{ug} / \mathrm{L} \\ <12 & \mathrm{ug} / \mathrm{L} \\ <10 & \mathrm{ug} / \mathrm{L} \\ <5.0 & \mathrm{ug} / \mathrm{L} \\ <10 & \mathrm{ug} / \mathrm{L} \\ <12 & \mathrm{ug} / \mathrm{L} \\ <10 & \mathrm{ug} / \mathrm{L} \\ <5.0 & \mathrm{ug} / \mathrm{L} \\ <20 & \mathrm{ug} / \mathrm{L} \\ <24 & \mathrm{ug} / \mathrm{L} \\ <20 & \mathrm{ug} / \mathrm{L} \\ <10.0 & \mathrm{ug} / \mathrm{L}\end{array}$


Storm Draln Data for SD-100-04

Location

$S D-104$

SD -104

SD -104

SD. 104

SD-104

SD-104

SD-104

SD-104

SD-104

SD- 104

SD- 104

SD- 104

SD -104

SD -104

SD- 104

SD- 104

SD- 104

SD-104

SD- 104

SD -104

SD -104

SD -104

SD -104

SD- 104

SD -104

SD-104

SD -104

SD -104

SD -104

SD-104

SD -104

SD -104

SD-104

SD-104

SD-104

SD-104

SD- 104

SD-104

SD -104

SD-104
Date

Compound

5-Mar-1987 4 6-DINITRO-2-METHYLPHENOL 11-Mar-1987 4 6-DINITRO-2-KETHYLPHENOL 12-May-1987 4 6-DINITRO-2-METHYLPHENOL 26-May-1987 4 6-DINTTRO-2-KETHYLPHENOL

5-Mar-1987 4-BROHOPHENYL-PHENYLETHER

11-Mar-1987 4-BROYOPHENT-PHENYLETHER

12-May-1987 4-BROMOPHRTYL-PHENYLETHER

26-MaY-1987 4-BROMOPHENYL-PAENYLETHER

5-MaI-1987 4-CHLORO-3-METHYLPHENOL

11-Mar-1987 4-CHLORO-3-KETHYLPHENOL

12-May-1987 4-CHLORO-3-BETHY.PHENOL

26-Mzy-1987 4-CHLORO-3-METHYLPHENOL

5-Mar-1987 4-CHLOROPHENYL-PHENYLETHER

11-Kar-1987 4-CHLOROPHENYL-PHENYLETHER

12-May-1987 4-CHLOROPHEWY-PHENYLETHER

26-May-1987 4-CHLOROPHENYL-PHENYLETHER

5-Mar-1987 4-NITROPHENOL

11-Mar-1987 4-NITROPHENOL

12-May-1987 4-NITROPHENOL

26-May-1987 4-NITROPHEROL

5-Mar-1987 ACENAPHTHENE

11-Mar-1987 ACENAPHTHEWTE

12-May-1987 ACENAPHTHENE

26-May-1987 ACENAPHTHEANE

5-MaY-1987 ACENAPHTHYLENE

11-Mar-1987 ACENAPHTHYLINE

12-May-1987 ACENAPHTHYLENE

26-May-1987 ACENAPHTHYIDNE

5-Mar-1987 ALKALINITY

11-Mar-1987 AIKAIINITY

18-Mar-1987 ALKALINITY

24-Mar-1987 ALKALINITY

3-Apr-1987 ALKALINITY

6-Apr-1987 AIKALINITY

12-May-1987 ALKALINITY

19-May-1987 ALKALINITY

26-May-1987 ALKALINITY

3-Jun-1987 ALRALINITY

11-Jun-1987 ALKALINITY

18-Jun-1987 AIKALINITY

* SD-104-D

SD-104-D 6-Apr-1987 ALKALINITY

SD-104-D 19-Kay-1987 ALKALINITY

* SD-104
SD-104
SD-104
SD-104
SD-104

5-Mar-1987 ALPHA

11- $\mathrm{Mar}-1987$ ALPHA

18-Mar-1987 ALPHA

24-Mar-1987 ALPHA

Results Units

$\begin{array}{ll}<50 & \mathrm{ug} / \mathrm{L} \\ <59 & \mathrm{ug} / \mathrm{L} \\ <50 & \mathrm{ug} / \mathrm{L} \\ <25.0 & \mathrm{ug} / \mathrm{L} \\ <10 & \mathrm{ug} / \mathrm{L} \\ <12 & \mathrm{ug} / \mathrm{L} \\ <10 & \mathrm{ug} / \mathrm{L} \\ <5.0 & \mathrm{ug} / \mathrm{L} \\ <10 & \mathrm{ug} / \mathrm{L} \\ <12 & \mathrm{ug} / \mathrm{L} \\ <10 & \mathrm{ug} / \mathrm{L} \\ <5.0 & \mathrm{ug} / \mathrm{L} \\ <10 & \mathrm{ug} / \mathrm{L} \\ <12 & \mathrm{ug} / \mathrm{L} \\ <10 & \mathrm{ug} / \mathrm{L} \\ <5.0 & \mathrm{ug} / \mathrm{L} \\ <50 & \mathrm{ug} / \mathrm{L} \\ <59 & \mathrm{ug} / \mathrm{L} \\ <50 & \mathrm{ug} / \mathrm{L} \\ <25.0 & \mathrm{ug} / \mathrm{L} \\ <10 & \mathrm{ug} / \mathrm{L} \\ <12 & \mathrm{ug} / \mathrm{L} \\ <10 & \mathrm{ug} / \mathrm{L} \\ <5.0 & \mathrm{ug} / \mathrm{L} \\ <10 & \mathrm{ug} / \mathrm{L} \\ <12 & \mathrm{ug} / \mathrm{L} \\ <10 & \mathrm{ug} / \mathrm{L} \\ <5.0 & \mathrm{ug} / \mathrm{L} \\ 88 & \mathrm{mg} / \mathrm{L} \\ 69 & \mathrm{mg} / \mathrm{L} \\ 98 & \mathrm{mg} / \mathrm{L} \\ 100 & \mathrm{mg} / \mathrm{L} \\ 76 & \mathrm{mg} / \mathrm{L} \\ 90 & \mathrm{mg} / \mathrm{L} \\ 98 & \mathrm{mg} / \mathrm{L} \\ 101 & \mathrm{mg} / \mathrm{L} \\ 100 & \mathrm{mg} / \mathrm{L} \\ 102 & \mathrm{mg} / \mathrm{L} \\ 99 & \mathrm{mg} / \mathrm{L} \\ 96 & \mathrm{mg} / \mathrm{L} \\ & \\ <5 & \end{array}$

90

101

$\mathrm{mg} / \mathrm{L}$

$\mathrm{mg} / \mathrm{L}$

$\begin{array}{ll}<2 & \mathrm{pCi} / \mathrm{L} \\ 2.1 & \mathrm{pCi} / \mathrm{L} \\ <2 & \mathrm{pCi} / \mathrm{L} \\ 3.3 & \mathrm{pCi} / \mathrm{L}\end{array}$


Storn Drafn Date for SD-100-04

\begin{tabular}{|c|c|c|c|c|}
\hline Location & Date & Compound & Results & Units \\
\hline SD-104 & 3-Apr-1987 & ALPHA & 65.7 & $\mathrm{PC} i / L$ \\
\hline SD-104 & 6-Apr-1987 & ALPKA & 2.2 & $\mathrm{pCl} / \mathrm{L}$ \\
\hline SD. 104 & $12-$ Kay-1987 & ALPHA & 2 & $\mathrm{pCl} / \mathrm{L}$ \\
\hline SD- 104 & 19- -Lay-1987 & ALPHA & $<2$ & $\mathrm{pC1} / \mathrm{L}$ \\
\hline SD-104 & 26-Мау-1987 & ALPHA & $<2$ & $\mathrm{pCl} / \mathrm{L}$ \\
\hline SD-104 & 3-Jun-1987 & ALPHA & 13.2 & $\mathrm{pCi} / \mathrm{L}$ \\
\hline SD-104 & $11-J u n-1987$ & ALPHA & $<$ & $\mathrm{pCi} / \mathrm{L}$ \\
\hline SD- 104 & $18-J$ un- 1987 & ALPHA & 1.9 & $\mathrm{pCl} / \mathrm{L}$ \\
\hline *t SD 10 & & & & \\
\hline SD-104-D & 6-Apr $=1987$ & ALPHA & 2.2 & $\mathrm{pCi} / \mathrm{L}$ \\
\hline SD-104-D & 19-May- 1987 & ALPHA & 2.8 & $\mathrm{pCI} / \mathrm{L}$ \\
\hline * $\quad$ SD-10 & & & & \\
\hline SD- 104 & 5-Mar-1987 & ALUMINUM & $<0.10$ & $\mathrm{mg} / \mathrm{L}$ \\
\hline SD-104 & II-Mar-1987 & ALIMINUM & $<0.10$ & $\mathrm{mg} / \mathrm{L}$ \\
\hline SD- 104 & $18-\mathrm{Mar}-1987$ & ALUMINUM & 0.11 & $\mathrm{mg} / \mathrm{L}$ \\
\hline SD-104 & 24-Mar-1987 & ALUMINUM & 1.5 & $\mathrm{mg} / \mathrm{L}$ \\
\hline SD-104 & 3-Apr-1987 & AIJMINUM & 0.36 & $\mathrm{mg} / \mathrm{L}$ \\
\hline SD- 104 & 6-Apr-1987 & ALUMINUM & $<0.10$ & $\mathrm{mg} / \mathrm{L}$ \\
\hline SD- 104 & 12-May-1987 & ALIMINUY & $<0.10$ & $\mathrm{mg} / \mathrm{L}$ \\
\hline SD -104 & 19-Мay - 1987 & ALIMINUM & $<0.10$ & $\mathrm{mg} / \mathrm{L}$ \\
\hline SD- 104 & $26-$ May - 1987 & ALIRINUY & 0.025 & $\mathrm{mg} / \mathrm{L}$ \\
\hline SD-104 & 3-Jun-1987 & ALIUMTRUM & $<0.10$ & $\mathrm{mg} / \mathrm{L}$ \\
\hline SD- 104 & 11-Jun-1987 & ALOAINOTY & $<0.10$ & $\mathrm{mg} / \mathrm{L}$ \\
\hline SD-104 & $18-J$ un -1987 & ALOAINOH & $<0.10$ & $\operatorname{mg} / \mathrm{L}$ \\
\hline ** SD -10 & & & & \\
\hline SD-104-D & 6-Apr-1987 & ALUTINUM & $<0.10$ & $\mathrm{mg} / \mathrm{L}$ \\
\hline SD-104-D & $19-M_{a y}-1987$ & ALDIMINUM & $<0.10$ & $\mathrm{mg} / \mathrm{L}$ \\
\hline ** SD -10 & & & & \\
\hline SD-104 & 5-Mar-1987 & AMONLA & $<0.2$ & $\mathrm{mg} / \mathrm{L}$ \\
\hline SD-104 & 11- $\operatorname{Max}-1987$ & AMHONIA & 2.8 & $\mathrm{mg} / \mathrm{L}$ \\
\hline SD-104 & $18-\mathrm{Mar}-1987$ & AMATONIA & $<0.2$ & $\mathrm{mg} / \mathrm{L}$ \\
\hline SD- 104 & $24-\operatorname{Mar}-1987$ & AMONONA & $<0.2$ & $\mathrm{mg} / \mathrm{L}$ \\
\hline SD- 104 & 3 -Apr- 1987 & AMHONLA & $<0.2$ & $\mathrm{mg} / \mathrm{L}$ \\
\hline SD-104 & 6-Apr-1987 & AMMONIA & $<0.2$ & $\mathrm{mg} / \mathrm{L}$ \\
\hline SD-104 & 12-May-1987 & AMMONIA & $<0.2$ & $\mathrm{mg} / \mathrm{L}$ \\
\hline SD-104 & $19-$ Kay -1987 & AMMONIA & $<0.2$ & $\mathrm{mg} / \mathrm{L}$ \\
\hline SD-104 & $26-$ May -1987 & AMMONIA & $<0.2$ & $\mathrm{mg} / \mathrm{L}$ \\
\hline SD-104 & 3-Jun-1987 & AMMONIA & $<0.2$ & $\mathrm{mg} / \mathrm{L}$ \\
\hline SD-104 & $11-J$ un-1987 & AMMONIA & $<0.2$ & $\mathrm{mg} / \mathrm{L}$ \\
\hline SD- 104 & $18-J u n-1987$ & AMMOHLA & $<0.2$ & $\mathrm{mg} / \mathrm{L}$ \\
\hline$\#$ SD -10 & & & & \\
\hline$S D-104-D$ & $6-A p r-1987$ & AMMONIA & $<0.2$ & $\mathrm{mg} / \mathrm{L}$ \\
\hline SD-104-D & 19-May-1987 & AMMONIA & $<0.2$ & $\mathrm{mg} / \mathrm{L}$ \\
\hline
\end{tabular}


Storm Drain Data for SD-100-04

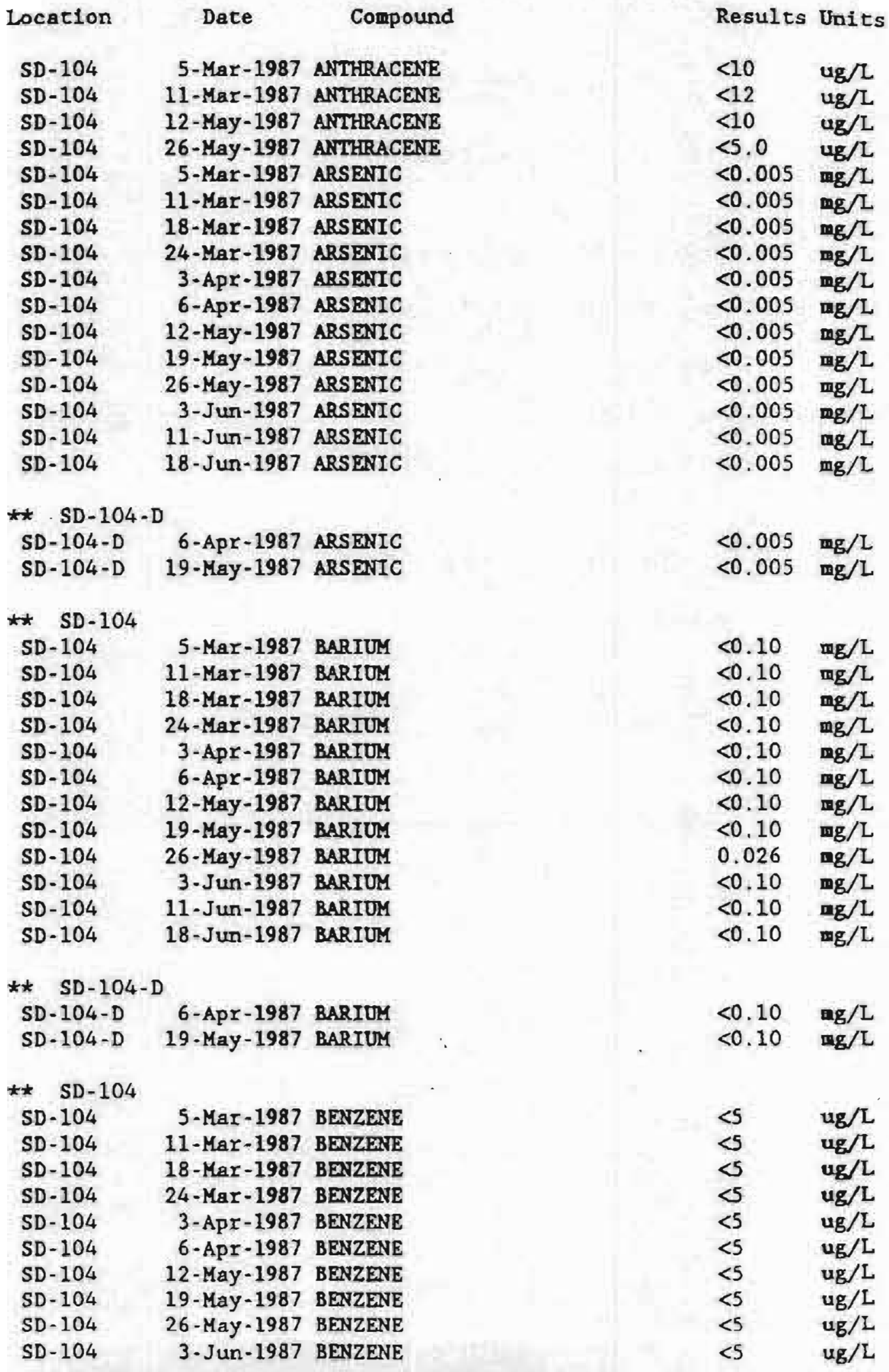


Storm Drain Data for SD-100-04

\begin{tabular}{|c|c|c|c|c|}
\hline Location & Date & Compound & Results & Units \\
\hline SD-104 & $11-J u n-1987$ & BENZENE & $<5$ & $u g / L$ \\
\hline SD-104 & $18-J u n-1987$ & BEIZENE & $<5$ & $\mathrm{ug} / \mathrm{L}$ \\
\hline$\star$ SD-104. & & & & \\
\hline SD-104-D & 6-Apr-1987 & BENZERE & $<$ & ug $/ \mathrm{L}$ \\
\hline SD-104-D & 19-May-1987 & BENZENE & $<5$ & $\operatorname{ug} / 2$ \\
\hline ** SD-104 & & & & \\
\hline SD-104 & 5-Mar-1987 & BENZIDINE & $<10$ & $\mathrm{ug} / \mathrm{L}$ \\
\hline SD- 204 & $11-\operatorname{Mar}-1987$ & BENZIDINE & $<12$ & ug $/ 1$ \\
\hline SD-104 & 12-Мay-1987 & BENZIDINE & $<10$ & $4 \mathrm{ug} / \mathrm{L}$ \\
\hline SD-104 & $26-$ Мay- 1987 & BENZIDINE & $<5.0$ & $u g / L$ \\
\hline SD-104 & 5-Mar-1987 & BENZO(A)ANTHRACENE & $<10$ & $u g / L$ \\
\hline SD-104 & $11-\operatorname{Mar}-1987$ & BENZO (A) ANTHRACENE & $<12$ & $4 g / L$ \\
\hline SD-104 & 12-May-1987 & BEN2O (A) ANTHRACENE & $<10$ & ug/L \\
\hline SD-104 & 26-May-1987 & BENZO (A) ANTHRACENE & $<5.0$ & $u g / 2$ \\
\hline SD - 104 & 5-Mar-1987 & BENZO (A) PYRENE & $<10$ & ug/L \\
\hline SD-104 & 11-Mar-1987 & BENZO (A) PYRENE & $<12$ & $\mathrm{ug} / \mathrm{L}$ \\
\hline SD-104 & $12-$ May -1987 & BENZO (A) PYRENE & $<10$ & ug/L \\
\hline SD - 104 & 26-May-1987 & BENZO (A) PYRENE & $<5.0$ & $\operatorname{ug} / \mathrm{L}$ \\
\hline SD-104 & $5-\operatorname{Mar}-1987$ & BENZO (B) FLUORANTHENE & $<10$ & $4 \mathrm{~g} / \mathrm{L}$ \\
\hline SD-104 & $11-\operatorname{Mar}-1987$ & BENZO (B) FLUORANTHENE & $<12$ & ug/L \\
\hline SD-104 & 12-May-1987 & BENZO (B) FLUORANTHENE & $<10$ & ug/L \\
\hline SD-104 & $26-$ May -1987 & BENZO (B) FLUORANTHENE & $<5.0$ & $\operatorname{ug} / \mathrm{L}$ \\
\hline SD-104 & 5-Mar-1987 & BENZO(G H I) PERYLENE & $<10$ & $\mathrm{ug} / \mathrm{L}$ \\
\hline SD-104 & 11-Mar-1987 & BENZO(G H I) PERYLENE & $<12$ & $48 / L$ \\
\hline SD-104 & 12-May-1987 & BENZO (G H I) PERYLENE & $<10$ & $u g / L$ \\
\hline SD-104 & 26-May-1987 & BENZO(G H I) PERYLENE & $<5.0$ & $u g / L$ \\
\hline SD-104 & 5-Mar-1987 & BENZO (K) FLUORANTHENE & $<10$ & $\mathrm{ug} / \mathrm{L}$ \\
\hline SD-104 & 11 -Mar - 1987 & BENZO(K) FLWORANTHENE & $<12$ & $48 / L$ \\
\hline SD-104 & 12-May-1987 & BENZO (K) FLUORANTHENE & $<10$ & $\mathrm{ug} / \mathrm{L}$ \\
\hline SD- 104 & $26-$ May -1987 & BENZO (K) FLUORANTHENE & $<5.0$ & $\mathrm{ug} / \mathrm{L}$ \\
\hline SD- 104 & 5-Mar-1987 & BERYLLIUK & $<0.0010$ & $\mathrm{mg} / \mathrm{L}$ \\
\hline SD- 104 & 11 -Mar-1987 & BERYLLIUK & $<0.0010$ & $\mathrm{mg} / \mathrm{L}$ \\
\hline SD- 104 & $18-\operatorname{Mar}-1987$ & BERYLLIUM & $<0.0010$ & $\mathrm{mg} / \mathrm{L}$ \\
\hline SD- 104 & 24-Mar-1987 & BERYLIIUM & $<0.0010$ & $\mathrm{mg} / \mathrm{L}$ \\
\hline SD- 104 & 3-Apr-1987 & BERYLLTUM & $<0.0010$ & $\mathrm{mg} / \mathrm{L}$ \\
\hline SD- 104 & 6-Apr-1987 & BERYLIIUK & $<0.0010$ & $\mathbf{m g} / \mathrm{L}$ \\
\hline SD- 104 & 12-May- 1987 & BERYLLIUM & $<0.0010$ & $\mathrm{mg} / \mathrm{L}$ \\
\hline SD- 104 & 19-May-1987 & BERYLLIUM & $<0.0010$ & $\operatorname{mg} / \mathrm{L}$ \\
\hline SD- 104 & 26-May- 1987 & BERYLLIUM & 0.0003 & $\operatorname{mg} / \mathrm{L}$ \\
\hline SD-104 & 3-Jun- 1987 & BERYLLIUM & $<0.0010$ & $m g / L$ \\
\hline SD-104 & 11-Jun-1987 & BERYLLIUY & $<0.0010$ & $\mathrm{mg} / \mathrm{L}$ \\
\hline SD-104 & $18-J$ un- 1987 & BERYLLIUM & $<0.0010$ & $\mathrm{mg} / \mathrm{L}$ \\
\hline $50-184$ & & & & \\
\hline SD-104-D & 6-Apr-1987 & BERYLLIUM & $<0.0010$ & $\mathrm{mg} / \mathrm{L}$ \\
\hline SD-104-D & 19-May-1987 & BERYLLIUY & $<0.0010$ & ㄸg $/ L$ \\
\hline
\end{tabular}


Storm Draln Data for SD-100-04

\begin{tabular}{lr} 
Locat1on & \multicolumn{1}{c}{ Date } \\
SD-104 & 5-Mar-1987 \\
BETA \\
SD-104 & 11-Mar-1987 BETA \\
SD-104 & 18 -Mar-1987 BETA \\
SD-104 & 24 -Mar-1987 BETA \\
SD-104 & 3-Apr-1987 BETA \\
SD-104 & 6-Apr-1987 BETA \\
SD-104 & 12 -May-1987 BETA \\
SD-104 & 19 -May-1987 BETA \\
SD-104 & 26-May-1987 BETA \\
SD-104 & 3-Jun-1987 BETA \\
SD-104 & 11 -Jun-1987 BETA \\
SD-104 & 18 -Jun-1987 BETA
\end{tabular}

* SD-104-D
SD-104-D
SD-104-D 19 -May-1987 BETA

Compound

Results Units

$\begin{array}{ll}<2 & \mathrm{pCI} / \mathrm{L} \\ 7.8 & \mathrm{pCI} / \mathrm{L} \\ <2 & \mathrm{pCI} / \mathrm{L} \\ 8.4 & \mathrm{pCI} / \mathrm{L} \\ 59.7 & \mathrm{pCI} / \mathrm{L} \\ 5.8 & \mathrm{pCI} / \mathrm{L} \\ 2 & \mathrm{pCI} / \mathrm{L} \\ 4.2 & \mathrm{pCI} / \mathrm{L} \\ <3 & \mathrm{pCI} / \mathrm{L} \\ 19.3 & \mathrm{pC1} / \mathrm{L} \\ <2 & \mathrm{pCI} / \mathrm{L} \\ 2.5 & \mathrm{pCI} / \mathrm{L}\end{array}$

$6.8 \mathrm{pCi} / \mathrm{L}$

$4.5 \mathrm{pCi} / \mathrm{L}$

$\star \star \quad$ SD-104

SD -104

5-Mar-1987 BIS (2-CHLOROETHOXY) METHANE

SD -104

11-Mar-1987 BIS (2-CHLOROETHOXY) METHANE

SD- 104

12-May- 1987 BIS (2 - CHLOROETHOXY) METHANE

SD -104

26-May-1987 BIS (2-CHLOROETHOXY) METHANE

SD -104

5-Mar-1987 BIS (2-CHLOROETHYL) ETHER

SD-104

11-MaY-1987 BIS (2 - CHLOROETHYL) ETHER

SD- 104

12-May-1987 BIS (2-CHLOROETHY) ETHBR

SD-104

SD -104

26-May-1987 BIS (2-CHLOROETHYL) ETHER

5-Mar-1987 BIS (2-CHLOROISOPROPYL) ETHER

SD- 104

11-Mar-1987 BIS (2 - CHLOROISOPROPYL) ETHER

SD- 104

SD -104

SD-104

12-May - 1987 BIS (2-CHIOROISOPROPYL) ETHER

26-May-1987 BIS (2-CHLOROISOPROPYL) ETHER

5-Mar-1987 BIS (2 - ETHYLHEXYL) PHTHALATE

SD-104

SD- 104

1I-Mar-1987 BIS (2-ETHYLHEXYL) PHTHALATE

12-May-1987 BIS (2-ETHYLHEXY) PHTHALATE

SD-104

SD -104

26-May-1987 BIS (2-ETHYLHEXYL) PHTHALATE

5- KaY-1987 BORON

SD -104

11- Kar-1987 BORON

SD -104

18-Mar-1987 BORON

SD -104

24-Mar-1987 BORON

SD-104

3-APr-1987 BORON

SD -104

6-Apr-1987 BORON

SD -104

12-May-1987 BORON

SD-104

19-May-1987 BORON

SD-104

26-May-1987 BORON

3 -Jun-1987 BORON

SD-104

11-Jur-1987 BORON

SD-104

18-Jun-1987 BORON

$\begin{array}{ll}<10 & \mathrm{ug} / \mathrm{L} \\ <12 & \mathrm{ug} / \mathrm{L} \\ <10 & \mathrm{ug} / \mathrm{L} \\ <5.0 & \mathrm{ug} / \mathrm{L} \\ <10 & \mathrm{ug} / \mathrm{L} \\ <12 & \mathrm{ug} / \mathrm{L} \\ <10 & \mathrm{ug} / \mathrm{L} \\ <5.0 & \mathrm{ug} / \mathrm{L} \\ <10 & \mathrm{ug} / \mathrm{L} \\ <12 & \mathrm{ug} / \mathrm{L} \\ <10 & \mathrm{ug} / \mathrm{L} \\ <5.0 & \mathrm{ug} / \mathrm{L} \\ <10 & \mathrm{ug} / \mathrm{L} \\ <12 & \mathrm{ug} / \mathrm{L} \\ <10 & \mathrm{ug} / \mathrm{L} \\ <5.0 & \mathrm{ug} / \mathrm{L} \\ 0.023 & \mathrm{mg} / \mathrm{L} \\ <0.0040 & \mathrm{mg} / \mathrm{L} \\ <0.0040 & \mathrm{mg} / \mathrm{L} \\ 0.034 & \mathrm{mg} / \mathrm{L} \\ 0.022 & \mathrm{mg} / \mathrm{L} \\ 0.014 & \mathrm{mg} / \mathrm{L} \\ 0.0055 & \mathrm{mg} / \mathrm{L} \\ 0.015 & \mathrm{mg} / \mathrm{L} \\ 0.031 & \mathrm{mg} / \mathrm{L} \\ 0.018 & \mathrm{mg} / \mathrm{L} \\ 0.011 & \mathrm{mg} / \mathrm{L} \\ 0.0081 & \mathrm{mg} / \mathrm{L}\end{array}$

$\star \star$ SD $-104-D$

SD-104-D 6-Apt-1987 BORON

$0.017 \mathrm{mg} / \mathrm{L}$

SD-104-D 19-May-1987 BORON

$0.015 \mathrm{mg} / \mathrm{L}$ 
Location

Date

Compound

Results Units

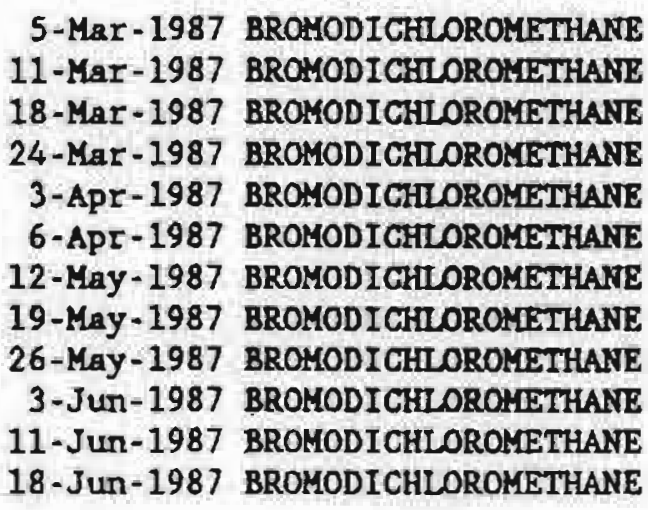

** SD-104-D

SD-104-D 6-APT-1987 BROMODICHLOROMETHANE

SD-I04-D 19-Kay-1987 BROMODICHLOROMETHANE

* SD-104

SD-104

SD- 104

SD-104

SD-104

SD-104

SD -104

SD -104

SD-104

SD-104

SD-104

SD- 104

SD-104
5-Mar-1987 BROMOFORM

11-Mar-1987 BROMOFORM

18-Mar-1987 BROMOFORM

24-Mar-1987 BROMOFORM

3-Apr-1987 BROMOFORM

6-Apr-1987 BROMOFORM

12- Kay-1987 BROMOFORM

19-May-1987 BROMOFORM

26-May- 1987 BROMOFORY

3-Jun-1987 BROMOFORM

11-Jun-1987 BROMOFORM

18-Jun-1987 BROKOFORM

** SD-104-D

SD-104-D 6-Apr-1987 BROMOFORM

SD-104-D 19-May-1987 BROMOFORY

$\star$ SD -104

SD-104

SD -104

SD- 104

SD-104

SD -104

SD -104

SD -104

SD -104

SD -104

SD -104

SD -104

SD- 104

5-MaY-1987 BROMOMETHANE

11-KaI-1987 BROMOMETHANE

18-Mar-1987 BROMOMETHANE

24-Mar-1987 BROMOMETKANE

3-APT-1987 BROMOMETHANE

6-Apr-1987 BROMOMETHANE

12-May-1987 BROMOMETHANE

19-Kay-1987 BROMOMETHANE

26-MaY-1987 BROMOMETHANE

3-JUn-1987 BROMOMETHANE

11-Jun-1987 BROMOMETHANE

18-JUn-1987 BROMOMETHANE

$\begin{array}{ll}6 & \mathrm{ug} / \mathrm{L} \\ <5 & \mathrm{ug} / \mathrm{L} \\ <5 & \mathrm{ug} / \mathrm{L} \\ 7 & \mathrm{ug} / \mathrm{L} \\ <5 & \mathrm{ug} / \mathrm{L} \\ 6 & \mathrm{ug} / \mathrm{L} \\ 9 & \mathrm{ug} / \mathrm{L} \\ 5 & \mathrm{ug} / \mathrm{L} \\ 5 & \mathrm{ug} / \mathrm{L} \\ 5 & \mathrm{ug} / \mathrm{L} \\ 5 & \mathrm{ug} / \mathrm{L} \\ 5 & \mathrm{ug} / \mathrm{L}\end{array}$

7

5

ug/ $/$

$u g / L$

$<5$

$<5$

$<5$

$<5$

$<5$

$<5$

$<5$

$<5$

$<5$

$<5$

$<5$

$<5$

$4 \mathrm{~g} / \mathrm{L}$

ug/L

$4 \mathrm{~g} / \mathrm{L}$

$u g / L$

$\mathrm{ug} / \mathrm{L}$

ug/L

$u g / L$

ug/L

$u g / L$

$u g / L$

ug/L

$\mathrm{ug} / \mathrm{L}$

$<5$

$<5$

ug/L

ug/L

$<10$

$<10$

$<10$

$<10$

$<10$

$<10$

$<10$

$<10$

$<10$

$<10$

$<10$

$<10$ ug/ $\mathrm{L}$ ug $/ 2$ ug/L $u g / L$ $4 \mathrm{~g} / \mathrm{L}$ ug $/$ L ug/L ug/L ug/L $\mathrm{ug} / \mathrm{L}$ $\mathrm{ug} / L$ $u g / L$ 
Storm Drala Data for SD-100-04

Location

SD-104-D 6-API-1987 BROMOMETHANE

SD-104-D 19-Kay-1987 BROKOHETHANE

$<10 \quad u g / L$

* SD-104

SD-104

SD. 104

5-Mar-1987 BUTYL CELLOSOLVE

SD-104

5-HaY-1987 BUTYLBENZYLPHTHALATE

SD-104

11-Kar-1987 BUTYLBENZYLPHTHAIATE

SD-104

12-May-1987 BUTYLBENZYLPHTHATATE

SD-104

26-KaY-1987 BUTYLBENZYLPHTHALATE

SD-104

5-Mar-1987 CADMTIM

SD-104

11-Mar-1987 CADHIUM

SD-104

18-Har-1987 GADMIUM

SD-104

24-Mar-1987 CADHIUM

SD-104

3-ApY-1987 CADMIUM

SD-104

6-Apr-1987 CADMIUM

SD-104

12-May-1987 CADHITUM

SD-104

19-May-1987 CADMIUM

SD-104

26-Msy-1987 CADMTUM

SD-104

3-Jun-1987 CADKTUM

SD-104

11-Jun-1987 CADYTUY

18-Jun-1987 CADMIUM

* SD-104-D

SD-104-D 6-Apr-1987 CADMTOM

SD-104-D 19-May-1987 CADMTUM

$<0.0030 \mathrm{mg} / \mathrm{L}$

$<0.0030 \mathrm{mg} / \mathrm{L}$

** SD -104

SD-104

5-Mar-1987 CALCIUM

SD-104 11-MaT-1987 GALCIUM

SD-104 18-Mar-1987 CALCIUM

SD-104 24-Mar-1987 CALCIUM

SD-104 3-APT-1987 CALCIUM

SD-104 6-API-1987 GALCIUM

SD-104 12-Kay-1987 CALCIUM

SD-104 19-May-1987 CALCIUM

SD-104 26-May-1987 CALCIUM

SD-104 3-JUn-1987 CALCIUM

SD-104 11-JUn-1987 CALCIUM

SD-104 18-Jun-1987 GALCIOM

$38 \quad \mathrm{ug} / \mathrm{L}$

$<10 \mathrm{ug} / \mathrm{L}$

$<12$ ug/L

$<10 \quad 4 \mathrm{~g} / \mathrm{L}$

$<.0 \mathrm{ug} / \mathrm{L}$

$<0.0030 \mathrm{mg} / \mathrm{L}$

$<0.0030 \mathrm{mg} / \mathrm{L}$

$<0.0030 \mathrm{mg} / \mathrm{L}$

$<0.0030 \mathrm{mg} / \mathrm{L}$

$0.0032 \mathrm{mg} / \mathrm{L}$

$<0.0030 \mathrm{mg} / \mathrm{L}$

$<0.0030 \mathrm{mg} / \mathrm{L}$

$<0.0030 \mathrm{mg} / \mathrm{L}$

$<0.0030 \mathrm{mg} / \mathrm{L}$

$<0.0030 \mathrm{mg} / \mathrm{L}$

$<0.0030 \mathrm{mg} / \mathrm{L}$

$<0.0030 \mathrm{mg} / \mathrm{L}$.

* SD-104-D

SD-104-D 6-APr-1987 GALCIUM

SD-104-D 19-May-1987 GALCIUM

29

34

$\operatorname{mg} / \mathrm{L}$

** SD-104

SD-104

SD-104

5-MaT-1987 CARBON TETRACHLORIDE

SD-104

11-Mar-1987 CARBON TETRACHLORIDE

18-MaY-1987 CARBON TETRACHLORIDE

$\begin{array}{ll}<5 & 4 g / L \\ <5 & 4 g / 2 \\ <5 & 4 g / 2\end{array}$ 
Storm Drain Daca for SD-100-04

Location

$$
\begin{aligned}
& \text { SD-104 } \\
& \text { SD-104 } \\
& \text { SD-104 } \\
& \text { SD-104 } \\
& \text { SD-104 } \\
& \text { SD-104 } \\
& \text { SD-104 } \\
& \text { SD-104 } \\
& \text { SD-104 }
\end{aligned}
$$

Date Compound

24-MaI-1987 CARBON TETRACHLORIDE

3-Apr-1987 GARBON TETRACHLORIDE

6-APr-1987 CARBON TEIRACHLORIDE

12-May-1987 CARBON TETRAGLLORIDE

19-May-1987 CARBON TETRACHLORIDE

26-May-1987 CARBON TETRACTIORTDE

3-JUM-1987 CARBON TETRACILORIDE

11-JUn-1987 CARBON TETRACKLORIDE

18-Jun-1987 CARBON TETRACHIORIDE

** SD-104-D

SD-104-D 6-APR-1987 CARBON TETRACHLORIDE

SD-104-D 19-May-1987 CARBON TETRACHLORIDE

** SD-104

SD-104

SD-104

SD -104

SD -104

SD-104

SD-104

SD-104

SD-104

SD-104

SD- 104

SD-104

SD-104

5-MaY-1987 CHEMICAL OXYGEN DEMAND (COD)

11-Mar-1987 CHEMICAL OXYGEN DEMAND (COD)

18-Mar-1987 CHEMICAL OXYGEN DEMAND (COD)

24-Mar-1987 CHEMICAL OXYGEN DEMAND (COD)

3-APr-1987 CHEMICAL OXYGEN DEMAND (COD)

6-API-1987 CHEMICAL OXYGEN DEMIAND (COD)

12-May-1987 CHEMICAL OXYGEN DEMAND (COD)

19-May-1987 CHEMICAL OXYGEN DEMAND (COD)

26-May-1987 CHEMICAL OXYGEN DEMAND (COD)

3-Jun-1987 CHEMICAL OXYGEN DEMAND (COD)

11-Jun-1987 CHEMICAL OXYGEN DEMAND (COD)

18-JUn-1987 CHEMICAL OXYGEN DEMAND (COD)

** SD-104-D

SD-104-D

$S D-104-D$

6-Apr-1987 CHEMICAL OXYGEN DEAAND (COD)

19-May-1987 CHEMICAL OXYGEN DEMAND (COD)

** SD-104

SD-104

5-Mar-1987 CHLORIDE

SD-104

11-Mar-1987 CHLORIDE

SD-104

18-Mar-1987 CHLORIDE

SD-104

24-Mar-1987 CHLORIDE

SD-104

3-Apr-1987 CHLORIDE

SD-104

6-Apr-1987 CHLORIDE

SD-104

12-May-1987 CHLORIDE

SD-104

19-May-1987 CHLORIDE

SD-104

SD- 104

26-May-1987 CHLORIDE

SD-104

3-Jun-1987 CHLORIDE

SD- 104

11-Jun-1987 CHLORIDE

18-Jun-1987 CHLORIDE

** SD-104-D

SD-104-D 6-APr-1987 CHLORIDE

SD-104-D 19-May-1987 CHLORIDE

Results Units

$\begin{array}{ll}<5 & u g / L \\ <5 & u g / L \\ <5 & u g / L \\ <5 & u g / L \\ <5 & u g / L \\ <5 & u g / L \\ <5 & u g / L \\ <5 & u g / L \\ <5 & u g / L\end{array}$

$<5 \quad$ ug/L

<s ug/L

6

$<5$

73

7

15

17

$<5$

$<5$

$<5^{\circ}$

11.0

4.5

$<5$

17

$<5$

$\operatorname{mg} / \mathrm{L}$

$\mathrm{mg} / \mathrm{L}$

$9.2 \mathrm{mg} / \mathrm{L}$

B.8 $\mathrm{mg} / \mathrm{L}$

$8.4 \mathrm{mg} / \mathrm{L}$

$120 \mathrm{mg} / \mathrm{L}$

$620 \mathrm{mg} / \mathrm{L}$

$8.6 \mathrm{mg} / \mathrm{L}$

$9.5 \mathrm{mg} / \mathrm{L}$

$9.9 \mathrm{mg} / \mathrm{L}$

$10.4 \mathrm{mg} / \mathrm{L}$

$9.6 \mathrm{mg} / \mathrm{L}$

$11.5 \mathrm{mg} / \mathrm{L}$

$13 \mathrm{mg} / \mathrm{L}$

$8.6 \mathrm{mg} / \mathrm{L}$

$9.9 \mathrm{mg} / \mathrm{L}$ 
Storm Drain Data for SD-100-04

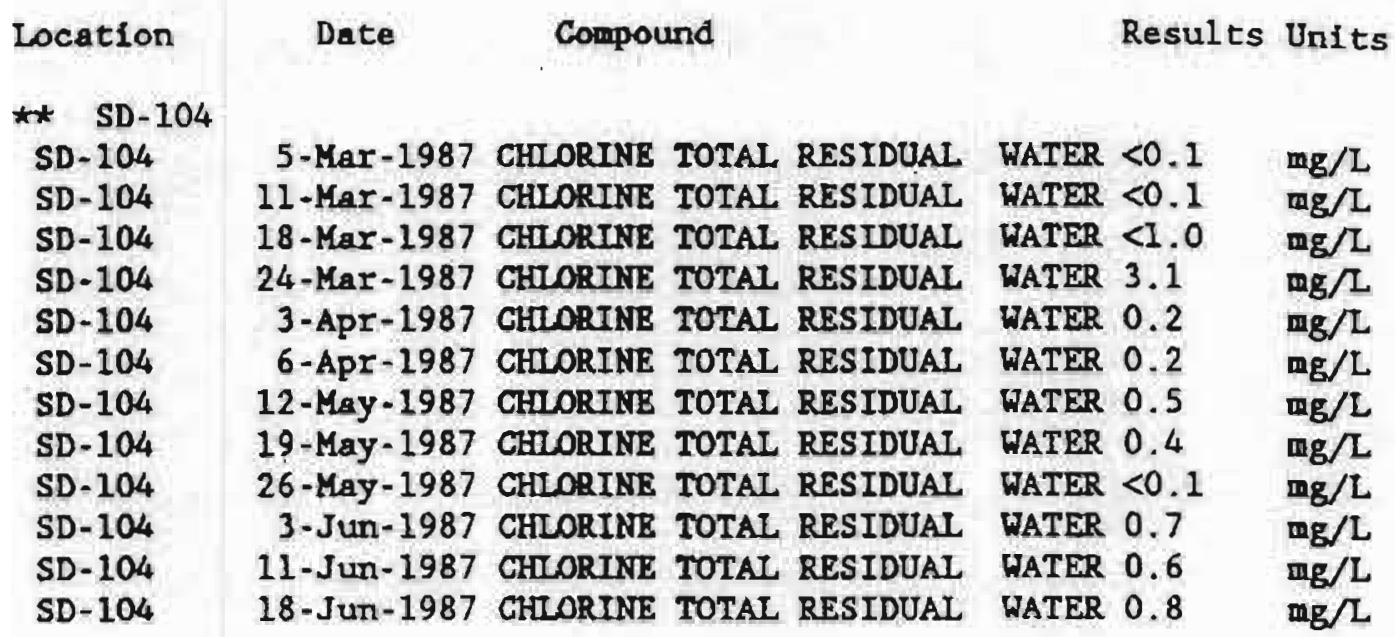

** SD-104-D

SD-104-D 6-APY-1987 CHLORINE TOTAL RESIDUAL WATER $0.2 \mathrm{mg} / \mathrm{L}$ SD-104-D 19-May-1987 CHLORINE TOTAL RESIDUAL WATER $0.5 \mathrm{mg} / \mathrm{L}$

\begin{tabular}{|c|c|c|c|c|}
\hline \multicolumn{5}{|l|}{ \#* SD-104 } \\
\hline$S D-104$ & 12-May-1987 & CHLORO CYCLOHEXANOL & 700 & $u g / L$ \\
\hline SD-104 & 12-Kay-1987 & CHLORO CYCLOHEXALTONE & 40 & $\mathrm{ug} / \mathrm{L}$ \\
\hline SD -104 & $12-$ May-1987 & CHLORO CYCLOHEXENE & 60 & $\mathrm{ug} / \mathrm{L}$ \\
\hline SD-104 & 5-Mar-1987 & CHLOROBEARENE & $<5$ & $\mathrm{ug} / \mathrm{L}$ \\
\hline SD -104 & 11-Mar-1987 & CHLOROBENZENE & $<5$ & $\operatorname{ug} / \mathrm{L}$ \\
\hline SD-104 & $18-\operatorname{Mar}-1987$ & CHLOROBENZENE & $<5$ & $\operatorname{ug} / \mathrm{L}$ \\
\hline SD-104 & $24-$ Mar -1987 & CHLOROBENZENE & $<5$ & ug $/ \mathrm{L}$ \\
\hline SD-104 & 3-Apr-1987 & CHLOROBENZENE & $<5$ & ug/L \\
\hline SD-104 & 6-Apt- 1987 & CHLOROBENZENE & $<5$ & $\lg / \mathrm{L}$ \\
\hline SD-104 & 12-May-1987 & CHLOROBERZENE & $<5$ & $u g / L$ \\
\hline SD-104 & $19-\mathrm{Kay}-1987$ & CHLOROBEMZEHE & $<5$ & $u g / L$ \\
\hline SD -104 & 26- - Kay- 1987 & CHLOROBEHZFNE & $<5$ & ug/L \\
\hline SD-104 & 3-Jun-1987 & CHLOROBENZENE & $<5$ & $\operatorname{ug} / \mathrm{L}$ \\
\hline SD-104 & $11-J u n-1987$ & CHLOROBENZENE & $<5$ & $\mathrm{ug} / \mathrm{L}$ \\
\hline SD-104 & $18-$ Jun- 1987 & CHLOROBENZENE & $<5$ & $\mathbf{u g} / \mathrm{L}$ \\
\hline \multicolumn{5}{|c|}{$\star \star \quad S D-104-D$} \\
\hline$S D-104-D$ & 6-Apr-1987 & CHLOROB EAZZENE & $<5$ & ug/L \\
\hline$S D-104-D$ & 19-May-1987 & CHLOROBEIZYENE & $<s$ & $\mathrm{ug} / \mathrm{L}$ \\
\hline \multicolumn{5}{|l|}{ * SD -104} \\
\hline SD-104 & $5-$ Mar - 1987 & CHLOROETHANE & $<10$ & $u g / 2$ \\
\hline SD-104 & 11-Mar-1987 & CHLOROETHANE & $<10$ & $\operatorname{ug} \Omega$ \\
\hline SD-104 & $18-$ Mar -1987 & CHLOROETHANE & $<10$ & $48 / 2$ \\
\hline SD -104 & 24 -Mar -1987 & CHLOROETHANE & $<10$ & $\operatorname{Lg} / 2$ \\
\hline SD- 104 & $3-A p r-1987$ & CHLOROETHANE & $<10$ & $\operatorname{ug} / \mathrm{L}$ \\
\hline SD - 104 & 6-Apr-1987 & CHLOROETHANE & $<10$ & ug/L \\
\hline SD. 104 & 12-May - 1987 & CHLOROETHANE & $<10$ & $\mathrm{ug} / \mathrm{L}$ \\
\hline SD-104 & 19-May - 1987 & GHLOROETRANE & $<10$ & $\mathrm{ug} / \mathrm{L}$ \\
\hline SD -104 & 26-May-1987 & CHLOROETHANE & $<10$ & $\mathrm{ug} / \mathrm{L}$ \\
\hline SD-104 & 3-Jun-1987 & CHLOROETHANE & $<10$ & $u g / L$ \\
\hline
\end{tabular}


Storm Drafn Data for SD-100-04

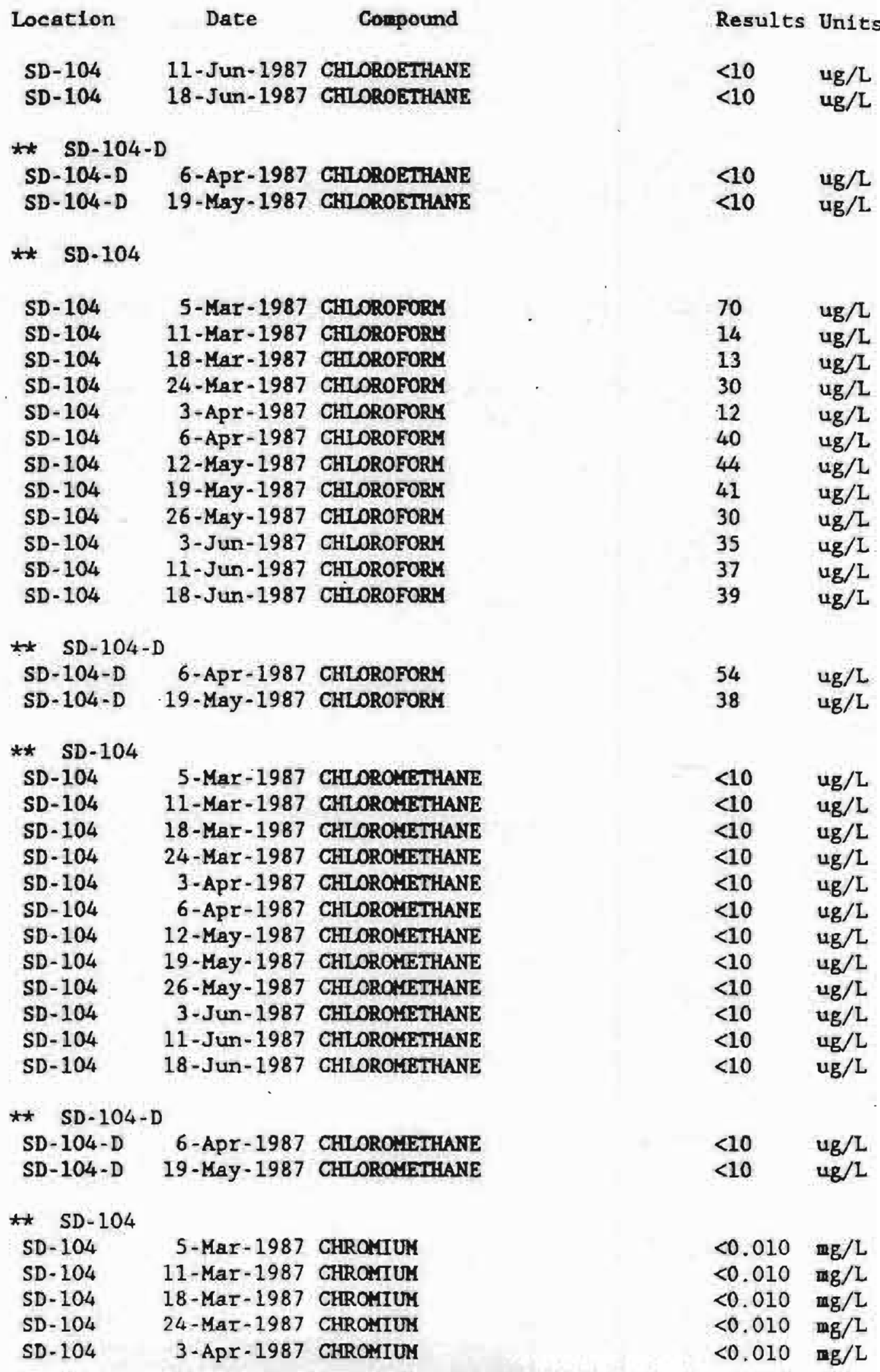


Storm Drain Data for SD-100-04

\begin{tabular}{|c|c|c|c|c|}
\hline Location & Date & Compound & Results & Units \\
\hline SD-104 & 6-Apr-1987 & CHROMIOM & $<0.010$ & $\mathbf{m g} / \mathrm{L}$ \\
\hline SD- 104 & 12-May-1987 & CEROTITOA & $<0.010$ & $\mathrm{mg} / \mathrm{L}$ \\
\hline SD-104 & $19-$ May-1987 & GEROMIIII & $<0.010$ & $\mathrm{mg} / \mathrm{x}$ \\
\hline$S D-104$ & $26-$ May - 1987 & CAROMIII & $<0.010$ & $\mathrm{mg} / \mathrm{L}$ \\
\hline SD-104 & 3-Jun-1987 & CAROMIOY & $<0.010$ & $\operatorname{mg} / \mathrm{L}$ \\
\hline SD-104 & $11 \cdot J u n \cdot 1987$ & CEROHIOM & $<0.010$ & $\mathrm{mg} / \mathrm{L}$ \\
\hline SD-104 & 18-Jun-1987 & CAROMIUY & $<0.010$ & $\mathrm{mg} / \mathrm{L}$ \\
\hline \multicolumn{5}{|c|}{$\star$ SD-104-D } \\
\hline SD-104-D & 6-Apr-1987 & CAROMIIM & $<0.010$ & $\mathrm{mg} / \mathrm{L}$ \\
\hline$S D-104-D$ & 19- - Мay-1987 & GRORITU & $<0.010$ & $\mathrm{mg} / \mathrm{L}$ \\
\hline \multicolumn{5}{|l|}{ * SD -104} \\
\hline SD-104 & $5-$ Mar-1987 & CARYSENE & $<10$ & $\mathrm{ug} / \mathrm{L}$ \\
\hline SD- 104 & 11-Mar-1987 & CHRYSENE & $<12$ & $\operatorname{lgg} / \mathrm{L}$ \\
\hline SD-104 & 12-May-1987 & CHRYSENE & $<10$ & $u g / L$ \\
\hline SD-104 & 26-May-1987 & CFRYSENE & $<5.0$ & $u g / L$ \\
\hline SD-104 & $5-\operatorname{Mar}-1987$ & CIS-1 3-DICHLOROPROPENE & $<5$ & $u g / L$ \\
\hline SD-104 & 11-Mar-1987 & CIS-1 3-DICHLOROPROPENE & $<5$ & $\mathrm{ug} / \mathrm{L}$ \\
\hline SD-104 & $18-\operatorname{Mar}-1987$ & CIS-1 3-DICHLOROPROPENE & $<5$ & $u g / L$ \\
\hline SD-104 & $24-\operatorname{Mar}-1987$ & GIS - 1 3-DICHLOROPROPENE & $<5$ & $4 \mathrm{~g} / \mathrm{L}$ \\
\hline SD- 104 & 3-Apr-1987 & CIS-1 3-DICHLOROPROPENE & $<5$ & $u g / L$ \\
\hline$S D-104$ & 6-Apr-1987 & CIS-1 3-DICHLOROPROPENE & $<5$ & ug/L \\
\hline SD-104 & 12-May-1987 & CIS-1 3-DICHLOROPROPENE & $<5$ & $u g / L$ \\
\hline SD-104 & 19-May-1987 & CIS-1 3-DICHLOROPROPENE & $<5$ & $4 g / L$ \\
\hline SD - 104 & $26-$ May -1987 & CIS-1 3-DICHLOROPROPENE & $<5$ & $\operatorname{ug} / \mathrm{L}$ \\
\hline SD-104 & 3-Jun-1987 & CIS-1 3-DICHLOROPROPENE & $<5$ & $u_{\mathrm{g} / \mathrm{L}}$ \\
\hline SD- 104 & 11-Jun-1987 & CIS - 1 3-DICHLOROPROPENE & $<5$ & $\mathrm{ug} / \mathrm{L}$ \\
\hline SD -104 & $18-J$ un -1987 & CIS-1 3-DICHLOROPROPENE & $<5$ & $u g / L$ \\
\hline \multicolumn{5}{|c|}{$\star$ SD-104-D } \\
\hline SD-104-D & 6-Apr-1987 & CIS-1 3-DICKLOROPROPENE & $<5$ & ug/L \\
\hline SD-104-D & 19-May-1987 & CIS-1 3-DICHLOROPROPENE & $<5$ & $4 \mathrm{~g} / \mathrm{L}$ \\
\hline \multicolumn{5}{|l|}{ ** SD-104 } \\
\hline SD-104 & 5-Mar- 1987 & COBALT & $<0.10$ & $\mathrm{mg} / \mathrm{L}$ \\
\hline SD -104 & $11-\operatorname{Mar}-1987$ & COBALT & $<0.10$ & $\mathrm{mg} / \mathrm{L}$ \\
\hline SD- 104 & $18-\operatorname{Mar}-1987$ & COBALT & $<0.10$ & $\mathrm{mg} / \mathrm{L}$ \\
\hline SD- 104 & $24-\operatorname{Mar}-1987$ & COBALT & $<0.10$ & $\mathrm{mg} / \mathrm{L}$ \\
\hline SD- 104 & 3-Apr-1987 & COBALT & $<0.10$ & $\mathrm{mg} / \mathrm{L}$ \\
\hline SD-104 & 6-Apr-1987 & COBALT & $<0.10$ & $\mathrm{mg} / \mathrm{L}$ \\
\hline SD-104 & 12-May-1987 & COBALT & $<0.10$ & $\mathrm{mg} / \mathrm{L}$ \\
\hline SD-104 & 19-May-1987 & COBALT & $<0.10$ & $\operatorname{mg} / \mathrm{L}$ \\
\hline SD-104 & 26-May-1987 & COBALI & $<0.0050$ & $\mathrm{mg} / \mathrm{L}$ \\
\hline SD-104 & 3-Jun-1987 & COBALT & $<0.10$ & $\mathrm{mg} / \mathrm{L}$ \\
\hline SD-104 & 11-Jun-1987 & COBALT & $<0.10$ & $\operatorname{mg} / \mathrm{L}$ \\
\hline SD- 104 & $18-J u n-1987$ & COBALT & $<0.10$ & $\mathrm{mg} / \mathrm{L}$ \\
\hline \multicolumn{5}{|c|}{ *t SD-104-D } \\
\hline$S D \cdot 104-D$ & 6-Apr-1987 & COBALI & $<0.10$ & $\operatorname{lgg} / \mathrm{L}$ \\
\hline SD-104-D & 19-May-1987 & COBALT & $<0.10$ & $\operatorname{mg} / \mathrm{L}$ \\
\hline
\end{tabular}




\begin{tabular}{|c|c|c|}
\hline Location & Date & Compound \\
\hline \multicolumn{3}{|l|}{$\star \star \quad$ SD-104 } \\
\hline SD-104 & 5-Maz-1987 & CONDUCTIVITY \\
\hline SD-104 & $11-\operatorname{Mar}-1987$ & CONDUGTIVITY \\
\hline SD-104 & $18-$ Mar -1987 & CONDUCTIVITY \\
\hline SD- 104 & 24 -Kar- 1987 & CONDUCTIVITY \\
\hline SD-104 & 3-Apr-1987 & CONDUCTIVITY \\
\hline SD- 104 & 6-ApL-1987 & CONDUCTIVITY \\
\hline SD- 104 & 12-Kay - 1987 & CONDUCTIVITY \\
\hline SD- 104 & 19-May-1987 & CONDOCTIVITY \\
\hline SD- 104 & 26-May - 1987 & CONDUCTIVITY \\
\hline SD- 104 & 3-Jun-1987 & CONDUCTIVITY \\
\hline SD- 104 & 11 - Jun- 1987 & CONDUCTIVITY \\
\hline SD-104 & 18 -Jun-1987 & CONDUCTIVITY \\
\hline
\end{tabular}

** SD-104-D

SD-104-D 6-Apr-1987 CONDUCTIVITY

SD-104-D 19-May-1987 CONDUCTIVITY

** SD -104
SD-104

SD-104

SD- 104

SD -104

SD -104

SD -104

SD -104

SD -104

SD-104

SD -104

SD-104

SD-104

5-Mar-1987 COPPER

11-Mar-1987 COPPER

18-Kar-1987 COPPER

24-Mar-1987 COPPER

3-Apr-1987 COPPER

6-APT-1987 COPPER

12-May-1987 COPPER

19-Mry-1987 COPPER

26-May-1987 COPPER

3-Jun-1987 COPPER

11-Jun-1987 COPPER

18-Jun-1987 COPPER

** SD-104-D

SD-104-D 6-API-1987 COPPER

SD-104-D 19-May-1987 COPPER

** SD -104

SD-104

SD-104

SD-104

SD-104

SD -104

SD-104

SD- 104

SD -104

SD -104

SD -104

SD-104

SD-104
5-Max-1987 CYANIDE

11-Mar-1987 CYANIDE

18-Mar-1987 CYANIDE

24-Mar-1987 CYANIDE

3-APr-1987 CYANIDE

6-Apr-1987 CYANIDE

12-May-1987 CYANIDE

19-May-1987 CYANIDE

26-May-1987 CYANIDE

3-Jun-1987 CYANIDE

11-JUn-1987 CYANIDE

18-Jun-1987 CYANIDE
Results Units

248 umho/cm

278 umho/cm

274 umho/cm

683 unho/cm

2150 umho/cm

254 umho/cu

297 unho/cm

291 umho/cm

301 umho/cm

303 umho/cm

313 umho/cm

281 unho/cm

254 umho/cm

290 waho/cm

$$
\begin{array}{ll}
0.013 \mathrm{mg} / \mathrm{L} \\
0.0050 \mathrm{mg} / \mathrm{L} \\
<0.0040 \mathrm{mg} / \mathrm{L} \\
0.0087 \mathrm{mg} / \mathrm{L} \\
0.0075 \mathrm{mg} / \mathrm{L} \\
0.013 \mathrm{mg} / \mathrm{L} \\
<0.0040 \mathrm{mg} / \mathrm{L} \\
<0.0040 \mathrm{mg} / \mathrm{L} \\
0.0042 \mathrm{mg} / \mathrm{L} \\
<0.0040 \mathrm{mg} / \mathrm{L} \\
<0.0040 \mathrm{mg} / \mathrm{L} \\
<0.0040 \mathrm{mg} / \mathrm{L}
\end{array}
$$

$0.013 \mathrm{mg} / \mathrm{L}$ $<0.0040 \mathrm{mg} / \mathrm{L}$

$\begin{array}{ll}0.005 & \mathrm{mg} / \mathrm{L} \\ <0.002 & \mathrm{mg} / \mathrm{L} \\ 0.003 & \mathrm{mg} / \mathrm{L} \\ 0.002 & \mathrm{mg} / \mathrm{L} \\ <0.002 & \mathrm{mg} / \mathrm{L} \\ 0.02 & \mathrm{mg} / \mathrm{L} \\ 0.002 & \mathrm{mg} / \mathrm{L} \\ 0.014 & \mathrm{mg} / \mathrm{L} \\ <0.002 & \mathrm{mg} / \mathrm{L} \\ <0.002 & \mathrm{mg} / \mathrm{L} \\ <0.002 & \mathrm{mg} / \mathrm{L} \\ <0.002 & \mathrm{mg} / \mathrm{L}\end{array}$




\begin{tabular}{|c|c|c|c|c|}
\hline Location & \multicolumn{2}{|r|}{ Compound } & Results & Units \\
\hline \multicolumn{5}{|c|}{ ** SD-104-D } \\
\hline SD-104-D & 6-Apr-1987 & CYANIDE & 0.02 & $\mathrm{mg} / \mathrm{L}$ \\
\hline$S D-104 \cdot D$ & $19-$-ay - 1987 & CYANIDE & 0.015 & $\mathrm{mg} / \mathrm{L}$ \\
\hline \multicolumn{5}{|l|}{$\star \star \quad S D-104$} \\
\hline SD-104 & 5-Mar-1987 & DI-N-BUTYLPHTHALATE & $<10$ & $\mathbf{u g} / \mathrm{L}$ \\
\hline SD- 104 & 11-Mar-1987 & DI-H-BUTYLPHTHALATE & $<12$ & $u g / L$ \\
\hline SD-104 & 12-May - 1987 & DI-N-BUTYLPHTHALATE & $<10$ & $4 \mathrm{~g} / \mathrm{L}$ \\
\hline SD-104 & $26-$ May-1987 & DI-H-BUTYLPHTHALATE & $<5.0$ & $u g / L$ \\
\hline SD-104 & 5-Mar-1987 & DI-N-OCTYLPHTHALATE & $1 \mathrm{~J}$ & $u g / L$ \\
\hline SD-104 & $11-\operatorname{Mar}-1987$ & DI-N-OCTYLPHTHALATE & $1 \mathrm{~J}$ & $u g / L$ \\
\hline SD-104 & 12-Kay- 1987 & DI-N-OCTYLPHTHALATE & $<10$ & $\mathrm{ug} / \mathrm{L}$ \\
\hline SD-104 & 26-May-1987 & DI-N-OCTYLPHTHALATE & $<5.0$ & $\mathrm{ug} / \mathrm{L}$ \\
\hline SD-104 & 11-Mat- 1987 & DIACETONE ALCOHOL & 67 & $u g / L$ \\
\hline SD-104 & 12-May- 1987 & DIACETONE ALCOHOL & 6 & $u g / L$ \\
\hline SD-104 & 5-Mar-1987 & DIBENZ (A H)ANTHRACENE & $<10$ & $\mathrm{ug} / \mathrm{L}$ \\
\hline SD-104 & $11-$ Mar - 1987 & DIBENZ (A H)ANTHRACENE & $<12$ & $u g / L$ \\
\hline SD-104 & 12-May-1987 & DIBENZ (A H) ANTHRACENE & $<10$ & $u g / L$ \\
\hline SD- 104 & $26-$ May - 1987 & DIBENZ (A H) ANTHRACENE & $<5.0$ & $\mathrm{ug} / \mathrm{L}$ \\
\hline SD-104 & 5-Mar-1987 & DIBROMOCHLOROMETHANE & $<5$ & $u g / L$ \\
\hline SD- 104 & $11-$ Mar- 1987 & DIBROMOCHLOROMETHANE & $<5$ & $\operatorname{ug} / \mathrm{L}$ \\
\hline SD- 104 & $18-\operatorname{Mar}-1987$ & DIBROMOCHLOROMETHANE & $<5$ & $u_{g} / L$ \\
\hline SD-104 & $24-$ Kar -1987 & DIBROMOCKLOROMETHANE & $<5$ & $u g / L$ \\
\hline$S D-104$ & 3-Apr-1987 & DIBROKOCHLOROMETHANE & $<5$ & $\operatorname{ag} / \mathrm{L}$ \\
\hline SD-104 & 6-Apr-1987 & DIBROMOCHLOROMETHANE & $<5$ & $4 \mathrm{~g} / \mathrm{L}$ \\
\hline SD-104 & 12-May - 1987 & DIBROMOGHLOROMETHANE & 5 & $\mathrm{ug} / \mathrm{L}$ \\
\hline SD-104 & 19-May-1987 & DIBROMOCHLOROKETHANE & $<5$ & $u g / L$ \\
\hline$S D-104$ & $26-$ May - 1987 & DIBROMOCHLOROMETHANE & $<5$ & $\mathrm{ug} / \mathrm{L}$ \\
\hline$S D-104$ & 3-Jun-1987 & DIBROMOCHLOROMETHANE & $<5$ & $u g / L$ \\
\hline SD-104 & $11-J u n-1987$ & DIBROMOCHLOROMETHANE & $<5$ & $u g / L$ \\
\hline SD-104 & $18-J u n-1987$ & DIBROMOCHLOROMETHANE & $<5$ & $u_{g} / L$ \\
\hline \multicolumn{5}{|c|}{$\star \star \quad S D-104-D$} \\
\hline$S D-104-D$ & 6-Apr-1987 & DIBROMOCHLOROMETHANE & $<5$ & $u_{g} / L$ \\
\hline SD-104-D & $19-$ May -1987 & DIBROMOCHLOROMETHANE & $<5$ & $u g / L$ \\
\hline \multicolumn{5}{|l|}{$\star \star \quad$ SD -104} \\
\hline SD -104 & 12-May-1987 & DICHLORO CYCLOHEXANE & 260 & $u g / L$ \\
\hline SD- 104 & $5-\operatorname{Mar}-1987$ & DIETHYLPHTHAIATE & $<10$ & ug/L \\
\hline SD- 104 & 11-Mar-1987 & DIETHYLPHTHALATE & $<12$ & $\mathbf{u g} / \mathrm{L}$ \\
\hline SD- 104 & 12-May-1987 & DIETHYLPKTHALATE & $<10$ & $\mathbf{u g} / \mathrm{L}$ \\
\hline$S D-104$ & $26-$ Мay-1987 & DIETHYLPHTHALATE & $<5.0$ & ug/L \\
\hline SD- 104 & 5-Mar-1987 & DIMETHYLPHTHALATE & $<10$ & $\mathbf{u g} / \mathrm{L}$ \\
\hline SD- 104 & 11-Mar-1987 & DIMETHYLPYTHALATE & $<12$ & $\lg / \mathrm{L}$ \\
\hline$S D-104$ & $12-$ May - 1987 & DIMETHYLPHTHALATE & $<10$ & $\mathbf{u g} / \mathrm{L}$ \\
\hline SD -104 & $26-$ Kay -1987 & DIMETHYLPHTHALATE & $<5.0$ & $\mathrm{ug} / \mathrm{L}$ \\
\hline SD-104 & $5-\operatorname{Mar}-1987$ & DISSOLYED OXYGER & $9.0 \mathrm{ppm}$ & \\
\hline SD- 104 & $11-\operatorname{Mar}-1987$ & DISSOLVED OXYGEN & $11.3 \mathrm{ppr}$ & \\
\hline SD -104 & $18-$ Mar -1987 & DISSOLVED OXYGEN & 7.2 ppm & \\
\hline
\end{tabular}


Storm Drala Data for SD-100-04

\begin{tabular}{rrl} 
Location & \multicolumn{1}{c}{ Date } & \multicolumn{1}{c}{ Compound } \\
SD-104 & 24-Mar-1987 & DISSOLVED OXYGEN \\
SD-104 & 3-Apr-1987 & DISSOLVED OXYGEN \\
SD-104 & 6-Apr-1987 & DISSOLVED OXYGEN \\
SD-104 & 12 -May-1987 & DISSOLVED OXYGEN \\
SD-104 & 19 -May-1987 & DISSOLVED OXYGEN \\
SD-104 & 26 -May-1987 DISSOLVED OXYGEN \\
SD-104 & 3-Jun-1987 & DISSOLVED OXYGEN \\
SD-104 & 11-Jun-1987 DISSOLVED OXYGEN \\
SD-104 & 18-Jun-1987 DISSOLVED OXYGEN
\end{tabular}

Results Un1ts

$7.8 \mathrm{ppm}$

$7.5 \mathrm{ppm}$

$8.0 \mathrm{ppm}$

$7.5 \mathrm{ppm}$

$10.0 \mathrm{pPm}$

8.9 PPI

$8.3 \mathrm{ppm}$

6.5 ppm

$8.5 \mathrm{ppm}$

* SD-104-D

$\begin{array}{lrl}\text { SD-104-D } & 6 \text {-Apr-1987 DISSOLVED OXYGEN } \\ \text { SD-104-D } & \text { 19-MaY-1987 DISSOLVED OXYGEN }\end{array}$

$8.0 \mathrm{ppm}$

** SD-104

SD -104

SD -104

5-MaI-1987 ETHYLBENZENE

SD -104

11-Mar-1987 ETHYLBENZENE

SD-104

18-Mar-1987 ETHYLBENZFEV

SD- 104

24-Mar-1987 ETHTLBENZENE

SD -104

3-Apr-1987 ETHYLBENZENE

6-Apr-1987 ETHYLBENZENE

SD-104

12-May-1987 ETHYLBENZERE

SD -104

19-May-1987 ETHYLBENZENE

SD-104

26-May-1987 ETHYLBENZENE

SD- 104

3-Jur-1987 ETHYLBENZENE

SD -104

11-Jun-1987 ETHYLBENZENE

SD -104

18-Jun-1987 BTHY BENZENE

\begin{tabular}{|c|c|c|}
\hline \multicolumn{3}{|c|}{$\star$ * SD-104-D } \\
\hline SD-104-D & 6-Apr-1987 & ETHYLBENZENE \\
\hline$S D-104-D$ & 19- Мay-1987 & ETKYLBENZERE \\
\hline \multicolumn{3}{|l|}{$\star \star S D-104$} \\
\hline SD-104 & 5-Mar-1987 & FLORANTHENE \\
\hline SD-104 & 11-Kar-1987 & FLUORANTHENE \\
\hline SD-104 & 12- May- 1987 & FLUORANTHERE \\
\hline SD- 104 & 26-May-1987 & FLUORANTHENE \\
\hline SD- 104 & S-Mar- 1987 & FLUORENE \\
\hline SD- 104 & $11-\operatorname{Mar}-1987$ & FLUYORENE \\
\hline SD- 104 & 12-May-1987 & FLUORENE \\
\hline SD-104 & 26-May-1987 & FLUORENE \\
\hline SD- 104 & 5-Kar - 1987 & FLUORIDB \\
\hline SD-104 & 11-Mar- 1987 & FLUORIDE \\
\hline SD- 104 & 18 -Mar- 1987 & FLUORIDE \\
\hline SD-104 & $24-\mathrm{Mar}+1987$ & FLUORIDE \\
\hline SD -104 & 3-Apr-1987 & FLUORIDE \\
\hline SD -104 & 6 -Арт-1987 & FLUORIDE \\
\hline SD. 104 & 12 - May - 1987 & FLUORIDE \\
\hline SD-104 & 19-May-1987 & FLUORIDE \\
\hline SD -104 & $26-$ May -1987 & FLUORIDE \\
\hline SD-104 & 3-Jun-1987 & FUUORIDE \\
\hline
\end{tabular}

$10.0 \mathrm{ppm}$

$\begin{array}{ll}<5 & u g / L \\ <5 & u g / L \\ <5 & u g / L \\ <5 & u g / L \\ <5 & u g / L \\ <5 & u g / L \\ <5 & u g / L \\ <5 & u g / L \\ <5 & u g / L \\ <5 & u g / L \\ <5 & u g / L \\ <5 & u g / L\end{array}$

$<5 \quad$ ug/t

$<$ ug/L

$<10$ ug/L

$<12$ ug/L

$<10 \quad u g / L$

$<5.0 \quad$ ug/L

$<10$ ug/L

$<12$ ug/L

$<10 \quad$ ug/L

$<5.0$ ug/L

$0.1 \mathrm{mg} / \mathrm{L}$

$.0 .1 \mathrm{mg} / \mathrm{L}$

$0.1 \mathrm{mg} / \mathrm{L}$

$0.1 \mathrm{mg} / \mathrm{L}$

$0.6 \mathrm{mg} / \mathrm{L}$

$0.1 \mathrm{mg} / \mathrm{L}$

$<0.1 \mathrm{mg} / \mathrm{L}$

$<0.1 \mathrm{mg} / \mathrm{L}$

$0.1 \mathrm{mg} / \mathrm{L}$

$<0.1 \mathrm{mg} / \mathrm{L}$ 
Storm Drain Data for SD-100-04

\begin{tabular}{|c|c|c|c|c|}
\hline Location & Date & Coupound & Results & Units \\
\hline SD- 104 & $11-J$ un - 1987 & FLUORIDE & $<0.1$ & $\mathrm{mg} / \mathrm{L}$ \\
\hline SD- 104 & $18-J$ un -1987 & FLUORIDE & $<0.1$ & $\mathrm{mg} / \mathrm{L}$ \\
\hline \multicolumn{5}{|c|}{$\star \quad$ SD-104-D } \\
\hline SD-104-D & 6-ApI-1987 & FLOORIDE & 0.1 & $\mathrm{mg} / \mathrm{L}$ \\
\hline SD-104-D & 19-May-1987 & FLUORIDE & $<0.1$ & $m g / L$ \\
\hline \multicolumn{5}{|l|}{ * SD-104 } \\
\hline SD-104 & $5-$ Kar-1987 & HARDNESS & 132 & $\mathbf{m g} / \mathrm{L}$ \\
\hline SD- 104 & 11-Max-1987 & HARDKESS & 123 & $m \mathrm{~g} / \mathrm{L}$ \\
\hline SD-104 & - 18-Mar - 1987 & HARDNESS & 130 & $\operatorname{mg} / \mathrm{L}$ \\
\hline SD-104 & 24-Mar-1987 & HARDNESS & 186 & $\mathrm{mg} / \mathrm{L}$ \\
\hline SD- 104 & 3-Apr-1987 & HARDNESS & 114 & mg $/ \mathrm{L}$ \\
\hline SD-104 & 6-Apr-1987 & HARDNESS & 114 & $\mathrm{ng} / \mathrm{L}$ \\
\hline SD-104 & 12- Kay - 1987 & HARDNESS & 164 & $\mathrm{mg} / \mathrm{L}$ \\
\hline SD- 104 & $19-$ May - 1987 & HARDNESS & 125. & $\operatorname{mg} / \mathrm{L}$ \\
\hline SD- 104 & 26-Hay- 1987 & HARDNESS & 132 & $\mathrm{ng} / \mathrm{L}$ \\
\hline SD-104 & 3-Jun-1987 & HARDNESS & 142 & $\mathrm{ng} / \mathrm{L}$ \\
\hline SD-104 & $11-J u n-1987$ & HARDNESS & 127 & $\mathbf{n g} / \mathrm{L}$ \\
\hline SD-104 & $18-J$ un -1987 & HARDNESS & 127 & $\mathrm{ng} / \mathrm{L}$ \\
\hline
\end{tabular}

\begin{tabular}{|c|c|c|c|c|}
\hline \multicolumn{5}{|c|}{ * SD-104-D } \\
\hline SD-104-D & 6-Apr-1987 & HARDNESS & 114 & $\mathrm{mg} / \mathrm{L}$ \\
\hline SD-104-D & 19-May-1987 & HARDNESS & 125 & $\mathrm{mg} / \mathrm{L}$ \\
\hline \multicolumn{5}{|l|}{$\star$ SD. 104} \\
\hline SD-104 & 5-Mar-1987 & HEXACHLOROBEYZENE & $<10$ & $u_{g} / 2$ \\
\hline SD-104 & 11-Mar-1987 & HEXACHLOROBENZENE & $<12$ & $u g / L$ \\
\hline SD-104 & 12-May-1987 & HEXACHLOROBETZENE & $<10$ & $\mathrm{ug} / \mathrm{L}$ \\
\hline SD-104 & $26-$ Hay -1987 & HEXACHLOROBENZENE & $<5.0$ & $4 \mathrm{~g} / \mathrm{L}$ \\
\hline SD- 104 & 5-Kar-1987 & HEXACHLOROBUTADIENE & $<10$ & ug/L \\
\hline SD-104 & 11-Mar-1987 & HEXACHLOROBUTADIENE & $<12$ & ug/L \\
\hline SD- 104 & 12-May-1987 & HEXAGHLOROBUTADIENE & $<10$ & $48 / 2$ \\
\hline SD-104 & $26-$ Kay -1987 & HEXACHLOROBUTADIERE & $<.0$ & ug/h \\
\hline SD-104 & 5-Mar-1987 & HEXACHLOROCYCLOPENTADIEN & $<10$ & ug/L \\
\hline SD-104 & $11-$ Mar -1987 & HEXACHLOROCYCLOPENTADIEN & $<12$ & $4 \mathrm{~g} / \mathrm{L}$ \\
\hline SD-104 & 12-Kay-1987 & HEXACHLOROCYGLOPENTADIEN & $<10$ & $48 / \mathrm{L}$ \\
\hline SD-104 & $26-$ May-1987 & HEXACHLOROCYCLOPENTADIEN & $<5.0$ & $\operatorname{ug} / \mathrm{L}$ \\
\hline SD -104 & $5-\operatorname{Mar}-1987$ & HEXACHLOROETHANE & $<10$ & ug $/ \mathrm{L}$ \\
\hline SD-104 & 11 - Mar - 1987 & HEXACHLOROETHANE & $<12$ & $\mathrm{ug} / \mathrm{L}$ \\
\hline SD-104 & 12-May-1987 & HEXACHLOROETHANE & $<10$ & ug $/ \mathrm{L}$ \\
\hline SD-104 & $26-$ May -1987 & HEXACHLOROETHANE & $<5.0$ & $48 / \mathrm{L}$ \\
\hline SD -104 & 5-Mar-1987 & INDENO(1 $\left.\begin{array}{lll}1 & 2 & 3-C D\end{array}\right)$ PYRENE & $<10$ & ug/L $/ \mathrm{L}$ \\
\hline SD-104 & $11-$ Mar-1987 & INDENO(1 2 ( 3-CD) PYRENE & $<12$ & ug/L $/ \mathrm{L}$ \\
\hline SD-104 & 12-Kny - 1987 & INDENO(1 2 3-CD) PYRENE & $<10$ & $u g / L$ \\
\hline SD-104 & 26-May- 1987 & INDENO(1 23 -CD) PYRENE & $<5.0$ & ug $/ 2$ \\
\hline SD-104 & 5-Mar-1987 & IRON & 0.11 & $\mathrm{gg} / \mathrm{L}$ \\
\hline SD -104 & $11-$ Mar-1987 & IRON & 0.15 & $\mathbf{n g} / \mathrm{L}$ \\
\hline SD - 104 & $18-\operatorname{Mar}-1987$ & IRON & 0.080 & $=\mathrm{g} / \mathrm{L}$ \\
\hline SD-104 & 24- $\mathrm{Mar}-1987$ & IRON & 1.8 & $\mathrm{ng} / \mathrm{L}$ \\
\hline
\end{tabular}


Storm Draín Data for SD-100-04

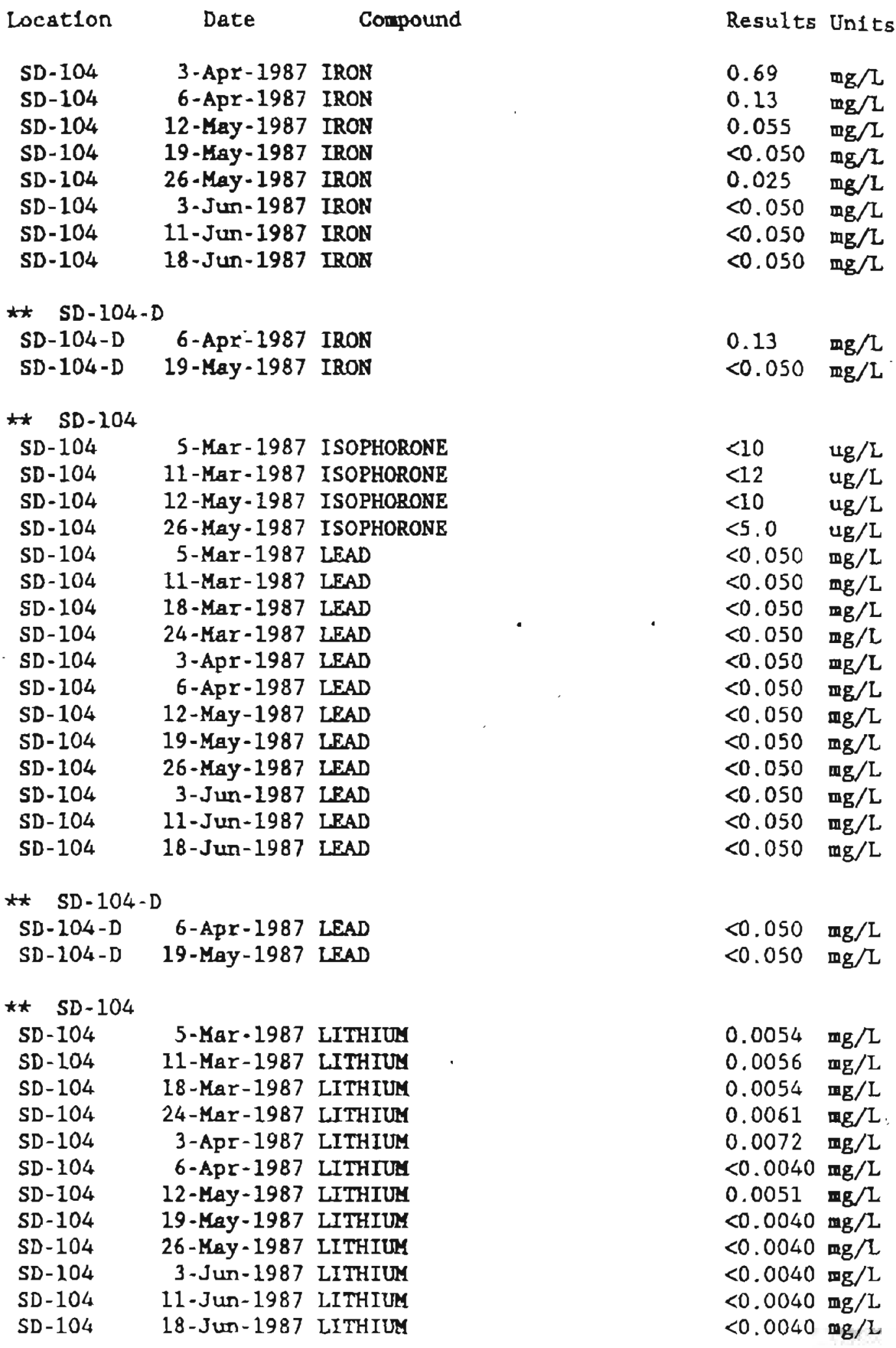


Storm Drain Data for SD-100-04

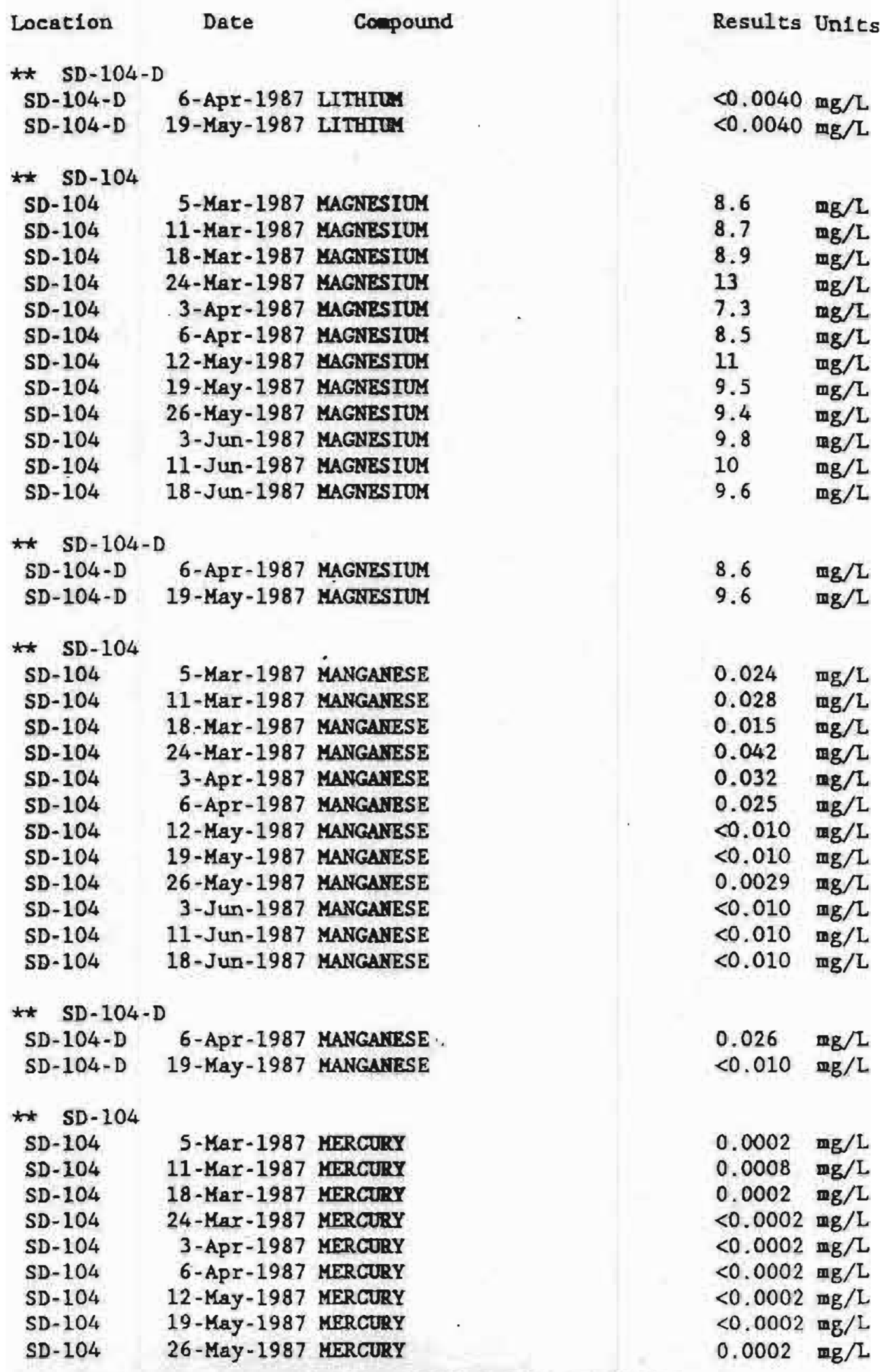


Storm Drain Data for SD-100-04

\begin{tabular}{|c|c|c|c|c|}
\hline Location & Date & Compound & Results & Units \\
\hline SD. 104 & 3-Jun- 1987 & MERCURY & $<0.0002$ & $\mathrm{mg} / \mathrm{L}$ \\
\hline SD-104 & 11-Jun-1987 & MERCURY & $<0.0002$ & $\mathrm{mg} / \mathrm{l}$ \\
\hline SD-104 & $18-J u n-1987$ & MERCURY & $<0.0002$ & $\operatorname{mg} / \mathrm{L}$ \\
\hline \multicolumn{5}{|c|}{ ** SD-104-D } \\
\hline SD-104-D & 6-Apr-1987 & LERCURY & $<0.0002$ & $\mathrm{mg} / \mathrm{L}$ \\
\hline SD-104-D & $19-\mathrm{May}-1987$ & MERCURY & $\infty 0.0002$ & $\mathrm{mg} / \mathrm{L}$ \\
\hline \multicolumn{5}{|l|}{ ** SD-104 } \\
\hline SD-104 & 5-Mar- 1987 & METHYLEME CHLORIDE & 5 & $\mathrm{ug} / \mathrm{I}$ \\
\hline SD-104 & 11-Mar- 1987 & MEIHYLENE CHLORIDE & $<5$ & $4 g / L$ \\
\hline SD-104 & $18 \cdot \operatorname{Mar}-1987$ & METHYLENE CHLORIDE. & 36 & $\operatorname{ug} / \mathrm{L}$ \\
\hline SD-104 & 24-Mar- 1987 & METHYLATE GHLORIDE & $\delta$ & $u g / L$ \\
\hline SD-104 & 3-APr-1987 & METHYLSAE CHLORIDE & 10 & $u g / h$ \\
\hline SD-104 & 6-Apr-1987 & METHYLENE CHLORIDE & $5 \mathrm{~J}$ & $4 g / 1$ \\
\hline SD-104 & 12-May- 1987 & METHYLENE CHLORIDE & $<5$ & $\mathrm{ug} / \mathrm{L}$ \\
\hline SD-104 & 19-May-1987 & LEIHYLEAV CHLORIDE & 14 & $\mathrm{ug} / \mathrm{L}$ \\
\hline SD-104 & 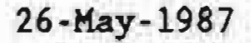 & METHYLENE CHLORIDE & 16 & $\mathrm{ug} / \mathrm{L}$ \\
\hline SD -104 & 3-Jun-1987 & METHYLENE CHLORIDE & 46 & $\mathrm{ug} / \mathrm{L}$ \\
\hline SD-104 & 11-Jun-1987 & METHYLENE CHLORIDE & 20 & ug $/ \mathrm{L}$ \\
\hline SD-104 & 18 -Jun- 1987 & METHYLENE CHLORIDE & 34 & ug/L \\
\hline \multicolumn{5}{|c|}{$\star \star \quad S D-104-D$} \\
\hline SD-104-D & 6-Apr-1987 & METHYLENE CHLORIDE & $<5$ & $4 g / L$ \\
\hline SD-104-D & 19-Kay - 1987 & METHYLENE CHLORIDE & 13 & $\mathrm{ug} / \mathrm{L}$ \\
\hline \multicolumn{5}{|l|}{ ** SD -104} \\
\hline SD-104 & 5-Mar-1987 & MOLYBDENUM & $<0.010$ & $\operatorname{mg} / \mathrm{L}$ \\
\hline SD-104 & $11-$ Kar- 1987 & MOLYBDENUY & $\infty 0.010$ & $\mathrm{mg} / \mathrm{L}$ \\
\hline SD-104 & 18-Mar-1987 & MOLYBDENUM & $<0.010$ & $\mathrm{mg} / \mathrm{L}$ \\
\hline SD-104 & 24-Kar-1987 & MOLYBDEAUM & $<0.010$ & mg/L \\
\hline SD-104 & 3-Apr-1987 & MOLYBDENOM & $<0.010$ & $\mathrm{mg} / \mathrm{L}$ \\
\hline SD- 104 & 6 -Apr- 1987 & MOLYBDEANUK & $<0.010$ & $\mathrm{mg} / \mathrm{L}$ \\
\hline SD-104 & 12- May $=1987$ & MOLYBDENUM & $<0.010$ & $\operatorname{mg} / \mathrm{L}$ \\
\hline SD-104 & 19-May-1987 & MOLYBDENUA & 0.016 & $\mathrm{mg} / \mathrm{L}$ \\
\hline SD-104 & $26-$ May -1987 & MOLYBDEIUUY & $<0.010$ & $\mathrm{mg} / \mathrm{L}$ \\
\hline SD-104 & 3-Jun-1987 & MOLYBDENOM & 0.023 & $\operatorname{ag} / 1$ \\
\hline SD-104 & 11-Jun-1987 & MOLYBDENUY & $<0.010$ & mg/L \\
\hline SD-104 & 18-Jun-1987 & MOLYBDENOM & $<0.010$ & $\operatorname{lng} / \mathrm{L}$ \\
\hline \multicolumn{5}{|c|}{$\star \star \quad S D-104-D$} \\
\hline SD-104-D & 6-Apr-1987 & MOLYBDENOK . & $<0.010$ & $\mathrm{mg} / \mathrm{L}$ \\
\hline$S D-104-D$ & 19 - May - 1987 & MOLYBDENOM & $<0.010$ & $\mathrm{mg} / \mathrm{L}$ \\
\hline \multicolumn{5}{|l|}{$\star$ SD-104 } \\
\hline SD-104 & 5-Mat-1987 & N-NIIROSO-DI-N-PROPYLAMINE & $<10$ & $\operatorname{Lg} / 1$ \\
\hline SD-104 & 11-Mar-1987 & N-NIIROSO-DI-N-PROPYLAMINE & $<12$ & $4 g / 2$ \\
\hline SD-104 & $12-\mathrm{May}-1987$ & N-NITROSO-DI -NT-PROPYLAMINE & $<10$ & ug $/ \mathrm{L}$ \\
\hline SD-104 & 26- - Маy-1987 & N-NITROSO-DI -N - PROPYLAMINE & $<5.0$ & ug/L \\
\hline SD-104 & $5-\operatorname{Mar}-1987$ & N-NITROSODIMETHYLAMINE & $<10$ & ug/L \\
\hline SD-104 & 11-Mar-1987 & N-NITROSODIMETHYLAMINE & $<12$ & $\operatorname{ug} / \mathrm{L}$ \\
\hline SD -104 & $12-\mathrm{Kay}-1987$ & N-NITROSODIMETHYLAMINE & $<10$ & $\mathrm{ug} / \mathrm{L}$ \\
\hline SD -104 & $26-\mathrm{May}-1987$ & N-NITROSODIMETHYLAMINE & $<.0$ & $\mathrm{ug} / \mathrm{L}$ \\
\hline SD -104 & $5-\operatorname{Mar}-1987$ & N-NITROSODIPHEVYLAMINE & $<10$ & $\mathrm{ug} / \mathrm{L}$ \\
\hline
\end{tabular}


Storm Drain Data for SD-100-04

\begin{tabular}{|c|c|c|c|c|}
\hline Location & Date & Compound & Results & Units \\
\hline SD-104 & 11 -Mar-1987 & N-NITROSODIPHENYLAKINE & $<12$ & $u g / L$ \\
\hline SD-104 & 12-May-1987 & N-NITROSODIPHENYLAMINE & $<10$ & ug/L \\
\hline SD- 104 & $26-$ - Мay -1987 & N-NITROSODIPHENYLAMINE & $<5.0$ & $\mathrm{ug} / \mathrm{L}$ \\
\hline$S D-104$ & 5-Mar-1987 & NAPHTHALERE & $<10$ & $\mathrm{ug} / \mathrm{L}$ \\
\hline SD-104 & 11 -Mar-1987 & NAPHTHALERE & $<12$ & ug/L \\
\hline SD-104 & 12- May-1987 & NAPHTHALENE & $<10$ & ug/L \\
\hline SO- 104 & 26-May-1987 & NAPHTHALENE & $<5.0$ & $\mathrm{ug} / \mathrm{L}$ \\
\hline SD- 104 & 5-Mar-1987 & NIGREL & $<0.050$ & $\mathrm{mg} / \mathrm{L}$ \\
\hline SD-104 & 11-Mar-1987 & NICKEL & $<0.050$ & $\operatorname{mg} / \mathrm{L}$ \\
\hline$S D-104$ & 18-Kar-1987 & NICKRL & $<0.050$ & $\mathbf{m g} / \mathrm{L}$ \\
\hline SD-104 & 24-Kar-1987 & NICKRL & $<0.050$ & $\mathrm{mg} / \mathrm{L}$ \\
\hline$S D-104$ & 3-Apr-1987 & NIGKEL & $<0.050$ & $\mathrm{mg} / \mathrm{I}$ \\
\hline SD-104 & 6 -Apr -1987 & NICKEL & $<0.050$ & $\operatorname{mg} / \mathrm{L}$ \\
\hline SD-104 & 12-May-1987 & NICKEL & $<0.050$ & $\mathbf{m g} / \mathrm{L}$ \\
\hline SD -104 & 19-Kay-1987 & NICKEL & $<0.050$ & $\mathrm{mg} / \mathrm{L}$ \\
\hline SD-104 & 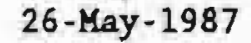 & NICKEL & $<0.010$ & $\mathrm{mg} / \mathrm{L}$ \\
\hline SD-104 & 3-Jun-1987 & NICKEL & $<0.050$ & $\mathrm{mg} / \mathrm{L}$ \\
\hline$S D-104$ & $11-J u n-1987$ & NIGKEL & $<0.050$ & $\mathbf{m g} / \mathrm{L}$ \\
\hline SD-104 & $18-J u n-1987$ & NICREL & $<0.050$ & $\mathrm{mg} / \mathrm{L}$ \\
\hline \multicolumn{5}{|c|}{ ** SD-104-D } \\
\hline SD-104-D & 6-Apr-1987 & NIGXEL & $<0.050$ & $\mathrm{mg} / \mathrm{L}$ \\
\hline SD-104-D. & 19-May-1987 & NICKEL & $<0.050$ & $\mathrm{mg} / \mathrm{L}$ \\
\hline \multicolumn{5}{|l|}{ ** SD-104 } \\
\hline SD-104 & $5-\operatorname{Mar}-1987$ & NIOBIUM & 0.0072 & $\mathrm{mg} / \mathrm{L}$ \\
\hline SD-104 & 11 - Kar -1987 & MIOBIOY & $<0.0070$ & $\mathrm{mg} / \mathrm{L}$ \\
\hline SD-104 & $28-\operatorname{Kan}-1987$ & NIOBIUK & $<0.0070$ & $\operatorname{mg} / \mathrm{L}$ \\
\hline SD-104 & $24-\mathrm{Mar}-1987$ & NIOBIUM & $<0.0070$ & $\mathrm{mg} / \mathrm{L}$ \\
\hline SD-104 & 3-Apr-1987 & NIOBIUK & $<0.0070$ & $\mathrm{mg} / \mathrm{L}$ \\
\hline SD-104 & 6-Apr-1987 & NIOBIUM & $<0.0070$ & $\mathrm{mg} / \mathrm{L}$ \\
\hline SD-104 & 12-May-1987 & NIOBIUM & $<0.0070$ & $\mathrm{mg} / \mathrm{L}$ \\
\hline SD-104 & 19-Мay-1987 & NIOBIUM & $<0.0070$ & $\mathrm{mg} / \mathrm{L}$ \\
\hline SD.104 & 26-May-1987 & NIOBIUM & $<0.0070$ & $\mathbf{m g} / \mathrm{L}$ \\
\hline SD-104 & 3 -Jun- 1987 & NIOBIUM & $<0.0070$ & $\mathrm{Igg} / \mathrm{L}$ \\
\hline SD -104 & $11-J u n-1987$ & NIOBIOM & $<0.0070$ & $\mathbf{m g} / \mathbf{L}$ \\
\hline SD-104 & $18-J$ un-1987 & NIOBIUK & $<0.0070$ & IIg/L \\
\hline \multicolumn{5}{|c|}{ ** SD-104-D } \\
\hline$S D-104-D$ & 6-Apr-1987 & NIOBIUM & $<0.0070$ & $\operatorname{mg} / \mathrm{L}$ \\
\hline SD-104-D & 19-May- 1987 & NIOBIUM & $<0.0070$ & mg $/ \mathrm{L}$ \\
\hline \multicolumn{5}{|l|}{ * SD-104 } \\
\hline SD-104 & $5-\operatorname{Mar}-1987$ & NITRATB. & 2.57 & $\mathrm{mg} / \mathrm{L}$ \\
\hline SD-104 & 11-Mar-1987 & NITRATE & 2.8 & $\mathrm{mg} / \mathrm{L}$ \\
\hline SD-104 & $18-\operatorname{Mar}-1987$ & NITRATE & 1.1 & $\mathrm{mg} / \mathrm{L}$ \\
\hline SD-104 & $24-\operatorname{Mar}-1987$ & NITRATE & 2.2 & $\mathrm{mg} / \mathrm{L}$ \\
\hline SD-104 & 3-Apr-1987 & NITRATE & 2.1 & $\mathrm{mg} / \mathrm{L}$ \\
\hline$S D-104$ & 6-Apr-1987 & NITRATE & 1.6 & $\operatorname{mg} / \mathrm{L}$ \\
\hline SD-104 & 12-May-1987 & NITRATE & 2.7 & $\mathrm{mg} / \mathrm{L}$ \\
\hline SD-104 & 19-May- 1987 & NITRATE & 2.2 & $\mathrm{mg} / \mathrm{L}$ \\
\hline SD-104 & 26-May - 1987 & NITRATE & 3.4 & 正 $/ \mathrm{L}$ \\
\hline SD-104 & 3-Jun-1987 & NITRATE & 2.7 & $\mathrm{mg} / \mathrm{L}$ \\
\hline SD-104 & $11-J u n-1987$ & NITRATE & 2.7 & $\mathrm{mg} / \mathrm{L}$ \\
\hline
\end{tabular}


Storm Drain Data for SD-100-04

\begin{tabular}{|c|c|c|c|c|}
\hline LOCAEION & Dete & Compound & Results & Units \\
\hline SD-104 & $18-J$ un- 1987 & NITRATE & 3.2 & $\mathbf{m g} / \mathrm{L}$ \\
\hline \multicolumn{5}{|c|}{ * SD-104-D } \\
\hline SD-104-D & 6-Apr-1987 & NITRATE & 1.6 & $\mathrm{mg} / \mathrm{L}$ \\
\hline$S D-104-D$ & 19-Hay- 1987 & NITRATE & 2.5 & $\mathrm{mg} / \mathrm{L}$ \\
\hline \multicolumn{5}{|l|}{ * SD-104 } \\
\hline SD- 104 & 5-Mar- 1987 & NITROBENZZENE & $<10$ & ug $/ \mathrm{L}$ \\
\hline SD-104 & 11- Mar- 1987 & NITROBENZERNE & $<12$ & $4 \mathrm{~g} / \mathrm{L}$ \\
\hline SD-104 & $12-$ May - 1987 & NITROBENZDENE & $<10$ & $u g / L$ \\
\hline SD- 104 & $26-$ May- 1987 & NITROBENZREN & $<.0$ & $u g / 2$ \\
\hline SD- 104 & 5-Mar -1987 & OIL \& GREASE & $<2$ & $\mathrm{mg} / \mathrm{L}$ \\
\hline SD-104 & 11- Max -1987 & OIL \& GREASE & $<2$ & $\mathrm{mg} / \mathrm{L}$ \\
\hline SD- 104 & 18 - Mar- 1987 & OIL \& GREASE & $<2$ & $\mathrm{mg} / \mathrm{L}$ \\
\hline SD- 104 & $24-$ Mar -1987 & OIL $\&$ GREASE & $<2$ & $\mathbf{m g} / \mathrm{L}$ \\
\hline SD-104 & 3-Apr-1987 & OIL \& GREASE & $<2$ & mg/L \\
\hline SD- 104 & 6-Apr-1987 & OIL \& GREASE & $<2$ & $\mathrm{mg} / \mathrm{L}$ \\
\hline SD-104 & 12-May - 1987 & OIL \& GREASE & $<2$ & $\mathrm{mg} / \mathrm{L}$ \\
\hline SD- 104 & $19-$ Мay- 1987 & OIL \& GREASE & $<2$ & $\mathrm{mg} / \mathrm{L}$ \\
\hline SD-104 & $26-\mathrm{May}-1987$ & OIL \& GREASE & $<2$ & $\mathrm{mg} / \mathrm{L}$ \\
\hline SD-104 & 3-Jun-1987 & OIL \& GREASE & $<2$ & $\mathrm{mg} / \mathrm{L}$ \\
\hline -SD- 104 & - 11-Jun-1987 & OIL \& GREASE & $\alpha$ & $\mathrm{mg} / \mathrm{L}$ \\
\hline SD- 104 & 18-Jun-1987 & OIL $\approx$ GREASE & $\alpha$ & $\operatorname{mog} / \mathrm{L}$ \\
\hline \multicolumn{5}{|c|}{ * SD-104-D } \\
\hline SD-104-D & 6-Apr-1987 & OIL \& GREASE & 2 & mg $/ \mathrm{L}$ \\
\hline SD-104-D & 19-May-1987 & OIL \& GREASE & $\alpha$ & $\mathbf{m g} / \mathbf{l}$ \\
\hline \multicolumn{5}{|l|}{ * SD- 104} \\
\hline SD-104 & $5-$ Mar- 1987 & PCB (AROCLOR-1016) & $<1$ & $\mathrm{ug} / \mathrm{L}$ \\
\hline SD-104 & 11-Mar- 1987 & PCB (AROCLOR - 1016) & INA & $u g / L$ \\
\hline SD-104 & 12-May- 1987 & PCB (AROCLOR - 1016) & $<0.5$ & $\mathrm{ug} / \mathrm{L}$ \\
\hline SD-104 & $26-\mathrm{May}-1987$ & PCB (AROCLOR - 1016) & $<0.5$ & $4 g / L$ \\
\hline$S D-104$ & 5-Mar-1987 & PCB (AROCLOR - 1221) & $<1$ & $\mathrm{ug} / \mathrm{L}$ \\
\hline$S D-104$ & $11-$ Mar-1987 & PCB (AROCLOR - 1221) & NA & $\mathrm{ug} / \mathrm{L}$ \\
\hline SD-104 & 12-May-1987 & PCB (AROCLOR-1221) & $<0.5$ & $4 g / L$ \\
\hline SD - 104 & 26-May- 1987 & PCB (AROCLOR-1221) & $<0.5$ & $\mathrm{ug} / \mathrm{L}$ \\
\hline SD -104 & 5 -Mar- 1987 & PCB (AROCLOR - 1232) & $<$ & $\mathrm{ug} / \mathrm{L}$ \\
\hline SD- 104 & 11- Мax-1987 & PCB (AROCLOR-1232) & NA & $\mathrm{ug} / \mathrm{L}$ \\
\hline SD-104 & 12-Мay-1987 & PCB (AROCLOR-1232) & $<0.5$ & $\mathrm{ug}_{2}$ \\
\hline SD-104 & 26-May-1987 & PCB (AROCLOR - 1232) & $<0.5$ & $u g / L$ \\
\hline SD- 104 & 5-Mar-1987 & PCB (AROCLOR - 1242) & $<1$ & $\mathrm{ug} / \mathrm{L}$ \\
\hline SD -104 & $11-\operatorname{Mar}-1987$ & PCB (AROCLOR - 1242) & MA & $u g / L$ \\
\hline SD-104 & 12-May-1987 & PCB (AROCLOR - 1242) & $<0.5$ & $u g / L$ \\
\hline SD- 104 & 26-May - 1987 & PCB (AROCLOR - 1242) & $<0.5$ & $\mathrm{ug} / \mathrm{L}$ \\
\hline SD- 104 & $5-\operatorname{Mar}-1987$ & PCB (AROCLOR - 1248) & $<1$ & $\mathrm{ug} / \mathrm{L}$ \\
\hline SD- 104 & 11-Mar-1987 & PCB (AROCLOR-1248) & NA & $4 \mathrm{~g} / \mathrm{L}$ \\
\hline SD -104 & 12-May-1987 & PCB (AROCLOR - 1248) & $<0.5$ & $u g / L$ \\
\hline SD-104 & $26-$ May- 1987 & PCB (AROCLOR - 1248) & $<0.5$ & ug/h \\
\hline SD - 104 & $5-\operatorname{Mar}-1987$ & PCB (AROCLOR-1254) & $<1$ & $\mathrm{ug} / \mathrm{L}$ \\
\hline
\end{tabular}


Storm Drain Data for SD-100-04

\begin{tabular}{|c|c|c|c|c|}
\hline Location & Date & Compound & Results & Units \\
\hline SD-104 & 11-Mar-1987 & PCB (AROCLOR-1254) & NA & $u g / L$ \\
\hline SD-104 & 12- Мay- 1987 & PCB (AROCLOR-1254) & $<1$ & $u g / L$ \\
\hline SD-104 & $26-$ May- 1987 & PCB (AROCLOR-1254) & $<1$ & $u g / L$ \\
\hline$S D-104$ & 5-Mar-1987 & PCB (AROCLOR-1260) & $<1$ & $\mathrm{ug} / \mathrm{L}$ \\
\hline SD-104 & $11-$ Mar -1987 & PCB (AROCLOR-1260) & $\mathbf{N A}$ & $1 \mathrm{~g} / \mathrm{L}$ \\
\hline SD-104 & 12-Мay-1987 & PCB (AROCLOR-1260) & $<1$ & ug/L \\
\hline SD-104 & 26-May-1987 & PCB (AROCLOR-1260) & $<1$ & $\mathrm{ug} / \mathrm{L}$ \\
\hline SD-104 & 5-Mar-1987 & PENTACHLOROPHEAOL & $<50$ & $\mathrm{ug} / \mathrm{L}$ \\
\hline SD- 104 & $11-$ Mar-1987 & PENTACHLOROPHENOL & $<59$ & $\mathrm{ug} / \mathrm{L}$ \\
\hline SD -104 & 12-May- 1987 & PEITACHLOROPHENOL & $<50$ & $\mathrm{ug} / \mathrm{L}$ \\
\hline SD-104 & 26-Кay- 1987 & PENTACHLOROPHEIYOL & $<25.0$ & $u g / L$ \\
\hline SD-104 & 5-Mar-1987 & PH & 7.5 & \\
\hline SD- 104 & 11-Mar-1987 & PH & 8.0 & \\
\hline SD-104 & $18-\mathrm{Mar}-1987$ & PH & $<1.0$ & \\
\hline SD-104 & 24-Mar-1987 & PH & 8.0 & \\
\hline SD-104 & 3-Apr-1987 & PH & 7.8 & \\
\hline SD-104 & 6-Apr-1987 & PH & B.1 & \\
\hline SD-104 & 12-May- 1987 & PH & 8.3 & \\
\hline SD- 104 & 19-May-1987 & PH & 8.2 & \\
\hline SD-104 & 26-May-1987 & PH & 8.1 & \\
\hline SD-104 & 3-Jun-1987 & PH & B. 3 & \\
\hline SD-104 & $11-J u n-1987$ & PH & 8.4 & \\
\hline SD -104 & $18 \cdot J u n-1987$ & PH & 8.6 & \\
\hline \multicolumn{5}{|c|}{$\star \star \quad S D-104-D$} \\
\hline SD-104-D & 6-Apr-1987 & PH & 8.1 & \\
\hline$S D-104-D$ & 19-Kay-1987 & PH & 7.8 & \\
\hline \multicolumn{5}{|l|}{$* *$ SD-104 } \\
\hline$S D-104$ & $5-$ Mar-1987 & PHENANTHRENE & $<10$ & ug/L \\
\hline SD- 104 & $11-$ Mar-1987 & PHENANTHRENE & $<12$ & $4 g / \mathrm{L}$ \\
\hline SD- 104 & $12-$-ay -1987 & PHENANTHRENE & $<10$ & $\mathrm{ug} / \mathrm{L}$ \\
\hline SD-104 & $26-$ Kay-1987 & PHENANTHRENE & $<5.0$ & $\mathrm{ug} / \mathrm{L}$ \\
\hline SD- 104 & 5 -Mar-1987 & PHENOL & $<10$ & $\mathrm{ug} / \mathrm{L}$ \\
\hline SD-104 & $11-\operatorname{Mar}-1987$ & PHENOI & $<12$ & ug/ \\
\hline SD-104 & 12-May-1987 & PHENOL & $<10$ & $\mathrm{ug} / \mathrm{L}$ \\
\hline SD-104 & 26-May-1987 & PHENOL & $<5.0$ & $\mathrm{Lg} / \mathrm{L}$ \\
\hline SD-104 & 5-Mar-1987 & PHOSPHORUS & 0.37 & $\mathrm{mg} / \mathrm{L}$ \\
\hline SD- 104 & $11-\mathrm{Max}-1987$ & PHOSPHORUS & 0.42 & $\mathrm{mg} / \mathrm{L}$ \\
\hline SD-104 & 18 -Mar-1987 & PHOSPHORUS & 0.88 & $\mathrm{mg} / \mathrm{L}$ \\
\hline SD-104 & 24-Mar -1987 & PHOSPHORUS & 0.30 & $\mathrm{mg} / \mathrm{L}$ \\
\hline SD-104 & 3-Apr-1987 & PHOSPHORUS & $<0.20$ & $\mathrm{mg} / \mathrm{L}$ \\
\hline SD- 104 & 6-Apr-1987 & PHOSPHORUS & 0.26 & $\mathrm{mg} / \mathrm{L}$ \\
\hline SD-104 & 12-May-1987 & PHOSPHORUS & 0.36 & $\mathrm{mg} / \mathrm{L}$ \\
\hline SD-104 & $19-$ May-1987 & PHOSPHORUS & 0.28 & $\mathrm{mg} / \mathrm{L}$ \\
\hline SD-104 & 26-May-1987 & PHOSPHORUS & 0.46 & $\mathrm{mg} / \mathrm{L}$ \\
\hline SD-104 & 3-Jun-1987 & PHOSPHORUS & 0.37 & $\mathrm{mg} / \mathrm{I}$ \\
\hline SD-104 & 11-Jun-1987 & PHOSPHORUS & 0.35 & $\mathrm{mg} / \mathrm{L}$ \\
\hline SD-104 & 18-Jun-1987 & PHOSPHORUS & 0.33 & $\mathrm{mg} / \mathrm{L}$ \\
\hline
\end{tabular}


Storm Drain Data for SD-100-04

Location

Date

Compound

** SD-104-D

SD-104-D

SD-104-D

6-Apr-1987 PHOSPHOROS

19-Kay-1987 PHOSPHORUS

** SD-104

SD-104

SD-104

SD- 104

SD-104

SD-104

SD- 104

SD-104

SD-104

SD-104

SD-104

SD-104

SD-104

5-Mar-1987 POTASSTUM

11-Mar-1987 POTASSIUA

18-KaT-1987 POTASSIUM

24-Mar-1987 POTASSIUM

3-Apr-1987 POTASSIUM

6-Apr-1987 POTASSIUM

12-May-1987 POTASSIUA

19-May-1987 POTASSIUM

26-Mey-1987 POTASSIUM

3-Jun-1987 POTASSIUR

11-Jun-1987 POTASSIUY

18-Jun-1987 POTASSIUM

** SD-104-D

$\begin{array}{lr}\text { SD-104-D } & \text { 6-Apr-1987 POTASSIUM } \\ \text { SD-104-D } & \text { 19-May-1987 POTASSIUI }\end{array}$

** SD-104

SD-104

SD- 104

SD- 104

SD-104

SD-104

SD-104

SD- 104

SD-104

SD-104

SD -104

SD-104

SD-104

SD- 104

SD- 104

SD- 104

SD-104
5-Mar-1987 PYRENE

11-Mar-1987 PYRENE

12-May-1987 PYRENE

26-May-1987 PYRENE

5-Mar-1987 SELENIUM

11-Mar-1987 SELENIUH

18-Mar-1987 SELENIJM

24-Mar-1987 SELENTUM

3-Apr-1987 SELENIUM

6-Apr-1987 SELENIUM

12-May-1987 SELENTUH

19- May-1987 SELENIUM

26-May-1987 SELENIUM

3-Jun- 1987 SELENIUM

11-Jun-1987 SELENIUM

18-JUR-1987 SELENIUL

** SD-104-D

SD-104-D 6-ApI-1987 SELENIUM

SD-104-D 19-May-1987 SELENIUM

$\begin{array}{crl}\text { ** SD-104 } & & \\ \text { SD-104 } & \text { 5-Mar-1987 } & \text { SILICON } \\ \text { SD-104 } & 11 \text {-Mar-1987 } & \text { SILICON } \\ \text { SD-104 } & 18 \text {-Mar-1987 } & \text { SILICON } \\ \text { SD-104 } & 24-\mathrm{Mar}-1987 & \text { SILICON } \\ \text { SD-104 } & \text { 3-Apr-1987 } & \text { SILICON }\end{array}$

Results Units

$0.22 \mathrm{mg} / \mathrm{L}$
$0.48 \mathrm{mg} / \mathrm{L}$

$1.5 \mathrm{mg} / \mathrm{L}$

$4.6 \mathrm{mg} / \mathrm{L}$

$2.0 \mathrm{mg} / \mathrm{L}$

$5.1 \mathrm{mg} / \mathrm{L}$

$1.7 \mathrm{mg} / \mathrm{L}$

$4.2 \mathrm{mg} / \mathrm{L}$

$<0.60 \mathrm{mg} / \mathrm{L}$

$2.0 \mathrm{mg} / \mathrm{L}$

$1.1 \mathrm{mg} / \mathrm{L}$

$1.7 \mathrm{mg} / \mathrm{L}$

$0.94 \mathrm{mg} / \mathrm{L}$

$2.7 \mathrm{mg} / \mathrm{L}$

$4.1 \mathrm{mg} / \mathrm{L}$

$1.9 \mathrm{mg} / \mathrm{L}$

$<10 \quad$ ug/L

$<12$ ug/L

$<10 \quad \mathrm{ug} / \mathrm{L}$

$<5.0 \quad \mathrm{ug} / \mathrm{L}$

$<0.005 \mathrm{mg} / \mathrm{L}$

$<0.005 \mathrm{mg} / \mathrm{h}$

$<0.005 \mathrm{mg} / \mathrm{L}$

$<0.005 \mathrm{mg} / \mathrm{L}$

$<0.005 \mathrm{mg} / \mathrm{L}$

$<0.005 \mathrm{mg} / \mathrm{L}$

$<0.005 \mathrm{mg} / \mathrm{L}$

$<0.005 \mathrm{mg} / \mathrm{L}$

$<0.005 \mathrm{mg} / \mathrm{L}$

$<0.005 \mathrm{mg} / \mathrm{L}$

$<0.005 \mathrm{mg} / \mathrm{L}$

$<0.005 \mathrm{mg} / \mathrm{L}$

$<0.005 \mathrm{mg} / \mathrm{L}$

$<0.005 \mathrm{mg} / \mathrm{L}$

$1.9 \mathrm{mg} / \mathrm{L}$

$1.8 \mathrm{mg} / \mathrm{L}$

$1.8 \mathrm{mg} / \mathrm{L}$

$3.4 \mathrm{mg} / \mathrm{L}$

$1.8 \mathrm{mg} / \mathrm{L}$ 
Storm Drain Data for SD-100-04

\begin{tabular}{|c|c|c|c|c|}
\hline Location & Dace & Compound & Results & Units \\
\hline$S D-104$ & 6-Apr -1987 & SILICON & 2.3 & $\operatorname{mg} / \mathrm{L}$ \\
\hline SD-104 & 12-May-1987 & SILICON & 1.6 & $\mathrm{mg} / \mathrm{L}$ \\
\hline SD -104 & 19-May - 1987 & SILICON & 1.5 & $\mathrm{mg} / \mathrm{L}$ \\
\hline SD-104 & 26-May-1987 & SILICON & 1.4 & $\mathrm{mg} / \mathrm{L}$ \\
\hline SD-104 & 3-Jun-1987 & SILICON & 1.5 & $\mathbf{m g} / \mathrm{L}$ \\
\hline SD -104 & $11-J u n-1987$ & SILICON & 1.4 & $\mathrm{mg} / \mathrm{L}$ \\
\hline SD-104 & $18-J u n-1987$ & SILICON & 1.6 & $\mathrm{mg} / \mathrm{L}$ \\
\hline \multicolumn{5}{|c|}{$\star \star \quad S D-104-D$} \\
\hline SD-104-D & 6-Apr-1987 & SILICON & 2.3 & $\mathrm{mg} / \mathrm{L}$ \\
\hline$S D-104-D$ & $19-$ May-1987 & SILICON & 1.5 & $\mathrm{mg} / \mathrm{L}$ \\
\hline \multicolumn{5}{|l|}{$\star \quad$ SD- 104} \\
\hline SD - 104 & 5-Mar-1987 & SILVER & $<0.010$ & $\mathrm{mg} / \mathrm{L}$ \\
\hline SD- 104 & $11-\operatorname{Mar}-1987$ & SILVER & 0.043 & $\mathrm{mg} / \mathrm{L}$ \\
\hline$S D-104$ & 18-Mar - 1987 & SILVER & $<0.010$ & $\mathrm{mg} / \mathrm{L}$ \\
\hline SD- 104 & $24-$ Mar -1987 & SILVER & $<0.010$ & $\mathrm{mg} / \mathrm{L}$ \\
\hline SD- 104 & 3-Арг-1987 & SILVER & $<0.010$ & $\mathrm{mg} / \mathrm{L}$ \\
\hline SD-104 & 6-Apr-1987 & SILVER & $<0.010$ & mg/L \\
\hline SD -104 & 12-May - 1987 & SILVER & $<0.010$ & $\mathrm{mg} / \mathrm{L}$ \\
\hline SD-104 & $19-$ May -1987 & SILVER & $<0.010$ & $\mathrm{mg} / \mathrm{l}$ \\
\hline SD- 104 & $26-$ May -1987 & SILVER & $<0.0060$ & $\mathrm{mg} / \mathrm{L}$ \\
\hline SD -104 & 3-Jun-1987 & SILVER & $<0.010$ & ming $/ \mathrm{L}$ \\
\hline$S D \cdot 104$ & $11-J$ un- 1987 & SILVER & $<0.010$ & $\operatorname{mg} / \mathrm{L}$ \\
\hline SD-104 & $18-J u n-1987$ & SILVER & $<0.010$ & $\log / \mathrm{L}$ \\
\hline \multicolumn{5}{|c|}{$\star S D-104-D$} \\
\hline$S D-104-D$ & 6-Apr-1987 & SILVER & $<0.010$ & $\mathrm{mg} / \mathrm{L}$ \\
\hline$S D-104-D$ & $19-$ May - 1987 & SILVER & $<0.010$ & mg/L \\
\hline \multicolumn{5}{|l|}{ ** SD-104 } \\
\hline SD-104 & 5-Mar-1987 & SODIUM & 6.9 & mg/L \\
\hline SD- 104 & 11-Kar-1987 & SODIUM & 8.7 & 표 $/ \mathrm{L}$ \\
\hline SD - 104 & $18-\operatorname{Mar}-1987$ & SODIUM & 7.2 & $\mathrm{mg} / \mathrm{L}$ \\
\hline -SD - 104 & $24-\operatorname{Mar}-1987$ & SODIUM & 75 & $\mathbf{m g} / \mathrm{L}$ \\
\hline$S D-104$ & $3-\mathrm{Apr}-1987$ & SODIUM & 380 & $\mathrm{mg} / \mathrm{L}$ \\
\hline SD -104 & 6-Apr-1987 & SODIUM & 6.0 & $\operatorname{mg} / \mathrm{L}$ \\
\hline$S D-104$ & 12 - -аay - 1987 & SODIUM & 6.6 & $\mathrm{mg} / \mathrm{L}$ \\
\hline SD - 104 & 19-May - 1987 & SODIUM & 6.4 & $\mathrm{mg} / \mathrm{l}$ \\
\hline SD - 104 & $26-$ Kay -1987 & SODIUM & 5.8 & $\mathbf{m g} / \mathrm{L}$ \\
\hline SD- 104 & 3-Jun-1987 & SODIUM & 5.7 & $\operatorname{mg} / \mathrm{L}$ \\
\hline SD- 104 & $11-J u n-1987$ & SODIUM & 5.7 & $m \mathrm{~g} / \mathrm{L}$ \\
\hline$S D-104$ & $18-J u n-1987$ & SODIUM & 5.8 & $\operatorname{lng} / \mathrm{L}$ \\
\hline \multicolumn{5}{|c|}{$\star \star S D-104-D$} \\
\hline SD-104-D & 6-Apr-1987 & SODIUM & 6.0 & $=g / L$ \\
\hline$S D-104-D$ & 19 - May - 1987 & SODIUM & 6.5 & $\mathrm{mg} / \mathrm{L}$ \\
\hline \multicolumn{5}{|l|}{$\star \star \quad S D-104$} \\
\hline SD - 104 & 5-Mar-1987 & STRONTIUM & 0.073 & $\operatorname{mg} / \mathrm{L}$ \\
\hline
\end{tabular}


Storm Drain Data for SD-100-04

\begin{tabular}{|c|c|c|c|c|}
\hline Location & Date & Compound & Results & Untes \\
\hline SD-104 & 11-Mar-1987 & STRONTIUM & 0.072 & $\mathrm{mg} / \mathrm{L}$ \\
\hline SD-104 & 18-MaI- 1987 & STRONTIUM & 0.083 & $\mathrm{mg} / \mathrm{L}$ \\
\hline SD -104 & $24-$ Kar - 1987 & STRONIIUA & 0.085 & $\mathrm{mg} / \mathrm{L}$ \\
\hline SD - 104 & 3-Apr-19B7 & STRONTTUK & 0.092 & $\mathrm{mg} / \mathrm{L}$ \\
\hline SD-104 & 6-Apr-1987 & STRONTIUM & 0.061 & $\mathrm{mg} / \mathrm{I}$ \\
\hline SD-104 & 12-Kay - 1987 & STRONIIUK & 0.069 & $\mathrm{mg} / \mathrm{l}$ \\
\hline SD-104 & $19-\mathrm{May}-1987$ & STRONIIUY & 0.067 & $\operatorname{mg} \Omega$ \\
\hline SD-104 & $26-\mathrm{May}-1987$ & STRONTTUY & 0.076 & $\mathrm{mg} / \mathrm{L}$ \\
\hline SD-104 & 3-Jun-1987 & STRONTIUM & 0.071 & $\mathrm{mg} / \mathrm{L}$ \\
\hline SD-104 & $11-J u n-1987$ & STRONTTUM & 0.075 & $\mathrm{mg} / \mathrm{L}$ \\
\hline SD-104 & $18-J$ un -1987 & STRONTIUM & 0.068 & $\mathrm{mg} / \mathrm{L}$ \\
\hline \multicolumn{5}{|c|}{$* \star S D \cdot 104-D$} \\
\hline SD-104-D & 6-Apr-1987 & STRONTIUM & 0.061 & $\mathrm{mg} / \mathrm{L}$ \\
\hline SD-104-D & 19-May - 1987 & STRONTIUM & 0.068 & mg/L \\
\hline \multicolumn{5}{|l|}{$x * \quad S D-104$} \\
\hline SD-104 & $5-\operatorname{Mar}-1987$ & SULFATE & 28 & $\mathrm{mg} / \mathrm{L}$ \\
\hline SD-104 & 11-Mar-1987 & SULFATE & 49 & $\operatorname{mg} / \mathrm{L}$ \\
\hline SD- 104 & $18-\operatorname{Mar}-1987$ & SULFAIE & 30.3 & $\mathrm{mg} / \mathrm{L}$ \\
\hline SD- 104 & 24-Mar-1987 & SULFAIE & 66 & $\mathrm{mg} / \mathrm{L}$ \\
\hline$S D \cdot 104$ & 3-Apr-1987 & SULFATE & 18 & $\mathrm{mg} / \mathrm{L}$ \\
\hline SD- 104 & 6-Apr-1987 & SULFATE & 23 & $\mathrm{Ig} / \mathrm{L}$ \\
\hline SD- 104 & 12-May-1987 & SULFATE & 26 & $\operatorname{mg} / \mathrm{L}$ \\
\hline SD-104 & 19-May-1987 & SULFATE & 27 & $\mathrm{mg} / \mathrm{L}$ \\
\hline SD-104 & $26-$ - Lay -1987 & SULFATE & 28.1 & $\mathrm{mg} / \mathrm{L}$ \\
\hline SD -104 & 3-Jun-1987 & SULFATE & 25 & $\mathrm{mg} / \mathrm{L}$ \\
\hline SD-104 & 11-Jun-1987 & SULFATE & 28 & $\mathrm{mg} / \mathrm{L}$ \\
\hline SD-104 & $18-J$ un - 1987 & SULFATE & 27 & $\mathrm{mg} / \mathrm{L}$ \\
\hline \multicolumn{5}{|c|}{$\star * \quad S D-104-D$} \\
\hline SD-104-D & $6-A p r-1987$ & SULFATE & 23 & $\mathbf{n g} / \mathrm{L}$ \\
\hline$S D=104-D$ & $19-$ May-1987 & SULFATE & 27 & $\mathrm{mg} / \mathrm{L}$ \\
\hline \multicolumn{5}{|l|}{ * SD -104} \\
\hline SD-104 & $5-\operatorname{Mar}-1987$ & TEMPERATURE & 13.0 & Deg. \\
\hline SD-104 & $11-\operatorname{Mar}-1987$ & TEMPERATURE & 11.0 & Deg. \\
\hline SD-204 & $18-\operatorname{Mar}-1987$ & TEMPERATURE & 12.0 & Deg. \\
\hline SD-104 & 24-Mar-1987 & TEMPERATURE & 13.0 & Deg. \\
\hline SD- 104 & 3-Apr-1987 & TEMPERATURE & 11.0 & Deg. \\
\hline SD-104 & 6-Apr-1987 & TEMPERATURE & 11.0 & Deg. \\
\hline SD-104 & 12-May-1987 & TEMPERATURE & 20.0 & Deg. \\
\hline SD-104 & 19-Mzy-1987 & TEMPERATURE & 21.0 & Deg. \\
\hline SD-104 & 26 - May-1987 & TEMPERATURE & 22.0 & Deg. \\
\hline SD- 104 & 3-Jun-1987 & TEMPERATURE & 22 & Deg. \\
\hline SD-104 & 11-Jun-1987 & TEMPERATURE & 22 & Deg. \\
\hline SD-104 & $18-J u n-1987$ & TEMPERATURE & 23.0 & Deg. \\
\hline \multicolumn{5}{|c|}{$* \star S D-104-D$} \\
\hline SD $-104-D$ & 6-Apr-1987 & TEMPERATURE & 11.0 & Deg. \\
\hline$S D-104-D$ & 19-May - 1987 & TEMPERATURE & 21.0 & Deg. \\
\hline
\end{tabular}


Storm Drain Data for SD-100-04

\begin{tabular}{|c|c|c|c|c|}
\hline Locacton & Date & Conpound & Results & Units \\
\hline$\star \star \quad S D-104$ & & & & \\
\hline SD-104 & 5-Mar-1987 & TETRACHLOROETHENE & $<$ & $u g / L$ \\
\hline SD-104 & 11-Mar-1987 & TETRACHLOROETHENE & $<5$ & $4 \mathrm{~g} / \mathrm{L}$ \\
\hline SD - 104 & 18-Kar-1987 & TETRACHLOROETHENE & $<5$ & ug $/ \mathrm{L}$ \\
\hline SD- 104 & $24-\operatorname{Mar}-1987$ & TEIRACHLOROETHENE & $<5$ & $\mathrm{ug} / \mathrm{L}$ \\
\hline SD-104 & 3-Арг-1987 & TETRAGILOROETHENS & $<$ & $\mu \mathrm{g} / \mathrm{L}$ \\
\hline SD-104 & 6-Apr-1987 & TETRACHLOROETHENE & $<5$ & $\mathrm{ug} / \mathrm{L}$ \\
\hline SD-104 & 12- - Lay-1987 & TETRACKLOROETHERE & $<5$ & $4 \mathrm{~g} / \mathrm{L}$ \\
\hline SD-104 & 19-May-1987 & TETRACHLOROETHENE & $<5$ & $u g / L$ \\
\hline SD-104 & $26-$-ay -1987 & TETRACHLOROETHETIE & $<5$ & $\mathrm{ug} / \mathrm{L}$ \\
\hline SD-104 & 3-Jun-1987 & TETRACHLOROETHENE & $<5$ & ug/L \\
\hline SD -104 & 11-Jun-1987 & TETRAGHLOROETHENE & $<5$ & $\mathrm{ug} / \mathrm{L}$ \\
\hline SD-104 & $18-J u n-1987$ & TETRACHLOROETHENE & $<5$ & $\mathrm{ug} / \mathrm{L}$ \\
\hline$\star \star \quad S D-104$ & & & & \\
\hline SD-104-D & 6-Apr-1987 & TETRACHLOROETHENE & $<5$ & $u g / L$ \\
\hline$S D-104-D$ & 19-May-1987 & TETRACHLOROETHENE & $<S$ & $\mathrm{ug} / \mathrm{L}$ \\
\hline$\star \star$ SD -104 & & & & \\
\hline SD-104 & $5-\operatorname{Max}-1987$ & THORIUI & $<0.20$ & mg $/ \mathrm{L}$ \\
\hline SD - 104 & $11-\operatorname{Max}-1987$ & THORTUM & $<0.20$ & $\mathrm{mg} / \mathrm{L}$ \\
\hline SD- 104 & $18-\operatorname{Mar}-1987$ & THORIUM & $<0.20$ & $\mathrm{mg} / \mathrm{L}$ \\
\hline SD-104 & 24-Mar-1987 & THORIUM & $<0.20$ & $\mathrm{mg} / \mathrm{L}$ \\
\hline SD -104 & 3-Apr-1987 & THORIUM & $<0.20$ & $\operatorname{mg} / \mathrm{L}$ \\
\hline SD-104 & 6-Apr-1987 & THORIUM & $<0.20$ & $\operatorname{mg} / L$ \\
\hline SD-104 & 12-Мay-1987 & THORIOM & $<0.20$ & $\operatorname{mg} / \mathrm{I}$ \\
\hline SD-104 & 19-May-1987 & THORIUM & $<0.20$ & $\operatorname{mg} / \mathrm{L}$ \\
\hline SD-104 & 26-May-1987 & THORIUM & $<0.20$ & $\mathrm{mg} / \mathrm{I}$ \\
\hline SD-104 & 3-Jun-1987 & THORIUM & $<0.20$ & $\mathrm{mg} / \mathrm{L}$ \\
\hline SD-104 & $11-J u n-1987$ & THORIUM & $<0.20$ & $\mathrm{mg} / \mathrm{L}$ \\
\hline SD 104 & $18-J u n-1987$ & THORIUM & $<0.20$ & $\mathrm{mg} / \mathrm{L}$ \\
\hline$\star \star$ SD-104 & & & & \\
\hline SD-104-D & 6-Apr-1987 & THORIUM & $<0.20$ & $\mathrm{mg} / \mathrm{L}$ \\
\hline SD-104-D & $19-$ May-1987 & THORIUM & $<0.20$ & $\mathrm{mg} / \mathrm{L}$ \\
\hline ** SD -104 & & & & \\
\hline SD -104 & 5-Max-1987 & TITANIUM & $<0.0030$ & $\operatorname{mg} / \mathrm{L}$ \\
\hline SD- 104 & 11 -Mar-1987 & TITANIUM & $<0.0030$ & $\mathrm{mg} / \mathrm{L}$ \\
\hline SD-104 & $18-\mathrm{Kar}=1987$ & TITANIUM & $<0.0030$ & $\mathbf{m g} / \mathrm{L}$ \\
\hline SD-104 & 24-Kar-1987 & TITANIUM & 0.022 & $\operatorname{mg} / \mathrm{L}$ \\
\hline SD- 104 & 3-Apr-1987 & TITANIUM & 0.016 & $\mathrm{mg} / \mathrm{L}$ \\
\hline SD -104 & $6-A p I-1987$ & TITANIUM & $<0.0030$ & $\mathrm{mg} / \mathrm{L}$ \\
\hline SD- 104 & 12-May-1987 & TITANIUM & $<0.0030$ & $\operatorname{mg} / \mathrm{L}$ \\
\hline SD-104 & 19-Кау - 1987 & TITANIUM & 0.0056 & $\operatorname{mg} / \mathrm{L}$ \\
\hline SD- 104 & 26-May-1987 & TITANIUM & 0.0078 & $\mathrm{mg} \Omega$ \\
\hline SD-104 & 3-Jun-1987 & TITANIUM & 0.010 & $\log / \mathrm{L}$ \\
\hline SD -104 & $11-J$ un -1987 & TITANIUM & 0.0052 & $\operatorname{mg} / \mathrm{L}$ \\
\hline SD- 104 & $18-J u n-1987$ & TITANIUM & 0.0077 & $\mathrm{mg} / \mathrm{L}$ \\
\hline
\end{tabular}


SD- 104

SD -104

5-Mar-1987 TOLUENE

SD -104

SD-104

SD-104

SD-104

SD-104

SD -104

SD -104

SD -104

SD -104

SD -104

** SD-104-D

SD-104-D

SD-104-D

** SD-104

SD-104

SD -104

SD -104

SD-104

SD-104

SD-104

SD -104

SD -104

SD -104

SD - 104

SD -104

SD -104

11- Mar-1987 TOLUENE

18-Mar-1987 TOUDERE

24-Mar-1987 TOLDENS

3-Apr-1987 TOLOERE

6-Apr-1987 TOLUENE

12-May-1987 TOLUENE

19-May-1987 TOLDENE

26-May-1987 TOLUENE

3-Jun-1987 TOLUENE

11-Jun-1987 TOLUENE

18-Jun-1987 TOLUENE

6-Aps-1987 TOLUENE

19-May-1987 TOLUENE
5-MaI-1987 TOTAL DISSOLVED SOLIDS

11-Mar-1987 TOTAL DISSOLVED SOLIDS

1B-Mar-1987 TOTAL DISSOLVED SOLIDS

24-Mar-1987 TOTAL DISSOLVED SOLIDS

3-APT-1987 TOTAL DISSOLVED SOLIDS

6-ApT-1987 TOTAL DISSOLVED SOLIDS

12-May-1987 TOTAL DISSOLVED SOLIDS

19-May-1987 TOTAL DISSOLVED SOLIDS

26-May-1987 TOTAL DISSOLVED SOLIDS

3-Jun-1987 TOTAL DISSOLVED SOLIDS

11-Jun-1987 TOTAL DISSOLVED SOLIDS

18-Jun-1987 TOTAL DISSOLVED SOLIDS

** SD-104-D

SD-104-D 6-Apr-1987 TOTAL DISSOLVED SOLIDS

SD - 104-D

19-May-1987 TOTAL DISSOLVED SOLIDS

** SD-104

SD-104

SD -104

SD-104

SD -104

SD-104

SD -104

SD -104

SD -104

5-MaT-1987 TOTAL ORGANIC CARBON (TOC)

11-Mar-1987 TOTAL ORGANIC CARBON (TOC)

18-Mar-1987 TOTAL ORGANIC CARBON (TOC)

24-Mar-1987 IOTAL ORGANIC CARBON (TOC)

3-APT-1987 TOTAL ORGANIC CARBON (TOC)

6-APY-1987 TOTAL ORGANIC CARBON (TOC)

12-May-1987 TOTAL ORGANIC CARBON (TOC)

19-May-1987 TOTAL ORGANIC CARBON (TOC)

$<5$

$<5$

$<5$

$<5$

$<5$

$<5$

$<9$

$<5$

$<5$

$<5$

$<5$

$<5$

$<5$

$<5$

188

414

126

426

1096

148

158

150

148

172

154

180

152

156

6

9

24

9

10

9

2.5

2. 2 $\mathrm{mg} / \mathrm{L}$

ng/L

$$
\begin{aligned}
& u g / L \\
& u g / L \\
& u g / L \\
& u g / L \\
& u g / L \\
& u g / L \\
& u g / L \\
& u g / L \\
& u g / L \\
& u g / L \\
& u g / L \\
& u g / L \\
& u g / L \\
& u g / L
\end{aligned}
$$

mg/L $\operatorname{mg} / \mathrm{L}$ mg $/ 2$

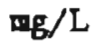
$\mathrm{ng} / \mathrm{L}$ $\mathrm{ng} / \mathrm{L}$ ng/L ng/L $\mathrm{mg} / \mathrm{L}$ $\mathrm{mg} / \mathrm{L}$ $\mathrm{ng} / \mathrm{L}$ $\mathrm{gg} / \mathrm{L}$

.

$$
\begin{aligned}
& \mathrm{mg} / \mathrm{L} \\
& \mathrm{gg} / \mathrm{L} \\
& \mathrm{mg} / \mathrm{L} \\
& \mathrm{mg} / \mathrm{L} \\
& \mathrm{mg} / \mathrm{L} \\
& \mathrm{mg} / \mathrm{L} \\
& \mathrm{gg} / \mathrm{L} \\
& \mathrm{ng} / \mathrm{L}
\end{aligned}
$$




\begin{tabular}{lrcrc} 
Location & \multicolumn{1}{c}{ Date } & Compound & \multicolumn{2}{c}{ Results Units } \\
SD-104 & 26-May-1987 TOTAL ORGANIC CARBON (TOC) & 2.4 & $\mathrm{mg} / \mathrm{L}$ \\
SD-104 & 3-Jun-1987 TOTAL ORGANIC CARBON (TOC) & 2.4 & $\mathrm{mg} / \mathrm{L}$ \\
SD-104 & 11-Jun-1987 TOTAL ORGANIC CARBON (TOC) & 1.9 & $\mathrm{mg} / \mathrm{L}$ \\
SD-104 & 18-Jun-19B7 TOTAL ORGANIC CARBON (TOC) & 2.3 & $\mathrm{mg} / \mathrm{L}$
\end{tabular}

** SD-104-D

SD-104-D 6-Apr-1987 TOTAL ORGANIC CARBON (TOC) SD-104-D 19-May-1987 TOTAL ORGANIC CARBON (TOC)
$11 \mathrm{mg} / \mathrm{L}$ $2.2 \mathrm{mg} / \mathrm{L}$

3

$<12 \mathrm{mg} / \mathrm{L}$

$2 \mathrm{mg} / \mathrm{L}$

$43 \mathrm{mg} / \mathrm{L}$

$13 \mathrm{mg} / \mathrm{L}$

$2 \mathrm{mg} / \mathrm{L}$.

$1 \mathrm{mg} / \mathrm{L}$

$1 \mathrm{mg} / \mathrm{L}$

$1 \mathrm{mg} / \mathrm{L}$

$<1 . \mathrm{mg} / \mathrm{L}$

$3 \mathrm{mg} / \mathrm{L}$

$<4 \quad \mathrm{mg} / \mathrm{L}$

$3 \mathrm{mg} / \mathrm{L}$

$<1 \quad \mathrm{mg} / \mathrm{L}$

SD-104-D 6-APr-1987 TOTAL SUSPENDED SOLIDS

SD-104-D 19-Kay-1987 TOTAL SUSPENDED SOLIDS

* SD -104

SD-104

SD-104

SD -104

SD -104

SD- 104

SD - 104

SD- 104

SD- 104

SD -104

SD-104

SD-104

SD-104
5-Mat-1987 TRANS-1 2-DICHLOROETHENE

11-Mar-1987 TRANS-1 2-DICHLOROETHENE

18-MaI-1987 TRANS-1 2-DICHLOROETHERE

24-Mar-1987 TRANS-1 2-DICHLOROETHENE

3-APT-1987 TRANS - 1 2-DICHLOROETHENE

6-Apr-1987 TRANS-1 2-DICHLOROETHENE

12-May-1987 TRANS-1 2-DICHLOROETHENE

19-May-1987 TRANS-1 2-DICHLOROETHENE

26-May-1987 TRANS - 1 2-DICHLOROETHENE

3-Jun-1987 TRANS - 1 2-DICHLOROETHENE

11-JUn-1987 TRANS-1 2-DICHLOROETHENE

18-Jun-1987 TRANS-1 2-DICHLOROETHENE

* SD-104-D

SD-104-D 6-APr-1987 TRANS-1 2-DICHLOROETHENE

SD-104-D 19-May-1987 TRANS-1 2-DIGHLOROETHENE

* SD-104

SD- 104

SD-104

SD- 104

SD- 104

5-Mar-1987 TRANS-1 3-DICHLOROPROPENE 11-Mar-1987 TRANS-1 3-DICHLOROPROPENE 18-Mat-1987 TRANS - 1 3-DICHLOROPROPENE 24-Mar-1987 TRANS-1 3-DICHLOROPROPENE

$<5$

$<5$

$<5$

$<5$

$<5$

$<5$

$<5$

$<5$

$<5$

$<5$

$<5$

$<5$

$<5$

$<5$

ug/L ug $/ L$

$<5$

$<5$

$<5$

$<5$

ug /

ug $/ L$

ug/t

ug/L

ug/L

ug/L

$u g / L$

ug/L

ug $/ \mathrm{L}$

ug/L

ug/ $/$

ug/L

$5 \quad u g / L$

ug/L

ug/L

ug/L

$u g /$ 
Storm Drain Data for SD-100-04

Location

SD -104

SD-104

SD- 104

SD -104

SD -104

SD -104

SD -104

SD -104
Date

Compound

3-Apt-1987 TRANS-1 3-DICLLOROPROPENE

6-Apr-1987 TRANS - 1 3-DICEIOROPROPENE

12-MRY-1987 TRANS-1 3-DICHLOROPROPENE

19-MaY-1987 IRANS - 1 3-DICHIOROPROPENE

26-MaY-1987 TRANS-1 3-DICHLOROPROPENE

3-JUn-1987 TRANS - 1 3-DICHLOROPROPENE

11-JUn-1987 TRANS - 1 3-DICHLOROPROPENE

18-JUn-1987 TRANS-1 3-DICHLDROPROPENE

* SD-104-D

$S D-104-D$

SD-104-D

6-Apr-1987 TRANS-1 3-DICHLOROPROPENE

19-NRy-1987 TRANS-1 3-DICHLOROPROPENE

** SD- 104

SD -104

SD-104

SD -104

SD- 104

SD -104

SD-104

SD-104

SD-104

SD-104

SD -104

SD -104

SD-104
5-Mar-1987 TRICHLOROETHENE

11-Kar-1987 TRICHLOROETHENE

18-Kar-1987 TRICHLOROETHENE

24-Mar-1987 TRICHLOROETHENE

3-Apr-1987 TRICHLOROETHENE

6-Apr-1987 TRICHLOROETHENE

12-Kay-1987 TRICHLOROETHENE

19-May-1987 TRICHLOROETHENE

26-May-1987 TRICHLOROETHENE

3-Jun-1987 TRICHLOROETHENE

11-Jun-1987 TRICHLOROETHENE

18-JWI-1987 TRICHLOROETHENE

** SD $-104-D$

$$
\text { SD-104-D 6-Apr-1987 TRICHLOROETHENE }
$$

SD-104-D 19-May-1987 TRICHLOROETHENE

* SD-104

SD -104

SD -104

SD-104

SD-104

SD-104

SD-104

SD- 104

SD -104

SD- 104

SD- 104

SD-104

SD-104
5-Kar-1987 TURBIDITY

11-Mar-1987 TURBIDITY

18-Mar-1987 TURBIDITY

24-Mar-1987 TURBIDITY

3-Apr-1987 TURBIDITY

6-ApI-1987 TURBIDITY

12-May-1987 TURBIDITY

19-May-1987 TURBIDITY

26-May-1987 TURBIDITY

3-Jun-1987 TURBIDITY

11-Jun-1987 TURBIDITY

18-Jun-1987 TURBIDITY

* SD-104-D

SD-104-D 6-Apr-1987 TURBIDITY

SD-104-D 19-May-1987 TURBIDITY

$\star$ SD -104

Results Undts

$\begin{array}{ll}<5 & u g / L \\ <5 & u g / L \\ <5 & u g / L \\ <5 & u g / L \\ <5 & u g / L \\ <5 & u g / L \\ <5 & u g / L \\ <5 & u g / L\end{array}$

$<5 \quad u g / L$

$<5 \quad u g / L$

$<5 \quad 4 g / L$

$<5 \quad u g / L$

$<5 \quad \mathrm{ug} / \mathrm{L}$

$<5 \quad$ ug $/ \mathrm{L}$

$<5 \quad$ ug/ $/ \mathrm{L}$

$<5.4 \mathrm{~g} / \mathrm{L}$

$<5 \quad u g / 2$

$<5 \quad u g / L$

$8 u g / L$

$<5 \quad u g / L$

$<5 \quad u g / L$

$<5 \quad$ ug/L

$<5 \quad$ ug $/ \mathrm{L}$

$<5 \quad$ ug $/ \mathrm{L}$

1 NTU

2 NTU

$<1 \quad$ NTU

36 NTU

31 NTU

2 NTU

0.70 NTU

0.45 NTU

0.75 NTU

0.35 NTU

0.50 NTU

0.55 NTU

3 NTU

$0.40 \mathrm{NTU}$ 
Storm Drain Data for SD-100-04

\begin{tabular}{|c|c|c|c|c|}
\hline Location & Date & Compound & Results & UnIts \\
\hline SD-104 & $5-\operatorname{Mar}-1987$ & UNKNNOWNS & 26 & $u g / L$ \\
\hline SD-104 & $5-$ Mar- 1987 & URANTUM & 0.005 & $\mathrm{mg} / \mathrm{L}$ \\
\hline $5 D-104$ & 11-Maz-1987 & URANTUM & 0.005 & $\mathrm{mg} / \mathrm{h}$ \\
\hline SD-104 & $18-\operatorname{Yar}-1987$ & URANIUM & 0.004 & $\log / 2$ \\
\hline SD-104 & $24-\operatorname{Mar}-1987$ & URANIUM & 0.008 & $\mathrm{mg} / \mathrm{L}$ \\
\hline SD-104 & $3-$ Apr -1987 & URANIUM & 0.003 & $\operatorname{mg} / \mathrm{L}$ \\
\hline SD-104 & $6-A p r-1987$ & URANIUM & 0.012 & $\mathrm{mg} / \mathrm{L}$ \\
\hline SD-104 & 12-May - 1987 & URANIUM & $<0.001$ & $\mathrm{mg} / \mathrm{L}$ \\
\hline SD-104 & 19-Kay - 1987 & URANIUM & 0.002 & $\mathrm{mg} / \mathrm{L}$ \\
\hline SD-104 & $26-\mathrm{Kay}-1987$ & URANIUM & $<0.001$ & $\lg / \mathrm{L}$ \\
\hline SD -104 & 3-Jun-1987 & IRANIUM & 0.002 & $\mathrm{mg} / \mathrm{L}$ \\
\hline SD- 104 & $11-J u n-1987$ & URANIUY & 0.002 & $\mathrm{mg} / \mathrm{L}$ \\
\hline SD-104 & $18-J u n-1987$ & URANIUM & 0.001 & $\mathrm{mg} / 1$ \\
\hline
\end{tabular}

\begin{tabular}{|c|c|c|}
\hline \multicolumn{3}{|c|}{$\star \quad$ SD-104-D } \\
\hline SD-104-D & 6-Apt-1987 & URANIUM \\
\hline SD-104-D & 19- - May-1987 & URANIUM \\
\hline * SD-102 & & \\
\hline$S D=104$ & 5-Mar-1987 & VARADIUM \\
\hline SD-104 & $11-\mathrm{Mar}-1987$ & VANADIUM \\
\hline-104 & 18-Mar-1987 & VANADIUM \\
\hline-104 & 24-Mar-1987 & VANADIUM \\
\hline SD- 104 & 3-Apr-1987 & VANADIUM \\
\hline SD- 104 & 6-Арт-1987 & VANADIUM \\
\hline SD- 104 & 12-Кay-1987 & VANADIUM \\
\hline SD -104 & 19-May -1987 & VANADIUM \\
\hline SD-104 & 26- Мay - 1987 & VANADIUM \\
\hline SD-104 & 3-Jun-1987 & VANADTUM \\
\hline SD-104 & $11-J u n-1987$ & VANADIUM \\
\hline D-104 & 18-Jun- 1987 & VANADIUY \\
\hline
\end{tabular}

$\begin{array}{ll}0.002 & \mathrm{mg} / \mathrm{L} \\ 0.004 & \mathrm{mg} / \mathrm{L} \\ <0.50 & \mathrm{mg} / \mathrm{L} \\ <0.50 & \mathrm{mg} / \mathrm{L} \\ <0.50 & \mathrm{mg} / \mathrm{L} \\ <0.50 & \mathrm{mg} / \mathrm{L} \\ <0.50 & \mathrm{mg} / \mathrm{L} \\ <0.50 & \mathrm{mg} / \mathrm{L} \\ <0.50 & \mathrm{mg} / \mathrm{L} \\ <0.50 & \mathrm{mg} / \mathrm{L} \\ <0.0050 \mathrm{mg} / \mathrm{L} \\ <0.50 \quad \mathrm{mg} / \mathrm{L} \\ <0.50 \quad \mathrm{mg} / \mathrm{L} \\ <0.50 \quad \mathrm{mg} / \mathrm{L}\end{array}$

** SD-104-D

$\begin{array}{lr}\text { SD-104-D } & \text { 6-Apt-1987 VANADIUM } \\ \text { SD-104-D } & 19-\text { May-1987 VANADIUM }\end{array}$

$<0.50 \mathrm{mg} / \mathrm{L}$

$<0.50 \mathrm{mg} / \mathrm{L}$

$\begin{array}{lrl}\text { ** SD-104 } & \\ \text { SD-104 } & \text { 5-Mar-1987 VINYL CHLORIDE } \\ \text { SD-104 } & \text { 11-Mar-1987 VINYL CHLORIDE } \\ \text { SD-104 } & \text { 18-Mar-1987 VINY CHLORIDE } \\ \text { SD-104 } & \text { 24-Mar-1987 VINYL CHLORIDE } \\ \text { SD-104 } & \text { 3-Apr-1987 VINYL CHLORIDE } \\ \text { SD-104 } & \text { 6-Apr-1987 VINYL CHLORIDE } \\ \text { SD-104 } & \text { 12-May-1987 VINY CHLORIDE } \\ \text { SD-104 } & \text { 19-May-1987 VINY CHLORIDE } \\ \text { SD-104 } & \text { 26-May-1987 VINYL CHLORIDE } \\ \text { SD-104 } & \text { 3-Jun-1987 VINI CHLORIDE } \\ \text { SD-104 } & \text { 11-Jun-1987 VINYL CHLORIDE } \\ \text { SD-104 } & \text { 18-Jun-1987 VINY CHLORIDE }\end{array}$

$\begin{array}{ll}<10 & \mathrm{ug} / \mathrm{L} \\ <10 & \mathrm{ug} / \mathrm{L} \\ <10 & \mathrm{ug} / \mathrm{L} \\ <10 & \mathrm{ug} / \mathrm{L} \\ <10 & \mathrm{ug} / \mathrm{L} \\ <10 & \mathrm{ug} / \mathrm{L} \\ <10 & \mathrm{ug} / \mathrm{L} \\ <10 & \mathrm{ug} / \mathrm{L} \\ <10 & \mathrm{ug} / \mathrm{L} \\ <10 & \mathrm{ug} / \mathrm{L} \\ <10 & \mathrm{ug} / \mathrm{L} \\ <10 & \mathrm{ug} / \mathrm{L}\end{array}$


Storm Drain Data for SD-100-04

\begin{tabular}{|c|c|c|}
\hline Location & Date & \\
\hline $\begin{array}{l}\text { SD-104-D } \\
\text { SD-104-D }\end{array}$ & $\begin{array}{r}6-\Delta p r-1987 \\
19-\text { Xey- } 1987\end{array}$ & \\
\hline \# SD-104 & & \\
\hline SD- 104 & 5-Mar- 1987 & ZINC \\
\hline SD -104 & 11-Mar-1987 & ZINC \\
\hline SD-104 & 18 - Мar - 1987 & ZINC \\
\hline SD-104 & 24-Mar- 1987 & ZINC \\
\hline SD- 104 & 3-Apr-1987 & ZINC \\
\hline SD- 104 & 6-Apr-1987 & ZINC \\
\hline SD -104 & 12-May-1987 & ZINC \\
\hline SD-104 & 19-May- 1987 & ZINC \\
\hline SD- 104 & 26 - May - 1987 & $\operatorname{ZINC}$ \\
\hline SD -104 & $3-J w-1987$ & ZINC \\
\hline SD- I04 & $11-J u n-1987$ & ZINC \\
\hline SD -104 & 18-Jun-1987 & ZINC \\
\hline
\end{tabular}

Results Units

$<10 \quad$ ug/L

$<10$ ug/

* SD-104-D

SD-104-D 6-Apx-1987 ZINC

SD-104-D 19-May-1987 ZINC

$0.042 \mathrm{mg} / \mathrm{L}$

$0.032 \mathrm{mg} / \mathrm{L}$

$0.049 \mathrm{mg} / \mathrm{L}$

$0.031 \mathrm{mg} / \mathrm{L}$

$0.094 \mathrm{mg} / \mathrm{L}$

$0.025 \mathrm{mg} / \mathrm{L}$

$0.021 \mathrm{mg} / \mathrm{L}$

$0.022 \mathrm{mg} / \mathrm{L}$

$0.029 \mathrm{mg} / \mathrm{L}$

$0.022 \mathrm{mg} / \mathrm{L}$

$0.032 \mathrm{mg} / \mathrm{L}$

$0.040 \mathrm{mg} / \mathrm{L}$

* SD-104

SD-104

5-Mar-1987 2IRCONTUM

. SD-104

11-Har-1987 2IRCONIUM

SD-104

18-Mar-I987 21RCONIUM

SD- 104

24-Mar-19B7 ZIRCONIUY

SD-104

3-Apr-1987 ZIRCONIUA

SD- 104

6-Apr-1987 ZIRCONIUM

SD -104

12-May-1987 2IRCONIUM

SD -104

19-May-1987 2IRCONIUM

SD-104

26-May-1987 ZIRCONIUM

SD -104

3-Jun-1987 ZIRCONIUM

SD- 104

11-Jun-1987 ZIRCONIU:

SD-104

18-Jun-1987 2IRCONIUM

$0.028 \mathrm{mg} / \mathrm{L}$

$0.023 \mathrm{mg} / \mathrm{L}$

* SD-104-D

SD-104-D 6-Apr-1987 ZIRCONIUM

SD-104-D 19-Kay-1987 ZIRCONIUM

$<0.0050 \mathrm{mg} / \mathrm{L}$

$<0.0050 \mathrm{mg} / \mathrm{L}$

$<0.0050 \mathrm{mg} / \mathrm{L}$

$<0.0050 \mathrm{mg} / \mathrm{L}$

$<0.0050 \mathrm{mg} / \mathrm{L}$

$<0.0050 \mathrm{mg} / \mathrm{L}$

$<0.0050 \mathrm{mg} / \mathrm{L}$

$<0.0050 \mathrm{mg} / \mathrm{L}$

$<0.0050 \mathrm{mg} / \mathrm{L}$

$<0.0050 \mathrm{mg} / \mathrm{L}$

$<0.0050 \mathrm{mg} / \mathrm{L}$

$<0.0050 \mathrm{mg} / \mathrm{L}$

$<0.0050 \mathrm{mg} / \mathrm{L}$

$<0.0050 \mathrm{mg} / \mathrm{L}$. 
$\sim$

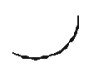

- - - - . 
